\title{
Coal Industry Annual 1996
}

\author{
November 1997
}

\section{Energy Information Administration}

Office of Coal, Nuclear, Electric and Alternate Fuels

U.S. Department of Energy

Washington, DC 20585

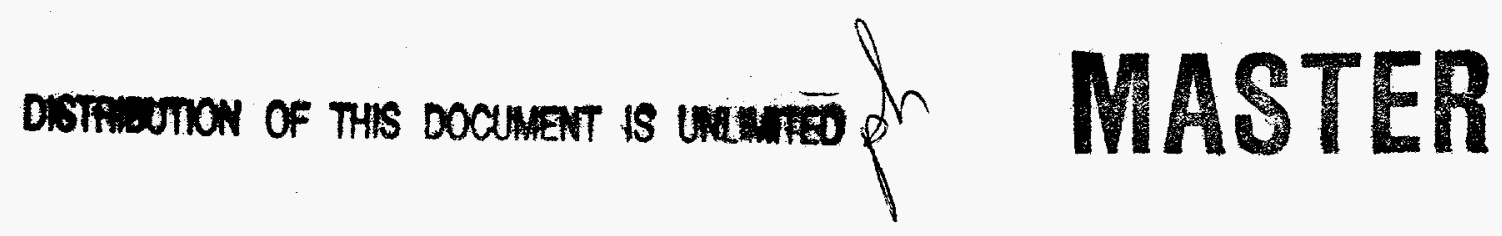




\section{Contacts}

This publication was prepared by Frederick L. Freme, Michelle Bowles, Stephen Scott, and Thomas Murphy under the direction of Mary K. Paull, Acting Chief, Coal Data Branch, Coal and Electric Data and Renewables Division, Office of Coal, Nuclear, Electric, and Alternate Fuels. Specific information about the data in this report can be obtained from Frederick L. Freme (202/426-1152), or e-mail FFREME @EIA.DOE.GOV. All other questions on coal statistics should be directed to the National Energy Information Center $(202 / 586-8800)$, or e-mail 


\section{DISCLAMMER}

This report was prepared as an account of work sponsored by an agency of the United States Government. Neither the United States Government nor any agency thereof, nor any of their employees, makes any warranty, express or implied, or assumes any legal liability or responsibility for the accuracy, completeness, or usefulness of any information, apparatus, product; or process disclosed, or represents that its use would not infringe privately owned rights. Reference herein to any specific commercial product, process, or service by trade name, trademark, manufacturer, or otherwise does not necessarily constitute or imply its endorsement, recommendation, or favoring by the United States Government or any agency thereof. The views and opinions of authors expressed herein do not necessarily state or reflect those of the United States Government or any agency thereof. 


\section{DISCLAmiER}

Portions of this docoment moy be illegible in electronic image products. Images are produced from the best availabie original docoment. 


\section{Preface}

Coal Industry Annual 1996 provides comprehensive information about U.S. coal production, number of mines, prices, productivity, employment, productive capacity, and recoverable reserves. U.S. coal production for 1996 and previous years is based on the annual survey EIA-7A, "Coal Production Report."

This report presents data on coal consumption, coal distribution, coal stocks, coal prices, coal quality, and emissions for Congress, Federal and State agencies, the coal industry, and the general public. Appendix A contains a compilation of coal statistics for the major coal-producing States. This report does not include coal consumption data for nonutility power producers that are not in the manufacturing, agriculture, mining, construction, or commercial sectors. Consumption for nonutility power producers not included in this report is estimated to be 24 million short tons for 1996 .

The data presented in the report were collected and published by the Energy Information Administration (EIA), to fulfill its data collection and dissemination responsibilities, as specified in the Federal Energy Administration Act of 1974 (Public Law 93-275), as amended. Data for the Demonstrated Reserve Base
(DRB) are now reported in U.S. Coal Reserves: $A$ Review and Update (DOE/EIA-0529). However, this report presents data on the recoverable portion of the DRB in Table 105.

The base year for the implicit price deflator, which is used to convert nominal figures to real figures is 1992, the same as the previous year (Table D3).

This report constitutes the 21st annual report on coal production published by EIA and continues the series formerly included in the Minerals Yearbook published by the Bureau of Mines.

The Office of Coal, Nuclear, Electric and Alternate Fuels gratefully acknowledges the cooperation of the respondents in supplying the information presented in this report and appreciates the valuable assistance of State coal mining agencies; the U.S. Department of the Interior: the Bureau of Land Management, the Minerals Management Service; the U.S. Department of Labor: the Mine Safety and Health Administration, the Bureau of Labor Statistics; the U.S. Department of Commerce: the Bureau of the Census; and the King Publishing Corporation. 


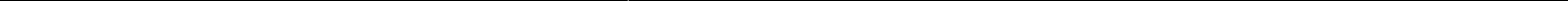




\section{Contents}

Page

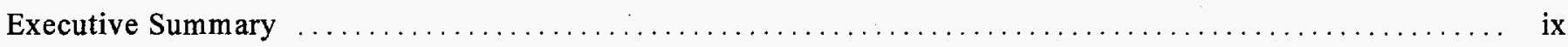

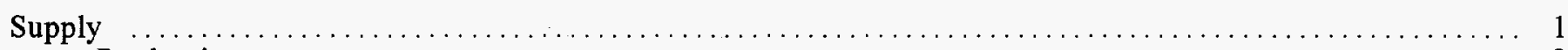

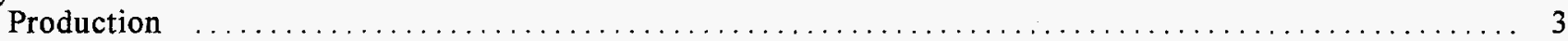

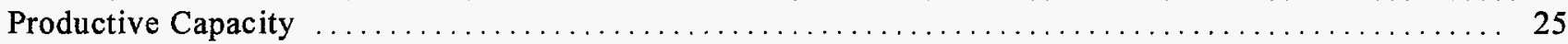

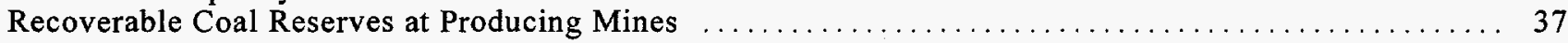

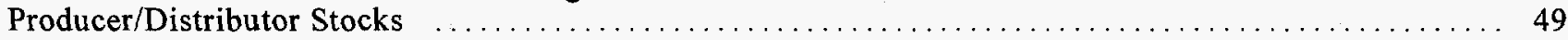

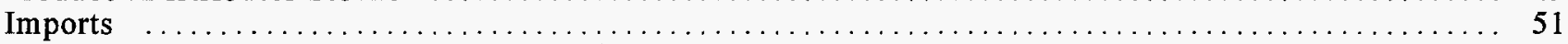

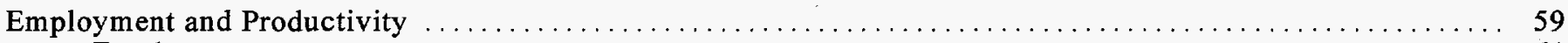

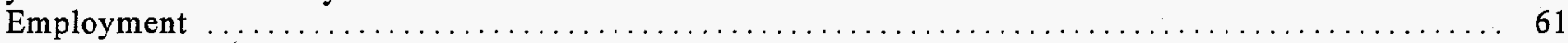

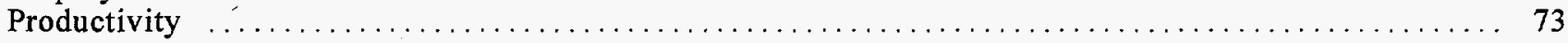

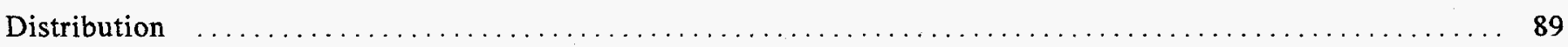

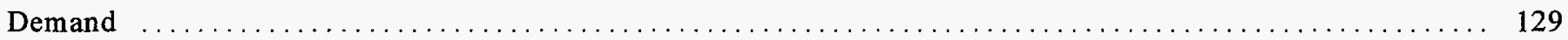

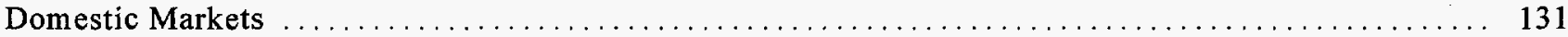

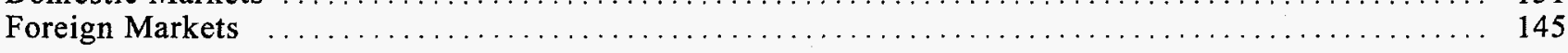

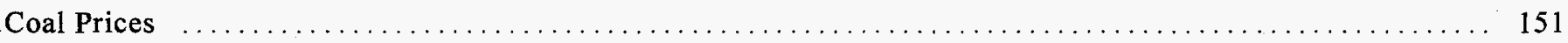

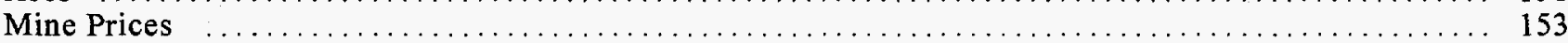

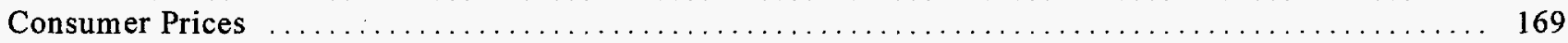

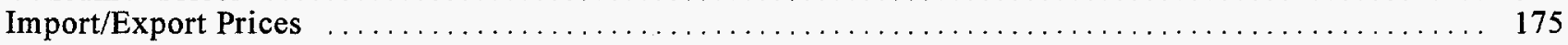

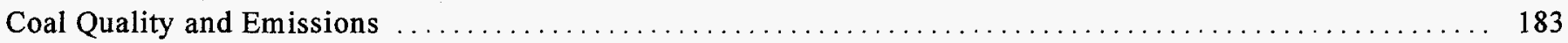

Appendices

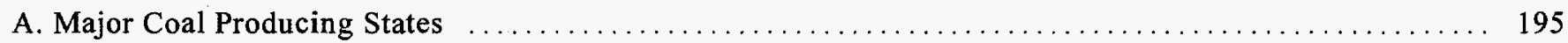

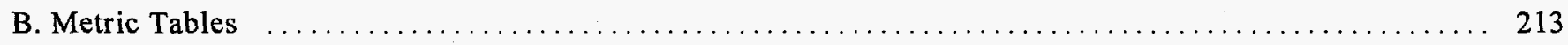

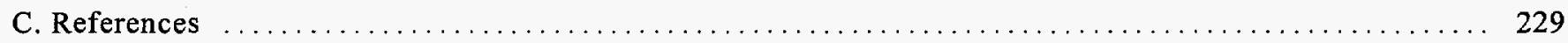

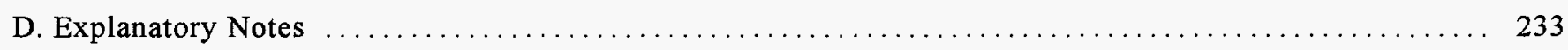

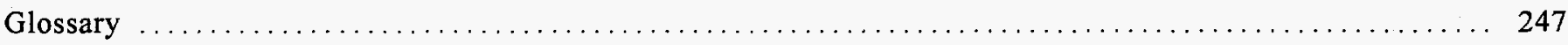




\section{Tables}

1. Coal Production by State, 1987, 1992-1996

2. Number of Coal Mines by State, 1987, 1992-1996

3. Coal Production and Number of Mires by State and Mine Type, 1996

4. Coal Production and Number of Mines by State, County, and Mine Type, $1996 \ldots \ldots \ldots \ldots$

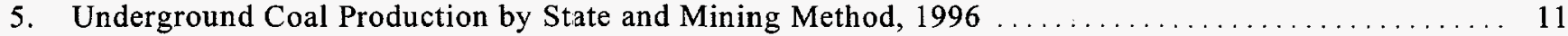

6. Coal Production and Number of Mines by State, Mine Type, and Mine Production Range, $1996 \ldots \ldots$

7. U.S. Coal Production by Coalbed Thickness and Mine Type, $1996 \ldots \ldots \ldots \ldots$

8. U.S. Coal Production and Coalbed Thickness by Major Coalbeds and Mine Type, $1996 \ldots \ldots \ldots$

9. Coal Production and Number of Mines by State and Coal Rank, $1996 \ldots \ldots \ldots$

10. Coal Production by State, Coal Rank, and Group, $1996 \ldots \ldots \ldots \ldots \ldots \ldots$

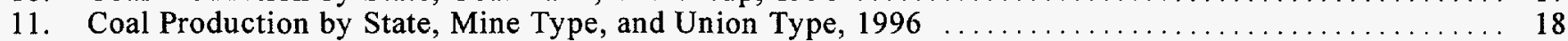

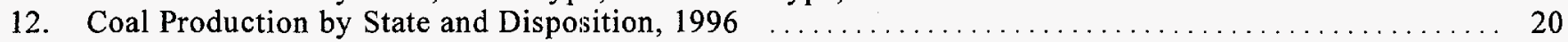

13. Coal Mining Acreage, Production and Royalties from Federal and Indian Leases by State, $1996 \ldots . . .21$

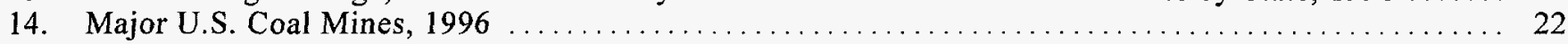

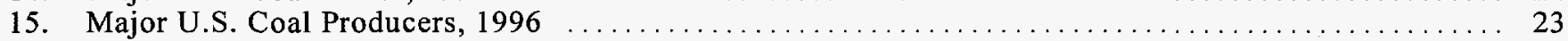

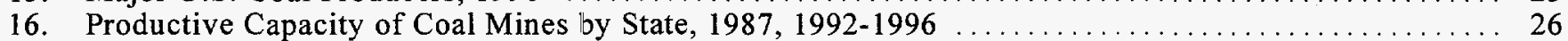

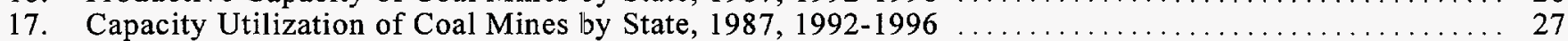

18. Production, Productive Capacity, and Capacity Utilization of Coal Mines by State and Mine Type, 199628

19. Productive Capacity and Capacity Utilization of Underground Coal Mines by State and Mining Method, 1996

20. Productive Capacity and Capacity Utilization of Coal Mines by State and Coal Rank, 1996 .

21. Productive Capacity and Capacity Utilization of Coal Mines by State and Mine Production Range, 199631

22. Productive Capacity and Productivity of Coal Mines by State and Capacity Utilization Range, $1996 . . .32$

23. Productive Capacity and Capacity Utilization of Coal Mines by State and Recoverable Reserves Range, 1996

24. Productive Capacity and Capacity Utilization of Coal Mines by State, Mine Type, and Union Type, 1996

25. Recoverable Coal Reserves at Producing Mines by State, 1987, 1992-1996

26. Average Recovery Percentage at Producing Coal Mines by State, 1987, 1992-1996 ...

Recoverable Coal Reserves and Average Recovery Percentage at Producing Mines by State and Mine Type, 1996

28. Recoverable Coal Reserves at Producing Underground Mines by State and Mining Method, 1996

29. Average Recovery Percentage at Producing Underground Coal Mines by State and Mining Method, 1996

30. Recoverable Coal Reserves and Average Recovery Percentage at Producing U.S. Mines by Mine Production Range and Mine Type, 1996

31. Recoverable Coal Reserves and Average Recovery Percentage at Producing U.S. Mines by Coalbed Thickness and Mine Type, 1996

32. Recoverable Coal Reserves and Average Recovery Percentage at Producing Mines by State, Mine Type, and Union Type, 1996

33. Status of Recoverable Coal Reserves and Coal Production from Producing Federal Coal Leases by State, 1996

34. Year-End Producer and Distributor Coal Stocks by State, 1992-1996

35. U.S. Coal Imports by Continent and Country of Origin, 1987, 1992-1996

36. Coal Imports by Customs District, 1987, 1992-1996

37. U.S. Receipts of Imported Coal by Country of Origin and Destination State, 1987, 1992-1996

38. Imported Coal Received at Electric Utilities by Country of Origin and Destination State, 1987, 1992-1996

39. Imported Coal Received at Manufacturing and Coke Plants by Country of Origin and Destination State, 1994-1996

40. Average Number of Miners by State, $1987,1992-1996$

41. Average Number of Miners at Underground Mines by State, 1987, 1992-1996

42. Average Number of Miners at Surface Mines by State, 1987, 1992-1996

43. Average Number of Miners by State and Mine Production Range, 1996

44. Average Number of Miners at Underground Mines by State and Mine Production Range, 1996

45. Average Number of Miners at Surface Mines by State and Mine Production Range, 1996 
46. Average Number of Miners by State, Mine Type, and Union Type, 1996

47. U.S. Coal Mine Injuries, 1987, 1992-1996

48. Coal Mining Productivity by State, 1987, 1992-1996

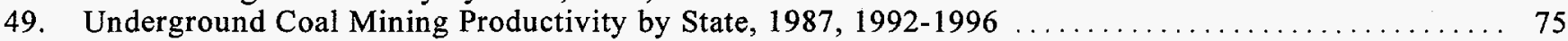

50. Surface Coal Mining Productivity by State, $1987,1992-1996 \ldots \ldots \ldots \ldots$

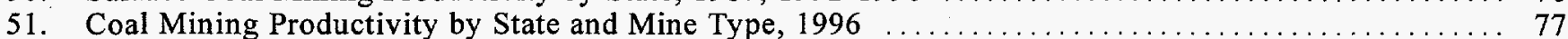

52. Weighted Average Number of Days Worked by State and Mine Type, 1987, 1992-1996 _....... 79

53. Weighted Average Number of Days Worked by Mine Production Range, $1996 \ldots \ldots \ldots$

54. Underground Coal Mining Productivity by State and Mining Method, $1996 \ldots \ldots \ldots \ldots$

55. U.S. Coal Mining Productivity by Coalbed Thickness and Mining Method, $1996 \ldots \ldots \ldots \ldots$

56. Coal Mining Productivity by State, Mine Type, and Mine Production Range, 1996 . . . . . . . . 84

57. Coal Mining Productivity by State, Mine Type, and Union Type, $1996 \ldots \ldots \ldots$

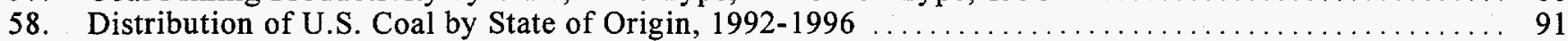

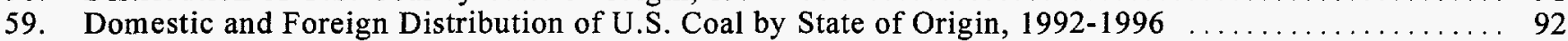

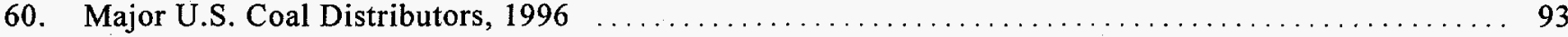

61. Domestic Distribution of U.S. Coal by Coal-Producing Region and State, and Destination Census

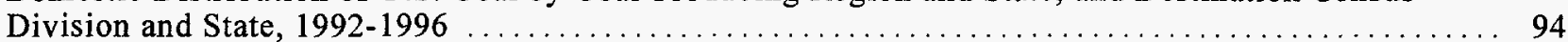

62. Foreign Distribution of U.S. Coal by Major Coal-Exporting States and Destination, 1992-1996 ..... 106

63. Foreign Distribution of U.S. Metallurgical Coal by Major Coal-Exporting States and Destination, 1992-1996

64. Foreign Distribution of U.S. Steam Coal by Major Coal-Exporting States and Destination, $1992-1996$

65. Distribution of U.S. Coal by Origin, Destination, and Method of Transportation, 1996

66. Major U.S. Coal Consumers, 1996

67. Coal Consumption by Census Division and State, $1987,1992-1996$

Year-End Consumer Coal Stocks by Census Division and State, 1987, 1992-1996

69. Coal Consumption at Electric Utility Plants by Census Division and State, 1987, 1992-1996 ...... 138 70. Year-End Coal Stocks at Electric Utility Plants by Census Division and State, 1987, 1992-1996 ... . 139

71. Coal Consumption at Other Industrial Plants by Census Division and State, $1987,1992-1996$. . . . . 140

72. Year-End Coal Stocks at Other Industrial Plants by Census Division and State, 1987, 1992-1996 .... 141

73. Coal Carbonized at Coke Plants by Census Division and State, 1987, 1992-1996

74. Year-End Coal Stocks at Coke Plants by Census Division and State, 1987, 1992-1996

75. Coal Consumption by Residential and Commercial Sector, by Census Division and State, 1987, 1992-1996

76. U.S. Coal Exports by Destination, 1987, 1992-1996

77. U.S. Metallurgical Coal Exports by Destination, 1987, 1992-1996.

78. U.S. Steam Coal Exports by Destination, 1987, 1992-1996

79. Coal Exports by Customs District, 1987, 1992-1996

80. Average Mine Price of Coal by State, 1987, 1992-1996

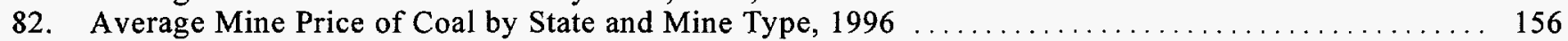

83. Average Mine Price of Coal by State and Underground Mining Method, $1996 \ldots \ldots \ldots \ldots$

84. Coal Production, Number of Mines, and Average Mine Price, by State and County, 1996 ........ 158

85. Average Mine Price by State and Coal Rank, 1996

86. Average Mine Price of U.S. Coal by Mine Production Range and Mine Type, 1996.

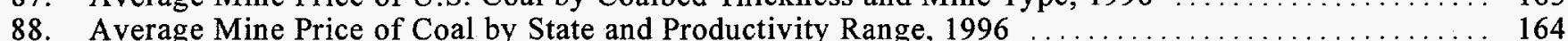

89. Average Mine Price of Underground Coal by State and Productivity Range, $1996 \ldots \ldots \ldots \ldots$

90. Average Mine Price of Surface Coal by State and Productivity Range, $1996 \ldots \ldots \ldots \ldots$

91. Average Mine Price by State and Disposition, 1996

92. Average Price of Coal Delivered to Electric Utilities by Census Division and State, 1987, $1992-1996$

93. Average Real Price of Coal Delivered to Electric Utilities by Census Division and State, 1987, 1992-1996

94. Average Price of Coal Delivered to Other Industrial Plants by Census Division and State, 1987, 1992-1996

95. Average Real Price of Coal Delivered to Other Industrial Plants by Census Division and State, 1987 , 1992-1996

96. Average Price of Coal Delivered to Coke Plants by Census Division and State, 1987, 1992-1996 ...

97. Average Real Price of Coal Delivered to Coke Plants by Census Division and State, 1987, 1992-1996

98. Average Price of U.S. Coal Imports by Continent and Country of Origin, 1987, 1992-1996 
107. Average Quality of Coal Received at Manufacturing and Coke Plants by Census Division and State, 1993-1996

A1. Alabama Coal Statistics, $1987,1992-1996$

A2. Arizona Coal Statistics, $1987,1992-1996$

A3. Colorado Coal Statistics, 1987, 1992-1996

A4. Illinois Coal Statistics, 1987, 1992-1996

A5. Indiana Coal Statistics, 1987, 1992-1996

A6. Kentucky Coal Statistics, 1987, 1992-1996

A7. Montana Coal Statistics, 1987, 1992-1996

A8. New Mexico Coal Statistics, 1987, 1992-1996

202

A9. North Dakota Coal Statistics, 1987, 1992-1996

A 10. Ohio Coal Statistics, 1987, 1992-1996

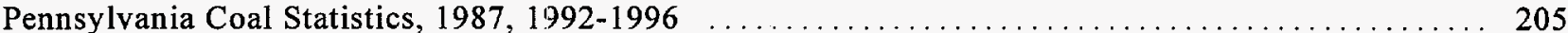

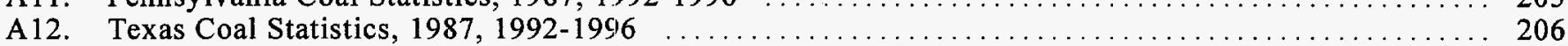

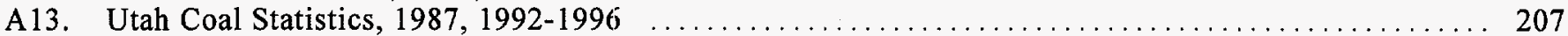

A14. Virginia Coal Statistics, $1987,1992-1996 \ldots \ldots \ldots \ldots \ldots \ldots \ldots \ldots \ldots \ldots \ldots \ldots \ldots \ldots \ldots \ldots \ldots$

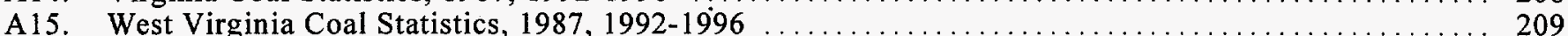

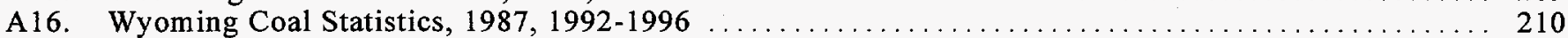

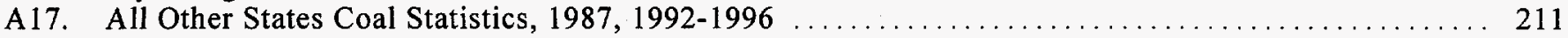

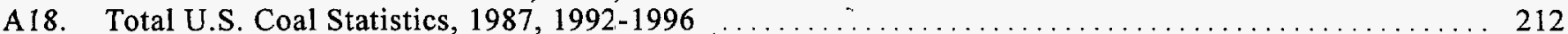

B1. Trends in U.S. Coal Production, Imports, Consumption, Exports, and Stocks, 1987, 1992-1996 _.... 213

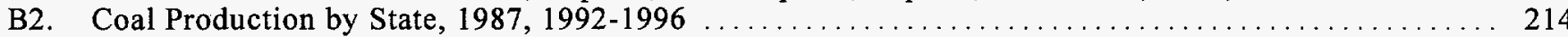

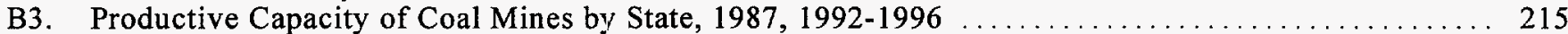

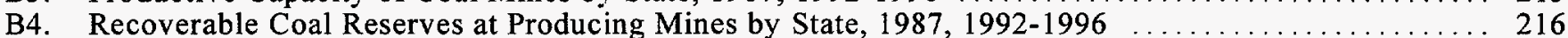

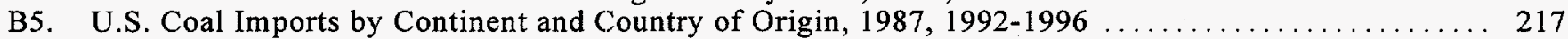

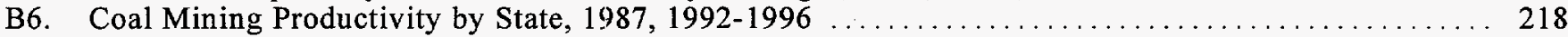

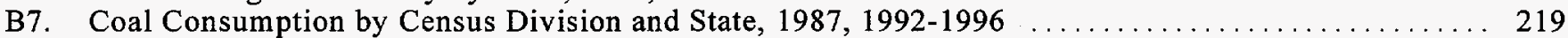

B8. Year-End Consumer Coal Stocks by Census Division and State, 1987, 1992-1996 _.............. 220

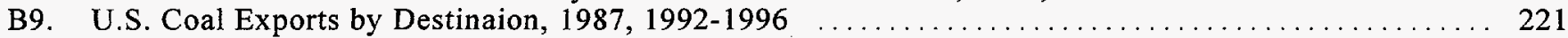

B10. Average Mine Price of Coal by State, 1987, 1992-1996

B11. Average Price of Coal Delivered to Electric Utilities by Census Division and State, 1987, 1992-1996 223

B12. Average Price of Coal Delivered to Other Industrial Plants by Census Division and State, 1987, 1992-1996

B13. Average Price of Coal Delivered to Coke Plants by Census Division and State, 1987, 1992-1996

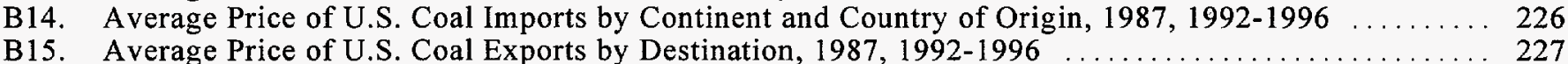

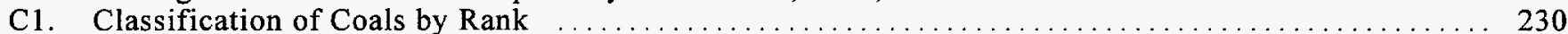

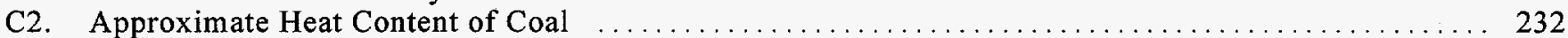

D1. Sources of Data for Total U.S. Coal Production and Number of Mining Operations . . . . . . . . . . ... 234

D2. Interquartile Range and Average Mine Price by State and Mine Type, $1995 \ldots \ldots \ldots \ldots \ldots \ldots \ldots .237$

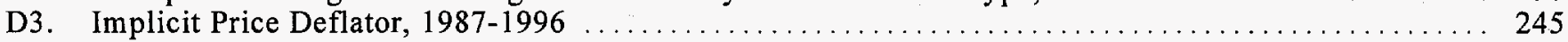

\section{Illustrations}

1. Recoverable Coal Reserves at Producing U.S. Mines by Mine Type and by Region, 1987-1996 ..... 38

2. Average Recovery Percentage at Proclucing U.S. Coal Mines by Mine Type and by Region, 1987-1996 38

3. U.S. Coal Imports, 1987-1996

4. Average Number of U.S. Miners by Mine Type and by Region, $1987-1996 \ldots \ldots \ldots \ldots \ldots \ldots \ldots \ldots 2$

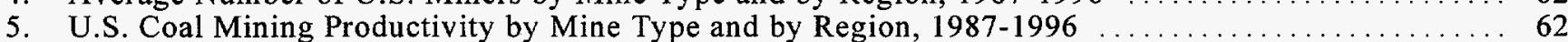

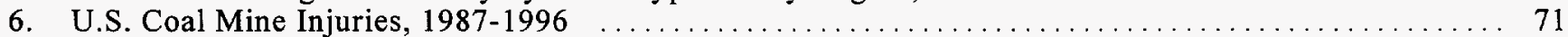

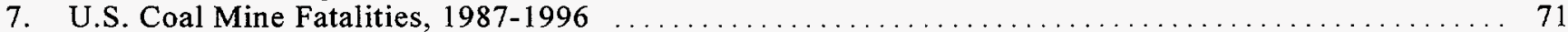

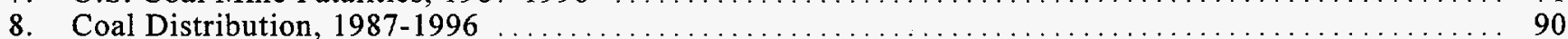

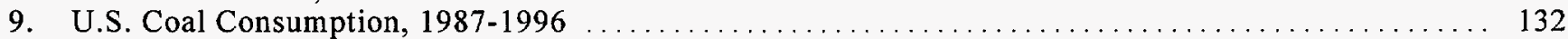

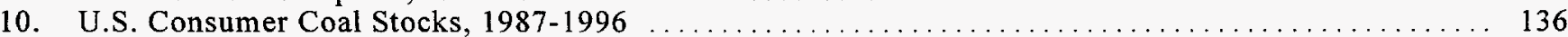

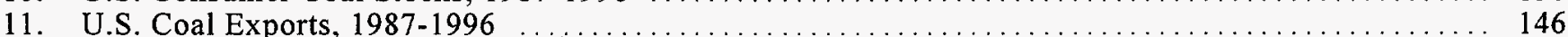

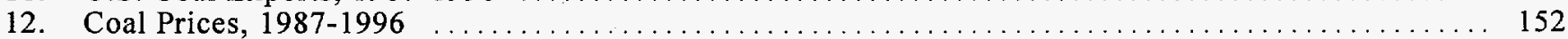

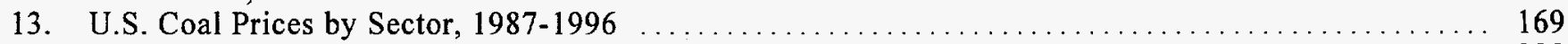

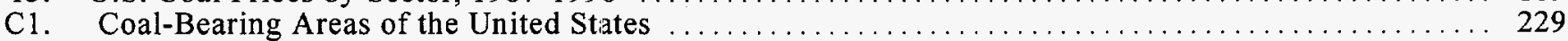




\section{Executive Summary}

\section{Coal's Role in the U.S. Energy Picture}

Total U.S. energy production in 1996 continued the upward swing begun in 1994, rising 2 percent to 72.61 quadrillion Btu. Coal production in Btu terms went up 3 percent to a record high of 22.61 quadrillion Btu (1,064 million short tons). Renewable energy sources, in particular hydroelectric power, and natural gas production went up from their 1995 levels, while oil and nuclear electric power production declined (Table ES1).

Total U.S. energy consumption reached a record level of 93.81 quadrillion Btu in 1996, the fifth consecutive year of growth. Coal consumption reached 20.99 quadrillion Btu, a 5-percent increase over the 1995 level. Consumption of natural gas, petroleum, hydroelectric power, and renewable energy sources also rose, while consumption of nuclear electric power declined slightly.

The electric utility sector accounted for 89 percent of the total coal consumed in 1996. Coal-fired units represented 57 percent of utility generation of electricity, 2 percent more than in 1995. Petroleum and hydroelectric power generation paralleled this increase, while electricity generated by natural gas and nuclear power decreased (Table ES2).

U.S. coal exports went up for the second consecutive year and retained its role as the primary U.S. energy export. In 1996, U.S. coal exports rose to 2.37 quadrillion Btu ( 90 million short tons), 2 percent more than in 1995. In comparison, coal imports, accounting for less than 1 percent of U.S. energy imports, remained about the same as in 1995 at 0.18 quadrillion Btu (7 million short tons).

\section{Production}

U.S. coal production totaled 1,064 million short tons, 31 million short tons higher than in 1995 (Table ES3). Wyoming and Utah set record high production levels. Wyoming remained the leading coal-producing State, followed by West Virginia, and Kentucky. In comparison, Kentucky and Arizona's production was at the lowest level since 1985; Washingtons's coal output was the lowest since 1987; Oklahoma's coal production was the lowest since 1990; and, New Mexico and North Dakota had the lowest production levels since 1991. On the other hand, Maryland had the highest production level since 1984; Texas had the highest coal output since 1990; and Tennessee had the highest coal production since 1991. (The total U.S. coal output does not include about 4 million short tons of low-Btu waste coal, which is recovered and used by independent power producers and reported to their respective State governments.)

Coal production for States east of the Mississippi River were 564 million short tons, 19 million short tons more than in 1995. The States with the largest increase in production were West Virginia, Pennsylvania, Indiana, and Ohio, which together had 20 million short tons more coal output than in 1995 . The eastern States had a combined net loss of 192 mines and 5,211 miners. However, miner productivity in this region rose 5 percent in 1996 to 3.63 short tons per miner per hour, the third consecutive year of substantial gain.

Coal production west of the Mississippi River reached a record 500 million short tons, despite 9 fewer mines in the region and 1,579 fewer miners. Wyoming's coal output, which was 15 million short tons higher than in 1995 , accounted for most of the growth. Miner productivity in the region rose 10 percent to 15.66 tons per miner per hour, in part due to the use of new, upgraded equipment at the mines.

\section{Coal Mine Prices}

The average price of U.S. coal in 1996 was $\$ 18.50$ per short ton, 2 percent less than in 1995. The price of coal from mines east of the Mississippi River fell 3 percent from the 1995 level, while coal prices at mines West of the Mississippi River also dipped, but by less than 1 percent.

\section{Coal Imports}

U.S. coal imports in 1996 were 7 million short tons, about the same as in 1995. An increase in shipments from Indonesia (500 thousand short tons) and Canada (100 thousand short tons) partially offset a decrease in shipment from Venezuela (400 thousand short tons) and Colombia (200 thousand short tons). Venezuela exported 7 percent less coal and Indonesia almost doubled its shipments to the U.S. electric utility sector. 
Table ES1. U.S. Energy Overview, Selected Years, 1987, 1992-1996 (Quadrillion Btu)

\begin{tabular}{|c|c|c|c|c|c|c|c|c|c|}
\hline \multirow{2}{*}{$\begin{array}{l}\text { Activity and } \\
\text { Energy Source }\end{array}$} & \multirow{2}{*}{$1996^{P}$} & \multirow{2}{*}{1995} & \multirow{2}{*}{1994} & \multirow{2}{*}{1993} & \multirow{2}{*}{1992} & \multirow{2}{*}{1987} & \multirow{2}{*}{$\begin{array}{c}\text { Percent } \\
\text { Change } \\
1995-1996\end{array}$} & \multicolumn{2}{|c|}{$\begin{array}{l}\text { Average Annual } \\
\text { Percent Change }\end{array}$} \\
\hline & & & & & & & & 1992-1996 & 1987-1996 \\
\hline 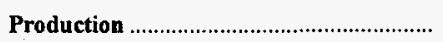 & 72.61 & R 71.12 & R 70.68 & 68.32 & 69.96 & 64.95 & 2.1 & 0.9 & 1.2 \\
\hline 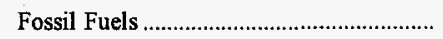 & 58.41 & R 57.41 & R 57.91 & 55.71 & 57.55 & 57.17 & 1.8 & .4 & .2 \\
\hline Coal & 22.61 & R 21.98 & 22.07 & 20.22 & 21.59 & 20.14 & 2.9 & 1.2 & 1.3 \\
\hline 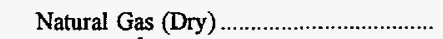 & 19.53 & R 19.10 & ${ }^{R} 19.35$ & 18.58 & 18.38 & 17.14 & 2.3 & 1.5 & 1.5 \\
\hline 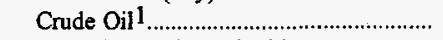 & 13.74 & R 13.89 & 14.10 & 14.49 & 15.22 & 17.67 & -1.1 & -2.5 & -2.8 \\
\hline Natural Gas Plant Liquids .......................... & 2.53 & 2.44 & 2.39 & 2.41 & 2.36 & 2.22 & 3.6 & 1.7 & 1.5 \\
\hline 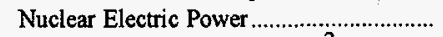 & 7.17 & R 7.18 & 6.84 & 6.52 & 6.61 & 4.91 & -.1 & 2.0 & 4.3 \\
\hline Hydroelectric Pumped Storage 2 ................... & -.03 & -.03 & -.03 & -.04 & -.04 & $(3)$ & - & - & - \\
\hline 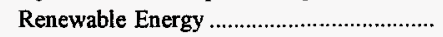 & 7.06 & R 6.56 & ${ }^{\mathrm{R}} 5.97$ & 6.13 & 5.84 & 2.88 & 7.6 & 4.8 & 10.5 \\
\hline Conventional Hydroelectric Power.......... & 3.59 & R $\quad 3.21$ & 2.67 & 2.88 & 2.61 & 2.63 & 12.0 & 8.3 & 3.5 \\
\hline 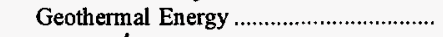 & .35 & .33 & .38 & .38 & .37 & .23 & 9.0 & -.9 & 4.9 \\
\hline Biofuels ${ }^{4}$ & 3.02 & $\mathrm{R}_{2.95}$ & ${ }^{\mathrm{R}} 2.84$ & 2.78 & 2.79 & .02 & 2.4 & 2.0 & 79.8 \\
\hline 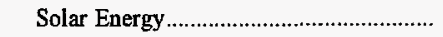 & .07 & .07 & .07 & .07 & .07 & - & - & - & - \\
\hline 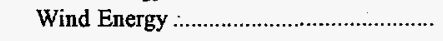 & .04 & R .03 & .04 & .03 & .03 & $*$ & 10.0 & 4.8 & 84.5 \\
\hline Imports & 23.68 & R 22.48 & 22.71 & 21.54 & 19.66 & 15.76 & 5.3 & 4.8 & 4.6 \\
\hline 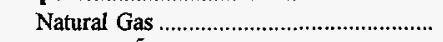 & 2.90 & ${ }^{R} 2.90$ & 2.68 & 2.40 & 2.16 & .99 & .1 & 7.7 & 12.7 \\
\hline Crude Oil 5 & 16.24 & $\mathrm{R}_{15.63}$ & 15.34 & 14.75 & 13.25 & 10.07 & 3.9 & 5.2 & 5.4 \\
\hline Petroleum Product ${ }^{6}$ & 3.86 & R 3.23 & 3.91 & 3.76 & 3.71 & 4.10 & 19.5 & .9 & -.7 \\
\hline Coal & .18 & .18 & .19 & .18 & .10 & .04 & -5.2 & 6.3 & 1.2 \\
\hline Other $^{7}$ & .50 & If .54 & .59 & .45 & .43 & .57 & -5.2 & 6.3 & 1.2 \\
\hline 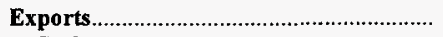 & 4.69 & 4.58 & 4.12 & 4.35 & 5.02 & 3.85 & - & - & - \\
\hline Coal & 2.37 & 2.32 & 1.88 & 1.96 & 2.68 & 2.09 & 2.2 & -3.0 & 1.4 \\
\hline 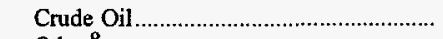 & 2.06 & 1.99 & 1.99 & 2.12 & 2.01 & 1.63 & 3.5 & .7 & 2.6 \\
\hline Other ${ }^{8}$ & .26 & .27 & .26 & .27 & .33 & .13 & -4.8 & -6.0 & 7.8 \\
\hline 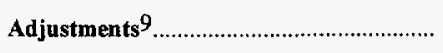 & 2.21 & $\mathrm{R}_{1.92}$ & ${ }^{\mathrm{R}}-.05$ & ${ }^{\mathrm{R}} 1.83$ & R .92 & .03 & 14.7 & 24.5 & 60.8 \\
\hline Consumption 10 & 93.81 & R 90.94 & R 89.21 & R 87.34 & ${ }^{R} 85.52$ & 76.89 & 3.1 & 2.3 & 2.2 \\
\hline 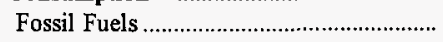 & 79.29 & R 76.94 & R 76.06 & R 74.51 & R 72.89 & 68.63 & 3.1 & 2.1 & 1.6 \\
\hline Coal & 20.99 & R 20.08 & ${ }^{\mathrm{R}} 20.02$ & ${ }^{R} 19.83$ & ${ }^{R} 19.21$ & 18.01 & 4.5 & 2.2 & 1.7 \\
\hline Coal Coke Net Imports............................... & $*$ & .03 & .02 & .02 & .03 & .01 & - & - & - \\
\hline 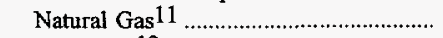 & 22.59 & R 22.16 & ${ }^{\mathrm{R}} 21.29$ & 20.83 & 20.13 & 17.74 & 1.9 & 2.9 & 2.7 \\
\hline 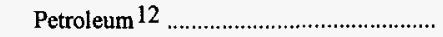 & 35.72 & R 34.66 & 34.73 & 33.84 & 33.53 & 32.87 & 3.0 & 1.6 & .9 \\
\hline Nuclear Electric Power & 7.17 & R 7.18 & 6.84 & 6.52 & 6.61 & 4.91 & -.1 & 2.0 & 4.3 \\
\hline Hydroelectric Pumped Storage 2 ................. & -.03 & -.03 & -.03 & -.04 & -.04 & $(3)$ & - & - & - \\
\hline 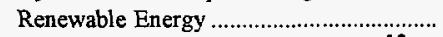 & 7.39 & R 6.85 & ${ }^{R} 6.28$ & 6.40 & 6.11 & 3.36 & 8.0 & 4.9 & 9.1 \\
\hline Conventional Hydroelectric Power 13 & 3.91 & $\mathrm{R} 3.47$ & 2.96 & 3.14 & 2.85 & 3.12 & 12.7 & 8.2 & 2.5 \\
\hline Geothermal Energy 14 & .35 & RR .33 & .38 & .38 & .37 & .23 & 9.0 & -.9 & 4.9 \\
\hline 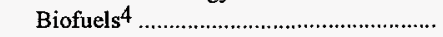 & 3.02 & R 2.95 & ${ }^{R} 2.84$ & 2.78 & 2,79 & .02 & 2.4 & 2.0 & 79.8 \\
\hline 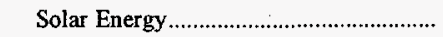 & .07 & .07 & .07 & .07 & .07 & - & - & - & - \\
\hline Wind Energy & .04 & $\mathrm{R} .03$ & .04 & .03 & .03 & * & 10.0 & 4.8 & 84.5 \\
\hline
\end{tabular}

1 Includes lease condensate.

2 Represents total pumped storage facility production minus energy used for pumping.

3 Pumped storage is included in conventional hydroelectric power.

4 Includes wood, wood waste, peat, wood liquors, railroad ties, pitch, wood sludge, municipal solid waste, agricultural waste, straw, tires, landfill gases,

fish oils, and/or other waste.

5 Includes imports of crude oil for the Stategic Petroleum Reserve, which began in 1977.

6 Includes imports of unfinished oils and natural gas plant liquids.

7 "Other" imports are electricity and coal coke.

8 "Other" exports are natural gas, petroleum products, electricity and coal coke.

9 A balancing item. Includes stock changes, losses, gains, miscellaneous blending components, and unaccounted for supply.

10 From 1990, the portion of net imports of electricity that is derived from nonrenewable energy sources is included directly in "Consumption."

11 Includes supplemental gaseous fuels.

12 Petroleum products supplied, including natural gas plant liquids and crude oil burned as fuel

13 Includes net imports of electricity.

14 Includes electricity imports from Mexico that are derived from geothermal energy.

* Data round to zero.

$\mathbf{R}$ Revised data.

P Preliminary data.

Notes: Coal Consumption does not include coal consumed by independent power producers. Total may not equal sum of components due to independent rounding.

Source: Energy Information Administration,Annual Energy Review 1996, DOE/EIA-0384(96), Table 1.1 
Table ES2. Share of U.S. Electric Utility Net Generation of Electricity by Source, 1987, 1992-1996 (Percent)

\begin{tabular}{|c|c|c|c|c|c|c|}
\hline Energy Source & 1996 & 1995 & 1994 & 1993 & 1992 & 1987 \\
\hline 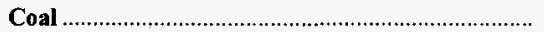 & 56.5 & 55.2 & 56.2 & 56.9 & 56.3 & 56.9 \\
\hline 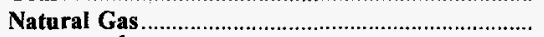 & 8.5 & 10.3 & 10.0 & 9.0 & 9.4 & 10.6 \\
\hline Petroleum 1 & 2.2 & 2.0 & 3.1 & 3.5 & 3.2 & 4.6 \\
\hline Nuclear Power & 21.9 & 22.5 & 22.0 & 21.2 & 22.1 & 17.7 \\
\hline Hydroelectric Power & 10.7 & 9.8 & 8.4 & 9.2 & 8.6 & 9.7 \\
\hline Geothermal and Other ${ }^{2}$ & .2 & .2 & .3 & .3 & .4 & .5 \\
\hline Total & 100.0 & 100.0 & 100.0 & 100.0 & 100.0 & 100.0 \\
\hline
\end{tabular}

1 Distillate fuel oil, residual fuel oil (including crude oil bumed as fuel), jet fuel, and petroleum coke.

2 Other is wood, waste, wind, photovoltaic, and solar thermal energy used to generate electricity for distribution. Note: Total may not equal sum of components due to independent rounding.

Source: Energy Information Administration, Form EIA-759, "Monthly Power Plant Report."

The average price of imported coal in 1996 declined 2 percent to $\$ 33.45$ per short ton. Coal imports were valued at $\$ 238$ million, down from $\$ 246$ million in 1995.

\section{Coal Consumption}

In 1996, domestic coal consumption rose to a record of 983 million short tons, a 4-percent increase over 1995. (This total does not include about 24 million short tons of coal consumed by independent power producers in 1996.) The increase is attributable to electric utility coal consumption, which rose by 6 percent to a record level of 875 million short tons, even though net generation at electric utilities rose by 3 percent. Coal consumption at electric utilities was indirectly affected by: a 10-percent increase in hydroelectric generation; a record level of nuclear power generation (up less than 1 percent); and, the lowest level of natural gas-fired generation since 1993 (down 14 percent from 1995).

Industrial plants consumed 71 million short tons of coal, 3 percent less than in 1995 . Coal carbonized at coke plants decreased by 4 percent to 32 million short tons, while the residential and commercial sectors consumed about 6 million short tons.

The average sulfur content (measured as percent sulfur by weight) of coal received at electric utilities in 1996 was 1.10 percent, up 2 percent from 1995. The quality of coal received at manufacturing and coke plants showed a sulfur content of 1.17 , a 2-percent increase.

Table ES3. Trends in U.S. Coal Production, Imports, Consumption, Exports, and Stocks, 1987, 1992-1996

(Million Short Tons)

\begin{tabular}{|c|c|c|c|c|c|c|c|c|c|}
\hline \multirow{2}{*}{ Activity } & \multirow{2}{*}{1996} & \multirow{2}{*}{1995} & \multirow{2}{*}{1994} & \multirow{2}{*}{1993} & \multirow{2}{*}{1992} & \multirow{2}{*}{1987} & \multirow{2}{*}{$\begin{array}{c}\text { Percent } \\
\text { Change } \\
1995-1996\end{array}$} & \multicolumn{2}{|c|}{ Average Annual Percent Change } \\
\hline & & & & & & & & $1992-1996$ & $1987-1996$ \\
\hline 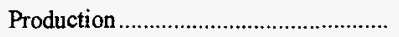 & 1,064 & 1,033 & 1,034 & 945 & 998 & 919 & 3.0 & 1.6 & 1.6 \\
\hline Imports & 7 & 7 & 8 & 7 & 4 & 2 & -1.0 & 17.0 & 16.9 \\
\hline Producer and Distributor Stocks 1 . & 29 & 34 & 33 & 25 & 34 & 34 & -16.8 & -4.2 & -1.9 \\
\hline 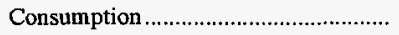 & 983 & 941 & 930 & 926 & 892 & 837 & 4.5 & 2.4 & 1.8 \\
\hline 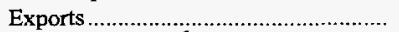 & 90 & 89 & 71 & 75 & 103 & 80 & 2.2 & -3.1 & 1.4 \\
\hline 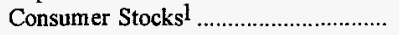 & 123 & 135 & 136 & 120 & 164 & 185 & -8.6 & -6.9 & -4.4 \\
\hline
\end{tabular}

1 Reported as of the last day of the quarter.

Note: Consumption does not include coal consumed by independent power producers.

Sources: - Production: Energy Information Administration (EIA), Form ELA-7A, "Coal Production Report"; U.S. Department of Labor, Mine Safety and Health Administration, Form 7000-2, "Quarterly Mine Employment and Coal Production Report"; and State Mining Agency Coal Production Reports. - Imports: U.S. Department of Commerce, Bureau of the Census, "Monthly Report IM 145." • Producer and Distributor Stocks: ELA, Form EIA-6, "Coal Distribution Report." - Exports: U.S. Department of Commerce, Bureau of the Census, "Monthly Report EM 545."

- Consumption and Consumer Stocks: EIA, Form EIA-759, "Monthly Power Plant Report"; Form EIA-3, Quarterly Coal Consumption Report - Manufacturing Plants"; Form EIA-5, "Coke Plant Report - Quarterly"; and Form EIA-6, "Coal Distribution Report." 


\section{Coal Stocks}

Coal stocks held by consumers on December 31, 1996, totaled 123 million short tons, 12 million short tons less than in 1995. Compared with 1995, year-end 1996 coal stocks at electric utility plants declined by 12 million short tons, while coal stocks at other industrial plants and coke plants remained about the same. Producer and distributor stocks at the end of 1996 were 29 million short tons, a decrease of 6 million short tons.

\section{Delivered Coal Prices}

The price of coal delivered to all consuming sectors declined in 1996. The delivered price of coal to the electric utility and other industrial sectors clropped by 2 percent and less than 1 percent, respectively, to $\$ 26.45$ per short ton and $\$ 32.32$ per short ton. The price of coal delivered to coke plants remained about the same at $\$ 47.33$ per short ton.

\section{Coal Exports}

U.S. coal exports went up 2 percent to 90 million short tons in 1996. An increase in shipments to Canada, the United Kingdom, Mexico, and the Republic of South Africa offset a decrease in shipments to Europe and Asia.

Compared with 1995 , steam coal exports rose 3 percent in 1996 to 38 million short tons. Steam coal exports to North America and Asia increased by 26 percent and 13 percent, respectively, while steam coal exports to Europe dropped by 11 percent to 19 million short tons.

U.S. metallurgical coal exports increased by 2 percent from the 1995 level, to 53 million short tons. Greater demand from foreign metallurgical coal markets, particularly in Canada, Italy, and the Republic of South Africa, were partially offset by lower dernand from Japan.

West Virginia continued to dominate coal exports in 1996, shipping over 42 million short tons, at 5-percent decrease from 1995. About 75 percent, or 32 million short tons, went to overseas metallurgical markets, while exports to overseas steam markets arnounted to 10 million short tons.

The average price of U.S. coal exports in 1996 was $\$ 40.76$ per short ton, a 1 -percent increase from 1995 . U.S. metallurgical coal export prices rose 3 percent to $\$ 45.49$ per short ton, while U.S. steam coal export prices went down slightly to $\$ 34.09$ per short ton. Coal exports in 1996 were valued at $\$ 3.7$ billion.

\section{Company Mergers \& Sales}

In the second largest railroad merger this decade, Conrail has been jointly acquired by CSX and Norfolk Southern. Conrail controls 11,000 miles of track in 12 States (including the District of Columbia) in the Northeast, Midwest, and Quebec, Canada. Conrail (for Consolidated Rail Corporation) was formed by the Federal government when Penn Central (which formerly merged with New York Central and five other Eastern lines) collapsed in bankruptcy in 1976. This consolidation of smaller lines allowed Conrail to have a monopoly of rail freight service along the eastern seaboard and west to Chicago.

Since the 1980 's, freight railroads have become more profitable, increasing freight traffic and operating more efficiently. These factors created the climate for recent mergers such as the one between Burlington Northern and Santa Fe Pacific in 1994. CSX and Norfolk Southern have each been trying to buy Conrail for some time, in a move to dominate the rail industry in the East. Under the agreement announced April 8, 1997, the two rail giants would divide the former Conrail lines with CSX getting 42 percent of the assets (for $\$ 4.3$ billion) and Norfolk Southern having the remaining assets (for $\$ 5.9$ billion). CSX would run the old New York Central's Hudson River route, which links New York City to Albany, then continues to Buffalo and Cleveland. In addition, they will serve the lines between New York and Philadelphia and Crestline, Ohio, and Chicago. Norfolk Southern will take over the Erie-Lackawanna route which runs through upstate New York, the old Pennsylvania Railroad line, and a section of the New York Central line from New Jersey to Chicago.

The joint merger application, filed with the U.S. Surface Transportation Board in June 1997, could take a year for final approval. If approved, the CSX-Norfolk Southern jointly-owned railroad would span 22 States and cover nearly 30,000 miles of track. Since Conrail's former rail lines will be divided between the two successors, competition will return to freight rail service in New York, Philadelphia, and other important Eastern ports after a nearly 30-year absence.

In January 1997, Nerox Energy Corp. acquired 1,410 acres of Alaskan coal-bearing lands from San Francisco-based Placer Dome U.S. Inc. Nerox set up offices in Anchorage prior to development of the site, located near Sutton, Alaska. Placer Dome's Evan Jones mine was renamed the Jonesville mine. The company negotiated a contract for an annual amount of 500 thousand metric tons of coal for the Asian export market and has submitted a bid with the Department of Defense to supply coal to military bases in Alaska.

Anker Energy took over Island Creek/Occidental Petroleum's jointly-owned coal properties from Glenn Springs Holdings in early 1996. The site, located in Upshur County, near Buckhannon, West Virginia, was not part of the large sale of Island Creek's assets to Consol in 1993. The property consists of haul roads, a prep plant and the Upshur deep mine, which has been 
idle since the middle 1980's. Spokespersons for Anker Energy said that the company will not mine the site, but instead will test its acid mine drainage techonology there.

Kerr-McGee Coal sold the stock of its subsidiary Pioneer Fuel to Ruhr American Coal Corp., in February 1996. Rhur American, owned by Germany's Ruhrkohl AG, purchased Pioneer's surface mine and the accompanying reserves, located in Wyoming County, West Virginia.

Cyprus AMAX Minerals Company, the second largest coal producing company in the U.S. purchased a 50 percent interest in an Australian mine in 1996. The Springvale mine, co-owned by Samsung Development, is a longwall-equipped deep mine which is currently producing at an annual rate of 2 million tons. Cyprus AMAX also successfully bid on the lease to 24 million tons of Federally-owned coal reserves at their Twentymile underground mine in Colorado. This addition brings the total to an estimated 140 million short tons of reserves at the mine. The Twentymile, which produced nearly 6 million tons in 1996, is estimated to produce 8 million tons in 1997.

Arch Minerals the 11th largest U.S. coal-producing company in 1995 sold land in Wyoming that included their Pilot Butte mine, to Union Pacific Corp., in 1996. The Pilot Butte, a small underground mine located near Rock Springs, Wyoming, had been supplying coal to the nearby Jim Bridger power plant. This arrangement did not prove profitable for Arch, and the mine has been idle for the past few years. Union Pacific, which already owned land near the mine, will have the lease for the coal reserves, including reserves which are controlled by the Bureau of Land Management. In August, 1996, Arch's subsidiary, Arch on the North Fork, closed its Ridgeline surface mine, located in Breathitt County, Kentucky. The mine was closed due to depletion of available reserves. In the West, Arch of Wyoming purchased an 80-thousand-acre tract of coal bearing land in Wyoming's Hanna Basin from Commonwealth Edison's Development. The site is located near Elk Mountain, Wyoming, and is estimated to have over 100 million tons in recoverable reserves.

Financially-troubled Morrison Knudsen agreed to merge with Washington Construction Group, a largeproject construction company located in Highland, California. Texas-based Morrison Knudsen intends to keep operating its contract mines for the near term. The merger is aimed at pooling the resources of several construction and mining-related interests that the two companies have in common.

Westmoreland Coal sold two of its idle Wise County, Virginia coal operations in 1996. The Wentz facilities (a mine and preparation plant) were transferred to Stonega Mining and Processing, and the Pine Branch Mine was sold to Roaring Fork Mining of Big Stone
Gap, Virginia. The deal was a non-cash sale; Stonega and Roaring Fork agreed to perform reclamation and be responsible for environmental liabilities and meet the UMWA-demands for medical benefit obligations to former Westmoreland employees affected by the sale.

In 1996, Jimmy Ryan, president of two Alabama coal companies, Kodiak Coal and Boone Resources opened the Kodiak No. 1 mine in Shelby County, despite union opposition. The UMWA claimed it had a right to representation at the mine since Ryan is leasing it from U.S. Steel, which is unionized. This was disputed by subsidiary U.S. Steel Mining, the actual operating company, which is non-union. The Kodiak No. 1 Mine produced just over 73 thousand tons of coal in 1996.

In September 1996, Beacon Group Energy Investment Fund purchased MAPCO Coal and MC Mining from MAPCO Inc. The terms of the sale included 100 percent of stock from these two companies. Other assets include mining operations in Kentucky, Maryland, Illinois, and Virgnia, a coal terminal on the Ohio River, and supply contracts with Delmarva Power, Seminole Electric, Virginia Power, Cogentrix, TVA, and U.S. Steel. Joe Craft III continues to serve as MAPCO Coal's President, and the company will maintain facilities at MAPCO Inc.'s headquarters in Tulsa, Oklahoma and in Lexington, Kentucky. MAPCO Coal was the 19th largest coal producer in 1996 , producing 12.8 million short tons.

In late December 1996, Coastal Corp. was finalizing the sale of its SUFCO, Skyline, and Soldier Creek mining operations in Utah to ARCO Coal and Japanese-owned ITOCHU Corp. The sale was concluded after several months of negotiations, including a period in which ANR Coal- Coastal's Appalachian subsidiary - was being considered as part of the purchase. The main sticking point in the negotiations was the future value of the long-term supply contracts held by Coastal with Intermountain Power Agency and Sierra Pacific Power. Coastal reassured ARCO and ITOCHU by offering to insure the contracts from devaluation due to the effects of arbitration or utility deregulation. The SUFCO, Skyline, and Soldier Creek controlled mines together produced nearly 9 million tons of high volatile, low - sulfur, bituminous coal in 1996. Ninety percent of this output went to U.S. electric utilities and 10 percent was exported to the Pacific Rim. There are an estimated 300 million tons of recoverable reserves on these properties, according to ARCO. Also included in the sale was Coastal's 9-percent share of the Los Angeles Export Terminal.

In November 1996, British-owned Costain PLC sold its U.S. coal company Costain Coal, to Rencoal, Inc., a subisdiary of New York-based Renco Group. Costain PLC had been trying to divest itself of its U.S. coal holdings since 1994. Costain Coal produced 9.3 million short tons in 1996. 
Figure ES1. Trends in U.S. Coal Production, Imports, Consumption, Exports, and Stocks, 1987-1996

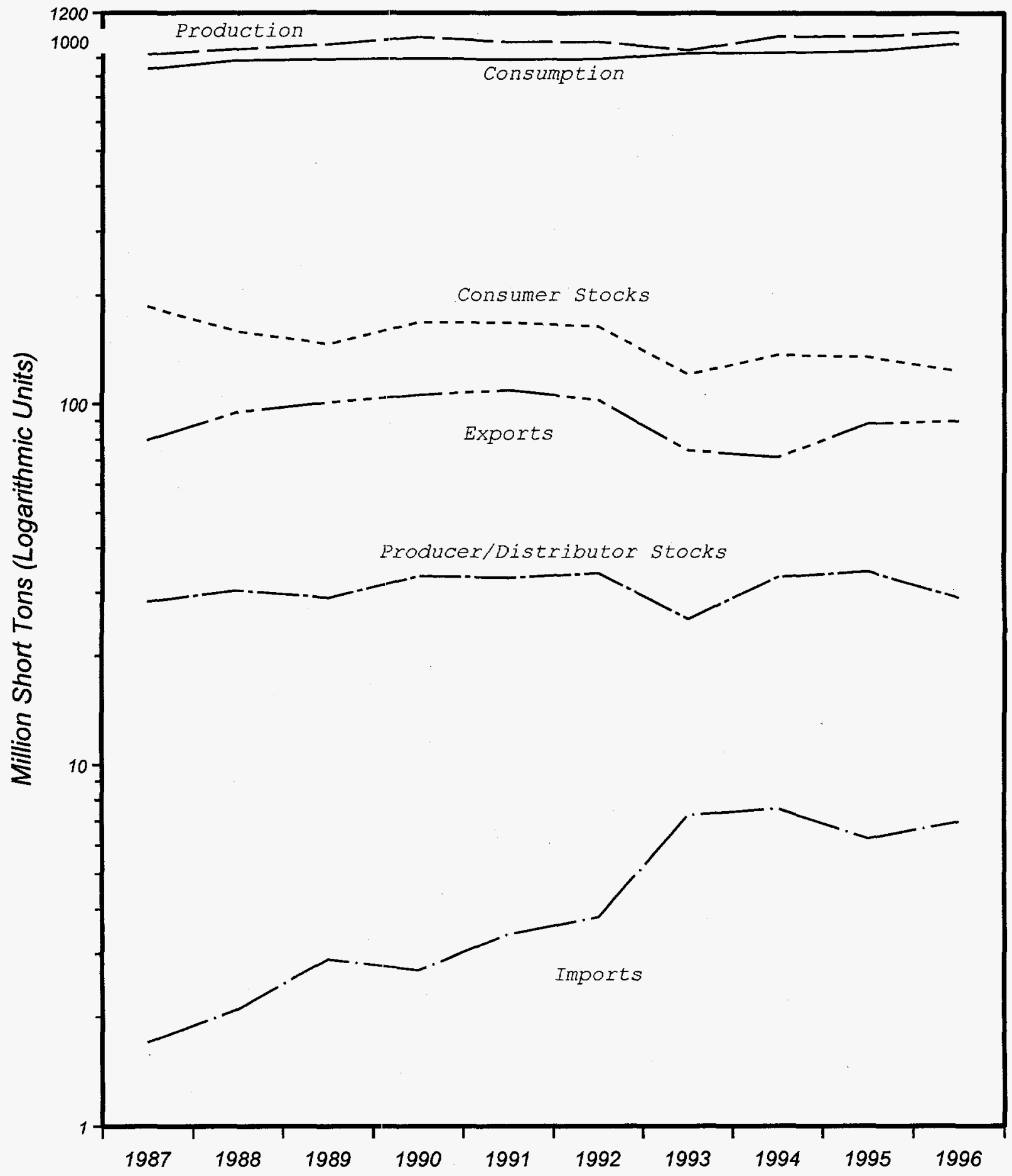

Note: Consumption does nol include cool consuned by independenl power producers.

Sources: Production: Energy Informotion Administrotion (EIA). Form EIA-TA, "Cool Production"; U.S. Department ol Lobor Mine Sofely and Health Administrolion, Form 7000-2. "Ouorlerly Mine Employment and Cool Produclion Report". and Stale Mining Agency Cool Production Reporls. Imporis: U.S. Deporiment ol Commerce, Bureou of ine Census, "Monthly Report IM 145. Cooproducer ond Distributor Stocks: EIA, Form EIA-6. "Cool Disiribution Report." Exports: U.S. Department of Commerce. Bureau of the Census. "Monthly Repori EM 545." Consumplion ond Consumer siocks: EiA. Form ElA-759. "Monthly Power Plant Report"; Form EiA-3. "Quorterly Coal Consumption Report - Monufocturing Pionts"; Form EIA-5. "Coke Plant Report - Quorterly"; ond Form EiA-6, "Cool Distribution Report." 
Table ES4. U.S. Coal Statistics, 1987, 1992-1996

\begin{tabular}{|c|c|c|c|c|c|c|c|c|c|}
\hline \multirow{2}{*}{ Category } & \multirow{2}{*}{1996} & \multirow{2}{*}{1995} & \multirow{2}{*}{1994} & \multirow{2}{*}{1993} & \multirow{2}{*}{1992} & \multirow{2}{*}{1987} & \multirow{2}{*}{$\begin{array}{c}\text { Percent } \\
\text { Change } \\
\text { 1995-1996 }\end{array}$} & \multicolumn{2}{|c|}{$\begin{array}{l}\text { Average Annual } \\
\text { Percent Change }\end{array}$} \\
\hline & & & & & & & & 1992-1996 & $1987-1996$ \\
\hline \multicolumn{10}{|l|}{ Supply (thousand short tons) } \\
\hline Recoverable Reserves........................... & $19,427,980$ & $20,105,197$ & $21,016,526$ & $21,535,283$ & $21,626,971$ & $24,241,218$ & -3.4 & -2.6 & -2.4 \\
\hline 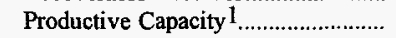 & $1,324,712$ & $1,299,054$ & $1,320,656$ & $1,261,873$ & $1,241,054$ & $1,183,404$ & 2.0 & 1.6 & 1.3 \\
\hline 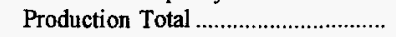 & $1,063,856$ & $1,032,974$ & $1,033,504$ & 945,424 & 997,545 & 918,762 & 3.0 & 1.6 & 1.6 \\
\hline 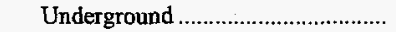 & 409,849 & 396,249 & 399,103 & 351,053 & 407,239 & 372,874 & 3.4 & .1 & 1.0 \\
\hline 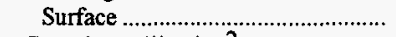 & 654,007 & 636,725 & 634,401 & 594,371 & 590,306 & 545,888 & 2.7 & 2.6 & 2.0 \\
\hline $\begin{array}{l}\text { Capacity Utilization } 2 \\
\text { Ratio of Recoverable }\end{array}$ & 80.21 & 79.40 & 78.11 & 74.77 & 80.20 & 77.29 & 1.0 & * & .4 \\
\hline Reserves to Production .......................... & 18.3 & 19.5 & 20.3 & 22.8 & 21.7 & 26.4 & -6.2 & -4.2 & -4.0 \\
\hline 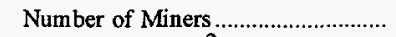 & 83,462 & 90,252 & 97,500 & 101,322 & 110,196 & 142,667 & -7.5 & -6.7 & -5.8 \\
\hline Productivity Total ${ }^{2} \ldots \ldots \ldots \ldots \ldots \ldots \ldots \ldots \ldots \ldots \ldots \ldots \ldots \ldots \ldots$ & 5.69 & 5.38 & 4.98 & 4.70 & 4.36 & 3.30 & 5.8 & 6.9 & 6.3 \\
\hline 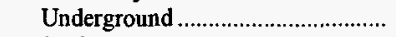 & 3.57 & 3.39 & 3.19 & 2.95 & 2.93 & 2.20 & 5.6 & 5.1 & 5.5 \\
\hline 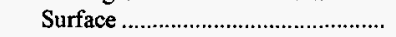 & 9.05 & 8.48 & 7.67 & 7.23 & 6.59 & 4.98 & 6.7 & 8.3 & 6.9 \\
\hline Producer/Distributor Stocks .............. & 28,648 & 34,444 & 33,219 & 25,284 & 33,993 & 34,090 & -16.8 & -4.2 & -1.9 \\
\hline 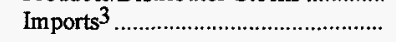 & 6,476 & 6,317 & 6,599 & 5,496 & 1,806 & 765 & 2.5 & 37.6 & 26.8 \\
\hline \multicolumn{10}{|l|}{ Distribution (thousand short tons) } \\
\hline 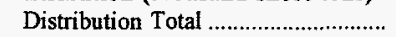 & $1,059,892$ & $1,030,330$ & $1,022,523$ & 959,445 & 998,647 & NA & 2.9 & 1.5 & NA \\
\hline Domestic Distribution Total........... & 967,693 & 940,423 & 949,843 & 883,934 & 897,267 & NA & 2.9 & 1.9 & NA \\
\hline 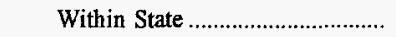 & 340,005 & 336,821 & 353,765 & 339,034 & 355,232 & $\mathrm{NA}$ & .9 & -1.1 & NA \\
\hline 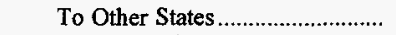 & 627,688 & 603,602 & 596,078 & 544,900 & 542,035 & NA & 4.0 & 3.7 & NA \\
\hline Foreign Distribution Total ............. & 92,199 & 89,907 & 72,680 & 75,510 & 101,380 & NA & 2.5 & -2.3 & NA \\
\hline Metallurgical ............................... & 56,162 & R 54,077 & R 52,206 & R 52,591 & R 61,432 & NA & 3.9 & -2.2 & NA \\
\hline 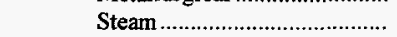 & 36,037 & R 35,830 & R 20,474 & R 22,919 & ${ }^{R} 39,948$ & NA & .6 & -2.5 & $\mathrm{NA}$ \\
\hline 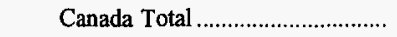 & 10,599 & 8,023 & 8,467 & 7,751 & 13,919 & $\mathrm{NA}$ & 32.1 & -6.6 & NA \\
\hline Metallurgical .............................. & 8,472 & R 6,986 & 7,464 & 6,666 & 9,394 & $\mathrm{NA}$ & 21.3 & -2.5 & $\mathrm{NA}$ \\
\hline Steam & 2,127 & 1,037 & 1,003 & 1,085 & 4,525 & NA & 105.0 & -17.2 & NA \\
\hline 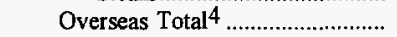 & 81,600 & 81,884 & 64,214 & 67,759 & 87,461 & NA & -.3 & -1.7 & $\mathrm{NA}$ \\
\hline 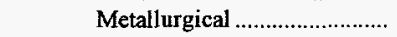 & 47,690 & R 47,091 & R 44,743 & R 45,925 & R 52,038 & NA & 1.3 & -2.2 & $\mathrm{NA}$ \\
\hline Steam & 33,910 & R 34,793 & R 19,471 & R 21,834 & R. 35,423 & NA & -2.5 & -1.1 & NA \\
\hline \multicolumn{10}{|l|}{ Demand (thousand short tons) } \\
\hline 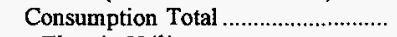 & 983,334 & 940,880 & 930,201 & 925,944 & 892,421 & 836,860 & 4.5 & 2.4 & 1.8 \\
\hline 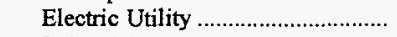 & 874,681 & 829,007 & 817,270 & 813,508 & 779,860 & 717,894 & 5.5 & 2.9 & 2.2 \\
\hline 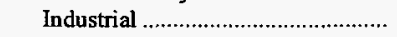 & 70,941 & 73,055 & 75,179 & 74,892 & 74,042 & 75,175 & -2.9 & -1.1 & -.6 \\
\hline 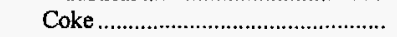 & 31,706 & 33,011 & 31,740 & 31,323 & 32,366 & 36,877 & -3.9 & -.5 & -1.7 \\
\hline Residential/Commercial .................. & 6,006 & 5,807 & 6,013 & 6,221 & 6,153 & 6,914 & 3.4 & -.6 & -1.5 \\
\hline Consumer Stocks Total ......................... & 123,024 & 134,639 & 136,139 & 120,458 & 163,692 & 185,459 & -8.6 & -6.9 & -4.4 \\
\hline 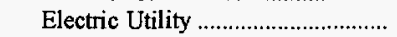 & 114,669 & 126,304 & 126,897 & 111,341 & 154,130 & 170,797 & -9.2 & -7.1 & -4.3 \\
\hline 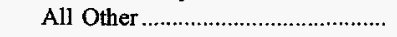 & 8,355 & 8,334 & 9,243 & 9,117 & 9,562 & 14,662 & .3 & -3.3 & -6.0 \\
\hline \multicolumn{10}{|c|}{ Coal Prices (nominal dollars per short ton) } \\
\hline Mine Total & $\$ 18.50$ & $\$ 18.83$ & $\$ 19.41$ & $\$ 19.85$ & $\$ 21.03$ & $\$ 23.07$ & -1.8 & -3.1 & -2.4 \\
\hline 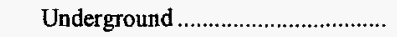 & 25.96 & 26.18 & 26.39 & 26.92 & 27.83 & 29.63 & -.9 & -1.7 & -1.5 \\
\hline Surface & 13.82 & 14.25 & 15.02 & 15.67 & 16.34 & 18.58 & -3.0 & -4.1 & -3.2 \\
\hline \multicolumn{10}{|l|}{ Consumer } \\
\hline 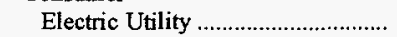 & 26.45 & 27.01 & 28.03 & 28.58 & 29.36 & 31.83 & -2.1 & -2.6 & -2.0 \\
\hline 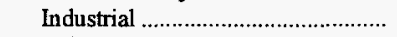 & 32.32 & 32.42 & 32.55 & 32.23 & 32.78 & 33.71 & -.3 & -.3 & -.5 \\
\hline Coke & 47.33 & 47.34 & 46.56 & 47.44 & 47.92 & 46.55 & $*$ & -.3 & .2 \\
\hline
\end{tabular}

1 For 1987, the Form EIA-7A solicited data on "Daily Productive Capacity." To obtain annual productive capacity for a mine in 1987, each mine's daily productive capacity was multiplied by the number of days worked during the year.

2 Capacity utilization (percent) is the ratio of total production to annual productive capacity as reported by mining companies on Form EIA-7A. Productivity (short tons per miner per hour) is calculated by dividing total coal production by the total direct labor hours worked by all employees engaged in production, preparation, processing, development, maintenance, repair, and shop or yard work at mining operations.

3 Imports for 1992 through 1996 include imports to electric utilities, manufacturing plants and coke plants. Imports for 1987 include only imports to electric utilities.

4 Includes Mexico.

* Data round to zero.

NA Not available.

R Revised Data.

Notes: Excludes silt, culm, refuse bank, slurry dam, and dredge operations except for Pennsylvania anthracite. Consumption Total does not include coal consumed by independent power producers. Totals may not equal sum of components due to independent rounding.

Sources: Energy Information Administration, Form EIA-3, "Quarterly Coal Consumption Report - Manufacturing Plants"; Form EIA-5, "Coke Plant Report - Quarterly"; Form EIA-6, "Coal Distribution Report"; Form EIA-7A, "Coal Production Report"; Form EIA-759, "Monthly Power Plant Report"; and U.S Department of Commerce, Bureau of the Census, "Monthly Report EM 545" and "Monthly Report IM 145." 


\section{Supply}

\section{Production}

U.S. coal production during 1996 increased 3 percent to 1,064 million short tons, setting a record level (Table 1). As in 1995, the largest coal-producing State was Wyoming, followed by West Virginia and Kentucky. Wyoming's coal production in 1996 increased 6 percent over its 1995 level to reach a record of 278 million short tons. West Virginina increased its coal production by 5 percent to a level of 170 million short tons, 3 percent less than the record level of 176 million short tons in 1947. Kentucky, the third largest coal-producing State, declined by 1 percent in 1996, to a level of 152 million short tons. Only 11 of the 25 coal producing States showed an increase in 1996.

Regionally, coal production increased in all regions. In Appalachia, increases in production in 1996 occured in Maryland, Ohio, Pennsylvania, Tennessee, Virginia, and West Virginia, resulting in the largest increase of any region, 17 million short tons (3.9 percent). The Interior Region registered increases in production in Indiana, Missouri, and Texas. Overall, the Interior Region increased in 1996 by 4.3 million short tons (2.6 percent). In the Western Region, two of the nine States (Utah and Wyoming) increased their coal production in 1996, with both States reaching record levels. Coal production in the Western Region increased 9.6 million short tons, an increase of 2.2 percent. Over the last decade, the Appalachian Region has only increased at an average annual rate of 0.2 percent, while the Western Region has increased 5.4 percent. During the same time period, the Interior Region has declined at an average annual rate of 1.7 percent.

U.S. coal producers operated 1,903 mines during 1996, including 885 underground mines and 1,018 surface mines (Table 3 ). Underground mines produced 410 million short tons and represented 39 percent of total coal production. Underground mines east of the Mississippi accounted for 89 percent of all underground coal production in 1996. Surface mines west of the Mississippi represented 69 percent of total surface coal production in 1996 . Of the top 20 coal mines in the United States, 18 are surface mines, all located west of the Mississippi; they accounted for 28.6 percent of total U.S. coal production. Nine of the top ten mines in the United States are in Wyoming and represent 19.4 percent of total U.S. coal production.

\section{Productive Capacity}

The total estimated productive capacity of U.S. coal mines in 1996 was 1,325 million short tons, an increase of 2.0 percent compared with the total estimated productive capacity of 1,299 million short tons in 1995 (Table 16). Following the same pattern as coal production, the total estimated productive capacity increased in all regions. Total coal mine capacity utilization, (defined as the ratio of actual production to productive capacity) rose to 80 percent in 1996 (Table 17). Capacity utilization increased for all three regions in 1996, with the Appalachian Region showing the largest increase.

\section{Recoverable Reserves}

Estimated recoverable U.S. coal reserves at producing mines in 1996 totaled 19 billion short tons. This 3 -percent decrease continued a decline that started in 1986 (Table 25). Recoverable reserves at producing mines in the Western Region accounted for 62.5 percent of total reserves, with reserves at producing mines in the Appalachian and Interior Regions representing 23.3 percent and 14.2 percent, respectively.

The average recovery percentage for all U.S. producing mines in 1996 increased slightly to 80.8 percent from the 80.1 percent in 1995 (Table 26). The estimated recovery percent for all underground mines in 1996 was 57.2 percent, an increase from the 54.5 percent in 1995 . The estimated recovery percent at surface mines remained unchanged in 1996 at 90 percent.

\section{Producers and Distributors Stocks}

Coal stocks held by U.S. coal producers and distributors at the end of 1996 totaled 29 million short tons, a decrease of 17 percent compared with stocks at year-end 1995 . 


\section{Coal Imports}

U.S. coal imports during 1996 totaled 7.1 million short tons, 1 percent less than the 7.2 million short tons imported during 1995 (Table 35). Since 1987, U.S. coal imports have increased at an average annual rate of 17 percent.

Colombia, Indonesia, Venezuela, and Canacla were the major sources of imported coal during 1996. They contributed 6.9 million short tons and accounted for 98 percent of total coal imports. 


\section{Production}

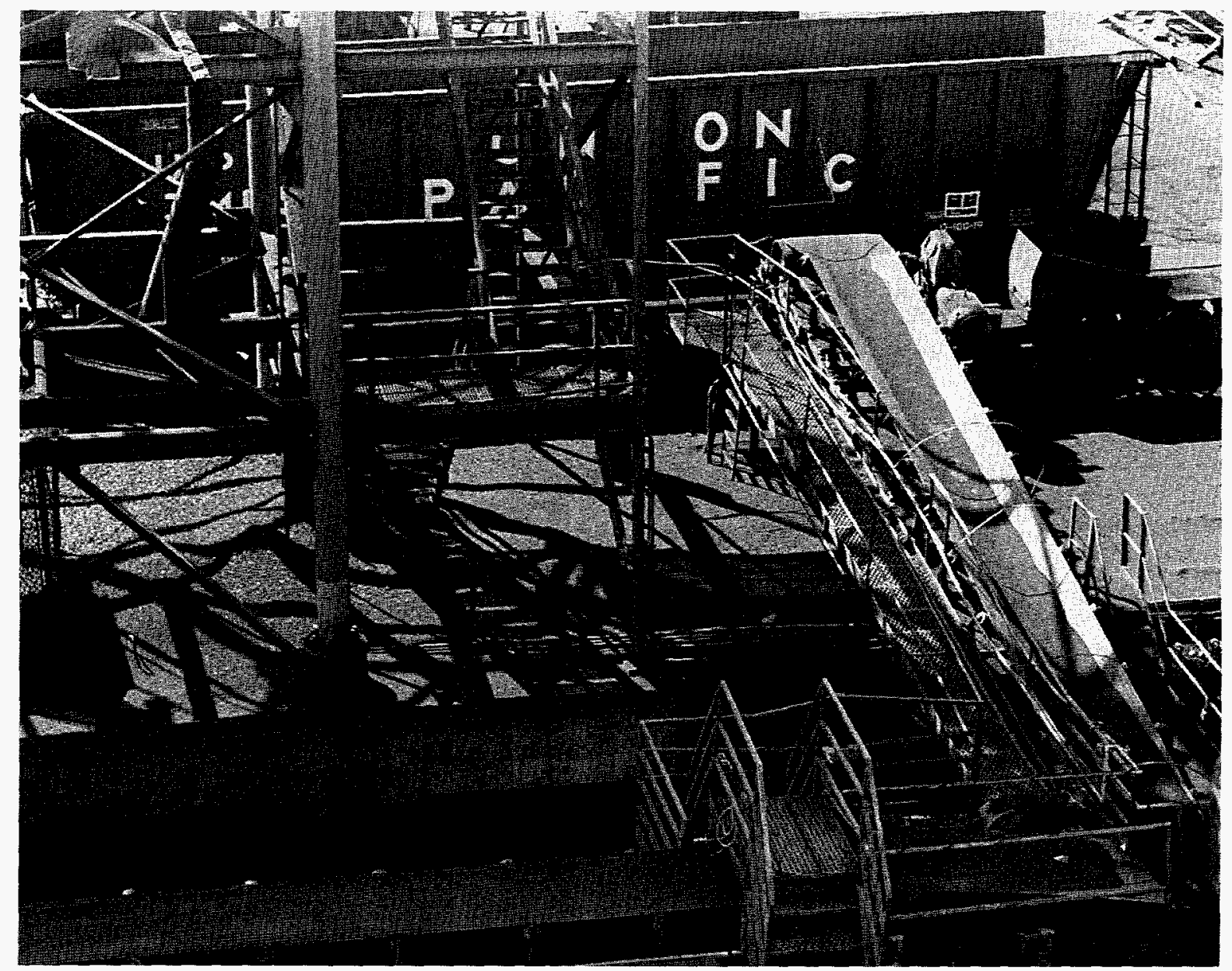

Virtually all aspects of the coal industry have been impacted by advanced technology. The Port of Los Angeles' dry bulk facility can stockpile over 170,000 metric tons of commodities including coal and petroleum coke. Petroleum coke is delivered by railcars or bottom dump trucks. 
Table 1. Coal Production by State, 1987, 1992-1996

(Thousand Short Tons)

\begin{tabular}{|c|c|c|c|c|c|c|c|c|c|}
\hline \multirow{2}{*}{$\begin{array}{l}\text { Coal-Producing } \\
\text { State and Region }\end{array}$} & \multirow{2}{*}{1996} & \multirow{2}{*}{1995} & \multirow{2}{*}{1994} & \multirow{2}{*}{1993} & \multirow{2}{*}{1992} & \multirow{2}{*}{1987} & \multirow{2}{*}{$\begin{array}{c}\text { Percent } \\
\text { Change } \\
1995-1996\end{array}$} & \multicolumn{2}{|c|}{ Average Annual Percent Change } \\
\hline & & & & & & & & 1992-1996 & 1987-1996 \\
\hline 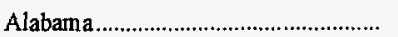 & 24,637 & 24,641 & 23,266 & 24,768 & 25,796 & 25,540 & * & -1.1 & -0.4 \\
\hline Alaska & 1,481 & $1,69: 8$ & 1,567 & 1,601 & 1,534 & 1,492 & -12.8 & -.9 & -.1 \\
\hline Arizona & 10,442 & 11,947 & 13,056 & 12,173 & 12,512 & 11,379 & -12.6 & -4.4 & -.9 \\
\hline 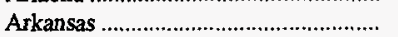 & 21 & 29 & 51 & 44 & 58 & 84 & -28.0 & -22.6 & -14.3 \\
\hline California & - & - & - & - & 103 & 46 & - & - & - \\
\hline 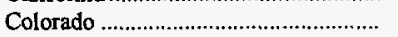 & 24,886 & 25,710 & 25,304 & 21,886 & 19,226 & 14,420 & -3.2 & 6.7 & 6.3 \\
\hline 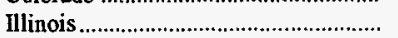 & 46,656 & 48,180 & 52,797 & 41,098 & 59,857 & 59,155 & -3.2 & -6.0 & -2.6 \\
\hline Indiana & 29,670 & 26,007 & 30,927 & 29,295 & 30,466 & 34,208 & 14.1 & -.6 & -1.6 \\
\hline Iowa & - & - & 46 & 175 & 289 & 468 & - & - & - \\
\hline 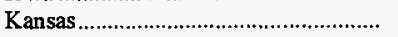 & 232 & 285 & 284 & 341 & 363 & 2,021 & -18.4 & -10.5 & -21.4 \\
\hline Kentucky Total & 152,425 & 153,739 & 161,642 & 156,299 & 161,068 & 165,192 & -.8 & -1.4 & -.9 \\
\hline 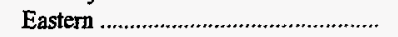 & 116,951 & 118,541 & 124,447 & 120,191 & 119,382 & 119,906 & -1.3 & -.5 & -.3 \\
\hline Western & 35,474 & 35,198 & 37,195 & 36,108 & 41,686 & 45,285 & .8 & -3.9 & -2.7 \\
\hline 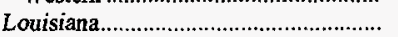 & 3,221 & 3,719 & 3,463 & 3,134 & 3,240 & 2,751 & -13.4 & -.1 & 1.8 \\
\hline 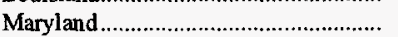 & 4,093 & 3,667 & 3,632 & 3,355 & 3,341 & 3,962 & 11.6 & 5.2 & .3 \\
\hline 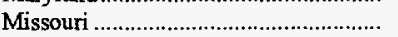 & 710 & 548 & 838 & 653 & 2,886 & 4,292 & 29.6 & -29.6 & -18.1 \\
\hline 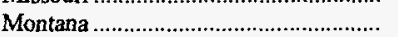 & 37,891 & 39,451 & 41,640 & 35,917 & 38,889 & 34,399 & -3.9 & -.6 & 1.1 \\
\hline 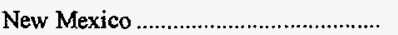 & 24,067 & 26,813 & 28,041 & 28,268 & 24,549 & 19,131 & -10.2 & -.5 & 2.6 \\
\hline 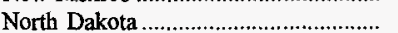 & 29,861 & 30,112 & 32,286 & 31,973 & 31,744 & 25,142 & -.8 & -1.5 & 1.9 \\
\hline Ohio & 28,572 & $26,1 ! 8$ & 29,897 & 28,816 & 30,403 & 35,788 & 9.4 & -1.5 & -2.5 \\
\hline Oklahoma & 1,701 & 1,876 & 1,911 & 1,758 & 1,741 & 2,870 & -9.3 & -.6 & -5.6 \\
\hline 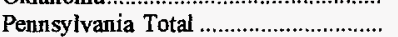 & 67,942 & 61,576 & 62,237 & 59,700 & 68,981 & 70,423 & 10.3 & -.4 & -.4 \\
\hline 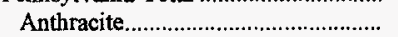 & 4,751 & 4,632 & 4,621 & 4,306 & 3,483 & 3,560 & 1.5 & 8.1 & 3.3 \\
\hline 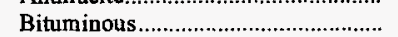 & 63,190 & 56,893 & 57,616 & 55,394 & 65,498 & 66,863 & 11.1 & -.9 & -.6 \\
\hline 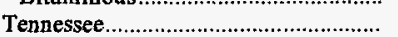 & 3,651 & $3,2.21$ & 2,987 & 3,047 & 3,476 & 6,442 & 13.3 & 1.2 & -6.1 \\
\hline Texas & 55,164 & 52,684 & 52,346 & 54,567 & 55,071 & 50,529 & 4.7 & * & 1.0 \\
\hline 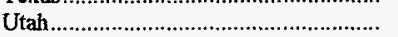 & 27,507 & 25,157 & 24,399 & 21,847 & 21,339 & 16,508 & 9.3 & 6.5 & 5.8 \\
\hline Virginia & 35,590 & 34,099 & 37,129 & 39,317 & 43,024 & 44,543 & 4.4 & -4.6 & -2.5 \\
\hline Washington & 4,565 & 4,868 & 4,893 & 4,739 & 5,251 & 4,449 & -6.2 & -3.4 & .3 \\
\hline 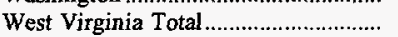 & 170,433 & 162,997 & 161,776 & 130,525 & 162,164 & 136,676 & 4.6 & 1.3 & 2.5 \\
\hline Northern & 45,910 & 46,114 & 49,316 & 33,802 & 50,022 & 52,003 & -4 & -2.1 & -1.4 \\
\hline Southern & 124,523 & 116,883 & 112,460 & 96,723 & 112,142 & 84,673 & 6.5 & 2.6 & 4.4 \\
\hline Wyoming & 278,440 & 263,822 & 237,092 & 210,129 & 190,172 & 146,850 & 5.5 & 10.0 & 7.4 \\
\hline Appalachian Total ${ }^{1}$............................. & 451,868 & 434,861 & 445,370 & 409,718 & 456,565 & 443,281 & 3.9 & -.3 & .2 \\
\hline Interior Total ${ }^{1}$ & 172,848 & 168,526 & 179,858 & 167,174 & 195,659 & 201,663 & 2.6 & -3.0 & -1.7 \\
\hline Western Total ${ }^{1} \ldots \ldots \ldots \ldots \ldots \ldots \ldots \ldots$ & 439,140 & 429,587 & 408,276 & 368,532 & $\mathbf{3 4 5 , 3 2 1}$ & 273,818 & 2.2 & 6.2 & 5.4 \\
\hline East of Miss. River................................ & 563,668 & $544,2,46$ & 566,289 & 516,219 & $\mathbf{5 8 8 , 5 7 5}$ & 581,929 & 3.6 & -1.1 & -.3 \\
\hline 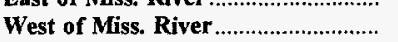 & 500,188 & 488,728 & 467,216 & 429,205 & 408,970 & 336,833 & 2.3 & 5.2 & 4.5 \\
\hline U.S. Total & $1,063,856$ & $1,032,974$ & $1,033,504$ & 945,424 & 997,545 & 918,762 & 3.0 & 1.6 & 1.6 \\
\hline
\end{tabular}

1 For a definition of coal-producing regions, see Appenclix C

* Data round to zero.

Notes: Coal production excludes silt, culm, refuse bank, slumy dam, and dredge operations except for Pennsylvania anthracite. Totals may not equal sum of components due to independent rounding.

Sources: Energy Information Administration, Form EIA-7A, "Coal Production Report"; State Mining Agency Coal Production Reports; and/or U.S. Department of Labor, Mine Safety and Health Administration, Form 7000-2, "Quarterly Mine Employment and Coal Production Report." 
Table 2. Number of Coal Mines by State, 1987, 1992-1996

\begin{tabular}{|c|c|c|c|c|c|c|c|c|c|}
\hline \multirow{2}{*}{$\begin{array}{l}\text { Coal-Producing } \\
\text { State and Region }\end{array}$} & \multirow{2}{*}{1996} & \multirow{2}{*}{1995} & \multirow{2}{*}{1994} & \multirow{2}{*}{1993} & \multirow{2}{*}{1992} & \multirow{2}{*}{1987} & \multirow{2}{*}{$\begin{array}{c}\text { Percent } \\
\text { Change } \\
1995-1996\end{array}$} & \multicolumn{2}{|c|}{ Average Annual Percent Change } \\
\hline & & & & & & & & $1992-1996$ & $1987-1996$ \\
\hline Alabama & 53 & 73 & 85 & 85 & 88 & 99 & -27.4 & -11.9 & -6.7 \\
\hline Alaska & 1 & 1 & 1 & 1 & 2 & 1 & - & -15.9 & - \\
\hline Arizona & 2 & 2 & 2 & 2 & 2 & 2 & - & - & - \\
\hline Arkansas & 5 & 3 & 6 & 6 & 6 & 8 & 66.7 & -4.4 & -5.1 \\
\hline 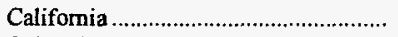 & - & - & - & - & 1 & 1 & - & - & - \\
\hline Colorado & 15 & 17 & 18 & 20 & 21 & 22 & -11.8 & -8.1 & -4.2 \\
\hline 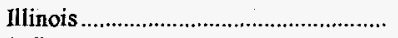 & 31 & 31 & 34 & 39 & 43 & 51 & - & -7.8 & -5.4 \\
\hline Indiana & 37 & 42 & 55 & 56 & 51 & 67 & -11.9 & -7.7 & -6.4 \\
\hline Iowa & - & - & 1 & 2 & 2 & 4 & - & - & - \\
\hline 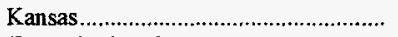 & 1 & 1 & 1 & 2 & 2 & 5 & - & -15.9 & -16.4 \\
\hline 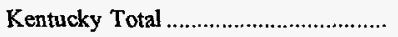 & 544 & 598 & 673 & 696 & 752 & 1,428 & -9.0 & -7.8 & -10.2 \\
\hline 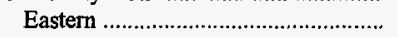 & 484 & 540 & 607 & 622 & 684 & 1,323 & -10.4 & -8.3 & -10.6 \\
\hline Western & 60 & 58 & 66 & 74 & 68 & 105 & 3.4 & -3.1 & -6.0 \\
\hline 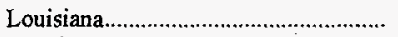 & 2 & 2 & 2 & 2 & 2 & 1 & - & - & 8.0 \\
\hline 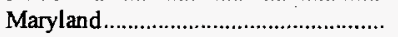 & 18 & 20 & 20 & 21 & 24 & 35 & -10.0 & -6.9 & -7.1 \\
\hline 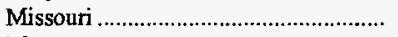 & 5 & 6 & 6 & 7 & 5 & 12 & -16.7 & - & -9.3 \\
\hline 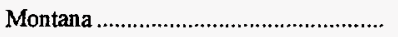 & 8 & 8 & 8 & 8 & 8 & 8 & - & - & - \\
\hline 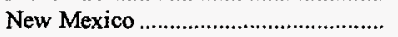 & 6 & 7 & 7 & 7 & 7 & 10 & -14.3 & -3.8 & -5.5 \\
\hline 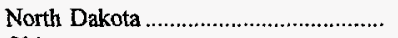 & 5 & 6 & 6 & 8 & 8 & 11 & -16.7 & -11.1 & -8.4 \\
\hline Ohio & 99 & 113 & 134 & 135 & 149 & 210 & -12.4 & -9.7 & -8.0 \\
\hline 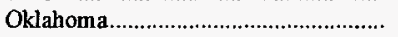 & 12 & 13 & 14 & 17 & 20 & 19 & -7.7 & -12.0 & -5.0 \\
\hline Pennsylvania Total & 402 & 459 & 505 & 524 & 578 & 683 & -12.4 & -8.7 & -5.7 \\
\hline Anthracite & 127 & 134 & 143 & 148 & 166 & 200 & -5.2 & -6.5 & -4.9 \\
\hline 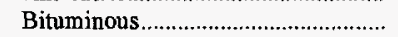 & 275 & 325 & 362 & 376 & 412 & 483 & -15.4 & -9.6 & -6.1 \\
\hline 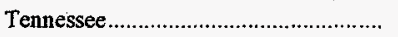 & 26 & 25 & 24 & 37 & 50 & 93 & 4.0 & -15.1 & -13.2 \\
\hline 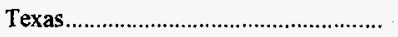 & 13 & 14 & 13 & 14 & 15 & 15 & -7.1 & -3.5 & -1.6 \\
\hline 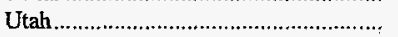 & 11 & 13 & 14 & 15 & 15 & 21 & -15.4 & -7.5 & -6.9 \\
\hline Virginia & 191 & 194 & 231 & 237 & 258 & 477 & -1.5 & -7.2 & -9.7 \\
\hline 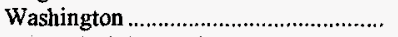 & 3 & 3 & 3 & 3 & 3 & 4 & - & - & -3.1 \\
\hline West Virginia Total .................................. & 386 & 424 & 462 & 502 & 604 & 778 & -9.0 & -10.6 & -7.5 \\
\hline Northern & 93 & 98 & 116 & 137 & 166 & 255 & -5.1 & -13.5 & -10.6 \\
\hline 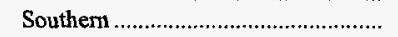 & 293 & 326 & 346 & 365 & 438 & 523 & -10.1 & -9.6 & -6.2 \\
\hline Wyoming & 27 & 29 & 29 & 29 & 30 & 29 & -6.9 & -2.6 & -.8 \\
\hline Appalachian Total ${ }^{1}$ & 1,659 & 1,848 & 2,068 & 2,163 & 2,435 & 3,698 & -10.2 & -9.1 & -8.5 \\
\hline Interior Total 1 & 166 & 170 & 198 & 219 & 214 & 287 & -2.3 & -6.1 & -5.9 \\
\hline Western Total ${ }^{l}$ & 78 & 86 & 88 & 93 & 97 & 109 & -9.3 & -5.3 & -3.6 \\
\hline East of Miss. River & 1,787 & 1,979 & 2,223 & 2,332 & 2,597 & 3,921 & -9.7 & -8.9 & -8.4 \\
\hline West of Miss. River............................... & 116 & 125 & 131 & 143 & 149 & 173 & -7.2 & -6.1 & -4.3 \\
\hline U.S. Total. & 1,903 & 2,104 & 2,354 & 2,475 & 2,746 & 4,094 & -9.5 & -8.8 & -8.1 \\
\hline
\end{tabular}

1 For a definition of coal-producing regions, see Appendix $C$.

Note: Excludes silt, culm, refuse bank, slurry dam, and dredge operations except for Pennsylvania anthracite.

Sources: Energy Information Administration, Form EIA-7A, "Coal Production Report"; State Mining Agency Coal Production Reports; and/or U.S. Department of Labor, Mine Safety and Health Administration, Form 7000-2, "Quarterly Mine Employment and Coal Production Report." 
Table 3. Coal Production and Number of Mines by State and Mine Type, 1996 (Thousand Short Tons)

\begin{tabular}{|c|c|c|c|c|c|c|}
\hline \multirow{2}{*}{$\begin{array}{l}\text { Coal-Producing } \\
\text { State and Region }\end{array}$} & \multicolumn{2}{|c|}{ Underground } & \multicolumn{2}{|c|}{ Surface } & \multicolumn{2}{|c|}{ Total } \\
\hline & Number of Mines & Production & Number of Mines & Production & Number of Mines & Production \\
\hline Alabama & 11 & 18,217 & 42 & 6,420 & 53 & 24,637 \\
\hline 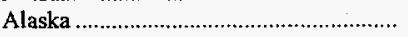 & - & - & 1 & 1,481 & 1 & 1,481 \\
\hline 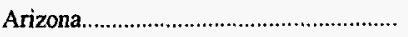 & - & - & 2 & 10,442 & 2 & 10,442 \\
\hline 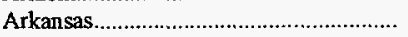 & - & - & 5 & 21 & 5 & 21 \\
\hline Colorado & 11 & 15,581 & 4 & 9,305 & 15 & 24,886 \\
\hline Illinois & 20 & 38,948 & 11 & 7,707 & 31 & 46,656 \\
\hline Indiana & 3 & 2,963 & 34 & 26,707 & 37 & 29,670 \\
\hline 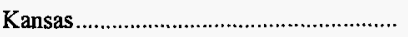 & - & - & 1 & 232 & 1 & 232 \\
\hline 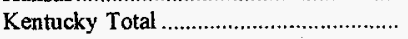 & 307 & 94,306 & 237 & 58,119 & 544 & 152,425 \\
\hline 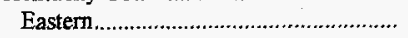 & 287 & 69,172 & 197 & 47,779 & 484 & 116,951 \\
\hline 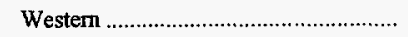 & 20 & 25,135 & 40 & 10,340 & 60 & 35,474 \\
\hline 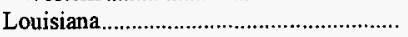 & - & - & 2 & 3,221 & 2 & 3,221 \\
\hline 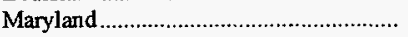 & 3 & 3,296 & 15 & 797 & 18 & 4,093 \\
\hline 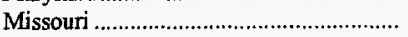 & - & - & 5 & 710 & 5 & 710 \\
\hline 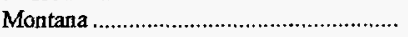 & 1 & 147 & 7 & 37,744 & 8 & 37,891 \\
\hline 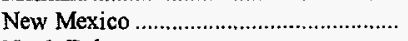 & - & - & 6 & 24,067 & 6 & 24,067 \\
\hline 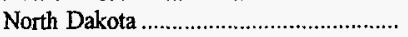 & - & - & 5 & 29,861 & 5 & 29,861 \\
\hline Ohio & 9 & 15,912 & 90 & 12,660 & 99 & 28,572 \\
\hline 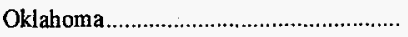 & 1 & 137 & 11 & 1,564 & 12 & 1,701 \\
\hline 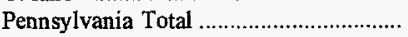 & 83 & 47,247 & 319 & 20,694 & 402 & 67,942 \\
\hline 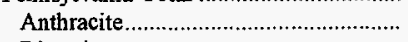 & 40 & 391 & 87 & 4,360 & 127 & 4,751 \\
\hline 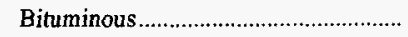 & 43 & 46,856 & 232 & 16,334 & 275 & 63,190 \\
\hline 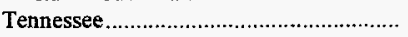 & 13 & 1,794 & 13 & 1,857 & 26 & 3,651 \\
\hline 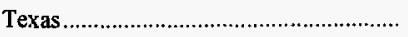 & - & - & 13 & 55,164 & 13 & 55,164 \\
\hline 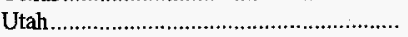 & 11 & 27,507 & - & - & 11 & 27,507 \\
\hline 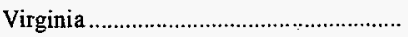 & 147 & 25,568 & 44 & 10,022 & 191 & 35,590 \\
\hline 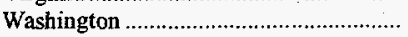 & - & - & 3 & 4,565 & 3 & 4,565 \\
\hline West Virginia Total ................................... & 264 & 115,585 & 122 & 54,848 & 386 & 170,433 \\
\hline 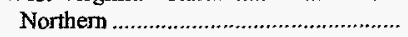 & 50 & 40,274 & 43 & 5,637 & 93 & 45,910 \\
\hline 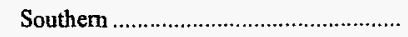 & 214 & 75,311 & 79 & 49,211 & 293 & 124,523 \\
\hline 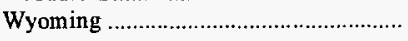 & 1 & 2,641 & 26 & 275,799 & 27 & 278,440 \\
\hline 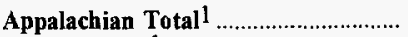 & 817 & 296,791 & 842 & 155,077 & 1,659 & 451,868 \\
\hline 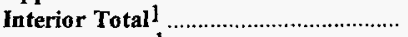 & 44 & 67,183 & 122 & 105,665 & 166 & 172,848 \\
\hline Western Total ${ }^{1}$ & 24 & 45,876 & 54 & 393,265 & $\mathbf{7 8}$ & 439,140 \\
\hline East of Miss. River & 860 & 363,837 & 927 & 199,831 & 1,787 & 563,668 \\
\hline 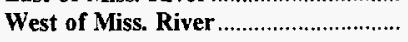 & 25 & 46,012 & 91 & 454,176 & 116 & 500,188 \\
\hline U.S. Total & 885 & 409,849 & 1,018 & 654,007 & 1,903 & $1,063,856$ \\
\hline
\end{tabular}

1 For a definition of coal-producing regions, see Appendix C.

Notes: Coal production excludes silt, culm, refuse bank, slurry dam, and dredge operations except for Pennsylvania anthracite. Totals may not equal sum of components due to independent rounding.

Sources: Energy Infornation Administration, Form EIA-7A, "Coal Production Report"; State Mining Agency Coal Production Reports; and/or U.S. Department of Labor, Mine Safety and Health Administration, Form 7000-2, "Quarterly Mine Employment and Coal Production Report." 
Table 4. Coal Production and Number of Mines by State, County, and Mine Type, 1996 (Thousand Short Tons)

\begin{tabular}{|c|c|c|c|c|c|c|}
\hline \multirow{2}{*}{$\begin{array}{l}\text { Coal-Producing } \\
\text { State and County }\end{array}$} & \multicolumn{2}{|c|}{ Underground } & \multicolumn{2}{|c|}{ Surface } & \multicolumn{2}{|c|}{ Total } \\
\hline & Number of Mines & Production & Number of Mines & Production & Number of Mines & Production \\
\hline 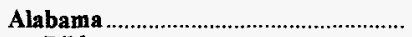 & 11 & 18,217 & 42 & 6,420 & 53 & 24,637 \\
\hline 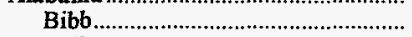 & - & - & 1 & 33 & 1 & 33 \\
\hline Cullman & - & - & 2 & 82 & 2 & 82 \\
\hline 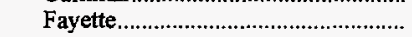 & 1 & 2,066 & - & - & l & 2,066 \\
\hline Jackson & - & - & 1 & 66 & 1 & 66 \\
\hline 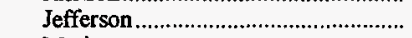 & 4 & 8,917 & 4 & 1,217 & 8 & 10,134 \\
\hline 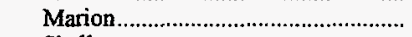 & - & - & 4 & 191 & 4 & 191 \\
\hline Shelby & 2 & 878 & - & - & 2 & 878 \\
\hline Tuscaloosa & 3 & 5,241 & 4 & 825 & 7 & 6,066 \\
\hline 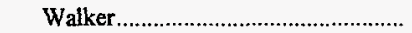 & 1 & 1,115 & 24 & 3,806 & 25 & 4,921 \\
\hline Winston & - & - & 2 & 201 & 2 & 201 \\
\hline 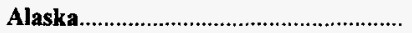 & - & - & 1 & 1,481 & 1 & 1,481 \\
\hline 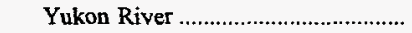 & - & - & 1 & 1,481 & 1 & 1,481 \\
\hline Arizona & - & - & 2 & 10,442 & 2 & 10,442 \\
\hline 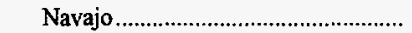 & - & - & 2 & 10,442 & 2 & 10,442 \\
\hline Arkansas $\ldots \ldots \ldots \ldots \ldots \ldots \ldots \ldots \ldots \ldots$ & - & - & 5 & 21 & 5 & 21 \\
\hline Johnson & - & - & 2 & 16 & 2 & 16 \\
\hline Scott & - & - & 1 & * & 1 & * \\
\hline Sebastian & - & - & 2 & 4 & 2 & 4 \\
\hline 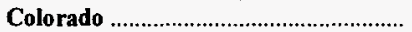 & 11 & 15,581 & 4 & 9,305 & $1 \overline{5}$ & 24,886 \\
\hline 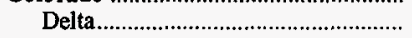 & 1 & 603 & - & - & 1 & 603 \\
\hline 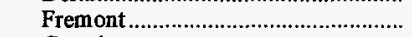 & 1 & 170 & - & - & 1 & 170 \\
\hline 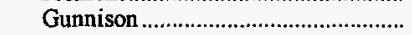 & 3 & 7,475 & - & - & 3 & 7,475 \\
\hline 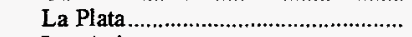 & 1 & 199 & - & - & 1 & 199 \\
\hline 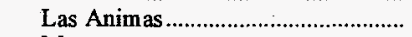 & 1 & 129 & - & - & 1 & 129 \\
\hline 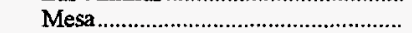 & 2 & 626 & - & - & 2 & 626 \\
\hline Moffat & - & - & 2 & 7,721 & $\overline{2}$ & 7,721 \\
\hline 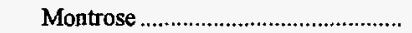 & - & - & 1 & 362 & 1 & 362 \\
\hline Rio Blanco & 1 & 543 & - & - & 1 & 543 \\
\hline 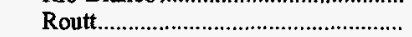 & 1 & 5,837 & 1 & 1,222 & 2 & 7,059 \\
\hline 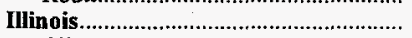 & 20 & 38,948 & 11 & 7,707 & 31 & 46,656 \\
\hline Clinton & 1 & 1,701 & - & - & 1 & 1,701 \\
\hline 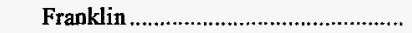 & 2 & 3,635 & - & - & 2 & 3,635 \\
\hline Fulton & - & - & 1 & 205 & 1 & 205 \\
\hline 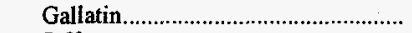 & 1 & 1,324 & - & - & 1 & 1,324 \\
\hline Jefferson & 2 & 4,299 & - & - & 2 & 4,299 \\
\hline 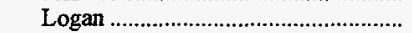 & $\overline{1}$ & 1,982 & - & - & $\overline{1}$ & 1,982 \\
\hline Macoupin & 3 & 5,454 & - & - & 3 & 5,454 \\
\hline 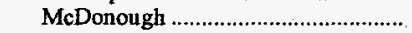 & - & - & 1 & 533 & 1 & 533 \\
\hline 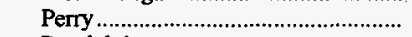 & 1 & 1,850 & 3 & 5,213 & 4 & 7,064 \\
\hline 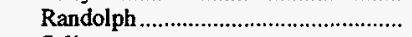 & 2 & 2,103 & - & - & 2 & 2,103 \\
\hline Saline & $\overline{3}$ & 8,660 & 1 & 673 & 4 & 9,333 \\
\hline Schuyler & - & - & 1 & 415 & 1 & 415 \\
\hline 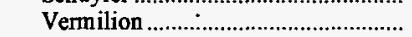 & 1 & 130 & - & - & 1 & 130 \\
\hline 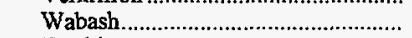 & 1 & 2,340 & - & - & 1 & 2,340 \\
\hline 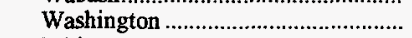 & 1 & 3,674 & - & - & 1 & 3,674 \\
\hline White & 1 & 1,796 & - & - & 1 & 1,796 \\
\hline William son & - & - & 4 & 668 & 4 & 668 \\
\hline Indiana & 3 & 2,963 & 34 & 26,707 & 37 & 29,670 \\
\hline Clay & - & - & 4 & 1,893 & 4 & 1,893 \\
\hline 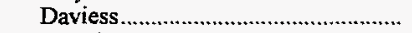 & - & - & 4 & 3,187 & 4 & 3,187 \\
\hline 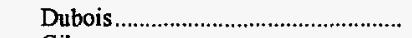 & - & - & I & 370 & 1 & 370 \\
\hline Gibson & 1 & 900 & 1 & 146 & 2 & 1,046 \\
\hline 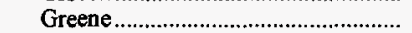 & - & - & 3 & 2,083 & 3 & 2,083 \\
\hline Knox & 2 & 2,063 & 3 & 1,662 & 5 & 3,725 \\
\hline Owen & - & - & 2 & 413 & 2 & 413 \\
\hline Pike & - & - & 6 & 4,359 & 6 & 4,359 \\
\hline Sullivan & - & - & 2 & 4,772 & 2 & 4,772 \\
\hline 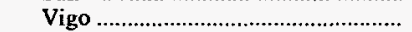 & - & - & 2 & 1,694 & 2 & 1,694 \\
\hline 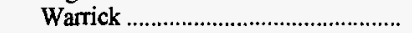 & - & - & 6 & 6,129 & 6 & 6,129 \\
\hline 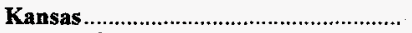 & - & - & 1 & 232 & 1 & 232 \\
\hline Crawford & - & - & 1 & 232 & 1 & 232 \\
\hline 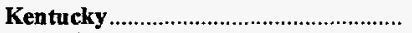 & 307 & 94,306 & 237 & 58,119 & 544 & 152,425 \\
\hline Bell & 13 & 2,654 & 12 & 2,391 & 25 & 5,044 \\
\hline 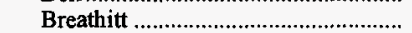 & - & - & 8 & 2,271 & 8 & 2,271 \\
\hline Butler & - & - & 2 & 244 & 2 & 244 \\
\hline Christian & - & - & 1 & 32 & 1 & 32 \\
\hline 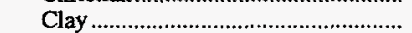 & - & - & 5 & 98 & 5 & 98 \\
\hline 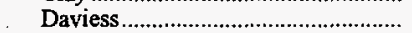 & - & - & 6 & 894 & 6 & 894 \\
\hline 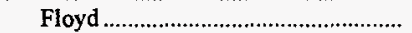 & 35 & 3,227 & 11 & 3,920 & 46 & 7,147 \\
\hline 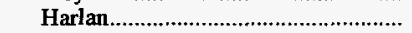 & 31 & 9,436 & 9 & 866 & 40 & 10,302 \\
\hline
\end{tabular}

See footnotes at end of table 
Table 4. Coal Production and Number of Mines by State, County, and Mine Type, 1996 (Continued) (Thousand Short Tons)

\begin{tabular}{|c|c|c|c|c|c|c|}
\hline \multirow{2}{*}{$\begin{array}{l}\text { Coal-Producing } \\
\text { State and County }\end{array}$} & \multicolumn{2}{|c|}{ Underground } & \multicolumn{2}{|c|}{ Surface } & \multicolumn{2}{|c|}{ Total } \\
\hline & Number of Mines & Production & Number of Mines & Production & Number of Mines & Production \\
\hline \multicolumn{7}{|l|}{ Kentucky (Continued) } \\
\hline Henderson & 1 & 631 & 2 & 1,453 & 3 & 2,084 \\
\hline 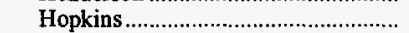 & 6 & 4,271 & $1 \overline{2}$ & 4,384 & 18 & 8,655 \\
\hline 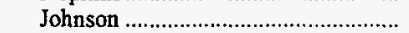 & 4 & 1,363 & 7 & 448 & 11 & 1,812 \\
\hline 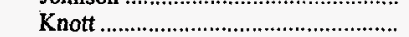 & 29 & 4,801 & 17 & 7,407 & 46 & 12,208 \\
\hline Knox & 11 & 417 & 4 & 50 & 15 & 467 \\
\hline 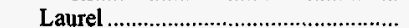 & - & - & 1 & 52 & 1 & 52 \\
\hline Lawrence. & - & - & 3 & 69 & 3 & 69 \\
\hline 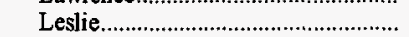 & 10 & 8,017 & 8 & 1,701 & 18 & 9,718 \\
\hline Letcher & 17 & 5,381 & 22 & 2,665 & 39 & 8,046 \\
\hline Magoffin & 1 & 34 & 7 & 944 & 8 & 978 \\
\hline Martin & 14 & 6,332 & 9 & 5,453 & 23 & 11,785 \\
\hline McLean & 1 & 80 & 2 & 114 & 3 & 194 \\
\hline Morgan & - & - & 3 & 5 & 3 & 5 \\
\hline Muhlenberg & 3 & 3,032 & 4 & 739 & 7 & 3,771 \\
\hline Ohio & 1 & 20 & 10 & 2,122 & 11 & 2,142 \\
\hline Owsley & - & - & 4 & 171 & 4 & 171 \\
\hline 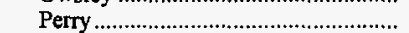 & 13 & 3,991 & 14 & 6,377 & 27 & 10,368 \\
\hline 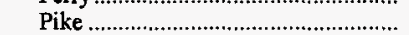 & 100 & 23,041 & 45 & 12,557 & 145 & 35,598 \\
\hline 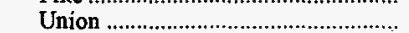 & 3 & 7,361 & - & - & 3 & 7,361 \\
\hline 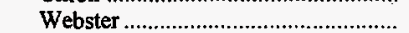 & 5 & 9,740 & 1 & 357 & 6 & 10,097 \\
\hline Whitley & 9 & 478 & 8 & 333 & 17 & 811 \\
\hline 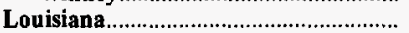 & - & - & 2 & 3,221 & 2 & 3,221 \\
\hline De Soto & _- & - & 1 & 2,434 & 1 & 2,434 \\
\hline Red River............................................ & - & - & 1 & 787 & 1 & 787 \\
\hline Maryland & 3 & 3,296 & 15 & 797 & 18 & 4,093 \\
\hline Allegany & - & - & 10 & 597 & 10 & 597 \\
\hline Garrett & 3 & 3,296 & 5 & 200 & 8 & 3,496 \\
\hline 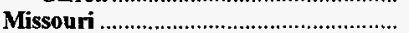 & - & - & 5 & 710 & 5 & 710 \\
\hline 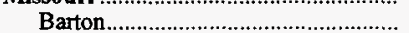 & - & - & 1 & 88 & 1 & 88 \\
\hline 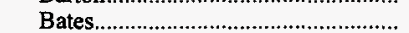 & - & - & 2 & 487 & 2 & 487 \\
\hline 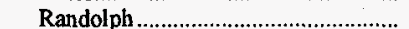 & - & - & 1 & 25 & 1 & 25 \\
\hline Vernon & - & - & 1 & 110 & i & 110 \\
\hline Montana & 1 & 147 & 7 & $\mathbf{3 7 , 7 4 4}$ & 8 & 37,891 \\
\hline Big Horn & - & - & 4 & 24,686 & 4 & 24,686 \\
\hline 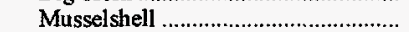 & 1 & 147 & - & - & 1 & 147 \\
\hline Richland & - & - & 1 & 256 & i & 256 \\
\hline Rosebud & - & - & 2 & 12,802 & 2 & 12,802 \\
\hline New Mexico & - & - & 6 & 24,067 & 6 & 24,067 \\
\hline Colfax & - & - & 1 & 1,259 & 1 & 1,259 \\
\hline McKinley & - & - & 2 & 9,527 & 2 & 9,527 \\
\hline San Juan & - & - & 3 & 13,281 & 3 & 13,281 \\
\hline North Dakota & - & - & $\mathbf{5}$ & 29,861 & 5 & 29,861 \\
\hline McLean & - & - & 1 & 7,024 & 1 & 7,024 \\
\hline Mercer & - & - & 2 & 16,908 & 2 & 16,908 \\
\hline Oliver & - & - & 2 & 5,929 & 2 & 5,929 \\
\hline Ohio & 9 & 15,912 & 90 & 12,660 & 99 & 28,572 \\
\hline Belmont & 1 & 4,741 & 9 & 1,175 & 10 & 5,916 \\
\hline Carroll & - & - & 6 & 329 & 6 & 329 \\
\hline 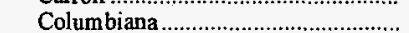 & 2 & 299 & 9 & 271 & 11 & 569 \\
\hline Coshocton & - & - & 5 & 757 & 5 & 757 \\
\hline 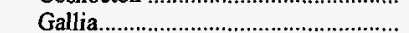 & - & - & 1 & 268 & 1 & 268 \\
\hline Guernsey & - & - & 6 & 302 & 6 & 302 \\
\hline 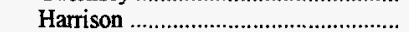 & 1 & 1,117 & 8 & 295 & 9 & 1,411 \\
\hline 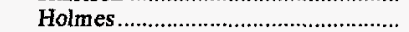 & - & - & 2 & 175 & 2 & 175 \\
\hline Jackson & - & - & 3 & 1,042 & 3 & 1,042 \\
\hline Jefferson & 2 & 422 & 9 & 412 & 11 & 833 \\
\hline Mahoning & - & - & 1 & 4 & 1 & 4 \\
\hline Meigs & 2 & 5,951 & - & - & 2 & 5,951 \\
\hline Monroe & 1 & 3,383 & - & - & 1 & 3,383 \\
\hline Morgan & - & - & 1 & 1,543 & 1 & 1,543 \\
\hline 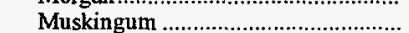 & - & - & 3 & 176 & 3 & 176 \\
\hline 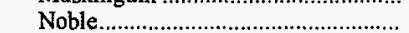 & - & - & 2 & 857 & 2 & 857 \\
\hline 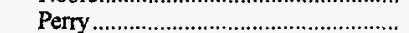 & - & - & 5 & 1,024 & 5 & 1,024 \\
\hline Stark & - & - & 7 & 606 & 7 & 606 \\
\hline 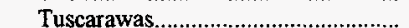 & - & - & 9 & 1,404 & 9 & 1,404 \\
\hline Vinton & - & - & 4 & 2,020 & 4 & 2,020 \\
\hline Oklahoma & 1 & 137 & 11 & 1,564 & 12 & 1,701 \\
\hline 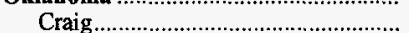 & - & - & 1 & 130 & 1 & 130 \\
\hline Latimer & - & - & 1 & 193 & 1 & 193 \\
\hline Le Flore & 1 & 137 & 6 & 1,047 & 7 & 1,184 \\
\hline
\end{tabular}

See footnotes at end of table 
Table 4. Coal Production and Number of Mines by State, County, and Mine Type, 1996 (Continued) (Thousand Short Tons)

\begin{tabular}{|c|c|c|c|c|c|c|}
\hline \multirow{2}{*}{$\begin{array}{l}\text { Coal-Producing } \\
\text { State and County }\end{array}$} & \multicolumn{2}{|c|}{ Underground } & \multicolumn{2}{|c|}{ Surface } & \multicolumn{2}{|c|}{ Total } \\
\hline & Number of Mines & Production & Number of Mines & Production & Number of Mines & Production \\
\hline \multicolumn{7}{|l|}{ Oklahoma (Continued) } \\
\hline 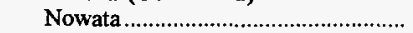 & - & - & 2 & 187 & 2 & 187 \\
\hline 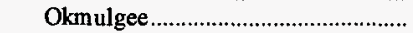 & - & - & $\bar{l}$ & 6 & 1 & 6 \\
\hline 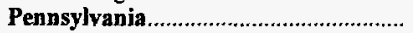 & 83 & 47,247 & 319 & 20,694 & 402 & 67,942 \\
\hline 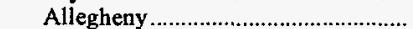 & - & - & 2 & 14 & 2 & 14 \\
\hline 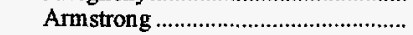 & 9 & 3,974 & 23 & 1,371 & 32 & 5,346 \\
\hline 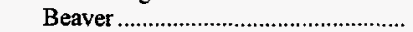 & - & - & 4 & 239 & 4 & 239 \\
\hline Blair & - & - & 1 & 59 & i & 59 \\
\hline Butler & - & - & 6 & 56 & 6 & 56 \\
\hline Cambria & 2 & 60 & 11 & 1,255 & 13 & 1,315 \\
\hline 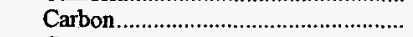 & - & - & 1 & 318 & 1 & 318 \\
\hline Centre & - & - & 3 & 328 & 3 & 328 \\
\hline 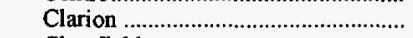 & - & - & 6 & 543 & 6 & 543 \\
\hline 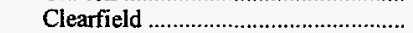 & 2 & 154 & 48 & 4,273 & 50 & 4,428 \\
\hline Columbia & 1 & 141 & 5 & 270 & 6 & 411 \\
\hline 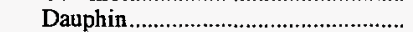 & 1 & 2 & 1 & 8 & 2 & 11 \\
\hline Elk & - & - & 6 & 460 & 6 & 460 \\
\hline 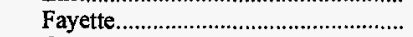 & - & - & 20 & 428 & 20 & 428 \\
\hline Greene & 9 & 29,746 & 2 & 48 & 11 & 29,794 \\
\hline Indiana & 8 & 4,190 & 19 & 952 & 27 & 5,143 \\
\hline 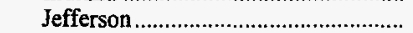 & 3 & 553 & 22 & 870 & 25 & 1,423 \\
\hline 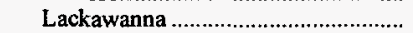 & - & - & 5 & 243 & 5 & 243 \\
\hline 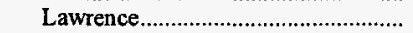 & - & - & 2 & 139 & 2 & 139 \\
\hline Luzerne & - & - & 17 & 1,030 & 17 & 1,030 \\
\hline 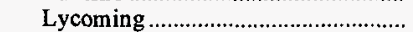 & - & - & 1 & 333 & 1 & 333 \\
\hline 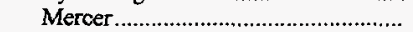 & - & - & 2 & 5 & 2 & 5 \\
\hline 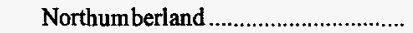 & 9 & 35 & 8 & 246 & 17 & 281 \\
\hline 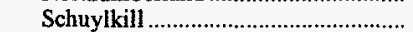 & 29 & 213 & 48 & 2,206 & 77 & 2,419 \\
\hline Snyder & - & - & 1 & 1 & 1 & 1 \\
\hline 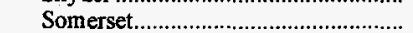 & 7 & 1,848 & 31 & 3,533 & 38 & 5,381 \\
\hline Sullivan & - & - & 1 & 37 & 1 & 37 \\
\hline 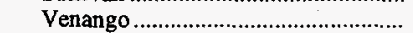 & - & - & 1 & 31 & $i$ & 31 \\
\hline 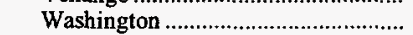 & 3 & 6,330 & 8 & 705 & 11 & 7,035 \\
\hline Westmoreland & - & - & 14 & 690 & 14 & 690 \\
\hline 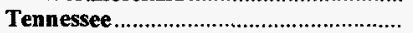 & 13 & 1,794 & 13 & 1,857 & 26 & 3,651 \\
\hline Anderson & 1 & 207 & 1 & 273 & 2 & 479 \\
\hline Bledsoe & - & - & 1 & 3 & 1 & 3 \\
\hline Campbell & 7 & 723 & 3 & 344 & 10 & 1,067 \\
\hline 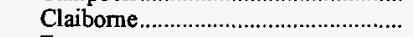 & 1 & 240 & 4 & 386 & 5 & 625 \\
\hline Fentress & - & - & 1 & 256 & 1 & 256 \\
\hline 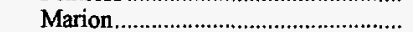 & - & - & 2 & 46 & 2 & 46 \\
\hline Morgan & 1 & 42 & - & - & 1 & 42 \\
\hline Scott & 1 & 155 & - & - & 1 & 155 \\
\hline Sequatchie & 2 & 428 & 1 & 550 & 3 & 978 \\
\hline Texas & - & - & 13 & 55,164 & 13 & 55,164 \\
\hline Atascosa & - & - & 1 & 3,324 & 1 & 3,324 \\
\hline Freestone & - & - & 1 & 5,196 & 1 & 5,196 \\
\hline 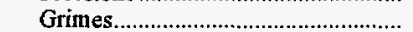 & - & - & 1 & 567 & 1 & 567 \\
\hline 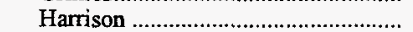 & - & - & 2 & 4,173 & 2 & 4,173 \\
\hline 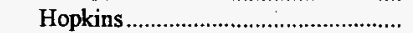 & - & - & 1 & 2,152 & 1 & 2,152 \\
\hline Leon & - & - & 1 & 8,865 & 1 & 8,865 \\
\hline Milam & - & - & 1 & 6,615 & 1 & 6,615 \\
\hline 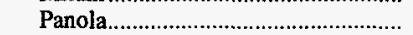 & - & - & 1 & 6,814 & 1 & 6,814 \\
\hline 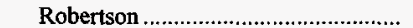 & - & - & 1 & 1,933 & 1 & 1,933 \\
\hline 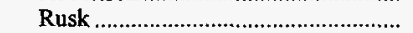 & - & - & i & 6,509 & 1 & 6,509 \\
\hline Titus & - & - & i & 8,569 & $i$ & 8,569 \\
\hline Webb & - & - & 1 & 446 & 1 & 446 \\
\hline Utah & 11 & $\mathbf{2 7 , 5 0 7}$ & - & - & 11 & 27,507 \\
\hline Carbon & 6 & 12,066 & - & - & 6 & 12,066 \\
\hline 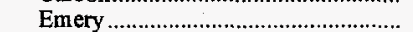 & 4 & 11,238 & - & - & 4 & 11,238 \\
\hline Sevier & 1 & 4,202 & - & - & i & 4,202 \\
\hline Virginia & 147 & 25,568 & 44 & 10,022 & 191 & $\mathbf{3 5 , 5 9 0}$ \\
\hline Buchanan & 65 & 13,364 & 9 & 1,419 & 74 & 14,784 \\
\hline 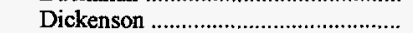 & 13 & 1,693 & 8 & 1,296 & 21 & 2,988 \\
\hline 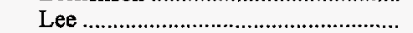 & 7 & 1,492 & 3 & 544 & 10 & 2,036 \\
\hline Russell & 6 & 915 & 1 & 430 & 7 & 1,346 \\
\hline Scott & 1 & 5 & - & - & 1 & 5 \\
\hline Tazewell & 16 & 1,717 & 1 & 122 & 17 & 1,840 \\
\hline Wise & 39 & 6,380 & 22 & 6,211 & 61 & 12,591 \\
\hline Washington & - & - & 3 & 4,565 & 3 & 4,565 \\
\hline King & - & - & 1 & 173 & 1 & 173 \\
\hline
\end{tabular}

See footnotes at end of table. 
Table 4. Coal Production and Number of Mines by State, County, and Mine Type, 1996 (Continued) (Thousand Short Tons)

\begin{tabular}{|c|c|c|c|c|c|c|}
\hline \multirow{2}{*}{$\begin{array}{l}\text { Coal-Producing } \\
\text { State and County }\end{array}$} & \multicolumn{2}{|c|}{ Underground } & \multicolumn{2}{|c|}{ Surface } & \multicolumn{2}{|c|}{ Total } \\
\hline & Number of Mines & Production & Number of Mines & Production & Number of Mines & Production \\
\hline \multicolumn{7}{|l|}{ Washington (Continued) } \\
\hline 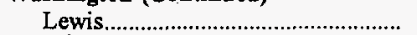 & - & - & 1 & 2,423 & 1 & 2,423 \\
\hline Thurston & - & - & 1 & 1,969 & 1 & 1,969 \\
\hline West Virginia & 264 & 115,585 & 122 & 54,848 & 386 & 170,433 \\
\hline 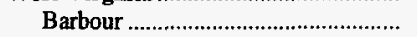 & 6 & 1,498 & 4 & 49 & 10 & 1,547 \\
\hline 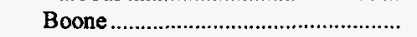 & 32 & 18,841 & 15 & 12,183 & 47 & 31,024 \\
\hline 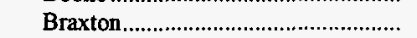 & 1 & 42 & - & - & 1 & 42 \\
\hline Brooke & 1 & 1,438 & 1 & 10 & 2 & 1,448 \\
\hline 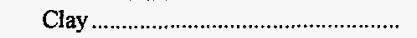 & - & - & 5 & 5,507 & 5 & 5,507 \\
\hline 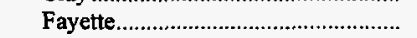 & 5 & 1,193 & 6 & 2,772 & 11 & 3,964 \\
\hline Gilmer & 1 & 30 & - & - & 1 & 30 \\
\hline Grant & 2 & 2,810 & 2 & 228 & 4 & 3,039 \\
\hline 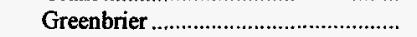 & 3 & 766 & 2 & 38 & 5 & 804 \\
\hline Harrison & 5 & 4,600 & 6 & 82 & 11 & 4,682 \\
\hline 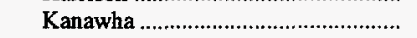 & 6 & 4,689 & 5 & 4,274 & 11 & 8,964 \\
\hline 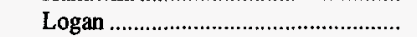 & 17 & 3,769 & 12 & 15,020 & 29 & 18,789 \\
\hline 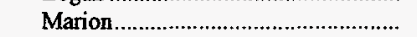 & 2 & 3,118 & 2 & 18 & 4 & 3,135 \\
\hline Marshall & 2 & 8,721 & - & - & 2 & 8,721 \\
\hline McDowell & 66 & 4,928 & 11 & 1,207 & 77 & 6,135 \\
\hline 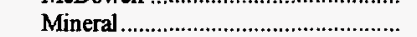 & - & - & 2 & 134 & 2 & 134 \\
\hline 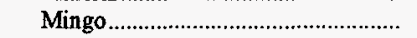 & 34 & 17,612 & 15 & 5,351 & 49 & 22,963 \\
\hline 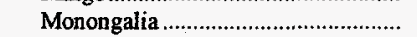 & 9 & 11,816 & 4 & 1,135 & 13 & 12,951 \\
\hline 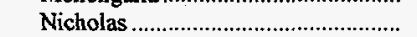 & 13 & 2,085 & 3 & 437 & 16 & 2,522 \\
\hline Preston & 7 & 1,771 & 8 & 158 & 15 & 1,929 \\
\hline Raleigh & 17 & 10,413 & 2 & 797 & 19 & 11,209 \\
\hline 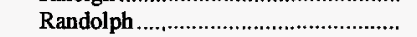 & 4 & 540 & 1 & 30 & 5 & 570 \\
\hline Tucker & - & - & 2 & 602 & 2 & 602 \\
\hline 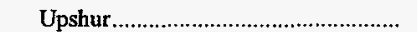 & 4 & 1,323 & 8 & 411 & 12 & 1,734 \\
\hline 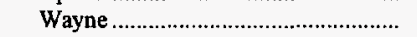 & 4 & 2,934 & 2 & 1,197 & 6 & 4,131 \\
\hline 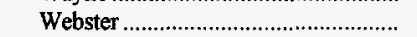 & 6 & 2,567 & 3 & 2,778 & 9 & 5,345 \\
\hline Wyoming & 17 & 8,082 & 1 & 428 & 18 & 8,510 \\
\hline Wyoming & 1 & 2,641 & 26 & 275,799 & 27 & 278,440 \\
\hline 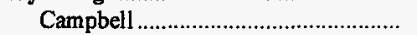 & - & - & 17 & 245,534 & 17 & 245,534 \\
\hline Carbon & 1 & 2,641 & 2 & 1,832 & 3 & 4,473 \\
\hline 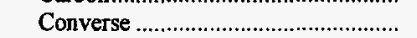 & - & - & 2 & 15,839 & 2 & 15,839 \\
\hline Lincoln & - & - & 2 & 4,419 & 2 & 4,419 \\
\hline 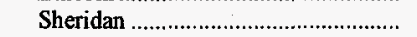 & - & - & 1 & 16 & 1 & 16 \\
\hline 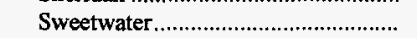 & - & - & 2 & 8,159 & 2 & 8,159 \\
\hline U.S. Total & 885 & 409,849 & 1,018 & 654,007 & 1,903 & $1,063,856$ \\
\hline
\end{tabular}

* Data round to zero.

Notes: Coal production excludes silt, culm, refuse bank, shurry dam, and dredge operations except for Pennsylvania anthracite. Totals may not equal sum of components due to independent rounding.

Sources: Energy Information Administration, Form EIA-7A, "Coal Production Report"; State Mining Agency Coal Production Reports; and/or U.S. Department of Labor, Mine Safety and Health Administration, Form 7000-2, "Quarterly Mine Employment and Coal Production Report." 
Table 5. Underground Coal Production by State and Mining Method, 1996 (Thousand Short Tons)

\begin{tabular}{|c|c|c|c|c|c|}
\hline $\begin{array}{l}\text { Coal-Producing } \\
\text { State and Region }\end{array}$ & Continuous 1 & Conventional ${ }^{2}$ & Longwall $^{3}$ & Other ${ }^{4}$ & Total \\
\hline Alabama & 1,410 & 73 & 16,734 & - & 18,217 \\
\hline 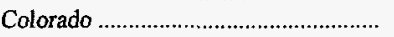 & 3,087 & 129 & 12,365 & - & 15,581 \\
\hline 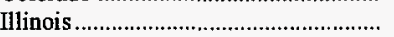 & 21,823 & 130 & 16,995 & - & 38,948 \\
\hline Indiana & 2,963 & - & - & - & 2,963 \\
\hline 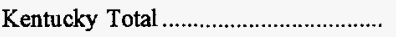 & 69,153 & 10,247 & 14,582 & 324 & 94,306 \\
\hline Eastern & 51,596 & 9,779 & 7,473 & 324 & 69,172 \\
\hline 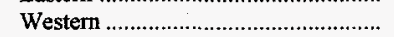 & 17,557 & 468 & 7,109 & - & 25,135 \\
\hline Maryland & 346 & - & 2,950 & - & 3,296 \\
\hline 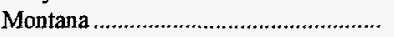 & 147 & - & - & - & 147 \\
\hline Ohio & 1,837 & - & 14,075 & - & 15,912 \\
\hline 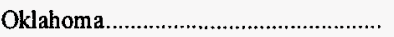 & 137 & - & - & - & 137 \\
\hline 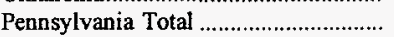 & 11,924 & 1,645 & 33,635 & 43 & 47,247 \\
\hline 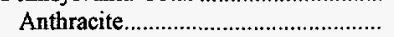 & 141 & 207 & - & 43 & 391 \\
\hline 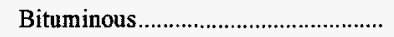 & 11,783 & 1,438 & 33,635 & - & 46,856 \\
\hline 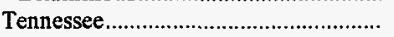 & 1,775 & 19 & - & - & 1,794 \\
\hline Utah & 2,069 & 588 & 24,849 & - & 27,507 \\
\hline 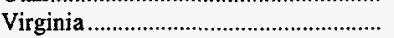 & 11,888 & 5,755 & 7,925 & - & 25,568 \\
\hline West Virginia Total & 54,974 & 12,507 & 47,592 & 512 & 115,585 \\
\hline Northern & 6,795 & 1,430 & 31,537 & 512 & 40,274 \\
\hline Southern & 48,179 & 11,078 & 16,055 & - & 75,311 \\
\hline 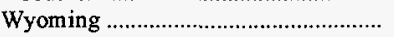 & - & - & 2,641 & - & 2,641 \\
\hline Appalachian Total ${ }^{5}$ & 135,750 & 29,778 & 130,384 & 878 & 296,791 \\
\hline Interior Total 5 & 42,480 & 598 & 24,104 & - & 67,183 \\
\hline 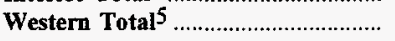 & 5,303 & 717 & 39,856 & - & 45,876 \\
\hline 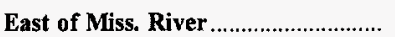 & 178,093 & $\mathbf{3 0 , 3 7 7}$ & 154,488 & 878 & 363,837 \\
\hline 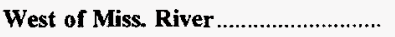 & 5,440 & 717 & 39,856 & - & 46,012 \\
\hline 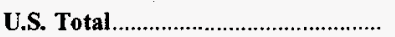 & 183,533 & 31,093 & 194,344 & 878 & 409,849 \\
\hline
\end{tabular}

1 Mines that produce greater than 50 percent of coal by continuous mining method.

2 Mines that produce greater than 50 percent of coal by conventional mining method.

3 Mines that have any production from longwall mining method. A typical longwall mining operation uses 80 percent longwall mining and 20 percent continuous mining.

4 Mines that produce coal using shortwall, scoop loading, hand loading, or other mining methods or a $50 / 50$ percent continuous/conventional split in mining method.

5 For a definition of coal-producing regions, see Appendix C.

Note: Totals may not equal sum of components due to independent rounding.

Source: Energy Information Administration, Form EIA-7A, "Coal Production Report." 
Table 6. Coal Production and Number of Mines by State, Mine Type, and Mine Production Range, 1996

(Thousand Short Tons)

\begin{tabular}{|c|c|c|c|c|c|c|c|c|c|c|c|c|}
\hline \multirow{3}{*}{$\begin{array}{l}\text { Coal-Producing State, Region } \\
\text { and Type of Mining }\end{array}$} & \multicolumn{6}{|c|}{ Number of Mines } & \multicolumn{6}{|c|}{ Production } \\
\hline & \multicolumn{12}{|c|}{$\begin{array}{l}\text { Mine Production Range } \\
\text { (thousand short tons) }\end{array}$} \\
\hline & $\begin{array}{c}1,000 \\
\text { and } \\
\text { over }\end{array}$ & $\begin{array}{c}500 \\
\text { to } \\
1,000\end{array}$ & $\begin{array}{c}200 \\
\text { to } \\
500\end{array}$ & $\begin{array}{c}100 \\
\text { to } \\
200\end{array}$ & $\begin{array}{c}10 \\
\text { to } \\
100\end{array}$ & $\begin{array}{c}\text { Less } \\
\text { than } \\
10\end{array}$ & $\begin{array}{r}1,000 \\
\text { and } \\
\text { over }\end{array}$ & $\begin{array}{c}500 \\
\text { to } \\
1,000\end{array}$ & $\begin{array}{c}200 \\
\text { to } \\
500\end{array}$ & $\begin{array}{c}100 \\
\text { to } \\
200\end{array}$ & $\begin{array}{r}10 \\
\text { to } \\
100\end{array}$ & $\begin{array}{c}\text { Less } \\
\text { than } \\
10\end{array}$ \\
\hline Alabama ................... & 8 & 6 & 4 & 6 & 25 & 4 & 17,488 & 3,866 & 1,036 & 977 & 1,256 & 14 \\
\hline Underground & 7 & 3 & - & - & 1 & - & 16,209 & 1,935 & - & - & 73 & - \\
\hline Surface & 1 & 3 & 4 & 6 & 24 & 4 & 1,279 & 1,931 & 1,036 & 977 & 1,182 & 14 \\
\hline 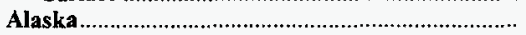 & 1 & - & - & - & - & - & 1,481 & - & - & - & - & - \\
\hline Surface & 1 & - & - & - & - & - & 1,481 & - & - & - & - & - \\
\hline Arizona & 2 & - & - & - & - & - & 10,442 & - & - & - & - & - \\
\hline Surface & 2 & - & - & - & - & - & 10,442 & - & - & - & - & - \\
\hline 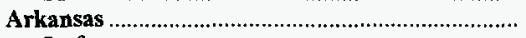 & - & - & - & - & - & 5 & - & - & - & - & - & 21 \\
\hline Surface & - & - & - & - & - & 5 & - & - & - & - & - & 21 \\
\hline 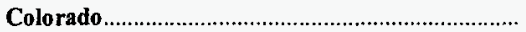 & 6 & 2 & 4 & 3 & - & - & 21,920 & 1,145 & 1,323 & 498 & - & - \\
\hline Underground & 3 & 2 & 3 & 3 & - & - & 12,976 & 1,145 & 962 & 498 & _ & - \\
\hline Surface & 3 & - & 1 & - & - & - & 8,944 & - & 362 & - & - & - \\
\hline 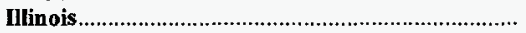 & 18 & 4 & 3 & 1 & 4 & 1 & 42,938 & 2,306 & 1,119 & 130 & 160 & 3 \\
\hline 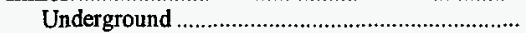 & 16 & 1 & 1 & 1 & 1 & - & 37,728 & 578 & 499 & 130 & 13 & - \\
\hline Surface & 2 & 3 & 2 & - & 3 & 1 & 5,210 & 1,727 & 620 & - & 147 & 3 \\
\hline Indiana & 10 & 11 & 8 & $\mathbf{3}$ & 4 & 1 & 18,426 & 7,335 & 3,326 & 381 & 199 & 3 \\
\hline Underground & 1 & 2 & - & - & - & - & 1,466 & 1,497 & - & - & - & - \\
\hline Surface & 9 & 9 & 8 & 3 & 4 & 1 & 16,960 & 5,839 & 3,326 & 381 & 199 & 3 \\
\hline 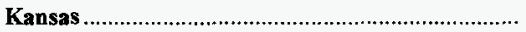 & - & - & 1 & - & - & - & - & - & 232 & - & - & - \\
\hline Surface & - & - & 1 & - & - & - & - & - & 232 & - & - & _ \\
\hline Kentucky Total & 31 & 62 & 102 & 83 & 195 & 71 & 54,592 & 43,633 & 33,111 & 12,013 & 8,742 & 333 \\
\hline Underground & 21 & 31 & 60 & 56 & 111 & 28 & 39,280 & 21,900 & 19,372 & 8,254 & 5,347 & 153 \\
\hline 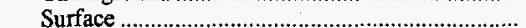 & 10 & 31 & 42 & 27 & 84 & 43 & 15,312 & 21,734 & 13,740 & 3,759 & 3,395 & 180 \\
\hline Eastern & 21 & 48 & 93 & 77 & 180 & 65 & 34,071 & 33,349 & 29,916 & 11,182 & 8,136 & 297 \\
\hline 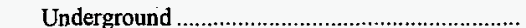 & 12 & 25 & 57 & 56 & 109 & 28 & 20,103 & 17,155 & 18,259 & 8,254 & 5,247 & 153 \\
\hline Surface & 9 & 23 & 36 & 21 & 71 & 37 & 13,968 & 16,194 & 11,657 & 2,928 & 2,889 & 144 \\
\hline Western & 10 & 14 & 9 & 6 & 15 & 6 & $\mathbf{2 0 , 5 2 1}$ & 10,285 & 3,196 & 831 & 606 & 36 \\
\hline Underground & 9 & 6 & 3 & - & 2 & - & 19,177 & 4,745 & 1,113 & - & 100 & - \\
\hline Surface & 1 & 8 & 6 & 6 & 13 & 6 & 1,343 & 5,540 & 2,083 & 831 & 506 & 36 \\
\hline 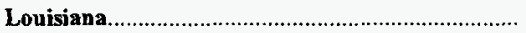 & 1 & $\mathbf{1}$ & - & - & - & - & 2,434 & 787 & - & - & - & 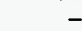 \\
\hline Surface & 1 & 1 & - & - & - & - & 2,434 & 787 & - & - & - & - \\
\hline Maryland & 1 & - & 2 & 1 & 10 & 4 & 2,950 & - & 520 & 160 & 437 & 25 \\
\hline Underground & 1 & - & 1 & - & 1 & - & 2,950 & - & 300 & - & 46 & - \\
\hline Surface & - & - & 1 & 1 & 9 & 4 & - & - & 221 & 160 & 391 & 25 \\
\hline 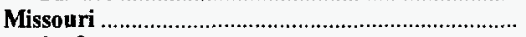 & - & - & 1 & 2 & 2 & - & - & - & 290 & 307 & 112 & - \\
\hline 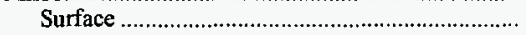 & - & - & 1 & 2 & 2 & - & - & - & 290 & 307 & 112 & - \\
\hline Montana & $\mathbf{5}$ & 1 & 1 & $\mathbf{1}$ & - & - & 36,886 & 602 & 256 & 147 & - & - \\
\hline 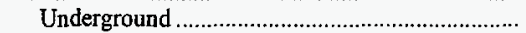 & - & - & - & $\bar{l}$ & - & - & - & - & - & 147 & $\rightarrow$ & - \\
\hline 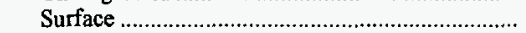 & 5 & 1 & 1 & - & - & - & 36,886 & 602 & 256 & - & - & - \\
\hline 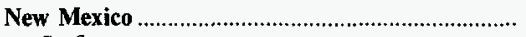 & 6 & - & - & - & - & - & 24,067 & - & - & - & - & - \\
\hline Surface & 6 & - & - & - & - & - & 24,067 & _ & - & _ & _ & _ \\
\hline 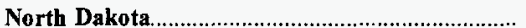 & 5 & - & - & _- & - & _ & 29,861 & _ & - & _ & - & - \\
\hline Surface & 5 & - & - & - & - & - & 29,861 & - & - & - & - & - \\
\hline Ohio & 7 & 5 & 11 & 16 & 47 & 13 & 17,826 & 3,170 & 3,248 & 2,483 & 1,792 & 53 \\
\hline 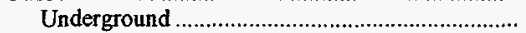 & 5 & - & 1 & 3 & - & - & 15,191 & - & 271 & 449 & - & - \\
\hline Surface & 2 & 5 & 10 & 13 & 47 & 13 & 2,635 & 3,170 & 2,977 & 2,034 & 1,792 & 53 \\
\hline Oklahoma & - & - & 3 & 5 & $\mathbf{2}$ & 2 & - & - & 909 & 738 & 40 & 14 \\
\hline 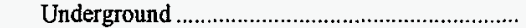 & - & - & - & 1 & - & - & - & - & - & 137 & - & - \\
\hline Surface & - & - & 3 & 4 & 2 & 2 & _ & - & 909 & 601 & 40 & 14 \\
\hline Pennsylvania Total & 12 & 8 & 33 & 39 & 172 & 138 & 39,573 & 5,698 & 10,399 & 5,597 & 6,143 & 530 \\
\hline Underground & 11 & 4 & 13 & 4 & 19 & 32 & 38,198 & 3,245 & 4,340 & 673 & 683 & 108 \\
\hline Surface & 1 & 4 & 20 & 35 & 153 & 106 & 1,375 & 2,453 & 6,059 & 4,924 & 5,460 & 423 \\
\hline Anthracite & - & 1 & 6 & 4 & 49 & 67 & - & $\mathbf{5 3 7}$ & 1,866 & 583 & 1,558 & 207 \\
\hline 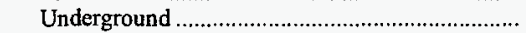 & - & - & - & 1 & 8 & 31 & - & - & - & 141 & 143 & 107 \\
\hline 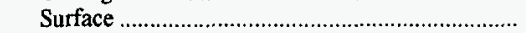 & - & 1 & 6 & 3 & 41 & 36 & - & 537 & 1,866 & 442 & 1,415 & 101 \\
\hline 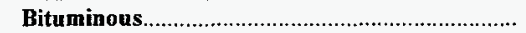 & 12 & 7 & 27 & 35 & 123 & 71 & 39,573 & 5,162 & $\mathbf{8 , 5 3 4}$ & 5,014 & 4,585 & 323 \\
\hline 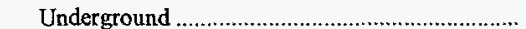 & 11 & 4 & 13 & 3 & 11 & 1 & 38,198 & 3,245 & 4,340 & 532 & 539 & 1 \\
\hline Surface & 1 & 3 & 14 & 32 & 112 & 70 & 1,375 & 1,917 & 4,193 & 4,482 & 4,045 & 322 \\
\hline 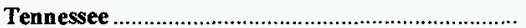 & - & 1 & 9 & 4 & 7 & 5 & - & $\mathbf{5 5 0}$ & 2,257 & 572 & 241 & 30 \\
\hline Underground & - & - & 5 & 3 & 4 & 1 & - & - & 1,224 & 415 & 152 & 4 \\
\hline Surface & - & 1 & 4 & 1 & 3 & 4 & - & 550 & 1,034 & 157 & 89 & 26 \\
\hline Texas & 10 & 1 & 2 & - & - & - & 53,869 & 567 & 727 & - & - & - \\
\hline 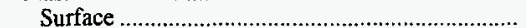 & 10 & 1 & 2 & - & - & - & 53,869 & 567 & 727 & - & - & - \\
\hline
\end{tabular}

See footnotes at end of table. 
Table 6. Coal Production and Number of Mines by State, Mine Type, and Mine Production Range, 1996 (Continued)

(Thousand Short Tons)

\begin{tabular}{|c|c|c|c|c|c|c|c|c|c|c|c|c|}
\hline \multirow{3}{*}{$\begin{array}{l}\text { Coal-Producing State, Region } \\
\text { and Type of Mining }\end{array}$} & \multicolumn{6}{|c|}{ Number of Mines } & \multicolumn{6}{|c|}{ Production } \\
\hline & \multicolumn{12}{|c|}{$\begin{array}{l}\text { Mine Production Range } \\
\text { (thousand short tons) }\end{array}$} \\
\hline & $\begin{array}{c}1,000 \\
\text { and } \\
\text { over }\end{array}$ & $\begin{array}{c}500 \\
\text { to } \\
1,000\end{array}$ & $\begin{array}{c}200 \\
\text { to } \\
500\end{array}$ & $\begin{array}{c}100 \\
\text { to } \\
200\end{array}$ & $\begin{array}{c}10 \\
\text { to } \\
100\end{array}$ & $\begin{array}{c}\text { Less } \\
\text { than } \\
10\end{array}$ & $\begin{array}{c}1,000 \\
\text { and } \\
\text { over }\end{array}$ & $\begin{array}{c}500 \\
\text { to } \\
1,000\end{array}$ & $\begin{array}{c}200 \\
\text { to } \\
500\end{array}$ & $\begin{array}{c}100 \\
\text { to } \\
200\end{array}$ & $\begin{array}{r}10 \\
\text { to } \\
100\end{array}$ & $\begin{array}{c}\text { Less } \\
\text { than } \\
10\end{array}$ \\
\hline Utah ..................... & 8 & 2 & - & - & - & 1 & 25,942 & 1,557 & - & - & - & 7 \\
\hline 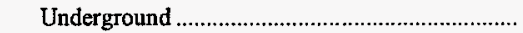 & 8 & 2 & - & - & - & 1 & 25,942 & 1,557 & - & - & - & 7 \\
\hline Virginia & 3 & 11 & 37 & 36 & 82 & 22 & 7,925 & 7,054 & 11,302 & 5,065 & 4,150 & 95 \\
\hline 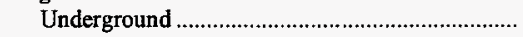 & 3 & 4 & 26 & 29 & 69 & 16 & 7,925 & 2,423 & 7,594 & 3,964 & 3,593 & 68 \\
\hline 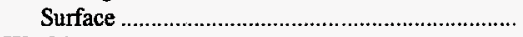 & - & 7 & 11 & 7 & 13 & 6 & - & 4,631 & 3,707 & 1,101 & 556 & 27 \\
\hline Washington & 2 & - & - & 1 & - & - & 4,393 & - & - & 173 & - & - \\
\hline Surface & 2 & - & - & 1 & - & - & 4,393 & - & - & 173 & - & - \\
\hline West Virginia Total & 42 & 43 & 73 & 44 & 147 & 37 & 101,588 & 32,292 & 24,001 & 5,910 & 6,482 & 160 \\
\hline Underground & 27 & 29 & 54 & 32 & 104 & 18 & 66,871 & 21,784 & 17,500 & 4,413 & 4,928 & 88 \\
\hline 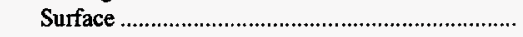 & 15 & 14 & 19 & 12 & 43 & 19 & 34,717 & 10,508 & 6,501 & 1,497 & 1,553 & 72 \\
\hline Northern & 12 & 5 & 10 & 10 & 38 & 18 & 35,632 & 4,023 & 3,311 & 1,299 & 1,573 & 73 \\
\hline 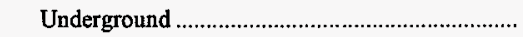 & 10 & 4 & 8 & 6 & 17 & 5 & 32,888 & 3,068 & 2,616 & 840 & 838 & 23 \\
\hline Surface & 2 & 1 & 2 & 4 & 21 & 13 & 2,744 & 955 & 695 & 459 & 735 & 50 \\
\hline Southern & 30 & 38 & 63 & 34 & 109 & 19 & 65,956 & 28,270 & 20,690 & 4,611 & 4,909 & 87 \\
\hline Underground & 17 & 25 & 46 & 26 & 87 & 13 & 33,982 & 18,716 & 14,884 & 3,573 & 4,090 & 65 \\
\hline Surface & 13 & 13 & 17 & 8 & 22 & 6 & 31,973 & 9,554 & 5,805 & 1,038 & 819 & 22 \\
\hline Wyoming & 22 & 2 & 1 & 1 & 1 & - & 276,349 & 1,599 & 278 & 198 & 16 & - - \\
\hline 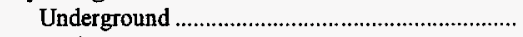 & 1 & - & - & - & - & - & 2,641 & - & - & - & - & - \\
\hline Surface & 21 & 2 & 1 & 1 & 1 & - & 273,708 & 1,599 & 278 & 198 & 16 & - \\
\hline Appalachian Total 1 . & 94 & 122 & 262 & 223 & 670 & 288 & 221,422 & 85,979 & 82,680 & 31,947 & 28,637 & 1,204 \\
\hline Underground & 66 & 65 & 157 & 127 & 307 & 95 & 167,447 & 46,542 & 49,488 & 18,168 & 14,724 & 421 \\
\hline Surface & 28 & 57 & 105 & 96 & 363 & 193 & 53,975 & 39,437 & 33,191 & 13,778 & 13,913 & 783 \\
\hline 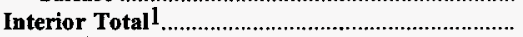 & 49 & 31 & 27 & 17 & 27 & 15 & 138,188 & 21,279 & 9,799 & 2,388 & 1,117 & 77 \\
\hline Underground & 26 & 9 & 4 & 2 & 3 & - & 58,371 & 6,820 & 1,613 & 267 & 113 & - \\
\hline 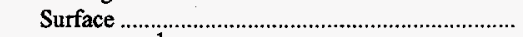 & 23 & 22 & 23 & 15 & 24 & 15 & 79,817 & 14,460 & 8,186 & 2,121 & 1,004 & 77 \\
\hline 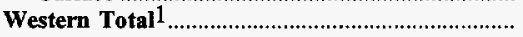 & 57 & 7 & 6 & 6 & 1 & $\mathbf{1}$ & 431,339 & 4,903 & 1,858 & 1,016 & 16 & 7 \\
\hline Underground & 12 & 4 & 3 & 4 & - & 1 & 41,558 & 2,703 & 962 & 645 & - & 7 \\
\hline Surface & 45 & 3 & 3 & 2 & 1 & - & 389,781 & 2,201 & 896 & 371 & 16 & - \\
\hline East of Miss. River & 132 & 151 & 282 & 233 & 693 & 296 & 303,306 & 105,904 & 90,320 & 33,289 & 29,601 & 1,246 \\
\hline Underground & 92 & 74 & 161 & 128 & 310 & 95 & 225,818 & 53,362 & 51,101 & 18,298 & 14,837 & 421 \\
\hline Surface & 40 & 77 & 121 & 105 & 383 & 201 & 77,488 & 52,543 & 39,219 & 14,991 & 14,764 & 825 \\
\hline West of Miss. River & 68 & 9 & 13 & 13 & 5 & 8 & 487,643 & 6,257 & 4,016 & 2,062 & 168 & 42 \\
\hline 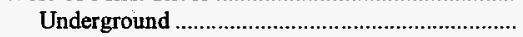 & 12 & 4 & 3 & 5 & - & 1 & 41,558 & 2,703 & 962 & 782 & - & 7 \\
\hline Surface & 56 & 5 & 10 & 8 & 5 & 7 & 446,085 & 3,554 & 3,054 & 1,280 & 168 & 35 \\
\hline U.S. Total & 200 & 160 & 295 & 246 & 698 & 304 & 790,949 & 112,162 & 94,336 & 35,351 & 29,769 & 1,288 \\
\hline 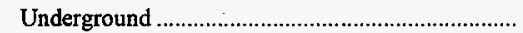 & 104 & 78 & 164 & 133 & 310 & 96 & 267,377 & 56,064 & 52,063 & 19,080 & 14,837 & 428 \\
\hline Surface & 96 & 82 & 131 & 113 & 388 & 208 & 523,573 & 56,097 & 42,274 & 16,271 & 14,932 & 860 \\
\hline
\end{tabular}

1 For a definition of coal-producing regions, see Appendix $\mathrm{C}$.

Notes: Coal production excludes silt, culm, refuse bank, slurry dam, and dredge operations except for Pennsylvania anthracite. Totals may not equal sum of components due to independent rounding.

Sources: Energy Information Administration, Form EIA-7A, "Coal Production Report"; State Mining Agency Coal Production Reports; and/or U.S. Department of Labor, Mine Safety and Health Administration, Form 7000-2, "Quarterly Mine Employment and Coal Production Report." 
Table 7. U.S. Coal Production by Coalbed Thickness and Mine Type, 1996 (Thousand Short Tons)

\begin{tabular}{|c|c|c|c|}
\hline $\begin{array}{c}\text { Coalbed Thickness } \\
\text { (inches) }\end{array}$ & Underground & Surface & Total \\
\hline$<7$ & - & 48 & 48 \\
\hline $7-12$ & - & 1,938 & 1,938 \\
\hline 13-18 & - & 8,602 & 8,602 \\
\hline $19-24$ & 385 & 19,274 & 19,659 \\
\hline $25-30$ & 5,997 & 22,801 & 28,798 \\
\hline 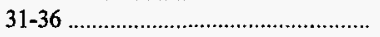 & 34,894 & 35,680 & 70,574 \\
\hline 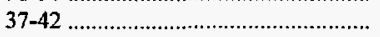 & 27,811 & 22,250 & 50,061 \\
\hline $43-48$ & 33,835 & 28,441 & 62,276 \\
\hline 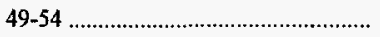 & 32,354 & 14,823 & 47,176 \\
\hline $55-60$ & 46,440 & 14,803 & 61,244 \\
\hline 61-66 & 46,723 & 12,354 & 59,077 \\
\hline 67-72 & 35,067 & 16,971 & 52,037 \\
\hline 73-78 & 21,643 & 6,771 & 28,413 \\
\hline $79-84$ & 38,309 & 11,885 & 50,195 \\
\hline $85-90$ & 8,000 & 8,917 & 16,917 \\
\hline $91-96$ & 28,244 & 6,785 & 35,029 \\
\hline $97-102$ & 5,926 & 12,426 & 18,352 \\
\hline $103-108$ & 3,881 & 5,982 & 9,863 \\
\hline $109-114$ & 5,837 & 1,400 & 7,237 \\
\hline $115-120$ & 9,870 & 5,546 & 15,415 \\
\hline$>\quad 120 \ldots \ldots$ & 24,205 & 395,452 & 419,657 \\
\hline Unknown ${ }^{1}$ & 428 & 860 & 1,288 \\
\hline 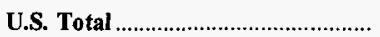 & 409,849 & 654,007 & $1,063,856$ \\
\hline
\end{tabular}

1 Includes mines with production of less than 10,000 short tons, which are required to provide only production data.

Notes: Coal production excludes silt, culm, refuse bank, slurry dam, and dredge operations except for Pennsylvania anthracite. Totals may not equal sum of components due to independent rounding.

Sources: Energy Information Administration, Form EIA-7A, "Coal Production Report"; State Mining Agency Coal Production Reports; and/or U.S. Department of Labor, Mine Safety and Health Administration, Form 7000-2, "Quarterly Mine Employment and Coal Production Report." 
Table 8. U.S. Coal Production and Coalbed Thickness by Major Coalbeds and Mine Type, 1996

\begin{tabular}{|c|c|c|c|c|c|c|}
\hline \multirow{2}{*}{$\begin{array}{l}\text { Coalbed ID Number } 1 \\
\text { Coalbed Name } 2\end{array}$} & \multicolumn{3}{|c|}{$\begin{array}{c}\text { Production } \\
\text { (thousand short tons) }\end{array}$} & \multicolumn{3}{|c|}{$\begin{array}{l}\text { Thickness } \\
\text { (inches) }\end{array}$} \\
\hline & Underground & Surface & Total & Average $^{3}$ & Low & High \\
\hline 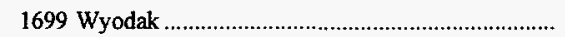 & - & 243,432 & 243,432 & 836 & 300 & 1,264 \\
\hline 0036 Pittsburgh & 71,962 & 1,957 & 73,919 & 74 & 18 & 108 \\
\hline 0489 No. 9 & 30,011 & 9,859 & 39,871 & 63 & 42 & 84 \\
\hline 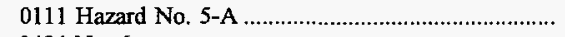 & 15,326 & 22,902 & 38,228 & 74 & 20 & 226 \\
\hline 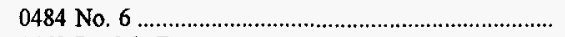 & 28,228 & 4,147 & 32,375 & 78 & 45 & 100 \\
\hline 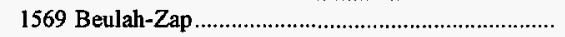 & - & 28,701 & 28,701 & 155 & 132 & 180 \\
\hline 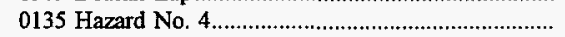 & 18,347 & 3,020 & 21,367 & 45 & 20 & 102 \\
\hline 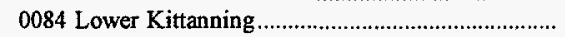 & 4,757 & 14,970 & 19,727 & 55 & 12 & 174 \\
\hline 0103 Stockton-Lewiston & 3,886 & 14,996 & 18,882 & 75 & 14 & 120 \\
\hline 0168 Lower Elkhorn & 14,983 & 1,636 & 16,619 & 58 & 18 & 96 \\
\hline 0071 Upper Freeport & 12,302 & 3,638 & 15,940 & 63 & 6 & 96 \\
\hline 1808 Rosebud. & - & 15,416 & 15,416 & 257 & 216 & 276 \\
\hline 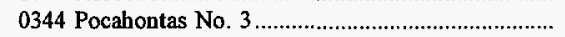 & 14,980 & 0 & 14,980 & 64 & 38 & 83 \\
\hline 0151 Elkhorn No. 1 & 10,856 & 3,871 & 14,727 & 49 & 12 & 96 \\
\hline 0154 Elkhorn No. 2 & 11,634 & 2,610 & 14,244 & 52 & 14 & 66 \\
\hline 0157 Elkhorn No. 3 & 9,388 & 2,970 & 12,359 & 72 & 15 & 120 \\
\hline 0121 Hazard No. 5 & 7,191 & 2,887 & 10,078 & 54 & 10 & 95 \\
\hline 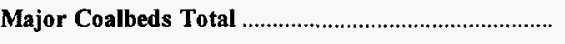 & 253,852 & 377,012 & 630,865 & 372 & 6 & 1,264 \\
\hline 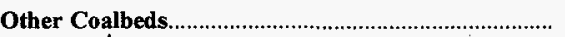 & $\mathbf{1 5 5 , 5 6 8}$ & 276,135 & 431,703 & 134 & 1 & 960 \\
\hline Unknown 4 & 428 & 860 & 1,288 & NA & $\mathbf{N A}$ & NA \\
\hline (1) & 409,849 & 654,007 & $1,063,856$ & 275 & 1 & 1,264 \\
\hline
\end{tabular}

1 The coalbed ID number is a unique code assigned by EIA to each correlated coalbed or to coal-bearing geologic formations, coal groups, or coal zones.

2 The coalbed name given is the name most commonly used in the State having the greatest production from that coalbed. The States having the greatest production for each coalbed are: Eastern Kentucky (coalbeds 0111, 0135, 0151, 0154, 0157, 0168); West Virginia (0036, 0084, 0103, 0344); Pennsylvania (0071,0076); Westem Kentucky (0489); Illinois (0484); North Dakota (1569); Montana (1808); Wyoming (1699). In some other States where these are major producing beds, the following alternate coalbed names are also used: 0084, No. 5 Block (Eastern Kentucky); 0111, Coalburg (West Virginia); 0135, Chilton (West Virginia); 0151, Jellico (Tennesee), Taggert (Virginia), Cedar Grove (West Virginia); 0154, Lower Cedar Grove (West Virginia); 0157, Upper Standiford (Virginia), Alma (West Virginia); 0168, No. 2 Gas (West Virginia); 0483, No. 12 (Western Kentucky); 0484, No. 11 (Western Kentucky); 0489, No. 5 (Illinois and Indiana).

3 Average thickness is the bed thickness weighted by bed production.

4 Includes mines with production of less than 10,000 short tons, which are required to provide only production data.

NA Not available.

Notes: Coal production excludes silt, culm, refuse bank, slurry dam, and dredge operations except for Pennsylvania anthracite. A major coalbed is defined here as a coalbed from which 10 million or more short tons were produced during the year. The category "Other Coalbeds" includes all coalbeds from which less than 10 million short tons were produced during the year. In some regions, coalbeds are characteristically discontinuous or uncorrelatable from one location to another, and production is identified by the geological formations, coal groups, or coal zones of the native rock where the coalbeds occur. These types of coalbeds are found primarily in the Rocky Mountain States and even in the Gulf Coast lignite belt. Coalbeds of these types are also included in "Other Coalbeds," even though production may exceed 10 million short tons. Totals may not equal sum of components due to independent rounding.

Sources: Energy Information Administration, Form EIA-7A, "Coal Production Report"; State Mining Agency Coal Production Reports; and/or U.S. Department of Labor, Mine Safety and Health Administration, Form 7000-2, "Quarterly Mine Employment and Coal Production Report." 
Table 9. Coal Production and Number of Mines by State and Coal Rank, 1996 (Thousand Short Tons)

\begin{tabular}{|c|c|c|c|c|c|c|c|c|c|c|}
\hline \multirow[b]{2}{*}{$\begin{array}{l}\text { Coal-Producing } \\
\text { State and Region }\end{array}$} & \multicolumn{2}{|c|}{ Bituminous } & \multicolumn{2}{|c|}{ Subbituminous } & \multicolumn{2}{|c|}{ Lignite } & \multicolumn{2}{|c|}{ Anthracite } & \multicolumn{2}{|c|}{ Total } \\
\hline & $\begin{array}{c}\text { Number } \\
\text { of } \\
\text { Mines }\end{array}$ & Production & $\begin{array}{c}\text { Number } \\
\text { of } \\
\text { Mines }\end{array}$ & Production & $\begin{array}{c}\text { Number } \\
\text { of } \\
\text { Mines }\end{array}$ & Production & $\begin{array}{c}\text { Number } \\
\text { of } \\
\text { Mines }\end{array}$ & Production & $\begin{array}{c}\text { Number } \\
\text { of } \\
\text { Mines }\end{array}$ & Production \\
\hline 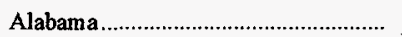 & 53 & 24,637 & - & - & - & - & - & - & 53 & 24,637 \\
\hline Alaska & - & - & 1 & 1,481 & - & - & - & - & 1 & 1,481 \\
\hline Arizona & 2 & 10,442 & - & - & - & - & - & - & 2 & 10,442 \\
\hline 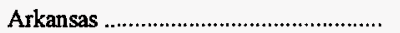 & 3 & 5 & - & - & - & - & 2 & 16 & 5 & 21 \\
\hline Colorado & 12 & 15,942 & 3 & 8,944 & - & - & - & - & 15 & 24,886 \\
\hline 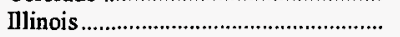 & 31 & 46,656 & - & - & - & - & - & - & 31 & 46,656 \\
\hline 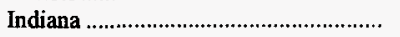 & 37 & 29,670 & - & - & - & - & - & - & 37 & 29,670 \\
\hline 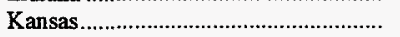 & 1 & 232 & - & - & - & - & - & - & 1 & 232 \\
\hline 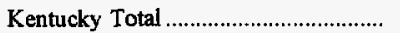 & 544 & 152,425 & - & - & - & - & - & - & 544 & 152,425 \\
\hline 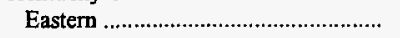 & 484 & 116,951 & - & - & - & - & - & - & 484 & 116,951 \\
\hline Western & 60 & 35,474 & - & - & - & - & - & - & 60 & 35,474 \\
\hline 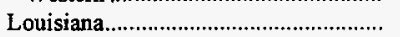 & - & - & - & - & 2 & 3,221 & - & - & 2 & 3,221 \\
\hline 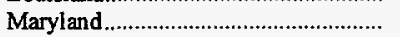 & 18 & 4,093 & - & - & - & - & - & - & 18 & 4,093 \\
\hline 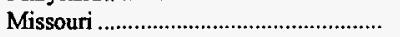 & 5 & 710 & - & - & - & - & - & - & 5 & 710 \\
\hline Montana & - & - & 7 & 37,635 & 1 & 256 & - & - & 8 & 37,891 \\
\hline New Mexico & 4 & 12,837 & 2 & 11,230 & - & - & - & - & 6 & 24,067 \\
\hline 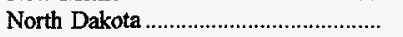 & - & - & - & - & 5 & 29,861 & - & - & 5 & 29,861 \\
\hline Ohio & 99 & 28,572 & - & - & - & - & - & - & 99 & 28,572 \\
\hline Oklahoma & 12 & 1,701 & - & - & - & - & - & - & 12 & 1,701 \\
\hline 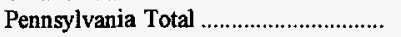 & 275 & 63,190 & - & - & - & - & 127 & 4,751 & 402 & 67,942 \\
\hline 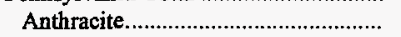 & - & - & - & - & - & - & 127 & 4,751 & 127 & 4,751 \\
\hline 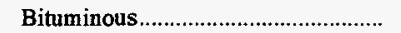 & 275 & 63,190 & - & - & - & - & - & - & 275 & 63,190 \\
\hline Tennessee & 26 & 3,651 & - & - & - & - & - & - & 26 & 3,651 \\
\hline Texas & 1 & 446 & - & - & 12 & 54,718 & - & - & 13 & 55,164 \\
\hline 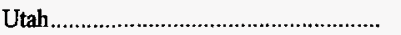 & 11 & 27,507 & - & - & - & - & - & - & 11 & 27,507 \\
\hline Virginia & 191 & 35,590 & - & - & - & - & - & - & 191 & 35,590 \\
\hline 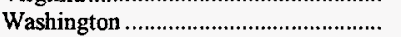 & 1 & 173 & 2 & 4,393 & - & - & - & - & 3 & 4,565 \\
\hline West Virginia Total ................................. & 386 & 170,433 & - & - & - & - & - & - & 386 & 170,433 \\
\hline Northern & 93 & 45,910 & - & - & - & - & - & - & 93 & 45,910 \\
\hline Southern & 293 & 124,523 & - & - & - & - & - & - & 293 & 124,523 \\
\hline 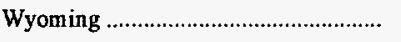 & 2 & 1,832 & 25 & 276,608 & - & - & - & - & 27 & 278,440 \\
\hline 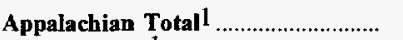 & 1,532 & 447,116 & - & - & - & - & 127 & 4,751 & 1,659 & 451,868 \\
\hline Interior Total $\mathbf{I}$ & 150 & 114,893 & - & - & 14 & $\mathbf{5 7 , 9 3 9}$ & 2 & 16 & 166 & 172,848 \\
\hline 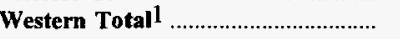 & 32 & 68,732 & 40 & 340,291 & 6 & 30,117 & - & - & 78 & 439,140 \\
\hline East of Miss. River & 1,660 & 558,916 & - & - & - & - & 127 & 4,751 & 1,787 & 563,668 \\
\hline 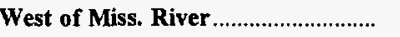 & 54 & 71,825 & 40 & 340,291 & 20 & 88,056 & 2 & 16 & 116 & 500,188 \\
\hline U.S. Total & 1,714 & 630,741 & 40 & 340,291 & 20 & 88,056 & 129 & 4,768 & 1,903 & $1,063,856$ \\
\hline
\end{tabular}

1 For a definition of coal-producing regions, see Appendix $C$

Notes: Coal production excludes silt, culm, refuse bank, slurry dam, and dredge operations except for Pennsylvania anthracite. Totals may not equal sum of components due to independent rounding.

Sources: Energy Information Administration, Form EIA-7A, "Coal Production Report"; State Mining Agency Coal Production Reports; and/or U.S. Department of Labor, Mine Safety and Health Administration, Form 7000-2, "Quarterly Mine Employment and Coal Production Report." 
Table 10. Coal Production by State, Coal Rank, and Group, 1996 (Thousand Short Tons)

\begin{tabular}{|c|c|c|c|c|c|c|c|c|}
\hline $\begin{array}{l}\text { Coal-Producing } \\
\text { State and Region }\end{array}$ & $\begin{array}{l}\text { Bituminous } \\
\text { Low Volatile }\end{array}$ & $\begin{array}{c}\text { Bituminous } \\
\text { Medium } \\
\text { Volatile }\end{array}$ & $\begin{array}{c}\text { Bituminous } \\
\text { High Volatile }\end{array}$ & $\begin{array}{l}\text { Bituminous } \\
\text { Total }^{1}\end{array}$ & $\begin{array}{l}\text { Subbitumi- } \\
\text { nous }\end{array}$ & Lignite & Anthracite & Total \\
\hline Alabama & 7,359 & 3,654 & 13,303 & 24,637 & - & - & - & 24,637 \\
\hline 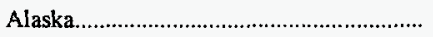 & - & - & - & - & 1,481 & - & - & 1,481 \\
\hline Arizona & - & - & 10,442 & 10,442 & - & - & - & 10,442 \\
\hline 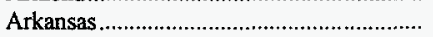 & 5 & - & - & 5 & - & - & 16 & 21 \\
\hline Colorado & - & 129 & 15,814 & 15,942 & 8,944 & - & - & 24,886 \\
\hline 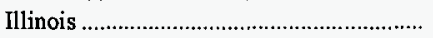 & - & - & 46,656 & 46,656 & - & - & - & 46,656 \\
\hline Indiana & - & - & 29,670 & 29,670 & - & - & - & 29,670 \\
\hline 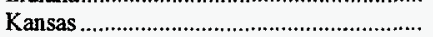 & - & - & 232 & 232 & - & - & - & 232 \\
\hline 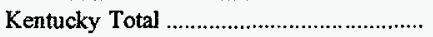 & 966 & 2,121 & 148,414 & 152,425 & - & - & - & 152,425 \\
\hline Eastern & 966 & 2,121 & 113,864 & 116,951 & - & - & - & 116,951 \\
\hline Western & - & - & 34,550 & 35,474 & - & - & - & 35,474 \\
\hline 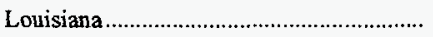 & - & 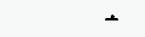 & - & - & - & 3,221 & - & 3,221 \\
\hline 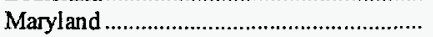 & 4,093 & - & - & 4,093 & - & - & - & 4,093 \\
\hline Missouri & - & - & 710 & 710 & - & - & - & 710 \\
\hline Montana & - & - & - & - & 37,635 & 256 & - & 37,891 \\
\hline 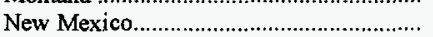 & - & - & $12,83.7$ & 12,837 & 11,230 & - & - & 24,067 \\
\hline North Dakota & - & $=$ & - & - & - & 29,861 & - & 29,861 \\
\hline 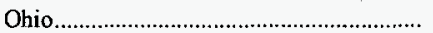 & 13 & 474 & 27,394 & 28,572 & - & - & - & 28,572 \\
\hline Oklahoma & 268 & 909 & 524 & 1,701 & - & - & - & 1,701 \\
\hline 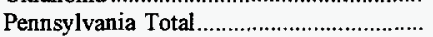 & 4,121 & 11,564 & 46,839 & 63,190 & - & - & 4,751 & 67,942 \\
\hline Anthracite & - & - & - & - & - & - & 4,751 & 4,751 \\
\hline Bituminous & 4,121 & 11,564 & 46,839 & 63,190 & - & - & - & 63,190 \\
\hline Tennessee & - & 1,219 & 2,432 & 3,651 & - & - & - & 3,651 \\
\hline Texas & - & 446 & - & 446 & - & 54,718 & - & 55,164 \\
\hline 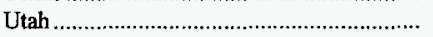 & - & - & 27,507 & 27,507 & - & - & - & 27,507 \\
\hline Virginia & 6,928 & 7,085 & 21,577 & 35,590 & - & - & - & 35,590 \\
\hline Washington & - & - & 173 & 173 & 4,393 & - & - & 4,565 \\
\hline West Virginia Total & 17,312 & 12,641 & 139,952 & 170,433 & - & - & - & 170,433 \\
\hline Northern & 3,927 & 1,803 & 39,669 & 45,910 & - & - & - & 45,910 \\
\hline Southem & 13,385 & 10,838 & 100,283 & 124,523 & - & - & - & 124,523 \\
\hline 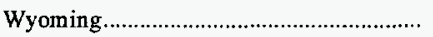 & - & - & 1,832 & 1,832 & 276,608 & - & - & 278,440 \\
\hline Appalachian Total ${ }^{2} \ldots \ldots \ldots \ldots \ldots \ldots \ldots \ldots \ldots \ldots \ldots$ & 40,792 & 38,758 & 365,361 & 447,116 & - & - & 4,751 & 451,868 \\
\hline Interior Total 2 & 273 & 1,354 & 112,342 & 114,893 & - & 57,939 & 16 & 172,848 \\
\hline 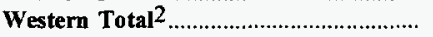 & - & 129 & 68,603 & 68,732 & 340,291 & 30,117 & - & 439,140 \\
\hline East of Miss. River ...................................... & 40,792 & 38,758 & 476,237 & 558,916 & - & - & 4,751 & 563,668 \\
\hline 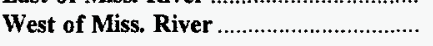 & 273 & 1,483 & 70,069 & 71,825 & 340,291 & 88,056 & 16 & 500,188 \\
\hline 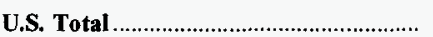 & 41,065 & 40,241 & 546,307 & 630,741 & 340,291 & 88,056 & 4,768 & $1,063,856$ \\
\hline
\end{tabular}

1 Includes bituminous production with volatile content not reported.

2 For a definition of coal-producing regions, see Appendix C.

Notes: Refer to the Classification of Coals by Rank table in Appendix C for coal group definitions. Coal production excludes silt, culm, refuse bank, slurry dam, and dredge operations except for Pennsylvania anthracite. Totals may not equal sum of components due to independent rounding.

Sources: Energy Information Administration, Form EIA-7A, "Coal Production Report"; State Mining Agency Coal Production Reports; and/or U.S. Department of Labor, Mine Safety and Health Administration, Form 7000-2, "Quarterly Mine Employment and Coal Production Report." 
Table 11. Coal Production by State, Mine Type, and Union Type, 1996 (Thousand Short Tons)

\begin{tabular}{|c|c|c|c|c|c|}
\hline $\begin{array}{l}\text { Coal-Producing } \\
\text { State and Region }\end{array}$ & UMWA & Other Unions & Union Total & Nonunion & Total \\
\hline Alabama & 17,060 & - & 17,060 & 7,563 & 24,623 \\
\hline Underground & 14,519 & - & 14,519 & 3,698 & 18,217 \\
\hline Surface & 2,541 & - & 2,541 & 3,865 & 6,406 \\
\hline 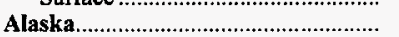 & - & 1,481 & 1,481 & - & 1,481 \\
\hline Surface & - & 1,481 & 1,481 & - & 1,481 \\
\hline Arizona & 10,442 & - & 10,442 & - & 10,442 \\
\hline Surface & 10,442 & - & 10,442 & - & 10,442 \\
\hline Colorado & $\mathbf{2 , 5 9 1}$ & 2,008 & 4,599 & 20,287 & 24,886 \\
\hline Underground & 1,007 & - & 1,007 & 14,574 & 15,581 \\
\hline Surface & 1,584 & 2,008 & 3,592 & 5,714 & 9,305 \\
\hline 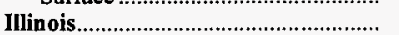 & 32,104 & 3,449 & $\mathbf{3 5 , 5 5 3}$ & 11,099 & 46,652 \\
\hline Underground & 25,635 & 2,776 & 28,411 & 10,538 & 38,948 \\
\hline Surface & 6,469 & 673 & 7,143 & 561 & 7,704 \\
\hline Indiana & 14,933 & - & 14,933 & 14,734 & 29,667 \\
\hline 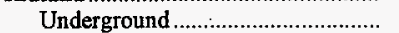 & 900 & - & 900 & 2,063 & 2,963 \\
\hline Surface & 14,033 & - & 14,033 & 12,672 & 26,704 \\
\hline 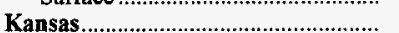 & 232 & - & 232 & - & 232 \\
\hline Surface & 232 & - & 232 & - & 232 \\
\hline 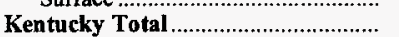 & 20,430 & 779 & 21,209 & 130,883 & 152,092 \\
\hline Underground & 15,903 & 245 & 16,148 & 78,005 & 94,153 \\
\hline Surface & 4,528 & 533 & 5,061 & 52,878 & 57,939 \\
\hline Eastern & 10,455 & 779 & 11,234 & 105,420 & 116,654 \\
\hline Underground & 7,149 & 245 & 7,395 & 61,624 & 69,018 \\
\hline Surface & 3,306 & 533 & 3,839 & 43,796 & 47,635 \\
\hline Western & 9,975 & - & 9,975 & 25,463 & $35, \mathbf{4 3 8}$ \\
\hline Underground & 8,754 & - & 8,754 & 16,381 & 25,135 \\
\hline Surface & 1,222 & - & 1,222 & 9,082 & 10,304 \\
\hline Louisiana & - & - & - & 3,221 & $\mathbf{3 , 2 2 1}$ \\
\hline Surface & - & - & - & 3,221 & 3,221 \\
\hline Maryland & - & - & - & 4,068 & 4,068 \\
\hline Underground & - & - & - & 3,296 & 3,296 \\
\hline 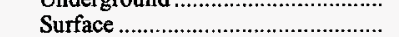 & - & - & - & 772 & 772 \\
\hline Missouri & - & - & - & 710 & 710 \\
\hline 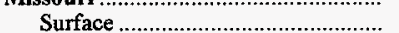 & - & - & - & 710 & 710 \\
\hline Montana & 16,241 & 12,476 & 28,717 & 9,174 & 37,891 \\
\hline Underground & - & - & - & 147 & 147 \\
\hline Surface & 16,241 & 12,476 & 28,717 & 9,027 & 37,744 \\
\hline New Mexico & 6,587 & 13,281 & 19,868 & 4,199 & 24,067 \\
\hline Surface & 6,587 & 13,281 & 19,868 & 4,199 & 24,067 \\
\hline North Dakota & 2,675 & 4,538 & 7,212 & 22,648 & 29,861 \\
\hline Surface & 2,675 & 4,538 & 7,212 & 22,648 & 29,861 \\
\hline Ohio & 15,673 & 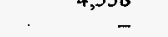 & 15,673 & 12,847 & $\mathbf{2 8 , 5 2 0}$ \\
\hline Underground & 14,075 & - & 14,075 & 1,837 & 15,912 \\
\hline Surface & 1,598 & - & 1,598 & 11,010 & 12,608 \\
\hline Oklahoma & 1 & - & - & 1,687 & 1,687 \\
\hline Underground & - & - & - & 137 & 137 \\
\hline Surface & - & - & - & 1,550 & 1,550 \\
\hline Pennsylvania Total & 25,899 & 65 & 25,964 & $\mathbf{4 1 , 4 4 7}$ & 67,411 \\
\hline Underground & 23,992 & - & 23,992 & 23,147 & 47,140 \\
\hline Surface & 1,907 & 65 & 1,972 & 18,300 & 20,272 \\
\hline Anthracite & 986 & 65 & 1,052 & 3,492 & 4,544 \\
\hline Underground & - & - & - & 284 & 284 \\
\hline Surface & 986 & 65 & 1,052 & 3,208 & 4,259 \\
\hline 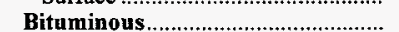 & 24,913 & - & 24,913 & 37,955 & 62,868 \\
\hline 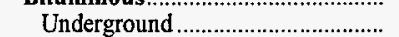 & 23,992 & - & 23,992 & 22,863 & 46,855 \\
\hline Surface & 920 & - & 920 & 15,092 & 16,012 \\
\hline Tennessee & - & - & - & 3,621 & 3,621 \\
\hline 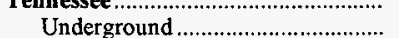 & - & - & - & 1,790 & 1,790 \\
\hline Surface & - & - & - & 1,830 & 1,830 \\
\hline Texas & - & 36,136 & 36,136 & 19,027 & 55,164 \\
\hline Surface & - & 36,136 & 36,136 & 19,027 & 55,164 \\
\hline Utah & 9,256 & - & 9,256 & 18,243 & 27,499 \\
\hline Underground & 9,256 & - & 9,256 & 18,243 & 27,499 \\
\hline Virginia & 7,309 & 707 & 8,017 & 27,478 & 35,495 \\
\hline Underground & 6,999 & - & 6,999 & 18,501 & 25,500 \\
\hline Surface & 310 & 707 & 1,018 & 8,978 & 9,995 \\
\hline 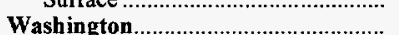 & - & 4,393 & 4,393 & 173 & 4,565 \\
\hline Surface & _ & 4,393 & 4,393 & 173 & 4,565 \\
\hline West Virginia Total & 89,208 & 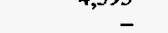 & 89,208 & 81,065 & 170,273 \\
\hline Underground & $\begin{array}{l}07,200 \\
65,086\end{array}$ & - & 65,086 & 50,411 & 115,497 \\
\hline Surface & 24,122 & - & 24,122 & 30,654 & 54,776 \\
\hline
\end{tabular}

See footnotes at end of table. 
Table 11. Coal Production by State, Mine Type, and Union Type, 1996 (Continued) (Thousand Short Tons)

\begin{tabular}{|c|c|c|c|c|c|}
\hline $\begin{array}{l}\text { Coal-Producing } \\
\text { State and Region }\end{array}$ & UMWA & Other Unions & Union Total & Nonunion & Total \\
\hline 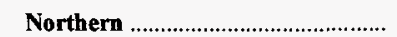 & 31,636 & - & 31,636 & 14,201 & 45,838 \\
\hline 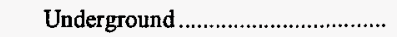 & 31,636 & - & 31,636 & 8,614 & 40,251 \\
\hline 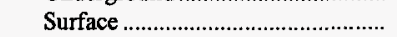 & - & - & - & 5,587 & 5,587 \\
\hline Southern & $\mathbf{5 7 , 5 7 2}$ & - & $\mathbf{5 7 , 5 7 2}$ & 66,863 & 124,435 \\
\hline 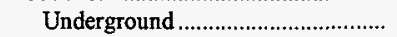 & 33,449 & - & 33,449 & 41,796 & 75,246 \\
\hline Surface & 24,122 & - & 24,122 & 25,067 & 49,189 \\
\hline 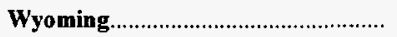 & 4,435 & 10,114 & 14,549 & 263,891 & 278,440 \\
\hline 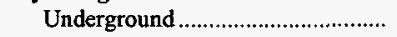 & - & - & - & 2,641 & 2,641 \\
\hline Surface & 4,435 & 10,114 & 14,549 & 261,250 & 275,799 \\
\hline Appalachian Total 1 .............................. & 165,605 & 1,551 & 167,156 & 283,508 & 450,664 \\
\hline Underground & 131,820 & 245 & 132,066 & 164,304 & 296,370 \\
\hline Surface & 33,785 & 1,306 & 35,090 & 119,204 & 154,294 \\
\hline Interior Total ${ }^{1}$ & $\mathbf{5 7 , 2 4 5}$ & 39,585 & 96,830 & 75,941 & 172,771 \\
\hline Underground & 35,289 & 2,776 & 38,064 & 29,118 & 67,183 \\
\hline Surface & 21,956 & 36,809 & 58,765 & 46,823 & 105,588 \\
\hline Western Total 1 & 52,227 & 48,290 & 100,517 & 338,616 & 439,133 \\
\hline 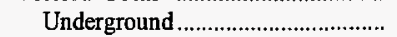 & 10,263 & - & 10,263 & 35,605 & 45,868 \\
\hline Surface & 41,963 & 48,290 & 90,253 & 303,011 & 393,265 \\
\hline East of Miss. River.................................. & 222,617 & 5,000 & 227,617 & 334,804 & 562,422 \\
\hline Underground & 167,109 & 3,021 & 170,130 & 193,286 & 363,416 \\
\hline Surface & 55,508 & 1,979 & 57,487 & 141,519 & 199,006 \\
\hline West of Miss. River & 52,459 & 84,426 & 136,885 & 363,261 & 500,146 \\
\hline 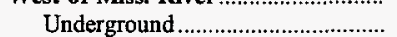 & 10,263 & - & 10,263 & 35,742 & 46,005 \\
\hline 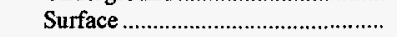 & 42,196 & 84,426 & 126,622 & 327,519 & 454,141 \\
\hline Unknown ${ }^{2}$ & NA & NA & $\mathbf{N A}$ & NA & 1,288 \\
\hline Underground & $\mathrm{NA}$ & $\mathrm{NA}$ & NA & $\mathrm{NA}$ & 428 \\
\hline Surface & $\mathrm{NA}$ & NA & NA & NA & 860 \\
\hline 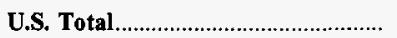 & 275,076 & 89,426 & 364,502 & 698,065 & $1,063,856$ \\
\hline 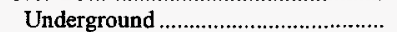 & 177,373 & 3,021 & 180,394 & 229,027 & 409,849 \\
\hline Surface & 97,704 & 86,405 & 184,109 & 469,038 & 654,007 \\
\hline
\end{tabular}

1 For a definition of coal-producing regions, see Appendix C.

2 Includes mines with production of less than 10,000 short tons, which are required to provide only production data.

NA Not available.

Notes: Coal production excludes silt, culm, refuse bank, slurry dam, and dredge operations except for Pennsylvania anthracite. Totals may not equal sum of components due to independent rounding. See Glossary for listing of other unions.

Sources: Energy Information Administration, Form EIA-7A, "Coal Production Report"; State Mining Agency Coal Production Reports; and/or U.S. Department of Labor, Mine Safety and Health Administration, Form 7000-2, "Quarterly Mine Employment and Coal Production Report." 
Table 12. Coal Production by State and Disposition, 1996

(Thousand Short Tons)

\begin{tabular}{|c|c|c|c|}
\hline $\begin{array}{l}\text { Coal-Producing } \\
\text { State and Region }\end{array}$ & Open Market ${ }^{1}$ & Captive ${ }^{2}$ & Total \\
\hline Alabama & 23,749 & 874 & 24,623 \\
\hline Alaska & 1,478 & 2 & 1,481 \\
\hline Arizona & 10,442 & 0 & 10,442 \\
\hline Colorado & 24,886 & 0 & 24,886 \\
\hline 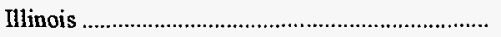 & 46,080 & 573 & 46,652 \\
\hline 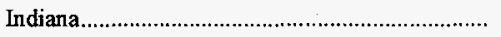 & 29,666 & 1 & 29,667 \\
\hline 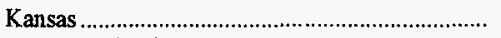 & 232 & 0 & 232 \\
\hline 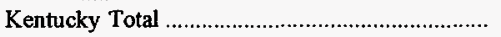 & 149,169 & 2,923 & 152,092 \\
\hline Eastern & 113,733 & 2,921 & 116,654 \\
\hline 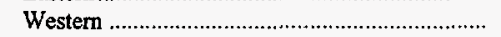 & 35,437 & 2 & 35,438 \\
\hline 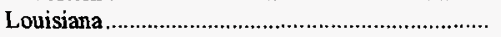 & 3,221 & 0 & 3,221 \\
\hline 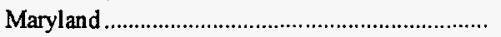 & 4,042 & 25 & 4,068 \\
\hline 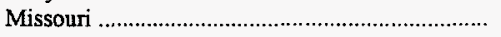 & 710 & 0 & 710 \\
\hline 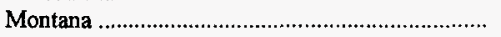 & 34,909 & 2,982 & 37,891 \\
\hline 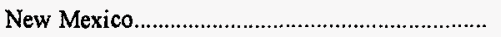 & 24,067 & 0 & 24,067 \\
\hline 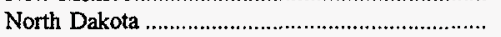 & 28,959 & 901 & 29,861 \\
\hline 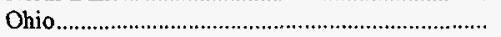 & 20,591 & 7,929 & 28,520 \\
\hline Oklahoma & 1,687 & 0 & 1,687 \\
\hline 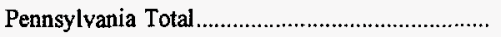 & 64,992 & 2,420 & 67,411 \\
\hline Anthracite & 2,853 & 1,691 & 4,544 \\
\hline 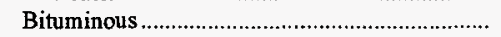 & 62,139 & 729 & 62,868 \\
\hline Tennessee & 3,603 & 17 & 3,621 \\
\hline 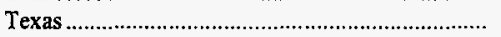 & 15,136 & 40,027 & 55,164 \\
\hline Utah & 19,294 & 8,205 & 27,499 \\
\hline 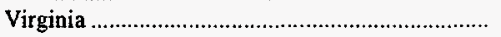 & 33,519 & 1,976 & 35,495 \\
\hline Washington & 173 & 4,393 & 4,565 \\
\hline 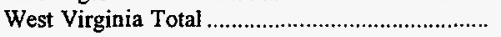 & 167,700 & 2,573 & 170,273 \\
\hline Northem & 44,756 & 1,082 & 45,838 \\
\hline Southern & 122,944 & 1,491 & 124,435 \\
\hline Wyoming & 262,384 & 16,056 & 278,440 \\
\hline 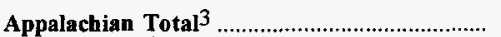 & 431,930 & 18,734 & 450,664 \\
\hline Interior Totak ${ }^{3}$ & 132,168 & 40,603 & 172,771 \\
\hline Western Total $\beta^{3}$ & 406,593 & 32,540 & 439,133 \\
\hline East of Miss. River & 543,112 & 19,310 & 562,422 \\
\hline West of Miss. River & 427,579 & 72,567 & 500,146 \\
\hline 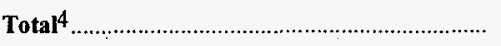 & 970,691 & 91,877 & $1,062,568$ \\
\hline 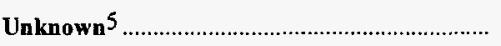 & NA & NA & 1,288 \\
\hline 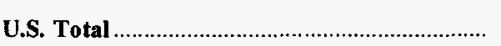 & NA & NA & $1,063,856$ \\
\hline
\end{tabular}

1 Open Market includes all coal sold on the open market to other coal companies or consumers.

2 Captive includes all coal used by the producing company or sold to affiliated or parent companies.

3 For a definition of coal-producing regions, see Appendix C.

4 Excludes mines producing less than 10,000 short tons, which are not required to provide these data.

5 Includes mines producing less than 10,000 short tons, which are required to provide only production data.

NA Not available.

Notes: Coal production excludes silt, culm, refuse bank, slurry dam, and dredge operations except for Pennsylvania anthracite.

Sources: Energy Information Administration, Form EIA-7A, "Coal Production Report"; State Mining Agency Coal Production Reports; and/or U.S. Department of Labor, Mine Safety and Health Administration, Form 7000-2, "Quarterly Mine Employment and Coal Production Report." 
Table 13. Coal Mining Acreage, Production and Royalties from Federal and Indian Leases by State, 1996

\begin{tabular}{|c|c|c|c|c|c|c|}
\hline \multirow{2}{*}{$\begin{array}{l}\text { Coal-Producing } \\
\text { State and Region }\end{array}$} & \multicolumn{3}{|c|}{ Federal Leases } & \multicolumn{3}{|c|}{ Indian Leases } \\
\hline & $\begin{array}{c}\text { Acres } \\
\text { Leased }\end{array}$ & $\begin{array}{c}\text { Production } \\
\text { (thousand short tons) }\end{array}$ & $\begin{array}{c}\text { Royalties } \\
\text { (thousand dollars) }\end{array}$ & $\begin{array}{l}\text { Acres } \\
\text { Leased }\end{array}$ & $\begin{array}{l}\text { Production } \\
\text { (thousand short tons) }\end{array}$ & $\begin{array}{c}\text { Royalties } \\
\text { (thousand dollars) }\end{array}$ \\
\hline 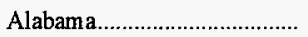 & 1,610 & 49 & 169 & - & - & - \\
\hline 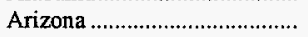 & - & - & - & 64,858 & 12,185 & 33,226 \\
\hline 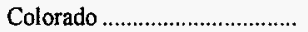 & 49,633 & 18,765 & 28,560 & - & - & - \\
\hline 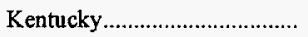 & 2,413 & 249 & 268 & - & - & - \\
\hline 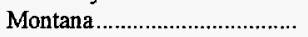 & 31,540 & 24,816 & 32,935 & 14,746 & 4,681 & 2,139 \\
\hline 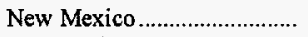 & 12,132 & 5,861 & 20,844 & 36,026 & 9,439 & 27,697 \\
\hline 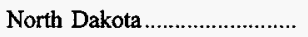 & 5,472 & 1,835 & 1,012 & - & - & - \\
\hline Oklahoma & 10,251 & 769 & 746 & - & - & - \\
\hline Utah & 44,215 & 26,403 & 39,456 & - & - & - \\
\hline 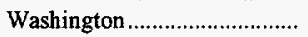 & 241 & 570 & 1,233 & - & - & - \\
\hline Wyoming & 118,403 & 248,866 & 176,655 & - & - & - \\
\hline Appalachian Total 1 .............. & 1,610 & 49 & 170 & - & - & - \\
\hline 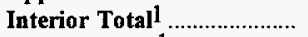 & 12,664 & 1,018 & 1,015 & - & - & - \\
\hline 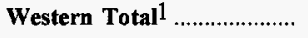 & 261,636 & 327,121 & 300,701 & 115,630 & 26,305 & 63,064 \\
\hline East of Miss. River................ & 4,023 & 298 & 438 & - & - & - \\
\hline West of Miss. River............... & 271,887 & 327,890 & 301,448 & 115,630 & 26,305 & 63,064 \\
\hline 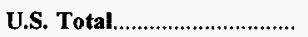 & 275,910 & 328,188 & 301,885 & 115,630 & 26,305 & 63,064 \\
\hline
\end{tabular}

1 For a definition of coal-producing regions, see Appendix $C$.

Notes: U.S. Total for this table represents Federal and Indian Leases only. Output from Federal and Indian Lands is reported as sales volume, the basis for royalties. It is approximately equivalent to production, which includes coal sold and coal added to stockpiles. Totals may not equal sum of components due to independent rounding.

Source: U.S. Department of the Interior, Minerals Management Service (MMS),Mineral Revenues, 1996, Report on Receipts from Federal and Indian Leases. 
Table 14. Major U.S. Coal Mines, 1996

\begin{tabular}{|c|c|c|c|c|}
\hline Rank & Mine Name/Company & Mine Type & State & $\begin{array}{l}\text { Production } \\
\text { (short tons) }\end{array}$ \\
\hline 1 & Black Thunder/Thunder Basin Coal & Surface & Wyoming & $39,258,024$ \\
\hline 2 & North Antelope/Powder River Coal & Surface & Wyoming & $28,623,169$ \\
\hline 3 & Rochelle/Powder River Coal & Surface & Wyoming & $26,248,241$ \\
\hline 4 & Jacobs Ranch/KerT-McGee Coal & Surface & Wyoming & $24,522,960$ \\
\hline 5 & Caballo/Caballo Coal & Surface & Wyoming & $22,003,064$ \\
\hline 6 & Belle Ayr/AMAX Coal West & Surface & Wyoming & $19,970,300$ \\
\hline 7 & Eagle Butte/AMAX Coal West & Surface & Wyoming & $15,642,744$ \\
\hline 8 & Freedom-Coteau/Coteau Properties & Surface & North Dakota & $15,624,702$ \\
\hline 9 & Caballo Rojo/Caballo Rojo & Surface & Wyoming & $15,082,891$ \\
\hline 10 & Rawhide/Caballo Coal & Surface & Wyoming & $15,068,358$ \\
\hline 11 & Cordero/Cordero Mining & Surface & Wyoming & $13,071,242$ \\
\hline 12 & Antelope/Antelope Coal & Surface & Wyoming & $12,045,854$ \\
\hline 13 & Buckskin/Triton Coal & Surface & Wyoming & $12,005,334$ \\
\hline 14 & West Decker/Decker Coal & Surface & Montana & $10,388,948$ \\
\hline 15 & Spring Creek/Spring Creek Coal & Surface & Montana & $9,026,789$ \\
\hline 16 & Jewett/Northwestern Resources & Surface & Texas & $8,865,214$ \\
\hline 17 & Enlow Fork/Enlow Fork Mining & Underground & Pennsylvania & $8,723,644$ \\
\hline 18 & Monticello-Winfield/Texas Utilities Mining & Surface & Texas & $8,568,801$ \\
\hline 19 & Rosebud No 6/Westem Energy & Surface & Montana & $7,807,347$ \\
\hline 20 & Bailey No 1/CONSOL & Underground & Pennsylvania & $7,469,255$ \\
\hline 21 & Navajo/BHP Minerals & Surface & New Mexico & $7,031,000$ \\
\hline 22 & Falkirk/Falkirk Mining & Surface & North Dakota & $7,023,633$ \\
\hline 23 & Martin Lake/Texas Utilities Mining & Surface & Texas & $6,813,996$ \\
\hline 24 & Galatia No $56 /$ Kerr-McGee Coal & Underground & Illinois & $6,629,282$ \\
\hline 25 & Sandow-Rockdale/ALCOA & Surface & Texas & $6,615,490$ \\
\hline 26 & Martin Lake-Oak Hill/Texas Utilities Mining & Surface & Texas & $6,508,728$ \\
\hline 27 & Jim Bridger/Bridger Coal & Surface & Wyoming & $6,321,161$ \\
\hline 28 & Kayenta/Peabody Westem & Surface & Arizona & $6,236,855$ \\
\hline 29 & Mount Gunnison/Mountain Coal & Underground & Colorado & $5,986,138$ \\
\hline 30 & Foidel Creek/Twenty Mile Coal & Underground & Colorado & $5,836,673$ \\
\hline 31 & Coal Creek/Thunder Basin Coal & Surface & Wyoming & $5,769,025$ \\
\hline 32 & Colowyo/Colowyo Coal & Surface & Colorado & $5,713,565$ \\
\hline 33 & Cumberland/Cyprus Cumberland & Underground & Pennsylvania & $5,327,908$ \\
\hline 34 & McKinley/Pittsburg \& Midway Coal & Surface & New Mexico & $5,327,649$ \\
\hline 35 & Mountaineer/Mingo Logan Coal & Underground & West Virginia & $5,245,650$ \\
\hline 36 & Big Brown/Texas Utilities Mining & Surface & Texas & $5,195,579$ \\
\hline 37 & Big Sky/Big Sky Coal & Surface & Montana & $4,994,234$ \\
\hline 38 & Peats Branch No 3/Hobet Mining & Surface & West Virginia & $4,847,375$ \\
\hline 39 & Powhatan No 6/Ohio Valley Coal & Underground & Ohio & $4,741,214$ \\
\hline 40 & Absaloka/Westmoreland Resources & Surface & Montana & $4,668,495$ \\
\hline 41 & Skyline/Coastal States Energy & Underground & Utah & $4,628,282$ \\
\hline 42 & Federal No 2/Eastern Associated Coal & Underground & West Virginia & $4,580,428$ \\
\hline 43 & No 37/Arch of Kentucky & Underground & Kentucky & $4,547,046$ \\
\hline 44 & Center/BNI Coal & Surface & North Dakota & $4,537,957$ \\
\hline 45 & Samples(Boone)/Catenary Coal & Surface & West Virginia & $4,493,734$ \\
\hline 46 & No 50/US Steel Mining & Underground & West Virginia & $4,492,196$ \\
\hline 47 & Shoemaker/CONSOL & Underground & West Virginia & $4,415,744$ \\
\hline 48 & Deer Creek/PacifiCorp & Underground & Utah & $4,337,999$ \\
\hline 49 & McElory/CONSOL & Underground & West Virginia & $4,305,682$ \\
\hline 50 & Robinson Run/CONSOL & Underground & West Virginia & $4,216,327$ \\
\hline 51 & Black Mesa/Peabody Western & Surface & Arizona & $4,204,908$ \\
\hline 52 & Southern Utah Fuel/Coastal States Energy & Underground & Utah & $4,202,439$ \\
\hline 53 & Lee Ranch/Lee Ranch Coal & Surface & New Mexico & $4,199,387$ \\
\hline 54 & No 13 Baker/Costain Coal & Underground & Kentucky & $3,986,028$ \\
\hline 55 & South Hallsville No 1/Sabine Mining & Surface & Texas & $3,892,053$ \\
\hline 56 & San Juan/San Juan Coal & Surface & New Mexico & $3,875,153$ \\
\hline 57 & Trail Mountain/PacifiCorp & Underground & Utah & $3,825,647$ \\
\hline 58 & Dave Johnston/PacifiCorp & Surface & Wyoming & $3,792,706$ \\
\hline 59 & Marissa(Washington)/Peabody Coal & Underground & Illinois & $3,673,750$ \\
\hline 60 & Kemmerer/Pittsburg \& Midway Coal & Surface & Wyoming & $3,650,865$ \\
\hline 61 & Dilworth/CONSOL & Underground & Pennsylvania & $3,632,018$ \\
\hline 62 & Buchanan No 1/CONSOL & Underground & Virginia & $3,556,890$ \\
\hline 63 & Blacksville No 2/CONSOL & Underground & West Virginia & $3,459,798$ \\
\hline 64 & Shoal Creek/Drummond Co & Underground & Alabama & $3,417,799$ \\
\hline 65 & Lynnville/Peabody Coal & Surface & Indiana & $3,404,172$ \\
\hline 66 & Powhatan No 4/CONSOL & Underground & Ohio & $3,382,895$ \\
\hline 67 & No 21/Hobet Mining & Surface & West Virginia & $3,366,564$ \\
\hline 68 & San Miguel/Atascosa Mining & Surface & Texas & $3,324,110$ \\
\hline 69 & Hawthom/Peabody Coal & Surface & Indiana & $3,317,693$ \\
\hline * & Subtotal & & & $\mathbf{5 5 1}, \mathbf{5 7 0 , 8 0 1}$ \\
\hline * & All Other Mines & & & $512,284,712$ \\
\hline * & U.S. Total & & & $1,063,855,513$ \\
\hline
\end{tabular}

Notes: Major mines are mines that produced more than 3.3 million short tons in 1996. The company is the firm operating the mine.

Sources: Energy Information Administration, Form EIA-7A., "Coal Production Report"; State Mining Agency Coal Production Reports; and/or U.S. Department of Labor, Mine Safety and Health Administration, Form 7000-2, "Quarterly Mine Employment and Production Report." 
Table 15. Major U.S. Coal Producers, 1996

\begin{tabular}{|c|c|c|c|}
\hline Rank & Company Name & $\begin{array}{l}\text { Production } \\
\text { (thousand short tons) }\end{array}$ & Percent of Total Production \\
\hline 1 & Peabody Holding Co., Inc. & 142,811 & 13.4 \\
\hline 2 & Cyprus AMAX Minerals Co. & 74,738 & 7.0 \\
\hline 3 & Consol Energy Inc. & 70,072 & 6.6 \\
\hline 4 & Kennecott Energy Co. & 62,527 & 5.9 \\
\hline 5 & ARCO Coal Co & 51,013 & 4.8 \\
\hline 6 & Kerr-McGee Coal Corp. & 31,350 & 2.9 \\
\hline 7 & Zeigler Coal Holding Co. & 31,001 & 2.9 \\
\hline 8 & Marrowbone Development Co. & 29,239 & 2.7 \\
\hline 9 & Texas Utilities Co. & 28,214 & 2.6 \\
\hline 10 & A.T. Massey Coal Co. & 27,327 & 2.6 \\
\hline 11 & North American Coal Corp. & 26,284 & 2.5 \\
\hline 12 & Arch Mineral Corp. & 20,153 & 1.9 \\
\hline 13 & Montana Power Co. & 19,623 & 1.8 \\
\hline 14 & Ashland Coal Inc. & 16,091 & 1.5 \\
\hline 15 & Marigold Land Co. & 14,731 & 1.4 \\
\hline 16 & Pittston Coal Group & 13,281 & 1.2 \\
\hline 17 & BHP Utah Minerals & 13,228 & 1.2 \\
\hline 18 & Pittsburg \& Midway Coal Mining Co. & 12,946 & 1.2 \\
\hline 19 & Mapco Coal Inc. & 12,844 & 1.2 \\
\hline 20 & Kiewit Coal Properties Inc. & 9,863 & .9 \\
\hline 21 & Costain Coal Inc. & 9,342 & .9 \\
\hline 22 & Coastal Corp (The) & 8,932 & .8 \\
\hline 23 & AEP Service Corp. & 8,652 & .8 \\
\hline 24 & Jamer River Coal Co. & 8,025 & .8 \\
\hline 25 & Anadalex Resources Inc. & 7,613 & .7 \\
\hline 26 & Drummond Company Inc. & 7,342 & .7 \\
\hline 27 & Rochester \& Pittsburgh Coal Co. & 7,315 & .7 \\
\hline 28 & Jim Walter Resources Inc. & 7,313 & .7 \\
\hline 29 & U S steele Mining Co., Inc. & 7,169 & .7 \\
\hline 30 & Black Beauty Coal Co. & 6,628 & 6 \\
\hline 31 & Teco Coal Corp. & 6,615 & .6 \\
\hline 32 & Aluminum Company of America & 5,379 & .5 \\
\hline 33 & Westmoreland Resources Inc. & 5,111 & .5 \\
\hline 34 & General Dynamics Corp. & 4,741 & .4 \\
\hline 35 & Ohio Valley Resources Inc. & 4,538 & .4 \\
\hline 36 & Minnesota Power \& Light & 4,199 & .4 \\
\hline 37 & Hanson PLC & 4,132 & .4 \\
\hline 38 & Lee Ranch Coal Company & 3,828 & .4 \\
\hline 39 & Exxon Corporation & 3,689 & 3 \\
\hline 40 & Sun Co., Inc. & 3,554 & .3 \\
\hline 41 & Kindill Mining Inc. & 3,324 & .3 \\
\hline 42 & Golden Oak Mining Co. LP & 3,243 & 3 \\
\hline 43 & San Miguel Electric CoOp & 3,209 & .3 \\
\hline 44 & Black Hills Corp. & 2,982 & 3 \\
\hline 45 & A N R Coal Co. & 2,948 & .3 \\
\hline 46 & Leslie Resources Inc. & 2,931 & 3 \\
\hline 47 & Addington Enterprises Inc. & 2,758 & .3 \\
\hline 48 & MDU Resources Group Inc. & 2,660 & .2 \\
\hline 49 & Pen Coal Corp. & 2,482 & .2 \\
\hline 50 & Fola Coal Co. & 2,455 & .2 \\
\hline 51 & Dorchester Coal Co., Inc. & 2,434 & .2 \\
\hline 52 & Mincorp Inc. & 2,306 & .2 \\
\hline 53 & Dolet Hills Mining Venture & 2,225 & .2 \\
\hline 54 & Anker Group Inc. & 2,056 & .2 \\
\hline 55 & Maple Creek Mining Inc. & 2,056 & 2 \\
\hline 56 & Appolo Fuels Inc. & 2,014 & 2 \\
\hline 57 & Waterloo Coal Co., Inc. & 2,008 & .2 \\
\hline * & Subtotal & 875,548 & 82.3 \\
\hline * & All other coal producers & 188,308 & 17.7 \\
\hline * & U.S. Total & $1,063,856$ & 100.0 \\
\hline
\end{tabular}

Notes: Major coal producers are companies that produced more than 2 million short tons in 1996 . The company is the firm owning the mineral rights to the mined coal.

Sources: Energy Information Administration, Form EIA-7A "Coal Production Report"; State Mining Agency Coal Production Reports; and/or U.S. Department of Labor, Mine Safety and Health Administration, Form 7000-2, "Quarterly Mine Employment and Production Report." 



\section{Productive Capacity}

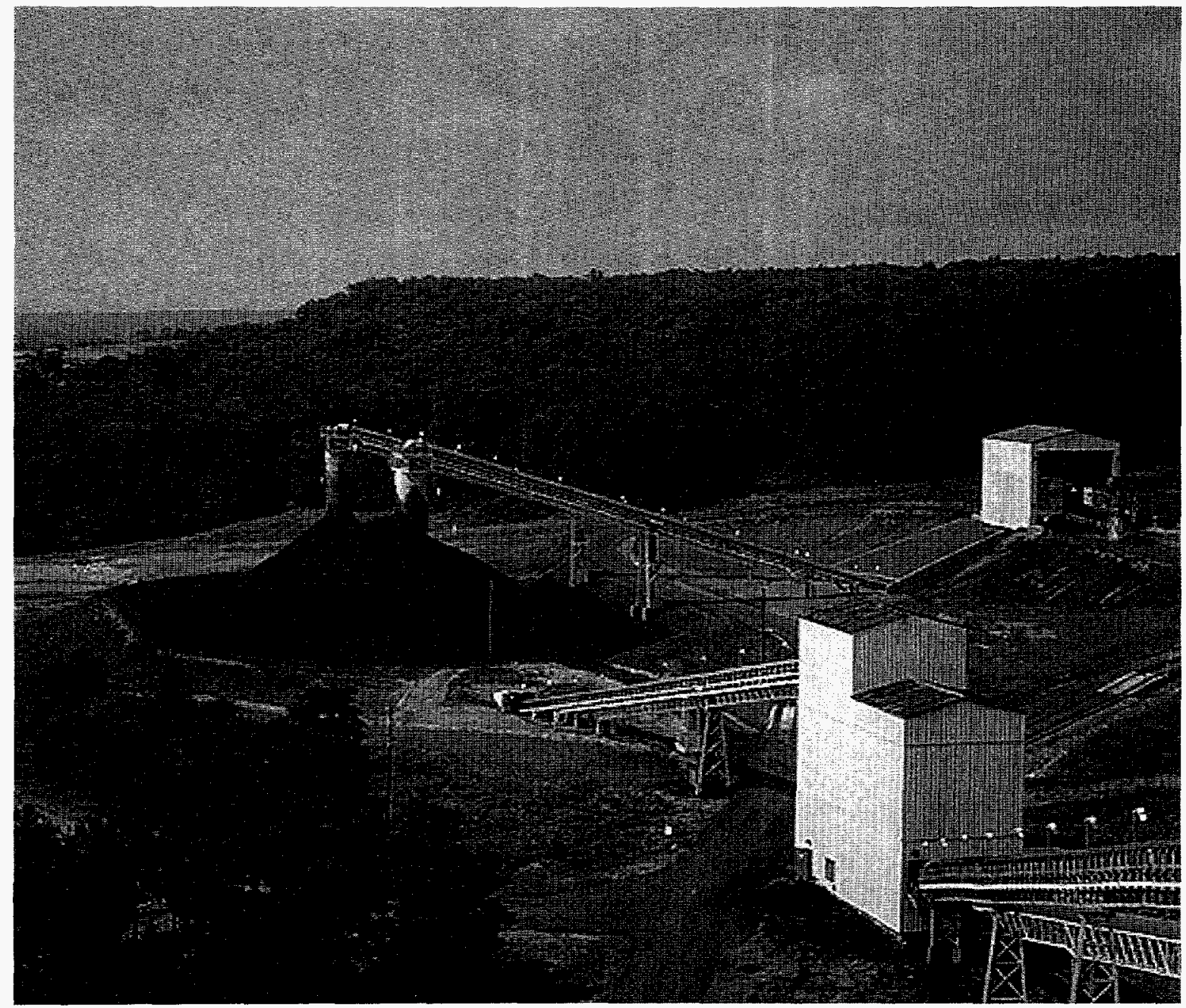

Total productivity for 1996 was 1,325 million short tons, a two percent increase since last year. Capacity utilization was 80.2 percent. 
Table 16. Productive Capacity of Coal Mines by State, 1987, 1992-1996

(Thousand Short Tons)

\begin{tabular}{|c|c|c|c|c|c|c|c|c|c|}
\hline \multirow{2}{*}{$\begin{array}{l}\text { Coal-Producing } \\
\text { State and Region }\end{array}$} & \multirow{2}{*}{1996} & \multirow{2}{*}{1995} & \multirow{2}{*}{1994} & \multirow{2}{*}{1993} & \multirow{2}{*}{1992} & \multirow{2}{*}{$1987^{1}$} & \multirow{2}{*}{$\begin{array}{c}\text { Percent } \\
\text { Change } \\
\text { 1995-1996 }\end{array}$} & \multicolumn{2}{|c|}{ Average Annual Percent Change } \\
\hline & & & & & & & & 1992-1996 & $1987-1996$ \\
\hline Alabama & 32,159 & 32,546 & 33,049 & 27,916 & 29,815 & 29,189 & -1.2 & 1.9 & 1.1 \\
\hline Alaska & w & w & $\mathrm{w}$ & w & $w$ & w & w & w & $\mathbf{w}$ \\
\hline Arizona & $\mathbf{w}$ & w & $w$ & $\mathbf{w}$ & $w$ & w & $w$ & $\mathrm{w}$ & w \\
\hline 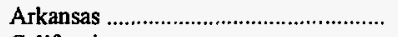 & - & $\mathbf{w}$ & $w$ & w & $\mathbf{w}$ & $\mathbf{w}$ & $\mathbf{w}$ & $\mathbf{w}$ & w \\
\hline 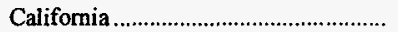 & - & - & - & - & w & w & w & $w$ & $\mathbf{w}$ \\
\hline Colorado & 29,330 & 32,435 & 31,075 & 30,040 & 25,848 & 21,321 & -9.6 & 3.2 & 3.6 \\
\hline 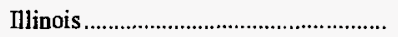 & 61,727 & 56,627 & 69,414 & 69,320 & 75,787 & 75,852 & 9.0 & -5.0 & -2.3 \\
\hline Indiana & 35,564 & 35,256 & 38,931 & 43,955 & 42,990 & 45,344 & .9 & -4.6 & -2.7 \\
\hline Iowa & - & - & w & w & w & w & w & w & w \\
\hline 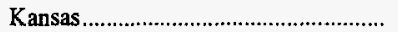 & w & $\mathbf{w}$ & $w$ & $\mathbf{w}$ & w & w & w & w & $\mathbf{w}$ \\
\hline 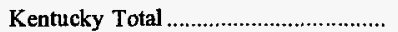 & 189,225 & 203,173 & 213,427 & 204,805 & 195,352 & 185,985 & -6.9 & -.8 & .2 \\
\hline Eastern & 145,691 & 152,111 & 161,731 & 157,318 & 149,046 & 128,874 & -4.2 & -.6 & 1.4 \\
\hline Western & 43,534 & 51,062 & 51,696 & 47,486 & 46,306 & 57,110 & -14.7 & -1.5 & -3.0 \\
\hline 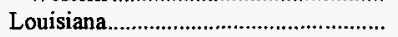 & $w$ & $\mathbf{w}$ & $\mathrm{w}$ & w & w & $w$ & w & $\mathbf{w}$ & w \\
\hline 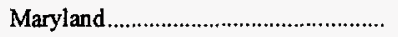 & 4,935 & 4,408 & 4,332 & 3,927 & 3,902 & 4,647 & 11.9 & 6.0 & .7 \\
\hline 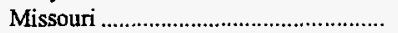 & 1,046 & 1,081 & 1,209 & w & $w$ & w & -3.2 & w & -16.6 \\
\hline Montana & 56,175 & 51,597 & 51,104 & 50,849 & 48,582 & 42,848 & 8.9 & 3.7 & 3.0 \\
\hline 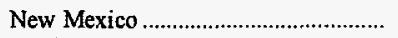 & 32,695 & 32,760 & 32,807 & 33,360 & 29,512 & 33,295 & -.2 & 2.6 & -.2 \\
\hline 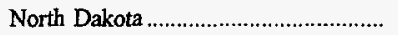 & 32,184 & 34,464 & 35,920 & 36,371 & 36,986 & 39,166 & -6.6 & -3.4 & -2.1 \\
\hline Ohio & 37,584 & 34,011 & 43,925 & 42,236 & 41,329 & 46,337 & 10.5 & -2.3 & -2.3 \\
\hline 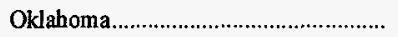 & 1,981 & 2,557 & 2,251 & 2,422 & 2,486 & 3,818 & -22.5 & -5.5 & -7.0 \\
\hline 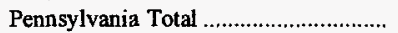 & 81,684 & 77,187 & 80,975 & 82,148 & 82,968 & 86,765 & 5.8 & -.4 & -.7 \\
\hline 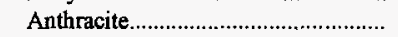 & 5,504 & 6,547 & 5,776 & 5,806 & 4,143 & 3,871 & -15.9 & 7.4 & 4.0 \\
\hline 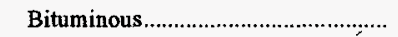 & 76,180 & 70,640 & 75,200 & 76,342 & 78,825 & 82,895 & 7.8 & -.8 & -.9 \\
\hline 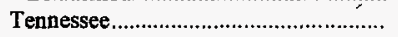 & 4,009 & 3,750 & 3,409 & 3,763 & 3,932 & 7,305 & 6.9 & .5 & -6.4 \\
\hline 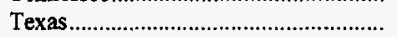 & 59,604 & 54,758 & 55,856 & 57,115 & 58,541 & 51,636 & 8.8 & .4 & 1.6 \\
\hline 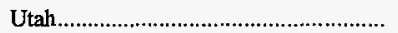 & 30,230 & 30,888 & 27,640 & 25,933 & 25,534 & 22,976 & -2.1 & 4.3 & 3.1 \\
\hline 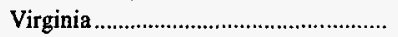 & 41,593 & 43,037 & 46,462 & 50,879 & 54,471 & 49,676 & -3.3 & -6.5 & -1.9 \\
\hline 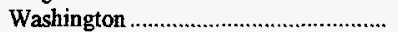 & $\mathbf{w}$ & $w$ & $\mathbf{w}$ & $w$ & $\mathbf{w}$ & $w$ & w & w & w \\
\hline 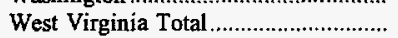 & 217,409 & 204,837 & 201,684 & 191,706 & 198,083 & 158,356 & 6.1 & 2.3 & 3.6 \\
\hline Northern & 54,602 & 56,355 & 59,295 & 60,015 & 62,811 & 59,630 & -3.1 & -3.4 & -1.0 \\
\hline Southern & 162,807 & 148,482 & 142,388 & 131,691 & 135,271 & 98,726 & 9.6 & 4.7 & 5.7 \\
\hline 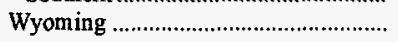 & 350,908 & 337,184 & 321,046 & 277,875 & 253,312 & 247,476 & 4.1 & 8.5 & 3.9 \\
\hline Appalachian Total ${ }^{2} \ldots \ldots \ldots \ldots$ & 565,064 & 551,888 & 575,568 & 559,893 & 563,545 & 511,150 & 2.4 & .1 & 1.1 \\
\hline Interior Total $2 \ldots \ldots \ldots \ldots \ldots \ldots \ldots \ldots \ldots \ldots$ & 207,658 & 205,393 & 223,897 & 225,938 & 235,040 & 245,624 & 1.1 & -3.0 & -1.8 \\
\hline Western Total ${ }^{2}$ & 551,990 & 541,773 & 521,191 & 476,042 & 442,469 & 426,630 & 1.9 & 5.7 & 2.9 \\
\hline East of Miss. River ................................ & 705,890 & 694,832 & 735,609 & $\mathbf{7 2 0 , 6 5 4}$ & 728,628 & 689,457 & 1.6 & -.8 & .3 \\
\hline 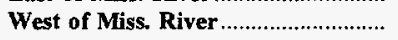 & 618,823 & 604,222 & 585,047 & 541,219 & 512,426 & 493,948 & 2.4 & 4.8 & 2.5 \\
\hline 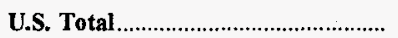 & $1,324,712$ & $1,299,054$ & $1,320,656$ & $1,261,873$ & $1,241,054$ & $1,183,404$ & $\mathbf{2 . 0}$ & 1.6 & 1.3 \\
\hline
\end{tabular}

1 For 1987, the Form ElA-7A solicited data on "Daily Productive Capacity." To obtain annual productive capacity for a mine in 1987, each mine's daily productive capacity was multiplied by the number of days worked during the year.

2 For a definition of coal-producing regions, see Appendix $C$.

Notes: Productive capacity is the maximum amount of coal that can be produced annually as reported by mining companies on Form EIA-7A. Excludes silt, culm, refuse bank, slurry dam, and dredge operations except for Pennsylvania anthracite. Excludes mines producing less than 10,000 short tons, which are not required to provide these data. Totals may not equal sum of components due to independent rounding. Source: Energy Information Administration, Form EIA-7A, "Coal Production Report." 
Table 17. Capacity Utilization of Coal Mines by State, 1987, 1992-1996

(Percent)

\begin{tabular}{|c|c|c|c|c|c|c|}
\hline $\begin{array}{l}\text { Coal-Producing } \\
\text { State and Region }\end{array}$ & 1996 & 1995 & 1994 & 1993 & 1992 & $1987^{1}$ \\
\hline Alabama & 76.57 & 75.52 & 70.19 & 88.46 & 86.26 & 87.23 \\
\hline 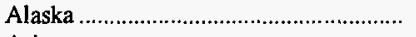 & $\mathbf{w}$ & w & $\mathbf{w}$ & w & $\mathbf{w}$ & w \\
\hline 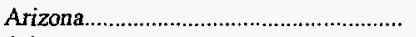 & $\mathbf{w}$ & $w$ & $w$ & $w$ & $w$ & $w$ \\
\hline 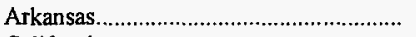 & - & $w$ & $\mathbf{w}$ & $\mathbf{w}$ & w & w \\
\hline 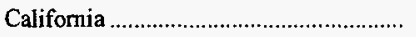 & - & - & - & - & w & w \\
\hline 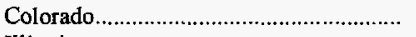 & 84.85 & 79.27 & 81.41 & 72.84 & 74.35 & 67.59 \\
\hline 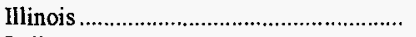 & 75.58 & 85.08 & 76.06 & 59.28 & 78.98 & 77.98 \\
\hline Indiana & 83.42 & 73.70 & 79.37 & 66.60 & 70.86 & 75.36 \\
\hline Iowa & - & - & w & w & w & w \\
\hline 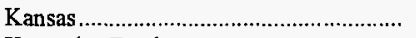 & w & w & w & w & w & w \\
\hline 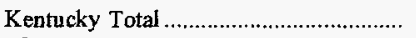 & 80.38 & 75.49 & 75.54 & 76.11 & 82.15 & 88.03 \\
\hline Eastern & 80.07 & 77.71 & 76.70 & 76.15 & 79.72 & 91.94 \\
\hline Western & 81.40 & 68.89 & 71.89 & 76.00 & 89.95 & 79.20 \\
\hline 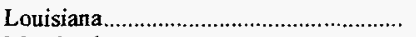 & w & w & w & w & w & $w$ \\
\hline Maryland & 82.42 & 82.65 & 83.07 & 85.01 & 84.50 & 84.48 \\
\hline 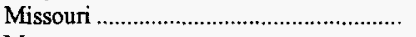 & 67.85 & 49.88 & 69.35 & $\mathbf{w}$ & w & w \\
\hline Montana & 67.45 & 76.44 & 81.47 & 70.64 & 80.03 & 80.28 \\
\hline 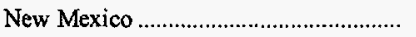 & 73.61 & 81.85 & 85.47 & 84.74 & 83.18 & 57.46 \\
\hline 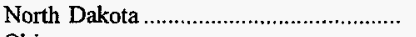 & 92.78 & 87,37 & 89.88 & 87.89 & 85.83 & 64.19 \\
\hline Ohio & 75.88 & 76.55 & 67.87 & 67.94 & 73.19 & 76.72 \\
\hline 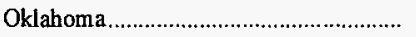 & 85.16 & 73.14 & 83.98 & 71.95 & 69.73 & 74.77 \\
\hline 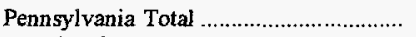 & 82.53 & 78.81 & 75.89 & 71.79 & 82.20 & 80.04 \\
\hline 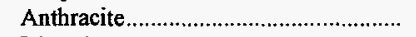 & 82.56 & 67.12 & 74.02 & 68.80 & 75.22 & 79.83 \\
\hline 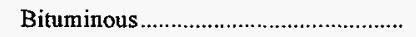 & 82.53 & 79.89 & 76.03 & 72.02 & 82.57 & 80.05 \\
\hline Tennessee & 90.32 & 85.51 & 87.27 & 79.73 & 86.50 & 86.94 \\
\hline 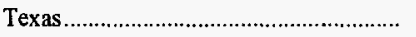 & 92.55 & 96.21 & 93.72 & 95.54 & 94.07 & 97.86 \\
\hline Utah & 90.97 & 81.48 & 88.27 & 84.22 & 83.57 & 71.85 \\
\hline Virginia & 85.34 & 79.07 & 79.61 & 77.07 & 78.70 & 88.93 \\
\hline 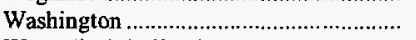 & w & $w$ & w & w & $\mathbf{w}$ & w \\
\hline 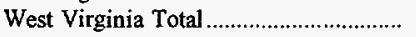 & 78.32 & 79.50 & 80.07 & 67.91 & 81.70 & 85.84 \\
\hline Northern & 83.95 & 81.70 & 83.04 & 56.15 & 79.44 & 86.72 \\
\hline Southern & 76.43 & 78.67 & 78.83 & 73.28 & 82.75 & 85.31 \\
\hline Wyoming & 79.35 & 78.24 & 73.85 & 75.62 & 75.07 & 59.34 \\
\hline 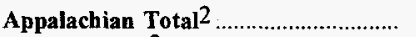 & 79.75 & $\mathbf{7 8 . 5 3}$ & 77.07 & 72.85 & 80.63 & 85.95 \\
\hline Interior Total ${ }^{2}$ & 83.20 & 82.01 & 80.29 & 73.95 & 83.22 & 82.05 \\
\hline 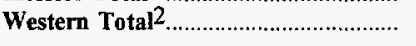 & 79.55 & 79.29 & 78.33 & 77.41 & 78.04 & 64.18 \\
\hline 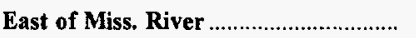 & 79.68 & 78.11 & 76.73 & 71.37 & 80.47 & 83.82 \\
\hline West of Miss. River .................................. & 80.82 & 80.88 & 79.85 & 79.29 & 79.80 & 68.18 \\
\hline U.S. Total & 80.21 & 79.40 & 78.11 & 74.77 & 80.20 & 77.29 \\
\hline
\end{tabular}

1 For 1987, the Form EIA-7A solicited data on "Daily Productive Capacity." To obtain annual productive capacity for a mine in 1987, each mine's daily productive capacity was multiplied by the number of days worked during the year.

2 For a definition of coal-producing regions, see Appendix $C$.

Notes: Capacity utilization is the ratio of total production to annual productive capacity as reported by mining companies on Form ElA-7A. Excludes silt, culm, refuse bank, slurry dam, and dredge operations except for Pennsylvania anthracite. Excludes mines producing less than 10,000 short tons, which are not required to provide these data.

Source: Energy Information Administration, Form EIA-7A, "Coal Production Report." 
Table 18. Production, Productive Capacity, and Capacity Utilization of Coal Mines by State and Mine Type, 1996

(Thousand Short Tons)

\begin{tabular}{|c|c|c|c|c|c|c|c|c|c|}
\hline \multirow[b]{2}{*}{$\begin{array}{l}\text { Coal-Producing } \\
\text { State and Region }\end{array}$} & \multicolumn{3}{|c|}{ Underground } & \multicolumn{3}{|c|}{ Surface } & \multicolumn{3}{|c|}{ Total } \\
\hline & Production & $\begin{array}{l}\text { Productive } \\
\text { Capacity }\end{array}$ & $\begin{array}{l}\text { Capacity } \\
\text { Utilization } \\
\text { (percent) }\end{array}$ & Production & $\begin{array}{l}\text { Productive } \\
\text { Capacity }\end{array}$ & $\begin{array}{l}\text { Capacity } \\
\text { Utilization } \\
\text { (percent) }\end{array}$ & Production & $\begin{array}{l}\text { Productive } \\
\text { Capacity }\end{array}$ & $\begin{array}{l}\text { Capacity } \\
\text { Utilization } \\
\text { (percent) }\end{array}$ \\
\hline 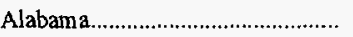 & 18,217 & 23,207 & 78.50 & 6,406 & 8,952 & 71.55 & 24,623 & 32,159 & 76.57 \\
\hline 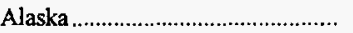 & - & - & - & 1,481 & $w$ & w & 1,481 & w & w \\
\hline 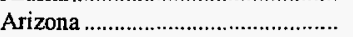 & - & - & - & 10,442 & $w$ & w & 10,442 & w & $\mathbf{w}$ \\
\hline Colorado & 15,581 & 18,530 & 84.08 & 9,305 & 10,800 & 86.16 & 24,886 & 29,330 & 84.85 \\
\hline 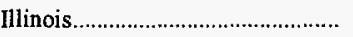 & 38,948 & 51,816 & 75.17 & 7,704 & 9,912 & 77.73 & 46,652 & 61,727 & 75.58 \\
\hline Indiana & 2,963 & w & $\mathbf{w}$ & 26,704 & w & w & 29,667 & 35,564 & 83.42 \\
\hline 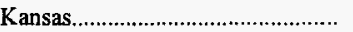 & - & - & - & 232 & $\mathbf{w}$ & $w$ & 232 & w & $w$ \\
\hline 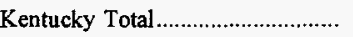 & 94,153 & 114,922 & 81.93 & 57,939 & 74,302 & 77.98 & 152,092 & 189,225 & 80.38 \\
\hline 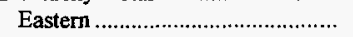 & 69,018 & 86,042 & 80.21 & 47,635 & 59,649 & 79.86 & 116,654 & 145,691 & 80.07 \\
\hline 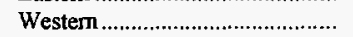 & 25,135 & 28,880 & 87.03 & 10,304 & 14,653 & 70.32 & 35,438 & 43,534 & 81.40 \\
\hline 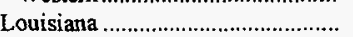 & - & - & - & 3,221 & w & w & 3,221 & w & w \\
\hline 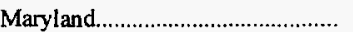 & 3,296 & $\mathrm{w}$ & $w$ & 772 & w & $\mathbf{w}$ & 4,068 & 4,935 & 82.42 \\
\hline 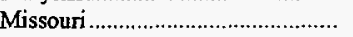 & - & - & - & 710 & 1,046 & 67.85 & 710 & 1,046 & 67.85 \\
\hline 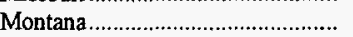 & 147 & w & $\mathbf{w}$ & 37,744 & w & w & 37,891 & 56,175 & 67.45 \\
\hline 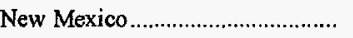 & - & - & - & 24,067 & 32,695 & 73.61 & 24,067 & 32,695 & 73.61 \\
\hline 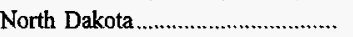 & - & - & - & 29,861 & 32,184 & 92.78 & 29,861 & 32,184 & 92.78 \\
\hline 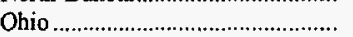 & 15,912 & 17,313 & 91.91 & 12,608 & 20,272 & 62.19 & 28,520 & 37,584 & 75.88 \\
\hline 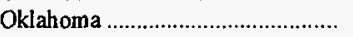 & 137 & $w$ & $w$ & 1,550 & w & w & 1,687 & 1,981 & 85.16 \\
\hline 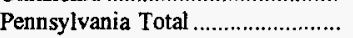 & 47,140 & 54,579 & 86.37 & 20,272 & 27,105 & 74.79 & 67,411 & 81,684 & 82.53 \\
\hline Anthracite & 284 & 459 & 61.96 & 4,259 & 5,045 & 84.44 & 4,544 & 5,504 & 82.56 \\
\hline 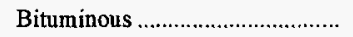 & 46,855 & 54,120 & 86.58 & 16,012 & 22,060 & 72.58 & 62,868 & 76,180 & 82.53 \\
\hline 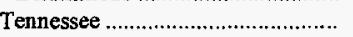 & 1,790 & w & w & 1,830 & w & $w$ & 3,621 & 4,009 & 90.32 \\
\hline 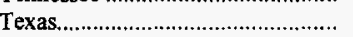 & - & - & - & 55,164 & 59,604 & 92.55 & 55,164 & 59,604 & 92.55 \\
\hline Utah & 27,499 & 30,230 & 90.97 & 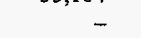 & - & - & 27,499 & 30,230 & 90.97 \\
\hline 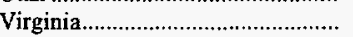 & 25,500 & 30,146 & 84.59 & 9,995 & 11,448 & 87.31 & 35,495 & 41,593 & 85.34 \\
\hline 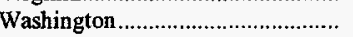 & - & - & - & 4,565 & $w$ & $w$ & 4,565 & w & w \\
\hline West Virginia Total ......................... & 115,497 & 143,797 & 80.32 & 54,776 & 73,612 & 74.41 & 170,273 & 217,409 & 78.32 \\
\hline Northem & 40,251 & 46,028 & 87.45 & 5,587 & 8,575 & 65.16 & 45,838 & 54,602 & 83.95 \\
\hline 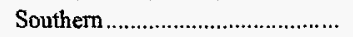 & 75,246 & 97,769 & 76.96 & 49,189 & 65,038 & 75.63 & 124,435 & 162,807 & 76.43 \\
\hline Wyoming & 2,641 & w & w & 275,799 & $\mathbf{w}$ & w & 278,440 & 350,908 & 79.35 \\
\hline Appalachian Totall ........................ & 296,370 & 361,103 & 82.07 & 154,294 & 203,961 & 75.65 & 450,664 & 565,064 & 79.75 \\
\hline 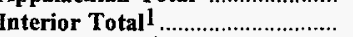 & 67,183 & 84,166 & 79.82 & 105,588 & 123,492 & 85.50 & 172,771 & 207,658 & 83.20 \\
\hline Western Total 1 .............................. & 45,868 & 52,160 & 87.94 & 393,265 & 499,830 & 78.68 & 439,133 & 551,990 & 79.55 \\
\hline East of Miss. River........................ & 363,416 & 444,969 & 81.67 & 199,006 & 260,920 & 76.27 & 562,422 & $\mathbf{7 0 5 , 8 9 0}$ & 79.68 \\
\hline West of Miss. River...................... & 46,005 & 52,460 & 87.70 & 454,141 & 566,363 & 80.19 & 500,146 & 618,823 & 80.82 \\
\hline 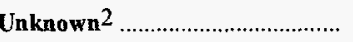 & 428 & NA & NA & 860 & NA & NA & 1,288 & NA & $\mathbf{N A}$ \\
\hline U.S. Total. & 409,849 & 497,429 & 82.31 & 654,007 & 827,283 & 78.95 & $1,063,856$ & $1,324,713$ & 80.21 \\
\hline
\end{tabular}

1 For a definition of coal-producing regions, see Appendix $C$.

2 Includes mines with production of less than 10,000 short tons, which are required to provide only production data.

NA Not available.

Notes: Productive capacity is the maximum amount of coal that can be produced annually as reported by mining companies on Form EIA-7A. Capacity utilization is the ratio of total production to annual productive capacity as reported by mining companies on Form EIA-7A. Coal production excludes silt culm, refuse bank, slurry dam, and dredge operations except for Pennsylvania anthracite. Totals may not equal sum of components due to independent rounding.

Sources: Energy Information Administration, Form EIA-7A, "Coal Production Report"; State Mining Agency Coal Production Reports; and/or U.S. Department of Labor, Mine Safety and Health Administration, Form 7000-2, "Quarterly Mine Employment and Coal Production Report." 
Table 19. Productive Capacity and Capacity Utilization of Underground Coal Mines by State and Mining Method, 1996

(Thousand Short Tons)

\begin{tabular}{|c|c|c|c|c|c|c|c|c|}
\hline \multirow[b]{2}{*}{$\begin{array}{l}\text { Coal-Producing } \\
\text { State and Region }\end{array}$} & \multicolumn{2}{|c|}{ Continuous ${ }^{1}$} & \multicolumn{2}{|c|}{ Conventional ${ }^{1}$} & \multicolumn{2}{|c|}{ Longwall 1} & \multicolumn{2}{|c|}{ Other $^{12}$} \\
\hline & $\begin{array}{l}\text { Productive } \\
\text { Capacity }\end{array}$ & $\begin{array}{l}\text { Capacity } \\
\text { Utilization } \\
\text { (percent) }\end{array}$ & $\begin{array}{l}\text { Productive } \\
\text { Capacity }\end{array}$ & $\begin{array}{l}\text { Capacity } \\
\text { Utilization } \\
\text { (percent) }\end{array}$ & $\begin{array}{c}\text { Productive } \\
\text { Capacity }\end{array}$ & $\begin{array}{l}\text { Capacity } \\
\text { Utilization } \\
\text { (percent) }\end{array}$ & $\begin{array}{l}\text { Productive } \\
\text { Capacity }\end{array}$ & $\begin{array}{l}\text { Capacity } \\
\text { Utilization } \\
\text { (percent) }\end{array}$ \\
\hline Alabama & $\mathbf{w}$ & $\mathbf{w}$ & $w$ & w & 16,277 & 80.11 & - & - \\
\hline Colorado & $\mathbf{w}$ & w & $\mathbf{w}$ & $\mathbf{w}$ & 9,677 & 96.60 & $\mathbf{w}$ & $\mathbf{w}$ \\
\hline 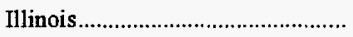 & 34,219 & 72.07 & $w$ & $w$ & $\mathbf{w}$ & w & - & - \\
\hline Indiana & w & w & - & - & - & - & - & - \\
\hline Kentucky Total & 87,876 & 82.75 & w & w & 12,450 & 87.52 & $\mathbf{w}$ & $\mathbf{w}$ \\
\hline Eastern & 65,449 & 81.61 & $\mathbf{w}$ & $\mathbf{w}$ & $w$ & w & $\mathbf{w}$ & $w$ \\
\hline 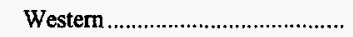 & 22,427 & 86.09 & $\mathbf{w}$ & $\mathbf{w}$ & w & w & - & - \\
\hline 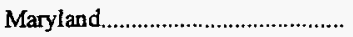 & w & $w$ & - & - & $w$ & $w$ & - & - \\
\hline Montana & $\mathbf{w}$ & w & - & - & - & - & - & - \\
\hline 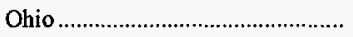 & 4,633 & 80.34 & - & - & 12,680 & 96.13 & - & - \\
\hline Oklahoma & w & $w$ & - & - & - & - & - & - \\
\hline 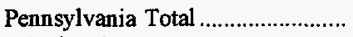 & 20,869 & 83.43 & w & $w$ & 31,250 & 90.08 & $w$ & $\mathbf{w}$ \\
\hline 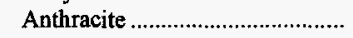 & $w$ & w & $\mathbf{w}$ & $\mathbf{w}$ & - & - & $w$ & w \\
\hline Bituminous & w & $\mathbf{w}$ & $w$ & $w$ & 31,250 & 90.08 & - & - \\
\hline 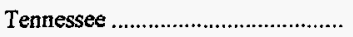 & $w$ & $w$ & $\mathbf{w}$ & $w$ & - & - & - & - \\
\hline Utah & w & w & $w$ & w & 21,763 & 91.55 & - & - \\
\hline 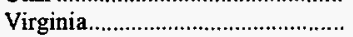 & 14,781 & 89.14 & w & w & $w$ & $w$ & - & - \\
\hline West Virginia Total ........................ & 82,132 & 76.69 & 16,700 & 76.71 & 44,965 & 86.42 & - & - \\
\hline Northem & 13,357 & 81.42 & 2,486 & 71.92 & 30,185 & 91.29 & - & - \\
\hline Southern & 68,776 & 75.78 & 14,213 & 77.55 & 14,780 & 76.49 & - & - \\
\hline 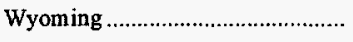 & w & w & - & - & $\mathrm{w}$ & w & - & - \\
\hline Appalachian Totak $3 \ldots \ldots \ldots \ldots \ldots$ & 198,144 & 79.93 & $\mathbf{w}$ & $\mathbf{w}$ & $\mathbf{w}$ & $\mathbf{w}$ & $\mathbf{w}$ & $\mathbf{w}$ \\
\hline 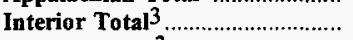 & 60,116 & 78.29 & $\mathbf{w}$ & $\mathbf{w}$ & $\mathbf{w}$ & $\mathbf{w}$ & - & - \\
\hline Western Total ${ }^{3} \ldots \ldots \ldots \ldots \ldots \ldots \ldots \ldots \ldots$ & 15,257 & 80.81 & $\mathbf{w}$ & $\mathbf{w}$ & 34,050 & 92.95 & $\mathbf{w}$ & $\mathbf{w}$ \\
\hline 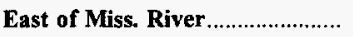 & 257,960 & 79.59 & $\mathbf{w}$ & $\mathbf{w}$ & 145,311 & 86.83 & $\mathbf{w}$ & $\mathbf{w}$ \\
\hline West of Miss. River.......................... & 15,557 & 80.13 & $\mathbf{w}$ & $\mathbf{w}$ & 34,050 & 92.95 & $\mathbf{w}$ & $\mathbf{w}$ \\
\hline U.S. Total. & 273,517 & 79.62 & 43,910 & 72.16 & 179,361 & 87.99 & 640 & 63.11 \\
\hline
\end{tabular}

1 Calculated by multiplying reported mining method percentages by the individual mine capacity.

2 Includes shortwall, scoop loading, hand loading and unknown.

3 For a definition of coal-producing regions, see Appendix $\mathrm{C}$.

Notes: Productive capacity is the maximum amount of coal that can be produced annually as reported by mining companies on Form EIA-7A. Capac-

ity utilization is the ratio of total production to annual productive capacity as reported by mining companies on Form EIA-7A. Excludes mines producing

less than 10,000 short tons, which are not required to provide these data. Totals may not equal sum of components due to independent rounding.

Source: Energy Information Administration, Form EIA-7A, "Coal Production Report." 
Table 20. Productive Capacity and Capacity Utilization of Coal Mines by State and Coal Rank, 1996

(Thousand Short Tons)

\begin{tabular}{|c|c|c|c|c|c|c|c|c|}
\hline \multirow[b]{2}{*}{$\begin{array}{l}\text { Coal-Producing } \\
\text { State and Region }\end{array}$} & \multicolumn{2}{|c|}{ Bituminous } & \multicolumn{2}{|c|}{ Subbituminous } & \multicolumn{2}{|c|}{ Lignite } & \multicolumn{2}{|c|}{ An thracite } \\
\hline & $\begin{array}{l}\text { Productive } \\
\text { Capacity }\end{array}$ & $\begin{array}{l}\text { Capacity } \\
\text { Utilization } \\
\text { (percent) }\end{array}$ & $\begin{array}{c}\text { Productive } \\
\text { Capacity }\end{array}$ & $\begin{array}{l}\text { Capacity } \\
\text { Utilization } \\
\text { (percent) }\end{array}$ & $\begin{array}{c}\text { Productive } \\
\text { Capacity }\end{array}$ & $\begin{array}{l}\text { Capacity } \\
\text { Utilization } \\
\text { (percent) }\end{array}$ & $\begin{array}{l}\text { Productive } \\
\text { Capacity }\end{array}$ & $\begin{array}{l}\text { Capacity } \\
\text { Utilization } \\
\text { (percent) }\end{array}$ \\
\hline 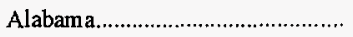 & 32,159 & 76.57 & - & - & - & - & - & - \\
\hline 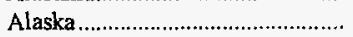 & - & - & $w$ & $w$ & - & - & - & - \\
\hline 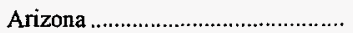 & w & w & - & - & - & - & - & - \\
\hline Colorado & 18,930 & 84.22 & 10,400 & 86.00 & - & - & - & - \\
\hline 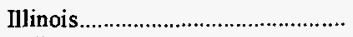 & 61,727 & 75.58 & - & - & - & - & - & - \\
\hline Indiana & 35,564 & 83.42 & - & - & - & - & - & - \\
\hline 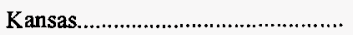 & $w$ & w & - & - & - & - & - & - \\
\hline 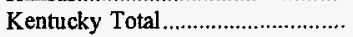 & 189,225 & 80.38 & - & - & - & - & - & - \\
\hline Eastern & 145,691 & 80.07 & - & - & - & - & - & - \\
\hline 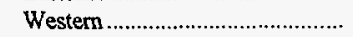 & 43,534 & 81.40 & - & - & - & - & - & - \\
\hline 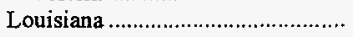 & - & - & - & - & w & $\mathrm{w}$ & - & - \\
\hline 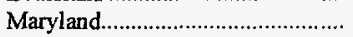 & 4,935 & 82.42 & - & - & - & - & - & - \\
\hline 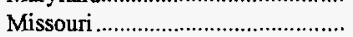 & 1,046 & 67.85 & - & - & - & - & - & - \\
\hline 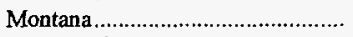 & - & - & w & $w$ & w & w & - & - \\
\hline New Mexico & w & w & w & w & - & - & - & - \\
\hline 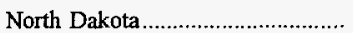 & - & - & - & - & 32,184 & 92.78 & - & - \\
\hline Ohio & 37,584 & 75.88 & - & - & - & - & - & - \\
\hline Oklahoma & 1,981 & 85.16 & - & - & - & - & - & - \\
\hline Pennsylvania Total ............................ & 76,180 & 82.53 & - & - & - & - & 5,504 & 82.56 \\
\hline Anthracite & - & - & - & - & - & - & 5,504 & 82.56 \\
\hline 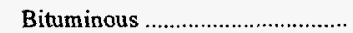 & 76,180 & 82.53 & - & - & - & - & - & - \\
\hline 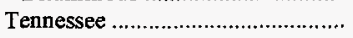 & 4,009 & 90.32 & - & - & - & - & - & - \\
\hline 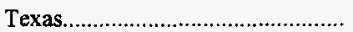 & $w$ & w & - & - & w & w & - & - \\
\hline Utah & 30,230 & 90.97 & - & - & - & - & - & - \\
\hline Virginia & 41,593 & 85.34 & - & - & - & - & - & - \\
\hline 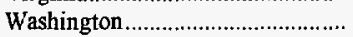 & w & w & $w$ & w & - & - & - & - \\
\hline 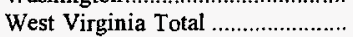 & 217,409 & 78.32 & - & - & - & - & - & - \\
\hline 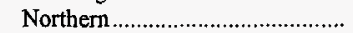 & 54,602 & 83.95 & - & - & - & - & - & - \\
\hline 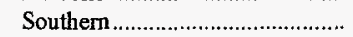 & 162,807 & 76.43 & - & - & - & - & - & - \\
\hline Wyoming & w & w & $\mathbf{w}$ & $\mathbf{w}$ & - & - & - & - \\
\hline Appalachian Total 1 ...................... & 559,560 & 79.73 & - & - & - & - & 5,504 & 82.56 \\
\hline Interior Total 1 & $\mathbf{w}$ & $\mathbf{w}$ & - & - & $\mathbf{w}$ & $\mathbf{w}$ & - & - \\
\hline Western Total ${ }^{1} \ldots \ldots \ldots \ldots \ldots \ldots \ldots .$. & $\mathbf{w}$ & $\mathbf{w}$ & 433,106 & 78.57 & $\mathbf{w}$ & $\mathbf{w}$ & - & - \\
\hline East of Miss. River........................ & 700,386 & 79.65 & - & - & - & - & 5,504 & 82.56 \\
\hline West of Miss. River....................... & 90,138 & 79.66 & 433,106 & 78.57 & 95,579 & 92.13 & - & - \\
\hline 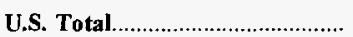 & 790,523 & 79.65 & 433,106 & 78.57 & 95,579 & 92.13 & 5,504 & 82.56 \\
\hline
\end{tabular}

1 For a definition of coal-producing regions, see Appendix C.

w Withbeld to avoid disclosure of individual company data.

Notes: Refer to the Classification of Coals by Rank table in Appendix $\mathrm{C}$ for coal rank definitions. Productive capacity is the maximum amount of coal that can be produced annually as reported by mining companies on Form EIA-7A. Capacity utilization is the ratio of total production to annual productive capacity as reported by mining companies on Form ElA-7A. Excludes silt, culm, refuse bank, slurry dam, and dredge operations except for Pennsylvania anthracite. Excludes mines producing less than 10,000 short tons, which are not required to provide these data. Totals may not equal sum of components due to independent rounding.

Source: Energy Information Administration, Form EIA-7A, "Coal Production Report." 
Table 21. Productive Capacity and Capacity Utilization of Coal Mines by State and Mine Production Range, 1996

(Thousand Short Tons, Percent)

\begin{tabular}{|c|c|c|c|c|c|c|c|c|c|c|}
\hline \multirow{3}{*}{$\begin{array}{l}\text { Coal-Producing } \\
\text { State and Region }\end{array}$} & \multicolumn{5}{|c|}{ Productive Capacity } & \multicolumn{5}{|c|}{$\begin{array}{c}\text { Capacity } \\
\text { Utilization } \\
\text { (percent) }\end{array}$} \\
\hline & \multicolumn{10}{|c|}{$\begin{array}{l}\text { Mine Production Range } \\
\text { (thousand short tons) }\end{array}$} \\
\hline & $\begin{array}{l}1,000 \\
\text { and } \\
\text { over }\end{array}$ & $\begin{array}{c}500 \\
\text { to } \\
1,000\end{array}$ & $\begin{array}{c}200 \\
\text { to } \\
500\end{array}$ & $\begin{array}{c}100 \\
\text { to } \\
200\end{array}$ & $\begin{array}{c}10 \\
\text { to } \\
100\end{array}$ & $\begin{array}{c}1,000 \\
\text { and } \\
\text { over }\end{array}$ & $\begin{array}{c}500 \\
\text { to } \\
1,000\end{array}$ & $\begin{array}{c}200 \\
\text { to } \\
500\end{array}$ & $\begin{array}{c}100 \\
\text { to } \\
200\end{array}$ & $\begin{array}{c}10 \\
\text { to } \\
100\end{array}$ \\
\hline 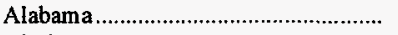 & 20,947 & 5,887 & $w$ & $\mathbf{w}$ & $w$ & 83.49 & 65.66 & $\mathbf{w}$ & $\mathbf{w}$ & w \\
\hline 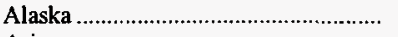 & $\mathbf{w}$ & - & - & - & - & w & - & - & - & - \\
\hline Arizona & w & - & - & - & - & w & - & - & - & - \\
\hline 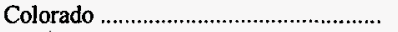 & 23,700 & $w$ & 1,420 & $\mathbf{w}$ & w & 92.49 & $\mathbf{w}$ & 93.19 & $\mathbf{w}$ & $\mathbf{w}$ \\
\hline 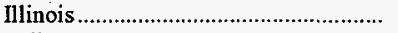 & 48,996 & w & $\mathbf{w}$ & $w$ & 3,954 & 87.64 & w & w & $\mathbf{w}$ & 4.04 \\
\hline Indiana & 19,988 & 10,305 & 4,187 & $w$ & w & 92.19 & 71.18 & 79.43 & $\mathbf{w}$ & w \\
\hline Kansas & - & - & $\mathbf{w}$ & - & - & - & - & w & - & - \\
\hline Kentucky Total ....................................... & 59,849 & 50,784 & 42,994 & 16,250 & 19,348 & 91.22 & 85.92 & 77.01 & 73.93 & 45.18 \\
\hline Eastern & 37,253 & 37,518 & 38,421 & 15,371 & 17,128 & 91.46 & 88.89 & 77.86 & 72.75 & 47.50 \\
\hline Western & 22,596 & 13,266 & 4,573 & 878 & 2,220 & 90.81 & 77.53 & 69.89 & 94.66 & 27.29 \\
\hline 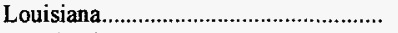 & w & w & - & - & - & $w$ & $\mathbf{w}$ & - & - & - \\
\hline Maryland & $\mathbf{w}$ & - & $\mathbf{w}$ & $\mathbf{w}$ & 850 & w & - & $\mathbf{w}$ & w & 51.42 \\
\hline 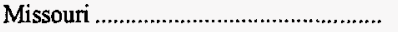 & - & - & $\mathbf{w}$ & $\mathbf{w}$ & $\mathbf{w}$ & - & - & $\mathbf{w}$ & $\mathbf{w}$ & $\mathbf{w}$ \\
\hline Montana & 51,234 & $w$ & $\mathbf{w}$ & $\mathbf{w}$ & - & 71.99 & $\mathbf{w}$ & w & w & - \\
\hline New Mexico & 32,695 & - & - & - & - & 73.61 & - & - & - & - \\
\hline North Dakota & 32,184 & - & - & - & - & 92.78 & - & - & - & - \\
\hline Obio & 19,851 & 4,421 & 3,974 & 3,824 & 5,515 & 89.80 & 71.69 & 81.73 & 64.94 & 32.50 \\
\hline Oklahoma & - & - & w & 1,150 & w & - & - & w & 64.17 & w \\
\hline 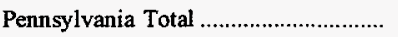 & 45,356 & 6,175 & 13,089 & 8,498 & 8,566 & 87.25 & 92.28 & 79.45 & 65.87 & 71.72 \\
\hline 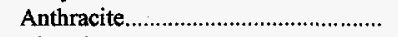 & - & $\mathbf{w}$ & 2,013 & 583 & 2,305 & - & w & 92.66 & 100.00 & 67.61 \\
\hline 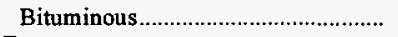 & 45,356 & $\mathbf{w}$ & 11,076 & 7,915 & 6,260 & 87.25 & w & 77.05 & 63.35 & 73.23 \\
\hline 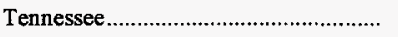 & - & $w$ & 2,303 & 717 & $w$ & - & $w$ & 98.03 & 79.73 & w \\
\hline Texas & 55,004 & $\mathbf{w}$ & $\mathbf{w}$ & - & - & 97.94 & $w$ & w & - & - \\
\hline Utah & 28,649 & $\mathbf{w}$ & - & - & - & 90.55 & $\mathbf{w}$ & - & - & - \\
\hline 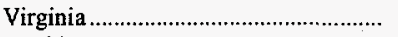 & w & $\mathbf{w}$ & 12,713 & 5,858 & 6,096 & w & $\mathbf{w}$ & 88.90 & 86.46 & 68.08 \\
\hline Washington & w & - & - & $w$ & - & $w$ & - & - & w & - \\
\hline West Virginia Total ................................. & 119,864 & 35,418 & 29,692 & 7,655 & 24,780 & 84.75 & 91.17 & 80.83 & 77.20 & 26.16 \\
\hline Northern . & 39,698 & 5,396 & 4,317 & 1,434 & 3,757 & 89.76 & 74.55 & 76.69 & 90.60 & 41.87 \\
\hline Southern & 80,166 & 30,022 & 25,375 & 6,221 & 21,023 & 82.27 & 94.16 & 81.54 & 74.12 & 23.35 \\
\hline Wyoming & 340,508 & $w$ & w & w & w & 81.16 & w & w & $w$ & w \\
\hline Appalachian Total ${ }^{1} \ldots \ldots \ldots \ldots \ldots \ldots \ldots$ & 255,770 & 97,396 & 102,284 & 44,458 & 65,156 & 86.57 & 88.28 & 80.83 & 71.86 & 43.95 \\
\hline Interior Total 1 . & 149,584 & 32,703 & 15,048 & $\mathbf{w}$ & $\mathbf{w}$ & 92.38 & 65.07 & 65.12 & $\mathbf{w}$ & $\mathbf{w}$ \\
\hline Western Total ${ }^{1} \ldots \ldots \ldots \ldots \ldots \ldots \ldots \ldots \ldots \ldots \ldots \ldots$ & 529,234 & 10,056 & 3,861 & $\mathbf{w}$ & $\mathbf{w}$ & 81.50 & 48.76 & 48.12 & $\mathbf{w}$ & $\mathbf{w}$ \\
\hline East of Miss. River & 347,351 & 125,649 & 115,009 & 45,848 & 72,033 & $\mathbf{8 7 . 3 2}$ & 84.29 & 78.53 & $\mathbf{7 2 . 6 1}$ & 41.09 \\
\hline 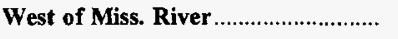 & 587,238 & 14,506 & 6,184 & 10,197 & 698 & 83.04 & 43.14 & 64.94 & 20.22 & 24.08 \\
\hline 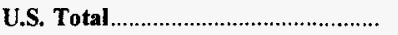 & 934,589 & 140,154 & 121,193 & 56,045 & $\mathbf{7 2 , 7 3 1}$ & 84.63 & 80.03 & 77.84 & 63.08 & 40.93 \\
\hline
\end{tabular}

1 For a definition of coal-producing regions, see Appendix C.

Notes: Productive capacity is the maximum amount of coal that can be produced annually as reported by mining companies on Form EIA-7A. Capacity utilization is the ratio of total production to annual productive capacity as reported by mining companies on Form EIA-7A. Excludes silt, culm, refuse bank, slurry dam, and dredge operations except for Pennsylvania anthracite. Excludes mines producing less than 10,000 short tons, which are not required to provide these data. Totals may not equal sum of components due to independent rounding.

Source: Energy Information Administration, Form ELA-7A, "Coal Production Report." 
Table 22. Productive Capacity and Productivity of Coal Mines by State and Capacity Utilization Range, 1996

(Thousand Short Tons, Short Tons per Miner per Hour)

\begin{tabular}{|c|c|c|c|c|c|c|c|c|c|c|c|c|}
\hline \multirow{3}{*}{$\begin{array}{l}\text { Coal-Producing } \\
\text { State and Region }\end{array}$} & \multicolumn{6}{|c|}{ Froductive Capacity } & \multicolumn{6}{|c|}{ Productivity } \\
\hline & \multicolumn{12}{|c|}{$\begin{array}{l}\text { Capacity Utilization Range } \\
\text { (percent) }\end{array}$} \\
\hline & $\begin{array}{c}90 \\
\text { and } \\
\text { over }\end{array}$ & $\begin{array}{l}80 \\
\text { to } \\
90\end{array}$ & $\begin{array}{l}70 \\
\text { to } \\
80\end{array}$ & $\begin{array}{l}60 \\
\text { to } \\
70\end{array}$ & $\begin{array}{c}\text { Less } \\
\text { than } \\
60\end{array}$ & Total & $\begin{array}{c}90 \\
\text { and } \\
\text { over }\end{array}$ & $\begin{array}{l}80 \\
\text { to } \\
90\end{array}$ & $\begin{array}{l}70 \\
\text { to } \\
80\end{array}$ & $\begin{array}{c}60 \\
\text { to } \\
70\end{array}$ & $\begin{array}{c}\text { Less } \\
\text { than } \\
60\end{array}$ & Total \\
\hline Alabama & 9,825 & 8,405 & w & $\mathbf{w}$ & 6,595 & 32,159 & 2.73 & 2.28 & 2.06 & 2.59 & 1.28 & 2.20 \\
\hline Alaska & - & - & - & $w$ & - & w & - & - & - & 6.81 & - & 6.81 \\
\hline 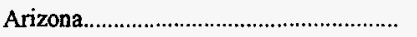 & - & $\mathbf{w}$ & $\mathbf{w}$ & - & - & w & - & 6.92 & 6.33 & - & - & 6.30 \\
\hline Colorado & 19,700 & $\mathbf{w}$ & w & $\mathbf{w}$ & $\mathbf{w}$ & 29,330 & 9.02 & 2.80 & 7.92 & 6.22 & 2.62 & 7.32 \\
\hline 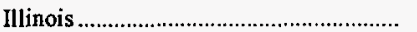 & 29,045 & $\mathbf{w}$ & 7,083 & $\mathbf{w}$ & 13,850 & 61,727 & 4.92 & 3.98 & 3.38 & 4.28 & 2.36 & 4.18 \\
\hline Indiana & 19,239 & $\mathbf{w}$ & w & $\mathbf{w}$ & 6,075 & 35,564 & 4.86 & 5.23 & 5.96 & 5.54 & 4.56 & 4.98 \\
\hline 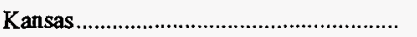 & $w$ & - & - & - & - & w & 2.17 & - & - & - & - & 2.17 \\
\hline 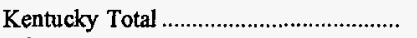 & 88,434 & 34,232 & 16,258 & 14,524 & 35,777 & 189,225 & 4.39 & 4.39 & 4.28 & 3.82 & 1.71 & 3.80 \\
\hline Eastern & 70,120 & 24,050 & $w$ & $w$ & 28,667 & 145,691 & 4.33 & 4.39 & 4.10 & 3.77 & 1.55 & 3.68 \\
\hline 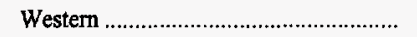 & 18,314 & 10,182 & w & $\mathbf{w}$ & 7,110 & 43,534 & 4.63 & 4.38 & 4.64 & 4.16 & 2.68 & 4.29 \\
\hline 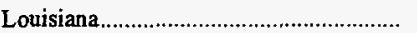 & - & w & - & - & - & w & - & 10.86 & - & - & - & 10.86 \\
\hline 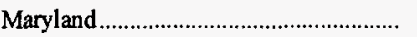 & 3,191 & $\mathbf{w}$ & - & $w$ & 1,039 & 4,935 & 5.22 & 2.98 & - & 2.26 & 2.26 & 4.13 \\
\hline 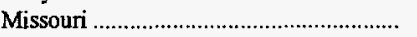 & w & - & - & - & w & 1,046 & 4.66 & - & - & - & 1.49 & 3.49 \\
\hline Montana & $\mathbf{w}$ & $w$ & $\mathbf{w}$ & - & 21,075 & 56,175 & 22.32 & 18.60 & 30.75 & - & 15.96 & 21.88 \\
\hline 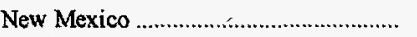 & w & $\mathbf{w}$ & $\mathbf{w}$ & $\mathbf{w}$ & - & 32,695 & 7.93 & 5.47 & 7.63 & 10.70 & - & 8.45 \\
\hline 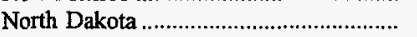 & w & - & - & w & - & 32,184 & 17.76 & - & - & 13.42 & - & 17.20 \\
\hline Obio & 18,220 & 3,838 & 2,772 & 1,900 & 10,854 & 37,584 & 4.11 & 4.51 & 4.56 & 4.19 & 2.95 & 3.95 \\
\hline Oklahoma & 841 & $w$ & - & $\mathbf{w}$ & $w$ & 1,981 & 2.99 & 4.49 & - & 2.88 & 1.98 & 2.61 \\
\hline Pennsylvania Total & 43,668 & 10,397 & 4,749 & 13,497 & 9,372 & 81,684 & 4.54 & 2.89 & 2.83 & 3.09 & .97 & 3.36 \\
\hline Anthracite & 2,522 & 1,732 & $\mathbf{w}$ & $w$ & 1,046 & 5,504 & 2.91 & 3.75 & .94 & 2.20 & .37 & 1.92 \\
\hline 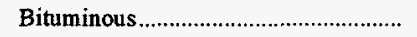 & 41,146 & 8,666 & $w$ & $w$ & 8,326 & 76,180 & 4.70 & 2.76 & 2.85 & 3.11 & 1.24 & 3.56 \\
\hline 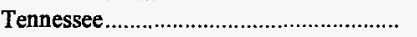 & 3,224 & - & w & $\mathbf{w}$ & 568 & 4,009 & 2.78 & - & 2.36 & 1.01 & .71 & 2.20 \\
\hline 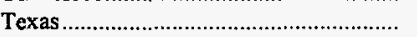 & 52,564 & - & $\mathbf{w}$ & - & $w$ & 59,604 & 10.36 & - & 7.16 & - & 8.40 & 10.13 \\
\hline Utah & 20,230 & w & w & - & w & 30,230 & 7.54 & 7.82 & 4.20 & - & - & 7.23 \\
\hline Virginia & 23,847 & 7,584 & 1,690 & 3,114 & 5,358 & 41,593 & 3.19 & 3.46 & 2.73 & 2.98 & .87 & 2.72 \\
\hline Washington & $\mathbf{w}$ & w & - & - & - & $\mathbf{w}$ & 4.21 & 1.63 & - & - & - & 3.97 \\
\hline West Virginia Total & 113,215 & 22,381 & 16,479 & 20,424 & 44,910 & 217,409 & 4.90 & 3.98 & 4.73 & 3.63 & 1.50 & 3.91 \\
\hline 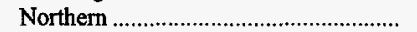 & 32,009 & 5,610 & 2,354 & 9,069 & 5,560 & 54,602 & 4.50 & 4.39 & 4.21 & 3.84 & 1.59 & 4.05 \\
\hline Southern & 81,205 & 16,771 & 14,125 & 11,355 & 39,350 & 162,807 & 5.08 & 3.86 & 4.84 & 3.48 & 1.48 & 3.86 \\
\hline Wyoming & 120,958 & 105,750 & w & w & 40,200 & 350,908 & 32.43 & 31.55 & 42.93 & 47.35 & 14.36 & 32.06 \\
\hline Appalachian Total 1 ................................. & 285,310 & 76,944 & 41,672 & 53,775 & 107,364 & 565,064 & 4.31 & 3.57 & 3.57 & 3.41 & 1.44 & 3.48 \\
\hline Interior Total 1 & 120,853 & 28,812 & 19,142 & 6,588 & 32,264 & 207,658 & 6.23 & 4.79 & 4.44 & 4.33 & 2.91 & 5.39 \\
\hline Western Total 1 & 207,123 & 127,864 & 81,860 & 70,568 & 64,575 & 551,990 & 17.08 & 20.73 & 15.40 & 20.53 & 11.27 & 17.41 \\
\hline East of Miss. River & 351,908 & 101,626 & 57,774 & 60,183 & 134,399 & 705,890 & 4.40 & 3.75 & 3.71 & 3.50 & 1.58 & 3.63 \\
\hline 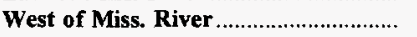 & 261,377 & 131,994 & 84,900 & $\mathbf{7 0 , 7 4 8}$ & 69,804 & 618,823 & 14.69 & 20.11 & 14.77 & 20.20 & 9.81 & 15.66 \\
\hline 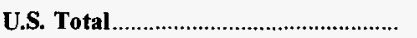 & 613,285 & 233,620 & 142,674 & 130,931 & 204,202 & $1,324,712$ & 6.25 & 6.92 & 6.71 & 6.34 & 2.23 & 5.69 \\
\hline
\end{tabular}

1 For a definition of coal-producing regions, see Appendix C.

Notes: Productivity is calculated by dividing total coal production by the total direct labor hours worked by all employees engaged in production, preparation, processing, development, maintenance, repair, and shop or yard work at mining operations. Excludes office workers. Includes mining operations management and all technical and engineering personnel. Productive capacity is the maximum amount of coal that can be produced annually as reported by mining companies on Form EIA-7A. Capacity utilization is the ratio of total production to annual productive capacity as reported by mining companies on Form EIA-7A. Excludes silt, culm, refuse bank, slurry dam, and dredge operations except for Pennsylvania anthracite. Excludes mines producing less than 10,000 short tons and preparation plants with less than 5,000 employee hours, which are not required to provide these data. Totals may not equal sum of components due to independent rounding.

Sources: Energy Information Administration, Form EIA-7A, "Coal Production Report"; State Mining Agency Coal Production Reports; and/or U.S. Department of Labor, Mine Safety and Health Administration, Form 7000-2, "Quarterly Mine Employment and Coal Production Report." 
Table 23. Productive Capacity and Capacity Utilization of Coal Mines by State and Recoverable Reserves Range, 1996

(Thousand Short Tons)

\begin{tabular}{|c|c|c|c|c|c|c|c|c|}
\hline \multirow{3}{*}{$\begin{array}{l}\text { Coal-Producing } \\
\text { State and Region }\end{array}$} & \multicolumn{8}{|c|}{$\begin{array}{l}\text { Recoverable Reserves Range } \\
\text { (million short tons) }\end{array}$} \\
\hline & \multicolumn{2}{|c|}{50 and over } & \multicolumn{2}{|c|}{10 to 50} & \multicolumn{2}{|c|}{0 to 10} & \multicolumn{2}{|c|}{ Total } \\
\hline & $\begin{array}{l}\text { Productive } \\
\text { Capacity }\end{array}$ & $\begin{array}{c}\text { Capacity } \\
\text { Utilization } \\
\text { (percent) }\end{array}$ & $\begin{array}{l}\text { Productive } \\
\text { Capacity }\end{array}$ & $\begin{array}{l}\text { Capacity } \\
\text { Utilization } \\
\text { (percent) }\end{array}$ & $\begin{array}{c}\text { Productive } \\
\text { Capacity }\end{array}$ & $\begin{array}{l}\text { Capacity } \\
\text { Utilization } \\
\text { (percent) }\end{array}$ & $\begin{array}{l}\text { Productive } \\
\text { Capacity }\end{array}$ & $\begin{array}{l}\text { Capacity } \\
\text { Utilization } \\
\text { (percent) }\end{array}$ \\
\hline 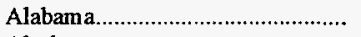 & 12,093 & 84.41 & 6,996 & 77.60 & 13,070 & 68.75 & 32,159 & 76.57 \\
\hline Alaska & $w$ & w & - & - & - & - & w & $\mathbf{w}$ \\
\hline Arizona & $\mathbf{w}$ & $\mathbf{w}$ & - & - & - & - & $\mathbf{w}$ & w \\
\hline Colorado & 19,500 & 90.59 & 8,795 & 69.98 & 1,035 & 103.06 & 29,330 & 84.85 \\
\hline Illinois & 16,361 & 88.87 & 30,808 & 64.03 & 14,559 & 85.08 & 61,727 & 75.58 \\
\hline Indiana & $\mathbf{w}$ & w & w & $w$ & 16,959 & 78.89 & 35,564 & 83.42 \\
\hline 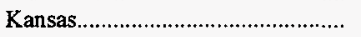 & - & - & - & - & $w$ & $w$ & w & $\mathbf{w}$ \\
\hline 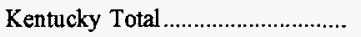 & 10,913 & 85.00 & 38,572 & 78.39 & 139,740 & 80.56 & 189,225 & 80.38 \\
\hline Eastern & w & w & w & w & 116,942 & 81.09 & 145,691 & 80.07 \\
\hline Western & $w$ & $w$ & $w$ & $w$ & 22,798 & 77.87 & 43,534 & 81.40 \\
\hline 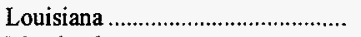 & $\mathbf{w}$ & $\mathbf{w}$ & w & w & - & - & w & w \\
\hline 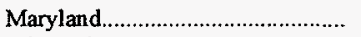 & - & - & $\mathbf{w}$ & $\mathbf{w}$ & w & w & 4,935 & 82.42 \\
\hline 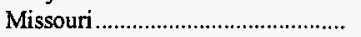 & - & - & - & - & 1,046 & 67.85 & 1,046 & 67.85 \\
\hline 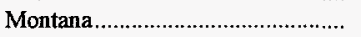 & w & $w$ & $\mathbf{w}$ & $w$ & - & - & 56,175 & 67.45 \\
\hline 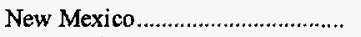 & $w$ & $w$ & $w$ & $\mathrm{w}$ & - & - & 32,695 & 73.61 \\
\hline 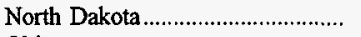 & 32,184 & 92.78 & - & - & - & - & 32,184 & 92.78 \\
\hline Ohio & w & w & $\mathbf{w}$ & $\mathbf{w}$ & 18,384 & 61.45 & 37,584 & 75.88 \\
\hline Oklahoma & - & - & w & w & w & w & 1,981 & 85.16 \\
\hline 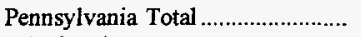 & 31,456 & 88.31 & 14,024 & 82.32 & 36,204 & 77.59 & 81,684 & 82.53 \\
\hline Anthracite & - & - & $\mathbf{w}$ & w & w & w & 5,504 & 82.56 \\
\hline 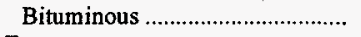 & 31,456 & 88.31 & w & $\mathbf{w}$ & $w$ & w & 76,180 & 82.53 \\
\hline Tennessee & - & - & w & $w$ & $w$ & $w$ & 4,009 & 90.32 \\
\hline 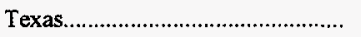 & 48,852 & 97.90 & w & w & $w$ & w & 59,604 & 92.55 \\
\hline Utah & w & w & 15,549 & 91.32 & w & w & 30,230 & 90.97 \\
\hline 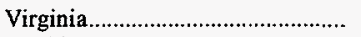 & - & - & 9,912 & 80.20 & 31,681 & 86.94 & 41,593 & 85.34 \\
\hline 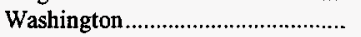 & - & - & w & w & $\mathbf{w}$ & $\mathbf{w}$ & $w$ & w \\
\hline 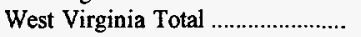 & 41,800 & 89.23 & 56,832 & 78.92 & 118,776 & 74.19 & 217,409 & 78.32 \\
\hline Northern & 24,400 & 89.89 & 13,903 & 79.87 & 16,300 & 78.54 & 54,602 & 83.95 \\
\hline Southern & 17,400 & 88.30 & 42,930 & 78.61 & 102,477 & 73.50 & 162,807 & 76.43 \\
\hline 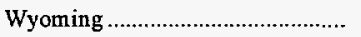 & 340,608 & 80.20 & - & - & 10,300 & 51.04 & 350,908 & 79.35 \\
\hline Appalachian Total ${ }^{1} \ldots \ldots \ldots \ldots \ldots \ldots \ldots$ & 99,399 & 89.19 & 125,237 & 78.81 & 340,428 & 77.35 & 565,064 & 79.75 \\
\hline 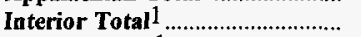 & 81,243 & 93.87 & 64,520 & 76.26 & 61,895 & 76.43 & 207,658 & 83.20 \\
\hline 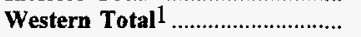 & 489,425 & 79.56 & 41,945 & 83.76 & 20,620 & 70.89 & 551,990 & 79.55 \\
\hline East of Miss. River............................ & 128,791 & 89.02 & 182,355 & 77.29 & 394,744 & $\mathbf{7 7 . 7 3}$ & 705,890 & 79.68 \\
\hline West of Miss. River......................... & 541,277 & 81.22 & 49,347 & 85.31 & 28,198 & 65.26 & 618,823 & 80.82 \\
\hline 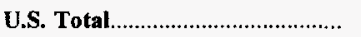 & 670,067 & 82.72 & 231,702 & 79.00 & 422,942 & 76.90 & $1,324,712$ & 80.21 \\
\hline
\end{tabular}

1 For a definition of coal-producing regions, see Appendix C.

Notes: Recoverable reserves represent the quantity of coal that can be recovered (i.e., mined) from existing coal reserves at reporting mines. Productive capacity is the maximum amount of coal that can be produced annually as reported by mining companies on Form EIA-7A. Capacity utilization is the ratio of total production to productive capacity as reported by mining companies on Form EIA-7A. Excludes silt, culm, refuse bank, slurry dam, and dredge operations except for Pennsylvania anthracite. Excludes mines producing less than 10,000 short tons, which are not required to provide these data. Totals may not equal sum of components due to independent rounding.

Sources: Energy Information Administration, Form EIA-7A "Coal Production Report"; State Mining Agency Coal Production Reports; and/or U.S. Department of Labor, Mine Safety and Health Administration, Form 7000-2, "Quarterly Mine Employment and Coal Production Report." 
Table 24. Productive Capacity and Capacity Utilization of Coal Mines by State, Mine Type, and Union Type, 1996

(Thousand Short Tons)

\begin{tabular}{|c|c|c|c|c|c|c|c|c|}
\hline \multirow[b]{2}{*}{$\begin{array}{l}\text { Coal-Producing } \\
\text { State and Region }\end{array}$} & \multicolumn{2}{|c|}{ UMWA } & \multicolumn{2}{|c|}{ Other Unions } & \multicolumn{2}{|c|}{ Nonunion } & \multicolumn{2}{|c|}{ Total } \\
\hline & $\begin{array}{l}\text { Productive } \\
\text { Capacity }\end{array}$ & $\begin{array}{c}\text { Capacity } \\
\text { Utilization } \\
\text { (percent) }\end{array}$ & $\begin{array}{l}\text { Productive } \\
\text { Capacity }\end{array}$ & $\begin{array}{l}\text { Capacity } \\
\text { Utilization } \\
\text { (percent) }\end{array}$ & $\begin{array}{l}\text { Productive } \\
\text { Capacity }\end{array}$ & $\begin{array}{l}\text { Capacity } \\
\text { Utilization } \\
\text { (percent) }\end{array}$ & $\begin{array}{l}\text { Productive } \\
\text { Capacity }\end{array}$ & $\begin{array}{l}\text { Capacity } \\
\text { Utilization } \\
\text { (percent) }\end{array}$ \\
\hline 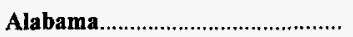 & 22,685 & 75.20 & - & - & 9,474 & 79.82 & 32,159 & 76.57 \\
\hline Underground & w & $\mathbf{w}$ & - & - & w & $\mathbf{w}$ & 23,207 & 78.50 \\
\hline 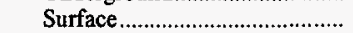 & $w$ & w & - & - & w & $\mathbf{w}$ & 8,952 & 71.55 \\
\hline Alaska & - & - & $\mathbf{w}$ & $\mathbf{w}$ & - & - & $\mathbf{w}$ & $\mathbf{w}$ \\
\hline 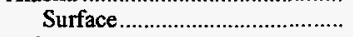 & - & - & $\mathbf{w}$ & $\mathbf{w}$ & - & - & $w$ & $w$ \\
\hline Arizona & $\mathbf{w}$ & $\mathbf{w}$ & - & - & - & - & $\mathbf{w}$ & $\mathbf{w}$ \\
\hline Surface & w & $\mathbf{w}$ & - & - & - & - & $\mathrm{w}$ & $w$ \\
\hline Colorado & $\mathbf{w}$ & $\mathbf{w}$ & $\mathbf{w}$ & $\mathbf{w}$ & 21,630 & 93.79 & 29,330 & 84.85 \\
\hline 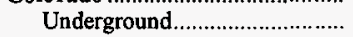 & w & $w$ & - & - & $w$ & $w$ & 18,530 & 84.08 \\
\hline Surface & $\mathbf{w}$ & $\mathbf{w}$ & $\mathbf{w}$ & $\mathbf{w}$ & w & $\mathbf{w}$ & 10,800 & 86.16 \\
\hline Illinois & 46,792 & 68.61 & $\mathbf{w}$ & $\mathbf{w}$ & $\mathbf{w}$ & $\mathbf{w}$ & 61,727 & $\mathbf{7 5 . 5 8}$ \\
\hline 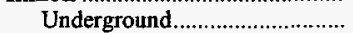 & 38,332 & 66.88 & $\mathbf{w}$ & $\mathrm{w}$ & w & $\mathrm{w}$ & 51,816 & 75.17 \\
\hline Surface & 8,460 & 76.47 & $w$ & $\mathbf{w}$ & w & $\mathbf{w}$ & 9,912 & 77.73 \\
\hline Indiana & 18,368 & 81.30 & - & - & 17,197 & 85.68 & 35,564 & 83.42 \\
\hline 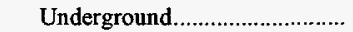 & w & w & - & - & w & $w$ & $\mathbf{w}$ & $w$ \\
\hline Surface & w & $\mathbf{w}$ & - & - & $\mathbf{w}$ & $\mathbf{w}$ & $\mathbf{w}$ & $\mathbf{w}$ \\
\hline 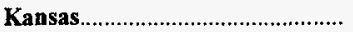 & $\mathbf{w}$ & $\mathbf{w}$ & - & - & - & - & $\mathbf{w}$ & $\mathbf{w}$ \\
\hline Surface ......................................... & $\mathbf{w}$ & $\mathbf{w}$ & - & - & - & - & w & $\mathbf{w}$ \\
\hline 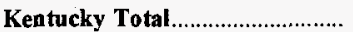 & $\mathbf{w}$ & $\mathbf{w}$ & $\mathbf{w}$ & $\mathbf{w}$ & 163,284 & 80.16 & 189,225 & 80.38 \\
\hline 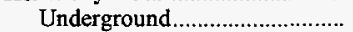 & w & w & $w$ & w & 97,216 & 80.24 & 114,922 & 81.93 \\
\hline 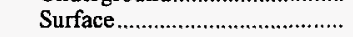 & $\mathrm{w}$ & w & $\mathbf{w}$ & $\mathbf{w}$ & 66,068 & 80.04 & 74,302 & 77.98 \\
\hline Eastern & $\mathbf{w}$ & $\mathbf{w}$ & $\mathbf{w}$ & $\mathbf{w}$ & 130,927 & 80.52 & 145,691 & 80.07 \\
\hline 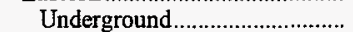 & $\mathbf{w}$ & $\mathbf{w}$ & $w$ & $w$ & $w$ & $w$ & 86,042 & 80.21 \\
\hline 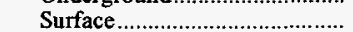 & $w$ & $\mathbf{w}$ & $\mathbf{w}$ & $w$ & $\mathrm{w}$ & $\mathbf{w}$ & 59,649 & 79.86 \\
\hline Western & $\mathbf{w}$ & $\mathbf{w}$ & - & - & 32,356 & 78.70 & 43,534 & 81.40 \\
\hline Underground & $\mathbf{w}$ & $\mathbf{w}$ & - & - & w & $\mathbf{w}$ & 28,880 & 87.03 \\
\hline Surface & $\mathbf{w}$ & $\mathbf{w}$ & - & - & $\mathbf{w}$ & $\mathbf{w}$ & 14,653 & 70.32 \\
\hline 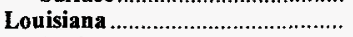 & - & - & - & - & $\mathbf{w}$ & $\mathbf{w}$ & $\mathbf{w}$ & $\mathbf{w}$ \\
\hline Surface & - & - & - & - & $\mathbf{w}$ & $\mathbf{w}$ & w & $w$ \\
\hline Maryland & - & - & - & - & 4,935 & 82.42 & 4,935 & 82.42 \\
\hline 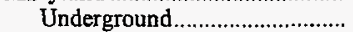 & - & - & - & - & $w$ & $w$ & $w$ & $w$ \\
\hline 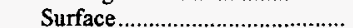 & _- & _ & - & - & $\mathbf{w}$ & w & w & w \\
\hline 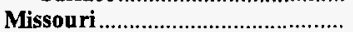 & - & - & - & - & 1,046 & 67.85 & 1,046 & 67.85 \\
\hline 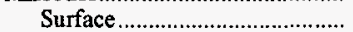 & - & - & - & - & 1,046 & 67.85 & 1,046 & 67.85 \\
\hline 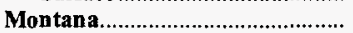 & 21,041 & 77.19 & $\mathbf{w}$ & $\mathbf{w}$ & $\mathbf{w}$ & $\mathbf{w}$ & 56,175 & 67.45 \\
\hline Underground & - & - & - & - & $\mathbf{w}$ & $\mathrm{w}$ & w & $w$ \\
\hline 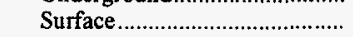 & 21,041 & 77.19 & $\mathbf{w}$ & $\mathbf{w}$ & $w$ & $\mathbf{w}$ & w & w \\
\hline 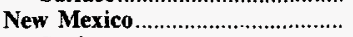 & $\mathbf{w}$ & $\mathbf{w}$ & $\mathbf{w}$ & $\mathbf{w}$ & $\mathbf{w}$ & $\mathbf{w}$ & 32,695 & 73.61 \\
\hline Surface & $\mathrm{w}$ & w & w & $\mathbf{w}$ & $\mathbf{w}$ & w & 32,695 & 73.61 \\
\hline North Dakota & $\mathbf{w}$ & $\mathbf{w}$ & $\mathbf{w}$ & $\mathbf{w}$ & $\mathbf{w}$ & $\mathbf{w}$ & 32,184 & 92.78 \\
\hline Surface & $\mathbf{w}$ & w & $\mathrm{w}$ & w & w & w & 32,184 & 92.78 \\
\hline 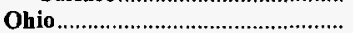 & $\mathbf{w}$ & $\mathbf{w}$ & $\mathbf{w}$ & $\mathbf{w}$ & 20,959 & 61.29 & $\mathbf{3 7 , 5 8 4}$ & $\mathbf{7 5 . 8 8}$ \\
\hline 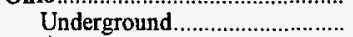 & $w$ & w & - & - & $w$ & $w$ & 17,313 & 91.91 \\
\hline Surface & $w$ & $w$ & - & - & $w$ & w & 20,272 & 62.19 \\
\hline Oklahoma & - & - & - & - & 1,981 & 85.16 & 1,981 & 85.16 \\
\hline Underground & - & - & - & - & $w$ & $w$ & $w$ & $w$ \\
\hline Surface & - & - & - & - & $\mathbf{w}$ & $\mathbf{w}$ & $\mathbf{w}$ & w \\
\hline 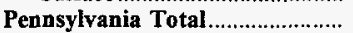 & $\mathbf{w}$ & $\mathbf{w}$ & $\mathbf{w}$ & $\mathbf{w}$ & $\mathbf{5 0 , 3 5 0}$ & 82.32 & 81,684 & 82.53 \\
\hline 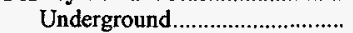 & $\mathbf{w}$ & w & - & - & $w$ & $w$ & 54,579 & 86.37 \\
\hline Surface & $w$ & $\mathbf{w}$ & $\mathbf{w}$ & $w$ & $\mathbf{w}$ & $\mathbf{w}$ & 27,105 & 74.79 \\
\hline Anthracite & $\mathbf{w}$ & $\mathbf{w}$ & $\mathbf{w}$ & $\mathbf{w}$ & 4,124 & 84.68 & 5,504 & 82.56 \\
\hline 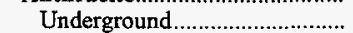 & - & - & - & - & $w$ & $w$ & 459 & 61.96 \\
\hline Surface & w & $\mathbf{w}$ & $w$ & w & w & w & 5,045 & 84.44 \\
\hline 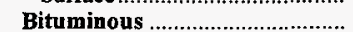 & 29,954 & 83.17 & - & - & 46,226 & 82.11 & 76,180 & $\mathbf{8 2 . 5 3}$ \\
\hline 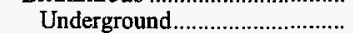 & 28,856 & 83.14 & - & - & 25,264 & 90.50 & 54,120 & 86.58 \\
\hline Surface & 1,097 & 83.85 & - & - & 20,963 & 72.00 & 22,060 & 72.58 \\
\hline Tennessee & - & - & - & - & 4,009 & 90.32 & 4,009 & 90.32 \\
\hline 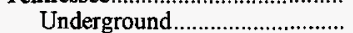 & - & - & - & - & 2,146 & 83.44 & 2,146 & 83.44 \\
\hline 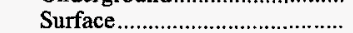 & - & - & - & - & 1,863 & 98.24 & 1,863 & 98.24 \\
\hline Texas & - & - & 36,664 & 98.56 & 22,940 & 82.94 & 59,604 & 92.55 \\
\hline Surface & - & - & 36,664 & 98.56 & 22,940 & 82.94 & 59,604 & 92.55 \\
\hline 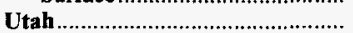 & $\mathbf{w}$ & $\mathbf{w}$ & - & - & $\mathbf{w}$ & $\mathbf{w}$ & 30,230 & 90.97 \\
\hline 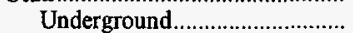 & w & $\mathrm{w}$ & - & - & $w$ & $\mathbf{w}$ & 30,230 & 90.97 \\
\hline Virginia & $\mathbf{w}$ & $\mathbf{w}$ & $\mathbf{w}$ & $\mathbf{w}$ & 31,305 & 87.78 & 41,593 & 85.34 \\
\hline Underground & $w$ & w & - & - & w & w & 30,146 & 84.59 \\
\hline Surface & $w$ & $\mathbf{w}$ & $\mathrm{w}$ & $\mathbf{w}$ & $w$ & $\mathrm{w}$ & 11,448 & 87.31 \\
\hline
\end{tabular}

See footnotes at end of table. 
Table 24. Productive Capacity and Capacity Utilization of Coal Mines by State, Mine Type, and Union Type, 1996 (Continued)

(Thousand Short Tons)

\begin{tabular}{|c|c|c|c|c|c|c|c|c|}
\hline \multirow[b]{2}{*}{$\begin{array}{l}\text { Coal-Producing } \\
\text { State and Region }\end{array}$} & \multicolumn{2}{|c|}{ UMWA } & \multicolumn{2}{|c|}{ Other Unions } & \multicolumn{2}{|c|}{ Nonunion } & \multicolumn{2}{|c|}{ Total } \\
\hline & $\begin{array}{l}\text { Productive } \\
\text { Capacity }\end{array}$ & $\begin{array}{c}\text { Capacity } \\
\text { Utilization } \\
\text { (percent) }\end{array}$ & $\begin{array}{l}\text { Productive } \\
\text { Capacity }\end{array}$ & $\begin{array}{l}\text { Capacity } \\
\text { Utilization } \\
\text { (percent) }\end{array}$ & $\begin{array}{l}\text { Productive } \\
\text { Capacity }\end{array}$ & $\begin{array}{l}\text { Capacity } \\
\text { Utilization } \\
\text { (percent) }\end{array}$ & $\begin{array}{l}\text { Productive } \\
\text { Capacity }\end{array}$ & $\begin{array}{l}\text { Capacity } \\
\text { Utilization } \\
\text { (percent) }\end{array}$ \\
\hline 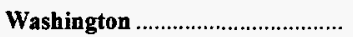 & - & - & $\mathbf{w}$ & $\mathbf{w}$ & $\mathbf{w}$ & $\mathbf{w}$ & $\mathbf{w}$ & $\mathbf{w}$ \\
\hline Surface & - & - & $\mathbf{w}$ & w & w & $\mathbf{w}$ & $w$ & w \\
\hline West Virginia Total ...................... & 116,539 & 76.55 & - & - & 100,870 & 80.37 & 217,409 & 78.32 \\
\hline 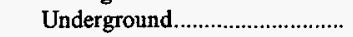 & 80,918 & 80.43 & - & - & 62,879 & 80.17 & 143,797 & 80.32 \\
\hline 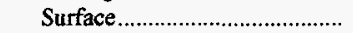 & 35,621 & 67.72 & - & - & 37,991 & 80.69 & 73,612 & 74.41 \\
\hline 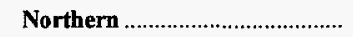 & $\mathbf{3 4 , 9 3 0}$ & 90.57 & - & - & 19,672 & 72.19 & 54,602 & 83.95 \\
\hline Underground & 34,930 & 90.57 & - & - & 11,098 & 77.62 & 46,028 & 87.45 \\
\hline 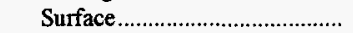 & - & - & - & - & 8,575 & 65.16 & 8,575 & 65.16 \\
\hline 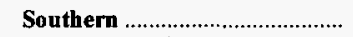 & 81,609 & 70.55 & - & - & 81,198 & 82.35 & 162,807 & 76.43 \\
\hline 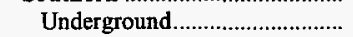 & 45,988 & 72.74 & - & - & 51,781 & 80.72 & 97,769 & 76.96 \\
\hline 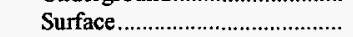 & 35,621 & 67.72 & - & - & 29,417 & 85.21 & 65,038 & 75.63 \\
\hline 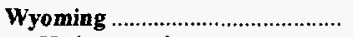 & $\mathbf{w}$ & $\mathbf{w}$ & $\mathbf{w}$ & $\mathbf{w}$ & 333,758 & 79.07 & 350,908 & 79.35 \\
\hline 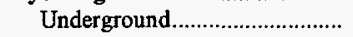 & - & - & - & - & $\mathrm{w}$ & w & $w$ & w \\
\hline Surface & $w$ & $w$ & $\mathbf{w}$ & w & w & w & $w$ & w \\
\hline 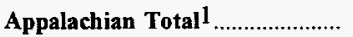 & 209,969 & $\mathbf{7 8 . 8 7}$ & 2,265 & 68.47 & 352,830 & 80.35 & 565,064 & 79.75 \\
\hline Underground & w & w & w & w & 200,634 & 81.89 & 361,103 & 82.07 \\
\hline Surface & w & w & $\mathbf{w}$ & w & 152,195 & 78.32 & 203,961 & 75.65 \\
\hline Interior Total ${ }^{1} \ldots \ldots \ldots \ldots \ldots \ldots \ldots \ldots$ & 76,589 & 74.74 & 40,372 & 98.05 & 90,698 & 83.73 & 207,658 & $\mathbf{8 3 . 2 0}$ \\
\hline 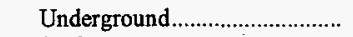 & w & $\mathbf{w}$ & w & w & 32,447 & 89.74 & 84,166 & 79.82 \\
\hline 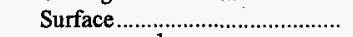 & w & w & $w$ & w & 58,251 & 80.38 & 123,492 & 85.50 \\
\hline 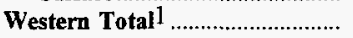 & 69,125 & 75.55 & 65,143 & 74.13 & 417,722 & 81.06 & $\mathbf{5 5 1 , 9 9 0}$ & 79.55 \\
\hline 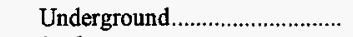 & 13,000 & 78.95 & - & - & 39,160 & 90.92 & 52,160 & 87.94 \\
\hline Surface & 56,125 & 74.77 & 65,143 & 74.13 & 378,562 & 80.04 & 499,830 & 78.68 \\
\hline 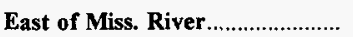 & 286,306 & $\mathbf{7 7 . 7 6}$ & 5,973 & 83.71 & 413,610 & 80.95 & 705,890 & 79.68 \\
\hline 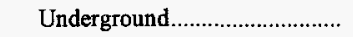 & 209,082 & 79.93 & 3,106 & 97.26 & 232,781 & 83.03 & 444,969 & 81.67 \\
\hline 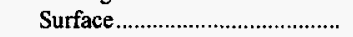 & 77,224 & 71.88 & 2,867 & 69.04 & 180,829 & 78.26 & 260,920 & 76.27 \\
\hline West of Miss. River. & 69,377 & 75.61 & 101,807 & 82.93 & 447,639 & 81.15 & 618,823 & 80.82 \\
\hline 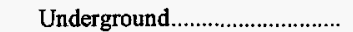 & 13,000 & 78.95 & - & - & 39,460 & 90.58 & 52,460 & 87.70 \\
\hline 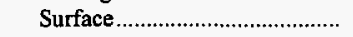 & 56,377 & 74.85 & 101,807 & 82.93 & 408,179 & 80.24 & 566,363 & 80.19 \\
\hline 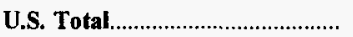 & 355,683 & 77.34 & 107,780 & 82.97 & 861,249 & 81.05 & $1,324,712$ & 80.21 \\
\hline 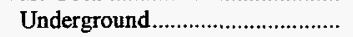 & 222,082 & 79.87 & 3,106 & 97.26 & 272,241 & 84.13 & 497,429 & 82.31 \\
\hline 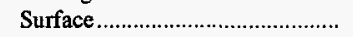 & 133,601 & 73.13 & 104,674 & 82.55 & 589,008 & 79.63 & 827,283 & 78.95 \\
\hline
\end{tabular}

1 For a definition of coal-producing regions, see Appendix C.

Notes: Productive capacity is the maximum amount of coal that can be produced annually as reported by mining companies on Form EIA-7A. Capacity utilization is the ratio of total production to annual productive capacity as reported by mining companies on Form EIA-7A. Excludes silt, culm, refuse bank, slurry dam, and dredge operations except for Pennsylvania anthracite. Excludes mines producing less than 10,000 short tons, which are not required to provide these data. Totals may not equal sum of components due to independent rounding. See Glossary for listing of other unions.

Source: Energy Information Administration, Form EIA-7A, "Coal Production Report." 



\section{Recoverable Coal Reserves at Producing Mines}

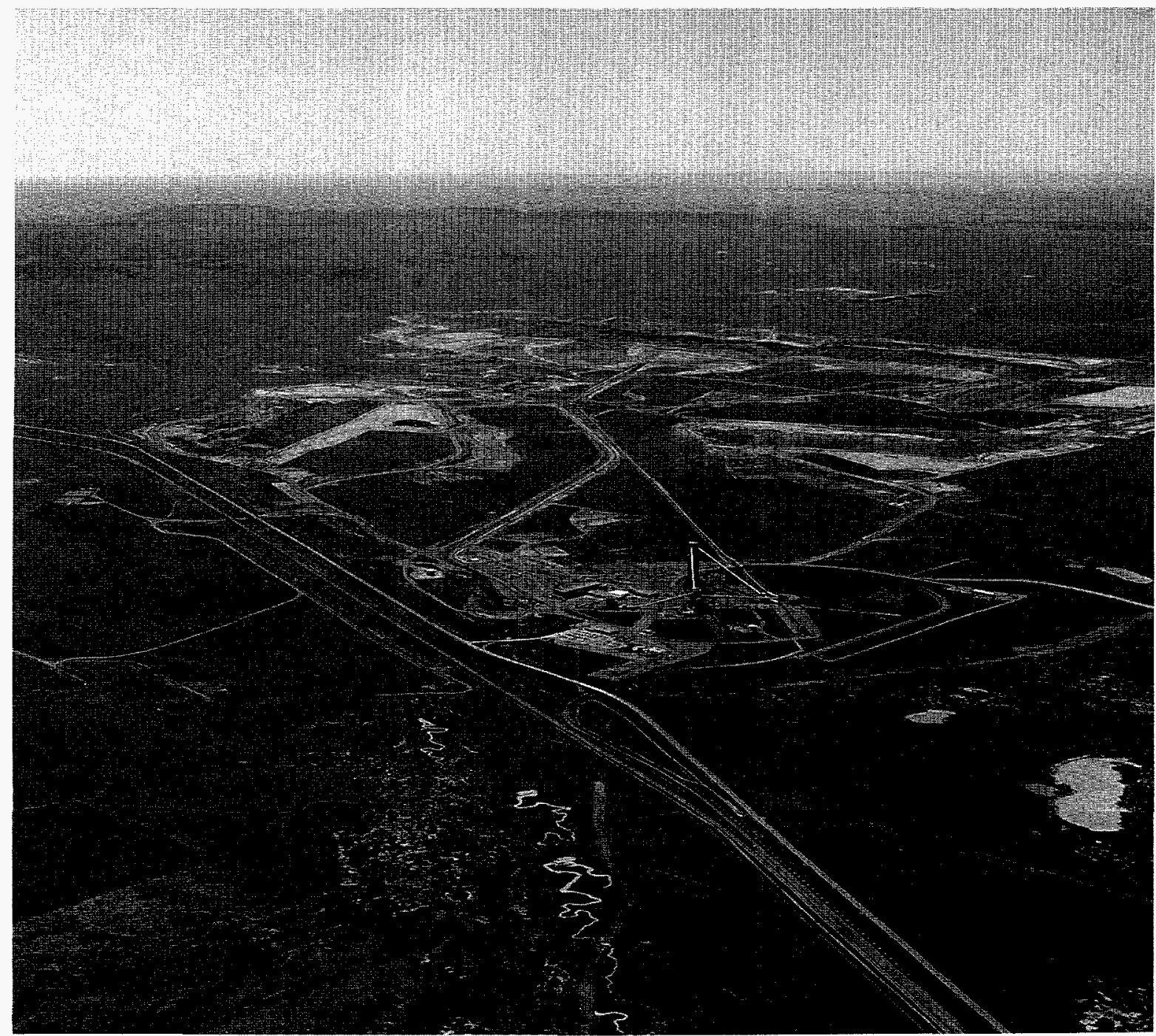

Black Thunder, the 2nd largest coal mine in the world shipped some 39 million short tons of coal in 1996. The mine has a total permit area of 15,943 acres. 
Figure 1. Recoverable Coal Reserves at Producing U.S. Mines by Mine Type and by Region, 1987 1996
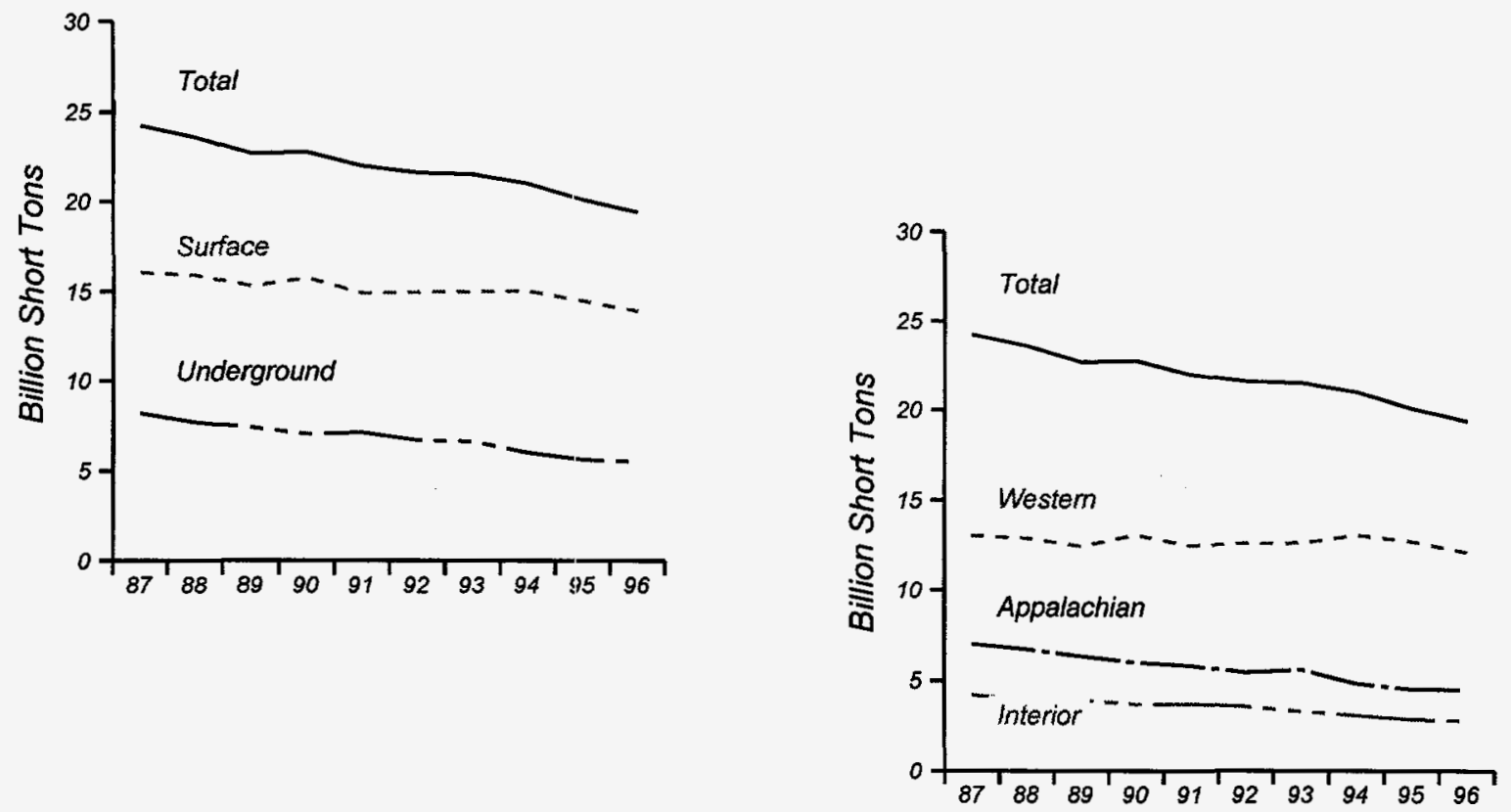

Figure 2. Average Recovery Percentage at Producing U.S. Coal Mines by Mine Type and by Region, $1987-1996$
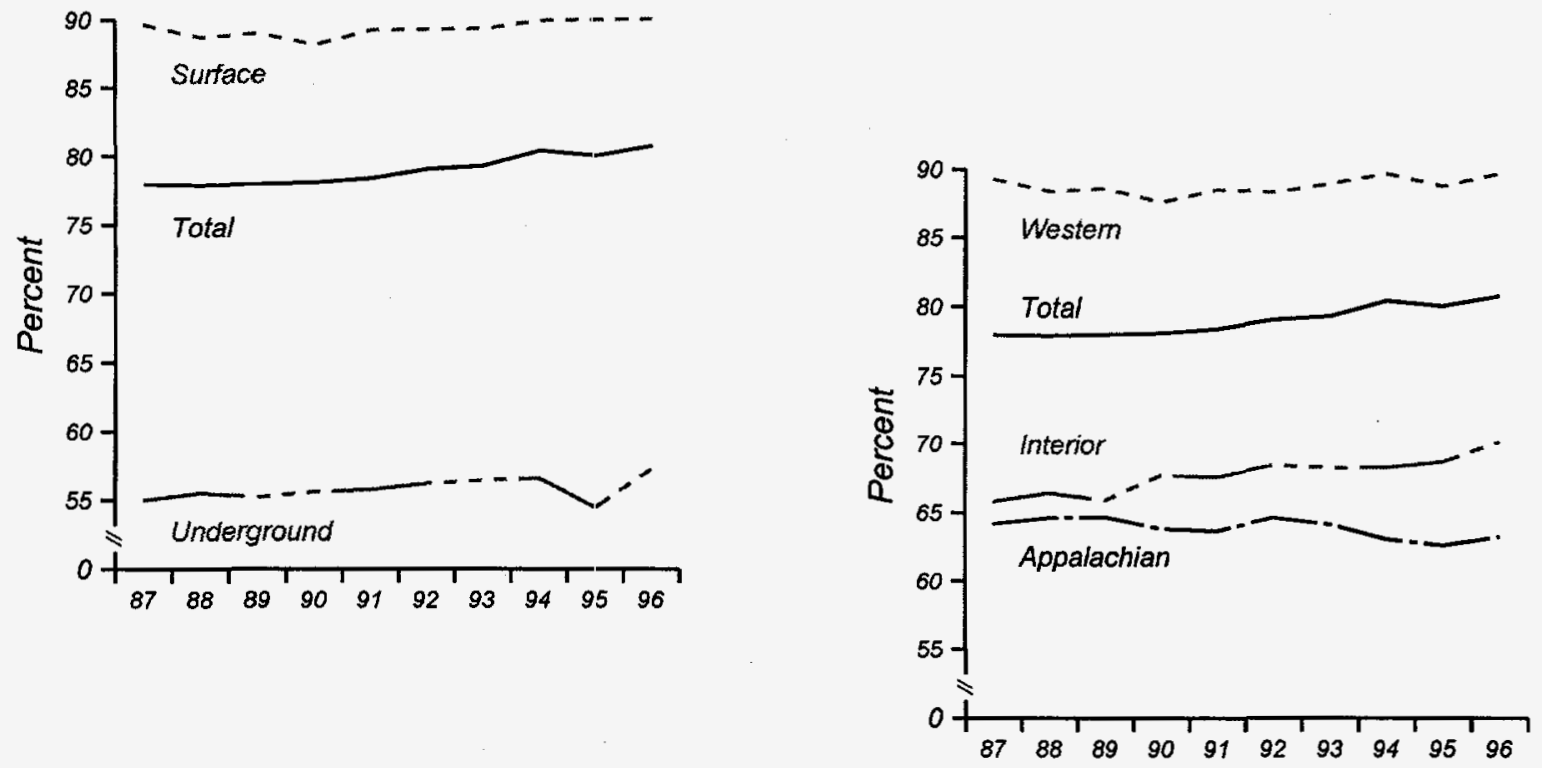

Sources: Energy Informotion Administrotion, Form ElA-7A, "Cool Production Report"; State Mining Agency Coal Production Reports and/or U.S. Deportment of Labor, Mine Safety ond Health Administration, Form $7000-2$,

"Quarterly Mine Employment and Coal Produclion Report." 
Table 25. Recoverable Coal Reserves at Producing Mines by State, 1987, 1992-1996 (Million Short Tons)

\begin{tabular}{|c|c|c|c|c|c|c|c|c|c|}
\hline \multirow{2}{*}{$\begin{array}{l}\text { Coal-Producing } \\
\text { State and Region }\end{array}$} & \multirow{2}{*}{1996} & \multirow{2}{*}{1995} & \multirow{2}{*}{1994} & \multirow{2}{*}{1993} & \multirow{2}{*}{1992} & \multirow{2}{*}{1987} & \multirow{2}{*}{$\begin{array}{c}\text { Percent } \\
\text { Change } \\
\text { 1995-1996 }\end{array}$} & \multicolumn{2}{|c|}{ Average Annual Percent Change } \\
\hline & & & & & & & & 1992-1996 & 1987-1996 \\
\hline Alabama & 452 & 510 & 457 & 427 & 468 & 538 & -11.5 & -0.9 & -1.9 \\
\hline Alaska & $\mathbf{w}$ & w & w & w & w & w & w & w & w \\
\hline 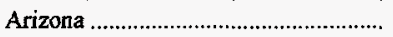 & $w$ & w & $w$ & $w$ & w & $w$ & $w$ & $w$ & $w$ \\
\hline Arkansas & - & $w$ & $w$ & $\mathbf{w}$ & w & $w$ & $w$ & $\mathbf{w}$ & $\mathbf{w}$ \\
\hline California & - & - & - & - & $\mathbf{w}$ & $\mathbf{w}$ & w & $w$ & w \\
\hline Colorado & 642 & 692 & 676 & 609 & 608 & 668 & -7.3 & 1.3 & -.4 \\
\hline 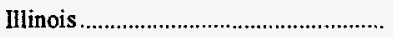 & 891 & 882 & 963 & 1,064 & 1,199 & 1,431 & 1.0 & -7.1 & -5.1 \\
\hline Indiana & 386 & 324 & 304 & 379 & 405 & 514 & 19.3 & -1.2 & -3.1 \\
\hline lowa & - & - & $\mathbf{w}$ & $w$ & w & 36 & w & $\mathbf{w}$ & w \\
\hline 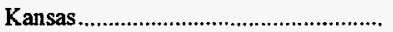 & $\mathbf{w}$ & $\mathbf{w}$ & $w$ & w & w & $\mathbf{w}$ & $\mathbf{w}$ & w & w \\
\hline 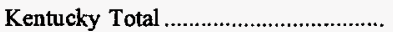 & 1,255 & 1,279 & 1,365 & 1,828 & 1,453 & 1,739 & -1.8 & -3.6 & -3.5 \\
\hline 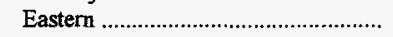 & 818 & 763 & 809 & 1,347 & 955 & 1,115 & 7.3 & -3.8 & -3.4 \\
\hline 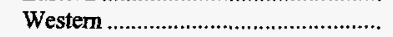 & 437 & 516 & 556 & 481 & 498 & 624 & -15.3 & -3.2 & -3.9 \\
\hline 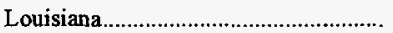 & $\mathbf{w}$ & $\mathbf{w}$ & $\mathbf{w}$ & w & w & w & w & w & $w$ \\
\hline 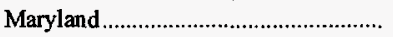 & 71 & 58 & 89 & 66 & 59 & 75 & 23.1 & 4.7 & -.7 \\
\hline 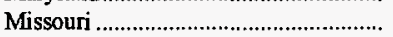 & 3 & 2 & 12 & w & $\mathbf{w}$ & 155 & 13.1 & w & -36.4 \\
\hline 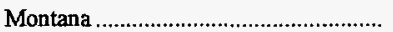 & 1,309 & 1,251 & 1,283 & 1,285 & 1,352 & 1,611 & 4.6 & -.8 & -2.3 \\
\hline 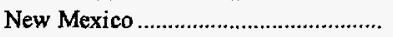 & 1,436 & 1,480 & 1,458 & 1,473 & 1,495 & 1,557 & -2.9 & -1.0 & -9 \\
\hline North Dakota & 1,301 & 1,668 & 1,695 & 1,411 & 1,335 & 1,384 & -21.9 & -6 & -7 \\
\hline 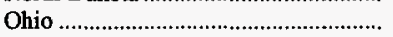 & 415 & 468 & 479 & 520 & 576 & 755 & -11.4 & -7.9 & -6.4 \\
\hline Oklahoma & 19 & 19 & 43 & 46 & 48 & 49 & -1.3 & -21.1 & -10.2 \\
\hline 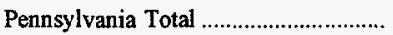 & 796 & 737 & 913 & 940 & 937 & 1,369 & 8.1 & -4.0 & -5.8 \\
\hline 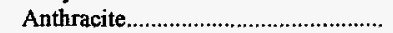 & 90 & 49 & 38 & 65 & 70 & 60 & 81.4 & 6.3 & 4.5 \\
\hline 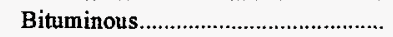 & 706 & 687 & 875 & 874 & 867 & 1,308 & 2.8 & -5.0 & -6.6 \\
\hline 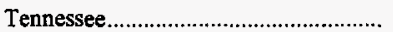 & 59 & 68 & 42 & 29 & 43 & 103 & -12.9 & 8.3 & -6.0 \\
\hline 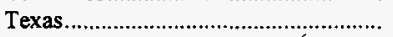 & 878 & 940 & 1,026 & 1,105 & 1,188 & 1,215 & -6.5 & -7.3 & -3.5 \\
\hline Utah & 284 & 375 & 423 & 447 & 488 & 490 & -24.1 & -12.6 & -5.8 \\
\hline 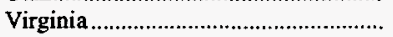 & 188 & 203 & 237 & 336 & 366 & 508 & -7.3 & -15.3 & -10.4 \\
\hline 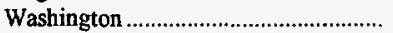 & w & $\mathbf{w}$ & $\mathbf{w}$ & $w$ & $\mathbf{w}$ & $\mathbf{w}$ & w & w & w \\
\hline 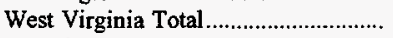 & 1,731 & 1,731 & 1,830 & 1,931 & 2,043 & 2,548 & $*$ & -4.0 & -4.2 \\
\hline Northem & 741 & 782 & 861 & 824 & 960 & 1,271 & -5.2 & -6.3 & -5.8 \\
\hline Southern & 990 & 949 & 969 & 1,107 & 1,083 & 1,277 & 4.3 & -2.2 & -2.8 \\
\hline 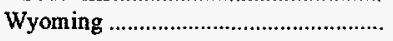 & 6,591 & 6,724 & 6,999 & 6,831 & 6,751 & 6,596 & -2.0 & -.6 & * \\
\hline Appalachian Total ${ }^{1}$ & 4,530 & 4,538 & 4,855 & 5,596 & 5,446 & 7,009 & -.2 & -4.5 & -4.7 \\
\hline Interior Total ${ }^{1}$ & 2,757 & 2,835 & 3,069 & 3,300 & $\mathbf{3 , 5 5 9}$ & 4,206 & -2.8 & -6.2 & -4.6 \\
\hline Western Total ${ }^{1}$ & 12,141 & 12,732 & 13,093 & 12,639 & 12,622 & 13,027 & -4.6 & -1.0 & -.8 \\
\hline East of Miss. River ............................... & 6,244 & 6,260 & 6,679 & $\mathbf{7 , 5 2 0}$ & $\mathbf{7 , 5 4 9}$ & 9,579 & -.2 & -4.6 & -4.6 \\
\hline West of Miss. River ................................ & 13,184 & 13,845 & 14,337 & 14,016 & 14,078 & 14,663 & -4.8 & -1.6 & -1.2 \\
\hline 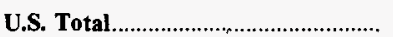 & 19,428 & 20,105 & 21,017 & 21,535 & 21,627 & 24,241 & -3.4 & -2.6 & -2.4 \\
\hline
\end{tabular}

1 For a definition of coal-producing regions, see Appendix C.

* Data round to zero.

Notes: Recoverable reserves represent the quantity of coal that can be recovered (i.e., mined) from existing coal reserves at reporting mines. Excludes silt, culm, refuse bank, slurry dam, and dredge operations except for Pennsylvania anthracite. Excludes mines producing less than 10,000 short tons, which are not required to provide these data. Totals may not equal sum of components due to independent rounding.

Sources: Energy Information Administration, Form ElA-7A, "Coal Production Report"; State Mining Agency Coal Production Reports; and/or U.S. Department of Labor, Mine Safety and Health Administration, Form 7000-2, "Quarteriy Mine Employment and Coal Production Report." 
Table 26. Average Recovery Percentage at Producing Coal Mines by State, 1987, 1992-1996

\begin{tabular}{|c|c|c|c|c|c|c|}
\hline $\begin{array}{l}\text { Coal-Producing } \\
\text { State and Region }\end{array}$ & 1996 & 1995 & 1994 & 1993 & 1992 & 1987 \\
\hline Alabama & 55.56 & 58.74 & 60.20 & 63.16 & 61.97 & 63.87 \\
\hline 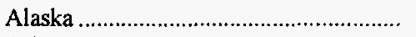 & w & w & $w$ & $\mathbf{w}$ & w & w \\
\hline 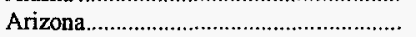 & $w$ & w & w & $\mathbf{w}$ & $w$ & w \\
\hline 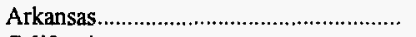 & - & w & $\mathbf{w}$ & $\mathbf{w}$ & $w$ & $w$ \\
\hline California & - & - & - & - & w & w \\
\hline 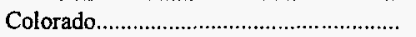 & 79.12 & 66.06 & 77.12 & 71.99 & 71.56 & 61.61 \\
\hline Illinois & 54.20 & 52.05 & 52.92 & 51.19 & 51.53 & 47.35 \\
\hline Indiana & 78.85 & 76.23 & 72.75 & 73.12 & 74.48 & 73.31 \\
\hline Iowa & - & - & w & w & w & 84.96 \\
\hline 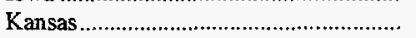 & $w$ & $\mathbf{w}$ & w & $\mathbf{w}$ & $w$ & w \\
\hline 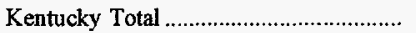 & 58.99 & 57.71 & 59.45 & 62.26 & 63.24 & 63.92 \\
\hline 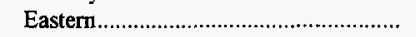 & 62.59 & 60.87 & 62.60 & 64.00 & 66.84 & 68.72 \\
\hline Western & 52.27 & 53.03 & 54.86 & 57.38 & 56.36 & 55.35 \\
\hline 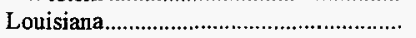 & w & w & w & w & w & $w$ \\
\hline 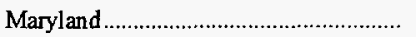 & 61.45 & 58.34 & 55.50 & 67.82 & 66.16 & 66.33 \\
\hline 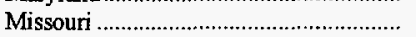 & 59.87 & 61.04 & 81.48 & w & $w$ & 90.17 \\
\hline Montana & 89.05 & 90.38 & 90.51 & 90.43 & 89.70 & 90.12 \\
\hline 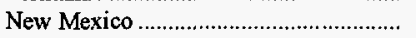 & 93.35 & 92.62 & 92.42 & 92.28 & 91.92 & 92.18 \\
\hline 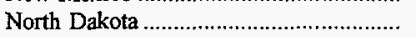 & 89.72 & 89.58 & 89.84 & 90.96 & 90.62 & 90.25 \\
\hline Ohio & 71.61 & 68.73 & 67.81 & 68.86 & 69.15 & 67.30 \\
\hline 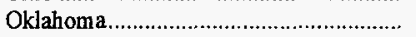 & 65.94 & 62.39 & 63.89 & 66.11 & 66.73 & 85.87 \\
\hline 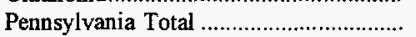 & 65.59 & 65.26 & 68.23 & 67.95 & 66.12 & 66.12 \\
\hline 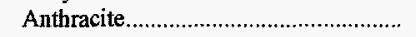 & 59.90 & 64.39 & 65.06 & 61.04 & 62.62 & 54.93 \\
\hline 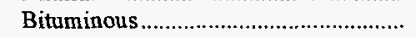 & 66.32 & 65.32 & 68.37 & 68.47 & 66.41 & 66.63 \\
\hline Tennessee & 63.33 & 64.14 & 65.18 & 68.92 & 69.80 & 69.65 \\
\hline 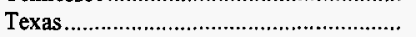 & 88.32 & 87.09 & 86.10 & 84.23 & 85.39 & 81.53 \\
\hline Utah & 45.61 & 46.10 & 46.10 & 47.51 & 49.04 & 58.44 \\
\hline 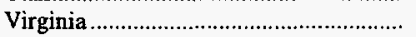 & 54.81 & 58.14 & 58.37 & 62.72 & 64.78 & 64.06 \\
\hline Washington & w & w & w & w & w & w \\
\hline 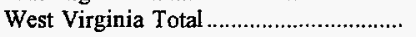 & 63.49 & 62.44 & 61.10 & 61.30 & 62.05 & 59.97 \\
\hline Northern & 58.91 & 55.10 & 53.06 & 52.17 & 54.04 & 52.42 \\
\hline Southern & 66.92 & 68.49 & 68.24 & 68.09 & 69.15 & 67.48 \\
\hline 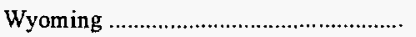 & 92.16 & 92.27 & 92.98 & 91.94 & 91.19 & 93.71 \\
\hline Appalachian Total ${ }^{1}$............................. & 63.25 & 62.65 & 63.07 & 64.11 & 64.62 & 64.16 \\
\hline Interior Total ${ }^{1}$ & 70.15 & 68.71 & 68.29 & 68.27 & 68.46 & 65.83 \\
\hline 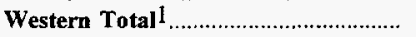 & 89.74 & 88.81 & 89.72 & 88.98 & 88.31 & 89.29 \\
\hline 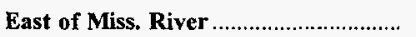 & 62.16 & 61.06 & 61.36 & 62.31 & 62.52 & 61.56 \\
\hline 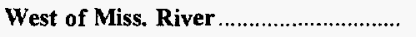 & 89.60 & 88.66 & 89.32 & 88.48 & 87.96 & 88.66 \\
\hline 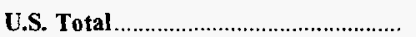 & 80.78 & 80.07 & 80.43 & $\mathbf{7 9 . 3 4}$ & 79.08 & 77.96 \\
\hline
\end{tabular}

1 For a definition of coal-producing regions, see Appendix $C$.

Notes: Average recovery percentage represents the percentage of coal that can be recovered from coal reserves at reporting mines, weighted for all mines in the geographic area. Excludes silt, culm, refuse bank, slurry dam, and dredge operations except for Pennsylvania anthracite. Excludes mines producing less than 10,000 short tons, which are not required to provide these data.

Sources: Energy Information Administration, Form EIA-"A, "Coal Production Report"; State Mining Agency Coal Production Reports; and/or U.S. Department of Labor, Mine Safety and Health Administration, Fom 7000-2, "Quarterly Mine Employment and Coal Production Report." 
Table 27. Recoverable Coal Reserves and Average Recovery Percentage at Producing Mines by State and Mine Type, 1996

(Million Short Tons)

\begin{tabular}{|c|c|c|c|c|c|c|}
\hline \multirow[b]{2}{*}{$\begin{array}{l}\text { Coal-Producing } \\
\text { State and Region }\end{array}$} & \multicolumn{2}{|c|}{ Underground } & \multicolumn{2}{|c|}{ Surface } & \multicolumn{2}{|c|}{ Total } \\
\hline & $\begin{array}{c}\text { Recoverable } \\
\text { Coal } \\
\text { Reserves }\end{array}$ & $\begin{array}{c}\text { Average } \\
\text { Recovery } \\
\text { Percentage }\end{array}$ & $\begin{array}{c}\text { Recoverable } \\
\text { Coal } \\
\text { Reserves }\end{array}$ & $\begin{array}{c}\text { Average } \\
\text { Recovery } \\
\text { Percentage }\end{array}$ & $\begin{array}{c}\text { Recoverable } \\
\text { Coal } \\
\text { Reserves }\end{array}$ & $\begin{array}{c}\text { Average } \\
\text { Recovery } \\
\text { Percentage }\end{array}$ \\
\hline Alabana & 417 & 52.98 & 35 & 86.25 & 452 & 55.56 \\
\hline 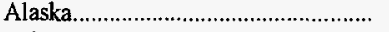 & - & - & $\mathrm{w}$ & w & $\mathbf{w}$ & $w$ \\
\hline Arizona & - & - & $w$ & $\mathbf{w}$ & $\mathbf{w}$ & w \\
\hline Colorado & 411 & 73.72 & 230 & 88.76 & 642 & 79.12 \\
\hline 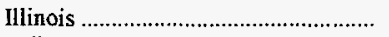 & 861 & 53.40 & 30 & 77.32 & 891 & 54.20 \\
\hline Indiana & w & $\mathbf{w}$ & $\mathbf{w}$ & w & 386 & 78.85 \\
\hline 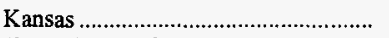 & - & - & $\mathrm{w}$ & w & $w$ & w \\
\hline Kentucky Total & 985 & 53.26 & 270 & 79.90 & 1,255 & 58.99 \\
\hline Eastern & 605 & 55.68 & 213 & 82.17 & 818 & 62.59 \\
\hline Western & 380 & 49.41 & 57 & 71.37 & 437 & 52.27 \\
\hline Louisiana & - & - & w & w & $\mathbf{w}$ & w \\
\hline 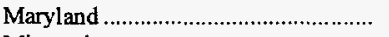 & $\mathbf{w}$ & $\mathbf{w}$ & w & w & 71 & 61.45 \\
\hline 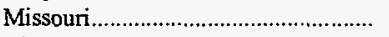 & - & - & 3 & 59.87 & 3 & 59.87 \\
\hline Montana & $\mathbf{w}$ & $\mathbf{w}$ & w & w & 1,309 & 89.05 \\
\hline 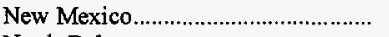 & - & - & 1,436 & 93.35 & 1,436 & 93.35 \\
\hline 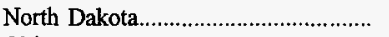 & - & - & 1,301 & 89.72 & 1,301 & 89.72 \\
\hline Ohio & 228 & 56.85 & 186 & 89.68 & 415 & 71.61 \\
\hline 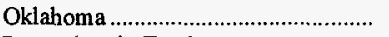 & $\mathbf{w}$ & w & $\mathbf{w}$ & $\mathbf{w}$ & 19 & 65.94 \\
\hline 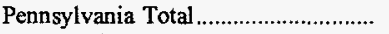 & 614 & 63.27 & 182 & 73.42 & 796 & 65.59 \\
\hline Anthracite & 23 & 73.55 & 66 & 55.13 & 90 & 59.90 \\
\hline 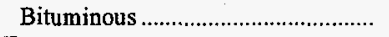 & 591 & 62.87 & 116 & 83.92 & 706 & 66.32 \\
\hline Tennessee & $\mathbf{w}$ & w & $w$ & $w$ & 59 & 63.33 \\
\hline 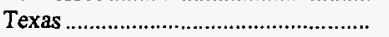 & - & - & 878 & 88.32 & 878 & 88.32 \\
\hline Utah & 284 & 45.61 & - & - & 284 & 45.61 \\
\hline 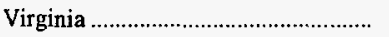 & 171 & 51.47 & 18 & 86.77 & 188 & 54.81 \\
\hline 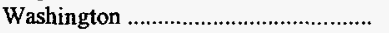 & $\rightarrow$ & - & $w$ & $\mathbf{w}$ & $w$ & $w$ \\
\hline West Virginia Total .............................. & 1,256 & 58.41 & 475 & 76.93 & 1,731 & 63.49 \\
\hline Northern & 641 & 57.81 & 100 & 65.96 & 741 & 58.91 \\
\hline Southern & 615 & 59.03 & 375 & 79.85 & 990 & 66.92 \\
\hline 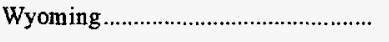 & w & w & w & w & 6,591 & 92.16 \\
\hline Appalachian Total 1 ............................ & 3,410 & 57.79 & 1,120 & 79.89 & 4,530 & 63.25 \\
\hline 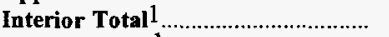 & 1,295 & 52.12 & 1,462 & 86.12 & 2,757 & 70.15 \\
\hline 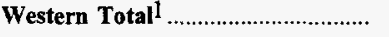 & 801 & 63.18 & 11,339 & 91.61 & 12,141 & 89.74 \\
\hline East of Miss. River ........................... & 4,691 & 56.22 & 1,554 & 80.10 & 6,244 & 62.16 \\
\hline West of Miss. River ............................. & 815 & 63.12 & 12,368 & 91.35 & 13,184 & 89.60 \\
\hline 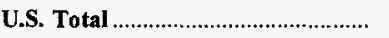 & 5,506 & 57.24 & 13,922 & 90.09 & 19,428 & 80.78 \\
\hline
\end{tabular}

1 For a definition of coal-producing regions, see Appendix $\mathrm{C}$.

Notes: Recoverable reserves represent the quantity of coal that can be recovered (i.e., mined) from existing coal reserves at reporting mines. Average recovery percentage represents the percentage of coal that can be recovered from coal reserves at reporting mines, weighted for all mines in the reported geographic area. Excludes silt, culm, refuse bank, slurry dam, and dredge operations except for Pennsylvania anthracite. Excludes mines producing less than 10,000 short tons, which are not required to provide these data. Totals may not equal sum of conponents due to independent rounding.

Sources: Energy Information Administration, Form EIA-7A, "Coal Production Report"; State Mining Agency Coal Production Reports; and/or U.S. Department of Labor, Mine Safety and Health Administration, Form 7000-2, "Quarterly Mine Employment and Coal Production Report." 
Table 28. Recoverable Coal Reserves at Producing Underground Mines by State and Mining Method, 1996

(Million Short Tons)

\begin{tabular}{|c|c|c|c|c|c|}
\hline $\begin{array}{l}\text { Coal-Producing } \\
\text { State and Region }\end{array}$ & Continuous 1 & Conventional 2 & Longwall 3 & Other 4 & Total \\
\hline Alabama & $\mathbf{w}$ & $\mathbf{w}$ & 372 & - & 417 \\
\hline 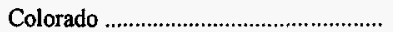 & $\mathbf{w}$ & w & 296 & - & 411 \\
\hline 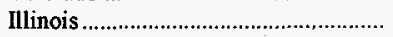 & 443 & - & 418 & - & 861 \\
\hline Indiana & $\mathbf{w}$ & - & - & - & w \\
\hline 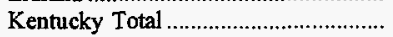 & 827 & $w$ & 121 & $\mathbf{w}$ & 985 \\
\hline Eastern & 558 & $\mathrm{w}$ & 12 & $\mathbf{w}$ & 605 \\
\hline 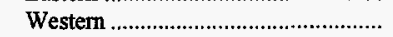 & 269 & 1 & 109 & - & 380 \\
\hline 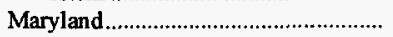 & w & - & w & - & w \\
\hline Montana & w & - & - & - & w \\
\hline Ohio & 5 & - & 223 & - & 228 \\
\hline 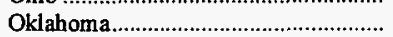 & $w$ & - & - & - & $w$ \\
\hline 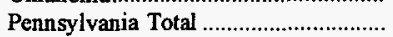 & 138 & w & 425 & $\mathbf{w}$ & 614 \\
\hline Anthracite & w & $\mathbf{w}$ & - & w & 23 \\
\hline 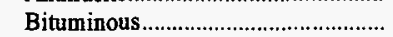 & $\mathbf{w}$ & w & 425 & - & 591 \\
\hline 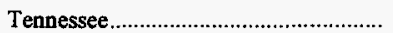 & $\mathbf{w}$ & $*$ & - & - & w \\
\hline 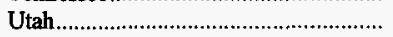 & $w$ & $\mathbf{w}$ & 190 & - & 284 \\
\hline Virginia & 84 & $w$ & $\mathbf{w}$ & - & 171 \\
\hline 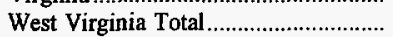 & 433 & 53 & 771 & - & 1,256 \\
\hline Northem & 54 & 18 & 570 & - & 641 \\
\hline Southern & 378 & 35 & 201 & - & 615 \\
\hline 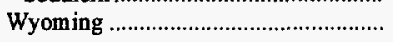 & - & w & w & - & w \\
\hline Appalachian Total 5 & 1,345 & $\mathbf{w}$ & $\mathbf{w}$ & 22 & $\mathbf{3 , 4 1 0}$ \\
\hline Interior Total 5 & $w$ & 1 & $\mathbf{w}$ & - & 1,295 \\
\hline Western Total ${ }^{5}$ & $\mathbf{w}$ & $\mathbf{w}$ & $\mathbf{w}$ & - & 801 \\
\hline East of Miss. River................................. & 2,096 & $\mathbf{w}$ & $\mathbf{w}$ & 22 & 4,691 \\
\hline West of Miss. River & 270 & $\mathbf{w}$ & $\mathbf{w}$ & - & 815 \\
\hline U.S. Total & 2,366 & 199 & 2,919 & 22 & 5,506 \\
\hline
\end{tabular}

1 Mines that produce greater than 50 percent of coal by continuous mining method.

2 Mines that produce greater than 50 percent of coal by conventional mining method.

3 Mines that have any production from longwall mining method. A typical longwall mining operation uses 80 percent longwall mining and 20 percent continuous mining.

4 Mines that produce coal using shortwall, scoop loading, hand loading, or other mining methods or a $50 / 50$ percent continuous/conventional split in mining method.

5 For a definition of coal-producing regions, see Appendix C.

* Data round to zero.

Notes: Recoverable reserves represent the quantity of coal that can be recovered (i.e., mined) from existing coal reserves at reporting mines. Excludes mines producing less than 10,000 short tons, which are not required to provide these data. Totals may not equal sum of components due to independent rounding.

Sources: Energy Information Administration, Form EIA-7A, "Coal Production Report”; State Mining Agency Coal Production Reports; and/or U.S. Department of Labor, Mine Safety and Health Administration, Fonn 7000-2, "Quarterly Mine Employment and Coal Production Report." 
Table 29. Average Recovery Percentage at Producing Underground Coal Mines by State and Mining Method, 1996

\begin{tabular}{|c|c|c|c|c|c|}
\hline $\begin{array}{l}\text { Coal-Producing } \\
\text { State and Region }\end{array}$ & Continuous 1 & Conventional $\left.\right|^{2}$ & Longwall $^{3}$ & Other 4 & Total \\
\hline Alabama & w & w & 54.16 & - & 52.98 \\
\hline 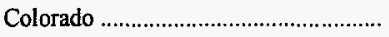 & w & $w$ & 83.66 & - & 73.72 \\
\hline Illinois & 50.30 & - & 56.69 & - & 53.40 \\
\hline Indiana & w & - & - & - & $w$ \\
\hline Kentucky Total & 53.22 & $w$ & 52.18 & w & 53.26 \\
\hline Eastern & 55.52 & w & 55.92 & w & 55.68 \\
\hline 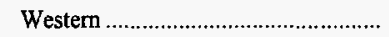 & 48.45 & 49.00 & 51.78 & - & 49.41 \\
\hline 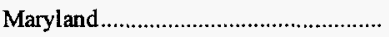 & $w$ & - & w & - & w \\
\hline 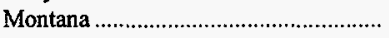 & w & - & - & - & $w$ \\
\hline Ohio & 54.90 & - & 56.90 & - & 56.85 \\
\hline Oklahoma & $\mathbf{w}$ & - & - & - & w \\
\hline Pennsylvania Total ................................. & 65.82 & $\mathbf{w}$ & 62,00 & $w$ & 63.27 \\
\hline Anthracite & $\mathbf{w}$ & $w$ & - & $\mathbf{w}$ & 73.55 \\
\hline 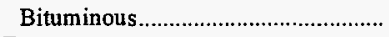 & $\mathrm{w}$ & $\mathbf{w}$ & 62.00 & - & 62.87 \\
\hline 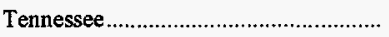 & $\mathbf{w}$ & 70.00 & - & - & w \\
\hline Utah & $w$ & w & 47.55 & - & 45.61 \\
\hline 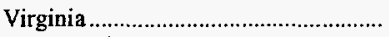 & 60.46 & w & w & - & 51.47 \\
\hline West Virginia Total.................................. & 55.78 & 54.31 & 60.16 & - & 58.41 \\
\hline 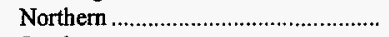 & 64.89 & 64.67 & 56.93 & - & 57.81 \\
\hline 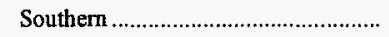 & 54.48 & 49.06 & 69.31 & - & 59.03 \\
\hline Wyoming & - & w & w & - & w \\
\hline 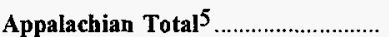 & 57.01 & $\mathbf{w}$ & $\mathbf{w}$ & 74.58 & $\mathbf{5 7 . 7 9}$ \\
\hline Interior Total 5 & w & 49.00 & $\mathbf{w}$ & - & 52.12 \\
\hline 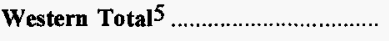 & 52.71 & $\mathbf{w}$ & $\mathbf{w}$ & - & 63.18 \\
\hline East of Miss. River & $\mathbf{5 4 . 3 1}$ & $\mathbf{w}$ & $\mathbf{w}$ & $\mathbf{7 4 . 5 8}$ & 56.22 \\
\hline West of Miss. River ............................. & 53.09 & $\mathbf{w}$ & $\mathbf{w}$ & - & 63.12 \\
\hline 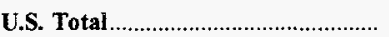 & 54.17 & 56.44 & 59.65 & 74.58 & 57.24 \\
\hline
\end{tabular}

1 Mines that produce greater than 50 percent of coal by continuous mining method.

2 Mines that produce greater than 50 percent of coal by conventional mining method.

3 Mines that have any production from longwall mining method. A typical longwall mining operation uses 80 percent longwall mining and 20 percent continuous mining.

4 Mines that produce coal using shortwall, scoop loading, hand loading, or other mining methods or a $50 / 50$ percent continuous/conventional split in mining method.

5 For a definition of coal-producing regions, see Appendix C.

Notes: Average recovery percentage represents the percentage of coal that can be recovered from coal reserves at reporting mines, weighted for all mines in the reported geographic area. Excludes mines producing less than 10,000 short tons, which are not required to provide these data.

Sources: Energy Information Administration, Form EIA-7A, "Coal Production Report”; State Mining Agency Coal Production Reports; and/or U.S. Department of Labor, Mine Safety and Health Administration, Form 7000-2, "Quarterly Mine Employment and Coal Production Report." 
Table 30. Recoverable Coal Reserves and Average Recovery Percentage at Producing U.S. Mines by Mine Production Range and Mine Type, 1996

(Million Short Tons)

\begin{tabular}{|c|c|c|c|c|c|c|}
\hline \multirow[b]{2}{*}{$\begin{array}{l}\text { Mine Production Range } \\
\text { (thousand short tons) }\end{array}$} & \multicolumn{2}{|c|}{ Underground } & \multicolumn{2}{|c|}{ Surface } & \multicolumn{2}{|c|}{ Total } \\
\hline & $\begin{array}{c}\text { Recoverable } \\
\text { Coal } \\
\text { Reserves }\end{array}$ & $\begin{array}{c}\text { Average } \\
\text { Recovery } \\
\text { Percentage }\end{array}$ & $\begin{array}{c}\text { Recoverable } \\
\text { Coal } \\
\text { Reserves }\end{array}$ & $\begin{array}{c}\text { Average } \\
\text { Recovery } \\
\text { Percentage }\end{array}$ & $\begin{array}{c}\text { Recoverable } \\
\text { Coal } \\
\text { Reserves }\end{array}$ & $\begin{array}{c}\text { Average } \\
\text { Recovery } \\
\text { Percentage }\end{array}$ \\
\hline 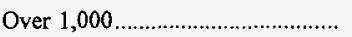 & 3,938 & 57.19 & 12,637 & 91.00 & 16,575 & 82.97 \\
\hline 500 to 1,000 & 653 & 53.04 & 651 & 82.54 & 1,304 & 67.77 \\
\hline 200 to 500 & 378 & 59.41 & 313 & 80.11 & 691 & 68.78 \\
\hline 100 to 200 & 262 & 62.64 & 211 & 83.34 & 473 & 71.88 \\
\hline 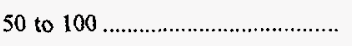 & 77 & 64.09 & 42 & 67.98 & 119 & 65.46 \\
\hline 10 to 50 & 199 & 58.18 & 68 & 74.46 & 267 & 62.32 \\
\hline 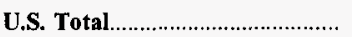 & 5,506 & 57.24 & 13,922 & 90.09 & 19,428 & 80.78 \\
\hline
\end{tabular}

Notes: Recoverable reserves represent the quantity of coal that can be recovered (i.e., mined) from existing coal reserves at reporting mines. Average recovery percentage represents the percentage of coal that can be recovered from coal reserves at reporting mines, weighted for all mines in the reported geographic area. Excludes silt, culm, refuse bank, slurry dam, and dredge operations except for Pennsylvania anthracite. Excludes mines producing less than 10,000 short tons, which are not required to provide these data. Totals may not equal sum of components due to independent rounding.

Sources: Energy Information Administration, Form EIA-7A, "Coal Production Report"; State Mining Agency Coal Production Reports; and/or U.S. Department of Labor, Mine Safety and Health Administration, Forna 7000-2, "Quarterly Mine Employment and Coal Production Report."

Table 31. Recoverable Coal Reserves and Average Recovery Percentage at Producing U.S. Mines by Coalbed Thickness and Mine Type, 1996 (Million Short Tons)

\begin{tabular}{|c|c|c|c|c|c|c|}
\hline \multirow[b]{2}{*}{$\begin{array}{c}\text { Coalbed Thickness } \\
\text { (inches) }\end{array}$} & \multicolumn{2}{|c|}{ Underground } & \multicolumn{2}{|c|}{ Surface } & \multicolumn{2}{|c|}{ Total } \\
\hline & $\begin{array}{c}\text { Recoverable } \\
\text { Coal } \\
\text { Reserves }\end{array}$ & $\begin{array}{c}\text { Average } \\
\text { Recovery } \\
\text { Percentage }\end{array}$ & $\begin{array}{c}\text { Recoverable } \\
\text { Coal } \\
\text { Reserves }\end{array}$ & $\begin{array}{c}\text { Average } \\
\text { Recovery } \\
\text { Percentage }\end{array}$ & $\begin{array}{c}\text { Recoverable } \\
\text { Coal } \\
\text { Reserves }\end{array}$ & $\begin{array}{c}\text { Average } \\
\text { Recovery } \\
\text { Percentage }\end{array}$ \\
\hline$<7$ & - & - & $*$ & 65.33 & $*$ & 65.33 \\
\hline $7-12$ & - & - & 23 & 87.13 & 23 & 87.13 \\
\hline $13-18$ & - & - & 56 & 88.49 & 56 & 88.49 \\
\hline $19-24$ & 1 & 55.68 & 213 & 84.18 & 214 & 84.00 \\
\hline $25-30$ & 61 & 57.07 & 176 & 84.38 & 238 & 77.33 \\
\hline 31-36 & 165 & 52.14 & 319 & 83.39 & 484 & 76.16 \\
\hline $37-42 \ldots \ldots \ldots \ldots \ldots \ldots \ldots \ldots \ldots \ldots$ & 269 & 54.41 & 186 & 79.70 & 454 & 64.75 \\
\hline $43-48$ & 460 & 152.61 & 552 & 85.86 & 1,012 & 75.30 \\
\hline $49-54$ & 435 & 56.82 & 264 & 88.62 & 699 & 68.81 \\
\hline $55-60$ & 508 & 56.65 & 126 & 84.19 & 634 & 62.12 \\
\hline 61-66 & 489 & 54.79 & 218 & 88.38 & 706 & 65.14 \\
\hline $67-72$ & 678 & 54.21 & 120 & 79.68 & 798 & 58.04 \\
\hline 73-78 & 293 & 56.26 & 162 & 88.16 & 455 & 67.61 \\
\hline 79-84 & 926 & 56.01 & 458 & 92.11 & 1,384 & 67.96 \\
\hline $85-90$ & 197 & 65.12 & 299 & 89.57 & 496 & 79.87 \\
\hline $91-96$ & 359 & 53.02 & 69 & 85.66 & 428 & 58.30 \\
\hline $97-102$ & 25 & 50.14 & 225 & 91.80 & 250 & 87.67 \\
\hline $103-108 \ldots \ldots \ldots \ldots$ & 112 & 46.86 & 115 & 80.98 & 227 & 64.16 \\
\hline $109-114$ & 106 & 62.00 & 7 & 85.52 & 113 & 63.49 \\
\hline $115-120$ & 64 & 47.08 & 43 & 79.45 & 107 & 59.97 \\
\hline$>\quad 120 \ldots \ldots \ldots \ldots \ldots \ldots \ldots \ldots$ & 360 & 88.67 & 10,290 & 91.32 & 10,650 & 90.55 \\
\hline 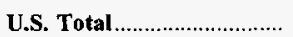 & 5,506 & $\$ 7.24$ & 13,922 & 90.09 & 19,428 & 80.78 \\
\hline
\end{tabular}

* Data round to zero

Notes: Recoverable reserves represent the quantity of coal that can be recovered (i.e., mined) from existing coal reserves at reparting mines. Average recovery percentage represents thet percentage of coal that cas be recovered from coal reserves at reporting mines, weighted for all mines in the reported geographic area. Excludes silt, culm, refuse bank, slurty dam, and dredge operations except for Pennsylvania anthracite. Excludes mines producing less than 10,000 short tons, which are not required to provide these data. Totals may not equal sum of components due to independent rounding

Sources: Energy Information Administration, Form EIA-7A, "Coal Production Report"; State Mining Agency Coal Production Reports; and/or U.S. Department of Labor, Mine Safety and Health Administration, Form 7000-2, "Quarterly Mine Employment and Coal Production Report." 
Table 32. Recoverable Coal Reserves and Average Recovery Percentage at Producing Mines by State, Mine Type, and Union Type, 1996 (Million Short Tons)

\begin{tabular}{|c|c|c|c|c|c|c|c|c|}
\hline \multirow[b]{2}{*}{$\begin{array}{l}\text { Coal-Producing } \\
\text { State and Region }\end{array}$} & \multicolumn{2}{|c|}{ UMWA } & \multicolumn{2}{|c|}{ Other Unions } & \multicolumn{2}{|c|}{ Nonunion } & \multicolumn{2}{|c|}{ Total } \\
\hline & $\begin{array}{c}\text { Recoverable } \\
\text { Coal } \\
\text { Reserves }\end{array}$ & $\begin{array}{c}\text { Average } \\
\text { Recovery } \\
\text { Percentage }\end{array}$ & $\begin{array}{c}\text { Recoverable } \\
\text { Coal } \\
\text { Reserves }\end{array}$ & $\begin{array}{c}\text { Average } \\
\text { Recovery } \\
\text { Percentage }\end{array}$ & $\begin{array}{c}\text { Recoverable } \\
\text { Coal } \\
\text { Reserves }\end{array}$ & $\begin{array}{c}\text { Average } \\
\text { Recovery } \\
\text { Percentage }\end{array}$ & $\begin{array}{c}\text { Recoverable } \\
\text { Coal } \\
\text { Reserves }\end{array}$ & $\begin{array}{c}\text { Average } \\
\text { Recovery } \\
\text { Percentage }\end{array}$ \\
\hline Alabama & 346 & 54.95 & - & - & 105 & $\mathbf{5 7 . 5 8}$ & 452 & 55.56 \\
\hline Underground & w & w & - & - & w & w & 417 & 52.98 \\
\hline Surface & $w$ & $w$ & - & - & $w$ & $\mathbf{w}$ & 35 & 86.25 \\
\hline Alaska. & - & - & $\mathbf{w}$ & $\mathbf{w}$ & - & - & $\mathbf{w}$ & $\mathbf{w}$ \\
\hline Surface & - & - & $\mathbf{w}$ & w & - & - & $\mathbf{w}$ & $w$ \\
\hline 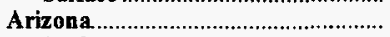 & $\mathbf{w}$ & $\mathbf{w}$ & - & - & - & - & $\mathbf{w}$ & $\mathbf{w}$ \\
\hline Surface & $w$ & $w$ & - & - & - & - & w & $\mathbf{w}$ \\
\hline Colorado & $\mathbf{w}$ & $\mathbf{w}$ & $\mathbf{w}$ & $\mathbf{w}$ & 504 & 79.94 & 642 & 79.12 \\
\hline 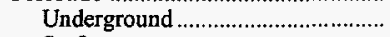 & w & $w$ & - & - & $\mathbf{w}$ & $w$ & 411 & 73.72 \\
\hline 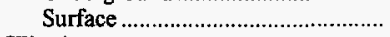 & w & w & $w$ & $\mathbf{w}$ & w & w & 230 & 88.76 \\
\hline 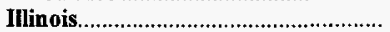 & 437 & 47.24 & $\mathbf{w}$ & $\mathbf{w}$ & $\mathbf{w}$ & $\mathbf{w}$ & 891 & 54.20 \\
\hline 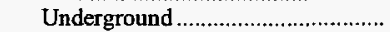 & 423 & 46.29 & w & w & $\mathbf{w}$ & $\mathbf{w}$ & 861 & 53.40 \\
\hline Surface & 14 & 75.15 & $\mathbf{w}$ & w & $w$ & $\mathbf{w}$ & 30 & 77.32 \\
\hline Indiana & 179 & $\mathbf{8 2 . 5 3}$ & - & - & 207 & 75.66 & 386 & 78.85 \\
\hline 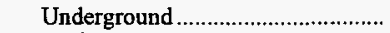 & $w$ & $\mathbf{w}$ & - & - & $w$ & w & $\mathbf{w}$ & w \\
\hline 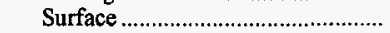 & $w$ & w & - & - & $w$ & w & w & $w$ \\
\hline 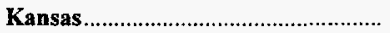 & $\mathbf{w}$ & $\mathbf{w}$ & - & - & - & - & $\mathbf{w}$ & $\mathbf{w}$ \\
\hline Surface & $\mathbf{w}$ & $\mathbf{w}$ & - & - & - & - & w & w \\
\hline Kentucky Total ...................................... & $\mathbf{w}$ & $\mathbf{w}$ & $\mathbf{w}$ & $\mathbf{w}$ & 1,062 & 59.03 & 1,255 & 58.99 \\
\hline 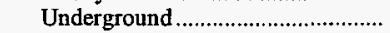 & $\mathbf{w}$ & w & $w$ & w & 843 & 54.13 & 985 & 53.26 \\
\hline Surface & w & w & $\mathbf{w}$ & w & 219 & 77.86 & 270 & 79.90 \\
\hline Eastern & $\mathbf{w}$ & $\mathbf{w}$ & $\mathbf{w}$ & $\mathbf{w}$ & 744 & 61.16 & 818 & 62.59 \\
\hline Underground & $\mathbf{w}$ & $\mathrm{w}$ & w & w & $w$ & $w$ & 605 & 55.68 \\
\hline 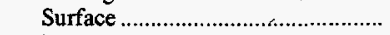 & w & w & $\mathbf{w}$ & $\mathbf{w}$ & w & $w$ & 213 & 82.17 \\
\hline 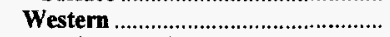 & $\mathbf{w}$ & $\mathbf{w}$ & - & - & 318 & 54.05 & 437 & 52.27 \\
\hline 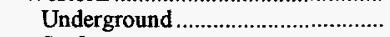 & $\mathbf{w}$ & w & - & - & w & w & 380 & 49.41 \\
\hline Surface & $w$ & w & - & - & $w$ & $\mathrm{w}$ & 57 & 71.37 \\
\hline 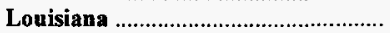 & - & - & - & - & $\mathbf{w}$ & $\mathbf{w}$ & $\mathbf{w}$ & $\mathbf{w}$ \\
\hline Surface & - & - & - & - & $w$ & w & $\mathbf{w}$ & $\mathbf{w}$ \\
\hline 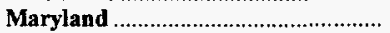 & - & - & - & - & 71 & 61.45 & 71 & 61.45 \\
\hline Underground & - & - & - & - & $\mathbf{w}$ & $\mathrm{w}$ & $w$ & w \\
\hline Surface & - & - & - & - & $\mathbf{w}$ & w & w & w \\
\hline 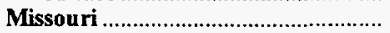 & - & - & - & - & 3 & 59.87 & 3 & 59.87 \\
\hline Surface & - & - & - & - & 3 & 59.87 & 3 & 59.87 \\
\hline 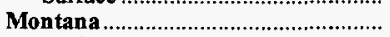 & 195 & 90.37 & $\mathbf{w}$ & $\mathbf{w}$ & $\mathbf{w}$ & $\mathbf{w}$ & 1,309 & 89.05 \\
\hline Underground & - & - & w & $w$ & $\mathrm{w}$ & $w$ & $w$ & w \\
\hline 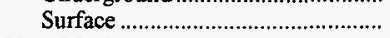 & 195 & 90.37 & $\mathbf{w}$ & $w$ & $w$ & w & w & w \\
\hline 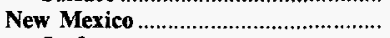 & $\mathbf{w}$ & $\mathbf{w}$ & $\mathbf{w}$ & $\mathbf{w}$ & $\mathbf{w}$ & $\mathbf{w}$ & 1,436 & 93.35 \\
\hline Surface & $w$ & w & w & w & $\mathbf{w}$ & $\mathrm{w}$ & 1,436 & 93.35 \\
\hline 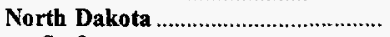 & $\mathbf{w}$ & $\mathbf{w}$ & $\mathbf{w}$ & $\mathbf{w}$ & $\mathbf{w}$ & $\mathbf{w}$ & 1,301 & 89.72 \\
\hline Surface & w & w & w & $\mathbf{w}$ & w & w & 1,301 & 89.72 \\
\hline Ohio & $\mathbf{w}$ & $\mathbf{w}$ & - & - & 115 & 84.48 & 415 & 71.61 \\
\hline 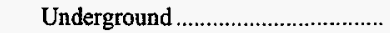 & $\mathbf{w}$ & w & - & - & $w$ & w & 228 & 56.85 \\
\hline Surface ............................................. & w & $\mathbf{w}$ & - & - & w & $\mathbf{w}$ & 186 & 89.68 \\
\hline Oklahoma & - & - & - & - & 19 & 65.94 & 19 & 65.94 \\
\hline 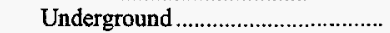 & - & - & - & - & 14 & 60.00 & 14 & 60.00 \\
\hline 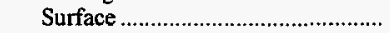 & - & - & - & - & 5 & 83.85 & 5 & 83.85 \\
\hline Pennsylvania Total & $\mathbf{w}$ & $\mathbf{w}$ & $\mathbf{w}$ & $\mathbf{w}$ & 367 & 72.99 & 796 & 65.59 \\
\hline 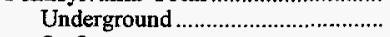 & w & w & - & - & w & w & 614 & 63.27 \\
\hline Surface & w & w & $w$ & w & $\mathbf{w}$ & $\mathbf{w}$ & 182 & 73.42 \\
\hline Anthracite & $\mathbf{w}$ & $\mathbf{w}$ & $\mathbf{w}$ & $\mathbf{w}$ & 29 & 69.11 & 90 & $\mathbf{5 9 . 9 0}$ \\
\hline Underground & - & - & - & - & $w$ & w & 23 & 73.55 \\
\hline Surface & w & $\mathbf{w}$ & $\mathbf{w}$ & $w$ & $w$ & w & 66 & 55.13 \\
\hline Bituminous & 368 & 59.89 & - & - & 338 & 73.32 & 706 & 66.32 \\
\hline Underground & 363 & 59.61 & - & - & 227 & 68.08 & 591 & 62.87 \\
\hline Surface & 5 & 80.11 & - & - & 110 & 84.10 & 116 & 83.92 \\
\hline 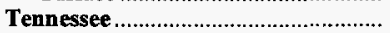 & - & - & - & - & 59 & 63.33 & 59 & 63.33 \\
\hline 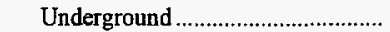 & - & - & - & - & 57 & 62.56 & 57 & 62.56 \\
\hline Surface & - & - & - & - & 2 & 85.10 & 2 & 85.10 \\
\hline 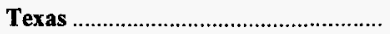 & - & - & 484 & 90.24 & 394 & 85.98 & 878 & 88.32 \\
\hline Surface & - & - & 484 & 90.24 & 394 & 85.98 & 878 & 88.32 \\
\hline Utah & $\mathbf{w}$ & $\mathbf{w}$ & - & - & $\mathbf{w}$ & $\mathbf{w}$ & 284 & 45.61 \\
\hline Underground & $w$ & $\mathrm{w}$ & - & - & w & $\mathbf{w}$ & 284 & 45.61 \\
\hline Virginia & $\mathbf{w}$ & $w$ & $\mathbf{w}$ & $\mathbf{w}$ & 126 & 59.18 & 188 & $\mathbf{5 4 . 8 1}$ \\
\hline 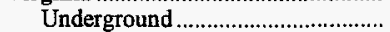 & $w$ & w & - & - & w & $w$ & 171 & 51.47 \\
\hline 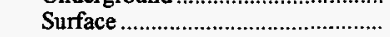 & $\mathrm{w}$ & w & $w$ & w & $\mathbf{w}$ & $\mathbf{w}$ & 18 & 86.77 \\
\hline
\end{tabular}

See footnotes at end of table. 
Table 32. Recoverable Coal Reserves and Average Recovery Percentage at Producing Mines by State, Mine Type, and Union Type, 1996 (Continued) (Million Short Tons)

\begin{tabular}{|c|c|c|c|c|c|c|c|c|}
\hline \multirow[b]{2}{*}{$\begin{array}{l}\text { Coal-Producing } \\
\text { State and Region }\end{array}$} & \multicolumn{2}{|c|}{ UMWA } & \multicolumn{2}{|c|}{ Other Unions } & \multicolumn{2}{|c|}{ Nonunion } & \multicolumn{2}{|c|}{ Total } \\
\hline & $\begin{array}{c}\text { Recoverable } \\
\text { Coal } \\
\text { Reserves }\end{array}$ & $\begin{array}{c}\text { Average } \\
\text { Recovery } \\
\text { Percentage }\end{array}$ & $\begin{array}{c}\text { Recoverable } \\
\text { Coal } \\
\text { Reserves }\end{array}$ & $\begin{array}{c}\text { Average } \\
\text { Recovery } \\
\text { Percentage }\end{array}$ & $\begin{array}{c}\text { Recoverable } \\
\text { Coal } \\
\text { Reserves }\end{array}$ & $\begin{array}{c}\text { Average } \\
\text { Recovery } \\
\text { Percentage }\end{array}$ & $\begin{array}{c}\text { Recoverable } \\
\text { Coal } \\
\text { Reserves }\end{array}$ & $\begin{array}{c}\text { Average } \\
\text { Recovery } \\
\text { Percentage }\end{array}$ \\
\hline 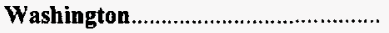 & - & - & $\mathbf{w}$ & $\mathbf{w}$ & $\mathbf{w}$ & $\mathbf{w}$ & $\mathbf{w}$ & $\mathbf{w}$ \\
\hline Surface & - & - & $\mathbf{w}$ & $\mathbf{w}$ & w & $\mathbf{w}$ & $w$ & $w$ \\
\hline West Virginia Total & 1,112 & 63.11 & - & - & 619 & 64.17 & 1,731 & 63.49 \\
\hline 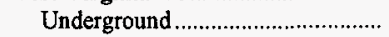 & 918 & $5 S .24$ & - & - & 338 & 56.15 & 1,256 & 58.41 \\
\hline Surface & 194 & 81.46 & - & - & 281 & 73.80 & 475 & 76.93 \\
\hline Northern & 570 & 56.92 & - & - & 172 & 65.50 & 741 & 58.91 \\
\hline 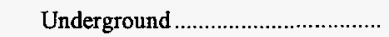 & 570 & 56.92 & - & - & 72 & 64.85 & 641 & 57.81 \\
\hline 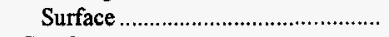 & - & - & - & - & 100 & 65.96 & 100 & 65.96 \\
\hline 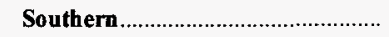 & 543 & 69.61 & - & - & 447 & 63.66 & 990 & 66.92 \\
\hline 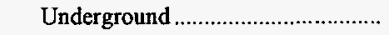 & 349 & 63.02 & - & - & 266 & 53.80 & 615 & 59.03 \\
\hline 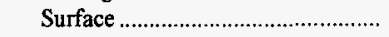 & 194 & 81.46 & - & - & 181 & 78.12 & 375 & 79.85 \\
\hline 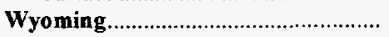 & $\mathbf{w}$ & $\mathbf{w}$ & $\mathbf{w}$ & $\mathbf{w}$ & 6,229 & 92.21 & 6,591 & 92.16 \\
\hline 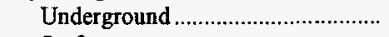 & - & - & - & - & $\mathbf{w}$ & $\mathbf{w}$ & w & $\mathbf{w}$ \\
\hline Surface & w & $w$ & w & w & $w$ & $\mathbf{w}$ & $w$ & w \\
\hline Appalachian Total ${ }^{1}$ & 2,314 & 61.64 & 11 & 58.22 & 2,206 & 64.97 & 4,530 & 63.25 \\
\hline 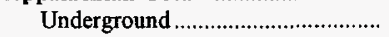 & $w$ & $w$ & $w$ & $\mathbf{w}$ & 1,495 & 58.25 & 3,410 & 57.79 \\
\hline Surface & $\mathbf{w}$ & $w$ & w & w & 711 & 79.09 & 1,120 & 79.89 \\
\hline Interior Total ${ }^{1}$ & 738 & 55.93 & 533 & 88.17 & 1,486 & 70.75 & 2,757 & 70.15 \\
\hline 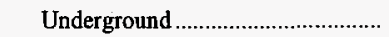 & w & w & $\mathbf{w}$ & w & 717 & 55.88 & 1,295 & 52.12 \\
\hline Surface & $\mathbf{w}$ & $\mathbf{w}$ & $\mathbf{w}$ & $w$ & 769 & 84.62 & 1,462 & 86.12 \\
\hline Western Total & 1,195 & 84.89 & 2,566 & 91.47 & 8,379 & 89.90 & 12,141 & 89.74 \\
\hline Underground & 167 & 55.08 & - & - & 635 & 65.31 & 801 & 63.18 \\
\hline Surface & 1,028 & 89.73 & 2,566 & 91.47 & 7,745 & 91.91 & 11,339 & 91.61 \\
\hline 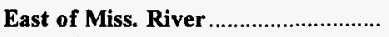 & 3,049 & 60.26 & 60 & 65.99 & 3,135 & 63.93 & 6,244 & 62.16 \\
\hline 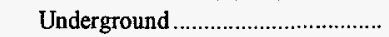 & 2,450 & 55.03 & 43 & 59.78 & 2,198 & 57.47 & 4,691 & 56.22 \\
\hline Surface & 600 & 81.61 & 17 & 82.15 & 937 & 79.09 & 1,554 & 80.10 \\
\hline West of Miss. River ............................... & 1,198 & 84.85 & 3,050 & 91.27 & 8,935 & 89.67 & 13,184 & 89.60 \\
\hline 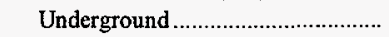 & 167 & 55.08 & - & - & 649 & 65.19 & 815 & 63.12 \\
\hline Surface & 1,031 & 89.68 & 3,050 & 91.27 & 8,287 & 91.59 & 12,368 & 91.35 \\
\hline 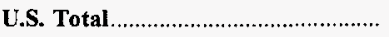 & 4,247 & 67.19 & 3,111 & 90.78 & 12,070 & 82.98 & 19,428 & 80.78 \\
\hline 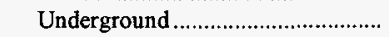 & 2,617 & 55.03 & 43 & 59.78 & 2,846 & 59.23 & 5,506 & 57.24 \\
\hline 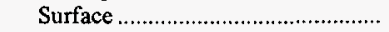 & 1,630 & 86.71 & 3,067 & 91,22 & 9,224 & 90.32 & 13,922 & 90.09 \\
\hline
\end{tabular}

1 For a definition of coal-producing regions, see Appendix C.

Notes: Recoverable reserves represent the quantity of coal that can be recovered (i.e., mined) from existing coal reserves at reporting mines. Average recovery percentage represents the percentage of coal that can be recovered from coal reserves at reporting mines, weighted for all mines in the reported geographic area. Excludes silt, culm, refuse bank, slurry clam, and dredge operations except for Pennsylvania anthracite. Excludes mines producing less than 10,000 short tons, which are not required to provide these data. Totals may not equal sum of components due to independent rounding. See Glossary for listing of other unions.

Sources: Energy Information Administration, Form EIA-7A, "Coal Production Report"; State Mining Agency Coal Production Reports; and/or U.S. Department of Labor, Mine Safety and Health Administration, Form 7000-2, "Quarterly Mine Employment and Coal Production Report." 
Table 33. Status of Recoverable Coal Reserves and Coal Production from Producing Federal Coal Leases by State, 1996

(Million Short Tons)

\begin{tabular}{|c|c|c|c|}
\hline Coal-Producing State & Number of Leases & Recoverable Coal Reserves & Production \\
\hline 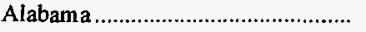 & 1 & 30 & * \\
\hline 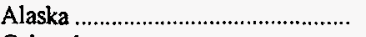 & - & 20 & - \\
\hline 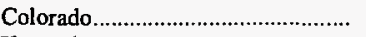 & 31 & 1,506 & 18.8 \\
\hline 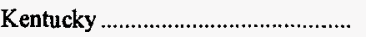 & 3 & 10 & .3 \\
\hline 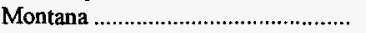 & 13 & 886 & 25.0 \\
\hline 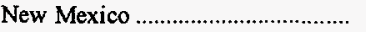 & 6 & 262 & 5.9 \\
\hline 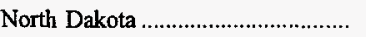 & 3 & 144 & 1.8 \\
\hline 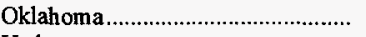 & 6 & 48 & .8 \\
\hline Utah & 31 & 2,987 & 26.4 \\
\hline 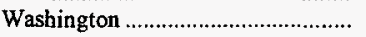 & 1 & - & .6 \\
\hline 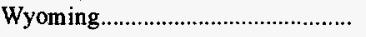 & 39 & 6,148 & 248.9 \\
\hline 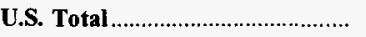 & 134 & 12,041 & 328.2 \\
\hline
\end{tabular}

* Less than 0.05 million short tons.

Notes: Output from Federal Lands is reported as sales volume, the basis for royalties. It is approximately equivalent to production, which includes coal sold and coal added to stockpiles. Totals may not equal sum of components because of independent rounding.

Source: U.S. Department of the Interior, Minerals Management Service,Mineral Revenues 1996 and Bureau of Land Management. 



\section{Producer/Distributor Stocks}

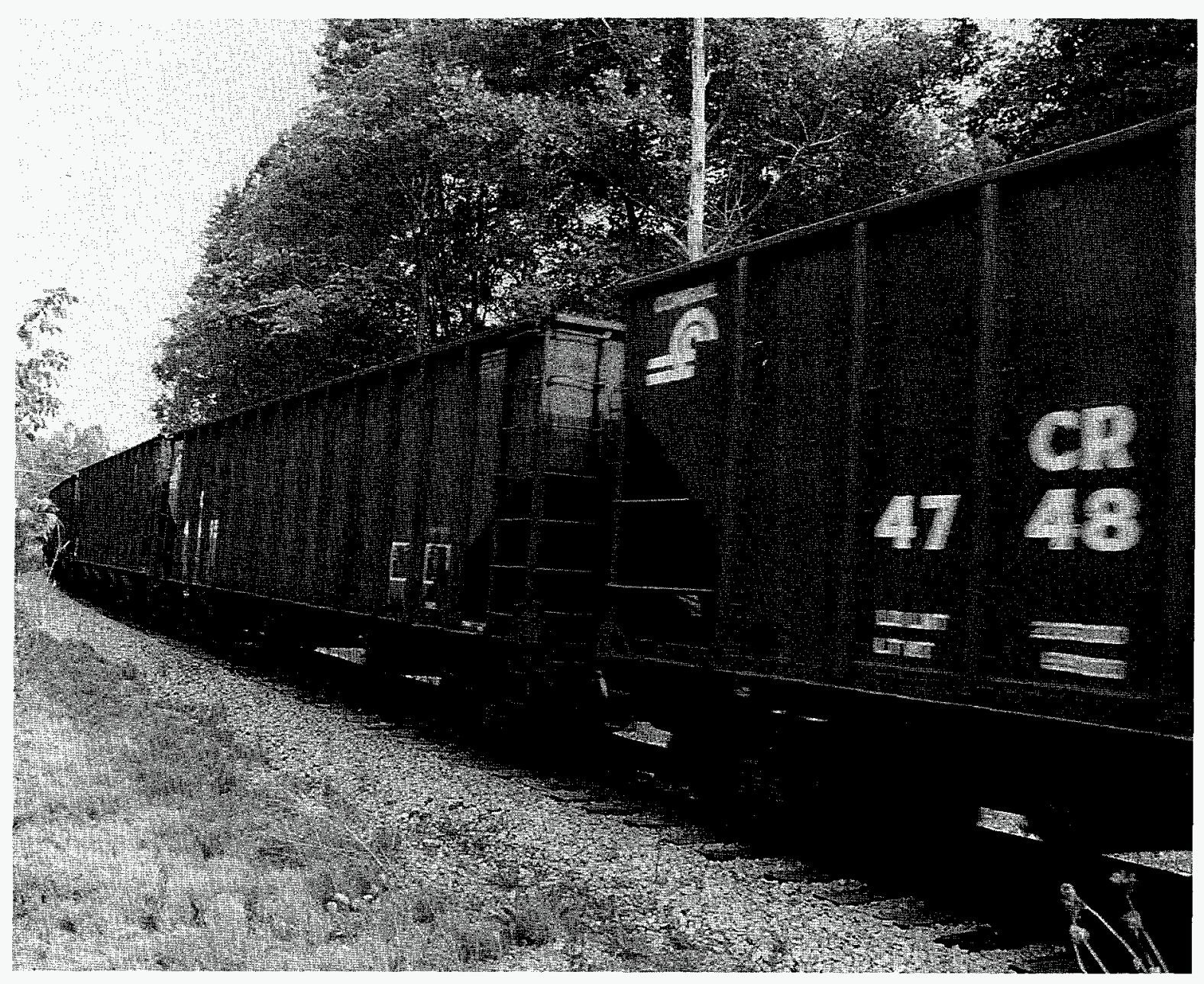

Coal cars in rural West Virginia. Rail is the primary mode of coal transportation. 
Table 34. Year-End Producer and Distributor Coal Stocks by State, 1992-1996 (Thousand Short Tons)

\begin{tabular}{|c|c|c|c|c|c|c|c|}
\hline \multirow{2}{*}{$\begin{array}{l}\text { Coal-Producing } \\
\text { State and Region }\end{array}$} & \multirow{2}{*}{1996} & \multirow{2}{*}{1995} & \multirow{2}{*}{1994} & \multirow{2}{*}{1993} & \multirow{2}{*}{1992} & \multirow{2}{*}{$\begin{array}{c}\text { Percent } \\
\text { Change } \\
1995-1996\end{array}$} & \multirow{2}{*}{$\begin{array}{c}\begin{array}{c}\text { Average Annual } \\
\text { Percent Change }\end{array} \\
1992-1996\end{array}$} \\
\hline & & & & & & & \\
\hline Alabama & 1,031 & 1,358 & 1,204 & 1,698 & 2,185 & -24.1 & -17.1 \\
\hline 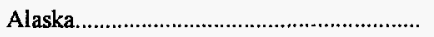 & 6 & 26 & 58 & 19 & 71 & -78.0 & -46.8 \\
\hline 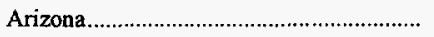 & 2,232 & 2,760 & 2,634 & 1,590 & 1,555 & -19.1 & 9.4 \\
\hline 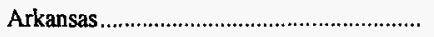 & 1 & 4 & 2 & 5 & 6 & -59.9 & -28.9 \\
\hline California & - & - & - & - & 36 & - & - \\
\hline 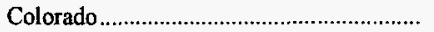 & 494 & 1,063 & 1,575 & 1,155 & 955 & -53.5 & -15.2 \\
\hline 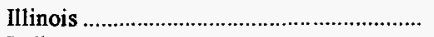 & 1,190 & 2,069 & 1,651 & 713 & 1,969 & -42.5 & -11.8 \\
\hline Indiana & 574 & 611 & 803 & 527 & 1,016 & -6.1 & -13.3 \\
\hline 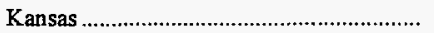 & 19 & 27 & 31 & 25 & 28 & -27.4 & -9.2 \\
\hline 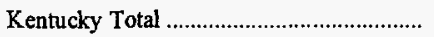 & 4,460 & 4,777 & 5,025 & 3,216 & 3,796 & -6.6 & 4.1 \\
\hline Eastern & 3,720 & 4,088 & 4,235 & 2,558 & 2,809 & -9.0 & 7.3 \\
\hline Western & 740 & 689 & 790 & 658 & 987 & 7.4 & -7.0 \\
\hline 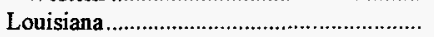 & 38 & 309 & 202 & 12 & 2 & -87.8 & 100.2 \\
\hline 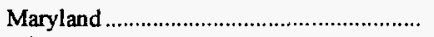 & 143 & 269 & 179 & 123 & 59 & -47.0 & 24.9 \\
\hline 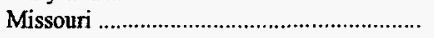 & - & - & - & 2 & - & - & - \\
\hline Montana & 580 & 718 & 635 & 876 & 694 & -19.3 & -4.4 \\
\hline 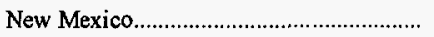 & 1,890 & 2,015 & 1,467 & 2,343 & 1,648 & -6.2 & 3.5 \\
\hline 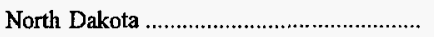 & 1,574 & 1,797 & 1,812 & 1,607 & 1,614 & -12.4 & -.6 \\
\hline Ohio & 532 & 1,374 & 833 & 550 & 1,087 & -61.3 & -16.4 \\
\hline 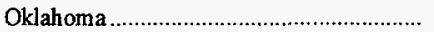 & 7 & 2 & 4 & 5 & 19 & 188.8 & -23.2 \\
\hline 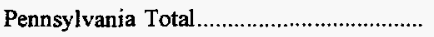 & 3,113 & 2,487 & 2,787 & 1,826 & 2,903 & 25.2 & 1.8 \\
\hline 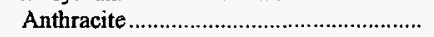 & 1,323 & 389 & 249 & 234 & 198 & 240.2 & 60.9 \\
\hline 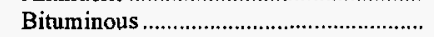 & 1,790 & 2,098 & 2,538 & 1,592 & 2,706 & -14.7 & -9.8 \\
\hline Tennessee & 23 & 88 & 57 & 35 & 21 & -73.7 & 2.4 \\
\hline 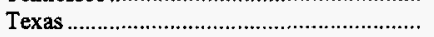 & 1,254 & 864 & 1,430 & 1,237 & 543 & 45.1 & 23.3 \\
\hline Utah & 1,337 & 1,946 & 1,301 & 1,203 & 1,827 & -31.3 & -7.5 \\
\hline 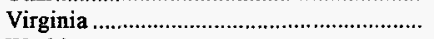 & 1,644 & 1,649 & 1,180 & 1,389 & 1,714 & -.3 & -1.0 \\
\hline 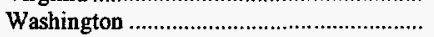 & 55 & 59 & 65 & 72 & 47 & -5.9 & 3.9 \\
\hline 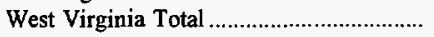 & 4,947 & 6,176 & 6,692 & 4,059 & 7,405 & -19.9 & -9.6 \\
\hline 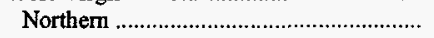 & 584 & 1,959 & 1,940 & 685 & 3,222 & -70.2 & -34.8 \\
\hline Southern & 4,362 & 4,217 & 4,752 & 3,374 & 4,183 & 3.4 & 1.1 \\
\hline 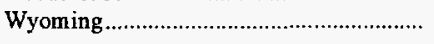 & 1,504 & 1,997 & 1,592 & 998 & 2,794 & -24.7 & -14.3 \\
\hline Appalachian Total ${ }^{l}$.................................... & 15,153 & 17,489 & 17,166 & 12,239 & 13,891 & -13.3 & -3.1 \\
\hline Interior Total 1 . & $\mathbf{3 , 8 2 3}$ & 4,575 & 4,913 & 3,182 & 4,570 & -16.4 & -4.4 \\
\hline 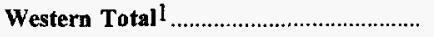 & 9,672 & 12,381 & 11,140 & 9,863 & 11,241 & -21.9 & -3.7 \\
\hline East of Miss. River & 17,657 & 20,858 & 20,410 & 14,137 & 17,680 & -15.3 & -5.4 \\
\hline 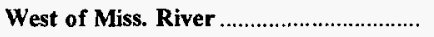 & 10,991 & 13,587 & 12,809 & 11,147 & 11,838 & -19.1 & -1.8 \\
\hline 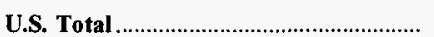 & 28,648 & 34,444 & 33,219 & 25,284 & 33,993 & -16.8 & -4.2 \\
\hline
\end{tabular}

1 For a definition of coal-producing regions, see Appendix C.

Note: Totals may not equal sum of components due to independent rounding.

Source: Energy Information Administration, Form EIA- 6 , "Coal Distribution Report." 


\section{Imports}

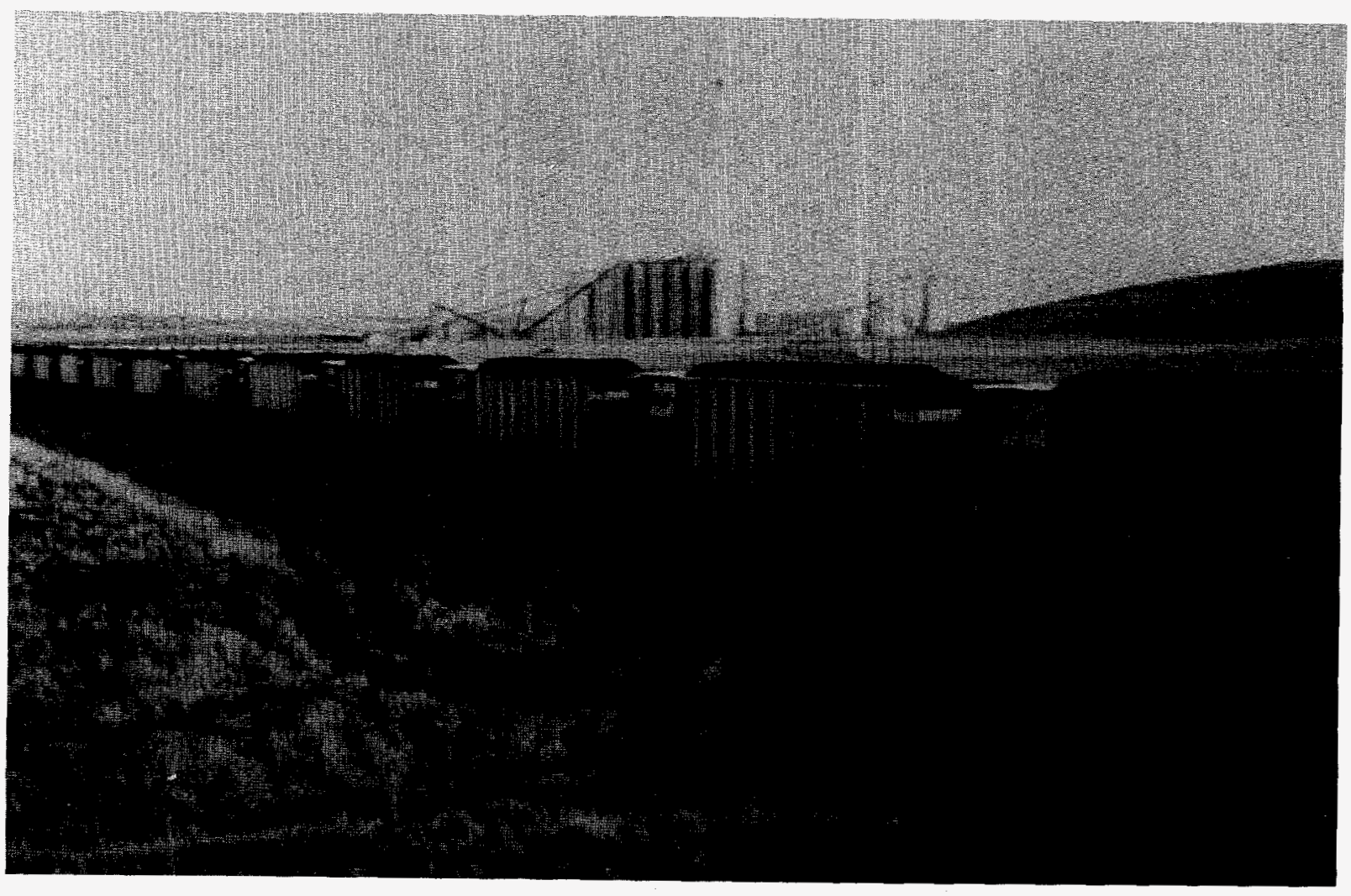

Coal is shipped by rail or barge where it is loaded abroad large ocean-going bulk carriers for shipment to Europe, Asia and other major markets. 
Figure 3. U.S. Coal Imports, 1987-1996

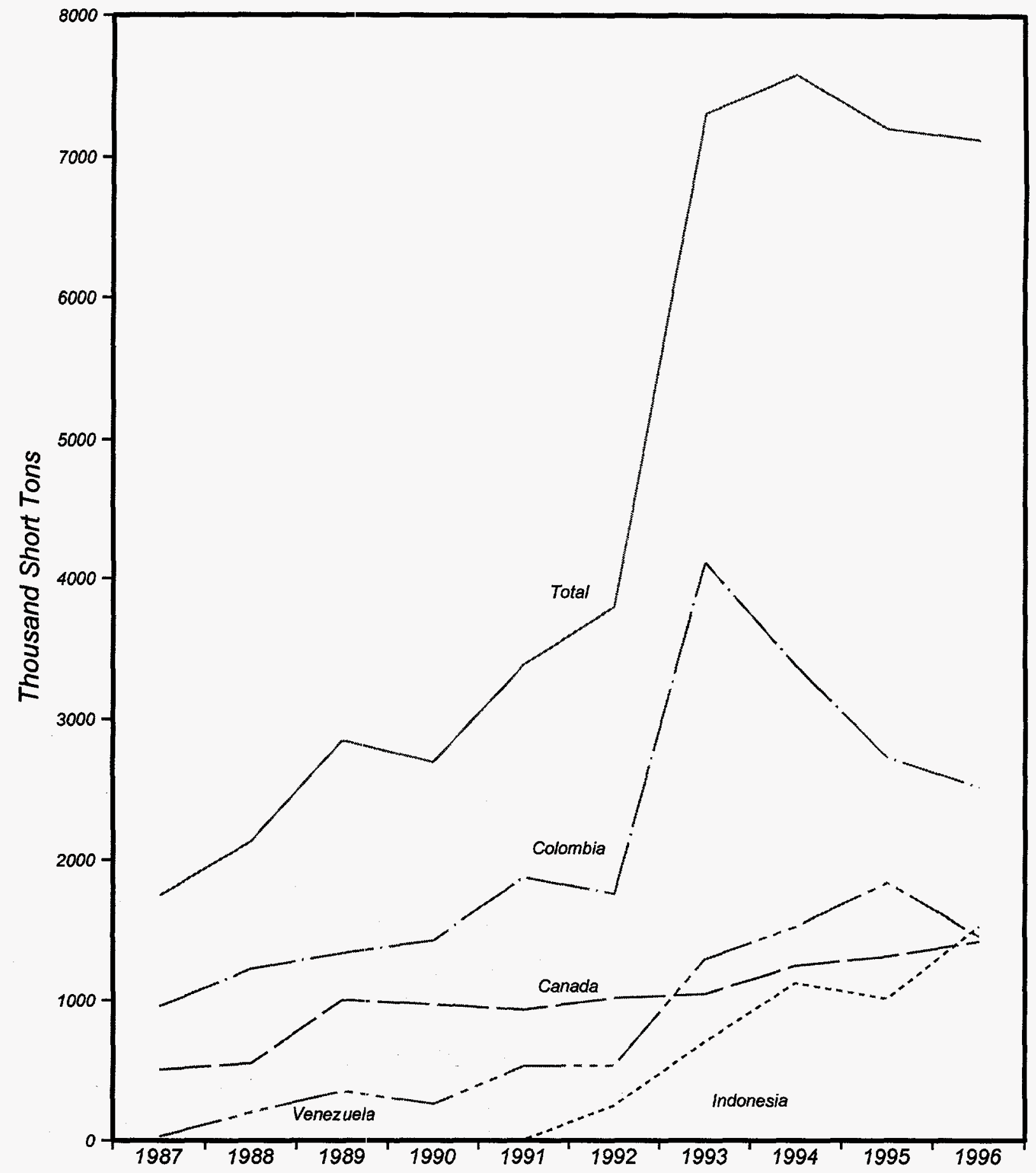

Source: U.S. Department of Commerce, Bureau of the Census, "Monthly Reporl IM 145." 
Table 35. U.S. Coal Imports by Continent and Country of Origin, 1987, 1992-1996 (Short Tons)

\begin{tabular}{|c|c|c|c|c|c|c|c|c|c|}
\hline \multirow{2}{*}{$\begin{array}{c}\text { Continent and Country } \\
\text { of Origin }\end{array}$} & \multirow{2}{*}{1996} & \multirow{2}{*}{1995} & \multirow{2}{*}{1994} & \multirow{2}{*}{1993} & \multirow{2}{*}{1992} & \multirow{2}{*}{1987} & \multirow{2}{*}{$\begin{array}{c}\text { Percent } \\
\text { Change } \\
\text { 1995-1996 }\end{array}$} & \multicolumn{2}{|c|}{ Average Annual Percent Change } \\
\hline & & & & & & & & $1992-1996$ & 1987-1996 \\
\hline North America Total ........................... & $1, \mathbf{4 3 3}, \mathbf{8 1 3}$ & $1,344,614$ & $1,253,417$ & $1,053,576$ & $\mathbf{1 , 0 2 0 , 8 5 2}$ & 549,292 & 6.6 & 8.9 & 11.2 \\
\hline 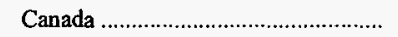 & $1,426,504$ & $1,319,742$ & $1,253,196$ & $1,051,273$ & $1,020,852$ & 506,760 & 8.1 & 8.7 & 12.2 \\
\hline 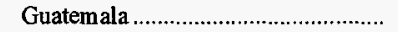 & - & - & - & 4 & - & - & - & - & - \\
\hline 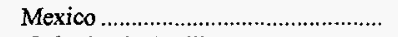 & 7,309 & 216 & 221 & 2,299 & - & 42,532 & NM & - & -17.8 \\
\hline Netherlands Antilles ........................... & - & 24,656 & - & $\cdot-$ & - & - & -100.0 & - & - \\
\hline 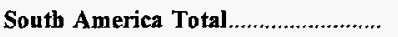 & $3,989,640$ & $4,583,283$ & $4,920,202$ & $5,415,318$ & $2,301,933$ & 986,967 & -12.9 & 14.7 & 16.8 \\
\hline 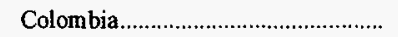 & $2,526,804$ & $2,736,933$ & $3,389,654$ & $4,117,036$ & $1,763,150$ & 956,899 & -7.7 & 9.4 & 11.4 \\
\hline 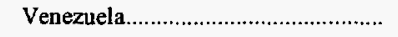 & $1,462,836$ & $1,846,350$ & $1,530,548$ & $1,298,282$ & 538,783 & 30,068 & -20.8 & 28.4 & 54.0 \\
\hline Europe Total & 2,613 & 522 & 40 & 62 & 89 & 240 & 400.6 & 132.8 & 30.4 \\
\hline Belgium \& Luxembourg ..................... & 2,473 & - & - & - & - & - & - & - & - \\
\hline 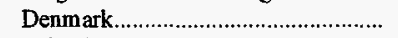 & - & 236 & - & 60 & - & - & -100.0 & - & - \\
\hline Poland & - & - & 40 & 2 & - & - & - & - & - \\
\hline 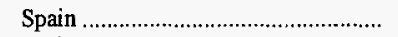 & 99 & - & - & - & - & 44 & - & - & 9.4 \\
\hline 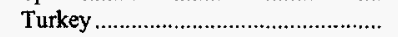 & 41 & - & - & - & - & - & - & - & - \\
\hline 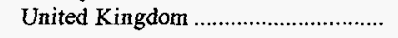 & - & 286 & - & - & 89 & 196 & -100.0 & -100.0 & -100.0 \\
\hline Asia Total & $1,534,989$ & $1,018,512$ & $1,153,561$ & 708,080 & 373,145 & - & 50.7 & 42.4 & - \\
\hline 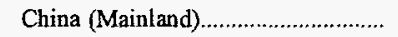 & - & 53 & 111 & - & 284 & - & -100.0 & -100.0 & - \\
\hline 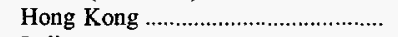 & 1 & - & - & - & 1 & - & - & - & - \\
\hline India & $\rightarrow$ & - & - & - & 66,154 & - & - & -100.0 & - \\
\hline Indonesia & $1,534,986$ & $1,018,433$ & $1,130,468$ & 708,080 & 253,287 & - & 50.7 & 56.9 & - \\
\hline Japan & 2 & 26 & 1 & - & - & - & -92.3 & - & - \\
\hline 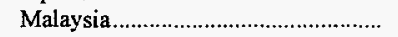 & - & - & - & - & 53,419 & - & - & -100.0 & - \\
\hline 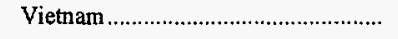 & - & - & 22,981 & - & - & - & - & - & - \\
\hline Oceania \& Australia Total ................... & 164,793 & 254,141 & 100,313 & 105,452 & 100,986 & 210,639 & -35.1 & 13.0 & -2.7 \\
\hline 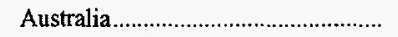 & 164,793 & 211,702 & 92,204 & 100,076 & 100,986 & 210,639 & -22.1 & 13.0 & -2.7 \\
\hline 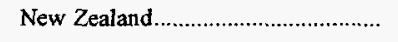 & - & 42,439 & 8,109 & 5,376 & - & - & -100.0 & - & - \\
\hline Africa Total & - & - & 156,452 & 26,419 & 5,762 & - & - & -100.0 & - \\
\hline 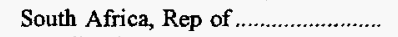 & - & - & 149,748 & 20,319 & 5,762 & - & - & -100.0 & - \\
\hline 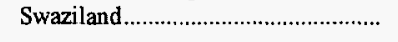 & - & - & 6,704 & 6,100 & - & - & - & - & - \\
\hline Total & $7,125,848$ & $7,201,072$ & $7,583,985$ & $7,308,907$ & $3,802,767$ & $1,747,138$ & -1.0 & 17.0 & 16.9 \\
\hline
\end{tabular}

NM Not meaningful as value is greater than 500 percent.

Note: Coal imports include coal to Puerto Rico and the Virgin Islands.

Source: U.S. Department of Commerce, Bureau of the Census, "Monthly Report IM 145." 
Table 36. Coal Imports by Customs District, 1987, 1992-1996 (Short Tons)

\begin{tabular}{|c|c|c|c|c|c|c|c|c|c|}
\hline \multirow{2}{*}{ Customs District } & \multirow{2}{*}{1996} & \multirow{2}{*}{1995} & \multirow{2}{*}{1994} & \multirow{2}{*}{1993} & \multirow{2}{*}{1992} & \multirow{2}{*}{1987} & \multirow{2}{*}{$\begin{array}{c}\text { Percent } \\
\text { Change } \\
\text { 1995-1996 }\end{array}$} & \multicolumn{2}{|c|}{ Average Annual Percent Change } \\
\hline & & & & & & & & 1992-1996 & $1987-1996$ \\
\hline 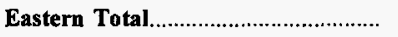 & $2,144,142$ & $1,935,4011$ & $1,554,466$ & $1,275,919$ & 340,317 & 127,308 & 10.8 & 58.4 & 36.8 \\
\hline 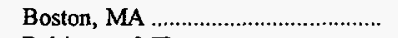 & $1,803,234$ & $1,484,886$ & 977,473 & 674,086 & 128,667 & 28,736 & 21.4 & 93.5 & 58.4 \\
\hline Baltimore, MD & 99 & $28,32: 8$ & 88,668 & 224,579 & - & - & -99.6 & - & - \\
\hline Portland, ME & 246,852 & 364,232 & 385,097 & 236,473 & 108,863 & 25,279 & -32.2 & 22.7 & 28.8 \\
\hline 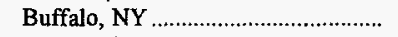 & 2,658 & 2,034 & - & - & - & 671 & 30.7 & - & 16.5 \\
\hline 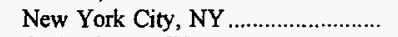 & 65 & 522 & - & 2 & - & 222 & -87.5 & - & -12.8 \\
\hline 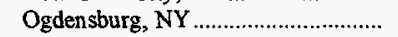 & 50 & - & - & - & - & - & - & - & - \\
\hline Philadelphia, PA & 91,184 & 55,399 & 78,387 & 140,779 & 102,787 & 72,400 & 64.6 & -2.9 & 2.6 \\
\hline Norfolk, VA & - & - & 24,841 & - & - & - & - & - & - \\
\hline 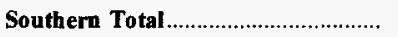 & $2,745,266$ & $3,101,069$ & $4,106,306$ & $4,321,336$ & $2,053,672$ & $1,040,055$ & -11.5 & 7.5 & 11.4 \\
\hline Mobile, $\mathrm{AL}$ & 288,484 & $1,108,555$ & $1,033,368$ & 935,232 & - & 155,173 & -74.0 & - & 7.1 \\
\hline Savannah, GA & 118,509 & - & 29,582 & - & - & - & - & - & - \\
\hline Miami, FL & - & 26,035 & 7,496 & - & - & - & -100.0 & - & - \\
\hline 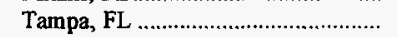 & $1,419,408$ & $1,284,109$ & $2,080,757$ & $2,263,893$ & $1,419,581$ & 96,743 & 10.5 & * & 34.8 \\
\hline 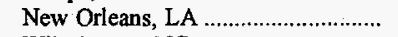 & 808,592 & 387,861 & 524,256 & 675,827 & 154,600 & 422,590 & 108.5 & 51.2 & 7.5 \\
\hline Wilmington, $\mathrm{NC}$ & - & - & 26,648 & - & - & - & - & - & - \\
\hline 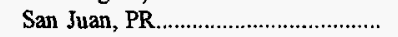 & 96,901 & 272,296 & 80,016 & 107,506 & 169,707 & 323,001 & -64.4 & -13.1 & -12.5 \\
\hline 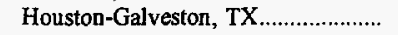 & 6,063 & - & 154,938 & 121,505 & 83,466 & 16 & - & -48.1 & 93.4 \\
\hline Laredo, TX & 7,309 & 167 & 221 & 2,299 & - & 42,532 & $\mathrm{NM}$ & - & -17.8 \\
\hline 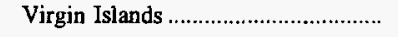 & - & 22,045 & 169,024 & 215,074 & 226,318 & - & -100.0 & -100.0 & - \\
\hline Western Total & 830,157 & 863,707 & 710,576 & 730,662 & 461,675 & $\mathbf{2 7 3}, \mathbf{7 7 2}$ & -3.9 & 15.8 & 13.1 \\
\hline 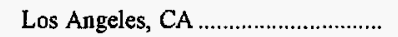 & 2 & - & 12 & - & - & - & - & - & - \\
\hline San Diego, CA & - & 49 & - & - & - & - & -100.0 & - & - \\
\hline San Francisco, CA & - & - & - & - & 284 & 22,048 & - & -100.0 & -100.0 \\
\hline 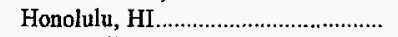 & 810,176 & 844,785 & 670,005 & 660,379 & 407,692 & 89,832 & -4.1 & 18.7 & 27.7 \\
\hline Great Falls, MT & 25 & 64.5 & 34,426 & 41,580 & 36,237 & 104,798 & -96.1 & -83.8 & -60.4 \\
\hline Seattle, WA & 19,954 & 18,2213 & 6,133 & 28,703 & 17,462 & 57,094 & 9.5 & 3.4 & -11.0 \\
\hline 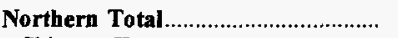 & $1,406,283$ & $1,300,894$ & $1,212,637$ & 980,990 & 947,103 & 306,003 & 8.1 & 10.4 & 18.5 \\
\hline 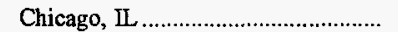 & 238,592 & 64,394 & 283,106 & 134,485 & 230,677 & 244,481 & 270.5 & .8 & -.3 \\
\hline Detroit, MI & 374,566 & 421,63 & 312,214 & 203,067 & 58,937 & 79 & -11.2 & 58.8 & 156.1 \\
\hline 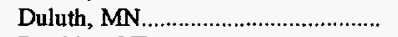 & 291,346 & 244,2713 & 77,355 & 12,811 & - & - & 19.3 & - & - \\
\hline 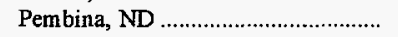 & 501,778 & 570,590 & 539,962 & 630,627 & 657,400 & 61,443 & -12.0 & -6.5 & 26.3 \\
\hline Cleveland, $\mathrm{OH}$ & - & - & - & - & 89 & - & - & -100.0 & - \\
\hline Total & $7,125,848$ & $7,201,072$ & $7,583,985$ & $7,308,907$ & $3,802,767$ & $1,747,138$ & -1.0 & 17.0 & 16.9 \\
\hline
\end{tabular}

* Data round to zero.

NM Not meaningful as value is greater than 500 percent.

Source: U.S. Department of Commerce, Bureau of the Census, "Monthly Report IM 145." 
Table 37. U.S. Receipts of Imported Coal by Country of Origin and Destination State, 1987, 1992-1996 (Short Tons)

\begin{tabular}{|c|c|c|c|c|c|c|c|}
\hline $\begin{array}{l}\text { Country of Origin } \\
\text { and Destination State }\end{array}$ & 1996 & 1995 & 1994 & 1993 & 1992 & 1987 & $\begin{array}{c}\text { Percent } \\
\text { Change } \\
\text { 1995-1996 }\end{array}$ \\
\hline 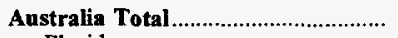 & 147,204 & 211,099 & 109,737 & 98,947 & - & 83,174 & -30.3 \\
\hline 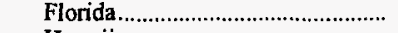 & - & - & - & - & - & 26,074 & - \\
\hline 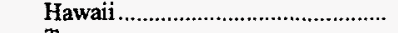 & 147,204 & 211,099 & 109,737 & 98,947 & - & - & -30.3 \\
\hline 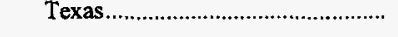 & - & - & - & - & - & 57,100 & - \\
\hline 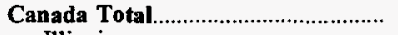 & $1,332,243$ & $1,401,960$ & $1,317,929$ & 664,835 & 48,090 & 15,600 & -5.0 \\
\hline 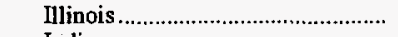 & 215,959 & 222,876 & 346,192 & 50,936 & - & - & -3.1 \\
\hline 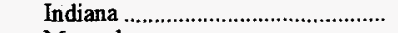 & 735,342 & 760,508 & 592,655 & 582,689 & - & - & -3.3 \\
\hline 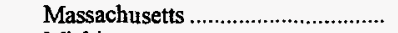 & - & - & - & - & 32,800 & - & - \\
\hline Michigan & 361,458 & 393,367 & 371,097 & - & - & - & -8.1 \\
\hline New Hampshire................................... & - & - & - & - & - & 15,600 & - \\
\hline 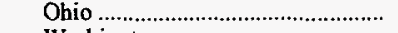 & 1,454 & 1,410 & 1,635 & 2,000 & - & - & 3.1 \\
\hline 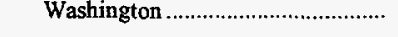 & 18,030 & 23,799 & 6,350 & 29,210 & 15,290 & - & -24.2 \\
\hline 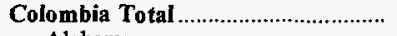 & $2,285,840$ & $2,202,005$ & $3,150,128$ & $3,642,728$ & $1,504,138$ & 568,116 & 3.8 \\
\hline Alabama & 160,675 & 161,950 & 178,330 & 57,602 & - & - & -.8 \\
\hline 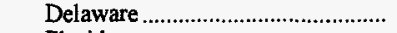 & - & 7,143 & 22,031 & - & - & - & -100.0 \\
\hline Florida & $1,417,220$ & $1,340,640$ & $2,348,550$ & $2,999,303$ & $1,418,580$ & 426,846 & 5.7 \\
\hline 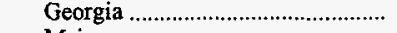 & - & - & 11,902 & - & - & - & - \\
\hline 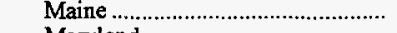 & 45,220 & - & - & - & - & - & - \\
\hline 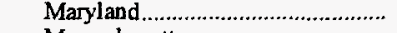 & $\overrightarrow{-}$ & - & 88,000 & 224,000 & - & - & - \\
\hline 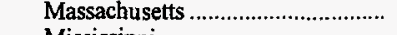 & 630,400 & 557,900 & 135,500 & 187,200 & - & 29,000 & 13.0 \\
\hline 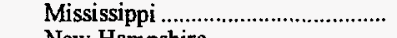 & - & - & - & $\vec{a}$ & - & 65,870 & $-\overline{-}$ \\
\hline 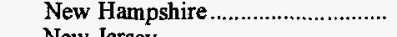 & 32,325 & 134,372 & 163,311 & 52,143 & 48,400 & 9,200 & -75.9 \\
\hline 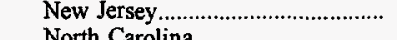 & - & - & 22,500 & - & - & - & - \\
\hline North Carolina ...................................... & - & - & 26,600 & - & - & - & - \\
\hline 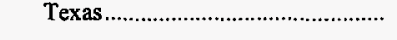 & - & - & 153,404 & 122,480 & 37,158 & 37,200 & - \\
\hline 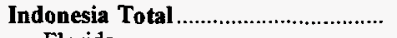 & 833,706 & 428,554 & 437,292 & 118,981 & 13,100 & - & 94.5 \\
\hline 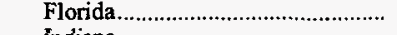 & 807,803 & 348,854 & 147,215 & - & - & - & 131.5 \\
\hline 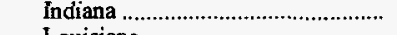 & - & - & - & 11,100 & - & - & - \\
\hline 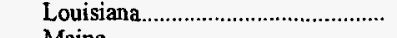 & - & - & 169,181 & - & - & - & - \\
\hline 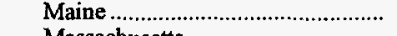 & - & - & - & 3,135 & - & - & - \\
\hline 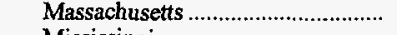 & - & - & 7,938 & - & - & - & - \\
\hline 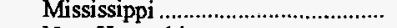 & - & - & - & 67,547 & - & - & - \\
\hline New Hampshire ................................ & 25,903 & 79,700 & 112,958 & 37,199 & - & - & -67.5 \\
\hline 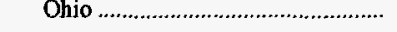 & - & - & - & - & 13,100 & - & - \\
\hline 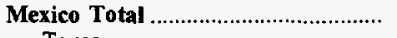 & 15,561 & - & - & 33,520 & - & - & - \\
\hline 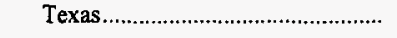 & 15,561 & - & - & 33,520 & - & - & - \\
\hline South Africa Total............................... & - & - & 127,300 & - & - & 79,300 & - \\
\hline Florida & - & - & 127,300 & - & - & 79,300 & - \\
\hline 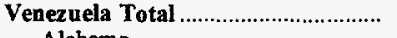 & $1,861,504$ & $2,073,645$ & $1,456,645$ & 936,945 & 240,584 & 19,266 & -10.2 \\
\hline Alabama & - & - & - & 30,278 & - & - & - \\
\hline 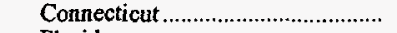 & 28,000 & - & - & - & - & - & - \\
\hline Florida & 298,200 & 891,400 & 421,674 & 312,193 & - & 19,266 & -66.5 \\
\hline 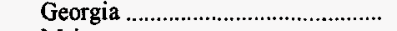 & 209,907 & - & 26,835 & - & - & - & - \\
\hline 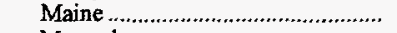 & 13,966 & 81,392 & 91,436 & 9,123 & - & - & -82.8 \\
\hline 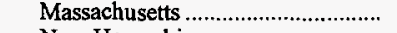 & $1,135,500$ & 903,700 & 916,700 & 476,100 & 163,800 & - & 25.6 \\
\hline 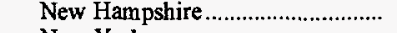 & 96,033 & 82,425 & - & 109,251 & 34,300 & - & 16.5 \\
\hline 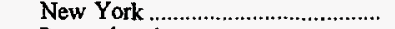 & - & 28,189 & - & - & - & - & -100.0 \\
\hline 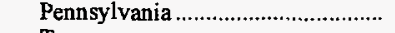 & 79,898 & 86,539 & - & - & - & - & -7.7 \\
\hline Texas & - & - & - & - & 42,484 & - & - \\
\hline Total & $6,476,058$ & $6,317,263$ & $6,599,031$ & $5,495,956$ & $1,805,912$ & 765,456 & 2.5 \\
\hline 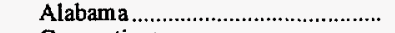 & 160,675 & 161,950 & 178,330 & 87,880 & - & - & -.8 \\
\hline 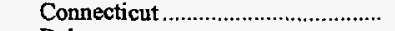 & 28,000 & - & - & - & - & - & - \\
\hline 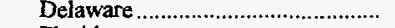 & - & 7,143 & 22,031 & - & - & - & -100.0 \\
\hline Florida & $2, \$ 23,223$ & $2,580,894$ & $3,044,739$ & $3,311,496$ & $1,418,580$ & 551,486 & -2.2 \\
\hline 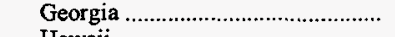 & 209,907 & $\vec{a}$ & 38,737 & $-\overline{7}$ & - & - & - \\
\hline 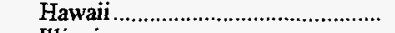 & 147,204 & 211,099 & 109,737 & 98,947 & - & - & -30.3 \\
\hline Illinois & 215,959 & 222,876 & 346,192 & 50,936 & - & - & -3.1 \\
\hline Indiana & 735,342 & 760,508 & 592,655 & 593,789 & - & - & -3.3 \\
\hline 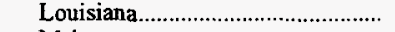 & - & - & 169,181 & - & - & - & - \\
\hline Maine & 59,186 & 81,392 & 91,436 & 12,258 & - & - & -27.3 \\
\hline Maryland & + & - & 88,000 & 224,000 & - & - & - \\
\hline 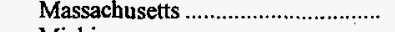 & $1,765,900$ & $1,461,600$ & $1,060,138$ & 663,300 & 196,600 & 29,000 & 20.8 \\
\hline 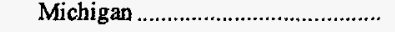 & 361,458 & 393,367 & 371,097 & - & - & - & -8.1 \\
\hline
\end{tabular}

See footnotes at end of table. 
Table 37. U.S. Receipts of Imported Coal by Country of Origin and Destination State, 1987, 1992-1996 (Continued)

(Short Tons)

\begin{tabular}{|c|c|c|c|c|c|c|c|}
\hline $\begin{array}{l}\text { Country of Origin } \\
\text { and Destination State }\end{array}$ & 1996 & 1995 & 1994 & 1993 & 1992 & 1987 & $\begin{array}{c}\text { Percent } \\
\text { Change } \\
\text { 1995-1996 }\end{array}$ \\
\hline \multicolumn{8}{|l|}{ Total (Continued) } \\
\hline 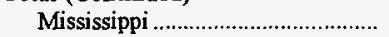 & - & - & - & 67,547 & - & 65,870 & - \\
\hline New Hampshire................................ & 154,261 & 296,497 & 276,269 & 198,593 & 82,700 & 24,800 & -48.0 \\
\hline 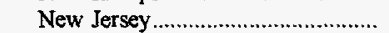 & - & - & 22,500 & - & - & 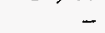 & - \\
\hline 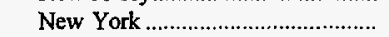 & - & 28,189 & - & - & - & - & -100.0 \\
\hline 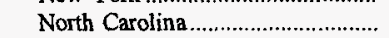 & - & - & 26,600 & - & - & - & - \\
\hline Ohio & 1,454 & 1,410 & 1,635 & 2,000 & 13,100 & - & 3.1 \\
\hline 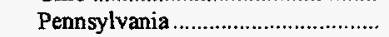 & 79,898 & 86,539 & - & - & - & - & -7.7 \\
\hline 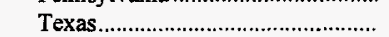 & 15,561 & - & 153,404 & 156,000 & 79,642 & 94,300 & - \\
\hline Washington & 18,030 & 23,799 & 6,350 & 29,210 & 15,290 & - & -24.2 \\
\hline
\end{tabular}

Notes: Data for 1987 are only for receipts at electric utilities. Data for 1992 through 1996 are for receipts at electric utilities, manufacturing plants and coke plants. See Table 38 and Table 39 for related data. See Technical Note 1 for the difference between receipts of imported coal and U.S. coal imports.

Sources: - 1987: Federal Energy Regulatory Commission (FERC), FERC Form 423, "Monthly Report of Cost and Quality of Fuels for Electric Plants." - 1992-1996: Energy Information Administration, Form EIA-3A, "Annual Coal Quality Report - Manufacturing Plants"; Form ElA-5A, "Annual Coal Quality Report - Coke Plants'; and FERC, FERC Form 423, "Monthly Report of Cost and Quality of Fuels for Electric Plants." 
Table 38. Imported Coal Received at Electric Utilities by Country of Origin and Destination State, 1987, 1992-1996

(Short Tons)

\begin{tabular}{|c|c|c|c|c|c|c|c|c|c|}
\hline \multirow{2}{*}{$\begin{array}{c}\text { Country of Origin } \\
\text { and Destination State }\end{array}$} & \multirow{2}{*}{1996} & \multirow{2}{*}{1995} & \multirow{2}{*}{1994} & \multirow{2}{*}{1993} & \multirow{2}{*}{1992} & \multirow{2}{*}{1987} & \multirow{2}{*}{$\begin{array}{c}\text { Percent } \\
\text { Change } \\
1995-1996\end{array}$} & \multicolumn{2}{|c|}{ Average Annual Percent Change } \\
\hline & & & & & & & & 1992-1996 & $1987-1996$ \\
\hline 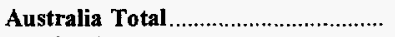 & - & - & - & - & - & 83,174 & - & - & -100.0 \\
\hline 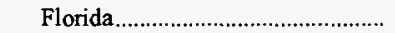 & - & - & - & - & - & 26,074 & - & - & -100.0 \\
\hline 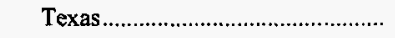 & - & - & - & - & - & 57,100 & $\sim$ & - & -100.0 \\
\hline Canada Total. & 18,030 & 23,799 & 63,350 & 29,210 & 48,090 & 15,600 & -24.2 & -21.7 & 1.6 \\
\hline 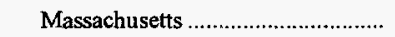 & - & - & - & - & 32,800 & - & - & -100.0 & - \\
\hline 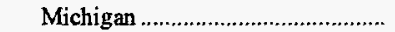 & - & - & 57,000 & - & - & - & - & - & - \\
\hline 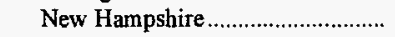 & - & - & - & - & - & 15,600 & - & - & -100.0 \\
\hline 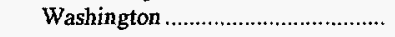 & 18,030 & 23,799 & 6,350 & 29,210 & 15,290 & - & -24.2 & 4.2 & - \\
\hline 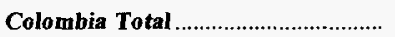 & $2,079,945$ & $2,040,055$ & $2,971,798$ & $3,585,126$ & $1,504,138$ & 568,116 & 1.9 & 8.4 & 15.5 \\
\hline Delaware & - & 7,143 & 22,031 & - & - & - & -100.0 & - & - \\
\hline Florida & $1,417,220$ & $1,340,640$ & $2,348,550$ & $2,999,303$ & $1,418,580$ & 426,846 & 5.7 & * & 14.3 \\
\hline 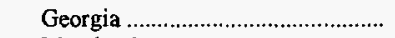 & - & - & 11,902 & - & - & - & - & - & - \\
\hline 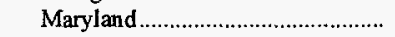 & - & - & 88,000 & 224,000 & - & - & - & - & - \\
\hline 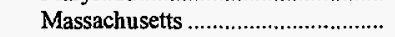 & 630,400 & 557,900 & 135,500 & 187,200 & - & 29,000 & 13.0 & - & 40.8 \\
\hline 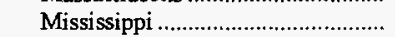 & - & - & - & - & - & 65,870 & - & - & -100.0 \\
\hline 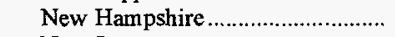 & 32,325 & 134,372 & 163,311 & 52,143 & 48,400 & 9,200 & -75.9 & -9.6 & 15.0 \\
\hline 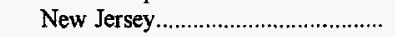 & - & - & 22,500 & - & - & - & - & - & - \\
\hline 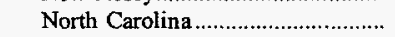 & - & - & 26,600 & - & - & - & - & - & - \\
\hline 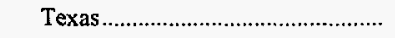 & - & - & 153,404 & 122,480 & 37,158 & 37,200 & - & -100.0 & -100.0 \\
\hline Indonesia Total & 833,706 & 428,554 & 437,292 & 115,846 & 13,100 & - & 94.5 & 182.4 & - \\
\hline 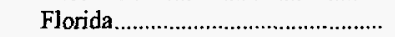 & 807,803 & 348,854 & 147,215 & - & - & - & 131.5 & - & - \\
\hline 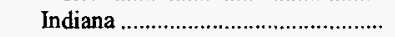 & - & - & - & 11,100 & - & - & - & - & - \\
\hline 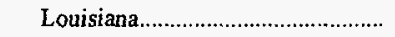 & - & - & 169,181 & - & - & - & - & - & - \\
\hline 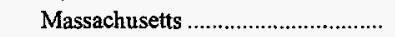 & - & - & 7,938 & - & - & - & - & - & - \\
\hline 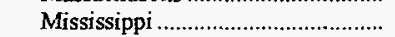 & - & - & - & 67,547 & - & - & - & - & - \\
\hline 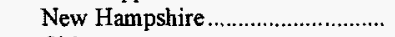 & 25,903 & 79,700 & 112,958 & 37,199 & - & - & -67.5 & - & - \\
\hline Ohio & - & - & - & - & 13,100 & - & - & -100.0 & - \\
\hline 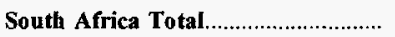 & - & - & 127,300 & - & - & 79,300 & - & - & -100.0 \\
\hline 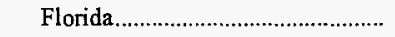 & - & $\rightarrow$ & 127,300 & - & - & 79,300 & - & - & -100.0 \\
\hline 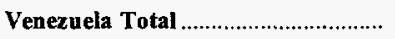 & $1,767,640$ & $1,905,714$ & $1,365,209$ & 897,544 & 240,584 & 19,266 & -7.2 & 64.6 & 65.2 \\
\hline 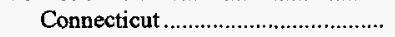 & 28,000 & - & - & - & - & - & - & - & - \\
\hline 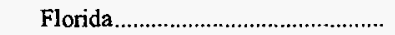 & 298,200 & 891,400 & 421,674 & 312,193 & - & 19,266 & -66.5 & - & 35.6 \\
\hline 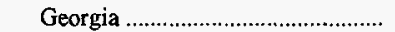 & 209,907 & - & 26,835 & - & - & - & - & - & - \\
\hline 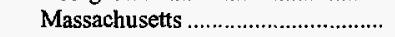 & $1,135,500$ & 903,700 & 916,700 & 476,100 & 163,800 & - & 25.6 & 62.3 & - \\
\hline New Hampshire................................... & 96,033 & 82,425 & - & 109,251 & 34,300 & - & 16.5 & 29.3 & - \\
\hline 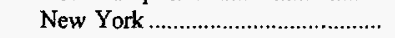 & - & 28,189 & - & - & - & - & -100.0 & - & - \\
\hline 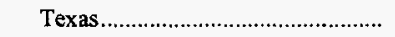 & - & - & - & - & 42,484 & - & - & -100.0 & - \\
\hline 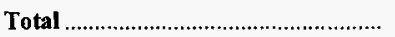 & $4,699,321$ & $4,398,122$ & $4,964,949$ & $4,627,726$ & $1,805,912$ & 765,456 & 6.8 & 27.0 & 22.3 \\
\hline Connecticut & 28,000 & - & - & - & - & - & - & - & - \\
\hline 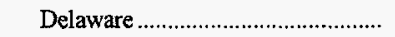 & - & 7,143 & 22,031 & - & - & - & -100.0 & - & - \\
\hline Florida & $2,523,223$ & $2,580,894$ & $3,044,739$ & $3,311,496$ & $1,418,580$ & 551,486 & -2.2 & 15.5 & 18.4 \\
\hline 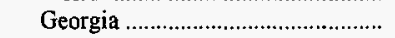 & 209,907 & - & 38,737 & - & - & - & - & - & - \\
\hline 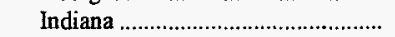 & - & - & - & 11,100 & - & - & - & - & - \\
\hline Louisiana & - & - & 169,181 & - & - & - & - & - & - \\
\hline 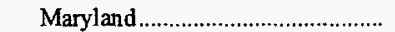 & - & - & 88,000 & 224,000 & - & - & - & - & - \\
\hline 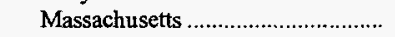 & $1,765,900$ & $1,461,600$ & $1,060,138$ & 663,300 & 196,600 & 29,000 & 20.8 & 73.1 & 57.9 \\
\hline 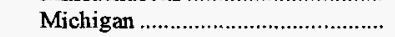 & - & - & 57,000 & - & - & - & - & - & - \\
\hline Mississippi & - & - & - & 67,547 & - & 65,870 & - & - & -100.0 \\
\hline 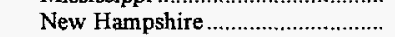 & 154,261 & 296,497 & 276,269 & 198,593 & 82,700 & 24,800 & -48.0 & 16.9 & 22.5 \\
\hline New Jersey & - & - & 22,500 & - & - & - & - & - & - \\
\hline 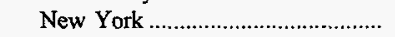 & - & 28,189 & - & - & - & - & -100.0 & - & - \\
\hline 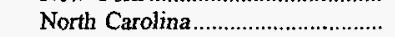 & - & - & 26,600 & - & - & - & - & - & - \\
\hline Ohio & - & - & - & - & 13,100 & - & - & -100.0 & - \\
\hline 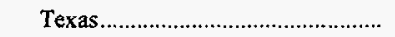 & - & - & 153,404 & 122,480 & 79,642 & 94,300 & - & -100.0 & -100.0 \\
\hline Washington & 18,030 & 23,799 & 6,350 & 29,210 & 15,290 & - & -24.2 & 4.2 & - \\
\hline
\end{tabular}

* Data round to zero.

Source: Federal Energy Regulatory Commission, FERC Form 423, "Monthly Report of Cost and Quality of Fuels for Electric Plants." 
Table 39. Imported Coal Received at Manufacturing and Coke Plants by Country of Origin and Destination State, 1994-1996

(Short Tons)

\begin{tabular}{|c|c|c|c|c|c|c|c|c|c|c|}
\hline \multirow{2}{*}{$\begin{array}{c}\text { Country } \\
\text { of Origin } \\
\text { and } \\
\text { Destination } \\
\text { State }\end{array}$} & \multicolumn{3}{|c|}{ Manufacturing } & \multicolumn{3}{|c|}{ Coke Plants } & \multicolumn{4}{|c|}{ Total } \\
\hline & 1996 & 1995 & 1994 & 1996 & 1995 & 1994 & 1996 & 1995 & 1994 & $\begin{array}{c}\text { Percent } \\
\text { Change } \\
\text { 1995-1996 }\end{array}$ \\
\hline 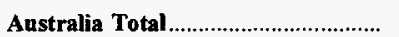 & 147,204 & 211,099 & 109,737 & - & - & - & 147,204 & 211,099 & 109,737 & -30.3 \\
\hline 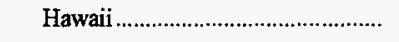 & 147,204 & 211,099 & 109,737 & - & - & - & 147,204 & 211,099 & 109,737 & -30.3 \\
\hline 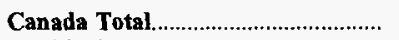 & 1,454 & 1,410 & 1,635 & $1,312,759$ & $1,376,751$ & $1,252,944$ & $1,314,213$ & $1,378,161$ & $1,254,579$ & -4.6 \\
\hline 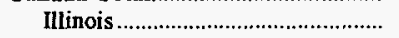 & - & - & - & 215,959 & 222,876 & 346,192 & 215,959 & 222,876 & 346,192 & -3.1 \\
\hline 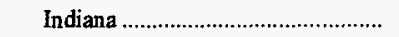 & - & $-\cdot$ & - & 735,342 & 760,508 & 592,655 & 735,342 & 760,508 & 592,655 & -3.3 \\
\hline Michigan & - & - & - & 361,458 & 393,367 & 314,097 & 361,458 & 393,367 & 314,097 & -8.1 \\
\hline Ohio & 1,454 & 1,410 & 1,635 & - & - & - & 1,454 & 1,410 & 1,635 & 3.1 \\
\hline 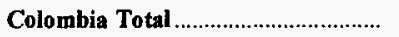 & 205,895 & 161,950 & 178,330 & - & - & - & 205,895 & 161,950 & 178,330 & 27.1 \\
\hline Alabama & 160,675 & 161,950 & 178,330 & - & - & - & 160,675 & 161,950 & 178,330 & -.8 \\
\hline Maine & 45,220 & - & - & - & - & - & 45,220 & - & - & - \\
\hline Indonesia Total & - & - & - & - & - & - & - & - & - & - \\
\hline 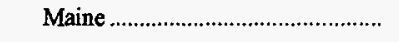 & - & - & - & - & - & - & - & - & - & - \\
\hline Mexico Total & 15,561 & - & - & - & - & - & 15,561 & - & - & - \\
\hline 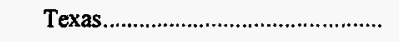 & 15,561 & - & - & - & - & - & 15,561 & - & - & - \\
\hline Venezuela Total ................................... & 93,864 & 167,931 & 91,436 & - & - & - & 93,864 & 167,931 & 91,436 & -44.1 \\
\hline Alabama & - & - & 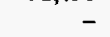 & - & - & - & 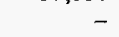 & - & 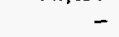 & - \\
\hline 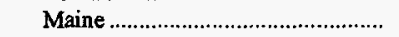 & 13,966 & 81,392 & 91,436 & - & - & - & 13,966 & 81,392 & 91,436 & -82.8 \\
\hline 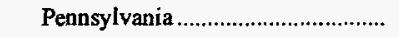 & 79,898 & 86,539 & - & - & - & - & 79,898 & 86,539 & - & -7.7 \\
\hline Total & 463,978 & 542,390 & 381,138 & $1,312,759$ & $1,376,751$ & $1,252,944$ & $1,776,737$ & $1,919,141$ & $1,634,082$ & -7.4 \\
\hline Alabama & 160,675 & 161,950 & 178,330 & - & - & - & 160,675 & 161,950 & 178,330 & -.8 \\
\hline 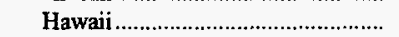 & 147,204 & 211,099 & 109,737 & - & - & - & 147,204 & 211,099 & 109,737 & -30.3 \\
\hline 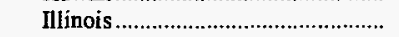 & - & - & - & 215,959 & 222,876 & 346,192 & 215,959 & 222,876 & 346,192 & -3.1 \\
\hline 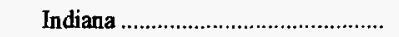 & - & - & - & 735,342 & 760,508 & 592,655 & 735,342 & 760,508 & 592,655 & -3.3 \\
\hline Maine & 59,186 & 81,392 & 91,436 & - & - & - & 59,186 & 81,392 & 91,436 & -27.3 \\
\hline 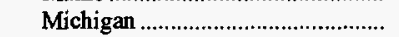 & - & - & - & 361,458 & 393,367 & 314,097 & 361,458 & 393,367 & 314,097 & -8.1 \\
\hline Ohio & 1,454 & 1,410 & 1,635 & - & - & - & 1,454 & 1,410 & 1,635 & 3.1 \\
\hline 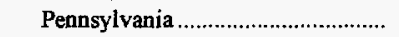 & 79,898 & 86,539 & - & - & - & - & 79,898 & 86,539 & - & -7.7 \\
\hline 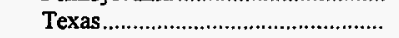 & 15,561 & - & - & - & - & - & 15,561 & - & - & - \\
\hline
\end{tabular}

Sources: Energy Information Administration, Form EIA-jA, “Annual Coal Quality Report - Manufacturing Plants"; and Form EIA-5A, “Annual Coal Quality Report - Coke Plants." 


\section{Employment and Productivity}

\section{Employment}

In 1996 , the average number of miners working daily at mines and/or preparation plants producing or processing 10,000 or more short tons of coal dropped 7.5 percent from the 1995 level, to 83,462 miners (Table 40). Of the 25 coal-producing States, 18 had a decrease in employment. Although all three regions experienced a decline in employment, the Western Region which declined 13.1 percent, accounted for the largest share of the decrease. The Appalachian Region had a 7 percent decline, while the Interior Region showed a decline of 8 percent.

The average number of miners at underground mines in 1996 totaled 53,796 miners, accounting for 64 percent of all U.S. miners (Table 41). Employment at underground mines decreased 7 percent since 1995.

In 1996, the average number of miners at surface mines dropped 8.4 percent from the 1995 level to 29,666 miners (Table 42). All three regions had decreases in surface employment in 1996, with the Interior Region showing only a slight decline of 0.2 percent.

Mines that produced 1 million short tons or more of coal in 1996, accounted for 74 percent of total pro- duction and employed 48 percent of the miners. Of this total, 32 percent worked in underground mines and 16 percent at surface mines. Over 36 thousand miners ( 43.8 percent) belong to a union.

The United Mine Workers of America (UMWA) represented 39 percent of the total coal mining workforce, while 4.6 percent belonged to "other unions" (Table 46).

\section{Productivity}

In 1996, coal miners working daily averaged 5.69 short tons per miner per hour, an increase of 5.8 percent from the 1995 level (Table 48). Increases occurred in all regions, with the Western Region showing the largest increase, 11 percent. Over the last decade, productivity has risen at an annual average rate of 6.3 percent, increasing both at underground and surface mines. Underground mines increased 5.5 percent over the last decade, while surface mines rose 6.9 percent during the same period. 



\section{Employment}

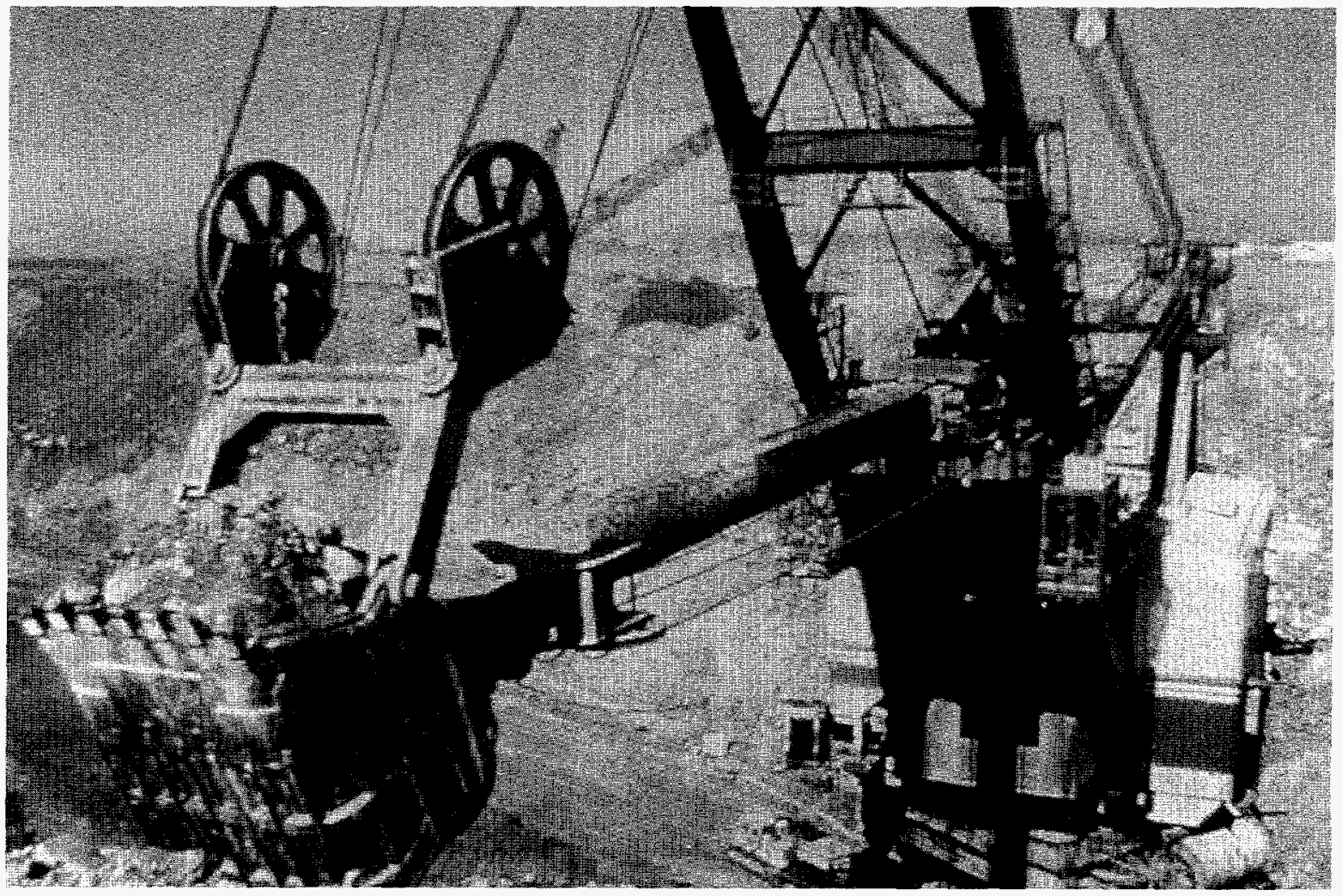

Overburden is removed by giant excavators, such as draglines and power shovels until large areas of coal is exposed. 
Figure 4. Average Number of U.S. Miners by Mine Type and by Region, 1987-1996
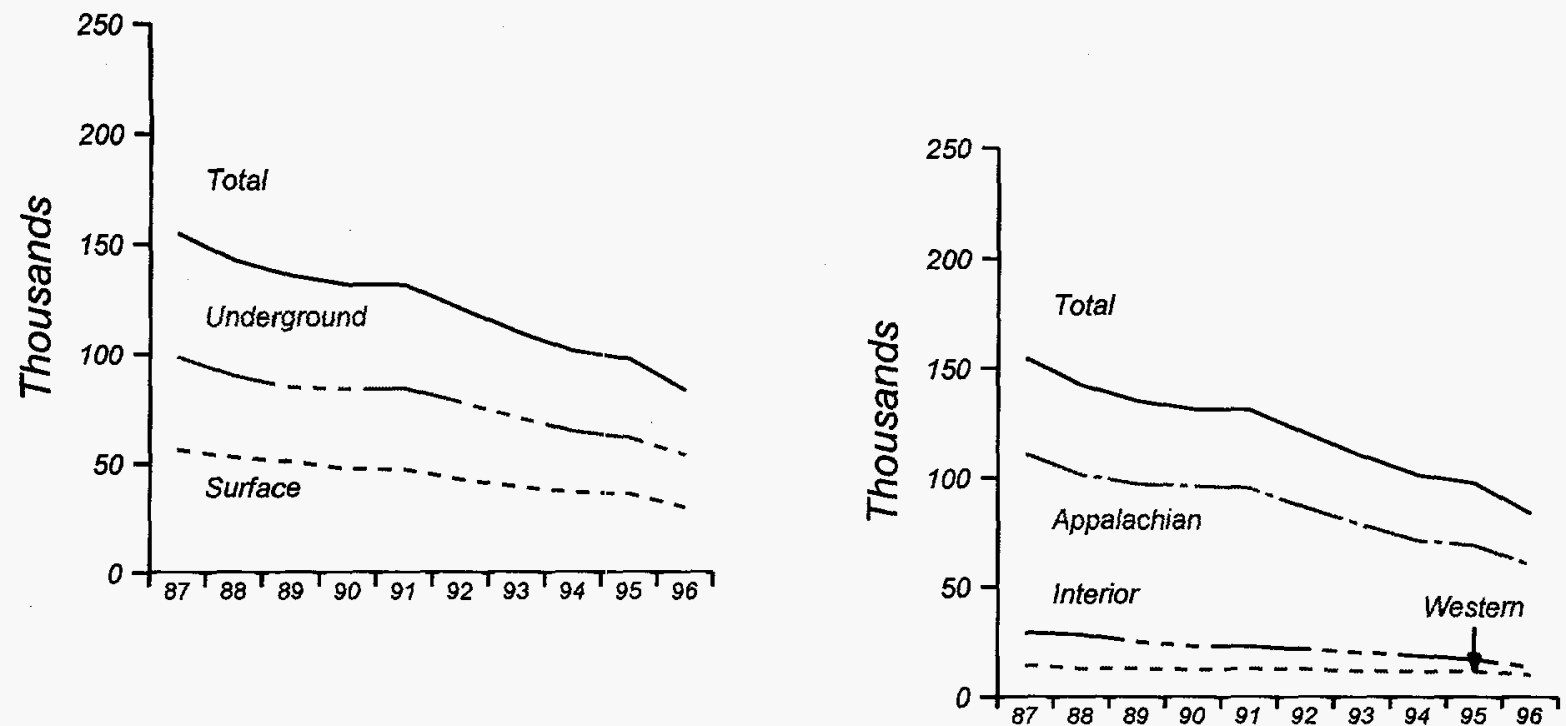

Figure 5. U.S. Coal Mining Productivity by Mine Type and by Region, 1987-1996
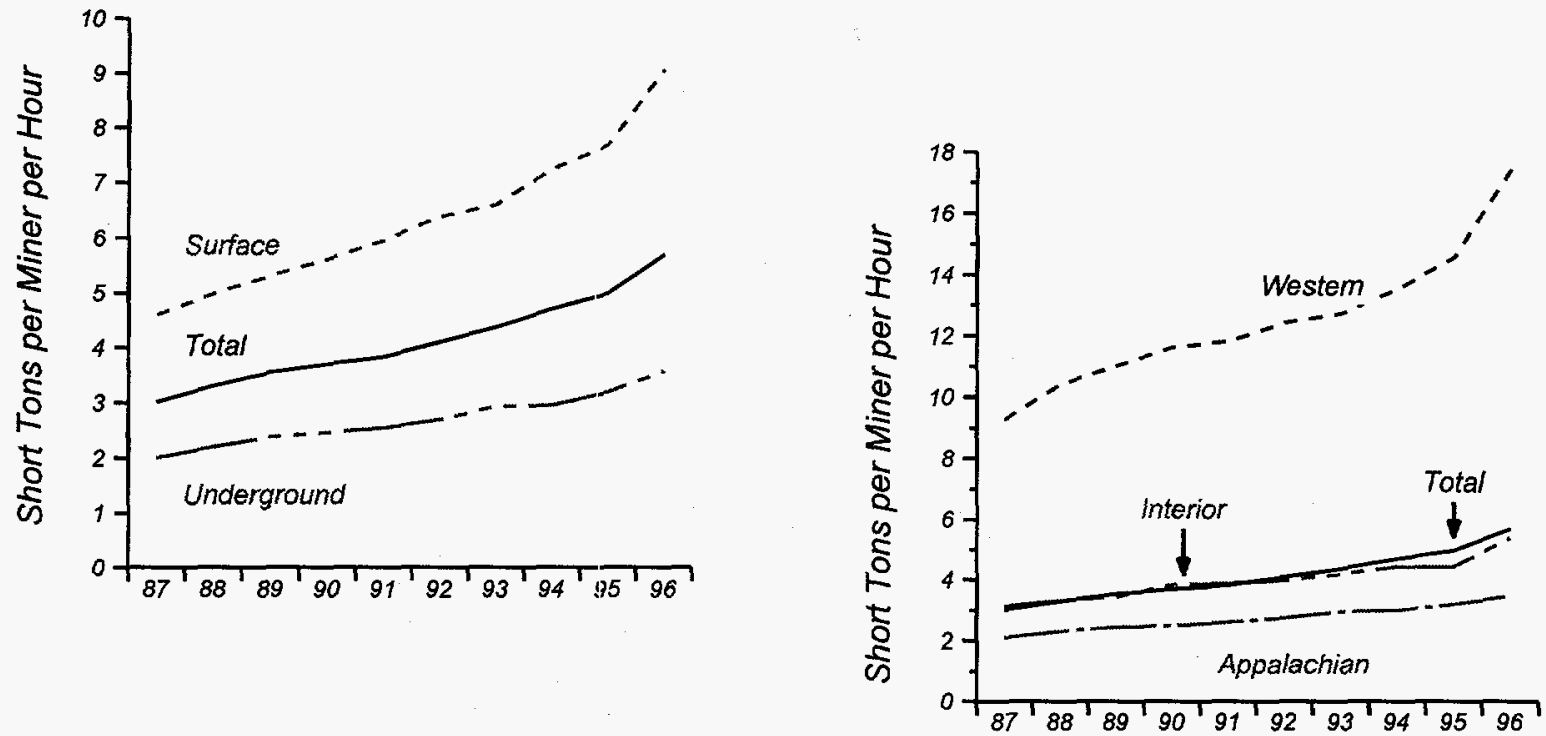

Nole: Scale hos been enlarged lo show detail in the shorl lons per miner per hour by type of mining plot. Because vertical scoles differ, grophs should not be compored. Excludes silt, culm, refuse bank, slurry dom, and dredge operalions except for Pennsylvanio onthrocite. Excludes mines producing less thon 10,000 short tons of coal during the yeor and preparotion plonls with less than 5.000 employe hours. Includes oll employees engaged in production, preparation, processing, development, mointenonce, repoir, shop or yord work ot mining operations. Excludes olfice workers. Includes mining operations monogement and all technical and engineering personnel. Shorl lons produced per miner per hour is calculated by dividing lotal coal produclion by the direct labor hours worked by oll mine employe es.

Sources: Energy Inlormation Administration, Form EIA-7A, "Cool Production Report"; Stale Mining Agency Cool

Production Reports: and/or U.S. Deportment of Lobor. Mine Sofety ond Health Administration, Form $7000-2$.

"Quorterly Mine Employment ond Cool Production Report." 
Table 40. Average Number of Miners by State, 1987, 1992-1996

\begin{tabular}{|c|c|c|c|c|c|c|c|c|c|}
\hline \multirow{2}{*}{$\begin{array}{l}\text { Coal-Producing } \\
\text { State and Region }\end{array}$} & \multirow{2}{*}{1996} & \multirow{2}{*}{1995} & \multirow{2}{*}{1994} & \multirow{2}{*}{1993} & \multirow{2}{*}{1992} & \multirow{2}{*}{1987} & \multirow{2}{*}{$\begin{array}{c}\text { Percent } \\
\text { Change } \\
1995-1996\end{array}$} & \multicolumn{2}{|c|}{ Average Annual Percent Change } \\
\hline & & & & & & & & $1992-1996$ & 1987-1996 \\
\hline Alabama & $5,03]$ & 5,567 & 5,418 & 5,399 & 5,386 & 6,718 & -9.6 & -1.7 & -3.2 \\
\hline 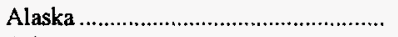 & 102 & 102 & 105 & 96 & 111 & 93 & - & -2.1 & 1.0 \\
\hline Arizona & 651 & 831 & 864 & 876 & 888 & 900 & -21.7 & -7.5 & -3.5 \\
\hline 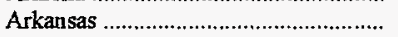 & - & 4 & 15 & 5 & 14 & 19 & -100.0 & - & - \\
\hline Califomia & - & - & - & - & 8 & 37 & - & - & - \\
\hline Colorado & 1,332 & 1,777 & 1,905 & 1,775 & 1,610 & 1,795 & -25.0 & -4.6 & $-3,3$ \\
\hline 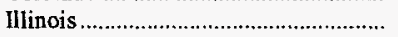 & 5,174 & 5,652 & 6,591 & 7,303 & 8,323 & 12,171 & -8.4 & -11.2 & -9.1 \\
\hline Indiana & 2,579 & 2,571 & 3,206 & 3,331 & 3,652 & 3,992 & .3 & -8.3 & -4.7 \\
\hline Iowa & - & - & 20 & 90 & 101 & 166 & - & - & - \\
\hline 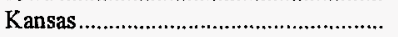 & 54 & 54 & 63 & 81 & 96 & 247 & - & -13.4 & -15.5 \\
\hline 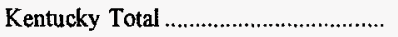 & 18,826 & 21,125 & 23,368 & 24,063 & 24,624 & 32,590 & -10.9 & -6.5 & -5.9 \\
\hline Eastern & 15,130 & 16,840 & 18,577 & 18,711 & 19,419 & 25,640 & -10.1 & -6.0 & -5.7 \\
\hline Western & 3,696 & 4,285 & 4,791 & 5,352 & 5,205 & 6,950 & -13.7 & -8.2 & -6.8 \\
\hline 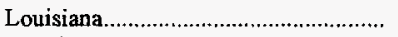 & 111 & 114 & 111 & 99 & 77 & 81 & -2.6 & 9.6 & 3.6 \\
\hline 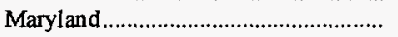 & 469 & 458 & 451 & 441 & 438 & 555 & 2.4 & 1.7 & -1.8 \\
\hline 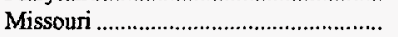 & 80 & 92 & 116 & 180 & 323 & 814 & -13.0 & -29.4 & -22.7 \\
\hline 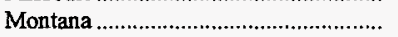 & 705 & 722 & 705 & 660 & 715 & 847 & -2.3 & -.3 & -2.0 \\
\hline 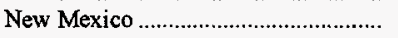 & 1,347 & 1,747 & 1,786 & 1,762 & 1,683 & 1,658 & -22.9 & -5.4 & -2.3 \\
\hline North Dakota & 640 & 716 & 645 & $\cdot 782$ & 744 & 961 & -10.6 & -3.7 & -4.4 \\
\hline Ohio & 3,232 & 3,386 & 3,983 & 3,866 & 4,515 & 7,827 & -4.5 & -8.0 & -9.4 \\
\hline 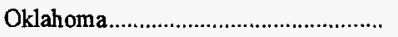 & 233 & 241 & 253 & 273 & 334 & 653 & -3.3 & -8.6 & -10.8 \\
\hline 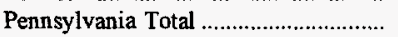 & 9,021 & 8,968 & 9,975 & 10,940 & 12,659 & 17,287 & .6 & -8.1 & -7.0 \\
\hline 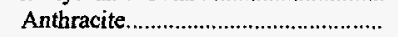 & 1,171 & 1,069 & 1,183 & 1,124 & 1,217 & 1,602 & 9.5 & -.9 & -3.4 \\
\hline 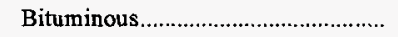 & 7,850 & 7,899 & 8,792 & 9,816 & 11,442 & 15,685 & -.6 & -9.0 & -7.4 \\
\hline Tennessee & 756 & 681 & 669 & 646 & 804 & 1,998 & 11.0 & -1.5 & -10.2 \\
\hline 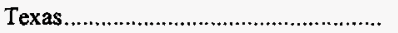 & 1,550 & 1,590 & 1,733 & 1,841 & 2,001 & 3,319 & -2.5 & -6.2 & -8.1 \\
\hline Utah & 1,804 & 1,893 & 1,675 & 1,769 & 1,997 & 2,544 & -4.7 & -2.5 & -3.7 \\
\hline 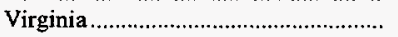 & 6,241 & 6,919 & 8,121 & 8,339 & 9,138 & 12,047 & -9.8 & -9.1 & -7.0 \\
\hline 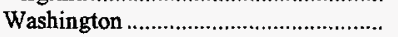 & 589 & 566 & 570 & 567 & 612 & 699 & 4.1 & -.9 & -1.9 \\
\hline West Virginia Total................................. & 20,121 & 21,334 & 21,861 & 22,979 & 26,017 & 29,458 & -5.7 & -6.2 & -4.1 \\
\hline 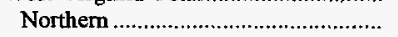 & 5,279 & 6,114 & 6,659 & 7,274 & 8,481 & 9,863 & -13.6 & -11.2 & -6.7 \\
\hline Southem & 14,842 & 15,220 & 15,202 & 15,705 & 17,536 & 19,595 & -2.5 & -4.1 & -3.0 \\
\hline 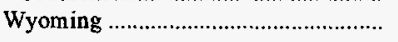 & 2,814 & 3,142 & 3,291 & 3,159 & 3,326 & 3,191 & -10.4 & -4.1 & -1.4 \\
\hline 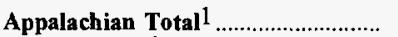 & 60,001 & 64,153 & 69,055 & $\mathbf{7 1 , 3 2 1}$ & 78,376 & 101,530 & -6.5 & -6.5 & -5.7 \\
\hline Interior Total 1 . & 13,477 & 14,603 & 16,899 & 18,555 & 20,126 & 28,412 & -7.7 & -9.5 & -7.9 \\
\hline Western Total ${ }^{1}$ & 9,984 & 11,496 & 11,546 & 11,446 & 11,694 & 12,725 & -13.1 & -3.9 & -2.6 \\
\hline 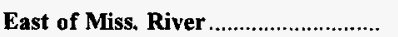 & 71,450 & 76,661 & 83,643 & 87,307 & 95,556 & 124,643 & -6.8 & -7.0 & -6.0 \\
\hline West of Miss. River ................................ & 12,012 & 13,591 & 13,857 & 14,015 & 14,640 & 18,024 & -11.6 & -4.8 & -4.4 \\
\hline 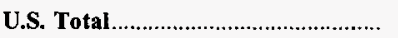 & 83,462 & 90,252 & 97,500 & 101,322 & 110,196 & 142,667 & -7.5 & -6.7 & -5.8 \\
\hline
\end{tabular}

1 For a definition of coal-producing regions, see Appendix C.

Notes: Includes all employees engaged in production, preparation, processing, development, maintenance, repair, shop or yard work at mining operations. Excludes office workers. Includes mining operations management and all technical and engineering personnel. Excludes silt, culm, refuse bank, slumy dam, and dredge operations except for Pennsylvania anthracite. Excludes mines producing less than 10,000 short tons and preparation plants with less than 5,000 employee hours, which are not required to provide these data.

Sources: Energy Information Administration, Form EIA-7A, "Coal Production Report"; State Mining Agency Coal Production Reports; and/or U.S. Department of Labor, Mine Safety and Health Administration, Form 7000-2, "Quarterly Mine Employment and Coal Production Report." 
Table 41. Average Number of Miners at Underground Mines by State, 1987, 1992-1996

\begin{tabular}{|c|c|c|c|c|c|c|c|c|c|}
\hline \multirow{2}{*}{$\begin{array}{l}\text { Coal-Producing } \\
\text { State and Region }\end{array}$} & \multirow{2}{*}{1996} & \multirow{2}{*}{1995} & \multirow{2}{*}{1994} & \multirow{2}{*}{1993} & \multirow{2}{*}{1992} & \multirow{2}{*}{1987} & \multirow{2}{*}{$\begin{array}{c}\text { Percent } \\
\text { Change } \\
\text { 1995-1996 }\end{array}$} & \multicolumn{2}{|c|}{ Average Annual Percent Chang } \\
\hline & & & & & & & & $1992-1996$ & $1987-1996$ \\
\hline Alabarna & 4,145 & 4.314 & 3,775 & 3,707 & 3,810 & 4,461 & -3.9 & 2.1 & -0.8 \\
\hline 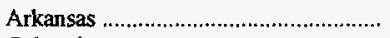 & - & - & 10 & - & - & - & - & - & - \\
\hline Colorado & 918 & 1,301 & 1,248 & 1,119 & 1,085 & 1,094 & -29.4 & -4.1 & -1.9 \\
\hline Illinois & 4,256 & 4,780 & 5,595 & 6,196 & 6,780 & 8,932 & -11.0 & -11.0 & -7.9 \\
\hline Indiana & 457 & 485 & 485 & 545 & 422 & 489 & -5.8 & 2.0 & -.7 \\
\hline Iowa & - & - & - & - & - & 87 & - & - & - \\
\hline 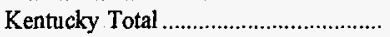 & 12,876 & 14,542 & $15,83 ?$ & 16,493 & 16,888 & 21,505 & -11.4 & -6.5 & -5.5 \\
\hline 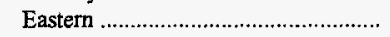 & 10,275 & 11,366 & 12,849 & 13,028 & 13,405 & 16,900 & -9.6 & -6.4 & -5.4 \\
\hline Western & 2,601 & 3,176 & 2,988 & 3,465 & 3,483 & 4,605 & -18.1 & -7.0 & -6.1 \\
\hline Maryland & 308 & 293 & 284 & 260 & 228 & 286 & 5.1 & 7.8 & 8 \\
\hline Montana & 18 & - & - & 7 & - & - & - & - & - \\
\hline New Mexico ............................................. & - & 132 & 168 & 246 & 141 & 192 & -100.0 & - & - \\
\hline Ohio & 1,706 & 1,670 & 1,694 & 1,601 & 1,926 & 3,552 & 2.1 & -3.0 & -7.8 \\
\hline 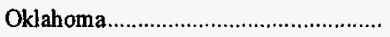 & 26 & 12 & 32 & 40 & 24 & - & 116.7 & 2.0 & - \\
\hline Pennsylvania Total .................................. & 5,599 & 5,659 & 6,192 & 6,853 & 8,113 & 10,402 & -1.1 & -8.8 & -6.6 \\
\hline Anthracite & 147 & 1152 & 149 & 194 & 147 & 254 & -3.3 & - & -5.9 \\
\hline Bituminous & 5,452 & 5,507 & 6,043 & 6,659 & 7,966 & 10,148 & -1.0 & -9.0 & -6.7 \\
\hline 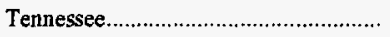 & 467 & 4.73 & 511 & 375 & 599 & 1,552 & -1.3 & -10 & -12.5 \\
\hline 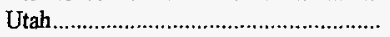 & 1,803 & 1,893 & 1,675 & 1,769 & 1,997 & 2,544 & -4.8 & -2.5 & -3.8 \\
\hline Virginia & 5,098 & 5,776 & 6,844 & 7,092 & 7,888 & 10,228 & -11.7 & -10.3 & -7.4 \\
\hline 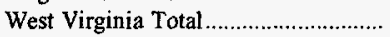 & 16,003 & $16,3,47$ & 16,956 & 18,040 & 20,738 & 24,513 & -2.1 & -6.3 & -4.6 \\
\hline Northem & 4,764 & 5,561 & 5,997 & 6,414 & 7,513 & 8,534 & -14.3 & -10.8 & -6.3 \\
\hline Southern & 11,239 & 10,786 & 10,959 & 11,626 & 13,225 & 15,979 & 4.2 & -4.0 & -3.8 \\
\hline 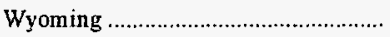 & 116 & 202 & 256 & 261 & 268 & 20 & -42.6 & -18.9 & 21.6 \\
\hline Appalachian Total ${ }^{1}$............................... & 43,601 & 45,898 & 49,105 & 50,956 & 56,707 & 71,894 & -5.0 & -6.3 & -5.4 \\
\hline Interior Total 1 & 7,340 & $8,4.53$ & 9,110 & 10,246 & 10,709 & 14,113 & -13.2 & -9.0 & -7.0 \\
\hline 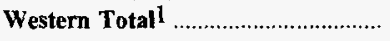 & 2,855 & 3,528 & 3,347 & 3,402 & 3,491 & 3,850 & -19.1 & -4.9 & -3.3 \\
\hline East of Miss. Rjver.................................. & 50,915 & 54,339 & 58,173 & 61,162 & 67,392 & 85,920 & -6.3 & -6.8 & -5.6 \\
\hline West of Miss. River ......................... & 2,881 & 3,540 & 3,389 & 3,442 & 3,515 & 3,937 & -18.6 & -4.8 & -3.4 \\
\hline 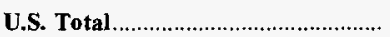 & 53,796 & 57,879 & 61,562 & 64,604 & 70,907 & 89,857 & -7.0 & -6.7 & -5.5 \\
\hline
\end{tabular}

1 For a definition of coal-producing regions, see Appendix $C$.

Notes: Includes all employees engaged in production, preparation, processing, development, maintenance, repair, shop or yard work at mining operations. Excludes office workers. Includes mining operations management and all technical and engineering personnel. Excludes silt, culm, refuse bank, slurty dam, and dredge operations except for Pennsylvania anthracite. Excludes mines producing less than 10,000 short tons and preparation plants with less than 5,000 employee hours, which are not required to provide these data.

Sources: Energy Information Administration, Form EIA-7A, "Coal Production Report"; State Mining Agency Coal Production Reports; and/or U.S. Department of Labor, Mine Safety and Health Administration, Form 7000-2, "Quarterly Mine Employment and Coal Production Report." 
Table 42. Average Number of Miners at Surface Mines by State, 1987, 1992-1996

\begin{tabular}{|c|c|c|c|c|c|c|c|c|c|}
\hline \multirow{2}{*}{$\begin{array}{l}\text { Coal-Producing } \\
\text { State and Region }\end{array}$} & \multirow{2}{*}{1996} & \multirow{2}{*}{1995} & \multirow{2}{*}{1994} & \multirow{2}{*}{1993} & \multirow{2}{*}{1992} & \multirow{2}{*}{1987} & \multirow{2}{*}{$\begin{array}{c}\text { Percent } \\
\text { Change } \\
1995-1996\end{array}$} & \multicolumn{2}{|c|}{ Average Annual Percent Change } \\
\hline & & & & & & & & $1992-1996$ & 1987-1996 \\
\hline Alabama & 886 & 1,253 & 1,643 & 1,692 & 1,576 & 2,257 & -29.3 & -13.4 & -9.9 \\
\hline 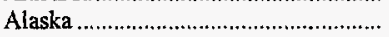 & 102 & 102 & 105 & 96 & 111 & 93 & - & -2.1 & 1.0 \\
\hline Arizona & 651 & 831 & 864 & 876 & 888 & 900 & -21.7 & -7.5 & -3.5 \\
\hline 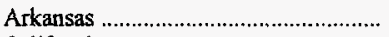 & - & 4 & 5 & 5 & 14 & 19 & -100.0 & - & - \\
\hline 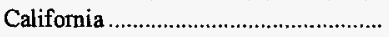 & - & - & - & - & 8 & 37 & - & - & - \\
\hline Colorado & 414 & 476 & 657 & 656 & 525 & 701 & -13.0 & -5.8 & -5.7 \\
\hline 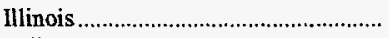 & 918 & 872 & 996 & 1,107 & 1,543 & 3,239 & 5.3 & -12.2 & -13.1 \\
\hline 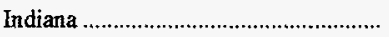 & 2,122 & 2,086 & 2,721 & 2,786 & 3,230 & 3,503 & 1.7 & -10.0 & -5.4 \\
\hline Iowa & - & - & 20 & 90 & 101 & 79 & - & - & - \\
\hline 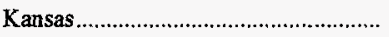 & 54 & 54 & 63 & 81 & 96 & 247 & - & -13.4 & -15.5 \\
\hline 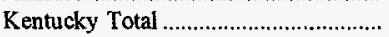 & 5,950 & 6,583 & 7,531 & 7,570 & 7,736 & 11,085 & -9.6 & -6.3 & -6.7 \\
\hline Eastern & 4,855 & 5,474 & 5,728 & 5,683 & 6,014 & 8,740 & -11.3 & -5.2 & -6.3 \\
\hline 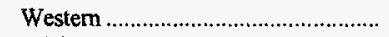 & 1,095 & 1,109 & 1,803 & 1,887 & 1,722 & 2,345 & -1.3 & -10.7 & -8.1 \\
\hline 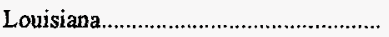 & 111 & 114 & 111 & 99 & 77 & 81 & -2.6 & 9.6 & 3.6 \\
\hline 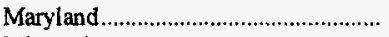 & 161 & 165 & 167 & 181 & 210 & 269 & -2.4 & -6.4 & -5.5 \\
\hline 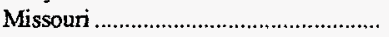 & 80 & 92 & 116 & 180 & 323 & 814 & -13.0 & -29.4 & -22.7 \\
\hline Montana & 687 & 722 & 705 & 653 & 715 & 847 & -4.8 & -1.0 & -2.3 \\
\hline 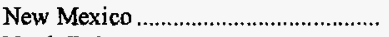 & 1,347 & 1,615 & 1,618 & 1,516 & 1,542 & 1,466 & -16.6 & -3.3 & -9 \\
\hline 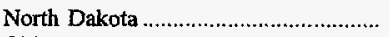 & 640 & 716 & 645 & 782 & 744 & 961 & -10.6 & -3.7 & -4.4 \\
\hline Ohio & 1,526 & 1,716 & 2,289 & 2,265 & 2,589 & 4,275 & -11.1 & -12.4 & -10.8 \\
\hline 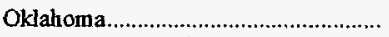 & 207 & 229 & 221 & 233 & 310 & 653 & -9.6 & -9.6 & -12.0 \\
\hline Pennsylvania Total ................................... & 3,422 & 3,309 & 3,783 & 4,087 & 4,546 & 6,885 & 3.4 & -6.8 & -7.5 \\
\hline 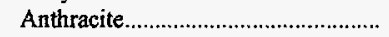 & 1,024 & 917 & 1,034 & 930 & 1,070 & 1,348 & 11.7 & -1.1 & -3.0 \\
\hline Bituminous & 2,398 & 2,392 & 2,749 & 3,157 & 3,476 & 5,537 & .3 & -8.9 & -8.9 \\
\hline 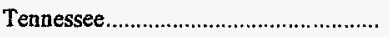 & 289 & 208 & 158 & 271 & 205 & 446 & 38.9 & 9.0 & -4.7 \\
\hline Texas & 1,550 & 1,590 & 1,733 & 1,841 & 2,001 & 3,319 & -2.5 & -6.2 & -8.1 \\
\hline Utah & 1 & - & - & - & - & - & - & - & - \\
\hline 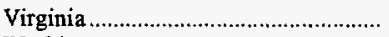 & 1,143 & 1,143 & 1,277 & 1,247 & 1,250 & 1,819 & - & -2.2 & -5.0 \\
\hline 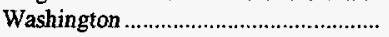 & 589 & 566 & 570 & 567 & 612 & 699 & 4.1 & -.9 & -1.9 \\
\hline West Virginia Total................................. & 4,118 & 4,987 & 4,905 & 4,939 & 5,279 & 4,945 & -17.4 & -6.0 & -2.0 \\
\hline 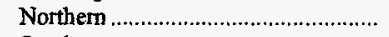 & 515 & 553 & 662 & 860 & 968 & 1,329 & -6.9 & -14.6 & -10.0 \\
\hline Southern & 3,603 & 4,434 & 4,243 & 4,079 & 4,311 & 3,616 & -18.7 & -4.4 & * \\
\hline 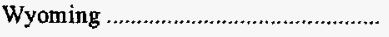 & 2,698 & 2,940 & 3,035 & 2,898 & 3,058 & 3,171 & -8.2 & -3.1 & $-1,8$ \\
\hline 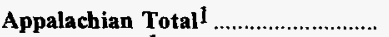 & 16,400 & 18,255 & 19,950 & 20,365 & 21,669 & 29,636 & -10.2 & -6.7 & -6.4 \\
\hline Interior Total 1 & 6,137 & 6,150 & 3,789 & 8,309 & 9,417 & 14,299 & -.2 & -10.1 & -9.0 \\
\hline Western Total 1 . & 7,129 & 7,968 & 8,199 & 8,044 & 8,203 & $\mathbf{8 , 8 7 5}$ & -10.5 & -3.4 & -2.4 \\
\hline East of Miss. River & 20,535 & 22,322 & 25,470 & 26,145 & 28,164 & 38,723 & -8.0 & -7.6 & -6.8 \\
\hline 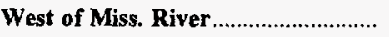 & 9,131 & 10,051 & 10,468 & 10,573 & 11,125 & 14,087 & -9.1 & -4.8 & -4.7 \\
\hline U.S. Total & 29,666 & 32,373 & 35,938 & 36,718 & 39,289 & 52,810 & -8.4 & -6.8 & -6.2 \\
\hline
\end{tabular}

1 For a definition of coal-producing regions, see Appendix C.

* Data round to zero.

Notes: Includes all employees engaged in production, preparation, processing, development, maintenance, repair, shop or yard work at mining operations. Excludes office workers. Includes mining operations management and all technical and engineering personnel. Excludes silt, culm, refuse bank slurry dam, and dredge operations except for Pennsylvania anthracite. Excludes mines producing less than 10,000 short tons and preparation plants with less than 5,000 employee hours, which are not required to provide these data.

Sources: Energy Information Administration, Form EIA-7A, "Coal Production Report"; State Mining Agency Coal Production Reports; and/or U.S. Department of Labor, Mine Safety and Health Administration, Form 7000-2, "Quarterly Mine Employment and Coal Production Report." 
Table 43. Average Number of Miners by State and Mine Production Range, 1996

\begin{tabular}{|c|c|c|c|c|c|c|c|c|}
\hline \multirow{2}{*}{$\begin{array}{l}\text { Coal-Producing } \\
\text { State and Region }\end{array}$} & \multicolumn{7}{|c|}{$\begin{array}{l}\text { Mine Production Range } \\
\text { (thousand short tons) }\end{array}$} & \multirow{2}{*}{ Total } \\
\hline & $\begin{array}{c}1,000 \\
\text { and over }\end{array}$ & $\begin{array}{c}51) 0 \\
\text { to } 1,000\end{array}$ & $\begin{array}{c}200 \\
\text { to } 500\end{array}$ & $\begin{array}{l}100 \\
\text { to } 200\end{array}$ & $\begin{array}{l}50 \\
\text { to } 100\end{array}$ & $\begin{array}{l}10 \\
\text { to } 50\end{array}$ & Zerol & \\
\hline Alabama & 3,624 & 765 & 148 & 133 & 142 & 115 & 104 & 5,031 \\
\hline 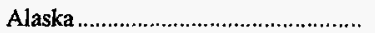 & 102 & - & - & - & - & - & - & 102 \\
\hline Arizona & 630 & - & - & - & - & - & 21 & 651 \\
\hline 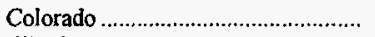 & 858 & 198 & 123 & 104 & - & - & 49 & 1,332 \\
\hline 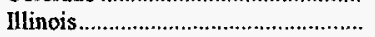 & 4,330 & 378 & 176 & 19 & $?$ & 92 & 172 & 5,174 \\
\hline 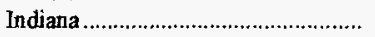 & 1,463 & 562 & 280 & 78 & 42 & 13 & 41 & 2,579 \\
\hline 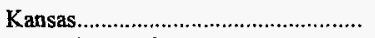 & - & - & 54 & - & - & - & - & 54 \\
\hline 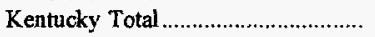 & 4,217 & 3,1555 & 3,705 & 2,233 & 1,588 & 1,498 & 1,930 & 18,826 \\
\hline Eastern & 2,429 & 2,672 & 3,391 & 2,138 & 1,438 & 1,392 & 1,670 & 15,130 \\
\hline Western & 1,788 & $\$, 83$ & 314 & 95 & 150 & 106 & 260 & 3,696 \\
\hline 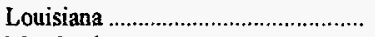 & 78 & 33 & - & - & - & - & - & 111 \\
\hline 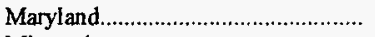 & 237 & - & 86 & 15 & 44 & 54 & 33 & 469 \\
\hline 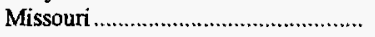 & - & - & 27 & 14 & 30 & 9 & - & 80 \\
\hline 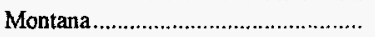 & 637 & 39 & 11 & 18 & - & - & - & 705 \\
\hline New Mexico ...................................... & 1,347 & - & - & - & - & - & - & 1,347 \\
\hline 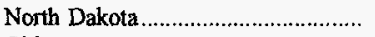 & 636 & - & - & - & - & - & 4 & 640 \\
\hline Ohio & 1,855 & 280 & 282 & 330 & 143 & 210 & 132 & 3,232 \\
\hline 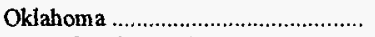 & - & - & 114 & 106 & - & 13 & - & 233 \\
\hline Pennsylvania Total .............................. & 3,704 & 1,041 & 1,384 & 656 & 506 & 765 & 965 & 9,021 \\
\hline Anthracite & - & 27 & 246 & 67 & 154 & 294 & 383 & 1,171 \\
\hline 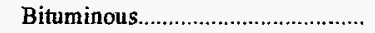 & 3,704 & 1,014 & 1,138 & 589 & 352 & 471 & 582 & 7,850 \\
\hline 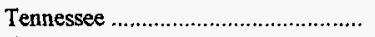 & - & 54 & 368 & 154 & 12 & 58 & 110 & 756 \\
\hline 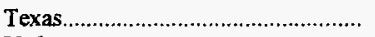 & 1,378 & 96 & 76 & - & - & - & - & 1,550 \\
\hline 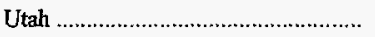 & 1,587 & 179 & - & - & - & - & 38 & 1,804 \\
\hline 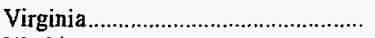 & 1,087 & 50.5 & 1,501 & 940 & 746 & 616 & 846 & 6,241 \\
\hline Washington $\ldots \ldots \ldots \ldots \ldots \ldots \ldots \ldots \ldots \ldots$ & 489 & - & - & 100 & - & - & - & 589 \\
\hline 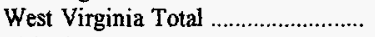 & 8,861 & 2,593 & 2,800 & 1,061 & 1,113 & 994 & 2,699 & 20,121 \\
\hline Northem & 3,630 & $32 \%$ & 445 & 164 & 160 & 201 & 352 & 5,279 \\
\hline Southern & 5,231 & 2,266 & 2,355 & 897 & 953 & 793 & 2,347 & 14,842 \\
\hline Wyoming & 2,658 & 106 & 13 & 24 & - & 13 & - & 2,814 \\
\hline Appalachian Total $2 \ldots \ldots \ldots \ldots \ldots \ldots \ldots$ & 21,797 & 7,910 & 9,960 & 5,427 & 4,144 & 4,204 & 6,559 & 60,001 \\
\hline 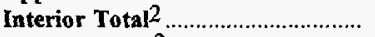 & 9,037 & 2,152 & 1,041 & 312 & 229 & 233 & 473 & 13,477 \\
\hline Western Total ${ }^{2} \ldots \ldots \ldots \ldots \ldots \ldots \ldots \ldots \ldots$ & 8,944 & 522 & 147 & 246 & - & 13 & 112 & 9,984 \\
\hline East of Miss. River............................ & 29,378 & 9,933 & 10,730 & 5,619 & 4,343 & 4,415 & 7,032 & 71,450 \\
\hline West of Miss. River ............................ & 10,400 & 651 & 418 & 366 & 30 & 35 & 112 & 12,012 \\
\hline U.S. Total & 39,778 & 10,584 & 11,148 & 5,985 & 4,373 & 4,450 & 7,144 & 83,462 \\
\hline
\end{tabular}

1 Includes all employees at preparation plants and tipples not co-located with a mine.

2 For a definition of coal-producing regions, see Appendix $C$.

Notes: Includes all employees engaged in production, preparation, processing, development, maintenance, repair, shop or yard work at mining operations. Excludes office workers. Includes mining operations management and all technical and engineering personnel. Excludes silt, culm refuse bank, slurry dam, and dredge operations except for Pennsylvania anthracite. Excludes mines producing less than 10,000 short tons and preparation plants with less than 5,000 employee hours, which are not required to provide these data.

Sources: Energy Information Administration, Form EIA-7A, "Coal Production Report"; State Mining Agency Coal Production Reports; and/or U.S. Department of Labor, Mine Safety and Health Administration, Form 7000-2, "Quarterly Mine Employment and Coal Production Report." 
Table 44. Average Number of Miners at Underground Mines by State and Mine Production Range, 1996

\begin{tabular}{|c|c|c|c|c|c|c|c|c|}
\hline \multirow{2}{*}{$\begin{array}{l}\text { Coal-Producing } \\
\text { State and Region }\end{array}$} & \multicolumn{7}{|c|}{$\begin{array}{l}\text { Mine Production Range } \\
\text { (thousand short tons) }\end{array}$} & \multirow{2}{*}{ Total } \\
\hline & $\begin{array}{c}1,000 \\
\text { and over }\end{array}$ & $\begin{array}{c}500 \\
\text { to } 1,000\end{array}$ & $\begin{array}{l}200 \\
\text { to } 500\end{array}$ & $\begin{array}{l}100 \\
\text { to } 200\end{array}$ & $\begin{array}{c}50 \\
\text { to } 100\end{array}$ & $\begin{array}{l}10 \\
\text { to } 50\end{array}$ & Zero 1 & \\
\hline Alabama & 3,471 & 567 & - & - & 32 & - & 75 & 4,145 \\
\hline 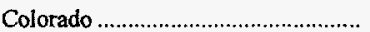 & 468 & 198 & 99 & 104 & - & - & 49 & 918 \\
\hline Illinais. & 3,829 & 78 & 105 & 19 & - & 81 & 144 & 4,256 \\
\hline Indiana & 174 & 267 & - & - & - & - & 16 & 457 \\
\hline 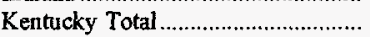 & 3,318 & 2,040 & 2,334 & 1,674 & 1,138 & 964 & 1,408 & 12,876 \\
\hline Eastern & 1,596 & 1,597 & 2,218 & 1,674 & 1,063 & 950 & 1,177 & 10,275 \\
\hline 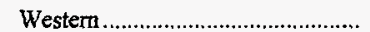 & 1,722 & 443 & 116 & - & 75 & 14 & 231 & 2,601 \\
\hline 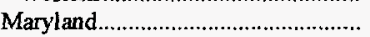 & 237 & - & 26 & - & - & 12 & 33 & 308 \\
\hline 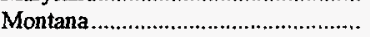 & - & - & - & 18 & - & - & - & 18 \\
\hline 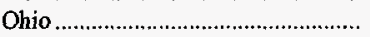 & 1,551 & - & 40 & 92 & - & - & 23 & 1,706 \\
\hline Oklahoma & - & - & - & 26 & - & - & - & 26 \\
\hline 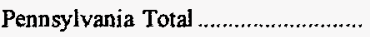 & 3,593 & 705 & 581 & 119 & 71 & 141 & 389 & 5,599 \\
\hline 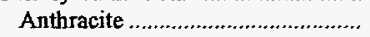 & - & - & - & 33 & - & 63 & 51 & 147 \\
\hline 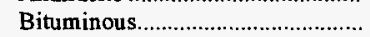 & 3,593 & 705 & 581 & 86 & 71 & 78 & 338 & 5,452 \\
\hline Tennessee & - & - & 248 & 100 & 12 & 34 & 73 & 467 \\
\hline Utah & 1,587 & 179 & - & - & - & - & 37 & 1,803 \\
\hline Virginia & 1,087 & 183 & 1,141 & 752 & 647 & 574 & 714 & 5,098 \\
\hline 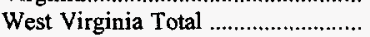 & 7,144 & 1,941 & 2,209 & 800 & 946 & 756 & 2,207 & 16,003 \\
\hline Northern & 3,535 & 267 & 350 & 99 & 113 & 132 & 268 & 4,764 \\
\hline 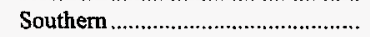 & 3,609 & 1,674 & 1,859 & 701 & 833 & 624 & 1,939 & 11,239 \\
\hline Wyoming & 116 & - & - & - & - & - & - & 116 \\
\hline Appalachian Total $2 \ldots \ldots \ldots \ldots \ldots \ldots$ & 18,679 & 4,993 & 6,463 & 3,537 & 2,771 & 2,467 & 4,691 & 43,601 \\
\hline Interior Tota 2 & 5,725 & 788 & 221 & 45 & 75 & 95 & 391 & 7,340 \\
\hline Western Total 2 & 2,171 & 377 & 99 & 122 & - & - & 86 & 2,855 \\
\hline East of Miss. River........................... & 24,404 & 5,781 & 6,684 & $\mathbf{3 , 5 5 6}$ & 2,846 & 2,562 & 5,082 & 50,915 \\
\hline West of Miss. River.......................... & 2,171 & 377 & 99 & 148 & - & - & 86 & 2,881 \\
\hline (n) & 26,575 & 6,158 & 6,783 & 3,704 & 2,846 & 2,562 & 5,168 & 53,796 \\
\hline
\end{tabular}

1 Includes all employees at preparation plants and tipples not co-located with a mine.

2 For a definition of coal-producing regions, see Appendix C.

Notes: Includes all employees engaged in production, preparation, processing, development, maintenance, repair, shop or yard work at mining operations. Excludes office workers. Includes mining operations management and all technical and engineering personnel. Excludes silt, culm refuse bank, slurry dam, and dredge operations except for Pennsylvania anthracite. Excludes mines producing less than 10,000 short tons and preparation plants with less than 5,000 employee hours, which are not required to provide these data.

Sources: Energy Information Administration, Form EIA-7A, "Coal Production Report"; State Mining Agency Coal Production Reports; and/or U.S. Department of Labor, Mine Safety and Health Administration, Form 7000-2, "Quarterly Mine Employment and Coal Production Report." 
Table 45. Average Number of Miners at Surface Mines by State and Mine Production Range, 1996

\begin{tabular}{|c|c|c|c|c|c|c|c|c|}
\hline \multirow{2}{*}{$\begin{array}{l}\text { Coal-Producing } \\
\text { State and Region }\end{array}$} & \multicolumn{7}{|c|}{$\begin{array}{l}\text { Mine Production Range } \\
\text { (thousand short tons) }\end{array}$} & \multirow{2}{*}{ Total } \\
\hline & $\begin{array}{c}1,000 \\
\text { and over }\end{array}$ & $\begin{array}{c}500 \\
\text { to } 1,000\end{array}$ & $\begin{array}{l}200 \\
\text { to } 500\end{array}$ & $\begin{array}{l}100 \\
\text { to } 200\end{array}$ & $\begin{array}{l}50 \\
\text { to } 100\end{array}$ & $\begin{array}{c}10 \\
\text { to } 50\end{array}$ & Zero 1 & \\
\hline 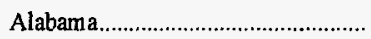 & 153 & 198 & 148 & 133 & 110 & 115 & 29 & 886 \\
\hline 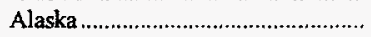 & 102 & - & - & - & - & - & - & 102 \\
\hline Arizona & 630 & - & - & - & - & - & 21 & 651 \\
\hline Colorado & 390 & - & 24 & - & - & - & - & 414 \\
\hline 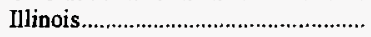 & 501 & 300 & 71 & - & 7 & 11 & 28 & 918 \\
\hline Indiana & 1,289 & 395 & 280 & 78 & 42 & 13 & 25 & 2,122 \\
\hline 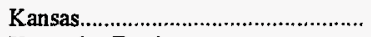 & - & - & 54 & - & - & - & - & 54 \\
\hline 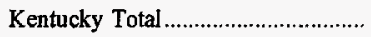 & 899 & 1,615 & 1,371 & 559 & 450 & 534 & 522 & 5,950 \\
\hline 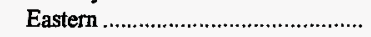 & 833 & 1,075 & 1,173 & 464 & 375 & 442 & 493 & 4,855 \\
\hline 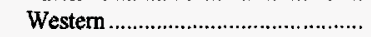 & 66 & 540 & 198 & 95 & 75 & 92 & 29 & 1,095 \\
\hline 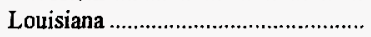 & 78 & 33 & - & - & - & - & - & 111 \\
\hline 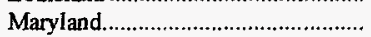 & - & - & 60 & 15 & 44 & 42 & - & 161 \\
\hline 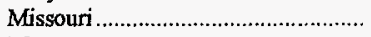 & - & - & 27 & 14 & 30 & 9 & - & 80 \\
\hline 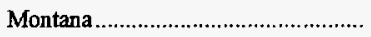 & 637 & 39 & 11 & - & - & - & - & 687 \\
\hline 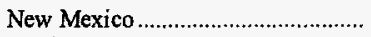 & 1,347 & - & - & - & - & - & - & 1,347 \\
\hline 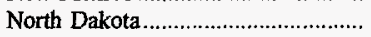 & 636 & - & - & - & - & - & 4 & 640 \\
\hline Ohio & 304 & 280 & 242 & 238 & 143 & 210 & 109 & 1,526 \\
\hline Oklahoma & - & - & 114 & 80 & - & 13 & - & 207 \\
\hline Pennsylvania Total ........................... & 111 & 336 & 803 & 537 & 435 & 624 & 576 & 3,422 \\
\hline 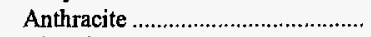 & - & 27 & 246 & 34 & 154 & 231 & 332 & 1,024 \\
\hline 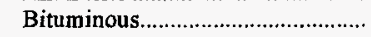 & 111 & 309 & 557 & 503 & 281 & 393 & 244 & 2,398 \\
\hline 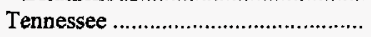 & - & 54 & 120 & 54 & - & 24 & 37 & 289 \\
\hline 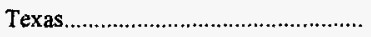 & 1,378 & 96 & 76 & - & - & - & - & 1,550 \\
\hline 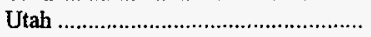 & - & - & - & - & - & - & 1 & 1 \\
\hline 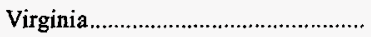 & - & 322 & 360 & 188 & 99 & 42 & 132 & 1,143 \\
\hline 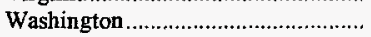 & 489 & - & - & 100 & - & - & - & 589 \\
\hline 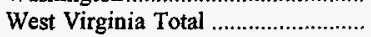 & 1,717 & 652 & 591 & 261 & 167 & 238 & 492 & 4,118 \\
\hline Northern & 95 & 60 & 95 & 65 & 47 & 69 & 84 & 515 \\
\hline 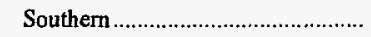 & 1,622 & 592 & 496 & 196 & 120 & 169 & 408 & 3,603 \\
\hline Wyoming & 2,542 & 106 & 13 & 24 & - & 13 & - & 2,698 \\
\hline Appalachian Total ${ }^{2} \ldots \ldots \ldots \ldots \ldots \ldots \ldots$ & 3,118 & 2,917 & 3,497 & 1,890 & 1,373 & 1,737 & 1,868 & 16,400 \\
\hline Interior Total $2 \ldots \ldots \ldots \ldots \ldots \ldots \ldots \ldots \ldots \ldots \ldots \ldots \ldots$ & 3,312 & 1,364 & 820 & 267 & 154 & 138 & 82 & 6,137 \\
\hline Western Total 2 & 6,773 & 145 & 48 & 124 & - & 13 & 26 & 7,129 \\
\hline East of Miss. River............................. & 4,974 & 4,152 & 4,046 & 2,063 & 1,497 & 1,853 & 1,950 & 20,535 \\
\hline West of Miss. River........................... & 8,229 & 274 & 319 & 218 & 30 & 35 & 26 & 9,131 \\
\hline 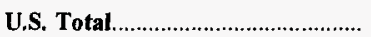 & 13,203 & 4,426 & 4,365 & 2,281 & 1,527 & 1,888 & 1,976 & 29,666 \\
\hline
\end{tabular}

1 Includes all employees at preparation plants and tipples not co-located with a mine.

2 For a definition of coal-producing regions, see Appendix $\mathrm{C}$.

Notes: Includes all employees engaged in production, preparation, processing, development, maintenance, repair, shop or yard work at mining operations. Excludes office workers. Includes mining operations management and all technical and engineering personnel. Excludes silt, culm refuse bank, slurry dam, and dredge operations except for Pennsylvania anthracite. Excludes mines producing less than 10,000 short tons and preparation plants with less than 5,000 employee hours, which are not required to provide these data.

Sources: Energy Information Administration, Form EIA-7A, "Coal Production Report"; State Mining Agency Coal Production Reports; and/or U.S. Department of Labor, Mine Safety and Health Administration, Forn 7000-2, "Quarterly Mine Employment and Coal Production Report." 
Table 46. Average Number of Miners by State, Mine Type, and Union Type, 1996

\begin{tabular}{|c|c|c|c|c|c|}
\hline $\begin{array}{l}\text { Coal-Producing } \\
\text { State and Region }\end{array}$ & UMWA & Other Unions & Union Total & Nonunion & Total \\
\hline 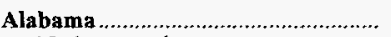 & 3,743 & - & 3,743 & 1,288 & 5,031 \\
\hline Underground & 3,423 & - & 3,423 & 722 & 4,145 \\
\hline 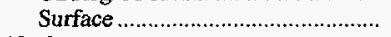 & 320 & - & 320 & 566 & 886 \\
\hline 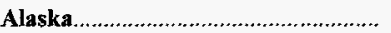 & - & 102 & 102 & - & 102 \\
\hline Surface & - & 102 & 102 & - & 102 \\
\hline Arizona. & 651 & - & 651 & - & 651 \\
\hline Surface & 651 & - & 651 & - & 651 \\
\hline Colorado & 350 & 129 & 479 & 853 & 1,332 \\
\hline 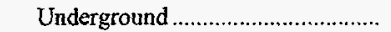 & 245 & - & 245 & 673 & 918 \\
\hline 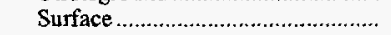 & 105 & 129 & 234 & 180 & 414 \\
\hline 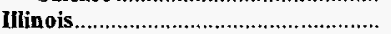 & 3,831 & 391 & 4,222 & 952 & 5,174 \\
\hline 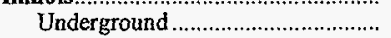 & 3,024 & 331 & 3,355 & 901 & 4,256 \\
\hline Surface & 807 & 60 & 867 & 51 & 918 \\
\hline Indiana & 1,317 & - & 1,317 & 1,262 & 2,579 \\
\hline 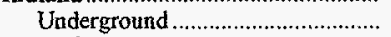 & 162 & - & 162 & 295 & 457 \\
\hline 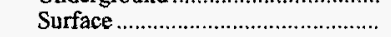 & 1,155 & - & 1,155 & 967 & 2,122 \\
\hline Kansas . & 54 & $\dot{-}$ & 54 & - & 54 \\
\hline Surface & 54 & - & 54 & - & 54 \\
\hline Kentucky Total & 2,096 & 106 & 2,202 & 16,624 & 18,826 \\
\hline Underground $\ldots \ldots \ldots \ldots \ldots \ldots \ldots \ldots \ldots$ & 1,777 & 50 & 1,827 & 11,049 & 12,876 \\
\hline Surface & 319 & 56 & 375 & 5,575 & 5,950 \\
\hline 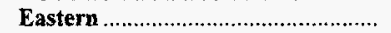 & 978 & 106 & 1,084 & 14,046 & 15,130 \\
\hline 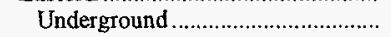 & 772 & 50 & 822 & 9,453 & 10,275 \\
\hline Surface & 206 & 56 & 262 & 4,593 & 4,855 \\
\hline 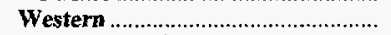 & 1,118 & - & 1,118 & 2,578 & 3,696 \\
\hline Underground & 1,005 & - & 1,005 & 1,596 & 2,601 \\
\hline Surface & 113 & - & 113 & 982 & 1,095 \\
\hline 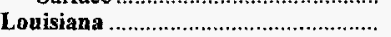 & - & - & - & 111 & 111 \\
\hline 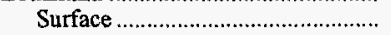 & - & - & - & 111 & 111 \\
\hline Maryland & - & - & - & 469 & 469 \\
\hline 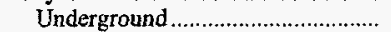 & - & - & - & 308 & 308 \\
\hline 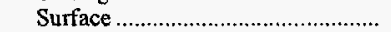 & - & - & - & 161 & 161 \\
\hline 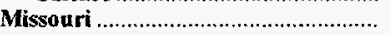 & - & - & - & 80 & 80 \\
\hline Surface & - & - & - & 80 & 80 \\
\hline 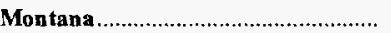 & 383 & 226 & 609 & 96 & 705 \\
\hline 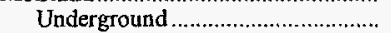 & - & - & - & 18 & 18 \\
\hline 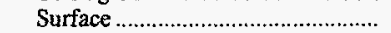 & 383 & 226 & 609 & 78 & 687 \\
\hline New Mexico & 405 & 750 & 1,155 & 192 & 1,347 \\
\hline 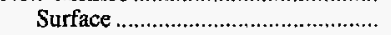 & 405 & 750 & 1,155 & 192 & 1,347 \\
\hline 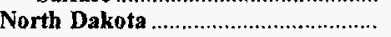 & 108 & 102 & 210 & 430 & 640 \\
\hline Surface & 108 & 102 & 210 & 430 & 640 \\
\hline Ohio & 1,700 & - & 1,700 & $\mathbf{1 , 5 3 2}$ & 3,232 \\
\hline 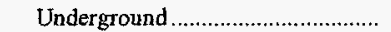 & 1,440 & - & 1,440 & 266 & 1,706 \\
\hline Surface & 260 & - & 260 & 1,266 & 1,526 \\
\hline Oklahoma & - & - & - & 233 & 233 \\
\hline 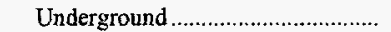 & - & - & - & 26 & 26 \\
\hline 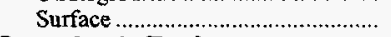 & - & - & - & 207 & 207 \\
\hline Pennsylvania Total ................................. & 4,119 & 25 & 4,144 & 4,877 & 9,021 \\
\hline Underground & 3,475 & 7 & 3,482 & 2,117 & 5,599 \\
\hline 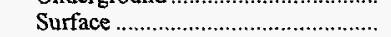 & 644 & 18 & 662 & 2,760 & 3,422 \\
\hline Anthracite & 502 & 19 & 521 & 650 & 1,171 \\
\hline 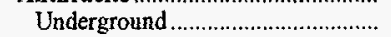 & - & 4 & 4 & 143 & 147 \\
\hline Surface & 502 & 15 & 517 & 507 & 1,024 \\
\hline 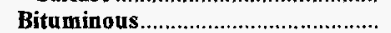 & 3,617 & 6 & 3,623 & 4,227 & 7,850 \\
\hline 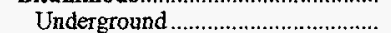 & 3,475 & 3 & 3,478 & 1,974 & 5,452 \\
\hline Surface & 142 & 3 & 145 & 2,253 & 2,398 \\
\hline 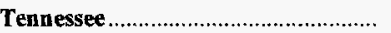 & - & - & - & 756 & 756 \\
\hline 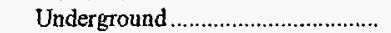 & - & - & - & 467 & 467 \\
\hline Surface & - & - & - & 289 & 289 \\
\hline 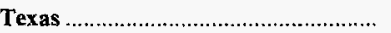 & - & 920 & 920 & 630 & 1,550 \\
\hline Surface & - & 920 & 920 & 630 & 1,550 \\
\hline Utah & 722 & - & 722 & 1,082 & 1,804 \\
\hline Underground & 722 & - & 722 & 1,081 & 1,803 \\
\hline Surface & - & - & - & 1 & 1 \\
\hline Virginia & 1,167 & 56 & 1,223 & 5,018 & 6,241 \\
\hline Underground & 1,089 & - & 1,089 & 4,009 & 5,098 \\
\hline Surface & 78 & 56 & 134 & 1,009 & 1,143 \\
\hline 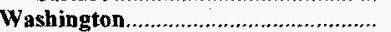 & - & 489 & 489 & 100 & 589 \\
\hline Surface & - & 489 & 489 & 100 & 589 \\
\hline West Virginia Total & 11,671 & 42 & 11,713 & 8,408 & 20,121 \\
\hline Underground ......................................... & 9,894 & 2 & 9,896 & 6,107 & 16,003 \\
\hline Surface & 1,777 & 40 & 1,817 & 2,301 & 4,118 \\
\hline
\end{tabular}

See footnotes at end of table.

9 
Table 46. Average Number of Miners by State, Mine Type, and Union Type, 1996 (Continued)

\begin{tabular}{|c|c|c|c|c|c|}
\hline $\begin{array}{l}\text { Coal-Producing } \\
\text { State and Region }\end{array}$ & UMWA & Other Unions & Union Total & Nonunion & Total \\
\hline 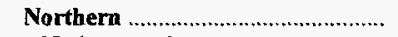 & $\mathbf{3 , 5 3 7}$ & - & $\mathbf{3 , 5 3 7}$ & 1,742 & 5,279 \\
\hline 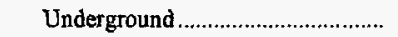 & 3,525 & - & 3,525 & 1,239 & 4,764 \\
\hline Surface & 12 & - & 12 & 503 & 515 \\
\hline Southern & 8,134 & 42 & 8,176 & 6,666 & 14,842 \\
\hline 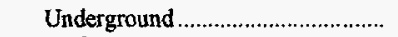 & 6,369 & 2 & 6,371 & 4,868 & 11,239 \\
\hline Surface & 1,765 & 40 & 1,805 & 1,798 & 3,603 \\
\hline Wyoming & 352 & 540 & 892 & 1,922 & 2,814 \\
\hline 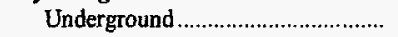 & - & - & - & 116 & 116 \\
\hline Surface ........................................ & 352 & 540 & 892 & 1,806 & 2,698 \\
\hline Appalachian Total ${ }^{1} \ldots \ldots \ldots \ldots \ldots \ldots \ldots \ldots$ & 23,378 & 229 & 23,607 & 36,394 & 60,001 \\
\hline 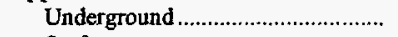 & 20,093 & 59 & 20,152 & 23,449 & 43,601 \\
\hline Surface $\ldots \ldots \ldots \ldots \ldots \ldots$ & 3,285 & 170 & 3,455 & 12,945 & 16,400 \\
\hline Interior Total ${ }^{1} \ldots \ldots \ldots \ldots \ldots \ldots \ldots \ldots$ & 6,320 & 1,311 & 7,631 & 5,846 & 13,477 \\
\hline 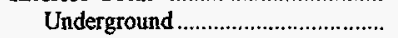 & 4,191 & 331 & 4,522 & 2,818 & 7,340 \\
\hline Surface & 2,129 & 980 & 3,109 & 3,028 & 6,137 \\
\hline Western Total 1 & 2,971 & 2,338 & 5,309 & 4,675 & 9,984 \\
\hline Underground & 967 & - & 967 & 1,888 & 2,855 \\
\hline 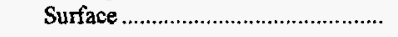 & 2,004 & 2,338 & 4,342 & 2,787 & 7,129 \\
\hline 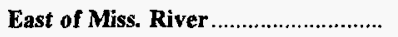 & 29,644 & 620 & 30,264 & 41,186 & 71,450 \\
\hline 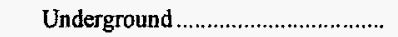 & 24,284 & 390 & 24,674 & 26,241 & 50,915 \\
\hline Surface & 5,360 & 230 & 5,590 & 14,945 & 20,535 \\
\hline West of Miss. River ............................... & 3,025 & 3,258 & 6,283 & 5,729 & 12,012 \\
\hline 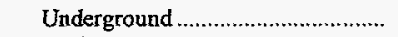 & 967 & - & 967 & 1,914 & 2,881 \\
\hline Surface & 2,058 & 3,258 & 5,316 & 3,815 & 9,131 \\
\hline U.S. Total. & 32,669 & 3,878 & 36,547 & 46,915 & 83,462 \\
\hline 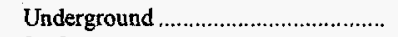 & 25,251 & 390 & 25,641 & 28,155 & 53,796 \\
\hline Surface & 7,418 & 3,488 & 10,906 & 18,760 & 29,666 \\
\hline
\end{tabular}

1 For a definition of coal-producing regions, see Appendix $C$.

Notes: Includes all employees engaged in production, preparation, processing, development, maintenance, repair, shop or yard work at mining operations. Excludes office workers. Includes mining operations management and all technical and engineering personnel. Excludes silt, culm, refuse bank, slurry dam, and dredge operations except for Pennsylvania anthracite. Excludes mines producing less than 10,000 short tons and preparation plants with less than 5,000 employee hours, which are not required to provide these data. See Glossary for listing of other unions.

Sources: Energy Information Administration, Form EIA-7A, "Coal Production Report"; State Mining Agency Coal Production Reports; and/or U.S. Department of Labor, Mine Safety and Health Administration, Form 7000-2, "Quarterly Mine Employment and Coal Production Report."

Table 47. U.S. Coal Mine Injuries, 1987, 1992-1996

\begin{tabular}{|c|c|c|c|c|c|c|c|c|c|}
\hline \multirow{2}{*}{ Injury Type } & \multirow{2}{*}{1996} & \multirow{2}{*}{1995} & \multirow{2}{*}{1994} & \multirow{2}{*}{1993} & \multirow{2}{*}{1992} & \multirow{2}{*}{1987} & \multirow{2}{*}{$\begin{array}{c}\text { Percent } \\
\text { Change } \\
\text { 1995-1996 }\end{array}$} & \multicolumn{2}{|c|}{ Average Annual Percent Change } \\
\hline & & & & & & & & 1992-1996 & $1987-1996$ \\
\hline Injuries Total ${ }^{1} \ldots \ldots \ldots \ldots \ldots \ldots \ldots$ & 5,896 & R $\mathbf{7 , 3 8 6}$ & $R_{8,765}$ & 8,446 & 10,110 & 12,417 & -20.2 & -12.6 & -7.9 \\
\hline Fatal & 38 & 47 & 45 & 47 & 55 & 63 & -19.1 & -8.8 & -5.5 \\
\hline Nonfatal 2 & 5,858 & R 7,339 & ${ }^{R} 8,720$ & 8,399 & 10,055 & 12,354 & -20.2 & -12.6 & -8.0 \\
\hline Injuries per 200,000 & & & & & & & & & \\
\hline Employee-Hours Total............ & 5.96 & ${ }^{R} 6.26$ & ${ }^{R} 6.89$ & 6.86 & 7.33 & 7.88 & -4.8 & -5.0 & -3.1 \\
\hline Fatal & .04 & .04 & 03 & .04 & .04 & .04 & 0 & 0 & .0 \\
\hline Nonfatal ${ }^{2} \ldots \ldots \ldots \ldots \ldots \ldots \ldots \ldots$ & 5.92 & ${ }^{R} 6.22$ & ${ }^{R} 6.86$ & 6.82 & 7.29 & 7.84 & -4.8 & -5.1 & -3.1 \\
\hline
\end{tabular}

1 Includes contractors and office workers.

2 Inchudes only non-fatal injuries that resulted in absence from work.

Revised.

Note: Calculations of growth rate are based using unrounded values.

Source: U.S. Department of Labor, Mine Safety and Health Administration,Mine Injuries and Worktime, Quarterly, various issues. 
Figure 6. U.S. Coal Mine Injuries, 1987-1996

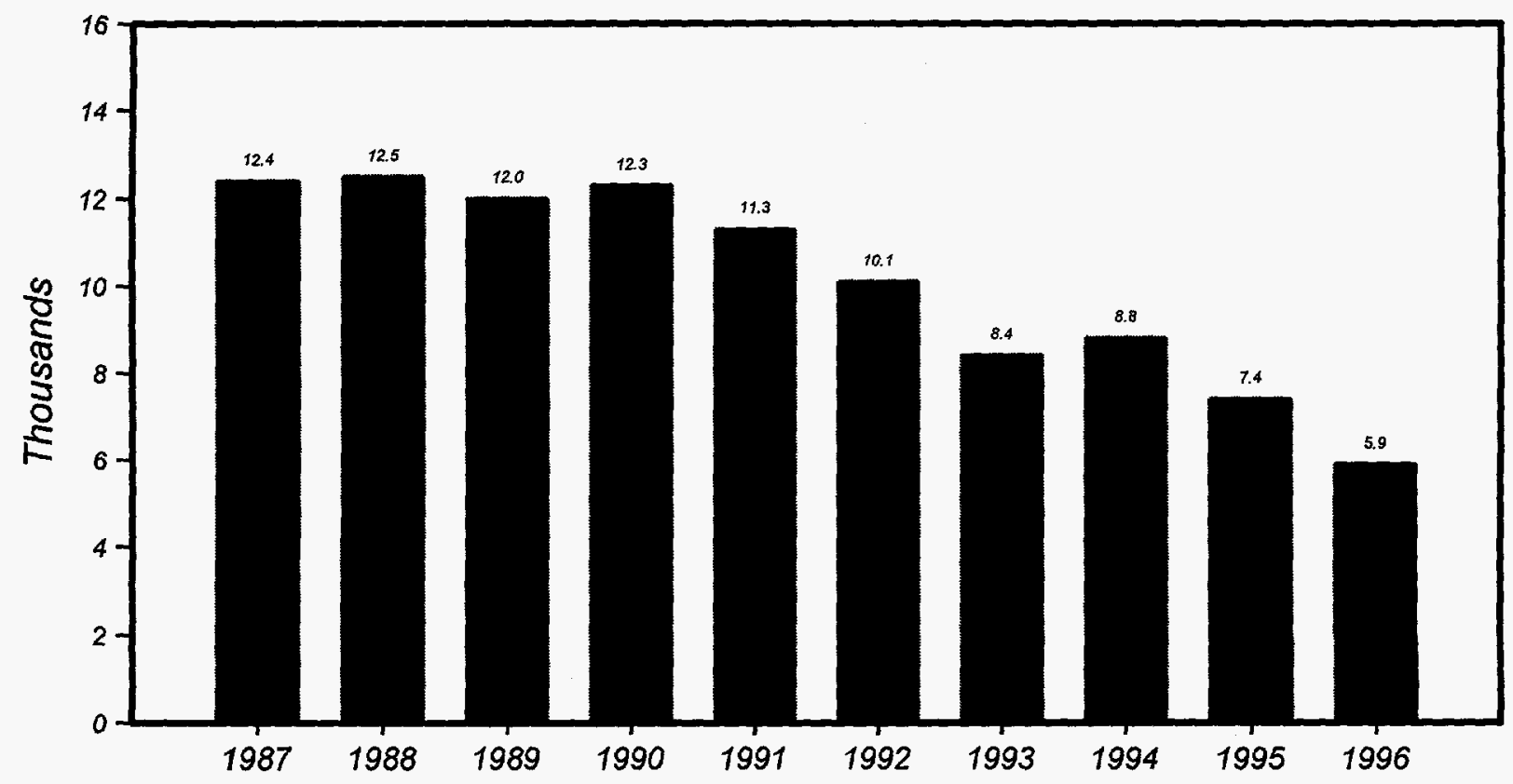

Figure 7. U.S. Coal Mine Fatalities, 1987-1996

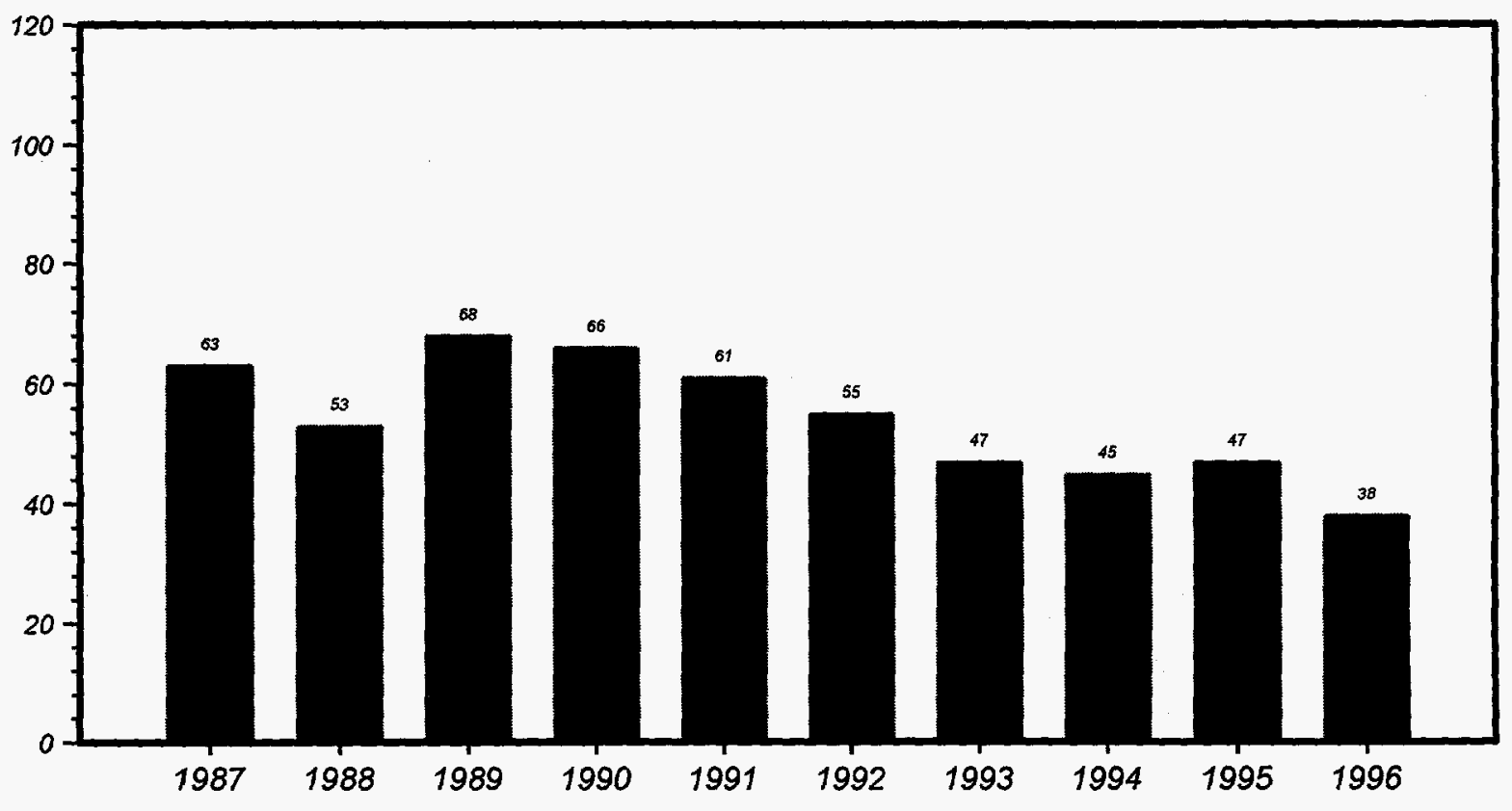

Sources: U.S. Deportment of Labor, Mine Sofety and Health Administration, Mine Injuries and Worklime, Ouorterly, various issues. 



\section{Productivity}

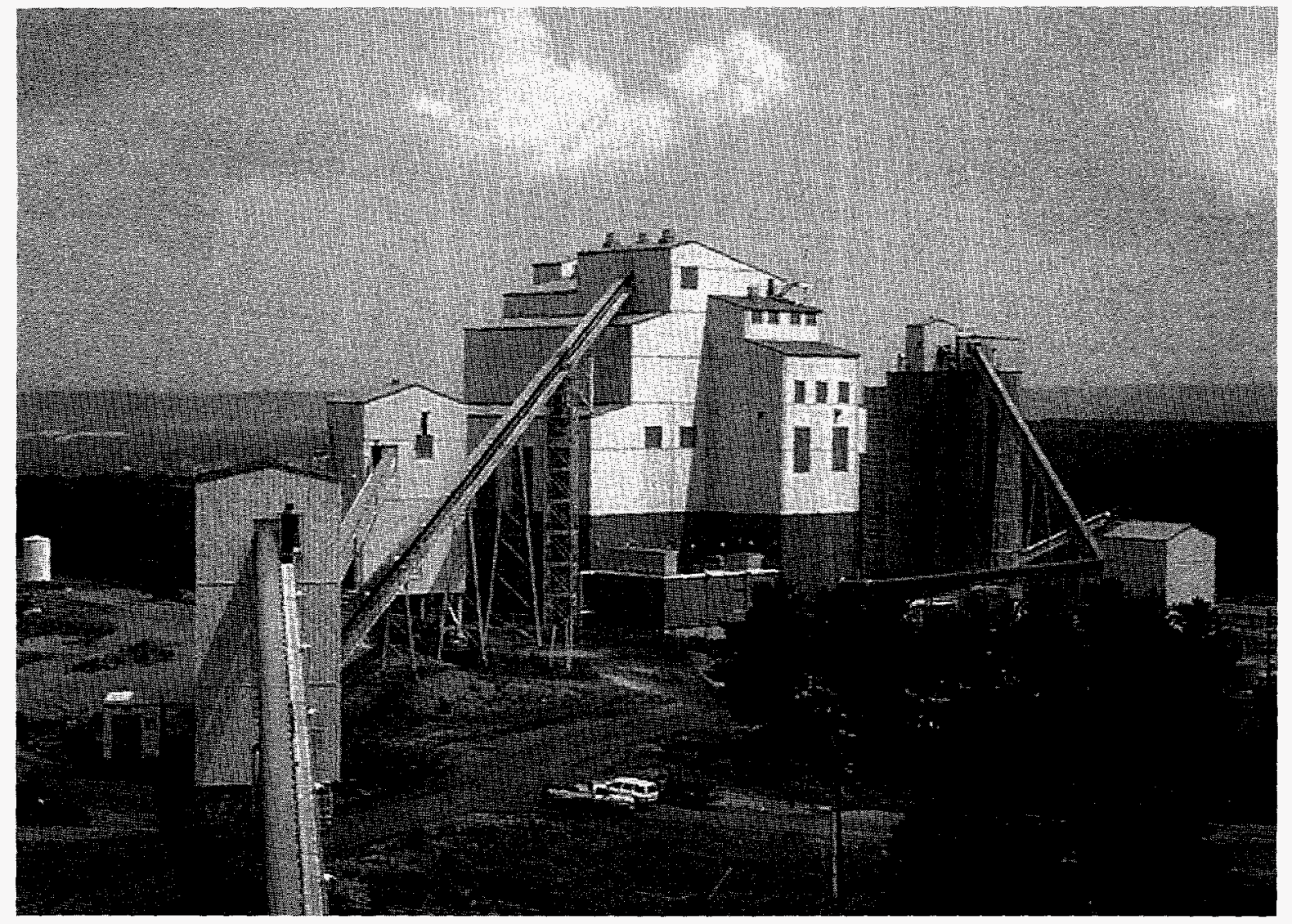

In 1996, the average coal mining productivity exceeded its 1995 record high of 5.4 short tons of coal per miner per hour to 5.7 short tons of coal per miner per hour (inset, coal cleaning facility located in Central City, PA).

Productivity at surface mines was 9.1 and 3.6 at underground mines. 
Table 48. Coal Mining Productivity by State, 1987, 1992-1996 (Short Tons of Coal Produced per Miner per Hour)

\begin{tabular}{|c|c|c|c|c|c|c|c|c|c|}
\hline \multirow{2}{*}{$\begin{array}{l}\text { Coal-Producing } \\
\text { State and Region }\end{array}$} & \multirow{2}{*}{1996} & \multirow{2}{*}{1995} & \multirow{2}{*}{1994} & \multirow{2}{*}{1993} & \multirow{2}{*}{1992} & \multirow{2}{*}{1987} & \multirow{2}{*}{$\begin{array}{c}\text { Percent } \\
\text { Change } \\
\text { 1995-1996 }\end{array}$} & \multicolumn{2}{|c|}{ Average Annual Percent Change } \\
\hline & & & & & & & & $1992-1996$ & $1987-1996$ \\
\hline Alabama & 2.20 & 2.24 & 2.25 & 2.35 & 2.49 & 1.97 & -1.6 & -3.0 & 1.3 \\
\hline Alaska & 6.81 & 7.46 & 6.94 & 7.40 & 6.93 & 6.84 & -8.8 & -.4 & $*$ \\
\hline Arizona & 6.30 & 6.34 & 6.71 & 6.21 & 6.29 & 6.76 & -.6 & * & -.8 \\
\hline Arkansas & - & 1.47 & 1.52 & 1.39 & 1.68 & 1.80 & -100.0 & - & - \\
\hline California & - & - & - & - & 11.12 & 5.19 & - & - & - \\
\hline Colorado & 7.32 & 6.14 & 6.20 & 5.85 & 5.27 & 4.22 & 19.2 & 8.5 & 6.3 \\
\hline Illinois & 4.18 & 3.87 & 3.59 & 3.23 & 3.42 & 2.52 & 8.2 & 5.2 & 5.8 \\
\hline Indiana & 4.98 & 4.68 & 4.28 & 4.46 & 4.09 & 3.57 & 6.4 & 5.0 & 3.8 \\
\hline Iowa & - & - & 1.52 & .72 & 1.14 & 1.39 & - & - & - \\
\hline 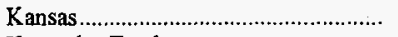 & 2.17 & 2.22 & 1.93 & 2.30 & 2.21 & 3.75 & -2.4 & -.5 & -5.9 \\
\hline 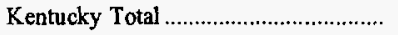 & 3.80 & 3.57 & 3.25 & 3.25 & 3.20 & 2.69 & 6.6 & 4.4 & 3.9 \\
\hline 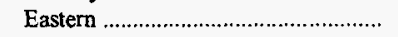 & 3.68 & 3.47 & 3.24 & 3.18 & 3.10 & 2.59 & 6.1 & 4.3 & 4.0 \\
\hline 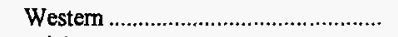 & 4.29 & 3.97 & 3.28 & 3.49 & 3.49 & 2.98 & 7.9 & 5.3 & 4.1 \\
\hline 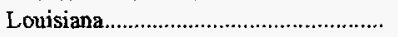 & 10.86 & 13.25 & 13.00 & 12.14 & 12.43 & 15.26 & -18.0 & -3.3 & -3.7 \\
\hline Maryland & 4.13 & 3.82 & 3.68 & 3.41 & 2.91 & 3.38 & 8.2 & 9.1 & 2.2 \\
\hline 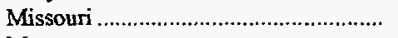 & 3.49 & 2.55 & 3.59 & 1.84 & 3.10 & 2.28 & 36.9 & 3.0 & 4.8 \\
\hline 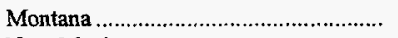 & 21.88 & 21.106 & 21.92 & 19.49 & 20.16 & 18.70 & 3.9 & 2.1 & 1.8 \\
\hline 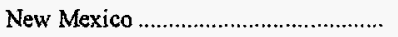 & 8.45 & 6.92 & 6.77 & 6.68 & 6.68 & 5.74 & 22.1 & 6.0 & 4.4 \\
\hline North Dakota & 17.20 & 16.80 & 18.84 & 17.66 & 18.12 & 13.46 & 2.4 & -1.3 & 2.8 \\
\hline Ohio & 3.95 & 3.62 & 3.42 & 3.46 & 3.04 & 2.28 & 9.1 & 6.8 & 6.3 \\
\hline 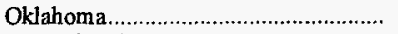 & 2.61 & 2.97 & 2.68 & 2.80 & 2.17 & 1.90 & -12.1 & 4.7 & 3.6 \\
\hline 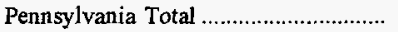 & 3.36 & 3.23 & 2.98 & 2.80 & 2.67 & 1.97 & 4.1 & 5.9 & 6.1 \\
\hline 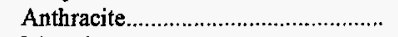 & 1.92 & 2.18 & 1.93 & 1.85 & 1.33 & 1.13 & -8.0 & 9.5 & 6.0 \\
\hline 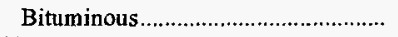 & 3.56 & 3.37 & 3.11 & 2.91 & 2.81 & 2.04 & 5.4 & 6.0 & 6.3 \\
\hline Tennessee & 2.20 & 2.36 & 2.23 & 2.47 & 2.19 & 1.67 & -6.8 & .1 & 3.1 \\
\hline 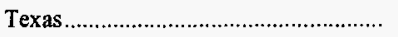 & 10.13 & 9.10 & 8.82 & 8.42 & 7.34 & 6.45 & 11.3 & 8.4 & 5.1 \\
\hline 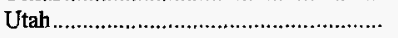 & 7.23 & 7.02 & 6.59 & 5.96 & 5.46 & 3.86 & 3.0 & 7.2 & 7.2 \\
\hline Virginia & 2.72 & 2.50 & 2.51 & 2.41 & 2.37 & 2.08 & 8.4 & 3.5 & 3.0 \\
\hline Washington & 3.97 & 4.04 & 4.11 & 4,00 & 4.51 & 3.06 & -1.8 & -3.1 & 2.9 \\
\hline West Virginia Total ............................. & 3.91 & 3.74 & 3.69 & 3.27 & 3.27 & 2.47 & 4.4 & 4.5 & 5.3 \\
\hline 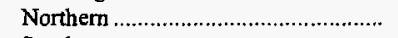 & 4.05 & 3.72 & 3.63 & 2.98 & 3.17 & 2.61 & 8.9 & 6.3 & 5.0 \\
\hline Southem & 3.86 & 3.75 & 3.72 & 3.39 & 3.32 & 2.38 & 2.8 & 3.8 & 5.5 \\
\hline 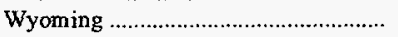 & 32.06 & 30.05 & 26.05 & 24.46 & 21.50 & 17.91 & 6.6 & 10.5 & 6.7 \\
\hline 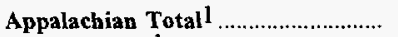 & 3.48 & 3.3i & 3.20 & 3.00 & 2.95 & 2.30 & 4.8 & 4.3 & 4.7 \\
\hline 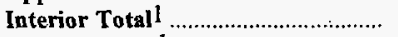 & 5.39 & 4.97 & 4.43 & 4.43 & 4.18 & 3.33 & 8.4 & 6.6 & 5.5 \\
\hline 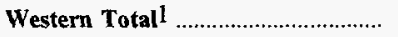 & 17.41 & 15.68 & 14.58 & 13.53 & 12.73 & 10.42 & 11.0 & 8.1 & 5.9 \\
\hline East of Miss. River................................ & 3.63 & 3.45 & 3.28 & 3.11 & 3.07 & 2.42 & 5.3 & 4.3 & 4.6 \\
\hline West of Miss. River ............................. & 15.66 & 14.18 & 13.22 & 12.14 & 11.03 & 8.73 & 10.4 & 9.2 & 6.7 \\
\hline U.S. Total & 5.69 & 5.38 & 4.98 & 4.70 & 4.36 & $\mathbf{3 . 3 0}$ & 5.8 & 6.9 & 6.3 \\
\hline
\end{tabular}

1 For a definition of coal-producing Jegions, see Appendix C.

* Data round to zero.

Notes: Productivity is calculated by dividing total coal production by the total direct labor hours worked by all employees engaged in production, preparation, processing, development, maintenance, repair, shop or yard work at mining operations, but excludes office workers. Excludes silt, culm, refuse bank, slurry dam, and dredge operations except for Pennsylvaniz anthracite. Excludes mines producing less than 10,000 short tons, which are not required to provide these data.

Sources: Energy Information Administration, Form EIA-7A, "Coal Production Report"; State Mining Agency Coal Production Reports; and/or U.S. Department of Labor, Mine Safety and Health Administration, Forn 7000-2, "Quarterly Mine Employment and Coal Production Report." 
Table 49. Underground Coal Mining Productivity by State, 1987, 1992-1996 (Short Tons of Coal Produced per Miner per Hour)

\begin{tabular}{|c|c|c|c|c|c|c|c|c|c|}
\hline \multirow{2}{*}{$\begin{array}{l}\text { Coal-Producing } \\
\text { State and Region }\end{array}$} & \multirow{2}{*}{1996} & \multirow{2}{*}{1995} & \multirow{2}{*}{1994} & \multirow{2}{*}{1993} & \multirow{2}{*}{1992} & \multirow{2}{*}{1987} & \multirow{2}{*}{$\begin{array}{c}\text { Percent } \\
\text { Change } \\
1995-1996\end{array}$} & \multicolumn{2}{|c|}{ Average Annual Percent Change } \\
\hline & & & & & & & & 1992-1996 & 1987-1996 \\
\hline 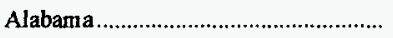 & 1.95 & 2.02 & 1.94 & 2.09 & 2.17 & 1.68 & -3.6 & -2.6 & 1.7 \\
\hline 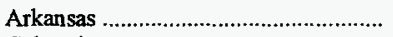 & - & - & 1.62 & - & - & - & - & - & - \\
\hline 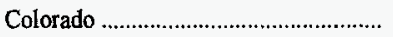 & 6.67 & 5.86 & 5.81 & 5.21 & 4.52 & 2.75 & 13.7 & 10.2 & 10.3 \\
\hline 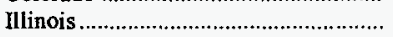 & 4.10 & 3.86 & 3.49 & 3.11 & 3.21 & 2.20 & 6.1 & 6.3 & 7.1 \\
\hline Indiana & 3.09 & 3.22 & 2.82 & 2.49 & 2.80 & 2.05 & -4.1 & 2.5 & 4.6 \\
\hline lowa & - & - & - & - & - & 1.22 & - & - & - \\
\hline Kentucky Total & 3.53 & 3.25 & 2.89 & 2.93 & 2.91 & 2.40 & 8.6 & 5.0 & 4.4 \\
\hline 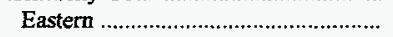 & 3.37 & 3.12 & 2.87 & 2.89 & 2.86 & 2.28 & 8.2 & 4.2 & 4.4 \\
\hline Western & 4.05 & 3.70 & 2.96 & 3.10 & 3.05 & 2.74 & 9.4 & 7.3 & 4.4 \\
\hline 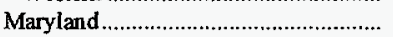 & 4.82 & 4.77 & 4.52 & 4.34 & 3.50 & 4.10 & 1.0 & 8.3 & 1.8 \\
\hline Montana & 3.50 & - & - & 1.06 & - & - & - & - & - \\
\hline 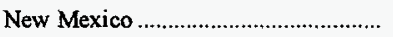 & - & 2.68 & 2.57 & 1.63 & .40 & 1.74 & -100.0 & - & - \\
\hline Ohio & 4.19 & 3.81 & 3.51 & 3.27 & 3.01 & 1.80 & 9.9 & 8.6 & 9.8 \\
\hline Oklahoma & 1.75 & .74 & 1.70 & 1.03 & 1.04 & - & 138.0 & 13.9 & - \\
\hline 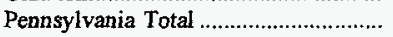 & 3.74 & 3.49 & 3.18 & 2.91 & 2.81 & 1.81 & 7.1 & 7.4 & 8.4 \\
\hline 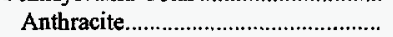 & .94 & .86 & .64 & .74 & .84 & .84 & 8.7 & 2.9 & 1.2 \\
\hline 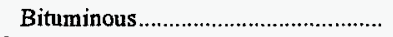 & 3.81 & 3.56 & 3.25 & 2.98 & 2.84 & 1.83 & 6.9 & 7.6 & 8.5 \\
\hline 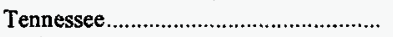 & 1.76 & 2.02 & 1.90 & 2.34 & 1.81 & 1.58 & -13.0 & -.8 & 1.2 \\
\hline Utah. & 7.24 & 7.02 & 6.59 & 5.96 & 5.46 & 3.86 & 3.1 & 7.3 & 7.2 \\
\hline 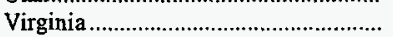 & 2.44 & 2.25 & 2.27 & 2.19 & 2.20 & 2.01 & 8.6 & 2.7 & 2.2 \\
\hline West Virginia Total ................................ & 3.50 & 3.40 & 3.38 & 2.92 & 2.99 & 2.31 & 2.9 & 4.0 & 4.7 \\
\hline 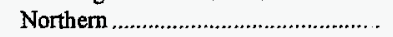 & 3.98 & 3.66 & 3.61 & 2.84 & 3.12 & 2.54 & 8.7 & 6.2 & 5.1 \\
\hline Southern & 3.29 & 3.27 & 3.25 & 2.96 & 2.92 & 2.17 & .7 & 3.0 & 4.7 \\
\hline 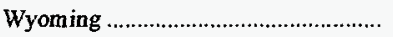 & 9.18 & 5.97 & 5.07 & 3.56 & 4.19 & 2.71 & 53.6 & 21.7 & 14.5 \\
\hline 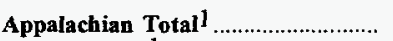 & 3.24 & 3.08 & 2.96 & 2.75 & 2.76 & 2.11 & 5.3 & 4.2 & 4.9 \\
\hline Interior Total 1 & 4.01 & 3.76 & 3.26 & 3.06 & 3.14 & 2.38 & 6.7 & 6.3 & 6.0 \\
\hline Western Total 1 & 7.09 & 6.35 & 5.98 & 5.23 & 4.88 & 3.40 & 11.8 & 9.8 & 8.5 \\
\hline East of Miss. River. & 3.36 & 3.19 & 3.02 & 2.81 & 2.82 & 2.16 & 5.3 & 4.5 & 5.1 \\
\hline West of Miss. River............................. & 7.03 & 6.32 & 5.93 & 5.18 & 4.85 & 3.39 & 11.2 & 9.7 & 8.4 \\
\hline 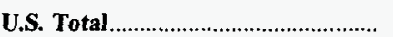 & 3.57 & 3.39 & 3.19 & 2.95 & 2.93 & 2.20 & 5.6 & 5.1 & 5.5 \\
\hline
\end{tabular}

1 For a definition of coal-producing regions, see Appendix C.

Notes: Productivity is calculated by dividing total coal production by the total direct labor hours worked by all mine employees engaged in production, preparation, processing, development, maintenance, repair, shop or yard work at mining operations, but excludes office workers. Excludes silt, culm, refuse bank, slurry dam, and dredge operations except for Pennsylvania anthracite. Excludes mines producing less than 10,000 short tons, which are not required to provide these data.

Sources: Energy Information Administration, Form EIA-7A, “Coal Production Report”; State Mining Agency Coal Production Reports; and/or U.S. Department of Labor, Mine Safety and Health Administration, Form 7000-2, "Quarterly Mine Employment and Coal Production Report." 
Table 50. Surface Coal Mining Productivity by State, 1987, 1992-1996 (Short Tons of Coal Produced per Miner per Hour)

\begin{tabular}{|c|c|c|c|c|c|c|c|c|c|}
\hline \multirow{2}{*}{$\begin{array}{l}\text { Coal-Producing } \\
\text { State and Region }\end{array}$} & \multirow{2}{*}{1996} & \multirow{2}{*}{1995} & \multirow{2}{*}{1994} & \multirow{2}{*}{1993} & \multirow{2}{*}{1992} & \multirow{2}{*}{1987} & \multirow{2}{*}{$\begin{array}{c}\text { Percent } \\
\text { Change } \\
1995-1996\end{array}$} & \multicolumn{2}{|c|}{ Average Annual Percent Change } \\
\hline & & & & & & & & 1992-1996 & $1987-1996$ \\
\hline 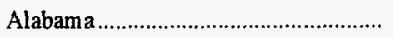 & 3.50 & 3.07 & 3.07 & 3.01 & 3.28 & 2.53 & 14.2 & 1.6 & 3.7 \\
\hline Alaska & 6.81 & 7.46 & 6.94 & 7.40 & 6.93 & 6.84 & -8.8 & -.4 & $*$ \\
\hline 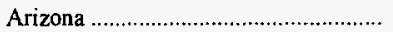 & 6.30 & 6.34 & 6.71 & 6.21 & 6.29 & 6.76 & -.6 & $*$ & -.8 \\
\hline 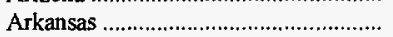 & - & 1.47 & 1.46 & 1.39 & 1.68 & 1.80 & -100.0 & - & - \\
\hline 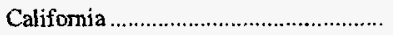 & - & - & - & - & 11.12 & 5.19 & - & - & - \\
\hline 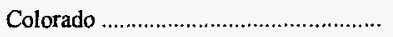 & 8.76 & 6.79 & 7.06 & 7.07 & 6.52 & 6.44 & 29.0 & 7.7 & 3.5 \\
\hline 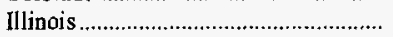 & 4.67 & 3.89 & 4.12 & 3.86 & 4.47 & 3.37 & 19.9 & 1.1 & 3.7 \\
\hline Indiana & 5.34 & 5.04 & 4.56 & 4.82 & 4.28 & 3.79 & 6.0 & 5.7 & 3.9 \\
\hline Lowa & - & - & 1.52 & .72 & 1.14 & 1.43 & - & - & - \\
\hline 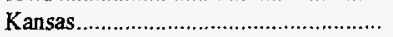 & 2.17 & 2.22 & 1.93 & 2.30 & 2.21 & 3.75 & -2.4 & -.5 & -5.9 \\
\hline 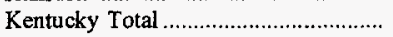 & 4.35 & 4.23 & 3.96 & 3.84 & 3.75 & 3.18 & 2.9 & 3.8 & 3.5 \\
\hline 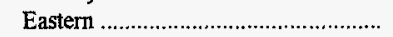 & 4.23 & 4.13 & 3.97 & 3.74 & 3.57 & 3.12 & 2.6 & 4.3 & 3.5 \\
\hline Western & 5.02 & 4.77 & 3.93 & 4.14 & 4.31 & 3.37 & 5.1 & 3.9 & 4.5 \\
\hline 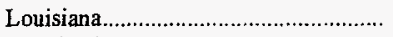 & 10.86 & 13.25 & 13.00 & 12.14 & 12.43 & 15.26 & -18.0 & -3.3 & -3.7 \\
\hline 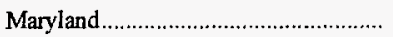 & 2.56 & 2.16 & 2.18 & 2.07 & 2.13 & 2.66 & 18.5 & 4.7 & -.4 \\
\hline 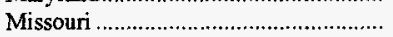 & 3.49 & 2.55 & 3.59 & 1.84 & 3.10 & 2.28 & 36.9 & 3.0 & 4.8 \\
\hline Montana & 22.34 & 21.06 & 21.92 & 19.59 & 20.16 & 18.70 & 6.1 & 2.6 & 2.0 \\
\hline 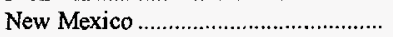 & 8.45 & 7.19 & 7.18 & 7.26 & 7.11 & 6.23 & 17.4 & 4.4 & 3.4 \\
\hline 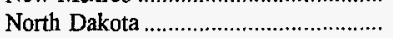 & 17.20 & 16.80 & 18.84 & 17.66 & 18.12 & 13.46 & 2.4 & -1.3 & 2.8 \\
\hline 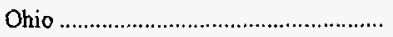 & 3.69 & 3.46 & 3.34 & 3.58 & 3.06 & 2.67 & 6.9 & 4.8 & 3.7 \\
\hline 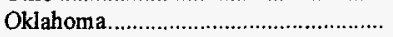 & 2.73 & 3.10 & 2.80 & 3.12 & 2.26 & 1.90 & -12.0 & 4.8 & 4.1 \\
\hline 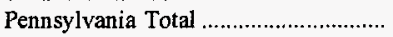 & 2.72 & 2.79 & 2.67 & 2.63 & 2.45 & 2.23 & -2.4 & 2.6 & 2.3 \\
\hline 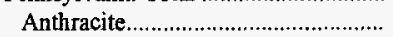 & 2.06 & 2.30 & 2.13 & 2.09 & 1.39 & 1.19 & -10.3 & 10.3 & 6.3 \\
\hline 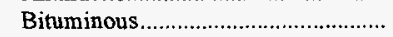 & 2.97 & 2.515 & 2.84 & 2.78 & 2.74 & 2.43 & .6 & 2.0 & 2.3 \\
\hline 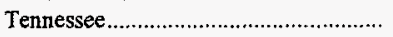 & 2.91 & 3.20 & 3.19 & 2.71 & 3.06 & 2.04 & -9.1 & -1.3 & 4.0 \\
\hline 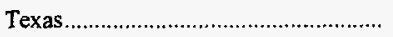 & 10.13 & 9.10 & 8.82 & 8.42 & 7.34 & 6.45 & 11.3 & 8.4 & 5.1 \\
\hline Virginia & 3.79 & 3.73 & 3.73 & 3.55 & 3.50 & 2.52 & 1.7 & 2.0 & 4.6 \\
\hline Washington & 3.97 & 4.04 & 4.11 & 4.00 & 4.51 & 3,06 & -1.8 & -3.1 & 2.9 \\
\hline West Virginia Total.................................. & 5.18 & 4.74 & 4.62 & 4.35 & 4.27 & 3.29 & 9.4 & 5.0 & 5.2 \\
\hline Northern & 4.72 & 4.31 & 3.78 & 3.70 & 3.52 & 3.16 & 9.5 & 7.6 & 4.5 \\
\hline Southem & 5.24 & 4.79 & 4.75 & 4.49 & 4.43 & 3.33 & 9.4 & 4.3 & 5.2 \\
\hline 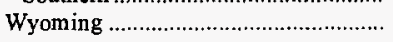 & 32.84 & 31.02 & 27.37 & 26.03 & 22.76 & 17.98 & 5.9 & 9.6 & 6.9 \\
\hline Appalachian Total ${ }^{1}$............................... & 4.05 & 3.88 & 3.72 & 3.55 & 3.40 & 2.76 & 4.3 & 4.5 & 4.3 \\
\hline 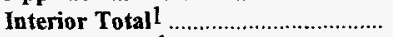 & 6.89 & 6.39 & 5.71 & 5.71 & 5.21 & 4.14 & 7.8 & 7.2 & 5.8 \\
\hline Western Total ${ }^{1}$ & 20.96 & 18.93 & 17.68 & 16.49 & 15.46 & 12.82 & 10.7 & 7.9 & 5.6 \\
\hline East of Miss. River & 4.25 & 4.03 & 3.85 & 3.74 & 3.61 & 2.97 & 5.4 & 4.2 & 4.1 \\
\hline West of Miss. River & 17.89 & 16.23 & 15.19 & 13.94 & 12.49 & 9.86 & 10.2 & 9.4 & 6.8 \\
\hline U.S. Total & 9.05 & 8.48 & 7.67 & 7.23 & 6.59 & 4.98 & 6.7 & 8.3 & 6.9 \\
\hline
\end{tabular}

1 For a definition of coal-producing regions, see Appendix C

* Data round to zero.

Notes: Productivity is calculated by dividing total coal production by the total direct labor hours worked by all mine employees engaged in production, preparation, processing, development, maintenance, repair, shop or yard work at mining operations, but excludes office workers. Excludes silt, culm, refuse bank, slurry dam, and dredge operations except for Pennsylvania anthracite. Excludes mines producing less than 10,000 short tons, which are not required to provide these data.

Sources: Energy Information Administration, Form EIA-7A, "Coal Production Report"; State Mining Agency Coal Production Reports; and/or U.S. Department of Labor, Mine Safety and Health Administration, Forn 7000-2, "Quarterly Mine Employment and Coal Production Report." 
Table 51. Coal Mining Productivity by State and Mine Type, 1996

\begin{tabular}{|c|c|c|c|c|}
\hline $\begin{array}{l}\text { Coal-Producing } \\
\text { State and Region }\end{array}$ & $\begin{array}{c}\text { Number of } \\
\text { Mining } \\
\text { Operations } 1\end{array}$ & $\begin{array}{c}\text { Average Number } \\
\text { of Miners } \\
\text { Working Daily } 2\end{array}$ & $\begin{array}{l}\text { Average Production } \\
\text { per Miner per Hour } \\
\quad \text { (short tons) }\end{array}$ & $\begin{array}{c}\text { Average Production } \\
\text { per Miner per Shift } \\
\text { (short tons) }\end{array}$ \\
\hline Alabama & 58 & 5,031 & 2.20 & 18.77 \\
\hline 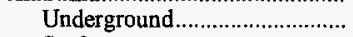 & 16 & 4,145 & 1.95 & 16.39 \\
\hline Surface & 42 & 886 & 3.50 & 30.02 \\
\hline Alaska & 1 & 102 & 6.81 & 61.29 \\
\hline Surface & 1 & 102 & 6.81 & 61.29 \\
\hline Arizona & 3 & 651 & 6.30 & 55.27 \\
\hline Surface & 3 & 651 & 6.30 & 55.27 \\
\hline Colorado & 18 & 1,332 & 7.32 & 66.07 \\
\hline Underground & 14 & 918 & 6.67 & 60.39 \\
\hline 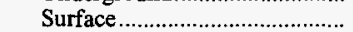 & 4 & 414 & 8.76 & 78.07 \\
\hline 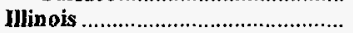 & 36 & 5,174 & 4.18 & 36.10 \\
\hline Underground & 24 & 4,256 & 4.10 & 34.91 \\
\hline 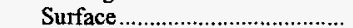 & 12 & 918 & 4.67 & 41.37 \\
\hline Indiana & 40 & 2,579 & 4.98 & 47.11 \\
\hline Underground ............................... & 5 & 457 & 3.09 & 26.24 \\
\hline 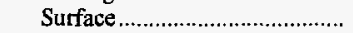 & 35 & 2,122 & 5.34 & 51.28 \\
\hline 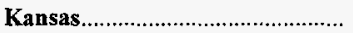 & 1 & 54 & 2.17 & 17.33 \\
\hline 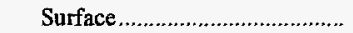 & 1 & 54 & 2.17 & 17.33 \\
\hline 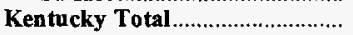 & 631 & 18,826 & $\mathbf{3 . 8 0}$ & 34.78 \\
\hline Underground & 365 & 12,876 & 3.53 & 31.04 \\
\hline Surface & 266 & 5,950 & 4.35 & 41.87 \\
\hline Eastern & 560 & 15,130 & 3.68 & 33.73 \\
\hline 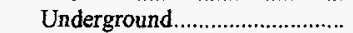 & 334 & 10,275 & 3.37 & 29.71 \\
\hline Surface & 226 & 4,855 & 4.23 & 41.08 \\
\hline Western & 71 & 3,696 & 4.29 & 38.22 \\
\hline 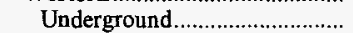 & 31 & 2,601 & 4.05 & 34.95 \\
\hline 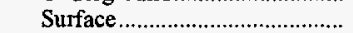 & 40 & 1,095 & 5.02 & 45.79 \\
\hline 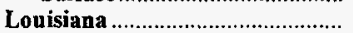 & 2 & 111 & 10.86 & 92.35 \\
\hline Surface & 2 & 111 & 10.86 & 92.35 \\
\hline 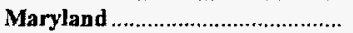 & 15 & 469 & 4.13 & 35.39 \\
\hline 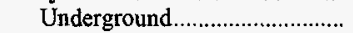 & 4 & 308 & 4.82 & 39.15 \\
\hline Surface & 11 & 161 & 2.56 & 22.36 \\
\hline 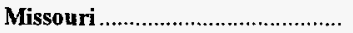 & 5 & 80 & 3.49 & 33.38 \\
\hline Surface & 5 & 80 & 3.49 & 33.38 \\
\hline 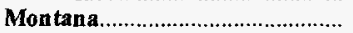 & 8 & 705 & 21.88 & 186.71 \\
\hline 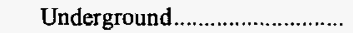 & 1 & 18 & 3.50 & 35.04 \\
\hline 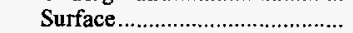 & 7 & 687 & 22.34 & 185.93 \\
\hline 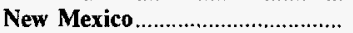 & 6 & 1,347 & 8.45 & 65.71 \\
\hline Surface & 6 & 1,347 & 8.45 & 65.71 \\
\hline North Dakota & 6 & 640 & 17.20 & 144.58 \\
\hline Surface & 6 & 640 & 17.20 & 144.58 \\
\hline 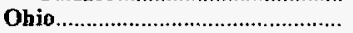 & 104 & 3,232 & 3.95 & 36.99 \\
\hline Underground & 15 & 1,706 & 4.19 & 36.70 \\
\hline 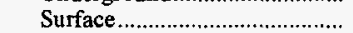 & 89 & 1,526 & 3.69 & 34.93 \\
\hline 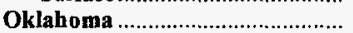 & 10 & 233 & 2.61 & 24.48 \\
\hline 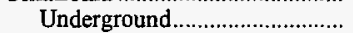 & 1 & 26 & 1.75 & 16.20 \\
\hline Surface & 9 & 207 & 2.73 & 25.63 \\
\hline 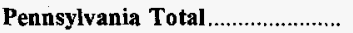 & 359 & 9,021 & 3,36 & 28.96 \\
\hline 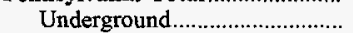 & 91 & 5,599 & 3.74 & 31.24 \\
\hline Surface & 268 & 3,422 & 2.72 & 23.68 \\
\hline 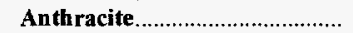 & 95 & 1,171 & 1.92 & 15.49 \\
\hline 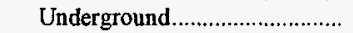 & 19 & 147 & .94 & 7.31 \\
\hline Surface & 76 & 1,024 & 2.06 & 16.79 \\
\hline 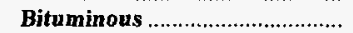 & 264 & 7,850 & 3.56 & 31.31 \\
\hline Underground & 72 & 5,452 & 3.81 & 32.36 \\
\hline Surface & 192 & 2,398 & 2.97 & 26.53 \\
\hline 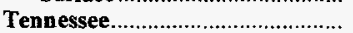 & 33 & 756 & 2.20 & 20.50 \\
\hline Underground & 18 & 467 & 1.76 & 15.51 \\
\hline Surface & 15 & 289 & 2.91 & 28.88 \\
\hline Texas & 13 & 1,550 & 10.13 & 103.71 \\
\hline Surface & 13 & 1,550 & 10.13 & 103.71 \\
\hline 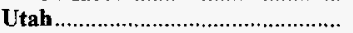 & 15 & 1,804 & 7.23 & 63.84 \\
\hline Underground .................................. & 14 & 1,803 & 7.24 & 63.80 \\
\hline Surface ...................................... & 1 & 1 & .00 & .00 \\
\hline Virginia & 225 & 6,241 & 2.72 & 24.35 \\
\hline 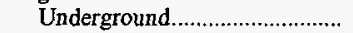 & 163 & 5,098 & 2.44 & 21.57 \\
\hline Surface & 62 & 1,143 & 3.79 & 35.42 \\
\hline Washington & 3 & 589 & 3.97 & 33.75 \\
\hline Surface & 3 & 589 & 3.97 & 33.75 \\
\hline West Virginia Total ....................... & 479 & 20,121 & 3.91 & 34.22 \\
\hline 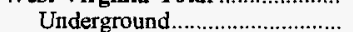 & 323 & 16,003 & 3.50 & 29.87 \\
\hline Surface & 156 & 4,118 & 5.18 & 47.77 \\
\hline
\end{tabular}

See footnotes at end of table 
Table 51. Coal Mining Productivity by State and Mine Type, 1996 (Continued)

\begin{tabular}{|c|c|c|c|c|}
\hline $\begin{array}{l}\text { Coal-Producing } \\
\text { State and Region }\end{array}$ & $\begin{array}{l}\text { Number of } \\
\text { Mining } \\
\text { Operations } 1\end{array}$ & $\begin{array}{c}\text { Average Number } \\
\text { of Miners } \\
\text { Working Daily } 2\end{array}$ & $\begin{array}{l}\text { Average Production } \\
\text { per Miner per Hour } \\
\text { (short tons) }^{3}\end{array}$ & $\begin{array}{l}\text { Average Production } \\
\text { per Miner per Shift } \\
\text { (short tons) } 4\end{array}$ \\
\hline Northern & 102 & 5,279 & 4.05 & 34.70 \\
\hline 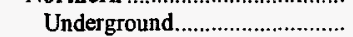 & 59 & 4,764 & 3.98 & 33.14 \\
\hline 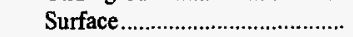 & 43 & 515 & 4.72 & 41.82 \\
\hline Southern & 377 & 14,842 & 3.86 & 33.98 \\
\hline Underground & 264 & 11,239 & 3.29 & 28.21 \\
\hline 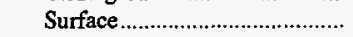 & 113 & 3,603 & 5.24 & 49.01 \\
\hline Wyoming & 27 & 2,814 & 32.06 & 332.43 \\
\hline 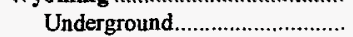 & 1 & 116 & 9.18 & 91.80 \\
\hline 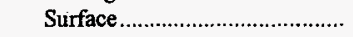 & 26 & 2,698 & 32.84 & 341.03 \\
\hline Appalachian Total ${ }^{S} \ldots \ldots \ldots \ldots \ldots \ldots$ & 1,833 & 60,001 & 3.48 & 31.04 \\
\hline 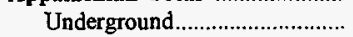 & 964 & 43,601 & 3.24 & 28.12 \\
\hline Surface & 869 & 16,400 & 4.05 & 37.25 \\
\hline Interior Totals & 178 & 13,477 & 5.39 & 49.08 \\
\hline 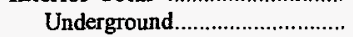 & 61 & 7,340 & 4.01 & 34.44 \\
\hline Surface & 117 & 6,137 & 6.89 & 64.65 \\
\hline 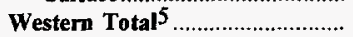 & 87 & 9,984 & 17.41 & 160.26 \\
\hline Underground & 30 & 2,855 & 7.09 & 63.89 \\
\hline Surface & 57 & 7,129 & 20.96 & 195.20 \\
\hline 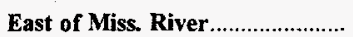 & 1,980 & 71,450 & 3.63 & 32.41 \\
\hline 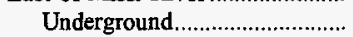 & 1,024 & 50,915 & 3.36 & 29.14 \\
\hline Surface & 956 & 20,535 & 4.25 & 39.14 \\
\hline West of Miss. River....................... & 118 & 12,012 & 15.66 & 146.13 \\
\hline 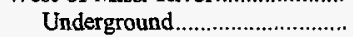 & 31 & 2,881 & 7.03 & 63.37 \\
\hline Surface & 87 & 9,131 & 17.89 & 168.90 \\
\hline 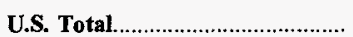 & 2,098 & 83,462 & 5.69 & 50.90 \\
\hline 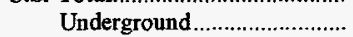 & 1,055 & 53,796 & 3.57 & 30.99 \\
\hline 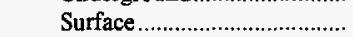 & 1,043 & 29,666 & 9.05 & 83.46 \\
\hline
\end{tabular}

1 Mining operations that consist of a mine and preparation plant, or a preparation plant only processing both underground and surface coal will be counted as two operations.

2 Includes all employees engaged in production, preparation, processing, development, maintenance, repair, shop or yard work at mining operations. Excludes office workers. Includes mining operations management and all technical and engineering employees.

3 Calculated by dividing total coal production by the total direct labor hours worked by all employees engaged in production, preparation, processing, development, maintenance, repair, shop or yard work at mining operations, but excludes office workers.

4 Calculated by multiplying average production per miner per hour by the average length of a miner shift.

5 For a definition of coal-producing regions, see Appendix $C$.

Notes: Excludes silt, culm, refuse bank, slurry dam, and dredge operations except for Pennsylvania anthracite. Excludes mines producing less than 10,000 short tons of coal during the year, and preparation plants with less than 5,000 employee hours, which are not required to provide these data.

Sources: Energy Information Administration, Form EIA-7A, "Coal Production Report"; State Mining Agency Coal Production Reports; and/or U.S. Department of Labor, Mine Safety and Health Administration, Form 7000-2, "Quarterly Mine Employment and Coal Production Report." 
Table 52. Weighted Average Number of Days Worked by State and Mine Type, 1987, 1992-1996

\begin{tabular}{|c|c|c|c|c|c|c|c|c|c|}
\hline \multirow{2}{*}{$\begin{array}{l}\text { Coal-Producing } \\
\text { State and Region }\end{array}$} & \multirow{2}{*}{1996} & \multirow{2}{*}{1995} & \multirow{2}{*}{1994} & \multirow{2}{*}{1993} & \multirow{2}{*}{1992} & \multirow{2}{*}{1987} & \multirow{2}{*}{$\begin{array}{c}\text { Percent } \\
\text { Change } \\
\text { 1995-1996 }\end{array}$} & \multicolumn{2}{|c|}{ Average Annual Percent Change } \\
\hline & & & & & & & & $1992-1996$ & $1987-1996$ \\
\hline Alabama & 267 & 260 & 240 & 250 & 245 & 250 & 2.8 & 2.3 & 0.7 \\
\hline Underground ......................................... & 268 & 259 & 246 & 249 & 236 & 237 & 3.4 & 3.2 & 1.4 \\
\hline Surface ……………………... & 265 & 262 & 231 & 253 & 258 & 267 & 1.1 & .6 & -.1 \\
\hline Alaska . & 239 & 251 & 246 & 250 & 224 & 250 & -4.8 & 1.6 & -.5 \\
\hline Surface & 239 & 251 & 246 & 250 & 224 & 250 & -4.8 & 1.6 & -.5 \\
\hline Arizona & 281 & 211 & 217 & 211 & 225 & 196 & 32.9 & 5.7 & 4.1 \\
\hline Surface & 281 & 211 & 217 & 211 & 225 & 196 & 32.9 & 5.7 & 4.1 \\
\hline 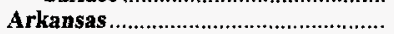 & - & 240 & 176 & 240 & 228 & 206 & -100.0 & - & - \\
\hline 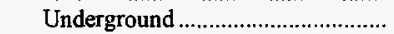 & - & - & 85 & - & - & - & - & - & - \\
\hline 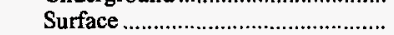 & - & 240 & 240 & 240 & 228 & 206 & -100.0 & - & - \\
\hline California ………………………… & - & - & - & - & 116 & 30 & - & - & - \\
\hline 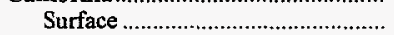 & - & - & - & - & 116 & 30 & - & - & _ \\
\hline Colorado & 316 & 287 & 258 & 243 & 278 & 266 & 10.2 & 3.3 & 1.9 \\
\hline Underground …………...................... & 325 & 287 & 288 & 282 & 268 & 249 & 13.2 & 5.0 & 3.0 \\
\hline 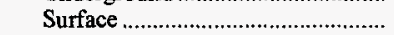 & 301 & 286 & 204 & 188 & 290 & 277 & 5.1 & .9 & .9 \\
\hline Illinois........... & 276 & 260 & 253 & 220 & 253 & 246 & 6.1 & 2.2 & 1.3 \\
\hline Underground ………………………...... & 277 & 264 & 257 & 222 & 258 & 240 & 4.7 & 1.7 & 1.6 \\
\hline Surface ………………………… & 270 & 233 & 231 & 212 & 233 & 256 & 15.7 & 3.8 & .6 \\
\hline Indiana & 276 & 266 & 274 & 254 & 250 & 242 & 3.9 & 2.4 & 1.5 \\
\hline 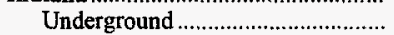 & 279 & 277 & 285 & 246 & 263 & 241 & .6 & 1.4 & 1.6 \\
\hline 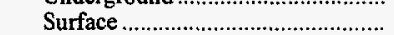 & 276 & 264 & 272 & 255 & 249 & 242 & 4.5 & 2.5 & 1.4 \\
\hline Iowa …………………………... & - & - & 150 & 260 & 259 & 250 & - & - & - \\
\hline Underground & - & _- & - & - & - & 142 & - & - & _- \\
\hline Surface & - & - & 150 & 260 & 259 & 267 & - & - & - \\
\hline 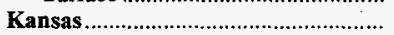 & 246 & 265 & 269 & 259 & 242 & 256 & -7.2 & .4 & -.4 \\
\hline Sufface …… & 246 & 265 & 269 & 259 & 242 & 256 & -7.2 & .4 & -4 \\
\hline Kentucky Total .......................... & 261 & 254 & 261 & 255 & 259 & 239 & 2.5 & .1 & .9 \\
\hline Underground ....................................... & 257 & 252 & 257 & 247 & 254 & 236 & 2.1 & 3 & 9 \\
\hline 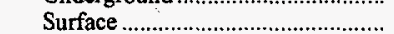 & 266 & 258 & 268 & 267 & 267 & 243 & 3.3 & * & 1.0 \\
\hline Eastern ...................................... & 258 & 250 & 257 & 253 & 250 & 231 & 3.3 & .8 & 1.2 \\
\hline 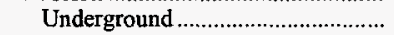 & 249 & 246 & 249 & 244 & 246 & 223 & .9 & .2 & 1.2 \\
\hline 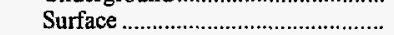 & 271 & 254 & 268 & 266 & 255 & 240 & 6.6 & 1.5 & 1.3 \\
\hline 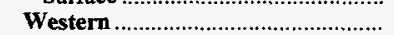 & 270 & 269 & 276 & 263 & 286 & 262 & .2 & -1.4 & .3 \\
\hline 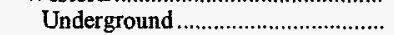 & 279 & 267 & 283 & 256 & 278 & 270 & 4.8 & .1 & .4 \\
\hline 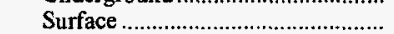 & 247 & 276 & 266 & 270 & 296 & 251 & $-10,4$ & -4.4 & -.2 \\
\hline 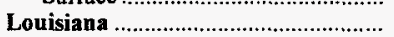 & 305 & 299 & 290 & 293 & 335 & 286 & 2.1 & -2.3 & .7 \\
\hline 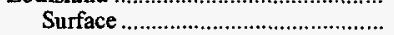 & 305 & 299 & 290 & 293 & 335 & 286 & 2.1 & -2.3 & .7 \\
\hline Maryland …… & 261 & 237 & 237 & 249 & 311 & 239 & 10.1 & -4.3 & 1.0 \\
\hline 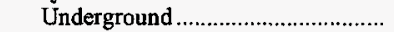 & 271 & 241 & 236 & 249 & 339 & 240 & 12.5 & -5.4 & 1.3 \\
\hline 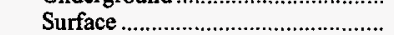 & 219 & 223 & 243 & 250 & 251 & 236 & -1.5 & -3.3 & -8 \\
\hline 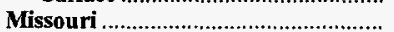 & 290 & 278 & 263 & 181 & 345 & 248 & 4.1 & -4.3 & 1.8 \\
\hline Surface ……………………………… & 290 & 278 & 263 & 181 & 345 & 248 & 4.1 & -4.3 & 1.8 \\
\hline 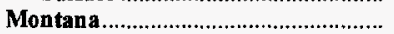 & 322 & 341 & 341 & 341 & 344 & 301 & -5.4 & -1.6 & .8 \\
\hline 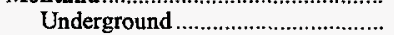 & 223 & - & - & 158 & - & - & - & - & - \\
\hline 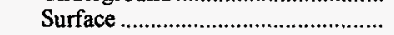 & 323 & 341 & 341 & 341 & 344 & 301 & -5.2 & -1.6 & .8 \\
\hline New Mexico ………………………… & 275 & 282 & 283 & 297 & 284 & 242 & -2.7 & -.8 & 1.4 \\
\hline 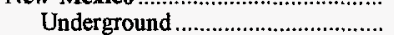 & - & 241 & 241 & 241 & 241 & 217 & -100.0 & - & - \\
\hline 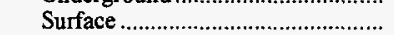 & 275 & 283 & 284 & 299 & 284 & 243 & -3.0 & -8 & 1.4 \\
\hline 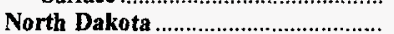 & 314 & 289 & 303 & 307 & 287 & 248 & 8.5 & 2.2 & 2.6 \\
\hline 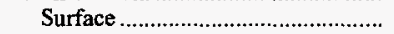 & 314 & 289 & 303 & 307 & 287 & 248 & 8.5 & 2.2 & 2.6 \\
\hline Ohio & 264 & 242 & 257 & 266 & 268 & 240 & 9.0 & -.4 & 1.0 \\
\hline Underground …………………….......... & 271 & 239 & 265 & 266 & 268 & 238 & 13.4 & .3 & 1.4 \\
\hline Surface ……………………… & 254 & 245 & 251 & 266 & 267 & 242 & 3.8 & -1.2 & .6 \\
\hline Oklahoma & 296 & 296 & 305 & 294 & 282 & 260 & -.1 & 1.2 & 1.4 \\
\hline 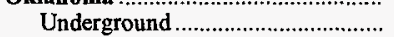 & 308 & 309 & 310 & 300 & 260 & - & -3 & 4.3 & - \\
\hline Surface ……………………..... & 295 & 296 & 305 & 293 & 283 & 260 & -.4 & 1.0 & 1.4 \\
\hline Pennsylvania Total & 268 & 262 & 257 & 246 & 249 & 245 & 2.4 & 1.9 & 1.0 \\
\hline Underground ……………………....... & 272 & 266 & 256 & 238 & 245 & 236 & 2.2 & 2.6 & 1.6 \\
\hline 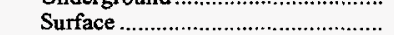 & 259 & 253 & 259 & 258 & 257 & 255 & 2.4 & .2 & .2 \\
\hline 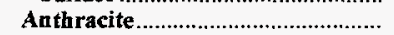 & 269 & 253 & 264 & 247 & 246 & 237 & 6.3 & 2.2 & 1.4 \\
\hline 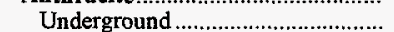 & 266 & 256 & 264 & 263 & 227 & 226 & 4.2 & 4.1 & 1.8 \\
\hline 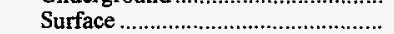 & 269 & 252 & 264 & 246 & 247 & 239 & 6.4 & 2.1 & 1.3 \\
\hline 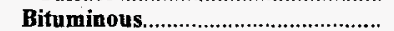 & 268 & 263 & 256 & 246 & 249 & 245 & 2.1 & 1.8 & I.o \\
\hline 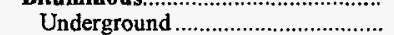 & 272 & 266 & 256 & 238 & 245 & 236 & 2.2 & 2.6 & 1.6 \\
\hline 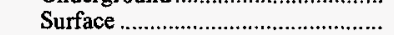 & 257 & 253 & 258 & 261 & 258 & 256 & 1.3 & -.1 & $*$ \\
\hline 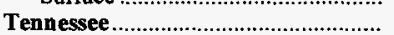 & 243 & 236 & 230 & 223 & 243 & 238 & 2.8 & * & .2 \\
\hline Underground & 254 & 245 & 233 & 254 & 241 & 241 & 3.7 & 1.4 & 6 \\
\hline 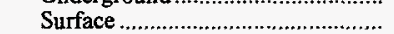 & 231 & 221 & 223 & 173 & 246 & 229 & 4.5 & -1.5 & .1 \\
\hline
\end{tabular}

See footnotes at end of table. 
Table 52. Weighted Average Number of Days Worked by State and Mine Type, 1987, 1992-1996 (Continued)

\begin{tabular}{|c|c|c|c|c|c|c|c|c|c|}
\hline \multirow{2}{*}{$\begin{array}{l}\text { Coal-Producing } \\
\text { State and Region }\end{array}$} & \multirow{2}{*}{1996} & \multirow{2}{*}{1995} & \multirow{2}{*}{1994} & \multirow{2}{*}{1993} & \multirow{2}{*}{1992} & \multirow{2}{*}{1987} & \multirow{2}{*}{$\begin{array}{c}\text { Percent } \\
\text { Change } \\
\text { 1995-1996 }\end{array}$} & \multicolumn{2}{|c|}{ Average Annual Percent Change } \\
\hline & & & & & & & & $1992-1996$ & $1987-1996$ \\
\hline Texas & 349 & 345 & 346 & 350 & 348 & 268 & 1.3 & * & 3.0 \\
\hline Surface & 349 & 345 & 346 & 350 & 348 & 268 & 1.3 & * & 3.0 \\
\hline Utah & 251 & 241 & 257 & 249 & 246 & 239 & 4.1 & 0.5 & .5 \\
\hline 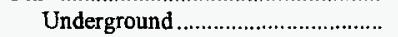 & 251 & 241 & 257 & 249 & 246 & 239 & 4.1 & .5 & .5 \\
\hline Virginia & 260 & 254 & 245 & 246 & 253 & 227 & 2.3 & .7 & 1.5 \\
\hline 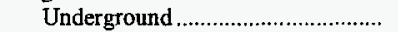 & 258 & 254 & 243 & 245 & 256 & 228 & 1.5 & .2 & 1.4 \\
\hline Surface & 266 & 255 & 251 & 247 & 241 & 220 & 4.2 & 2.5 & 2.1 \\
\hline Washington & 231 & 262 & 263 & 252 & 231 & 255 & -11.7 & * & -1.1 \\
\hline 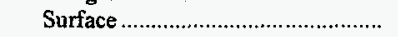 & 231 & 262 & 263 & 252 & 231 & 255 & -11.7 & * & -1.1 \\
\hline West Virginia Total............................... & 272 & 255 & 252 & 227 & 246 & 235 & 6.9 & 2.6 & 1.6 \\
\hline 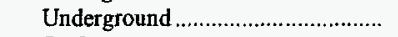 & 257 & 244 & 244 & 217 & 243 & 235 & 5.3 & 1.4 & 1.0 \\
\hline 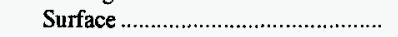 & 306 & 278 & 270 & 248 & 255 & 234 & 10.0 & 4.7 & 3.0 \\
\hline 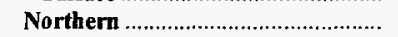 & 263 & 242 & 246 & 194 & 235 & 243 & 8.7 & 2.9 & .9 \\
\hline 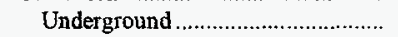 & 261 & 237 & 242 & 175 & 235 & 246 & 9.9 & 2.6 & .6 \\
\hline 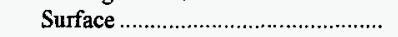 & 284 & 283 & 278 & 271 & 236 & 226 & .6 & 4.7 & 2.6 \\
\hline 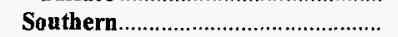 & 276 & 260 & 255 & 238 & 251 & 230 & 6.1 & 2.3 & 2.0 \\
\hline 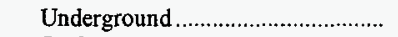 & 255 & 248 & 246 & 235 & 248 & 228 & 2.8 & .7 & 1.2 \\
\hline Surface & 308 & 277 & 268 & 243 & 258 & 236 & 11.1 & 4.6 & 3.0 \\
\hline Wyoming & 351 & 352 & 341 & 345 & 339 & 316 & -.2 & .9 & 1.2 \\
\hline 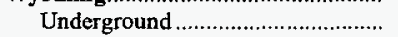 & 248 & 245 & 243 & 258 & 238 & 208 & 1.2 & 1.0 & 2.0 \\
\hline 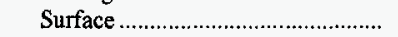 & 352 & 353 & 342 & 346 & 341 & 316 & -.1 & .8 & 1.2 \\
\hline 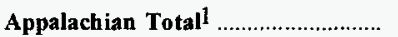 & 266 & 254 & 253 & 243 & 250 & 236 & 4.8 & 1.5 & 1.3 \\
\hline 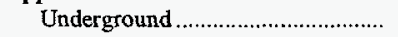 & 259 & 249 & 248 & 235 & 247 & 232 & 3.9 & 1.2 & 1.2 \\
\hline 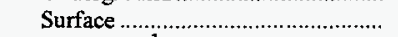 & 279 & 261 & 262 & 257 & 256 & 243 & 6.7 & 2.2 & 1.5 \\
\hline Interior Total ${ }^{1}$ & 299 & 291 & 290 & 280 & 289 & 255 & 2.8 & .8 & 1.8 \\
\hline Underground & 278 & 266 & 267 & 236 & 265 & 252 & 4.5 & 1.2 & 1.1 \\
\hline Surface & 312 & 308 & 304 & 302 & 304 & 257 & 1.4 & .6 & 2.2 \\
\hline Western Total 1 & 330 & 326 & 319 & 320 & 316 & 289 & 1.3 & 1.1 & 1.5 \\
\hline 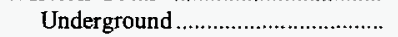 & 276 & 2.59 & 267 & 261 & 252 & 241 & 6.6 & 2.3 & 1.5 \\
\hline Surface & 337 & 334 & 325 & 327 & 323 & 293 & .8 & 1.1 & 1.5 \\
\hline 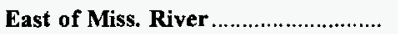 & 267 & 256 & 256 & 243 & 253 & 239 & 4.6 & 1.4 & 1.2 \\
\hline 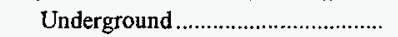 & 262 & 253 & 252 & 235 & 250 & 236 & 3.9 & 1.2 & 1.2 \\
\hline Surface & 277 & 261 & 262 & 256 & 257 & 245 & 5.8 & 1.8 & 1.4 \\
\hline West of Miss. River................................ & 332 & 328 & 321 & 323 & 320 & 285 & 1.3 & .9 & 1.7 \\
\hline Underground & 276 & 259 & 267 & 261 & 252 & 240 & 6.6 & 2.3 & 1.5 \\
\hline Surface & 338 & 335 & 327 & 329 & 326 & 288 & .8 & .9 & 1.8 \\
\hline U.S. Total & 298 & 290 & 285 & 280 & 280 & 256 & 2.7 & 1.5 & 1.7 \\
\hline 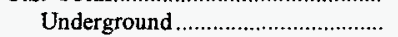 & 264 & $2: 53$ & 253 & 238 & 251 & 236 & 4.2 & 1.3 & 1.2 \\
\hline Surface & 319 & 313 & 305 & 304 & 301 & 270 & 2.0 & 1.4 & 1.9 \\
\hline
\end{tabular}

1 For a definition of coal-producing regions, see Appendix $C$.

* Data round to zero.

Notes: Weighted average number of days worked is cilculated by multiplying average days worked for each mine times its production and then summing these products over all mines in the region/State and then dividing the sum by the total production for the region/State. Excludes silt, culm, refuse bank, slurry dam, and dredge operations except for Pennsylvania anthracite. Excludes mines producing less than 10,000 short tons, which are not required to provide these data.

Sources: Energy Information Administration, Form EIA-7A, "Coal Production Report"; State Mining Agency Coal Production Reports; and/or U.S. Department of Labor, Mine Safety and Health Administration, Form 7000-2, "Quarterly Mine Employment and Coal Production Report." 
Table 53. Weighted Average Number of Days Worked by Mine Production Range, 1996

\begin{tabular}{|c|c|c|c|c|c|c|c|}
\hline \multirow{2}{*}{$\begin{array}{l}\text { Coal-Producing } \\
\text { State and Region }\end{array}$} & \multicolumn{6}{|c|}{$\begin{array}{l}\text { Mine Production Range } \\
\text { (thousand short tons) }\end{array}$} & \multirow{2}{*}{ Total 1} \\
\hline & $\begin{array}{l}1,000 \\
\text { and over }\end{array}$ & $\begin{array}{c}500 \\
\text { to } 1,000\end{array}$ & $\begin{array}{l}200 \\
\text { to } 500\end{array}$ & $\begin{array}{l}100 \\
\text { to } 200\end{array}$ & $\begin{array}{l}50 \\
\text { to } 100\end{array}$ & $\begin{array}{l}10 \\
\text { to } 50\end{array}$ & \\
\hline 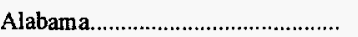 & 268 & 278 & 280 & 261 & 237 & 176 & 267 \\
\hline 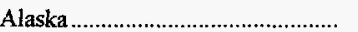 & 239 & - & - & - & - & - & 239 \\
\hline Arizona & 281 & - & - & - & - & - & 281 \\
\hline Colorado & 326 & 225 & 271 & 218 & - & - & 316 \\
\hline 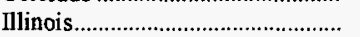 & 278 & 263 & 207 & 277 & 211 & 196 & 276 \\
\hline 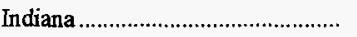 & 292 & 256 & 253 & 175 & 64 & 119 & 276 \\
\hline 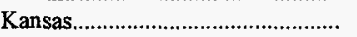 & - & - & 246 & - & - & - & 246 \\
\hline Kentucky Total & 286 & 278 & 246 & 203 & 166 & 125 & 261 \\
\hline Eastern & 290 & 276 & 249 & 204 & 172 & 127 & 258 \\
\hline 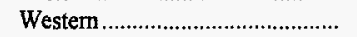 & 280 & 283 & 217 & 201 & 78 & 102 & 270 \\
\hline 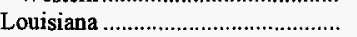 & 323 & 250 & - & - & - & - & 305 \\
\hline 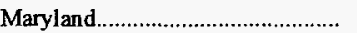 & 273 & - & 213 & 291 & 208 & 258 & 261 \\
\hline 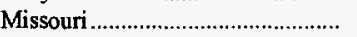 & - & - & 300 & 304 & 229 & 204 & 290 \\
\hline 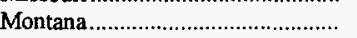 & 325 & 230 & 245 & 223 & - & - & 322 \\
\hline 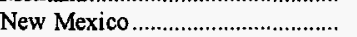 & 275 & - & - & - & - & - & 275 \\
\hline 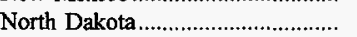 & 314 & - & - & - & - & - & 314 \\
\hline 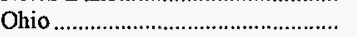 & 271 & 283 & 269 & 225 & 231 & 172 & 264 \\
\hline Oklahoma & - & - & 306 & 288 & - & 214 & 296 \\
\hline 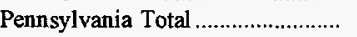 & 275 & 265 & 270 & 261 & 243 & 221 & 268 \\
\hline 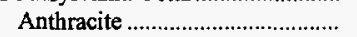 & - & 261 & 296 & 278 & 259 & 220 & 269 \\
\hline 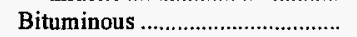 & 275 & 265 & 264 & 259 & 239 & 221 & 268 \\
\hline Tennessee & - & 247 & 254 & 192 & 325 & 211 & 243 \\
\hline 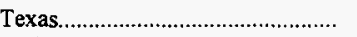 & 353 & 55 & 301 & - & - & - & 349 \\
\hline Utah & 253 & 212 & - & - & - & - & 251 \\
\hline 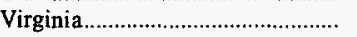 & 246 & 306 & 273 & 234 & 219 & 173 & 260 \\
\hline 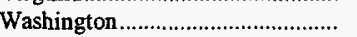 & 235 & - & - & 141 & - & - & 231 \\
\hline West Virginia Total ............................ & 289 & 276 & 238 & 209 & 192 & 141 & 272 \\
\hline Northem & 268 & 264 & 251 & 248 & 237 & 167 & 263 \\
\hline Southern & 301 & 278 & 236 & 198 & 178 & 131 & 276 \\
\hline 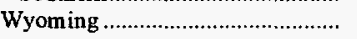 & 352 & 280 & 189 & 88 & - & 239 & 351 \\
\hline Appalachian Total $2 \ldots \ldots \ldots \ldots \ldots \ldots$ & 282 & 278 & 253 & 223 & 204 & 170 & 266 \\
\hline Interior Total 2 & 310 & 264 & 245 & 241 & 110 & 139 & 299 \\
\hline Western Total ${ }^{2} \ldots \ldots \ldots \ldots \ldots \ldots \ldots \ldots \ldots$ & 332 & 239 & 255 & 181 & - & 239 & 330 \\
\hline East of Miss. River............................ & 282 & 277 & 251 & 222 & 201 & 169 & 267 \\
\hline West of Miss. River.......................... & 334 & 224 & 278 & 237 & 229 & 216 & 332 \\
\hline 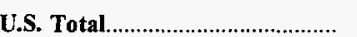 & 314 & 274 & 252 & 223 & 201 & 169 & 298 \\
\hline
\end{tabular}

1 Includes stand alone preparation plants.

2 For a definition of coal-producing regions, see Appendix C

Notes: Weighted average number of days worked is calculated by multiplying average days worked for each mine times its production and then summing these products over all mines in the region/State and then dividing the sum by the total production for the region/State. Excludes silt, culm refuse bank, slurry dam, and dredge operations except for Pennsylvania anthracite. Excludes mines producing less than 10,000 short tons and preparation plants with less than 5,000 employee hours, which are not required to provide these data.

Sources: Energy Information Administration, Form EIA-7A, "Coal Production Report"; State Mining Agency Coal Production Reports; and/or U.S. Department of Labor, Mine Safety and Health Administration, Form 7000-2, "Quarterly Mine Employment and Coal Production Report." 
Table 54. Underground Coal Mining Productivity by State and Mining Method, 1996 (Short Tons of Coal Produced per Miner per Hour)

\begin{tabular}{|c|c|c|c|c|c|}
\hline $\begin{array}{l}\text { Coal-Producing } \\
\text { State and Region }\end{array}$ & Continuous 1 & Conventional $^{2}$ & Longwall ${ }^{3}$ & Other 4 & Total \\
\hline Alabana & 1.83 & 1.24 & 1.96 & - & 1.95 \\
\hline Colorado & 3.93 & 4.30 & 8.13 & - & 6.67 \\
\hline 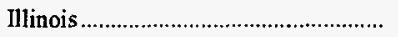 & 3.77 & 2.89 & 4.62 & - & 4.10 \\
\hline Indiana & 3.09 & - & - & - & 3.09 \\
\hline 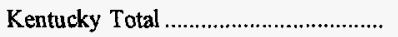 & 3.58 & 2.60 & 4.44 & 1.66 & 3.53 \\
\hline Eastern & 3.44 & 2.55 & 4.98 & 1.66 & 3.37 \\
\hline Western & 4.02 & 3.42 & 4.18 & - & 4.05 \\
\hline Maryland. & 4.35 & - & 4.88 & - & 4.82 \\
\hline 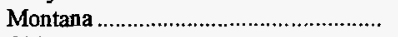 & 3.50 & - & - & - & 3.50 \\
\hline 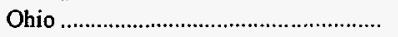 & 4.01 & - & 4.21 & - & 4.19 \\
\hline 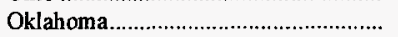 & 1.75 & - & - & - & 1.75 \\
\hline Pennsylvania Total .................................... & 2.50 & 2.39 & 4.74 & .87 & 3.74 \\
\hline 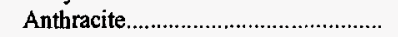 & 1.03 & .85 & - & .87 & .94 \\
\hline 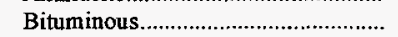 & 2.54 & 2.59 & 4.74 & - & 3.81 \\
\hline 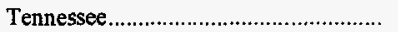 & 1.76 & 2.50 & - & - & 1.76 \\
\hline 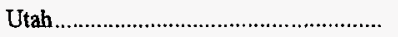 & 4.58 & 2.82 & 7.91 & - & 7.24 \\
\hline 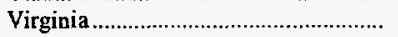 & 2.22 & 2.19 & 3.19 & - & 2.44 \\
\hline West Virginia Total................................ & 3.28 & 3.02 & 3.99 & 3.36 & 3.50 \\
\hline Northem & 3.41 & 2.70 & 4.23 & 3.36 & 3.98 \\
\hline 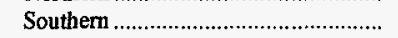 & 3.25 & 3.06 & 3.58 & - & 3.29 \\
\hline 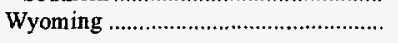 & - & - & 9.18 & - & 9.18 \\
\hline Appalachian Total ${ }^{5} \ldots \ldots \ldots \ldots \ldots \ldots \ldots \ldots$ & 3.08 & 2.66 & 3.64 & 1.80 & 3.24 \\
\hline Interior Total 5 & 3.80 & 3.27 & 4.48 & - & 4.01 \\
\hline Western Total ${ }^{5}$ & 4.14 & 3.00 & 8.05 & - & 7.09 \\
\hline East of Miss. River ................................ & 3.23 & 2.68 & 3.75 & 1.80 & 3.36 \\
\hline West of Miss. River............................... & 4.00 & 3.00 & 8.06 & - & 7.03 \\
\hline U.S. Total & 3.26 & 2.70 & 4.18 & 2.30 & 3.57 \\
\hline
\end{tabular}

1 Mines that produce greater than 50 percent of coal by continuous mining method.

2 Mines that produce greater than 50 percent of coal by conventional mining method.

3 Mines that have any production from longwall mining method. A typical longwall mining operation uses 80 percent longwall mining and 20 percent continuous mining.

4 Mines that produce coal using shortwall, scoop loading hand loading, or other mining methods or a $50 / 50$ percent continuous/conventional split in mining method.

5 For a definition of coal-producing regions, see Appendix C

Notes: For each State, stand alone preparation plant hours are distributed across the mining methods by the proportion of production for all stand alone mines. Productivity is calculated by dividing total coal production by the total direct labor hours worked by all employees engaged in production, preparation, processing, development, maintenance, repair, shop or yard work at mining operations, but excludes office workers. Excludes mines producing less than 10,000 short tons of coal during the year, and preparation plants with less than 5,000 employee hours, which are not required to provide these data.

Sources: Energy Information Administration, Form EIA-7A, "Coal Production Report"; State Mining Agency Coal Production Reports; and/or U.S. Department of Labor, Mine Safety and Health Administration, Form 7000-2, "Quarterly Mine Employment and Coal Production Report." 
Table 55. U.S. Coal Mining Productivity by Coalbed Thickness and Mining Method, 1996

(Short Tons of Coal Produced per Miner per Hour)

\begin{tabular}{|c|c|c|c|c|c|c|}
\hline \multirow{2}{*}{$\begin{array}{c}\text { Coalbed Thickness } \\
\text { (inches) }\end{array}$} & \multicolumn{4}{|c|}{ Underground } & \multirow{2}{*}{ Surface } & \multirow{2}{*}{ Total } \\
\hline & Continuous 1 & Conventional 2 & Longwall 3 & Other 4 & & \\
\hline$<7$ & - & - & - & - & 3.85 & 3.85 \\
\hline $7-12$ & - & - & - & - & 4.69 & 4.69 \\
\hline 13-18 & - & - & - & - & 5.06 & 5.06 \\
\hline 19-24 & - & 1.53 & - & 1.82 & 5.03 & 4.97 \\
\hline $25-30$ & 2.55 & 2.35 & - & - & 4.48 & 4.19 \\
\hline 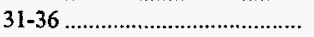 & 3.11 & 3.16 & - & 1.69 & 4.99 & 4.23 \\
\hline $37-42$ & 3.32 & 2.59 & - & - & 5.13 & 4.24 \\
\hline $43-48$ & 3.31 & 3.01 & 2.99 & - & 5.78 & 4.49 \\
\hline $49-54$ & 4.02 & 3.20 & 2.77 & - & 6.23 & 4.71 \\
\hline $55-60$ & 4.14 & 3.60 & 3.53 & - & 5.71 & 4.63 \\
\hline 61-66 & 3.43 & 2.95 & 5.73 & - & 6.00 & 5.11 \\
\hline $67-72 \ldots \ldots$ & 3.96 & 2.82 & 3.11 & 4.08 & 5.98 & 4.48 \\
\hline 73-78 & 4.23 & - & 3.89 & - & 7.07 & 4.76 \\
\hline 79-84 & 5.09 & 4.44 & 4.38 & - & 6.85 & 5.37 \\
\hline $85-90 \ldots \ldots$ & 7.77 & - & 6.23 & - & 6.22 & 6.27 \\
\hline $91-96 \ldots$ & 4.83 & 2.41 & 4.19 & 1.22 & 5.25 & 4.58 \\
\hline 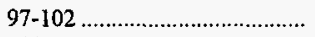 & 3.64 & - & 5.76 & - & 7.14 & 6.32 \\
\hline $103-108$ & 4.36 & - & 6.80 & - & 12.10 & 8.19 \\
\hline $109-114$ & - & - & 7.61 & - & 4.82 & 6.10 \\
\hline $115-120$ & 6.29 & - & 6.63 & - & 5.59 & 6.10 \\
\hline$>\quad 120 \ldots$ & 4.46 & 2.15 & 9.77 & - & 19.16 & 17.89 \\
\hline U.S. Total $5 \ldots$ & 3.26 & 2.70 & 4.18 & 2.30 & 9.05 & 5.69 \\
\hline
\end{tabular}

1 Mines that produce greater than 50 percent of coal by continuous mining method.

2 Mines that produce greater than 50 percent of coal by conventional mining method.

3 Mines that have any production from longwall mining method. A typical longwall mining operation uses 80 percent longwall mining and 20 percent continuous mining.

4 Mines that produce coal using shortwall, scoop loading, hand loading, or other mining methods or a 50/50 percent continuous/conventional split in mining method.

5 Includes stand alone preparation plants.

Notes: Productivity is calculated by dividing total coal production by the total direct labor hours worked by all employees engaged in production, preparation, processing, development, maintenance, repair, shop or yard work at mining operations, but excludes office workers. Excludes silt, culm, refuse bank, slurry dam, and dredge operations except for Pennsylvania anthracite. Excludes mines producing less than 10,000 short tons and preparation plants with less than 5,000 employee hours, which are not required to provide these data.

Sources: Energy Information Administration, Form EIA-7A, "Coal Production Report"; State Mining Agency Coal Production Reports; and/or U.S. Department of Labor, Mine Safety and Health Administration, Form 7000-2, "Quarterly Mine Employment and Coal Production Report." 
Table 56. Coal Mining Productivity by State, Mine Type, and Mine Production Range, 1996

(Short Tons of Coal Produced per Miner per Hour)

\begin{tabular}{|c|c|c|c|c|c|c|c|}
\hline \multirow{2}{*}{$\begin{array}{l}\text { Coal-Producing } \\
\text { State and Region }\end{array}$} & \multicolumn{6}{|c|}{$\begin{array}{l}\text { Mine Production Range } \\
\text { (thousand short tons) }\end{array}$} & \multirow{2}{*}{ Total 1} \\
\hline & $\begin{array}{c}1,000 \\
\text { and over }\end{array}$ & $\begin{array}{c}500 \\
\text { to } 1,000\end{array}$ & $\begin{array}{c}200 \\
\text { to } 500\end{array}$ & $\begin{array}{l}100 \\
\text { to } 200\end{array}$ & $\begin{array}{c}50 \\
\text { to } 100\end{array}$ & $\begin{array}{c}10 \\
\text { to } 50\end{array}$ & \\
\hline Alabama & 2.20 & 2.01 & 2.72 & 3.40 & 3.41 & 2.94 & 2.20 \\
\hline Underground & 2.13 & 1.31 & - & - & 1.30 & - & 1.95 \\
\hline Surface & 3.72 & 4.35 & 2.72 & 3.40 & 3.95 & 2.94 & 3.50 \\
\hline Alaska & 6.81 & - & - & - & - & - & 6.81 \\
\hline Surface & 6.81 & - & - & - & - & - & 6.81 \\
\hline 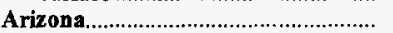 & 6.56 & - & - & - & - & - & 6.30 \\
\hline 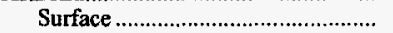 & 6.56 & - & - & - & - & - & 6.30 \\
\hline Colorado & 8.88 & 2.84 & 4.60 & 2.61 & - & - & $\mathbf{7 . 3 2}$ \\
\hline 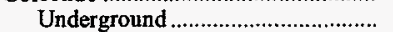 & 8.99 & 2.84 & 3.84 & 2.61 & - & - & 6.67 \\
\hline Surface & 8.73 & - & 9.61 & - & - & - & 8.76 \\
\hline Illinois & 4.43 & 3.51 & 3.98 & 2.89 & 7.18 & 1.11 & 4.18 \\
\hline Underground & 4.31 & 3.19 & 3.76 & 2.89 & - & .27 & 4.10 \\
\hline Surface & 5.67 & 3.63 & 4.18 & - & 7.18 & 2.60 & 4.67 \\
\hline Indiana & 5.32 & 4.67 & 4.82 & 3.42 & 4.85 & 5.14 & 4.98 \\
\hline Underground & 3.52 & 2.90 & - & - & - & - & 3.09 \\
\hline Surface & 5.56 & 5,53 & 4.82 & 3.42 & 4.85 & 5.14 & 5.34 \\
\hline Kansas. & - & - & 2.17 & - & - & - & 2.17 \\
\hline Surface & - & - & 2.17 & - & - & - & 2.17 \\
\hline 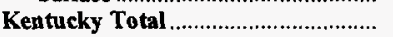 & 4.97 & 4.67 & 4.14 & 3.13 & 2.73 & 2.58 & 3.80 \\
\hline Underground & 4.75 & 4.40 & 3.87 & 2.90 & 2.39 & 2.24 & 3.53 \\
\hline Surface & 5.61 & 4.97 & 4.59 & 3.78 & 3.77 & 3.14 & 4.35 \\
\hline Eastern & 5.20 & 4.76 & 4.00 & 3.06 & 2.66 & 2.55 & 3.68 \\
\hline 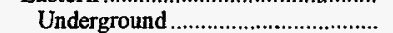 & 5.04 & 4.47 & 3.81 & 2.90 & 2.39 & 2.28 & 3.37 \\
\hline 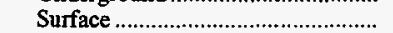 & 5.45 & 5.12 & 4.35 & 3.66 & 3.53 & 3.03 & 4.23 \\
\hline Western & 4.62 & 4.39 & 6.12 & 4.26 & 4.43 & 3.08 & 4.29 \\
\hline Underground & 4.49 & 4.17 & 5.25 & - & 2.32 & .92 & 4.05 \\
\hline Surface & 8.05 & 4.60 & 6.71 & 4.26 & 5.84 & 3.99 & 5.02 \\
\hline Louisiana & 10.72 & 11.35 & - & - & - & - & 10.86 \\
\hline Surface & 10.72 & 11.35 & - & - & - & - & 10.86 \\
\hline Maryland & 5.48 & - & 4.34 & 3.22 & 2.98 & 1.56 & 4.13 \\
\hline 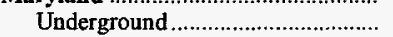 & 5.48 & - & 6.34 & - & - & 1.89 & 4.82 \\
\hline 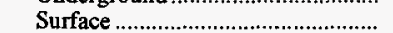 & - & - & 3.04 & 3.22 & 2.98 & 1.47 & 2.56 \\
\hline 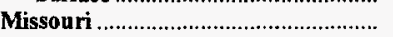 & - & - & 3.40 & 7.17 & 1.51 & 1.42 & 3.49 \\
\hline Surface & - & - & 3,40 & 7.17 & 1.51 & 1.42 & 3.49 \\
\hline Montana & 23.01 & 8.74 & 14.37 & 3.50 & - & - & 21.88 \\
\hline 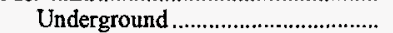 & - & - & - & 3.50 & - & - & 3.50 \\
\hline 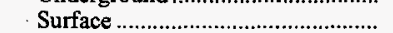 & 23.01 & 8.74 & 14.37 & - & - & - & 22.34 \\
\hline New Mexico & 8.45 & - & - & - & - & - & 8.45 \\
\hline Surface & 8.45 & - & - & - & - & - & 8.45 \\
\hline 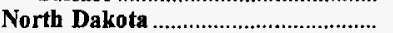 & 17.26 & - & - & - & - & - & 17.20 \\
\hline 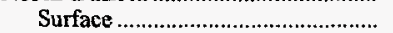 & 17.26 & - & - & - & - & - & 17.20 \\
\hline Ohio & 4.26 & 4.41 & 4.41 & 3.94 & 2.86 & 2.66 & 3.95 \\
\hline Underground & 4.30 & - & 3.65 & 3.34 & - & - & 4,19 \\
\hline Surface & 4.06 & 4.41 & 4.50 & 4.11 & 2.86 & 2.66 & 3.69 \\
\hline Oklahoma & - & - & 2.75 & 2.46 & - & 2.60 & 2.61 \\
\hline Underground & - & - & - & 1.75 & - & - & 1.75 \\
\hline Surface & - & - & 2.75 & 2.70 & - & 2.60 & 2.73 \\
\hline 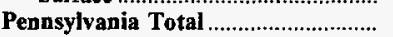 & 4.57 & 2.48 & 3.30 & 3.82 & 2.55 & 2.48 & 3.36 \\
\hline Underground & 4.57 & 2.18 & 3.37 & 2.53 & 2.41 & 1.26 & 3.74 \\
\hline Surface & 4.59 & 3.03 & 3.25 & 4.10 & 2.57 & 2.76 & 2.72 \\
\hline Anth racite & - & 9.14 & 3.58 & 3.49 & 1.74 & 1.99 & 1.92 \\
\hline 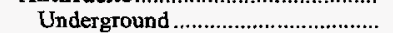 & - & - & - & 1.59 & - & 1.21 & .94 \\
\hline 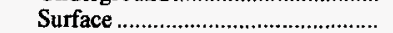 & - & 9.14 & 3.58 & 5.66 & 1.74 & 2.24 & 2.06 \\
\hline 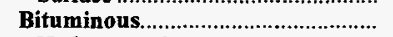 & 4.57 & 2.31 & 3.24 & 3.86 & 2.90 & 2.77 & 3.56 \\
\hline 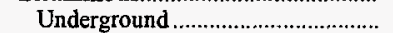 & 4.57 & 2.18 & 3.37 & 3.00 & 2.41 & 1.32 & 3.81 \\
\hline Surface & 4.59 & 2.56 & 3.12 & 4.00 & 3.02 & 3.02 & 2.97 \\
\hline 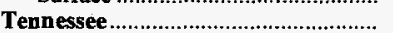 & - & 4.15 & 2.64 & 2.48 & 1.58 & 1.53 & 2.20 \\
\hline Underground & - & - & 2.25 & 2.25 & 1.58 & 1.27 & 1.76 \\
\hline Surface & - & 4.15 & 3.32 & 3.41 & - & 1.83 & 2.91 \\
\hline Texas & 10.42 & 11.11 & 3.23 & - & - & - & 10.13 \\
\hline Surface & 10.42 & 11.11 & 3.23 & - & - & - & 10.13 \\
\hline Utah & 7.76 & 4.03 & - & - & - & - & 7.23 \\
\hline Underground & 7.76 & 4.03 & - & - & - & - & 7.24 \\
\hline 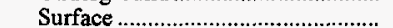 & - & - & - & - & - & - & .00 \\
\hline Virginia & 3.55 & 4.46 & 3.09 & 2.68 & 2.18 & 2.04 & 2.72 \\
\hline Underground & 3.55 & 3.71 & 2.76 & 2.59 & 2.07 & 1.90 & 2.44 \\
\hline Surface & - & 4.99 & 4.06 & 3.04 & 3.36 & 3.44 & 3.79 \\
\hline
\end{tabular}

See footnotes at end of table. 
Table 56. Coal Mining Productivity by State, Mine Type, and Mine Production Range, 1996

(Continued)

(Short Tons of Coal Produced per Miner per Hour)

\begin{tabular}{|c|c|c|c|c|c|c|c|}
\hline \multirow{2}{*}{$\begin{array}{l}\text { Coal-Producing } \\
\text { State and Region }\end{array}$} & \multicolumn{6}{|c|}{$\begin{array}{l}\text { Mine Production Range } \\
\text { (thousand short tons) }\end{array}$} & \multirow{2}{*}{ Total 1} \\
\hline & $\begin{array}{c}1,000 \\
\text { and over }\end{array}$ & $\begin{array}{c}500 \\
\text { to } 1,000\end{array}$ & $\begin{array}{l}200 \\
\text { to } 500\end{array}$ & $\begin{array}{l}100 \\
\text { to } 200\end{array}$ & $\begin{array}{c}50 \\
\text { to } 100\end{array}$ & $\begin{array}{l}10 \\
\text { to } 50\end{array}$ & \\
\hline 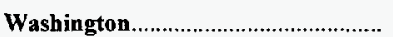 & 4.21 & - & - & 1.63 & - & - & 3.97 \\
\hline Surface & 4.21 & - & . - & 1.63 & - & - & 3.97 \\
\hline 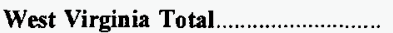 & 4.82 & 5.21 & 4.17 & 3.19 & 2.55 & 2.34 & 3.91 \\
\hline 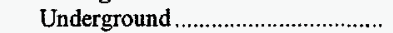 & 4.17 & 4.86 & 4.04 & 3.19 & 2.64 & 2.48 & 3.50 \\
\hline 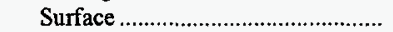 & 6.88 & 6.12 & 4.57 & 3.16 & 2.22 & 2.10 & 5.18 \\
\hline Northern & 4.47 & $\mathbf{5 . 5 1}$ & 3.48 & 3.69 & 2.88 & 2.69 & 4.05 \\
\hline 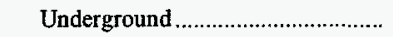 & 4.28 & 5.12 & 3.60 & 4.06 & 2.29 & 2.39 & 3.98 \\
\hline 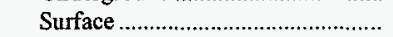 & 9.70 & 7.28 & 3.10 & 3.16 & 4.15 & 3.11 & 4.72 \\
\hline 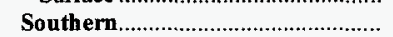 & 5.04 & 5.17 & 4.31 & 3.07 & 2.47 & 2.23 & 3.86 \\
\hline 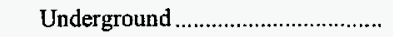 & 4.08 & 4.83 & 4.13 & 3.04 & 2.71 & 2.50 & 3.29 \\
\hline Surface & 6.71 & 6.03 & 4.85 & 3.16 & 1.43 & 1.72 & 5.24 \\
\hline Wyoming & 33.26 & $\mathbf{5 . 3 4}$ & 10.52 & 9.52 & - & .50 & 32.06 \\
\hline Underground & 9.18 & - & - & - & - & - & 9.18 \\
\hline Surface & 34.13 & 5.34 & 10.52 & 9.52 & - & .50 & 32.84 \\
\hline Appalachian Total ${ }^{2} \ldots \ldots \ldots \ldots \ldots \ldots \ldots \ldots \ldots$ & 4.32 & 4.33 & 3.73 & 3.18 & 2.56 & 2.39 & 3.48 \\
\hline 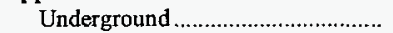 & 3.96 & 3.90 & 3.58 & 2.86 & 2.35 & 2.10 & 3.24 \\
\hline 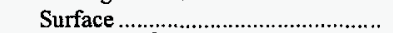 & 6.06 & 4.98 & 3.99 & 3.71 & 2.91 & 2.65 & 4.05 \\
\hline Interior Total 2 & 6.01 & 4.53 & 4.37 & 3.44 & 3.71 & 2.27 & 5.39 \\
\hline Underground & 4.34 & 3.72 & 4.68 & 2.17 & 2.32 & .47 & 4.01 \\
\hline Surface & 8.37 & 5.06 & 4.32 & 3.71 & 4.04 & 3.28 & 6.89 \\
\hline Western Total 2 & 18.63 & 4.24 & 5.59 & 2.82 & - & .50 & 17.41 \\
\hline 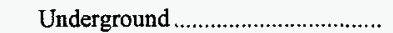 & 8.19 & 3.43 & 3.84 & 2.77 & - & - & 7.09 \\
\hline 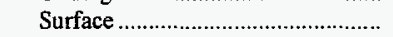 & 21.56 & 5.98 & 10.94 & 2.92 & - & .50 & 20.96 \\
\hline 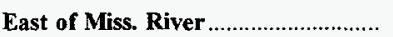 & 4.41 & 4.34 & 3.82 & 3.20 & 2.60 & 2.38 & 3.63 \\
\hline 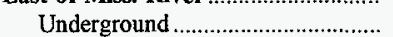 & 4.05 & 3.87 & 3.60 & 2.86 & 2.35 & 2.05 & 3.36 \\
\hline 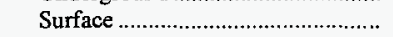 & 5.94 & 4.93 & 4.15 & 3.73 & 3.00 & 2.69 & 4.25 \\
\hline West of Miss. River ................................ & 17.08 & 4.90 & 3.72 & 2.93 & 1.51 & 1.26 & 15.66 \\
\hline Underground & 8.19 & 3.43 & 3.84 & 2.51 & - & - & 7.03 \\
\hline 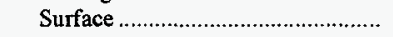 & 19,00 & 7.28 & 3.68 & 3.26 & 1.51 & 1.26 & 17.89 \\
\hline 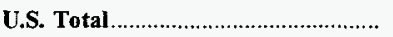 & 8.13 & 4.36 & 3.82 & 3.18 & 2.59 & 2.37 & 5.69 \\
\hline 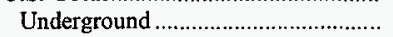 & 4.40 & 3.85 & 3.61 & 2.85 & 2.35 & 2.05 & 3.57 \\
\hline Surface & 14.34 & 5.04 & 4.11 & 3.69 & 2.97 & 2.65 & 9.05 \\
\hline
\end{tabular}

1 Includes stand alone preparation plants.

2 For a definition of coal-producing regions, see Appendix C.

Notes: Productivity is calculated by dividing total coal production by the total direct labor hours worked by all employees engaged in production, prepa-

ration, processing, development, maintenance, repair, shop or yard work at mining operations, but excludes office workers. Excludes silt, culm, refuse

bank, slurry dam, and dredge operations except for Pennsylvania anthracite. Excludes mines producing less than 10,000 short tons and preparation plants with less than 5,000 employee hours, which are not required to provide these data.

Sources: Energy Information Administration, Form EIA-7A, "Coal Production Report"; State Mining Agency Coal Production Reports; and/or U.S. Department of Labor, Mine Safety and Health Administration, Form 7000-2, "Quarterly Mine Employment and Coal Production Report." 
Table 57. Coal Mining Productivity by State, Mine Type, and Union Type,1996 (Short Tons of Coal Produced per Miner per Hour)

\begin{tabular}{|c|c|c|c|c|c|}
\hline $\begin{array}{l}\text { Coal-Producing } \\
\text { State and Region }\end{array}$ & UMWA & Other Unions & Union Total & Nonunion & Total \\
\hline 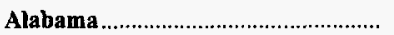 & 2.03 & - & 2.03 & 2.71 & 2.20 \\
\hline 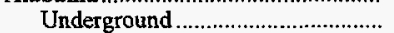 & 1.89 & - & 1.89 & 2.21 & 1.95 \\
\hline Surface & 3.55 & - & 3.55 & 3.48 & 3.50 \\
\hline 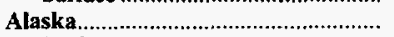 & - & 6.81 & 6.81 & - & 6.81 \\
\hline 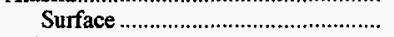 & - & 6.81 & 6.81 & - & 6.81 \\
\hline 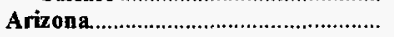 & 6.30 & - & 6.30 & - & 6.30 \\
\hline 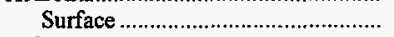 & 6.30 & - & 6.30 & - & 6.30 \\
\hline 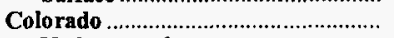 & 4.78 & 7.92 & 5.78 & 7.79 & $\mathbf{7 . 3 2}$ \\
\hline Underground & 2.77 & - & 2.77 & 7.39 & 6.67 \\
\hline Surface & 8.90 & 7.92 & 8.32 & 9.05 & 8.76 \\
\hline 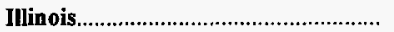 & 4.05 & 4.33 & 4.08 & 4.56 & 4.18 \\
\hline 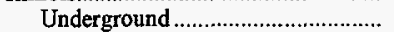 & 3.91 & 4.28 & 3.95 & 4.57 & 4.10 \\
\hline Surface & 4.71 & 4.55 & 4.70 & 4.36 & 4.67 \\
\hline Indiana & 5.18 & - & 5.18 & 4.79 & 4.98 \\
\hline 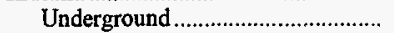 & 2.80 & - & 2.80 & 3.23 & 3.09 \\
\hline 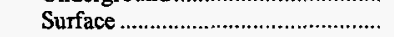 & 5.48 & - & 5.48 & 5.20 & 5.34 \\
\hline 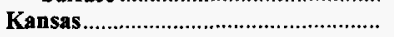 & 2.17 & - & 2.17 & - & 2.17 \\
\hline Surface & 2.17 & - & 2.17 & - & 2.17 \\
\hline 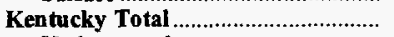 & 4.25 & 3.74 & 4.23 & 3.74 & 3.80 \\
\hline 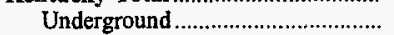 & 4.00 & 2.67 & 3.97 & 3.45 & 3.53 \\
\hline Surface & 5.44 & 4.58 & 5.33 & 4.28 & 4.35 \\
\hline Eastern & 4.66 & 3.74 & 4.59 & 3.60 & 3.68 \\
\hline 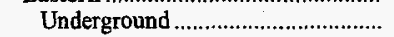 & 4.28 & 2.67 & 4.20 & 3.30 & 3.37 \\
\hline Surface & 5.79 & 4.58 & 5.58 & 4.15 & 4.23 \\
\hline Western & 3.89 & - & 3.89 & 4.47 & 4.29 \\
\hline 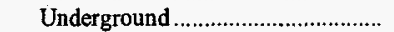 & 3.80 & - & 3.80 & 4.20 & 4.05 \\
\hline Surface & 4.68 & - & 4.68 & 5.07 & 5.02 \\
\hline 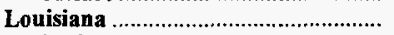 & - & - & - & 10.86 & 10.86 \\
\hline 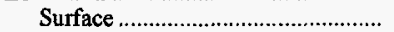 & - & - & - & 10.86 & 10.86 \\
\hline 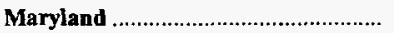 & - & - & - & 4.13 & 4.13 \\
\hline 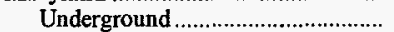 & - & - & - & 4.82 & 4.82 \\
\hline Surface & - & - & - & 2.56 & 2.56 \\
\hline Missouri & - & - & - & 3.49 & 3.49 \\
\hline Surface & - & - & - & 3.49 & 3.49 \\
\hline Montana & 19.79 & 20.38 & 20.04 & 30.70 & 21.88 \\
\hline 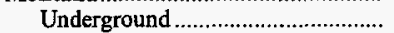 & - & - & - & 3.50 & 3.50 \\
\hline Surface & 19.79 & 20.38 & 20.04 & 35.16 & 22.34 \\
\hline New Mexico & 8.91 & 7.68 & 8.05 & 11.00 & 8.45 \\
\hline Surface & 8.91 & 7.68 & 8.05 & 11.00 & 8.45 \\
\hline 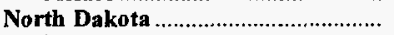 & 13.42 & 17.14 & 15.55 & 17.81 & 17.20 \\
\hline Surface & 13.42 & 17.14 & 15.55 & 17.81 & 17.20 \\
\hline Ohio & 4.14 & - & 4.14 & 3.75 & 3.95 \\
\hline Underground & 4.25 & - & 4.25 & 3.73 & 4.19 \\
\hline Surface & 3.32 & - & 3.32 & 3.76 & 3.69 \\
\hline Oklahoma & - & - & - & 2.61 & 2.61 \\
\hline Underground & - & - & - & 1.75 & 1.75 \\
\hline Surface & - & - & - & 2.73 & 2.73 \\
\hline 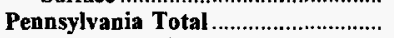 & 2.76 & 1.38 & 2.76 & 3.90 & 3.36 \\
\hline 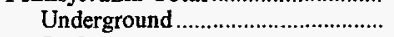 & 2.98 & - & 2.97 & 5.11 & 3.74 \\
\hline 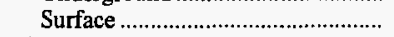 & 1.45 & 2.06 & 1.47 & 3.00 & 2.72 \\
\hline Anth racite & .98 & 2.07 & 1.01 & 2.62 & 1.92 \\
\hline 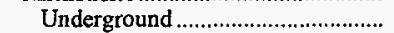 & - & - & - & .95 & .94 \\
\hline Surface & .98 & 2.57 & 1.02 & 3.09 & 2.06 \\
\hline Bituminous & 2.98 & - & 2.97 & 4.08 & 3.56 \\
\hline 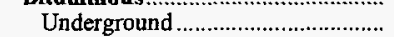 & 2.98 & - & 2.97 & 5.40 & 3.81 \\
\hline 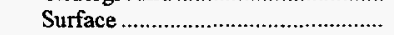 & 2.98 & - & 2.92 & 2.98 & 2.97 \\
\hline 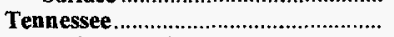 & - & - & - & 2.20 & 2.20 \\
\hline Underground ......................................... & - & - & - & 1.76 & 1.76 \\
\hline Surface & - & - & - & 2.91 & 2.91 \\
\hline Texas & - & 9.71 & 9.71 & 11.03 & 10.13 \\
\hline Surface & - & 9.71 & 9.71 & 11.03 & 10.13 \\
\hline Utah & 7.05 & - & 7.05 & 7.32 & 7.23 \\
\hline 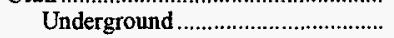 & 7.05 & - & 7.05 & 7.33 & 7.24 \\
\hline Surface & - & - & - & - & - \\
\hline Virginia & 2.90 & 4.76 & 3.00 & 2.64 & 2.72 \\
\hline Underground & 2.97 & - & 2.97 & 2.29 & 2.44 \\
\hline 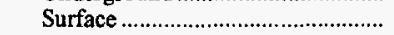 & 1.93 & 4.76 & 3.29 & 3.86 & 3.79 \\
\hline 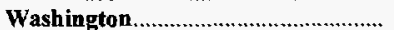 & - & 4.21 & 4.21 & 1.63 & 3.97 \\
\hline Surface & - & 4.21 & 4.21 & 1.63 & 3.97 \\
\hline 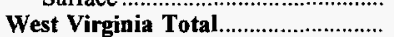 & 3.54 & - & 3.52 & 4.44 & 3.91 \\
\hline Underground & 3.13 & - & 3.13 & 4.13 & 3.50 \\
\hline 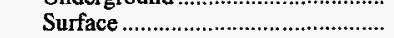 & 5.43 & - & 5.33 & 5.07 & 5.18 \\
\hline
\end{tabular}

See footnotes at end of table 
Table 57. Coal Mining Productivity by State, Mine Type, and Union Type,1996 (Continued) (Short Tons of Coal Produced per Miner per Hour)

\begin{tabular}{|c|c|c|c|c|c|}
\hline $\begin{array}{l}\text { Coal-Producing } \\
\text { State and Region }\end{array}$ & UMWA & Other Unions & Union Total & Nonunion & Total \\
\hline 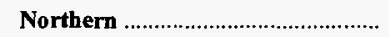 & 4.20 & - & 4.20 & 3.77 & 4.05 \\
\hline 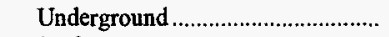 & 4.20 & - & 4.20 & 3.33 & 3.98 \\
\hline 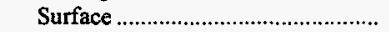 & - & - & - & 4.74 & 4.72 \\
\hline 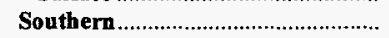 & 3.26 & - & 3.24 & 4.61 & 3.86 \\
\hline 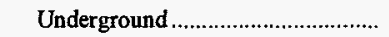 & 2.53 & - & 2.52 & 4.34 & 3.29 \\
\hline Surface & 5.44 & - & 5.34 & 5.15 & 5.24 \\
\hline 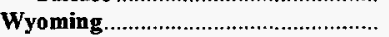 & 5.98 & 10.17 & 8.38 & 37.97 & 32.06 \\
\hline Underground & - & - & - & 9.18 & 9.18 \\
\hline Surface & 5.98 & 10.17 & 8.38 & 39.22 & 32.84 \\
\hline 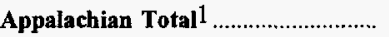 & 3.21 & 3.15 & 3.21 & 3.66 & 3.48 \\
\hline 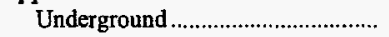 & 3.01 & 2.21 & 3.00 & 3.47 & 3.24 \\
\hline Surface & 4.40 & 3.42 & 4.35 & 3.97 & 4.05 \\
\hline Interior Total 1 & 4.25 & 8.76 & 5.38 & 5.39 & 5.39 \\
\hline 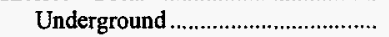 & 3.85 & 4.28 & 3.87 & 4.20 & 4.01 \\
\hline Surface & 5.11 & 9.51 & 7.19 & 6.55 & 6.89 \\
\hline 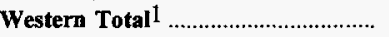 & 8.69 & 9.44 & 9.03 & 24.01 & 17.41 \\
\hline 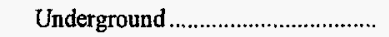 & 6.12 & - & 6.12 & 7.43 & 7.09 \\
\hline 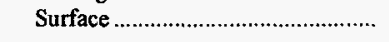 & 9.68 & 9.44 & 9.55 & 32.54 & 20.96 \\
\hline East of Miss. River ................................. & 3.43 & 3.88 & 3.44 & 3.78 & 3.63 \\
\hline 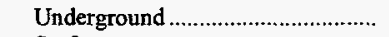 & 3.15 & 3.97 & 3.16 & 3.56 & 3.36 \\
\hline Surface & 4.67 & 3.73 & 4.63 & 4.12 & 4.25 \\
\hline West of Miss. River............................. & 8.57 & 9.55 & 9.15 & 21.40 & 15.66 \\
\hline 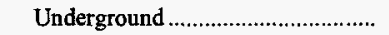 & 6.12 & - & 6.12 & 7.34 & 7.03 \\
\hline Surface & 9.50 & 9.55 & 9.54 & 27.05 & 17.89 \\
\hline 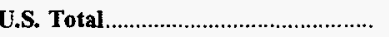 & 3.87 & 8.83 & 4.49 & 6.61 & 5.69 \\
\hline 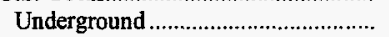 & 3.24 & 3.97 & 3.25 & 3.88 & 3.57 \\
\hline 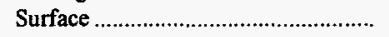 & 5.99 & 9.22 & 7.17 & 10.09 & 9.05 \\
\hline
\end{tabular}

1 For a definition of coal-producing regions, see Appendix $C$.

Notes: Productivity is calculated by dividing total coal production by the total direct labor hours worked by all employees engaged in production, preparation, processing, development, maintenance, repair, shop or yard work at mining operations, but excludes office workers. Excludes silt, culm, refuse bank, slurry dam, and dredge operations except for Pennsylvania anthracite. Excludes mines producing less than 10,000 short tons and preparation plants with less than 5,000 employee hours, which are not required to provide these data. See Glossary for listing of other unions.

Sources: Energy Information Administration, Form EIA-7A, "Coal Production Report"; State Mining Agency Coal Production Reports; and/or U.S. Department of Labor, Mine Safety and Health Administration, Form 7000-2, "Quarterly Mine Employment and Coal Production Report." 


\section{Distribution}

The amount of U.S. coal distributed during 1996 reached a record $1,059.9$ million short tons, 2.9 percent more than the $1,030.3$ million short tons distributed during 1995. Compared with 1995, distribution of U.S. coal to domestic consumers rose 2.9 percent to 968 million short tons, while foreign distribution rose 2.5 to 92.2 million short tons.

Texas was the leading destination for U.S. coal distributed domestically during 1996 with shipments totaling 95.4 million short tons, representing 9.9 percent of the total domestic distributions. Fifty-two percent of the U.S. coal distributed in Texas during 1996 was indigenous, with virtually all of the remainder arriving from Wyoming and Colorado.

Other major destination States included Indiana, Ohio, and Pennsylvania, which collectively received 183.6 million short tons, or 19 percent of total 1996 domestic distributions. As with distributions in Texas, a substantial portion of the coal distributed in these three States was indigenous.
West Virginia was the leading source of U.S. coal distributed abroad during 1996, with foreign shipments totaling 42 million short tons, representing 45.6 percent of total foreign distributions. Other leading sources of U.S. coal distributed abroad during 1996 were Virginia, Pennsylvania, and Kentucky. Collectively, foreign distributions of coal mined in these three States totaled 31.8 million short tons, accounting for 34.5 percent of the U.S. coal shipped abroad during 1996.

Rail continued to be the primary method of transporting the U.S. coal distributed domestically during 1996, accounting for 611.7 million short tons or 63 percent of domestic coal shipments. Coal transported by water (including shipment by river, shipments on the Great Lakes, and shipments through tidewater ports) accounted for 247.9 million short tons or 23.4 percent of the U.S. coal distributed. Distributions of coal by truck totaled 99.9 million short tons while distributions by tramway and conveyor totaled 98.9 million short tons. 
Figure 8. Coal Distribution, 1987-1996

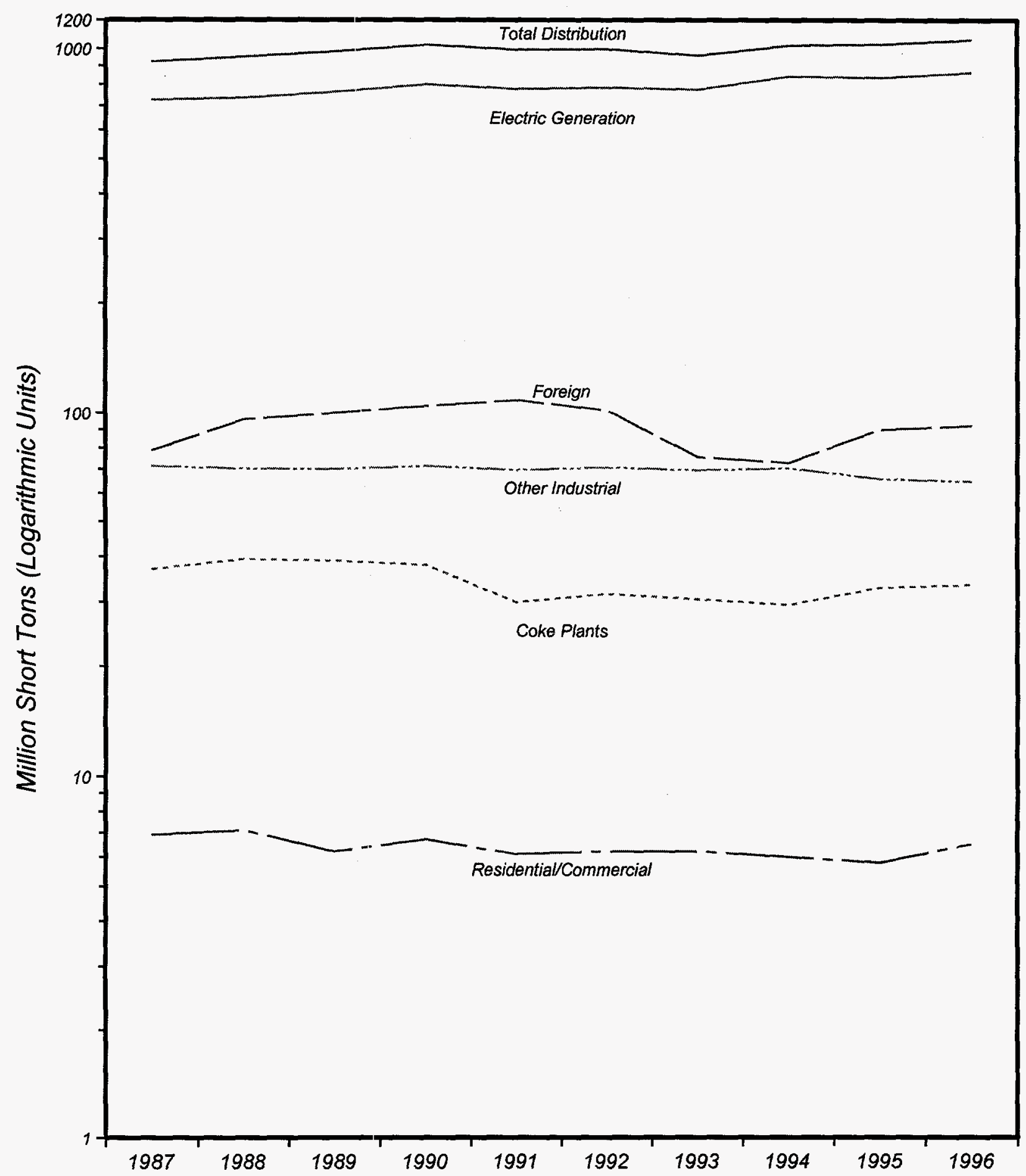

Source: Energy Information Administration, Form ElA-6, "Cool Distribution Report." 
Table 58. Distribution of U.S. Coal by State of Origin, 1992-1996 (Thousand Short Tons)

\begin{tabular}{|c|c|c|c|c|c|c|c|}
\hline \multirow{2}{*}{$\begin{array}{l}\text { Coal-Producing } \\
\text { State and Region }\end{array}$} & \multirow{2}{*}{1996} & \multirow{2}{*}{1995} & \multirow{2}{*}{1994} & \multirow{2}{*}{1993} & \multirow{2}{*}{1992} & \multirow{2}{*}{$\begin{array}{c}\text { Percent } \\
\text { Change } \\
\text { 1995-1996 }\end{array}$} & \multirow{2}{*}{$\begin{array}{c}\begin{array}{c}\text { Average Annual } \\
\text { Percent Change }\end{array} \\
1992-1996\end{array}$} \\
\hline & & & & & & & \\
\hline Alabama & 24,636 & 25,159 & 23,750 & 25,556 & 25,491 & -2.1 & -0.8 \\
\hline 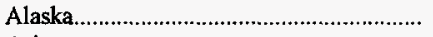 & 1,473 & 1,670 & 1,505 & 1,598 & 1,531 & -11.8 & -1.0 \\
\hline Arizona & 10,970 & 11,783 & 12,011 & 12,138 & 12,418 & -6.9 & -3.0 \\
\hline 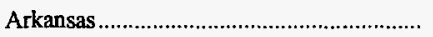 & 7 & 11 & 28 & 25 & 32 & -39.2 & -32.0 \\
\hline California & - & - & - & - & 142 & - & - \\
\hline Colorado & 25,405 & 25,635 & 24,810 & 21,465 & 18,864 & -.9 & 7.7 \\
\hline 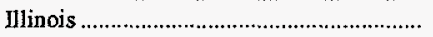 & 47,076 & 47,869 & 51,973 & 42,000 & 58,913 & -1.7 & -5.4 \\
\hline 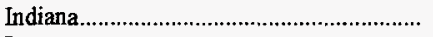 & 29,674 & 25,695 & 30,684 & 29,664 & 31,393 & 15.5 & -1.4 \\
\hline 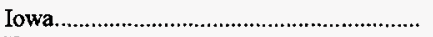 & - & - & 46 & 175 & 287 & - & - \\
\hline 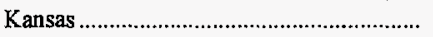 & 245 & 291 & 282 & 345 & 354 & -15.9 & -8.8 \\
\hline Kentucky Total & 152,891 & 151,466 & 159,130 & 160,395 & 161,860 & 9 & -1.4 \\
\hline Eastern & 117,404 & 117,831 & 124,257 & 125,041 & 120,186 & -.4 & -.6 \\
\hline 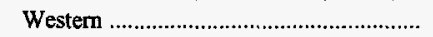 & 35,487 & 33,635 & 34,873 & 35,354 & 41,674 & 5.5 & -3.9 \\
\hline 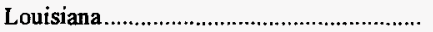 & 3,222 & 3,426 & 3,463 & 3,103 & 3,208 & -6.0 & .1 \\
\hline Maryland & 4,199 & 3,570 & 3,460 & 3,572 & 3,480 & 17.6 & 4.8 \\
\hline 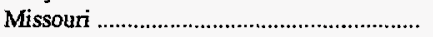 & 846 & 464 & 679 & 638 & 2,795 & 82.3 & -25.8 \\
\hline Montana & 38,288 & 39,620 & 41,916 & 35,916 & 38,866 & -3.4 & -.4 \\
\hline 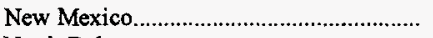 & 25,043 & 26,154 & 28,570 & 27,942 & 24,827 & -4.3 & .2 \\
\hline North Dakota & 30,025 & 30,118 & 32,056 & 32,372 & 31,702 & -.3 & -1.3 \\
\hline 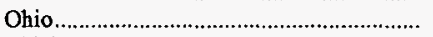 & 28,881 & 24,345 & 28,749 & 28,315 & 29,550 & 18.6 & -.6 \\
\hline Oklahoma & 2,216 & 2,158 & 1,925 & 2,309 & 1,954 & 2.7 & 3.2 \\
\hline 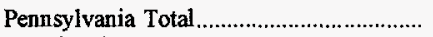 & 69,128 & 62,240 & 61,508 & 58,990 & 67,649 & 11.1 & .5 \\
\hline Anthracite & 4,836 & 3,994 & 4,700 & 3,331 & 3,554 & $21 . \mathrm{I}$ & 8.0 \\
\hline 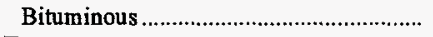 & 64,291 & 58,246 & 56,808 & 55,659 & 64,095 & 10.4 & .1 \\
\hline 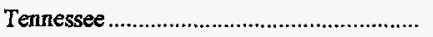 & 3,052 & 2,627 & 2,547 & 2,577 & 2,837 & 16.2 & 1.8 \\
\hline 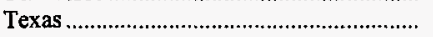 & 49,655 & 52,832 & 52,256 & 54,224 & 54,447 & -6.0 & -2.3 \\
\hline Utah & 23,868 & 25,521 & 23,225 & 22,243 & 21,052 & -6.5 & 3.2 \\
\hline 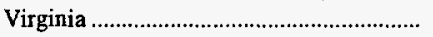 & 36,208 & 34,024 & 38,548 & 41,639 & 45,728 & 6.4 & -5.7 \\
\hline 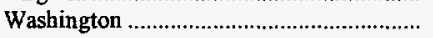 & 4,569 & 4,863 & 4,877 & 4,714 & 5,283 & -6.1 & -3.6 \\
\hline 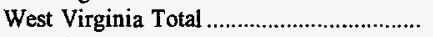 & 169,200 & 165,187 & 158,985 & 135,818 & 163,723 & 2.4 & .8 \\
\hline 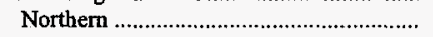 & 46,436 & 42,615 & 45,535 & 37,100 & 50,646 & 9.0 & -2.1 \\
\hline 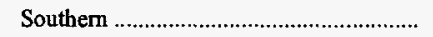 & 122,764 & 122,572 & 113,449 & 98,718 & 113,077 & .2 & 2.1 \\
\hline 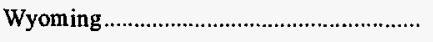 & 279,117 & 263,601 & 235,540 & 211,713 & 190,260 & 5.9 & 10.0 \\
\hline Appalachian Total ${ }^{1}$ & 452,707 & 434,984 & 441,805 & 421,508 & 458,645 & 4.1 & -.3 \\
\hline Interior Total l & 168,427 & 166,380 & 176,208 & 167,836 & 195,057 & 1.2 & -3.6 \\
\hline Western Total ${ }^{1}$ & 438,758 & 428,966 & 404,510 & 370,102 & 344,945 & 2.3 & 6.2 \\
\hline 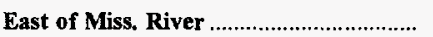 & 564,944 & 542,182 & 559,334 & 528,525 & 590,624 & 4.2 & -1.1 \\
\hline 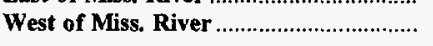 & 494,948 & 488,148 & 463,190 & 430,920 & 408,023 & 1.4 & 4.9 \\
\hline U.S. Total & $1,059,892$ & $1,030,330$ & $1,022,523$ & 959,445 & 998,647 & 2.9 & 1.5 \\
\hline
\end{tabular}

1 For a definition of coal-producing regions, see Appendix C. rounding.

Notes: See Technical Note 1 for the difference between production and distribution. Totals may not equal sum of components due to independent Source: Energy Information Administration, Form EIA-6, "Coal Distribution Report." 
Table 59. Domestic and Foreign Distribution of U.S. Coal by State of Origin, 1992-1996 (Thousand Short Tons)

\begin{tabular}{|c|c|c|c|c|c|c|c|}
\hline \multirow{2}{*}{$\begin{array}{l}\text { Coal-Producing } \\
\text { State and Region }\end{array}$} & \multirow[t]{2}{*}{1996} & \multirow[t]{2}{*}{1995} & \multirow{2}{*}{1994} & \multirow{2}{*}{1993} & \multirow{2}{*}{1992} & \multirow{2}{*}{$\begin{array}{c}\text { Percent } \\
\text { Change } \\
\text { 1995-1996 }\end{array}$} & \multirow{2}{*}{$\begin{array}{c}\begin{array}{c}\text { Average Annual } \\
\text { Percent Change }\end{array} \\
1992-1996\end{array}$} \\
\hline & & & & & & & \\
\hline & \multicolumn{7}{|c|}{ Domestic } \\
\hline 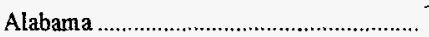 & 19,772 & 19,127 & 19,220 & 19,668 & 19,560 & 3.4 & 0.3 \\
\hline 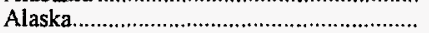 & 697 & 815 & 789 & 855 & 797 & -14.5 & -3.3 \\
\hline Arizona & 10,970 & 11,783 & 12,011 & 12,138 & 12,418 & -6.9 & -3.0 \\
\hline 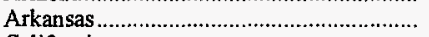 & 7 & 11 & 28 & 25 & 29 & -39.2 & -30.4 \\
\hline 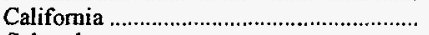 & - & - & - & - & 142 & - & - \\
\hline 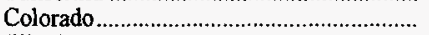 & 23,990 & 24,734 & 24,059 & 20,338 & 18,195 & -3.0 & 7.2 \\
\hline 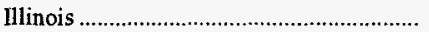 & 45,190 & 45,170 & 51,737 & 41,330 & 57,670 & * & -5.9 \\
\hline Indiana & 29,664 & 25,625 & 30,477 & 29,475 & 31,216 & 15.8 & -1.3 \\
\hline 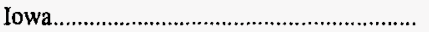 & - & - & 46 & 175 & 287 & - & - \\
\hline 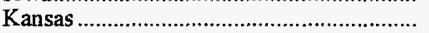 & 245 & 291 & 282 & 345 & 354 & -15.9 & -8.8 \\
\hline 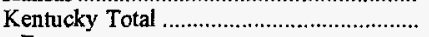 & 143,748 & 141,771 & 151,963 & 150,874 & 147,825 & 1.4 & -.7 \\
\hline Eastern & 108,927 & 108,781 & 117,234 & 115,723 & 106,372 & .1 & .6 \\
\hline Western & 34,821 & 32,990 & 34,729 & 35,151 & 41,453 & 5.5 & -4.3 \\
\hline 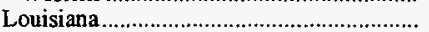 & 3,222 & 3,426 & 3,463 & 3,103 & 3,208 & -6.0 & .1 \\
\hline 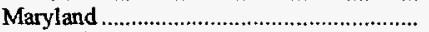 & 3,555 & 3,382 & 3,277 & 3,278 & 3,246 & 5.1 & 2.3 \\
\hline 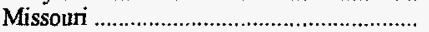 & 846 & 464 & 679 & 638 & 2,795 & 82.3 & -25.8 \\
\hline Montana & 37,770 & 39,362 & 41,672 & 35,795 & 38,804 & -4.0 & -.7 \\
\hline 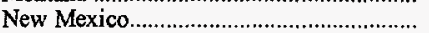 & 25,035 & 25,640 & 28,540 & 27,942 & 24,823 & -2.4 & .2 \\
\hline 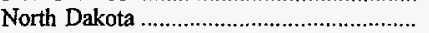 & 30,025 & 30,118 & 32,056 & 32,372 & 31,702 & -3 & -1.3 \\
\hline 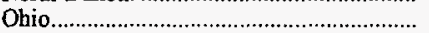 & 28,609 & 24,318 & 28,688 & 28,315 & 29,549 & 17.6 & -8 \\
\hline 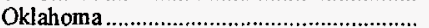 & 2,136 & 2,158 & 1,925 & 2,297 & 1,940 & -1.0 & 2.4 \\
\hline Pennsylvania Total & 59,882 & 53,961 & 55,207 & 53,482 & 61,208 & 11.0 & -.5 \\
\hline Anthracite & 4,330 & 3,497 & 4,346 & 3,015 & 3,230 & 23.8 & 7.6 \\
\hline 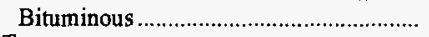 & 55,552 & 50,464 & 50,861 & 50,467 & 57,979 & 10.1 & -1.1 \\
\hline 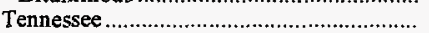 & 3,052 & 2,627 & 2,547 & 2,577 & 2,835 & 16.2 & 1.9 \\
\hline 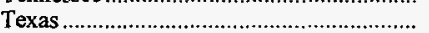 & 49,538 & 52,812 & 52,256 & 54,224 & 54,447 & -6.2 & -2.3 \\
\hline 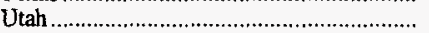 & 18,563 & 21,591 & 20,527 & 19,283 & 18,792 & -14.0 & -.3 \\
\hline Virginia & 22,776 & 24,283 & 26,866 & 27,388 & 28,504 & -6.2 & -5.4 \\
\hline 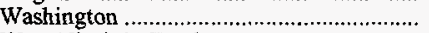 & 4,526 & 4,756 & 4,731 & 4,621 & 5,020 & -4.8 & -2.6 \\
\hline West Virginia Total ................................... & 127,156 & 120,866 & 122,779 & 102,659 & 112,917 & 5.2 & 30 \\
\hline Northern & 40,398 & 36,073 & 39,985 & 34,573 & 44,093 & 12.0 & -2.2 \\
\hline Southern & 86,757 & 84,793 & 82,794 & 68,086 & 68,824 & 2.3 & 6.0 \\
\hline 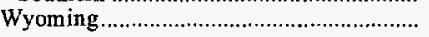 & 276,723 & 261,333 & 234,016 & 210,739 & 188,983 & 5.9 & 10.0 \\
\hline 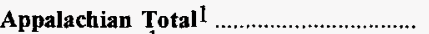 & 373,728 & 357,344 & 375,819 & 353,089 & 364,191 & 4.6 & .6 \\
\hline Interior Total 1 & 165,668 & 162,947 & 175,622 & 166,763 & 193,400 & 1.7 & -3.8 \\
\hline 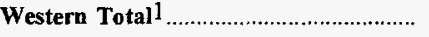 & 428,297 & 420,132 & 398,402 & 364,083 & 339,676 & 1.9 & 6.0 \\
\hline 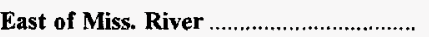 & 483,402 & 461,128 & 492,762 & 459,045 & 494,530 & 4.8 & -.6 \\
\hline West of Miss. River .................................... & 484,291 & 479,294 & 457,081 & 424,890 & 402,737 & 1.0 & 4.7 \\
\hline \multirow[t]{2}{*}{ 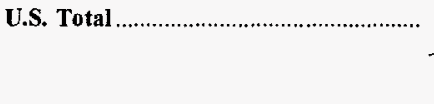 } & 967,693 & 940,423 & 949,843 & 883,934 & 897,267 & 2.9 & 1.9 \\
\hline & \multicolumn{7}{|c|}{ Foreign } \\
\hline Alabama & 4,864 & 6,032 & 4,529 & 5,888 & 5,931 & -19.4 & -4.8 \\
\hline 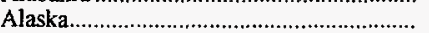 & 776 & 855 & 716 & 743 & 734 & -9.2 & 1.4 \\
\hline 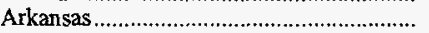 & - & - & - & - & 3 & - & - \\
\hline 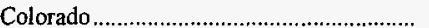 & 1,415 & 900 & 752 & 1,128 & 669 & 57.2 & 20.6 \\
\hline 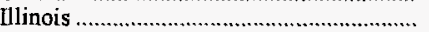 & 1,886 & 2,699 & 236 & 670 & 1,242 & -30.1 & 11.0 \\
\hline 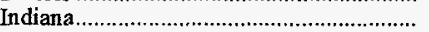 & 11 & 70 & 206 & 188 & 177 & -84.7 & -50.4 \\
\hline Kentucky Total & 9,143 & 9,695 & 7,167 & 9,521 & 14,036 & -5.7 & -10.2 \\
\hline 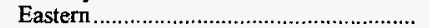 & 8,477 & 9,051 & 7,023 & 9,318 & 13,815 & -6.3 & -11.5 \\
\hline Western & 666 & 645 & 144 & 204 & 221 & 3.3 & 31.8 \\
\hline 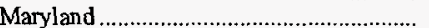 & 645 & 188 & 184 & 295 & 234 & 242.0 & 28.8 \\
\hline 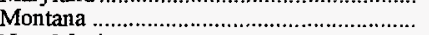 & 518 & 259 & 243 & 121 & 62 & 100.2 & 70.2 \\
\hline 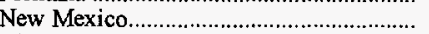 & 9 & 514 & 30 & - & 5 & -98.3 & 16.5 \\
\hline 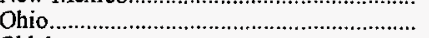 & 271 & 28 & 61 & - & 2 & NM & 257.2 \\
\hline Oklahoma & 80 & - & - & 11 & 14 & - & 54.5 \\
\hline Pennsylvania Total ....................................... & 9,246 & 8,279 & 6,301 & 5,508 & 6,440 & 11.7 & 9.5 \\
\hline Anthracite & 506 & 497 & 354 & 316 & 324 & 1.9 & 11.8 \\
\hline 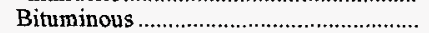 & 8,740 & 7,782 & 5,947 & 5,192 & 6,116 & 12.3 & 9.3 \\
\hline 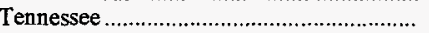 & - & - & - & - & 2 & - & - \\
\hline 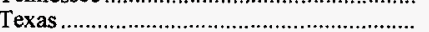 & 117 & 20 & - & - & - & 494.0 & - \\
\hline 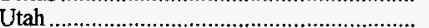 & 5,305 & 3,930 & 2,698 & 2,959 & 2,260 & 35.0 & 23.8 \\
\hline 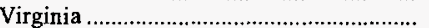 & 13,432 & 9,742 & 11,683 & 14,251 & 17,224 & 37.9 & -6.0 \\
\hline Washington & 43 & 107 & 146 & 94 & 264 & -59.8 & -36.4 \\
\hline 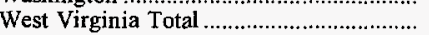 & 42,044 & 44,321 & 36,205 & 33,159 & 50,806 & -5.1 & -4.6 \\
\hline Northern & 6,038 & 6,542 & 5,550 & 2,527 & 6,553 & -7.7 & -2.0 \\
\hline Southern & 36,006 & 37,779 & 30,655 & 30,632 & 44,254 & -4.7 & -5.0 \\
\hline Wyoming & 2,395 & 2,269 & 1,524 & 974 & 1,277 & 5.5 & 17.0 \\
\hline
\end{tabular}

See footnotes at end of table 
Table 59. Domestic and Foreign Distribution of U.S. Coal by State of Origin, 1992-1996 (Continued) (Thousand Short Tons)

\begin{tabular}{|c|c|c|c|c|c|c|c|}
\hline \multirow{2}{*}{$\begin{array}{l}\text { Coal-Producing } \\
\text { State and Region }\end{array}$} & \multirow{2}{*}{1996} & \multirow[t]{2}{*}{1995} & \multirow{2}{*}{1994} & \multirow{2}{*}{1993} & \multirow{2}{*}{1992} & \multirow{2}{*}{$\begin{array}{c}\text { Percent } \\
\text { Change } \\
1995-1996\end{array}$} & \multirow{2}{*}{$\begin{array}{c}\begin{array}{c}\text { Average Annual } \\
\text { Percent Change }\end{array} \\
1992-1996\end{array}$} \\
\hline & & & & & & & \\
\hline & \multicolumn{7}{|c|}{ Foreign } \\
\hline 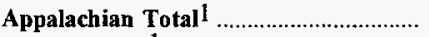 & 78,979 & 77,640 & 65,986 & 68,419 & 94,454 & 1.7 & -4.4 \\
\hline Interior Total ${ }^{1} \ldots \ldots \ldots$ & 2,759 & 3,433 & 586 & 1,073 & 1,657 & -19.6 & 13.6 \\
\hline Western Total ${ }^{1}$ & 10,460 & 8,834 & 6,108 & 6,018 & 5,269 & 18.4 & 18.7 \\
\hline 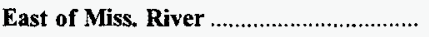 & 81,542 & 81,054 & 66,572 & 69,481 & 96,094 & .6 & -4.0 \\
\hline 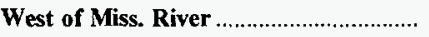 & 10,657 & 8,854 & 6,108 & 6,030 & 5,286 & 20.4 & 19.2 \\
\hline 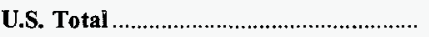 & 92,199 & 89,907 & 72,680 & 75,510 & 101,380 & 2.5 & -2.3 \\
\hline
\end{tabular}

1 For a definition of coal-producing regions, see Appendix C.

* Data round to zero.

NM Not meaningful as value is greater than 500 percent.

Note: Totals may not equal sum of components due to independent rounding.

Source: Energy Information Administration, Form EIA-6, "Coal Distribution Report."

Table 60. Major U.S. Coal Distributors, 1996

\begin{tabular}{|c|c|}
\hline \multicolumn{2}{|c|}{ Company Name } \\
\hline \multicolumn{2}{|c|}{ Top Ten Distributors } \\
\hline $\begin{array}{l}\text { Atlantic Richfield Co. } \\
\text { A.T. Massey Coal Co., Inc. } \\
\text { Consol Energy Inc. } \\
\text { Cyprus AMAX Minerals Co. } \\
\text { Kennecott Energy Co. }\end{array}$ & $\begin{array}{l}\text { Kerr-McGee Coal Corp. } \\
\text { Kiewit Coal Properties } \\
\text { North American Coal Corp. } \\
\text { Peabody Holding Co. } \\
\text { Zeigler Coal Holding Co. }\end{array}$ \\
\hline \multicolumn{2}{|c|}{ Other Major Distributors } \\
\hline $\begin{array}{l}\text { Addington Enterprises Inc. } \\
\text { AEP Service Corp. } \\
\text { Aluminum Co. of America } \\
\text { American Coal Sales, Inc. } \\
\text { American Metals \& Coal }\end{array}$ & $\begin{array}{l}\text { Knife River Coal Mining } \\
\text { Mapco Coal Co. } \\
\text { Marigold Land Corp. } \\
\text { Montana Power Co. } \\
\text { Monterey Coal Co. }\end{array}$ \\
\hline $\begin{array}{l}\text { AMVEST Minerals } \\
\text { Andalex Resources Inc. } \\
\text { Anker Energy Corp. } \\
\text { Arch Mineral Corp. } \\
\text { Ashland Coal Inc. }\end{array}$ & $\begin{array}{l}\text { Mincorp, Inc. } \\
\text { Minnesota Power \& Light } \\
\text { Orion Resources Inc. } \\
\text { Pacificorp Electric } \\
\text { Pardee Coal Co., Inc. }\end{array}$ \\
\hline $\begin{array}{l}\text { BHP Minerals Int ’ } \\
\text { Black Beauty Coal Co. } \\
\text { Blue Diamond Coal Co. } \\
\text { Central Coal Co. } \\
\text { Chevron Corp. }\end{array}$ & $\begin{array}{l}\text { Pen Holdings } \\
\text { Phibro Energy Inc. } \\
\text { Quaker Coal Co. } \\
\text { Rochester \& Pittsburgh Co. } \\
\text { San Miguel Electric CoOp. }\end{array}$ \\
\hline $\begin{array}{l}\text { Coal Arbed Int '1 Trading Co. } \\
\text { Costain America Inc. } \\
\text { Dolet Hills Mining Venture } \\
\text { Drummond Co. } \\
\text { Electric Fuels Corp. }\end{array}$ & $\begin{array}{l}\text { Smoky Mountain Coal } \\
\text { Sun Coal Co. } \\
\text { Teco Coal Corp. } \\
\text { Texas Utilities Co. } \\
\text { The Coastal Corp. }\end{array}$ \\
\hline $\begin{array}{l}\text { General Dynamics Corp. } \\
\text { Golden Oak Mining Co. } \\
\text { James River Coal Co. } \\
\text { Jim Walter Resources, Inc. } \\
\text { Kindill Mining }\end{array}$ & $\begin{array}{l}\text { The Pittston Co. } \\
\text { United Coal Co. } \\
\text { USX Corp. } \\
\text { Westmoreland Coal Co. } \\
\text { Wyodak Resources Dev 't. }\end{array}$ \\
\hline
\end{tabular}

Notes: The top 10 distribution companies accounted for 51 percent of the total distribution. Companies are listed in alphabetical order to ensure nondisclosure of company data.

Source: Energy Information Administration, Form EIA-6A, "Coal Distribution Report." 
Table 61. Domestic Distribution of U.S. Coal by Coal-Producing Region and State, and Destination Census Division and State, 1992-1996 (Thousand Short Tons)

\begin{tabular}{|c|c|c|c|c|c|c|c|}
\hline \multirow{2}{*}{$\begin{array}{c}\text { Coal-Producing Region and State, } \\
\text { and Destination Census Division } \\
\text { and State }\end{array}$} & \multirow{2}{*}{1996} & \multirow{2}{*}{1995} & \multirow{2}{*}{1994} & \multirow{2}{*}{1993} & \multirow{2}{*}{1992} & \multirow{2}{*}{$\begin{array}{c}\text { Percent } \\
\text { Change } \\
1995-1996\end{array}$} & \multirow{2}{*}{$\begin{array}{c}\begin{array}{c}\text { Average Annual } \\
\text { Percent Change }\end{array} \\
1992-1996\end{array}$} \\
\hline & & & & & & & \\
\hline Appalachian Total & 373,728 & 357,344 & 375,819 & 353,089 & 364,191 & 4.6 & 0.6 \\
\hline Alabama & 19,772 & 19,127 & 19,220 & 19,668 & 19,560 & 3.4 & .3 \\
\hline 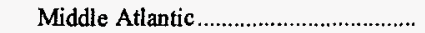 & 579 & 616 & 496 & 492 & 228 & -6.0 & 26.3 \\
\hline 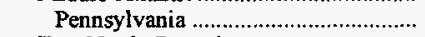 & 579 & 616 & 496 & 492 & 228 & -6.0 & 26.3 \\
\hline East North Central ................................... & 108 & - & - & - & - & - & - \\
\hline 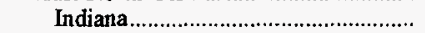 & 57 & - & - & - & - & - & - \\
\hline 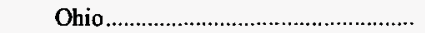 & 51 & - & - & - & - & - & - \\
\hline West North Central.................................. & $*$ & - & - & - & 1 & - & -17.9 \\
\hline Minnesota & * & - & - & - & - & - & - \\
\hline 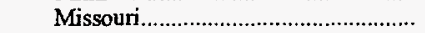 & $*$ & - & - & - & - & - & - \\
\hline 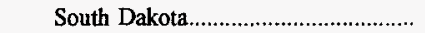 & - & - & - & - & 1 & - & - \\
\hline 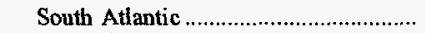 & 385 & 253 & 89 & 207 & 287 & 52.1 & 7.7 \\
\hline Florida & 8 & 115 & 85 & 202 & 185 & -92.7 & -53.8 \\
\hline 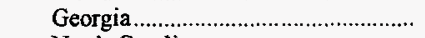 & 373 & 134 & $*$ & 1 & 102 & 178.4 & 38.3 \\
\hline 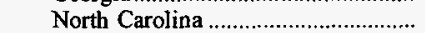 & 4 & - & - & - & - & - & - \\
\hline 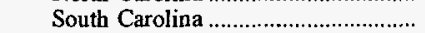 & - & 4 & 3 & 4 & - & -100.0 & - \\
\hline 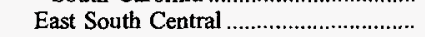 & 18,628 & 18,182 & 18,484 & 18,851 & 18,918 & 2.4 & -.4 \\
\hline Alabama & 18,503 & 18,024 & 18,351 & 18,716 & 18,849 & 2.7 & -.5 \\
\hline 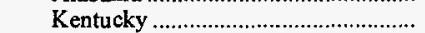 & 15 & - & - & - & - & - & - \\
\hline 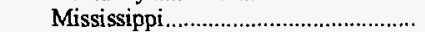 & 110 & 156 & 129 & 132 & 68 & -29.5 & 12.7 \\
\hline 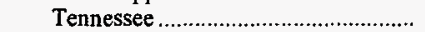 & 1 & 2 & 4 & 3 & $*$ & -65.5 & 21.3 \\
\hline West South Central ................................... & 39 & 24 & 63 & 18 & 33 & 57.8 & 3.9 \\
\hline 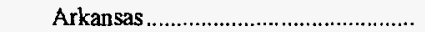 & 39 & 24 & 60 & 9 & 12 & 57.8 & 33.2 \\
\hline 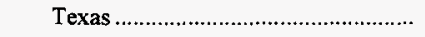 & - & - & 3 & 8 & 21 & - & - \\
\hline 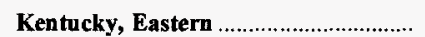 & 108,927 & 108,781 & 117,234 & 115,723 & 106,372 & .1 & .6 \\
\hline New England & 1,337 & 1,764 & 1,447 & 1,106 & 2,337 & -24.2 & -13.0 \\
\hline 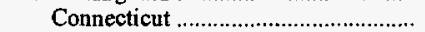 & 659 & 811 & 787 & 576 & 837 & -18.7 & -5.8 \\
\hline Maine & 271 & 258 & 433 & 380 & 771 & 5.3 & -23.0 \\
\hline 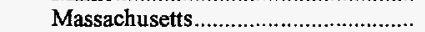 & 407 & 695 & 227 & 71 & 729 & -41.5 & -13.6 \\
\hline New Hampshire ................................... & - & - & - & 79 & - & - & - \\
\hline Middle Atlantic & 3,977 & 4,145 & 4,522 & 3,679 & 3,901 & -4.0 & .5 \\
\hline New Jersey ......................................... & 29 & 381 & 63 & 61 & 204 & -92.3 & -38.5 \\
\hline 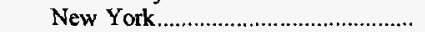 & 1,227 & 996 & 1,288 & 1,188 & 1,288 & 23.2 & -1.2 \\
\hline 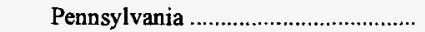 & 2,721 & 2,767 & 3,171 & 2,429 & 2,409 & -1.7 & 3.1 \\
\hline 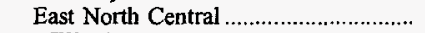 & 20,340 & 22,832 & 24,669 & 25,309 & 21,902 & -10.9 & -1.8 \\
\hline 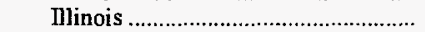 & 1,478 & 1,442 & 1,555 & 2,333 & 1,575 & 2.5 & -1.6 \\
\hline Indiana & 1,962 & 2,397 & 2,109 & 2,074 & 2,645 & -18.1 & -7.2 \\
\hline Michigan & 6,671 & 6,977 & 9,524 & 8,857 & 8,083 & -4.4 & -4.7 \\
\hline Ohio & 9,490 & 11,200 & 10,532 & 11,294 & 8,648 & -15.3 & 2.3 \\
\hline 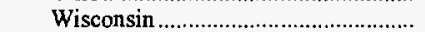 & 738 & 816 & 949 & 751 & 951 & -9.5 & -6.1 \\
\hline West North Central .................................. & 889 & 611 & 564 & 482 & 444 & 45.4 & 19.0 \\
\hline Iowa & 439 & 160 & 40 & 25 & 65 & 174.5 & 61.4 \\
\hline Kansas & - & - & - & - & 5 & - & - \\
\hline 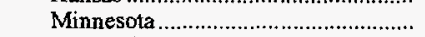 & 135 & 211 & 172 & 124 & 125 & -36.1 & 2.0 \\
\hline 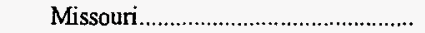 & 315 & 238 & 351 & 334 & 249 & 32.5 & 6.0 \\
\hline 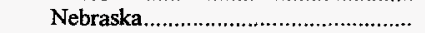 & - & 3 & - & - & - & -100.0 & - \\
\hline 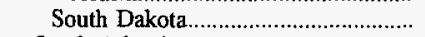 & - & - & 1 & - & - & - & - \\
\hline 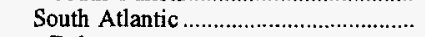 & 63,554 & 57,820 & 60,914 & 57,532 & 52,997 & 9.9 & 4.6 \\
\hline 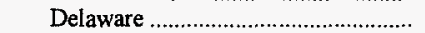 & - & - & 37 & 27 & - & - & - \\
\hline District of Columbia & - & - & 13 & 16 & 18 & - & - \\
\hline Florida & 14,015 & 12,121 & 12,069 & 11,311 & 12,584 & 15.6 & 2.7 \\
\hline Georgia & 14,689 & 15,803 & 15,649 & 14,163 & 11,770 & -7.0 & 5.7 \\
\hline 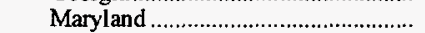 & 105 & 29 & 423 & 958 & 296 & 265.6 & -22.8 \\
\hline 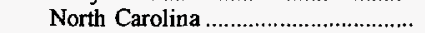 & 17,240 & 12,902 & 13,590 & 13,491 & 12,517 & 33.6 & 8.3 \\
\hline 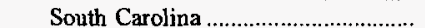 & 11,417 & 10,007 & 11,443 & 9,962 & 9,847 & 14.1 & 3.8 \\
\hline 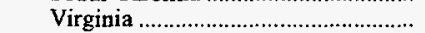 & 5,662 & 6,129 & 6,539 & 6,672 & 5,246 & -7.6 & 1.9 \\
\hline 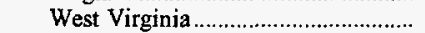 & 426 & 829 & 1,151 & 932 & 721 & -48.6 & -12.3 \\
\hline 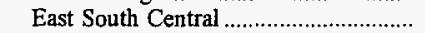 & 18,182 & 20,332 & 22,813 & 26,008 & 22,917 & -10.6 & -5.6 \\
\hline Alabama & 727 & 1,434 & 2,402 & 2,320 & 2,703 & -49.3 & -28.0 \\
\hline 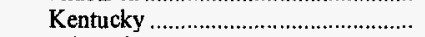 & 9,326 & 9,653 & 10,317 & 10,145 & 10,486 & -3.4 & -2.9 \\
\hline 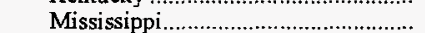 & 928 & 815 & 1,006 & 1,731 & 1,616 & 13.8 & -12.9 \\
\hline Tennessee & 7,202 & 8,430 & 9,088 & 11,811 & 8,112 & -14.6 & -2.9 \\
\hline West South Central .................................. & 71 & 513 & 802 & 104 & 95 & -86.1 & -7.1 \\
\hline 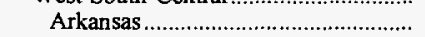 & - & - & $*$ & - & - & - & - \\
\hline Louisiana & 44 & 500 & 791 & 104 & 95 & -91.2 & -17.6 \\
\hline Oklahoma & 2 & 4 & - & - & - & -62.3 & - \\
\hline Texas & 26 & 8 & 10 & - & - & 201.0 & - \\
\hline 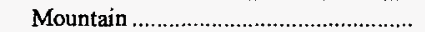 & - & 2 & 10 & - & 2 & -100.0 & - \\
\hline
\end{tabular}

See footnotes at end of table. 
Table 61. Domestic Distribution of U.S. Coal by Coal-Producing Region and State, and Destination Census Division and State, 1992-1996 (Continued) (Thousand Short Tons)

\begin{tabular}{|c|c|c|c|c|c|c|c|}
\hline \multirow{2}{*}{$\begin{array}{c}\text { Coal-Producing Region and State, } \\
\text { and Destination Census Division } \\
\text { and State }\end{array}$} & \multirow[t]{2}{*}{1996} & \multirow[t]{2}{*}{1995} & \multirow[t]{2}{*}{1994} & \multirow[t]{2}{*}{1993} & \multirow[t]{2}{*}{1992} & \multirow{2}{*}{$\begin{array}{c}\text { Percent } \\
\text { Change } \\
\text { 1995-1996 }\end{array}$} & \multirow{2}{*}{$\begin{array}{c}\begin{array}{c}\text { Average Annual } \\
\text { Percent Change }\end{array} \\
1992-1996\end{array}$} \\
\hline & & & & & & & \\
\hline \multicolumn{8}{|l|}{ Kentucky, Eastern (Continued) } \\
\hline Idaho & - & 2 & - & - & 2 & -100.0 & - \\
\hline 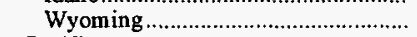 & - & - & 10 & - & - & - & - \\
\hline 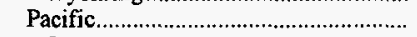 & 24 & 15 & 15 & 4 & - & 58.9 & - \\
\hline 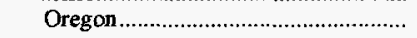 & 24 & 15 & 15 & 4 & - & 58.9 & - \\
\hline 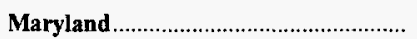 & 3,555 & 3,382 & 3,277 & 3,278 & 3,246 & 5.1 & 2.3 \\
\hline New England & 3 & 32 & - & 11 & - & -90.2 & - \\
\hline 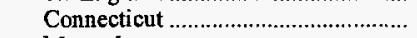 & 3 & 32 & - & - & - & -90.9 & - \\
\hline 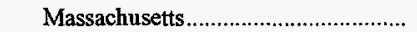 & * & - & - & 11 & - & - & - \\
\hline Middle Atlantic & 4 & 45 & - & 19 & 6 & -91.9 & -11.7 \\
\hline New York & - & - & $\dot{-}$ & - & 4 & - & - \\
\hline 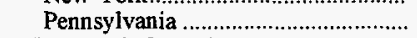 & 4 & 45 & - & 19 & 2 & -91.9 & 11.4 \\
\hline 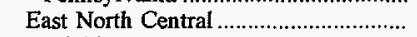 & 19 & - & - & - & - & - & - \\
\hline 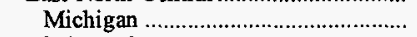 & 15 & - & - & - & - & - & - \\
\hline Wisconsin & 3 & - & $-\quad-$ & $\cdot-$ & - & - & - \\
\hline 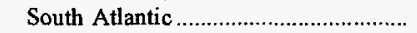 & 3,518 & 3,297 & 3,277 & 3,230 & 3,166 & 6.7 & 2.7 \\
\hline 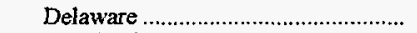 & 130 & 97 & 125 & 37 & - & 33.9 & - \\
\hline 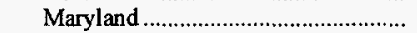 & 1,147 & 1,216 & 1,034 & 820 & 1,159 & -5.7 & -.2 \\
\hline 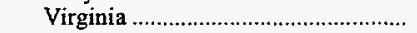 & 27 & 12 & 1 & - & 1 & 119.9 & 111.4 \\
\hline 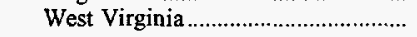 & 2,213 & 1,971 & 2,116 & 2,373 & 2,006 & 12.3 & 2.5 \\
\hline Ohio & 28,609 & 24,318 & 28,688 & 28,315 & 29,549 & 17.6 & -.8 \\
\hline 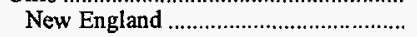 & - & - & $*$ & 17 & 4 & - & - \\
\hline 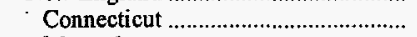 & - & - & $*$ & $*$ & 1 & - & - \\
\hline 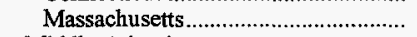 & - & - & - & 17 & 4 & - & - \\
\hline 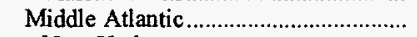 & 1,168 & 1,568 & 2,443 & 1,388 & 556 & -25.5 & 20.4 \\
\hline 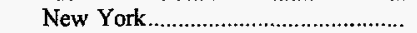 & 125 & 25 & 124 & 52 & 47 & 400.3 & 27.5 \\
\hline 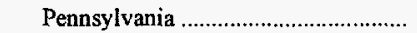 & 1,043 & 1,543 & 2,318 & 1,336 & 508 & -32.4 & 19.7 \\
\hline 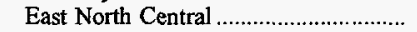 & 25,201 & 20,912 & 24,810 & 25,119 & 27,497 & 20.5 & -2.2 \\
\hline Illinois & 5 & - & 17 & * & 2 & - & 20.2 \\
\hline Indiana & 464 & 243 & 345 & 274 & 134 & 91.1 & 36.3 \\
\hline 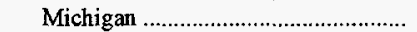 & 246 & 431 & 522 & 474 & 419 & -43.0 & -12.5 \\
\hline Ohio & 24,478 & 20,228 & 23,907 & 24,370 & 26,941 & 21.0 & -2.4 \\
\hline 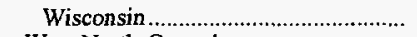 & 9 & 10 & 18 & - & - & -9.1 & - \\
\hline 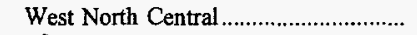 & - & 10 & 33 & 83 & - & -100.0 & - \\
\hline 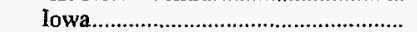 & - & - & 15 & 67 & - & - & - \\
\hline 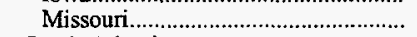 & - & 10 & 18 & 16 & - & -100.0 & - \\
\hline 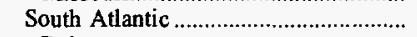 & 2,036 & 1,620 & 971 & 1,041 & 1,385 & 25.7 & 10.1 \\
\hline 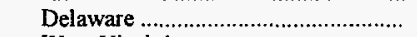 & - & - & 29 & 48 & - & - & - \\
\hline 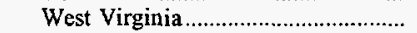 & 2,036 & 1,620 & 942 & 993 & 1,385 & 25.7 & 10.1 \\
\hline 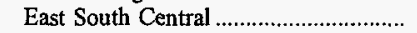 & 137 & 53 & 130 & 261 & - & 160.5 & - \\
\hline 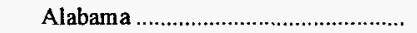 & 103 & 18 & 37 & 151 & - & 468.5 & - \\
\hline 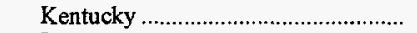 & 30 & 14 & 93 & 29 & - & 118.8 & - \\
\hline 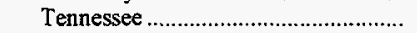 & 4 & 21 & - & 81 & - & -82.9 & - \\
\hline 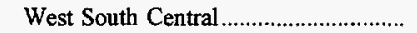 & - & - & - & - & * & - & - \\
\hline 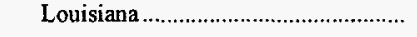 & - & - & - & - & $*$ & - & - \\
\hline \multicolumn{8}{|l|}{ Pennsylvania, } \\
\hline Anthracite & 4,330 & 3,497 & 4,346 & 3,015 & 3,230 & 23.8 & 7.6 \\
\hline 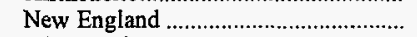 & 31 & 37 & 54 & 64 & 73 & -15.6 & -19.0 \\
\hline 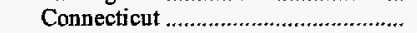 & 5 & 8 & 11 & 13 & 14 & -34.1 & -20.9 \\
\hline 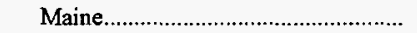 & 4 & 3 & 6 & 8 & 12 & 22.9 & -25.0 \\
\hline 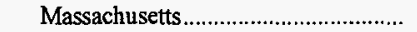 & 14 & 16 & 20 & 24 & 22 & -12.7 & -11.3 \\
\hline 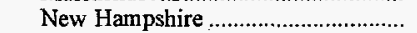 & 4 & 5 & 8 & 10 & 14 & -24.7 & -28.6 \\
\hline 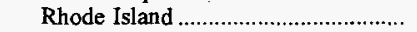 & 3 & 3 & 3 & 3 & 5 & 21.2 & -9.6 \\
\hline Vermont. & 2 & 3 & 5 & 6 & 7 & -39.0 & -28.9 \\
\hline Middle Atlantic & 3,985 & 2,922 & 3,227 & 2,618 & 2,916 & 36.4 & 8.1 \\
\hline 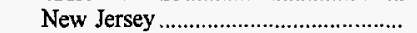 & 14 & 15 & 17 & 20 & 17 & -7.1 & -5.4 \\
\hline New York & 151 & 140 & 121 & 179 & 215 & 7.8 & -8.5 \\
\hline 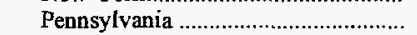 & 3,821 & 2,768 & 3,089 & 2,420 & 2,685 & 38.0 & 9.2 \\
\hline 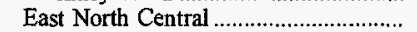 & 41 & 37 & 36 & 39 & 40 & 9.3 & .2 \\
\hline 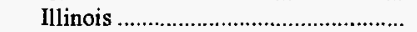 & 7 & 9 & 8 & 14 & 8 & -21.7 & -3.4 \\
\hline Indiana & 6 & 6 & 4 & 5 & 5 & 9.6 & 6.2 \\
\hline Michigan & 3 & 9 & 2 & * & 1 & -67.3 & 21.0 \\
\hline 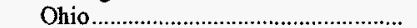 & 19 & 8 & 21 & 19 & 26 & 137.7 & -6.8 \\
\hline 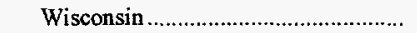 & 5 & 6 & 2 & 1 & $*$ & -11.2 & 215.3 \\
\hline 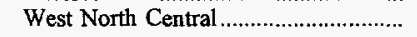 & 64 & 46 & 31 & 34 & 35 & 38.5 & 15.8 \\
\hline
\end{tabular}

See footnotes at end of table. 
Table 61. Domestic Distribution of U.S. Coal by Coal-Producing Region and State, and Destination Census Division and State, 1992-1996 (Continued) (Thousand Short Tons)

\begin{tabular}{|c|c|c|c|c|c|c|c|}
\hline \multirow{2}{*}{$\begin{array}{c}\text { Coal-Producing Region and State, } \\
\text { and Destination Census Division } \\
\text { and State }\end{array}$} & \multirow{2}{*}{1996} & \multirow{2}{*}{1995} & \multirow{2}{*}{1994} & \multirow{2}{*}{1993} & \multirow{2}{*}{1992} & \multirow{2}{*}{$\begin{array}{c}\text { Percent } \\
\text { Change } \\
\text { 1995-1996 }\end{array}$} & \multirow{2}{*}{$\begin{array}{c}\text { Average Annua } \\
\text { Percent Change } \\
1992-1996\end{array}$} \\
\hline & & & & & & & \\
\hline \multicolumn{8}{|l|}{$\begin{array}{l}\text { Pennsylvania, } \\
\text { Anthracite (Continued) }\end{array}$} \\
\hline 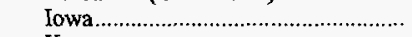 & 54 & 39 & 26 & 28 & 27 & 39.3 & 18.5 \\
\hline 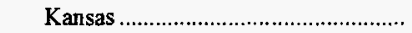 & * & - & - & $*$ & 6 & - & -63.6 \\
\hline 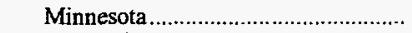 & 7 & 7 & 3 & 3 & $*$ & -5.3 & 270.6 \\
\hline 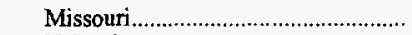 & $*$ & $*$ & 2 & 2 & $*$ & -88.1 & 38.4 \\
\hline 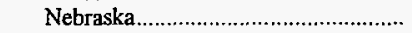 & 1 & $*$ & * & $\overline{2}$ & 2 & NM & -21.2 \\
\hline 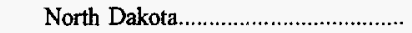 & 2 & $*$ & * & * & * & $\mathbf{N M}$ & 260.2 \\
\hline 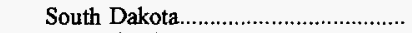 & - & - & $*$ & - & $*$ & - & - \\
\hline 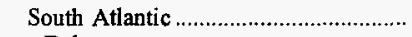 & 56 & 91 & 79 & 64 & 51 & -38.7 & 2.3 \\
\hline 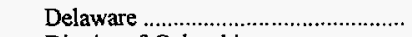 & 10 & 11 & 11 & 11 & 7 & -6.1 & 10.0 \\
\hline District of Columbia............................ & $*$ & $*$ & * & $*$ & $*$ & -62.0 & 3.1 \\
\hline Florida & 6 & 9 & 8 & 6 & 7 & -37.4 & -4.1 \\
\hline 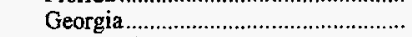 & $*$ & * & $*$ & $*$ & 1 & -25.4 & -23.9 \\
\hline Maryland & 1 & 27 & 6 & 4 & 3 & -95.8 & -22.4 \\
\hline 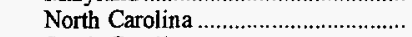 & $*$ & * & * & $*$ & $*$ & -41.4 & -6.2 \\
\hline 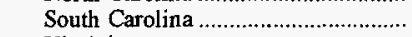 & * & 3 & 8 & 11 & * & -91.4 & 14.0 \\
\hline 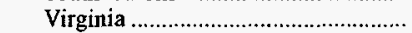 & 6 & 9 & 11 & 14 & 13 & -35.2 & -17.9 \\
\hline 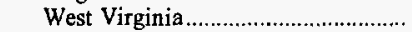 & 32 & 31 & 35 & 18 & 20 & 2.2 & 12.9 \\
\hline 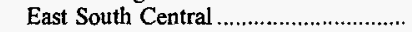 & 50 & 44 & 28 & 34 & 42 & 14.3 & 4.8 \\
\hline Alabama & 2 & 1 & 1 & 3 & 6 & 58.3 & -23.2 \\
\hline 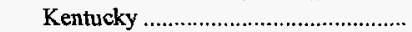 & 22 & 21 & 12 & 18 & 24 & 5.2 & -1.9 \\
\hline 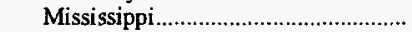 & * & * & * & * & * & 17.9 & -6.4 \\
\hline 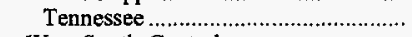 & 26 & 22 & 15 & 14 & 12 & 20.2 & 22.1 \\
\hline 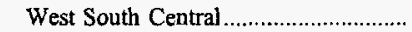 & 8 & 12 & 8 & 11 & 10 & -34.7 & -4.9 \\
\hline Arkansas & $*$ & 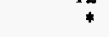 & 1 & $*$ & 4 & 14.3 & -53.3 \\
\hline 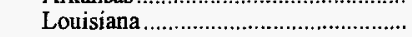 & 6 & 10 & 3 & 8 & $*$ & -38.8 & 132.1 \\
\hline Oklahoma & $*$ & $*$ & * & * & 1 & 20.7 & -14.9 \\
\hline 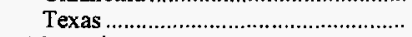 & 1 & 2 & 3 & 3 & 5 & -21.1 & -30.2 \\
\hline 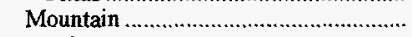 & 21 & 13 & 18 & 3 & $*$ & 60.9 & 162.1 \\
\hline 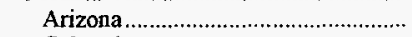 & 1 & $*$ & $*$ & * & * & $\mathrm{NM}$ & 105.3 \\
\hline Colorado & 16 & 12 & 15 & 3 & $*$ & 32.1 & 165.8 \\
\hline 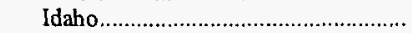 & $*$ & $*$ & 3 & $*$ & - & -94.8 & - \\
\hline 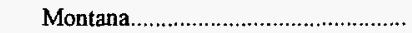 & 2 & - & $*$ & * & - & - & - \\
\hline 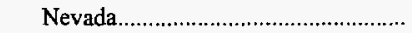 & * & - & - & - & - & - & - \\
\hline 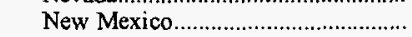 & * & * & * & $*$ & $*$ & -71.7 & 5.0 \\
\hline Utah & * & $*$ & * & $*$ & - & -93.1 & - \\
\hline 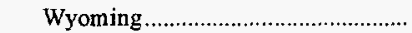 & 1 & - & $*$ & * & $*$ & - & 172.1 \\
\hline 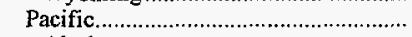 & 12 & 12 & 7 & 10 & $*$ & 2.1 & 246.9 \\
\hline 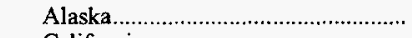 & - & - & $*$ & - & - & - & - \\
\hline 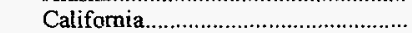 & * & * & $*$ & * & * & NM & 53.5 \\
\hline Oregon & 12 & 12 & 7 & 9 & - & -1.4 & - \\
\hline 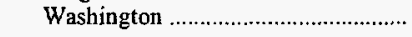 & - & $*$ & - & - & * & -100.0 & - \\
\hline \multicolumn{8}{|l|}{ Pennsylvania, } \\
\hline 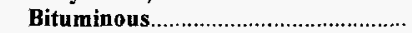 & 55,552 & 50,464 & 50,861 & 50,467 & 57,979 & 10.1 & -1.1 \\
\hline 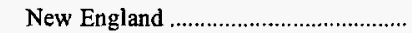 & 1,021 & 1,009 & 1,025 & 989 & 1,124 & 1.1 & -2.4 \\
\hline 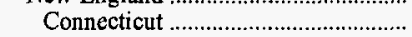 & 227 & 516 & 12 & $*$ & 131 & -56.0 & 14.8 \\
\hline Maine & - & 32 & 24 & 2 & 19 & -100.0 & - \\
\hline 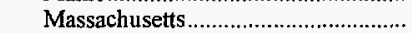 & 202 & 4 & 292 & 330 & 294 & NM & -8.9 \\
\hline 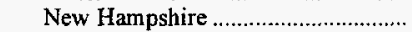 & 592 & 458 & 698 & 656 & 681 & 29.3 & -3.5 \\
\hline 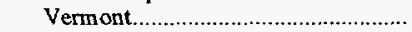 & * & - & - & * & - & - & - \\
\hline 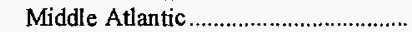 & 40,063 & 37,612 & 38,188 & 37,417 & 43,559 & 6.5 & -2.1 \\
\hline 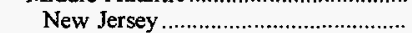 & 538 & 558 & 537 & 153 & 15 & -3.6 & 143.8 \\
\hline 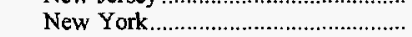 & 4,125 & 3,675 & 5,551 & 6,228 & 8,532 & 12.3 & -16.6 \\
\hline 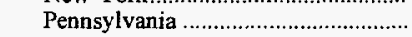 & 35,400 & 33,379 & 32,100 & 31,036 & 35,012 & 6.0 & .3 \\
\hline 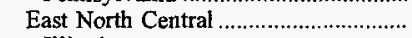 & 8,460 & 6,682 & 6,288 & 6,502 & 7,466 & 26.6 & 3.2 \\
\hline Illinois & - & - & 206 & 43 & 69 & - & - \\
\hline 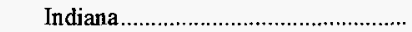 & 559 & 222 & 629 & 505 & 380 & 152.1 & 10.2 \\
\hline 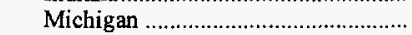 & 2,075 & 2,650 & 1,756 & 1,607 & 1,862 & -21.7 & 2.7 \\
\hline Ohio & 4,463 & 2,707 & 2,769 & 3,848 & 3,567 & 64.9 & 5.8 \\
\hline 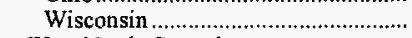 & 1,362 & 1,103 & 928 & 500 & 1,589 & 23.5 & -3.8 \\
\hline 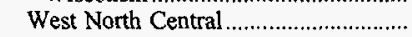 & 248 & 228 & 46 & 205 & 191 & 8.7 & 6.8 \\
\hline 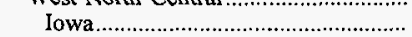 & 225 & 227 & 46 & 103 & 162 & -.9 & 8.5 \\
\hline Minnesota & 23 & - & - & - & 28 & - & -4.9 \\
\hline Missouri & - & 1 & - & 102 & 1 & -100.0 & - \\
\hline 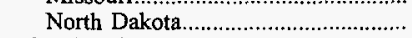 & - & - & - & $*$ & - & - & - \\
\hline South Atlantic & 3,968 & 3,377 & 3,783 & 3,955 & 4,090 & 17.5 & -8 \\
\hline
\end{tabular}

See footnotes at end of table. 
Table 61. Domestic Distribution of U.S. Coal by Coal-Producing Region and State, and Destination Census Division and State, 1992-1996 (Continued) (Thousand Short Tons)

\begin{tabular}{|c|c|c|c|c|c|c|c|}
\hline \multirow{2}{*}{$\begin{array}{l}\text { Coal-Producing Region and State, } \\
\text { and Destination Census Division } \\
\text { and State }\end{array}$} & \multirow[t]{2}{*}{1996} & \multirow[t]{2}{*}{1995} & \multirow[t]{2}{*}{1994} & \multirow[t]{2}{*}{1993} & \multirow[t]{2}{*}{1992} & \multirow{2}{*}{$\begin{array}{c}\text { Percent } \\
\text { Change } \\
\text { 1995-1996 }\end{array}$} & \multirow{2}{*}{$\begin{array}{c}\begin{array}{c}\text { Average Annual } \\
\text { Percent Change }\end{array} \\
1992-1996\end{array}$} \\
\hline & & & & & & & \\
\hline \multicolumn{8}{|l|}{$\begin{array}{l}\text { Pennsylvania, } \\
\text { Bituminous (Continued) }\end{array}$} \\
\hline 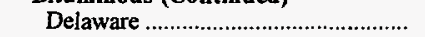 & 528 & 452 & 314 & 252 & 226 & 16.7 & 23.6 \\
\hline 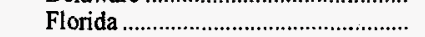 & - & - & 135 & - & - & - & - \\
\hline 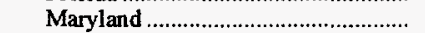 & 1,602 & 1,741 & 2,278 & 2,372 & 2,437 & -8.0 & -9.9 \\
\hline 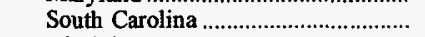 & - & 6 & - & - & 1 & -100.0 & - \\
\hline 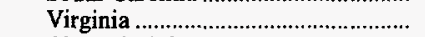 & 5 & 20 & 55 & 29 & 5 & -74.2 & -1.3 \\
\hline 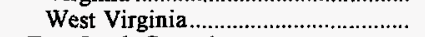 & 1,833 & 1,157 & 1,002 & 1,302 & 1,421 & 58.5 & 6.6 \\
\hline 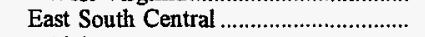 & 1,144 & 1,078 & 993 & 772 & 83 & 6.1 & 92.5 \\
\hline 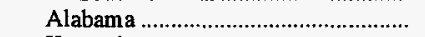 & 6 & 39 & 34 & 46 & 1 & -85.3 & 83.1 \\
\hline 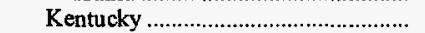 & 500 & 363 & 460 & 225 & 31 & 37.5 & 99.7 \\
\hline Mississippi & - & - & - & 3 & 6 & - & - \\
\hline 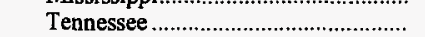 & 639 & 675 & 500 & 498 & 46 & -5.4 & 93.5 \\
\hline 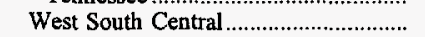 & 195 & 6 & - & $\neq$ & $*$ & $\mathrm{NM}$ & NM \\
\hline Louisiana & 195 & - & - & - & - & - & - \\
\hline 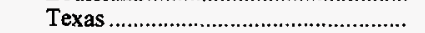 & $*$ & 6 & - & * & * & -94.2 & 65.8 \\
\hline 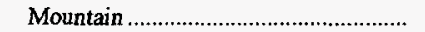 & 230 & 215 & 183 & 240 & 290 & 7.3 & -5.6 \\
\hline Utah & 230 & 215 & 183 & 240 & 290 & 7.3 & -5.6 \\
\hline Tennessee & 3,052 & 2,627 & 2,547 & 2,577 & 2,835 & 16.2 & 1.9 \\
\hline 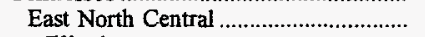 & 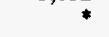 & $*$ & 1 & $*$ & 24 & -33.5 & -72.3 \\
\hline 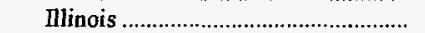 & - & - & 1 & - & - & - & - \\
\hline Indiana & - & - & - & $*$ & 3 & - & - \\
\hline Michigan & - & $*$ & - & - & 21 & -100.0 & - \\
\hline Ohio & $*$ & $*$ & - & - & - & -5.4 & - \\
\hline 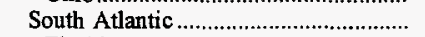 & 141 & 251 & 301 & 303 & 387 & -43.5 & -22.2 \\
\hline Florida & - & 39 & 40 & - & - & -100.0 & - \\
\hline 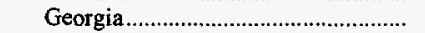 & 141 & 189 & 202 & 204 & 259 & -25.4 & -14.1 \\
\hline North Carolina ...................................... & 1 & 23 & 58 & 96 & 114 & -97.2 & -72.8 \\
\hline 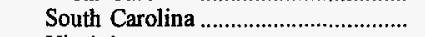 & - & - & - & 3 & 13 & - & - \\
\hline Virginia & - & - & - & $*$ & 1 & - & - \\
\hline 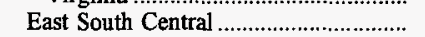 & 2,902 & 2,363 & 2,223 & 2,215 & 2,376 & 22.8 & 5.1 \\
\hline Alabama & 331 & 936 & 710 & 592 & 734 & -64.6 & -18.0 \\
\hline Kentucky & 23 & 5 & 135 & 122 & 39 & 367.4 & -12.9 \\
\hline Tennessee & 2,548 & 1,422 & 1,378 & 1,501 & 1,603 & 79.2 & 12.3 \\
\hline 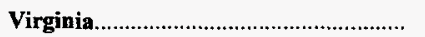 & 22,776 & 24,283 & 26,866 & 27,388 & 28,504 & -6.2 & -5.4 \\
\hline New England & 18 & 19 & - & - & 281 & -6.4 & -49.8 \\
\hline Maine & - & - & - & - & 44 & - & - \\
\hline 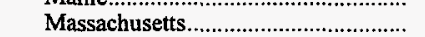 & 8 & - & - & - & 237 & - & -57.4 \\
\hline New Hampshire ............................... & 10 & 19 & - & - & - & -47.4 & - \\
\hline Middle Atlantic & 2,045 & 2,311 & 1,490 & 2,073 & 2,957 & -11.5 & -8.8 \\
\hline 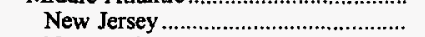 & 601 & 635 & 190 & 359 & 734 & -5.4 & -4.9 \\
\hline New York & 146 & 362 & 156 & 108 & 96 & -59.8 & 10.9 \\
\hline 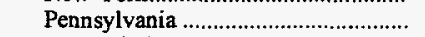 & 1,299 & 1,314 & 1,143 & 1,607 & 2,127 & -1.2 & -11.6 \\
\hline 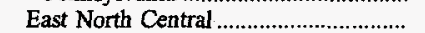 & 3,237 & 3,557 & 2,455 & 3,475 & 3,794 & -9.0 & -3.9 \\
\hline 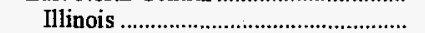 & 583 & 578 & 302 & 260 & 478 & .8 & 5.1 \\
\hline 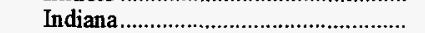 & 2,290 & 2,395 & 1,202 & 2,045 & 2,240 & -4.4 & .5 \\
\hline Michigan & 25 & 83 & 376 & 188 & 68 & -69.9 & -22.3 \\
\hline 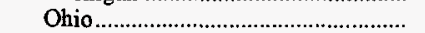 & 331 & 493 & 488 & 875 & 940 & -32.8 & -23.0 \\
\hline Wisconsin & 9 & 9 & 86 & 108 & 68 & 1.0 & -39.4 \\
\hline 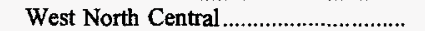 & - & 8 & - & - & $*$ & -100.0 & - \\
\hline 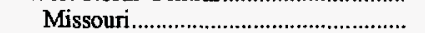 & - & 8 & - & - & - & -100.0 & - \\
\hline 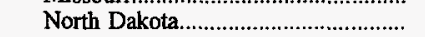 & - & - & - & - & * & - & - \\
\hline 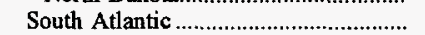 & 13,507 & 14,600 & 18,898 & 18,553 & 16,839 & -7.5 & -5.4 \\
\hline Delaware & 166 & 152 & 203 & 155 & 208 & 9.3 & -5.5 \\
\hline Florida & 549 & 377 & 531 & 457 & 451 & 45.4 & 5.0 \\
\hline Georgia & 1,785 & 2,064 & 3,038 & 3,496 & 2,779 & -13.5 & -10.5 \\
\hline 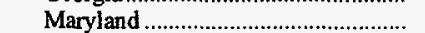 & 1 & 392 & 90 & 8 & 79 & -99.8 & -70.3 \\
\hline North Carolina & 1,883 & 4,056 & 5,634 & 5,867 & 5,207 & -53.6 & -22.4 \\
\hline 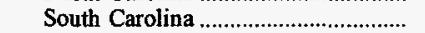 & 1,605 & 1,468 & 1,604 & 1,663 & 1,356 & 9.4 & 4.3 \\
\hline Virginia & 7,231 & 5,657 & 6,867 & 6,076 & 6,082 & 27.8 & 4.4 \\
\hline 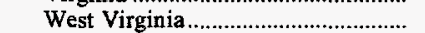 & 287 & 433 & 930 & 832 & 677 & -33.8 & -19.3 \\
\hline 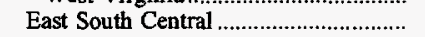 & 3,581 & 3,413 & 3,658 & 3,026 & 3,432 & 4.9 & 1.1 \\
\hline Alabama & 1,036 & 1,083 & 1,156 & 887 & 1,017 & -4.3 & 5 \\
\hline Kentucky & 3 & 142 & 41 & 1 & $*$ & -98.2 & 266.9 \\
\hline 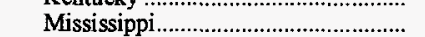 & 13 & - & - & - & - & - & - \\
\hline Tennessee & 2,529 & 2,187 & 2,462 & 2,138 & 2,415 & 15.6 & 1.2 \\
\hline
\end{tabular}

See footnotes at end of table. 
Table 61. Domestic Distribution of U.S. Coal by Coal-Producing Region and State, and Destination Censuls Division and State, 1992-1996 (Continued) (Thousand Short Tons)

\begin{tabular}{|c|c|c|c|c|c|c|c|}
\hline \multirow{2}{*}{$\begin{array}{c}\text { Coal-Producing Region and State, } \\
\text { and Destination Census Division } \\
\text { and State }\end{array}$} & \multirow{2}{*}{1996} & \multirow{2}{*}{1995} & \multirow{2}{*}{1994} & \multirow{2}{*}{1993} & \multirow{2}{*}{1992} & \multirow{2}{*}{$\begin{array}{c}\text { Percent } \\
\text { Change } \\
1995-1996\end{array}$} & \multirow{2}{*}{$\begin{array}{c}\begin{array}{c}\text { Average Annual } \\
\text { Percent Change }\end{array} \\
1992-1996\end{array}$} \\
\hline & & & & & & & \\
\hline \multicolumn{8}{|l|}{ Virginia (Continued) } \\
\hline West South Central ................................. & 13 & 21 & - & 2 & 2 & -38.9 & 55.8 \\
\hline 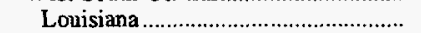 & - & 21 & $\tau$ & - & - & -100.0 & - \\
\hline 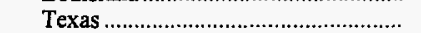 & 13 & - & - & 2 & 2 & - & 55.8 \\
\hline 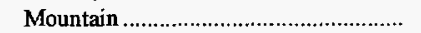 & 332 & 313 & 320 & 103 & 192 & 6.3 & 14.7 \\
\hline 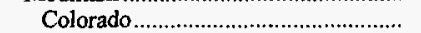 & - & - & - & $*$ & - & - & - \\
\hline Utah & 332 & 313 & 320 & 103 & 192 & 6.3 & 14.7 \\
\hline West Virginia, Northern ........................ & 40,398 & 36,073 & 39,985 & 34,573 & 44,093 & 12.0 & -2.2 \\
\hline New England & 1,070 & 918 & 1,086 & 1,002 & 2,776 & 16.6 & -21.2 \\
\hline 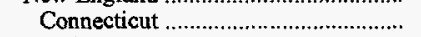 & 683 & 572 & 166 & - & $*$ & 19.3 & NM \\
\hline Maine & 13 & 9 & - & - & - & 41.7 & - \\
\hline 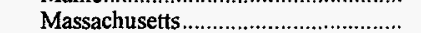 & 53 & 113 & 648 & 613 & 2,379 & -53.0 & -61.4 \\
\hline 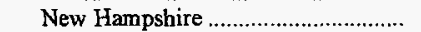 & 322 & 225 & 272 & 389 & 397 & 43.4 & -5.1 \\
\hline 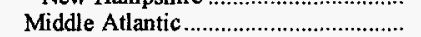 & 14,276 & 13,740 & 12,566 & 9,769 & 13,804 & 3.9 & .8 \\
\hline 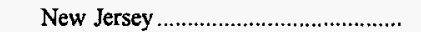 & 1,530 & 1,182 & 1,096 & 1,234 & 1,213 & 29.4 & 6.0 \\
\hline 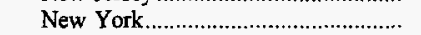 & 4,140 & 4,040 & 3,079 & 1,336 & 2,530 & 2.5 & 13.1 \\
\hline 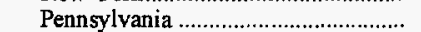 & 8,606 & 8,518 & 8,391 & 7,200 & 10,061 & 1.0 & -3.8 \\
\hline 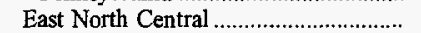 & 5,410 & 2,887 & 5,924 & 4,220 & 5,803 & 87.4 & -1.7 \\
\hline 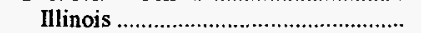 & 9 & 51 & 23 & 44 & - & -82.4 & - \\
\hline Indiana & 479 & 38 & 1,060 & 216 & 70 & $\mathrm{NM}$ & 61.6 \\
\hline 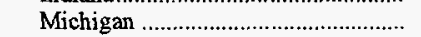 & 640 & 437 & 392 & 113 & 114 & 46.4 & 54.0 \\
\hline Ohio & 3,966 & 1,977 & 4,098 & 3,659 & 5,365 & 100.6 & -7.3 \\
\hline Wisconsin & 317 & 384 & 350 & 188 & 254 & -17.6 & 5.7 \\
\hline 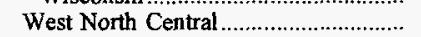 & $*$ & 3 & 12 & 54 & 9 & -99.3 & -77.6 \\
\hline Iowa & - & - & 2 & - & - & - & - \\
\hline Minnesota & - & 3 & 10 & - & 9 & -100.0 & - \\
\hline 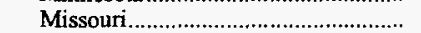 & - & - & - & 54 & - & - & - \\
\hline 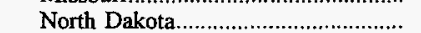 & $*$ & - & - & - & - & - & - \\
\hline 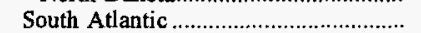 & 17,820 & 17,213 & 19,270 & 17,772 & 20,988 & 3.5 & -4.0 \\
\hline Delaware & 449 & 737 & 969 & 1,096 & 1,111 & -39.0 & -20.3 \\
\hline 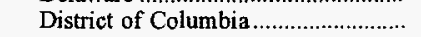 & 6 & 5 & 10 & 12 & 6 & 12.9 & 2.1 \\
\hline 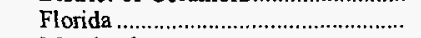 & 551 & 259 & 449 & 580 & 452 & 112.5 & 5.1 \\
\hline Maryland & 4,773 & 3,341 & 3,443 & 4,314 & 3,466 & 42.9 & 8.3 \\
\hline North Carolina & 13 & - & 7 & - & $*$ & - & 143.0 \\
\hline South Carolina & 3 & - & 1 & - & - & - & - \\
\hline Virginia & 66 & 30 & 75 & 306 & 243 & 121.3 & -27.8 \\
\hline 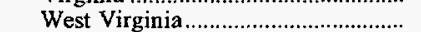 & 11,959 & 12,841 & 14,316 & 11,464 & 15,710 & -6.9 & -6.6 \\
\hline East South Central & 1,522 & 1,178 & 492 & 749 & 167 & 29.2 & 73.9 \\
\hline Alabama & 419 & 604 & 34 & 27 & - & -30.7 & - \\
\hline 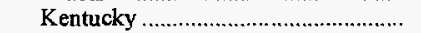 & 1,026 & 527 & 160 & 130 & 4 & 94.4 & 293.8 \\
\hline 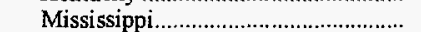 & - & - & 5 & 5 & 4 & - & - \\
\hline 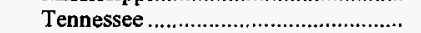 & 78 & 46 & 293 & 589 & 159 & 67.9 & -16.3 \\
\hline 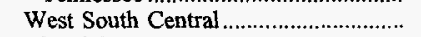 & 203 & - & 368 & 154 & - & - & - \\
\hline 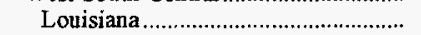 & 203 & - & 368 & 154 & - & - & - \\
\hline Mountain & - & $*$ & - & - & - & -100.0 & - \\
\hline 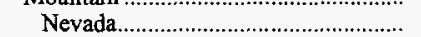 & - & * & - & - & - & -100.0 & - \\
\hline 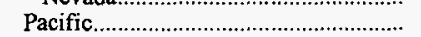 & - & - & - & $*$ & * & - & - \\
\hline 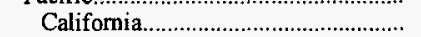 & - & - & - & $*$ & * & - & - \\
\hline \multicolumn{8}{|l|}{ West Virginia, } \\
\hline 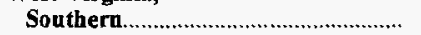 & 86,757 & 84,793 & 82,794 & 68,086 & 68,824 & 2.3 & 6.0 \\
\hline 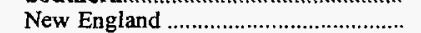 & 1,853 & 1,404 & 1,351 & 917 & 1,109 & 32.0 & 13.7 \\
\hline Connecticut & 24 & - & - & 106 & 21 & - & 4.1 \\
\hline 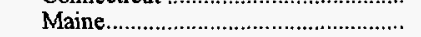 & 13 & - & * & 14 & 20 & - & -10.5 \\
\hline 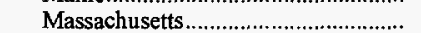 & 1,792 & 1,330 & 1,351 & 739 & 971 & 34.8 & 16.5 \\
\hline 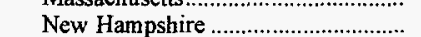 & 24 & 74 & - & 58 & 97 & -67.7 & -29.5 \\
\hline Rhode Island & - & - & - & $*$ & - & - & - \\
\hline 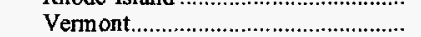 & - & - & * & - & - & - & - \\
\hline Middle Atlantic & 7,391 & 7,170 & 6,738 & 6,965 & 5,160 & 3.1 & 9.4 \\
\hline 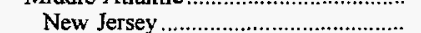 & 216 & 165 & 260 & 343 & 250 & 30.7 & -3.6 \\
\hline 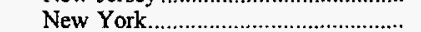 & 1,545 & 1,466 & 1,345 & 1,860 & 1,265 & 5.4 & 5.1 \\
\hline 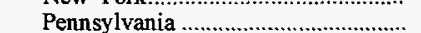 & 5,630 & 5,538 & 5,133 & 4,763 & 3,644 & 1.6 & 11.5 \\
\hline 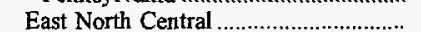 & 30,404 & 28,905 & 27,503 & 22,906 & 25,560 & 5.2 & 4.4 \\
\hline Illinois & 1,841 & 1,400 & 1,403 & 1,971 & 1,472 & 31.5 & 5.8 \\
\hline Indiana & 4,809 & 5,142 & 4,918 & 3,401 & 4,920 & -6.5 & -.6 \\
\hline Michigan & $\begin{array}{l}4,809 \\
4,869\end{array}$ & 4,416 & 5,903 & $\begin{array}{l}5,048 \\
4,048\end{array}$ & 6,120 & 10.3 & -5.6 \\
\hline Ohio & $\begin{array}{r}4,005 \\
18,770\end{array}$ & 17,566 & 14,802 & 12,485 & 12,457 & 6.8 & 10.8 \\
\hline
\end{tabular}

See footnotes at end of table. 
Table 61. Domestic Distribution of U.S. Coal by Coal-Producing Region and State, and Destination Census Division and State, 1992-1996 (Continued) (Thousand Short Tons)

\begin{tabular}{|c|c|c|c|c|c|c|c|}
\hline \multirow{2}{*}{$\begin{array}{l}\text { Coal-Producing Region and State, } \\
\text { and Destination Census Division } \\
\text { and State }\end{array}$} & \multirow{2}{*}{1996} & \multirow{2}{*}{1995} & \multirow{2}{*}{1994} & \multirow{2}{*}{1993} & \multirow{2}{*}{1992} & \multirow{2}{*}{$\begin{array}{c}\text { Percent } \\
\text { Change } \\
1995-1996\end{array}$} & \multirow{2}{*}{$\begin{array}{c}\text { Average Annua } \\
\text { Percent Change } \\
1992-1996\end{array}$} \\
\hline & & & & & & & \\
\hline \multicolumn{8}{|l|}{$\begin{array}{l}\text { West Virginia, } \\
\text { Southern (Continued) }\end{array}$} \\
\hline Wisconsin & 115 & 381 & 478 & 1,002 & 592 & -69.7 & -33.5 \\
\hline West North Central ................................. & 113 & 313 & 237 & 223 & 364 & -64.0 & -25.4 \\
\hline Iowa & 44 & 119 & 70 & 69 & 77 & -63.1 & -13.0 \\
\hline 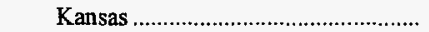 & - & - & - & - & 34 & - & - \\
\hline 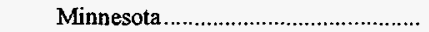 & 32 & 107 & 92 & 11 & 24 & -70.0 & 7.3 \\
\hline 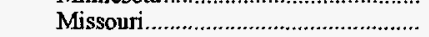 & 36 & 85 & 70 & 142 & 228 & -58.3 & -37.2 \\
\hline 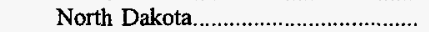 & - & $*$ & $*$ & - & - & -100.0 & - \\
\hline 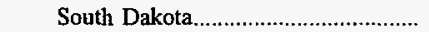 & 1 & 2 & 6 & - & - & -37.7 & - \\
\hline 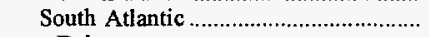 & 37,986 & 36,164 & 36,045 & 26,459 & 28,512 & 5.0 & 7.4 \\
\hline 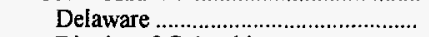 & 551 & 485 & 621 & 617 & 361 & 13.7 & 11.1 \\
\hline 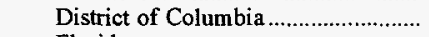 & 17 & - & 24 & 23 & 27 & - & -10.8 \\
\hline Florida & 1,123 & 1,341 & 1,372 & 817 & 1,010 & -16.3 & 2.7 \\
\hline Georgia & 4,064 & 4,159 & 4,106 & 2,958 & 2,477 & -2.3 & 13.2 \\
\hline Maryland & 3,132 & 3,209 & 2,855 & 1,376 & 2,296 & -2.4 & 8.1 \\
\hline 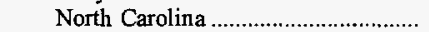 & 8,274 & 7,169 & 6,910 & 6,167 & 7,963 & 15.4 & 1.0 \\
\hline South Carolina & 347 & 257 & 394 & 107 & 121 & 34.9 & 30.0 \\
\hline 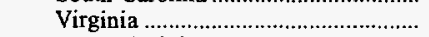 & 3,217 & 3,367 & 3,188 & 2,971 & 3,323 & -4.4 & -.8 \\
\hline 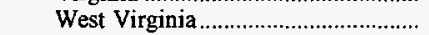 & 17,261 & 16,177 & 16,575 & 11,423 & 10,934 & 6.7 & 12.1 \\
\hline 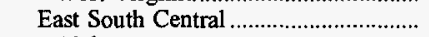 & 8,677 & 10,433 & 10,551 & 10,095 & 7,054 & -16.8 & 5.3 \\
\hline Alabama & 2,922 & 3,487 & 4,392 & 3,736 & 2,362 & -16.2 & 5.5 \\
\hline 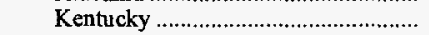 & 4,250 & 5,330 & 4,744 & 4,704 & 3,755 & -20.3 & 3.1 \\
\hline 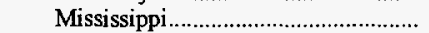 & 24 & 44 & 93 & 87 & 34 & -45.9 & -8.3 \\
\hline 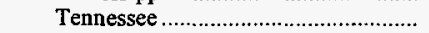 & 1,482 & 1,571 & 1,322 & 1,569 & 904 & -5.7 & 13.1 \\
\hline 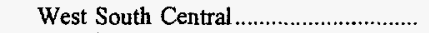 & 81 & 48 & 60 & 49 & 22 & 67.7 & 37.8 \\
\hline 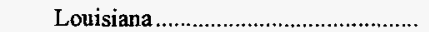 & - & - & 4 & 2 & - & - & - \\
\hline Oklahoma & 77 & 48 & 56 & 48 & 22 & 58.4 & 35.9 \\
\hline 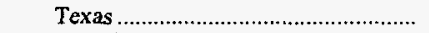 & 4 & - & 1 & - & - & - & - \\
\hline 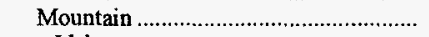 & 18 & 206 & 212 & 135 & - & -91.0 & - \\
\hline Idaho & - & - & $*$ & - & - & - & - \\
\hline Utah & 18 & 206 & 211 & 135 & - & -91.0 & - \\
\hline 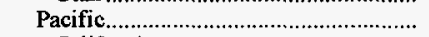 & 28 & 2 & 1 & * & - & $\mathrm{NM}$ & - \\
\hline 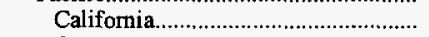 & - & - & - & $*$ & - & - & - \\
\hline 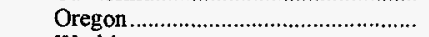 & 2 & 2 & 1 & - & - & -17.2 & - \\
\hline 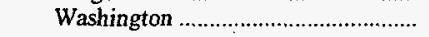 & 26 & - & - & - & - & - & - \\
\hline 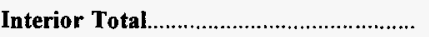 & 165,668 & 162,947 & 175,622 & 166,763 & 193,400 & 1.7 & -3.8 \\
\hline 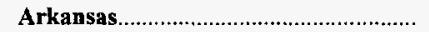 & 7 & 11 & 28 & 25 & 29 & -39.2 & -30.4 \\
\hline 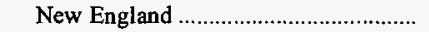 & - & - & - & - & 13 & - & - \\
\hline Vermont & - & - & - & - & 13 & - & - \\
\hline West North Central ................................... & - & - & 4 & 8 & 11 & - & - \\
\hline 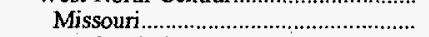 & - & - & 4 & 8 & 11 & - & - \\
\hline West South Central & 7 & 11 & 24 & 17 & 5 & -39.2 & 7.1 \\
\hline Arkansas & 7 & 11 & 13 & 12 & - & -39.2 & - \\
\hline 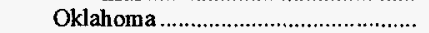 & - & - & $*$ & 5 & 5 & - & - \\
\hline 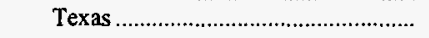 & - & - & 12 & - & - & - & - \\
\hline Illinois .................. & 45,190 & 45,170 & $\mathbf{5 1 , 7 3 7}$ & 41,330 & 57,670 & * & -5.9 \\
\hline New England & 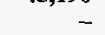 & * & - & - & 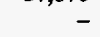 & -100.0 & - \\
\hline 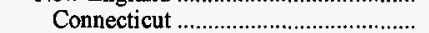 & - & * & - & - & - & -100.0 & - \\
\hline 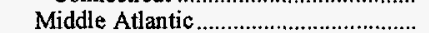 & * & * & * & * & * & -80.0 & -9.9 \\
\hline New Jersey & * & * & - & - & $*$ & -63.6 & -35.4 \\
\hline New York & $*$ & * & $*$ & * & * & -91.5 & -29.5 \\
\hline 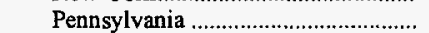 & * & * & * & - & - & -50.0 & - \\
\hline 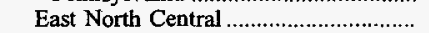 & 25,316 & 25,629 & 28,299 & 20,483 & 28,821 & -1.2 & -3.2 \\
\hline 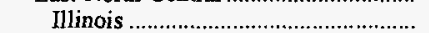 & 16,052 & 15,587 & 17,517 & 15,206 & 18,167 & 3.0 & -3.0 \\
\hline 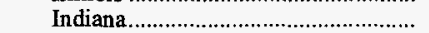 & 8,178 & 8,559 & 9,574 & 4,541 & 9,595 & -4.4 & -3.9 \\
\hline Michigan & 59 & 70 & 51 & - & 6 & -15.0 & 76.9 \\
\hline Ohio & 18 & 1 & 18 & - & - & $\mathrm{NM}$ & - \\
\hline Wisconsin & 1,008 & 1,412 & 1,139 & 736 & 1,053 & -28.6 & -1.1 \\
\hline 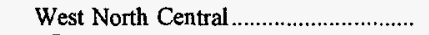 & 5,347 & 6,270 & 9,448 & 7,783 & 13,499 & -14.7 & -20.7 \\
\hline 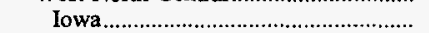 & 694 & 1,216 & 1,535 & 1,534 & 1,175 & -42.9 & -12.3 \\
\hline 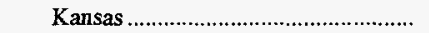 & 149 & 128 & 193 & 179 & 640 & 16.3 & -30.5 \\
\hline 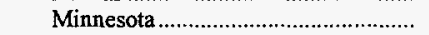 & 100 & 111 & 179 & 43 & 58 & -9.8 & 14.4 \\
\hline 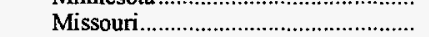 & 4,403 & 4,815 & 7,541 & 6,027 & 11,625 & -8.5 & -21.5 \\
\hline 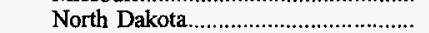 & - & - & - & * & - & - & - \\
\hline South Atlantic & 7,255 & 6,651 & 8,403 & 8,137 & 10,485 & 9.1 & -8.8 \\
\hline
\end{tabular}

See footnotes at end of table. 
Table 61. Domestic Distribution of U.S. Coal by Coal-Producing Region and State, and Destination Census Division and State, 1992-1996 (Continued) (Thousand Short Tons)

\begin{tabular}{|c|c|c|c|c|c|c|c|}
\hline \multirow{2}{*}{$\begin{array}{l}\text { Coal-Producing Region and State, } \\
\text { and Destination Census Division } \\
\text { and State }\end{array}$} & \multirow{2}{*}{1996} & \multirow{2}{*}{1995} & \multirow{2}{*}{1994} & \multirow{2}{*}{1993} & \multirow{2}{*}{1992} & \multirow{2}{*}{$\begin{array}{c}\text { Percent } \\
\text { Change } \\
\text { 1995-1996 }\end{array}$} & \multirow{2}{*}{$\begin{array}{c}\begin{array}{c}\text { Average Annua } \\
\text { Percent Change }\end{array} \\
1992-1996\end{array}$} \\
\hline & & & & & & & \\
\hline \multicolumn{8}{|l|}{ Illinois (Continued) } \\
\hline 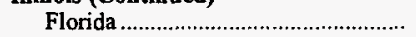 & 6,052 & 6,056 & 5,846 & 4,782 & 5,529 & -0.1 & 2.3 \\
\hline 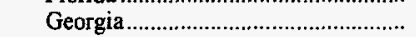 & 1,204 & 584 & 2,557 & 3,355 & 4,955 & 106.0 & -29.8 \\
\hline 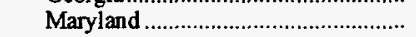 & - & 5 & - & 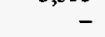 & - & -100.0 & - \\
\hline 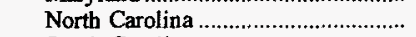 & - & $*$ & - & - & - & -100.0 & - \\
\hline 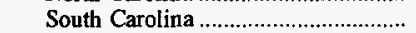 & - & - & - & - & 1 & - & - \\
\hline 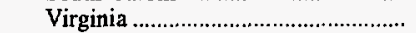 & * & $*$ & - & - & - & -81.8 & - \\
\hline West Virginia & - & 6 & $*$ & - & - & -100.0 & - \\
\hline 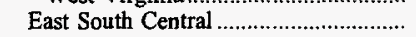 & 7,130 & 6,510 & 5,453 & 4,823 & 4,780 & 9.5 & 10.5 \\
\hline 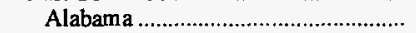 & 2,155 & 1,146 & 750 & 401 & 632 & 88.1 & 35.9 \\
\hline 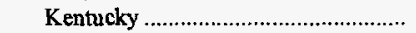 & 1 & 274 & 343 & 535 & 7 & -99.7 & -39.8 \\
\hline 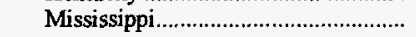 & 1,749 & 1,304 & 1,164 & 1,106 & 1,879 & 34.1 & -1.8 \\
\hline 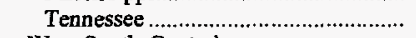 & 3,225 & 3,787 & 3,195 & 2,780 & 2,261 & -14.8 & 9.3 \\
\hline 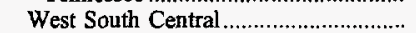 & 86 & 86 & 46 & 58 & 81 & .5 & 1.4 \\
\hline Arkansas & 76 & 76 & 30 & 37 & 81 & 1 & -1.7 \\
\hline 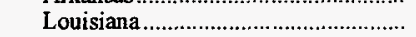 & - & - & - & 21 & - & - & - \\
\hline Oklahoma & 10 & 10 & 16 & - & - & 3.1 & - \\
\hline Mountain & 40 & - & - & - & - & - & - \\
\hline 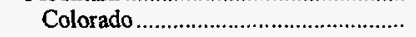 & 40 & - & - & - & - & - & - \\
\hline Indiana & 29,664 & 25,625 & 30,477 & 29,475 & 31,216 & 15.8 & -1.3 \\
\hline 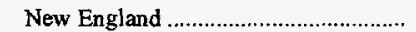 & * & - & $*$ & 21 & - & - & - \\
\hline 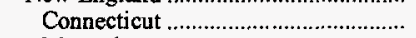 & * & - & $*$ & - & - & - & - \\
\hline 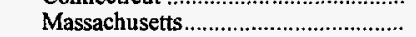 & - & - & - & 21 & - & - & - \\
\hline 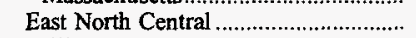 & 26,318 & 22,461 & 27,088 & 26,347 & 27,677 & 17.2 & -1.3 \\
\hline 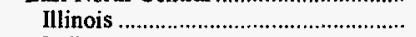 & 1,444 & 963 & 1,597 & 1,562 & 1,021 & 50.0 & 9.1 \\
\hline 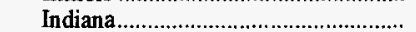 & 24,309 & 21,185 & 24,733 & 23,913 & 24,655 & 14.8 & -.3 \\
\hline 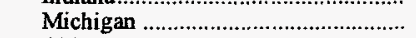 & 181 & 180 & 135 & 184 & 338 & .3 & -14.5 \\
\hline Ohio & 34 & 26 & 36 & 60 & 135 & 30.3 & -29.0 \\
\hline Wisconsin & 350 & 106 & 587 & 628 & 1,528 & 228.4 & -30.8 \\
\hline 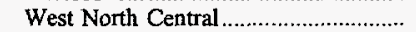 & 655 & 454 & 973 & 1,249 & 756 & 44.1 & -3.5 \\
\hline 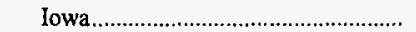 & 638 & 435 & 426 & 646 & 746 & 46.8 & -3.8 \\
\hline 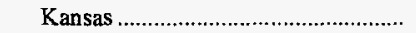 & - & - & - & 18 & - & - & - \\
\hline Minnesota & - & - & 43 & - & - & - & - \\
\hline 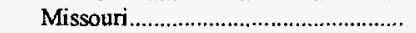 & 17 & 19 & 504 & 585 & 11 & -14.7 & 11.7 \\
\hline 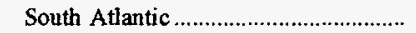 & - & - & 19 & 264 & 548 & - & - \\
\hline Florida & - & - & - & 96 & - & - & - \\
\hline 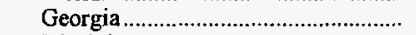 & - & - & 19 & 153 & 548 & - & - \\
\hline Virginia & - & - & - & 15 & - & - & - \\
\hline 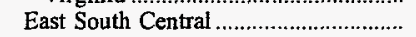 & 2,677 & 2,586 & 2,313 & 1,506 & 2,071 & 3.5 & 6.6 \\
\hline Alabama & 26 & 1 & 56 & 60 & 127 & NM & -32.5 \\
\hline 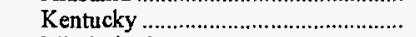 & 2,610 & 2,466 & 2,219 & 1,313 & 1,694 & 5.8 & 11.4 \\
\hline 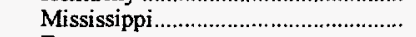 & - & - & - & - & 3 & - & - \\
\hline 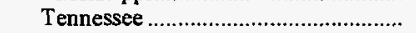 & 41 & 119 & 38 & 132 & 247 & -65.6 & -36.2 \\
\hline 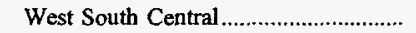 & 6 & 1 & 4 & 1 & - & NM & - \\
\hline Oklahoma & 4 & - & 3 & 1 & - & - & - \\
\hline Texas & 2 & 1 & $*$ & - & - & 140.9 & - \\
\hline Iowa & - & - & 46 & 175 & 287 & - & - \\
\hline 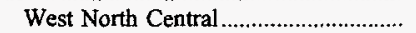 & - & - & 46 & 175 & 287 & - & - \\
\hline 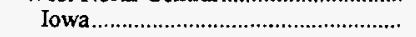 & - & - & 46 & 175 & 287 & - & - \\
\hline 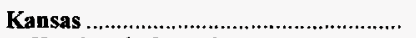 & 245 & 291 & 282 & 345 & 354 & -15.9 & -8.8 \\
\hline 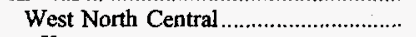 & 233 & 291 & 282 & 345 & 345 & -19.9 & -9.3 \\
\hline 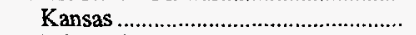 & 164 & 160 & 165 & 167 & 165 & 2.3 & -1 \\
\hline 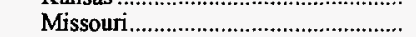 & 69 & 131 & 117 & 178 & 181 & -47.1 & -21.3 \\
\hline 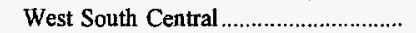 & 12 & - & - & - & - & - & - \\
\hline Oklahoma & 12 & - & - & - & - & - & - \\
\hline Kentucky, Western ................................... & 34,821 & 32,990 & 34,729 & 35,151 & 41,453 & 5.5 & -4.3 \\
\hline 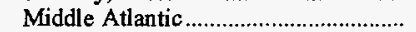 & - & - & $*$ & $*$ & $*$ & - & - \\
\hline Pennsylvania & - & - & $*$ & $*$ & * & - & - \\
\hline East North Central ................................... & 1,016 & 542 & 2,762 & 4,169 & 4,346 & 87.7 & -30.5 \\
\hline 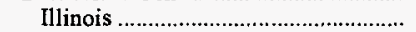 & 119 & - & 192 & 659 & 214 & - & -13.7 \\
\hline Indiana & 680 & 243 & 2,214 & 3,229 & 3,275 & 180.3 & -32.5 \\
\hline 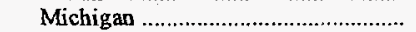 & - & - & 12 & 28 & 8 & - & - \\
\hline Ohio & 31 & 103 & 180 & 146 & 514 & -70.3 & -50.6 \\
\hline Wisconsin & 187 & 196 & 165 & 108 & 336 & -4.7 & -13.7 \\
\hline
\end{tabular}

See footnotes at end of table. 
Table 61. Domestic Distribution of U.S. Coal by Coal-Producing Region and State, and Destination Census Division and State, 1992-1996 (Continued) (Thousand Short Tons)

\begin{tabular}{|c|c|c|c|c|c|c|c|}
\hline \multirow{2}{*}{$\begin{array}{c}\text { Coal-Producing Region and State, } \\
\text { and Destination Census Division } \\
\text { and State }\end{array}$} & \multirow[t]{2}{*}{1996} & \multirow[t]{2}{*}{1995} & \multirow[t]{2}{*}{1994} & \multirow[t]{2}{*}{1993} & \multirow[t]{2}{*}{1992} & \multirow{2}{*}{$\begin{array}{c}\text { Percent } \\
\text { Change } \\
1995-1996\end{array}$} & \multirow{2}{*}{$\begin{array}{c}\begin{array}{c}\text { Average Annua } \\
\text { Percent Change }\end{array} \\
1992-1996\end{array}$} \\
\hline & & & & & & & \\
\hline \multicolumn{8}{|l|}{ Kentucky, Western (Continued) } \\
\hline 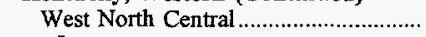 & 237 & 101 & 1,170 & 352 & 896 & 136.0 & -28.3 \\
\hline Iowa & 211 & 75 & 377 & 217 & 271 & 180.3 & -6.1 \\
\hline 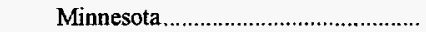 & 22 & 19 & 26 & 32 & 27 & 13.0 & -5.7 \\
\hline 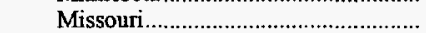 & 5 & 6 & 766 & 102 & 598 & -16.6 & -69.4 \\
\hline 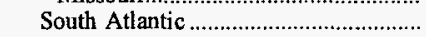 & 3,254 & 2,375 & 2,709 & 3,040 & 5,260 & 37.0 & -11.3 \\
\hline Florida & 3,254 & 2,375 & 2,706 & 3,039 & 3,910 & 37.0 & -4.5 \\
\hline Georgia & - & - & - & - & 1,350 & - & - \\
\hline 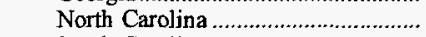 & - & - & * & - & - & - & - \\
\hline 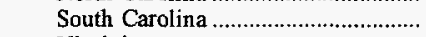 & - & - & * & - & - & - & - \\
\hline 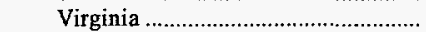 & $*$ & - & - & - & * & - & -40.8 \\
\hline 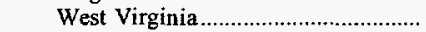 & - & - & $\dot{3}$ & 1 & * & - & - \\
\hline 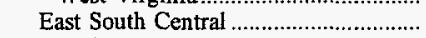 & 29,605 & 29,927 & 28,048 & 27,512 & 30,682 & -1.1 & -.9 \\
\hline Alabama & 3,142 & 1,717 & 2,038 & 1,761 & 2,310 & 83.0 & 8.0 \\
\hline 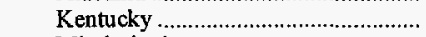 & 16,375 & 17,488 & 16,401 & 17,990 & 17,455 & -6.4 & -1.6 \\
\hline Mississippi & 107 & - & - & -10 & 190 & - & -13.4 \\
\hline 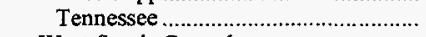 & 9,981 & 10,723 & 9,609 & 7,751 & 10,727 & -6.9 & -1.8 \\
\hline 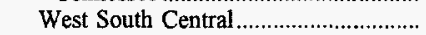 & 657 & 13 & 8 & 12 & 204 & $\mathrm{NM}$ & 34.0 \\
\hline 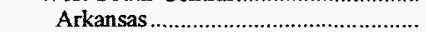 & 11 & 13 & 8 & 12 & 8 & -14.9 & 8.2 \\
\hline 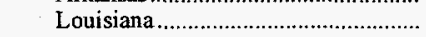 & 646 & - & - & - & 196 & - & 34.8 \\
\hline 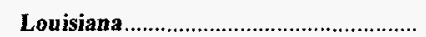 & 3,222 & 3,426 & 3,463 & 3,103 & 3,208 & -6.0 & .1 \\
\hline West North Central & - & - & - & $*$ & - & - & - \\
\hline 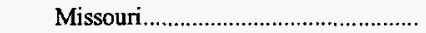 & - & - & - & $*$ & - & - & - \\
\hline West South Central & 3,222 & 3,426 & 3,463 & 3,103 & 3,208 & -6.0 & .1 \\
\hline 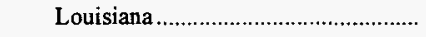 & 3,222 & 3,426 & 3,463 & 3,103 & 3,208 & -6.0 & .1 \\
\hline 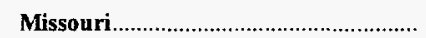 & 846 & 464 & 679 & 638 & 2,795 & 82.3 & -25.8 \\
\hline West North Central & 846 & 464 & 679 & 614 & 2,783 & 82.3 & -25.8 \\
\hline 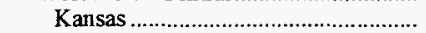 & 345 & 91 & 77 & 23 & 54 & 278.3 & 59.0 \\
\hline 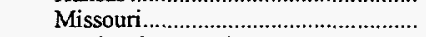 & 501 & 373 & 602 & 592 & 2,729 & 34.3 & -34.6 \\
\hline 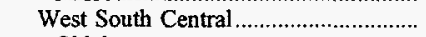 & - & - & - & 2 & - & - & - \\
\hline Oklahoma & - & - & - & 2 & - & - & - \\
\hline Oklahoma & 2,136 & 2,158 & 1,925 & 2,297 & 1,940 & -1.0 & 2.4 \\
\hline 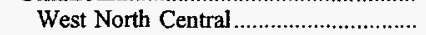 & 63 & 31 & 27 & 57 & 83 & 98.6 & -6.8 \\
\hline 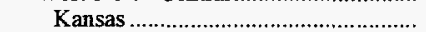 & 63 & 31 & 22 & 41 & 73 & 100.1 & -3.7 \\
\hline Missouri & - & $*$ & 5 & 16 & 10 & -100.0 & -3.8 \\
\hline East South Central & - & 2 & - & - & - & -100.0 & - \\
\hline Kentucky & - & 2 & - & - & - & -100.0 & - \\
\hline West South Central ................................ & 2,066 & 2,121 & 1,896 & 2,240 & 1,857 & -2.6 & 2.7 \\
\hline Arkansas & 170 & 159 & 205 & 196 & 130 & 7.1 & 6.9 \\
\hline Oklahoma & 1,712 & 1,790 & 1,532 & 1,869 & 1,601 & -4.4 & 1.7 \\
\hline 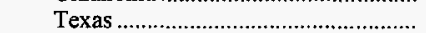 & 184 & 171 & 159 & 175 & 126 & 7.5 & 10.0 \\
\hline 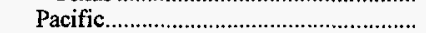 & - & - & $*$ & * & - & - & - \\
\hline 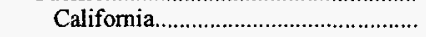 & - & - & * & * & - & - & - \\
\hline 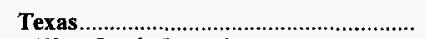 & 49,538 & 52,812 & 52,256 & 54,224 & 54,447 & -6.2 & -2.3 \\
\hline West South Central & 49,538 & 52,812 & 52,256 & 54,224 & 54,447 & -6.2 & -2.3 \\
\hline 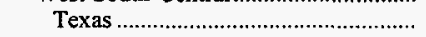 & 49,538 & 52,812 & 52,256 & 54,224 & 54,447 & -6.2 & -2.3 \\
\hline Western Total & 428,297 & 420,132 & 398,402 & 364,083 & 339,676 & 1.9 & 6.0 \\
\hline Alaska & 697 & 815 & 789 & 855 & 797 & -14.5 & -3.3 \\
\hline Pacific & 697 & 815 & 789 & 855 & 797 & -14.5 & -3.3 \\
\hline 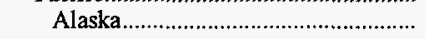 & 697 & 815 & 789 & 855 & 797 & -14.5 & -3.3 \\
\hline 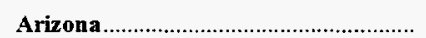 & 10,970 & 11,783 & 12,011 & 12,138 & 12,418 & -6.9 & -3.0 \\
\hline Mountain & 10,970 & 11,783 & 12,011 & 12,138 & 12,418 & -6.9 & -3.0 \\
\hline Arizona & 6,499 & 6,956 & 7,580 & 7,566 & 7,441 & -6.6 & -3.3 \\
\hline 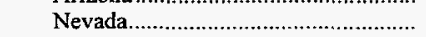 & 4,470 & 4,827 & 4,431 & 4,572 & 4,977 & -7.4 & -2.6 \\
\hline 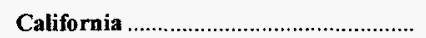 & - & - & - & - & 142 & - & - \\
\hline Pacific & - & - & - & - & 142 & - & - \\
\hline California & - & - & - & - & 142 & - & - \\
\hline Colorado & 23,990 & 24,734 & 24,059 & 20,338 & 18,195 & $-\mathbf{3 . 0}$ & 7.2 \\
\hline East North Central & 1,366 & 2,333 & 2,357 & 2,471 & 1,322 & -41.4 & .8 \\
\hline 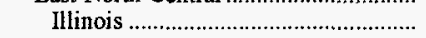 & 640 & 1,628 & 1,439 & 1,246 & 518 & -60.7 & 5.4 \\
\hline
\end{tabular}

See footnotes at end of table. 
Table 61. Domestic Distribution of U.S. Coal by Coal-Producing Region and State, and Destination Census Division and State, 1992-1996 (Continued) (Thousand Short Tons)

\begin{tabular}{|c|c|c|c|c|c|c|c|}
\hline \multirow{2}{*}{$\begin{array}{c}\text { Coal-Producing Region and State, } \\
\text { and Destination Census Division } \\
\text { and State }\end{array}$} & \multirow[t]{2}{*}{1996} & \multirow[t]{2}{*}{1995} & \multirow[t]{2}{*}{1994} & \multirow[t]{2}{*}{1993} & \multirow[t]{2}{*}{1992} & \multirow{2}{*}{$\begin{array}{c}\text { Percent } \\
\text { Change } \\
\text { 1995-1996 }\end{array}$} & \multirow{2}{*}{$\begin{array}{c}\begin{array}{c}\text { Average Annual } \\
\text { Percent Change }\end{array} \\
1992-1996\end{array}$} \\
\hline & & & & & & & \\
\hline \multicolumn{8}{|l|}{ Colorado (Continued) } \\
\hline 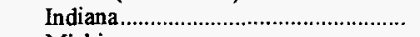 & - & 20 & 457 & 978 & 794 & -100.0 & - \\
\hline Michigan & - & 44 & - & - & - & -100.0 & - \\
\hline Wisconsin & 726 & 641 & 462 & 246 & 10 & 13.3 & 189.8 \\
\hline West North Central ..................................... & 3,218 & 3,109 & 2,194 & 871 & 1,013 & 3.5 & 33.5 \\
\hline Iowa & 591 & 550 & 171 & 135 & 122 & 7.6 & 48.3 \\
\hline Kansas & 1,493 & 1,436 & 1,148 & 90 & 298 & 3.9 & 49.6 \\
\hline 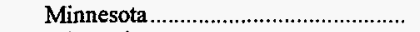 & - & 13 & 23 & 8 & - & -100.0 & - \\
\hline 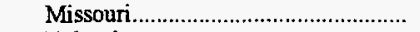 & 1,077 & 1,005 & 775 & 566 & 526 & 7.2 & 19.6 \\
\hline 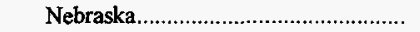 & 56 & 104 & 77 & 72 & 67 & -46.6 & -4.3 \\
\hline South Atlantic & 136 & 811 & 435 & 44 & 181 & -83.2 & -6.9 \\
\hline Florida & 136 & 811 & 423 & - & 181 & -83.2 & -6.9 \\
\hline 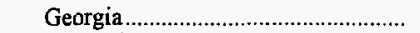 & - & - & 11 & 44 & - & - & - \\
\hline 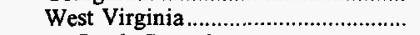 & - & - & 2 & - & - & - & - \\
\hline 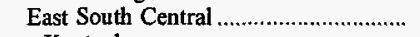 & 3,817 & 2,797 & 2,038 & 711 & - & 36.5 & - \\
\hline 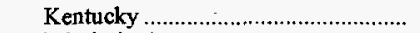 & 260 & 1,098 & 710 & - & - & -76.3 & - \\
\hline Mississippi & 519 & 963 & 735 & 170 & - & -46.1 & - \\
\hline Tennessee & 3,038 & 736 & 593 & 541 & - & 312.6 & - \\
\hline 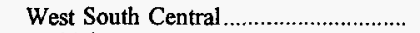 & 2,443 & 2,258 & 2,563 & 2,628 & 2,250 & 8.2 & 2.1 \\
\hline 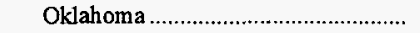 & - & 31 & 26 & 121 & - & -100.0 & - \\
\hline Texas & 2,443 & 2,228 & 2,537 & 2,507 & 2,250 & 9.6 & 2.1 \\
\hline 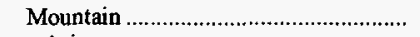 & 12,861 & 13,353 & 14,362 & 13,497 & 13,369 & -3.7 & -1.0 \\
\hline 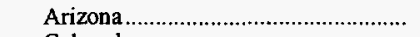 & 355 & 105 & 219 & 117 & 226 & 237.0 & 12.0 \\
\hline Colorado & 10,704 & 11,820 & 12,035 & 11,181 & 11,241 & -9.4 & -1.2 \\
\hline Idaho & - & 3 & - & - & - & -100.0 & - \\
\hline 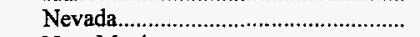 & 132 & 161 & 306 & 514 & 169 & -18.0 & -5.9 \\
\hline 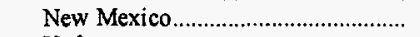 & 88 & 97 & 84 & 84 & 69 & -9.9 & 6.0 \\
\hline 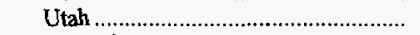 & 1,204 & 1,113 & 1,714 & 1,598 & 1,604 & 8.1 & -6.9 \\
\hline 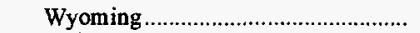 & 378 & 53 & 4 & 3 & 60 & NM & 58.2 \\
\hline Pacific & 131 & 37 & 73 & 80 & 60 & 251.8 & 21.4 \\
\hline Califomia & - & 1 & 22 & 33 & 24 & -100.0 & - \\
\hline Oregon & 94 & - & - & - & - & - & - \\
\hline 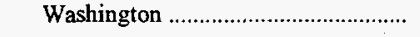 & 37 & 36 & 52 & 47 & 37 & 2.3 & .4 \\
\hline 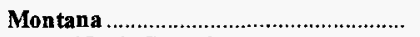 & 37,770 & 39,362 & 41,672 & 35,795 & 38,804 & -4.0 & -.7 \\
\hline East North Central ...................................... & 15,814 & 16,582 & 17,875 & 15,841 & 15,717 & -4.6 & .1 \\
\hline 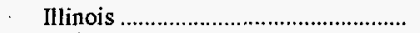 & 2,162 & 2,713 & 4,338 & 3,295 & 3,013 & -20.3 & -8.0 \\
\hline 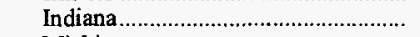 & 869 & 720 & 749 & 433 & 451 & 20.6 & 17.8 \\
\hline 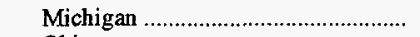 & 9,806 & 11,014 & 10,481 & 10,055 & 10,376 & -11.0 & -1.4 \\
\hline 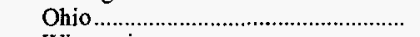 & 26 & - & - & - & - & - & - \\
\hline 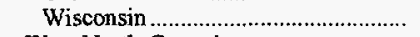 & 2,950 & 2,135 & 2,307 & 2,057 & 1,878 & 38.2 & 12.0 \\
\hline West North Central .................................... & 11,622 & 11,338 & 10,668 & 9,411 & 9,152 & 2.5 & 6.1 \\
\hline 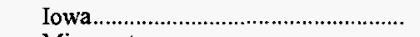 & - & 2 & $*$ & 1 & - & -100.0 & - \\
\hline Minnesota & 9,791 & 10,199 & 10,038 & 8,852 & 8,566 & -4.0 & 3.4 \\
\hline 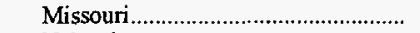 & - & 6 & - & - & - & -100.0 & - \\
\hline 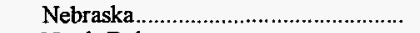 & 113 & 205 & 71 & 136 & 142 & -44.9 & -5.5 \\
\hline 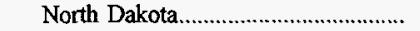 & 417 & 469 & 559 & 422 & 444 & -11.0 & -1.6 \\
\hline 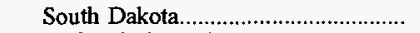 & 1,301 & 457 & - & - & - & 184.6 & - \\
\hline 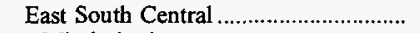 & 2,226 & 1,234 & 1,314 & 178 & 84 & 80.4 & 127.0 \\
\hline Mississippi & 2,226 & 1,234 & 1,314 & 178 & 82 & 80.4 & 128.1 \\
\hline 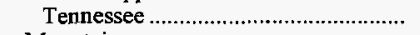 & - & - & - & - & 2 & - & - \\
\hline 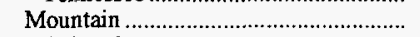 & 7,995 & 9,611 & 10,718 & 9,233 & $11,27 \overline{6}$ & -16.8 & -8.2 \\
\hline 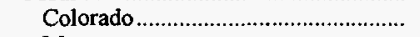 & 26 & 63 & 89 & 86 & 106 & -59.1 & -29.8 \\
\hline 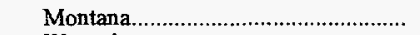 & 7,844 & 9,477 & 10,581 & 9,115 & 11,159 & -17.2 & -8.4 \\
\hline 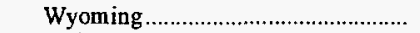 & 125 & 71 & 49 & 31 & 11 & 75.0 & 82.5 \\
\hline 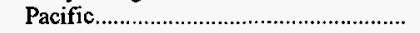 & 113 & 583 & 1,097 & 1,108 & 2,549 & -80.7 & -54.2 \\
\hline Oregon & - & - & - & 355 & 1,835 & - & - \\
\hline 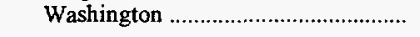 & 113 & 583 & 1,097 & 753 & 715 & -80.7 & -37.0 \\
\hline 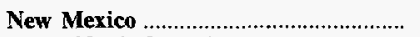 & 25,035 & 25,640 & 28,540 & 27,942 & 24,823 & -2.4 & .2 \\
\hline East North Central ...................................... & 732 & 1,591 & 1,495 & 1,392 & 590 & -54.0 & 5.5 \\
\hline 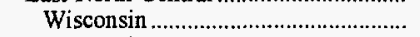 & 732 & $1 ; 591$ & 1,495 & 1,392 & 590 & -54.0 & 5.5 \\
\hline 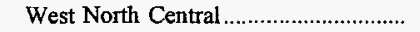 & 92 & - & - & - & - & - & - \\
\hline 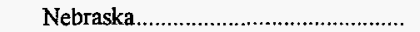 & 92 & - & - & - & - & - & - \\
\hline 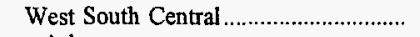 & 334 & 160 & 296 & 350 & 216 & 109.1 & 11.5 \\
\hline Arkansas & 1 & - & - & - & - & - & - \\
\hline Oklahoma & 17 & - & - & 5 & - & - & - \\
\hline 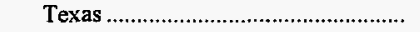 & 316 & 160 & 296 & 345 & 216 & 97.7 & 10.0 \\
\hline
\end{tabular}

See footnotes at end of table. 
Table 61. Domestic Distribution of U.S. Coal by Coal-Producing Region and State, and Destination Census Division and State, 1992-1996 (Continued) (Thousand Short Tons)

\begin{tabular}{|c|c|c|c|c|c|c|c|}
\hline \multirow{2}{*}{$\begin{array}{l}\text { Coal-Producing Region and State, } \\
\text { and Destination Census Division } \\
\text { and State }\end{array}$} & \multirow[t]{2}{*}{1996} & \multirow[t]{2}{*}{1995} & \multirow[t]{2}{*}{1994} & \multirow[t]{2}{*}{1993} & \multirow[t]{2}{*}{1992} & \multirow{2}{*}{$\begin{array}{c}\text { Percent } \\
\text { Change } \\
1995-1996\end{array}$} & \multirow{2}{*}{$\begin{array}{c}\begin{array}{c}\text { Average Annua } \\
\text { Percent Change }\end{array} \\
1992-1996\end{array}$} \\
\hline & & & & & & & \\
\hline \multicolumn{8}{|l|}{ New Mexico (Continued) } \\
\hline 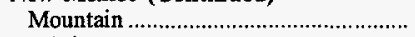 & 23,877 & 23,889 & 26,749 & 26,201 & 24,005 & * & -0.1 \\
\hline 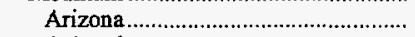 & 8,860 & 9,259 & {$[1,284$} & 11,263 & 9,175 & -4.3 & -9 \\
\hline 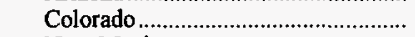 & 9 & - & - & - & - & - & - \\
\hline New Mexico........................................... & 15,009 & 14,630 & 15,464 & 14,938 & 14,829 & 2.6 & .3 \\
\hline 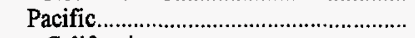 & - & - & - & - & 12 & - & - \\
\hline 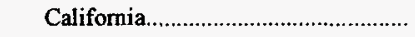 & - & - & - & - & 12 & - & - \\
\hline 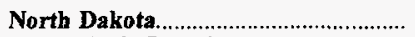 & 30,025 & 30,118 & 32,056 & 32,372 & 31,702 & -.3 & -1.3 \\
\hline 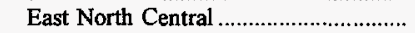 & - & - & * & * & - & - & - \\
\hline 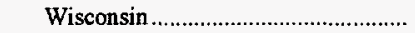 & - & - & $*$ & * & - & - & - \\
\hline West North Central ................................ & 30,025 & 30,113 & 32,055 & 32,367 & 31,702 & -.3 & -1.3 \\
\hline 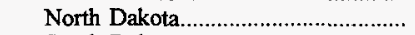 & 30,025 & 28,838 & 29,731 & 30,215 & 29,573 & 4.1 & .4 \\
\hline 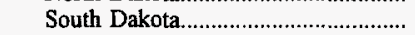 & - & 1,276 & 2,325 & 2,153 & 2,129 & -100.0 & -7 \\
\hline 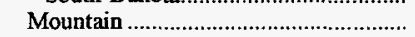 & - & - & - & 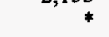 & - & - & - \\
\hline Montana & - & - & - & - & - & - & - \\
\hline Utah & 18,563 & 21,591 & 20,527 & 19,283 & 18,792 & -14.0 & -.3 \\
\hline 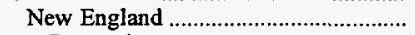 & - & 17 & - & - & - & -100.0 & - \\
\hline 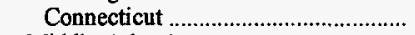 & - & 17 & - & - & - & -100.0 & - \\
\hline 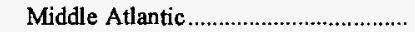 & - & 20 & 68 & - & - & -100.0 & - \\
\hline 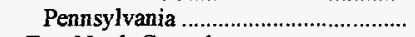 & - & 20 & 68 & - & - & -100.0 & - \\
\hline 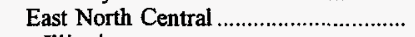 & 2,650 & 1,932 & 656 & 421 & 233 & 37.1 & 83.6 \\
\hline 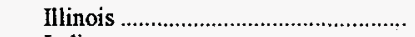 & 2,473 & 1,776 & 369 & 207 & 233 & 39.2 & 80.4 \\
\hline 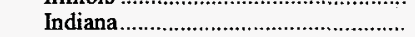 & - & - & 178 & 204 & - & - & - \\
\hline 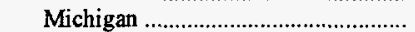 & 44 & 76 & 66 & - & - & -41.8 & - \\
\hline Ohio & - & - & - & * & - & - & - \\
\hline Wisconsin & 133 & 81 & 43 & 10 & - & 65.0 & - \\
\hline 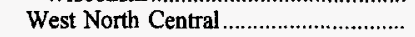 & 330 & 395 & 414 & 382 & 80 & -16.4 & 42.7 \\
\hline 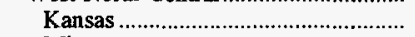 & - & $*$ & 2 & - & - & -100.0 & - \\
\hline Minnesota & - & 1 & - & - & - & -100.0 & - \\
\hline 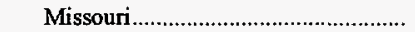 & 330 & 393 & 412 & 382 & 79 & -16.2 & 42.7 \\
\hline 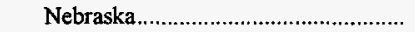 & - & - & - & 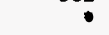 & $*$ & - & - \\
\hline South Atlantic & - & - & - & 159 & 32 & - & - \\
\hline Florida & - & - & - & 155 & 32 & - & - \\
\hline 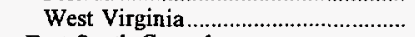 & - & - & - & 4 & - & - & - \\
\hline 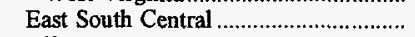 & 1,421 & 1,095 & 218 & - & - & 29.7 & - \\
\hline 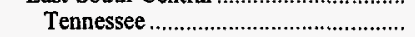 & 1,421 & 1,095 & 218 & - & - & 29.7 & - \\
\hline 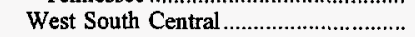 & - & 4 & 33 & - & - & -100.0 & - \\
\hline 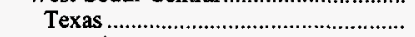 & - & 4 & 33 & - & - & -100.0 & - \\
\hline 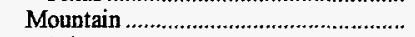 & 11,791 & 15,163 & 15,793 & 15,456 & 15,289 & -22.2 & -6.3 \\
\hline Arizona & 69 & 80 & 86 & 89 & 101 & -13.6 & -9.2 \\
\hline 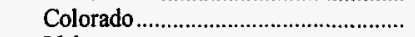 & 2 & 6 & 4 & 14 & 34 & -61.6 & -50.0 \\
\hline 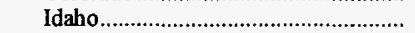 & $6 \overline{5}$ & 141 & 59 & 95 & 61 & -54.1 & 1.8 \\
\hline 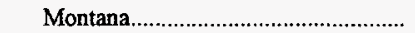 & - & 9 & 29 & 42 & 42 & -100.0 & - \\
\hline 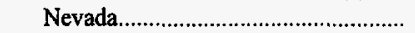 & 2,265 & 2,150 & 2,027 & 1,781 & 1,979 & 5.4 & 3.4 \\
\hline 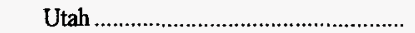 & 9,389 & 12,755 & 13,586 & 13,418 & 13,035 & -26.4 & -7.9 \\
\hline Wyoming & $*$ & 22 & 2 & 18 & 37 & -98.5 & -69.3 \\
\hline Pacific. & 2,366 & 2,965 & 3,317 & 2,843 & 3,143 & -20.2 & -6.8 \\
\hline 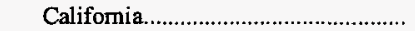 & 2,240 & 2,838 & 3,074 & 2,575 & 2,777 & -21.1 & -5.2 \\
\hline 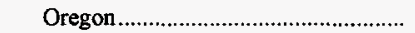 & $*$ & 2 & 127 & 123 & 113 & -71.9 & -75.2 \\
\hline 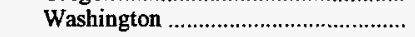 & 125 & $12 \overline{6}$ & 115 & 145 & 253 & -.4 & -16.1 \\
\hline Washington & 4,526 & 4,756 & 4,731 & 4,621 & 5,020 & -4.8 & -2.6 \\
\hline 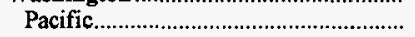 & 4,526 & 4,756 & 4,731 & 4,621 & 5,020 & -4.8 & -2.6 \\
\hline Oregon & 3 & 2 & - & - & - & 19.1 & - \\
\hline 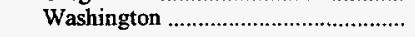 & 4,523 & 4,754 & 4,731 & 4,621 & 5,020 & -4.9 & -2.6 \\
\hline Wyoming & 276,723 & 261,333 & 234,016 & 210,739 & 188,983 & 5.9 & 10.0 \\
\hline New England & - & - & * & 14 & - & - & - \\
\hline 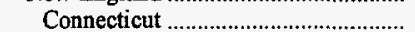 & - & - & * & - & - & - & - \\
\hline 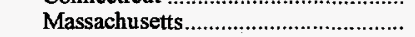 & - & - & - & 14 & - & - & - \\
\hline Middle Atlantic & - & - & - & - & $*$ & - & - \\
\hline 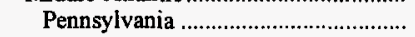 & - & - & - & - & * & - & - \\
\hline 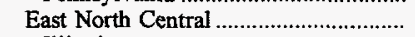 & 62,041 & 55,223 & 42,685 & 37,648 & 30,920 & 12.3 & 19.0 \\
\hline 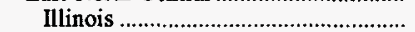 & 17,734 & 15,480 & 9,779 & 7,593 & 4,417 & 14.6 & 41.5 \\
\hline Indiana & 18,079 & 18,306 & 15,417 & 12,635 & 11,804 & -1.2 & 11.3 \\
\hline 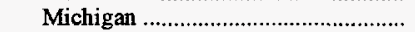 & 8,551 & 7,543 & 4,267 & 4,487 & 2,948 & 13.4 & 30.5 \\
\hline
\end{tabular}

See footnotes at end of table 
Table 61. Domestic Distribution of U.S. Coal by Coal-Producing Region and State, and Destination Census Division and State, 1992-1996 (Continued) (Thousand Short Tons)

\begin{tabular}{|c|c|c|c|c|c|c|c|}
\hline \multirow{2}{*}{$\begin{array}{l}\text { Coal-Producing Region and State, } \\
\text { and Destination Census Division } \\
\text { and State }\end{array}$} & \multirow{2}{*}{1996} & \multirow[t]{2}{*}{1995} & \multirow[t]{2}{*}{1994} & \multirow[t]{2}{*}{1993} & \multirow[t]{2}{*}{1992} & \multirow{2}{*}{$\begin{array}{c}\text { Percent } \\
\text { Change } \\
\text { 1995-1996 }\end{array}$} & \multirow{2}{*}{$\begin{array}{c}\begin{array}{c}\text { Average Annual } \\
\text { Percent Change }\end{array} \\
1992-1996\end{array}$} \\
\hline & & & & & & & \\
\hline \multicolumn{8}{|l|}{ Wyoming (Continued) } \\
\hline Ohio & 37 & - & - & - & 124 & - & -26.2 \\
\hline 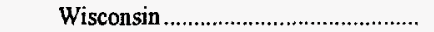 & 17,640 & 13,895 & 13,221 & 12,934 & 11,627 & 27.0 & 11.0 \\
\hline 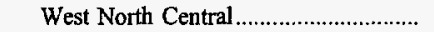 & 82,593 & 77,051 & 67,523 & 61,641 & 52,269 & 7.2 & 12.1 \\
\hline 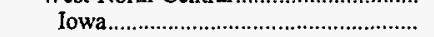 & 18,121 & 16,955 & 15,505 & 15,950 & 14,056 & 6.9 & 6.6 \\
\hline 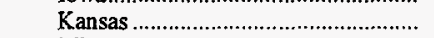 & 11,772 & 14,243 & 16,490 & 16,484 & 13,526 & -17.3 & -3.4 \\
\hline 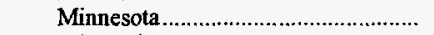 & 8,569 & 8,816 & 9,911 & 9,093 & 8,385 & -2.8 & 5 \\
\hline 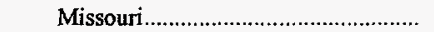 & 33,312 & 25,731 & 16,112 & 10,815 & 7,958 & 29.5 & 43.0 \\
\hline 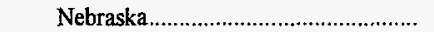 & 10,464 & 10,065 & 8,908 & 8,801 & 7,882 & 4.0 & 7.3 \\
\hline 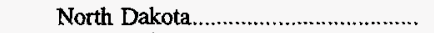 & $*$ & $*$ & - & 5 & 5 & -61.7 & -68.5 \\
\hline 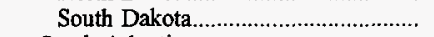 & 355 & 1,241 & 597 & 493 & 456 & -71.4 & -6.1 \\
\hline 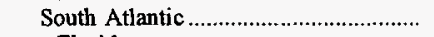 & 7,409 & 7,432 & 5,836 & 938 & 12 & -.3 & 395.6 \\
\hline 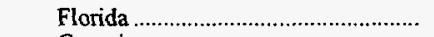 & 591 & - & 93 & - & 12 & - & 163.3 \\
\hline Georgia & 6,818 & 6,796 & 4,914 & 726 & - & .3 & - \\
\hline 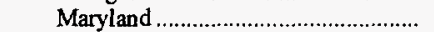 & - & 636 & 829 & 213 & - & -100.0 & - \\
\hline 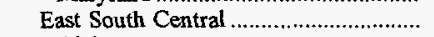 & 4,010 & 2,970 & 594 & 317 & 156 & 35.0 & 125.3 \\
\hline 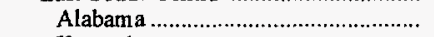 & 3,686 & 2,950 & 251 & - & - & 25.0 & - \\
\hline 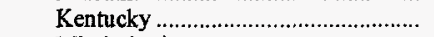 & - & - & - & 248 & 1 & - & - \\
\hline 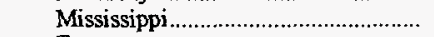 & 26 & - & - & - & 83 & - & -25.0 \\
\hline 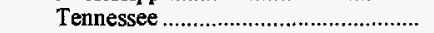 & 298 & 20 & 342 & 68 & 72 & NM & 42.8 \\
\hline West South Central ....................................... & 86,413 & 82,918 & 80,246 & 76,692 & 72,339 & 4.2 & 4.5 \\
\hline 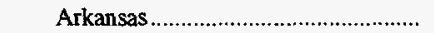 & 14,614 & 14,033 & 12,184 & 10,826 & 11,571 & 4.1 & 6.0 \\
\hline 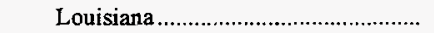 & 9,209 & 10,309 & 11,215 & 11,133 & 10,039 & -10.7 & -2.1 \\
\hline Oklahoma & 19,751 & 20,326 & 17,577 & 16,726 & 16,733 & -2.8 & 4.2 \\
\hline 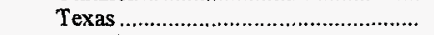 & 42,839 & 38,250 & 39,270 & 38,007 & 33,995 & 12.0 & 5.9 \\
\hline 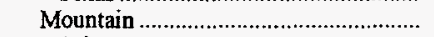 & 33,363 & 32,950 & 34,935 & 32,195 & 33,165 & 1.3 & .1 \\
\hline 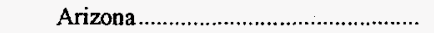 & - & - & - & 5 & - & - & - \\
\hline Colorado & 6,124 & 5,602 & 5,132 & 5,538 & 4,952 & 9.3 & 5.4 \\
\hline 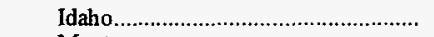 & 268 & 293 & 337 & 343 & 393 & -8.4 & -9.1 \\
\hline 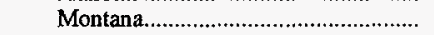 & 513 & 193 & 119 & 37 & 44 & 165.6 & 84.6 \\
\hline 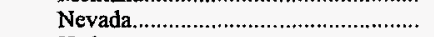 & 204 & 342 & 1,014 & 753 & 802 & -40.3 & -29.0 \\
\hline Utah & 1 & $*$ & - & - & 586 & $\mathrm{NM}$ & -78.9 \\
\hline 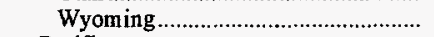 & 26,253 & 26,521 & 28,334 & 25,519 & 26,388 & -1.0 & -.1 \\
\hline 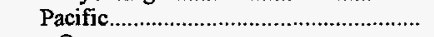 & 894 & 2,775 & 2,198 & 1,271 & 120 & -67.8 & 65.3 \\
\hline Oregon & 894 & 1,485 & 2,197 & 1,270 & 114 & -39.8 & 67.2 \\
\hline 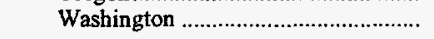 & 1 & 1,290 & 1 & 1 & 6 & -99.9 & -39.4 \\
\hline U.S. Total & 967,693 & 940,423 & 949,843 & $\mathbf{8 8 3 , 9 3 4}$ & 897,267 & 2.9 & 1.9 \\
\hline 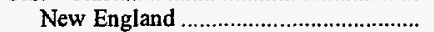 & 5,334 & 5,199 & 4,963 & 4,141 & 7,717 & 2.6 & -8.8 \\
\hline 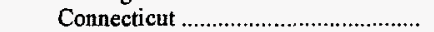 & 1,602 & 1,955 & 976 & 695 & 1,003 & -18.1 & 12.4 \\
\hline Maine & 301 & 302 & 463 & 405 & 866 & -.4 & -23.2 \\
\hline 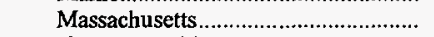 & 2,475 & 2,157 & 2,538 & 1,840 & 4,634 & 14.8 & -14.5 \\
\hline 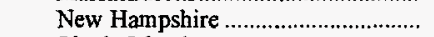 & 951 & 780 & 978 & 1,192 & 1,189 & 21.9 & -5.4 \\
\hline 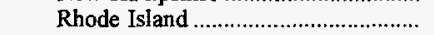 & 3 & 3 & 3 & 3 & 5 & 21.2 & -9.6 \\
\hline 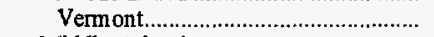 & 2 & 3 & 5 & 6 & 20 & -38.2 & -45.8 \\
\hline 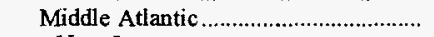 & 73,489 & 70,149 & 69,737 & 64,421 & 73,087 & 4.8 & .1 \\
\hline 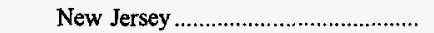 & 2,927 & 2,936 & 2,163 & 2,169 & 2,433 & -.3 & 4.7 \\
\hline 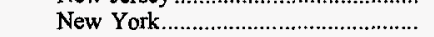 & 11,459 & 10,705 & 11,664 & 10,950 & 13,977 & 7.0 & -4.8 \\
\hline 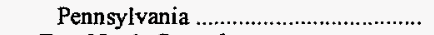 & 59,103 & 56,509 & 55,910 & 51,302 & 56,677 & 4.6 & 1.0 \\
\hline 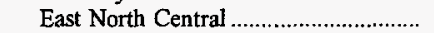 & 228,473 & 212,105 & 214,903 & 196,343 & 201,713 & 7.7 & 3.2 \\
\hline 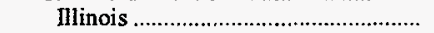 & 44,547 & 41,626 & 38,745 & 34,433 & 31,186 & 7.0 & 9.3 \\
\hline 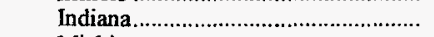 & 62,741 & 59,476 & 63,589 & 54,452 & 60,970 & 5.5 & .7 \\
\hline 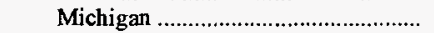 & 33,186 & 33,928 & 33,487 & 30,041 & 30,363 & -2.2 & 2.3 \\
\hline Ohio & 61,713 & 54,310 & 56,850 & 56,755 & 58,716 & 13.6 & 1.3 \\
\hline 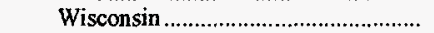 & 26,285 & 22,766 & 22,231 & 20,662 & 20,477 & 15.5 & 6.4 \\
\hline 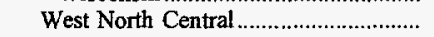 & 136,573 & 130,836 & 126,407 & 116,337 & 113,921 & 4.4 & 4.6 \\
\hline lowa & 21,017 & 19,777 & 18,259 & 18,950 & 16,988 & 6.3 & 5.5 \\
\hline 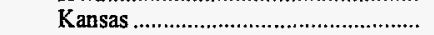 & 13,987 & 16,091 & 18,097 & 17,002 & 14,801 & -13.1 & -1.4 \\
\hline 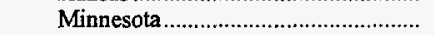 & 18,679 & 19,488 & 20,498 & 18,166 & 17,224 & -4.1 & 2.0 \\
\hline 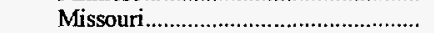 & 40,064 & 32,821 & 27,278 & 19,921 & 24,207 & 22.1 & 13.4 \\
\hline 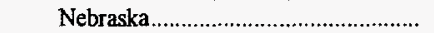 & 10,726 & 10,377 & 9,055 & 9,011 & 8,093 & 3.4 & 7.3 \\
\hline 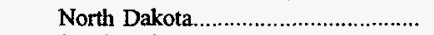 & 30,444 & 29,307 & 30,290 & 30,642 & 30,022 & 3.9 & .3 \\
\hline 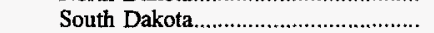 & 1,657 & 2,976 & 2,929 & 2,646 & 2,586 & -44.3 & -10.5 \\
\hline 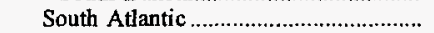 & 161,026 & 151,954 & 161,029 & 141,701 & 145,219 & 6.0 & 2.6 \\
\hline 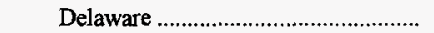 & 1,834 & 1,933 & 2,309 & 2,242 & 1,913 & -5.1 & -1.0 \\
\hline District of Columbia & 23 & 6 & 47 & 51 & 50 & 309.2 & -17.5 \\
\hline Florida & 26,285 & 23,505 & 23,757 & 21,446 & 24,351 & 11.8 & 1.9 \\
\hline Georgia & 29,074 & 29,730 & 30,497 & 25,101 & 24,242 & -2.2 & 4.6 \\
\hline
\end{tabular}

See footnotes at end of table. 
Table 61. Domestic Distribution of U.S. Coal by Coal-Producing Region and State, and Destination Census Division and State, 1992-1996 (Continued) (Thousand Short Tons)

\begin{tabular}{|c|c|c|c|c|c|c|c|}
\hline \multirow{2}{*}{$\begin{array}{c}\text { Coal-Producing Region and State, } \\
\text { and Destination Census Division } \\
\text { and State }\end{array}$} & \multirow[t]{2}{*}{1996} & \multirow[t]{2}{*}{1995} & \multirow[t]{2}{*}{1994} & \multirow[t]{2}{*}{1993} & \multirow[t]{2}{*}{1992} & \multirow{2}{*}{$\begin{array}{c}\text { Percent } \\
\text { Change } \\
\text { 1995-1996 }\end{array}$} & \multirow{2}{*}{$\begin{array}{c}\begin{array}{c}\text { Average Annual } \\
\text { Percent Change }\end{array} \\
1992-1996\end{array}$} \\
\hline & & & & & & & \\
\hline \multicolumn{8}{|l|}{ U.S. Total (Continued) } \\
\hline 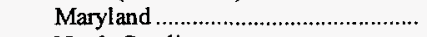 & 10,762 & 10,596 & 10,959 & 10,063 & 9,735 & 1.6 & 2.5 \\
\hline 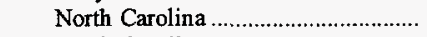 & 27,414 & 24,149 & 26,199 & 25,621 & 25,801 & 13.5 & 1.5 \\
\hline 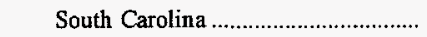 & 13,373 & 11,745 & 13,454 & 11,749 & 11,338 & 13.9 & 4.2 \\
\hline 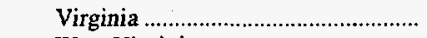 & 16,214 & 15,225 & 16,735 & 16,084 & 14,915 & 6.5 & 2.1 \\
\hline 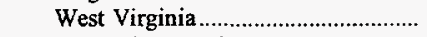 & 36,047 & 35,065 & 37,071 & 29,343 & 32,874 & 2.8 & 2.3 \\
\hline East South Central ................................ & 105,708 & 104,194 & 99,350 & 97,057 & 92,761 & 1.4 & 3.3 \\
\hline Alabama & 33,057 & 31,440 & 30,210 & 28,698 & 28,741 & 5.1 & 3.6 \\
\hline 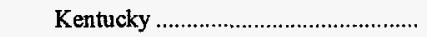 & 34,438 & 37,382 & 35,636 & 35,461 & 33,497 & -7.9 & .7 \\
\hline 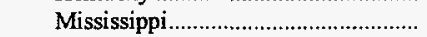 & 5,702 & 4,516 & 4,446 & 3,421 & 3,965 & 26.3 & 9.5 \\
\hline 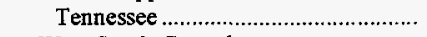 & 32,510 & 30,856 & 29,057 & 29,477 & 26,559 & 5.4 & 5.2 \\
\hline West South Central & 145,394 & 144,435 & 142,136 & 139,664 & 134,770 & .7 & 1.9 \\
\hline 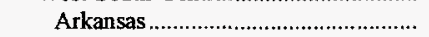 & 14,918 & 14,317 & 12,501 & 11,094 & 11,807 & 4.2 & 6.0 \\
\hline 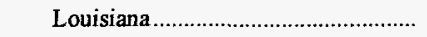 & 13,526 & 14,267 & 15,844 & 14,524 & 13,539 & -5.2 & $*$ \\
\hline 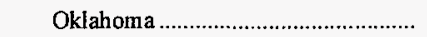 & 21,584 & 22,210 & 19,210 & 18,777 & 18,362 & -2.8 & 4.1 \\
\hline Texas & 95,367 & 93,642 & 94,581 & 95,269 & 91,062 & 1.8 & 1.2 \\
\hline Mountain & 101,497 & 107,497 & 115,311 & 109,200 & 110,007 & -5.6 & -2.0 \\
\hline 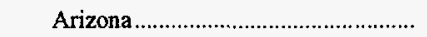 & 15,785 & 16,401 & 19,169 & 19,039 & 16,944 & -3.8 & -1.8 \\
\hline Colorado & 16,920 & 17,502 & 17,274 & 16,821 & 16,333 & -3.3 & .9 \\
\hline 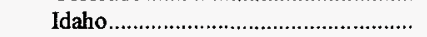 & 333 & 440 & 400 & 438 & 456 & -24.2 & -7.5 \\
\hline 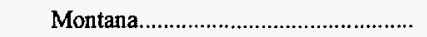 & 8,359 & 9,678 & 10,729 & 9,194 & 11,245 & -13.6 & -7.1 \\
\hline 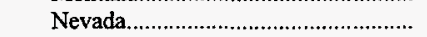 & 7,072 & 7,479 & 7,777 & 7,621 & 7,926 & -5.4 & -2.8 \\
\hline 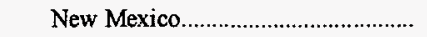 & 15,097 & 14,727 & 15,549 & 15,022 & 14,899 & 2.5 & .3 \\
\hline Utah & 11,175 & 14,602 & 16,015 & 15,493 & 15,707 & -23.5 & -8.2 \\
\hline 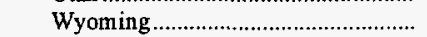 & 26,757 & 26,668 & 28,399 & 25,571 & 26,497 & 3 & .2 \\
\hline 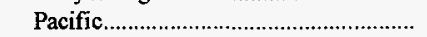 & 8,790 & 11,962 & 12,228 & 10,791 & 11,844 & -26.5 & -7.2 \\
\hline Alaska & 697 & 815 & 789 & 855 & 797 & -14.5 & -3.3 \\
\hline 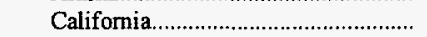 & 2,240 & 2,839 & 3,096 & 2,608 & 2,955 & -21.1 & -6.7 \\
\hline 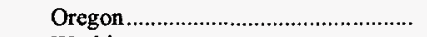 & 1,028 & 1,518 & 2,347 & 1,761 & 2,062 & -32.3 & -16.0 \\
\hline Washington & 4,825 & 6,790 & 5,996 & 5,567 & 6,030 & -28.9 & -5.4 \\
\hline
\end{tabular}

* Data round to zero.

NM Not meaningful as value is greater than 500 percent.

Note: Totals may not equal sum of components due to independent rounding.

Source: Energy Information Administration, Form EIA-6, "Coal Distribution Report." 
Table 62. Foreign Distribution of U.S. Coal by Major Coal-Exporting States and Destination, 1992-1996

(Thousand Short Tons)

\begin{tabular}{|c|c|c|c|c|c|c|c|}
\hline \multirow{2}{*}{$\begin{array}{l}\text { Coal-Exporting State } \\
\text { and Destination }\end{array}$} & \multirow{2}{*}{1996} & \multirow{2}{*}{1995} & \multirow{2}{*}{1994} & \multirow{2}{*}{1993} & \multirow{2}{*}{1992} & \multirow{2}{*}{$\begin{array}{l}\text { Percent } \\
\text { Change } \\
\text { 1995-1996 }\end{array}$} & \multirow{2}{*}{$\begin{array}{c}\begin{array}{c}\text { Average Annual } \\
\text { Percent Change }\end{array} \\
1992-1996\end{array}$} \\
\hline & & & & & & & \\
\hline Alabama & 4,864 & 6,032 & 4,529 & 5,888 & 5,931 & -19.4 & -4.8 \\
\hline Albania & - & - & - & - & 21 & - & - \\
\hline Argentina & 216 & 306 & 268 & 319 & 157 & -29.5 & 8.3 \\
\hline Belgium \& Luxembourg ............................. & 703 & 574 & 627 & 866 & 861 & 22.3 & -5.0 \\
\hline 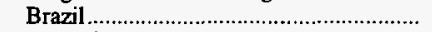 & 566 & 564 & 42 & 193 & - & .3 & - \\
\hline 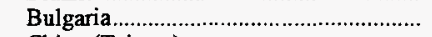 & 208 & 128 & 35 & 109 & - & 63.2 & - \\
\hline 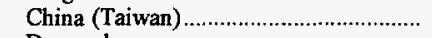 & - & - & - & 42 & 45 & - & - \\
\hline 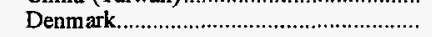 & - & 26 & - & - & - & -100.0 & - \\
\hline 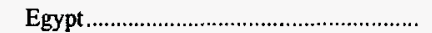 & - & 111 & - & - & - & -100.0 & - \\
\hline 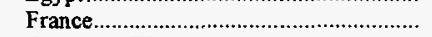 & - & - & $*$ & - & - & - & - \\
\hline Germany, FR & 184 & 201 & - & - & - & -8.0 & - \\
\hline Italy & 659 & 930 & 565 & 429 & 506 & -29.2 & 6.8 \\
\hline Japan & 861 & 1,358 & 1,266 & 2,202 & 2,343 & -36.6 & -22.1 \\
\hline Morocco & - & - & - & 39 & - & - & - \\
\hline 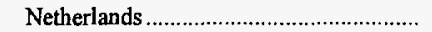 & 73 & 276 & 88 & - & - & -73.4 & - \\
\hline Romania & 170 & 492 & 602 & 171 & 183 & -65.5 & -2.0 \\
\hline 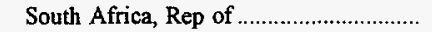 & 57 & - & - & - & 212 & - & -28.0 \\
\hline Spain & 52 & 48 & 27 & - & - & 8.4 & - \\
\hline 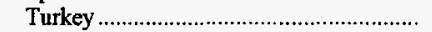 & 326 & 302 & 137 & 253 & 424 & 8.0 & -6.4 \\
\hline 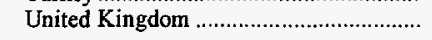 & 789 & 717 & 872 & 1,266 & 1,179 & 10.1 & -9.6 \\
\hline 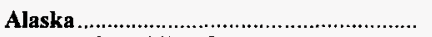 & 776 & 855 & 716 & 743 & 734 & -9.2 & 1.4 \\
\hline 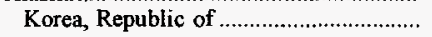 & 776 & 855 & 716 & 743 & 734 & -9.2 & 1.4 \\
\hline Colorado & 1,415 & 900 & 752 & 1,128 & 669 & $\mathbf{5 7 . 2}$ & 20.6 \\
\hline China (Taiwan) & 219 & 235 & 134 & - & - & -6.5 & - \\
\hline 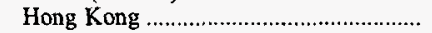 & - & - & 46 & - & - & - & - \\
\hline 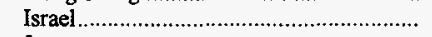 & 30 & - & - & - & - & - & - \\
\hline Japan & 343 & 651 & 395 & 918 & 669 & -47.3 & -15.3 \\
\hline Korea, Republic of & 65 & - & 177 & 209 & - & - & - \\
\hline Mexico & 758 & - & - & - & - & - & - \\
\hline 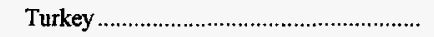 & - & 14 & - & - & - & -100.0 & - \\
\hline Illinois . . & 1,886 & 2,699 & 236 & 670 & 1,242 & -30.1 & 11.0 \\
\hline Belgium \& Luxembourg ............................ & 76 & - & - & - & 58 & - & 6.9 \\
\hline 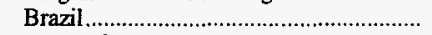 & 1 & - & - & - & 298 & - & -74.2 \\
\hline Denmark & 364 & 516 & - & - & 45 & -29.4 & 68.2 \\
\hline 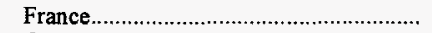 & - & 57 & - & - & - & -100.0 & - \\
\hline 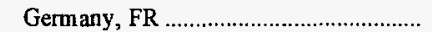 & 325 & 722 & - & - & - & -54.9 & - \\
\hline Ireland & - & - & - & 108 & 213 & - & - \\
\hline Italy & - & 42 & - & - & - & -100.0 & - \\
\hline Japan & 66 & 49 & 236 & 109 & 53 & 35.0 & 5.8 \\
\hline Korea, Republic of & - & - & - & - & 50 & - & - \\
\hline Morocco & 103 & 775 & - & 452 & 350 & -86.7 & -26.3 \\
\hline 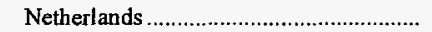 & 120 & - & - & - & - & - & - \\
\hline Sweden & 25 & - & - & - & - & - & - \\
\hline 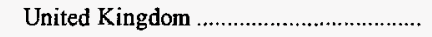 & 805 & 538 & - & - & 175 & 49.7 & 46.4 \\
\hline Kentucky & 9,143 & 9,695 & 7,167 & 9,521 & 14,036 & -5.7 & -10.2 \\
\hline 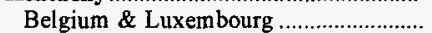 & 67 & 366 & 472 & 493 & 1,242 & -81.7 & -51.8 \\
\hline 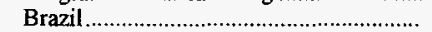 & - & 52 & 23 & 392 & 98 & -100.0 & - \\
\hline Canada & 1,178 & 777 & 1,099 & 1,416 & 2,078 & 51.5 & -13.2 \\
\hline China (Taiwan) & 1,978 & 2,397 & 2,643 & 3,625 & 3,333 & -17.5 & -12.2 \\
\hline 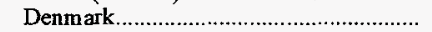 & - & - & - & 33 & 172 & - & - \\
\hline Finland & 4 & - & - & 2 & - & - & - \\
\hline France. & 548 & 262 & 146 & $66 \overline{5}$ & 1,812 & 108.6 & -25.9 \\
\hline 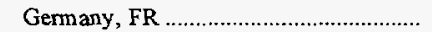 & - & 187 & - & - & - & -100.0 & - \\
\hline 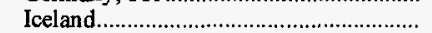 & 119 & 76 & 7 & - & 31 & 56.3 & 40.0 \\
\hline Ireland & - & 58 & - & 16 & 336 & -100.0 & - \\
\hline Israel & - & 217 & - & - & - & -100.0 & - \\
\hline 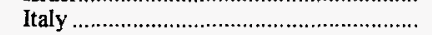 & 1,745 & 1,714 & 805 & 719 & 805 & 1.8 & 21.3 \\
\hline 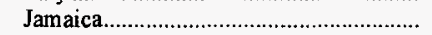 & 17 & 62 & 26 & 39 & 35 & -73.0 & -16.4 \\
\hline Japan & 93 & 53 & 100 & 269 & 303 & 74.0 & -25.6 \\
\hline 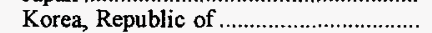 & 1,876 & 1,523 & 1,163 & 1,256 & 1,191 & 23.2 & 12.0 \\
\hline 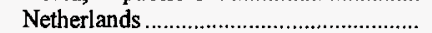 & 581 & 621 & 268 & 241 & 1,312 & -6.5 & -18.4 \\
\hline 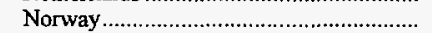 & 140 & 142 & 74 & 91 & 98 & -1.4 & 9.3 \\
\hline 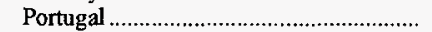 & 229 & - & 24 & - & - & - & - \\
\hline 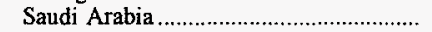 & 22 & - & - & - & - & - & - \\
\hline Spain & - & 231 & - & - & 98 & -100.0 & - \\
\hline
\end{tabular}

See footnotes at end of table 
Table 62. Foreign Distribution of U.S. Coal by Major Coal-Exporting States and Destination, 1992-1996 (Continued)

(Thousand Short Tons)

\begin{tabular}{|c|c|c|c|c|c|c|c|}
\hline \multirow{2}{*}{$\begin{array}{l}\text { Coal-Exporting State } \\
\text { and Destination }\end{array}$} & \multirow[t]{2}{*}{1996} & \multirow[t]{2}{*}{1995} & \multirow[t]{2}{*}{1994} & \multirow[t]{2}{*}{1993} & \multirow[t]{2}{*}{1992} & \multirow{2}{*}{$\begin{array}{c}\text { Percent } \\
\text { Change } \\
1995-1996\end{array}$} & $\begin{array}{l}\text { Average Annual } \\
\text { Percent Change }\end{array}$ \\
\hline & & & & & & & 1992-1996 \\
\hline \multicolumn{8}{|l|}{ Kentucky (Continued) } \\
\hline Sweden . & - & - & 16 & 195 & 456 & - & - \\
\hline 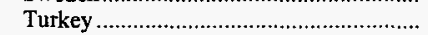 & - & 197 & - & - & 151 & -100.0 & - \\
\hline United Kingdom ………………………........... & 548 & 758 & 301 & 70 & 447 & -27.7 & 5.2 \\
\hline 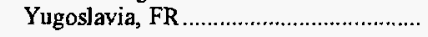 & - & - & - & - & 38 & - & - \\
\hline Pennsylvania & 9,246 & $\mathbf{8 , 2 7 9}$ & 6,301 & 5,508 & 6,440 & 11.7 & 9.5 \\
\hline Argentina & - & - & - & 46 & - & - & - \\
\hline 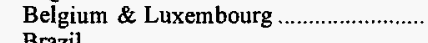 & - & - & 29 & - & 90 & - & - \\
\hline & 261 & 380 & 338 & 156 & 148 & -31.3 & 15.1 \\
\hline & 1050 & $7 \overline{3}$ & $\overrightarrow{844}$ & 71 & 73 & - & - \\
\hline & 1,050 & 713 & 844 & 597 & 1,599 & 47.4 & -10.0 \\
\hline $\begin{array}{l}\text { Chile } \\
\text { Costa Rica }\end{array}$ & - & - & - & - & 37 & - & - \\
\hline & - & - & - & $\overline{159}$ & * & - & - \\
\hline & $8 \overline{1}$ & & $50 \overline{0}$ & 159 & - & - & - \\
\hline & $\begin{array}{r}801 \\
50\end{array}$ & $\begin{array}{r}1,589 \\
18\end{array}$ & $\begin{array}{r}508 \\
65\end{array}$ & 187 & 1,441 & -49.6 & -13.7 \\
\hline & $\begin{array}{r}50 \\
283\end{array}$ & 18 & 65 & 59 & 157 & 172.7 & -24.8 \\
\hline & 283 & 544 & 71 & 56 & - & -48.1 & - \\
\hline 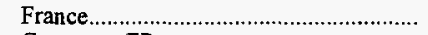 & 89 & 9 & - & 188 & 184 & NM & -16.7 \\
\hline Germany, FR & 256 & 383 & 197 & 102 & 151 & -33.3 & 14.0 \\
\hline Greece & 491 & - & - & - & - & - & - \\
\hline Indonesia & - & & - & - & * & - & - \\
\hline Ireland & 1,067 & 1,161 & 1,015 & 911 & - & -8.0 & - \\
\hline 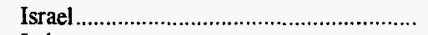 & 1,068 & 995 & 922 & 940 & 1,090 & 7.4 & -.5 \\
\hline Italy & 89 & - & 601 & 50 & - & - & - \\
\hline Japan & 1,057 & 916 & 834 & 1,384 & 921 & 15.3 & 3.5 \\
\hline 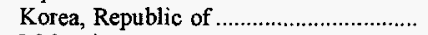 & 195 & 109 & 214 & 91 & - & 78.4 & - \\
\hline Malaysia & - & - & - & - & $*$ & - & - \\
\hline Mexico & - & - & - & - & 18 & - & - \\
\hline Morocco & 173 & - & - & - & - & - & - \\
\hline 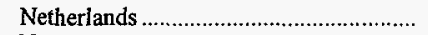 & 732 & 593 & 261 & - & 2 & 23.5 & 349.7 \\
\hline Norway & 30 & 28 & 14 & 30 & 48 & 5.1 & -11.5 \\
\hline 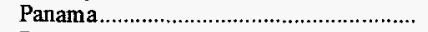 & - & - & - & - & * & - & - \\
\hline 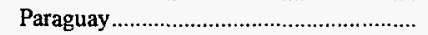 & - & - & - & - & 3 & - & - \\
\hline 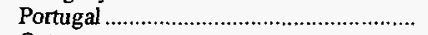 & 592 & 472 & 378 & 467 & 302 & 25.5 & 18.3 \\
\hline 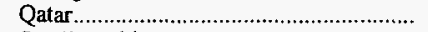 & - & - & - & - & * & - & - \\
\hline 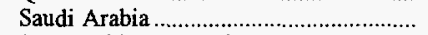 & - & - & - & * & - & - & - \\
\hline South Africa, Rep of & 112 & - & - & - & - & - & - \\
\hline Spain & - & 18 & - & - & - & -100.0 & - \\
\hline 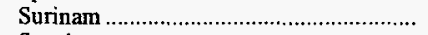 & - & - & - & * & * & - & - \\
\hline Sweden & - & - & - & - & 1 & - & - \\
\hline Trinidad \& Tobago & - & - & - & 1 & 2 & - & - \\
\hline 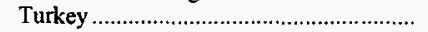 & - & 43 & - & - & - & -100.0 & - \\
\hline 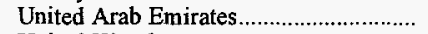 & - & - & - & * & - & - & - \\
\hline United Kingdom ................................................. & 851 & 299 & - & * & - & 184.9 & - \\
\hline Venezuela.................... & 1 & 9 & 11 & 15 & 39 & -88.6 & -59.5 \\
\hline 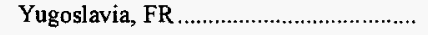 & - & - & - & - & 132 & - & - \\
\hline Utah & 5,305 & 3,930 & 2,698 & 2,959 & 2,260 & 35.0 & 23.8 \\
\hline Canada & - & - & - & 346 & - & - & - \\
\hline Chile & 445 & 170 & - & - & - & 162.1 & - \\
\hline 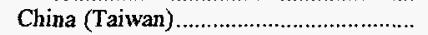 & 648 & 323 & 321 & 849 & 721 & 100.4 & -2.6 \\
\hline 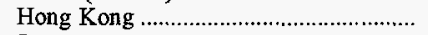 & - & - & & & 359 & - & \\
\hline 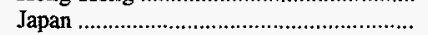 & 4,058 & 3,000 & 2,377 & 1,764 & 1,180 & 35.3 & 36.2 \\
\hline Korea, Republic of .............................. & 154 & 438 & - & - & - & -64.7 & - \\
\hline Mexico & - & - & - & * & - & - & - \\
\hline Virginia & 13,432 & $\mathbf{9 , 7 4 2}$ & 11,683 & 14,251 & 17,224 & 37.9 & -6.0 \\
\hline Algeria & 206 & 166 & 269 & 344 & 534 & 24.1 & -21.2 \\
\hline Argentina & & - & 53 & 52 & & - & \\
\hline Belgium \& Luxembourg ............................... & 1,078 & 764 & 884 & 1,147 & 2,284 & 41.2 & -17.1 \\
\hline 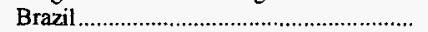 & 1,228 & 1,091 & 1,218 & 1,741 & 2,252 & 12.5 & -14.1 \\
\hline Bulgaria & - & - & - & 242 & 172 & - & - \\
\hline 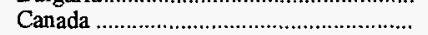 & 387 & 445 & 786 & 1,229 & 1,331 & -13.0 & -26.5 \\
\hline 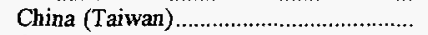 & - & - & 15 & $*$ & - & - & - \\
\hline 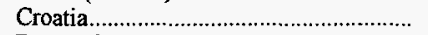 & - & - & - & 251 & - & - & - \\
\hline 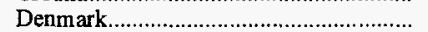 & - & - & - & - & 60 & - & - \\
\hline Egypt & 835 & 333 & 436 & 234 & 239 & 150.7 & 36.8 \\
\hline
\end{tabular}

See footnotes at end of table. 
Table 62. Foreign Distribution of U.S. Coal by Major Coal-Exporting States and Destination, 1992-1996 (Continued)

(Thousand Short Tons)

\begin{tabular}{|c|c|c|c|c|c|c|c|}
\hline \multirow{2}{*}{$\begin{array}{l}\text { Coal-Exporting State } \\
\text { and Destination }\end{array}$} & \multirow{2}{*}{1996} & \multirow{2}{*}{1995} & \multirow{2}{*}{1994} & \multirow{2}{*}{1993} & \multirow{2}{*}{1992} & \multirow{2}{*}{$\begin{array}{c}\text { Percent } \\
\text { Change } \\
1995-1996\end{array}$} & \multirow{2}{*}{$\begin{array}{c}\begin{array}{l}\text { Average Annual } \\
\text { Percent Change }\end{array} \\
1992-1996\end{array}$} \\
\hline & & & & & & & \\
\hline \multicolumn{8}{|l|}{ Virginia (Continued) } \\
\hline 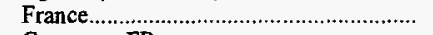 & 910 & 625 & 563 & 737 & 915 & 45.7 & -0.1 \\
\hline Germany, FR & - & 68 & 9 & 50 & 37 & -100.0 & - \\
\hline Italy & 2,198 & 1,474 & 1,804 & 1,364 & 1,345 & 49.1 & 13.1 \\
\hline 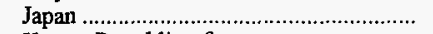 & 2,300 & 1,796 & 2,114 & 2,410 & 2,754 & 28.0 & -4.4 \\
\hline Korea, Republic of & 466 & 589 & 539 & 853 & 1,244 & -20.9 & -21.8 \\
\hline 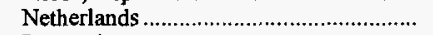 & 1,193 & 793 & 766 & 1,009 & 1,192 & 50.4 & * \\
\hline 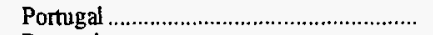 & 145 & 105 & 91 & - & 284 & 38.0 & -15.5 \\
\hline Romania & 32 & - & 223 & - & 145 & - & -31.3 \\
\hline South Africa, Rep of & 76 & - & - & - & - & - & - \\
\hline Spain & 1,370 & 847 & 1,297 & 1,682 & 1,339 & 61.7 & 6 \\
\hline Sweden & 185 & 115 & 37 & - & - & 60.9 & - \\
\hline 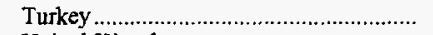 & 24 & - & - & - & - & - & - \\
\hline 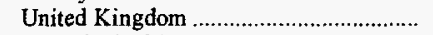 & 798 & 531 & 579 & 905 & 1,054 & 50.3 & -6.7 \\
\hline Yugoslavia, FR & - & - & - & - & 44 & - & - \\
\hline West Virginia & 42,044 & 44,321 & 36,205 & 33,159 & 50,806 & -5.1 & -4.6 \\
\hline 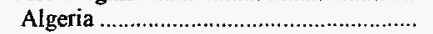 & - & - & $*$ & - & - & - & - \\
\hline Argentina & - & - & 35 & 132 & 169 & - & - \\
\hline 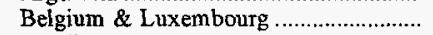 & 2,182 & 2,020 & 2,312 & 2,209 & 1,785 & 8.0 & 5.1 \\
\hline 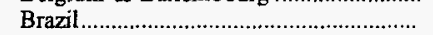 & 4,256 & 4,329 & 4,109 & 2,496 & 2,907 & -1.7 & 10.0 \\
\hline Bulgaria & 1,214 & 1,360 & 1,571 & 644 & 619 & -10.8 & 18.3 \\
\hline 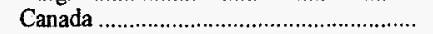 & 7,222 & 5,784 & 5,644 & 4,108 & 8,911 & 24.9 & -5.1 \\
\hline Chile & 195 & 118 & - & - & - & 66.0 & - \\
\hline China (Taiwan) & 353 & 355 & 284 & 141 & 283 & -.7 & 5.7 \\
\hline Croatia & - & 72 & - & 63 & 245 & -100.0 & - \\
\hline Denmark & - & 189 & - & 168 & 2,397 & -100.0 & - \\
\hline 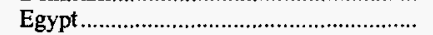 & 303 & 714 & 593 & 601 & 557 & -57.5 & -14.1 \\
\hline 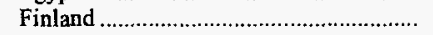 & 507 & 792 & 375 & 212 & 207 & -36.0 & 25.0 \\
\hline 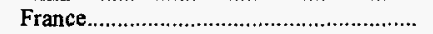 & 3,676 & 4,408 & 3,514 & 3,400 & 6,311 & -16.6 & -12.6 \\
\hline Germany, FR & 943 & 1,107 & 382 & 635 & 959 & -14.9 & -.4 \\
\hline Iceland & - & - & - & - & 8 & - & - \\
\hline India & 11 & - & - & - & - & - & - \\
\hline Ireland & - & - & - & 117 & 822 & - & - \\
\hline 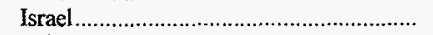 & 375 & - & - & - & 210 & - & 15.6 \\
\hline 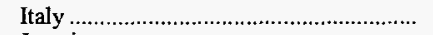 & 4,965 & 5,138 & 3,634 & 3,815 & 6,299 & -3.4 & -5.8 \\
\hline 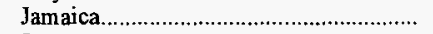 & 36 & - & & & & & - \\
\hline Japan & 2,062 & 3,431 & 2,595 & 2,691 & 3,313 & -39.9 & -11.2 \\
\hline 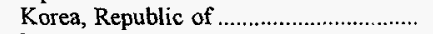 & 1,050 & 1,013 & 523 & 318 & - & 3.7 & - \\
\hline Morocco & 1,111 & 275 & 101 & 79 & 170 & 304.0 & 59.9 \\
\hline Netherlands & 1,636 & 3,628 & 3,340 & 3,205 & 3,804 & -54.9 & -19.0 \\
\hline Nigeria & - & - & - & 43 & - & - & - \\
\hline 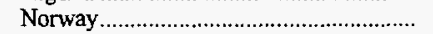 & - & - & - & - & 15 & - & - \\
\hline 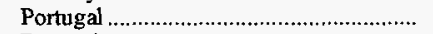 & 1,128 & 1,390 & 674 & 1,144 & 962 & -18.9 & 4.0 \\
\hline Romania & 1,315 & 1,623 & 925 & 820 & 376 & -19.0 & 36.7 \\
\hline Slovenia & - & - & - & - & 69 & - & - \\
\hline South Africa, Rep of ................................. & 947 & 946 & 771 & 577 & 408 & .1 & 23.4 \\
\hline Spain & 887 & 1,084 & 1,255 & $1,2 ! 1$ & 1,501 & -18.1 & -12.3 \\
\hline 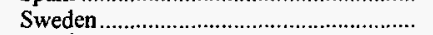 & 882 & 1,352 & 886 & 603 & 819 & -34.8 & 1.9 \\
\hline 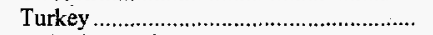 & 1,655 & 1,560 & 1,468 & 1,370 & 1,429 & 6.1 & 3.7 \\
\hline 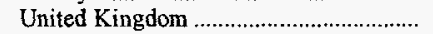 & 3,133 & 1,633 & 1,212 & 2,359 & 4,816 & 91.9 & -10.2 \\
\hline Yugoslavia, FR & - & - & - & - & 436 & - & - \\
\hline Wyoming & 2,395 & 2,269 & 1,524 & 974 & 1,277 & $\mathbf{5 . 5}$ & 17.0 \\
\hline 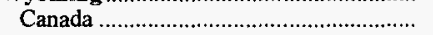 & 443 & 32 & - & - & - & NM & - \\
\hline Japan & - & - & - & * & - & - & - \\
\hline 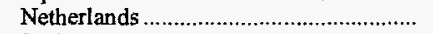 & 63 & - & - & - & - & - & - \\
\hline 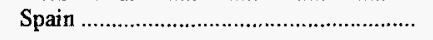 & 1,889 & 2,237 & 1,524 & 974 & 1,277 & -15.6 & 10.3 \\
\hline Major States Total & 90,506 & 88,722 & 71,811 & 74,801 & 100,619 & 2.0 & -2.6 \\
\hline 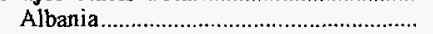 & - & & & & 21 & - & - \\
\hline Algeria & 206 & 166 & 269 & 344 & 534 & 24.1 & -21.2 \\
\hline Argentina & 216 & 306 & 356 & 548 & 326 & -29.5 & -9.8 \\
\hline 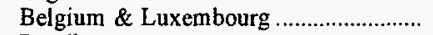 & 4,106 & 3,724 & 4,324 & 4,715 & 6,320 & 10.2 & -10.2 \\
\hline 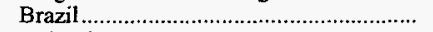 & 6,312 & 6,416 & 5,730 & 4,977 & 5,704 & -1.6 & 2.6 \\
\hline 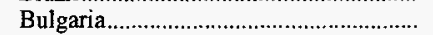 & 1,422 & 1,488 & 1,607 & 1,065 & 863 & -4.4 & 13.3 \\
\hline Canada & 10,280 & 7,750 & 8,373 & 7,696 & 13,919 & 32.6 & -7.3 \\
\hline Chile & 640 & 287 & - & 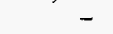 & 37 & 122.8 & 103.4 \\
\hline
\end{tabular}

See footnotes at end of table. 
Table 62. Foreign Distribution of U.S. Coal by Major Coal-Exporting States and Destination, 1992-1996 (Continued)

(Thousand Short Tons)

\begin{tabular}{|c|c|c|c|c|c|c|c|}
\hline \multirow{2}{*}{$\begin{array}{l}\text { Coal-Exporting State } \\
\text { and Destination }\end{array}$} & \multirow[t]{2}{*}{1996} & \multirow[t]{2}{*}{1995} & \multirow[t]{2}{*}{1994} & \multirow[t]{2}{*}{1993} & \multirow[t]{2}{*}{1992} & \multirow{2}{*}{$\begin{array}{c}\text { Percent } \\
\text { Change } \\
1995-1996\end{array}$} & \multirow{2}{*}{$\begin{array}{c}\begin{array}{c}\text { Average Annua } \\
\text { Percent Change }\end{array} \\
1992-1996\end{array}$} \\
\hline & & & & & & & \\
\hline \multicolumn{8}{|l|}{ Major States Total (Continued) } \\
\hline 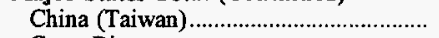 & 3,197 & 3,310 & 3,397 & 4,656 & 4,382 & -3.4 & -7.6 \\
\hline Costa Rica ... & - & 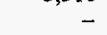 & - & - & $*$ & - & - \\
\hline 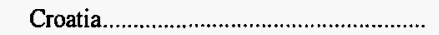 & - & 72 & - & 473 & 245 & -100.0 & - \\
\hline 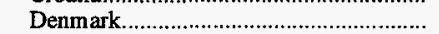 & 1,165 & 2,320 & 508 & 388 & 4,116 & -49.8 & -27.1 \\
\hline Dominican Republic .................................... & 50 & 18 & 65 & 59 & 157 & 172.7 & -24.8 \\
\hline 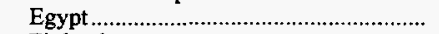 & 1,138 & 1,158 & 1,029 & 834 & 796 & -1.7 & 9.4 \\
\hline 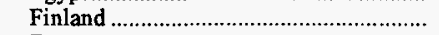 & 794 & 1,337 & 446 & 269 & 207 & -40.6 & 39.9 \\
\hline 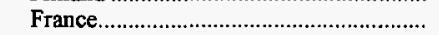 & 5,223 & 5,362 & 4,223 & 4,990 & 9,222 & -2.6 & -13.3 \\
\hline 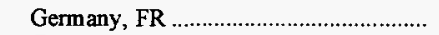 & 1,708 & 2,668 & 588 & 787 & 1,147 & -36.0 & 10.5 \\
\hline 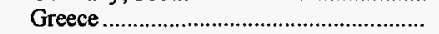 & 491 & - & - & - & 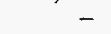 & - & - \\
\hline 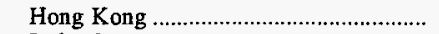 & - & - & 46 & - & 359 & - & - \\
\hline Iceland & 119 & 76 & 7 & - & 39 & 56.3 & 32.3 \\
\hline India & 11 & - & - & - & - & - & - \\
\hline Indonesia & - & - & - & - & * & - & - \\
\hline 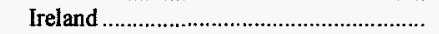 & 1,067 & 1,219 & 1,015 & 1,152 & 1,371 & -12.4 & -6.1 \\
\hline 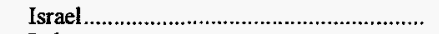 & 1,473 & 1,212 & 922 & 940 & 1,300 & 21.5 & 3.2 \\
\hline 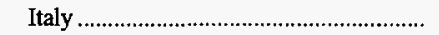 & 9,656 & 9,298 & 7,409 & 6,377 & 8,955 & 3.8 & 1.9 \\
\hline 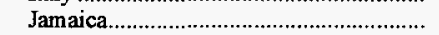 & 53 & 62 & 26 & 39 & 35 & -15.6 & 11.1 \\
\hline 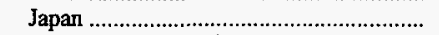 & 10,840 & 11,254 & 9,918 & 11,747 & 11,537 & -3.7 & -1.5 \\
\hline Korea, Republic of & 4,582 & 4,526 & 3,332 & 3,470 & 3,219 & 1.2 & 9.2 \\
\hline 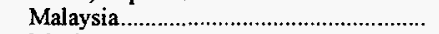 & & - & 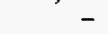 & - & * & - & - \\
\hline 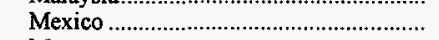 & 758 & - & - & $*$ & 18 & - & 156.3 \\
\hline Morocco & 1,388 & 1,050 & 101 & 571 & 520 & 32.2 & 27.8 \\
\hline Netherlands & 4,398 & 5,911 & 4,723 & 4,455 & 6,310 & -25.6 & -8.6 \\
\hline 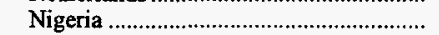 & - & - & - & 43 & 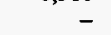 & - & - \\
\hline 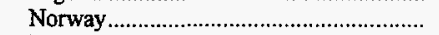 & 169 & 170 & 88 & 121 & 161 & -.3 & 1.2 \\
\hline Panama & - & - & - & - & * & - & - \\
\hline Paraguay & - & - & - & - & 3 & - & - \\
\hline 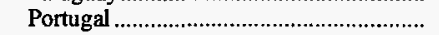 & 2,094 & 1,967 & 1,167 & 1,611 & 1,548 & 6.4 & 7.8 \\
\hline 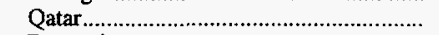 & - & & - & - & $*$ & - & - \\
\hline Romania & 1,517 & 2,115 & 1,750 & 991 & 705 & -28.3 & 21.1 \\
\hline 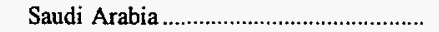 & 22 & - & - & 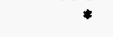 & - & - & - \\
\hline 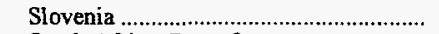 & - & - & - & - & 69 & - & - \\
\hline 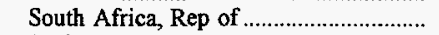 & 1,192 & 946 & 771 & 577 & 620 & 26.0 & 17.8 \\
\hline 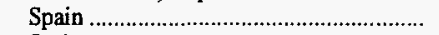 & 4,197 & 4,465 & 4,103 & 3,867 & 4,215 & -6.0 & -.1 \\
\hline 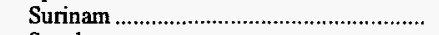 & & & & * & & - & - \\
\hline Sweden & 1,091 & 1,466 & 939 & 798 & 1,276 & -25.6 & -3.8 \\
\hline Trinidad \& Tobago & 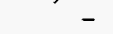 & - & - & 1 & 2 & - & - \\
\hline 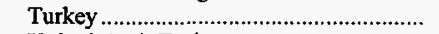 & 2,005 & 2,116 & 1,606 & 1,623 & 2,003 & -5.2 & * \\
\hline 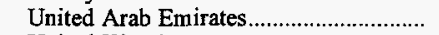 & - & - & - & * & - & - & - \\
\hline 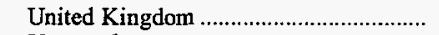 & 6,925 & 4,476 & 2,963 & 4,600 & 7,672 & 54.7 & -2.5 \\
\hline Venezuela & 1 & 9 & 11 & 15 & 39 & -88.6 & -59.5 \\
\hline 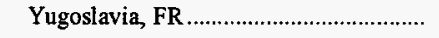 & - & - & - & - & 650 & - & - \\
\hline Other States Total & 1,693 & 1,185 & 870 & 709 & 761 & 42.8 & 22.1 \\
\hline 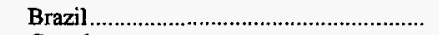 & - & 188 & 184 & 295 & - & -100.0 & - \\
\hline Canada & 319 & 273 & 93 & 55 & * & 16.9 & 426.7 \\
\hline France & - & - & - & - & 9 & - & - \\
\hline 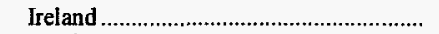 & 80 & - & - & - & 10 & - & 68.5 \\
\hline Mexico & - & 498 & - & 11 & - & -100.0 & - \\
\hline Spain & - & - & 153 & - & 53 & - & - \\
\hline 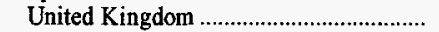 & - & - & - & - & 4 & - & - \\
\hline Unknown & 1,294 & 226 & 439 & 348 & 685 & 471.6 & 17.3 \\
\hline U.S. Total & 92,199 & 89,907 & $\mathbf{7 2 , 6 8 0}$ & 75,510 & 101,380 & 2.5 & -2.3 \\
\hline Albania & - & - & & - & 21 & - & - \\
\hline Algeria & 206 & 166 & 269 & 344 & 534 & 24.1 & -21.2 \\
\hline Argentina & 216 & 306 & 356 & 548 & 326 & -29.5 & -9.8 \\
\hline 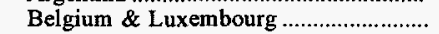 & 4,106 & 3,724 & 4,324 & 4,715 & 6,320 & 10.2 & -10.2 \\
\hline 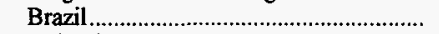 & 6,312 & 6,605 & 5,914 & 5,271 & 5,704 & -4.4 & 2.6 \\
\hline Bulgaria & 1,422 & 1,488 & 1,607 & 1,065 & 863 & -4.4 & 13.3 \\
\hline Canada & 10,599 & 8,023 & 8,467 & 7,751 & 13,919 & 32.1 & -6.6 \\
\hline 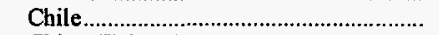 & 640 & 287 & - & - & 37 & 122.8 & 103.4 \\
\hline China (Taiwan) & 3,197 & 3,310 & 3,397 & 4,656 & 4,382 & -3.4 & -7.6 \\
\hline 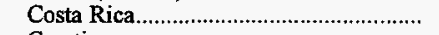 & - & - & - & - & 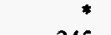 & - & - \\
\hline 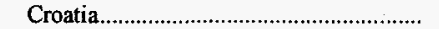 & - & 72 & - & 473 & 245 & -100.0 & - \\
\hline 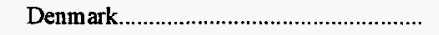 & 1,165 & 2,320 & 508 & 388 & 4,116 & -49.8 & -27.1 \\
\hline
\end{tabular}

See footnotes at end of table. 
Table 62. Foreign Distribution of U.S. Coal by Major Coal-Exporting States and Destination, 1992-1996 (Continued) (Thousand Short Tons)

\begin{tabular}{|c|c|c|c|c|c|c|c|}
\hline \multirow{2}{*}{$\begin{array}{l}\text { Coal-Exporting State } \\
\text { and Destination }\end{array}$} & \multirow{2}{*}{1996} & \multirow{2}{*}{1995} & \multirow{2}{*}{1994} & \multirow{2}{*}{1993} & \multirow{2}{*}{1992} & \multirow{2}{*}{$\begin{array}{c}\text { Percent } \\
\text { Change } \\
\text { 1995-1996 }\end{array}$} & \multirow{2}{*}{$\begin{array}{c}\begin{array}{c}\text { Average Annual } \\
\text { Percent Change }\end{array} \\
1992-1996\end{array}$} \\
\hline & & & & & & & \\
\hline \multicolumn{8}{|l|}{ U.S. Total (Continued) } \\
\hline 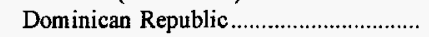 & 50 & 18 & 65 & 59 & 157 & 172.7 & -24.8 \\
\hline Egypt & 1,138 & 1,158 & 1,029 & 834 & 796 & -1.7 & 9.4 \\
\hline Finland & 794 & 1,337 & 446 & 269 & 207 & -40.6 & 39.9 \\
\hline 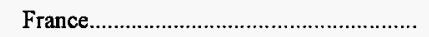 & 5,223 & 5,362 & 4,223 & 4,990 & 9,230 & -2.6 & -13.3 \\
\hline Germany, FR & 1,708 & 2,668 & 588 & 787 & 1,147 & -36.0 & 10.5 \\
\hline 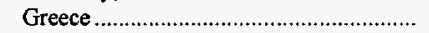 & 491 & - & - & - & - & - & - \\
\hline 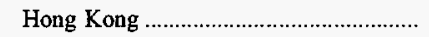 & - & - & 46 & - & 359 & - & - \\
\hline 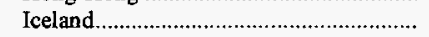 & 119 & 76 & 7 & - & 39 & 56.3 & 32.3 \\
\hline India & 11 & - & - & - & - & - & - \\
\hline 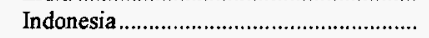 & - & - & - & - & * & - & - \\
\hline Ireland & 1,147 & 1,219 & 1,015 & 1,152 & 1,381 & -5.8 & -4.5 \\
\hline 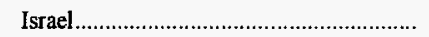 & 1,473 & 1,212 & 922 & 940 & 1,300 & 21.5 & 3.2 \\
\hline Italy & 9,656 & 9,298 & 7,409 & 6,377 & 8,955 & 3.8 & 1.9 \\
\hline 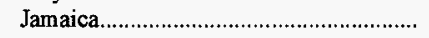 & 53 & 62 & 26 & 39 & 35 & -15.6 & 11.1 \\
\hline Japan & 10,840 & 11,254 & 9,918 & 11,747 & 11,537 & -3.7 & -1.5 \\
\hline 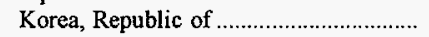 & 4,582 & 4,526 & 3,332 & 3,470 & 3,219 & 1.2 & 9.2 \\
\hline 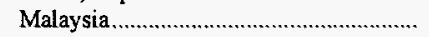 & - & - & - & - & $*$ & - & - \\
\hline Mexico & 758 & 498 & - & 11 & 18 & 52.2 & 156.3 \\
\hline Morocco & 1,388 & 1,050 & 101 & 571 & 520 & 32.2 & 27.8 \\
\hline 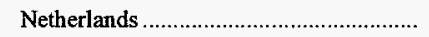 & 4,398 & 5,911 & 4,723 & 4,455 & 6,310 & -25.6 & -8.6 \\
\hline Nigeria & - & - & - & 43 & - & - & - \\
\hline 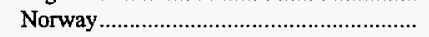 & 169 & 170 & 88 & 121 & 161 & -.3 & 1.2 \\
\hline 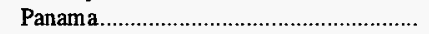 & - & - & - & - & $*$ & - & - \\
\hline 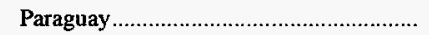 & - & - & - & - & 3 & - & - \\
\hline 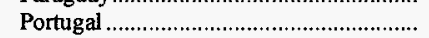 & 2,094 & 1,967 & 1,167 & 1,611 & 1,548 & 6.4 & 7.8 \\
\hline 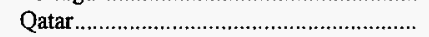 & - & - & - & - & $*$ & - & - \\
\hline Romania & 1,517 & 2,115 & 1,750 & 991 & 705 & -28.3 & 21.1 \\
\hline 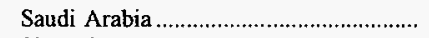 & 22 & - & - & $*$ & - & - & - \\
\hline Slovenia & - & - & - & - & 69 & - & - \\
\hline 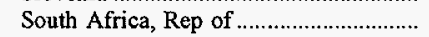 & 1,192 & 946 & 771 & 577 & 620 & 26.0 & 17.8 \\
\hline Spain & 4,197 & 4,465 & 4,256 & 3,867 & 4,268 & -6.0 & -.4 \\
\hline Surinam & - & - & - & $*$ & $\bullet$ & - & - \\
\hline Sweden & 1,091 & 1,466 & 939 & 798 & 1,276 & -25.6 & -3.8 \\
\hline Trinidad \& Tobago & - & - & - & 1 & 2 & - & - \\
\hline 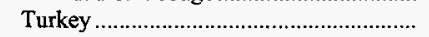 & 2,005 & 2,116 & 1,606 & 1,623 & 2,003 & -5.2 & $*$ \\
\hline United Arab Emirates................................. & - & - & - & * & - & - & - \\
\hline United Kingdom & 6,925 & 4,476 & 2,963 & 4,600 & 7,676 & 54.7 & -2.5 \\
\hline Venezuela & 1 & 9 & 11 & 15 & 39 & -88.6 & -59.5 \\
\hline Yugoslavia, FR & - & - & - & - & 650 & - & - \\
\hline Unknown & 1,294 & 226 & 439 & 348 & 685 & 471.6 & 17.3 \\
\hline
\end{tabular}

* Data round to zero.

NM Not meaningful as value is greater than 500 percent.

Notes: Major coal-exporting States are those with total coal exports of over 600,000 short tons in 1996. Totals may not equal sum of components due to independent rounding. Foreign distribution to some countries for 1992 through 1995 have been revised.

Sources: Values shown for destinations other than Canada and Mexico are estimates based upon information reported on Energy Information Administration Form EIA-6, "Coal Distribution Report," and coal export data presented in King's COALBASE (King Publishing Corporation, Knoxville, Tennessee). See the Explanatory Notes for a complete description of the methodology used to develop these estimates. Values shown for Canada and Mexico are based upon Form EIA-6. 
Table 63. Foreign Distribution of U.S. Metallurgical Coal by Major Coal-Exporting States and Destination, 1992-1996

(Thousand Short Tons)

\begin{tabular}{|c|c|c|c|c|c|c|c|}
\hline \multirow{2}{*}{$\begin{array}{l}\text { Coal-Exporting State } \\
\text { and Destination }\end{array}$} & \multirow{2}{*}{1996} & \multirow{2}{*}{1995} & \multirow{2}{*}{1994} & \multirow{2}{*}{1993} & \multirow{2}{*}{1992} & \multirow{2}{*}{$\begin{array}{c}\text { Percent } \\
\text { Change } \\
1995-1996\end{array}$} & \multirow{2}{*}{$\begin{array}{c}\text { Average Annua } \\
\text { Percent Change }\end{array}$} \\
\hline & & & & & & & \\
\hline Alabama & 4,523 & $\mathbf{5 , 3 3 0}$ & 4,359 & 5,846 & 5,800 & -15.1 & -6.0 \\
\hline Albania & & & - & - & 21 & & - \\
\hline Argentina & 216 & 306 & 268 & 319 & 157 & -29.5 & 8.3 \\
\hline 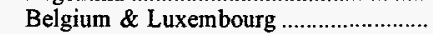 & 703 & 574 & 627 & 866 & 861 & 22.3 & -5.0 \\
\hline 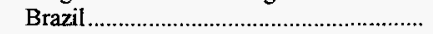 & 566 & 564 & 42 & 193 & - & .3 & - \\
\hline Bulgaria & 208 & 128 & 35 & 109 & - & 63.2 & - \\
\hline 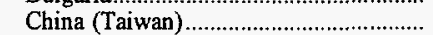 & - & - & - & 42 & 45 & - & - \\
\hline Egypt t.1.1. & - & 111 & - & - & - & -100.0 & - \\
\hline France & - & - & * & - & - & - & - \\
\hline Germany, FR . . & 184 & 201 & - & - & - & -8.0 & - \\
\hline 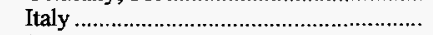 & 318 & 314 & 421 & 429 & 378 & 1.5 & -4.2 \\
\hline 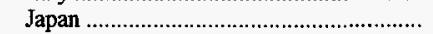 & 861 & 1,358 & 1,266 & 2,199 & 2,340 & -36.6 & -22.1 \\
\hline 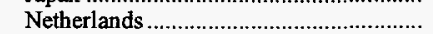 & 73 & 217 & 88 & - & 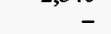 & -66.1 & - \\
\hline Romania & 170 & 492 & 602 & 171 & 183 & -65.5 & -2.0 \\
\hline South Africa, Rep of ............................ & 57 & - & - & - & 212 & - & -28.0 \\
\hline Spain & 52 & 48 & - & - & - & 8.4 & - \\
\hline 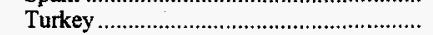 & 326 & 302 & 137 & 253 & 424 & 8.0 & -6.4 \\
\hline United Kingdom ............................................. & 789 & 717 & 872 & 1,266 & 1,179 & 10.1 & -9.6 \\
\hline Colorado & 30 & - & - & - & - & - & - \\
\hline Japan & 30 & - & - & - & - & - & - \\
\hline 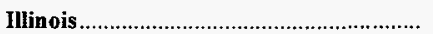 & - & 49 & 236 & 109 & 614 & -100.0 & -100.0 \\
\hline Belgium \& Luxembourg ............................ & - & - & - & - & 58 & - & - \\
\hline 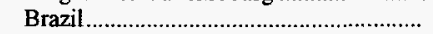 & - & - & - & - & 298 & - & - \\
\hline 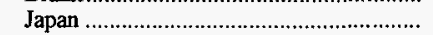 & - & 49 & 236 & 109 & 32 & -100.0 & - \\
\hline 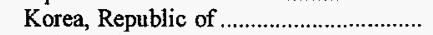 & - & - & - & - & 50 & - & - \\
\hline 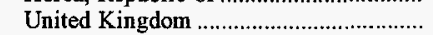 & - & - & - & - & 175 & - & - \\
\hline Kentucky & 5,303 & 3,640 & 3,120 & 4,415 & 5,916 & 45.7 & -2.7 \\
\hline Belgium \& Luxembourg ……………........... & 67 & - & 66 & 56 & 665 & - & -43.7 \\
\hline 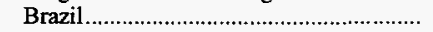 & - & 52 & 23 & 392 & 98 & -100.0 & - \\
\hline 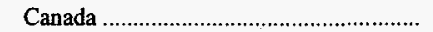 & 1,178 & 777 & 1,073 & 1,356 & 1,373 & 51.5 & -3.8 \\
\hline China (Taiwan) & - & 76 & 98 & 140 & 123 & -100.0 & - \\
\hline 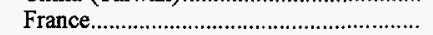 & 548 & 262 & 146 & 400 & 427 & 108.6 & 6.4 \\
\hline 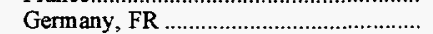 & - & 93 & - & - & - & -100.0 & - \\
\hline 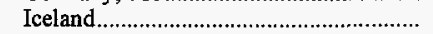 & 119 & 76 & 7 & - & 31 & 56.3 & 40.0 \\
\hline Italy & 132 & - & 42 & - & - & - & - \\
\hline 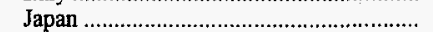 & 93 & 53 & 67 & 219 & 303 & 74.0 & -25.6 \\
\hline 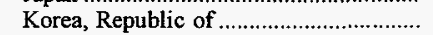 & 1,876 & 1,523 & 1,163 & 1,256 & 1,191 & 23.2 & 12.0 \\
\hline 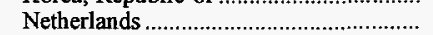 & 581 & 102 & 45 & 241 & 766 & 468.8 & -6.7 \\
\hline 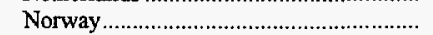 & 140 & 142 & 74 & 91 & 98 & -1.4 & 9.3 \\
\hline 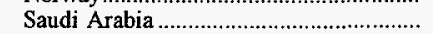 & 22 & - & - & - & - & - & - \\
\hline 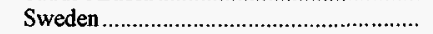 & - & - & 16 & 195 & 456 & - & - \\
\hline 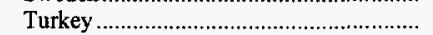 & - & - & - & & 151 & - & - \\
\hline 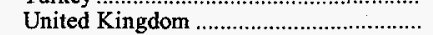 & 548 & 483 & 301 & 70 & 197 & 13.5 & 29.1 \\
\hline 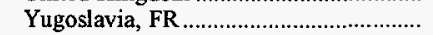 & - & - & - & - & 38 & - & - \\
\hline 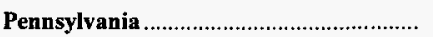 & 1,642 & 1,467 & 1,624 & 1,919 & 1,301 & 11.9 & 6.0 \\
\hline 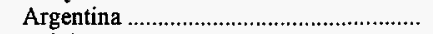 & - & - & - & 46 & - & - & - \\
\hline Belgium \& Luxembourg ...................... & - & - & 29 & - & - & - & - \\
\hline 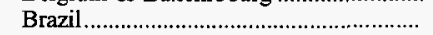 & 258 & 371 & 333 & 148 & 137 & -30.4 & 17.2 \\
\hline 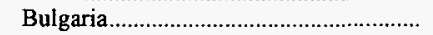 & - & - & - & 71 & 73 & - & - \\
\hline Canada ....................... & - & 4 & - & 10 & - & -100.0 & - \\
\hline Chile & - & - & - & - & 37 & - & - \\
\hline 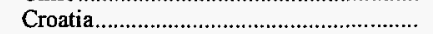 & - & - & - & $\cdot 114$ & - & - & - \\
\hline 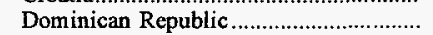 & - & - & 23 & - & - & - & - \\
\hline Finland & _- & - & - & 56 & - & - & - \\
\hline 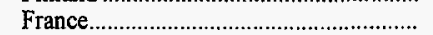 & 89 & - & - & - & - & - & - \\
\hline 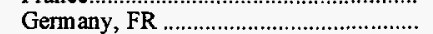 & - & 66 & 174 & - & - & -100.0 & - \\
\hline Japan ... & 920 & 916 & 834 & 1,384 & 921 & .4 & * \\
\hline Korea, Republic of ........................... & 101 & 109 & 214 & 91 & - & -7.8 & - \\
\hline Netherlands ......................................... & 162 & - & 17 & - & - & - & - \\
\hline 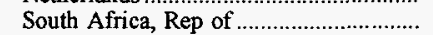 & 112 & - & - & - & - & - & - \\
\hline Yugoslavia, FR & - & - & - & - & 132 & - & - \\
\hline Utah & 187 & - & - & - & - & - & - \\
\hline 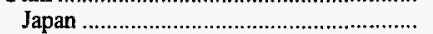 & 187 & - & - & - & - & - & - \\
\hline
\end{tabular}

See footnotes at end of table. 
Table 63. Foreign Distribution of U.S. Metallurgical Coal by Major Coal-Exporting States and Destination, 1992-1996 (Continued) (Thousand Short Tons)

\begin{tabular}{|c|c|c|c|c|c|c|c|}
\hline \multirow{2}{*}{$\begin{array}{l}\text { Coal-Exporting State } \\
\text { and Destination }\end{array}$} & \multirow[t]{2}{*}{1996} & \multirow[t]{2}{*}{1995} & \multirow[t]{2}{*}{1994} & \multirow[t]{2}{*}{1993} & \multirow[t]{2}{*}{1992} & \multirow{2}{*}{$\begin{array}{c}\text { Percent } \\
\text { Change } \\
\text { 1995-1996 }\end{array}$} & \multirow{2}{*}{$\begin{array}{c}\text { Average Annual } \\
\text { Percent Change } \\
1992-1996\end{array}$} \\
\hline & & & & & & & \\
\hline Virginia & 12,760 & 8,921 & 11,155 & 13,747 & 14,389 & 43.0 & $-\mathbf{3 . 0}$ \\
\hline Algeria & 206 & 166 & 269 & 344 & 534 & 24.1 & -21.2 \\
\hline Argentina & - & - & 53 & 52 & - & - & - \\
\hline 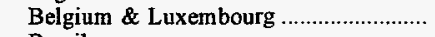 & 1,078 & 764 & 884 & 1,147 & 1,972 & 41.2 & -14.0 \\
\hline 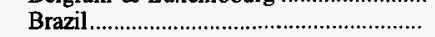 & 1,228 & 1,091 & 1,218 & 1,741 & 2,252 & 12.5 & -14.1 \\
\hline 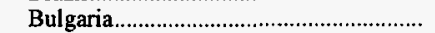 & - & - & - & 242 & 172 & - & - \\
\hline Canada & 387 & 445 & 786 & 1,229 & - & -13.0 & - \\
\hline 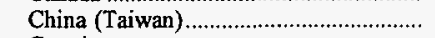 & - & - & 15 & $*$ & - & - & - \\
\hline Croatia & - & - & - & 251 & - & - & - \\
\hline Egypt & 835 & 333 & 436 & 234 & 239 & 150.7 & 36.8 \\
\hline 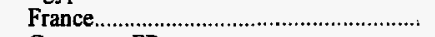 & 910 & 625 & 563 & 737 & 846 & 45.7 & 1.8 \\
\hline Germany, FR & - & 68 & 9 & 50 & 37 & -100.0 & - \\
\hline Italy & 1,696 & 758 & 1,445 & 951 & 622 & 123.8 & 28.5 \\
\hline Japan & 2,300 & 1,796 & 2,037 & 2,355 & 2,689 & 28.0 & -3.8 \\
\hline 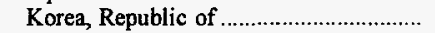 & 466 & 589 & 539 & 853 & 1,244 & -20.9 & -21.8 \\
\hline 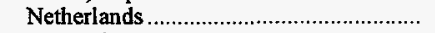 & 1,193 & 793 & 766 & 973 & 1,192 & 50.4 & * \\
\hline Portugal & - & - & - & - & 73 & - & - \\
\hline 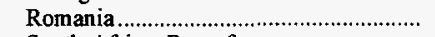 & 32 & - & 223 & - & 145 & - & -31.3 \\
\hline 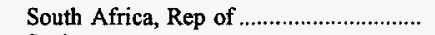 & 76 & - & - & - & - & - & - \\
\hline Spain & 1,370 & 847 & 1,297 & 1,682 & 1,274 & 61.7 & 1.8 \\
\hline 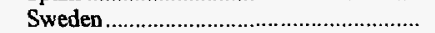 & 185 & 115 & 37 & - & - & 60.9 & - \\
\hline United Kingdom & 798 & 531 & 579 & 905 & 1,054 & 50.3 & -6.7 \\
\hline Yugoslavia, FR & - & - & - & - & 44 & - & - \\
\hline West Virginia & 31,717 & 34,633 & 31,603 & 26,504 & 33,413 & -8.4 & -1.3 \\
\hline Algeria & - & - & * & - & - & - & - \\
\hline Argentina & - & - & 35 & 132 & 169 & - & - \\
\hline Belgium \& Luxembourg .......................... & 1,261 & 1,175 & 1,302 & 1,396 & 538 & 7.3 & 23.7 \\
\hline 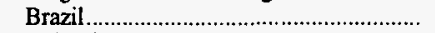 & 4,247 & 4,329 & 4,109 & 2,496 & 2,907 & -1.9 & 9.9 \\
\hline 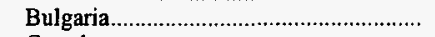 & 1,152 & 1,360 & 1,571 & 644 & 619 & -15.3 & 16.8 \\
\hline 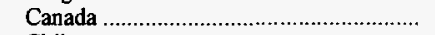 & 6,907 & 5,759 & 5,605 & 4,071 & 8,021 & 19.9 & -3.7 \\
\hline 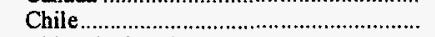 & 43 & - & - & - & - & - & - \\
\hline China (Taiwan) & 353 & 355 & 284 & 141 & 283 & -.7 & 5.7 \\
\hline 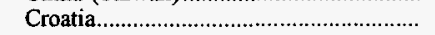 & - & - & - & 63 & 245 & - & - \\
\hline Egypt & 303 & 714 & 593 & 601 & 557 & -57.5 & -14.1 \\
\hline Finland & 507 & 683 & 375 & 212 & 207 & -25.8 & 25.0 \\
\hline 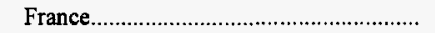 & 2,859 & 3,594 & 3,514 & 2,864 & 3,216 & -20.4 & -2.9 \\
\hline Germany, FR & 584 & 254 & 382 & 286 & 314 & 130.4 & 16.8 \\
\hline Iceland & - & - & - & - & 8 & - & - \\
\hline India & 11 & - & - & - & - & - & - \\
\hline 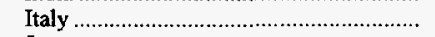 & 2,361 & 2,873 & 2,927 & 3,111 & 4,899 & -17.8 & -16.7 \\
\hline Japan & 2,062 & 3,222 & 2,148 & 2,260 & 2,895 & -36.0 & -8.1 \\
\hline Korea, Republic of & 1,050 & 1,013 & 523 & 318 & - & 3.7 & - \\
\hline 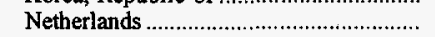 & 1,223 & 1,523 & 1,717 & 2,014 & 2,486 & -19.7 & -16.3 \\
\hline 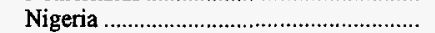 & - & - & - & 43 & - & - & - \\
\hline 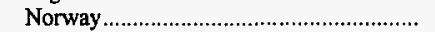 & - & - & - & - & 15 & - & - \\
\hline 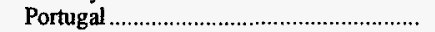 & 164 & 33 & - & 151 & 108 & 389.9 & 10.9 \\
\hline 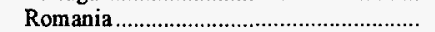 & 1,315 & 1,623 & 925 & 820 & 376 & -19.0 & 36.7 \\
\hline 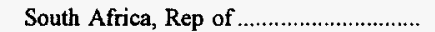 & 947 & 946 & 771 & 577 & 408 & .1 & 23.4 \\
\hline Spain & 818 & 1,084 & 1,255 & 1,071 & 1,232 & -24.5 & -9.7 \\
\hline Sweden & 882 & 1,352 & 886 & 603 & 819 & -34.8 & 1.9 \\
\hline Turkey & 1,643 & 1,560 & 1,468 & 1,370 & 1,429 & 5.3 & 3.5 \\
\hline United Kingdom & 1,024 & 1,182 & 1,212 & 1,261 & 1,225 & -13.3 & -4.4 \\
\hline 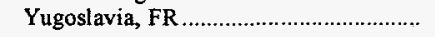 & - & - & - & - & 436 & - & - \\
\hline Major States Total & 56,162 & 54,039 & 52,098 & 52,541 & 61,432 & 3.9 & -2.2 \\
\hline Albania & - & - & - & - & 21 & - & - \\
\hline 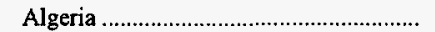 & 206 & 166 & 269 & 344 & 534 & 24.1 & -21.2 \\
\hline 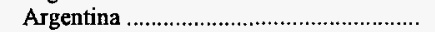 & 216 & 306 & 356 & 548 & 326 & -29.5 & -9.8 \\
\hline 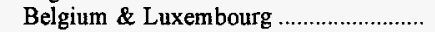 & 3,109 & 2,513 & 2,908 & 3,465 & 4,094 & 23.7 & -6.6 \\
\hline 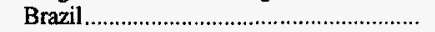 & 6,298 & 6,407 & 5,725 & 4,969 & 5,692 & -1.7 & 2.6 \\
\hline 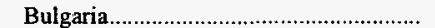 & 1,361 & 1,488 & 1,607 & 1,065 & 863 & -8.6 & 12.0 \\
\hline Canada & 8,472 & 6,986 & 7,464 & 6,666 & 9,394 & 21.3 & -2.5 \\
\hline 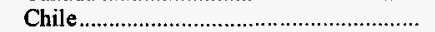 & 43 & - & - & - & 37 & - & 3.4 \\
\hline 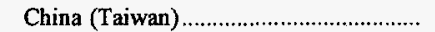 & 353 & 431 & 397 & 323 & 451 & -18.2 & -6.0 \\
\hline Croatia & - & - & - & 428 & 245 & - & - \\
\hline 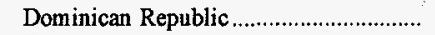 & - & - & 23 & - & - & - & - \\
\hline 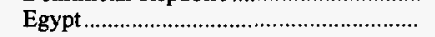 & 1,138 & 1,158 & 1,029 & 834 & 796 & -1.7 & 9.4 \\
\hline
\end{tabular}

See footnotes at end of table. 
Table 63. Foreign Distribution of U.S. Metallurgical Coal by Major Coal-Exporting States and Destination, 1992-1996 (Continued)

(Thousand Short Tons)

\begin{tabular}{|c|c|c|c|c|c|c|c|}
\hline \multirow{2}{*}{$\begin{array}{l}\text { Coal-Exporting State } \\
\text { and Destination }\end{array}$} & \multirow{2}{*}{1996} & \multirow{2}{*}{1995} & \multirow{2}{*}{1994} & \multirow{2}{*}{1993} & \multirow{2}{*}{1992} & \multirow{2}{*}{$\begin{array}{c}\text { Percent } \\
\text { Change } \\
1995-1996\end{array}$} & \multirow{2}{*}{$\begin{array}{c}\begin{array}{c}\text { Average Annual } \\
\text { Percent Change }\end{array} \\
1992-1996\end{array}$} \\
\hline & & & & & & & \\
\hline \multicolumn{8}{|l|}{ Major States Total (Continued) } \\
\hline Finland & 507 & 683 & 375 & 267 & 207 & -25.8 & 25.0 \\
\hline France & 4,406 & 4,481 & 4,223 & 4,001 & 4,489 & -1.7 & -.5 \\
\hline 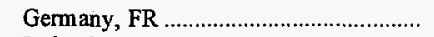 & 769 & 681 & 565 & 336 & 352 & 12.9 & 21.6 \\
\hline 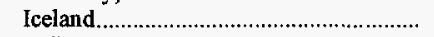 & 119 & 76 & 7 & - & 39 & 56.3 & 32.3 \\
\hline India & 11 & - & - & - & - & - & - \\
\hline Italy & 4,507 & 3,944 & 4,836 & 4,491 & 5,899 & 14.3 & -6.5 \\
\hline Japan & 6,453 & 7,395 & 6,588 & 8,525 & 9,181 & -12.7 & -8.4 \\
\hline Korea, Republic of & 3,493 & 3,234 & 2,438 & 2,517 & 2,485 & 8.0 & 8.9 \\
\hline 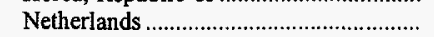 & 3,233 & 2,635 & 2,633 & 3,228 & 4,444 & 22.7 & -7.6 \\
\hline Nigeria & - & - & - & 43 & - & - & - \\
\hline Norway & 140 & 142 & 74 & 91 & 113 & -1.4 & 5.4 \\
\hline Portugal & 164 & 33 & - & 151 & 181 & 389.9 & -2.5 \\
\hline Romania & 1,517 & 2,115 & 1,750 & 991 & 705 & -28.3 & 21.1 \\
\hline Saudi Arabia & 22 & - & - & - & - & 20. & - \\
\hline 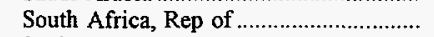 & 1,192 & 946 & 771 & 577 & 620 & 26.0 & 17.8 \\
\hline Spain & 2,240 & 1,979 & 2,552 & 2,754 & 2,505 & 13.2 & -2.8 \\
\hline Sweden & 1,066 & 1,466 & 939 & 798 & 1,276 & -27.3 & -4.4 \\
\hline Turkey & 1,969 & 1,862 & 1,606 & 1,623 & 2,003 & 5.7 & -.4 \\
\hline United Kingdom & 3,160 & 2,913 & 2,963 & 3,503 & 3,831 & 8.5 & -4.7 \\
\hline 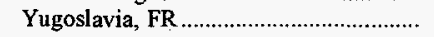 & - & - & - & - & 650 & - & - \\
\hline 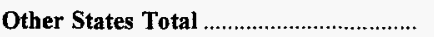 & - & 188 & 184 & 295 & - & -100.0 & - \\
\hline 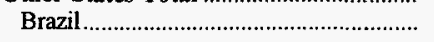 & - & 188 & 184 & 295 & - & -100.0 & - \\
\hline 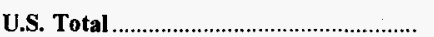 & 56,162 & 54,228 & 52,282 & 52,835 & 61,432 & 3.6 & -2.2 \\
\hline 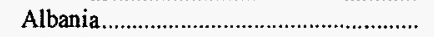 & - & - & - & - & 21 & - & - \\
\hline Algeria & 206 & 166 & 269 & 344 & 534 & 24.1 & -21.2 \\
\hline Argentina & 216 & 306 & 356 & 548 & 326 & -29.5 & -9.8 \\
\hline 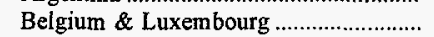 & 3,109 & 2,513 & 2,908 & 3,465 & 4,094 & 23.7 & -6.6 \\
\hline 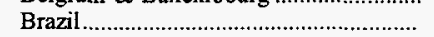 & 6,298 & 6,596 & 5,909 & 5,264 & 5,692 & -4.5 & 2.6 \\
\hline 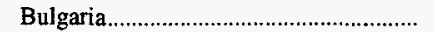 & 1,361 & 1,488 & 1,607 & 1,065 & 863 & -8.6 & 12.0 \\
\hline Canada & 8,472 & 6,986 & 7,464 & 6,666 & 9,394 & 21.3 & -2.5 \\
\hline 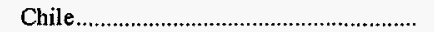 & 43 & - & - & - & 37 & - & 3.4 \\
\hline China (Taiwan) & 353 & 431 & 397 & 323 & 451 & -18.2 & -6.0 \\
\hline Croatia & - & - & - & 428 & 245 & - & - \\
\hline 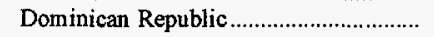 & - & - & 23 & - & - & - & - \\
\hline Egypt & 1,138 & 1,158 & 1,029 & 834 & 796 & -1.7 & 9.4 \\
\hline Finland & 507 & 683 & 375 & 267 & 207 & -25.8 & 25.0 \\
\hline France. & 4,406 & 4,481 & 4,223 & 4,001 & 4,489 & -1.7 & -.5 \\
\hline Germany, FR & 769 & 681 & 565 & 336 & 352 & 12.9 & 21.6 \\
\hline 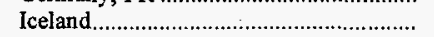 & 119 & 76 & 7 & - & 39 & 56.3 & 32.3 \\
\hline India & 11 & - & - & - & - & - & - \\
\hline Italy & 4,507 & 3,944 & 4,836 & 4,491 & 5,899 & 14.3 & -6.5 \\
\hline Japan & 6,453 & 7,395 & 6,588 & 8,525 & 9,181 & -12.7 & -8.4 \\
\hline 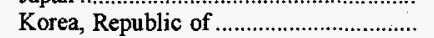 & 3,493 & 3,234 & 2,438 & 2,517 & 2,485 & 8.0 & 8.9 \\
\hline Netherlands & 3,233 & 2,635 & 2,633 & 3,228 & 4,444 & 22.7 & -7.6 \\
\hline Nigeria & - & - & - & 43 & - & - & - \\
\hline Norway & 140 & 142 & 74 & 91 & 113 & -1.4 & 5.4 \\
\hline Portugal & 164 & 33 & - & 151 & 181 & 389.9 & -2.5 \\
\hline 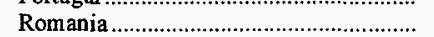 & 1,517 & 2,115 & 1,750 & 991 & 705 & -28.3 & 21.1 \\
\hline Saudi Arabia & 22 & - & - & - & - & - & - \\
\hline South Africa, Rep of & 1,192 & 946 & 771 & 577 & 620 & 26.0 & 17.8 \\
\hline Spain & 2,240 & 1,979 & 2,552 & 2,754 & 2,505 & 13.2 & -2.8 \\
\hline Sweden & 1,066 & 1,466 & 939 & 798 & 1,276 & -27.3 & -4.4 \\
\hline Turkey & 1,969 & 1,862 & 1,606 & 1,623 & 2,003 & 5.7 & -.4 \\
\hline United Kingdom & 3,160 & 2,913 & 2,963 & 3,503 & 3,831 & 8.5 & -4.7 \\
\hline Yugoslavia, FR & - & - & - & 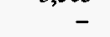 & 650 & - & - \\
\hline
\end{tabular}

\footnotetext{
* Data round to zero.
}

Notes: Major coal-exporting States are those with total coal exports of over 600,000 short tons in 1996 . Totals may not equal sum of components due to independent rounding. Foreign distribution to some countries for 1992 through 1995 have been revised.

Sources: Values shown for destinations other than Canada and Mexico are estimates based upon information reported on Energy Information Administration Form EIA-6, "Coal Distribution Report," and coal export data presented in King's COALBASE (King Publishing Corporation, Knoxville, Tennessee). See the Explanatory Notes for a complete description of the methodology used to develop these estimates. Values shown for Canada and Mexico are based upon Form EIA-6. 
Table 64. Foreign Distribution of U.S. Steam Coal by Major Coal-Exporting States and Destination, 1992-1996

(Thousand Short Tons)

\begin{tabular}{|c|c|c|c|c|c|c|c|}
\hline \multirow{2}{*}{$\begin{array}{l}\text { Coal-Exporting State } \\
\text { and Destination }\end{array}$} & \multirow{2}{*}{1996} & \multirow[t]{2}{*}{1995} & \multirow[t]{2}{*}{1994} & \multirow{2}{*}{1993} & \multirow[t]{2}{*}{1992} & \multirow{2}{*}{$\begin{array}{c}\text { Percent } \\
\text { Change } \\
\text { 1995-1996 }\end{array}$} & \multirow{2}{*}{$\begin{array}{c}\begin{array}{c}\text { Average Annual } \\
\text { Percent Change }\end{array} \\
1992-1996\end{array}$} \\
\hline & & & & & & & \\
\hline Alabama & 341. & 702 & 170 & 43 & 131 & -51.5 & 27.0 \\
\hline Denmark & - & 26 & - & - & - & -100.0 & - \\
\hline Italy & 341. & 617 & 144 & - & 128 & -44.8 & 27.8 \\
\hline Japan & - & - & - & 3 & 3 & - & - \\
\hline 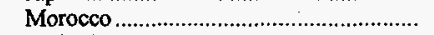 & - & - & - & 39 & - & - & - \\
\hline 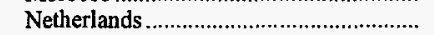 & - & 59 & - & - & - & -100.0 & - \\
\hline Spain & - & - & 27 & - & - & - & - \\
\hline Alaska & 776 & 855 & 716 & 743 & 734 & -9.2 & 1.4 \\
\hline Korea, Republic of & 776 & 855 & 716 & 743 & 734 & -9.2 & 1.4 \\
\hline Colorado & 1,385 & 900 & 752 & 1,128 & 669 & 53.9 & 20.0 \\
\hline 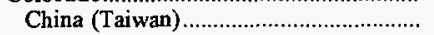 & 219 & 235 & 134 & - & - & -6.5 & - \\
\hline 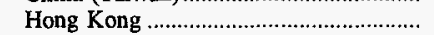 & - & - & 46 & - & - & - & - \\
\hline 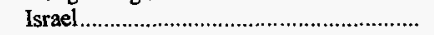 & 30 & - & - & - & - & - & - \\
\hline Japan .....1. & 314 & 651 & 395 & 918 & 669 & -51.9 & -17.3 \\
\hline 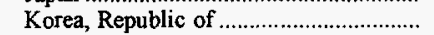 & 65 & - & 177 & 209 & - & - & - \\
\hline 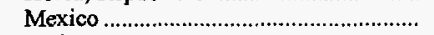 & 758 & - & - & - & - & - & - \\
\hline 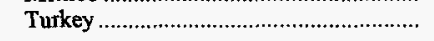 & - & 14 & - & - & - & -100.0 & - \\
\hline 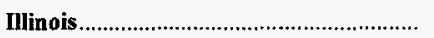 & 1,886 & 2,650 & - & 561 & 629 & -28.8 & 31.6 \\
\hline 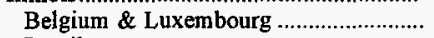 & 76 & - & - & - & - & - & - \\
\hline 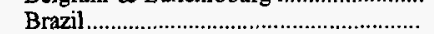 & 1 & - & - & - & - & - & - \\
\hline 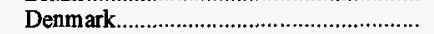 & 364 & 516 & - & - & 45 & -29.4 & 68.2 \\
\hline 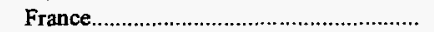 & - & 57 & - & _- & - & -100.0 & - \\
\hline Germany, FR & 325 & 722 & - & - & - & -54.9 & - \\
\hline lreland & - & - & - & 108 & 213 & - & - \\
\hline Italy ...... & - & 42 & - & - & - & -100.0 & - \\
\hline 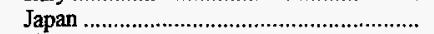 & 66 & - & - & - & 21 & & 33.8 \\
\hline Morocco & 103 & 775 & - & 452 & 350 & -86.7 & -26.3 \\
\hline 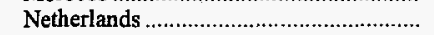 & 120 & - & - & - & - & - & - \\
\hline 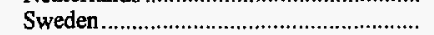 & 25 & - & - & - & - & - & - \\
\hline 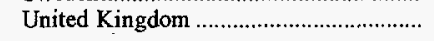 & 805 & 538 & - & - & - & 49.7 & - \\
\hline Kentucky & 3,841 & 6,055 & 4,047 & 5,106 & 8,120 & -36.6 & -17.1 \\
\hline Belgium \& Luxembourg ……………....... & - & 366 & 406 & 437 & 577 & -100.0 & - \\
\hline 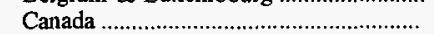 & - & - & 26 & 60 & 705 & - & - \\
\hline 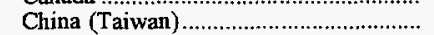 & 1,978 & 2,321 & 2,545 & 3,485 & 3,211 & -14.8 & -11.4 \\
\hline 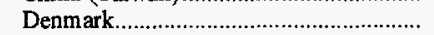 & 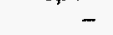 & - & - & 33 & 172 & - & - \\
\hline 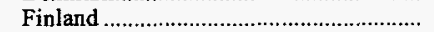 & 4 & - & - & 2 & - & - & - \\
\hline 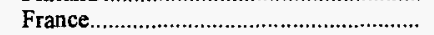 & - & - & - & $26 \overline{6}$ & 1,385 & - & - \\
\hline 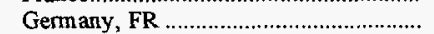 & - & 95 & - & - & - & -100.0 & - \\
\hline Ireland & _ & 58 & - & 16 & 336 & -100.0 & - \\
\hline 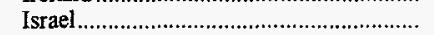 & - & 217 & - & - & - & -100.0 & - \\
\hline 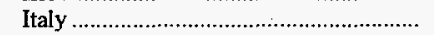 & 1,613 & 1,714 & 763 & 719 & 805 & -5.9 & 19.0 \\
\hline Jamaica........ & 17 & 62 & 26 & 39 & 35 & -73.0 & -16.4 \\
\hline Japan & - & - & 33 & 50 & - & - & - \\
\hline 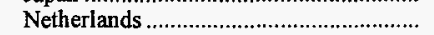 & - & 519 & 223 & - & 546 & -100.0 & - \\
\hline 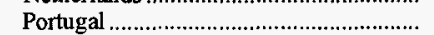 & 229 & & 24 & - & - & - & - \\
\hline 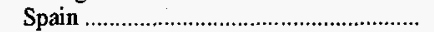 & - & 231 & - & - & 98 & -100.0 & - \\
\hline 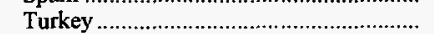 & - & 197 & - & - & - & -100.0 & - \\
\hline United Kingdom .................................... & - & 275 & - & - & 250 & -100.0 & - \\
\hline Pennsylvania & 7,604 & 6,812 & 4,677 & 3,589 & 5,139 & 11.6 & 10.3 \\
\hline Belgium \& Luxembourg ............................ & - & - & - & - & 90 & - & - \\
\hline 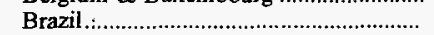 & 3 & 9 & 5 & 7 & 12 & -71.1 & -31.2 \\
\hline 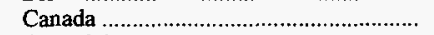 & 1,050 & 708 & 844 & 587 & 1,599 & 48.2 & -10.0 \\
\hline 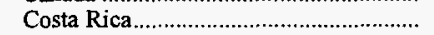 & - & - & - & - & $*$ & - & - \\
\hline 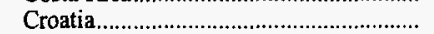 & - & - & - & 45 & - & - & - \\
\hline 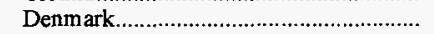 & 801 & 1,589 & 508 & 187 & 1,441 & -49.6 & -13.7 \\
\hline Dominican Republic & 50 & 18 & 42 & 59 & 157 & 172.7 & -24.8 \\
\hline 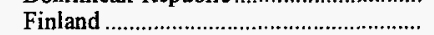 & 283 & 544 & 71 & - & - & -48.1 & - \\
\hline 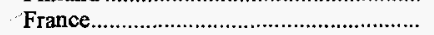 & - & 9 & - & 188 & 184 & -100.0 & - \\
\hline Germany, FR ..... & 256 & 317 & 23 & 102 & 151 & -19.3 & 14.0 \\
\hline 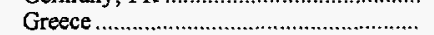 & 491 & - & - & - & - & - & - \\
\hline Indonesia & - & - & - & - & * & - & - \\
\hline Ireland & 1,067 & 1,161 & 1,015 & 911 & - & -8.0 & - \\
\hline Israel & 1,068 & 995 & 922 & 940 & 1,090 & 7.4 & -.5 \\
\hline Italy ...1-1 & 89 & - & 601 & 50 & - & - & - \\
\hline
\end{tabular}

See footnotes at end of table 
Table 64. Foreign Distribution of U.S. Steam Coal by Major Coal-Exporting States and Destination, 1992-1996 (Continued)

(Thousand Short Tons)

\begin{tabular}{|c|c|c|c|c|c|c|c|}
\hline \multirow{2}{*}{$\begin{array}{l}\text { Coal-Exporting State } \\
\text { and Destination }\end{array}$} & \multirow{2}{*}{1996} & \multirow{2}{*}{1995} & \multirow{2}{*}{1994} & \multirow{2}{*}{1993} & \multirow{2}{*}{1992} & \multirow{2}{*}{$\begin{array}{c}\text { Percent } \\
\text { Change } \\
\text { 1995-1996 }\end{array}$} & \multirow{2}{*}{$\begin{array}{c}\begin{array}{c}\text { Average Annual } \\
\text { Percent Change }\end{array} \\
1992-1996\end{array}$} \\
\hline & & & & & & & \\
\hline \multicolumn{8}{|l|}{ Pennsylvania (Continued) } \\
\hline 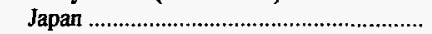 & 136 & - & - & - & - & - & - \\
\hline Korea, Republic of ................................... & 94 & - & - & - & - & - & - \\
\hline Malaysia & - & - & - & - & * & - & - \\
\hline Mexico & - & - & - & - & 18 & - & - \\
\hline Morocco & 173 & - & - & - & - & - & - \\
\hline Netherlands & 570 & 593 & 244 & - & 2 & -3.8 & 322.5 \\
\hline Norway & 30 & 28 & 14 & 30 & 48 & 5.1 & -11.5 \\
\hline Panama & - & - & - & - & * & - & - \\
\hline 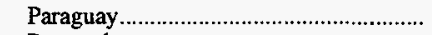 & - & - & - & - & 3 & - & - \\
\hline 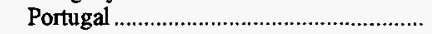 & 592 & 472 & 378 & 467 & 302 & 25.5 & 18.3 \\
\hline 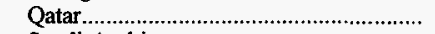 & - & - & - & - & * & - & - \\
\hline 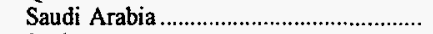 & - & - & - & * & - & - & - \\
\hline Spain & - & 18 & - & - & - & -100.0 & - \\
\hline Surinam & - & - & - & * & * & - & - \\
\hline Sweden & - & - & - & - & 1 & - & - \\
\hline Trinidad \& Tobago.......................................... & - & - & - & 1 & 2 & - & - \\
\hline 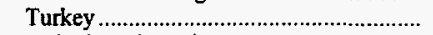 & - & 43 & - & - & - & -100.0 & - \\
\hline United Arab Emirates................................... & - & - & - & * & - & - & - \\
\hline United Kingdom ............................................ & 851 & 299 & - & * & - & 184.9 & - \\
\hline 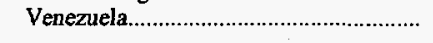 & 1 & 9 & 11 & 15 & 39 & -88.6 & -59.5 \\
\hline Utah & 5,118 & 3,930 & 2,698 & 2,959 & 2,260 & 30.2 & 22.7 \\
\hline Canada & - & - & - & 346 & - & - & - \\
\hline 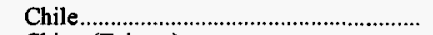 & 445 & 170 & - & - & - & 162.1 & - \\
\hline 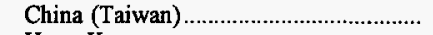 & 648 & 323 & 321 & 849 & 721 & 100.4 & -2.6 \\
\hline 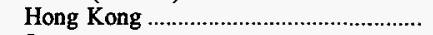 & - & & - & - & 359 & - & - \\
\hline Japan & 3,871 & 3,000 & 2,377 & 1,764 & 1,180 & 29.1 & 34.6 \\
\hline 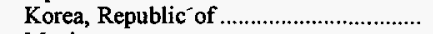 & 154 & 438 & - & - & - & -64.7 & - \\
\hline 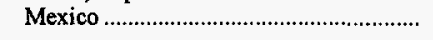 & - & - & - & * & - & - & - \\
\hline Virginia & 671 & 821 & 527 & 503 & 2,835 & -18.2 & -30.2 \\
\hline 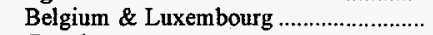 & - & - & - & - & 312 & - & - \\
\hline Canada & - & - & - & - & 1,331 & - & - \\
\hline 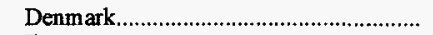 & - & - & - & - & 60 & - & - \\
\hline 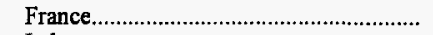 & - & - & - & - & 68 & - & - \\
\hline Italy & 502 & 716 & 359 & 413 & 723 & -29.9 & -8.7 \\
\hline 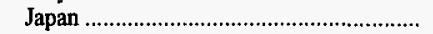 & - & - & 77 & 55 & 65 & - & - \\
\hline Netherlands & - & - & - & 36 & - & - & - \\
\hline Portugal & 145 & 105 & 91 & - & 210 & 38.0 & -8.9 \\
\hline Spain & - & - & - & - & 66 & - & - \\
\hline 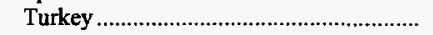 & 24 & - & - & - & - & - & - \\
\hline 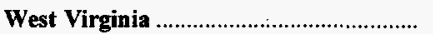 & 10,327 & 9,688 & 4,602 & 6,655 & 17,393 & 6.6 & -12.2 \\
\hline Belgium \& Luxembourg ............................. & 921 & 845 & 1,010 & 813 & 1,247 & 8.9 & -7.3 \\
\hline 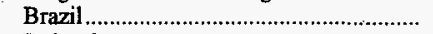 & 10 & * & - & - & - & NM & - \\
\hline Bulgaria & 62 & - & - & - & - & - & - \\
\hline Canada & 315 & 25 & 40 & 37 & 890 & NM & -22.8 \\
\hline Chile & 152 & 118 & - & - & - & 29.7 & - \\
\hline 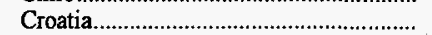 & - & 72 & - & - & - & -100.0 & - \\
\hline 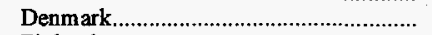 & - & 189 & - & 168 & 2,397 & -100.0 & - \\
\hline 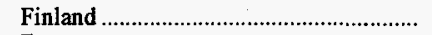 & - & 109 & - & - & - & -100.0 & - \\
\hline 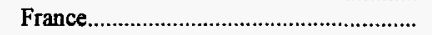 & 817 & 815 & - & 536 & 3,095 & .3 & -28.3 \\
\hline Germany, FR & 358 & 854 & - & 349 & 644 & -58.0 & -13.6 \\
\hline 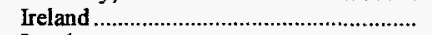 & - & - & - & 117 & 822 & - & - \\
\hline 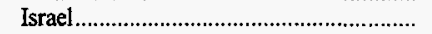 & 375 & - & - & - & 210 & - & 15.6 \\
\hline Italy & 2,604 & 2,266 & 707 & 705 & 1,400 & 14.9 & 16.8 \\
\hline Jamaica & 36 & - & - & - & - & - & - \\
\hline 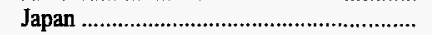 & & 209 & 448 & 431 & 418 & -100.0 & - \\
\hline Morocco & 1,111 & 275 & 101 & 79 & 170 & 304.0 & 59.9 \\
\hline 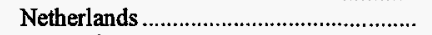 & 413 & 2,105 & 1,623 & 1,191 & 1,318 & -80.4 & -25.2 \\
\hline Portugal .................... & 964 & 1,357 & 674 & 993 & 854 & -29.0 & 3.1 \\
\hline Slovenia & - & 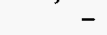 & - & - & 69 & - & - \\
\hline 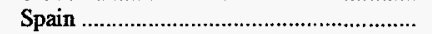 & 69 & - & - & 140 & 269 & - & -28.9 \\
\hline 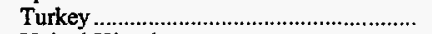 & 12 & - & - & - & - & - & - \\
\hline 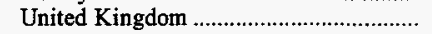 & 2,109 & 451 & - & 1,097 & 3,590 & 367.9 & -12.5 \\
\hline
\end{tabular}

See footnotes at end of table. 
Table 64. Foreign Distribution of U.S. Steam Coal by Major Coal-Exporting States and Destination, 1992-1996 (Continued)

(Thousand Short Tons)

\begin{tabular}{|c|c|c|c|c|c|c|c|}
\hline \multirow{2}{*}{$\begin{array}{l}\text { Coal-Exporting State } \\
\text { and Destination }\end{array}$} & \multirow{2}{*}{1996} & \multirow{2}{*}{1995} & \multirow{2}{*}{1994} & \multirow{2}{*}{1993} & \multirow{2}{*}{1992} & \multirow{2}{*}{$\begin{array}{c}\text { Percent } \\
\text { Change } \\
1995-1996\end{array}$} & \multirow{2}{*}{$\begin{array}{c}\begin{array}{c}\text { Average Annua } \\
\text { Percent Change }\end{array} \\
1992-1996\end{array}$} \\
\hline & & & & & & & \\
\hline Wyoming & 2,395 & 2,269 & 1,524 & 974 & 1,277 & 5.5 & 17.0 \\
\hline 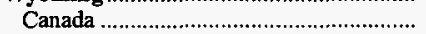 & 443 & 32 & - & - & - & NM & - \\
\hline Japan & - & - & - & * & - & - & - \\
\hline 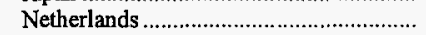 & 63 & - & - & - & - & - & - \\
\hline Spain & 1,889 & 2,237 & 1,524 & 974 & 1,277 & -15.6 & $10 . \overline{3}$ \\
\hline Major States Total & 34,344 & 34,683 & 19,713 & 22,261 & 39,187 & -1.0 & -3.2 \\
\hline Belgium \& Luxembourg .............................. & 997 & 1,211 & 1,416 & 1,250 & 2,226 & -17.7 & -18.2 \\
\hline 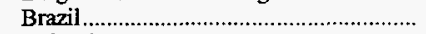 & 14 & 9 & 5 & 7 & 12 & 51.8 & 4.2 \\
\hline Bulgaria & 62 & - & - & - & - & - & - \\
\hline Canada & 1,808 & 765 & 910 & 1,030 & 4,525 & 136.4 & -20.5 \\
\hline Chile & 597 & 287 & - & - & - & 107.9 & - \\
\hline 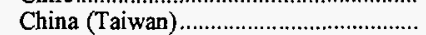 & 2,845 & 2,879 & 3,000 & 4,334 & 3,931 & -1.2 & -7.8 \\
\hline Costa Rica & 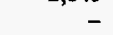 & - & - & - & 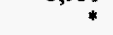 & - & - \\
\hline Croatia & - & 72 & - & 45 & - & -100.0 & - \\
\hline Denmark & 1,165 & 2,320 & 508 & 388 & 4,116 & -49.8 & -27.1 \\
\hline Dominican Republic ................................ & 50 & 18 & 42 & 59 & 157 & 172.7 & -24.8 \\
\hline 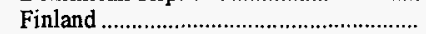 & 287 & 654 & 71 & 2 & - & -56.1 & - \\
\hline 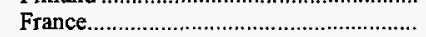 & 817 & 882 & - & 989 & 4,732 & -7.3 & -35.5 \\
\hline 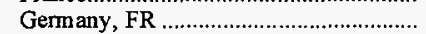 & 939 & 1,987 & 23 & 451 & 796 & -52.7 & 4.2 \\
\hline 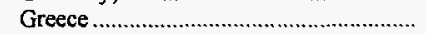 & 491 & 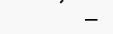 & - & - & - & - & - \\
\hline Hong Kong …….............................. & - & - & 46 & - & 359 & - & - \\
\hline Indonesia & - & - & - & - & * & - & - \\
\hline Ireland & 1,067 & 1,219 & 1,015 & 1,152 & 1,371 & -12.4 & -6.1 \\
\hline Israel & 1,473 & 1,212 & 922 & 940 & 1,300 & 21.5 & 3.2 \\
\hline Italy & 5,149 & 5,354 & 2,573 & 1,886 & 3,056 & -3.8 & 13.9 \\
\hline 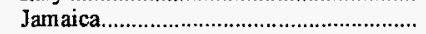 & 53 & 62 & 26 & 39 & 35 & -15.6 & 11.1 \\
\hline Japan & 4,387 & 3,860 & 3,330 & 3,222 & 2,356 & 13.7 & 16.8 \\
\hline 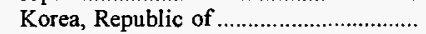 & 1,089 & 1,292 & 894 & 952 & 734 & -15.7 & 10.4 \\
\hline 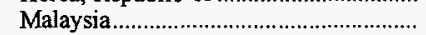 & - & 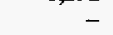 & - & - & $*$ & - & - \\
\hline Mexico & 758 & - & - & * & 18 & - & 156.3 \\
\hline Morocco & 1,388 & 1,050 & 101 & 571 & 520 & 32.2 & 27.8 \\
\hline 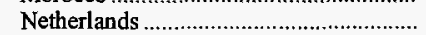 & 1,165 & 3,276 & 2,090 & 1,227 & 1,866 & -64.4 & -11.1 \\
\hline 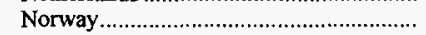 & 30 & 28 & 14 & 30 & 48 & 5.1 & -11.5 \\
\hline 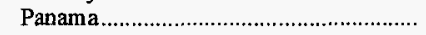 & - & - & - & - & * & - & - \\
\hline Paraguay & - & - & - & - & 3 & - & - \\
\hline Portugal .............. & 1,930 & 1,933 & 1,167 & 1,460 & 1,366 & -.2 & 9.0 \\
\hline 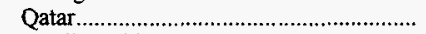 & - & - & - & - & * & - & - \\
\hline 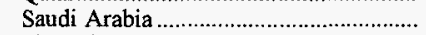 & - & - & - & * & - & - & - \\
\hline Slovenia & - & - & - & - & 69 & - & - \\
\hline 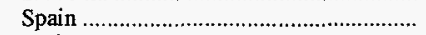 & 1,957 & 2,486 & 1,550 & 1,113 & 1,710 & -21.3 & 3.4 \\
\hline 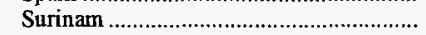 & - & - & - & $*$ & * & - & - \\
\hline 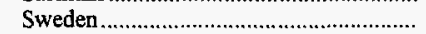 & 25 & - & - & - & 1 & - & 129.1 \\
\hline Trinidad \& Tobago & - & - & - & 1 & 2 & - & - \\
\hline 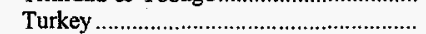 & 37 & 254 & _- & - & - & -85.6 & - \\
\hline United Arab Emirates................................... & - & - & - & * & - & - & - \\
\hline United Kingdom & 3,765 & 1,563 & - & 1,098 & 3,840 & 140.9 & -.5 \\
\hline Venezuela & l & 9 & 11 & 15 & 39 & -88.6 & -59.5 \\
\hline Other States Total & 1,693 & 997 & 686 & 414 & 761 & 69.9 & 22.1 \\
\hline Canada & 319 & 273 & 93 & 55 & * & 16.9 & 426.7 \\
\hline France & - & - & - & - & 9 & - & - \\
\hline Ireland & 80 & - & - & - & 10 & - & 68.5 \\
\hline 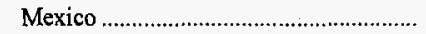 & - & 498 & - & 11 & - & -100.0 & - \\
\hline Spain & - & - & 153 & - & 53 & - & - \\
\hline United Kingdom ……… & - & - & - & - & 4 & - & - \\
\hline 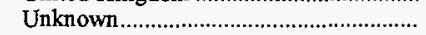 & 1,294 & 226 & 439 & 348 & 685 & 471.6 & 17.3 \\
\hline U.S. Total & 36,037 & 35,680 & 20,399 & 22,675 & 39,948 & 1.0 & -2.5 \\
\hline Belgium \& Luxembourg ................................ & 997 & 1,211 & 1,416 & 1,250 & 2,226 & -17.7 & -18.2 \\
\hline Brazil............................................... & 14 & 9 & 5 & 7 & 12 & 51.8 & 4.2 \\
\hline Bulgaria & 62 & - & - & - & - & - & - \\
\hline Canada & 2,127 & 1,037 & 1,003 & 1,085 & 4,525 & 105.0 & -17.2 \\
\hline Chile & 597 & 287 & - & - & - & 107.9 & - \\
\hline 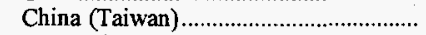 & 2,845 & 2,879 & 3,000 & 4,334 & 3,931 & -1.2 & -7.8 \\
\hline Costa Rica & - & - & - & - & * & & - \\
\hline Croatia & - & 72 & - & 45 & - & -100.0 & - \\
\hline 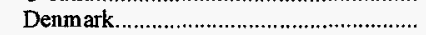 & 1,165 & 2,320 & 508 & 388 & 4,116 & -49.8 & -27.1 \\
\hline
\end{tabular}

See footnotes at end of table. 
Table 64. Foreign Distribution of U.S. Steam Coal by Major Coal-Exporting States and Destination, 1992-1996 (Continued)

(Thousand Short Tons)

\begin{tabular}{|c|c|c|c|c|c|c|c|}
\hline \multirow{2}{*}{$\begin{array}{l}\text { Coal-Exporting State } \\
\text { and Destination }\end{array}$} & \multirow[t]{2}{*}{1996} & \multirow[t]{2}{*}{1995} & \multirow[t]{2}{*}{1994} & \multirow[t]{2}{*}{1993} & \multirow[t]{2}{*}{1992} & \multirow{2}{*}{$\begin{array}{c}\text { Percent } \\
\text { Change } \\
\text { 1995-1996 }\end{array}$} & \multirow{2}{*}{$\begin{array}{c}\begin{array}{c}\text { Average Annual } \\
\text { Percent Change }\end{array} \\
1992-1996\end{array}$} \\
\hline & & & & & & & \\
\hline \multicolumn{8}{|l|}{ U.S. Total (Continued) } \\
\hline 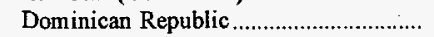 & 50 & 18 & 42 & 59 & 157 & 172.7 & -24.8 \\
\hline Finland & 287 & 654 & 71 & 2 & - & -56.1 & - \\
\hline 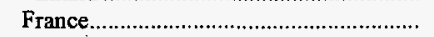 & 817 & 882 & - & 989 & 4,741 & -7.3 & -35.6 \\
\hline 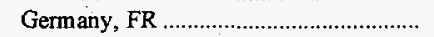 & 939 & 1,987 & 23 & 451 & 796 & -52.7 & 4.2 \\
\hline Greece & 491 & - & - & - & - & - & - \\
\hline 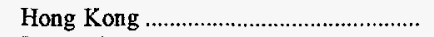 & - & - & 46 & - & 359 & - & - \\
\hline 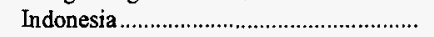 & - & - & - & - & * & - & - \\
\hline Ireland & 1,147 & 1,219 & 1,015 & 1,152 & 1,381 & -5.8 & -4.5 \\
\hline 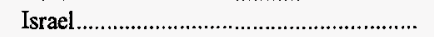 & 1,473 & 1,212 & 922 & 940 & 1,300 & 21.5 & 3.2 \\
\hline Italy & 5,149 & 5,354 & 2,573 & 1,886 & 3,056 & -3.8 & 13.9 \\
\hline 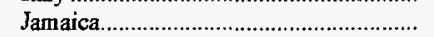 & 53 & 62 & 26 & 39 & 35 & -15.6 & 11.1 \\
\hline Japan & 4,387 & 3,860 & 3,330 & 3,222 & 2,356 & 13.7 & 16.8 \\
\hline 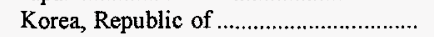 & 1,089 & 1,292 & 894 & 952 & 734 & -15.7 & 10.4 \\
\hline 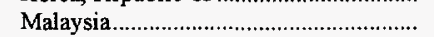 & - & - & - & - & $*$ & - & - \\
\hline Mexico & 758 & 498 & - & 11 & 18 & 52.2 & 156.3 \\
\hline Morocco & 1,388 & 1,050 & 101 & 571 & 520 & 32.2 & 27.8 \\
\hline 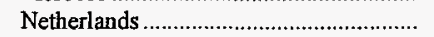 & 1,165 & 3,276 & 2,090 & 1,227 & 1,866 & -64.4 & -11.1 \\
\hline Norway & 30 & 28 & 14 & 30 & 48 & 5.1 & -11.5 \\
\hline 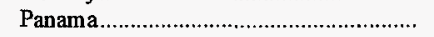 & - & - & - & - & $*$ & - & - \\
\hline 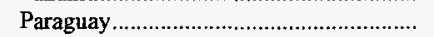 & - & - & - & - & 3 & - & - \\
\hline Portugal & 1,930 & 1,933 & 1,167 & 1,460 & 1,366 & -.2 & 9.0 \\
\hline 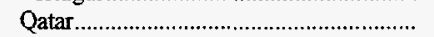 & - & - & - & - & $*$ & - & - \\
\hline Saudi Arabia & - & - & - & * & - & - & - \\
\hline Slovenia & - & - & - & - & 69 & - & - \\
\hline Spain & 1,957 & 2,486 & 1,704 & 1,113 & 1,763 & -21.3 & 2.6 \\
\hline Surinam & - & - & - & + & $*$ & - & - \\
\hline Sweden & 25 & - & - & - & 1 & - & 129.1 \\
\hline 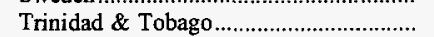 & - & - & - & 1 & 2 & - & - \\
\hline Turkey & 37 & 254 & - & - & - & -85.6 & - \\
\hline 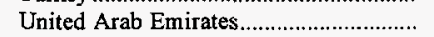 & - & - & - & $*$ & - & - & - \\
\hline United Kingdom & 3,765 & 1,563 & - & 1,098 & 3,844 & 140.9 & -.5 \\
\hline 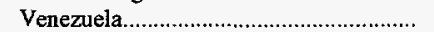 & 1 & 9 & 11 & 15 & 39 & -88.6 & -59.5 \\
\hline Unknown & 1,294 & 226 & 439 & 348 & 685 & 471.6 & 17.3 \\
\hline
\end{tabular}

* Data round to zero.

NM Not meaningful as value is greater than 500 percent.

Notes: Major coal-exporting States are those with total coal exports of over 600,000 short tons in 1996. Totals may not equal sum of components due to independent rounding. Foreign distribution to some countries for 1992 through 1995 have been revised.

Sources: Values shown for destinations other than Canada and Mexico are estimates based upon information reported on Energy Information Administration Form EIA-6, "Coal Distribution Report," and coal export data presented in King's COALBASE (King Publishing Corporation, Knoxville, Tennessee). See the Explanatory Notes for a complete description of the methodology used to develop these estimates. Values shown for Canada and Mexico are based upon Form EIA-6. 
Table 65. Distribution of U.S. Coal by Origin, Destination, and Method of Transportation, 1996 (Thousand Short Tons)

\begin{tabular}{|c|c|c|c|c|c|c|c|c|c|}
\hline \multirow{2}{*}{$\begin{array}{l}\text { Origin State and } \\
\text { Destination State }\end{array}$} & \multirow{2}{*}{ Railroad } & \multicolumn{4}{|c|}{ Water } & \multirow{2}{*}{ Truck } & \multirow{2}{*}{$\begin{array}{l}\text { Tramway, } \\
\text { Conveyor, } \\
\text { and Slurry } \\
\text { Pipeline }\end{array}$} & \multirow{2}{*}{ Unknown } & \multirow{2}{*}{ Total } \\
\hline & & River & $\begin{array}{l}\text { Great } \\
\text { Lakes }\end{array}$ & Tidewater & Total & & & & \\
\hline Alabama & 7,875 & 4,613 & - & 4,864 & 9,477 & 6,143 & 1,108 & 32 & 24,636 \\
\hline Alabama & 7,323 & 4,012 & - & - & 4,012 & 6,059 & 1,108 & - & 18,503 \\
\hline 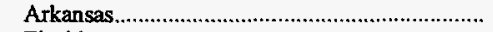 & 29 & - & - & - & - & 9 & - & - & 39 \\
\hline Florida & - & - & - & - & - & 8 & - & - & 8 \\
\hline 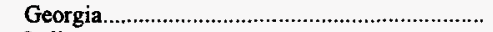 & 373 & - & - & - & - & - & - & - & 373 \\
\hline Indiana & 57 & - & - & - & - & - & - & - & 57 \\
\hline Kentucky & 9 & - & - & - & - & 5 & - & - & 15 \\
\hline 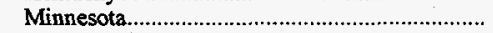 & - & - & - & - & - & * & - & - & * \\
\hline 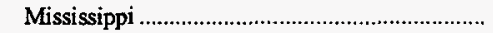 & 80 & 22 & - & - & 22 & 9 & - & - & 110 \\
\hline 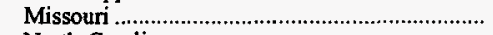 & - & - & - & - & - & * & - & - & * \\
\hline 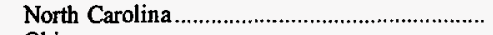 & 4 & - & - & - & - & - & - & - & 4 \\
\hline Ohio & - & - & - & - & - & 51 & - & - & 51 \\
\hline Pennsylvania & - & 579 & - & - & 579 & - & - & - & 579 \\
\hline 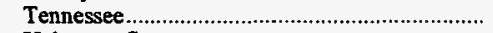 & - & - & - & - & - & 1 & - & - & 1 \\
\hline Unknown State & - & - & - & - & - & - & - & 32 & 32 \\
\hline 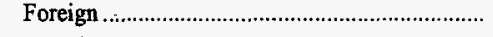 & - & - & - & 4,864 & 4,864 & - & - & - & 4,864 \\
\hline Alaska & 519 & - & - & 776 & 776 & 177 & - & - & 1,473 \\
\hline Alaska & 519 & - & - & - & - & 177 & - & - & 697 \\
\hline Foreign & - & - & - & 776 & 776 & - & - & - & 776 \\
\hline Arizona & 6,499 & - & - & - & - & - & 4,470 & - & 10,970 \\
\hline 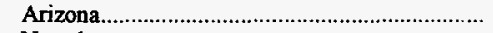 & 6,499 & - & - & - & - & - & - & - & 6,499 \\
\hline Nevada & - & - & - & - & - & - & 4,470 & - & 4,470 \\
\hline 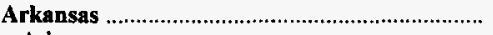 & - & - & - & - & - & 7 & - & - & 7 \\
\hline 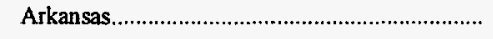 & - & - & - & - & - & 7 & - & - & 7 \\
\hline Colorado & 18,817 & 1,030 & 73 & 793 & 1,896 & 4,673 & - & 18 & 25,405 \\
\hline 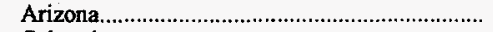 & 355 & - & - & - & - & $*$ & - & - & 355 \\
\hline 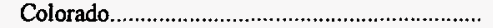 & 6,566 & - & - & - & - & 4,138 & - & - & 10,704 \\
\hline Florida & - & - & - & 136 & 136 & - & - & - & 136 \\
\hline Illinois & 221 & 349 & - & - & 349 & 69 & - & - & 640 \\
\hline Iowa & 587 & 5 & - & - & 5 & - & - & - & 591 \\
\hline 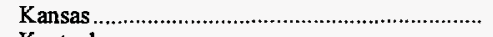 & 1,493 & - & - & - & - & - & - & - & 1,493 \\
\hline 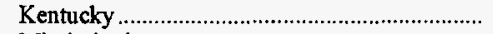 & 260 & - & - & - & - & - & - & - & 260 \\
\hline 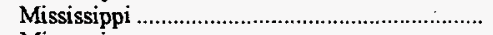 & 519 & $\overline{-}$ & - & - & - & - & - & - & 519 \\
\hline 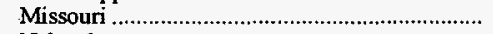 & 1,055 & 22 & - & - & 22 & - & - & - & 1,077 \\
\hline Nebraska & 56 & - & - & - & - & - & - & - & 56 \\
\hline 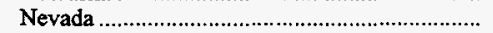 & 132 & - & - & - & - & - & - & - & 132 \\
\hline New Mexico & - & - & - & - & - & 88 & - & - & 88 \\
\hline Oregon & 94 & - & - & - & - & - & - & - & 94 \\
\hline Tennessee & 3,038 & - & - & - & - & - & - & - & 3,038 \\
\hline Texas & 2,443 & - & - & - & - & - & - & - & 2,443 \\
\hline Utah & 1,204 & - & - & - & - & - & - & - & 1,204 \\
\hline Washington & 37 & - & - & - & - & - & - & - & 37 \\
\hline Wisconsin & - & 653 & 73 & - & 726 & - & - & - & 726 \\
\hline 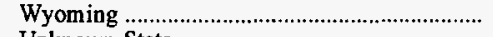 & - & - & - & - & - & 378 & - & - & 378 \\
\hline Unknown State & - & - & - & - & - & - & - & 18 & 18 \\
\hline Foreign & 758 & - & - & 657 & 657 & - & - & - & 1,415 \\
\hline Mlinois & 26,181 & 12,736 & 47 & 1,886 & 14,668 & 6,187 & 23 & 16 & 47,076 \\
\hline Alabama & - & 2,155 & - & - & 2,155 & - & - & - & 2,155 \\
\hline 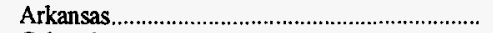 & 76 & - & - & - & - & - & - & - & 76 \\
\hline 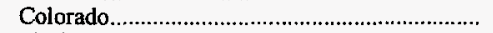 & 40 & - & - & - . & - & - & - & - & 40 \\
\hline Florida & 1,790 & 4,262 & - & - & 4,262 & - & - & - & 6,052 \\
\hline 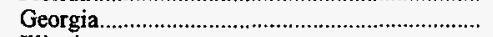 & 1,204 & - & - & - & - & - & - & - & 1,204 \\
\hline 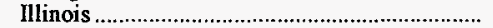 & 10,145 & 878 & - & - & 878 & 5,006 & 23 & - & 16,052 \\
\hline Indiana & 7,806 & 109 & - & - & 109 & 263 & - & - & 8,178 \\
\hline lowa & 29 & 524 & - & - & 524 & 141 & - & - & 694 \\
\hline 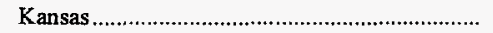 & 149 & - & - & - & - & - & - & - & 149 \\
\hline Kentucky & - & - & - & - & - & 1 & - & - & 1 \\
\hline 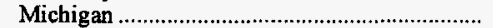 & 40 & - & 19 & - & 19 & - & - & - & 59 \\
\hline Minnesota & 100 & - & - & - & - & - & - & - & 100 \\
\hline 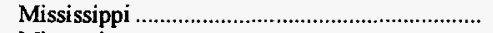 & 1,262 & 469 & - & - & 469 & 17 & - & - & 1,749 \\
\hline 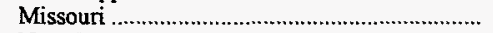 & 3,038 & 660 & - & - & 660 & 705 & - & - & 4,403 \\
\hline 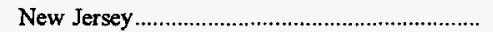 & - & - & - & - & - & $*$ & - & - & * \\
\hline New York & - & - & - & - & - & $*$ & - & - & $*$ \\
\hline Ohio & 17 & - & - & - & - & 1 & - & - & 18 \\
\hline
\end{tabular}

See footnotes at end of table. 
Table 65. Distribution of U.S. Coal by Origin, Destination, and Method of Transportation, 1996 (Continued)

(Thousand Short Tons)

\begin{tabular}{|c|c|c|c|c|c|c|c|c|c|}
\hline \multirow{2}{*}{$\begin{array}{l}\text { Origin State and } \\
\text { Destination State }\end{array}$} & \multirow[b]{2}{*}{ Railroad } & \multicolumn{4}{|c|}{ Water } & \multirow{2}{*}{ Truck } & \multirow{2}{*}{$\begin{array}{c}\text { Tramway, } \\
\text { Conveyor, } \\
\text { and Slurry } \\
\text { Pipeline }\end{array}$} & \multirow[b]{2}{*}{ Unknown } & \multirow[b]{2}{*}{ Total } \\
\hline & & River & $\begin{array}{l}\text { Great } \\
\text { Lakes }\end{array}$ & Tidewater & Total & & & & \\
\hline \multicolumn{10}{|l|}{ Illinois (Continued) } \\
\hline 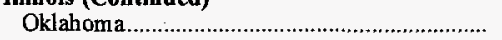 & - & 10 & - & - & 10 & - & - & - & 10 \\
\hline 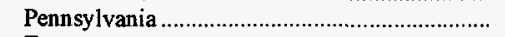 & - & - & - & - & - & * & - & - & $*$ \\
\hline 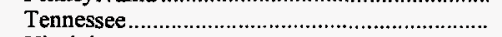 & 203 & 3,021 & - & - & 3,021 & - & - & - & 3,225 \\
\hline 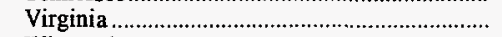 & - & - & - & - & - & $*$ & - & - & $*$ \\
\hline Wisconsin & 282 & 647 & 28 & - & 675 & 52 & - & - & 1,008 \\
\hline Unknown State & - & - & - & - & - & - & - & 16 & 16 \\
\hline 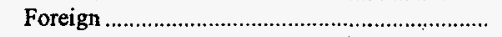 & - & - & - & 1,886 & 1,886 & - & - & - & 1,886 \\
\hline Indiana & 16,570 & 2,756 & 447 & 11 & 3,214 & 9,378 & 505 & 8 & 29,674 \\
\hline Alabama & - & 26 & - & - & 26 & - & - & - & 26 \\
\hline 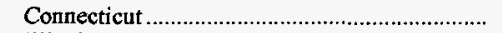 & - & - & - & - & - & * & - & - & $*$ \\
\hline lllinois & 662 & 206 & - & - & 206 & 575 & - & - & 1,444 \\
\hline Indiana & 15,161 & - & 199 & - & 199 & 8,445 & 505 & - & 24,309 \\
\hline lowa & 504 & 134 & - & - & 134 & - & - & - & 638 \\
\hline Kentucky & - & 2,297 & - & - & 2,297 & 312 & - & - & 2,610 \\
\hline 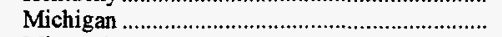 & - & - & 175 & - & 175 & 6 & - & - & 181 \\
\hline 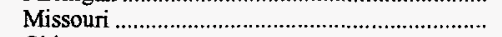 & - & 12 & - & - & 12 & 4 & - & - & 17 \\
\hline Ohio & - & 34 & - & - & 34 & - & - & - & 34 \\
\hline 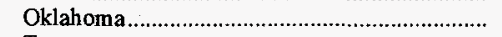 & - & 4 & - & - & 4 & - & - & - & 4 \\
\hline 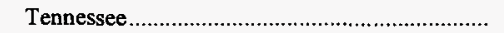 & - & 41 & - & - & 41 & - & - & - & 41 \\
\hline 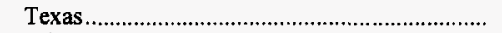 & - & - & - & - & - & 2 & - & - & 2 \\
\hline Wisconsin & 243 & - & 73 & - & 73 & 34 & - & - & 349 \\
\hline 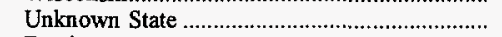 & - & - & - & - & - & - & - & 8 & 8 \\
\hline 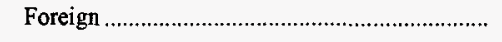 & - & - & - & 11 & 11 & - & - & - & 11 \\
\hline Kansas & - & - & - & - & - & 245 & - & - & 245 \\
\hline 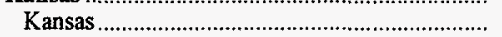 & - & - & - & - & - & 164 & - & - & 164 \\
\hline 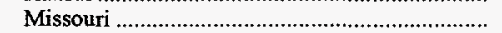 & - & - & - & - & - & 69 & - & - & 69 \\
\hline Oklahoma & - & - & - & - & - & 12 & - & - & 12 \\
\hline 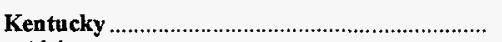 & 95,648 & 32,375 & 2,043 & 10,061 & 44,479 & 12,161 & - & 603 & 152,891 \\
\hline Alabama & 1,472 & 2,374 & - & - & 2,374 & 23 & - & - & 3,869 \\
\hline 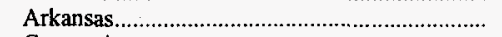 & - & - & - & - & - & 11 & - & - & 11 \\
\hline 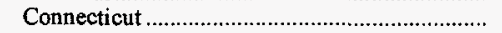 & - & - & - & 659 & 659 & - & - & - & 659 \\
\hline Florida & 12,949 & 3,372 & - & 949 & 4,321 & - & - & - & 17,270 \\
\hline 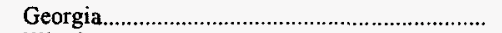 & 14,569 & - & - & - & - & 120 & - & - & 14,689 \\
\hline 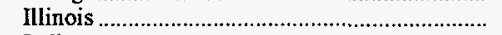 & 1,205 & 274 & - & - & 274 & 118 & - & - & 1,597 \\
\hline Indiana & 2,113 & 430 & - & - & 430 & 99 & - & - & 2,642 \\
\hline Iowa & 63 & 586 & - & - & 586 & - & - & - & 650 \\
\hline 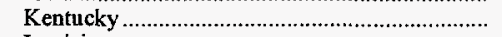 & 8,208 & 8,280 & - & - & 8,280 & 9,213 & - & - & 25,700 \\
\hline 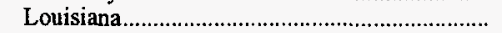 & - & 690 & - & - & 690 & - & $\rightarrow$ & - & 690 \\
\hline Maine & - & - & - & 271 & 271 & - & - & - & 271 \\
\hline 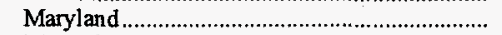 & 97 & 8 & - & - & 8 & - & - & - & 105 \\
\hline 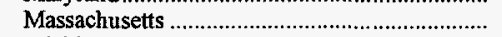 & 255 & - & - & 151 & 151 & - & - & - & 407 \\
\hline Michigan & 5,717 & 310 & 537 & - & 846 & 108 & - & - & 6,671 \\
\hline 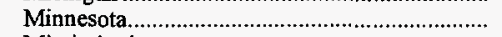 & - & 113 & 43 & - & 156 & $\bullet$ & - & - & 156 \\
\hline 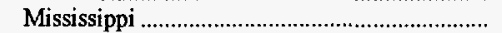 & 928 & 107 & - & - & 107 & - & - & - & 1,035 \\
\hline 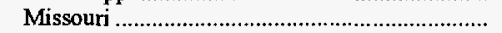 & 101 & 220 & - & - & 220 & - & - & - & 320 \\
\hline 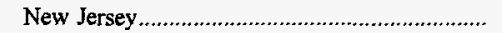 & - & - & - & 29 & 29 & - & - & - & 29 \\
\hline 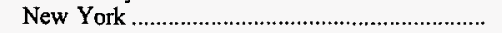 & 1,184 & 8 & - & 36 & 43 & - & - & - & 1,227 \\
\hline North Carolina & 16,885 & 52 & - & - & 52 & 302 & - & - & 17,240 \\
\hline Ohio & 3,885 & 4,528 & 38 & - & 4,566 & 1,070 & - & - & 9,520 \\
\hline Oklahoma & - & 2 & - & - & 2 & - & - & - & 2 \\
\hline Oregon & 24 & - & - & - & - & - & - & - & 24 \\
\hline 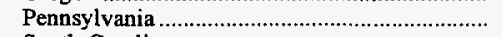 & 1,079 & 1,622 & - & - & 1,622 & 20 & - & - & 2,721 \\
\hline 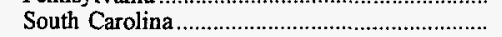 & 11,385 & - & - & - & - & 33 & - & - & 11,417 \\
\hline 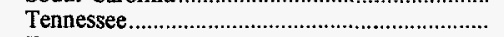 & 7,376 & 8,972 & - & - & 8,972 & 834 & - & - & 17,183 \\
\hline 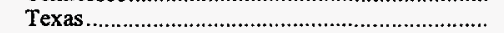 & 2 & 23 & - & - & 23 & - & - & - & 26 \\
\hline Virginia & 5,535 & - & - & - & - & 126 & - & - & 5,662 \\
\hline 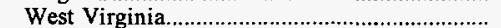 & 189 & 187 & - & - & 187 & 50 & - & - & 426 \\
\hline 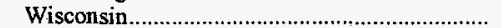 & 273 & 218 & 403 & - & 621 & 31 & - & - & 925 \\
\hline 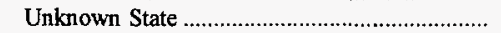 & - & - & - & - & - & - & - & 603 & 603 \\
\hline Foreign . & 154 & - & 1,022 & 7,966 & 8,987 & 2 & - & - & 9,143 \\
\hline Kentucky, Eastern & 87,381 & 11,122 & 2,043 & 9,395 & 22,560 & 6,911 & - & 553 & 117,404 \\
\hline
\end{tabular}

See footnotes at end of table. 
Table 65. Distribution of U.S. Coal by Origin, Destination, and Method of Transportation, 1996 (Continued)

(Thousand Short Tons)

\begin{tabular}{|c|c|c|c|c|c|c|c|c|c|}
\hline \multirow{2}{*}{$\begin{array}{l}\text { Origin State and } \\
\text { Destination State }\end{array}$} & \multirow[b]{2}{*}{ Railroad } & \multicolumn{4}{|c|}{ Water } & \multirow[b]{2}{*}{ Truck } & \multirow{2}{*}{$\begin{array}{c}\text { Tramway, } \\
\text { Conveyor, } \\
\text { and Slurry } \\
\text { Pipeline }\end{array}$} & \multirow[b]{2}{*}{ Unknown } & \multirow[b]{2}{*}{ Total } \\
\hline & & River & $\begin{array}{l}\text { Great } \\
\text { Lakes }\end{array}$ & Tidewater & Total & & & & \\
\hline
\end{tabular}

Kentucky, Eastern (Continued)

Alabama

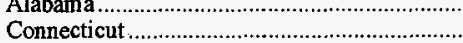

Florida .........

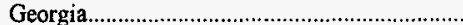

Indiana

Iowa ....

Kentucky

Louisiana

Maine .

Massachusetts

Michigan

Minnesot

Mississippi

Missouri ...

New Jersey

New York

North Carolina.

Ohio

Oklahoma..

Oregon ....

Pennsylvania

South Carolina

Tennessee.

Texas....

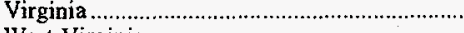

West Virgin

Unknown State

Foreign

Kentucky, Western

Alabama

Arkansas.

Florida .

Illinois

Indiana

Iowa ...

Kentucky

Louisiana..

Minnesota.

Mississippi

Missouri .

Ohio

Tennessee

Virginia

Wisconsin.

Unknown State

.

Ereign

Louisiana.

Louisiana.

Maryland.

Connecticu

Delaware

Maryland...

Massachusetts

Michigan ...

Pennsylvan

Virginia.

West Virginia

Wisconsin

Unknown State

\begin{tabular}{|c|c|c|c|}
\hline 522 & 182 & - & - \\
\hline - & - & - & 659 \\
\hline 12,848 & 218 & - & 949 \\
\hline 14,569 & - & - & - \\
\hline 1,205 & 207 & - & - \\
\hline 1,737 & 126 & - & - \\
\hline 63 & 376 & - & - \\
\hline 3,270 & 1,642 & - & - \\
\hline- & 44 & - & - \\
\hline - & - & - & 271 \\
\hline 97 & 8 & - & - \\
\hline 255 & - & - & 151 \\
\hline 5,717 & 310 & 537 & - \\
\hline - & 91 & 43 & - \\
\hline 928 & - & - & - \\
\hline 101 & 214 & - & - \\
\hline- & - & - & 29 \\
\hline 1,184 & 8 & - & 36 \\
\hline 16,885 & 52 & - & - \\
\hline 3,885 & 4,497 & 38 & - \\
\hline- & 2 & - & - \\
\hline 24 & - & - & - \\
\hline 1,079 & 1,622 & - & - \\
\hline 11,385 & - & - & - \\
\hline 5,649 & 1,107 & - & - \\
\hline & 23 & - & - \\
\hline 5,535 & - & - & - \\
\hline 189 & 187 & - & - \\
\hline 97 & 207 & 403 & - \\
\hline - & - & - & - \\
\hline 154 & _ & 1,022 & 7,299 \\
\hline
\end{tabular}

- 666

$8,267 \quad 21,253$

$950 \quad 2,192$

$10 \overline{1} \quad 3,154$

376

4,938

68
304
211

211
6,638

646
22
107

107
5

31

1,727

7,865

175

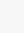

$1 \overline{1}$

$6 \overline{6}$

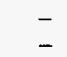

2,728

143

-
-
-
-
-
-
-
-
182

659
1,167

$20 \overline{7}$

207
126
376

376
1,642

1,642
44

27

8
151

846
134

214

29
43

52
4,535

2

$1,62 \overline{2}$

1,107

23

187

610

$8,32 \overline{1}$

21,919

21,919
2,192

3,154

68

304
211

211
6,638

646

22
107

5
31

7,865

,

$1 \overline{1}$

666

$\begin{array}{lll}645 & 787 & 672\end{array}$

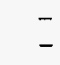

$\begin{array}{lll}645 & 787 & 672\end{array}$

$\begin{array}{rrrr}23 & - & - & 727 \\ - & - & - & 659 \\ 120 & - & - & 14,015 \\ 66 & - & - & 14,689\end{array}$

727
659

14,689

1,478

1,962
439

439
9326

$4,41 \overline{4}$

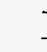

$10 \overline{8}$

*

-

302

1,070

$\begin{array}{llll}- & - & - & - \\ 24\end{array}$

20 - $\quad-\quad 2,721$

11,417

7,202

26

5,662

426

738
553

8,477

$\mathbf{5 , 2 5 0}$

11

35,487

3,142

3254

119

680

211
16,375

4,799

646

22

107

31

9,981

187
51

51

3,222

3,222

672

4,199

3
130

1,147

143

See footnotes at end of table. 
Table 65. Distribution of U.S. Coal by Origin, Destination, and Method of Transportation, 1996 (Continued)

(Thousand Short Tons)

\begin{tabular}{|c|c|c|c|c|c|c|c|c|c|}
\hline \multirow{2}{*}{$\begin{array}{l}\text { Origin State and } \\
\text { Destination State }\end{array}$} & \multirow[b]{2}{*}{ Railroad } & \multicolumn{4}{|c|}{ Water } & \multirow[b]{2}{*}{ Truck } & \multirow{2}{*}{$\begin{array}{l}\text { Tramway, } \\
\text { Conveyor, } \\
\text { and Slurry } \\
\text { Pipeline }\end{array}$} & \multirow[b]{2}{*}{ Unknown } & \multirow[b]{2}{*}{ Total } \\
\hline & & River & $\begin{array}{l}\text { Great } \\
\text { Lakes }\end{array}$ & Tidewater & Total & & & & \\
\hline $\begin{array}{l}\text { Maryland (Continued) } \\
\text { Foreign }\end{array}$ & - & - & - & 645 & 645 & - & - & - & 645 \\
\hline 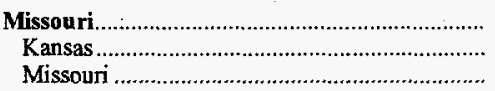 & $\begin{array}{r}184 \\
-\overline{184}\end{array}$ & $\overline{-}$ & $\overline{-}$ & $\bar{z}$ & $\begin{array}{l}- \\
-\end{array}$ & $\begin{array}{l}662 \\
345 \\
316\end{array}$ & $\begin{array}{l}- \\
- \\
-\end{array}$ & $\begin{array}{l}- \\
-\end{array}$ & $\begin{array}{l}846 \\
345 \\
501\end{array}$ \\
\hline 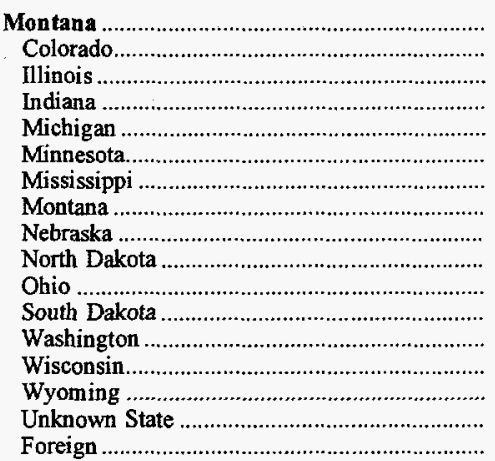 & $\begin{array}{r}23,181 \\
26 \\
2,162 \\
869 \\
3,361 \\
9,688 \\
2,226 \\
125 \\
113 \\
417 \\
1,301 \\
113 \\
2,758 \\
10 \\
- \\
11\end{array}$ & $\begin{array}{l}- \\
\overline{-} \\
\overline{-} \\
\overline{-} \\
\overline{-} \\
\overline{-} \\
\overline{-} \\
\overline{-} \\
\overline{-} \\
\overline{-}\end{array}$ & $\begin{array}{r}6,948 \\
- \\
- \\
6,445 \\
49 \\
- \\
= \\
\overline{-} \\
26 \\
- \\
- \\
165 \\
- \\
- \\
264\end{array}$ & $\begin{array}{r}243 \\
= \\
= \\
= \\
= \\
= \\
= \\
= \\
= \\
= \\
= \\
\end{array}$ & $\begin{array}{r}7,192 \\
\overline{-} \\
\overline{-} \\
6,445 \\
49 \\
- \\
- \\
- \\
- \\
26 \\
- \\
\overline{-} \\
16 \overline{-} \\
- \\
50 \overline{7}\end{array}$ & $\begin{array}{r}611 \\
- \\
- \\
- \\
\overline{5} \\
5 \overline{-} \\
416 \\
\overline{-} \\
- \\
- \\
\overline{-} \\
27 \\
115 \\
- \\
-\end{array}$ & $\begin{array}{r}7,303 \\
- \\
- \\
- \\
- \\
- \\
\overline{-} \\
7,303 \\
- \\
- \\
- \\
- \\
- \\
- \\
- \\
-\end{array}$ & $\begin{array}{l}\text { E } \\
- \\
- \\
- \\
- \\
- \\
- \\
- \\
- \\
- \\
- \\
- \\
- \\
\text { E } \\
-\end{array}$ & $\begin{array}{r}38,288 \\
26 \\
2,162 \\
869 \\
9,806 \\
9,791 \\
2,226 \\
7,844 \\
113 \\
417 \\
26 \\
1,301 \\
113 \\
2,950 \\
125 \\
* \\
518\end{array}$ \\
\hline $\begin{array}{l}\text { New Mexico } \\
\text { Arizona } \\
\text { Arkansas } \text { Colorado } \\
\text { Nebraska a } \\
\text { New Mexico } \\
\text { Oklahoma a } \\
\text { Texas } \\
\text { Wisconsin } \\
\text { Foreign }\end{array}$ & $\begin{array}{r}18,448 \\
8,859 \\
1 \\
9 \\
92 \\
8,422 \\
17 \\
316 \\
732 \\
-\end{array}$ & $\begin{array}{l}\overline{-} \\
\overline{-} \\
\overline{-} \\
\overline{-} \\
\overline{-} \\
-\end{array}$ & $\begin{array}{l}- \\
\bar{z} \\
\overline{-} \\
\bar{z} \\
\overline{-} \\
\overline{-}\end{array}$ & $\begin{array}{l}9 \\
= \\
= \\
= \\
= \\
= \\
9\end{array}$ & $\begin{array}{l}9 \\
- \\
- \\
\overline{-} \\
- \\
- \\
\overline{-}\end{array}$ & $\begin{array}{l}7 \\
* \\
- \\
- \\
\overline{7} \\
- \\
- \\
-\end{array}$ & $\begin{array}{r}6,580 \\
- \\
- \\
- \\
- \\
6,580 \\
- \\
- \\
- \\
-\end{array}$ & $\begin{array}{l}- \\
\bar{z} \\
\bar{z} \\
\bar{z} \\
\bar{z} \\
\overline{-} \\
-\end{array}$ & $\begin{array}{r}25,043 \\
8,860 \\
1 \\
9 \\
92 \\
15,009 \\
17 \\
316 \\
732 \\
9\end{array}$ \\
\hline $\begin{array}{l}\text { North Dakota } \\
\text { North Dakota }\end{array}$ & $\begin{array}{l}336 \\
336\end{array}$ & - & - & $\overline{-}$ & - & $\begin{array}{l}4,581 \\
4,581\end{array}$ & $\begin{array}{l}25,107 \\
25,107\end{array}$ & - & $\begin{array}{l}30,025 \\
30,025\end{array}$ \\
\hline $\begin{array}{l}\text { Ohio } \\
\text { Alabama } \\
\text { Illinois } \\
\text { Indiana } \text { Kentucky . } \\
\text { Michigan } \\
\text { New York } \\
\text { Ohio } \\
\text { Pennsylvania } \\
\text { Tennessee } \\
\text { West Virginia } \\
\text { Wisconsin } \\
\text { Unknown State } \\
\text { Foreign }\end{array}$ & $\begin{array}{r}1,759 \\
\overline{-} \\
\overline{23} \\
\overline{5} \\
123 \\
1,607 \\
1 \\
\overline{-} \\
- \\
- \\
-\end{array}$ & $\begin{array}{r}9,370 \\
103 \\
- \\
338 \\
22 \\
- \\
\overline{-} \\
6,302 \\
598 \\
4 \\
2,004 \\
- \\
- \\
-\end{array}$ & $\begin{array}{l}97 \\
\overline{-} \\
\overline{-} \\
\overline{7} \\
\overline{87} \\
\overline{-} \\
\overline{-} \\
\overline{-} \\
\overline{3}\end{array}$ & $\begin{array}{r}269 \\
= \\
= \\
= \\
= \\
= \\
= \\
= \\
= \\
=\end{array}$ & $\begin{array}{r}9,736 \\
103 \\
- \\
338 \\
22 \\
7 \\
- \\
6,390 \\
598 \\
4 \\
2,004 \\
- \\
\overline{-} \\
271\end{array}$ & $\begin{array}{r}8,890 \\
\overline{5} \\
102 \\
9 \\
234 \\
2 \\
8,057 \\
444 \\
- \\
28 \\
9 \\
- \\
-\end{array}$ & $\begin{array}{r}8,429 \\
= \\
= \\
= \\
= \\
8,424 \\
= \\
\overline{4} \\
= \\
=\end{array}$ & $\begin{array}{l}67 \\
- \\
- \\
- \\
- \\
- \\
- \\
- \\
- \\
- \\
- \\
67 \\
-\end{array}$ & $\begin{array}{r}28,881 \\
103 \\
5 \\
464 \\
30 \\
246 \\
125 \\
24,478 \\
1,043 \\
4 \\
2,036 \\
9 \\
67 \\
271\end{array}$ \\
\hline 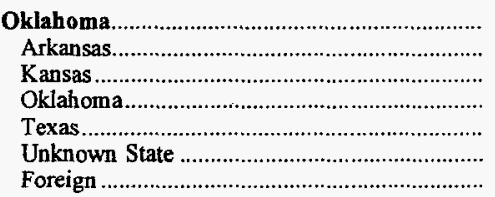 & $\begin{array}{l}\bar{z} \\
\bar{z} \\
\bar{z} \\
\bar{z}\end{array}$ & $\begin{array}{l}\overline{-} \\
\overline{-} \\
\bar{z}\end{array}$ & $\begin{array}{l}\bar{z} \\
\bar{z} \\
\bar{z}\end{array}$ & $\begin{array}{l}80 \\
= \\
= \\
= \\
80\end{array}$ & $\begin{array}{l}80 \\
\overline{-} \\
\overline{-} \\
\overline{-} \\
\overline{80}\end{array}$ & $\begin{array}{r}2,129 \\
170 \\
63 \\
1,712 \\
184 \\
- \\
-\end{array}$ & $\begin{array}{l}- \\
- \\
- \\
- \\
-\end{array}$ & $\begin{array}{l}7 \\
\overline{-} \\
- \\
\bar{z} \\
\overline{7} \\
-\end{array}$ & $\begin{array}{r}2,216 \\
170 \\
63 \\
1,712 \\
184 \\
7 \\
80\end{array}$ \\
\hline $\begin{array}{l}\text { Pennsylvania } \\
\text { Alabama } \\
\text { Arizona } \\
\text { Arkansas. } \\
\text { California } \\
\text { Colorado }\end{array}$ & $\begin{array}{r}24,146 \\
1 \\
1 \\
* \\
* \\
16\end{array}$ & $\begin{array}{r}11,285 \\
6 \\
- \\
= \\
-\end{array}$ & $\begin{array}{r}2,648 \\
- \\
- \\
- \\
-\end{array}$ & $\begin{array}{r}8,581 \\
- \\
= \\
-\end{array}$ & $\begin{array}{r}22,514 \\
6 \\
- \\
- \\
- \\
-\end{array}$ & $\begin{array}{r}17,008 \\
1 \\
* \\
* \\
* \\
-\end{array}$ & $\begin{array}{r}5,175 \\
- \\
- \\
- \\
-\end{array}$ & $\begin{array}{r}284 \\
- \\
= \\
-\end{array}$ & $\begin{array}{r}69,128 \\
8 \\
1 \\
* \\
* \\
16\end{array}$ \\
\hline
\end{tabular}

See footnotes at end of table. 
Table 65. Distribution of U.S. Coal by Origin, Destination, and Method of Transportation, 1996 (Continued)

(Thousand Short Tons)

\begin{tabular}{|c|c|c|c|c|c|c|c|c|c|}
\hline \multirow{2}{*}{$\begin{array}{l}\text { Origin State and } \\
\text { Destination State }\end{array}$} & \multirow{2}{*}{ Railroad } & \multicolumn{4}{|c|}{ Water } & \multirow{2}{*}{ Truck } & \multirow{2}{*}{$\begin{array}{l}\text { Tramway, } \\
\text { Conveyor, } \\
\text { and Slurry } \\
\text { Pipeline }\end{array}$} & \multirow{2}{*}{ Unknown } & \multirow{2}{*}{ Total } \\
\hline & & River & $\begin{array}{l}\text { Great } \\
\text { Lakes }\end{array}$ & Tidewater & Total & & & & \\
\hline \multicolumn{10}{|l|}{ Pennsylvania (Continued) } \\
\hline 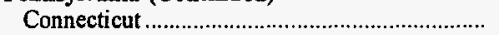 & 217 & 9 & - & - & 9 & 6 & - & - & 232 \\
\hline Delaware & 470 & 53 & - & - & 53 & 15 & - & - & 538 \\
\hline 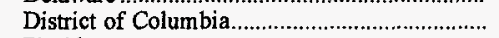 & - & - & - & - & - & $*$ & - & - & $*$ \\
\hline 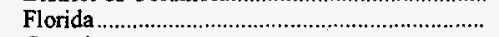 & 5 & - & - & - & - & * & - & - & 6 \\
\hline 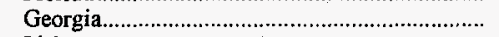 & $*$ & - & - & - & - & * & - & - & * \\
\hline Idaho & $*$ & - & - & - & - & - & - & - & * \\
\hline 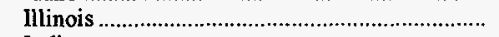 & 6 & - & - & - & - & 2 & - & - & 7 \\
\hline Indiana & * & 559 & - & - & 559 & 6 & - & - & 565 \\
\hline Iowa & 53 & 225 & - & - & 225 & 1 & - & - & 279 \\
\hline 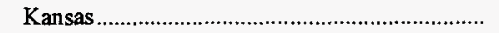 & $*$ & - & - & - & - & - & - & - & * \\
\hline 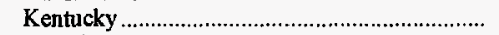 & 488 & 25 & - & - & 25 & 9 & - & - & 522 \\
\hline 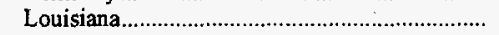 & 6 & 195 & - & - & 195 & - & - & - & 201 \\
\hline Maine & 1 & - & - & - & - & 3 & - & - & 4 \\
\hline 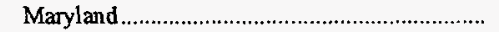 & 1,415 & - & - & - & - & 189 & - & - & 1,604 \\
\hline 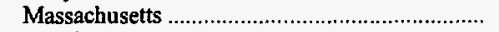 & 197 & - & - & - & - & 19 & - & - & 216 \\
\hline 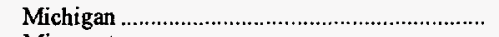 & 1,945 & - & 128 & - & 128 & 5 & - & - & 2,078 \\
\hline 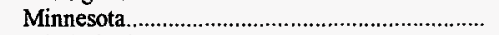 & 6 & 23 & - & - & 23 & 1 & - & - & 30 \\
\hline 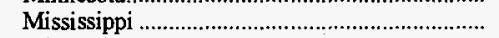 & * & - & - & - & - & - & - & - & $*$ \\
\hline 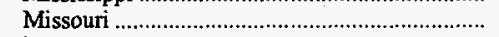 & - & - & - & - & - & * & - & - & * \\
\hline Montana & 2 & - & - & - & - & * & - & - & 2 \\
\hline 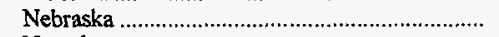 & $*$ & - & - & - & - & 1 & - & - & 1 \\
\hline Nevada & - & - & - & - & - & * & - & - & * \\
\hline 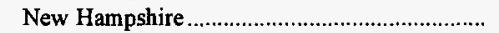 & 527 & - & - & 60 & 60 & 8 & - & - & 595 \\
\hline 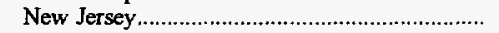 & 538 & - & - & - & - & 14 & - & - & 551 \\
\hline 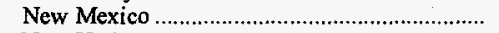 & - & - & - & - & - & * & - & - & * \\
\hline New York & 3,066 & 36 & 538 & - & 574 & 635 & - & - & 4,276 \\
\hline 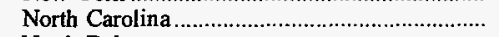 & 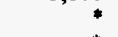 & - & - & - & - & * & - & - & * \\
\hline 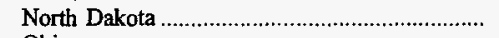 & * & - & - & - & - & 2 & - & - & 2 \\
\hline 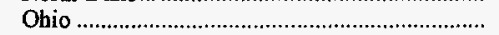 & 1,499 & 2,259 & 608 & - & 2,867 & $11 \overline{7}$ & - & - & 4,483 \\
\hline 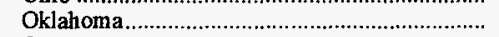 & 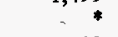 & - & - & - & - & $*$ & - & - & * \\
\hline Oregon & 12 & - & - & - & - & * & - & - & 12 \\
\hline 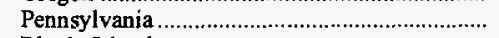 & 12,727 & 5,537 & - & - & 5,537 & 15,783 & 5,175 & - & 39,222 \\
\hline 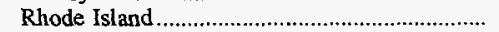 & - & - & - & - & - & 3 & - & - & 3 \\
\hline South Carolina & $*$ & - & - & - & - & $*$ & - & - & * \\
\hline 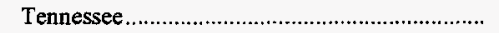 & 11 & 639 & - & - & 639 & 16 & - & - & 665 \\
\hline Texas & 1 & - & - & - & - & * & - & - & 2 \\
\hline Utah & 230 & - & - & - & - & - & - & - & 230 \\
\hline 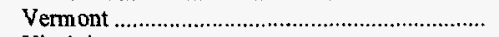 & * & - & - & - & - & 2 & - & - & 2 \\
\hline 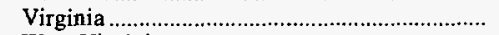 & $*$ & - & - & - & - & 11 & - & - & 11 \\
\hline 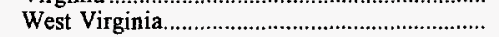 & 6 & 1,719 & - & - & 1,719 & 140 & - & - & 1,865 \\
\hline Wisconsin. & 546 & - & 812 & - & 812 & 9 & - & - & 1,367 \\
\hline Wyoming & 1 & - & - & - & - & - & - & - & 1 \\
\hline 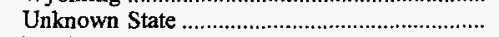 & - & - & - & - & - & - & - & 284 & 284 \\
\hline Foreign & 152 & - & 562 & 8,521 & 9,083 & 11 & - & - & 9,246 \\
\hline Pennsylvania Anthracite & 1,313 & - & - & 343 & 343 & 3,119 & - & 62 & 4,836 \\
\hline Alabama & 1 & - & - & - & - & 1 & - & - & 2 \\
\hline 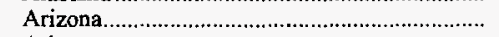 & 1 & - & - & - & - & * & - & - & 1 \\
\hline 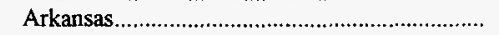 & $*$ & - & - & - & - & * & - & - & * \\
\hline California & $*$ & - & - & - & - & * & - & - & * \\
\hline Colorado & 16 & - & - & - & - & - & - & - & 16 \\
\hline Connecticut & $*$ & - & - & - & - & 5 & - & - & 5 \\
\hline Delaware & $*$ & - & - & - & - & 10 & - & - & 10 \\
\hline 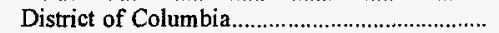 & - & - & - & - & - & * & - & - & $\bullet$ \\
\hline Florida & 5 & - & - & - & - & * & - & - & 6 \\
\hline 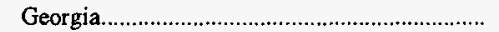 & $*$ & - & - & - & - & * & - & - & $*$ \\
\hline Idaho & $\bullet$ & - & - & - & - & - & - & - & * \\
\hline 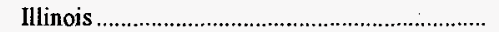 & 6 & - & - & - & - & 2 & - & - & 7 \\
\hline Indiana & $*$ & - & - & - & - & 6 & - & - & 6 \\
\hline Iowa & 53 & - & - & - & - & 1 & - & - & 54 \\
\hline 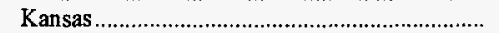 & $*$ & - & - & - & - & - & - & - & $*$ \\
\hline Kentucky & 13 & - & - & - & - & 9 & - & - & 22 \\
\hline Louisiana & 6 & - & - & - & - & - & - & - & 6 \\
\hline Maine & I & - & - & - & - & 3 & - & - & 4 \\
\hline Maryland & $*$ & - & - & - & - & 1 & - & - & 1 \\
\hline 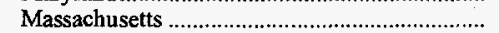 & * & - & - & - & - & 13 & - & - & 14 \\
\hline 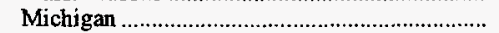 & $*$ & - & - & - & - & 3 & - & - & 3 \\
\hline
\end{tabular}

See footnotes at end of table. 
Table 65. Distribution of U.S. Coal by Origin, Destination, and Method of Transportation, 1996 (Continued)

(Thousand Short Tons)

\begin{tabular}{|c|c|c|c|c|c|c|c|c|c|}
\hline \multirow{2}{*}{$\begin{array}{l}\text { Origin State and } \\
\text { Destination State }\end{array}$} & \multirow[b]{2}{*}{ Railroad } & \multicolumn{4}{|c|}{ Water } & \multirow{2}{*}{ Truck } & \multirow{2}{*}{$\begin{array}{c}\text { Tramway, } \\
\text { Conveyor, } \\
\text { and Slurry } \\
\text { Pipeline }\end{array}$} & \multirow[b]{2}{*}{ Unknown } & \multirow[b]{2}{*}{ Total } \\
\hline & & River & $\begin{array}{l}\text { Great } \\
\text { Lakes }\end{array}$ & Tidewater & Total & & & & \\
\hline
\end{tabular}

Pennsylvania Anthracite (Continued)

Minnesota.

Mississippi

Missouri .......

Montana

Nebraska .

Nevada

New Hampshire

New Jersey.

New Mexico

New York

North Carolina

North Dakota .

Ohio

Oklahoma

Oregon ..........

Pennsylvania

Rhode Island...

South Carolina

Tennessee.

Texas

Utah...

Vermont

Virginia

West Virginia

Wisconsin.

Wyoming

Unknown State

Foreign.

Pennsylvania Bituminous.

Alabama...

Connecticut

Delaware...

Indiana

Iowa.

Kentucky

Louisiana.

Maryland...

Massachusetts

Michigan .

Minnesota.

New Hampshire.

New Jersey.

New York

Ohio

Pennsylvania

Tennessee.

Texas

Utah

Vermont

Virginia

West Virginia

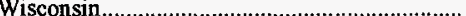

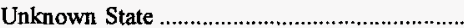

Foreign.

Tennessee

Alabama....

Georgia...

Kentucky

Ohio

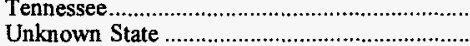

Texas.

See footnotes at end of table.

\begin{tabular}{|c|c|c|c|c|c|c|c|c|}
\hline 6 & - & - & - & - & 1 & - & - & $?$ \\
\hline$*$ & - & - & - & - & - & - & - & * \\
\hline- & - & - & - & - & * & - & - & * \\
\hline 2 & - & - & - & - & * & - & - & 2 \\
\hline$*$ & - & - & - & - & 1 & - & - & 1 \\
\hline- & - & - & - & - & * & - & - & * \\
\hline$*$ & - & - & - & - & 3 & - & - & 4 \\
\hline$*$ & - & - & - & - & 13 & - & - & 14 \\
\hline- & - & - & - & - & * & - & - & * \\
\hline 18 & - & - & - & - & 133 & - & - & 151 \\
\hline * & - & - & - & - & * & - & - & * \\
\hline * & - & - & - & - & 2 & - & - & 2 \\
\hline- & - & - & - & - & 19 & - & - & 19 \\
\hline * & - & - & - & - & * & - & - & * \\
\hline 12 & - & - & - & - & * & - & - & 12 \\
\hline 1,006 & - & - & - & - & 2,815 & - & - & 3,821 \\
\hline * & - & - & - & - & 3 & - & - & 3 \\
\hline * & - & - & - & - & * & - & - & * \\
\hline 11 & - & - & - & - & 16 & - & - & 26 \\
\hline 1 & - & - & - & - & * & - & - & 1 \\
\hline$*$ & - & - & - & - & - & - & - & * \\
\hline * & - & - & - & - & 2 & - & - & 2 \\
\hline$*$ & - & - & - & - & 6 & - & - & 6 \\
\hline- & - & - & - & - & 32 & - & - & 32 \\
\hline$*$ & - & - & - & - & 5 & - & - & 5 \\
\hline 1 & - & - & - & - & - & - & - & 1 \\
\hline- & - & - & - & - & - & - & 62 & 62 \\
\hline 152 & - & - & 343 & 343 & 11 & - & - & 506 \\
\hline 22,834 & 11,285 & 2,648 & 8,238 & 22,171 & 13,889 & 5,175 & 222 & 64,291 \\
\hline- & 6 & - & - & 6 & - & - & - & 6 \\
\hline 217 & 9 & - & - & 9 & * & - & - & 227 \\
\hline 470 & 53 & - & - & 53 & 5 & - & - & 528 \\
\hline- & 559 & - & - & 559 & - & - & - & 559 \\
\hline- & 225 & - & - & 225 & - & - & - & 225 \\
\hline 475 & 25 & - & - & 25 & - & - & - & 500 \\
\hline- & 195 & - & - & 195 & - & - & - & 195 \\
\hline 1,415 & - & - & - & - & 188 & - & - & 1,602 \\
\hline 196 & - & - & - & - & 6 & - & - & 202 \\
\hline 1,945 & - & 128 & - & 128 & 2 & - & - & 2,075 \\
\hline- & 23 & - & - & 23 & - & - & - & 23 \\
\hline 527 & - & - & 60 & 60 & 4 & - & - & 592 \\
\hline 538 & - & - & - & - & * & - & - & 538 \\
\hline 3,049 & 36 & 538 & - & 574 & 502 & - & - & 4,125 \\
\hline 1,499 & 2,259 & 608 & - & 2,867 & 97 & - & - & 4,463 \\
\hline 11,721 & 5,537 & - & - & 5,537 & 12,968 & 5,175 & - & 35,400 \\
\hline- & 639 & - & - & 639 & - & - & - & 639 \\
\hline * & - & - & - & - & * & - & - & ${ }^{*}$ \\
\hline 230 & - & - & - & - & - & - & - & 230 \\
\hline- & - & - & - & - & * & - & - & * \\
\hline- & - & - & - & - & 5 & - & - & 5 \\
\hline 6 & 1,719 & - & - & 1,719 & 108 & - & - & 1,833 \\
\hline 546 & - & 812 & - & 812 & 4 & - & - & 1,362 \\
\hline- & - & - & - & - & - & - & 222 & 222 \\
\hline- & - & 562 & 8,177 & 8,740 & - & - & - & 8,740 \\
\hline 1,605 & 458 & - & - & 458 & 980 & - & 9 & 3,052 \\
\hline$\overline{-}$ & 331 & - & - & 331 & - & - & - & 331 \\
\hline 140 & - & - & - & - & 1 & - & - & 141 \\
\hline - & - & - & - & - & 23 & - & - & 23 \\
\hline - & - & - & - & - & 1 & - & - & 1 \\
\hline - & - & - & - & - & * & - & - & * \\
\hline 1,466 & 127 & - & - & 127 & 956 & - & - & 2,548 \\
\hline- & - & - & - & - & - & - & 9 & 9 \\
\hline 24,044 & - & - & 117 & 117 & 9,854 & 15,641 & - & 49,655 \\
\hline
\end{tabular}


Table 65. Distribution of U.S. Coal by Origin, Destination, and Method of Transportation, 1996 (Continued)

(Thousand Short Tons)

\begin{tabular}{|c|c|c|c|c|c|c|c|c|c|}
\hline \multirow{2}{*}{$\begin{array}{l}\text { Origin State and } \\
\text { Destination State }\end{array}$} & \multirow[b]{2}{*}{ Railroad } & \multicolumn{4}{|c|}{ Water } & \multirow[b]{2}{*}{ Truck } & \multirow{2}{*}{$\begin{array}{l}\text { Tramway, } \\
\text { Conveyor, } \\
\text { and SIurry } \\
\text { Pipeline }\end{array}$} & \multirow[b]{2}{*}{ Unknown } & \multirow[b]{2}{*}{ Total } \\
\hline & & River & $\begin{array}{l}\text { Great } \\
\text { Lakes }\end{array}$ & Tidewater & Total & & & & \\
\hline \multicolumn{10}{|l|}{ Texas (Continued) } \\
\hline 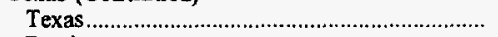 & 24,044 & - & - & - & - & 9,854 & 15,641 & - & 49,538 \\
\hline 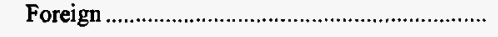 & - & - & - & 117 & 117 & - & - & - & 117 \\
\hline Utah & 13,409 & - & 44 & 5,305 & 5,349 & 5,104 & - & 6 & 23,868 \\
\hline 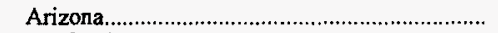 & 69 & - & - & - & - & - & - & - & 69 \\
\hline California & 2,240 & - & - & - & - & $*$ & - & - & 2,240 \\
\hline 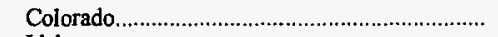 & 1 & - & - & - & - & 1 & - & - & 2 \\
\hline Idaho & 33 & - & - & - & - & 32 & - & - & 65 \\
\hline 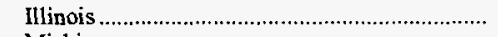 & 2,473 & - & - & - & - & - & - & - & 2,473 \\
\hline 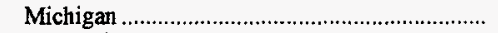 & - & - & 44 & - & 44 & - & - & - & 44 \\
\hline 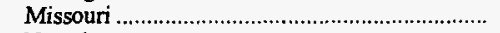 & 330 & - & - & - & - & - & - & - & 330 \\
\hline Nevada & 2,185 & - & - & - & - & 81 & - & - & 2,265 \\
\hline Oregon & - & - & - & - & - & $*$ & - & - & $*$ \\
\hline 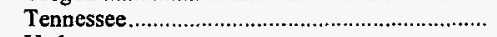 & 1,421 & - & - & - & - & - & - & - & 1,421 \\
\hline Utah & 4,402 & - & - & - & - & 4,988 & - & - & 9,389 \\
\hline Washington & 124 & - & - & - & - & 1 & - & - & 125 \\
\hline Wisconsin & 133 & - & - & - & - & $\overrightarrow{-}$ & - & - & 133 \\
\hline 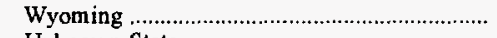 & - & - & - & - & - & $*$ & - & - & $*$ \\
\hline 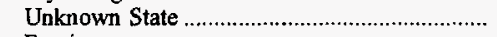 & - & - & - & - & - & - & - & 6 & 6 \\
\hline Foreign & - & - & - & 5,305 & 5,305 & - & - & - & 5,305 \\
\hline Virginia & 17,635 & 2,517 & - & 13,645 & 16,162 & 1,361 & 1,007 & 43 & 36,208 \\
\hline 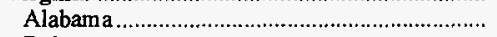 & 1,022 & 5 & - & - & 5 & 9 & - & - & 1,036 \\
\hline 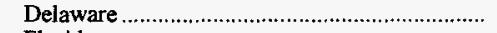 & 166 & - & - & - & - & - & - & - & 166 \\
\hline Florida & 549 & - & - & - & - & - & - & - & 549 \\
\hline 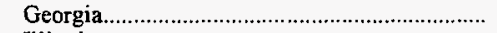 & 1,785 & - & - & - & $\vec{a}$ & * & - & - & 1,785 \\
\hline 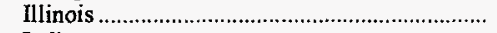 & - & 547 & - & - & 547 & 35 & - & - & 583 \\
\hline Indiana & 1,338 & 951 & - & - & 951 & - & - & - & 2,290 \\
\hline 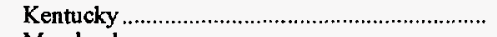 & $*$ & 2 & - & - & 2 & - & - & - & 3 \\
\hline 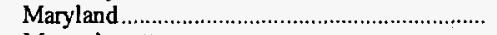 & $*$ & - & - & - & - & * & - & - & 1 \\
\hline 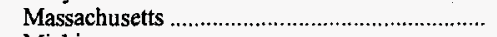 & 8 & - & - & - & - & - & - & - & 8 \\
\hline 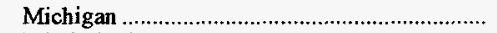 & 25 & - & - & - & - & $*$ & - & - & 25 \\
\hline 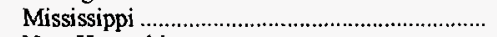 & 13 & - & - & - & - & - & - & - & 13 \\
\hline 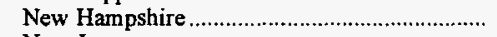 & 10 & - & - & - & - & - & - & - & 10 \\
\hline 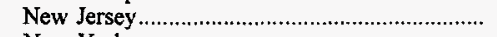 & - & - & - & 601 & 601 & - & - & - & 601 \\
\hline New York & 146 & - & - & - & - & - & - & - & 146 \\
\hline North Carolina & 1,816 & - & - & - & - & 68 & - & - & 1,883 \\
\hline Ohio & 228 & 103 & - & - & 103 & $*$ & - & - & 331 \\
\hline 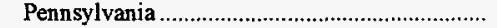 & 403 & 896 & - & - & 896 & * & - & - & 1,299 \\
\hline South Carolina & 1,591 & - & - & - & - & 14 & - & - & 1,605 \\
\hline Tennessee & 2,525 & - & - & - & - & 4 & - & - & 2,529 \\
\hline 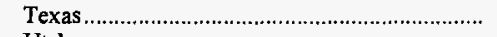 & 13 & - & - & - & - & - & - & - & 13 \\
\hline Utah & 332 & - & - & - & - & - & - & - & 332 \\
\hline Virginia & 5,357 & - & - & - & - & 866 & 1,007 & - & 7,231 \\
\hline 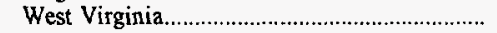 & 275 & 8 & - & - & 8 & 4 & - & - & 287 \\
\hline Wisconsin & 5 & 4 & - & - & 4 & - & - & - & 9 \\
\hline 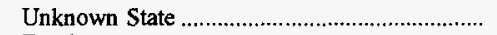 & - & - & - & - & - & - & - & 43 & 43 \\
\hline Foreign & 27 & - & - & 13,044 & 13,044 & 360 & - & - & 13,432 \\
\hline Washington & 2 & - & - & 43 & 43 & 132 & 4,393 & - & 4,569 \\
\hline Oregon & 2 & - & - & - & - & 1 & - & - & 3 \\
\hline Washington & - & - & - & - & - & 131 & 4,393 & - & 4,523 \\
\hline 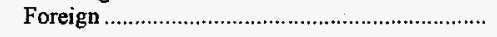 & - & - & - & 43 & 43 & $*$ & - & - & 43 \\
\hline 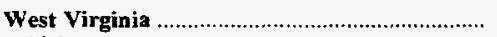 & 64,267 & 44,560 & 8,137 & 41,487 & 94,185 & 5,335 & 5,110 & 302 & 169,200 \\
\hline Alabama & 1,986 & 1,348 & - & - & 1,348 & 6 & - & - & 3,340 \\
\hline 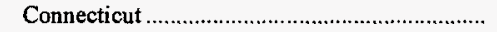 & 17 & - & - & 690 & 690 & - & - & - & 707 \\
\hline Delaware & 996 & - & - & - & - & 5 & - & - & 1,000 \\
\hline 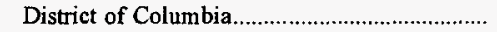 & 3 & 14 & - & - & 14 & 6 & - & - & 23 \\
\hline Florida & 658 & 25 & - & 992 & 1,016 & - & - & - & 1,674 \\
\hline 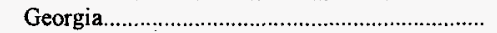 & 4,050 & - & - & - & - & 14 & - & - & 4,064 \\
\hline Illinois & 973 & 773 & 105 & - & 877 & - & - & - & 1,850 \\
\hline Indiana & 4,358 & 930 & - & - & 930 & - & - & - & 5,287 \\
\hline Iowa & 27 & 17 & - & - & 17 & - & - & - & 44 \\
\hline Kentucky & 1,956 & 3,146 & - & - & 3,146 & 174 & - & - & 5,276 \\
\hline 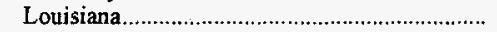 & - & 203 & - & - & 203 & - & - & - & 203 \\
\hline Maine & 26 & - & - & - & - & - & - & - & 26 \\
\hline
\end{tabular}

See footnotes at end of table. 
Table 65. Distribution of U.S. Coal by Origin, Destination, and Method of Transportation, 1996 (Continued)

(Thousand Short Tons)

\begin{tabular}{|c|c|c|c|c|c|c|c|c|c|}
\hline \multirow{2}{*}{$\begin{array}{l}\text { Origin State and } \\
\text { Destination State }\end{array}$} & \multirow[b]{2}{*}{ Railroad } & \multicolumn{4}{|c|}{ Water } & \multirow[b]{2}{*}{ Truck } & \multirow{2}{*}{$\begin{array}{c}\text { Tramway, } \\
\text { Conveyor, } \\
\text { and Sturry } \\
\text { Pipeline }\end{array}$} & \multirow[b]{2}{*}{ Unknown } & \multirow[b]{2}{*}{ Total } \\
\hline & & River & $\begin{array}{l}\text { Great } \\
\text { Lakes }\end{array}$ & Tidewater & Total & & & & \\
\hline \multicolumn{10}{|l|}{ West Virginia (Continued) } \\
\hline Maryland & 4,611 & - & - & 2,719 & 2,719 & 574 & - &. & 7,905 \\
\hline 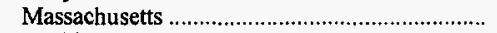 & 443 & - & - & 1,401 & 1,401 & - & - & - & 1,845 \\
\hline Michigan & 4,887 & 282 & 294 & - & 575 & 47 & $\rightarrow$ & - & 5,509 \\
\hline 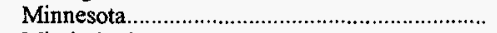 & - & 22 & 10 & - & 32 & * & - & - & 32 \\
\hline 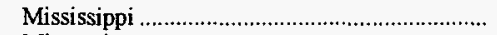 & 19 & 4 & - & - & 4 & 1 & - & - & 24 \\
\hline 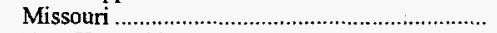 & - & 36 & - & - & 36 & - & - & - & 36 \\
\hline New Hampshire & 269 & - & - & 77 & 77 & - & - & - & 346 \\
\hline 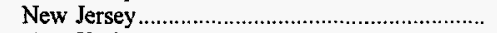 & 1,108 & 156 & - & 482 & 638 & - & - & - & 1,746 \\
\hline 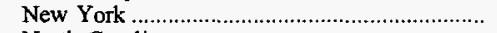 & 5,433 & 253 & - & - & 253 & - & - & - & 5,685 \\
\hline North Carolina & 8,287 & - & - & - & - & - & - & - & 8,287 \\
\hline 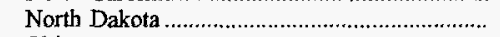 & - & - & - & - & - & * & - & - & * \\
\hline Ohio …… & 5,792 & 15,200 & 1,426 & - & 16,626 & 317 & - & - & 22,735 \\
\hline 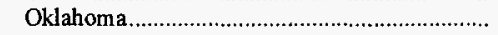 & 2 & 25 & - & 50 & 75 & - & - & - & 77 \\
\hline Oregon & 2 & - & - & - & - & - & - & - & 2 \\
\hline 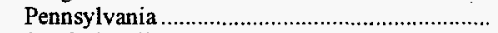 & 3,204 & 10,781 & - & - & 10,781 & 251 & - & - & 14,236 \\
\hline South Carolina & 350 & - & - & - & - & - & - & - & 350 \\
\hline South Dakota & - & 1 & - & - & 1 & - & - & - & 1 \\
\hline 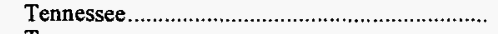 & 98 & 1,461 & - & - & 1,461 & * & - & - & 1,560 \\
\hline Texas & - & - & - & 4 & 4 & - & - & - & 4 \\
\hline Utah & 18 & - & - & - & - & - & - & - & 18 \\
\hline Virginia & 3,040 & 46 & - & - & 46 & 197 & - & - & 3,283 \\
\hline 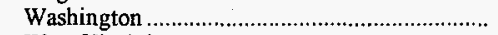 & 26 & - & - & - & - & - & - & - & 26 \\
\hline 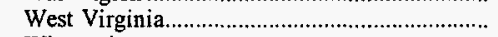 & 10,647 & 9,726 & - & - & 9,726 & 3,737 & 5,110 & - & 29,220 \\
\hline Wisconsin & 67 & 10 & 351 & - & 360 & 5 & - & - & 432 \\
\hline Unknown State & - & - & - & - & - & - & - & 302 & 302 \\
\hline Foreign & 916 & 105 & 5,953 & 35,071 & 41,128 & - & - & - & 42,044 \\
\hline 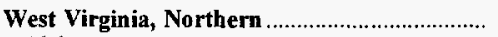 & 15,640 & 14,521 & 2,103 & $\mathbf{6 , 5 7 2}$ & 23,196 & 2,898 & 4,606 & 96 & 46,436 \\
\hline 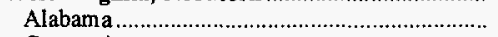 & - & 419 & - & - & 419 & - & - & - & 419 \\
\hline 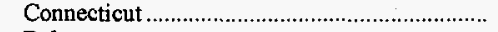 & 8 & - & - & 674 & 674 & - & - & - & 683 \\
\hline 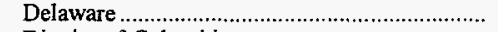 & 445 & - & - & - & - & 5 & - & - & 449 \\
\hline District of Columbia & - & - & - & - & - & 6 & - & - & 6 \\
\hline 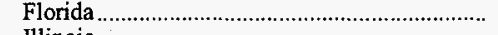 & 551 & - & - & - & - & - & - & - & 551 \\
\hline 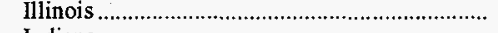 & 9 & - & - & - & - & - & - & - & 9 \\
\hline Indiana & 469 & 10 & - & - & 10 & - & - & - & 479 \\
\hline 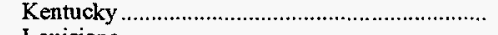 & 87 & 938 & - & - & 938 & * & - & - & 1,026 \\
\hline Louisiana & $\overline{-}$ & 203 & - & - & 203 & - & - & - & 203 \\
\hline 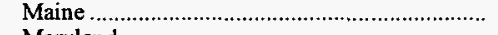 & 13 & - & - & - & - & - & - & - & 13 \\
\hline Maryland & 3,972 & - & - & 622 & 622 & 178 & - & - & 4,773 \\
\hline 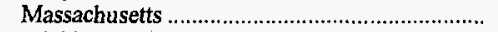 & 53 & - & - & - & - & - & - & - & 53 \\
\hline 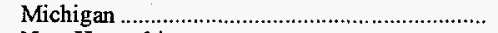 & 622 & - & 18 & - & 18 & - & - & - & 640 \\
\hline 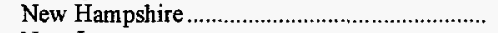 & 269 & - & - & 53 & 53 & - & - & - & 322 \\
\hline 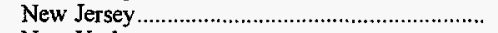 & 893 & 156 & - & 482 & 638 & - & - & - & 1,530 \\
\hline 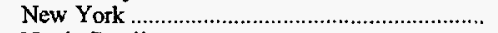 & 4,140 & - & - & - & - & - & - & - & 4,140 \\
\hline 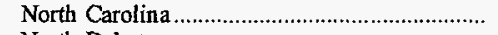 & 13 & - & - & - & - & - & - & - & 13 \\
\hline North Dakota & - & - & - & - & - & * & - & - & * \\
\hline Ohio & 1,073 & 2,130 & 759 & - & 2,889 & 4 & - & - & 3,966 \\
\hline 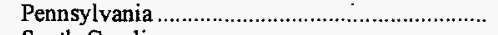 & 1,702 & 6,824 & - & - & 6,824 & 81 & - & - & 8,606 \\
\hline South Carolina & 3 & - & - & - & - & - & - & - & 3 \\
\hline Tennessee & - & 78 & - & - & 78 & $*$ & - & - & 78 \\
\hline Virginia & 66 & - & - & - & - & * & - & - & 66 \\
\hline 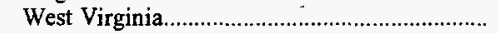 & 965 & 3,764 & - & - & 3,764 & 2,624 & 4,606 & - & 11,959 \\
\hline Wisconsin & 2 & - & 315 & - & 315 & - & - & - & 317 \\
\hline Unknown State & - & - & - & - & - & - & - & 96 & 96 \\
\hline Foreign & 286 & - & 1,011 & 4,740 & 5,751 & - & - & - & 6,038 \\
\hline West Virginia, Southern & 48,627 & 30,040 & 6,034 & 34,916 & 70,989 & 2,437 & 504 & 206 & 122,764 \\
\hline Alabama & 1,986 & 929 & - & - & 929 & 6 & - & - & 2,922 \\
\hline 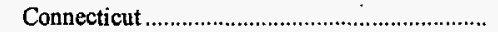 & 8 & - & - & 16 & 16 & - & - & - & 24 \\
\hline Delaware & 551 & - & - & - & - & - & - & - & 551 \\
\hline District of Columbia & 3 & 14 & - & - & 14 & - & - & - & 17 \\
\hline Florida & 107 & 25 & - & 992 & 1,016 & - & - & - & 1,123 \\
\hline Georgia & 4,050 & - & - & - & - & 14 & - & - & 4,064 \\
\hline Illinois & 964 & 773 & 105 & - & 877 & - & - & - & 1,841 \\
\hline Indiana & 3,889 & 920 & - & - & 920 & - & - & - & 4,809 \\
\hline Iowa & 27 & 17 & - & - & 17 & - & - & - & 44 \\
\hline Kentucky & 1,868 & 2,208 & - & - & 2,208 & 174 & - & - & 4,250 \\
\hline
\end{tabular}

See footnotes at end of table. 
Table 65. Distribution of U.S. Coal by Origin, Destination, and Method of Transportation, 1996 (Continued)

(Thousand Short Tons)

\begin{tabular}{|c|c|c|c|c|c|c|c|c|c|}
\hline \multirow{2}{*}{$\begin{array}{l}\text { Origin State and } \\
\text { Destination State }\end{array}$} & \multirow{2}{*}{ Railroad } & \multicolumn{4}{|c|}{ Water } & \multirow{2}{*}{ Truck } & \multirow{2}{*}{$\begin{array}{c}\text { Tramway, } \\
\text { Conveyor, } \\
\text { and Slurry } \\
\text { Pipeline }\end{array}$} & \multirow[b]{2}{*}{ Unknown } & \multirow[b]{2}{*}{ Total } \\
\hline & & River & $\begin{array}{l}\text { Great } \\
\text { Lakes }\end{array}$ & Tidewater & Total & & & & \\
\hline
\end{tabular}

West Virginia, Southern (Continued)

Maine ...

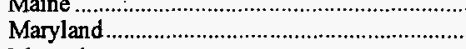

Massachusetts

Michigan

Minnesota.

Mississippi

Missouri

New Jersey

New York.

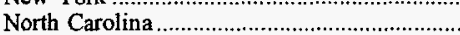

Ohio

Oklahoma.

Oregon

Pennsylvania

South Carolina.

South Dakota

Tennesse

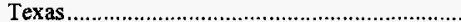

Utah...

Virginia ........

Washington ....

West Virginia

Wisconsin.

Unknown State

Foreign.

Wyoming.

Alabama.

Arkansas.

Colorado.

Florida.

Georgia.

Idaho...

Illinois

Indiana

Iowa .

Kansas....

Louisiana

Michigan

Minnesota.

Mississippi

Missouri

Montana

Nebraska

Nevada

(a)

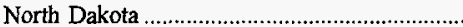

Ohio

Oklahoma

Oregon

South Dakota

Tennessee.

Texas...

Utah.

Washington

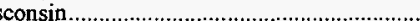

Wyoming

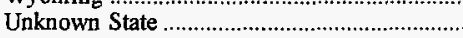

Foreign.

U.S. Total

Alabama.

Alaska

Arizona...

Arkansas.

California

Colorado.

See footnotes at end of table.
13

$\begin{array}{r}639 \\ 491 \\ \hline 265\end{array}$

4,265

19

216

1,293

8,274

4,720

2
2

1,502

347

98

18
2,974
26

9,682

65

629

$\mathbf{2 4 7 , 8 2 0}$

3,686

14,614

6,124

591
6,818

6,818
237

17,734

18,079

17,580

11,772

4,173

8,551

8,115

26
24,971

24,9713
10,464

10,464

204

19,751

894

298

42,839

1
17,640

12,145

-

611,674

611,674
15,491

519
15,784

15,784
14,720

2,240
12,781

-
-
-
282
22
4
36
-
-
253
-
13,070
25
-
3,958
-
1
1,383
-
-
46
-
5,962
10
-
105

13,918

-

$5,03 \overline{6}$

$-$

8,341

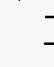

$=$

-
-

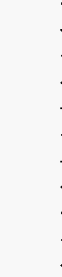

135,760

10,360

10,360
-

\section{$-$}

924

\section{2,097}

2,097
1,401

275

10

-

- 24

$6 \overline{7}$

-

-

-

36

$4,94 \overline{1}$

30,331

1,952

2,097

$$
\begin{array}{r}
2,097 \\
1,401 \\
557
\end{array}
$$

1,401
557

4
36
24

253

13,736

75

3,958

$\overrightarrow{1}$
1383

1,383
4

$4 \overline{6}$

5,962

45

35,377

16,793

$$
\text { - }
$$$$
\text { - }
$$

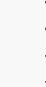

54

$5,03 \overline{6}$

444

$8,34 \overline{1}$

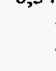

-

$$
37
$$

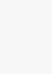$$
\begin{aligned}
& - \\
& -
\end{aligned}
$$$$
44 \overline{3}
$$$$
1,95 \overline{2}
$$

21,408

90,767

247,935 $\begin{array}{llll}396 & - & - & \\ \overline{7} & - & - & 3,132\end{array}$

3,132
1,792

4,869

32

24

36
24

216

1,545

8,274

18,770

77
2

5,629

347

1,482

1,482
4

18
3,217

26

17,261

I15

206
36,006

279,117

3,686

14,614

6,124

6,818

268

17,734

18,079

18,121

11,772

9,209

8,551

8,569

33,312

513

10,464

204

37

19,751

894

355

298

42,839

17.640

26,253

2,395

99,941

98,934

98,934
1,108

1,108

1,059,892

33,057

697

15,785

14,918

2,240

16,920 
Table 65. Distribution of U.S. Coal by Origin, Destination, and Method of Transportation, 1996 (Continued)

(Thousand Short Tons)

\begin{tabular}{|c|c|c|c|c|c|c|c|c|c|}
\hline \multirow{2}{*}{$\begin{array}{l}\text { Origin State and } \\
\text { Destination State }\end{array}$} & \multirow{2}{*}{ Railroad } & \multicolumn{4}{|c|}{ Water } & \multirow{2}{*}{ Truck } & \multirow{2}{*}{$\begin{array}{c}\text { Tramway, } \\
\text { Conveyor, } \\
\text { and Slurry } \\
\text { Pipeline }\end{array}$} & \multirow{2}{*}{ Unknown } & \multirow{2}{*}{ Total } \\
\hline & & River & $\begin{array}{l}\text { Great } \\
\text { Lakes }\end{array}$ & Tidewater & Total & & & & \\
\hline \multicolumn{10}{|l|}{ U.S. Total (Continued) } \\
\hline 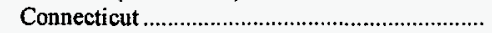 & 237 & 9 & - & 1,350 & 1,359 & 6 & - & - & 1,602 \\
\hline Delaware & 1,762 & 53 & - & - & 53 & 20 & - & - & 1,834 \\
\hline District of Columbia & 3 & 14 & - & - & 14 & 6 & - & - & 23 \\
\hline Florida & 16,541 & 7,659 & - & 2,076 & 9,735 & 9 & - & - & 26,285 \\
\hline 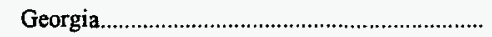 & 28,938 & - & - & - & - & 135 & - & - & 29,074 \\
\hline Idaho & 270 & - & - & - & - & 63 & - & - & 333 \\
\hline Illinois & 35,581 & 3,028 & 105 & - & 3,133 & 5,810 & 23 & - & 44,547 \\
\hline Indiana & 49,804 & 3,317 & 199 & - & 3,516 & 8,916 & 505 & - & 62,741 \\
\hline lowa & 18,843 & 2,032 & - & - & 2,032 & 142 & - & - & 21,017 \\
\hline 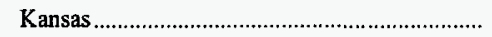 & 13,415 & - & - & - & - & 572 & - & - & 13,987 \\
\hline 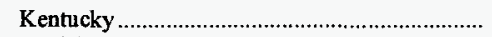 & 10,921 & 13,771 & - & - & 13,771 & 9,746 & - & - & 34,438 \\
\hline 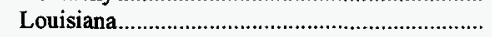 & 4,179 & 6,124 & - & - & 6,124 & 787 & 2,435 & - & 13,526 \\
\hline 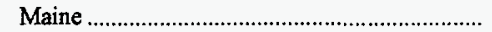 & 26 & - & - & 271 & 271 & 3 & - & - & 301 \\
\hline 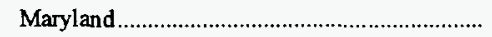 & 6,863 & 8 & - & 2,719 & 2,727 & 1,172 & - & - & 10,762 \\
\hline 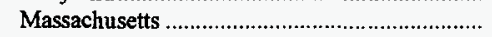 & 903 & - & - & 1,553 & 1,553 & 19 & - & - & 2,475 \\
\hline 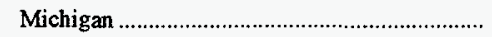 & 24,546 & 591 & 7,648 & - & 8,240 & 401 & - & - & 33,186 \\
\hline Minnesota & 17,909 & 158 & 546 & - & 705 & 65 & - & - & 18,679 \\
\hline 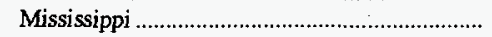 & 5,073 & 602 & - & - & 602 & 27 & - & - & 5,702 \\
\hline 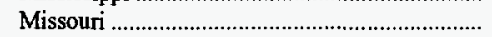 & 29,678 & 9,291 & - & - & 9,291 & 1,095 & - & - & 40,064 \\
\hline Montana & 640 & - & - & - & - & 416 & 7,303 & - & 8,359 \\
\hline 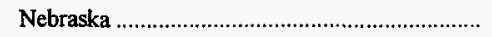 & 10,725 & - & - & - & - & 1 & - & - & 10,726 \\
\hline 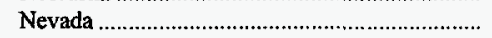 & 2,520 & - & - & - & - & 81 & 4,470 & - & 7,072 \\
\hline 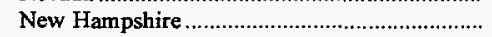 & 806 & - & - & 137 & 137 & 8 & - & - & 951 \\
\hline 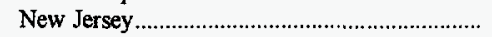 & 1,646 & 156 & - & 1,112 & 1,268 & 14 & - & - & 2,927 \\
\hline 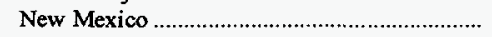 & 8,422 & - & - & - & - & 95 & 6,580 & - & 15,097 \\
\hline 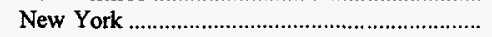 & 9,951 & 297 & 538 & 36 & 870 & 637 & - & - & 11,459 \\
\hline 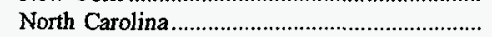 & 26,992 & 52 & - & - & 52 & 370 & - & - & 27,414 \\
\hline 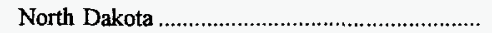 & 753 & - & - & - & - & 4,583 & 25,107 & - & 30,444 \\
\hline Ohio & 13,028 & 28,426 & 2,221 & - & 30,648 & 9,613 & 8,424 & - & 61,713 \\
\hline 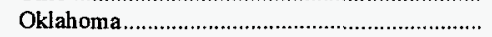 & 19,769 & 40 & - & 50 & 91 & 1,724 & - & - & 21,584 \\
\hline Oregon & 1,027 & - & - & - & - & 1 & - & - & 1,028 \\
\hline Pennsylvania & 17,414 & 20,013 & - & - & 20,013 & 16,501 & 5,175 & - & 59,103 \\
\hline 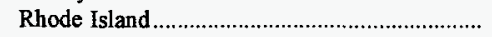 & - & - & - & - & - & 3 & - & - & 3 \\
\hline 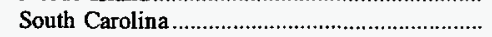 & 13,326 & - & - & - & - & 47 & - & - & 13,373 \\
\hline 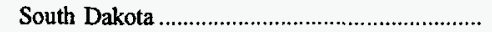 & 1,301 & 1 & - & - & 1 & 355 & - & - & 1,657 \\
\hline 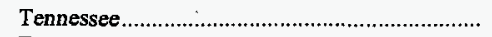 & 16,436 & 14,264 & - & - & 14,264 & 1,810 & - & - & 32,510 \\
\hline 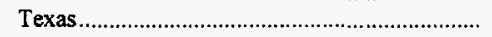 & 69,657 & 23 & - & 4 & 28 & 10,041 & 15,641 & - & 95,367 \\
\hline 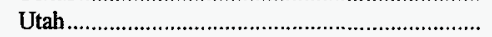 & 6,188 & - & - & - & - & 4,988 & - & - & 11,175 \\
\hline Vermont & $*$ & - & - & - & - & 2 & - & - & 2 \\
\hline 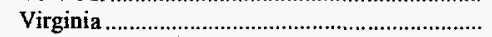 & 13,933 & 46 & - & - & 46 & 1,228 & 1,007 & - & 16,214 \\
\hline Washington & 301 & - & - & - & - & 132 & 4,393 & - & 4,825 \\
\hline 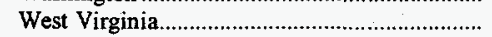 & 12,955 & 13,787 & - & - & 13,787 & 4,191 & 5,115 & - & 36,047 \\
\hline Wisconsin & 22,683 & 1,532 & 1,904 & - & 3,436 & 167 & - & - & 26,285 \\
\hline 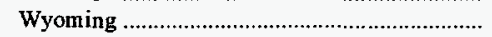 & 12,156 & - & - & - & - & 2,954 & 11,647 & - & 26,757 \\
\hline 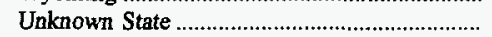 & - & - & - & - & - & - & - & 1,408 & 1,408 \\
\hline Foreign & 2,017 & 105 & 8,246 & 81,457 & 89,808 & 374 & - & - & 92,199 \\
\hline
\end{tabular}

* Data round to zero.

Note: Totals may not equal sum of components due to independent rounding. Source: Energy Information Administration, Form EIA-6, "Coal Distribution Report." 


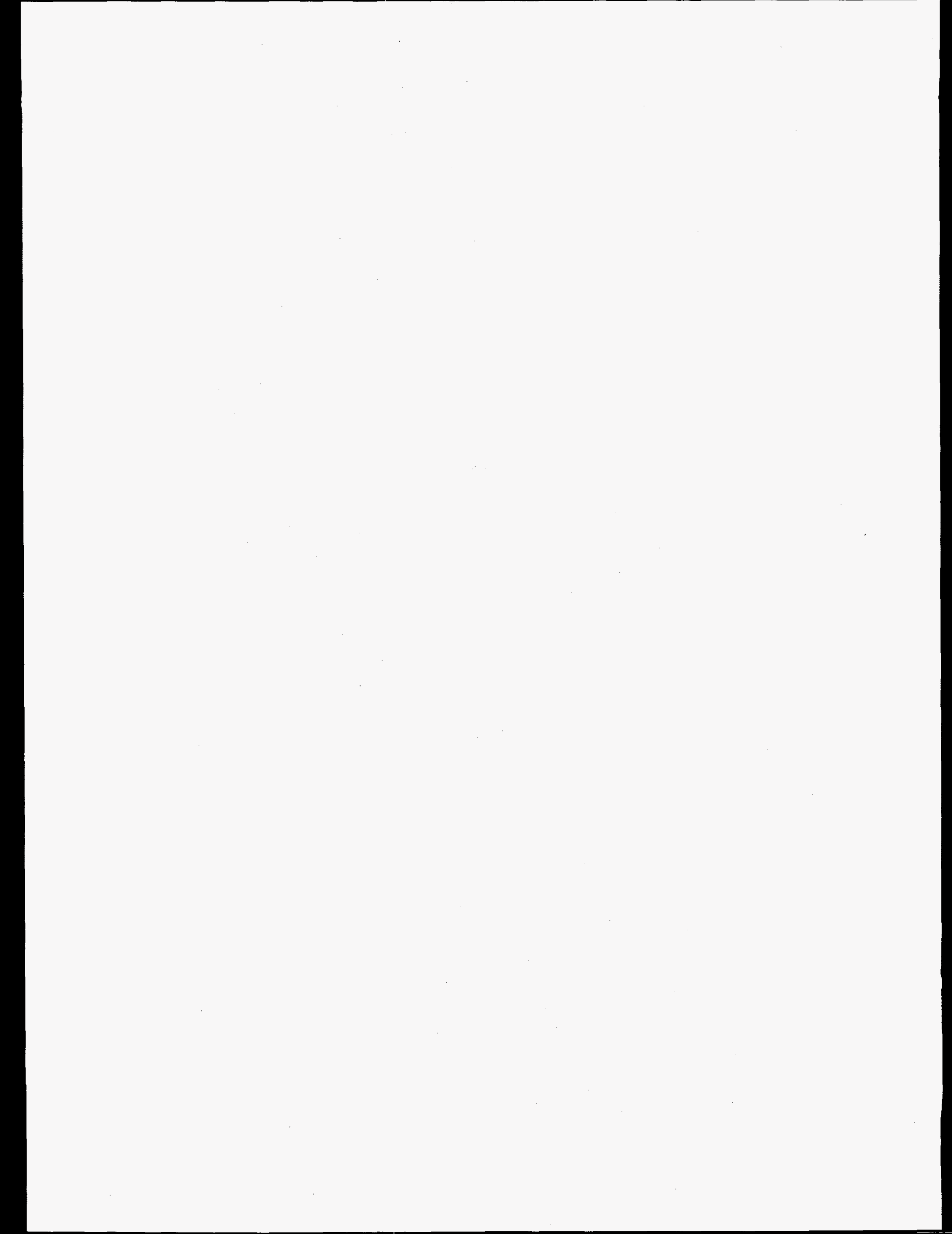




\section{Demand}

\section{Domestic Markets}

U.S. coal consumption during 1996 reached a record 983.3 million short tons, surpassing by 5.0 percent the previous record of 940.8 million short tons consumed during 1995. Compared with 1995, the amount of coal consumed by U.S. electric utilities during 1996 increased 5.5 percent to a record 874.7 million short tons, accounting for 89 percent of total domestic coal consumption.

In contrast, compared with 1995 , coal consumption at U.S. coke plants declined 3.9 percent to 31.7 million short tons, while coal consumption at other industrial plants declined 2.9 percent to 70.9 million short tons.

\section{Electric Utility Use}

Nearly one-half of the 1996 growth in total electric utility coal consumption was attributable to increases in the amount of coal consumed by electric utility generators located in the East North Central and South Atlantic Census Divisions. Collectively, electric utilities in these two Divisions accounted for 39.8 percent of the coal consumed by electric utilities during 1996.

In the East North Central Division, electric utiltiy coal consumption rose 6.1 percent to 198.9 million short tons as utilities in Illinois and Ohio relied upon coal-fired generating units to compensate for reduced generation by nuclear and gas-fired units. Similarly, in the South Atlantic Division, electric utility coal consumption rose 8.1 percent to 149.4 million short tons as coal-fired generating units accounted for increased shares of the electricity produced in the Carolinas and Virginia.

Coal consumption by electric utilities in the West South Central Division increased by 5.9 percent to 140.5 million short tons in response to increased electricity demand coupled with a decline in the share of generation supplied by natural gas.

Coal consumption in the West North Central Division increased 4.9 percent to 122.4 million short tons as coal-fired generation rose in response to increased electricity demand. In the East South Central Division, the amount of coal consumed by electric utilities increased 4.9 percent to 96.8 million short tons as increased demand for electricity offset a decline in the coal-fired share of the Divison's total generation.

\section{Coke Plant Use}

The decrease in the total amount of coal consumed by U.S. coke plants during 1996 was attributable primarily to coke plants in Ohio, where consumption of coking coal declined 33.7 percent to 1.8 million short tons. During 1996, coke plants in Pennsylvania and Indiana remained the Nation's leading consumers of coking coke as they collectively consumed 16.5 million short tons, accounting for 52.1 percent of total domestic coal consumption at coke plants.

\section{Other Uses}

Compared with 1995, coal consumption in the other industrial sector declined 2.9 percent to 70.9 million short tons, reflecting decreased coal consumption by this sector in all Census Divisions except the Middle Atlantic and East North Central Divisions. Coal consumption in the residential and commercial sectors in 1996 totaled 6.0 million short tons, an increase of 3.4 percent compared with 1995.

\section{Coal Stocks}

Stocks of coal held by domestic consumers at the close of 1996 totaled 123.0 million short tons, a decline of 8.6 percent compared with the 134.6 million short tons held in stock at the end of 1995. Compared with 1995, coal stocks at electric utility plants declined 9.2 percent to 114.7 million short tons, while stocks held at coke plants increased slightly to 2.7 million short tons. Coal stocks at other industrial plants remained relatively constant at 5.7 million short tons.

\section{Foreign Markets}

U.S. coal exports during 1996 totaled 90.5 million short tons, 2.2 percent more than the 88.5 million short tons exported during 1995. Compared with 1995, exports of metallurgical coal during 1996 increased 1.6 percent to 52.9 million short tons, accounting for 58.5 percent of total 1996 coal exports. Exports of steam coal during 1996 totaled 37.5 million short tons, an increase of 2.9 percent compared with 1995.

During 1996, Brazil replaced Japan as the leading destination for U.S. metallurgical coal exports. Compared with 1995, metallurgical coal shipments to Brazil increased 1.7 percent to 6.4 million short tons, while shipments of metallurgical coal to Japan declined 30.0 percent to 5.6 million short tons. 
Exports of metallurgical coal to Europe during 1996 totaled 28.3 million short tons, an increase of 3.5 percent compared with 1995. Shipments to Italy, the leading European consumer of U.S. metallurgical coal, increased 17.5 percent to 5.3 million short tons. In North America, shipments of U.S. metallurgical coal to Canada increased 35.4 percent to 6.0 million short tons.

Compared with 1995, U.S. steam coal exports to Europe during 1996 declined 11.2 percent to 18.9 million short tons as significant increases in shipments to France and the United Kingdom were offset by decreases in shipments to other European destinations. In North America, the amount of steam coal exported to Canada increased 20.6 percent to 6.0 million short tons and steam coal exports to Mexico increased 90 percent to over 1 million short tons. Exports of steam coal to Asia increased 13.4 percent to 9.2 million short tons as increases in exports to Japan and Israel offset decreased shipments to Taiwan and Korea. 


\section{Domestic Markets}

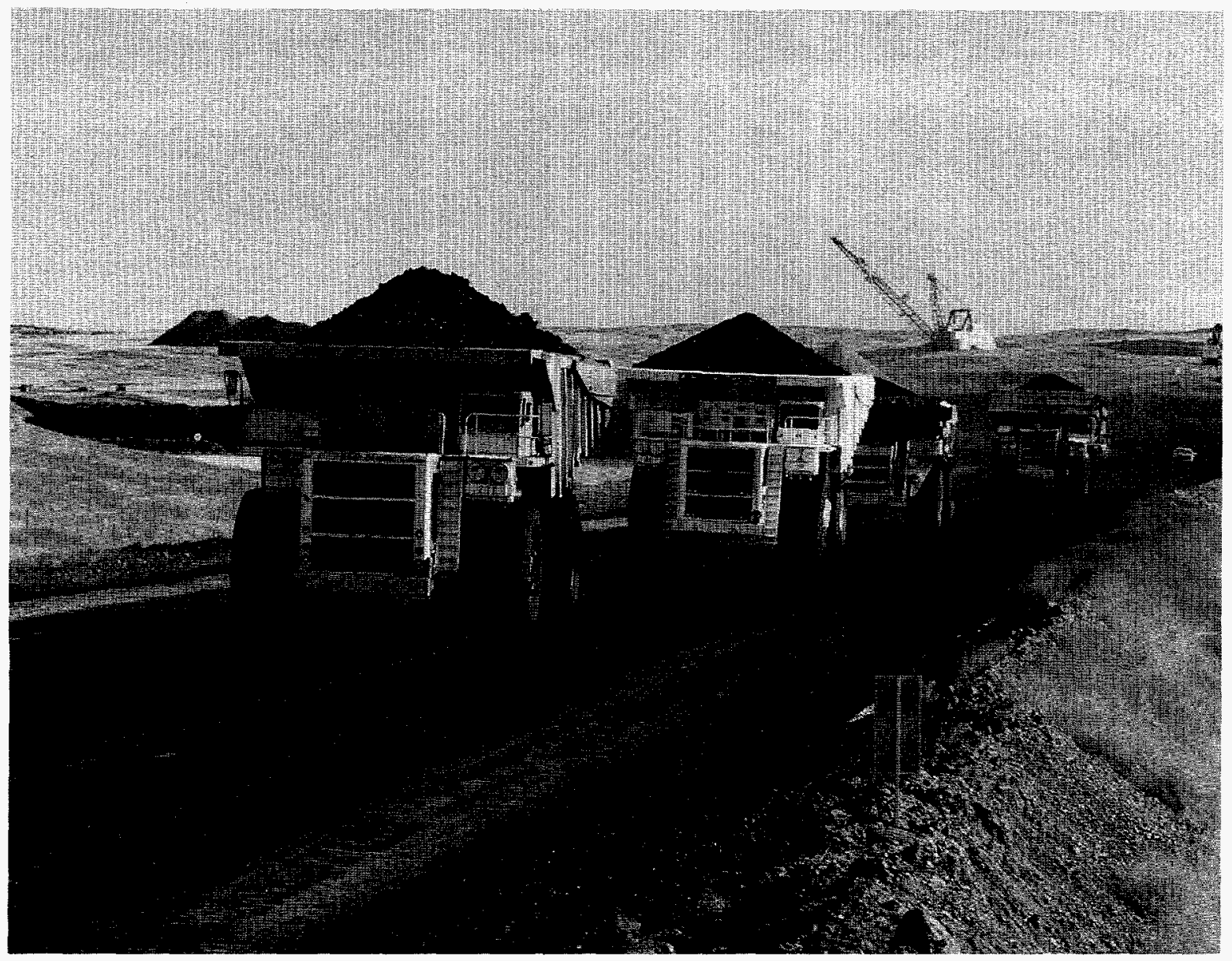

In 1996, consumption of U.S. coal increased 4.5 percent to 983 million short tons. Consumption at electric utility plants accounted for 89 percent of total consumption. Distribution by truck accounted for 9 percent of total coal disbursed. 
Figure 9. U.S. Coal Consumption, 1987-1996

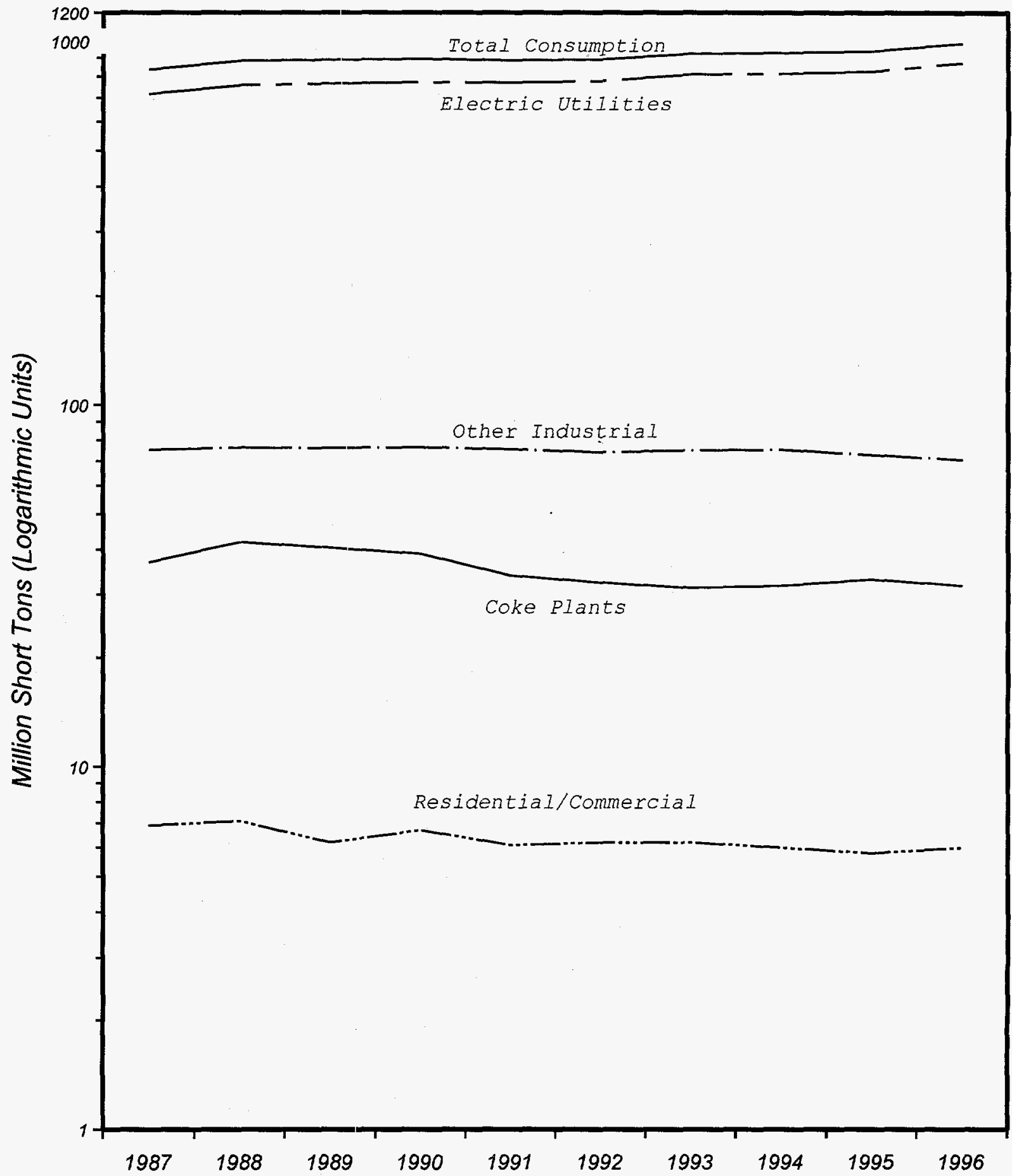

Note: Tolol consumption does not include coal.consumed by independent power producers.

Sources: Energy informotion Administrotion. Electric Utilities: Form ElA-759, "Monthly Power Plant Report."

Coke Plonis: Form EIA-5. "Coke Plont Report -Quorlerly." Other Industrial: Form ElA- 3, "Quorlerly Coa

Consumption Report - Manufocluring Plonts" and Form ElA-6. "Cool Dislribution Report." Riesidenlial and

Commerciol: Form EIA-6. "Cool Distribution Report." 
Table 66. Major U.S. Coal Consumers, 1996

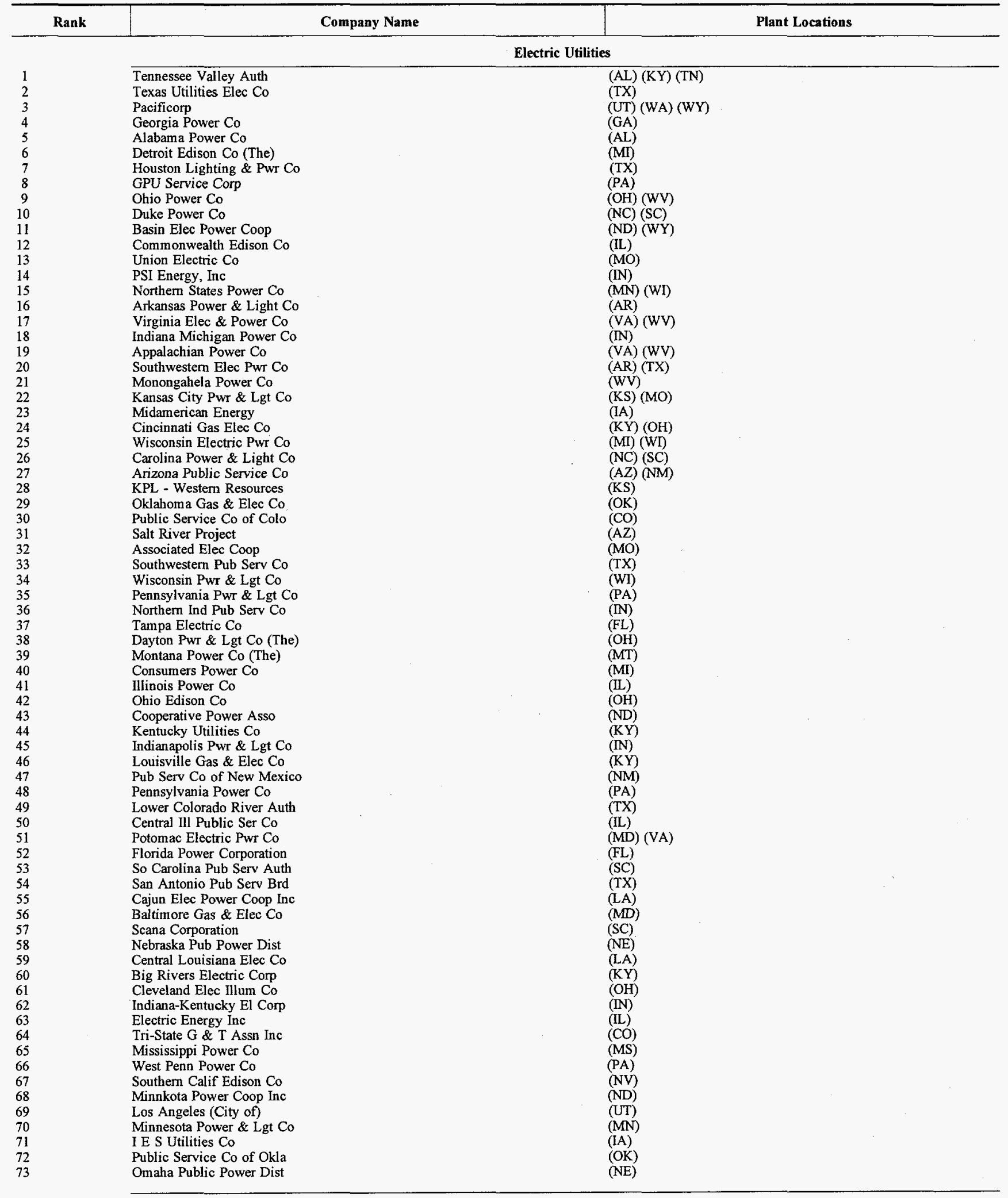

See footnotes at end of table. 
Table 66. Major U.S. Coal Consumers, 1996 (Continued)

\begin{tabular}{|c|c|}
\hline Company Name & Plant Location \\
\hline \multicolumn{2}{|c|}{ Top Ten Manufacturers } \\
\hline $\begin{array}{l}\text { Aluminum Company of America } \\
\text { Archer Daniels Midland Co } \\
\text { Basin Electric Power Coop } \\
\text { Champion International Corp } \\
\text { E I Du Pont De Nemours \& Co } \\
\text { Eastman Chemical Company } \\
\text { Hoechst AG } \\
\text { Holnam Inc } \\
\text { Inland Steel Industries Inc } \\
\text { Westvaco Corporation }\end{array}$ & $\begin{array}{l}\text { (IN) (OH) (TN) (TX) } \\
\text { (IA) (IL) (MN) } \\
\text { (ND) } \\
\text { (AL) (FL) (ME) (MI) (MN) (NC) (NY) (OH) } \\
\text { (DE) (MS) (NC) (SC) (TN) (VA) (WV) } \\
\text { (AR) (NY) (SC) (TN) (TX) } \\
\text { (SC) (TX) (VA) } \\
\text { (AL) (CO) (LA) (MI) (MO) (MS) (SC) (UT) (WA) } \\
\text { (IN) } \\
\text { (MD) (PA) (SD) (VA) }\end{array}$ \\
\hline \multicolumn{2}{|c|}{ Other Major Manufacturers } \\
\hline $\begin{array}{l}\text { A E Staley Manufacturing Co } \\
\text { American Crystal Sugar Co } \\
\text { Applied Energy Services Inc } \\
\text { Ash Grove Cement Company } \\
\text { Blue Circle Inc } \\
\text { Cargill Incorporated } \\
\text { CarMeuse/Marblehead Lime Co. } \\
\text { Dravo Corporation } \\
\text { Elkem A/S } \\
\text { Florida Crushed Stone } \\
\text { Fort Howard Corporation } \\
\text { FMC Corporation } \\
\text { G E Company } \\
\text { General Chemical Corporation } \\
\text { General Motors Corporation } \\
\text { Georgia-Pacific Corp } \\
\text { Heidelberger Zement Ag-Heidelb } \\
\text { International Paper Company } \\
\text { Jefferson Smurfit Corp } \\
\text { Kerr-McGee Corporation } \\
\text { Kimberly Clark Corporation } \\
\text { Lafarge Corporation } \\
\text { Lone Star Industries, Inc. } \\
\text { Mead Corporation The } \\
\text { Monsanto Company } \\
\text { P H Glatfelter Co } \\
\text { PPG Industries Inc } \\
\text { Southdown Inc } \\
\text { Stone Container Corporation } \\
\text { Union Camp Corporation }\end{array}$ & $\begin{array}{l}\text { (IL) (IN) (TN) } \\
\text { (MN) (ND) } \\
\text { (PA) } \\
\text { (AR) (KS) (MT) (NE) (OR) (TX) (UT) } \\
\text { (AL) (GA) (NY) (OK) (SC) } \\
\text { (GA) (IA) (MN) (NC) (OH) (TN) (VA) } \\
\text { (IL) (IN) (MI) } \\
\text { (AL) (KY) } \\
\text { (OH) (WV) } \\
\text { (FL) } \\
\text { (GA) (OK) (WI) } \\
\text { (NC) (WV) (WY) } \\
\text { (IN) (KY) (PA) } \\
\text { (WY) } \\
\text { (AL) (IL) (IN) (MI) (MO) (OH) (WI) } \\
\text { (AR) (GA) (MI) (VA) } \\
\text { (AL) (IA) (IN) (MD) } \\
\text { (AL) (LA) (PA) (SC) (WI) } \\
\text { (AL) (FL) (IL) (IN) (OH) (PA) } \\
\text { (CA) } \\
\text { (AL) (MI) (PA) (WI) } \\
\text { (IA) (IL) (KS) (MI) (MO) (PA) } \\
\text { (FL) (IL) (IN) (MO) (OK) (TX) } \\
\text { (MI) (OH) (TN) } \\
\text { (AL) (IA) (ID) (IL) (MA) (WV) } \\
\text { (NC) (PA) } \\
\text { (WV) } \\
\text { (CA) (CO) (FL) (OH) (TN) } \\
\text { (AZ) (FL) (MI) (SC) (VA) } \\
\text { (AL) (GA) (OH) (SC) (VA) } \\
\text { (IN) }\end{array}$ \\
\hline \multicolumn{2}{|c|}{ Top Ten Coke Producers } \\
\hline $\begin{array}{l}\text { AK Steel Corp } \\
\text { Bethlehem Steel Corp } \\
\text { Citizens Gas \& Coke Utility } \\
\text { Drummond Company Inc } \\
\text { Geneva Steel Company } \\
\text { LTV Steel Company Inc } \\
\text { National Steel Corp } \\
\text { Sun Coal Co } \\
\text { U S Steel Mining Company LLC } \\
\text { USX Corporation }\end{array}$ & $\begin{array}{l}(\mathrm{KY})(\mathrm{OH}) \\
(\mathrm{IN})(\mathrm{MD})(\mathrm{NY})(\mathrm{PA}) \\
(\mathrm{IN}) \\
\text { (AL) } \\
\text { (UT) } \\
\text { (IL) (IN) (OH) (PA) } \\
\text { (IL) (MI) } \\
\text { (VA) } \\
\text { (PA) } \\
\text { (IN) }\end{array}$ \\
\hline
\end{tabular}

Note: Major electric utility coal consumers are companies that consumed more than 3.6 million short tons of coal in 1996 . Major manufacturers are the top 40 coal consumers in the manufacturing sector. Major coke producers are the top 10 coal consumers in the coke plant sector. Electric utilities are ranked by consumption and manufacturers and coke producers are listed in alphabetical order.

Sources: Energy Information Administration, • Electric Utilities: Form EIA-759, "Monthly Power Plant Report." • Manufacturers: Form EIA-3, "Quarterly Coal Consumption Report - Manufacturing Plants." - Coke Plants: Form EIA-5, "Coke Plant Report - Quarterly." 
Table 67. Coal Consumption by Census Division and State, 1987, 1992-1996 (Thousand Short Tons)

\begin{tabular}{|c|c|c|c|c|c|c|c|c|c|}
\hline \multirow{2}{*}{ Census Division and State } & \multirow{2}{*}{1996} & \multirow{2}{*}{1995} & \multirow{2}{*}{1994} & \multirow{2}{*}{1993} & \multirow{2}{*}{1992} & \multirow{2}{*}{1987} & \multirow{2}{*}{$\begin{array}{c}\text { Percent } \\
\text { Change } \\
1995-1996\end{array}$} & \multicolumn{2}{|c|}{ Average Annual Percent Change } \\
\hline & & & & & & & & 1992-1996 & 1987-1996 \\
\hline 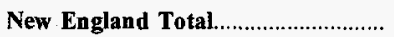 & 7,024 & 6,662 & 6,553 & 6,485 & 7,298 & 6,767 & 5.4 & -0.9 & 0.4 \\
\hline Connecticut & 931 & 906 & 862 & 788 & 849 & 815 & 2.7 & 2.3 & 1.5 \\
\hline Maine & 234 & 282 & 464 & 449 & 856 & 273 & -17.0 & -27.7 & -1.7 \\
\hline 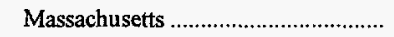 & 4,477 & 4,113 & 3,932 & 3,811 & 4,257 & 4,487 & 8.8 & 1.3 & * \\
\hline New Hampshire................................... & 1,377 & 1,355 & 1,287 & 1,428 & 1,311 & 1,176 & 1.6 & 1.2 & 1.8 \\
\hline 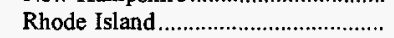 & 3 & 3 & 3 & 3 & 5 & 5 & 21.1 & -9.6 & -3.9 \\
\hline 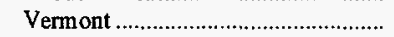 & 2 & 3 & 5 & $\cdot 6$ & 20 & 12 & -38.2 & -45.8 & -19.8 \\
\hline 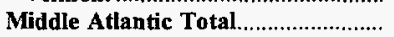 & 70,965 & 68,462 & 67,536 & 70,389 & 71,418 & 70,211 & 3.6 & -.1 & .1 \\
\hline 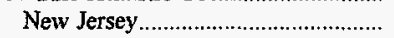 & 2,402 & 2,074 & 1,969 & 2,353 & 2,348 & 3,434 & 15.8 & .6 & -3.9 \\
\hline New York & 11,337 & 11,062 & 11,474 & 11,878 & 12,996 & 11,471 & 2.5 & -3.3 & -1 \\
\hline 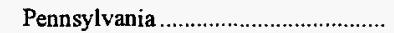 & 57,226 & 55,326 & 54,094 & 56,158 & 56,074 & 55,305 & 3.4 & .5 & .4 \\
\hline East North Central Total ................... & 229,000 & 217,702 & 213,188 & 210,632 & 200,660 & 201,833 & 5.2 & 3.3 & 1.4 \\
\hline 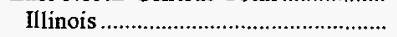 & 44,431 & 39,623 & 39,077 & 38,135 & 31,599 & 35,581 & 12.1 & 8.9 & 2.5 \\
\hline 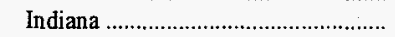 & 64,021 & 62,631 & 59,996 & 60,353 & 58,765 & 51,385 & 2.2 & 2.2 & 2.5 \\
\hline 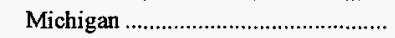 & 36,694 & 35,802 & 35,674 & 32,217 & 31,554 & 35,865 & 2.5 & 3.8 & .3 \\
\hline 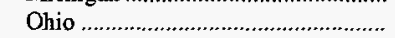 & 59,835 & 56,580 & 56,711 & 59,031 & 58,671 & 59,350 & 5.8 & .5 & .1 \\
\hline Wisconsin & 24,019 & 23,066 & 21,731 & 20,897 & 20,071 & 19,652 & 4.1 & 4.6 & 2.3 \\
\hline West North Central Total .................. & 136,643 & 131,028 & 125,591 & 120,940 & 115,505 & 101,599 & 4.3 & 4.3 & 3.3 \\
\hline Iowa & 21,171 & 20,636 & 19,341 & 19,188 & 17,992 & 15,191 & 2.6 & 4.1 & 3.8 \\
\hline 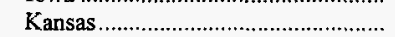 & 19,084 & 16,521 & 17,158 & 17,386 & 14,227 & 15,194 & 15.5 & 7.6 & 2.6 \\
\hline 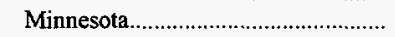 & 19,264 & 18,947 & 18,729 & 18,321 & 16,924 & 14,504 & 1.7 & 3.3 & 3.2 \\
\hline 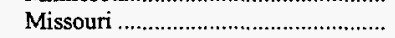 & 34,382 & 31,753 & 27,663 & 23,381 & 25,180 & 24,764 & 8.3 & 8.1 & 3.7 \\
\hline 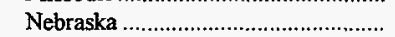 & 10,379 & 10,396 & 9,300 & 9,666 & 8,212 & 6,744 & -.2 & 6.0 & 4.9 \\
\hline 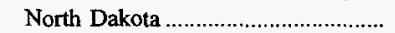 & 30,511 & 30,237 & 30,363 & 30,302 & 30,301 & 24,101 & .9 & .2 & 2.6 \\
\hline 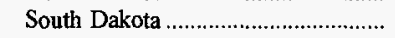 & 1,852 & 2,537 & 3,036 & 2,696 & 2,670 & 1,101 & -27.0 & -8.7 & 5.9 \\
\hline 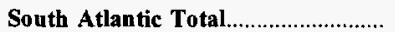 & 165,545 & 155,259 & 151,935 & 150,580 & 144,178 & 146,569 & 6.6 & 3.5 & 1.4 \\
\hline 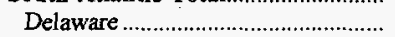 & 1,956 & 2,011 & 2,226 & 2,446 & 1,770 & 2,710 & -2.7 & 2.5 & -3.5 \\
\hline District of Columbia. & 23 & 6 & 47 & 51 & 50 & 70 & 309.0 & -17.6 & -11.5 \\
\hline 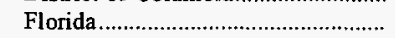 & 28,443 & 26,526 & 26,082 & 26,430 & 26,368 & 23,644 & 7.2 & 1.9 & 2.1 \\
\hline 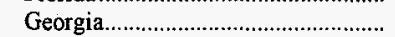 & 31,158 & 31,288 & 29,254 & 27,081 & 25,481 & 29,126 & -.4 & 5.1 & .8 \\
\hline 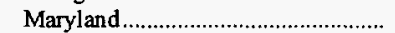 & 11,366 & 11,198 & 10,491 & 10,268 & 9,713 & 11,311 & 1.5 & 4.0 & $*$ \\
\hline 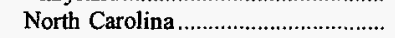 & 27,624 & 24,084 & 23,282 & 25,760 & 24,075 & 19,965 & 14.7 & 3.5 & 3.7 \\
\hline 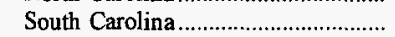 & 13,852 & 12,279 & 12,993 & 12,914 & 11,285 & 11,701 & 12.8 & 5.3 & 1.9 \\
\hline 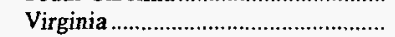 & 14,983 & 13,378 & 12,792 & 13,584 & 13,418 & 13,227 & 12.0 & 2.8 & 1.4 \\
\hline 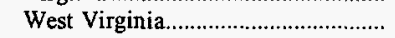 & 36,139 & 34,489 & 34,767 & 32,046 & 32,019 & 34,815 & 4.8 & 3.1 & .4 \\
\hline East South Central Total...................... & 110,450 & 105,830 & 99,289 & 104,027 & 93,804 & 88,203 & 4.4 & 4.2 & 2.5 \\
\hline Alabama & 37,052 & 34,309 & 31,473 & 33,047 & 31,510 & 26,632 & 8.0 & 4.1 & 3.7 \\
\hline 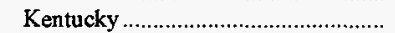 & 40,863 & 39,516 & 38,090 & 39,095 & 34,704 & 32,023 & 3.4 & 4.2 & 2.7 \\
\hline 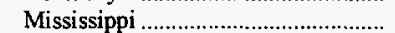 & 5,791 & 4,606 & 4,285 & 4,030 & 3,485 & 4,846 & 25.7 & 13.5 & 2.0 \\
\hline 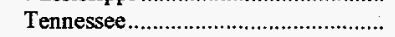 & 26,744 & 27,399 & 25,440 & 27,854 & 24,106 & 24,702 & -2.4 & 2.6 & .9 \\
\hline West South Central Total .................. & 146,472 & 139,106 & 138,251 & 140,797 & 135,210 & 118,847 & 5.3 & 2.0 & 2.3 \\
\hline 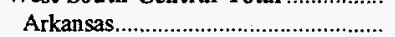 & 14,816 & 13,540 & 12,596 & 11,447 & 12,538 & 12,066 & 9.4 & 4.3 & 2.3 \\
\hline 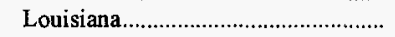 & 12,534 & $13,35 ?$ & 14,100 & 13,676 & 13,674 & 10,391 & -6.2 & -2.1 & 2.1 \\
\hline 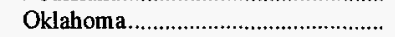 & 20,125 & 19,596 & 17,726 & 18,866 & 17,430 & 13,476 & 2.7 & 3.7 & 4.5 \\
\hline Texas & 98,997 & 92,612 & 93,829 & 96,809 & 91,568 & 82,915 & 6.9 & 2.0 & 2.0 \\
\hline 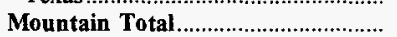 & 107,226 & 107,923 & 115,695 & 110,673 & 112,163 & 94,126 & -.6 & -1.1 & 1.4 \\
\hline 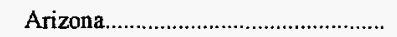 & 16,792 & 16,682 & 19,580 & 18,991 & 17,915 & 13,375 & .6 & -1.6 & 2.6 \\
\hline 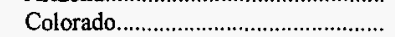 & 17,222 & 16,971 & 17,475 & 17,070 & 16,696 & 15,007 & 1.5 & .8 & 1.5 \\
\hline Idaho & 397 & 465 & 534 & 528 & 535 & 494 & -14.5 & -7.2 & -2.4 \\
\hline 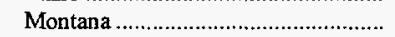 & 8,032 & 10,005 & 11,089 & 9,247 & 11,040 & 7,730 & -19.7 & -7.6 & 4 \\
\hline Nevada & 7,604 & 7,340 & 7,968 & 7,806 & 8,088 & 6,920 & 3.6 & -1.5 & 1.0 \\
\hline New Mexico & 15,297 & 15,221 & 15,374 & 15,012 & 14,832 & 14,395 & .5 & .8 & .7 \\
\hline 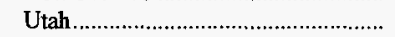 & 15,237 & 15,307 & 16,216 & 15,848 & 15,719 & 11,807 & -.4 & -.8 & 2.9 \\
\hline 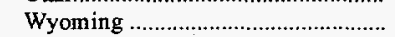 & 26,646 & 25,933 & 27,459 & 26,171 & 27,339 & 24,399 & 2.8 & -.6 & 1.0 \\
\hline Pacific Total & 10,008 & $\mathbf{8 , 9 0 8}$ & 12,162 & 11,422 & 12,186 & 8,294 & 12.3 & -4.8 & 2.1 \\
\hline Alaska & 706 & 815 & 796 & 863 & 792 & 274 & -13.4 & -2.8 & 11.1 \\
\hline California & 2,317 & 2,618 & 2,498 & 2,453 & 2,821 & 1,934 & -11.5 & -4.8 & 2.0 \\
\hline Hawaii & 169 & 192 & 86 & 73 & 47 & 63 & -11.6 & 37.9 & 11.6 \\
\hline 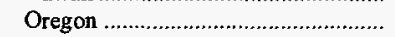 & 1,134 & 1,125 & 2,479 & 2,099 & 2,124 & 205 & .8 & -14.5 & 20.9 \\
\hline 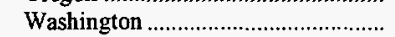 & 5,682 & 4,158 & 6,303 & 5,934 & 6,402 & 5,819 & 36.6 & -2.9 & -.3 \\
\hline 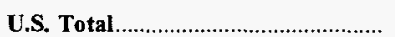 & 983,334 & 940,880 & 930,201 & 925,944 & 892,421 & 836,860 & 4.5 & 2.4 & 1.8 \\
\hline
\end{tabular}

* Data round to zero. rounding.

Notes: U.S. Total does not include coal consumed by independent power producers. Totals may not equal sum of components due to independent

Sources: Energy Information Administration, Form EIA-759, "Monthly Power Plant Report"; Form EIA-3, "Quarterly Coal Consumption Report - Manufacturing Plants"; Form EIA-5, "Coke Plant Report - Quarterly"; and Form EIA-6, "Coal Distribution Report." 
Figure 10. U.S. Consumer Coal Stocks, 1987-1996

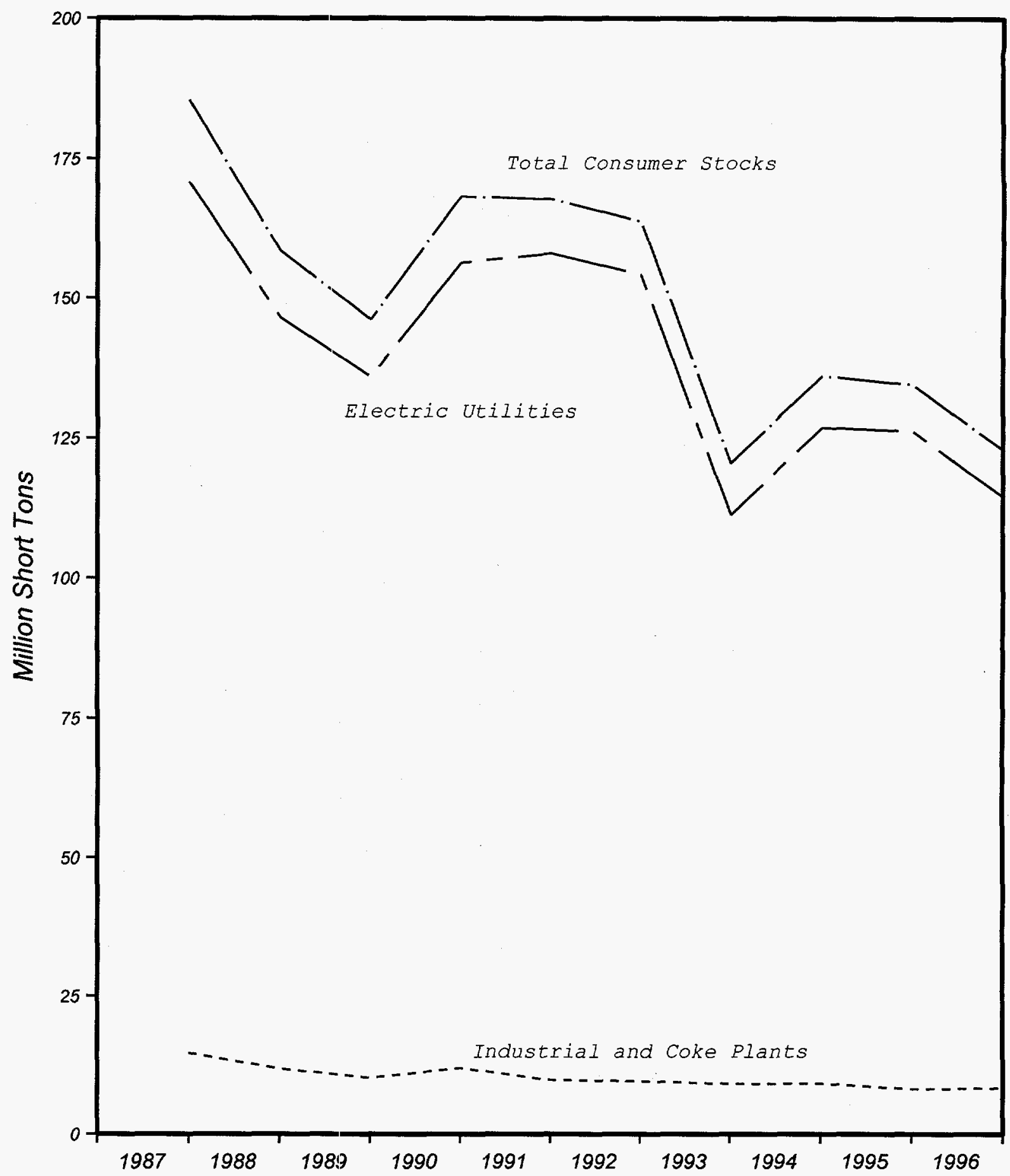

Nole: Eoch increment represents end-of yeoc dato. Industrial slocks reflect monulocluring plonts ond coke plonts. - Sources: Energy Informotion Administrotión. Electric Utilities: Form ElA-759. "Monthly Power Plant Reporl." Industriol: Form EIA-5. "Coke PIonI Report - Ouorterly" and Form EIA-3, "Ouorterly Coal Consumption Report Monufocturing Plonts. 
Table 68. Year-End Consumer Coal Stocks by Census Division and State, 1987, 1992-1996 (Thousand Short Tons)

\begin{tabular}{|c|c|c|c|c|c|c|c|c|c|}
\hline \multirow{2}{*}{ Census Division and State } & \multirow{2}{*}{1996} & \multirow{2}{*}{1995} & \multirow{2}{*}{1994} & \multirow{2}{*}{1993} & \multirow{2}{*}{1992} & \multirow{2}{*}{1987} & \multirow{2}{*}{$\begin{array}{c}\text { Percent } \\
\text { Change } \\
\text { 1995-1996 }\end{array}$} & \multicolumn{2}{|c|}{ Average Annual Percent Change } \\
\hline & & & & & & & & 1992-1996 & $1987-1996$ \\
\hline 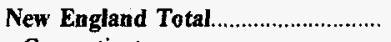 & 1,297 & 969 & $\mathbf{1}, 117$ & 989 & 1,253 & 1,056 & 33.9 & 0.9 & 2.3 \\
\hline 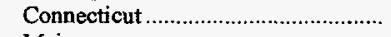 & w & $\mathbf{w}$ & w & $\mathbf{w}$ & w & w & $\mathbf{w}$ & w & w \\
\hline 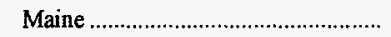 & w & $w$ & $\mathbf{w}$ & $w$ & $w$ & w & $\mathbf{w}$ & w & $\mathbf{w}$ \\
\hline 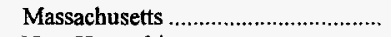 & w & $\mathbf{w}$ & $\mathrm{w}$ & $w$ & $w$ & $\mathbf{w}$ & $\mathbf{w}$ & $\mathbf{w}$ & $\mathbf{w}$ \\
\hline 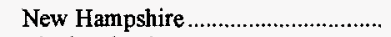 & w & w & $\mathbf{w}$ & $\mathbf{w}$ & w & w & w & $w$ & $\mathbf{w}$ \\
\hline 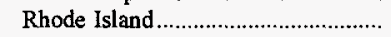 & - & - & - & - & - & $w$ & - & - & - \\
\hline 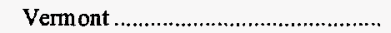 & - & - & - & - & - & w & - & - & - \\
\hline Middle Atlantic Total.......................... & 10,899 & 12,404 & 14,068 & 14,060 & 19,486 & 19,598 & -12.1 & -13.5 & -6.3 \\
\hline 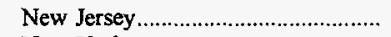 & $w$ & $w$ & w & $\mathbf{w}$ & w & $w$ & w & w & w \\
\hline 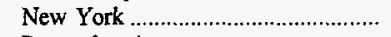 & w & w & $\mathbf{w}$ & $w$ & w & $w$ & w & w & w \\
\hline Pennsylvania & 8,860 & 10,303 & 12,060 & 12,265 & 15,976 & 16,121 & -14.0 & -13.7 & -6.4 \\
\hline East North Central Total ..................... & 30,823 & 33,818 & 35,833 & 30,162 & 43,661 & 51,835 & -8.8 & -8.3 & -5.6 \\
\hline 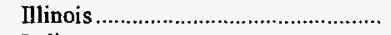 & $\mathbf{w}$ & w & $w$ & $\mathbf{w}$ & w & w & $\mathbf{w}$ & w & w \\
\hline Indiana & 7,958 & 9,298 & 11,707 & 7,798 & 12,507 & 14,998 & -14.4 & -10.7 & -6.8 \\
\hline 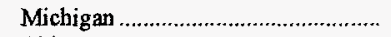 & $\mathbf{w}$ & $w$ & w & w & $w$ & $w$ & $\mathbf{w}$ & $w$ & w \\
\hline Ohio & 5,431 & 5,936 & 7,815 & 7,630 & 10,804 & 11,164 & -8.5 & -15.8 & -7.7 \\
\hline Wisconsin & 4,458 & 3,656 & $w$ & $w$ & $w$ & $w$ & 21.9 & $w$ & $w$ \\
\hline West North Central Total .................. & 18,335 & 18,713 & $\mathbf{w}$ & $\mathbf{w}$ & $\mathbf{w}$ & $\mathbf{w}$ & -2.0 & $\mathbf{w}$ & $w$ \\
\hline Iowa & 4,614 & 4,447 & 4,178 & 3,819 & 4,857 & 5,094 & 3.8 & -1.3 & -1.1 \\
\hline 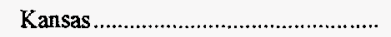 & 2,986 & 3,860 & 2,623 & 2,024 & 2,759 & 3,327 & -22.6 & 2.0 & -1.2 \\
\hline 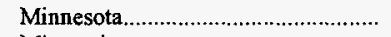 & 1,739 & 1,985 & 2,234 & 1,250 & 2,252 & 3,427 & -12.4 & -6.3 & -7.3 \\
\hline 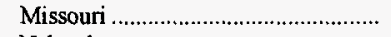 & 5,320 & 4,779 & w & w & $w$ & w & 11.3 & w & w \\
\hline Nebraska & w & $\mathbf{w}$ & w & $\mathbf{w}$ & w & $\mathbf{w}$ & w & $\mathbf{w}$ & $w$ \\
\hline 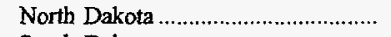 & $w$ & $\mathbf{w}$ & $w$ & $w$ & $w$ & $w$ & $w$ & $\mathbf{w}$ & $\mathbf{w}$ \\
\hline 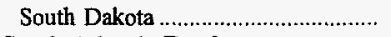 & w & w & w & w & $w$ & w & w & w & w \\
\hline South Atlantic Total............................ & 19,584 & 19,707 & 24,427 & 19,092 & 29,282 & 32,040 & -.6 & -9.6 & -5.3 \\
\hline Delaware & $w$ & $w$ & w & w & w & w & w & w & w \\
\hline Florida & 3,440 & 3,268 & 3,914 & 3,541 & 4,121 & 3,298 & 5.3 & -4.4 & .5 \\
\hline 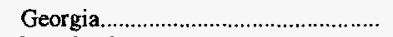 & 3,848 & 3,786 & 4,843 & 2,926 & 4,836 & 6,712 & 1.6 & -5.5 & -6.0 \\
\hline 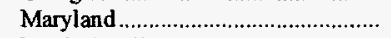 & w & w & w & w & $w$ & $w$ & w & w & w \\
\hline North Carolina & 2,672 & 2,855 & 4,318 & 3,059 & 4,715 & 4,794 & -6.4 & -13.2 & -6.3 \\
\hline South Carolina & 2,178 & 2,194 & 2,533 & 1,893 & 2,451 & 2,376 & -.7 & -2.9 & -1.0 \\
\hline 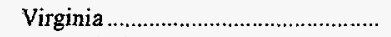 & $w$ & $w$ & $w$ & $w$ & $w$ & $w$ & $w$ & $w$ & $w$ \\
\hline 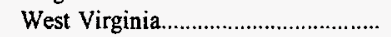 & w & w & w & w & w & w & w & w & w \\
\hline East South Central Total...................... & 9,311 & 10,940 & 11,267 & 9,277 & 14,400 & 19,574 & -14.9 & -10.3 & -7.9 \\
\hline 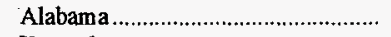 & 2,860 & 3,648 & 4,132 & 2,797 & 4,529 & 5,012 & -21.6 & -10.8 & -6.0 \\
\hline 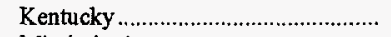 & w & $w$ & w & w & w & w & w & $\mathbf{w}$ & w \\
\hline 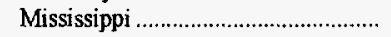 & w & $\mathbf{w}$ & $\mathbf{w}$ & $\mathbf{w}$ & $\mathbf{w}$ & $w$ & $\mathrm{w}$ & $\mathbf{w}$ & $w$ \\
\hline 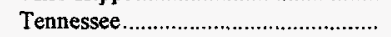 & 1,501 & 1,884 & w & w & $w$ & $\mathbf{w}$ & -20.3 & $w$ & $\mathrm{w}$ \\
\hline West South Central Total ................... & 19,894 & 20,564 & 15,959 & 15,105 & $\mathbf{w}$ & $\mathbf{w}$ & -3.3 & $\mathbf{w}$ & $\mathbf{w}$ \\
\hline 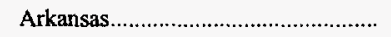 & 2,720 & 2,820 & 1,777 & 1,881 & 1,591 & 2,359 & -3.5 & 14.3 & 1.6 \\
\hline 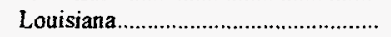 & 2,481 & 2,669 & 1,922 & 2,000 & 1,755 & 2,820 & -7.0 & 9.0 & -1.4 \\
\hline Oklahoma & 4,212 & 4,246 & 2,467 & 2,052 & 3,161 & 3,787 & -.8 & 7.4 & 1.2 \\
\hline 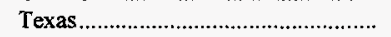 & 10,481 & 10,829 & 9,793 & 9,172 & $w$ & $\mathbf{w}$ & -3.2 & w & w \\
\hline Mountain Total & $\mathbf{w}$ & $\mathbf{w}$ & $\mathbf{w}$ & $\mathbf{w}$ & $\mathbf{w}$ & $\mathbf{w}$ & $\mathbf{w}$ & $\mathbf{w}$ & $\mathbf{w}$ \\
\hline 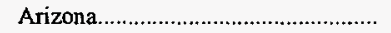 & 2,025 & 3,032 & 3,242 & 3,717 & 3,596 & 3,840 & -33.2 & -13.4 & -6.9 \\
\hline Colorado & 3,057 & 3,682 & 3,145 & 3,454 & 3,439 & w & -17.0 & -2.9 & w \\
\hline Idaho & 77 & 118 & 78 & 86 & 101 & 116 & -34.4 & -6.5 & -4.4 \\
\hline Montana & $\mathbf{w}$ & $w$ & $w$ & $w$ & w & $\mathbf{w}$ & $\mathbf{w}$ & $\mathbf{w}$ & $w$ \\
\hline 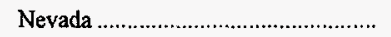 & $\mathrm{w}$ & $w$ & w & $w$ & w & $\mathbf{w}$ & $\mathbf{w}$ & w & $w$ \\
\hline 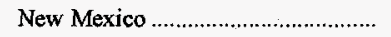 & $w$ & $\mathbf{w}$ & $\mathbf{w}$ & $\mathbf{w}$ & w & $\mathbf{w}$ & $\mathbf{w}$ & $\mathbf{w}$ & $\mathbf{w}$ \\
\hline Utah & $w$ & w & $\mathbf{w}$ & w & $\mathbf{w}$ & $w$ & $w$ & w & w \\
\hline Wyoming & 2,269 & 2,936 & 2,553 & 1,841 & 2,242 & 2,867 & -22.7 & .3 & -2.6 \\
\hline 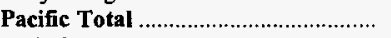 & 1,275 & 2,586 & 877 & 918 & 1,529 & 1,470 & -50.7 & -4.4 & -1.6 \\
\hline 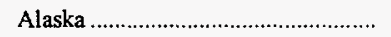 & 1 & 1 & 2 & 5 & $w$ & $w$ & - & w & w \\
\hline 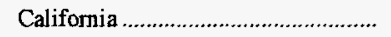 & 150 & 133 & 126 & 93 & 109 & 174 & 12.4 & 8.3 & -1.6 \\
\hline 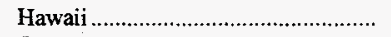 & w & $w$ & $\mathbf{w}$ & $\mathbf{w}$ & $\mathbf{w}$ & w & $\mathbf{w}$ & w & w \\
\hline Oregon & $w$ & w & $w$ & $w$ & $\mathbf{w}$ & $w$ & $w$ & $w$ & $w$ \\
\hline Washington & 857 & 1,969 & 569 & 459 & 736 & 681 & -56.4 & 3.9 & 2.6 \\
\hline U.S. Total & 123,024 & 134,639 & 136,139 & 120,458 & 163,692 & 185,459 & -8.6 & -6.9 & -4.4 \\
\hline
\end{tabular}

withheld to avoid disclosure of individual company data.

Notes: Stocks for the residential and commercial sector are not included. Totals may not equal sum of components due to independent rounding.

Sources: Energy Information Administration, Form EIA-759, "Monthly Power Plant Report"; Form EIA-5, "Coke Plant Report - Quarterly"; and Form

EIA-3, "Quarterly Coal Consumption Report - Manufacturing Plants." 
Table 69. Coal Consumption at Electric Utility Plants by Census Division and State, 1987, 1992-1996

(Thousand Short Tons)

\begin{tabular}{|c|c|c|c|c|c|c|c|c|c|}
\hline \multirow{2}{*}{ Census Division and State } & \multirow{2}{*}{1996} & \multirow{2}{*}{1996} & \multirow{2}{*}{1994} & \multirow{2}{*}{1993} & \multirow{2}{*}{1992} & \multirow{2}{*}{1987} & \multirow{2}{*}{$\begin{array}{c}\text { Percent } \\
\text { Change } \\
\text { 1995-1996 }\end{array}$} & \multicolumn{2}{|c|}{ Average Annual Percent Change } \\
\hline & & & & & & & & 1992-1996 & $1987-1996$ \\
\hline New England Total.............................. & 6,701 & 6,272 & 5,945 & 5,736 & 6,112 & 6,217 & 6.8 & 2.3 & 0.8 \\
\hline 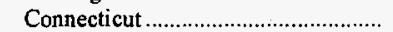 & 925 & 881 & 821 & 745 & 817 & 787 & 5.0 & 3.1 & 1.8 \\
\hline 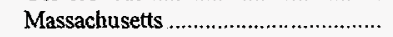 & 4,406 & 4,044 & 3,845 & 3,652 & 4,044 & 4,267 & 8.9 & 2.2 & .3 \\
\hline New Hampshire .................................... & 1,369 & 1,346 & 1,279 & 1,339 & 1,251 & 1,163 & 1.7 & 2.3 & 1.8 \\
\hline Middle Atlantic Total .......................... & 51,718 & 49,357 & 48,326 & 51,079 & 52,488 & 52,172 & 4.8 & -.4 & -.1 \\
\hline 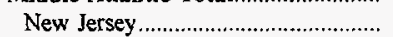 & 2,387 & 2,054 & 1,887 & 2,123 & 2,118 & 3,081 & 16.2 & 30 & -2.8 \\
\hline 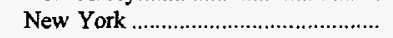 & 8,254 & 8,051 & 8,395 & 8,699 & 9,963 & 7,828 & 2.5 & -4.6 & 6 \\
\hline 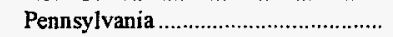 & 41,076 & 39,252 & 38,044 & 40,257 & 40,407 & 41,263 & 4.6 & .4 & * \\
\hline East North Central Total ................... & 198,900 & 187,490 & 183,282 & 179,833 & 169,029 & 161,833 & 6.1 & 4.1 & 2.3 \\
\hline 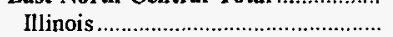 & 38,090 & 33,463 & 32,599 & 31,744 & 25,264 & 28,894 & 13.8 & 10.8 & 3.1 \\
\hline 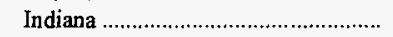 & 52,855 & 52,089 & 50,554 & 48,836 & 46,937 & 36,987 & 1.5 & 3.0 & 4.0 \\
\hline 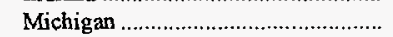 & 32,175 & 31,165 & 31,106 & 28,749 & 28,238 & 30,854 & 3.2 & 3.3 & .5 \\
\hline Ohio & 53,543 & 49,785 & 49,326 & 51,456 & 50,358 & 47,520 & 7.5 & 1.5 & 1.3 \\
\hline 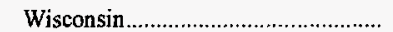 & 22,236 & 20,1987 & 19,696 & 19,049 & 18,231 & 17,579 & 5.9 & 5.1 & 2.6 \\
\hline West North Central Total ................. & 122,419 & 116,720 & 111,672 & 107,584 & 102,557 & 89,174 & 4.9 & 4.5 & 3.6 \\
\hline Iowa & 17,864 & 17,185 & 16,565 & 16,623 & 15,357 & 12,997 & .4 & 3.8 & 3.6 \\
\hline 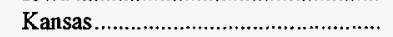 & 18,853 & 16,345 & 16,989 & 17,226 & 14,068 & 14,942 & 15.3 & 7.6 & 2.6 \\
\hline 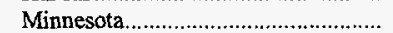 & 17,459 & 17,282 & 17,046 & 16,844 & 15,841 & 13,495 & 1.0 & 2.5 & 2.9 \\
\hline 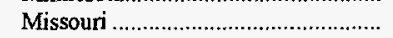 & 33,059 & 30,440 & 26,375 & 21,945 & 23,815 & 23,012 & 8.6 & 8.5 & 4.1 \\
\hline 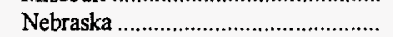 & 10,091 & 10,0148 & 8,879 & 9,297 & 7,881 & 6,428 & .4 & 6.4 & 5.1 \\
\hline 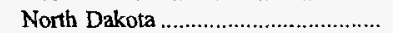 & 23,640 & 22,680 & 23,248 & 23,290 & 23,192 & 17,434 & 4.2 & .5 & 3.4 \\
\hline 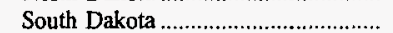 & 1,453 & 2,137 & 2,570 & 2,360 & 2,402 & 865 & -32.0 & -11.8 & 5.9 \\
\hline 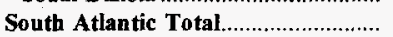 & 149,354 & 138,134 & 133,984 & 132,885 & 126,093 & 125,582 & 8.1 & 4.3 & 1.9 \\
\hline Delaware & 1,787 & 1,816 & 2,007 & 2,223 & 1,628 & 2,449 & -1.6 & 2.4 & -3.4 \\
\hline Florida & 27,172 & 25,200 & 24,758 & 25,108 & 25,016 & 22,598 & 7.8 & 2.1 & 2.1 \\
\hline 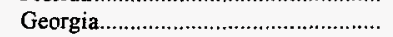 & 29,171 & 29,280 & 27,293 & 25,339 & 23,656 & 27,130 & -.4 & 5.4 & .8 \\
\hline 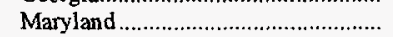 & 10,540 & 10,141 & 9,717 & 9,521 & 8,993 & 8,228 & 3.9 & 4.0 & 2.8 \\
\hline 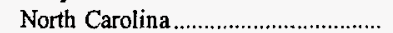 & 25,083 & 21,424 & 20,624 & 23,055 & 21,011 & 17,255 & 17.1 & 4.5 & 4.2 \\
\hline 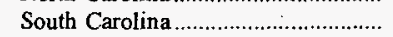 & 11,833 & 10,074 & 10,597 & 10,410 & 9,078 & 9,019 & 17.5 & 6.8 & 3.1 \\
\hline Virginia & 10,994 & 9,543 & 8,670 & 9,447 & 8,661 & 8,297 & 15.2 & 6.1 & 3.2 \\
\hline West Virginia & 32,775 & 30,657 & 30,318 & 27,782 & 28,050 & 30,605 & 6.9 & 4.0 & .8 \\
\hline East South Central Total................... & 96,809 & 92,262 & 85,622 & 90,365 & 80,203 & 74,573 & 4.9 & 4.8 & 2.9 \\
\hline Alabama & 31,216 & $28,7,59$ & 25,817 & 27,533 & 24,988 & 20,746 & 8.5 & 5.7 & 4.6 \\
\hline Kentucky & 37,072 & $35,71) 7$ & 34,564 & 35,264 & 31,715 & 28,569 & 3.8 & 4.0 & 2.9 \\
\hline 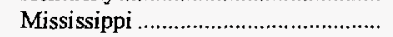 & 5,558 & $4,3: 9$ & 3,989 & 3,767 & 3,237 & 4,562 & 28.7 & 14.5 & 2.2 \\
\hline Tennessee & 22,964 & $23,4: 7$ & 21,253 & 23,801 & 20,263 & 20,697 & -2.2 & 3.2 & 1.2 \\
\hline West South Central Total & 140,493 & 132,633 & 131,168 & 134,009 & 129,351 & 113,455 & 5.9 & 2.1 & 2.4 \\
\hline 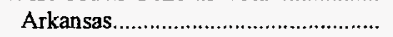 & 14,467 & 13,216 & 12,250 & 11,116 & 12,241 & 11,764 & 9.5 & 4.3 & 2.3 \\
\hline 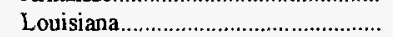 & 12,450 & 12,950 & 13,479 & 13,089 & 13,077 & 10,029 & -3.7 & -1.2 & 2.4 \\
\hline Oklahoma & 19,386 & $18,1 \equiv 0$ & 16,961 & 17,668 & 16,699 & 12,861 & 6.9 & 3.8 & 4.7 \\
\hline 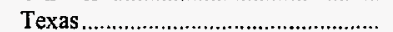 & 94,189 & 88,358 & 88,479 & 92,135 & 87,333 & 78,802 & 6.6 & 1.9 & 2.0 \\
\hline Mountain Total. & 101,507 & 101,013 & 108,651 & 104,093 & 105,609 & 89,145 & .5 & -1.0 & 1.4 \\
\hline 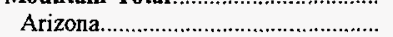 & 16,117 & 16,021 & 18,853 & 18,316 & 17,280 & 12,706 & .6 & -1.7 & 2.7 \\
\hline 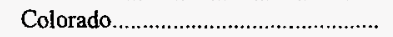 & 16,841 & 16,222 & 16,596 & 16,252 & 15,902 & 14,178 & 3.8 & 1.4 & 1.9 \\
\hline 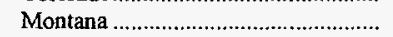 & 7,897 & 9,373 & 10,513 & 8,869 & 10,768 & 7,530 & -15.8 & -7.4 & .5 \\
\hline Nevada & 7,424 & 7,084 & 7,772 & 7,608 & 7,914 & 6,807 & 4.8 & -1.6 & 1.0 \\
\hline 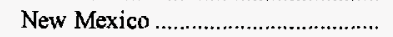 & 15,215 & 15,137 & 15,297 & 14,942 & 14,775 & 14,340 & .5 & .7 & 6 \\
\hline Utah & 13,584 & 13,325 & 14,269 & 13,995 & 13,857 & 11,175 & 1.9 & -.5 & 2.2 \\
\hline Wyoming & 24,430 & 23,850 & 25,350 & 24,111 & 25,114 & 22,408 & 2.4 & -.7 & 1.0 \\
\hline 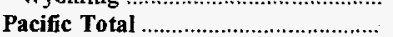 & 6,780 & 5,127 & 8,621 & 7,924 & 8,419 & $\mathbf{5 , 7 4 2}$ & 32.2 & -5.3 & 1.9 \\
\hline Alaska & 229 & 29.3 & 271 & 298 & 277 & 274 & -21.6 & -4.6 & -2.0 \\
\hline Oregon & 1,044 & 977 & 2,333 & 1,981 & 1,994 & - & 6.8 & -14.9 & - \\
\hline 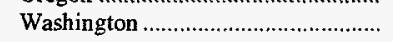 & 5,507 & 3,857 & 6,016 & 5,646 & 6,148 & 5,468 & 42.8 & -2.7 & .1 \\
\hline U.S. Total & 874,681 & $829,00^{77}$ & 817,270 & 813,508 & 779,860 & $\mathbf{7 1 7 , 8 9 4}$ & 5.5 & 2.9 & 2.2 \\
\hline
\end{tabular}

* Data round to zero.

Note: Totals may not equal sum of components due to independent rounding.

Source: Energy Information Administration, Form EIA-759, "Monthly Power Plant Report." 
Table 70. Year-End Coal Stocks at Electric Utility Plants by Census Division and State, 1987, 1992-1996

(Thousand Short Tons)

\begin{tabular}{|c|c|c|c|c|c|c|c|c|c|}
\hline \multirow{2}{*}{ Census Division and State } & \multirow{2}{*}{1996} & \multirow{2}{*}{1995} & \multirow{2}{*}{1994} & \multirow{2}{*}{1993} & \multirow{2}{*}{1992} & \multirow{2}{*}{1987} & \multirow{2}{*}{$\begin{array}{c}\text { Percent } \\
\text { Change } \\
\text { 1995-1996 }\end{array}$} & \multicolumn{2}{|c|}{ Average Annual Percent Change } \\
\hline & & & & & & & & 1992-1996 & 1987-1996 \\
\hline New England Total................................. & 1,237 & 908 & 1,079 & 967 & 1,237 & 1,012 & 36.2 & - & 2.3 \\
\hline 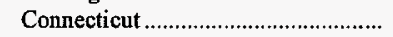 & 173 & 164 & 202 & 160 & 150 & - & 5.5 & 3.7 & - \\
\hline 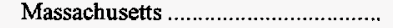 & 704 & 425 & 629 & 449 & 727 & 677 & 65.7 & -.8 & .4 \\
\hline 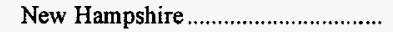 & 360 & 319 & 248 & 358 & 361 & 307 & 12.6 & -.1 & 1.8 \\
\hline 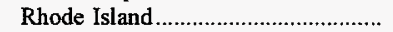 & - & - & - & - & - & 28 & - & - & - \\
\hline Middle Atlantic Total.......................... & 9,609 & 11,064 & 12,687 & 12,564 & 17,743 & 17,764 & -13.1 & -14.2 & -6.6 \\
\hline 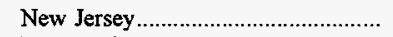 & 824 & 804 & 688 & 501 & 771 & 842 & 2.4 & 1.7 & -.2 \\
\hline 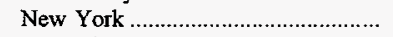 & 905 & 1,015 & 999 & 953 & 2,106 & 1,889 & -10.8 & -19.0 & -7.8 \\
\hline 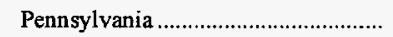 & 7,880 & 9,244 & 11,000 & 11,110 & 14,866 & 15,034 & -14.8 & -14.7 & -6.9 \\
\hline East North Central Total ...................... & 27,626 & 30,505 & 32,088 & 27,296 & 40,250 & 45,855 & -9.4 & -9.0 & -5.5 \\
\hline Illinois & 4,581 & 5,331 & 4,526 & 4,019 & 7,399 & 8,861 & -14.1 & -11.3 & -7.1 \\
\hline Indiana & 7,105 & 8,435 & 10,449 & 6,935 & 11,294 & $\cdot 13,185$ & -15.8 & -10.9 & -6.6 \\
\hline 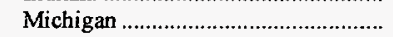 & 6,531 & 7,708 & 6,505 & 6,206 & 7,402 & 9,751 & -15.3 & -3.1 & -4.3 \\
\hline Ohio & 5,232 & 5,661 & 7,499 & 7,249 & 10,395 & 9,959 & -7.6 & -15.8 & -6.9 \\
\hline Wisconsin & 4,177 & 3,371 & 3,109 & 2,887 & 3,760 & 4,099 & 23.9 & 2.7 & .2 \\
\hline West North Central Total ...................... & 17,115 & 17,732 & 16,739 & 14,123 & 19,712 & 21,904 & -3.5 & -3.5 & -2.7 \\
\hline Iowa & 4,044 & 3,923 & 3,642 & 3,401 & 4,301 & 4,507 & 3.1 & -1.5 & -1.2 \\
\hline Kansas & 2,970 & 3,850 & 2,610 & 2,008 & 2,747 & 3,286 & -22.9 & 2.0 & -1.1 \\
\hline Minnesota & 1,462 & 1,898 & 2,134 & 1,182 & 2,175 & 3,346 & -23.0 & -9.5 & -8.8 \\
\hline 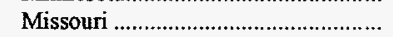 & 5,162 & 4,641 & 4,410 & 3,555 & 6,211 & 4,958 & 11.2 & -4.5 & .4 \\
\hline 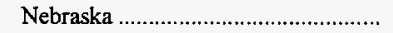 & 1,692 & 1,409 & 1,276 & 1,272 & 1,798 & 1,880 & 20.1 & -1.5 & -1.2 \\
\hline 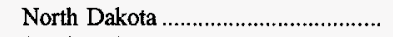 & 1,642 & 1,858 & 2,406 & 2,417 & 2,194 & 3,586 & -11.6 & -7.0 & -8.3 \\
\hline 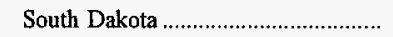 & 143 & 153 & 259 & 287 & 285 & 341 & -6.5 & -15.8 & -9.2 \\
\hline South Atlantic Total............................... & 18,669 & 18,851 & 23,226 & 17,877 & 27,977 & 29,878 & -1.0 & -9.6 & -5.1 \\
\hline Delaware & 322 & 363 & 470 & 192 & 361 & 438 & -11.3 & -2.8 & -3.3 \\
\hline Florida & 3,350 & 3,204 & 3,813 & 3,451 & 4,021 & 3,196 & 4.5 & -4.5 & 5 \\
\hline 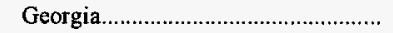 & 3,727 & 3,657 & 4,699 & 2,825 & 4,692 & 6,505 & 1.9 & -5.6 & -6.0 \\
\hline 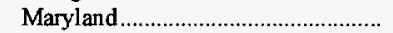 & 1,347 & 1,038 & 1,306 & 1,455 & 2,400 & 1,604 & 29.8 & -13.4 & -1.9 \\
\hline North Carolina ........................................ & 2,560 & 2,715 & 4,139 & 2,887 & 4,512 & 4,465 & -5.7 & -13.2 & -6.0 \\
\hline 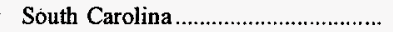 & 1,980 & 2,033 & 2,255 & 1,648 & 2,206 & 1,968 & -2.6 & -2.6 & .1 \\
\hline 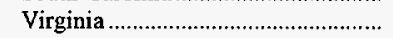 & 1,010 & 1,098 & 2,064 & 1,418 & 1,922 & 2,300 & -7.9 & -14.8 & -8.7 \\
\hline 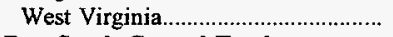 & 4,372 & 4,744 & 4,479 & 4,001 & 7,863 & 9,401 & -7.8 & -13.6 & -8.1 \\
\hline East South Central Total................... & 8,518 & 10,148 & 10,317 & 8,370 & 13,401 & 18,183 & -16.0 & -10.7 & -8.1 \\
\hline Alabama & 2,528 & 3,282 & 3,652 & 2,331 & 4,071 & 4,450 & -23.0 & -11.2 & $-6,1$ \\
\hline 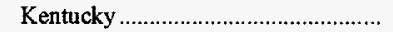 & 4,121 & 4,472 & 4,466 & 3,990 & 5,415 & 7,679 & -7.8 & -6.6 & -6.7 \\
\hline 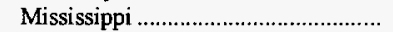 & 603 & 724 & 690 & 417 & 899 & 837 & -16.8 & -9.5 & -3.6 \\
\hline Tennessee & 1,267 & 1,670 & 1,509 & 1,632 & 3,016 & 5,217 & -24.1 & -19.5 & -14.5 \\
\hline West South Central Total ...................... & 19,533 & 20,195 & 15,520 & 13,867 & 16,483 & 17,757 & -3.3 & 4.3 & 1.1 \\
\hline 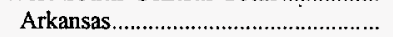 & 2,702 & 2,790 & 1,751 & 1,866 & 1,572 & 2,329 & -3.2 & 14.5 & 1.7 \\
\hline 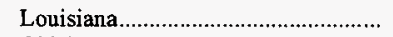 & 2,472 & 2,659 & 1,872 & 1,932 & 1,701 & 2,803 & -7.0 & 9.8 & -1.4 \\
\hline Oklahoma & 4,069 & 4,118 & 2,319 & 1,944 & 3,066 & 3,634 & -1.2 & 7.3 & 1.3 \\
\hline 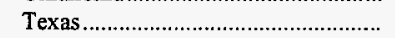 & 10,290 & 10,628 & 9,578 & 8,125 & 10,143 & 8,992 & -3.2 & .4 & 1.5 \\
\hline Mountain Total & 11,309 & 14,562 & 14,559 & 15,529 & 16,009 & 17,265 & -22.3 & -8.3 & -4.6 \\
\hline Arizona & 1,993 & 2,998 & 3,197 & 3,687 & 3,543 & 3,813 & -33.5 & -13.4 & -6.9 \\
\hline 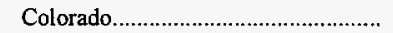 & 3,030 & 3,622 & 3,118 & 3,428 & 3,410 & 3,635 & -16.3 & -2.9 & -2.0 \\
\hline 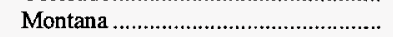 & 509 & 511 & 517 & 721 & 735 & 851 & -.5 & -8.8 & -5.6 \\
\hline Nevada & 1,240 & 1,356 & 1,034 & 1,195 & 1,447 & 1,782 & -8.6 & -3.8 & -3.9 \\
\hline 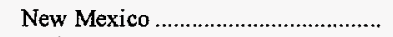 & 814 & 967 & 1,462 & 1,506 & 1,570 & 1,246 & -15.8 & -15.1 & -4.6 \\
\hline Utah & 1,526 & 2,250 & 2,753 & 3,264 & 3,153 & 3,133 & -32.2 & -16.6 & -7.7 \\
\hline Wyoming & 2,198 & 2,857 & 2,476 & 1,728 & 2,153 & 2,805 & -23.1 & .5 & -2.7 \\
\hline Pacific Total & 1,052 & 2,341 & 683 & 748 & 1,317 & 1,180 & $-\mathbf{5 5 . 0}$ & -5.4 & -1.3 \\
\hline 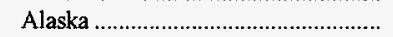 & 1 & 1 & 2 & 5 & 6 & 4 & - & -38.3 & -15.1 \\
\hline Oregon & 203 & 399 & 150 & 312 & 615 & 561 & -49.2 & -24.2 & -10.7 \\
\hline 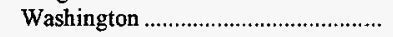 & 849 & 1,941 & 531 & 431 & 697 & 615 & -56.3 & 5.0 & 3.6 \\
\hline U.S. Total & 114,669 & 126,304 & 126,897 & 111,341 & 154,130 & 170,797 & -9.2 & -7.1 & -4.3 \\
\hline
\end{tabular}

* Data round to zero.

Note: Totals may not equal sum of components due to independent rounding.

Source: Energy Information Administration, Form EIA-759, "Monthly Power Plant Report." 
Table 71. Coal Consumption at Other Industrial Plants by Census Division and State, 1987, 1992-1996

(Thousand Short Tons)

\begin{tabular}{|c|c|c|c|c|c|c|c|c|c|}
\hline \multirow{2}{*}{ Census Division and State } & \multirow{2}{*}{1996} & \multirow{2}{*}{1995} & \multirow{2}{*}{1994} & \multirow{2}{*}{1993} & \multirow{2}{*}{1992} & \multirow{2}{*}{1987} & \multirow{2}{*}{$\begin{array}{c}\text { Percent } \\
\text { Change } \\
1995-1996\end{array}$} & \multicolumn{2}{|c|}{ Average Annual Percent Change } \\
\hline & & & & & & & & 1992-1996 & $1987-1996$ \\
\hline 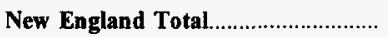 & 268 & 321 & 553 & 647 & 1,045 & 398 & -16.4 & -28.8 & -4.3 \\
\hline 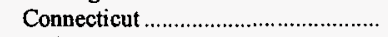 & - & w & $w$ & w & $w$ & w & w & w & $w$ \\
\hline 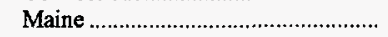 & $w$ & $\mathbf{w}$ & $w$ & w & $\mathbf{w}$ & $w$ & w & $w$ & $w$ \\
\hline 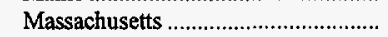 & $w$ & $\mathbf{w}$ & $w$ & $\mathbf{w}$ & $\mathbf{w}$ & $\mathbf{w}$ & $\mathbf{w}$ & $\mathbf{w}$ & w \\
\hline New Hampshire .................................. & - & w & - & w & $\mathbf{w}$ & $\mathbf{w}$ & $w$ & $w$ & $\mathbf{w}$ \\
\hline 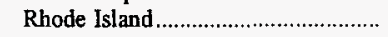 & - & - & - & w & - & $\mathbf{w}$ & $w$ & $w$ & $\mathbf{w}$ \\
\hline Vermont & - & - & - & w & $\mathbf{w}$ & $\mathbf{w}$ & w & $w$ & $w$ \\
\hline 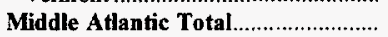 & $\mathbf{w}$ & $\mathbf{w}$ & $\mathbf{w}$ & $\mathbf{w}$ & $\mathbf{w}$ & $\mathbf{w}$ & $\mathbf{w}$ & $\mathbf{w}$ & $\mathbf{w}$ \\
\hline 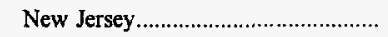 & w & $w$ & w & w & $w$ & w & w & w & w \\
\hline New York & 1,449 & 1,408 & 1,559 & 1,704 & 1,610 & 1,823 & 2.9 & -2.6 & -2.5 \\
\hline Pennsylvania & 4,466 & 4,027 & 4,044 & 4,311 & 4,173 & 4,112 & 10.9 & 1.7 & .9 \\
\hline East North Central Total .................. & 17,113 & 16,566 & 17,098 & 17,699 & 16,931 & 20,380 & 3.3 & .3 & -1.9 \\
\hline 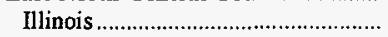 & 3,740 & 3,653 & 4,187 & 3,970 & 3,736 & 3,900 & 2.4 & $*$ & -.5 \\
\hline Indiana & 4,987 & 4,373 & 4,244 & 4,587 & 4,263 & 5,016 & 14.0 & 4.0 & -1 \\
\hline Michigan & 2,914 & 2,983 & 2,890 & 3,230 & 3,127 & 3,826 & -2.3 & -1.7 & -3.0 \\
\hline 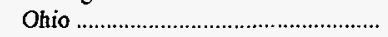 & 3,794 & 3,609 & 3,794 & 4,100 & 3,970 & 5,657 & 5.1 & -1.1 & -4.3 \\
\hline Wisconsin & 1,678 & 1,949 & 1,984 & 1,811 & 1,835 & 1,980 & -13.9 & -2.2 & -1.8 \\
\hline West North Central Total ..................... & 13,415 & 13,581 & 13,238 & 12,753 & 12,505 & 11,479 & -1.2 & 1.8 & 1.7 \\
\hline Jowa & 3,085 & 2,761 & 2,735 & 2,494 & 2,571 & 1,857 & 11.7 & 4.7 & 5.8 \\
\hline 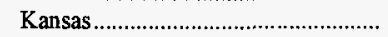 & 154 & 138 & 137 & 137 & 158 & 252 & 11.6 & -.7 & -5.3 \\
\hline Minnesota & 1,649 & 1,401 & 1,455 & 1,370 & 1,059 & 838 & 17.7 & 11.7 & 7.8 \\
\hline 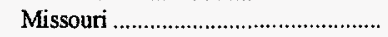 & 1,118 & 1,102 & 1,070 & 1,177 & 1,137 & 1,427 & 1.5 & -.4 & -2.7 \\
\hline Nebraska & w & $\mathbf{w}$ & w & $\mathbf{w}$ & w & $\mathbf{w}$ & $w$ & w & w \\
\hline 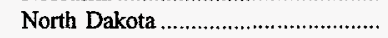 & w & w & $w$ & $\mathrm{w}$ & $\mathbf{w}$ & w & w & w & $\mathbf{w}$ \\
\hline 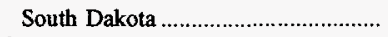 & $w$ & $w$ & $\mathbf{w}$ & $\mathbf{w}$ & $\mathbf{w}$ & $\mathbf{w}$ & $\mathbf{w}$ & $\mathbf{w}$ & $\mathbf{w}$ \\
\hline South Atlantic Total .............................. & $w$ & $\mathbf{w}$ & $\mathbf{w}$ & $\mathbf{w}$ & $\mathbf{w}$ & $\mathbf{w}$ & $\mathbf{w}$ & $\mathbf{w}$ & $\mathbf{w}$ \\
\hline 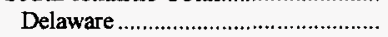 & w & w & w & w & $w$ & $\mathbf{w}$ & w & w & w \\
\hline Florida & 1,270 & 1,325 & 1,303 & 1,307 & 1,335 & 993 & -4.1 & -1.2 & 2.8 \\
\hline 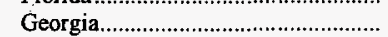 & 1,985 & 1,949 & 1,933 & 1,720 & 1,787 & 1,960 & 1.8 & 2.7 & .1 \\
\hline 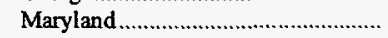 & 785 & 760 & 738 & 731 & 706 & 972 & 3.2 & 2.7 & -2.3 \\
\hline North Carolina & 2,336 & 2,437 & 2,396 & 2,476 & 2,860 & 2,548 & -4.1 & -4.9 & -1.0 \\
\hline 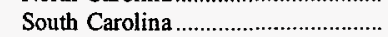 & 2,000 & 2,188 & 2,334 & 2,395 & 2,177 & 2,562 & -8.6 & -2.1 & -2.7 \\
\hline 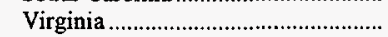 & 2,613 & 2,585 & 2,838 & 2,863 & 3,592 & 3,662 & 1.1 & -7.6 & -3.7 \\
\hline 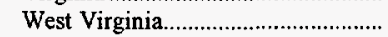 & 1,630 & 1,984 & 2,637 & 2,406 & 2,266 & 2,326 & -17.8 & -7.9 & -3.9 \\
\hline East South Central Total....................... & $\mathbf{w}$ & $\mathbf{w}$ & $w$ & $\mathbf{w}$ & $\mathbf{w}$ & $\mathbf{w}$ & $\mathbf{w}$ & $\mathbf{w}$ & $\mathbf{w}$ \\
\hline 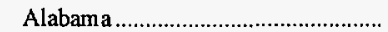 & 2,545 & 2,286 & 2,394 & 2,268 & 3,136 & 2,614 & 11.3 & -5.1 & -.3 \\
\hline 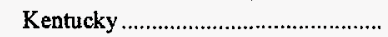 & 2,322 & 2,250 & 1,994 & 2,392 & 1,648 & 1,869 & 3.2 & 8.9 & 2.4 \\
\hline 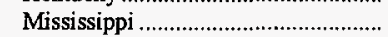 & $w$ & $w$ & $\mathbf{w}$ & W & $W$ & $w$ & w & w & w \\
\hline Tennessee & 3,670 & 3,777 & 4,097 & 3,942 & 3,686 & 3,842 & -2.8 & -.1 & -.5 \\
\hline West South Central Total .................... & 5,978 & 6,456 & 7,082 & 6,780 & 5,846 & $\mathbf{w}$ & -7.4 & .5 & $\mathbf{w}$ \\
\hline 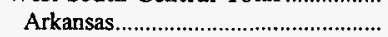 & 348 & 325 & 346 & 330 & 295 & 302 & 7.2 & 4.3 & 1.6 \\
\hline 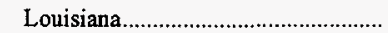 & $w$ & w & w & $w$ & $w$ & w & w & $\mathbf{w}$ & w \\
\hline 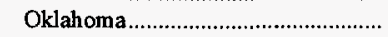 & w & w & w & w & w & 613 & w & $w$ & $\mathrm{w}$ \\
\hline 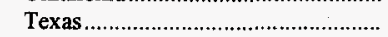 & 4,808 & 4,255 & 5,350 & 4,667 & 4,225 & 4,083 & 13.0 & 3.3 & 1.8 \\
\hline 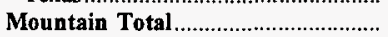 & 4,141 & 5,615 & 5,614 & 5,163 & 4,973 & 4,634 & -26.3 & -4.5 & -1.2 \\
\hline 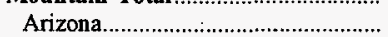 & 675 & 657 & 727 & 674 & 632 & 669 & 2.8 & 1.7 & .1 \\
\hline 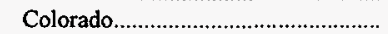 & 367 & 729 & 857 & 780 & 735 & 748 & -49.6 & -15.9 & -7.6 \\
\hline Idaho & 369 & 426 & 494 & 486 & 484 & 430 & -13.3 & -6.5 & -2.7 \\
\hline Montana & w & w & w & $w$ & $w$ & $\mathbf{w}$ & $w$ & $w$ & $w$ \\
\hline Nevada & w & $w$ & $\mathbf{w}$ & $w$ & $\mathbf{w}$ & $\mathbf{w}$ & $\mathbf{w}$ & $\mathrm{w}$ & $\mathrm{w}$ \\
\hline New Mexico & $\mathbf{w}$ & $\mathbf{w}$ & $\mathbf{w}$ & $\mathbf{w}$ & $\mathbf{w}$ & w & $\mathbf{w}$ & w & w \\
\hline 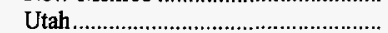 & 512 & 915 & 835 & 727 & 525 & 507 & -44.1 & -.6 & .1 \\
\hline Wyoming & 1,835 & 1,937 & 1,867 & 1,873 & 2,126 & 1,887 & -5.3 & -3.6 & -3 \\
\hline Pacific Total & 2,553 & 3,047 & 2,769 & 2,677 & 3,161 & 2,496 & -16.2 & -5.2 & $\mathbf{3}$ \\
\hline 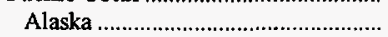 & $w$ & - & 5 & 2 & - & - & w & w & $w$ \\
\hline Califomia & 2,140 & 2,485 & 2,332 & 2,311 & 2,821 & 1,933 & -13.9 & -6.7 & 1.1 \\
\hline Hawaii & w & w & $\mathrm{w}$ & $w$ & $\mathrm{w}$ & $w$ & W & w & w \\
\hline Oregon & w & w & w & w & $\mathrm{w}$ & $\mathrm{w}$ & w & $w$ & $w$ \\
\hline 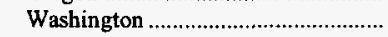 & 152 & 223 & 201 & 174 & 163 & 298 & -31.9 & -1.8 & -7.2 \\
\hline U.S. Total & 70,941 & 73,055 & 75,179 & 74,892 & 74,042 & 75,175 & -2.9 & -1.1 & -.6 \\
\hline
\end{tabular}

* Data round to zero.

Withheld to avoid disclosure of individual company data.

Note: Totals may not equal sum of components due to independent rounding. bution Report."

Sources: Energy Information Administration, Form ElA-3, "Quarterly Coal Consumption Report - Manufacturing Plants"; and Form ElA-6, "Coal Distri- 
Table 72. Year-End Coal Stocks at Other Industrial Plants by Census Division and State, 1987, 1992-1996

(Thousand Short Tons)

\begin{tabular}{|c|c|c|c|c|c|c|c|c|c|}
\hline \multirow{2}{*}{ Census Division and State } & \multirow{2}{*}{1996} & \multirow{2}{*}{1995} & \multirow{2}{*}{1994} & \multirow{2}{*}{1993} & \multirow{2}{*}{1992} & \multirow{2}{*}{1987} & \multirow{2}{*}{$\begin{array}{c}\text { Percent } \\
\text { Change } \\
\text { 1995-1996 }\end{array}$} & \multicolumn{2}{|c|}{ Average Annual Percent Change } \\
\hline & & & & & & & & 1992-1996 & 1987-1996 \\
\hline New England Total............................... & 60 & 60 & 38 & 21 & 16 & 44 & * & 39.7 & 3.5 \\
\hline 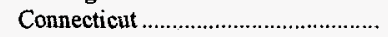 & - & - & - & - & - & $\mathbf{w}$ & $w$ & $w$ & w \\
\hline Maine & $w$ & $\mathbf{w}$ & $w$ & $\mathbf{w}$ & $w$ & $w$ & w & $w$ & $\mathbf{w}$ \\
\hline 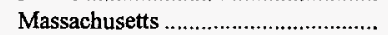 & $\mathrm{w}$ & $\mathrm{w}$ & $w$ & $w$ & $\mathbf{w}$ & $w$ & $w$ & $w$ & $w$ \\
\hline 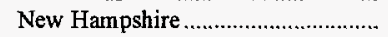 & - & - & - & - & - & $w$ & $w$ & $\mathbf{w}$ & $\mathbf{w}$ \\
\hline 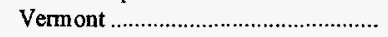 & - & - & - & - & - & w & $\mathrm{w}$ & w & $\mathbf{w}$ \\
\hline Middle Atlantic Total............................ & $\mathbf{w}$ & $\mathbf{w}$ & $\mathbf{w}$ & $\mathbf{w}$ & $\mathbf{w}$ & $\mathbf{w}$ & $\mathbf{w}$ & $\mathbf{w}$ & $\mathbf{w}$ \\
\hline 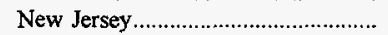 & $\mathbf{w}$ & $\mathbf{w}$ & w & $\mathbf{w}$ & w & $w$ & $\mathbf{w}$ & $\mathbf{w}$ & $\mathbf{w}$ \\
\hline 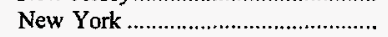 & 192 & 203 & 250 & 321 & 402 & 452 & -5.4 & -16.8 & -9.1 \\
\hline Pennsylvania & 231 & 218 & 298 & 287 & 306 & 465 & 6.2 & -6.7 & -7.4 \\
\hline 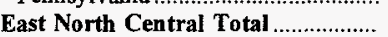 & 1,862 & 2,031 & 2,462 & 2,044 & 2,505 & 3,884 & -8.3 & -7.1 & -7.8 \\
\hline 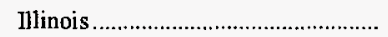 & 252 & 333 & 426 & 368 & 389 & 598 & -24.2 & -10.2 & -9.1 \\
\hline Indiana & 384 & 451 & 690 & 470 & 732 & 902 & -14.9 & -14.9 & -9.0 \\
\hline Michigan & 827 & 822 & 865 & 702 & 882 & 1,387 & .5 & -1.6 & -5.6 \\
\hline Ohio & 118 & 138 & 153 & 198 & 228 & 445 & -14.9 & -15.2 & -13.7 \\
\hline 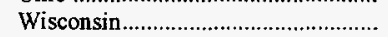 & 281 & 286 & 328 & 306 & 274 & 553 & -1.8 & .6 & -7.3 \\
\hline West North Central Total .................. & 1,220 & 981 & 978 & 775 & 1,020 & 1,196 & 24.3 & 4.6 & .2 \\
\hline Iowa & 570 & 524 & 535 & 418 & 556 & 587 & 8.7 & .6 & -.3 \\
\hline 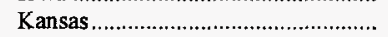 & 16 & 10 & 13 & 16 & 11 & 41 & 64.6 & 8.6 & -9.9 \\
\hline 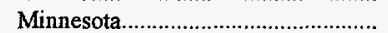 & 277 & 87 & 99 & 68 & 77 & 81 & 218.1 & 37.9 & 14.7 \\
\hline 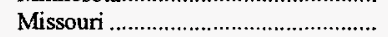 & 158 & 138 & 159 & 148 & 227 & 293 & 14.5 & -8.7 & -6.6 \\
\hline 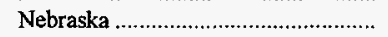 & w & $w$ & $w$ & $w$ & w & w & w & w & $w$ \\
\hline 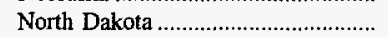 & w & w & w & $w$ & $w$ & $\mathbf{w}$ & $w$ & $\mathbf{w}$ & $w$ \\
\hline South Dakota & w & $w$ & $w$ & w & $w$ & $w$ & $w$ & $w$ & $w$ \\
\hline South Atlantic Total.............................. & $\mathbf{w}$ & $\mathbf{w}$ & $\mathbf{w}$ & $\mathbf{w}$ & $\mathbf{w}$ & $\mathbf{w}$ & $\mathbf{w}$ & $\mathbf{w}$ & $\mathbf{w}$ \\
\hline Delaware & $w$ & $\mathbf{w}$ & w & $w$ & $\mathbf{w}$ & w & $w$ & w & $\mathrm{w}$ \\
\hline Florida & 89 & 64 & 101 & 90 & 100 & 102 & 40.2 & -2.7 & -1.4 \\
\hline 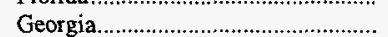 & 121 & 129 & 144 & 101 & 144 & 207 & -6.3 & -4.3 & -5.8 \\
\hline 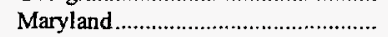 & 30 & 24 & 36 & 41 & 35 & 49 & 27.1 & -3.7 & -5.1 \\
\hline North Carolina...................................... & 112 & 140 & 179 & 172 & 203 & 329 & -19.8 & -13.7 & -11.3 \\
\hline South Carolina & 198 & 160 & 278 & 245 & 245 & 409 & 23.5 & -5.2 & -7.7 \\
\hline Virginia & 133 & 177 & 217 & 216 & 275 & 440 & -25.0 & -16.6 & -12.4 \\
\hline 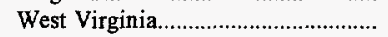 & 136 & 105 & 130 & 167 & 156 & 268 & 30.0 & -3.3 & -7.3 \\
\hline East South Central Total...................... & $\mathbf{w}$ & $\mathbf{w}$ & $\mathbf{w}$ & $\mathbf{w}$ & $\mathbf{w}$ & $\mathbf{w}$ & $\mathbf{w}$ & $\mathbf{w}$ & $\mathbf{w}$ \\
\hline Alabama & 135 & 133 & 183 & 132 & 127 & 183 & 1.2 & 1.6 & -3.3 \\
\hline 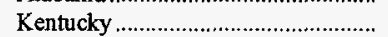 & 83 & 120 & 112 & 73 & 127 & 178 & -30.3 & -10.0 & -8.1 \\
\hline 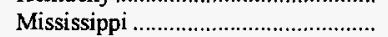 & $\mathbf{w}$ & w & w & w & $w$ & w & $w$ & w & w \\
\hline Tennessee & 234 & 215 & 256 & 246 & 270 & 434 & 9.0 & -3.5 & -6.6 \\
\hline West South Central Total .................. & 361 & 370 & 439 & 1,218 & 395 & 1,419 & -2.3 & -2.2 & -14.1 \\
\hline Arkansas. & 18 & 29 & 26 & 15 & 19 & 30 & -37.9 & -1.2 & -5.5 \\
\hline 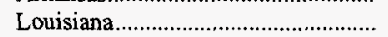 & $w$ & $w$ & w & $w$ & $w$ & 18 & w & w & $w$ \\
\hline Okdahoma & w & w & $\mathbf{w}$ & $\mathbf{w}$ & w & 154 & w & w & $\mathbf{w}$ \\
\hline 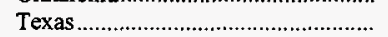 & 190 & 201 & 215 & 1,026 & 228 & 1,218 & -5.6 & -4.4 & -18.6 \\
\hline Mountain Total & 231 & 313 & 267 & 332 & 360 & 324 & -26.2 & -10.4 & -3.7 \\
\hline 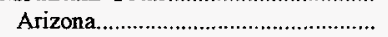 & 32 & 34 & 45 & 30 & 53 & 26 & -5.8 & -11.7 & 2.3 \\
\hline 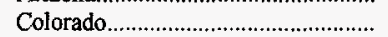 & 27 & 59 & 26 & 25 & 29 & 56 & -54.2 & -1.6 & -7.7 \\
\hline Idaho & $w$ & 118 & 78 & 86 & 101 & 116 & w & w & w \\
\hline 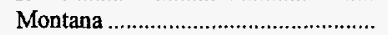 & $w$ & w & $w$ & $\mathbf{w}$ & $w$ & w & $\mathbf{w}$ & $w$ & $\mathbf{w}$ \\
\hline Nevada & $w$ & $\mathbf{w}$ & $w$ & $w$ & $\mathrm{w}$ & $w$ & $w$ & $w$ & $\mathbf{w}$ \\
\hline 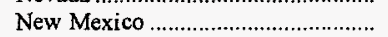 & $w$ & $\mathbf{w}$ & $w$ & $w$ & $w$ & $w$ & w & $\mathbf{w}$ & w \\
\hline Utah & 5 & 7 & 13 & 20 & 26 & 39 & -19.6 & -32.3 & -19.8 \\
\hline 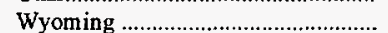 & 71 & 79 & 77 & 113 & 89 & 62 & -10.7 & -5.6 & 1.5 \\
\hline Pacific Total & 222 & 245 & 194 & 170 & 211 & 290 & -9.2 & 1.3 & -2.9 \\
\hline California & 150 & 133 & 126 & 93 & 109 & 174 & 12.4 & 8.3 & -1.6 \\
\hline Hawaii & $w$ & w & $\mathrm{w}$ & $w$ & w & w & $w$ & $w$ & w \\
\hline Oregon & $w$ & $w$ & $\mathbf{w}$ & $w$ & $w$ & $\mathrm{w}$ & w & w & w \\
\hline Washington & 8 & 28 & 38 & 27 & 39 & 66 & -69.2 & -31.5 & -20.4 \\
\hline 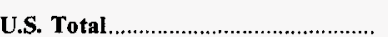 & 5,688 & 5,702 & 6,585 & 6,716 & 6,965 & 10,777 & -.2 & -4.9 & -6.8 \\
\hline
\end{tabular}

- Data round to zero.

w Witheld to avoid disclosure of individual company data.

Notes: Other industrial plants include manufacturing plants only. Totals may not equal sum of components due to independent rounding

Source: Energy Information Administration, Form EIA-3, "Quarterly Coal Consumption Report - Manufacturing Plants." 
Table 73. Coal Carbonized at Coke Plants by Census Division and State, 1987, 1992-1996

(Thousand Short Tons)

\begin{tabular}{|c|c|c|c|c|c|c|c|c|c|}
\hline \multirow{2}{*}{ Census Division and State } & \multirow{2}{*}{1996} & \multirow{2}{*}{1994} & \multirow{2}{*}{1994} & \multirow{2}{*}{1993} & \multirow{2}{*}{1992} & \multirow{2}{*}{1987} & \multirow{2}{*}{$\begin{array}{c}\text { Percent } \\
\text { Change } \\
1995-1996\end{array}$} & \multicolumn{2}{|c|}{ Average Annual Percent Change } \\
\hline & & & & & & & & $1992-1996$ & $1987-1996$ \\
\hline Middle Atlantic Total & $\mathbf{w}$ & $\mathbf{w}$ & $\mathbf{w}$ & $\mathbf{w}$ & $\mathbf{w}$ & $\mathbf{w}$ & $\mathbf{w}$ & $\mathbf{w}$ & $\mathbf{w}$ \\
\hline 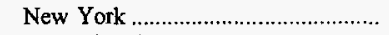 & $\mathrm{w}$ & $\mathbf{w}$ & w & $w$ & w & w & w & $\mathbf{w}$ & $\mathbf{w}$ \\
\hline 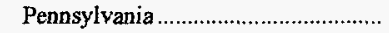 & 10,689 & 10,858 & 10,849 & 10,333 & 9,868 & 8,395 & -1.5 & 2.0 & 2.7 \\
\hline East North Central Total .................... & 11,414 & 12,345 & 11,356 & 11,643 & 13,224 & 17,782 & -7.5 & -3.6 & -4.8 \\
\hline 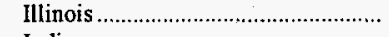 & w & w & w & w & $w$ & $w$ & w & w & w \\
\hline Indiana & 5,823 & 5,883 & 4,841 & 6,591 & 7,153 & 8,841 & -1.0 & -5.0 & -4.5 \\
\hline 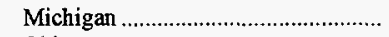 & w & w & w & - & - & $\mathbf{w}$ & $\mathbf{w}$ & $\mathbf{w}$ & $\mathbf{w}$ \\
\hline Ohio & 1,842 & 2,777 & 3,092 & 2,892 & 3,755 & 5,361 & -33.7 & -16.3 & -11.2 \\
\hline West North Central Total ..................... & - & - & - & - & - & $\mathbf{w}$ & - & $\mathbf{w}$ & $\mathbf{w}$ \\
\hline 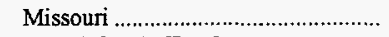 & - & - & - & - & - & $\mathbf{w}$ & - & $\mathbf{w}$ & $\mathbf{w}$ \\
\hline South Atlantic Total........................... & $\mathbf{w}$ & $\mathbf{w}$ & $\mathbf{w}$ & $\mathbf{w}$ & $\mathbf{w}$ & $\mathbf{w}$ & $\mathbf{w}$ & $\mathbf{w}$ & $\mathbf{w}$ \\
\hline 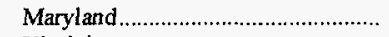 & - & - & - & - & - & $w$ & $w$ & $w$ & $\mathbf{w}$ \\
\hline 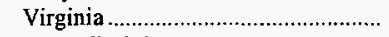 & w & w & w & w & $\mathbf{w}$ & $\mathbf{w}$ & $w$ & $\mathbf{w}$ & $\mathbf{w}$ \\
\hline West Virginia & $w$ & $w$ & w & $\mathbf{w}$ & $w$ & $\mathbf{w}$ & w & $\mathbf{w}$ & w \\
\hline East South Central Total & $\mathbf{w}$ & $\mathbf{w}$ & $\mathbf{w}$ & $\mathbf{w}$ & $w$ & $\mathbf{w}$ & $w$ & $\mathbf{w}$ & $\mathbf{w}$ \\
\hline 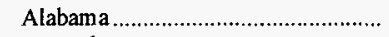 & 3,247 & $3 i, 257$ & 3,253 & 3,206 & 3,297 & 3,150 & -.3 & -.4 & .3 \\
\hline 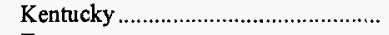 & $\mathbf{w}$ & w & $\mathbf{w}$ & w & w & $\mathbf{w}$ & w & $\mathbf{w}$ & $w$ \\
\hline 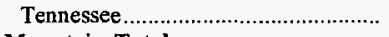 & - & - & - & - & - & w & - & $w$ & w \\
\hline Mountain Total & $\mathbf{w}$ & $\mathbf{w}$ & $\mathbf{w}$ & $\mathbf{w}$ & $\mathbf{w}$ & - & $\mathbf{w}$ & $\mathbf{w}$ & $\mathbf{w}$ \\
\hline Utah & w & w & $w$ & $w$ & $\mathbf{w}$ & - & $\mathbf{w}$ & $w$ & w \\
\hline 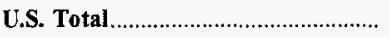 & 31,706 & 33,011 & 31,740 & 31,323 & 32,366 & 36,877 & -3.9 & -.5 & -1.7 \\
\hline
\end{tabular}

w Withbeld to avoid disclosure of individual company data

Note: Totals may not equal sum of components due to independent rounding

Source: Energy Information Administration, Form EIA-5, "Coke Plant Report - Quarterly."

Table 74. Year-End Coal Stocks at Coke Plants by Census Division and State, 1987, 1992-1996

(Thousand Short Tons)

\begin{tabular}{|c|c|c|c|c|c|c|c|c|c|}
\hline \multirow{2}{*}{ Census Division and State } & \multirow{2}{*}{1996} & \multirow{2}{*}{1995} & \multirow{2}{*}{1994} & \multirow{2}{*}{1993} & \multirow{2}{*}{1992} & \multirow{2}{*}{1987} & \multirow{2}{*}{$\begin{array}{c}\text { Percent } \\
\text { Change } \\
\text { 1995-1996 }\end{array}$} & \multicolumn{2}{|c|}{ Average Annual Percent Change } \\
\hline & & & & & & & & 1992-1996 & $1987-1996$ \\
\hline Middle Atlantic Total......................... & $\mathbf{w}$ & $\mathbf{w}$ & $\mathbf{w}$ & $\mathbf{w}$ & $\mathbf{w}$ & $\mathbf{w}$ & $\mathbf{w}$ & $\mathbf{w}$ & $\mathbf{w}$ \\
\hline New York & $w$ & $\mathbf{w}$ & $\mathbf{w}$ & w & w & w & w & w & w \\
\hline 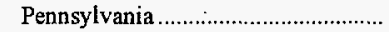 & 748 & 841 & 762 & 868 & 804 & 622 & -11.0 & -1.8 & 2.1 \\
\hline East North Central Total .................. & 1,335 & $1, \mathbf{2 8 2}$ & 1,282 & 822 & 906 & 2,095 & 4.1 & 10.2 & -4.9 \\
\hline 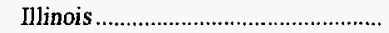 & $w$ & $w$ & $\mathbf{w}$ & $w$ & $w$ & $\mathbf{w}$ & $w$ & w & w \\
\hline Indiana & 469 & 412 & 567 & 394 & 481 & 912 & 13.7 & -.6 & -7.1 \\
\hline 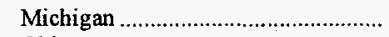 & w & w & w & - & - & w & w & $\mathbf{w}$ & w \\
\hline Ohio & 81 & 136 & 163 & 183 & 180 & 760 & -40.5 & -18.1 & -22.0 \\
\hline 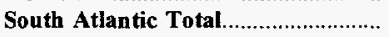 & $\mathbf{w}$ & $\mathbf{w}$ & $\mathbf{w}$ & $\mathbf{w}$ & $\mathbf{w}$ & $\mathbf{w}$ & $\mathbf{w}$ & $\mathbf{w}$ & $\mathbf{w}$ \\
\hline 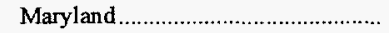 & - & - & - & - & - & $\mathbf{w}$ & w & w & w \\
\hline 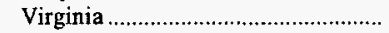 & - & - & - & - & $\mathbf{w}$ & - & $\mathbf{w}$ & $\mathbf{w}$ & $\mathbf{w}$ \\
\hline West Virginia & w & $\mathbf{w}$ & $\mathbf{w}$ & $\mathbf{w}$ & $\mathbf{w}$ & $\mathbf{w}$ & $\mathbf{w}$ & $\mathbf{w}$ & $\mathbf{w}$ \\
\hline East South Central Total....................... & $\mathbf{w}$ & $\mathbf{w}$ & $\mathbf{w}$ & $\mathbf{w}$ & $\mathbf{w}$ & $\mathbf{w}$ & $\mathbf{w}$ & $\mathbf{w}$ & $\mathbf{w}$ \\
\hline Alabama & 197 & 233 & 297 & 333 & 332 & 379 & -15.4 & -12.2 & -7.0 \\
\hline 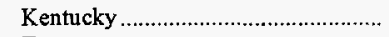 & w & w & $\mathbf{w}$ & $\mathbf{w}$ & $w$ & w & w & w & w \\
\hline 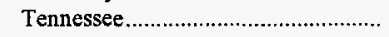 & - & - & - & - & - & w & $w$ & $\mathbf{w}$ & $w$ \\
\hline West South Central Total .................. & - & - & - & 21 & $\mathbf{w}$ & $\mathbf{w}$ & - & $\mathbf{w}$ & $\mathbf{w}$ \\
\hline 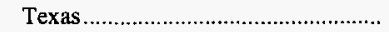 & - & - & - & 21 & $w$ & w & - & $\mathbf{w}$ & w \\
\hline 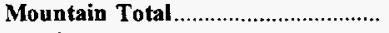 & $\mathbf{w}$ & $\mathbf{w}$ & $\mathbf{w}$ & $\mathbf{w}$ & $\mathbf{w}$ & - & $\mathbf{w}$ & $\mathbf{w}$ & $\mathbf{w}$ \\
\hline Utah & $\mathbf{w}$ & $\mathbf{w}$ & $w$ & w & w & - & $w$ & w & $\mathbf{w}$ \\
\hline 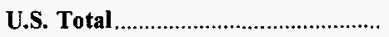 & 2,667 & 2,632 & 2,657 & 2,401 & 2,597 & 3,884 & 1.3 & .6 & -4.1 \\
\hline
\end{tabular}

withheld to avoid disclosure of individual company data

Note: Totals may not equal sum of components due to independent rounding.

Source: Energy Information Administration, Form EIA-5, "Coke Plant Report - Quarterly." 
Table 75. Coal Consumption by Residential and Commercial Sector, by Census Division and State, 1987, 1992-1996

(Thousand Short Tons)

\begin{tabular}{|c|c|c|c|c|c|c|c|c|c|}
\hline \multirow{2}{*}{ Census Division and State } & \multirow{2}{*}{1996} & \multirow{2}{*}{1995} & \multirow{2}{*}{1994} & \multirow{2}{*}{1993} & \multirow{2}{*}{1992} & \multirow{2}{*}{$198 ?$} & \multirow{2}{*}{$\begin{array}{c}\text { Percent } \\
\text { Change } \\
\text { 1995-1996 }\end{array}$} & \multicolumn{2}{|c|}{ Average Annual Percent Change } \\
\hline & & & & & & & & 1992-1996 & $1987-1996$ \\
\hline New England Total.............................. & 55 & 69 & 56 & 102 & 141 & 152 & -20.3 & -20.8 & -10.6 \\
\hline 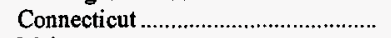 & $w$ & w & $w$ & w & w & $w$ & w & w & w \\
\hline Maine & $w$ & w & $w$ & w & $w$ & w & $w$ & w & w \\
\hline 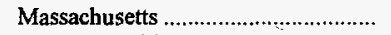 & $w$ & $w$ & $w$ & w & $w$ & w & $\mathbf{w}$ & w & w \\
\hline 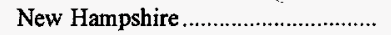 & $w$ & $\mathbf{w}$ & $w$ & $w$ & $\mathbf{w}$ & w & w & $\mathbf{w}$ & $w$ \\
\hline 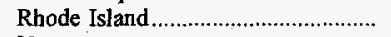 & $w$ & $w$ & w & w & $w$ & w & $w$ & $w$ & w \\
\hline Verm ont & w & $\mathbf{w}$ & $\mathbf{w}$ & $\mathbf{w}$ & w & $w$ & w & w & $\mathbf{w}$ \\
\hline Middle Atlantic Total........................... & 1,285 & 1,416 & 1,351 & 1,498 & 1,916 & 1,935 & -9.2 & -9.5 & -4.4 \\
\hline 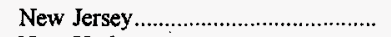 & w & $w$ & w & w & $w$ & $\mathbf{w}$ & $w$ & $w$ & w \\
\hline 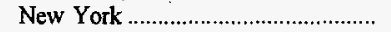 & $w$ & $w$ & $w$ & $w$ & w & $\mathbf{w}$ & w & w & w \\
\hline 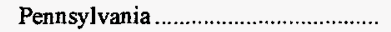 & 995 & 1,188 & 1,156 & 1,257 & 1,626 & 1,535 & -16.3 & -11.6 & -4.7 \\
\hline East North Central Total .................... & 1,574 & 1,301 & 1,452 & 1,458 & 1,476 & 1,838 & 21.0 & 1.6 & -1.7 \\
\hline 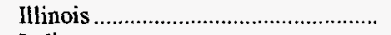 & w & $\mathbf{w}$ & $\mathbf{w}$ & w & w & w & w & $\mathbf{w}$ & w \\
\hline Indiana & 356 & 287 & 356 & 339 & 411 & 541 & 24.3 & -3.5 & -4.5 \\
\hline 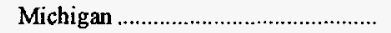 & $\mathbf{w}$ & $\mathbf{w}$ & w & w & $\mathbf{w}$ & $w$ & w & w & w \\
\hline Ohio & 656 & 409 & 498 & 584 & 588 & 812 & 60.4 & 2.8 & -2.3 \\
\hline 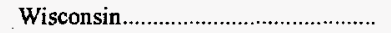 & w & $\mathbf{w}$ & w & w & $w$ & w & $w$ & $w$ & $\mathbf{w}$ \\
\hline West North Central Total .................. & $\mathbf{w}$ & $\mathbf{w}$ & $\mathbf{w}$ & $\mathbf{w}$ & $\mathbf{w}$ & $\mathbf{w}$ & $\mathbf{w}$ & $\mathbf{w}$ & $\mathbf{w}$ \\
\hline lowa & 222 & 90 & 40 & 70 & 64 & 337 & 147.0 & 36.5 & -4.5 \\
\hline 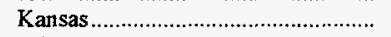 & 78 & 38 & 32 & 23 & $*$ & 1 & 107.2 & 359.7 & 66.2 \\
\hline 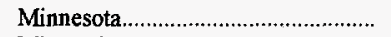 & 156 & 264 & 229 & 107 & 25 & 171 & -40.9 & 58.4 & -1.0 \\
\hline 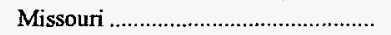 & $w$ & w & w & w & $w$ & w & $\mathbf{w}$ & w & $w$ \\
\hline 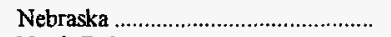 & w & $\mathbf{w}$ & $w$ & $w$ & $w$ & w & $w$ & w & $w$ \\
\hline North Dakota & w & $\mathbf{w}$ & $\mathbf{w}$ & w & w & $w$ & $w$ & $\mathbf{w}$ & $\mathbf{w}$ \\
\hline 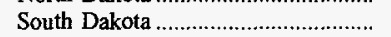 & $w$ & w & w & $w$ & $w$ & w & $\mathbf{w}$ & $\mathrm{w}$ & $\mathbf{w}$ \\
\hline 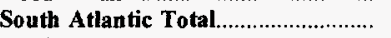 & 803 & 954 & 887 & 904 & 632 & 1,107 & -15.9 & 6.2 & -3.5 \\
\hline 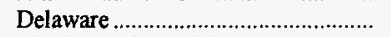 & $w$ & $w$ & $w$ & $w$ & w & w & $\mathrm{NM}$ & w & $w$ \\
\hline 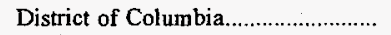 & 23 & 6 & 47 & 51 & 50 & 70 & 309.0 & -17.6 & -11.5 \\
\hline 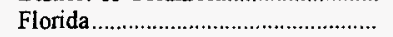 & 1 & 1 & 20 & 16 & 16 & 53 & -24.2 & -50.5 & -35.8 \\
\hline Georgia & 3 & 59 & 28 & 22 & 38 & 37 & -94.8 & -46.9 & -24.2 \\
\hline Maryland & w & $w$ & w & $w$ & $\mathbf{w}$ & $w$ & $w$ & w & $\mathbf{w}$ \\
\hline North Carolina ....................................... & 206 & 224 & 263 & 229 & 204 & 162 & -8.1 & .2 & 2.7 \\
\hline 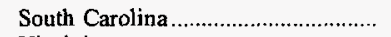 & 19 & 17 & 61 & 109 & 31 & 120 & 12.2 & -11.3 & -18.6 \\
\hline Virginia & $w$ & $\mathbf{w}$ & $w$ & w & $w$ & $\mathbf{w}$ & $w$ & w & $w$ \\
\hline 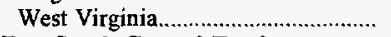 & w & w & w & w & w & w & w & w & $w$ \\
\hline East South Central Total .................. & 272 & 283 & 386 & 417 & 458 & 488 & -3.8 & -12.2 & -6.3 \\
\hline 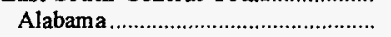 & 44 & 7 & 11 & 40 & 89 & 121 & NM & -16.0 & -10.7 \\
\hline 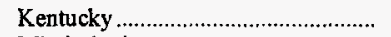 & $\mathrm{w}$ & $\mathbf{w}$ & $\mathbf{w}$ & w & w & $\mathbf{w}$ & $w$ & w & w \\
\hline 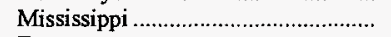 & - & - & - & $w$ & $\mathbf{w}$ & $w$ & $w$ & $w$ & $w$ \\
\hline 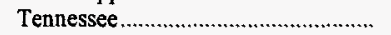 & w & $\mathbf{w}$ & w & $w$ & $w$ & $w$ & w & $\mathbf{w}$ & $\mathbf{w}$ \\
\hline West South Central Total .................. & $\mathbf{w}$ & 17 & 1 & 8 & 13 & 32 & $\mathbf{w}$ & $\mathbf{w}$ & $\mathbf{w}$ \\
\hline 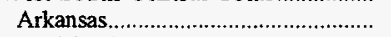 & - & - & * & 1 & 2 & $*$ & - & - & - \\
\hline 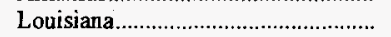 & - & $\mathbf{w}$ & - & $w$ & - & - & $w$ & $w$ & $w$ \\
\hline Oklahoma & w & w & w & w & 1 & 2 & $w$ & $\mathbf{w}$ & w \\
\hline 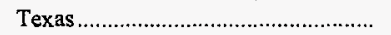 & - & - & $*$ & 6 & w & w & - & $\mathbf{w}$ & $\mathrm{w}$ \\
\hline 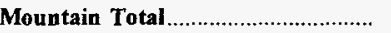 & $w$ & $\mathbf{w}$ & $w$ & $\mathbf{w}$ & $\mathbf{w}$ & $\mathbf{w}$ & $\mathbf{w}$ & $\mathbf{w}$ & $\mathbf{w}$ \\
\hline Arizona & $*$ & 5 & $*$ & 1 & $w$ & $\mathbf{w}$ & -96.3 & w & $\mathbf{w}$ \\
\hline 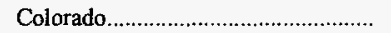 & 13 & 20 & 23 & 38 & $w$ & $w$ & -32.2 & $w$ & $w$ \\
\hline Idaho & 28 & 39 & 40 & 43 & 51 & 24 & -27.5 & -13.8 & 2.0 \\
\hline 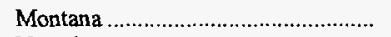 & $w$ & $w$ & $w$ & $\mathrm{w}$ & $w$ & $w$ & w & w & $w$ \\
\hline 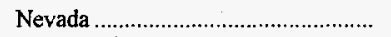 & w & $w$ & $w$ & w & $\mathrm{w}$ & $w$ & $w$ & $w$ & $w$ \\
\hline New Mexico .......................................... & $w$ & w & $w$ & $w$ & $\mathrm{w}$ & $w$ & $w$ & $w$ & $w$ \\
\hline Utah & $\mathbf{w}$ & $\mathbf{w}$ & $\mathbf{w}$ & w & $w$ & $w$ & w & $w$ & w \\
\hline 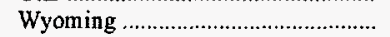 & 382 & 146 & 242 & 187 & w & $w$ & 162.0 & w & $w$ \\
\hline Pacific Total & 675 & 734 & 373 & 821 & $\mathbf{w}$ & $\mathbf{w}$ & -8.0 & $\mathbf{w}$ & $\mathbf{w}$ \\
\hline 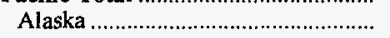 & 474 & 523 & 520 & 563 & $\mathrm{w}$ & $w$ & -9.3 & w & $\mathrm{w}$ \\
\hline 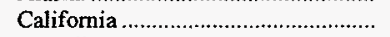 & 177 & 133 & 166 & 142 & $w$ & $w$ & 33.2 & $\mathbf{w}$ & $\mathrm{w}$ \\
\hline 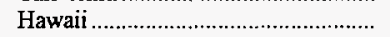 & w & $w$ & $w$ & w & $w$ & $w$ & $w$ & w & $w$ \\
\hline Oregon & $\mathbf{w}$ & $\mathbf{w}$ & $\mathrm{w}$ & $w$ & $w$ & $w$ & w & w & w \\
\hline 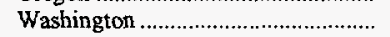 & 23 & 78 & 86 & 114 & 91 & 52 & -70.0 & -28.8 & -8.5 \\
\hline U.S. Total. & 6,006 & 5,807 & 6,013 & 6,221 & 6,153 & 6,914 & 3.4 & -.6 & -1.5 \\
\hline
\end{tabular}

* Data round to zero.

w Withheld to avoid disclosure of individual company data.

NM Not meaningful as value is greater than 500 percent.

Note: Totals may not equal sum of components due to independent rounding.

Source: Energy Information Administration, Form EIA-6, "Coal Distribution Report." 


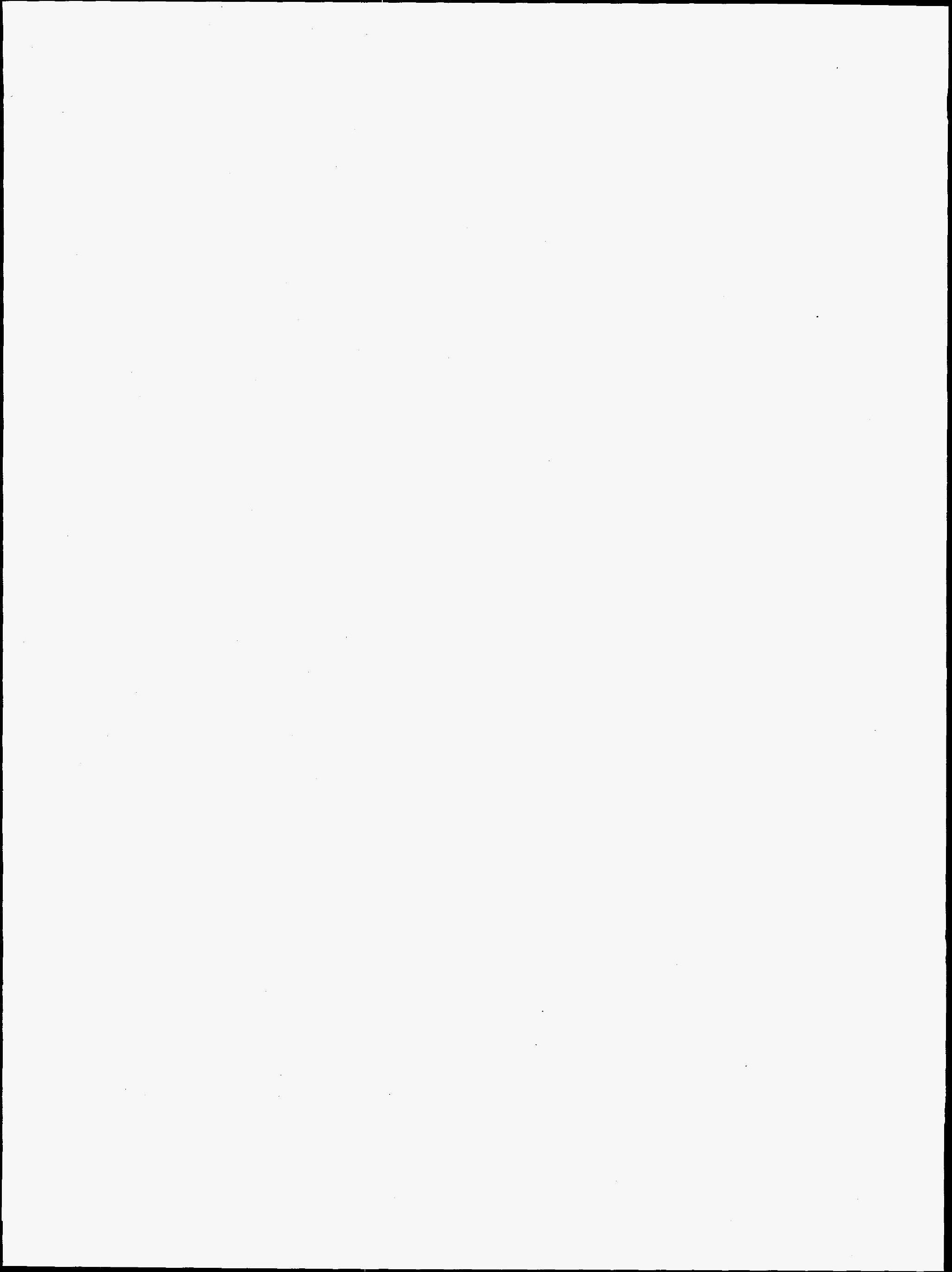




\section{Foreign Markets}

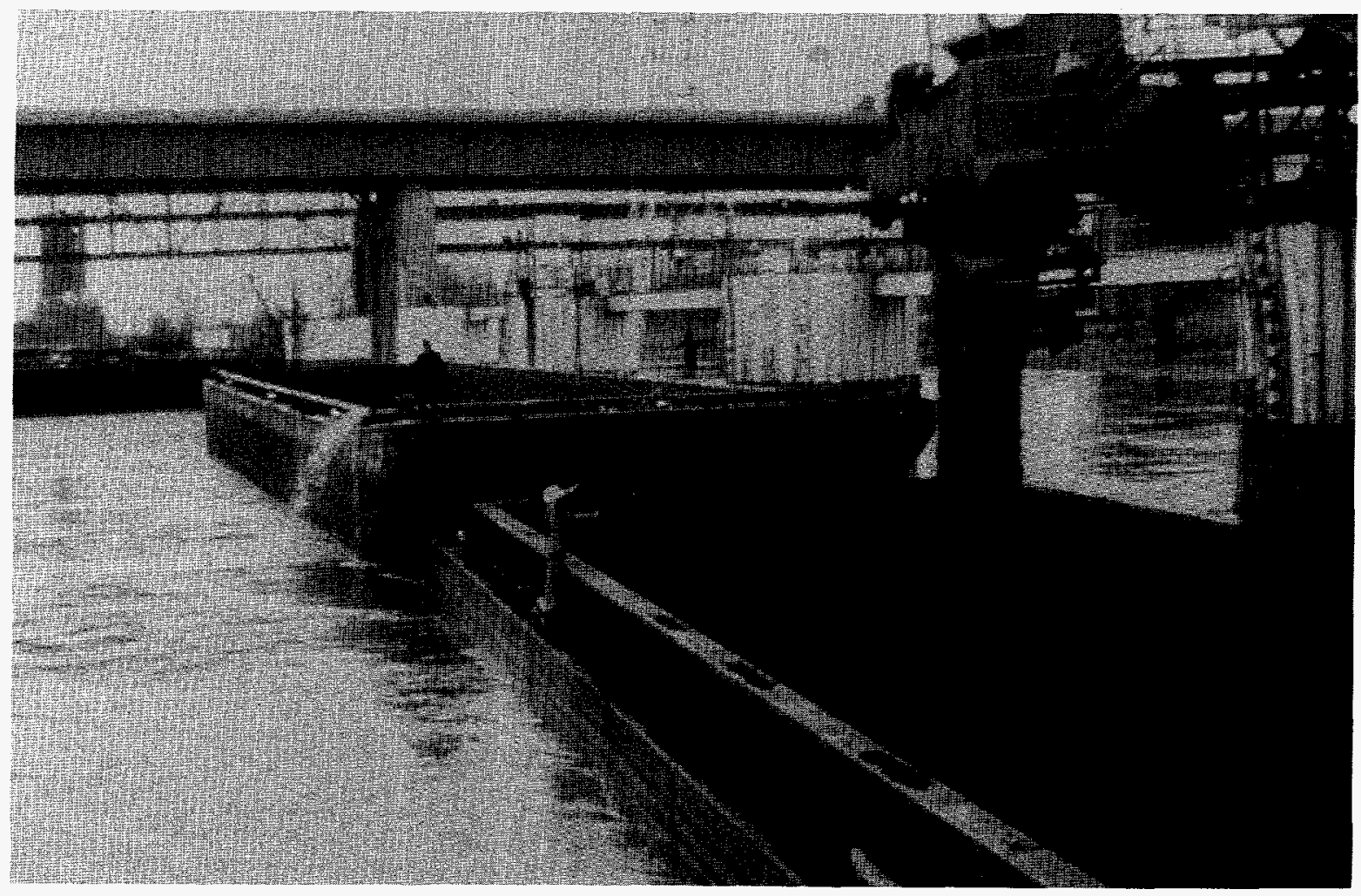

Japan, Canada, and Italy continues to be the principal export markets for U.S. coal. Historically, Canada has been the principal purchaser of American coal. 
Figure 11. U.S. Coal Exports, 1987-1996

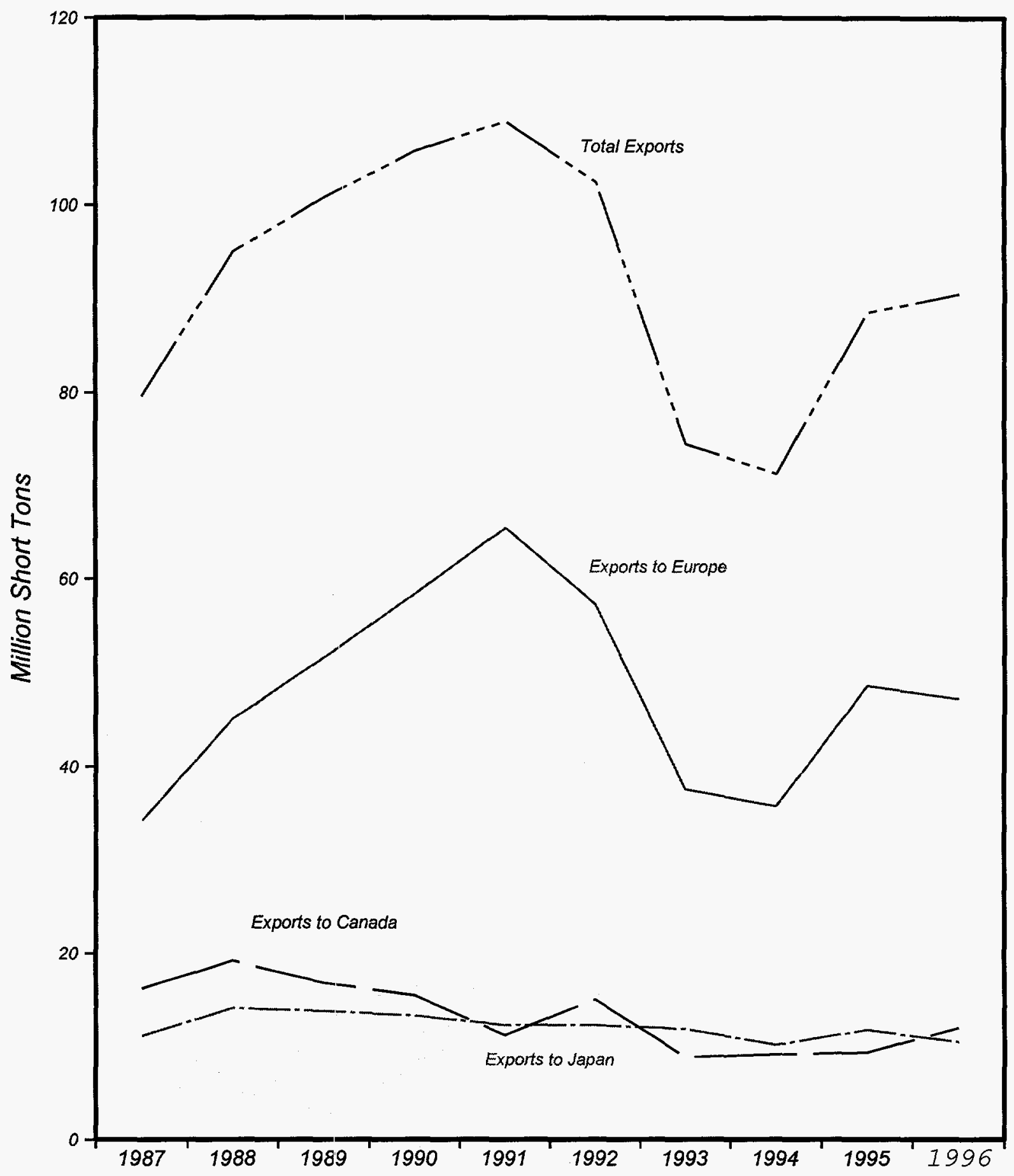

Source: U.S. Deportment of Commerce, Bureou of the Census, "Monthly Report EM 545." 
Table 76. U.S. Coal Exports by Destination, 1987, 1992-1996 (Thousand Short Tons)

\begin{tabular}{|c|c|c|c|c|c|c|c|c|c|}
\hline \multirow{2}{*}{$\begin{array}{c}\text { Continent and Country } \\
\text { of Destination }\end{array}$} & \multirow{2}{*}{1996} & \multirow{2}{*}{1995} & \multirow{2}{*}{1994} & \multirow{2}{*}{1993} & \multirow{2}{*}{1992} & \multirow{2}{*}{1987} & \multirow{2}{*}{$\begin{array}{c}\text { Percent } \\
\text { Change } \\
\text { 1995-1996 }\end{array}$} & \multicolumn{2}{|c|}{ Average Annual Percent Change } \\
\hline & & & & & & & & $1992-1996$ & 1987-1996 \\
\hline North America Total. & 13,609 & 10,411 & 9,505 & 9,219 & 15,331 & 16,464 & 30.7 & -2.9 & -2.1 \\
\hline Canada ${ }^{1}$ & 12,029 & 9,427 & 9,193 & 8,889 & 15,140 & 16,227 & 27.6 & -5.6 & -3.3 \\
\hline 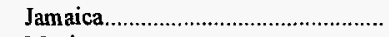 & 24 & 83 & 26 & 29 & 27 & - & -71.1 & -2.9 & - \\
\hline 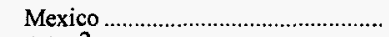 & 1,509 & 871 & 241 & 250 & 77 & 106 & 73.3 & 110.6 & 34.3 \\
\hline Other ${ }^{2}$ & 48 & 30 & 46 & 52 & 88 & 132 & 62.5 & -13.8 & -10.5 \\
\hline 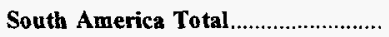 & 7,505 & 6,968 & 5,946 & 5,750 & 6,769 & 6,720 & 7.7 & 2.6 & 1.2 \\
\hline Argentina & 304 & 342 & 453 & 524 & 335 & 748 & -11.1 & -2.4 & -9.5 \\
\hline 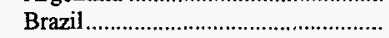 & 6,540 & 6,351 & 5,482 & 5,197 & 6,370 & 5,830 & 3.0 & .7 & 1.3 \\
\hline 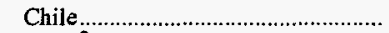 & 574 & 227 & * & * & 27 & 93 & 152.9 & 114.5 & 22.4 \\
\hline 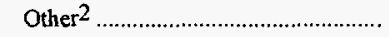 & 87 & 49 & 12 & 28 & 37 & 48 & 78.2 & 23.7 & 6.8 \\
\hline 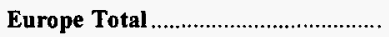 & 47,193 & 48,620 & 35,825 & 37,575 & $\mathbf{5 7 , 2 5 5}$ & 34,159 & -2.9 & -4.7 & 3.6 \\
\hline 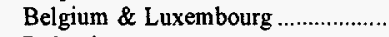 & 4,569 & 4,501 & 4,911 & 5,229 & 7,196 & 4,578 & 1.5 & -10.7 & $*$ \\
\hline 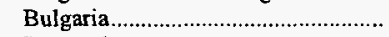 & 1,387 & 1,339 & 1,238 & 906 & 602 & - & 3.6 & 23.2 & - \\
\hline 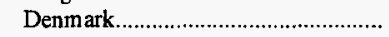 & 1,316 & 2,100 & 477 & 336 & 3,832 & 934 & -37.3 & -23.4 & 3.9 \\
\hline Finland & 704 & 1,308 & 377 & 252 & 205 & 172 & -46.2 & 36.1 & 17.0 \\
\hline 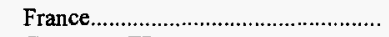 & 3,852 & 3,659 & 2,875 & 3,972 & 8,060 & 2,886 & 5.3 & -16.8 & 3.3 \\
\hline 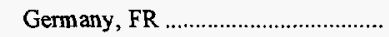 & 1,055 & 1,953 & 323 & 508 & 1,003 & 477 & -46.0 & 1.3 & 9.2 \\
\hline Ireland & 765 & 914 & 974 & 985 & 1,449 & 1,410 & -16.3 & -14.8 & -6.6 \\
\hline Italy & 9,204 & 9,063 & 7,543 & 6,918 & 9,344 & 9,537 & 1.6 & -.4 & -.4 \\
\hline 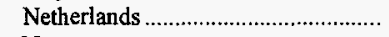 & 7,058 & 7,301 & 4,874 & 5,562 & 9,148 & 4,093 & -3.3 & -6.3 & 6.2 \\
\hline Norway & 85 & 120 & 87 & 101 & 118 & 173 & -29.2 & -7.9 & -7.6 \\
\hline 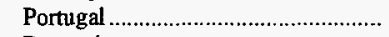 & 1,803 & 1,752 & 1,057 & 1,491 & 1,479 & 1,287 & 2.8 & 5.1 & 3.8 \\
\hline 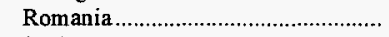 & 1,512 & 1,984 & 1,553 & 720 & 753 & 1,098 & -23.8 & 19.0 & 3.6 \\
\hline Spain & 4,093 & 4,653 & 4,132 & 4,064 & 4,535 & 2,454 & -12.0 & -2.5 & 5.8 \\
\hline Sweden & 1,070 & 1,117 & 702 & 736 & 1,165 & 668 & -4.3 & -2.1 & 5.4 \\
\hline 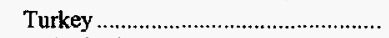 & 2,167 & 2,011 & 1,335 & 1,605 & 1,990 & 772 & 7.7 & 2.1 & 12.1 \\
\hline 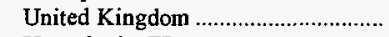 & 6,196 & 4,726 & 3,363 & 4,111 & 5,595 & 2,589 & 31.1 & 2.6 & 10.2 \\
\hline Yugoslavia, FR & - & 65 & - & 78 & 726 & 889 & -100.0 & -100.0 & -100.0 \\
\hline Other $^{2}$ & 357 & 52 & 8 & $*$ & 54 & 142 & NM & 60.1 & 10.8 \\
\hline 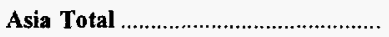 & 17,980 & 19,095 & 17,957 & 19,500 & 20,540 & 20,206 & -5.8 & $-\mathbf{3 . 3}$ & -1.3 \\
\hline China (Taiwan) & 2,441 & 2,533 & 3,374 & 3,435 & 3,560 & 4,769 & -3.6 & -9.0 & -7.2 \\
\hline 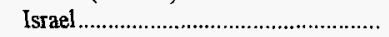 & 1,202 & 760 & 864 & 849 & 824 & 243 & 58.0 & 9.9 & 19.4 \\
\hline Japan & 10,529 & 11,787 & 10,158 & 11,878 & 12,304 & 11,082 & -10.7 & -3.8 & -.6 \\
\hline 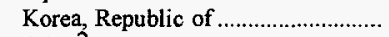 & 3,773 & 4,012 & 3,558 & 3,316 & 3,352 & 4,025 & -6.0 & 3.0 & -.7 \\
\hline Other ${ }^{2}$ & 36 & 2 & 3 & 22 & 500 & 87 & NM & -48.3 & -9.3 \\
\hline Oceania \& Australia Total................. & 1 & * & 1 & 1 & * & 2 & 322.7 & 31.5 & -5.9 \\
\hline Other ${ }^{2}$ & 1 & $*$ & 1 & 1 & * & 2 & 322.7 & 31.5 & -5.9 \\
\hline Africa Total & 4,184 & 3,453 & 2,124 & 2,474 & 2,621 & 2,056 & 21.2 & 12.4 & 8.2 \\
\hline Algeria & 177 & 220 & 355 & 409 & 611 & 910 & -19.8 & -26.7 & -16.6 \\
\hline Egypt & 1,038 & 1,235 & 1,048 & 868 & 848 & 467 & -16.0 & 5.2 & 9.3 \\
\hline Morocco & 1,650 & 1,212 & 83 & 587 & 737 & 504 & 36.2 & 22.3 & 14.1 \\
\hline 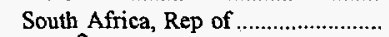 & 1,320 & 786 & 638 & 567 & 425 & 62 & 67.9 & 32.8 & 40.5 \\
\hline Other $^{2}$ & - & - & - & 42 & - & 114 & - & - & -100.0 \\
\hline Total & 90,473 & 88,547 & 71,359 & 74,519 & 102,516 & 79,607 & 2.2 & -3.1 & 1.4 \\
\hline
\end{tabular}

1 Based on the U.S. - Canada Free Trade Agreement, as of January 1990, the U.S. Department of Commerce began reporting statistics on U.S. exports to Canada based on information on imports provided monthly by the Canadian government.

2 Includes countries with exports less than or equal to 50,000 short tons in 1995

* Data round to zero.

NM Not meaningful as value is greater than 500 percent.

Note: Totals may not equal sum of components due to independent rounding.

Source: U.S. Department of Commerce, Bureau of the Census, "Monthly Report EM 545." 
Table 77. U.S. Metallurgical Coal Exports by Destination, 1987, 1992-1996 (Thousand Short Tons)

\begin{tabular}{|c|c|c|c|c|c|c|c|c|c|}
\hline \multirow{2}{*}{$\begin{array}{l}\text { Continent and Country } \\
\text { of Destination }\end{array}$} & \multirow{2}{*}{1996} & \multirow{2}{*}{1995} & \multirow{2}{*}{1994} & \multirow{2}{*}{1993} & \multirow{2}{*}{1992} & \multirow{2}{*}{1987} & \multirow{2}{*}{$\begin{array}{c}\text { Percent } \\
\text { Change } \\
\text { 1995-1996 }\end{array}$} & \multicolumn{2}{|c|}{ Average Annual Percent Change } \\
\hline & & & & & & & & 1992-1996 & $1987-1996$ \\
\hline North America Total & 6,500 & 4,776 & 4,246 & 4,853 & 4,957 & 6,553 & 36.1 & 7.0 & -0.1 \\
\hline Canada 1 & 6,030 & 4,452 & 4,032 & 4,663 & 4,920 & 6,413 & 35.4 & 5.2 & -.7 \\
\hline Mexico & 470 & 324 & 214 & 190 & 36 & 9 & 45.0 & 89.7 & 55.5 \\
\hline Other ${ }^{2}$ & - & - & - & $*$ & $*$ & 131 & - & -100.0 & -100.0 \\
\hline 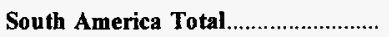 & 6,814 & 6,778 & 5,926 & 5,514 & 6,524 & 6,521 & $\mathbf{5}$ & 1.1 & .5 \\
\hline Argentina & 291 & 336 & 449 & 487 & 332 & 747 & -13.4 & -3.3 & -9.9 \\
\hline 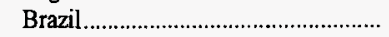 & 6,445 & 6,336 & 5,477 & 5,027 & 6,165 & 5,681 & 1.7 & 1.1 & 1.4 \\
\hline Chile & 78 & 106 & - & - & 27 & 93 & -27.0 & 30.2 & -2.0 \\
\hline Other ${ }^{2}$ & $*$ & - & - & - & - & - & - & - & - \\
\hline 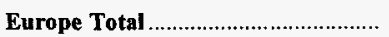 & 28,253 & 27,282 & 25,245 & 26,791 & 33,365 & 23,895 & 3.5 & -4.1 & 1.9 \\
\hline Belgium \& Luxembourg .................... & 3,445 & 3,468 & 3,706 & 4,030 & 5,165 & 3,937 & -.7 & -9.6 & -1.5 \\
\hline Bulgaria & 1,214 & 1,339 & 1,184 & 849 & 602 & - & -9.3 & 19.2 & - \\
\hline 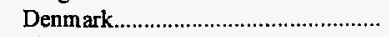 & - & - & - & 106 & - & - & - & - & - \\
\hline Finland & 540 & 724 & 311 & 252 & 205 & 172 & -25.5 & 27.3 & 13.6 \\
\hline France & 3,084 & 3,155 & 2,816 & 3,101 & 4,314 & 2,768 & -2.3 & -8.0 & 1.2 \\
\hline Germany, FR & 538 & 231 & 288 & 203 & 247 & 324 & 133.1 & 21.4 & 5.8 \\
\hline 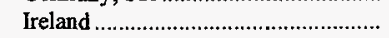 & - & - & - & - & - & 90 & - & - & -100.0 \\
\hline Italy & 5,293 & 4,504 & 5,045 & 4,965 & 6,180 & 5,265 & 17.5 & -3.8 & * \\
\hline 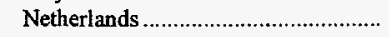 & 4,142 & 3,978 & 3,231 & 3,743 & 4,836 & 2,601 & 4.1 & -3.8 & 5.3 \\
\hline 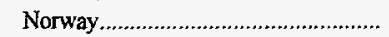 & 61 & 92 & 73 & 70 & 91 & 135 & -33.6 & -9.5 & -8.5 \\
\hline 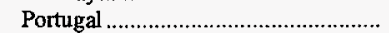 & 174 & 30 & - & 149 & 135 & 197 & 476.1 & 6.6 & -1.3 \\
\hline 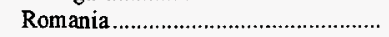 & 1,512 & 1,685 & 663 & 373 & 753 & 1,098 & -10.2 & 19.0 & 3.6 \\
\hline Spain & 2,103 & 2,178 & 2,656 & 2,994 & 2,795 & 2,354 & -3.5 & -6.9 & -1.2 \\
\hline 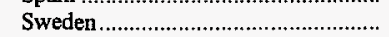 & 987 & 1,109 & 702 & 736 & 1,165 & 668 & -11.0 & -4.1 & 4.4 \\
\hline Turkey & 2,027 & 1,806 & 1,335 & 1,604 & 1,989 & 772 & 12.3 & 5 & 11.3 \\
\hline United Kingdom & 3,081 & 2,932 & 3,228 & 3,573 & 4,175 & 2,586 & 5.1 & -7.3 & 2.0 \\
\hline 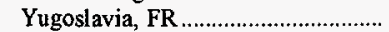 & - & - & - & 43 & 658 & 828 & - & -100.0 & -100.0 \\
\hline Other ${ }^{2}$ & 54 & 52 & 7 & - & 54 & 100 & 4.4 & * & -6.6 \\
\hline Asia Total & 8,814 & 11,014 & 9,877 & 10,608 & 12,655 & 13,158 & -20.0 & -8.6 & -4.3 \\
\hline China (Taiwan) & 376 & 370 & 296 & 285 & 424 & 483 & 1.4 & -3.0 & -2.8 \\
\hline Israel & 265 & 141 & - & - & 143 & - & 88.9 & 16.7 & - \\
\hline Japan & 5,552 & 7,929 & 7,195 & 8,028 & 9,480 & 10,110 & -30.0 & -12.5 & -6.4 \\
\hline Korea, Republic of & 2,597 & 2,574 & 2,386 & 2,276 & 2,608 & 2,479 & .9 & -.1 & .5 \\
\hline Other $^{2}$ & 24 & - & - & 19 & - & 86 & - & - & -13.4 \\
\hline 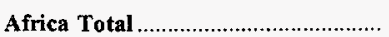 & 2,570 & 2,239 & 2,040 & 1,886 & 1,925 & 1,553 & 14.8 & 7.5 & 5.8 \\
\hline Algeria & 177 & 220 & 355 & 409 & 611 & 910 & -19.8 & -26.7 & -16.6 \\
\hline Egypt & 1,037 & 1,233 & 1,047 & 868 & 848 & 467 & -15.9 & 5.2 & 9.3 \\
\hline Moracco & 37 & - & - & - & 41 & - & - & -2.8 & - \\
\hline 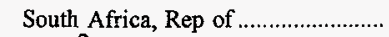 & 1,320 & 786 & 638 & 567 & 425 & 62 & 68.0 & 32.8 & 40.5 \\
\hline Other ${ }^{2}$ & - & - & - & 42 & - & 114 & - & - & -100.0 \\
\hline 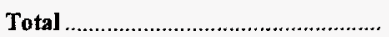 & 52,950 & 52,089 & 47,334 & 49,652 & $\mathbf{5 9 , 4 2 6}$ & 51,679 & 1.6 & -2.8 & .3 \\
\hline
\end{tabular}

1 Based on the U.S. - Canada Free Trade Agreement, as of January 1990, the U.S. Department of Commerce began reporting statistics on U.S. exports to Canada based on information on imports provided monthly by the Canadian government.

2 Includes countries with exports less than or equal to 50,000 short tons in 1995 .

* Data round to zero.

Note: Totals may not equal sum of components due to independent rounding.

Source: U.S. Department of Commerce, Bureau of the Census, "Monthly Report EM 545." 
Table 78. U.S. Steam Coal Exports by Destination, 1987, 1992-1996 (Thousand Short Tons)

\begin{tabular}{|c|c|c|c|c|c|c|c|c|c|}
\hline \multirow{2}{*}{$\begin{array}{c}\text { Continent and Country } \\
\text { of Destination }\end{array}$} & \multirow{2}{*}{1996} & \multirow{2}{*}{1995} & \multirow{2}{*}{1994} & \multirow{2}{*}{1993} & \multirow{2}{*}{1992} & \multirow{2}{*}{1987} & \multirow{2}{*}{$\begin{array}{c}\text { Percent } \\
\text { Change } \\
1995-1996\end{array}$} & \multicolumn{2}{|c|}{ Average Annual Percent Change } \\
\hline & & & & & & & & 1992-1996 & $1987-1996$ \\
\hline 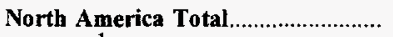 & 7,110 & 5,635 & 5,259 & 4,366 & 10,374 & 9,912 & 26.2 & -9.0 & -3.6 \\
\hline Canada 1 & 5,999 & 4,975 & 5,161 & 4,225 & 10,219 & 9,814 & 20.6 & -12.5 & -5.3 \\
\hline 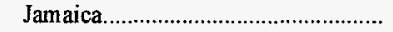 & 24 & 83 & 26 & 29 & 27 & - & -71.1 & -2.9 & - \\
\hline 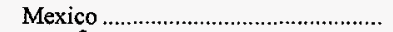 & 1,039 & 547 & 26 & 60 & 40 & 97 & 90.0 & 125.2 & 30.1 \\
\hline Other ${ }^{2}$ & 48 & 30 & 46 & 52 & 88 & 1 & 62.5 & -13.8 & 53.1 \\
\hline South America Total ............................ & 691 & 190 & 20 & 236 & 245 & 199 & 263.3 & 29.6 & 14.8 \\
\hline Argentina & 13 & 6 & 4 & 38 & 3 & 2 & 114.1 & 47.2 & 24.6 \\
\hline 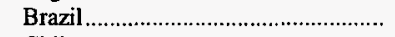 & 95 & 15 & 5 & 170 & 205 & 149 & NM & -17.5 & -4.9 \\
\hline 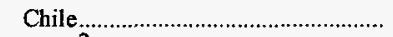 & 496 & 121 & $*$ & $*$ & $*$ & * & 311.6 & $\mathrm{NM}$ & 211.4 \\
\hline Other ${ }^{2}$ & 87 & 49 & 12 & 28 & 37 & 48 & 77.8 & 23.6 & 6.7 \\
\hline Europe Total & 18,940 & 21,338 & 10,580 & 10,784 & 23,891 & 10,264 & -11.2 & -5.6 & 7.0 \\
\hline 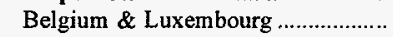 & 1,125 & 1,033 & 1,205 & 1,199 & 2,031 & 641 & 8.8 & -13.7 & 6.4 \\
\hline 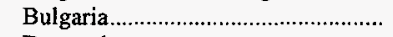 & 173 & - & 54 & 57 & - & - & - & - & - \\
\hline Denmark & 1,316 & 2,100 & 477 & 230 & 3,832 & 934 & -37.3 & -23.4 & 3.9 \\
\hline Finland & 164 & 584 & 66 & 1 & - & - & -71.9 & - & - \\
\hline 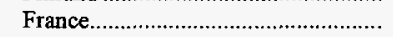 & 769 & 503 & 58 & 870 & 3,745 & 118 & 52.7 & -32.7 & 23.1 \\
\hline 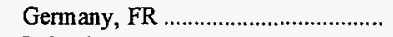 & 517 & 1,722 & 35 & 305 & 756 & 153 & -70.0 & -9.1 & 14.5 \\
\hline Ireland & 765 & 914 & 974 & 985 & 1,449 & 1,319 & -16.3 & -14.8 & -5.9 \\
\hline Italy & 3,911 & 4,559 & 2,498 & 1,954 & 3,164 & 4,272 & -14.2 & 5.4 & -1.0 \\
\hline 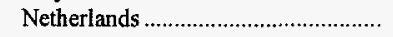 & 2,917 & 3,323 & 1,643 & 1,819 & 4,312 & 1,492 & -12.2 & -9.3 & 7.7 \\
\hline 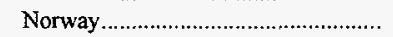 & 24 & 28 & 13 & 31 & 27 & 37 & -14.7 & -3.4 & -4.9 \\
\hline 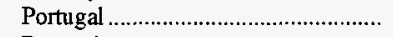 & 1,628 & 1,722 & 1,057 & 1,342 & 1,344 & 1,090 & -5.4 & 4.9 & 4.6 \\
\hline 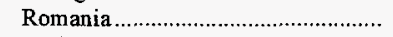 & - & 299 & 890 & 347 & - & - & -100.0 & - & - \\
\hline Spain & 1,990 & 2,475 & 1,476 & 1,070 & 1,740 & 101 & -19.6 & 3.4 & 39.3 \\
\hline 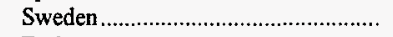 & 83 & 9 & - & - & $*$ & - & NM & 327.7 & - \\
\hline Turkey & 140 & 206 & - & * & * & - & -31.8 & 359.0 & - \\
\hline 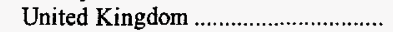 & 3,115 & 1,795 & 135 & 538 & 1,421 & 3 & 73.6 & 21.7 & 115.3 \\
\hline 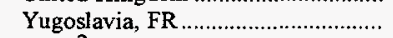 & - & 65 & - & 35 & 68 & 61 & -100.0 & -100.0 & -100.0 \\
\hline Other 2 & 303 & $*$ & $*$ & * & * & 42 & NM & NM & 24.5 \\
\hline 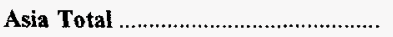 & 9,166 & 8,081 & 8,080 & 8,892 & 7,885 & 7,048 & 13.4 & 3.8 & 3.0 \\
\hline China (Taiwan) & 2,066 & 2,163 & 3,078 & 3,150 & 3,136 & 4,286 & -4.5 & -9.9 & -7.8 \\
\hline 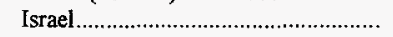 & 936 & 620 & 864 & 849 & 681 & 243 & 51.0 & 8.3 & 16.2 \\
\hline Japan & 4,976 & 3,858 & 2,963 & 3,850 & 2,823 & 972 & 29.0 & 15.2 & 19.9 \\
\hline Korea, Republic of & 1,175 & 1,438 & 1,172 & 1,040 & 744 & 1,546 & -18.3 & 12.1 & -3.0 \\
\hline Other ${ }^{2} \ldots \ldots \ldots \ldots \ldots \ldots \ldots \ldots \ldots$ & 12 & 2 & 3 & 3 & 500 & 1 & 431.2 & -60.4 & 32.2 \\
\hline Oceania \& Australia Total............... & 1 & * & 1 & 1 & * & 2 & 322.7 & 31.5 & -5.9 \\
\hline Other ${ }^{2}$ & 1 & * & 1 & 1 & * & 2 & 322.7 & 31.5 & -5.9 \\
\hline 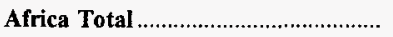 & 1,615 & 1,214 & 85 & 588 & 696 & 504 & 33.0 & 23.4 & 13.8 \\
\hline Egypt & 1 & 2 & 1 & 1 & 1 & * & -41.1 & 5.4 & 48.4 \\
\hline Morocco & 1,614 & 1,212 & 83 & 587 & 695 & 504 & 33.2 & 23.4 & 13.8 \\
\hline 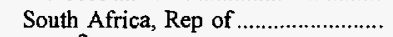 & - & $*$ & - & - & - & - & -100.0 & - & - \\
\hline Other ${ }^{2}$ & - & - & - & - & - & $*$ & - & - & -100.0 \\
\hline Total & $\mathbf{3 7 , 5 2 2}$ & 36,458 & 24,025 & 24,867 & 43,090 & 27,928 & 2.9 & -3.4 & 3.3 \\
\hline
\end{tabular}

1 Based on the U.S. - Canada Free Trade Agreement, as of January 1990, the U.S. Department of Commerce began reporting statistics on U.S. exports to Canada based on information on imports provided monthly by the Canadian government.

2 Includes countries with exports less than or equal to 50,000 short tons in 1995.

* Data round to zero.

NM Not meaningful as value is greater than 500 percent.

Note: Totals may not equal sum of components due to independent rounding

Source: U.S. Department of Commerce, Bureau of the Census, "Monthly Report EM 545." 
Table 79. Coal Exports by Customs District, 1987, 1992-1996

(Thousand Short Tons)

\begin{tabular}{|c|c|c|c|c|c|c|c|c|c|}
\hline \multirow{2}{*}{ Customs District } & \multirow{2}{*}{1996} & \multirow{2}{*}{1995} & \multirow{2}{*}{1994} & \multirow{2}{*}{1993} & \multirow{2}{*}{1992} & \multirow{2}{*}{1987} & \multirow{2}{*}{$\begin{array}{c}\text { Percent } \\
\text { Change } \\
\text { 1995-1996 }\end{array}$} & \multicolumn{2}{|c|}{ Average Annual Percent Change } \\
\hline & & & & & & & & 1992-1996 & $1987-1996$ \\
\hline 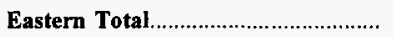 & 58,161 & 55,374 & 43,474 & 45,138 & 64,826 & 46,077 & $\mathbf{5 . 0}$ & -2.7 & 2.6 \\
\hline Boston, MA & - & 33 & - & 33 & - & * & -100.0 & - & -100.0 \\
\hline 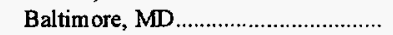 & 11,221 & 11,313 & 7,912 & 7,354 & 9,450 & 6,500 & -.8 & 4.4 & 6.3 \\
\hline 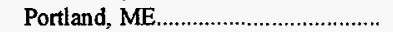 & $*$ & 57 & 1 & 1 & $*$ & 1 & -99.8 & -5.7 & -18.4 \\
\hline 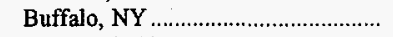 & 2,263 & 1,574 & 166 & 67 & 1,971 & 41 & 43.7 & 3.5 & 56.2 \\
\hline 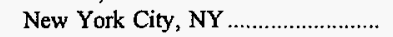 & 6 & 87 & 1 & 1 & 4 & 249 & -92.6 & 12.5 & -33.4 \\
\hline Ogdensburg, NY & 116 & 163 & 337 & 44 & 66 & 9 & -28.8 & 15.3 & 33.4 \\
\hline Philadelphia, PA & 406 & 339 & 213 & 190 & 252 & 1,975 & 19.9 & 12.6 & -16.1 \\
\hline 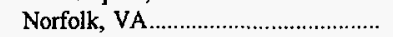 & 44,148 & 41,808 & 34,845 & 37,448 & 53,083 & 37,303 & 5.6 & -4.5 & 1.9 \\
\hline St. Albans, VT & 1 & $*$ & * & $*$ & $*$ & - & NM & 30.5 & - \\
\hline 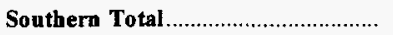 & 16,077 & 19,936 & 15,607 & 16,658 & 21,343 & 15,706 & -19.3 & -6.8 & .3 \\
\hline Mobile, AL & 5,897 & 8,283 & 4,997 & 6,262 & 6,853 & 7,085 & -28.8 & -3.7 & -2.0 \\
\hline 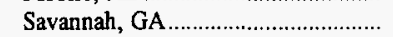 & - & 4 & 1 & - & 37 & $*$ & -100.0 & -100.0 & -100.0 \\
\hline Miami, FL & 2 & 2 & 3 & 2 & 2 & * & 15.0 & 5.4 & 32.3 \\
\hline Tampa, FL & - & 1 & * & * & - & * & -100.0 & - & -100.0 \\
\hline 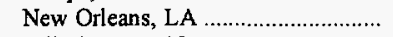 & 8,669 & 10,522 & 9,475 & 9,705 & 13,480 & 7,733 & -17.6 & -10.4 & 1.3 \\
\hline Wilmington, $\mathrm{NC}$ & 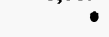 & - & - & - & - & 121 & - & - & -60.5 \\
\hline 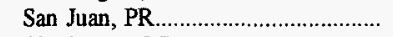 & $*$ & $*$ & 26 & * & - & * & 420.0 & - & 39.6 \\
\hline 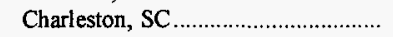 & 154 & 401 & 957 & 475 & 791 & 566 & -61.6 & -33.6 & -13.5 \\
\hline El Paso, TX & - & $*$ & - & $*$ & 1 & $*$ & -100.0 & -100.0 & -100.0 \\
\hline Houston-Galveston, TX & 297 & 179 & 121 & 155 & 145 & 106 & 65.4 & 19.7 & 12.1 \\
\hline Laredo, TX & 1,057 & 542 & 26 & 59 & 36 & 62 & 94.9 & 133.3 & 36.9 \\
\hline Port Arthur, TX & - & - & - & - & - & 32 & - & - & -100.0 \\
\hline 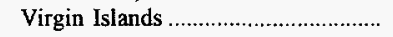 & - & - & - & - & $*$ & - & - & -100.0 & - \\
\hline 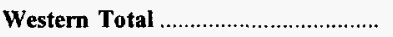 & 6,832 & 5,527 & 3,813 & 4,201 & 3,651 & 2,105 & 23.6 & 16.9 & 14.0 \\
\hline Anchorage, AK & 784 & 919 & 719 & 733 & 728 & 839 & -14.7 & 1.9 & -.7 \\
\hline Nogales, A.Z & $*$ & - & $*$ & $*$ & $*$ & - & - & -42.3 & - \\
\hline Los Angeles, CA & 5,899 & 4,475 & 2,963 & 3,358 & 2,721 & 1,233 & 31.8 & 21.3 & 19.0 \\
\hline 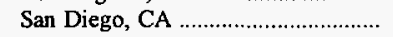 & - & $*$ & 1 & 1 & 4 & 1 & -100.0 & -100.0 & -100.0 \\
\hline San Francisco, CA & $\mathbf{1}$ & $*$ & 1 & - & 60 & 1 & 72.2 & -67.8 & -2.6 \\
\hline Great Falls, MT & $*$ & * & 1 & $*$ & - & 1 & 79.0 & - & -4.3 \\
\hline Portland, OR & - & - & - & 2 & - & - & - & - & - \\
\hline Seattle, WA & 147 & 132 & 128 & 106 & 137 & 30 & 11.4 & 1.8 & 19.1 \\
\hline 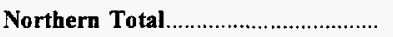 & 9,358 & 7,688 & 8,437 & 8,495 & 12,557 & 15,720 & 21.7 & -7.1 & -5.6 \\
\hline 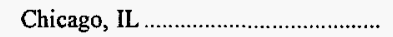 & - & - & 24 & - & * & - & - & -100.0 & - \\
\hline Detroit, MI & 3,804 & 1,845 & 2,600 & 609 & 2,181 & 49 & 106.2 & 14.9 & 62.3 \\
\hline 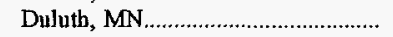 & 247 & 210 & 161 & 134 & 119 & * & 17.6 & 20.1 & 149.9 \\
\hline Pembina, ND & 1 & 19 & 10 & 1 & * & 6 & -95.7 & 58.2 & -19.5 \\
\hline Cleveland, $\mathrm{OH}$ & 5,306 & 5.614 & 5,642 & 7,751 & 10,258 & 15,665 & -5.5 & -15.2 & -11.3 \\
\hline 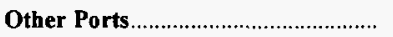 & 45 & 22 & 28 & 26 & 138 & - & 100.3 & -24.5 & - \\
\hline Total & 90,473 & 88,547 & 71,359 & 74,519 & 102,516 & 79,607 & 2.2 & -3.1 & 1.4 \\
\hline
\end{tabular}

* Data round to zero.

Mot meaningful as value is greater than 500 percent.

Note: Totals may not equal sum of components due to independent rounding.

Source: U.S. Department of Commerce, Bureau of the Census, "Monthly Report EM 545." 


\section{Coal Prices}

\section{Mine Prices}

The average mine price of U.S. coal in 1996 was $\$ 18.50$ per short ton, a drop of 2 percent from 1995 and the 14th straight year of decline (Table 80). Since 1987 the average mine price of U.S. coal has declined at an average annual rate of 2.4 percent. During this period, the average mine price of coal east of the Mississippi River decreased at an annual average rate of 1.2 percent, whereas west of the Mississippi River the decline was 2.9 percent. In real dollars, the decline over this period for the United States was $\mathbf{5 . 4}$ percent (Table 81).

Compared with 1995 , the average mine price of coal in all regions decreased. The decline in Appalachia was 2.4 percent; in the Interior, 2.1 percent; and in the West, 1.1 percent. In the three largest coal-producing States, prices fell more than the decline of the U.S. price. Kentucky's coal price dropped 3.6 percent, Wyoming's price fell 2.6 percent, and West Vírginia's price declined 2.2 percent.

\section{Consumer Prices}

The average price of coal delivered to electric utilities during 1996 was $\$ 26.45$ per short ton, 2.1 percent less than the average price of $\$ 27.01$ per short ton reported for 1995 (Table 92). Similarly, compared with 1995, the average price of coal delivered to industrial consumers other than coke plants declined slightly to $\$ 32.32$ per short ton, while the average price of coal delivered to coke plants remained essentially unchanged at $\$ 47.33$ per short ton (Tables 94 and 96 ).

The average price of U.S. coal imports in 1996 was $\$ 33.45$ per short ton, a 2 percent decrease compared with the 1995 average of $\$ 34.13$ per short ton.

The average price of U.S. coal exported during 1996 was $\$ 40.76$ per short ton, 1.2 percent above the average of $\$ 40.27$ per short ton reported for 1995 (Table 99). Compared with 1995, the average price of metallurgical coal exported during 1996 increased 2.7 percent to $\$ 45.49$ per short ton, while the average price of steam coal dropped 1.2 percent to $\$ 34.09$ per short ton (Tables 101 and 103). 
Figure 12. Coal Prices, 1987-1996

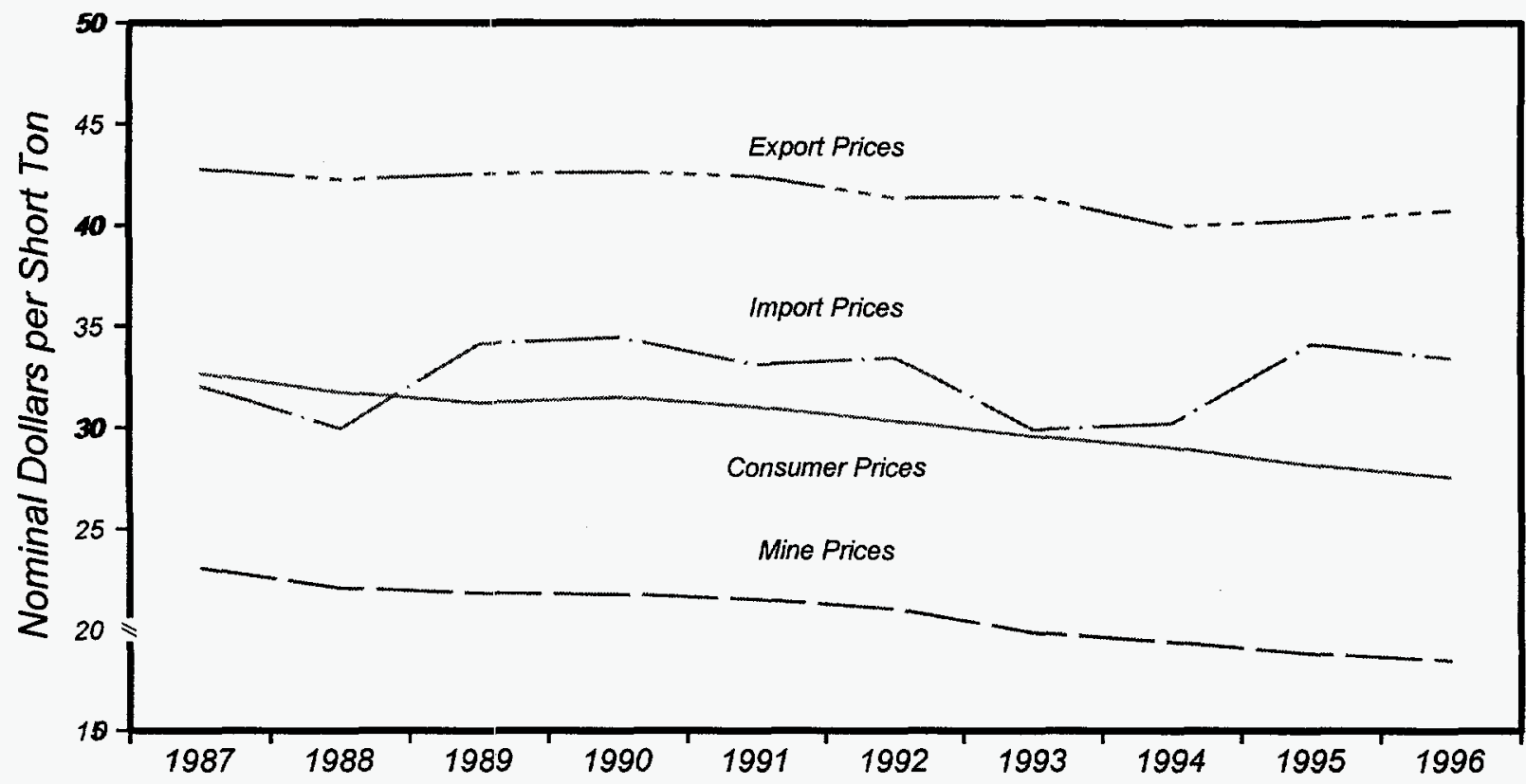

Note: Averoge mine prices exclude mines producing less thon 10,000 short tons of cool during the yeor. Mine Price is calculoted by dividing the lotol free on boord (f.o.b.) mine yolue of the coal produced by the lotal produclion. Consumer Price is bosed on the cost including insurance ond freight (c.i.f. cost) for electric ulilities, ond insuronce, Ireight ond laxes lor monufocturing and coke plonts, and does nol include the residential ond commerciol sector. Export Price
is bosed on the lree olongside ship (I.o.s.) valje. Import Price is bosed on the customs import value.

Sources: "Mine Prices: Energy Informotion Administrotion (EIA), Form EIA-7A. "Cool Produclion Report": Stote Mining Agency Cool Production Reports; ond/or U.S. Department oL Lobor, Mine Solety and Heallh Administrotion, Form $7000-2$. "Qugrterly Mine Employment and Cool Production Report." Consumer Prices: Federal Regulotory Commission (FERC). FERC Form 423. "Monthly Report of Cost and Quality of Fuels lor Electric Plonls":; ElA, Form EJA-5. "Coke Plont Report"Quorlerly" ond Form EIA-3. "Quarterly Cool Consumption Reporl - Manufocturing Plants." Export Prices: U.S. Department of Commerce, Bureau of the Census, "Monthly Report EM 545." Import Prices: U.S. Deportment of Commerce, Bureou of the Census, "Monthly Report IM 145." 


\section{Mine Prices}

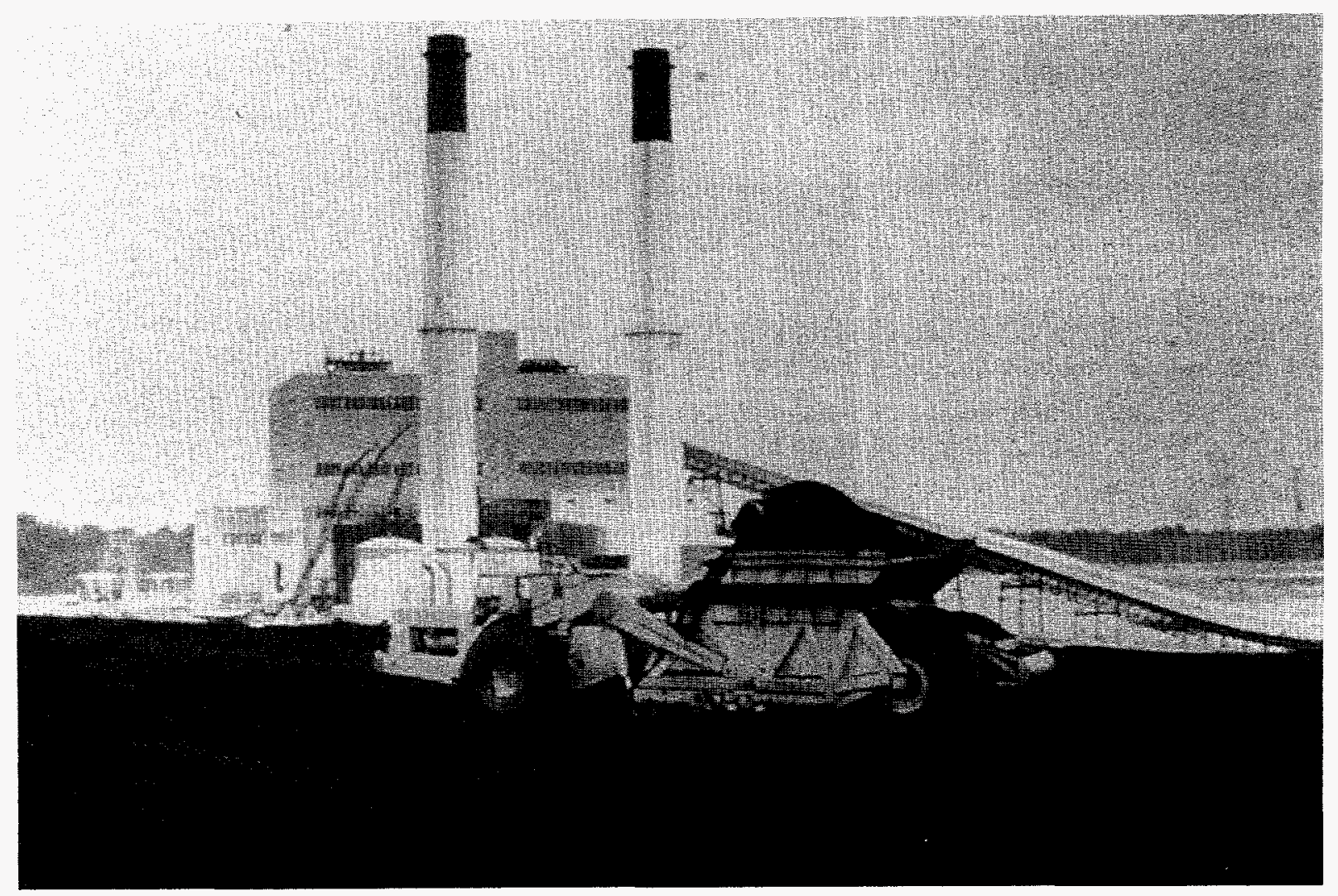

In 1996, the average mine price of U.S. coal was $\$ 18.50$, a 2-percent decrease since last year. 
Table 80. Average Mine Price of Coal by State, 1987, 1992-1996 (Nominal Dollars per Short Ton)

\begin{tabular}{|c|c|c|c|c|c|c|c|c|c|}
\hline \multirow{2}{*}{$\begin{array}{l}\text { Coal-Producing } \\
\text { State and Region }\end{array}$} & \multirow{2}{*}{1996} & \multirow{2}{*}{1995} & \multirow{2}{*}{1994} & \multirow{2}{*}{1993} & \multirow{2}{*}{1992} & \multirow{2}{*}{1987} & \multirow{2}{*}{$\begin{array}{c}\text { Percent } \\
\text { Change } \\
1995-1996\end{array}$} & \multicolumn{2}{|c|}{ Average Annual Percent Change } \\
\hline & & & & & & & & 1992-1996 & $1987-1996$ \\
\hline Alabama & $\$ 39.48$ & $\$ 38.44$ & $\$ 40.12$ & $\$ 42.34$ & $\$ 40.82$ & $\$ 41.42$ & 2.7 & -0.8 & -0.5 \\
\hline 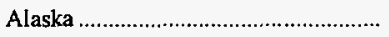 & $w$ & w & w & w & w & $w$ & w & w & w \\
\hline Arizona & $\mathbf{w}$ & $\mathbf{w}$ & $\mathbf{w}$ & $\mathbf{w}$ & $\mathbf{w}$ & $w$ & $\mathbf{w}$ & w & $w$ \\
\hline 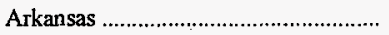 & - & $w$ & $\mathbf{w}$ & $\mathbf{w}$ & w & $w$ & $\mathbf{w}$ & w & $\mathbf{w}$ \\
\hline 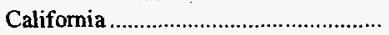 & - & - & - & - & $\mathbf{w}$ & w & $w$ & $\mathbf{w}$ & $w$ \\
\hline Colorado & $\$ 17.94$ & $\$ 19: 26$ & $\$ 19.76$ & $\$ 20.35$ & $\$ 21.33$ & $\$ 23.58$ & -6.9 & -4.2 & -3.0 \\
\hline 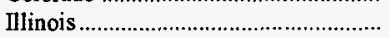 & 22.74 & 23.05 & 23.14 & 25.27 & 27.66 & 29.56 & -1.3 & -4.8 & -2.9 \\
\hline Indiana & 20.24 & 21.71 & 22.28 & 22.89 & 23.41 & 24.57 & -6.8 & -3.6 & -2.1 \\
\hline Iowa & - & - & w & $\mathbf{w}$ & w & 25.89 & w & w & w \\
\hline Kansas & w & w & w & w & $\mathbf{w}$ & 24.54 & w & $w$ & $\mathbf{w}$ \\
\hline 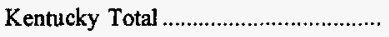 & $\$ 23.91$ & $\$ 24.79$ & $\$ 24.88$ & $\$ 24.77$ & $\$ 24.50$ & 26.15 & -3.6 & -.6 & -1.0 \\
\hline Eastern & 24.98 & 26.00 & 25.25 & 25.50 & 25.00 & 26.71 & -3.9 & $*$ & -.7 \\
\hline Western & 20.38 & 20.75 & 23.63 & 22.36 & 23.10 & 24.68 & -1.8 & -3.1 & -2.1 \\
\hline 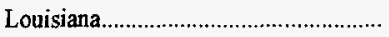 & w & w & w & w & w & w & $w$ & w & w \\
\hline 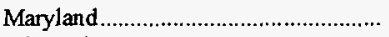 & $\$ 24.40$ & $\$ 24.69$ & $\$ 26.34$ & $\$ 25.21$ & $\$ 25.39$ & $\$ 24.3 \mathrm{I}$ & -1.2 & -1.0 & * \\
\hline 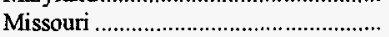 & 23.31 & 18.91 & 21.78 & w & w & 28.92 & 23.3 & w & -2.4 \\
\hline 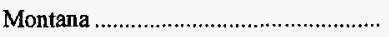 & 9.96 & 9.62 & 10.39 & $\$ 11.05$ & $\$ 10.20$ & 12.43 & 3.5 & -.6 & -2.4 \\
\hline 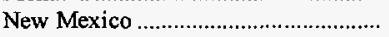 & 24.66 & 23.80 & 23.29 & 22.96 & 23.14 & 21.78 & 3.6 & 1.6 & 1.4 \\
\hline North Dakota & 8.01 & 7.99 & 7.62 & 7.63 & 7.48 & 7.91 & 2 & 1.7 & .1 \\
\hline Ohio & 24.85 & 25.97 & 29.13 & 28.04 & 26.93 & 30.80 & -4.3 & -2.0 & -2.3 \\
\hline 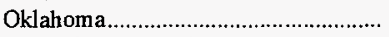 & 26.54 & 24.13 & 25.57 & 24.91 & 25.76 & 31.83 & 9.9 & .7 & -2.0 \\
\hline Pennsylvania Total .................................. & 25.78 & 26.78 & 26.18 & 26.50 & 28.61 & 29.81 & -3.7 & -2.6 & -1.6 \\
\hline 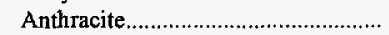 & 36.78 & 39.78 & 36.07 & 32.94 & 34.24 & 43.65 & -7.5 & 1.8 & -1.9 \\
\hline 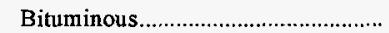 & 24.98 & 25.77 & 25.45 & 26.03 & 28.34 & 29.16 & -3.0 & -3.1 & -1.7 \\
\hline 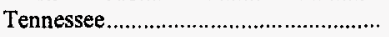 & 27.79 & 26.94 & 27.17 & 27.23 & 27.11 & 27.65 & 3.1 & .6 & * \\
\hline 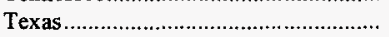 & 12.17 & 12.16 & 12.38 & 12.87 & 12.42 & 12.10 & $*$ & -.5 & .1 \\
\hline Utah & 21.63 & 19.10 & 19.27 & 20.81 & 21.11 & 25.70 & 13.3 & .6 & -1.9 \\
\hline 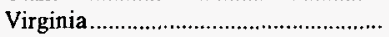 & 28.45 & 28.47 & 26.84 & 26.80 & 27.55 & 27.42 & -.1 & .8 & .4 \\
\hline Washington & $w$ & w & w & w & w & $w$ & $w$ & w & w \\
\hline West Virginia Total.............................. & $\$ 26.58$ & $\$ 27.18$ & $\$ 27.42$ & $\$ 27.58$ & $\$ 28.15$ & $\$ 29.15$ & -2.2 & -1.4 & -1.0 \\
\hline Northem & 24.86 & 24.91 & 26.77 & 28.09 & 29.03 & 28.67 & -.2 & -3.8 & -1.6 \\
\hline Southern & 27.21 & 28.07 & 27.71 & 27.40 & 27.76 & 29.44 & -3.1 & -.5 & -.9 \\
\hline 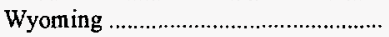 & 6.41 & 6.58 & 6.83 & 7.32 & 8.14 & 9.80 & -2.6 & -5.8 & -4.6 \\
\hline Appalachian Total ${ }^{1} \ldots \ldots \ldots \ldots \ldots \ldots \ldots \ldots \ldots \ldots \ldots \ldots \ldots$ & 26.78 & 27.45 & 27.36 & 27.64 & 27.95 & 29.20 & -2.4 & -1.1 & -.9 \\
\hline Interior Total 1 & 18.41 & 18.81 & 19.87 & 20.03 & 21.61 & 23.02 & -2.1 & -3.9 & -2.4 \\
\hline Western Total ${ }^{1}$ & 10.03 & 10.15 & 10.57 & 11.14 & 11.60 & 13.25 & -1.1 & -3.5 & $-\mathbf{3 . 0}$ \\
\hline 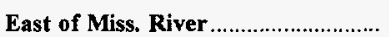 & 25.70 & 26.35 & 26.44 & 26.81 & 27.34 & 28.61 & -2.5 & -1.5 & -1.2 \\
\hline 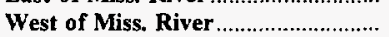 & 10.40 & 10.48 & 10.91 & 11.50 & 11.98 & 13.55 & -.8 & -3.5 & -2.9 \\
\hline 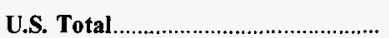 & 18.50 & 18.83 & 19.41 & 19.85 & 21.03 & 23.07 & -1.8 & -3.1 & -2.4 \\
\hline
\end{tabular}

1 For a definition of coal-producing regions, see Appendix C.

* Data round to zero.

Notes: Average mine price is calculated by dividing the total free on board (f.o.b.) mine value of the coal produced by the total production. A measure of dispersion of these average prices at the State level (interquartile range) is given in Appendix D, Table D2. Excludes silt, culm, refuse bank, slurry dam, and dredge operations except for Pennsylvania anthracite. Excludes mines producing less than 10,000 short tons, which are not required to provide these data.

Sources: Energy Information Administration, Form EIA-7A, "Coal Production Report"; State Mining Agency Coal Production Reports; and/or U.S. Department of Labor, Mine Safety and Health Administration, Form 7000-2, "Quarterly Mine Employment and Coal Production Report." 
Table 81. Average Real Mine Price of Coal by State, 1987, 1992-1996 (Real Dollars per Short Ton)

\begin{tabular}{|c|c|c|c|c|c|c|c|c|c|}
\hline \multirow{2}{*}{$\begin{array}{l}\text { Coal-Producing } \\
\text { State and Region }\end{array}$} & \multirow{2}{*}{1996} & \multirow{2}{*}{1995} & \multirow{2}{*}{1994} & \multirow{2}{*}{1993} & \multirow{2}{*}{1992} & \multirow{2}{*}{1987} & \multirow{2}{*}{$\begin{array}{c}\text { Percent } \\
\text { Change } \\
1995-1996\end{array}$} & \multicolumn{2}{|c|}{ Average Annual Percent Change } \\
\hline & & & & & & & & $1992-1996$ & $1987-1996$ \\
\hline Alabama & $\$ 35.99$ & $\$ 35.73$ & $\$ 38.24$ & $\$ 41.27$ & $\$ 40.82$ & $\$ 49.85$ & 0.7 & -3.1 & -3.5 \\
\hline Alaska & w & $\mathbf{w}$ & $\mathbf{w}$ & $\mathbf{w}$ & $\mathbf{w}$ & w & $\mathbf{w}$ & w & $w$ \\
\hline Arizona & $w$ & $w$ & $\mathbf{w}$ & $w$ & $w$ & w & $\mathbf{w}$ & $w$ & $w$ \\
\hline Arkansas & - & $\mathbf{w}$ & $\mathbf{w}$ & $\mathbf{w}$ & w & $w$ & $\mathbf{w}$ & $\mathbf{w}$ & $w$ \\
\hline California & - & - & - & - & $\mathbf{w}$ & $\mathbf{w}$ & $w$ & $w$ & $w$ \\
\hline 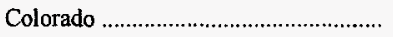 & $\$ 16.35$ & $\$ 17.90$ & $\$ 18.84$ & $\$ 19.84$ & $\$ 21.33$ & $\$ 28.37$ & -8.6 & -6.4 & -5.9 \\
\hline 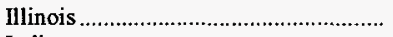 & 20.73 & 21.43 & 22.05 & 24.63 & 27.66 & 35.57 & -3.2 & -6.9 & -5.8 \\
\hline Indiana & 18.45 & 20.18 & 21.24 & 22.31 & 23.41 & 29.57 & -8.5 & -5.8 & -5.1 \\
\hline lowa & - & - & $w$ & $w$ & w & 31.15 & w & w & w \\
\hline 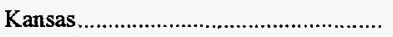 & $\mathbf{w}$ & w & w & w & w & 29.53 & w & w & w \\
\hline Kentucky Total & $\$ 21.80$ & $\$ 23.04$ & $\$ 23.72$ & $\$ 24.15$ & $\$ 24.50$ & 31.47 & -5.4 & -2.9 & -4.0 \\
\hline Eastern & 22.77 & 24.16 & 24.07 & 24.86 & 25.00 & 32.15 & -5.7 & -2.3 & -3.8 \\
\hline Western & 18.58 & 19.29 & 22.53 & 21.79 & 23.10 & 29.70 & -3.6 & -5.3 & -5.1 \\
\hline Louisiana & $w$ & w & $w$ & w & $w$ & $w$ & $w$ & w & $\mathbf{w}$ \\
\hline 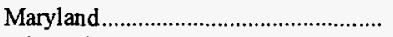 & $\$ 22.24$ & $\$ 22.95$ & $\$ 25.11$ & $\$ 24.57$ & $\$ 25.39$ & $\$ 29.25$ & -3.1 & -3.3 & -3.0 \\
\hline 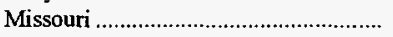 & 21.25 & 17.57 & 20.76 & w & w & 34.80 & 20.9 & w & -5.3 \\
\hline 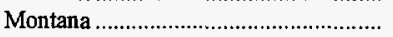 & 9.08 & 8.94 & 9.91 & $\$ 10.77$ & $\$ 10.20$ & 14.96 & 1.5 & -2.8 & -5.4 \\
\hline New Mexico & 22.48 & 22.12 & 22.20 & 22.38 & 23.14 & 26.21 & 1.6 & -.7 & -1.7 \\
\hline 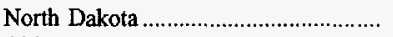 & 7.30 & 7.43 & 7.26 & 7.44 & 7.48 & 9.52 & -1.7 & -.6 & -2.9 \\
\hline Ohio & 22.65 & 24.13 & 27.77 & 27.33 & 26.93 & 37.07 & -6.1 & -4.2 & -5.3 \\
\hline 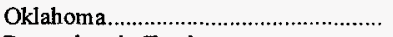 & 24.19 & 22.43 & 24.37 & 24.28 & 25.76 & 38.30 & 7.8 & -1.6 & -5.0 \\
\hline Pennsylvania Total & 23.50 & 24.89 & 24.96 & 25.83 & 28.61 & 35.87 & -5.6 & -4.8 & -4.6 \\
\hline 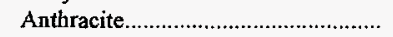 & 33.53 & 36.97 & 34.38 & 32.11 & 34.24 & 52,52 & -9.3 & -.5 & -4.9 \\
\hline 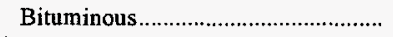 & 22.78 & 23.95 & 24.26 & 25.37 & 28.34 & 35.09 & -4.9 & -5.3 & -4.7 \\
\hline 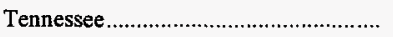 & 25.33 & 25.04 & 25.90 & 26.54 & 27.11 & 33.27 & 1.2 & -1.7 & -3.0 \\
\hline Texas & 11.09 & 11.30 & 11.81 & 12.54 & 12.42 & 14.56 & -1.9 & -2.8 & -3.0 \\
\hline 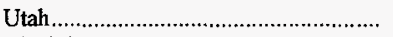 & 19.72 & 17.75 & 18.37 & 20.28 & 21.11 & 30.93 & 11.1 & -1.7 & -4.9 \\
\hline 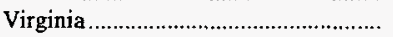 & 25.93 & 26.46 & 25.59 & 26.13 & 27.55 & 33.00 & -2.0 & -1.5 & -2.6 \\
\hline 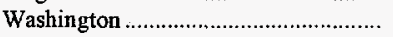 & $\mathbf{w}$ & w & w & $\mathbf{w}$ & w & w & w & $\mathbf{w}$ & w \\
\hline West Virginia Total & $\$ 24.23$ & $\$ 25.26$ & $\$ 26.14$ & $\$ 26.88$ & $\$ 28.15$ & $\$ 35.08$ & -4.1 & -3.7 & -4.0 \\
\hline 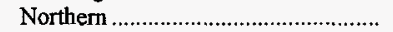 & 22.66 & 23.16 & 25.52 & 27.38 & 29.03 & 34,50 & -2.1 & -6.0 & -4.6 \\
\hline 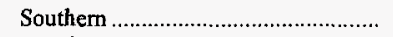 & 24.81 & 26.09 & 26.41 & 26.70 & 27.76 & 35.43 & -4.9 & -2.8 & -3.9 \\
\hline Wyoming & 5.84 & 6.11 & 6.51 & 7.13 & 8.14 & 11.80 & -4.5 & -8.0 & -7.5 \\
\hline 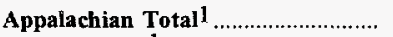 & $\mathbf{2 4 . 4 1}$ & $\mathbf{2 5 . 5 1}$ & 26.08 & 26.94 & 27.95 & 35.14 & -4.3 & -3.3 & -4.0 \\
\hline 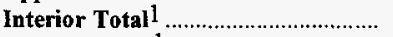 & 16.78 & 17.48 & 18.94 & 19.52 & 21.61 & 27.71 & -4.0 & -6.1 & -5.4 \\
\hline Western Total ${ }^{1} \ldots \ldots \ldots \ldots \ldots \ldots \ldots \ldots \ldots \ldots$ & 9.14 & 9.43 & 10.08 & 10.86 & 11.60 & 15.94 & $-\mathbf{3 . 0}$ & -5.8 & -6.0 \\
\hline 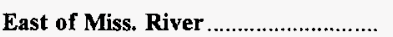 & 23.42 & 24.49 & 25.21 & 26.13 & 27.34 & 34.43 & -4.3 & -3.8 & -4.2 \\
\hline 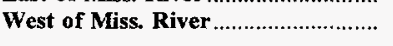 & 9.48 & 9.74 & 10.40 & 11.21 & 11.98 & 16.30 & -2.7 & -5.7 & -5.8 \\
\hline 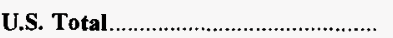 & 16.86 & 17.50 & 18.50 & 19.35 & 21.03 & 27.76 & -3.6 & -5.4 & -5.4 \\
\hline
\end{tabular}

1 For a definition of coal-producing regions, see Appendix C.

Notes: Real prices are in 1992 dollars, calculated using implicit Gross Domestic Product price deflators. See Appendix D, Table D3. Average mine price is calculated by dividing the total free on board (f.o.b.) mine value of the coal produced by the total production. Excludes silt, culm, refuse bank, slurry dam, and dredge operations except for Pennsylvania anthracite. Excludes mines producing less than 10,000 short tons, which are not required to provide these data.

Sources: Energy Information Administration, Form EIA-7A, "Coal Production Report"; State Mining Agency Coal Production Reports; and/or U.S. Department of Labor, Mine Safety and Health Administration, Form 7000-2, "Quarterly Mine Employment and Coal Production Report," 
Table 82. Average Mine Price of Coal by State and Mine Type, 1996 (Dollars per Short Ton)

\begin{tabular}{|c|c|c|c|c|c|c|}
\hline \multirow{2}{*}{$\begin{array}{l}\text { Coal-Producing } \\
\text { State and Region }\end{array}$} & \multicolumn{2}{|c|}{ Underground } & \multicolumn{2}{|c|}{ Surface } & \multicolumn{2}{|c|}{ Total } \\
\hline & Nominal & Real ${ }^{1}$ & Nominal & Real ${ }^{1}$ & Nominal & Real ${ }^{l}$ \\
\hline Alabama & $\$ 40.75$ & $\$ 37.15$ & $\$ 35.87$ & $\$ 32.69$ & $\$ 39.48$ & $\$ 35.99$ \\
\hline Alaska & - & - & w & $\mathbf{w}$ & w & w \\
\hline 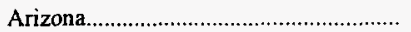 & - & - & w & w & w & w \\
\hline Colorado & 17.73 & 16.16 & $\$ 18.28$ & $\$ 16.66$ & $\$ 17.94$ & $\$ 16.35$ \\
\hline 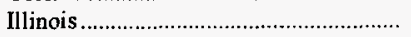 & 23.12 & 21.07 & 20.86 & 19.02 & 22.74 & 20.73 \\
\hline Indiana & $w$ & w & $w$ & w & 20.24 & 18.45 \\
\hline 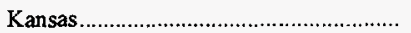 & - & - & w & w & w & w \\
\hline 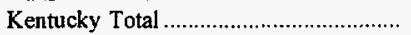 & $\$ 24.66$ & $\$ 22.48$ & $\$ 22.68$ & $\$ 20.68$ & $\$ 23.91$ & $\$ 21.80$ \\
\hline 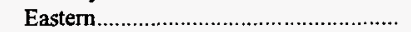 & 25.98 & 23.69 & 23.53 & 21.45 & 24.98 & 22.77 \\
\hline 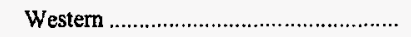 & 21.04 & 19.18 & 18.79 & 17.12 & 20.38 & 18.58 \\
\hline 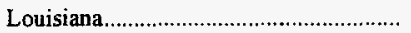 & - & - & $w$ & $\mathbf{w}$ & w & w \\
\hline 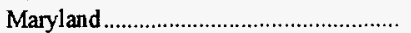 & $\mathbf{w}$ & $\mathbf{w}$ & w & w & $\$ 24.40$ & $\$ 22.24$ \\
\hline 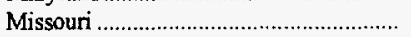 & - & - & $\$ 23,31$ & $\$ 21.25$ & 23.31 & 21.25 \\
\hline Montana & $w$ & $w$ & w & $w$ & 9.96 & 9.08 \\
\hline 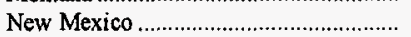 & - & - & 24.66 & 22.94 & 24.66 & 22.48 \\
\hline 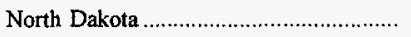 & - & - & $\$ 8.01$ & $\$ 7.30$ & 8.01 & 7.30 \\
\hline Ohio & $\$ 25.98$ & $\$ 23.68$ & 23.43 & 21.35 & 24.85 & 22.65 \\
\hline Oklahoma & $w$ & w & $w$ & w & 26.54 & 24.19 \\
\hline 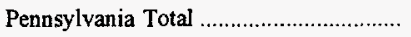 & $\$ 25.79$ & $\$ 23.51$ & $\$ 25.76$ & $\$ 23.49$ & 25.78 & 23.50 \\
\hline Anthracite & 27.99 & 25.52 & 37.37 & 34.06 & 36.78 & 33.53 \\
\hline 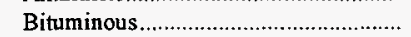 & 25.77 & 23.49 & 22.68 & 20.67 & 24.98 & 22.78 \\
\hline 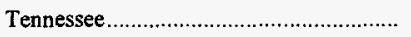 & w & $\mathbf{w}$ & $w$ & w & 27.79 & 25.33 \\
\hline 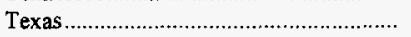 & - & - & $\$ 12.17$ & $\$ 11.09$ & 12.17 & 11.09 \\
\hline Utah & $\$ 21.63$ & $\$ 19.72$ & - & - & 21.63 & 19.72 \\
\hline Virginia & 29.46 & 26.85 & 25.88 & 23.59 & 28.45 & 25.93 \\
\hline Washington & - & - & w & w & w & w \\
\hline 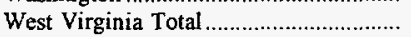 & 27.31 & 24.90 & $\$ 25.04$ & $\$ 22.82$ & $\$ 26.58$ & $\$ 24.23$ \\
\hline Northern & 25.21 & 22.98 & 22.37 & 20.39 & 24.86 & 22.66 \\
\hline Southern & 28.44 & 25.92 & 25.34 & 23.10 & 27.21 & 24.81 \\
\hline 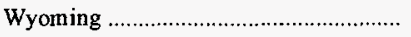 & $\mathbf{w}$ & $w$ & w & w & 6.41 & 5.84 \\
\hline 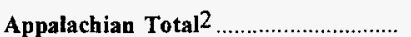 & 27.67 & 25.22 & 25.07 & 22.85 & 26.78 & 24.41 \\
\hline Interior Total 2 & 22.43 & 20.45 & 15.85 & 14.45 & 18.41 & 16.78 \\
\hline 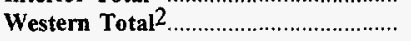 & 20.04 & 18.27 & 8.86 & 8.08 & 10.03 & 9.14 \\
\hline East of Miss. River & 26.70 & 24.34 & 23.86 & 21.75 & 25.70 & 23.42 \\
\hline 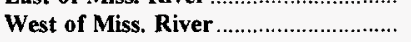 & 20.07 & 18.30 & 9.42 & 8.59 & 10.40 & 9.48 \\
\hline U.S. Total. & 25.96 & 23.66 & 13.82 & 12.60 & 18.50 & 16.86 \\
\hline
\end{tabular}

1 Real prices are in 1992 dollars, calculated using implicit Gross Domestic Product price deflators. See Appendix D, Table D3.

2 For a definition of coal-producing regions, see Appendix $C$.

Notes: Average mine price is calculated by dividing the total free on board (f.o.b.) mine value of the coal produced by the total production. A measure of dispersion of these average nominal prices at the State level (interquartile range) is given in Appendix D, Table D2. Excludes silt, culm, refuse bank, slurry dam, and dredge operations except for Pennsylvania anthracite. Excludes mines producing less than 10,000 short tons, which are not required to provide these data.

Sources: Energy Information Administration, Form ElA-7.A, "Coal Production Report'; State Mining Agency Coal Production Reports; and/or U.S. Department of Labor, Mine Safety and Health Administration, Form 7000-2, "Quarterly Mine Employment and Coal Production Report." 
Table 83. Average Mine Price of Coal by State and Underground Mining Method, 1996 (Nominal Dollars per Short Ton)

\begin{tabular}{|c|c|c|c|c|c|}
\hline $\begin{array}{l}\text { Coal-Producing } \\
\text { State and Region }\end{array}$ & Continuous 1 & Conventional 2 & Longwall ${ }^{3}$ & Other 4 & Total \\
\hline Alabama & $\mathbf{w}$ & $w$ & $\$ 40.84$ & - & $\$ 40.75$ \\
\hline 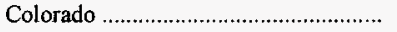 & $w$ & $\mathbf{w}$ & 17.76 & - & 17.73 \\
\hline 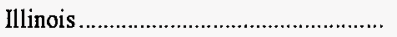 & $\$ 23.34$ & w & w & - & 23.12 \\
\hline Indiana & w & - & - & - & $w$ \\
\hline 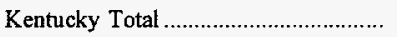 & $\$ 24.88$ & $\$ 24.73$ & $\$ 23.46$ & $\mathbf{w}$ & $\$ 24.66$ \\
\hline 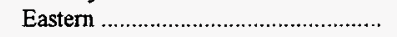 & 26.36 & w & w & $\mathrm{w}$ & 25.98 \\
\hline 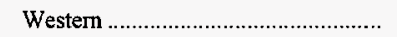 & 20.53 & $\mathbf{w}$ & w & - & 21.04 \\
\hline 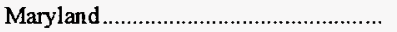 & $w$ & - & w & - & w \\
\hline Montana & w & - & - & - & w \\
\hline Ohio & $\$ 21.22$ & - & $\$ 26.60$ & - & $\$ 25.98$ \\
\hline Oklahoma & w & - & - & - & w \\
\hline 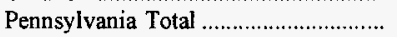 & w & $\$ 24.06$ & $w$ & w & $\$ 25.79$ \\
\hline Anthracite $\ldots \ldots \ldots \ldots \ldots \ldots \ldots \ldots \ldots \ldots \ldots$ & $\mathbf{w}$ & 34.80 & - & $w$ & 27.99 \\
\hline 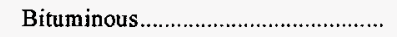 & w & 23.31 & $w$ & - & 25.77 \\
\hline 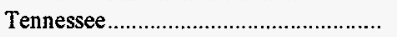 & $w$ & w & - & - & $w$ \\
\hline Utah & w & $w$ & $\$ 21.69$ & - & $\$ 21.63$ \\
\hline Virginia & $\$ 27.32$ & w & 32.90 & w & 29.46 \\
\hline West Virginia Total ............................... & 27.56 & $\$ 27.70$ & 27.04 & $\$ 16.50$ & 27.31 \\
\hline Northern & 21.69 & 25.65 & 26.09 & 16.50 & 25.21 \\
\hline Southern & 28.38 & 27.96 & 28.92 & - & 28.44 \\
\hline Wyoming & - & - & $w$ & - & w \\
\hline Appalachian Total ${ }^{5}$ & 27.14 & 26.95 & 28.42 & 22.25 & 27.67 \\
\hline Interior Total 5 . & 22.30 & $\mathbf{w}$ & $\mathbf{w}$ & - & 22.43 \\
\hline Western Total ${ }^{5}$ & 19.24 & $\mathbf{w}$ & $\mathbf{w}$ & - & 20.04 \\
\hline 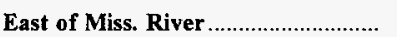 & 25.98 & $\mathbf{w}$ & $\mathbf{w}$ & 22.25 & 26.70 \\
\hline 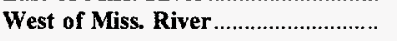 & 19.51 & $\mathbf{w}$ & $\mathbf{w}$ & - & 20.07 \\
\hline 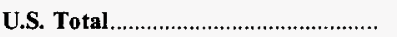 & 25.79 & 26.55 & 26.03 & 22.25 & 25.96 \\
\hline
\end{tabular}

1 Mines that produce greater than 50 percent of coal by continuous mining method.

2 Mines that produce greater than 50 percent of coal by conventional mining method.

3 Mines that have any production from longwall mining method. A typical longwall mining operation uses 80 percent longwall mining and 20 percent continuous mining.

4 Mines that produce coal using shortwall, scoop loading, hand loading, or other mining methods or a $50 / 50$ percent continuous/conventional split in mining method.

5 For a definition of coal-producing regions, see Appendix $C$.

Notes: Average mine price is calculated by dividing the total free on board (f.o.b.) mine value of the coal produced by the total production. Excludes mines producing less than 10,000 short tons, which are not required to provide these data.

Sources: Energy Information Administration, Form EIA-7A, "Coal Production Report"; State Mining Agency Coal Production Reports; and/or U.S. Department of Labor, Mine Safety and Health Administration, Form 7000-2, "Quarterly Mine Employment and Coal Production Report." 
Table 84. Coal Production, Number of Mines, and Average Mine Price, by State and County, 1996

(Thousand Short Tons, Nominal Dollars per Short Ton)

\begin{tabular}{|c|c|c|c|}
\hline $\begin{array}{l}\text { Coal-Producing } \\
\text { State and County }\end{array}$ & Number of Mines & Production & Average Mine Price \\
\hline Alabama & $\mathbf{5 3}$ & 24,637 & 39.48 \\
\hline 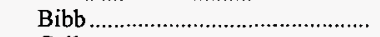 & 1 & 33 & $\mathbf{w}$ \\
\hline 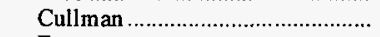 & 2 & 82 & $w$ \\
\hline 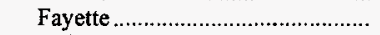 & 1 & 2,066 & $\mathbf{w}$ \\
\hline 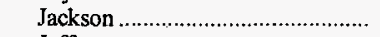 & 1 & 66 & $\mathbf{w}$ \\
\hline 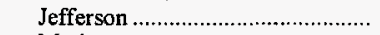 & 8 & 10,134 & $\$ 42.94$ \\
\hline 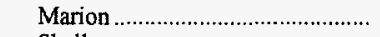 & 4 & 191 & 27.13 \\
\hline 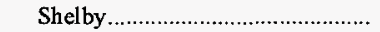 & 2 & 878 & w \\
\hline 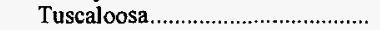 & 7 & 6,066 & $\mathbf{w}$ \\
\hline 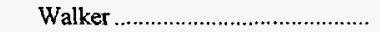 & 25 & 4,921 & $\$ 37.33$ \\
\hline Winston & 2 & 201 & w \\
\hline Alaska & 1 & 1,481 & $\mathbf{w}$ \\
\hline 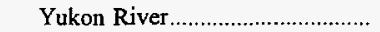 & 1 & 1,481 & w \\
\hline 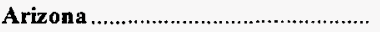 & 2 & 10,442 & $\mathbf{w}$ \\
\hline 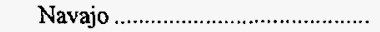 & 2 & 10,442 & w \\
\hline 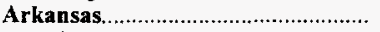 & 5 & 21 & - \\
\hline 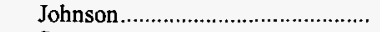 & 2 & 16 & - \\
\hline 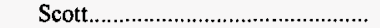 & 1 & * & - \\
\hline Sebastian & 2 & 4 & - \\
\hline 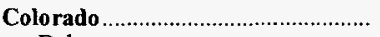 & 15 & 24,886 & 17.94 \\
\hline Delta & 1 & 603 & $w$ \\
\hline Fremont & 1 & 170 & w \\
\hline 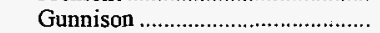 & 3 & 7,475 & $\mathbf{w}$ \\
\hline 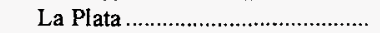 & 1 & 199 & $w$ \\
\hline 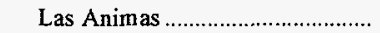 & 1 & 129 & w \\
\hline 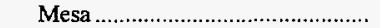 & 2 & 626 & $\mathbf{w}$ \\
\hline 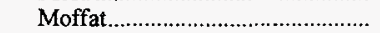 & 2 & 7,721 & $w$ \\
\hline Montrose $\ldots \ldots \ldots \ldots \ldots \ldots \ldots \ldots \ldots \ldots \ldots \ldots \ldots$ & 1 & 362 & $\mathbf{w}$ \\
\hline 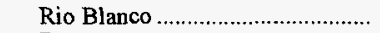 & 1 & 543 & $\mathbf{w}$ \\
\hline 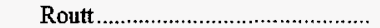 & 2 & 7,059 & $\mathbf{w}$ \\
\hline Illinois & 31 & 46,656 & 22.74 \\
\hline 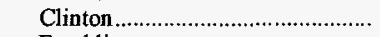 & 1 & 1,701 & $w$ \\
\hline 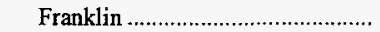 & 2 & 3,635 & w \\
\hline Fulton & 1 & 205 & $w$ \\
\hline 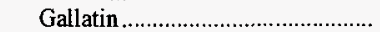 & 1 & 1,324 & w \\
\hline 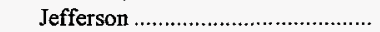 & 2 & 4,299 & $\mathbf{w}$ \\
\hline Logan & 1 & 1,982 & $\mathrm{w}$ \\
\hline 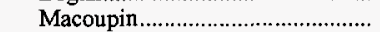 & 3 & 5,454 & $w$ \\
\hline 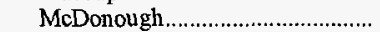 & 1 & 533 & w \\
\hline 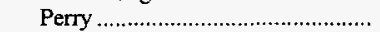 & 4 & 7,064 & $w$ \\
\hline Randolph & 2 & 2,103 & w \\
\hline Saline & 4 & 9,333 & $\$ 23.54$ \\
\hline 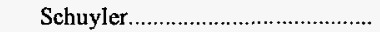 & 1 & 415 & $\mathbf{w}$ \\
\hline Vermilion & 1 & 130 & w \\
\hline 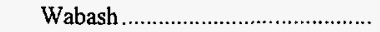 & 1 & 2,340 & w \\
\hline 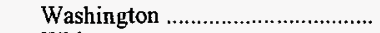 & 1 & 3,674 & $\mathbf{w}$ \\
\hline 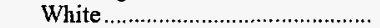 & 1 & 1,796 & w \\
\hline 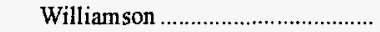 & 4 & 668 & w \\
\hline 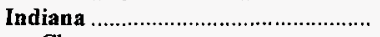 & 37 & 29,670 & 20.24 \\
\hline 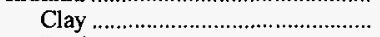 & 4 & 1,893 & $\$ 20.28$ \\
\hline 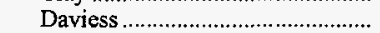 & 4 & 3,187 & 18.21 \\
\hline 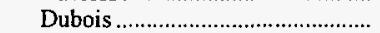 & 1 & 370 & w \\
\hline Gibson & 2 & 1,046 & w \\
\hline 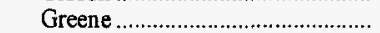 & 3 & 2,083 & w \\
\hline 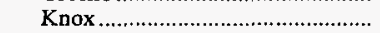 & 5 & 3,725 & 21.04 \\
\hline 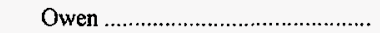 & 2 & 413 & w \\
\hline 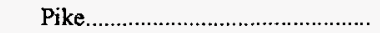 & 6 & 4,359 & $\$ 16.34$ \\
\hline Sullivan & 2 & 4,772 & w \\
\hline Vigo & 2 & 1,694 & $\mathbf{w}$ \\
\hline Warrick & 6 & 6,129 & w \\
\hline 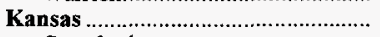 & 1 & 232 & $\mathbf{w}$ \\
\hline 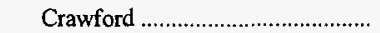 & 1 & 232 & w \\
\hline 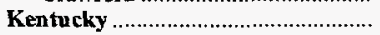 & 544 & 152,425 & 23.91 \\
\hline Bell & 25 & 5,044 & $\$ 25.53$ \\
\hline 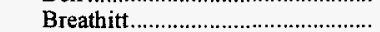 & 8 & 2,271 & 20.86 \\
\hline 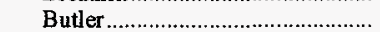 & 2 & 244 & $\mathbf{w}$ \\
\hline 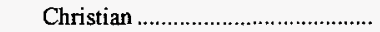 & 1 & 32 & w \\
\hline 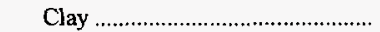 & 5 & 98 & w \\
\hline 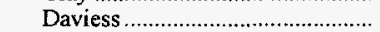 & 6 & 894 & 18.60 \\
\hline 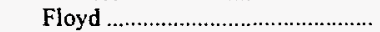 & 46 & 7,147 & $\$ 23.14$ \\
\hline 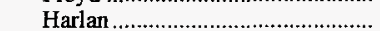 & 40 & 10,302 & 27.06 \\
\hline
\end{tabular}

See footnotes at end of table. 
Table 84. Coal Production, Number of Mines, and Average Mine Price, by State and County, 1996 (Continued)

(Thousand Short Tons, Nominal Dollars per Short Ton)

\begin{tabular}{|c|c|c|c|}
\hline $\begin{array}{l}\text { Coal-Producing } \\
\text { State and County }\end{array}$ & Number of Mines & Production & Average Mine Price \\
\hline \multicolumn{4}{|l|}{ Kentucky (Continued) } \\
\hline Henderson ..................................... & 3 & 2,084 & w \\
\hline Hopkins & 18 & 8,655 & $\$ 19.98$ \\
\hline 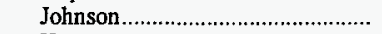 & 11 & 1,812 & 22.73 \\
\hline 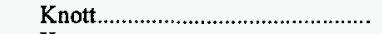 & 46 & 12,208 & 22.87 \\
\hline Knox & 15 & 467 & 30.77 \\
\hline Laurel ………….............................. & 1 & 52 & w \\
\hline 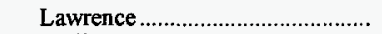 & 3 & 69 & $\mathbf{w}$ \\
\hline Leslie & 18 & 9,718 & $\$ 22.98$ \\
\hline 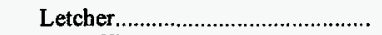 & 39 & 8,046 & 24.52 \\
\hline 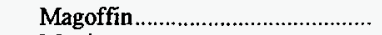 & 8 & 978 & 21.59 \\
\hline Martin & 23 & 11,785 & $\$ 28.25$ \\
\hline McLean & 3 & 194 & w \\
\hline Morgan & 3 & 5 & - \\
\hline 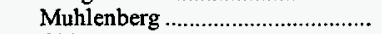 & 7 & 3,771 & $\$ 15.44$ \\
\hline Ohio & 11 & 2,142 & 17.78 \\
\hline 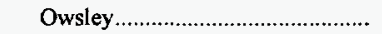 & 4 & 171 & 21.18 \\
\hline 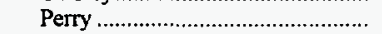 & 27 & 10,368 & $\$ 24.03$ \\
\hline Pike & 145 & 35,598 & 25.64 \\
\hline 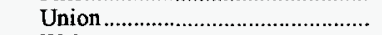 & 3 & 7,361 & w \\
\hline 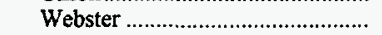 & 6 & 10,097 & $\$ 22.50$ \\
\hline Whitley & 17 & 811 & 26.34 \\
\hline Louisiana & 2 & $\mathbf{3 , 2 2 1}$ & $\mathbf{w}$ \\
\hline De Soto & 1 & 2,434 & $w$ \\
\hline 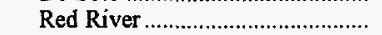 & 1 & 787 & w \\
\hline Maryland & 18 & 4,093 & 24.40 \\
\hline Allegany & 10 & 597 & w \\
\hline 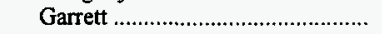 & 8 & 3,496 & w \\
\hline 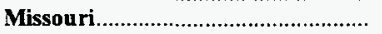 & 5 & 710 & 23.31 \\
\hline Barton & 1 & 88 & w \\
\hline Bates & 2 & 487 & $w$ \\
\hline 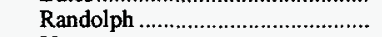 & 1 & 25 & $\mathrm{w}$ \\
\hline Vernon & 1 & 110 & w \\
\hline 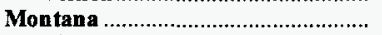 & 8 & 37,891 & 9.96 \\
\hline Big Hom …………………............ & 4 & 24,686 & $\$ 10.64$ \\
\hline 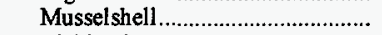 & 1 & 147 & w \\
\hline 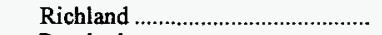 & 1 & 256 & w \\
\hline Rosebud.......................................... & 2 & 12,802 & w \\
\hline New Mexico & 6 & 24,067 & 24.66 \\
\hline Colfax & 1 & 1,259 & w \\
\hline 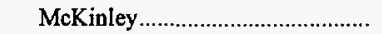 & 2 & 9,527 & w \\
\hline 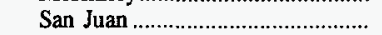 & 3 & 13,281 & w \\
\hline North Dakota............................................. & 5 & 29,861 & 8.01 \\
\hline McLean ........................................... & 1 & 7,024 & w \\
\hline 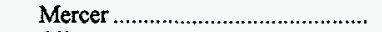 & 2 & 16,908 & w \\
\hline 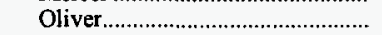 & 2 & 5,929 & $\mathrm{w}$ \\
\hline 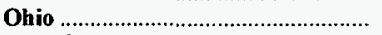 & 99 & 28,572 & 24.85 \\
\hline 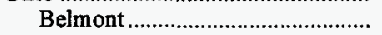 & 10 & 5,916 & w \\
\hline Carroll & 6 & 329 & $\$ 18.59$ \\
\hline 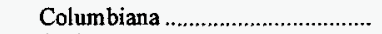 & 11 & 569 & 18.28 \\
\hline 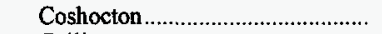 & 5 & 757 & w \\
\hline 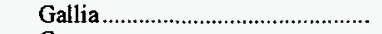 & 1 & 268 & $w$ \\
\hline 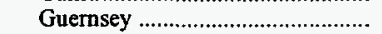 & 6 & 302 & $\$ 22.91$ \\
\hline 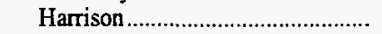 & 9 & 1,411 & 20.72 \\
\hline Holmes & 2 & 175 & w \\
\hline Jackson & 3 & 1,042 & w \\
\hline 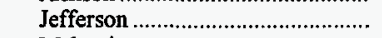 & 11 & 833 & $\$ 21.33$ \\
\hline 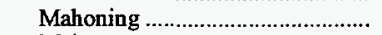 & 1 & 4 & - \\
\hline Meigs & 2 & 5,951 & w \\
\hline 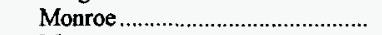 & 1 & 3,383 & w \\
\hline 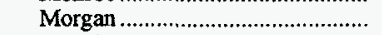 & i & 1,543 & w \\
\hline 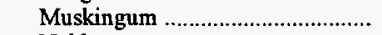 & 3 & 176 & w \\
\hline Noble & 2 & 857 & $w$ \\
\hline 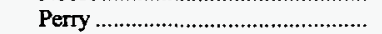 & 5 & 1,024 & $\$ 22.59$ \\
\hline Stark & 7 & 606 & 16.11 \\
\hline 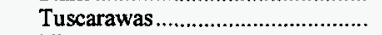 & 9 & 1,404 & 21.15 \\
\hline Vinton................................................... & 4 & 2,020 & 20.61 \\
\hline 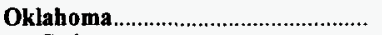 & 12 & 1,701 & 26.54 \\
\hline Craig & 1 & 130 & $\mathbf{w}$ \\
\hline 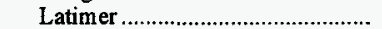 & 1 & 193 & $\mathbf{w}$ \\
\hline 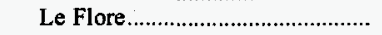 & 7 & 1,184 & $\$ 26.44$ \\
\hline
\end{tabular}

See footnotes at end of table. 
Table 84. Coal Production, Number of Mines, and Average Mine Price, by State and County, 1996 (Continued)

(Thousand Short Tons, Nominal Dollars per Short Ton)

\begin{tabular}{|c|c|c|c|}
\hline $\begin{array}{l}\text { Coal-Producing } \\
\text { State and County }\end{array}$ & Number of Mines & Production & Average Mine Price \\
\hline \multicolumn{4}{|l|}{ Oklahoma (Continued) } \\
\hline Nowata & 2 & 187 & $\mathbf{w}$ \\
\hline Okmulgee & 1 & 6 & - \\
\hline Pennsylvania & 402 & 67,942 & 25.78 \\
\hline Allegheny & 2 & 14 & $w$ \\
\hline 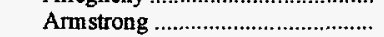 & 32 & 5,346 & $\$ 25.92$ \\
\hline 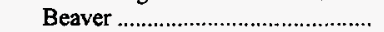 & 4 & 239 & w \\
\hline 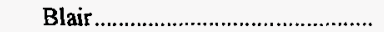 & 1 & 59 & $w$ \\
\hline 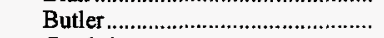 & 6 & 56 & $\mathrm{w}$ \\
\hline 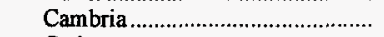 & 13 & 1,315 & $\$ 26.61$ \\
\hline Carbon & 1 & 318 & w \\
\hline 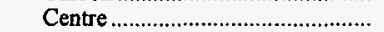 & 3 & 328 & w \\
\hline 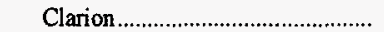 & 6 & 543 & 27.08 \\
\hline 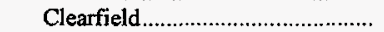 & 50 & 4,428 & $\$ 25.12$ \\
\hline 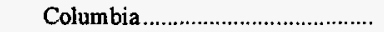 & 6 & 411 & 25.11 \\
\hline 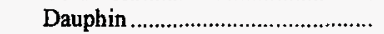 & 2 & 11 & - \\
\hline Elk & 6 & 460 & $\$ 25.01$ \\
\hline 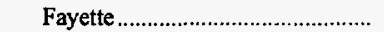 & 20 & 428 & 23.59 \\
\hline 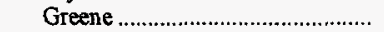 & 11 & 29,794 & 24.51 \\
\hline Indiana & 27 & 5,143 & 30.33 \\
\hline Jefferson & 25 & 1,423 & 25.15 \\
\hline 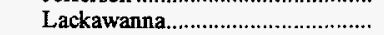 & 5 & 243 & w \\
\hline Lawrence & 2 & 139 & $\mathrm{w}$ \\
\hline 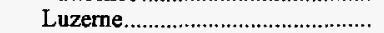 & 17 & 1,030 & $\$ 41.09$ \\
\hline Lycoming & 1 & 333 & w \\
\hline 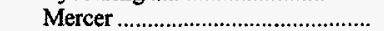 & 2 & 5 & - \\
\hline 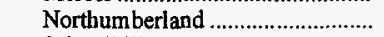 & 17 & 281 & 23.22 \\
\hline 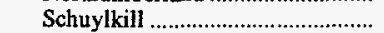 & 77 & 2,419 & $\$ 42.50$ \\
\hline 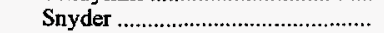 & 1 & 1 & - \\
\hline 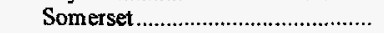 & 38 & 5,381 & 19.13 \\
\hline 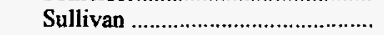 & 1 & 37 & w \\
\hline 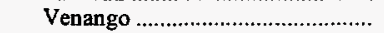 & 1 & 31 & w \\
\hline 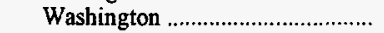 & 11 & 7,035 & $\$ 26.63$ \\
\hline 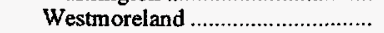 & 14 & 690 & 23.40 \\
\hline Tenn essee & 26 & 3,651 & 27.79 \\
\hline 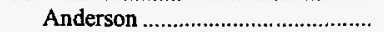 & 2 & 479 & $w$ \\
\hline Bledsoe & $\overline{1}$ & 3 & - \\
\hline 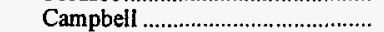 & 10 & 1,067 & $\$ 30.69$ \\
\hline 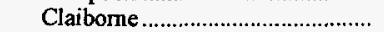 & 5 & 625 & w \\
\hline 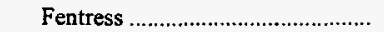 & 1 & 256 & $w$ \\
\hline Marion & 2 & 46 & $\mathbf{w}$ \\
\hline Morgan & 1 & 42 & $\mathbf{w}$ \\
\hline 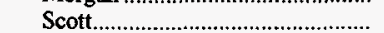 & 1 & 155 & $w$ \\
\hline Sequatchie & 3 & 978 & $\mathbf{w}$ \\
\hline 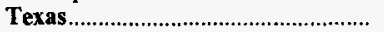 & 13 & 55,164 & 12.17 \\
\hline Atascosa & 1 & 3,324 & w \\
\hline Freestone & 1 & 5,196 & $\mathbf{w}$ \\
\hline Grimes & 1 & 567 & $w$ \\
\hline 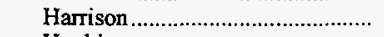 & 2 & 4,173 & w \\
\hline 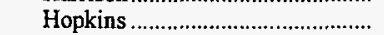 & 1 & 2,152 & $w$ \\
\hline Leon & i & 8,865 & $w$ \\
\hline Milam & 1 & 6,615 & $w$ \\
\hline 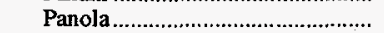 & $i$ & 6,814 & w \\
\hline 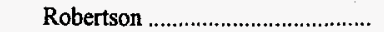 & 1 & 1,933 & $\mathbf{w}$ \\
\hline 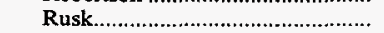 & 1 & 6,509 & $\mathbf{w}$ \\
\hline 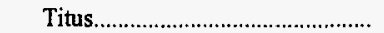 & 1 & 8,569 & w \\
\hline 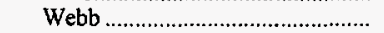 & 1 & 446 & $w$ \\
\hline Utah & 11 & 27,507 & 21.63 \\
\hline Carbon & 6 & 12,066 & $\$ 22,52$ \\
\hline 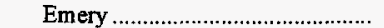 & 4 & 11,238 & $\mathbf{w}$ \\
\hline 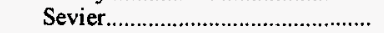 & 1 & 4,202 & $w$ \\
\hline 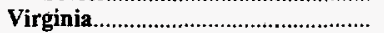 & 1191 & 35,590 & 28.45 \\
\hline 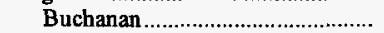 & 74 & 14,784 & $\$ 29.62$ \\
\hline Dickenson & 21 & 2,988 & 28.71 \\
\hline 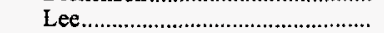 & 10 & 2,036 & 28.23 \\
\hline 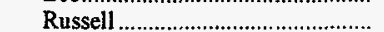 & 7 & 1,346 & 29.40 \\
\hline 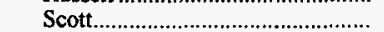 & 1 & 5 & - \\
\hline 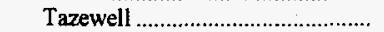 & 17 & 1,840 & $\$ 29.68$ \\
\hline 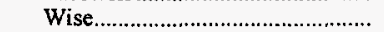 & 61 & 12,591 & 26.77 \\
\hline
\end{tabular}

See footnotes at end of table. 
Table 84. Coal Production, Number of Mines, and Average Mine Price, by State and County, 1996 (Continued)

(Thousand Short Tons, Nominal Dollars per Short Ton)

\begin{tabular}{|c|c|c|c|}
\hline $\begin{array}{l}\text { Coal-Producing } \\
\text { State and County }\end{array}$ & Number of Mines & Production & Average Mine Price \\
\hline 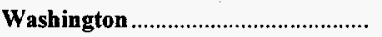 & 3 & 4,565 & w \\
\hline King & 1 & 173 & $\mathbf{w}$ \\
\hline Lewis & 1 & 2,423 & w \\
\hline Thurston ...................................... & 1 & 1,969 & $\mathbf{w}$ \\
\hline West Virginia & 386 & 170,433 & 26.58 \\
\hline 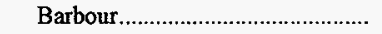 & 10 & 1,547 & w \\
\hline Boone & 47 & 31,024 & $\$ 28.15$ \\
\hline Braxton & 1 & 42 & w \\
\hline 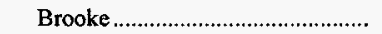 & 2 & 1,448 & w \\
\hline 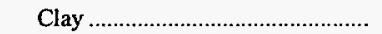 & 5 & 5,507 & w \\
\hline Fayette ............................................ & 11 & 3,964 & $\$ 28.91$ \\
\hline 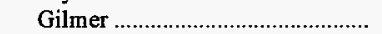 & 1 & 30 & w \\
\hline Grant & 4 & 3,039 & w \\
\hline 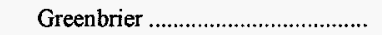 & 5 & 804 & $\$ 29.01$ \\
\hline 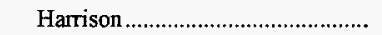 & 11 & 4,682 & w \\
\hline Kanawha & 11 & 8,964 & $\$ 22.88$ \\
\hline 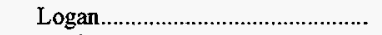 & 29 & 18,789 & 24.31 \\
\hline 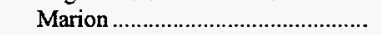 & 4 & 3,135 & w \\
\hline 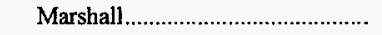 & 2 & 8,721 & w \\
\hline 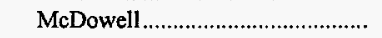 & 77 & 6,135 & $\$ 25.77$ \\
\hline Mineral & 2 & 134 & $\mathbf{w}$ \\
\hline 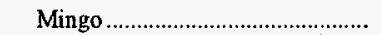 & 49 & 22,963 & $\$ 28.46$ \\
\hline 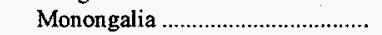 & 13 & 12,951 & 23.69 \\
\hline Nicholas........................................ & 16 & 2,522 & $\$ 27.16$ \\
\hline Preston & 15 & 1,929 & 24.02 \\
\hline Raleigh & 19 & 11,209 & 29.10 \\
\hline 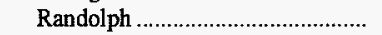 & 5 & 570 & 27.30 \\
\hline Tucker ……….................................... & 2 & 602 & w \\
\hline 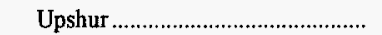 & 12 & 1,734 & $\$ 18.89$ \\
\hline 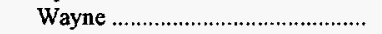 & 6 & 4,131 & 24.14 \\
\hline Webster & 9 & 5,345 & $\$ 21.68$ \\
\hline Wyoming .......................................... & 18 & 8,510 & 30.66 \\
\hline Wyoming & 27 & $\mathbf{2 7 8 , 4 4 0}$ & 6.41 \\
\hline Campbell ......................................... & 17 & 245,534 & $\$ 5.55$ \\
\hline 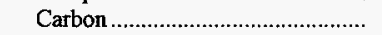 & 3 & 4,473 & $\mathbf{w}$ \\
\hline Converse & 2 & 15,839 & $\mathbf{w}$ \\
\hline Lincoln & 2 & 4,419 & w \\
\hline Sheridan & 1 & 16 & w \\
\hline 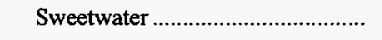 & 2 & 8,159 & w \\
\hline U.S. Total & 1,903 & $1,063,856$ & 18.50 \\
\hline
\end{tabular}

* Data round to zero.

Notes: Average mine price is calculated by dividing the total free on board (f.o.b.) mine value of the coal produced by the total production and excludes mines producing less than 10,000 short tons, which are not required to provide these data. Coal production excludes silt, culm, refuse bank, slurry dam, and dredge operations except for Pennsylvania anthracite. Totals may not equal sum of components due to independent rounding.

Sources: Energy Information Administration, Form EIA-7A, "Coal Production Report"; State Mining Agency Coal Production Reports; and/or U.S. Department of Labor, Mine Safety and Health Administration, Form 7000-2, "Quarterly Mine Employment and Coal Production Report." 
Table 85. Average Mine Price by State and Coal Rank, 1996

(Nominal Dollars per Short Ton)

\begin{tabular}{|c|c|c|c|c|c|}
\hline $\begin{array}{l}\text { Coal-Producing } \\
\text { State and Region }\end{array}$ & Bituminous & Subbitumịnous & Lignite & Anthracite & Total \\
\hline Alabama & $\$ 39.48$ & - & - & - & $\$ 39.48$ \\
\hline Alaska & - & w & - & - & $w$ \\
\hline 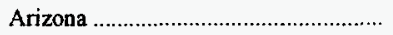 & w & - & - & - & w \\
\hline 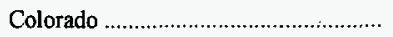 & 17.72 & $\$ 18.32$ & - & - & 17.94 \\
\hline Illinois & 22.74 & - & - & - & 22.74 \\
\hline Indiana & 20.24 & - & - & - & 20.24 \\
\hline 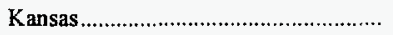 & $\mathbf{w}$ & - & - & - & w \\
\hline 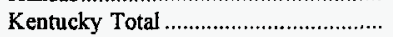 & 23.91 & - & - & - & 23.91 \\
\hline Eastern & 24.98 & - & - & - & 24.98 \\
\hline Western & 20.38 & - & - & - & 20.38 \\
\hline Louisiana & - & - & $w$ & - & $w$ \\
\hline 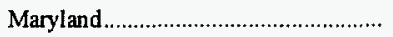 & 24.40 & - & - & - & 24.40 \\
\hline 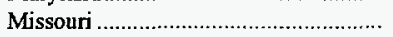 & 23.31 & - & - & - & 23.31 \\
\hline 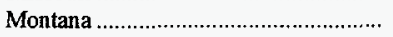 & - & $\mathbf{w}$ & $w$ & - & 9.96 \\
\hline New Mexico & w & $w$ & - & - & 24.66 \\
\hline 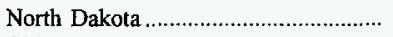 & - & - & $\$ 8.01$ & - & 8.01 \\
\hline Ohio & $\$ 24.85$ & - & - & - & 24.85 \\
\hline 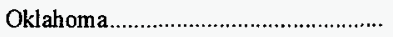 & 26.54 & - & - & - & 26.54 \\
\hline Pennsylvania Total ................................. & 24.98 & - & - & $\$ 36.78$ & 25.78 \\
\hline Anthracite. & - & - & - & 36.78 & 36.78 \\
\hline 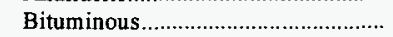 & 24.98 & - & - & - & 24.98 \\
\hline Tennessee & 27.79 & - & - & - & 27.79 \\
\hline Texas & $w$ & - & $\mathrm{w}$ & - & 12.17 \\
\hline 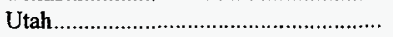 & $\$ 21.63$ & - & - & - & 21.63 \\
\hline 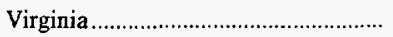 & 28.45 & - & - & - & 28.45 \\
\hline 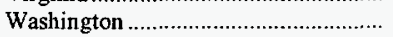 & w & $\mathrm{w}$ & - & - & w \\
\hline 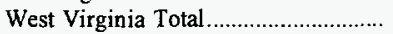 & 26.58 & - & - & - & 26.58 \\
\hline 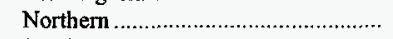 & 24.86 & - & - & - & 24.86 \\
\hline 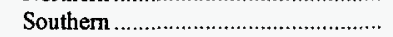 & 27.21 & - & - & - & 27.21 \\
\hline Wyoming & w & $\mathbf{w}$ & - & - & 6.41 \\
\hline 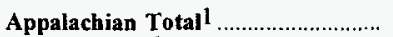 & 26.68 & - & - & 36.78 & 26.78 \\
\hline Interior Total 1 . & 21.43 & - & 12.42 & - & 18.41 \\
\hline Western Total ${ }^{1}$ & 21.61 & 7.87 & 8.04 & - & 10.03 \\
\hline 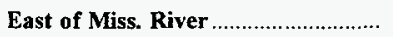 & 25.61 & - & - & 36.78 & 25.70 \\
\hline 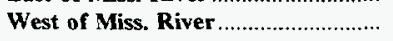 & 21.75 & 7.87 & 10.92 & - & 10.40 \\
\hline 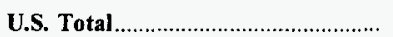 & 25.17 & 7.87 & 10.92 & 36.78 & 18.50 \\
\hline
\end{tabular}

1 For a definition of coal-producing regions, see Appendix C

Withheld to avoid disclosure of individual company data.

Notes: Average mine price is calculated by dividing the total free on board (f.o.b.) mine value of the coal produced by the total production. Excludes silt, culm, refuse bank, slurry dam, and dredge operations except for Pennsylvania anthracite. Excludes mines producing less than 10,000 short tons, which are not required to provide these data.

Sources: Energy Information Administration, Form EIA-7A, "Coal Production Report"; State Mining Agency Coal Production Reports; and/or U.S. Department of Labor, Mine Safety and Health Administration, Form 7000-2, "Quarterly Mine Employment and Coal Production Report." 
Table 86. Average Mine Price of U.S. Coal by Mine Production Range and Mine Type, 1996 (Nominal Dollars per Short Ton)

\begin{tabular}{|c|c|c|c|}
\hline $\begin{array}{l}\text { Mine Production Range } \\
\text { (thousand short tons) }\end{array}$ & Underground & Surface & Total \\
\hline Over 1,000 & $\$ 25.81$ & $\$ 11.44$ & $\$ 16.30$ \\
\hline 500 to 1,000 & 27.74 & 22.77 & 25.25 \\
\hline 200 to 500 & 25.27 & 24.09 & 24.74 \\
\hline 100 to 200 & 24.73 & 23.40 & 24.12 \\
\hline 50 to 100 & 25.71 & 25.02 & 25.40 \\
\hline 10 to 50 & 26.14 & 23.44 & 24.54 \\
\hline U.S. Total & 25.96 & 13.82 & 18.50 \\
\hline
\end{tabular}

Notes: Average mine price is calculated by dividing the total free on board (f.o.b.) mine value of the coal produced by the total production. Excludes silt, culm, refuse bank, slurry dam, and dredge operations except for Pennsyivania anthracite. Excludes mines producing less than 10,000 short tons, which are not required to provide these data.

Sources: Energy Information Administration, Form EIA-7A, “Coal Production Report"; State Mining Agency Coal Production Reports; and/or U.S. Department of Labor, Mine Safety and Health Administration, Form 7000-2, "Quarterly Mine Employment and Coal Production Report."

Table 87. Average Mine Price of U.S. Coal by Coalbed Thickness and Mine Type, 1996 (Nominal Dollars per Short Ton)

\begin{tabular}{|c|c|c|c|}
\hline $\begin{array}{c}\text { Coalbed Thickness } \\
\text { (inches) }\end{array}$ & Underground & Surface & Total \\
\hline$<7$ & - & $\$ 25.61$ & $\$ 25.61$ \\
\hline 7-12 & - & 25.32 & 25.32 \\
\hline 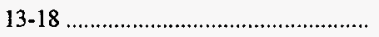 & - & 23.69 & 23.69 \\
\hline $19-24$ & $\$ 33.30$ & 20.57 & 20.82 \\
\hline $25-30$ & 25.41 & 24.10 & 24.37 \\
\hline $31-36$ & 27.26 & 23.16 & 25.19 \\
\hline 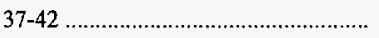 & 26.91 & 23.29 & 25.30 \\
\hline $43-48$ & 27.91 & 23.37 & 25.84 \\
\hline 49-54 & 26.51 & 23.93 & 25.70 \\
\hline $55-60$ & 27.63 & 22.25 & 26.33 \\
\hline 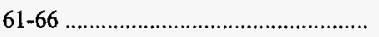 & 26.14 & 21.79 & 25.23 \\
\hline $67-72$ & 28.22 & 18.98 & 25.21 \\
\hline 73-78 & 26.03 & 22.21 & 25.12 \\
\hline 79-84 & 24.99 & 22.22 & 24.34 \\
\hline $85-90$ & 23.75 & 20.91 & 22.26 \\
\hline 91-96 & 25.14 & 26.21 & 25.34 \\
\hline $97-102$ & 21.68 & 17.19 & 18.64 \\
\hline $103-108$ & 27.36 & 12.07 & 18.09 \\
\hline $109-114$ & 15.90 & 23.47 & 17.36 \\
\hline $115-120$ & 25.40 & 26.37 & 25.75 \\
\hline$>\quad 120 \ldots \ldots$ & 19.28 & 8.34 & 8.97 \\
\hline 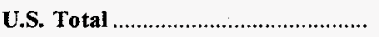 & 25.96 & 13.82 & 18.50 \\
\hline
\end{tabular}

Notes: Average mine price is calculated by dividing the total free on board (f.o.b.) mine value of the coal produced by the total production. Excludes silt, culm, refuse bank, slurry dam, and dredge operations except for Pennsylvania anthracite. Excludes mines producing less than 10,000 short tons, which are not required to provide these data.

Sources: Energy Information Administration, Form EIA-7A, "Coal Production Report"; State Mining Agency Coal Production Reports; and/or U.S. Department of Labor, Mine Safety and Health Administration, Form 7000-2, "Quarterly Mine Employment and Coal Production Report." 
Table 88. Average Mine Price of Coal by State and Productivity Range, 1996 (Nominal Dollars per Short Ton)

\begin{tabular}{|c|c|c|c|c|c|c|}
\hline \multirow{2}{*}{$\begin{array}{l}\text { Coal-Producing } \\
\text { State and Region }\end{array}$} & \multicolumn{6}{|c|}{$\begin{array}{c}\text { Productivity Range } \\
\text { (short tons per miner per hour) }\end{array}$} \\
\hline & $>=16$ & 8 to 16 & 4 to 8 & 2 to 4 & 0 to 2 & Total \\
\hline 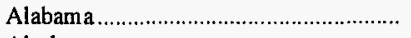 & $\mathbf{w}$ & $w$ & $\$ 29.38$ & $\$ 40.87$ & $\$ 42.84$ & $\$ 39.48$ \\
\hline 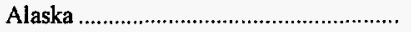 & - & - & w & - & - & w \\
\hline Arizona & - & - & w & - & - & w \\
\hline 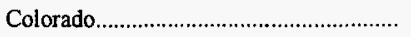 & - & $\$ 17.02$ & $\$ 16.78$ & w & $w$ & $\$ 17.94$ \\
\hline 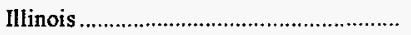 & - & $\mathbf{w}$ & 21.82 & $\$ 26.31$ & $\mathbf{w}$ & 22.74 \\
\hline Indiana & - & - & 19.76 & 23.20 & - & 20.24 \\
\hline 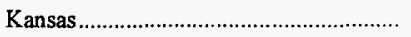 & - & - & - & w & - & w \\
\hline 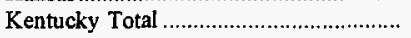 & $\$ 16.37$ & $\$ 22.48$ & 23.57 & $\$ 24.67$ & $\$ 26.84$ & $\$ 23.91$ \\
\hline Eastern & w & 24.77 & 24.66 & 25.45 & w & 24.98 \\
\hline 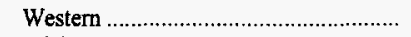 & $\mathbf{w}$ & 19.90 & 20.41 & 20.59 & $\mathbf{w}$ & 20.38 \\
\hline 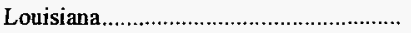 & - & w & - & - & - & w \\
\hline 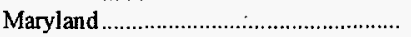 & - & - & w & 19.90 & $w$ & $\$ 24.40$ \\
\hline Missouri & - & $w$ & w & w & $w$ & 23.31 \\
\hline 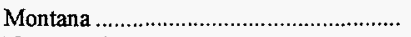 & $\$ 9.83$ & $\mathbf{w}$ & - & $w$ & - & 9.96 \\
\hline 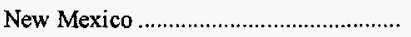 & - & $\mathbf{w}$ & $\mathrm{w}$ & - & - & 24.66 \\
\hline North Dakota & $\mathbf{w}$ & w & - & - & - & 8.01 \\
\hline Ohio & - & $\$ 23.94$ & $\$ 24.12$ & $\$ 27.19$ & $\$ 18.65$ & 24.85 \\
\hline 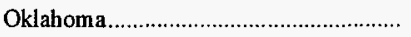 & - & - & w & 26.02 & w & 26.54 \\
\hline 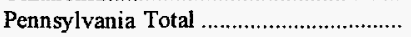 & $\$ 21.06$ & 24.84 & $\$ 23.59$ & 25.72 & $\$ 36.80$ & 25.78 \\
\hline 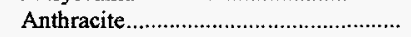 & 21.06 & 38.25 & 23.30 & 26.74 & 54.61 & 36.78 \\
\hline 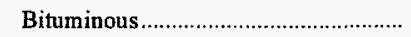 & - & 23.47 & 23.59 & 25.68 & 32.08 & 24.98 \\
\hline Tennessee & - & - & w & 25.57 & w & 27.79 \\
\hline 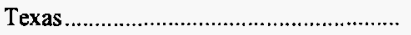 & - & 12.04 & w & w & - & 12.17 \\
\hline Utah & - & $\mathbf{w}$ & $\$ 21.49$ & w & - & 21.63 \\
\hline Virginia & - & $w$ & $\mathrm{w}$ & $\$ 29.17$ & $\$ 29.13$ & 28.45 \\
\hline Washington & - & - & w & - & w & w \\
\hline 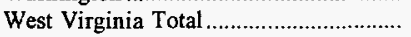 & w & $\mathbf{w}$ & $\$ 26.11$ & 28.03 & $\$ 32.72$ & $\$ 26.58$ \\
\hline 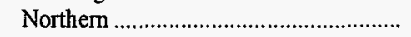 & - & $w$ & 24.69 & 25.71 & w & 24.86 \\
\hline Southern & $w$ & $\$ 25.30$ & 26.59 & 29.32 & $\mathbf{w}$ & 27.21 \\
\hline 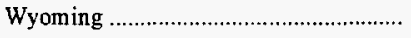 & $\mathrm{w}$ & 16.25 & w & - & w & 6.41 \\
\hline 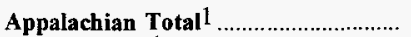 & w & 24.83 & 25.27 & 28.26 & $\mathbf{w}$ & 26.78 \\
\hline 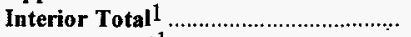 & w & 12.81 & 20.49 & 24.31 & $\mathbf{w}$ & 18.41 \\
\hline 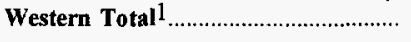 & 6.14 & 18.26 & 21.35 & 25.26 & 26.38 & 10.03 \\
\hline 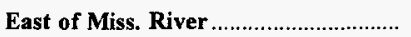 & 20.26 & 23.64 & 24.12 & 27.67 & 35.52 & 25.70 \\
\hline 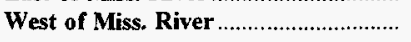 & 6.14 & 15.64 & 21.02 & 24.95 & 27.66 & 10.40 \\
\hline 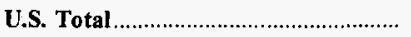 & 6.19 & 17.80 & 23.65 & 27.60 & 35.24 & 18.50 \\
\hline
\end{tabular}

1 For a definition of coal-producing regions, see Appendix $C$.

Notes: Average mine price is calculated by dividing the total free on board (f.o.b.) mine value of the coal produced by the total production. Excludes silt, culm, refuse bank, slurry dam, and dredge operations except for Pennsylvania anthracite. Excludes mines producing less than 10,000 short tons, which are not required to provide these data.

Sources: Energy Information Administration, Form EIA-7A, "Coal Production Report"; State Mining Agency Coal Production Reports; and/or U.S. Department of Labor, Mine Safety and Health Administration, Form 7000-2, "Quarterly Mine Employment and Coal Production Report." 
Table 89. Average Mine Price of Underground Coal by State and Productivity Range, 1996 (Nominal Dollars per Short Ton)

\begin{tabular}{|c|c|c|c|c|c|c|}
\hline \multirow{2}{*}{$\begin{array}{l}\text { Coal-Producing } \\
\text { State and Region }\end{array}$} & \multicolumn{6}{|c|}{$\begin{array}{c}\text { Productivity Range } \\
\text { (short tons per miner per hour) }\end{array}$} \\
\hline & $>=16$ & 8 to 16 & 4 to 8 & 2 to 4 & 0 to 2 & Total \\
\hline Alabama & - & - & $w$ & $\$ 42.30$ & $\mathbf{w}$ & $\$ 40.75$ \\
\hline Colorado & - & $w$ & w & 28.87 & $\mathbf{w}$ & 17.73 \\
\hline 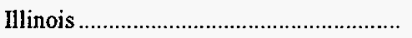 & - & - & $\$ 21.65$ & $\mathbf{w}$ & $\mathbf{w}$ & 23.12 \\
\hline Indiana & - & - & - & $\mathbf{w}$ & - & $w$ \\
\hline Kentucky Total & - & $\$ 27.06$ & 23.96 & $\$ 25.43$ & $\$ 27.42$ & $\$ 24.66$ \\
\hline 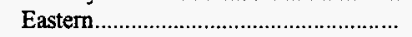 & - & 27.06 & 25.60 & $w$ & w & 25.98 \\
\hline Western & - & - & 20.99 & $w$ & $w$ & 21.04 \\
\hline 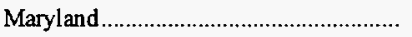 & - & - & w & - & $w$ & w \\
\hline 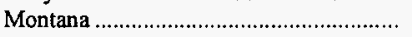 & - & - & - & w & - & w \\
\hline Ohio & - & - & $\$ 23.71$ & $\$ 33.81$ & - & $\$ 25.98$ \\
\hline 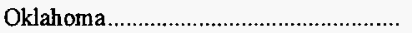 & - & - & - & - & $w$ & w \\
\hline 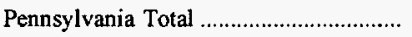 & - & $\mathbf{w}$ & 24.50 & $\mathbf{w}$ & $\$ 37.44$ & $\$ 25.79$ \\
\hline 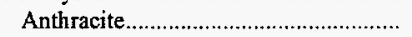 & - & - & - & $\mathbf{w}$ & $w$ & 27.99 \\
\hline 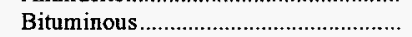 & - & $\mathbf{w}$ & 24.50 & w & $\mathbf{w}$ & 25.77 \\
\hline Tennessee & - & - & - & 26.57 & 27.65 & w \\
\hline Utah & - & w & 21.49 & w & - & $\$ 21.63$ \\
\hline 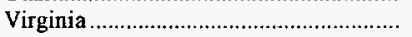 & - & - & 26.11 & $\$ 29.92$ & $\$ 28.81$ & 29.46 \\
\hline 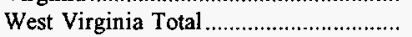 & - & $\$ 27.13$ & 26.49 & 28.11 & 34.15 & 27.31 \\
\hline Northem & - & $w$ & 24.83 & 25.77 & w & 25.21 \\
\hline 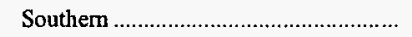 & - & $w$ & 27.52 & 29.42 & $w$ & 28.44 \\
\hline Wyoming & - & w & - & - & - & $\mathbf{w}$ \\
\hline Appalachian Total ${ }^{1} \ldots \ldots \ldots \ldots \ldots \ldots \ldots \ldots \ldots \ldots$ & - & $\mathbf{w}$ & $\mathbf{w}$ & 28.99 & 36.31 & 27.67 \\
\hline Interior Total ${ }^{1}$ & - & - & 21.37 & 24.62 & 28.41 & $\mathbf{2 2 . 4 3}$ \\
\hline 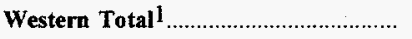 & - & $\mathbf{w}$ & $\mathbf{w}$ & 25.26 & 22.37 & 20.04 \\
\hline East of Miss. River................................... & - & $\mathbf{w}$ & $\mathbf{w}$ & 28.34 & 36.28 & 26.70 \\
\hline 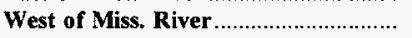 & - & $\mathbf{w}$ & $\mathbf{w}$ & 25.26 & 25.44 & 20.07 \\
\hline 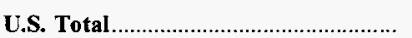 & - & 22.56 & 24.08 & 28.29 & 36.07 & 25.96 \\
\hline
\end{tabular}

1 For a definition of coal-producing regions, see Appendix $C$.

Notes: Average mine price is calculated by dividing the total free on board (fo.b.) mine value of the coal produced by the total production. Excludes silt, culm, refuse bank, slurry dam, and dredge operations except for Pennsylvania anthracite. Excludes mines producing less than 10,000 short tons, which are not required to provide these data.

Sources: Energy Information Administration, Form EIA-7A, "Coal Production Report"; State Mining Agency Coal Production Reports; and/or U.S. Department of Labor, Mine Safety and Health Administration, Form 7000-2, "Quarterly Mine Employment and Coal Production Report." 
Table 90. Average Mine Price of Surface Coal by State and Productivity Range, 1996 (Nominal Dollars per Short Ton)

\begin{tabular}{|c|c|c|c|c|c|c|}
\hline \multirow{2}{*}{ Coal-Producing State and Region } & \multicolumn{6}{|c|}{$\begin{array}{c}\text { Productivity Range } \\
\text { (short tons per miner per hour) }\end{array}$} \\
\hline & $>=16$ & 8 to 16 & 4 to 8 & 2 to 4 & 0 to 2 & Total \\
\hline Alabama & w & w & $\$ 33.12$ & $\$ 37.62$ & $w$ & $\$ 35.87$ \\
\hline 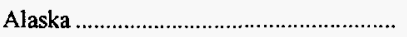 & - & - & w & - & - & w \\
\hline 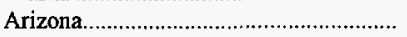 & - & - & $w$ & - & - & $\mathrm{w}$ \\
\hline 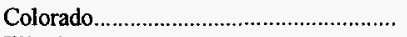 & - & w & $w$ & - & - & $\$ 18.28$ \\
\hline 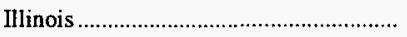 & - & $\mathbf{w}$ & $w$ & 27.66 & $w$ & 20.86 \\
\hline Indiana & - & - & $\mathbf{w}$ & w & - & $w$ \\
\hline 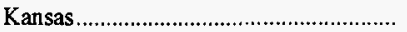 & - & - & - & w & - & w \\
\hline 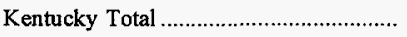 & $\$ 16.37$ & $\$ 19.88$ & $\$ 23.05$ & $\$ 22.56$ & $\$ 23.31$ & $\$ 22.68$ \\
\hline 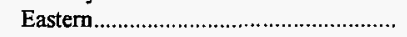 & w & 19.83 & 23.74 & w & w & 23.53 \\
\hline 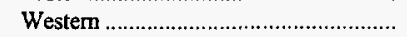 & w & 19.90 & 18.10 & $\mathbf{w}$ & $w$ & 18.79 \\
\hline 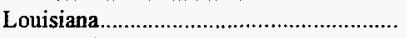 & - & w & - & - & - & w \\
\hline 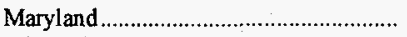 & - & - & $\mathbf{w}$ & w & $\mathbf{w}$ & w \\
\hline 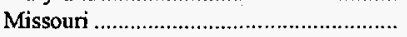 & - & w & $w$ & w & $w$ & $\$ 23.31$ \\
\hline 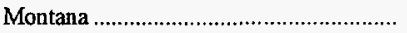 & $w$ & $\$ 15.62$ & - & - & - & 9.97 \\
\hline 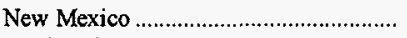 & - & w & $\mathbf{w}$ & - & - & 24.66 \\
\hline 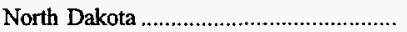 & w & w & - & - & - & $\$ 8.01$ \\
\hline Ohio & - & $\$ 23.94$ & $\$ 24.80$ & $\$ 20.98$ & $\$ 18.65$ & 23.43 \\
\hline 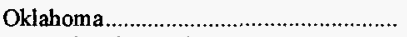 & - & - & $w$ & w & w & $\mathbf{w}$ \\
\hline 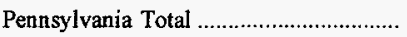 & $\$ 21.06$ & w & $\$ 21.20$ & w & $\$ 36.33$ & $\$ 25.76$ \\
\hline 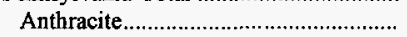 & 21.06 & $\$ 38.25$ & 23.30 & $\$ 26.66$ & w & 37.37 \\
\hline 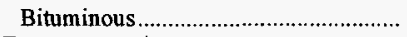 & - & 21.46 & 21.05 & 23.35 & $\mathbf{w}$ & 22.68 \\
\hline 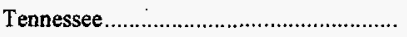 & - & - & w & w & $\mathbf{w}$ & $w$ \\
\hline 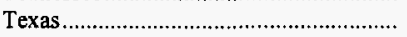 & - & 12.04 & $\mathbf{w}$ & w & - & $\$ 12.17$ \\
\hline Virginia & - & w & $\mathbf{w}$ & $\$ 24.01$ & $\$ 33.38$ & 25.88 \\
\hline Washington & - & - & w & - & w & $w$ \\
\hline 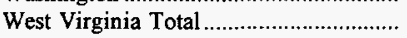 & $w$ & $w$ & $\$ 25.47$ & 27.21 & $\$ 22.22$ & $\$ 25.04$ \\
\hline Northern & - & $w$ & 22.36 & 25.06 & $\mathbf{w}$ & 22.37 \\
\hline Southern & $\mathbf{w}$ & $\$ 23.94$ & 25.59 & 28.26 & $w$ & 25.34 \\
\hline Wyoming & $\mathbf{w}$ & w & w & - & $\mathbf{w}$ & $\mathbf{w}$ \\
\hline Appalachian Total ${ }^{l}$ & 20.80 & 23.97 & 24.72 & 25.47 & 33.17 & 25.07 \\
\hline Interior Total ${ }^{1}$ & 16.03 & 12.81 & 19.34 & 23.49 & 28.80 & 15.85 \\
\hline Western Total ${ }^{1} \ldots \ldots \ldots \ldots \ldots \ldots \ldots \ldots \ldots \ldots \ldots$ & 6.14 & 17.53 & 22.43 & - & 30.62 & 8.86 \\
\hline East of Miss. River & 20.26 & 22.07 & 23.48 & 25.11 & 33.16 & 23.86 \\
\hline 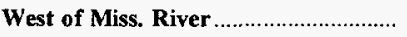 & 6.14 & 14.74 & 21.82 & 24.54 & 29.14 & 9.42 \\
\hline U.S. Total & 6.19 & 16.22 & 23.12 & 25.08 & $\mathbf{3 2 . 8 3}$ & 13.82 \\
\hline
\end{tabular}

1 For a definition of coal-producing regions, see Appendix $\mathrm{C}$

Notes: Average mine price is calculated by dividing the total free on board (f.o.b.) mine value of the coal produced by the total production. Excludes silt, culm, refuse bank, slurry dam, and dredge operations except for Pennsylvania anthracite. Excludes mines producing less than 10,000 short tons, which are not required to provide these data.

Sources: Energy Information Administration, Form EIA-7A "Coal Production Report"; State Mining Agency Coal Production Reports; and/or U.S. Department of Labor, Mine Safety and Health Administration, Form 7000-2, "Quarterly Mine Employment and Coal Production Report." 
Table 91. Average Mine Price by State and Disposition, 1996

(Nominal Dollars per Short Ton)

\begin{tabular}{|c|c|c|c|}
\hline $\begin{array}{l}\text { Coal-Producing } \\
\text { State and Region }\end{array}$ & Open Market ${ }^{1}$ & Captive $^{2}$ & Total \\
\hline Alabama & w & $\mathbf{w}$ & 39.48 \\
\hline Alaska & $\mathbf{w}$ & $\mathbf{w}$ & w \\
\hline Arizona & w & - & w \\
\hline 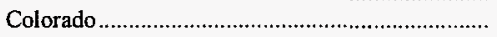 & 17.94 & - & 17.94 \\
\hline Illinois & w & $\mathbf{w}$ & 22.74 \\
\hline 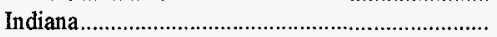 & $\mathbf{w}$ & w & 20.24 \\
\hline 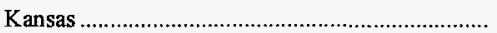 & $w$ & - & w \\
\hline 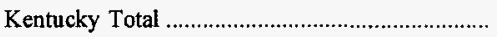 & 23.81 & 29.04 & 23.91 \\
\hline Eastern & $\mathbf{w}$ & w & 24.98 \\
\hline 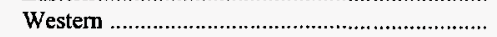 & $\mathbf{w}$ & $\mathbf{w}$ & 20.38 \\
\hline 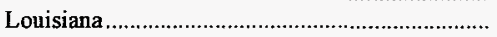 & $\mathbf{w}$ & - & w \\
\hline Maryland & $\mathbf{w}$ & w & 24.40 \\
\hline 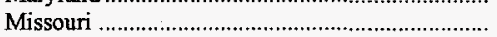 & 23.31 & - & 23.31 \\
\hline Montana & $w$ & $w$ & 9.96 \\
\hline 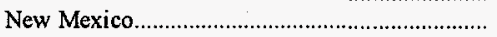 & 24.66 & - & 24.66 \\
\hline North Dakota & w & w & 8.01 \\
\hline Ohio & 20.17 & 37.01 & 24.85 \\
\hline Oklahoma & 26.54 & - & 26.54 \\
\hline 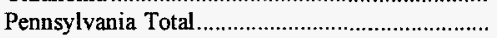 & 25.80 & 25.25 & 25.78 \\
\hline Anthracite & 42.53 & 27.08 & 36.78 \\
\hline 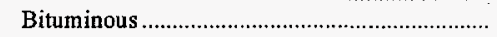 & 25.03 & 21.02 & 24.98 \\
\hline Tennessee & $w$ & $\mathbf{w}$ & 27.79 \\
\hline Texas & 13.09 & 11.82 & 12.17 \\
\hline Utah & 24.05 & 15.96 & 21.63 \\
\hline Virginia & 28.54 & 26.97 & 28.45 \\
\hline Washington & $\mathbf{w}$ & w & w \\
\hline 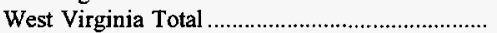 & 26.39 & 39.09 & 26.58 \\
\hline Northern & 24.30 & 48.30 & 24.86 \\
\hline Southern & 27.15 & 32.41 & 27.21 \\
\hline Wyoming & 6.03 & 12.48 & 6.41 \\
\hline 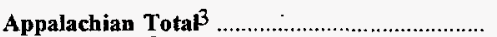 & 26.48 & 33.56 & 26.78 \\
\hline Interior Tota $\beta$ & 20.35 & 12.07 & 18.41 \\
\hline Western Tota $\beta^{3}$ & 9.65 & 14.82 & 10.03 \\
\hline 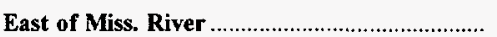 & 25.42 & 33.43 & 25.70 \\
\hline 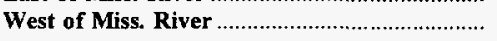 & 9.93 & 13.16 & 10.40 \\
\hline U.S. Total & 18.60 & 17.42 & 18.50 \\
\hline
\end{tabular}

1 Open Market includes all coal sold on the open market to other coal companies or consumers.

2 Captive includes all coal used by the producing company or sold to affiliated or parent companies.

3 For a definition of coal-producing regions, see Appendix $\mathrm{C}$.

Notes: Average mine price is calculated by dividing the total free on board (f.o.b.) mine value of the coal produced by the total production. Excludes silt, culm, refuse bank, slurry dam, and dredge operations except for Pennsylvania anthracite. Excludes mines producing less than 10,000 short tons, which are not required to provide these data.

Sources: Energy Information Administration, Form EIA-7A, "Coal Production Report"; State Mining Agency Coal Production Reports; and/or U.S. Department of Labor, Mine Safety and Health Administration, Form 7000-2, "Quarterly Mine Employment and Coal Production Report." 


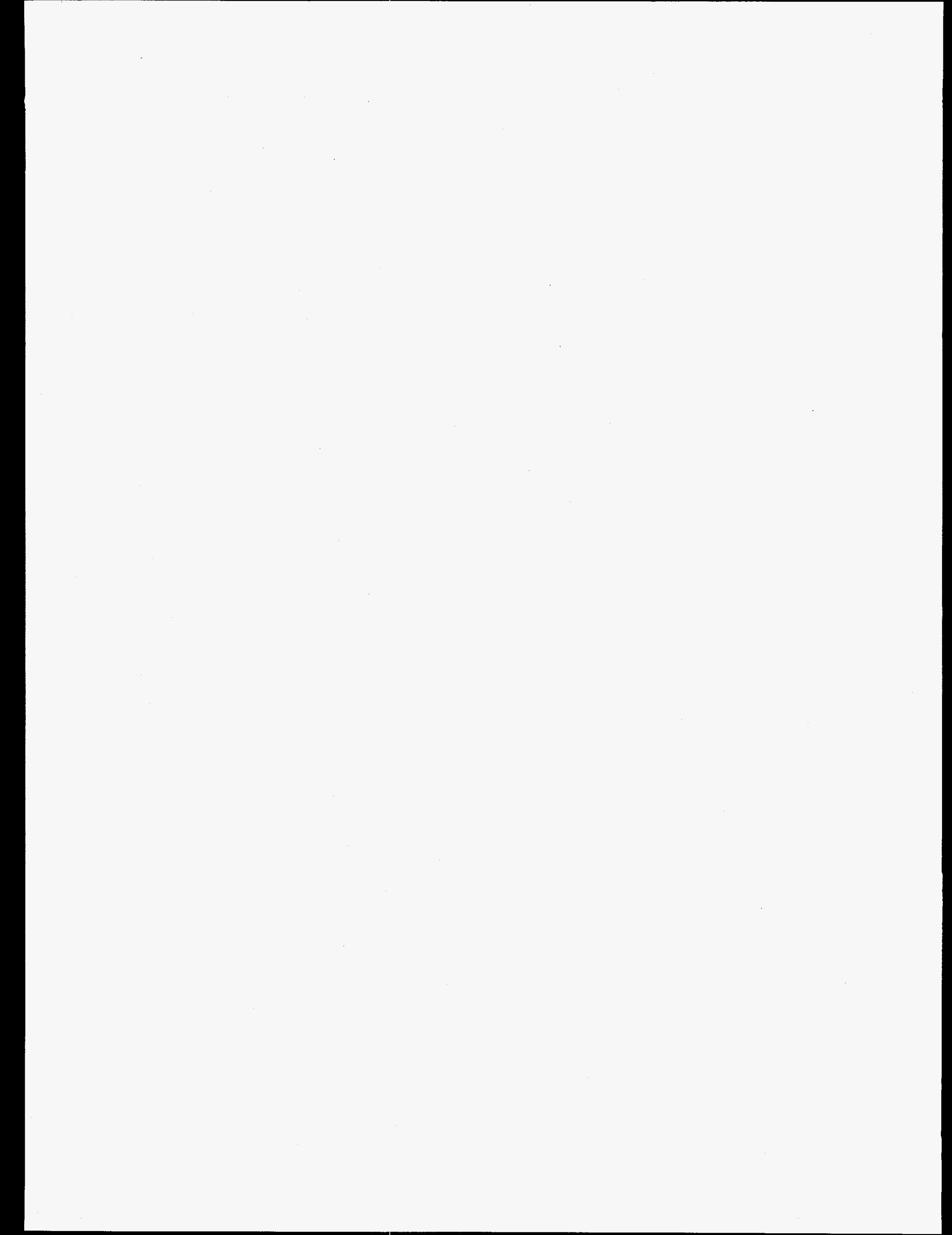




\section{Consumer Prices}

Figure 13. U.S. Coal Prices by Sector, 1987-1996

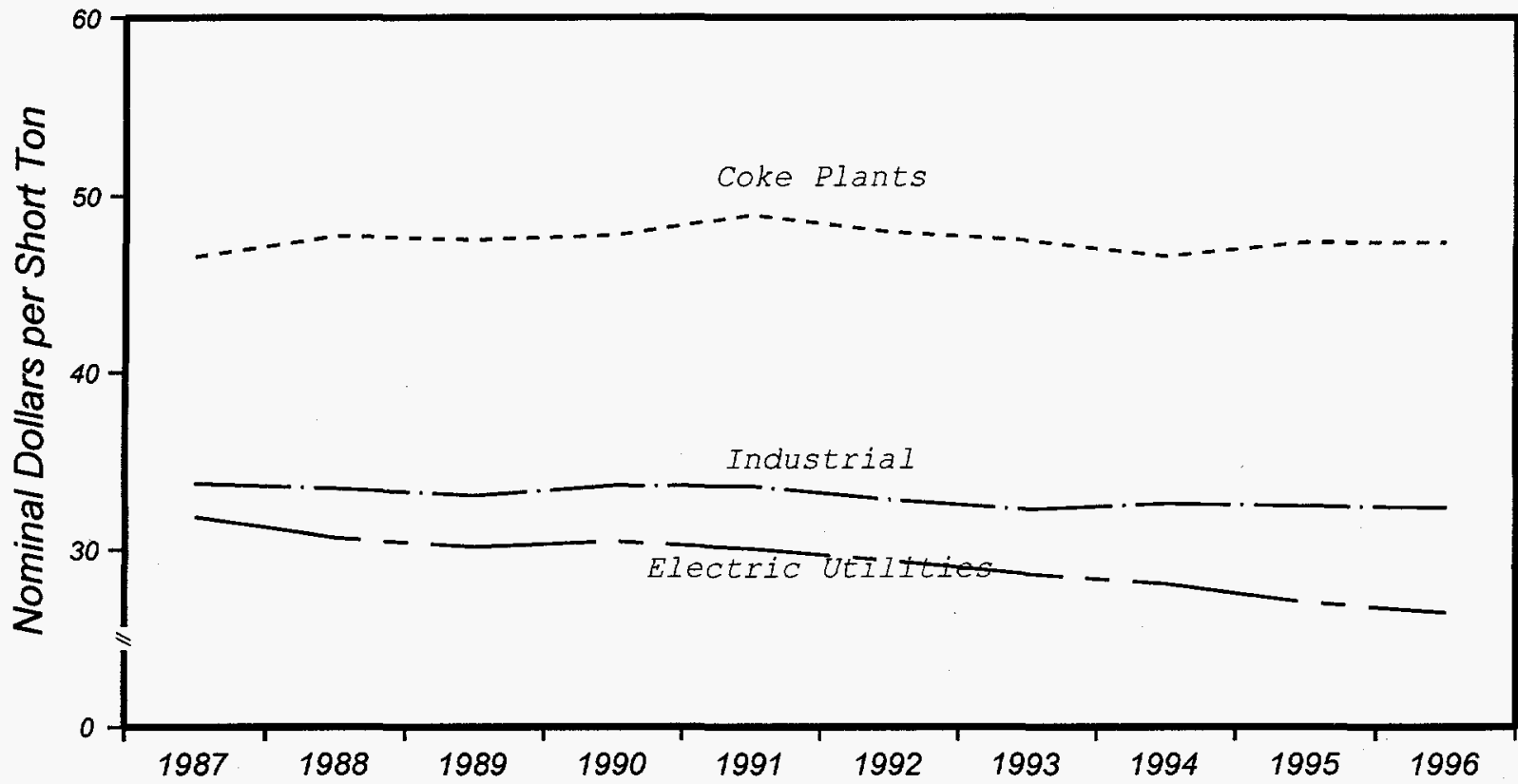

Sources: "Electric Utililies: Federal Energy Regulalory Commission(FERC), FERC Form 423 , "Monthly Report of Cost and Quality of Fuels for Electcic Plants." Coke Plonis: Energy Informolion Administrotion (EiA), Form EIA-5. "Coke Plonl Report - Quorterly." Industrial: ElA, Form ElA-3, "Quarlerly Cool Consumplion Report - Manufocluring Plonts." 
Table 92. Average Price of Coal Delivered to Electric Utilities by Census Division and State, 1987, 1992-1996

(Nominal Dollars per Short Ton)

\begin{tabular}{|c|c|c|c|c|c|c|c|c|c|}
\hline \multirow{2}{*}{ Census Division and State } & \multirow{2}{*}{1996} & \multirow{2}{*}{ 1995: } & \multirow{2}{*}{1994} & \multirow{2}{*}{1993} & \multirow{2}{*}{1992} & \multirow{2}{*}{1987} & \multirow{2}{*}{$\begin{array}{c}\text { Percent } \\
\text { Change } \\
1995-1996\end{array}$} & \multicolumn{2}{|c|}{ Average Annual Percent Change } \\
\hline & & & & & & & & $1992-1996$ & $1987-1996$ \\
\hline 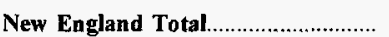 & $\$ 43.55$ & $\$ 4.3 .34$ & $\$ 42.81$ & $\$ 43.34$ & $\$ 45.14$ & $\$ 46.95$ & 0.5 & -0.9 & -0.8 \\
\hline 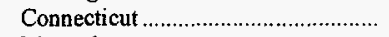 & 50.05 & 49.33 & 46.45 & 44.80 & 51.30 & 63.56 & 1.5 & -.6 & -2.6 \\
\hline 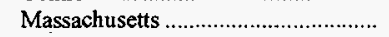 & 42.64 & 42.63 & 43.00 & 43.39 & 44.11 & 42.37 & $*$ & -.8 & .1 \\
\hline 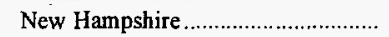 & 42.23 & 41.67 & 39.66 & 42.39 & 44.69 & 51.47 & 1.3 & -1.4 & -2.2 \\
\hline Middle Atlantic Total. & 35.08 & 34.63 & 36.33 & 36.66 & 37.56 & 36.60 & 1.3 & -1.7 & -.5 \\
\hline 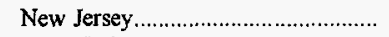 & 45.53 & 47.17 & 48.49 & 47.50 & 46.62 & 46.80 & -3.5 & -.6 & -.3 \\
\hline 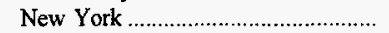 & 37.15 & 36.86 & 37.63 & 38.63 & 38.62 & 39.46 & .8 & -1.0 & -.7 \\
\hline 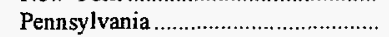 & 34.06 & 3.3 .48 & 35.39 & 35.73 & 36.81 & 35.43 & 1.7 & -1.9 & -.4 \\
\hline East North Central Total .................. & 28.29 & 29.67 & 30.56 & 30.98 & 32.05 & 36.62 & -4.6 & -3.1 & -2.8 \\
\hline 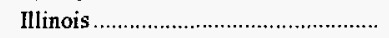 & 32.14 & 32.58 & 32.69 & 35.30 & 37.06 & 42.80 & -1.3 & -3.5 & -3.1 \\
\hline Indiana & 24.67 & 25.94 & 26.79 & 26.73 & 27.89 & 31.93 & -4.9 & -3.0 & -2.8 \\
\hline 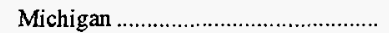 & 29.34 & 30.95 & 32.90 & .33 .17 & 34.23 & 40.55 & -5.2 & -3.8 & -3.5 \\
\hline Ohio & 32,31 & 34.44 & 34.70 & 34.05 & 34.40 & 37.15 & -6.2 & -1.5 & -1.5 \\
\hline 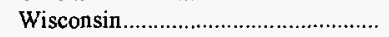 & 19.55 & 21.23 & 23.13 & 22.96 & 25.92 & 28.35 & -7.9 & -6.8 & -4.0 \\
\hline West North Central Total ..................... & 15.53 & 16.10 & 16.76 & 16.88 & 18.92 & 21.23 & -3.6 & -4.8 & -3.4 \\
\hline Iowa & 16.30 & 17.13 & 17.39 & 17.53 & 19.58 & 22.81 & -4.8 & -4.5 & -3.7 \\
\hline 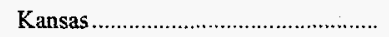 & 17.51 & 17.83 & 17.85 & 17.69 & 20.99 & 22.22 & -1.8 & -4.4 & -2.6 \\
\hline 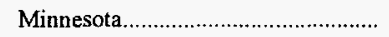 & 18.99 & 20.12 & 20.09 & 20.07 & 20.96 & 21.09 & -5.6 & -2.4 & -1.1 \\
\hline 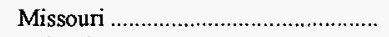 & 17.31 & 18.14 & 21.39 & 24.40 & 27.57 & 29.96 & -4.6 & -11.0 & -5.9 \\
\hline 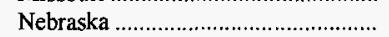 & 12.37 & 12.86 & 13.11 & 12.92 & 12.77 & 16.26 & -3.8 & -.8 & -3.0 \\
\hline 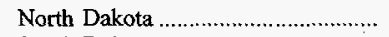 & 9.72 & 9.65 & 9.28 & 9.38 & 9.45 & 10.46 & .7 & .7 & -.8 \\
\hline South Dakota & 16.94 & 14.35 & 13.10 & 13.30 & 13.68 & 14.95 & 18.0 & 5.5 & 1.4 \\
\hline South Atlantic Total & 36.68 & $38: .25$ & 39.53 & 40.80 & 41.28 & 41.51 & -4.1 & -2.9 & -1.4 \\
\hline Delaware & 41.51 & $42: 27$ & 41.98 & 44.02 & 45.31 & 47.33 & -1.8 & -2.2 & $-1,4$ \\
\hline Florida & 42.40 & 43.93 & 43.71 & 43.58 & 45.03 & 44.98 & -3.5 & -1.5 & -.6 \\
\hline 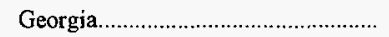 & 36.54 & $38: 62$ & 39.82 & 43.29 & 43.36 & 43.56 & -5.4 & -4.2 & -1.9 \\
\hline 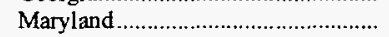 & 38.49 & 39.00 & 39.84 & 40.78 & 40.68 & 39.97 & -1.3 & -1.4 & -.4 \\
\hline 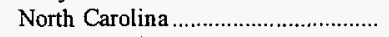 & 36.87 & 40.57 & 41.77 & 42.36 & 43.00 & 44.92 & -9.1 & -3.8 & -2.2 \\
\hline 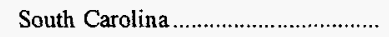 & 37.54 & $38: 86$ & 39.84 & 40.17 & 39.13 & 44.01 & -3.4 & -1.0 & -1.8 \\
\hline 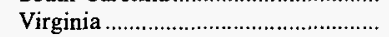 & 35.73 & 36.90 & 37.05 & 37.57 & 37.81 & 40.52 & -3.2 & -1.4 & -1.4 \\
\hline 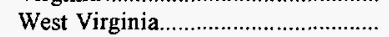 & 30.93 & 31.61 & 34.70 & 35.42 & 36.88 & 67.41 & -2.1 & -4.3 & -101.5 \\
\hline East South Central Total ................... & 29.35 & 30.08 & 32.43 & 33.30 & 33.05 & 35.95 & -2.4 & -2.9 & -2.2 \\
\hline 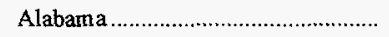 & 36.39 & 37.00 & 40.42 & 42.56 & 41.67 & 47.04 & -1.6 & -3.3 & -2.8 \\
\hline 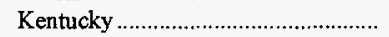 & 24.43 & 25.71 & 27.16 & 27.29 & 27.01 & 28.94 & -5.0 & -2.5 & -1.9 \\
\hline Mississippi & 33.31 & 34.40 & 35.54 & 40.51 & 39.94 & 49.56 & -3.2 & -4.4 & -4.3 \\
\hline 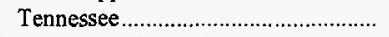 & 27.64 & 27.94 & 30.61 & 30.94 & 31.01 & 32.74 & -1.1 & -2.8 & -1.9 \\
\hline West South Central Total ...................... & 20.13 & 20.66 & 20.79 & 22.14 & 22.55 & 23.92 & -2.6 & -2.8 & -1.9 \\
\hline 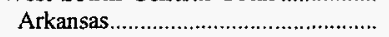 & 26.15 & 27.99 & 27.91 & 29.50 & 28.84 & 26.94 & -6.5 & -2.4 & -.3 \\
\hline 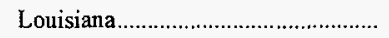 & 24.74 & 25.13 & 25.04 & 25.65 & 24.93 & 26.84 & -1.5 & -.2 & -.9 \\
\hline 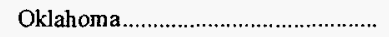 & 16.79 & 17.00 & 17.50 & 21.32 & 21.47 & 27.56 & -1.2 & -5.9 & -5.3 \\
\hline 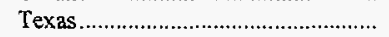 & 19.26 & 19.65 & 19.84 & 20.91 & 21.58 & 22.48 & -1.9 & -2.8 & -1.7 \\
\hline 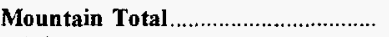 & 21.82 & 21.51 & 21.83 & 22.11 & 21.64 & 22.01 & 1.5 & .2 & -.1 \\
\hline Arizona & 29.55 & 28.65 & 28.26 & 27.78 & 28.31 & 27.56 & 3.1 & 1.1 & .8 \\
\hline Colorado & 20.24 & 20.73 & 21.01 & 21.59 & 21.67 & 22.01 & -2.4 & -1.7 & -.9 \\
\hline 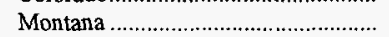 & 11.90 & 11.47 & 11.79 & 11.78 & 12.14 & 11.12 & 3.8 & -.5 & .7 \\
\hline 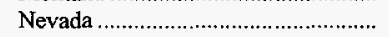 & 30.44 & 29.02 & 32.37 & 32.34 & 32.32 & 31.28 & 4.9 & -1.5 & -.3 \\
\hline 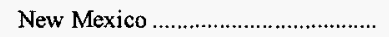 & 26.04 & 25.59 & 25.48 & 24.61 & 23.83 & 21.87 & 1.8 & 2.2 & 2.0 \\
\hline 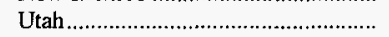 & 24.66 & 25.27 & 26.10 & 27.34 & 27.54 & 29.09 & -2.4 & -2.7 & -1.8 \\
\hline 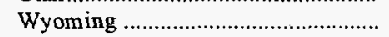 & 14.30 & 14.29 & 14.09 & 14.03 & 13.42 & 15.27 & 1 & 1.6 & -.7 \\
\hline Pacific Total & 23.96 & 22.83 & 21.93 & 21.55 & 22.17 & 26.82 & 5.0 & 2.0 & -1.2 \\
\hline Oregon & 18.81 & 18.79 & 19.18 & 19.75 & 21.23 & 23.78 & .1 & -3.0 & -2.6 \\
\hline 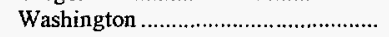 & 24.91 & 23.74 & 22.93 & 22.09 & 22.48 & 27.08 & 4.9 & 2.6 & -.9 \\
\hline U.S. Total & 26.45 & 27.01 & 28.03 & 28.58 & 29.36 & 31.83 & -2.1 & -2.6 & -2.0 \\
\hline
\end{tabular}

* Data round to zero.

Note: Average prices are based on the cost including insurance and freight

Source: Federal Energy Regulatory Commission, FERC Form 423, "Monthly Report of Cost and Quality of Fuels for Electric Plants." 
Table 93. Average Real Price of Coal Delivered to Electric Utilities by Census Division and State, 1987, 1992-1996

(Real Dollars per Short Ton)

\begin{tabular}{|c|c|c|c|c|c|c|c|c|c|}
\hline \multirow{2}{*}{ Census Division and State } & \multirow{2}{*}{1996} & \multirow{2}{*}{1995} & \multirow{2}{*}{1994} & \multirow{2}{*}{1993} & \multirow{2}{*}{1992} & \multirow{2}{*}{1987} & \multirow{2}{*}{$\begin{array}{c}\text { Percent } \\
\text { Change } \\
\text { 1995-1996 }\end{array}$} & \multicolumn{2}{|c|}{ Average Annual Percent Change } \\
\hline & & & & & & & & 1992-1996 & 1987-1996 \\
\hline 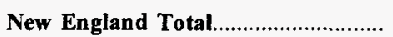 & $\$ 39.70$ & $\$ 40.28$ & $\$ 40.81$ & $\$ 42.24$ & $\$ 45.14$ & $\$ 56.50$ & -1.4 & -3.1 & -3.8 \\
\hline 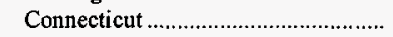 & 45.63 & 45.84 & 44.28 & 43.66 & 51.30 & 76.48 & -.5 & -2.9 & -5.6 \\
\hline 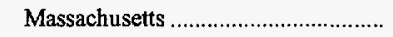 & 38.87 & 39.62 & 40.99 & 42.29 & 44.11 & 50.99 & -1.9 & -3.1 & -3.0 \\
\hline New Hampshire .................................... & 38.49 & 38.72 & 37.81 & 41.32 & 44.69 & 61.93 & -6 & -3.7 & -5.1 \\
\hline Middle Atlantic Total & 31.98 & 32.18 & 34.63 & 35.73 & 37.56 & 44.04 & -.6 & -3.9 & -3.5 \\
\hline 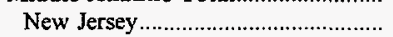 & 41.50 & 43.84 & 46.22 & 46.30 & 46.62 & 56.32 & -5.3 & -2.9 & -3.3 \\
\hline 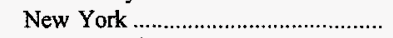 & 33.87 & 34.25 & 35.88 & 37.65 & 38.62 & 47.48 & -1.1 & -3.2 & -3.7 \\
\hline Pennsylvania & 31.05 & 31.12 & 33.73 & 34.82 & 36.81 & 42,63 & -.2 & -4.2 & -3.5 \\
\hline East North Central Total ...................... & 25.79 & 27.57 & 29.13 & 30.20 & 32.05 & 44.07 & -6.5 & -5.3 & -5.8 \\
\hline 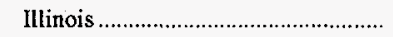 & 29.30 & 30.28 & 31.17 & 34.41 & 37.06 & 51.50 & -3.2 & -5.7 & -6.1 \\
\hline Indiana & 22.49 & 24.11 & 25.54 & 26.06 & 27.89 & 38.42 & -6.7 & -5.2 & -5.8 \\
\hline Michigan & 26.74 & 28.76 & 31.36 & 32.33 & 34.23 & 48.79 & -7.0 & -6.0 & -6.5 \\
\hline Ohio & 29.45 & 32.00 & 33.08 & 33.19 & 34.40 & 44.70 & -8.0 & -3.8 & -4.5 \\
\hline 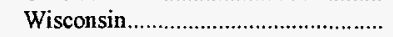 & 17.82 & 19.73 & 22.05 & 22.38 & 25.92 & 34.11 & -9.7 & -8.9 & -7.0 \\
\hline West North Central Total ................. & 14.15 & 14.97 & 15.97 & 16.45 & 18.92 & 25.55 & -5.4 & -7.0 & -6.3 \\
\hline Iowa & 14.86 & 15.92 & 16.58 & 17.08 & 19.58 & 27.45 & -6.7 & -6.7 & -6.6 \\
\hline Kansas & 15.96 & 16.57 & 17.02 & 17.24 & 20.99 & 26.74 & -3.7 & -6.6 & -5.6 \\
\hline 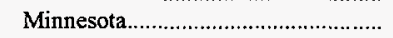 & 17.32 & 18.70 & 19.15 & 19.56 & 20.96 & 25.37 & -7.4 & -4.6 & -4.1 \\
\hline 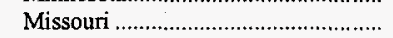 & 15.78 & 16.86 & 20.39 & 23.78 & 27.57 & 36.05 & -6.4 & -13.0 & -8.8 \\
\hline Nebraska & 11.28 & 11.96 & 12.50 & 12.59 & 12.77 & 19.56 & -5.7 & -3.0 & -5.9 \\
\hline 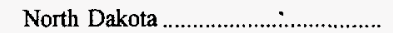 & 8.86 & 8.97 & 8.85 & 9.14 & 9.45 & 12.59 & -1.2 & -1.6 & -3.8 \\
\hline South Dakota & 15.44 & 13.34 & 12.49 & 12.96 & 13.68 & 18.00 & 15.7 & 3.1 & -1.7 \\
\hline 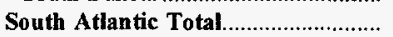 & 33.44 & 35.55 & 37.68 & 39.77 & 41.28 & 49.95 & -5.9 & -5.1 & -4.4 \\
\hline 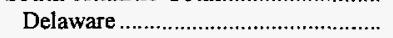 & 37.84 & 39.29 & 40.02 & 42.91 & 45.31 & 56.96 & -3.7 & -4.4 & -4.4 \\
\hline Florida & 38.65 & 40.83 & 41.67 & 42.48 & 45.03 & 54.13 & -5.3 & -3.7 & -3.7 \\
\hline 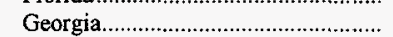 & 33.31 & 35.89 & 37.96 & 42.19 & 43.36 & 52.42 & -7.2 & -6.4 & -4.9 \\
\hline Maryland. & 35.09 & 36.24 & 37.98 & 39.74 & 40.68 & 48.10 & -3.2 & -3.6 & -3.4 \\
\hline 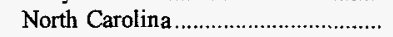 & 33.61 & 37.70 & 39.82 & 41.28 & 43.00 & 54.05 & -10.8 & -6.0 & -5.1 \\
\hline 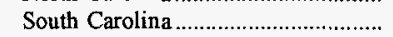 & 34.22 & 36.11 & 37.98 & 39.15 & 39.13 & 52.96 & -5.2 & -3.3 & -4.7 \\
\hline 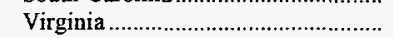 & 32.57 & 34.29 & 35.32 & 36.62 & 37.81 & 48.76 & -5.0 & -3.6 & -4.4 \\
\hline 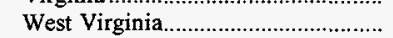 & 28.19 & 29.38 & 33.08 & 34.53 & 36.88 & 81.12 & -4.0 & -6.5 & -104.5 \\
\hline 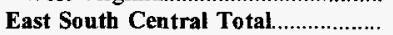 & 26.75 & 27.96 & 30.92 & 32.46 & 33.05 & 43.26 & -4.3 & -5.1 & -5.2 \\
\hline 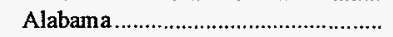 & 33.17 & 34.39 & 38.53 & 41.48 & 41.67 & 56.61 & -3.5 & -5.5 & -5.8 \\
\hline Kentucky & 22.27 & 23.89 & 25.89 & 26.60 & 27.01 & 34.82 & -6.8 & -4.7 & -4.8 \\
\hline 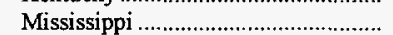 & 30.36 & 31.97 & 33.88 & 39.48 & 39.94 & 59.64 & -5.0 & -6.6 & -7.2 \\
\hline Tennessee & 25.19 & 25.97 & 29.18 & 30.16 & 31.01 & 39.40 & -3.0 & -5.1 & -4.8 \\
\hline West South Central Total ................. & 18.35 & 19.20 & 19.82 & 21.58 & 22.55 & 28.79 & -4.4 & -5.0 & -4.9 \\
\hline 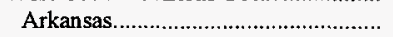 & 23.84 & 26.01 & 26.60 & 28.75 & 28.84 & 32.42 & -8.3 & -4.6 & -3.3 \\
\hline 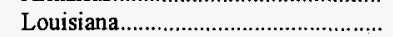 & 22.55 & 23.35 & 23.87 & 25.00 & 24.93 & 32.30 & -3.4 & -2.5 & -3.9 \\
\hline 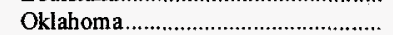 & 15.31 & 15.80 & 16.68 & 20.78 & 21.47 & 33.16 & -3.1 & -8.1 & -8.2 \\
\hline 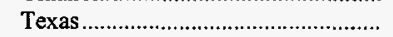 & 17.56 & 18.26 & 18.91 & 20.38 & 21.58 & 27.05 & -3.8 & -5.0 & -4.7 \\
\hline 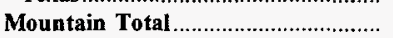 & 19.89 & 19.99 & 20.81 & 21.55 & 21.64 & 26.48 & -.5 & -2.1 & -3.1 \\
\hline Arizona & 26.93 & 26.63 & 26.94 & 27.08 & 28.31 & 33.16 & 1.1 & -1.2 & -2.3 \\
\hline 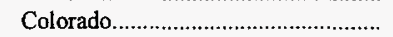 & 18.45 & 19.27 & 20.03 & 21.04 & 21.67 & 26.49 & -4.2 & -3.9 & -3.9 \\
\hline 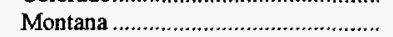 & 10.85 & 10.66 & 11.24 & 11.48 & 12.14 & 13.39 & 1.8 & $-2,8$ & -2.3 \\
\hline Nevada & 27.75 & 26.97 & 30.85 & 31.52 & 32.32 & 37.64 & 2.9 & -3.7 & -3.3 \\
\hline 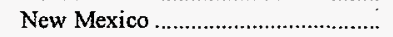 & 23.74 & 23.78 & 24.29 & 23.98 & 23.83 & 26.32 & -.2 & -1 & -1.1 \\
\hline 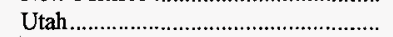 & 22.48 & 23.48 & 24.88 & 26.64 & 27.54 & 35.01 & -4.3 & -4.9 & -4.8 \\
\hline Wyoming & 13.04 & 13.28 & 13.43 & 13.68 & 13.42 & 18.38 & -1.8 & -.7 & -3.7 \\
\hline 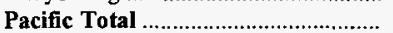 & 21.85 & 21.22 & 20.91 & 21.00 & 22.17 & 32.27 & 3.0 & -.4 & -4.2 \\
\hline Oregon & 17.14 & 17.47 & 18.28 & 19.25 & 21.23 & 28.62 & -1.8 & -5.2 & -5.5 \\
\hline 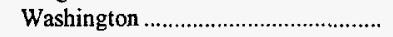 & 22.71 & 22.06 & 21.85 & 21.53 & 22.48 & 32.58 & 2.9 & .2 & -3.9 \\
\hline U.S. Total & 24.11 & 25.10 & 26.72 & 27.86 & 29.36 & 38.30 & -3.9 & -4.8 & -5.0 \\
\hline
\end{tabular}

Notes: Real prices are in 1992 dollars, calculated using implicit Gross Domestic Product price deflators. See Appendix D, Table D3. Average prices are based on the cost including insurance and freight.

Source: Federal Energy Regulatory Commission, FERC Form 423, "Monthly Report of Cost and Quality of Fuels for Electric Plants." 
Table 94. Average Price of Coal Delivered to Other Industrial Plants By Census Division and State, 1987, 1992-1996

(Nominal Dollars per Short Ton)

\begin{tabular}{|c|c|c|c|c|c|c|c|c|c|}
\hline \multirow{2}{*}{ Census Division and State } & \multirow{2}{*}{1996} & \multirow{2}{*}{1995} & \multirow{2}{*}{1994} & \multirow{2}{*}{1993} & \multirow{2}{*}{1992} & \multirow{2}{*}{1987} & \multirow{2}{*}{$\begin{array}{c}\text { Percent } \\
\text { Change } \\
\text { 1995-1996 }\end{array}$} & \multicolumn{2}{|c|}{ Average Annual Percent Change } \\
\hline & & & & & & & & $1992-1996$ & $1987-1996$ \\
\hline 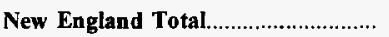 & $\$ 57.36$ & $\$ 56.90$ & $\$ 55.73$ & $\$ 57.83$ & $\$ 65.71$ & $\$ 59.23$ & 0.8 & -3.3 & -0.3 \\
\hline 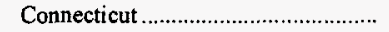 & - & - & - & - & - & $\mathbf{w}$ & $w$ & $\mathbf{w}$ & $w$ \\
\hline 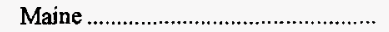 & w & $w$ & $\mathbf{w}$ & $w$ & w & $w$ & $\mathbf{w}$ & $w$ & w \\
\hline 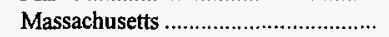 & $w$ & $w$ & w & $\mathbf{w}$ & w & $w$ & $w$ & $\mathbf{w}$ & $w$ \\
\hline 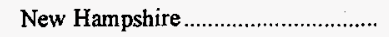 & - & - & - & - & - & $w$ & $w$ & $w$ & $\mathbf{w}$ \\
\hline 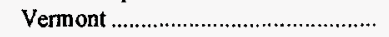 & - & - & - & - & - & $w$ & $w$ & $w$ & w \\
\hline Middle Atlantic Total......................... & $\mathbf{w}$ & $\mathbf{w}$ & $\mathbf{w}$ & $\mathbf{w}$ & $\mathbf{w}$ & $\mathbf{w}$ & $\mathbf{w}$ & $\mathbf{w}$ & $\mathbf{w}$ \\
\hline 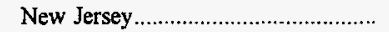 & w & w & $\mathbf{w}$ & $w$ & w & $w$ & w & w & w \\
\hline New York & 40.11 & 41.91 & 42.20 & 42.15 & 43.95 & 44.14 & -4.3 & -2.3 & -1.0 \\
\hline 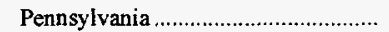 & 33.84 & 34.07 & 33.66 & 34.04 & 35.70 & 36.62 & -.7 & -1.3 & -.9 \\
\hline East North Central Total ................... & 34.44 & 34.89 & 34.72 & 34.54 & 35.05 & 35.72 & -1.3 & -.4 & -.4 \\
\hline 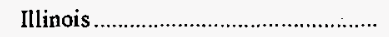 & 29.69 & 29.03 & 29.13 & 29.42 & 29.24 & 33.15 & 2.3 & 4 & -1.2 \\
\hline Indiana & 31.76 & 33.14 & 31.35 & 30.91 & 31.58 & 30.94 & -4.2 & .1 & .3 \\
\hline Michigan & 41.28 & 41.18 & 41.20 & 41.46 & 42.17 & 43.09 & .2 & -.5 & -.5 \\
\hline Ohio & 35.28 & 35.18 & 35.75 & 34.82 & 35.24 & 33.98 & 3 & $*$ & .4 \\
\hline 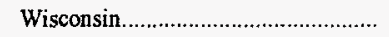 & 40.02 & 40.21 & 41.23 & 40.85 & 42.27 & 43.99 & -.4 & -1.3 & -1.0 \\
\hline West North Central Total ................ & 19.05 & 18.92 & 18.61 & 18.00 & 17.76 & 17.62 & .6 & 1.8 & .9 \\
\hline lowa & 29.32 & 29.24 & 28.52 & 28.01 & 27.17 & 34.87 & .3 & 1.9 & -1.9 \\
\hline Kansas & 32.46 & 32.42 & 32.25 & 33.06 & 31.96 & 34.49 & .1 & .4 & -.7 \\
\hline Minnesota. & 28.85 & 34.40 & 35.66 & 35.81 & 35.63 & 37.40 & -16.1 & -5.1 & -2.8 \\
\hline 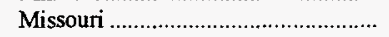 & 31.37 & 32.81 & 32.87 & 32.12 & 31.48 & 29.32 & -4.4 & -.1 & .8 \\
\hline 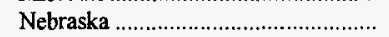 & $w$ & $w$ & $w$ & $w$ & $w$ & $w$ & w & $w$ & w \\
\hline 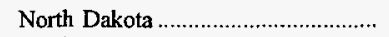 & $\mathbf{w}$ & w & $w$ & $\mathbf{w}$ & $w$ & $\mathbf{w}$ & $\mathbf{w}$ & $w$ & $w$ \\
\hline 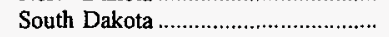 & $\mathbf{w}$ & $w$ & $\mathbf{w}$ & $w$ & $w$ & $\mathbf{w}$ & $\mathbf{w}$ & $\mathbf{w}$ & w \\
\hline South Atlantic Total. & $\mathbf{w}$ & $\mathbf{w}$ & $\mathbf{w}$ & $\mathbf{w}$ & $\mathbf{w}$ & $\mathbf{w}$ & $\mathbf{w}$ & $\mathbf{w}$ & $\mathbf{w}$ \\
\hline Delaware & w & w & $\mathbf{w}$ & w & $\mathrm{w}$ & w & $w$ & w & w \\
\hline Florida & 45.69 & 46.63 & 46.60 & 48.28 & 48.27 & 46.69 & -2.0 & -1.4 & -.2 \\
\hline 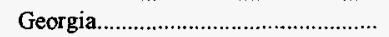 & 44.21 & 44.64 & 45.71 & 45.20 & 45.18 & 43.67 & -1.0 & -.5 & .1 \\
\hline 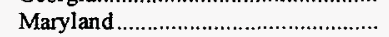 & 32.52 & 31.66 & 32.18 & 32.18 & 32.83 & 32.37 & 2.7 & -.2 & $*$ \\
\hline 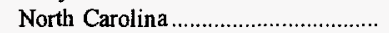 & 43.36 & 43.29 & 43.62 & 43.44 & 43.46 & 43.78 & .2 & $*$ & -.1 \\
\hline 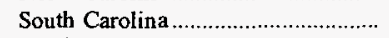 & 44.08 & 43.16 & 43.84 & 43.35 & 43.31 & 42.62 & 2.1 & .4 & .4 \\
\hline 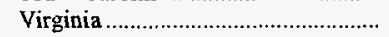 & 43.51 & 42.50 & 41.56 & 41.27 & 40.97 & 38.92 & 2.4 & 1.5 & 1.2 \\
\hline 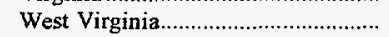 & 33.37 & 33.61 & 32.73 & 32.91 & 31.93 & 31.39 & -.7 & 1.1 & .7 \\
\hline East South Central Total.................... & $\mathbf{w}$ & $\mathbf{w}$ & $\mathbf{w}$ & $\mathbf{w}$ & $\mathbf{w}$ & $\mathbf{w}$ & $\mathbf{w}$ & $\mathbf{w}$ & $\mathbf{w}$ \\
\hline 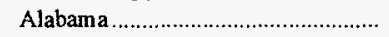 & 40.15 & 39.53 & 38.74 & 39.01 & 39.76 & 40.38 & 1.5 & .2 & -.1 \\
\hline 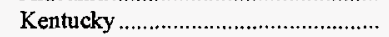 & 44.02 & 44.09 & 43.22 & 42.30 & 43.78 & 43.12 & -.2 & .1 & .2 \\
\hline 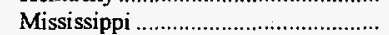 & $\mathbf{w}$ & $w$ & w & $w$ & $w$ & w & w & $\mathbf{w}$ & w \\
\hline Tennessee & 35.21 & 35.68 & 35.34 & 35.41 & 35.52 & 35.48 & -1.3 & -.2 & -.1 \\
\hline West South Central Total ................. & 21.79 & 22.04 & 22.95 & 21.38 & 22.80 & $\mathbf{w}$ & -1.1 & -1.1 & $\mathbf{w}$ \\
\hline 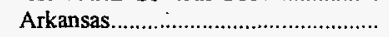 & 43.24 & 43.52 & 44.28 & 44.06 & 44.61 & 45.19 & -.6 & -.8 & -.5 \\
\hline Louisiana & $\mathbf{w}$ & $w$ & $\mathbf{w}$ & $w$ & $w$ & w & w & $w$ & w \\
\hline Oklahoma & w & w & $\mathbf{w}$ & $\mathbf{w}$ & w & 41.32 & $\mathbf{w}$ & w & $\mathrm{w}$ \\
\hline Texas & 18.99 & 18.76 & 19.54 & 17.58 & 18.14 & 19.19 & 1.2 & 1.1 & -.1 \\
\hline Mountain Total & 26.70 & 27.06 & 28.78 & 28.51 & 28.66 & 28.73 & -1.3 & -1.7 & -.8 \\
\hline 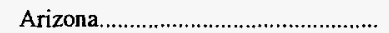 & 39.27 & 40.46 & 41.35 & 40.51 & 40.95 & 38.66 & -2.9 & -1.0 & .2 \\
\hline 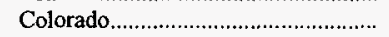 & 23.17 & 26.11 & 28.96 & 28.63 & 30.34 & 28.79 & -11.2 & -6.5 & -2.4 \\
\hline Idaho & 36.39 & 34.11 & 33.35 & 32.78 & 33.29 & 35.03 & 6.7 & 2.2 & .4 \\
\hline 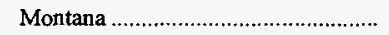 & w & $w$ & w & w & w & w & $w$ & w & $\mathrm{w}$ \\
\hline 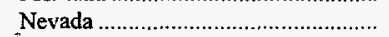 & $w$ & $\mathrm{w}$ & $\mathbf{w}$ & $\mathbf{w}$ & w & $w$ & $w$ & $w$ & $w$ \\
\hline 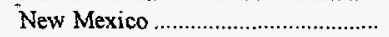 & w & w & $\mathbf{w}$ & $\mathbf{w}$ & w & w & w & $w$ & w \\
\hline Utah & 19.10 & 19.74 & 26.57 & 26.51 & 25.35 & 23.85 & -3.2 & -6.8 & -2.4 \\
\hline Wyoming & 22.32 & 22.72 & 22.87 & 23.43 & 23.67 & 23.61 & -1.8 & -1.4 & -.6 \\
\hline Pacific Total & $\mathbf{4 2 . 4 5}$ & 43.158 & 44.92 & 43.83 & 43.37 & 47.73 & -2.8 & -.5 & -1.3 \\
\hline 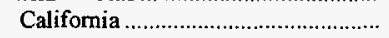 & 39.54 & 41.11 & 43.39 & 42.86 & 42.05 & 48.63 & -3.8 & -1.5 & -2.3 \\
\hline 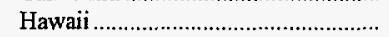 & w & $w$ & $w$ & $w$ & $w$ & w & w & w & $w$ \\
\hline Oregon & w & $\mathbf{w}$ & w & $\mathbf{w}$ & $w$ & $w$ & w & $\mathbf{w}$ & $w$ \\
\hline 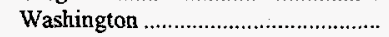 & 58.81 & 59.15 & 58.86 & 53.11 & 56.25 & 46.43 & -.6 & 1.1 & 2.7 \\
\hline U.S. Total & $\mathbf{3 2 . 3 2}$ & 32.42 & 32.55 & 32.23 & 32.78 & 33.71 & -.3 & -.3 & -.5 \\
\hline
\end{tabular}

* Data round to zero.

w Withheld to avoid disclosure of individual company data.

Notes: Price data are for manufacturing plants only. Average prices are based on the cost including insurance, freight, and taxes.

Source: Energy Information Administration, Form EIA.-3, "Quarterly Coal Consumption - Manufacturing Plants." 
Table 95. Average Real Price of Coal Delivered to Other Industrial Plants by Census Division and State, 1987, 1992-1996

(Real Dollars per Short Ton)

\begin{tabular}{|c|c|c|c|c|c|c|c|c|c|}
\hline \multirow{2}{*}{ Census Division and State } & \multirow{2}{*}{1996} & \multirow{2}{*}{1995} & \multirow{2}{*}{1994} & \multirow{2}{*}{1993} & \multirow{2}{*}{1992} & \multirow{2}{*}{1987} & \multirow{2}{*}{$\begin{array}{c}\text { Percent } \\
\text { Change } \\
1995-1996\end{array}$} & \multicolumn{2}{|c|}{ Average Annual Percent Change } \\
\hline & & & & & & & & 1992-1996 & $1987-1996$ \\
\hline New England Total.............................. & $\$ 52.28$ & $\$ 52.88$ & $\$ 53.13$ & $\$ 56.37$ & $\$ 65.71$ & $\$ 71.27$ & -1.1 & -5.5 & -3.4 \\
\hline 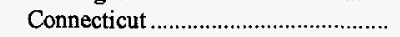 & - & - & - & - & - & w & $w$ & $w$ & w \\
\hline 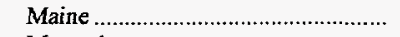 & w & $w$ & $w$ & - $w$ & $w$ & $w$ & $w$ & $w$ & $w$ \\
\hline 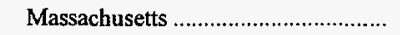 & w & $w$ & $\mathbf{w}$ & w & $w$ & w & $\mathbf{w}$ & $\mathrm{w}$ & $\mathbf{w}$ \\
\hline 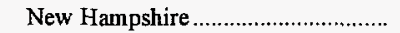 & - & - & - & - & - & $w$ & w & w & $w$ \\
\hline Vermont & - & - & - & - & - & $w$ & w & w & $w$ \\
\hline Middle Atlantic Total............................ & $\mathbf{w}$ & $\mathbf{w}$ & $\mathbf{w}$ & $\mathbf{w}$ & $\mathbf{w}$ & $\mathbf{w}$ & $\mathbf{w}$ & $\mathbf{w}$ & $\mathbf{w}$ \\
\hline 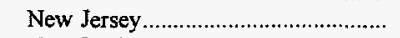 & $\mathbf{w}$ & w & w & w & $w$ & w & w & $w$ & w \\
\hline 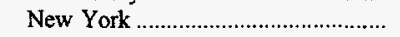 & 36.56 & 38.95 & 40.23 & 41.09 & 43.95 & 53.12 & -6.1 & -4.5 & -4.1 \\
\hline 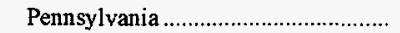 & 30.85 & 31.66 & 32.09 & 33.18 & 35.70 & 44.06 & -2.6 & -3.6 & -3.9 \\
\hline East North Central Total .................. & 31.40 & 32.42 & 33.10 & 33.66 & 35.05 & 42.99 & -3.2 & -2.7 & -3.4 \\
\hline 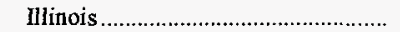 & 27.06 & 26.98 & 27.77 & 28.67 & 29.24 & 39.89 & .3 & -1.9 & -4.2 \\
\hline 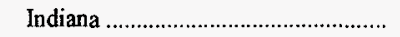 & 28.95 & 30.80 & 29.89 & 30.13 & 31.58 & 37.24 & -6.0 & -2.1 & -2.8 \\
\hline 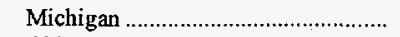 & 37.63 & 38.27 & 39.28 & 40.41 & 42.17 & 51.85 & -1.7 & -2.8 & -3.5 \\
\hline 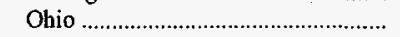 & 32.16 & 32.70 & 34.08 & 33.94 & 35.24 & 40.89 & -1.6 & -2.3 & -2.6 \\
\hline Wisconsin & 36.49 & 37.37 & 39.31 & 39.81 & 42.27 & 52.93 & -2.4 & -3.6 & -4.0 \\
\hline West North Central Total .................. & 17.36 & 17.59 & 17.74 & 17.54 & 17.76 & 21.20 & -1.3 & -.6 & -2.2 \\
\hline Iowa & 26.73 & 27.17 & 27.19 & 27.30 & 27.17 & 41.96 & -1.6 & -.4 & -4.9 \\
\hline 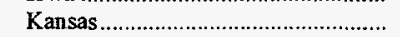 & 29.59 & 30.13 & 30.74 & 32.22 & 31.96 & 41.50 & -1.8 & -1.9 & -3.7 \\
\hline 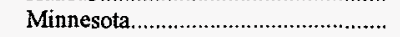 & 26.30 & 31.97 & 33.99 & 34.90 & 35.63 & 45.01 & -17.7 & -7.3 & -5.8 \\
\hline 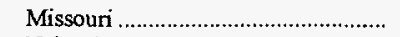 & 28.60 & 30.49 & 31.34 & 31.30 & 31.48 & 35.28 & -6.2 & -2.4 & -2.3 \\
\hline 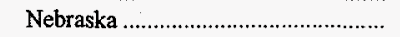 & w & w & $w$ & w & w & w & w & w & $\mathbf{w}$ \\
\hline 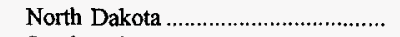 & $\mathrm{w}$ & $w$ & $\mathbf{w}$ & $\mathrm{w}$ & $w$ & $\mathbf{w}$ & w & w & $w$ \\
\hline 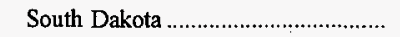 & $\mathbf{w}$ & $w$ & $\mathbf{w}$ & $w$ & w & $\mathrm{w}$ & $w$ & $w$ & w \\
\hline South Atlan tic Total............................ & $\mathbf{w}$ & $\mathbf{w}$ & $\mathbf{w}$ & $\mathbf{w}$ & $\mathbf{w}$ & $\mathbf{w}$ & $\mathbf{w}$ & $\mathbf{w}$ & $\mathbf{w}$ \\
\hline Delaware & w & w & $w$ & w & $w$ & w & w & w & w \\
\hline Florida & 41.65 & 43.34 & 44.43 & 47.06 & 48.27 & 56.19 & -3.9 & -3.6 & -3.3 \\
\hline 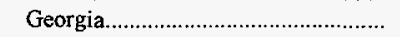 & 40.30 & 41.49 & 43.58 & 44.05 & 45.18 & 52.55 & -2.8 & -2.8 & -2.9 \\
\hline 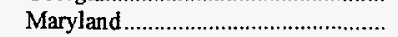 & 29.65 & 29.42 & 30.67 & 31.36 & 32.83 & 38.95 & .8 & -2.5 & -3.0 \\
\hline 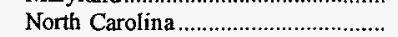 & 39.53 & 40.23 & 41.58 & 42.34 & 43.46 & 52.68 & -1.8 & -2.3 & -3.1 \\
\hline South Carolina & 40.18 & 40.11 & 41.79 & 42.25 & 43.31 & 51.29 & .2 & -1.8 & -2.7 \\
\hline 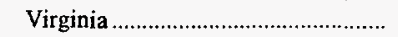 & 39.66 & 39.50 & 39.62 & 40.23 & 40.97 & 46.83 & .4 & -.8 & -1.8 \\
\hline 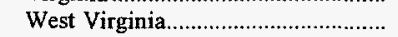 & 30.42 & 31.24 & 31.20 & 32.08 & 31.93 & 37.77 & -2.6 & -1.2 & -2.4 \\
\hline East South Central Total .................... & $\mathbf{w}$ & $\mathbf{w}$ & $\mathbf{w}$ & $\mathbf{w}$ & $\mathbf{w}$ & $\mathbf{w}$ & $\mathbf{w}$ & $\mathbf{w}$ & $\mathbf{w}$ \\
\hline 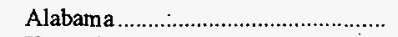 & 36.60 & 36.74 & 36.93 & 38.02 & 39.76 & 48.59 & -.4 & -2.0 & -3.1 \\
\hline 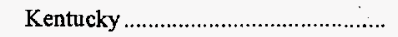 & 40.12 & 40.97 & 41.20 & 41.23 & 43.78 & 51.89 & -2.1 & -2.1 & -2.8 \\
\hline 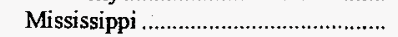 & w & w & w & w & w & $w$ & $w$ & $w$ & w \\
\hline Tennessee & 32.10 & 33.16 & 33.69 & 34.52 & 35.52 & 42.69 & -3.2 & -2.5 & -3.1 \\
\hline West South Central Total ................. & 19.86 & 20.48 & 21.87 & 20.84 & 22.80 & $\mathbf{w}$ & -3.0 & -3.4 & $\mathbf{w}$ \\
\hline 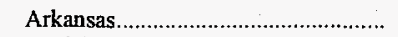 & 39.42 & 40.44 & 42.21 & 42.95 & 44.61 & 54.38 & -2.5 & -3.0 & -3.5 \\
\hline 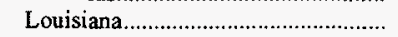 & w & $w$ & w & w & w & $w$ & w & $w$ & w \\
\hline Oklahoma & w & w & $\mathbf{w}$ & $\mathbf{w}$ & $\mathbf{w}$ & 49.72 & w & w & w \\
\hline 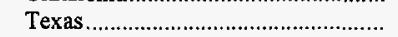 & 17.31 & 17.43 & 18.63 & 17.13 & 18.14 & 23.10 & -.7 & -1.2 & -3.1 \\
\hline 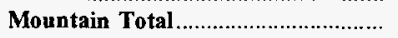 & 24.34 & 25.15 & 27.44 & 27.79 & 28.66 & 34.57 & -3.2 & -4.0 & -3.8 \\
\hline 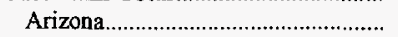 & 35.80 & 37.60 & 39.42 & 39.48 & 40.95 & 46.52 & -4.8 & -3.3 & -2.9 \\
\hline 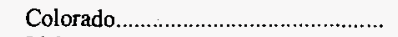 & 21.12 & 24.26 & 27.61 & 27.90 & 30.34 & 34.64 & -12.9 & -8.6 & -5.3 \\
\hline Idaho & 33.17 & 31.70 & 31.79 & 31.95 & 33.29 & 42.16 & 4.6 & -.1 & -2.6 \\
\hline 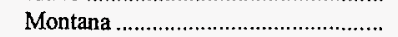 & w & $w$ & $w$ & w & $w$ & w & w & w & $w$ \\
\hline 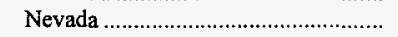 & $w$ & w & $w$ & w & $w$ & w & $w$ & $w$ & $w$ \\
\hline 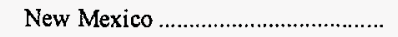 & w & $\mathbf{w}$ & w & w & w & $w$ & w & w & w \\
\hline 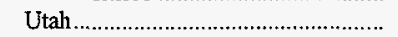 & 17.41 & 18.34 & 25.33 & 25.84 & 25.35 & 28.70 & -5.1 & -8.9 & -5.4 \\
\hline 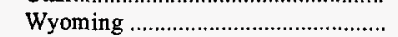 & 20.35 & 21.12 & 21.81 & 22.84 & 23.67 & 28.41 & -3.6 & -3.7 & -3.6 \\
\hline 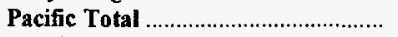 & 38.69 & 40.60 & 42.82 & 42.72 & 43.37 & 57.44 & -4.7 & -2.8 & -4.3 \\
\hline 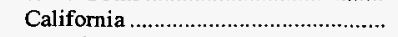 & 36.04 & 38.21 & 41.37 & 41.78 & 42.05 & 58.52 & -5.7 & -3.8 & -5.2 \\
\hline 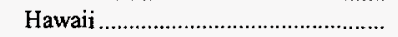 & w & w & w & w & $w$ & w & w & $w$ & w \\
\hline Oregon & w & $\mathbf{w}$ & w & w & w & w & w & w & $\mathrm{w}$ \\
\hline Washington & 53.61 & 54.97 & 56.11 & 51.76 & 56.25 & 55.87 & -2.5 & -1.2 & -.4 \\
\hline U.S. Total & 29.46 & 30.13 & 31.03 & 31.41 & 32.78 & 40.56 & -2.2 & -2.6 & -3.5 \\
\hline
\end{tabular}

w Withheld to avoid disclosure of individual company data.

Notes: Price data are for manufacturing plants only. Real prices are in 1992 dollars, caiculated using implicit Gross Domestic Product price deflators. See Appendix D, Table D3. Average prices are based on the cost including insurance, freight, and taxes.

Source: Energy Information Administration, Form EIA-3, "Quarterly Coal Consumption - Manufacturing Plants." 
Table 96. Average Price of Coal Delivered to Coke Plants by Census Division and State, 1987, 1992-1996

(Nominal Dollars per Short Ton)

\begin{tabular}{|c|c|c|c|c|c|c|c|c|c|}
\hline \multirow{2}{*}{ Census Division and State } & \multirow{2}{*}{1996} & \multirow{2}{*}{1995} & \multirow{2}{*}{1994} & \multirow{2}{*}{1993} & \multirow{2}{*}{1992} & \multirow{2}{*}{1987} & \multirow{2}{*}{$\begin{array}{c}\text { Percent } \\
\text { Change } \\
1995-1996\end{array}$} & \multicolumn{2}{|c|}{ Average Annual Percent Change } \\
\hline & & & & & & & & $1992-1996$ & 1987-1996 \\
\hline Middle Atlantic Total......................... & $\mathbf{w}$ & $\mathbf{w}$ & $\mathbf{w}$ & $\mathbf{w}$ & $\mathbf{w}$ & $\mathbf{w}$ & $\mathbf{w}$ & $\mathbf{w}$ & $\mathbf{w}$ \\
\hline 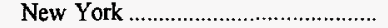 & w & w & $\mathbf{w}$ & w & w & $\mathbf{w}$ & w & $w$ & $\mathbf{w}$ \\
\hline Pennsylvania & $\$ 45.16$ & $\$ 46.11$ & $\$ 46.25$ & $\$ 46.41$ & $\$ 46.49$ & $\$ 42.54$ & -2.0 & -0.7 & 0.7 \\
\hline East North Central Total .................... & 49.54 & 49.09 & 47.23 & 49.52 & 50.37 & 49.00 & .9 & -.4 & .1 \\
\hline 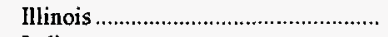 & w & $\mathbf{w}$ & w & $\mathbf{w}$ & $\mathbf{w}$ & w & $\mathbf{w}$ & w & $w$ \\
\hline Indiana & 51.93 & 52.74 & 50.90 & 52.29 & 53.72 & 51.44 & -1.5 & -.8 & .1 \\
\hline 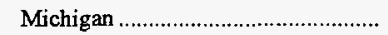 & $w$ & $\mathbf{w}$ & w & - & - & $w$ & $\mathbf{w}$ & $w$ & w \\
\hline Ohio & 44.98 & 42.18 & 42.02 & 45.07 & 46.68 & 46.04 & 6.6 & -.9 & -.3 \\
\hline West North Central Total ................ & - & - & - & - & - & $\mathbf{w}$ & - & $\mathbf{w}$ & $\mathbf{w}$ \\
\hline 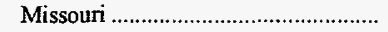 & - & - & - & - & - & $\mathbf{w}$ & - & w & $\mathbf{w}$ \\
\hline South Atlantic Total. & $\mathbf{w}$ & $\mathbf{w}$ & $\mathbf{w}$ & $\mathbf{w}$ & $\mathbf{w}$ & $\mathbf{w}$ & $\mathbf{w}$ & $\mathbf{w}$ & $\mathbf{w}$ \\
\hline 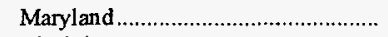 & - & - & - & - & - & $\mathbf{w}$ & $w$ & $w$ & $\mathbf{w}$ \\
\hline 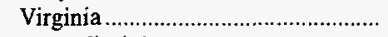 & w & $w$ & $w$ & $w$ & $w$ & w & $\mathrm{w}$ & $w$ & $\mathbf{w}$ \\
\hline West Virginia & $w$ & $w$ & w & $w$ & $w$ & w & $\mathbf{w}$ & $\mathbf{w}$ & $\mathbf{w}$ \\
\hline East South Central Total...................... & $\mathbf{w}$ & $\mathbf{w}$ & $\mathbf{w}$ & $\mathbf{w}$ & $\mathbf{w}$ & $\mathbf{w}$ & $\mathbf{w}$ & $\mathbf{w}$ & $\mathbf{w}$ \\
\hline Alabama & 49.37 & 48.42 & 47.45 & 47.50 & 47.80 & 45.89 & 1.9 & .8 & .8 \\
\hline 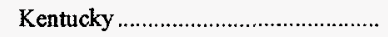 & w & w & w & w & w & w & $\mathbf{w}$ & $\mathbf{w}$ & $w$ \\
\hline 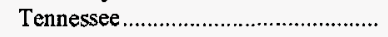 & - & - & - & - & - & $\mathbf{w}$ & - & $\mathbf{w}$ & $\mathbf{w}$ \\
\hline 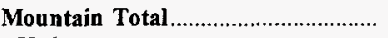 & $\mathbf{w}$ & $\mathbf{w}$ & $\mathbf{w}$ & $\mathbf{w}$ & $\mathbf{w}$ & - & $\mathbf{w}$ & $\mathbf{w}$ & $\mathbf{w}$ \\
\hline 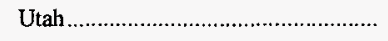 & w & w & w & w & w & - & w & $\mathbf{w}$ & $w$ \\
\hline U.S. Total. & 47.33 & 47.34 & 46.56 & 47.44 & 47.92 & 46.55 & - & -.3 & .2 \\
\hline
\end{tabular}

* Data round to zero.

Withheld to avoid disclosure of individual company data.

Note: Average prices are based on the cost including insurance, freight, and taxes.

Source: Energy Information Administration, Form EIA-5, "Coke Plant Report - Quarterly."

Table 97. Average Real Price of Coal Delivered to Coke Plants by Census Division and State, 1987, 1992-1996

(Real Dollars per Short Ton)

\begin{tabular}{|c|c|c|c|c|c|c|c|c|c|}
\hline \multirow{2}{*}{ Census Division and State } & \multirow{2}{*}{1996} & \multirow{2}{*}{1995} & \multirow{2}{*}{1994} & \multirow{2}{*}{1993} & \multirow{2}{*}{1992} & \multirow{2}{*}{1987} & \multirow{2}{*}{$\begin{array}{c}\text { Percent } \\
\text { Change } \\
1995-1996\end{array}$} & \multicolumn{2}{|c|}{ Average Annual Percent Change } \\
\hline & & & & & & & & $1992-1996$ & $1987-1996$ \\
\hline Middle Atlantic Total ............................ & $\mathbf{w}$ & $\mathbf{w}$ & $\mathbf{w}$ & $\mathbf{w}$ & $\mathbf{w}$ & $\mathbf{w}$ & $\mathbf{w}$ & $\mathbf{w}$ & $\mathbf{w}$ \\
\hline New York & w & w & $\mathbf{w}$ & w & w & $\mathbf{w}$ & $\mathbf{w}$ & w & $\mathbf{w}$ \\
\hline 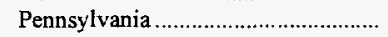 & $\$ 41.17$ & $\$ 42.85$ & $\$ 44.09$ & $\$ 45.24$ & $\$ 46.49$ & $\$ 51.19$ & -3.9 & -3.0 & -2.4 \\
\hline East North Central Total ..................... & 45.16 & 45.63 & 45.02 & 48.26 & 50.37 & 58.96 & -1.0 & -2.7 & -2.9 \\
\hline Illinois & w & w & w & w & $\mathbf{w}$ & $w$ & w & w & w \\
\hline Indiana & 47.34 & 49.01 & 48.52 & 50.97 & 53.72 & 61.90 & -3.4 & -3.1 & -2.9 \\
\hline 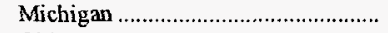 & w & w & w & - & - & w & w & w & w \\
\hline 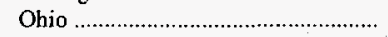 & 41.00 & 39.21 & 40.05 & 43.92 & 46.68 & 55.40 & 4.6 & -3.2 & -3.3 \\
\hline West North Central Total ................... & - & - & - & - & - & $\mathbf{w}$ & - & $\mathbf{w}$ & $\mathbf{w}$ \\
\hline 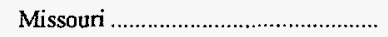 & - & - & - & - & - & $w$ & - & w & w \\
\hline South Atlantic Total & $\mathbf{w}$ & $\mathbf{w}$ & $\mathbf{w}$ & $\mathbf{w}$ & $\mathbf{w}$ & $\mathbf{w}$ & $\mathbf{w}$ & $\mathbf{w}$ & $\mathbf{w}$ \\
\hline 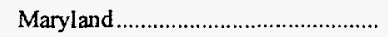 & - & - & - & - & - & w & $w$ & w & $w$ \\
\hline 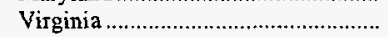 & $\mathbf{w}$ & w & $w$ & $w$ & $\mathbf{w}$ & $\mathbf{w}$ & $\mathbf{w}$ & $\mathbf{w}$ & $w$ \\
\hline 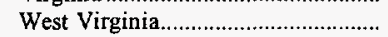 & $\mathbf{w}$ & $w$ & $\mathbf{w}$ & w & $\mathrm{w}$ & $w$ & w & w & $w$ \\
\hline East South Central Total..................... & $\mathbf{w}$ & $\mathbf{w}$ & $\mathbf{w}$ & $\mathbf{w}$ & $\mathbf{w}$ & $\mathbf{w}$ & $\mathbf{w}$ & $\mathbf{w}$ & $\mathbf{w}$ \\
\hline Alabama & 45.00 & 45.00 & 45.23 & 46.30 & 47.80 & 55.23 & $*$ & -1.5 & -2.2 \\
\hline 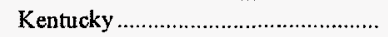 & w & w & w & $\mathbf{w}$ & w & w & w & w & w \\
\hline 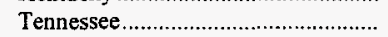 & - & - & - & - & - & $\mathbf{w}$ & - & w & w \\
\hline Mountain Total & $\mathbf{w}$ & $\mathbf{w}$ & w & $\mathbf{w}$ & $\mathbf{w}$ & - & $\mathbf{w}$ & $w$ & $\mathbf{w}$ \\
\hline Utah & w & w & $\mathbf{w}$ & w & $\mathbf{w}$ & - & $\mathbf{w}$ & $\mathbf{w}$ & $\mathbf{w}$ \\
\hline 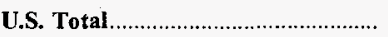 & 43.15 & 44.00 & 44.38 & 46.24 & 47.92 & 56.01 & -1.9 & -2.6 & -2.8 \\
\hline
\end{tabular}

* Data round to zero.

Withheld to avoid disclosure of individual company data

Notes: Real prices are in 1992 dollars, calculated using implicit Gross Domestic Product price deflators. See Appendix D, Table D3. Average prices are based on the cost including insurance, freight, and taxes.

Source: Energy Information Administration, Form EIA-5, "Coke Plant Report - Quarterly." 


\section{Import/Export Prices}

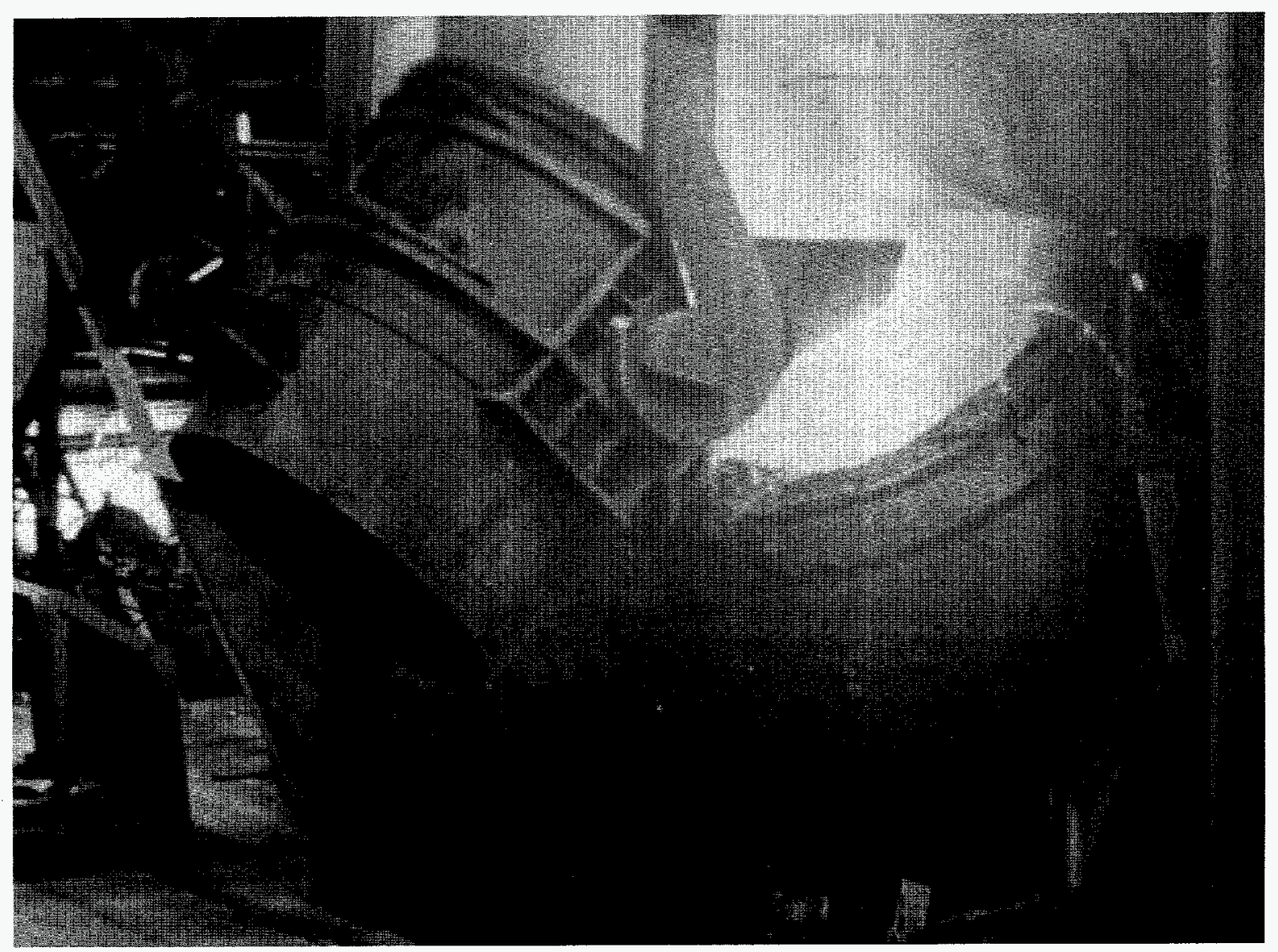

The iron and steel industry is the primary user of coke, which is generally made from bituminous coal. 
Table 98. Average Price of U.S. Coal IImports by Continent and Country of Origin, 1987, 1992-1996

(Nominal Dollars per Short Ton)

\begin{tabular}{|c|c|c|c|c|c|c|c|c|c|}
\hline \multirow{2}{*}{$\begin{array}{c}\text { Continent and Country } \\
\text { of Origin }\end{array}$} & \multirow{2}{*}{1996} & \multirow{2}{*}{1995} & \multirow{2}{*}{1994} & \multirow{2}{*}{1993} & \multirow{2}{*}{1992} & \multirow{2}{*}{1987} & \multirow{2}{*}{$\begin{array}{c}\text { Percent } \\
\text { Change } \\
\text { 1995-1996 }\end{array}$} & \multicolumn{2}{|c|}{ Average Annual Percent Change } \\
\hline & & & & & & & & 1992-1996 & $1987-1996$ \\
\hline North America Total & $\$ 34.89$ & $\$ 35.12$ & $\$ 30.81$ & $\$ 29.60$ & $\$ 29.56$ & $\$ 32.53$ & -0.7 & 4.2 & 0.8 \\
\hline Canada & 34.90 & 35.12 & 30.81 & 29.62 & 29.56 & 33.19 & -.6 & 4.2 & .5 \\
\hline Mexico & 33.43 & - & - & 21.19 & - & 25.57 & - & $\leftarrow$ & 3.0 \\
\hline South America Total............................ & 31.24 & 32.76 & 29.00 & 27.64 & 33.04 & 32.96 & -4.6 & -1.4 & -.6 \\
\hline Colombia & 31.40 & 31.15 & 27.46 & 27.26 & 32.25 & 32.89 & .8 & -.7 & -.5 \\
\hline 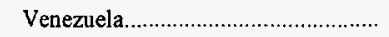 & 30.97 & 35.14 & 32.41 & 28.87 & 35.61 & 35.22 & -11.9 & -3.4 & -1.4 \\
\hline 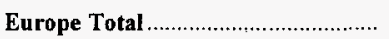 & - & 25.70 & - & 32.08 & 34.12 & 28.70 & -100.0 & -100.0 & -100.0 \\
\hline Denmark & - & - & - & 32.08 & - & - & - & - & - \\
\hline Spain & - & - & - & - & - & 28.70 & - & - & -100.0 \\
\hline 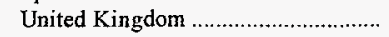 & - & 25.70 & - & - & 34.12 & - & -100.0 & -100.0 & - \\
\hline Asia Total & 32.45 & 35.13 & 34.09 & 42.70 & 38.75 & - & -7.6 & -4.3 & - \\
\hline India & - & - & - & - & 23.65 & - & - & -100.0 & - \\
\hline Indonesia & 32.45 & 35.13 & 33.80 & 42.70 & 40.94 & - & -7.6 & -6 & - \\
\hline 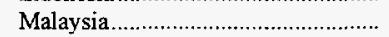 & - & - & - & - & 47.06 & - & - & -100.0 & - \\
\hline Vietnam & - & - & 48.08 & - & - & - & - & - & - \\
\hline Oceania \& Australia Total................. & 33.41 & 33.57 & 31.16 & 31.56 & 36.07 & 27.17 & -.5 & -1.9 & 2.3 \\
\hline 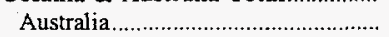 & 33.41 & 30.99 & 30.02 & 31.56 & 36.07 & 27.17 & 7.8 & -1.9 & 2.3 \\
\hline 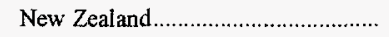 & - & 45.42 & 44.15 & - & - & - & -100.0 & $\rightarrow$ & - \\
\hline Africa Total & - & - & 25.33 & 27.81 & 49.29 & - & - & -100.0 & - \\
\hline 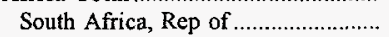 & - & - & 25.33 & - & 49.29 & - & - & -100.0 & - \\
\hline 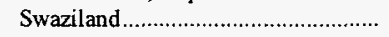 & - & - & - & 27.81 & - & - & - & - & - \\
\hline 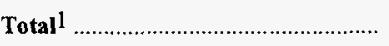 & 32.17 & 33.54 & 30.01 & 29.44 & 32.83 & 32.08 & -4.1 & -.5 & * \\
\hline U.S. Total ${ }^{2}$ & 33.45 & 3.4 .13 & 30.21 & 29.89 & 33.46 & 32.04 & -2.0 & * & .5 \\
\hline
\end{tabular}

1 The average prices presented in this table, with the exception of U.S. Total, are considered to be representative prices for coal imports and fall within the range of $\$ 20$ to $\$ 55$ per short ton, inclusively.

2 U.S. Total is the average price of all coal imports.

* Data round to zero.

Notes: Average price is based on the customs import value. Coal imports include coal to Puerto Rico and the Virgin Islands.

Source: U.S. Department of Commerce, Bureau of the Census, "Monthly Report IM 145." 
Table 99. Average Price of U.S. Coal Exports by Destination, 1987, 1992-1996 (Nominal Dollars per Short Ton)

\begin{tabular}{|c|c|c|c|c|c|c|c|c|c|}
\hline \multirow{2}{*}{$\begin{array}{c}\text { Continent and Country } \\
\text { of Destination }\end{array}$} & \multirow{2}{*}{1996} & \multirow{2}{*}{1995} & \multirow{2}{*}{1994} & \multirow{2}{*}{1993} & \multirow{2}{*}{1992} & \multirow{2}{*}{1987} & \multirow{2}{*}{$\begin{array}{c}\text { Percent } \\
\text { Change } \\
\text { 1995-1996 }\end{array}$} & \multicolumn{2}{|c|}{ Average Annual Percent Change } \\
\hline & & & & & & & & $1992-1996$ & $1987-1996$ \\
\hline North America Total... & $\$ 33.09$ & $\$ 34.05$ & $\$ 32.42$ & $\$ 34.05$ & $\$ 33.05$ & $\$ 41.16$ & -2.8 & $\bullet$ & -2.4 \\
\hline 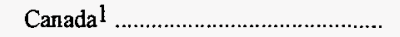 & 32.23 & 33.49 & 32.08 & 33.76 & 32.98 & 41.19 & -3.8 & -0.6 & -2.7 \\
\hline 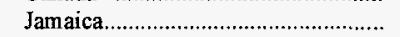 & 34.78 & 32.67 & 33.22 & 28.44 & 26.82 & - & 6.5 & 6.7 & - \\
\hline 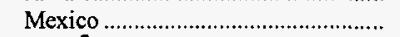 & 39.70 & 39.96 & 44.28 & 44.04 & 44.69 & 39.20 & -.6 & -2.9 & .1 \\
\hline Other 2 & 39.65 & 39.04 & 36.98 & 36.80 & 36.54 & 38.77 & 1.6 & 2.1 & .2 \\
\hline South America Total & 43.81 & 43.46 & 42.28 & 43.77 & 45.49 & 44.03 & .8 & -.9 & * \\
\hline Argentina & 46.36 & 43.40 & 42.48 & 43.21 & 45.41 & 43.27 & 6.8 & .5 & .8 \\
\hline 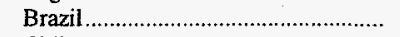 & 44.67 & 43.88 & 42.26 & 43.84 & 45.57 & 44.06 & 1.8 & -.5 & .1 \\
\hline 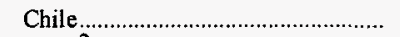 & 32.37 & 31.70 & 34.47 & 46.78 & 47.38 & 45.97 & 2.1 & -9.1 & -3.8 \\
\hline 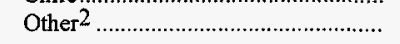 & 39.19 & 43.42 & 40.88 & 39.46 & 31.19 & 50.88 & -9.8 & 5.9 & -2.9 \\
\hline Europe Total & 42.10 & 40.92 & 41.86 & 43.05 & 42.40 & 42.37 & 2.9 & -.2 & -.1 \\
\hline Belgium \& Luxembourg ....................... & 45.73 & 43.47 & 42.23 & 43.23 & 44.01 & 41.61 & 5.2 & 1.0 & 1.0 \\
\hline 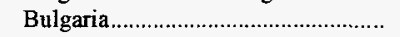 & 44.26 & 44.04 & 42.09 & 41.96 & 41.70 & - & .5 & 1.5 & - \\
\hline Denmark & 29.30 & 29.37 & 29.23 & 34.95 & 32.69 & 33.01 & -.2 & -2.7 & -1.3 \\
\hline 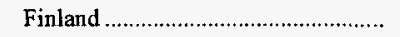 & 42.11 & 39.47 & 41.14 & 39.61 & 40.81 & 41.11 & 6.7 & 8 & 3 \\
\hline France & 44.94 & 43.71 & 44.23 & 42.24 & 39.43 & 43.12 & 2.8 & 3.3 & .4 \\
\hline 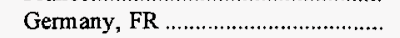 & 41.08 & 34.99 & 45.33 & 38.98 & 38.27 & 41.76 & 17.4 & 1.8 & -.2 \\
\hline Ireland & 37.35 & 36.07 & 33.82 & 35.84 & 36.81 & 43.58 & 3.6 & 4 & -1.7 \\
\hline Italy & 45.05 & 44.14 & 43.00 & 44.33 & 45.43 & 41.58 & 2.0 & -.2 & .9 \\
\hline 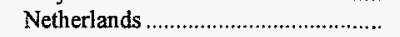 & 41.36 & 41.97 & 41.93 & 44.22 & 43.34 & 43.31 & -1.4 & -1.2 & -.5 \\
\hline 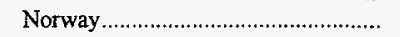 & 57.05 & 56.42 & 54.57 & 53.48 & 50.12 & 47.26 & 1.1 & 3.3 & 2.1 \\
\hline 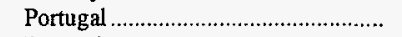 & 36.53 & 36.46 & 36.25 & 37.70 & 40.74 & 42.91 & .2 & -2.7 & -1.8 \\
\hline 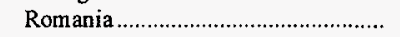 & 46.95 & 42.43 & 38.10 & 38.54 & 42.66 & 42.92 & 10.6 & 2.4 & 1.0 \\
\hline Spain & 37.56 & 34.75 & 40.12 & 42.66 & 41.95 & 43.79 & 8.1 & -2.7 & -1.7 \\
\hline Sweden & 47.50 & 48.21 & 45.56 & 45.96 & 46.66 & 43.34 & -1.5 & .4 & 1.0 \\
\hline 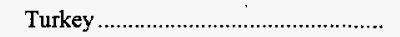 & 44.33 & 42.61 & 41.28 & 42.58 & 45.54 & 44.15 & 4.0 & -.7 & $*$ \\
\hline United Kingdom ................................ & 38.90 & 40.92 & 45.06 & 45.94 & 45.59 & 44.26 & -4.9 & -3.9 & -1.4 \\
\hline Yugoslavia, FR & - & 38.06 & - & 39.60 & 42.83 & 42.83 & -100.0 & -100.0 & -100.0 \\
\hline Other 2 & 38.03 & 55.95 & 38.14 & 40.67 & 41.89 & 42.89 & -32.0 & -2.4 & -1.3 \\
\hline Asia Total & 39.57 & 39.10 & 38.63 & 40.49 & 42.65 & 43.38 & 1.2 & -1.8 & -1.0 \\
\hline China (Taiwan) & 36.86 & 36.95 & 38.65 & 39.49 & 41.23 & 39.94 & -.2 & -2.8 & -.9 \\
\hline 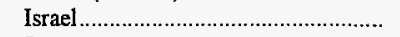 & 36.40 & 35.79 & 33.23 & 34.79 & 39.11 & 36.56 & 1.7 & -1.8 & * \\
\hline 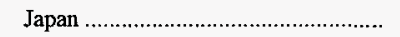 & 39.41 & 39.14 & 38.52 & 40.72 & 42.91 & 45.61 & .7 & -2.1 & -1.6 \\
\hline 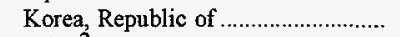 & 42.72 & 40.97 & 40.24 & 42.12 & 44.93 & 41.53 & 4.3 & -1.3 & 3 \\
\hline Other $^{2}$ & 48.89 & 30.41 & 37.24 & 44.23 & 37.06 & 54.29 & 60.8 & 7.2 & -1.1 \\
\hline Oceania \& Australia Total................. & 40.71 & 39.87 & 39.99 & 34.46 & 34.50 & 38.13 & 2.1 & 4.2 & .7 \\
\hline Other ${ }^{2}$ & 40.71 & 39.87 & 39.99 & 34.46 & 34.50 & 38.13 & 2.1 & 4.2 & .7 \\
\hline Africa Total & 44.36 & 43.07 & 43.59 & 42.55 & 42.97 & 41.32 & 3.0 & .8 & .8 \\
\hline 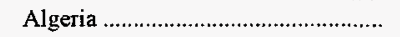 & 50.23 & 47.80 & 43.24 & 44.32 & 46.35 & 43.72 & 5.1 & 2.0 & 1.5 \\
\hline 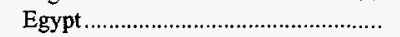 & 53.37 & 49.36 & 43.14 & 44.86 & 46.08 & 42.08 & 8.1 & 3.7 & 2.7 \\
\hline Morocco & 33.93 & 33.00 & 35.03 & 33.86 & 33.80 & 35.55 & 2.8 & .1 & -.5 \\
\hline South Africa, Rep of & 49.55 & 47.38 & 45.67 & 46.87 & 47.79 & 48.25 & 4,6 & .9 & .3 \\
\hline Other $^{2}$ & - & - & - & 40.53 & - & 40.82 & - & - & -100.0 \\
\hline 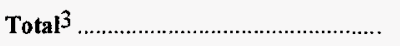 & 40.53 & 40.03 & 39.90 & 41.33 & 41.29 & 42.49 & 1.2 & -.5 & -.5 \\
\hline 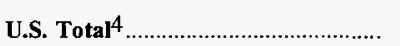 & 40.76 & 40.27 & 39.93 & 41.41 & 41.34 & 42.77 & 1.2 & -.3 & -.5 \\
\hline
\end{tabular}

1 Based on the U.S. - Canada Free Trade Agreement, as of January 1990, the U.S. Department of Commerce began reporting statistics on U.S. exports to Canada based on information on imports provided monthly by the Canadian government.

2 Includes countries with exports less than or equal to 50,000 short tons in 1995.

3 The average prices presented in this table, with the exception of U.S. Total, are considered to be representative prices for coal exports and fall within the range of $\$ 20$ to $\$ 60$ per short ton, inclusively.

4 U.S. Total is the average price of all coal exports.

* Data round to zero.

Note: Average price is based on the free alongside ship (f.a.s.) value.

Source: U.S. Department of Commerce, Bureau of the Census, "Monthly Report EM 545." 
Table 100. Average Real Price of U.S. Coal Exports by Destination, 1987, 1992-1996

(Real Dollars per Short Ton)

\begin{tabular}{|c|c|c|c|c|c|c|c|c|c|}
\hline \multirow{2}{*}{$\begin{array}{c}\text { Continent and Country } \\
\text { of Destination }\end{array}$} & \multirow{2}{*}{1996} & \multirow{2}{*}{1995} & \multirow{2}{*}{1994} & \multirow{2}{*}{1993} & \multirow{2}{*}{1992} & \multirow{2}{*}{1987} & \multirow{2}{*}{$\begin{array}{c}\text { Percent } \\
\text { Change } \\
1995-1996\end{array}$} & \multicolumn{2}{|c|}{ Average Annual Percent Change } \\
\hline & & & & & & & & 1992-1996 & $1987-1996$ \\
\hline North America Total ............................. & $\$ 30.17$ & $\$ 31.64$ & $\$ 30.90$ & $\$ 33.19$ & $\$ 33.05$ & $\$ 49.53$ & -4.7 & -2.3 & -5.4 \\
\hline Canada 1 & 29.38 & 31.12 & 30.58 & 32.91 & 32.98 & 49.56 & -5.6 & -2.8 & -5.6 \\
\hline Jamaica & 31.71 & 30.36 & 31.67 & 27.72 & 26.82 & - & 4.4 & 4.3 & - \\
\hline 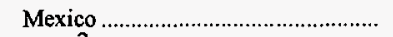 & 36.19 & 37.13 & 42.21 & 42.93 & 44.69 & 47.17 & -2.5 & -5.1 & -2.9 \\
\hline Other $^{2}$ & 36.14 & 36.28 & 35.26 & 35.87 & 36.54 & 46.65 & -.4 & -.3 & -2.8 \\
\hline South America Total............................... & 39.93 & 40.39 & 40.31 & 42.66 & 45.49 & 52.98 & -1.1 & -3.2 & -3.1 \\
\hline 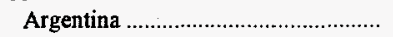 & 42.26 & 40.34 & 40.50 & 42.12 & 45.41 & 52.07 & 4.8 & -1.8 & -2.3 \\
\hline 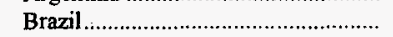 & 40.72 & 40.78 & 40.29 & 42.73 & 45.57 & 53.02 & -.1 & -2.8 & -2.9 \\
\hline Chile & 29.51 & 29.46 & 32.86 & 45.59 & 47.38 & 55.32 & .2 & -11.2 & -6.7 \\
\hline Other $^{2}$ & 35.72 & 40.36 & 38.97 & 38.46 & 31.19 & 61.23 & -11.5 & 3.4 & -5.8 \\
\hline Europe Total & 38.38 & 34.03 & 39.90 & 41.96 & 42.40 & 50.99 & .9 & -2.5 & -3.1 \\
\hline Belgium \& Iuxembourg .................... & 41.69 & 40.40 & 40.26 & 42.13 & 44.01 & 50.07 & 3.2 & -1.3 & -2.0 \\
\hline Bulgaria & 40.35 & 40.93 & 40.13 & 40.90 & 41.70 & - & -1.4 & -.8 & - \\
\hline Denmark & 26.71 & 27.29 & 27.86 & 34.07 & 32.69 & 39.72 & -2.1 & -4.9 & -4.3 \\
\hline 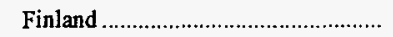 & 38.39 & 36.68 & 39.21 & 38.61 & 40.81 & 49.47 & 4.6 & -1.5 & -2.8 \\
\hline France. & 40.96 & 40.63 & 42.17 & 41.17 & 39.43 & 51.89 & .8 & .9 & -2.6 \\
\hline 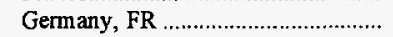 & 37.44 & 32.52 & 43.21 & 37.99 & 38.27 & 50.25 & 15.1 & -.5 & -3.2 \\
\hline Ireland & 34.05 & 33.52 & 32.24 & 34.93 & 36.81 & 52.44 & 1.6 & -1.9 & -4.7 \\
\hline Italy & 41.07 & 41.02 & 40.99 & 43.20 & 45.43 & 50.04 & .1 & -2.5 & -2.2 \\
\hline 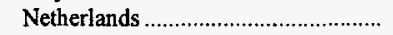 & 37.70 & 39.00 & 39.97 & 43.10 & 43.34 & 52.12 & -3.3 & -3.4 & -3.5 \\
\hline 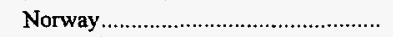 & 52.01 & 52.44 & 52.02 & 52.12 & 50.12 & 56.87 & -.8 & .9 & -1.0 \\
\hline 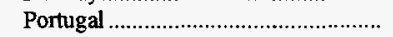 & 33.30 & 33.88 & 34.56 & 36.74 & 40.74 & 51.64 & -1.7 & -4.9 & -4.8 \\
\hline 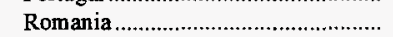 & 42.80 & 39.43 & 36.32 & 37.56 & 42.66 & 51.65 & 8.5 & .1 & -2.1 \\
\hline Spain & 34.24 & 32.29 & 38.25 & 41.58 & 41.95 & 52.69 & 6.0 & -4.9 & -4.7 \\
\hline Sweden & 43.30 & 44.80 & 43.43 & 44.80 & 46.66 & 52.15 & -3.3 & -1.8 & -2.0 \\
\hline 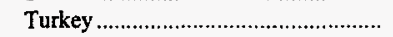 & 40.41 & 39.60 & 39.35 & 41.50 & 45.54 & 53.13 & 2.0 & -2.9 & -3.0 \\
\hline 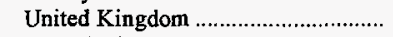 & 35.46 & $3: 3.03$ & 42.95 & 44.77 & 45.59 & 53.26 & -6.8 & -6.1 & -4.4 \\
\hline Yugoslavia, FR & - & 35.37 & - & 38.59 & 42.83 & 51.54 & -100.0 & -100.0 & -100.0 \\
\hline Other $^{2}$ & 34.66 & 51.99 & 36.36 & 39.64 & 41.89 & 51.61 & -33.3 & -4.6 & -4.3 \\
\hline 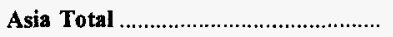 & 36.07 & 36.34 & 36.83 & 39.46 & 42.65 & $\mathbf{5 2 . 2 0}$ & -.7 & -4.1 & -4.0 \\
\hline China (Taiwan) & 33.60 & 34.34 & 36.84 & 38.49 & 41.23 & 48.07 & -2.1 & -5.0 & -3.9 \\
\hline 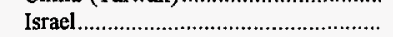 & 33.18 & 33.26 & 31.68 & 33.91 & 39.11 & 44.00 & -.2 & -4.0 & -3.1 \\
\hline Japan & 35.93 & 36.38 & 36.73 & 39.68 & 42.91 & 54.89 & -1.2 & -4.3 & -4.6 \\
\hline Korea Republic of ............................... & 38.95 & 38.08 & 38.36 & 41.05 & 44.93 & 49.98 & 2.3 & -3.5 & -2.7 \\
\hline Other ${ }^{2}$ & 44.57 & 28.26 & 35.50 & 43.11 & 37.06 & 65.33 & 57.7 & 4.7 & -4.1 \\
\hline Oceania \& Australia Total ............... & 37.11 & 37.05 & 38.12 & 33.59 & 34.50 & 45.89 & .1 & 1.8 & -2.3 \\
\hline Other ${ }^{2}$ & 37.11 & 37.05 & 38.12 & 33.59 & 34.50 & 45.89 & .1 & 1.8 & -2.3 \\
\hline 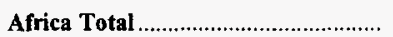 & 40.44 & 40.03 & 41.56 & 41.47 & 42.97 & 49.73 & 1.0 & -1.5 & -2.3 \\
\hline Algeria & 45.79 & 44.42 & 41.22 & 43.20 & 46.35 & 52.61 & 3.1 & -.3 & -1.5 \\
\hline 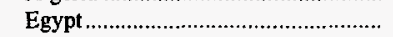 & 48.65 & 45.88 & 41.12 & 43.72 & 46.08 & 50.64 & 6.0 & 1.4 & -.4 \\
\hline Morocco & 30.93 & 30.67 & 33.40 & 33.00 & 33.80 & 42.78 & .9 & -2.2 & -3.5 \\
\hline 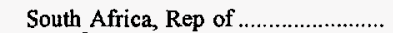 & 45.16 & 44.03 & 43.53 & 45.69 & 47.79 & 58.06 & 2.6 & -1.4 & -2.8 \\
\hline Other 2 & - & - & - & 39.51 & - & 49.13 & - & - & -100.0 \\
\hline Total $^{3}$ & 36.94 & $3 \% .20$ & 38.03 & 40.28 & 41.29 & 51.13 & -.7 & -2.7 & -3.5 \\
\hline U.S. Total ${ }^{4}$ & 37.16 & 37.43 & 38.07 & 40.36 & 41.34 & 51.47 & -.7 & -2.6 & -3.5 \\
\hline
\end{tabular}

1 Based on the U.S. - Canada Free Trade Agreement, as of January 1990, the U.S. Department of Commerce began reporting statistics on U.S. exports to Canada based on information on imports provided monthly by the Canadian government.

2 Includes countries with exports less than or equal to 50,000 short tons in 1995

3 The average prices presented in this table, with the exception of U.S. Total, are considered to be representative prices for coal exports and fall within the range of $\$ 20$ to $\$ 60$ (nominal) per short ton, inclusively.

4 U.S. Total is the average price of all coal exports.

Notes: Real prices are in 1992 dollars, calculated using implicit Gross Domestic Product price deflators. See Appendix D, Table D3. Average prices are based on the free alongside ship (f.a.s.) value.

Source: U.S. Department of Commerce, Bureau of the Census, "Monthly Report EM 545." 
Table 101. Average Price of U.S. Metallurgical Coal Exports by Destination, 1987, 1992-1996

(Nominal Dollars per Short Ton)

\begin{tabular}{|c|c|c|c|c|c|c|c|c|c|}
\hline \multirow{2}{*}{$\begin{array}{c}\text { Continent and Country } \\
\text { of Destination }\end{array}$} & \multirow{2}{*}{1996} & \multirow{2}{*}{1995} & \multirow{2}{*}{1994} & \multirow{2}{*}{1993} & \multirow{2}{*}{1992} & \multirow{2}{*}{1987} & \multirow{2}{*}{$\begin{array}{c}\text { Percent } \\
\text { Change } \\
\text { 1995-1996 }\end{array}$} & \multicolumn{2}{|c|}{ Average Annual Percent Change } \\
\hline & & & & & & & & $1992-1996$ & $1987-1996$ \\
\hline North America Total........................... & $\$ 36.79$ & $\$ 37.25$ & $\$ 35.08$ & $\$ 36.03$ & $\$ 36.39$ & $\$ 44.53$ & $-1,3$ & 0.3 & -2.1 \\
\hline 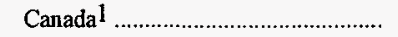 & 35.99 & 36.55 & 34.58 & 35.66 & 36.31 & 44.65 & -1.5 & -.2 & -2.4 \\
\hline 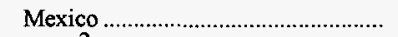 & 47.36 & 46.90 & 44.54 & 45.17 & 47.95 . & - & 1.0 & -.3 & - \\
\hline Other ${ }^{2}$ & - & - & - & - & - & 38.77 & - & - & -100.0 \\
\hline South America Total ........................ & 44.61 & 43.66 & 42.29 & 43.91 & 45.65 & 43.99 & 2.2 & -.6 & .1 \\
\hline 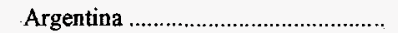 & 46.87 & 43.54 & 42.56 & 43.63 & 45.45 & 43.27 & 7.6 & .8 & .9 \\
\hline 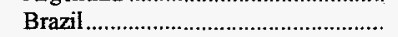 & 44.73 & 43.89 & 42.26 & 43.94 & 45.65 & 44.05 & 1.9 & -.5 & .2 \\
\hline 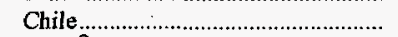 & 30.26 & 30.26 & - & - & 47.40 & 45.97 & $*$ & -10.6 & -4.5 \\
\hline Other ${ }^{2}$ & 50.76 & - & - & - & - & - & - & - & - \\
\hline 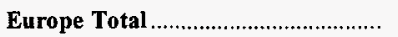 & 47.68 & 46.02 & 44.56 & 45.62 & 46.61 & 43.68 & 3.6 & .6 & 1.0 \\
\hline Belgium \& Luxembourg ..................... & 48.68 & 45.97 & 45.07 & 45.95 & 47.02 & 42.58 & 5.9 & .9 & 1.5 \\
\hline 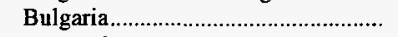 & 43.36 & 44.04 & 42.13 & 41.99 & 41.70 & - & -1.5 & 1.0 & - \\
\hline 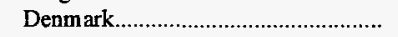 & - & - & - & 36.29 & - & - & - & - & - \\
\hline Finland & 44.21 & 42.65 & 42.34 & 39.61 & 40.81 & 41.11 & 3.6 & 2.0 & .8 \\
\hline France. & 47.13 & 45.08 & 44.36 & 45.17 & 44.45 & 43.40 & 4.5 & 1.5 & .9 \\
\hline 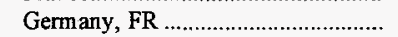 & 49.87 & 47.52 & 45.88 & 46.17 & 45.79 & 43.50 & 4.9 & 2.1 & 1.5 \\
\hline Ireland & - & - & - & - & - & 41.64 & - & - & -100.0 \\
\hline Italy & 47.90 & 46.61 & 45.32 & 45.93 & 47.54 & 44.37 & 2.8 & .2 & .8 \\
\hline 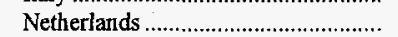 & 47.29 & 46.56 & 44.96 & 46.17 & 47.00 & 44.04 & 1.6 & .1 & 8 \\
\hline 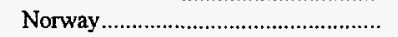 & 57.05 & 56.42 & 54.57 & 53.48 & 50.12 & 46.23 & 1.1 & 3.3 & 2.4 \\
\hline Portugal & 45.20 & 46.45 & - & 38.43 & 44.33 & 44.50 & -2.7 & .5 & .2 \\
\hline Romania & 46.95 & 43.02 & 34.71 & 40.64 & 42.66 & 42.92 & 9.1 & 2.4 & 1.0 \\
\hline Spain & 50.92 & 49.02 & 46.36 & 47.47 & 48.58 & 44.28 & 3.9 & 1.2 & 1.6 \\
\hline Sweden & 48.20 & 48.20 & 45.56 & 45.96 & 46.67 & 43.34 & $*$ & .8 & 1.2 \\
\hline Turkey & 44.54 & 43.28 & 41.28 & 42.58 & 45.54 & 44.15 & 2.9 & -.5 & .1 \\
\hline 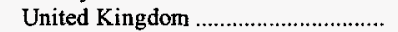 & 49.08 & 47.22 & 45.15 & 46.64 & 47.72 & 44.27 & 3.9 & .7 & 1.1 \\
\hline Yugoslavia, FR & - & - & - & 48.31 & 45.04 & 42.43 & - & -100.0 & -100.0 \\
\hline Other ${ }^{2}$ & 57.43 & 56.04 & 38.00 & - & 41.89 & 40.43 & 2.5 & 8.2 & 4.0 \\
\hline 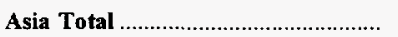 & 43.45 & 42.38 & 41.45 & 43.81 & 45.39 & 46.00 & 2.5 & -1.1 & -.6 \\
\hline China (Taiwan) & 45.24 & 44.48 & 42.48 & 44.86 & 45.63 & 42.15 & 1.7 & -.2 & 8 \\
\hline Israel & 40.91 & 40.91 & - & - & 40.13 & - & $*$ & .5 & - \\
\hline Japan & 42.19 & 41.14 & 40.57 & 43.24 & 44.98 & 46.40 & 2.5 & -1.6 & -1.0 \\
\hline Korea, Republic of ................................ & 46.08 & 45.98 & 44.00 & 45.67 & 47.13 & 44.82 & .2 & -6 & .3 \\
\hline Other ${ }^{2}$ & 59.36 & - & - & 45.13 & - & 54.43 & - & - & 1.0 \\
\hline 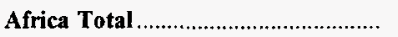 & 50.87 & 48.52 & 43.95 & 45.25 & 46.29 & 43.20 & 4.8 & 2.4 & 1.8 \\
\hline Algeria & 50.23 & 47.80 & 43.24 & 44.32 & 46.35 & 43.72 & 5.1 & 2.0 & 1.5 \\
\hline Egypt & 53.38 & 49.38 & 43.14 & 44.86 & 46.09 & 42.08 & 8.1 & 3.7 & 2.7 \\
\hline Morocco & 30.30 & - & - & - & 34.22 & - & - & -3.0 & - \\
\hline 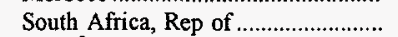 & 49.55 & 47.38 & 45.67 & 46.87 & 47.79 & 48.25 & 4.6 & .9 & .3 \\
\hline Other $^{2}$ & - & $\begin{array}{r}47.50 \\
-\end{array}$ & - & 40.53 & 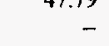 & 40.82 & $\begin{array}{l}4.0 \\
-\end{array}$ & - & -100.0 \\
\hline Total $^{3}$ & 45.40 & 44.24 & 42.75 & 44.09 & $\mathbf{4 5 . 3 8}$ & $\mathbf{4 4 . 4 0}$ & 2.6 & * & .2 \\
\hline U.S. Total ${ }^{4}$ & 45.49 & 44.30 & 42.77 & 44.11 & $\mathbf{4 5 . 4 1}$ & 44.49 & 2.7 & * & .2 \\
\hline
\end{tabular}

1 Based on the U.S. - Canada Free Trade Agreement, as of January 1990, the U.S. Department of Commerce began reporting statistics on U.S. exports to Canada based on information on imports provided monthly by the Canadian government.

2 Includes countries with exports less than or equal to 50,000 short tons in 1995 .

3 The average prices presented in this table, with the exception of U.S. Total, are considered to be representative prices for coal exports and fall within the range of $\$ 20$ to $\$ 60$ per short ton, inclusively.

4 U.S. Total is the average price of all coal exports.

* Data round to zero.

Note: Average price is based on the free alongside ship (f.a.s.) value

Source: U.S. Department of Commerce, Bureau of the Census, "Monthly Report EM 545." 
Table 102. Average Real Price of U.S. Metallurgical Coal Exports by Destination, 1987, 1992-1996

(Real Dollars per Short Ton)

\begin{tabular}{|c|c|c|c|c|c|c|c|c|c|}
\hline \multirow{2}{*}{$\begin{array}{c}\text { Continent and Country } \\
\text { of Destination }\end{array}$} & \multirow{2}{*}{1996} & \multirow{2}{*}{1995} & \multirow{2}{*}{1994} & \multirow{2}{*}{1993} & \multirow{2}{*}{1992} & \multirow{2}{*}{1987} & \multirow{2}{*}{$\begin{array}{c}\text { Percent } \\
\text { Change } \\
\text { 1995-1996 }\end{array}$} & \multicolumn{2}{|c|}{ Average Annual Percent Change } \\
\hline & & & & & & & & 1992-1996 & 1987-1996 \\
\hline North America Total............................ & $\$ 33.53$ & $\$ 34.62$ & $\$ 33.44$ & $\$ 35.12$ & $\$ 36.39$ & $\$ 53.58$ & -3.1 & -2.0 & -5.1 \\
\hline 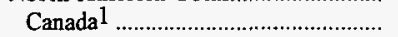 & 32.81 & 33.97 & 32.96 & 34.76 & 36.31 & 53.72 & -3.4 & -2.5 & -5.3 \\
\hline Mexico & 43.17 & 43.59 & 42.46 & 44.03 & 47.95 & - & -1.0 & -2.6 & - \\
\hline Other 2 & - & - & - & - & - & 46.65 & - & - & -100.0 \\
\hline South America Total............................. & 40.67 & 40.58 & 40.31 & 42.80 & 45.65 & 52.94 & .2 & -2.8 & -2.9 \\
\hline 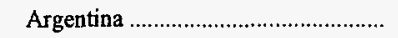 & 42.73 & 40.46 & 40.57 & 42.52 & 45.45 & 52.07 & 5.6 & -1.5 & -2.2 \\
\hline Brazil & 40.78 & 40.79 & 40.29 & 42.83 & 45.65 & 53.01 & $*$ & -2.8 & -2.9 \\
\hline Chile & 27.59 & 28.13 & - & - & 47.40 & 55.32 & -1.9 & -12.6 & -7.4 \\
\hline Other ${ }^{2}$ & 46.27 & - & - & - & - & - & - & - & - \\
\hline 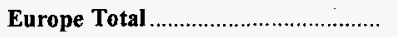 & 43.46 & 42.77 & 42.48 & 44.47 & 46.61 & 52.56 & 1.6 & -1.7 & -2.1 \\
\hline 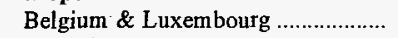 & 44.37 & 42.72 & 42.97 & 44.78 & 47.02 & 51.24 & 3.9 & -1.4 & -1.6 \\
\hline Bulgaria & 39.53 & 40.93 & 40.16 & 40.93 & 41.70 & - & -3.4 & -1.3 & - \\
\hline 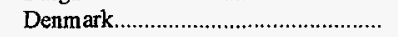 & - & - & - & 35.37 & - & - & - & - & - \\
\hline Finland & 40.30 & 39.64 & 40.37 & 38.60 & 40.81 & 49.47 & 1.7 & -.3 & -2.3 \\
\hline France & 42.96 & 41.90 & 42.29 & 44.02 & 44.45 & 52.23 & 2.5 & -.8 & -2.1 \\
\hline Germany, FR & 45.46 & 44.16 & 43.74 & 45.00 & 45.79 & 52.35 & 2.9 & -.2 & -1.5 \\
\hline Ireland & - & - & - & - & - & 50.11 & - & - & -100.0 \\
\hline Italy & 43.66 & 43.32 & 43.21 & 44.76 & 47.54 & 53.39 & .8 & -2.1 & -2.2 \\
\hline 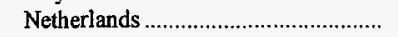 & 43.11 & 43.27 & 42.86 & 45.00 & 47.00 & 53.00 & -.4 & -2.1 & -2.3 \\
\hline 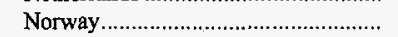 & 52.01 & 52.44 & 52.02 & 52.12 & 50.12 & 55.63 & -.8 & .9 & -.7 \\
\hline 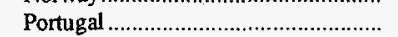 & 41.21 & 43.17 & - & 37.46 & 44.33 & 53.55 & -4.5 & -1.8 & -2.9 \\
\hline 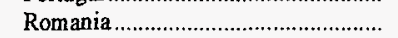 & 42.80 & 39.99 & 33.09 & 39.61 & 42.66 & 51.65 & 7.0 & .1 & -2.1 \\
\hline 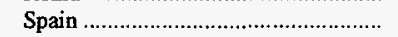 & 46.42 & 45.56 & 44.19 & 46.27 & 48.58 & 53.29 & 1.9 & -1.1 & -1.5 \\
\hline 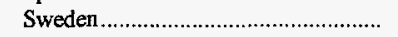 & 43.93 & 44.80 & 43.43 & 44.80 & 46.67 & 52.15 & -1.9 & -1.5 & -1.9 \\
\hline Turkey & 40.60 & 40.22 & 39.35 & 41.51 & 45.54 & 53.13 & .9 & -2.8 & -2.9 \\
\hline 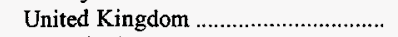 & 44.74 & 43.88 & 43.04 & 45.46 & 47.72 & 53.27 & 2.0 & -1.6 & -1.9 \\
\hline Yugoslavia, FR & - & - & - & 47.08 & 45.04 & 51.05 & - & -100.0 & -100.0 \\
\hline Other ${ }^{2}$ & 52.35 & $5: 2.09$ & 36.23 & - & 41.89 & 48.65 & .5 & 5.7 & .8 \\
\hline 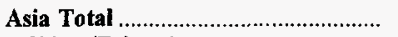 & 39.61 & $3 ! 9.39$ & 39.52 & 42.70 & 45.39 & 55.35 & .6 & -3.3 & -3.6 \\
\hline China (Taiwan) & 4124 & 41.34 & 40.50 & 43.73 & 45.63 & 50.72 & -.2 & -2.5 & -2.3 \\
\hline 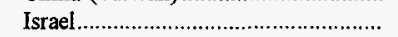 & 37.30 & 33.02 & - & - & 40.13 & - & -1.9 & -1.8 & - \\
\hline Japan & 38.46 & 33.23 & 38.67 & 42.15 & 44.98 & 55.84 & .6 & -3.8 & -4.0 \\
\hline 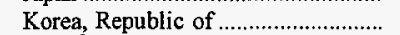 & 42.00 & 42.74 & 41.94 & 44.52 & 47.13 & 53.94 & -1.7 & -2.8 & -2.7 \\
\hline Other ${ }^{2}$ & 54.11 & - & - & 43.99 & - & 65.50 & - & - & -2.1 \\
\hline Africa Total & 46.37 & 45.09 & 41.89 & 44.11 & 46.29 & 51.98 & 2.8 & * & -1.3 \\
\hline Algeria & 45.79 & 44.42 & 41.22 & 43.20 & 46.35 & 52.61 & 3.1 & -.3 & -1.5 \\
\hline Egypt & 48.66 & 45.89 & 41.12 & 43.73 & 46.09 & 50.64 & 6.0 & 1.4 & -.4 \\
\hline Morocco & 27.62 & - & - & - & 34.22 & - & - & -5.2 & - \\
\hline 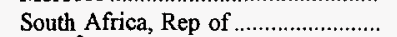 & 45.16 & 44.04 & 43.53 & 45.69 & 47.79 & 58.06 & 2.5 & -1.4 & -2.8 \\
\hline Other $^{2}$ & - & - & - & 39.51 & - & 49.13 & - & - & -100.0 \\
\hline Total $^{3}$ & 41.39 & 41.12 & 40.75 & 42.98 & 45.38 & $\mathbf{5 3 . 4 3}$ & .6 & -2.3 & -2.8 \\
\hline 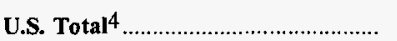 & 41.46 & 41.17 & 40.77 & 42.99 & 45.41 & 53.54 & .7 & -2.2 & -2.8 \\
\hline
\end{tabular}

1 Based on the U.S. - Canada Free Trade Agreement, as of January 1990, the U.S. Department of Commerce began reporting statistics on U.S. exports to Canada based on information on imports provided monthly by the Canadian government.

2 Includes countries with exports less than or equal to 50,000 short tons in 1995 .

3 The average prices presented in this table, with the exception of U.S. Total, are considered to be representative prices for coal exports and fall within the range of $\$ 20$ to $\$ 60$ (nominal) per short ton, inclusively

4 U.S. Total is the average price of all coal exports.

* Data round to zero.

Notes: Real prices are in 1992 dollars, calculated using implicit Gross Domestic Product price deflators. See Appendix D, Table D3. Average prices are based on the free alongside ship (f.a.s.) value.

Source: U.S. Department of Commerce, Bureau of the Census, "Monthly Report EM 545." 
Table 103. Average Price of U.S. Steam Coal Exports by Destination, 1987, 1992-1996

(Nominal Dollars per Short Ton)

\begin{tabular}{|c|c|c|c|c|c|c|c|c|c|}
\hline \multirow{2}{*}{$\begin{array}{l}\text { Continent and Country } \\
\text { of Destination }\end{array}$} & \multirow{2}{*}{1996} & \multirow{2}{*}{1995} & \multirow{2}{*}{1994} & \multirow{2}{*}{1993} & \multirow{2}{*}{1992} & \multirow{2}{*}{1987} & \multirow{2}{*}{$\begin{array}{c}\text { Percent } \\
\text { Change } \\
1995-1996\end{array}$} & \multicolumn{2}{|c|}{ Average Annual Percent Change } \\
\hline & & & & & & & & 1992-1996 & $1987-1996$ \\
\hline North America Total........................... & $\$ 29.41$ & $\$ 31.06$ & $\$ 30.09$ & $\$ 31.68$ & $\$ 31.41$ & $\$ 38.83$ & -5.3 & -1.6 & -3.0 \\
\hline 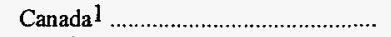 & 28.06 & 30.46 & 29.97 & 31.50 & 31.33 & 38.83 & -7.9 & -2.7 & -3.5 \\
\hline 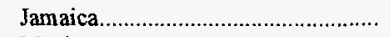 & 34.78 & 32.67 & 33.22 & 28.44 & 26.82 & - & 6.5 & 6.7 & - \\
\hline 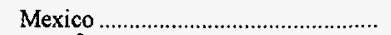 & 36.21 & 35.60 & 41.42 & 40.40 & 41.59 & 39.20 & 1.7 & -3.4 & -.9 \\
\hline Other ${ }^{2}$ & 39.65 & 39.04 & 36.98 & 36.80 & 36.54 & 40.02 & 1.6 & 2.1 & -.1 \\
\hline South America Total............................. & 34.94 & 35.66 & 36.52 & 40.05 & 41.06 & $\mathbf{4 5 . 4 2}$ & -2.0 & -3.9 & -2.9 \\
\hline Argentina & 40.53 & 36.09 & 33.11 & 37.81 & 30.13 & 39.98 & 12.3 & 7.7 & .1 \\
\hline 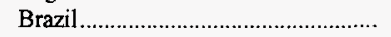 & 40.57 & 34.81 & - & 40.61 & 42.95 & 44.22 & 16.5 & -1.4 & -.9 \\
\hline Chile & 32.76 & 32.96 & 34.47 & 46.78 & 40.96 & - & -.6 & -5.4 & - \\
\hline Other ${ }^{2}$ & 39.16 & 43.42 & 40.88 & 39.46 & 31.19 & 50.88 & -9.8 & 5.8 & -2.9 \\
\hline 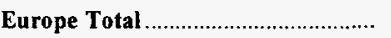 & 33.71 & 34.33 & 35.01 & 36.50 & 36.44 & 39.30 & -1.8 & -1.9 & -1.7 \\
\hline Belgium \& Luxembourg ...................... & 36.69 & 35.07 & 33.49 & 34.09 & 36.35 & 35.65 & 4.6 & .2 & .3 \\
\hline 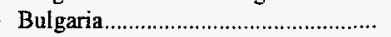 & 50.55 & - & 41.40 & 41.51 & - & - & - & - & - \\
\hline 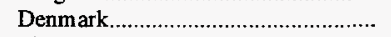 & 29.30 & 29.37 & 29.23 & 34.34 & 32.69 & 33.01 & -.2 & -2.7 & -1.3 \\
\hline Finland & 35.23 & 35.53 & 35.47 & 40.85 & - & - & -.9 & - & - \\
\hline France & 36.14 & 35.13 & 38.11 & 31.83 & 33.64 & 36.50 & 2.9 & 1.8 & -.1 \\
\hline 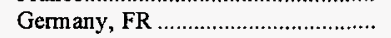 & 31.92 & 33.31 & 40.67 & 34.19 & 35.82 & 38.06 & -4.2 & -2.8 & -1.9 \\
\hline Ireland & 37.35 & 36.07 & 33.82 & 35.84 & 36.81 & 43.72 & 3.6 & .4 & -1.7 \\
\hline Italy & 41.20 & 41.70 & 38.30 & 40.26 & 41.32 & 38.15 & -1.2 & -.1 & .8 \\
\hline 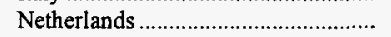 & 32.94 & 36.45 & 35.95 & 40.21 & 39.24 & 42.04 & -9.6 & -4.3 & -2.7 \\
\hline 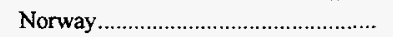 & - & - & - & - & - & 57.76 & - & - & -100.0 \\
\hline 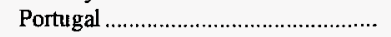 & 35.60 & 36.28 & 36.25 & 37.62 & 40.38 & 42.62 & -1.9 & -3.1 & -2.0 \\
\hline 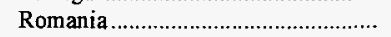 & - & 39.08 & 40.63 & 36.29 & - & - & -100.0 & - & - \\
\hline Spain & 22.14 & 21.37 & 21.19 & 25.21 & 28.38 & 30.45 & 3.6 & -6.0 & -3.5 \\
\hline Sweden & 39.21 & 48.54 & - & - & 34.53 & - & -19.2 & 3.2 & - \\
\hline Turkey & 41.28 & 30.98 & - & 40.84 & 40.72 & - & 33.2 & .3 & - \\
\hline 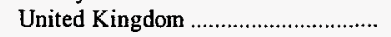 & 28.82 & 30.63 & 42.71 & 41.26 & 39.34 & 35.22 & -5.9 & -7.5 & -2.2 \\
\hline Yugoslavia, FR & - & 38.06 & - & 29.03 & 22.35 & 50.42 & -100.0 & -100.0 & -100.0 \\
\hline Other 2 & 34.56 & 40.69 & 40.92 & 40.67 & 42.07 & 50.08 & -15.1 & -4.8 & -4.0 \\
\hline Asia Total & 35.84 & 34.63 & 35.18 & 36.52 & 38.27 & 38.53 & 3.5 & -1.6 & -.8 \\
\hline China (Taiwan) & 35.33 & 35.66 & 38.28 & 39.01 & 40.63 & 39.70 & -.9 & -3.4 & -1.3 \\
\hline 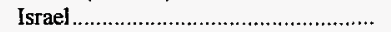 & 35.12 & 34.63 & 33.23 & 34.79 & 38.89 & 36.56 & 1.4 & -2.5 & -.4 \\
\hline Japan & 36.31 & 35.03 & 33.57 & 35.45 & 35.98 & 37.48 & 3.6 & .2 & -.3 \\
\hline 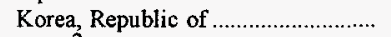 & 35.32 & 32.01 & 32.56 & 34.34 & 37.24 & 36.26 & 10.3 & -1.3 & -.3 \\
\hline Other $^{2}$ & 38.26 & 30.41 & 37.24 & 37.31 & 37.06 & 39.11 & 25.8 & .8 & -.2 \\
\hline Oceania \& Australia Total ................ & 40.71 & 39.87 & 39.99 & 34.46 & 34.50 & 38.13 & 2.1 & 4.2 & .7 \\
\hline Other 2 & 40.71 & 39.87 & 39.99 & 34.46 & 34.50 & 38.13 & 2.1 & 4.2 & .7 \\
\hline Africa Total & 34.02 & 33.01 & 35.12 & 33.86 & 33.78 & 35.55 & 3.1 & .2 & -.5 \\
\hline Egypt & 40.78 & 40.81 & 40.89 & 40.88 & 39.92 & 58.65 & -.1 & .5 & -3.9 \\
\hline Morocco & 34.01 & 33.00 & 35.03 & 33.86 & 33.77 & 35.55 & 3.1 & .2 & -.5 \\
\hline 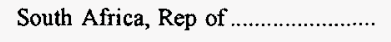 & - & 39.80 & - & - & - & - & -100.0 & - & - \\
\hline 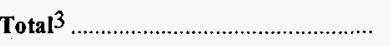 & 33.51 & 33.89 & 34.03 & 35.67 & 35.57 & 38.91 & -1.1 & -1.5 & -1.6 \\
\hline 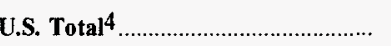 & 34.09 & 34.51 & 34.34 & 36.03 & 35.73 & 39.60 & -1.2 & -1.2 & -1.6 \\
\hline
\end{tabular}

1 Based on the U.S. - Canada Free Trade Agreement, as of January 1990, the U.S. Department of Commerce began reporting statistics on U.S. exports to Canada based on information on imports provided monthly by the Canadian govemment.

2 Includes countries with exports less than or equal to 50,000 short tons in 1995 .

3 The average prices presented in this table, with the exception of U.S. Total, are considered to be representative prices for coal exports and fall within the range of $\$ 20$ to $\$ 60$ per short ton, inclusively.

4 U.S. Total is the average price of all coal exports.

Notes: Average price is based on the free alongside ship (f.a.s.) value. Steam coal includes bituminous, subbituminous, lignite, and anthracite. Source: U.S. Department of Commerce, Bureau of the Census, "Monthly Report EM 545." 
Table 104. Average Real Price of U.S. Steam Coal Exports by Destination, 1987, 1992-1996

(Real Dollars per Short Ton)

\begin{tabular}{|c|c|c|c|c|c|c|c|c|c|}
\hline \multirow{2}{*}{$\begin{array}{l}\text { Continent and Country } \\
\text { of Destination }\end{array}$} & \multirow{2}{*}{1996} & \multirow{2}{*}{1995} & \multirow{2}{*}{1994} & \multirow{2}{*}{1993} & \multirow{2}{*}{1992} & \multirow{2}{*}{1987} & \multirow{2}{*}{$\begin{array}{c}\text { Percent } \\
\text { Change } \\
1995-1996\end{array}$} & \multicolumn{2}{|c|}{ Average Annual Percent Change } \\
\hline & & & & & & & & 1992-1996 & 1987-1996 \\
\hline North America Total............................. & $\$ 26.81$ & $\$ 48.87$ & $\$ 28.69$ & $\$ 30.87$ & $\$ 31.41$ & $\$ 46.73$ & -7.1 & -3.9 & -6.0 \\
\hline Canada 1 & 25.58 & 28.31 & 28.57 & 30.70 & 31.33 & 46.73 & -9.7 & -4.9 & -6.5 \\
\hline 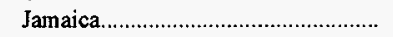 & 31.71 & 30.36 & 31.67 & 27.72 & 26.82 & - & 4.4 & 4.3 & - \\
\hline 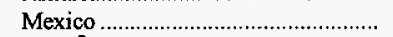 & 33.00 & 33.09 & 39.48 & 39.37 & 41.59 & 47.17 & -.3 & -5.6 & -3.9 \\
\hline Other ${ }^{2}$ & 36.14 & 36.28 & 35.26 & 35.87 & 36.54 & 48.16 & -.4 & -.3 & -3.1 \\
\hline South America Total............................. & 31.85 & 33.14 & 34.81 & 39.04 & 41.06 & 54.65 & -3.9 & -6.1 & -5.8 \\
\hline 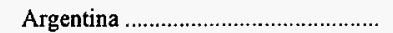 & 36.95 & 33.54 & 31.56 & 36.85 & 30.13 & 48.11 & 10.1 & 5.2 & -2.9 \\
\hline 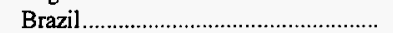 & 36.99 & 32.36 & - & 39.58 & 42.95 & 53.21 & 14.3 & -3.7 & -3.9 \\
\hline Chile & 29.86 & 30.63 & 32.86 & 45.59 & 40.96 & - & -2.5 & -7.6 & - \\
\hline Other ${ }^{2}$ & 35.69 & 40.36 & 38.97 & 38.46 & 31.19 & 61.23 & -11.5 & 3.4 & -5.8 \\
\hline Europe Total & 30.73 & 31.90 & 33.38 & 35.57 & 36.44 & 47.29 & -3.7 & -4.2 & -4.7 \\
\hline 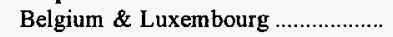 & 33.45 & 32.59 & 31.92 & 33.23 & 36.35 & 42.90 & 2.6 & -2.0 & -2.7 \\
\hline 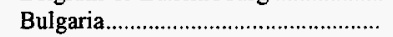 & 46.08 & - & 39.46 & 40.46 & - & - & - & - & - \\
\hline Denmark & 26.71 & 27.29 & 27.86 & 33.47 & 32.69 & 39.72 & -2.1 & -4.9 & -4.3 \\
\hline Finland & 32.11 & 33.02 & 33.81 & 39.81 & - & - & -2.8 & - & - \\
\hline 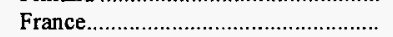 & 32.94 & 32.65 & 36.33 & 31.02 & 33.64 & 43.92 & .9 & -.5 & -3.1 \\
\hline Germany, FR & 29.10 & 30.96 & 38.77 & 33.33 & 35.82 & 45.81 & -6.0 & -5.0 & -4.9 \\
\hline Ireland & 34.05 & 33.52 & 32.24 & 34.93 & 36.81 & 52.61 & 1.6 & -1.9 & -4.7 \\
\hline Italy & 37.55 & 38.76 & 36.51 & 39.24 & 41.32 & 45.91 & -3.1 & -2.4 & -2.2 \\
\hline 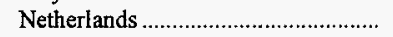 & 30.03 & 33.88 & 34.27 & 39.19 & 39.24 & 50.59 & -11.4 & -6.5 & -5.6 \\
\hline 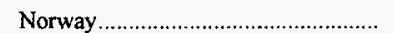 & - & - & - & - & - & 69.51 & - & - & -100.0 \\
\hline Portugal & 32.45 & 33.72 & 34.56 & 36.67 & 40.38 & 51.29 & -3.8 & -5.3 & -4.9 \\
\hline Romania & - & 36.32 & 38.74 & 35.37 & - & - & -100.0 & - & - \\
\hline 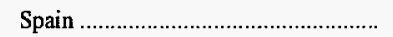 & 20.19 & 19.86 & 20.20 & 24.57 & 28.38 & 36.64 & 1.6 & -8.2 & -6.4 \\
\hline Sweden & 35.74 & 45.11 & - & - & 34.53 & - & -20.8 & .9 & - \\
\hline Turkey & 37.63 & 28.80 & - & 39.80 & 40.72 & - & 30.7 & -1.9 & - \\
\hline United Kingdom ................................. & 26.27 & 28.47 & 40.71 & 40.22 & 39.34 & 42.38 & -7.7 & -9.6 & -5.2 \\
\hline Yugoslavia, FR & - & 35.37 & - & 28.29 & 22.35 & 60.67 & -100.0 & -100.0 & -100.0 \\
\hline Other $^{2}$ & 31.51 & 37.82 & 39.01 & 39.64 & 42.07 & 60.27 & -16.7 & -7.0 & -6.9 \\
\hline 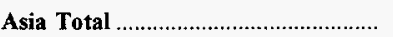 & 32.67 & 32.18 & 33.54 & 35.59 & 38.27 & 46.36 & 1.5 & -3.9 & -3.8 \\
\hline China (Taiwan) & 32.21 & 33.14 & 36.49 & 38.02 & 40.63 & 47.77 & -2.8 & -5.6 & $-4,3$ \\
\hline Israel & 32.01 & 32.18 & 31.68 & 33.91 & 38.89 & 44.00 & -.5 & -4.8 & -3.5 \\
\hline Japan & 33.10 & 32.55 & 32.00 & 34.55 & 35.98 & 45.10 & 1.7 & -2.1 & -3.4 \\
\hline 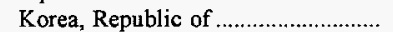 & 32.20 & 29.75 & 31.04 & 33.47 & 37.24 & 43.63 & 8.2 & -3.6 & -3.3 \\
\hline Other ${ }^{2}$ & 34.87 & 28.26 & 35.50 & 36.37 & 37.06 & 47.06 & 23.4 & -1.5 & -3.3 \\
\hline Oceania \& Australia Total................ & 37.11 & 37.05 & 38.12 & 33.59 & 34.50 & 45.89 & .1 & 1.8 & -2.3 \\
\hline Other ${ }^{2}$ & 37.11 & 37.05 & 38.12 & 33.59 & 34.50 & 45.89 & 1 & 1.8 & -2.3 \\
\hline 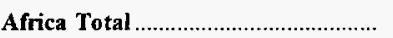 & 31.01 & 30.68 & 33.48 & 33.01 & 33.78 & 42.78 & 1.1 & -2.1 & -3.5 \\
\hline 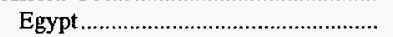 & 37.17 & 37.93 & 38.98 & 39.85 & 39.92 & 70.58 & -2.0 & -1.8 & -6.9 \\
\hline Morocco & 31.01 & 30.67 & 33.40 & 33.00 & 33.77 & 42.78 & 1.1 & -2.1 & -3.5 \\
\hline 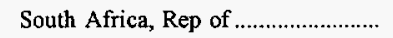 & - & 36.99 & - & - & - & - & -100.0 & - & - \\
\hline Total $^{3}$ & 30.55 & 31.49 & 32.44 & 34.76 & 35.57 & 46.82 & -3.0 & -3.7 & -4.6 \\
\hline 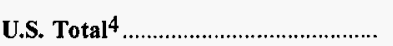 & 31.08 & 32.08 & 32.74 & 35.12 & 35.73 & 47.65 & -3.1 & -3.4 & -4.6 \\
\hline
\end{tabular}

1 Based on the U.S. - Canada Free Trade Agreement, as of January 1990, the U.S. Department of Commerce began reporting statistics on U.S. exports to Canada based on information on imports provided monthly by the Canadian government.

2 Includes countries with exports less than or equal to 50,000 short tons in 1995.

3 The average prices presented in this table, with the exception of U.S. Total, are considered to be representative prices for coal exports and fall within the range of $\$ 20$ to $\$ 60$ (nominal) per short ton, inclusively.

4 U.S. Total is the average price of all coal exports.

Notes: Real prices are in 1992 dollars, calculated using implicit Gross Domestic Product price deflators. See Appendix D, Table D3. Average prices are based on the free alongside ship (f.a.s.) value. Steam coal includes bituminous, subbituminous, lignite, and anthracite.

Source: U.S. Department of Commerce, Bureau of the Census, "Monthly Report EM 545." 


\section{Coal Quality and Emissions}

As of January 1, 1995, recoverable U.S. coal reserves were estimated to total 274 billion short tons (Table 105). Reserves of low sulfur coal (defined as coal containing from 0 to 0.60 pounds of sulfur per million Btu) are estimated to represent 37 percent of all reserves, with medium sulfur coal (containing 0.61 to 1.67 pounds sulfur per million Btu) and high sulfur coal (containing more than 1.67 pounds sulfur per million Btu) each accounting for 32 percent of total reserves.

Compared with 1995, the average sulfur content of coal delivered to the Nation's electric utilities during
1996 increased 1.6 percent to 1.10 percent sulfur by weight, while the average ash content remained about the same at 9.22 percent by weight (Table 106). The average heat content of electric utility coal totaled 10,263 Btu per pound.

By comparison, the average heat content of coal delivered to manufacturing and coke plants during 1996 increased slightly ( 0.3 percent) to 11,405 Btu per pound, while the average ash content and average sulfur content of industrial coal was 7.58 percent and 1.17 percent, respectively. 
Table 105. Estimate of Recoverable Reserves of Coal by Sulfur Range, State, and Mine Type

(Million Short Tons Remaining as of January 1, 1995)

\begin{tabular}{|c|c|c|c|c|c|c|c|}
\hline \multirow{2}{*}{ State and Type of Mining } & \multicolumn{6}{|c|}{$\begin{array}{l}\text { Sulfur Content } \\
\text { (pounds of sulfur per milion Btu) }\end{array}$} & \multirow{2}{*}{ Total } \\
\hline & $<=0.40$ & $0.41-0.60$ & $0.61-0.83$ & $0.84-1.67$ & $1.68-2.50$ & $>2.50$ & \\
\hline Alabama .......... & - & 413 & 374 & 2,036 & 194 & - & 3,018 \\
\hline Surface & - & 255 & 269 & 1,670 & 137 & - & 2,332 \\
\hline 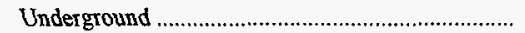 & - & 158 & 105 & 366 & 57 & - & 686 \\
\hline Alaska, Southern & $\therefore, 453$ & 94 & - & - & - & - & 2,548 \\
\hline Surface & 386 & 41 & - & - & - & - & 427 \\
\hline Underground & 2,067 & 53 & - & - & - & - & 2,120 \\
\hline Alaska, Northern & - & - & - & - & - & - & - \\
\hline Surface & - & - & - & - & - & - & - \\
\hline Underground & - & - & - & - & $\rightarrow$ & - & - \\
\hline Arizona & - & 121 & - & - & - & - & 121 \\
\hline Surface & - & 69 & - & - & - & - & 69 \\
\hline Underground & - & 51 & - & - & - & - & 51 \\
\hline 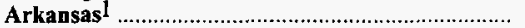 & - & 8 & 163 & 44 & 6 & 7 & 228 \\
\hline Surface & - & 2 & 73 & 24 & 1 & 1 & 101 \\
\hline Underground & - & 7 & 90 & 20 & 5 & 6 & 127 \\
\hline 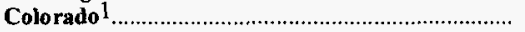 & 3,674 & 2,316 & 3,684 & 326 & 96 & - & 10,096 \\
\hline Surface & 273 & 120 & 3,325 & 41 & 14 & - & 3,772 \\
\hline Underground & 3,400 & 2,196 & 359 & 285 & 82 & - & 6,323 \\
\hline 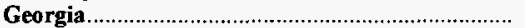 & 1 & 1 & * & * & * & * & 2 \\
\hline 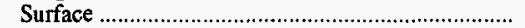 & * & * & * & * & * & * & 1 \\
\hline Underground & * & - & * & * & * & * & 1 \\
\hline Idaho & * & 1 & 1 & 1 & - & - & 2 \\
\hline Surface & - & - & - & - & - & - & - \\
\hline 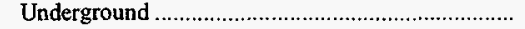 & * & 1 & 1 & 1 & - & - & 2 \\
\hline 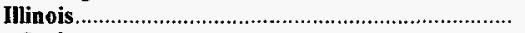 & 5 & 113 & 350 & 1,456 & 1,567 & 30,477 & 33,969 \\
\hline Surface & - & - & 1 & 43 & 291 & 9,031 & 9,366 \\
\hline Underground & 5 & 113 & 349 & 1,413 & 1,276 & 21,445 & 24,603 \\
\hline Indiana & - & 312 & 185 & 674 & 1,125 & 2,033 & 4,329 \\
\hline Surface & - & 63 & 27 & 107 & 149 & 307 & 654 \\
\hline Underground & - & 248 & 158 & 567 & 976 & 1,727 & 3,675 \\
\hline Iowa & - & - & - & - & 407 & 720 & 1,127 \\
\hline Surface & - & - & - & - & 320 & - & 320 \\
\hline Underground & - & - & - & - & 87 & 720 & 807 \\
\hline Kansas & - & - & - & - & 226 & 457 & 683 \\
\hline Surface & - & - & - & - & 226 & 457 & 683 \\
\hline 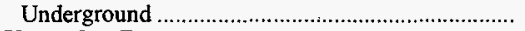 & - & - & - & - & - & - & - \\
\hline Ken tucky, Eastern & 175 & 2,077 & 1,434 & 1,769 & 860 & 657 & 6,973 \\
\hline Surface & 140 & 1,657 & 1,144 & 1,412 & 686 & 525 & 5,563 \\
\hline Underground & 35 & 420 & 290 & 358 & 174 & 133 & 1,410 \\
\hline Ken tucky, Western & - & - & - & 156 & 2,723 & 6,406 & 9,285 \\
\hline Surface & - & - & - & 125 & 926 & 1,276 & 2,327 \\
\hline 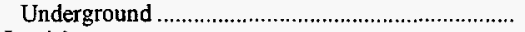 & - & - & - & 32 & 1,798 & 5,130 & 6,959 \\
\hline Louisiana. & - & - & - & 349 & - & - & 349 \\
\hline Surface & - & - & - & 349 & - & - & 349 \\
\hline 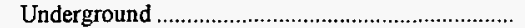 & - & - & - & - & - & - & - \\
\hline Maryland & - & 31 & 58 & 118 & 205 & - & 412 \\
\hline Surface & - & 3 & 8 & 13 & 32 & - & 56 \\
\hline Underground & - & 28 & 50 & 105 & 173 & - & 356 \\
\hline Michigan & - & - & 8 & 23 & 16 & 11 & 59 \\
\hline Surface & - & - & 1 & 2 & 1 & $*$ & 3 \\
\hline 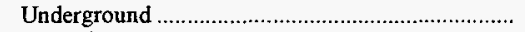 & - & - & 8 & 21 & 16 & 11 & 55 \\
\hline 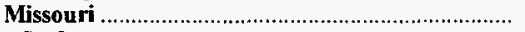 & - & - & - & - & 170 & 3,681 & $\mathbf{3 , 8 5 1}$ \\
\hline Surface & - & - & - & - & 150 & 3,012 & 3,162 \\
\hline Underground & - & - & - & - & 20 & 670 & 689 \\
\hline Montana & 33,577 & 16,828 & 16,776 & 4,815 & 2,022 & 1,371 & 75,389 \\
\hline 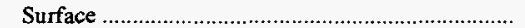 & 18,014 & 6,995 & 9,499 & 2,444 & 1,610 & 906 & 39,466 \\
\hline Underground & 15,563 & 9,833 & 7,277 & 2,371 & 413 & 466 & 35,923 \\
\hline New Mexico ${ }^{1}$ & 62 & 2,638 & 1,890 & $\mathbf{3 , 6 2 5}$ & - & - & 8,215 \\
\hline Surface & 37 & 1,229 & 1,348 & 2,587 & - & - & 5,202 \\
\hline Underground & 25 & 1,409 & 542 & 1,038 & - & - & 3,013 \\
\hline North Carolina. & - & - & * & 2 & 2 & 1 & 5 \\
\hline Surface & - & - & - & - & - & - & - \\
\hline 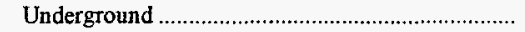 & - & - & * & 2 & 2 & 1 & 5 \\
\hline North Dakota. & 436 & 750 & 1,375 & 3,438 & 857 & 369 & 7,224 \\
\hline 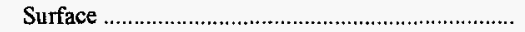 & 436 & 750 & 1,375 & 3,438 & 857 & 369 & 7,224 \\
\hline Underground & - & - & - & - & - & - & - \\
\hline Ohio & 82 & 169 & 335 & 1,049 & 2,636 & $\mathbf{7 , 4 4 7}$ & 11,718 \\
\hline Surface & 20 & 96 & 168 & 416 & 863 & 2,304 & 3,867 \\
\hline Underground & 62 & 73 & 168 & 634 & 1,773 & 5,143 & 7,852 \\
\hline
\end{tabular}

See footnotes at end of table. 
Table 105. Estimate of Recoverable Reserves of Coal by Sulfur Range, State, and Mine Type (Continued)

(Million Short Tons Remaining as of January 1, 1995)

\begin{tabular}{|c|c|c|c|c|c|c|c|}
\hline \multirow{2}{*}{ State and Type of Mining } & \multicolumn{6}{|c|}{$\begin{array}{c}\text { Sulfur Content } \\
\text { (pounds of sulfur per million Btu) }\end{array}$} & \multirow{2}{*}{ Total } \\
\hline & $<=0.40$ & $0.41-0.60$ & $0.61-0.83$ & $0.84-1.67$ & $1.68-2.50$ & $>2.50$ & \\
\hline 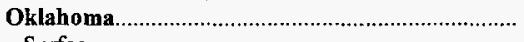 & - & 220 & 123 & 178 & 114 & 181 & 816 \\
\hline Surface & - & 66 & 25 & 34 & 33 & 82 & 240 \\
\hline Underground & - & 154 & 98 & 145 & 80 & 100 & 577 \\
\hline Oregon & 4 & 1 & 3 & - & 1 & 1 & 9 \\
\hline Surface & 1 & * & 1 & - & $*$ & $*$ & 2 \\
\hline 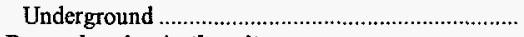 & 3 & 1 & 2 & - & 1 & 1 & 7 \\
\hline Pennsylvania, Anth racite & 180 & 468 & 96 & 17 & 2 & * & 763 \\
\hline Surface & 85 & 263 & 62 & 11 & 1 & * & 422 \\
\hline 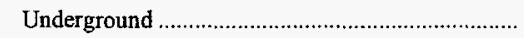 & 95 & 205 & 35 & 6 & 1 & - & 341 \\
\hline 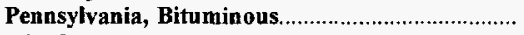 & - & 283 & 807 & 5,001 & 4,151 & 1,517 & 11,757 \\
\hline Surface & - & 22 & 64 & 307 & 227 & 135 & 756 \\
\hline Underground & - & 260 & 742 & 4,694 & 3,923 & 1,381 & 11,001 \\
\hline 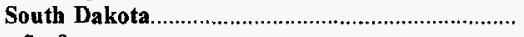 & - & - & 104 & 1 & 172 & - & 277 \\
\hline Surface & - & - & 104 & 1 & 172 & - & 277 \\
\hline 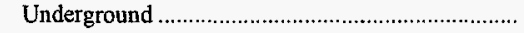 & - & - & - & - & - & - & - \\
\hline 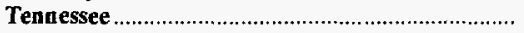 & - & 106 & 62 & 224 & 99 & - & 491 \\
\hline Surface & - & 39 & 23 & 93 & 40 & - & 195 \\
\hline 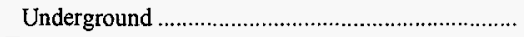 & - & 67 & 38 & 131 & 59 & - & 295 \\
\hline 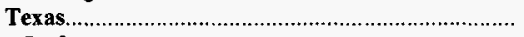 & - & - & 590 & 5,681 & 3,409 & 376 & 10,057 \\
\hline Surface & - & - & 590 & 5,681 & 3,409 & 376 & 10,057 \\
\hline 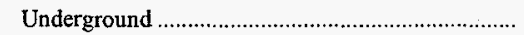 & - & - & - & - & - & - & - \\
\hline Utah & 378 & 678 & 554 & 864 & 225 & 301 & 3,001 \\
\hline Surface & 8 & 32 & 19 & 85 & 35 & 34 & 212 \\
\hline Underground & 370 & 646 & 535 & 780 & 190 & 267 & 2,788 \\
\hline 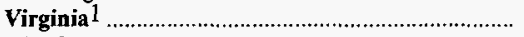 & 195 & 632 & 403 & 133 & - & - & 1,362 \\
\hline Surface & 43 & 197 & 161 & 46 & - & - & 447 \\
\hline Underground & 152 & 435 & 241 & 87 & - & - & 915 \\
\hline Washington & 63 & 92 & 86 & 489 & - & - & 729 \\
\hline 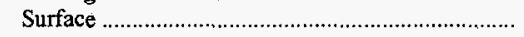 & - & - & - & 54 & - & - & 54 \\
\hline Underground & 63 & 92 & 86 & 434 & - & - & 675 \\
\hline West Virginia & 653 & 6,497 & 2,736 & 4,036 & 2,516 & 3,211 & 19,649 \\
\hline 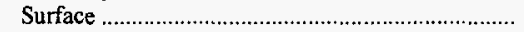 & 116 & 1,464 & 568 & 447 & 180 & 107 & 2,881 \\
\hline 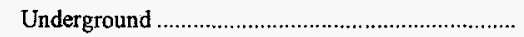 & 537 & 5,034 & 2,168 & 3,589 & 2,337 & 3,104 & 16,769 \\
\hline Wyoming & 6,786 & 17,586 & 10,186 & $\mathbf{7 , 4 3 8}$ & 1,168 & 2,241 & 45,403 \\
\hline Surface & 4,434 & 8,822 & 4,134 & 2,314 & 527 & 2,208 & 22,439 \\
\hline Underground & 2,352 & 8,764 & 6,052 & 5,123 & 641 & 33 & 22,964 \\
\hline U.S. Total & 48,723 & 52,434 & 42,380 & 43,943 & 24,968 & 61,466 & 273,913 \\
\hline Surface & 23,993 & 22,185 & 22,987 & 21,742 & 10,888 & 21,130 & 122,925 \\
\hline Underground & 24,730 & 30,249 & 19,393 & 22,201 & 14,080 & 40,336 & 150,988 \\
\hline
\end{tabular}

1 Data include minor amounts of anthracite (all occurring in heat content categories greater than 23.00 million short tons) as follows: Arkansas 52.2 , Colorado 13.4, New Mexico 1.2, and Virginia 70.5, expressed in million short tons.

* Data round to zero.

Note: Totals may not equal sum of components due to independent rounding.

Source: Energy Information Administration, U.S. Coal Reserves: A Review and Update (DOE/EIA-0529(95)), August, 1996. 
Table 106. Average Quality of Coal Received at Electric Utilities by Census Division and State, 1987, 1992-1996

\begin{tabular}{|c|c|c|c|c|c|c|c|c|c|}
\hline \multirow{2}{*}{$\begin{array}{l}\text { Census Division and State } \\
\text { and Qualityl }\end{array}$} & \multirow{2}{*}{1996} & \multirow{2}{*}{1995} & \multirow{2}{*}{1994} & \multirow{2}{*}{1993} & \multirow{2}{*}{1992} & \multirow{2}{*}{1987} & \multirow{2}{*}{$\begin{array}{c}\text { Percent } \\
\text { Change } \\
\text { 1995-1996 }\end{array}$} & \multicolumn{2}{|c|}{ Average Annual Percent Change } \\
\hline & & & & & & & & $1992-1996$ & 1987-1996 \\
\hline \multicolumn{10}{|l|}{ New England } \\
\hline 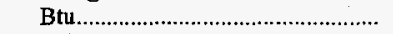 & 12,793 & 12,848 & 12,897 & 13,033 & 13,120 & 13,185 & * & -1 & * \\
\hline 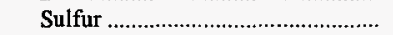 & .85 & .84 & .98 & 1.11 & 1.18 & 1.29 & 0.9 & -7.8 & -4.5 \\
\hline Ash & 7.75 & 7.48 & 7.49 & 7.62 & 7.66 & 7.37 & 3.6 & 3 & $*$ \\
\hline \multicolumn{10}{|l|}{ Connecticut } \\
\hline 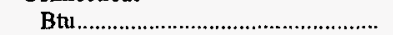 & 13,100 & 13,110 & 13,094 & 13,144 & 13,167 & 13,134 & * & * & * \\
\hline Sulfur & .54 & .56 & .54 & .55 & .55 & .49 & -3.4 & -.5 & 1.3 \\
\hline Ash & 7.14 & $\because .05$ & 7.38 & 6.95 & 6.25 & 6.74 & 1.3 & 3.4 & .6 \\
\hline Massachusetts & & & & & & & & & \\
\hline 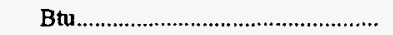 & 12,633 & 12,598 & 12,814 & 12,951 & 13,070 & 13,128 & -1 & -1 & * \\
\hline Sulfur & .71 & .71 & .91 & 1.03 & 1.17 & 1.18 & -.3 & -11.7 & -5.5 \\
\hline Ash & 8.07 & 7.83 & 7.85 & 8.10 & 8.26 & 8.07 & 3.1 & -.5 & * \\
\hline New Hampshire & & & & & & & & & \\
\hline Btu & 13,146 & 13,111 & 13,032 & 13,179 & 13,260 & 13,416 & * & * & * \\
\hline Sulfur & 1.56 & 1.38 & 1.52 & 1.62 & 1.61 & 2.20 & 13.2 & -.7 & -3.7 \\
\hline Ash & 7.02 & 6.74 & 6.40 & 6.75 & 6.52 & 7.44 & 4.1 & 1.9 & -.6 \\
\hline Middle Atlantic & & & & & & & & & \\
\hline 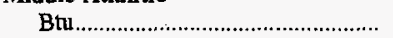 & 12,460 & 12,474 & 12,509 & 12,556 & 12,555 & 12,401 & * & * & - \\
\hline 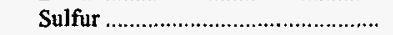 & 2.01 & 2.03 & 2.01 & 1.96 & 1.99 & 2.00 & -.8 & .2 & .1 \\
\hline 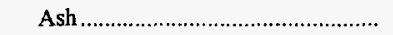 & 11.80 & 11.93 & 11.52 & 11.29 & 11.45 & 12.64 & -1.1 & .8 & -.8 \\
\hline New Jersey & & & & & & & & & \\
\hline Btu... & 12,993 & 13,282 & 13,341 & 13,397 & 13,465 & 13,236 & -2 & -1 & * \\
\hline 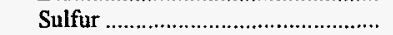 & 1.36 & 1.21 & 1.29 & 1.29 & 1.29 & 1.32 & 12.2 & 1.3 & .3 \\
\hline 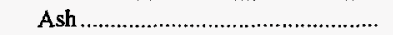 & 9.02 & 7.51 & 7.44 & 7.21 & 6.84 & 8.05 & 20.1 & 7.2 & 1.3 \\
\hline New York & & & & & & & & & \\
\hline Btu & 13,013 & 13,051 & 12,959 & 12,914 & 12,978 & 12,787 & * & * & * \\
\hline 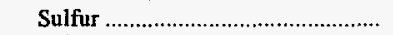 & 1.80 & 1.79 & 1.71 & 1.55 & 1.65 & 1.84 & .3 & 2.1 & -.3 \\
\hline Ash & 7.91 & 7.90 & 7.98 & 8.15 & 8.02 & 9.69 & .2 & -.3 & -2.2 \\
\hline Pennsylvania & & & & & & & & & \\
\hline 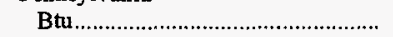 & 12,321 & 12,315 & 12,368 & 12,443 & 12,399 & 12,276 & * & * & * \\
\hline 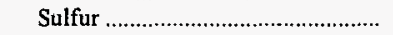 & 2.09 & 2.12 & 2.11 & 2.07 & 2.12 & 2.07 & -1.4 & -.3 & .1 \\
\hline Ash & 12.72 & 12.97 & 12.49 & 12.11 & 12.56 & 13.48 & -2.0 & .3 & -.6 \\
\hline East North Central & & & & & & & & & \\
\hline Btu. & 10,611 & 10,676 & 10,837 & 10,885 & 11,005 & 11,151 & -1 & -1 & -1 \\
\hline Sulfur & 1.36 & 1.28 & 1.55 & 1.61 & 1.77 & 1.86 & 5.8 & -6.4 & -3.4 \\
\hline Ash & 8.07 & 8.00 & 8.34 & 8.41 & 8.72 & 9.04 & .8 & -1.9 & -1.3 \\
\hline Illinois & & & & & & & & & \\
\hline Btu & 9,878 & 9,970 & 10,181 & 10,362 & 10,666 & 10,698 & -1 & -2 & -1 \\
\hline 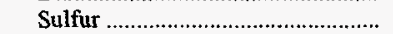 & 1.16 & 1.14 & 1.46 & 1.63 & 1.91 & 1.93 & 2.5 & -11.6 & -5.4 \\
\hline 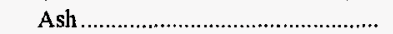 & 6.98 & 7.01 & 7.44 & 7.51 & 8.19 & 8.56 & -.5 & -3.9 & -2.2 \\
\hline Indiana & & & & & & & & & \\
\hline Btu & 10,357 & 10,338 & 10,535 & 10,539 & 10,628 & 10,878 & * & -1 & -1 \\
\hline 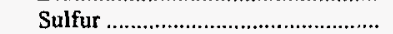 & 1.59 & 1.57 & 1.76 & 1.78 & 1.88 & 2.39 & 1.7 & -4.0 & -4.4 \\
\hline 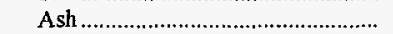 & 7.76 & 7.65 & 8.09 & 8.23 & 8.43 & 9.14 & 1.5 & -2.0 & -1.8 \\
\hline Michigan & & & & & & & & & \\
\hline Btu. & 10,504 & 10,677 & 10,925 & 10,853 & 10,995 & 11,564 & -2 & -1 & -1 \\
\hline Sulfur …………………………........ & .63 & .63 & .68 & .68 & .69 & .76 & • & -2.1 & -2.0 \\
\hline Ash & 6.59 & 6.66 & 6.97 & 6.61 & 6.76 & 6.97 & -1.0 & -.6 & -.6 \\
\hline Ohio & & & & & & & & & \\
\hline Btu & 12,056 & 12,122 & 12,052 & 12,049 & 11,983 & 11,904 & -1 & * & * \\
\hline 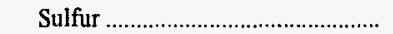 & 2.08 & 1.89 & 2.34 & 2.39 & 2.57 & 2.44 & 9.9 & -5.1 & -1.8 \\
\hline Ash & 11.01 & 10.84 & 10.91 & 11.01 & 11.24 & 11.39 & 1.5 & -.5 & -.4 \\
\hline Wisconsin & & & & & & & & & \\
\hline 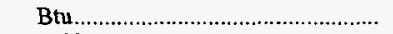 & 9,222 & 9,351 & 9,565 & 9,490 & 9,725 & 9,630 & -1 & -1 & * \\
\hline 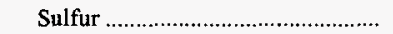 & .46 & .46 & .51 & .49 & .71 & .85 & -.7 & -10.5 & -6.6 \\
\hline Ash & 5.74 & 6.03 & 6.27 & 6.11 & 6.18 & 6.47 & -4.8 & -1.8 & -1.3 \\
\hline West North Central & & & & & & & & & \\
\hline 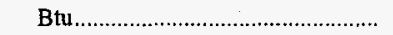 & 8,430 & 8,418 & 8,480 & 8,366 & 8,602 & 8,810 & * & -1 & * \\
\hline 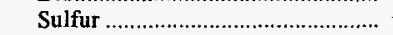 & .53 & .54 & .68 & .63 & .91 & 1.14 & -1.0 & -12.5 & -8.1 \\
\hline Ash & 6.38 & 6.41 & 6.82 & 6.74 & 7.18 & 7.84 & -.4 & -2.9 & -2.2 \\
\hline Iowa & & & & & & & & & \\
\hline Btu & 8,658 & 8,678 & 8,783 & 8,660 & 8,867 & 9,152 & * & -1 & -1 \\
\hline 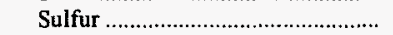 & .45 & .49 & .57 & .52 & .67 & .86 & -9.0 & -9.8 & -7.0 \\
\hline Ash & 5.61 & 5.50 & 5.59 & 5.47 & 5.78 & 6.24 & .2 & -.7 & -1.2 \\
\hline
\end{tabular}

See footnotes at end of table. 
Table 106. Average Quality of Coal Received at Electric Utilities by Census Division and State, 1987, 1992-1996 (Continued)

\begin{tabular}{|c|c|c|c|c|c|c|c|c|c|}
\hline \multirow{2}{*}{$\begin{array}{c}\text { Census Division and State } \\
\text { and Qualityl }\end{array}$} & \multirow{2}{*}{1996} & \multirow{2}{*}{1995} & \multirow{2}{*}{1994} & \multirow{2}{*}{1993} & \multirow{2}{*}{1992} & \multirow{2}{*}{1987} & \multirow{2}{*}{$\begin{array}{c}\text { Percent } \\
\text { Change } \\
1995-1996\end{array}$} & \multicolumn{2}{|c|}{ Average Annual Percent Change } \\
\hline & & & & & & & & $1992-1996$ & 1987-1996 \\
\hline Kansas & & & & & & & & & \\
\hline Btu & 8,827 & 8,730 & 8,708 & 8,654 & 8,900 & 8,764 & 1 & * & * \\
\hline Sulfur & .49 & .43 & .49 & .43 & .49 & .96 & 14.3 & 0.1 & -7.2 \\
\hline Ash & 5.52 & 5.46 & 5.63 & 5.19 & 5.74 & 7.52 & 1.1 & -1.0 & -3.4 \\
\hline Minnesota & & & & & & & & & \\
\hline 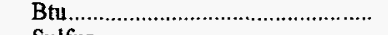 & 8,914 & 8,828 & 8,821 & 8,844 & 8,838 & 8,742 & I & * & * \\
\hline Sulfur & .45 & .47 & .46 & .44 & .45 & .53 & -3.9 & * & -1.9 \\
\hline 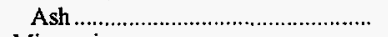 & 6.32 & 6.71 & 6.64 & 6.40 & 6.57 & 7.10 & -5.8 & -1.0 & -1.3 \\
\hline Missouri & & & & & & & & & \\
\hline 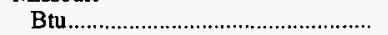 & 9,063 & 9,216 & 9,718 & 9,860 & 10,321 & 10,598 & -2 & -3 & -2 \\
\hline Sulfur & .58 & .57 & 1.03 & 1.02 & 1.80 & 2.36 & .3 & -24.8 & -14.5 \\
\hline 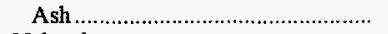 & 5.62 & 5.69 & 6.65 & 6.54 & 7.71 & 9.37 & -1.2 & -7.6 & -5.5 \\
\hline Nebraska & & & & & & & & & \\
\hline Btu & 8,599 & 8,594 & 8,571 & 8,561 & 8,553 & 8,601 & * & * & * \\
\hline 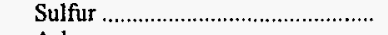 & .34 & .33 & .35 & .35 & .37 & .35 & 2.1 & -1.9 & -.4 \\
\hline Ash & 5.11 & 5.16 & 5.17 & 5.11 & 5.00 & 5.13 & -1.1 & .5 & * \\
\hline North Dakota & & & & & & & & & \\
\hline Btu & 6,597 & 6,585 & 6,593 & 6,570 & 6,558 & 6,601 & * & * & * \\
\hline Sulfur & .72 & .74 & .75 & .74 & .87 & .71 & -2.7 & -4.6 & .1 \\
\hline Ash & 9.32 & 9.29 & 9.39 & 9.47 & 9.29 & 8.83 & 3 & .1 & .6 \\
\hline South Dakota & & & & & & & & & \\
\hline Btu & 9,034 & 6,972 & 6,049 & 6,057 & 6,034 & 6,062 & 30 & 11 & 5 \\
\hline Sulfur & .52 & .87 & .91 & .90 & .92 & .87 & -40.7 & -13.4 & -5.6 \\
\hline Ash & 6.66 & 4.96 & 8.81 & 8.82 & 9.34 & 8.22 & 34.3 & -8.1 & -2.3 \\
\hline South Atlantic & & & & & & & & & \\
\hline Btu & 12,285 & 12,324 & 12,362 & 12,465 & 12,461 & 12,461 & * & * & * \\
\hline Sulfur & 1.27 & 1.27 & 1.33 & 1.39 & 1.52 & 1.53 & .2 & -4.4 & -2.0 \\
\hline Ash & 9.75 & 9.71 & 9.72 & 9.81 & 9.95 & 10.10 & .4 & -.5 & -.4 \\
\hline Delaware & & & & & & & & & \\
\hline Btu & 13,020 & 13,085 & 12,954 & 13,027 & 13,064 & 13,065 & * & * & * \\
\hline 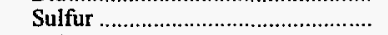 & 1.01 & 1.00 & .92 & .94 & 1.03 & 1.01 & 1.2 & -.5 & * \\
\hline 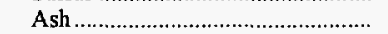 & 8.72 & 8.56 & 9.09 & 9.08 & 8.83 & 8.72 & 1.9 & -3 & * \\
\hline Florida & & & & & & & & & \\
\hline 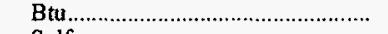 & 12,193 & 12,296 & 12,293 & 12,332 & 12,370 & 12,399 & -1 & * & * \\
\hline Sulfur & 1.55 & 1.47 & 1.60 & 1.57 & 1.68 & 1.85 & 5.5 & -2.0 & -1.9 \\
\hline Ash & 7.96 & 8.09 & 8.19 & 8.04 & 8.33 & 8.74 & -1.7 & -1.1 & -1.0 \\
\hline Georgia & & & & & & & & & \\
\hline Btu. & 11,581 & 11,576 & 11,774 & 12,148 & 12,039 & 12,175 & * & -1 & -1 \\
\hline Sulfur & .83 & .81 & 1.05 & 1.37 & 1.68 & 1.73 & 1.8 & -16.2 & -7.8 \\
\hline Ash & 8.84 & 8.87 & 8.99 & 9.94 & 10.29 & 9.92 & -.3 & -3.7 & -1.3 \\
\hline Maryland & & & & & & & & & \\
\hline 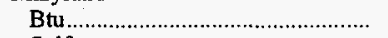 & 12,879 & 12,965 & 12,824 & 12,752 & 12,753 & 12,676 & -1 & * & * \\
\hline Sulfur & 1.11 & 1.06 & 1.16 & 1.31 & 1.36 & 1.47 & 5.0 & -4.9 & -3.1 \\
\hline Ash $\ldots \ldots \ldots \ldots \ldots$ & 9.49 & 9.32 & 9.91 & 10.02 & 10.47 & 10.99 & 1.8 & -2.4 & -1.6 \\
\hline North Carolina & & & & & & & & & \\
\hline Btu & 12,422 & 12,461 & 12,416 & 12,465 & 12,456 & 12,550 & * & * & * \\
\hline 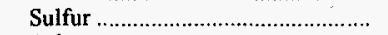 & .89 & .86 & .95 & .96 & .92 & .92 & 3.7 & -.8 & -.4 \\
\hline 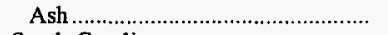 & 10.16 & 10.20 & 10.27 & 10.12 & 10.17 & 9.71 & -.4 & $*$ & .5 \\
\hline South Carolina & & & & & & & & & \\
\hline Btu & 12,757 & 12,852 & 12,771 & 12,802 & 12,817 & 12,649 & -1 & * & * \\
\hline Sulfur & 1.21 & 1.19 & 1.21 & 1.17 & 1.14 & 1.22 & 1.9 & 1.4 & -.1 \\
\hline Ash & 8.90 & 8.53 & 8.87 & 8.92 & 8.77 & 9.50 & 4.3 & .3 & -.7 \\
\hline Virginia & & & & & & & & & \\
\hline Btu & 12,597 & 12,743 & 12,778 & 12,817 & 12,830 & 12,814 & -1 & * & * \\
\hline Sulfur & .99 & 1.03 & .99 & 1.00 & 1.03 & .91 & -3.6 & -.9 & .9 \\
\hline Ash & 11.02 & 10.21 & 9.91 & 9.60 & 9.48 & 9.33 & 8.0 & 3.8 & 1.9 \\
\hline West Virginia & & & & & & & & & \\
\hline $\mathrm{Btu}$ & 12,378 & 12,418 & 12,468 & 12,489 & 12,524 & 24,792 & * & * & -100 \\
\hline 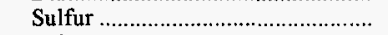 & 1.93 & 1.98 & 1.87 & 1.94 & 2.05 & 4.12 & -2.5 & -1.5 & -99.1 \\
\hline Ash & 11.78 & 11.88 & 11.50 & 11.61 & 11.32 & 23.77 & -.8 & 1.0 & -99.8 \\
\hline East South Central & & & & & & & & & \\
\hline Btu & 11,714 & 11,808 & 11,909 & 11,988 & 11,933 & 11,886 & -1 & * & * \\
\hline Sulfur & 1.86 & 1.87 & 1.88 & 1.91 & 1.99 & 2.09 & -.6 & -1.7 & -1.3 \\
\hline Ash & 10.60 & 10.58 & 10.66 & 10.92 & 10.70 & 11.16 & .2 & -.2 & -.6 \\
\hline Alabama & & & & & & & & & \\
\hline $\mathrm{B} u$ & 11,794 & 11,861 & 12,088 & 12,092 & 12,061 & 12,223 & -1 & -1 & * \\
\hline Sulfur & 1.24 & 1.20 & 1.30 & 1.33 & 1.43 & 1.52 & 3.3 & -3.6 & -2.3 \\
\hline Ash & 10.71 & 10.74 & 11.54 & 11.79 & 11.80 & 11.75 & -.3 & -2.4 & -1.0 \\
\hline
\end{tabular}

See footnotes at end of table. 
Table 106. Average Quality of Coal Received at Electric Utilities by Census Division and State, 1987, 1992-1996 (Continued)

\begin{tabular}{|c|c|c|c|c|c|c|c|c|c|}
\hline \multirow{2}{*}{$\begin{array}{l}\text { Census Division and State } \\
\text { and Quality } 1\end{array}$} & \multirow{2}{*}{1996} & \multirow{2}{*}{1995} & \multirow{2}{*}{1994} & \multirow{2}{*}{1993} & \multirow{2}{*}{1992} & \multirow{2}{*}{1987} & \multirow{2}{*}{$\begin{array}{c}\text { Percent } \\
\text { Change } \\
\text { 1995-1996 }\end{array}$} & \multicolumn{2}{|c|}{ Average Annual Percent Change } \\
\hline & & & & & & & & $1992-1996$ & $1987-1996$ \\
\hline \multicolumn{10}{|l|}{ Kentucky } \\
\hline 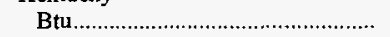 & 11,536 & 11,625 & 11,683 & 11,697 & 11,620 & 11,496 & -1 & $*$ & $*$ \\
\hline Sulfur & 2.47 & 2.42 & 2.34 & 2.39 & 2.44 & 2.59 & 2.1 & 0.3 & -0.5 \\
\hline Ash & 12.15 & 11.91 & 11.35 & 11.64 & 11.60 & 12.26 & 2.0 & 1.2 & -.1 \\
\hline \multicolumn{10}{|l|}{ Mississippi } \\
\hline 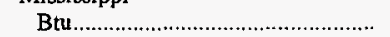 & 11,023 & 11,221 & 11,312 & 12,338 & 12,507 & 12,674 & -2 & -3 & -2 \\
\hline Sulfur & .93 & 1.04 & 1.02 & 1.41 & 1.69 & 1.36 & -10.5 & -13.9 & -4.1 \\
\hline Ash & 6.44 & 7.81 & 7.88 & 8.52 & 8.31 & 7.74 & -17.5 & -6.2 & -2.0 \\
\hline Tennessee & & & & & & & & & \\
\hline 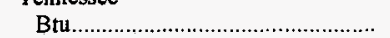 & 12,062 & 12,130 & 12,186 & 12,268 & 12,182 & 11,979 & -1 & $\bullet$ & * \\
\hline Sulfur & 1.87 & 1.97 & 2.00 & 1.92 & 2.02 & 2.05 & -5.3 & -1.9 & -1.0 \\
\hline Ash & 8.89 & 8.83 & 8.94 & 9.14 & 8.31 & 9.63 & .7 & 1.7 & -9 \\
\hline West South Central & & & & & & & & & \\
\hline 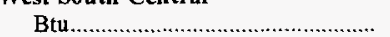 & 7,798 & 7,733 & 7,709 & 7,646 & 7,648 & 7,657 & 1 & * & * \\
\hline Sulfur & .60 & .64 & .62 & .64 & .65 & .60 & -6.1 & -1.9 & .1 \\
\hline Ash & 9.19 & 9.53 & 9.50 & 10.06 & 10.25 & 10.15 & -3.6 & -2.7 & -1.1 \\
\hline Arkansas & & & & 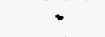 & & & & & \\
\hline 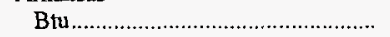 & 8,703 & 8,687 & 8,707 & 8,665 & 8,724 & 8,682 & * & $*$ & $*$ \\
\hline Sulfur & .33 & .33 & .32 & .32 & .32 & .32 & .4 & .3 & .1 \\
\hline Ash & 5.20 & 5.10 & 4.92 & 5.06 & 5.12 & 5.47 & 1.9 & .3 & -.6 \\
\hline Louisiana & & & & & & & & & \\
\hline 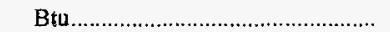 & 8,171 & 8,110 & 8,136 & 8,092 & 8,122 & 8,160 & 1 & * & * \\
\hline 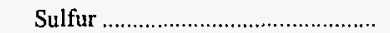 & .57 & .58 & .51 & .52 & .50 & .49 & -2.6 & 3.3 & 1.7 \\
\hline Ash & 7.13 & 7.42 & 7.16 & 7.13 & 7.20 & 7.59 & -4.0 & -.3 & -.7 \\
\hline Oklahoma & & & & & & & & & \\
\hline 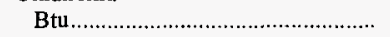 & 8,600 & 8,557 & 8,573 & 8,621 & 8,700 & 8,851 & 1 & * & * \\
\hline 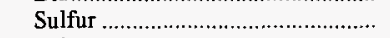 & .33 & .36 & .35 & .37 & .42 & .44 & -9.3 & -6.0 & -3.1 \\
\hline Ash & 4.93 & 5.20 & 5.07 & 5.21 & 5.24 & 5.46 & -5.3 & -1.5 & -1.1 \\
\hline Texas & & & & & & & & & \\
\hline 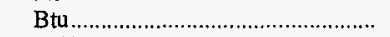 & 7,440 & 7,346 & 7,346 & 7,284 & 7,234 & 7,242 & 1 & 1 & * \\
\hline 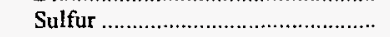 & .71 & .77 & .73 & .75 & .76 & .68 & -7.2 & -1.8 & .4 \\
\hline 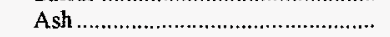 & 10.98 & 11.50 & 11.31 & 11.95 & 12.33 & 11.97 & -4.5 & -2.9 & -.9 \\
\hline Mountain & & & & & & & & & \\
\hline 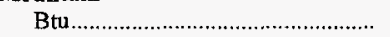 & 9,741 & 9,736 & 9,755 & 9,751 & 9,722 & 9,820 & $*$ & * & * \\
\hline 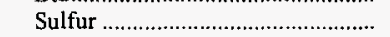 & .55 &.$\$ 4$ & .55 & .54 & .55 & .54 & 1.4 & .2 & .1 \\
\hline Ash & 11.37 & 11.16 & 11.11 & 11.19 & 11.15 & 10.93 & 1.9 & .5 & 4 \\
\hline Arizona & & & & & & & & & \\
\hline 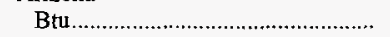 & 10,232 & $10,2: 4$ & 10,281 & 10,271 & 10,303 & 10,608 & $*$ & * & $*$ \\
\hline Sulfur & .55 & .53 & .51 & .49 & .51 & .51 & 4.7 & 2.0 & .8 \\
\hline Ash & 12.41 & 12.13 & 11.97 & 12.08 & 12.19 & 11.29 & 2.3 & .5 & 1.1 \\
\hline Colorado & & & & & & & & & \\
\hline 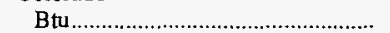 & 9,858 & $9,8 \leq 5$ & 9,946 & 9,888 & 9,920 & 9,842 & * & * & $*$ \\
\hline Sulfur & .39 & .39 & .40 & .38 & .38 & .37 & -.7 & .4 & .6 \\
\hline Ash & 6.94 & 7.16 & 7.12 & 6.97 & 7.01 & 6.94 & -3.0 & -.2 & * \\
\hline Montana & & & & & & & & & \\
\hline 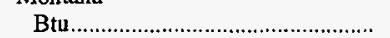 & 8,439 & 8,520 & 8,500 & 8,496 & 8,576 & 8,590 & -1 & * & $*$ \\
\hline Sulfur & .68 & .68 & .66 & .65 & .66 & .62 & 2 & .8 & 1.1 \\
\hline Ash & 9.00 & 9.15 & 9.05 & 8.99 & 8.92 & 8.82 & -1.6 & .2 & .2 \\
\hline Nevada & & & & & & & & & \\
\hline 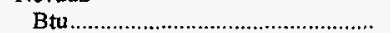 & 11,140 & 11,075 & 11,291 & 11,012 & 11,051 & 11,182 & 1 & $*$ & $*$ \\
\hline 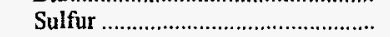 & .49 & .48 & .49 & .49 & .49 & .51 & 1.1 & -.3 & -.5 \\
\hline Ash & 9.71 & 9.70 & 9.57 & 9.73 & 9.67 & 9.51 & .1 & .1 & .2 \\
\hline New Mexico & & & & & & & & & \\
\hline 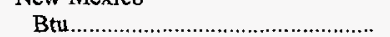 & 9,116 & 9,033 & 9,043 & 8,992 & 9,013 & 9,049 & 1 & $*$ & $*$ \\
\hline 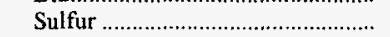 & .80 & .80 & .82 & .81 & .81 & .78 & -.3 & -.4 & .3 \\
\hline Ash & 22.78 & 22.51 & 22.44 & 22.77 & 22.49 & 21.64 & 1.2 & .3 & .6 \\
\hline Utah & & & & & & & & & \\
\hline 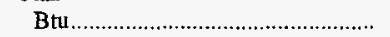 & 11,513 & 11,550 & 11,491 & 11,489 & 11,384 & 11,618 & $*$ & * & * \\
\hline Sulfur & 47 & .47 & .47 & .48 & .47 & .51 & -1.3 & $*$ & -.9 \\
\hline Ash & 10.90 & 10.27 & 10.25 & 10.47 & 11.24 & 9.91 & 6.0 & -.8 & 1.0 \\
\hline Wyoming & & & & & & & & & \\
\hline 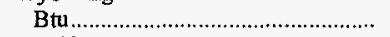 & 8,716 & 8,738 & 8,766 & 8,779 & 8,840 & 8,778 & $*$ & • & $*$ \\
\hline Sulfur & .52 & .50 & .52 & .51 & .52 & .52 & 5.0 & .2 & * \\
\hline Ash & 8.12 & 8.06 & 8.00 & 7.78 & 7.54 & 7.63 & .7 & 1.8 & .7 \\
\hline
\end{tabular}

See footnotes at end of table. 
Table 106. Average Quality of Coal Received at Electric Utilities by Census Division and State, 1987, 1992-1996 (Continued)

\begin{tabular}{|c|c|c|c|c|c|c|c|c|c|}
\hline \multirow{2}{*}{$\begin{array}{c}\text { Census Division and State } \\
\text { and Quality } 1\end{array}$} & \multirow{2}{*}{1996} & \multirow{2}{*}{1995} & \multirow{2}{*}{1994} & \multirow{2}{*}{1993} & \multirow{2}{*}{1992} & \multirow{2}{*}{1987} & \multirow{2}{*}{$\begin{array}{c}\text { Percent } \\
\text { Change } \\
\text { 1995-1996 }\end{array}$} & \multicolumn{2}{|c|}{ Average Annual Percent Change } \\
\hline & & & & & & & & 1992-1996 & $1987-1996$ \\
\hline \multicolumn{10}{|l|}{ Pacific } \\
\hline 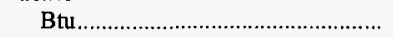 & 8,066 & 8,380 & 8,543 & 8,283 & 8,550 & 8,134 & -4 & -1 & * \\
\hline Sulfur & .64 & .62 & .57 & .63 & .59 & .66 & 3.4 & 1.8 & -0.3 \\
\hline Ash & 13.62 & 11.79 & 11.14 & 12.58 & 11.39 & 13.67 & 15.6 & 4.6 & * \\
\hline \multicolumn{10}{|l|}{ Oregon } \\
\hline 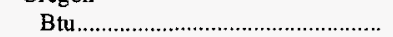 & 8,782 & 8,882 & 8,937 & 8,801 & 9,642 & 8,484 & -1 & -2 & * \\
\hline 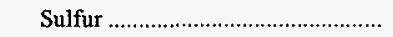 & .26 & .30 & .37 & .38 & .40 & .32 & -13.6 & -10.0 & -2.2 \\
\hline Ash & 4.79 & 5.52 & 5.89 & 4.98 & 4.42 & 4.68 & -13.2 & 2.0 & 2 \\
\hline \multicolumn{10}{|l|}{ Washington } \\
\hline 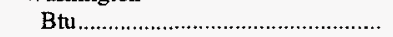 & 7,936 & 8,267 & 8,400 & 8,125 & 8,189 & 8,104 & -4 & -1 & $*$ \\
\hline Sulfur & .71 & .69 & 65 & .71 & .66 & .69 & 2.7 & 1.8 & .3 \\
\hline Ash & 15.24 & 13.20 & 13.04 & 14.90 & 13.69 & 14.43 & 15.4 & 2.7 & .6 \\
\hline \multicolumn{10}{|l|}{ U.S. Total } \\
\hline Btu & 10,263 & 10,248 & 10,338 & 10,315 & 10,395 & 10,569 & $*$ & * & * \\
\hline Sulfur & 1.10 & 1.08 & 1.17 & 1.18 & 1.29 & 1.38 & 1.6 & -4.0 & -2.5 \\
\hline Ash & 9.22 & 9.23 & 9.36 & 9.55 & 9.71 & 10.00 & -.2 & -1.3 & -.9 \\
\hline
\end{tabular}

1 Quality units are: Btu (per pound); sulfur (percent by weight); and ash (percent by weight)

* Data round to zero.

Source: Federal Energy Regulatory Commission, FERC Form 423, "Monthly Report of Cost and Quality of Fuels for Electric Plants." 
Table 107. Average Quality of Coal Received at Manufacturing and Coke Plants by Census Division and State, 1993-1996

\begin{tabular}{|c|c|c|c|c|c|}
\hline $\begin{array}{l}\text { Census Division and State } \\
\text { and Quality } 1\end{array}$ & 1996 & 1995 & 1994 & 1993 & $\begin{array}{c}\text { Percent } \\
\text { Change } \\
\text { 1995-1996 }\end{array}$ \\
\hline \multicolumn{6}{|l|}{ New England } \\
\hline Btu & 13,028 & 13,410 & 13,383 & 13,232 & -2.8 \\
\hline 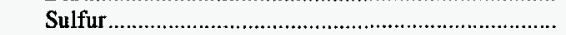 & 1.03 & 1.32 & .72 & 1.20 & -21.8 \\
\hline 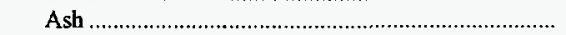 & 7.35 & 6.99 & 5.86 & 6.87 & 5.1 \\
\hline \multicolumn{6}{|l|}{ Maine } \\
\hline Btu & 12,935 & 13,392 & 13,162 & 13,062 & -3.4 \\
\hline Sulfur & 1.08 & 1.39 & .71 & 1.29 & -22.8 \\
\hline Ash & 7.31 & 6.93 & 5.30 & 7.18 & 5.6 \\
\hline \multicolumn{6}{|l|}{ Massachusetts } \\
\hline Btu & 13,697 & 13,556 & 13,939 & 13,992 & 1.0 \\
\hline Sulfur & .74 & .75 & .73 & .78 & -1.3 \\
\hline Ash & 7.65 & 7.55 & 7.26 & 5.48 & 1.3 \\
\hline \multicolumn{6}{|l|}{ Middle Atlantic } \\
\hline $\mathrm{Btu}$ & 12,780 & 12,559 & 12,549 & 12,367 & 1.8 \\
\hline Sulfur & 1.20 & 1.15 & 1.14 & 1.06 & 4.3 \\
\hline 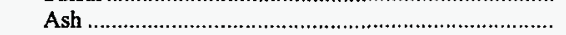 & 7.12 & 7.11 & 6.95 & 7.37 & .1 \\
\hline \multicolumn{6}{|l|}{ New Jersey } \\
\hline Btu & 12,474 & 12,575 & 12,515 & 11,685 & -.8 \\
\hline 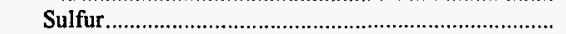 & 1.36 & .96 & .93 & .83 & 41.9 \\
\hline \multirow{2}{*}{\multicolumn{6}{|c|}{ New York ${ }^{2}$}} \\
\hline & & & & & \\
\hline Btu & 13,168 & 13,122 & 13,203 & 13,044 & .3 \\
\hline 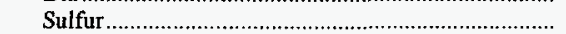 & 1.41 & 1.29 & 1.34 & 1.69 & 9.6 \\
\hline 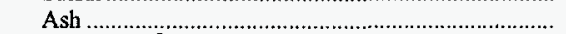 & 6.84 & 7.20 & 6.69 & 7.73 & -5.1 \\
\hline \multicolumn{6}{|l|}{ Pennsylvania ${ }^{2}$} \\
\hline Btu & 12,658 & 12,366 & 12,326 & 12,117 & 2.4 \\
\hline Sulfur .....1-1) & 1.16 & 1.12 & 1.10 & .99 & 3.3 \\
\hline 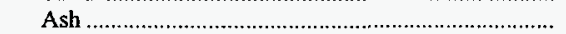 & 7.17 & 7.09 & 7.00 & 7.30 & 1.1 \\
\hline \multicolumn{6}{|l|}{ East North Central } \\
\hline Btu & 11,990 & 12,022 & 11,947 & 11,956 & -.3 \\
\hline 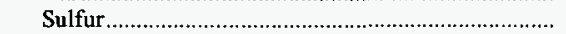 & 1.52 & 1.46 & 1.52 & 2.40 & 4.2 \\
\hline Ash & 7.34 & 7.59 & 7.61 & 6.98 & -3.3 \\
\hline \multicolumn{6}{|l|}{ 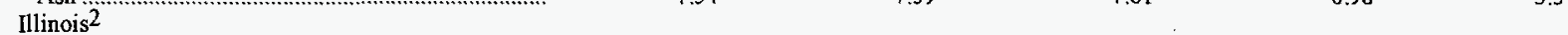 } \\
\hline Btu & 11,332 & 11,290 & 11,387 & 11,372 & .4 \\
\hline 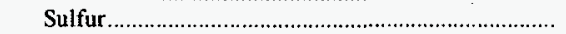 & 1.89 & 1.82 & 1.94 & 4.83 & 4.1 \\
\hline Ash & 7.41 & 7.49 & 7.66 & 7.03 & -1.1 \\
\hline \multicolumn{6}{|l|}{ Indiana ${ }^{2}$} \\
\hline Btu & 11,826 & 11,894 & 11,641 & 11,591 & -.6 \\
\hline Sulfur & 1.32 & 1.20 & 1.45 & 1.28 & 10.2 \\
\hline 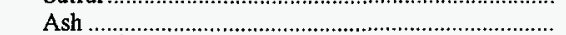 & 7.02 & 7.38 & 7.50 & 6.77 & -4.8 \\
\hline \multicolumn{6}{|l|}{ (1) } \\
\hline Btu & 12,440 & 12,386 & 12,470 & 12,547 & .4 \\
\hline 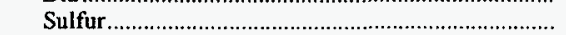 & .96 & .96 & 1.02 & 1.05 & -.7 \\
\hline Ash & 6.80 & 7.60 & 7.82 & 6.98 & -10.5 \\
\hline \multicolumn{6}{|l|}{$\mathrm{Ohi}^{2}$. } \\
\hline Btu & 12,415 & 12,424 & 12,429 & 12,476 & -.1 \\
\hline Sulfur & 1.82 & 1.69 & 1.60 & 1.40 & 7.9 \\
\hline Ash $\ldots \ldots \ldots \ldots \ldots \ldots \ldots \ldots \ldots$ & 8.27 & 7.79 & 7.66 & 7.10 & 6.2 \\
\hline \multicolumn{6}{|l|}{ Wisconsin } \\
\hline Btu & 12,330 & 12,450 & 11,873 & 11,745 & -1.0 \\
\hline 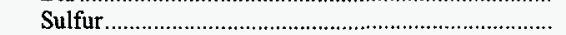 & 2.05 & 2.14 & 1.52 & 1.83 & -4.3 \\
\hline Ash & 7.72 & 8.40 & 7.30 & 7.25 & -8.0 \\
\hline \multicolumn{6}{|l|}{ West North Central } \\
\hline Btu & 8,703 & 8,669 & 8,710 & 8,589 & .4 \\
\hline 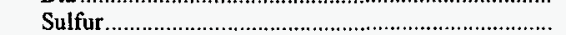 & .87 & .89 & .88 & .90 & -2.1 \\
\hline Ash & 6.40 & 6.54 & 6.56 & 6.46 & -2.2 \\
\hline Lowa & & & & & \\
\hline Btu & 10,373 & 10,332 & 10,480 & 10,344 & .4 \\
\hline 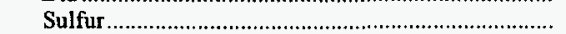 & 1.04 & .96 & .98 & 1.13 & 8.8 \\
\hline Ash & 6.40 & 6.44 & 6.38 & 6.41 & -.7 \\
\hline Kansas & & & & & \\
\hline Btu & 12,243 & 12,197 & 12,249 & 12,479 & .4 \\
\hline Sulfur & 3.07 & 3.41 & 3.11 & 3.14 & -10.2 \\
\hline Ash & 10.36 & 11.48 & 11.04 & 11.78 & -9.8 \\
\hline
\end{tabular}

See footnotes at end of table. 
Table 107. Average Quality of Coal Received at Manufacturing and Coke Plants by Census Division and State, 1993-1996 (Continued)

\begin{tabular}{|c|c|c|c|c|c|}
\hline $\begin{array}{l}\text { Census Division and State } \\
\text { and Quality } 1\end{array}$ & 1996 & 1995 & 1994 & 1993 & $\begin{array}{c}\text { Percent } \\
\text { Change } \\
1995-1996\end{array}$ \\
\hline \multicolumn{6}{|l|}{ Minnesota } \\
\hline Btu & 10,051 & 10,280 & 10,112 & 10,003 & -2.2 \\
\hline Sulfur & .61 & .56 & .54 & .51 & 9.6 \\
\hline \multirow{2}{*}{\multicolumn{6}{|c|}{ Missouri 2}} \\
\hline & & & & & \\
\hline Btu & 11,539 & 11,644 & 11,510 & 11,527 & -.9 \\
\hline Sulfur ....1. & 2.03 & 1.91 & 1.92 & 2.01 & 6.3 \\
\hline 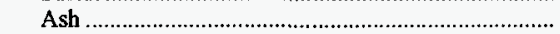 & 8.31 & 9.92 & 10.10 & 10.11 & -16.2 \\
\hline \multicolumn{6}{|l|}{ Nebraska } \\
\hline Btu & 10,622 & 10,096 & 9,931 & 9,979 & 5.2 \\
\hline Sulfur & .36 & .42 & 40 & .37 & -14.6 \\
\hline \multirow{2}{*}{\multicolumn{6}{|c|}{ North Dakota }} \\
\hline & & & & & \\
\hline Btu & 7,136 & 7,171 & 7,142 & 7,172 & -.5 \\
\hline Sulfur & .61 & .71 & .71 & .70 & -14.1 \\
\hline 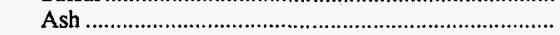 & 5.97 & 6.08 & 6.14 & 6.16 & -1.8 \\
\hline \multicolumn{6}{|l|}{ South Dakota } \\
\hline Btu & 9,849 & 9,504 & 9,418 & 8,159 & 3.6 \\
\hline 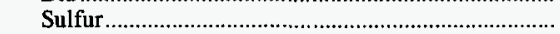 & .83 & .86 & .77 & .39 & -3.8 \\
\hline Ash & 7.55 & 7.72 & 7.15 & 5.42 & -2.1 \\
\hline \multicolumn{6}{|l|}{ South Atlantic } \\
\hline Btu & 12,972 & 12,992 & 13,043 & 12,973 & -.1 \\
\hline Sulfur & 1.09 & 1.10 & 1.15 & 1.12 & -1.7 \\
\hline Ash & 8.11 & 8.07 & 7.96 & 7.62 & .4 \\
\hline \multicolumn{6}{|l|}{ Delaware } \\
\hline Btu & 13,381 & 13,483 & 13,300 & 13,087 & -.8 \\
\hline 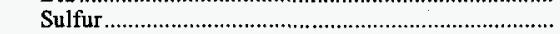 & 1.75 & 1.87 & 1.89 & 2.00 & -6.3 \\
\hline \multirow{2}{*}{\multicolumn{6}{|c|}{ Florida }} \\
\hline & & & & & \\
\hline Btu & 12,903 & 12,865 & 12,933 & 12,742 & .3 \\
\hline 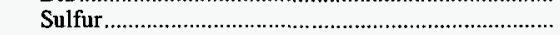 & .87 & .91 & .93 & .97 & -3.8 \\
\hline Ash & 8.07 & 8.14 & 8.93 & 9.46 & -.9 \\
\hline \multicolumn{6}{|l|}{ Georgia } \\
\hline Btu & 12,873 & 12,895 & 13,267 & 12,870 & -.2 \\
\hline Sulfur & 1.11 & 1.23 & 1.29 & 1.30 & -9.3 \\
\hline Ash & 8.79 & 8.78 & 8.90 & 7.73 & .1 \\
\hline \multicolumn{6}{|l|}{ Maryland ${ }^{2}$} \\
\hline Btu & 12,411 & 12,598 & 12,330 & 12,422 & -1.5 \\
\hline 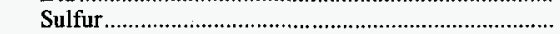 & 1.92 & 1.92 & 1.89 & 1.93 & .1 \\
\hline Ash & 14.19 & 14.52 & 10.90 & 13.32 & -2.3 \\
\hline \multicolumn{6}{|l|}{ North Carolina } \\
\hline Btu & 13,243 & 13,250 & 13,188 & 13,321 & * \\
\hline Sulfur & .93 & .97 & .91 & .92 & -3.9 \\
\hline Ash & 7.10 & 6.99 & 7.20 & 6.89 & 1.7 \\
\hline \multicolumn{6}{|l|}{ South Carolina } \\
\hline Btu & 13,076 & 13,051 & 12,994 & 12,955 & .2 \\
\hline 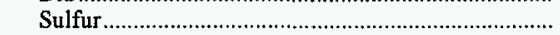 & 1.02 & 1.08 & 1.12 & 1.22 & -5.6 \\
\hline Ash & 8.09 & 8.24 & 7.95 & 8.67 & -1.8 \\
\hline Virginia $^{2}$ & & & & & \\
\hline Btu & 12,982 & 13,067 & 13,215 & 13,193 & -.6 \\
\hline 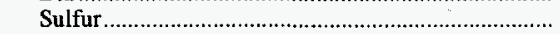 & 1.04 & 1.05 & 1.05 & .99 & -1.2 \\
\hline 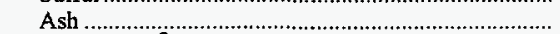 & 7.97 & 7.75 & 7.44 & 7.20 & 2.9 \\
\hline West Virginia ${ }^{2}$ & & & & & \\
\hline Btu & 12,809 & 12,765 & 12,780 & 12,721 & .3 \\
\hline Sulfur & 1.14 & 1.06 & 1.24 & 1.06 & 7.6 \\
\hline Ash & 7.24 & 7.28 & 7.54 & 6.32 & -.5 \\
\hline East South Central & & & & & \\
\hline Btu & 12,916 & 12,941 & 12,869 & 12,838 & -.2 \\
\hline 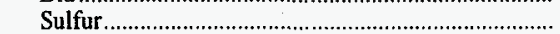 & 1.06 & 1.09 & 1.09 & .74 & -3.2 \\
\hline 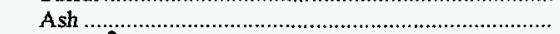 & 7.20 & 7.32 & 7.51 & 4.54 & -1.6 \\
\hline Alabama ${ }^{2}$ & & & & & \\
\hline Btu & 12,632 & 12,612 & 12,694 & 12,628 & .2 \\
\hline Sulfur & .98 & .94 & .98 & .71 & 4.8 \\
\hline Ash & 6.90 & 7.07 & 7.27 & 4.25 & -2.4 \\
\hline Kentucky2 2 & & & & & \\
\hline 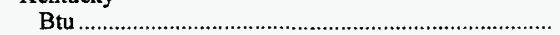 & 13,072 & 13,086 & 13,150 & 12,686 & -.1 \\
\hline 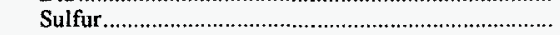 & 1.05 & 1.03 & .99 & .90 & 2.5 \\
\hline 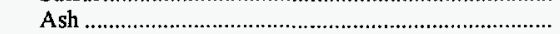 & 6.59 & 6.61 & 6.59 & 6.04 & -.4 \\
\hline
\end{tabular}

See footnotes at end of table. 
Table 107. Average Quality of Coal Received at Manufacturing and Coke Plants by Census Division and State, 1993-1996 (Continued)

\begin{tabular}{|c|c|c|c|c|c|}
\hline $\begin{array}{l}\text { Census Division and State } \\
\text { and Quality } 1\end{array}$ & 1996 & 1995 & 1994 & 1993 & $\begin{array}{c}\text { Percent } \\
\text { Change } \\
1995-1996\end{array}$ \\
\hline \multicolumn{6}{|l|}{ Mississippi } \\
\hline Btu & 11,911 & 11,897 & 11,786 & 12,013 & 0.1 \\
\hline Sulfur & 1.41 & 1.41 & 1.44 & 2.52 & .2 \\
\hline Ash & 9.73 & 10.66 & 9.98 & 10.33 & -8.7 \\
\hline \multicolumn{6}{|l|}{ Tennessee $^{2}$} \\
\hline Btu & 13,103 & 13,160 & 12,958 & 13,059 & -.4 \\
\hline Sulfur & 1.14 & 1.35 & 1.32 & 1.08 & -15.5 \\
\hline Ash & 7.94 & 8.04 & 8.47 & 8.38 & -1.1 \\
\hline \multicolumn{6}{|l|}{ West South Central } \\
\hline Btu & 9,176 & 9,116 & 8,925 & 9,143 & .7 \\
\hline Sulfur & 1.06 & 1.00 & 1.00 & 1.05 & 6.3 \\
\hline 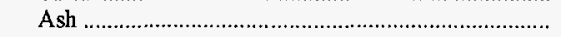 & 11.36 & 10.78 & 11.18 & $\mathrm{I} 1.55$ & 5.4 \\
\hline \multicolumn{6}{|l|}{ Arkansas } \\
\hline Btu & 12,474 & 12,573 & 12,646 & 12,850 & -.8 \\
\hline Sulfur & 2.03 & 2.02 & 2.10 & 1.97 & .7 \\
\hline Ash & 10.27 & 9.96 & 10.11 & 9.78 & 3.1 \\
\hline \multicolumn{6}{|l|}{ Louisiana } \\
\hline Btu & 12,627 & 9,292 & 9,051 & 9,255 & 35.9 \\
\hline Sulfur & 1.27 & .39 & .35 & .39 & 221.7 \\
\hline Ash & 10.23 & 5.29 & 5.09 & 5.83 & 93.4 \\
\hline \multicolumn{6}{|l|}{ Oklahoma } \\
\hline Btu & 9,835 & 9,995 & 10,118 & 11,263 & -1.6 \\
\hline Sulfur & .89 & .72 & .73 & .89 & 24.7 \\
\hline \multirow{2}{*}{\multicolumn{6}{|c|}{ Texas 2}} \\
\hline & & & & & \\
\hline Btu & 8,757 & 8,690 & 8,447 & 8,585 & .8 \\
\hline Sulfur . & 1.01 & 1.02 & 1.04 & 1.09 & -1.0 \\
\hline Ash & 12.37 & 12.20 & 12.72 & 12.98 & 1.3 \\
\hline \multicolumn{6}{|l|}{ Mountain } \\
\hline Btu ... & 10,699 & 10,698 & 10,601 & 10,443 & * \\
\hline 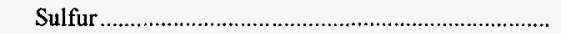 & .67 & .71 & 68 & .56 & -4.8 \\
\hline Ash & 7.79 & 7.28 & 7.05 & 6.75 & 6.9 \\
\hline \multicolumn{6}{|l|}{ Arizona } \\
\hline 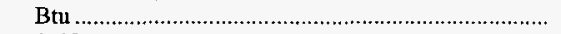 & 10,603 & 10,969 & 11,072 & 10,690 & -3.3 \\
\hline Sulfur & .53 & .54 & .48 & .46 & -2.1 \\
\hline 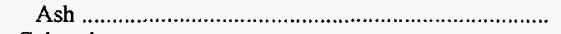 & 13.15 & 12.23 & 11.09 & 11.17 & 7.6 \\
\hline \multicolumn{6}{|l|}{ Colorado } \\
\hline Btu & 11,308 & 11,262 & 10,785 & 10,564 & .4 \\
\hline Sulfur & .54 & .61 & .58 & .54 & -12.6 \\
\hline Ash & 7.79 & 7.24 & 7.00 & 6.52 & 7.6 \\
\hline \multicolumn{6}{|l|}{ Idaho } \\
\hline Btu & 10,148 & 10,232 & 9,988 & 10,089 & -.8 \\
\hline Sulfur & .72 & .78 & .79 & .71 & -7.3 \\
\hline Ash & 6.40 & 6.22 & 5.72 & 6.03 & 2.8 \\
\hline \multicolumn{6}{|l|}{ Montana } \\
\hline Btu & 8,695 & 8,368 & 8,496 & 8,065 & 3.9 \\
\hline Sulfur & .44 & .59 & .57 & .46 & -25.1 \\
\hline Ash & 5.31 & 7.93 & 7.65 & 9.31 & -33.0 \\
\hline \multicolumn{6}{|l|}{ Nevada } \\
\hline Btu & 11,533 & 11,698 & 11,907 & 12,042 & -1.4 \\
\hline Sulfur & .51 & .48 & .26 & .28 & 4.8 \\
\hline Ash & 8.80 & 7.13 & 4.01 & 4.63 & 23.4 \\
\hline \multicolumn{6}{|l|}{ New Mexico } \\
\hline Btu & 12,302 & 12,518 & 12,688 & 12,776 & -1.7 \\
\hline 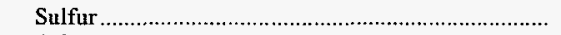 & .82 & .79 & .94 & .74 & 3.8 \\
\hline Ash & 11.67 & 10.26 & 9.87 & 9.95 & 13.7 \\
\hline $\mathrm{Utah}^{2}$ & & & & & \\
\hline Btu & 11,589 & 11,671 & 11,679 & 11,530 & -.7 \\
\hline Sulfur & .82 & .84 & .82 & .44 & -2.6 \\
\hline Ash & 8.01 & 8.08 & 7.68 & 7.12 & -.9 \\
\hline Wyoming & & & & & \\
\hline Btu & 10,365 & 10,170 & 10,098 & 10,074 & 1.9 \\
\hline 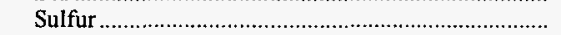 & .70 & .71 & .70 & .72 & -1.6 \\
\hline 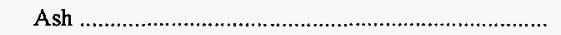 & 4.68 & 4.84 & 5.27 & 5.12 & -3.3 \\
\hline
\end{tabular}

See footnotes at end of table. 
Table 107. Average Quality of Coal Received at Manufacturing and Coke Plants by Census Division and State, 1993-1996 (Continued)

\begin{tabular}{|c|c|c|c|c|c|}
\hline $\begin{array}{l}\text { Census Division and State } \\
\text { and Quality } 1\end{array}$ & 1996 & 1995 & 1994 & 1993 & $\begin{array}{c}\text { Percent } \\
\text { Change } \\
\text { 1995-1996 }\end{array}$ \\
\hline \multicolumn{6}{|l|}{ Pacific } \\
\hline (1) & 11,677 & 11,551 & 11,749 & 12,218 & 1.1 \\
\hline Sulfur & .56 & .53 & .52 & .49 & 6.8 \\
\hline Ash & 9.15 & 9.17 & 8.98 & 8.65 & -.2 \\
\hline \multicolumn{6}{|l|}{ Califomia } \\
\hline Btu & 11,899 & 11,912 & 11,950 & 12,441 & -.1 \\
\hline Sulfur & .55 & .52 & .52 & .49 & 6.4 \\
\hline Ash & 8.72 & 8.62 & 8.93 & 8.35 & 1.2 \\
\hline \multicolumn{6}{|l|}{ Hawaii } \\
\hline Btu & 9,157 & 9,275 & 9,576 & 9,776 & -1.3 \\
\hline Sulfur & .53 & .53 & .51 & .52 & -.7 \\
\hline Ash & 14.99 & 15.37 & 16.72 & 15.96 & -2.5 \\
\hline \multicolumn{6}{|l|}{ Oregon } \\
\hline Btu & 11,159 & 10,188 & 10,704 & 10,216 & 9.5 \\
\hline Sulfur & .65 & .54 & .53 & .51 & 19.8 \\
\hline Ash & 7.03 & 5.98 & 6.48 & 5.94 & 17.7 \\
\hline \multicolumn{6}{|l|}{ Washington } \\
\hline 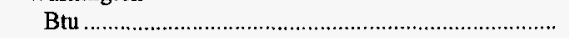 & 11,622 & 11,846 & 11,818 & 12,151 & -1.9 \\
\hline Sulfur & .65 & .57 & .58 & .56 & 13.3 \\
\hline Ash & 10.00 & 9.62 & 5.63 & 9.47 & 4.0 \\
\hline \multicolumn{6}{|l|}{ U.S. Total } \\
\hline Btu & 11,405 & 11,367 & 11,316 & 11,303 & 3 \\
\hline Sulfur & 1.17 & 1.15 & 1.16 & 1.23 & 1.8 \\
\hline Ash & 7.58 & 7.61 & 7.63 & 6.34 & -.4 \\
\hline
\end{tabular}

1 Quality units are: Btu (per pound); sulfur (percent by weight); and ash (percent by weight)

2 Includes sulfur and ash data for coke plants.

* Data round to zero.

Notes: Btu data are for manufacturing plants only. The national average of coke plant data ranges from .51 to 1.70 for sulfur and 2.6 to 10.1 for ash.

Sources: Energy Information Administration, Form EIA-3A, “Annual Coal Quality Report - Manufacturing Plants"; and Form EIA-5A, "Annual Coal Quality Report - Coke Plants." 


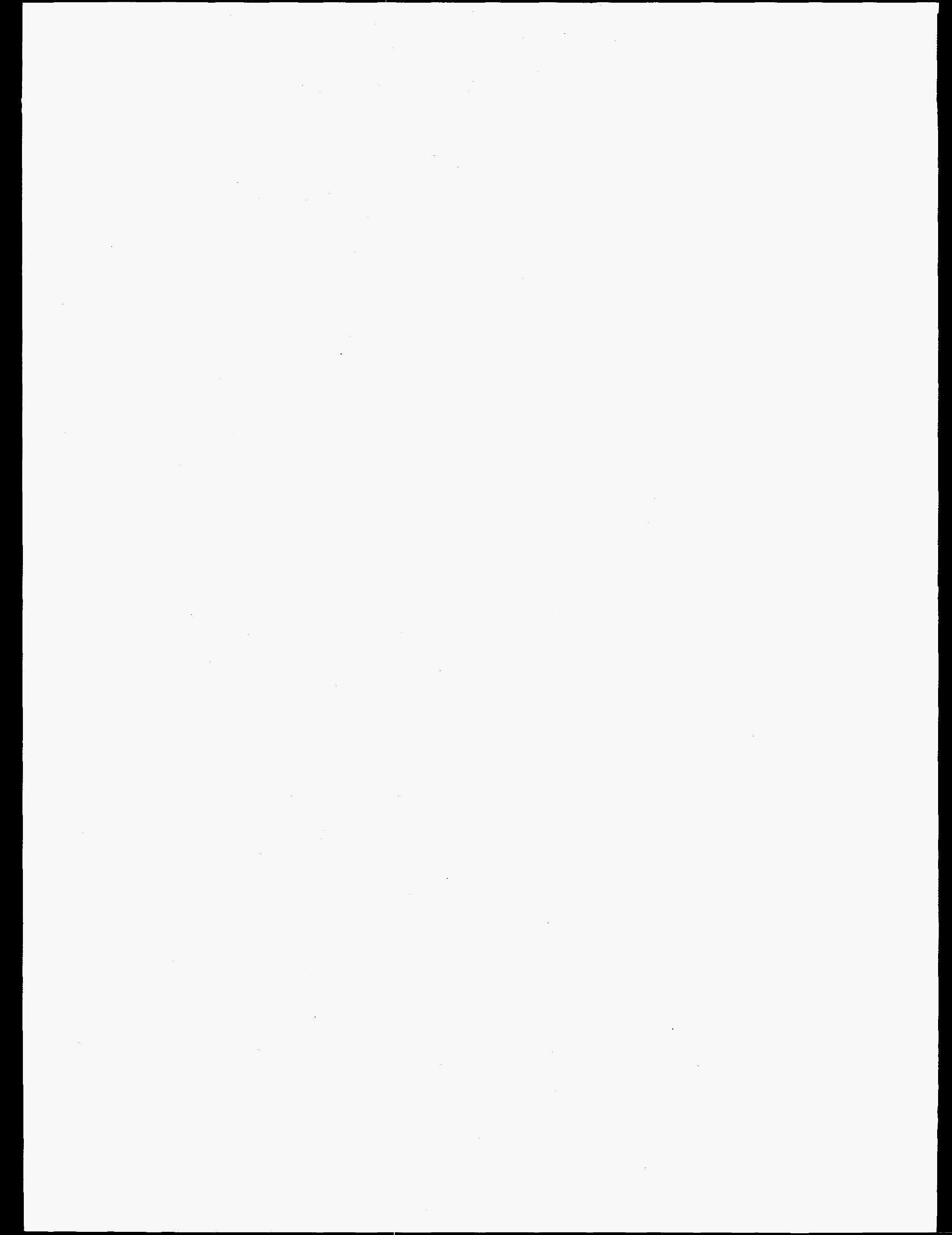


Major Coal Producing

States

Table A1. Alabama Coal Statistics, 1987, 1992-1996

\begin{tabular}{|c|c|c|c|c|c|c|c|c|c|}
\hline \multirow{2}{*}{ Category } & \multirow{2}{*}{1996} & \multirow{2}{*}{1995} & \multirow{2}{*}{1994} & \multirow{2}{*}{1993} & \multirow{2}{*}{1992} & \multirow{2}{*}{1987} & \multirow{2}{*}{$\begin{array}{c}\text { Percent } \\
\text { Change } \\
1995-1996\end{array}$} & \multicolumn{2}{|c|}{$\begin{array}{l}\text { Average Annual } \\
\text { Percent Change }\end{array}$} \\
\hline & & & & & & & & 1992-1996 & $1987-1996$ \\
\hline \multicolumn{10}{|l|}{ Supply (thousand short tons) } \\
\hline 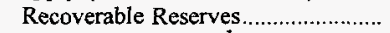 & 451,760 & 510,406 & 457,144 & 426,635 & 467,741 & 537,592 & -11.5 & -0.9 & -1.9 \\
\hline 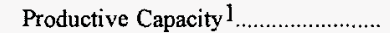 & 32,159 & 32,546 & 33,049 & 27,916 & 29,815 & 29,189 & -1.2 & 1.9 & 1.1 \\
\hline 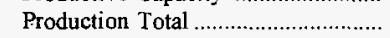 & 24,637 & 24,640 & 23,266 & 24,768 & 25,796 & 25,540 & $*$ & -1.1 & -.4 \\
\hline 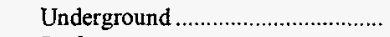 & 18,217 & 17,605 & 14,471 & 15,557 & 15,944 & 14,342 & 3.5 & 3.4 & 2.7 \\
\hline Surface & 6,420 & 7,036 & 8,795 & 9,211 & 9,852 & 11,198 & -8.7 & -10.1 & -6.0 \\
\hline 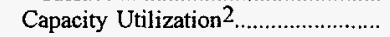 & 76.57 & 75.52 & 70.19 & 88.46 & 86.26 & 87.23 & 1.4 & -2.9 & -1.4 \\
\hline \multicolumn{10}{|l|}{ Ratio of Recoverable } \\
\hline Reserves to Production........................ & 18.3 & 20.7 & 19.6 & 17.2 & 18.1 & 21.0 & -11.5 & .3 & -1.5 \\
\hline 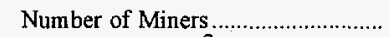 & 5,031 & 5,567 & 5,418 & 5,399 & 5,386 & 6,718 & -9.6 & -1.7 & -3.2 \\
\hline 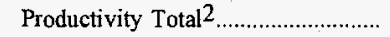 & 2.20 & 2.24 & 2.25 & 2.35 & 2.49 & 1.97 & -1.6 & -3.0 & 1.3 \\
\hline 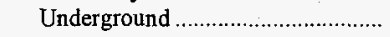 & 1.95 & 2.02 & 1.94 & 2.09 & 2.17 & 1.68 & -3.6 & -2.6 & 1.7 \\
\hline 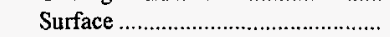 & 3.50 & 3.07 & 3.07 & 3.01 & 3.28 & 2.53 & 14.2 & 1.6 & 3.7 \\
\hline Producer/Distributor Stocks ............... & 1,031 & 1,358 & 1,204 & 1,698 & 2,185 & - & -24.1 & -17.1 & - \\
\hline Imports $^{3}$ & 161 & 162 & 178 & 88 & - & - & -.8 & - & - \\
\hline \multicolumn{10}{|l|}{ Distribution (thousand short tons) } \\
\hline 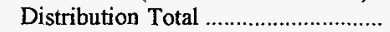 & 24,636 & 25,159 & 23,750 & 25,556 & 25,491 & NA & -2.1 & -.8 & $\mathrm{NA}$ \\
\hline Domestic Distribution Total ............ & 19,772 & 19,127 & 19,220 & 19,668 & 19,560 & NA & 3.4 & .3 & NA \\
\hline Within State & 18,503 & 18,024 & 18,351 & 18,716 & 18,849 & NA & 2.7 & -.5 & NA \\
\hline 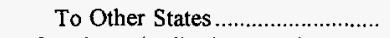 & 1,269 & 1,103 & 870 & 952 & 711 & NA & 15.1 & 15.6 & NA \\
\hline Foreign Distribution Total .............. & 4,864 & 6,032 & 4,529 & 5,888 & 5,931 & NA & -19.4 & -4.8 & NA \\
\hline Metallurgical ............................. & 4,523 & 5,330 & 4,359 & 5,846 & 5,800 & NA & -15.1 & -6.0 & NA \\
\hline Steam & 341 & 702 & 170 & 43 & 131 & NA & -51.5 & 27.0 & $\mathrm{NA}$ \\
\hline Overseas Total 4 & 4,864 & 6,032 & 4,529 & 5,888 & 5,931 & $\mathrm{NA}$ & -19.4 & -4.8 & NA \\
\hline 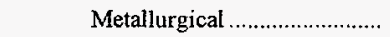 & 4,523 & 5,330 & 4,359 & 5,846 & 5,800 & NA & -15.1 & -6.0 & NA \\
\hline Steann & 341 & 702 & 170 & 43 & 131 & NA & -51.5 & 27.0 & NA \\
\hline \multicolumn{10}{|l|}{ Demand (thousand short tons) } \\
\hline 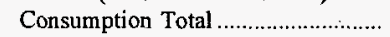 & 37,052 & 34,309 & 31,473 & 33,047 & 31,510 & 26,632 & 8.0 & 4.1 & 3.7 \\
\hline 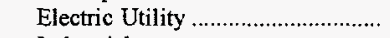 & 31,216 & 28,759 & 25,817 & 27,533 & 24,988 & 20,746 & 8.5 & 5.7 & 4.6 \\
\hline 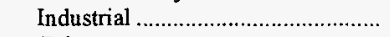 & 2,545 & 2,286 & 2,394 & 2,268 & 3,136 & 2,614 & 11.3 & -5.1 & -.3 \\
\hline Coke & 3,247 & 3,257 & 3,253 & 3,206 & 3,297 & 3,150 & -.3 & -.4 & .3 \\
\hline Residential/Commercial ................... & 44 & 7 & 11 & 40 & 89 & 121 & NM & -16.0 & -10.7 \\
\hline Consumer Stocks Total & 2,860 & 3,648 & 4,132 & 2,797 & 4,529 & 5,012 & -21.6 & -10.8 & -6.0 \\
\hline 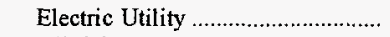 & 2,528 & 3,282 & 3,652 & 2,331 & 4,071 & 4,450 & -23.0 & -11.2 & -6.1 \\
\hline All Other & 332 & 366 & 480 & 466 & 458 & 562 & -9.4 & -7.7 & -5.7 \\
\hline \multicolumn{10}{|c|}{ Coal Prices (nominal dollars per short ton) } \\
\hline 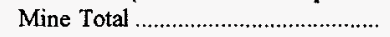 & $\$ 39.48$ & $\$ 38.44$ & $\$ 40.12$ & $\$ 42.34$ & $\$ 40.82$ & $\$ 41.42$ & 2.7 & -.8 & -.5 \\
\hline 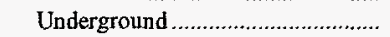 & 40.75 & 39.26 & 39.92 & 42.00 & 40.70 & 39.82 & 3.8 & * & .3 \\
\hline 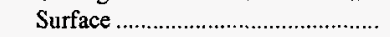 & 35.87 & 36.38 & 40.45 & 42.91 & 41.02 & 43.49 & -1.4 & -3.3 & -2.1 \\
\hline \multicolumn{10}{|l|}{ Consumer } \\
\hline 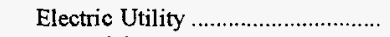 & 36.39 & 37.00 & 40.42 & 42.56 & 41.67 & 47.04 & -1.6 & -3.3 & -2.8 \\
\hline 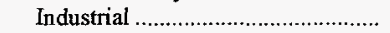 & 40.15 & 39.53 & 38.74 & 39.01 & 39.76 & 40.38 & 1.5 & .2 & -.1 \\
\hline Coke & 49.37 & 48.42 & 47.45 & 47.50 & 47.80 & 45.89 & 1.9 & .8 & .8 \\
\hline
\end{tabular}

1 For 1987, the Form EIA-7A solicited data on "Daily Productive Capacity." To obtain annual productive capacity for a mine in 1987, each mine's daily productive capacity was multiplied by the number of days worked during the year.

2 Capacity utilization (percent) is the ratio of total production to annual productive capacity as reported by mining companies on Form EIA-7A. Productivity (short tons per miner per hour) is calculated by dividing total coal production by the total direct labor hours worked by all employees engaged in production, preparation, processing, development, maintenance, repair, and shop or yard work at mining operations.

3 Imports for 1992 through 1996 include imports to electric utilities, manufacturing plants and coke plants. Imports for 1987 include only imports to electric utilities.

4 Includes Mexico.

* Data round to zero.

NM Not meaningful as value is greater than 500 percent.

NA Not available.

Notes: Excludes silt, culm, refuse bank, slurry dam, and dredge operations except for Pennsylvania anthracite. Consumption Total does not include coal consumed by independent power producers. Totals may not equal sum of components due to independent rounding.

Sources: Energy Information Administration, Form EIA-3, "Quarterly Coal Consumption Report - Manufacturing Plants"; Form EIA-5, "Coke Plant Report - Quarterly"; Form EIA-6, "Coal Distribution Report"; Form EIA-7A, "Coal Production Report"; Form EIA-759, "Monthly Power Plant Report"; and U.S. Department of Commerce, Bureau of the Census, "Monthly Report EM 545" and "Monthly Report IM 145." 
Table A2. Arizona Coal Statistics, 1987, 1992-1996

\begin{tabular}{|c|c|c|c|c|c|c|c|c|c|}
\hline \multirow{2}{*}{ Category } & \multirow{2}{*}{1996} & \multirow{2}{*}{1995} & \multirow{2}{*}{1994} & \multirow{2}{*}{1993} & \multirow{2}{*}{1992} & \multirow{2}{*}{1987} & \multirow{2}{*}{$\begin{array}{c}\text { Percent } \\
\text { Change } \\
1995-1996\end{array}$} & \multicolumn{2}{|c|}{$\begin{array}{l}\text { Average Annual } \\
\text { Percent Change }\end{array}$} \\
\hline & & & & & & & & $1992-1996$ & $1987-1996$ \\
\hline \multicolumn{10}{|l|}{ Supply (thousand short tons) } \\
\hline Recoverable Reserves........................... & $w$ & w & $w$ & $w$ & w & $w$ & w & w & w \\
\hline Productive Capacity 1 ......................... & w & $w$ & $\mathbf{w}$ & 13,800 & 13,800 & w & -4.3 & -1.1 & $\mathbf{w}$ \\
\hline 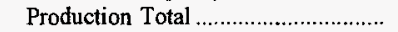 & 10,442 & 11,947 & 13,056 & 12,173 & 12,512 & 11,379 & -12.6 & -4.4 & -0.9 \\
\hline 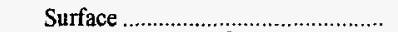 & 10,442 & 11,947 & 13,056 & 12,173 & 12,512 & 11,379 & -12.6 & -4.4 & -.9 \\
\hline $\begin{array}{l}\text { Capacity Utilization } 2 \\
\text { Ratio of Recoverable }\end{array}$ & w & $w$ & $w$ & 88.21 & 90.67 & w & -8.6 & -3.3 & w \\
\hline Reserves to Production......................... & $\mathbf{w}$ & $w$ & $\mathbf{w}$ & w & $\mathbf{w}$ & w & w & w & w \\
\hline Number of Miners & 651 & 831 & 864 & 876 & 888 & 900 & -21.7 & -7.5 & -3.5 \\
\hline Productivity Total $2 \ldots \ldots \ldots \ldots \ldots \ldots \ldots$ & 6.30 & 6.34 & 6.71 & 6.21 & 6.29 & 6.76 & -.6 & $*$ & -.8 \\
\hline 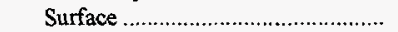 & 6.30 & 6.34 & 6.71 & 6.21 & 6.29 & 6.76 & -.6 & $*$ & -.8 \\
\hline Producer/Distributor Stocks .............. & 2,232 & 2,760 & 2,634 & 1,590 & 1,555 & - & -19.1 & 9.4 & - \\
\hline \multicolumn{10}{|l|}{ Distribution (thousand short tons) } \\
\hline 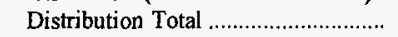 & 10,970 & 11,783 & 12,011 & 12,138 & 12,418 & NA & -6.9 & -3.0 & NA \\
\hline Domestic Distribution Total .......... & 10,970 & 11,783 & 12,011 & 12,138 & 12,418 & NA & -6.9 & -3.0 & NA \\
\hline 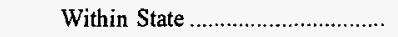 & 6,499 & 6,956 & 7,580 & 7,566 & 7,441 & NA & -6.6 & -3.3 & NA \\
\hline 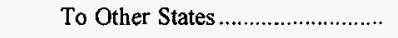 & 4,470 & 4,827 & 4,431 & 4,572 & 4,977 & NA & -7.4 & -2.6 & NA \\
\hline \multicolumn{10}{|l|}{ Demand (thousand short tons) } \\
\hline 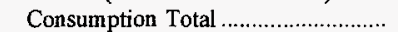 & 16,792 & 16,682 & 19,580 & 18,991 & 17,915 & 13,375 & .6 & -1.6 & 2.6 \\
\hline 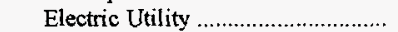 & 16,117 & 16,021 & 18,853 & 18,316 & 17,280 & 12,706 & 6 & -1.7 & 2.7 \\
\hline 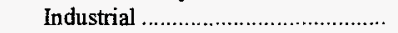 & 675 & 657 & 727 & 674 & 632 & $w$ & 2.8 & 1.7 & w \\
\hline Residential/Commercial ................. & * & 5 & * & 1 & $\mathbf{w}$ & $w$ & -96.3 & w & $\mathbf{w}$ \\
\hline 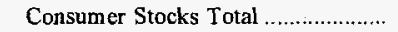 & 2,025 & 3,032 & 3,242 & 3,717 & 3,596 & $w$ & -33.2 & -13.4 & w \\
\hline 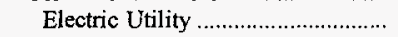 & 1,993 & 2,998 & 3,197 & 3,687 & 3,543 & 3,813 & -33.5 & -13.4 & -6.9 \\
\hline All Other & 32 & 34 & 45 & 30 & 53 & $w$ & -5.8 & -11.7 & w \\
\hline \multicolumn{10}{|c|}{ Coal Prices (nominal dollars per short ton) } \\
\hline 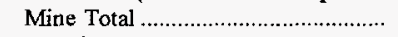 & $\mathbf{w}$ & $w$ & w & $\mathbf{w}$ & $\mathbf{w}$ & $w$ & $w$ & $w$ & $w$ \\
\hline 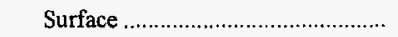 & $\mathbf{w}$ & $w$ & w & $\mathbf{w}$ & $\mathbf{w}$ & $w$ & w & $\mathbf{w}$ & w \\
\hline \multicolumn{10}{|l|}{ Consumer } \\
\hline 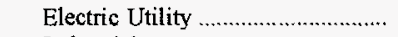 & $\$ 29.55$ & $\$ 28.65$ & $\$ 28.26$ & $\$ 27.78$ & $\$ 28.31$ & $\$ 27.56$ & 3.1 & 1.1 & .8 \\
\hline 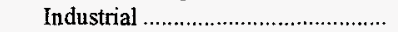 & 39.27 & 40.46 & 41.35 & 40.51 & 40.95 & w & -2.9 & -1.0 & w \\
\hline
\end{tabular}

1 For 1987, the Form EIA-7A solicited data on "Daily Productive Capacity." To obtain annual productive capacity for a mine in 1987, each mine's daily productive capacity was multiplied by the number of days worked during the year.

2 Capacity utilization (percent) is the ratio of total production to annual productive capacity as reported by mining companies on Form EIA-7A. Productivity (short tons per miner per hour) is calculated by dividing total coal production by the total direct labor hours worked by all employees engaged in production, preparation, processing, development, maintenance, repair, and shop or yard work at mining operations.

* Data round to zero.

Withheld to avoid disclosure of individual company data.

NA Not available.

Notes: Excludes silt, culm, refuse bank, slurry dam, and dredge operations except for Pennsylvania anthracite. Consumption Total does not include coal consumed by independent power producers. Totals may not equal sum of components due to independent rounding.

Sources: Energy Information Administration, Form EIA-3, "Quarterly Coal Consumption Report - Manufacturing Plants"; Form EIA-5, "Coke Plant Report - Quarterly"; Form EIA-6, “Coal Distribution Report"; Form EIA-7A, "Coal Production Report"; Form EIA-759, "Monthly Power Plant Report"; and U.S Department of Commerce, Bureau of the Census, "Monthly Report EM 545" and "Monthly Report IM 145." 
Table A3. Colorado Coal Statistics, 1987, 1992-1996

\begin{tabular}{|c|c|c|c|c|c|c|c|c|c|}
\hline \multirow{2}{*}{ Category } & \multirow{2}{*}{1996} & \multirow{2}{*}{1995} & \multirow{2}{*}{1994} & \multirow{2}{*}{1993} & \multirow{2}{*}{1992} & \multirow{2}{*}{1987} & \multirow{2}{*}{$\begin{array}{c}\text { Percent } \\
\text { Change } \\
1995-1996\end{array}$} & \multicolumn{2}{|c|}{$\begin{array}{l}\text { Average Annual } \\
\text { Percent Change }\end{array}$} \\
\hline & & & & & & & & 1992-1996 & 1987-1996 \\
\hline \multicolumn{10}{|l|}{ Supply (thousand short tons) } \\
\hline Recoverable Reserves......................... & 641,615 & 692,030 & 676,272 & 608,815 & 608,067 & 668,353 & -7.3 & 1.3 & -0.4 \\
\hline Productive Capacity 1 .......................... & 29,330 & 32,435 & 31,075 & 30,040 & 25,848 & 21,321 & -9.6 & 3.2 & 3.6 \\
\hline 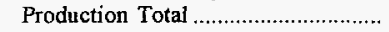 & 24,886 & 25,710 & 25,304 & 21,886 & 19,226 & 14,420 & -3.2 & 6.7 & 6.3 \\
\hline 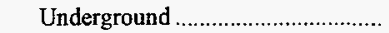 & 15,581 & 17,187 & 16,332 & 12,843 & 10,246 & 5,645 & -9.3 & 11.0 & 11.9 \\
\hline 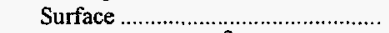 & 9,305 & 8,523 & 8,972 & 9,043 & 8,981 & 8,775 & 9.2 & .9 & .6 \\
\hline 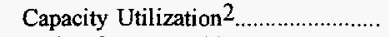 & 84.85 & 79.27 & 81.41 & 72.84 & 74.35 & 67.59 & 7.0 & 3.3 & 2.5 \\
\hline \multicolumn{10}{|l|}{ Ratio of Recoverable } \\
\hline Reserves to Production ......................... & 25.8 & 26.9 & 26.7 & 27.8 & 31.6 & 46.3 & -4.2 & -5.0 & -6.3 \\
\hline Number of Miners & 1,332 & 1,777 & 1,905 & 1,775 & 1,610 & 1,795 & -25.0 & -4.6 & -3.3 \\
\hline 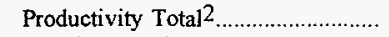 & 7.32 & 6.14 & 6.20 & 5.85 & 5.27 & 4.22 & 19.2 & 8.5 & 6.3 \\
\hline 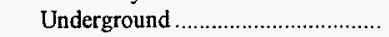 & 6.67 & 5.86 & 5.81 & 5.21 & 4.52 & 2.75 & 13.7 & 10.2 & 10.3 \\
\hline 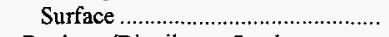 & 8.76 & 6.79 & 7.06 & 7.07 & 6.52 & 6.44 & 29.0 & 7.7 & 3.5 \\
\hline Producer/Distributor Stocks .................. & 494 & 1,063 & 1,575 & 1,155 & 955 & - & -53.5 & -15.2 & - \\
\hline \multicolumn{10}{|l|}{ Distribution (thousand short tons) } \\
\hline 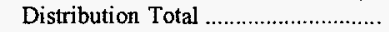 & 25,405 & 25,635 & 24,810 & 21,465 & 18,864 & NA & -.9 & 7.7 & NA \\
\hline Domestic Distribution Total........... & 23,990 & 24,734 & 24,059 & 20,338 & 18,195 & NA & -3.0 & 7.2 & NA \\
\hline 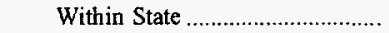 & 10,704 & 11,820 & 12,035 & 11,181 & 11,241 & NA & -9.4 & -1.2 & NA \\
\hline 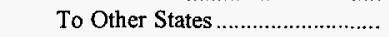 & 13,286 & 12,915 & 12,024 & 9,157 & 6,954 & $\mathrm{NA}$ & 2.9 & 17.6 & NA \\
\hline Foreign Distribution Total ............... & 1,415 & 900 & 752 & 1,128 & 669 & NA & 57.2 & 20.6 & NA \\
\hline 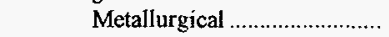 & 30 & - & - & - & - & NA & - & - & NA \\
\hline Steam & 1,385 & 900 & 752 & 1,128 & 669 & NA & 53.9 & 20.0 & NA \\
\hline Overseas Total ${ }^{3}$ & 1,415 & 900 & 752 & 1,128 & 669 & NA & 57.2 & 20.6 & NA \\
\hline 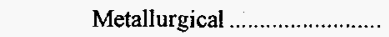 & 30 & - & - & - & - & NA & - & - & NA \\
\hline 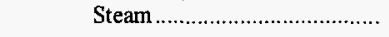 & 1,385 & 900 & 752 & 1,128 & 669 & NA & 53.9 & 20.0 & NA \\
\hline \multicolumn{10}{|l|}{ Demand (thousand short tons) } \\
\hline 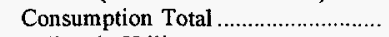 & 17,222 & 16,971 & 17,475 & 17,070 & 16,696 & 15,007 & 1.5 & .8 & 1.5 \\
\hline 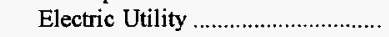 & 16,841 & 16,222 & 16,596 & 16,252 & 15,902 & 14,178 & 3.8 & 1.4 & 1.9 \\
\hline 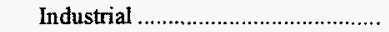 & 367 & 729 & 857 & 780 & 735 & 748 & -49.6 & -15.9 & -7.6 \\
\hline Residential/Commercial................. & 13 & 20 & 23 & 38 & w & w & -32.2 & $w$ & w \\
\hline Consumer Stocks Total ........................ & 3,057 & 3,682 & 3,145 & 3,454 & 3,439 & w & -17.0 & -2.9 & $w$ \\
\hline 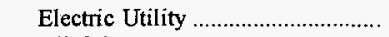 & 3,030 & 3,622 & 3,118 & 3,428 & 3,410 & 3,635 & -16.3 & -2.9 & -2.0 \\
\hline 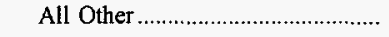 & 27 & 59 & 26 & 25 & 29 & $\mathbf{w}$ & -54.2 & -1.6 & w \\
\hline \multicolumn{10}{|c|}{ Coal Prices (nominal dollars per short ton) } \\
\hline 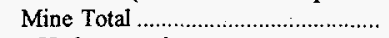 & $\$ 17.94$ & $\$ 19.26$ & $\$ 19.76$ & $\$ 20.35$ & $\$ 21.33$ & $\$ 23.58$ & -6.9 & -4.2 & -3.0 \\
\hline 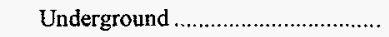 & 17.73 & 18.58 & 19.05 & 20.53 & 21.80 & 28.16 & -4.5 & -5.0 & -5.0 \\
\hline Surface & 18.28 & 20.63 & 21.05 & 20.10 & 20.80 & 20.63 & -11.4 & -3.2 & -1.3 \\
\hline \multicolumn{10}{|l|}{ Consumer } \\
\hline 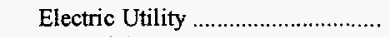 & 20.24 & 20.73 & 21.01 & 21.59 & 21.67 & 22.01 & -2.4 & -1.7 & -.9 \\
\hline 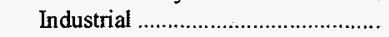 & 23.17 & 26.11 & 28.96 & 28.63 & 30.34 & 28.79 & -11.2 & -6.5 & -2.4 \\
\hline
\end{tabular}

1 For 1987, the Form EIA-7A solicited data on "Daily Productive Capacity." To obtain annual productive capacity for a mine in 1987, each mine's daily product:ve capacity was multiplied by the number of days worked during the year.

2 Capacity utilization (percent) is the ratio of total production to annual productive capacity as reported by mining companies on Form EIA-7A. Productivity (short tons per miner per hour) is calculated by dividing total coal production by the total direct labor hours worked by all employees engaged in production, preparation, processing, development, maintenance, repair, and shop or yard work at mining operations.

3 Includes Mexico.

withheld to avoid disclosure of individual company data.

NA Not available.

Notes: Excludes silt, culm, refuse bank, slurry dam, and dredge operations except for Pennsylvania anthracite. Consumption Total does not include coal consumed by independent power producers. Totals may not equal sum of components due to independent rounding.

Sources: Energy Information Administration, Form EIA-3, "Quarterly Coal Consumption Report - Manufacturing Plants"; Form EIA-5, "Coke Plant Report - Quarterly"; Form EIA-6, "Coal Distribution Report"; Form EIA-7A, "Coal Production Report"; Form EIA-759, "Monthly Power Plant Report"; and U.S Department of Commerce, Bureau of the Census, "Monthly Report EM 545" and "Monthly Report IM 145." 


\begin{tabular}{|c|c|c|c|c|c|c|c|c|c|}
\hline \multirow{2}{*}{ Category } & \multirow{2}{*}{1996} & \multirow{2}{*}{1995} & \multirow{2}{*}{1994} & \multirow{2}{*}{1993} & \multirow{2}{*}{1992} & \multirow{2}{*}{1987} & \multirow{2}{*}{$\begin{array}{c}\text { Percent } \\
\text { Change } \\
\text { 1995-1996 }\end{array}$} & \multicolumn{2}{|c|}{$\begin{array}{l}\text { Average Annual } \\
\text { Percent Change }\end{array}$} \\
\hline & & & & & & & & 1992-1996 & 1987-1996 \\
\hline \multicolumn{10}{|l|}{ Supply (thousand short tons) } \\
\hline Recoverable Reserves.......................... & 891,109 & 882,323 & 963,470 & $1,063,832$ & $1,199,313$ & $1,431,240$ & 1.0 & -7.1 & -5.1 \\
\hline 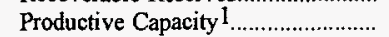 & 61,727 & 56,627 & 69,414 & 69,320 & 75,787 & 75,852 & 9.0 & -5.0 & -2.3 \\
\hline Production Total & 46,656 & 48,180 & 52,797 & 41,098 & 59,857 & 59,155 & -3.2 & -6.0 & -2.6 \\
\hline Underground & 38,948 & 41,118 & 43,281 & 33,096 & 46,965 & 37,521 & -5.3 & -4.6 & .4 \\
\hline Surface & 7,707 & 7,062 & 9,516 & 8,002 & 12,892 & 21,634 & 9.1 & -12.1 & -10.8 \\
\hline $\begin{array}{l}\text { Capacity Utilization }{ }^{2} \\
\text { Ratio of Recoverable }\end{array}$ & 75.58 & 85.08 & 76.06 & 59.28 & 78.98 & 77.98 & -11.2 & -1.1 & -.3 \\
\hline Reserves to Production ....................... & 19.1 & 18.3 & 18.3 & 25.9 & 20.0 & 24.2 & 4.3 & -1.2 & -2.6 \\
\hline Number of Miners & 5,174 & 5,652 & 6,591 & 7,303 & 8,323 & 12,171 & -8.4 & -11.2 & -9.1 \\
\hline Productivity Total ${ }^{2}$ & 4.18 & 3.87 & 3.59 & 3.23 & 3.42 & 2.52 & 8.2 & 5.2 & 5.8 \\
\hline 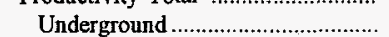 & 4.10 & 3.86 & 3.49 & 3.11 & 3.21 & 2.20 & 6.1 & 6.3 & 7.1 \\
\hline 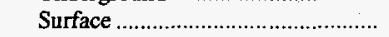 & 4.67 & 3.89 & 4.12 & 3.86 & 4.47 & 3.37 & 19.9 & 1.1 & 3.7 \\
\hline Producer/Distributor Stocks .............. & 1,190 & 2,069 & 1,651 & 713 & 1,969 & - & -42.5 & -11.8 & - \\
\hline Imports $^{3} \ldots \ldots \ldots \ldots \ldots \ldots \ldots \ldots \ldots \ldots \ldots \ldots \ldots$ & 216 & 223 & 346 & 51 & - & - & -3.1 & - & - \\
\hline \multicolumn{10}{|l|}{ Distribution (thousand short tons) } \\
\hline 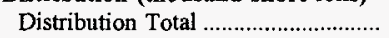 & 47,076 & 47,869 & 51,973 & 42,000 & 58,913 & NA & -1.7 & -5.4 & NA \\
\hline 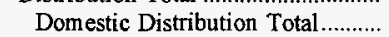 & 45,190 & 45,170 & 51,737 & 41,330 & 57,670 & $\mathrm{NA}$ & * & -5.9 & NA \\
\hline 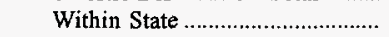 & 16,052 & 15,587 & 17,517 & 15,206 & 18,167 & $\mathrm{NA}$ & 3.0 & -3.0 & $\mathrm{NA}$ \\
\hline To Other States & 29,137 & 29,582 & 34,220 & 26,124 & 39,503 & $\mathrm{NA}$ & -1.5 & -7.3 & $\mathrm{NA}$ \\
\hline 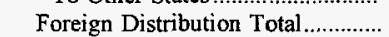 & 1,886 & 2,699 & 236 & 670 & 1,242 & $\mathrm{NA}$ & -30.1 & 11.0 & $\mathrm{NA}$ \\
\hline 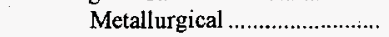 & - & 49 & 236 & 109 & 614 & $\mathrm{NA}$ & -100.0 & -100.0 & $\mathrm{NA}$ \\
\hline Steam & 1,886 & 2,650 & - & 561 & 629 & $\mathrm{NA}$ & -28.8 & 31.6 & $\mathrm{NA}$ \\
\hline Overseas Total 4 & 1,886 & 2,699 & 236 & 670 & 1,242 & NA & -30.1 & 11.0 & NA \\
\hline 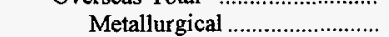 & - & 49 & 236 & 109 & 614 & NA & -100.0 & - & $\mathrm{NA}$ \\
\hline Steam & 1,886 & 2,650 & - & 561 & 629 & NA & -28.8 & 31.6 & $\mathrm{NA}$ \\
\hline \multicolumn{10}{|l|}{ Demand (thousand short tons) } \\
\hline 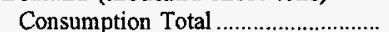 & 44,431 & 39,623 & 39,077 & 38,135 & 31,599 & 35,581 & 12.1 & 8.9 & 2.5 \\
\hline 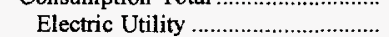 & 38,090 & 33,463 & 32,599 & 31,744 & 25,264 & 28,894 & 13.8 & 10.8 & 3.1 \\
\hline Industrial & 3,740 & 3,653 & 4,187 & 3,970 & 3,736 & 3,900 & 2.4 & $*$ & -.5 \\
\hline Coke & $w$ & w & $\mathrm{w}$ & w & $w$ & 2,515 & w & w & w \\
\hline Residential/Commercial .................. & $w$ & w & $\mathrm{w}$ & w & w & 273 & $w$ & $w$ & w \\
\hline Consumer Stocks Total ........................ & w & w & $w$ & w & w & 9,676 & w & w & $\mathbf{w}$ \\
\hline 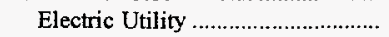 & 4,581 & 5,331 & 4,526 & 4,019 & 7,399 & 8,861 & -14.1 & -11.3 & -7.1 \\
\hline All Other & W & w & $w$ & $w$ & w & 815 & $\mathrm{w}$ & w & w \\
\hline \multicolumn{10}{|c|}{ Coal Prices (nominal dollars per short ton) } \\
\hline 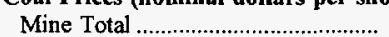 & $\$ 22.74$ & $\$ 23.05$ & $\$ 23.14$ & $\$ 25.27$ & $\$ 27.66$ & $\$ 29.56$ & -1.3 & -4.8 & -2.9 \\
\hline 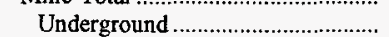 & 23.12 & 22.88 & 23.18 & 25.54 & 27.93 & 30.39 & 1.0 & -4.6 & -3.0 \\
\hline Surface & 20.86 & 24.04 & 22.92 & 24.18 & 26.69 & 28.12 & -13.2 & -6.0 & -3.3 \\
\hline \multicolumn{10}{|l|}{ Consumer } \\
\hline 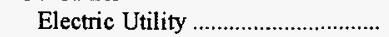 & 32.14 & 32.58 & 32.69 & 35.30 & 37.06 & 42.80 & $-1: 3$ & -3.5 & -3.1 \\
\hline 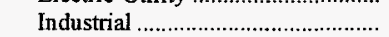 & 29.69 & 29.03 & 29.13 & 29.42 & 29.24 & 33.15 & 2.3 & .4 & -1.2 \\
\hline Coke & w & w & w & $w$ & w & 44.19 & $w$ & $\ddot{w}$ & $\mathrm{w}$ \\
\hline
\end{tabular}

1 For 1987, the Form EIA-7A solicited data on "Daily Productive Capacity." To obtain annual productive capacity for a mine in 1987, each mine's daily productive capacity was multiplied by the number of days vrorked during the year.

2 Capacity utilization (percent) is the ratio of total prodıction to annual productive capacity as reported by mining companies on Form EIA-7A. Productivity (short tons per miner per hour) is calculated by dividing total coal production by the total direct labor hours worked by all employees engaged in production, preparation, processing, development, maintenance, repair, and shop or yard work at mining operations.

3 Imports for 1992 through 1996 include imports to electric utilities, manufacturing plants and coke plants. Imports for 1987 include only imports to electric utilities.

4 Includes Mexico.

* Data round to zero.

Withheld to avoid disclosure of individual company data.

NA Not available.

Notes: Excludes silt, culm, refuse bank, slurry dam, and dredge operations except for Pennsylvania anthracite. Consumption Total does not include coal consumed by independent power producers. Totals may not equal sum of components due to independent rounding.

Sources: Energy Information Administration, Form EIA-3, "Quarterly Coal Consumption Report - Manufacturing Plants"; Form EIA-5, "Coke Plant Report - Quarterly", Form EIA-6, "Coal Distribution Report"; Form ElA-7A, "Coal Production Report"; Form EIA-759, "Monthly Power Plant Report"; and U.S. Department of Commerce, Bureau of the Census, "Monthly Report EM 545" and "Monthly Report IM 145." 
Table A5. Indiana Coal Statistics, 1987, 1992-1996

\begin{tabular}{|c|c|c|c|c|c|c|c|c|c|}
\hline \multirow{2}{*}{ Category } & \multirow{2}{*}{1996} & \multirow{2}{*}{1995} & \multirow{2}{*}{1994} & \multirow{2}{*}{1993} & \multirow{2}{*}{1992} & \multirow{2}{*}{1987} & \multirow{2}{*}{$\begin{array}{c}\text { Percent } \\
\text { Change } \\
\text { 1995-1996 }\end{array}$} & \multicolumn{2}{|c|}{$\begin{array}{l}\text { Average Annual } \\
\text { Percent Change }\end{array}$} \\
\hline & & & & & & & & 1992-1996 & 1987-1996 \\
\hline \multicolumn{10}{|l|}{ Supply (thousand short tons) } \\
\hline 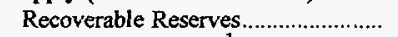 & 386,063 & 323,667 & 304,225 & 379,294 & 404,549 & 514,341 & 19.3 & $-1,2$ & -3.1 \\
\hline 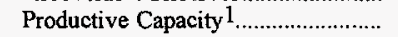 & 35,564 & 35,256 & 38,931 & 43,955 & 42,990 & 45,344 & .9 & -4.6 & -2.7 \\
\hline 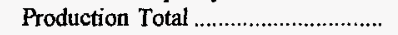 & 29,670 & 26,007 & 30,927 & 29,295 & 30,466 & 34,208 & 14.1 & -.6 & -1.6 \\
\hline 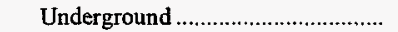 & 2,963 & 3,540 & 3,324 & 2,583 & 2,641 & 2,447 & -16.3 & 2.9 & 2.1 \\
\hline 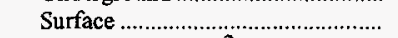 & 26,707 & 22,467 & 27,603 & 26,713 & 27,825 & 31,761 & 18.9 & -1.0 & -1.9 \\
\hline 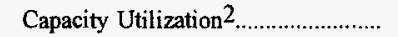 & 83.42 & 73.70 & 79.37 & 66.60 & 70.86 & 75.36 & 13.2 & 4.2 & 1.1 \\
\hline Ratio of Recoverable & & & & & & & & & \\
\hline Reserves to Production .......................... & 13.0 & 12.4 & 9.8 & 12.9 & 13.3 & 15.0 & 4.5 & -.5 & -1.6 \\
\hline 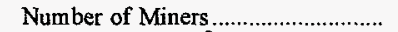 & 2,579 & 2,571 & 3,206 & 3,331 & 3,652 & 3,992 & .3 & -8.3 & -4.7 \\
\hline 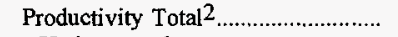 & 4.98 & 4.68 & 4.28 & 4.46 & 4.09 & 3.57 & 6.4 & 5.0 & 3.8 \\
\hline 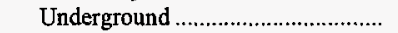 & 3.09 & 3.22 & 2.82 & 2.49 & 2.80 & 2.05 & -4.1 & 2.5 & 4.6 \\
\hline Surface & 5.34 & 5.04 & 4.56 & 4.82 & 4.28 & 3.79 & 6.0 & 5.7 & 3.9 \\
\hline Producer/Distributor Stocks ............... & 574 & 611 & 803 & 527 & 1,016 & - & -6.1 & -13.3 & - \\
\hline 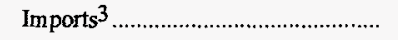 & 735 & 761 & 593 & 594 & - & - & -3.3 & - & - \\
\hline \multicolumn{10}{|l|}{ Distribution (thousand short tons) } \\
\hline 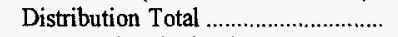 & 29,674 & 25,695 & 30,684 & 29,664 & 31,393 & NA & 15.5 & -1.4 & NA \\
\hline Domestic Distribution Total........... & 29,664 & 25,625 & 30,477 & 29,475 & 31,216 & NA & 15.8 & -1.3 & NA \\
\hline 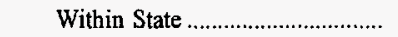 & 24,309 & 21,185 & 24,733 & 23,913 & 24,655 & NA & 14.8 & -.3 & NA \\
\hline 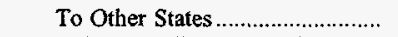 & 5,354 & 4,439 & 5,744 & 5,562 & 6,561 & NA & 20.6 & -4.9 & NA \\
\hline Foreign Distribution Total.............. & 11 & 70 & 206 & 188 & 177 & NA & -84.7 & -50.4 & NA \\
\hline 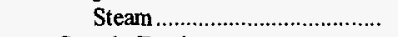 & 11 & 70 & 206 & 188 & 177 & NA & -84.7 & -50.4 & NA \\
\hline 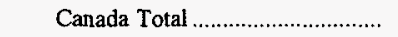 & - & $*$ & - & - & - & NA & -100.0 & - & NA \\
\hline 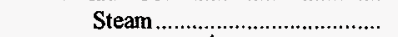 & - & $*$ & - & - & - & $\mathrm{NA}$ & -100.0 & - & NA \\
\hline Overseas Total ${ }^{4} \ldots \ldots \ldots \ldots \ldots \ldots \ldots \ldots \ldots$ & 11 & 69 & 206 & 188 & 177 & NA & -84.6 & -50.4 & $\mathrm{NA}$ \\
\hline Steam & 11 & 69 & 206 & 188 & 177 & NA & -84.6 & -50.4 & NA \\
\hline \multicolumn{10}{|l|}{ Demand (thousand short tons) } \\
\hline Consumption Total .............................. & 64,021 & 62,631 & 59,996 & 60,353 & 58,765 & 51,385 & 2.2 & 2.2 & 2.5 \\
\hline 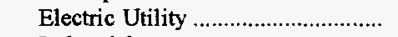 & 52,855 & 52,089 & 50,554 & 48,836 & 46,937 & 36,987 & 1.5 & 3.0 & 4.0 \\
\hline 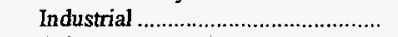 & 4,987 & 4,373 & 4,244 & 4,587 & 4,263 & 5,016 & 14.0 & 4.0 & -.1 \\
\hline Coke & 5,823 & 5,883 & 4,841 & 6,591 & 7,153 & 8,841 & -1.0 & -5.0 & -4.5 \\
\hline Residential/Commercial................... & 356 & 287 & 356 & 339 & 411 & 541 & 24.3 & -3.5 & -4.5 \\
\hline Consumer Stocks Total ......................... & 7,958 & 9,298 & 11,707 & 7,798 & 12,507 & 14,998 & -14.4 & -10.7 & -6.8 \\
\hline 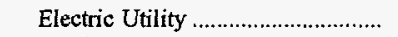 & 7,105 & 8,435 & 10,449 & 6,935 & 11,294 & 13,185 & -15.8 & -10.9 & -6.6 \\
\hline 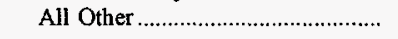 & 853 & 863 & 1,258 & 863 & 1,214 & 1,813 & -1.2 & -8.4 & -8.0 \\
\hline \multicolumn{10}{|c|}{ Coal Prices (nominal dollars per short ton) } \\
\hline 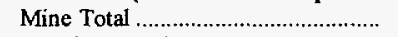 & $\$ 20.24$ & $\$ 21.71$ & $\$ 22.28$ & $\$ 22.89$ & $\$ 23.41$ & $\$ 24.57$ & -6.8 & -3.6 & -2.1 \\
\hline 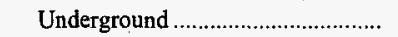 & w & w & w & w & w & w & w & w & w \\
\hline Surface & $w$ & w & $w$ & $w$ & $w$ & w & $\mathbf{w}$ & $\mathbf{w}$ & w \\
\hline \multicolumn{10}{|l|}{ Consumer } \\
\hline 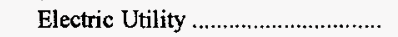 & $\$ 24.67$ & $\$ 25.94$ & $\$ 26.79$ & $\$ 26.73$ & $\$ 27.89$ & $\$ 31.93$ & -4.9 & -3.0 & -2.8 \\
\hline 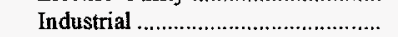 & 31.76 & 33.14 & 31.35 & 30.91 & 31.58 & 30.94 & -4.2 & .1 & .3 \\
\hline 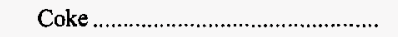 & 51.93 & 52.74 & 50.90 & 52.29 & 53.72 & 51.44 & -1.5 & -.8 & .1 \\
\hline
\end{tabular}

1 For 1987, the Form EIA-7A solicited data on "Daily Productive Capacity." To obtain annual productive capacity for a mine in 1987, each mine's daily productive capacity was multiplied by the number of days worked during the year.

2 Capacity utilization (percent) is the ratio of total production to annual productive capacity as reported by mining companies on Form EIA-7A. Productivity (short tons per miner per hour) is calculated by dividing total coal production by the total direct labor hours worked by all employees engaged in production, preparation, processing, development, maintenance, repair, and shop or yard work at mining operations.

3 Imports for 1992 through 1996 include imports to electric utilities, manufacturing plants and coke plants. Imports for 1987 include only imports to electric utilities.

4 Includes Mexico.

* Data round to zero.

withheld to avoid disclosure of individual company data

NA Not available.

Notes: Excludes silt, culm, refuse bank, slurry dam, and dredge operations except for Pennsylvania anthracite. Consumption Total does not include coal consumed by independent power producers. Totals may not equal sum of components due to independent rounding.

Sources: Energy Information Administration, Form ElA-3, "Quarterly Coal Consumption Report - Manufacturing Plants"; Form EIA-5, "Coke Plant Report - Quarterly"; Form EIA-6, "Coal Distribution Report"; Form EIA-7A, "Coal Production Report"; Form EIA-759, "Monthly Power Plant Report"; and U.S. Department of Commerce, Bureau of the Census, "Monthly Report EM 545" and "Monthly Report IM 145." 
Table A6. Kentucky Coal Statistics, 1987, 1992-1996

\begin{tabular}{|c|c|c|c|c|c|c|c|c|c|}
\hline \multirow{2}{*}{ Category } & \multirow{2}{*}{1996} & \multirow{2}{*}{1995} & \multirow{2}{*}{1994} & \multirow{2}{*}{1993} & \multirow{2}{*}{1992} & \multirow{2}{*}{1987} & \multirow{2}{*}{$\begin{array}{c}\text { Percent } \\
\text { Change } \\
\text { 1995-1996 }\end{array}$} & \multicolumn{2}{|c|}{$\begin{array}{l}\text { Average Annual } \\
\text { Percent Change }\end{array}$} \\
\hline & & & & & & & & $1992-1996$ & $1987-1996$ \\
\hline \multicolumn{10}{|l|}{ Supply (thousand short tons) } \\
\hline Recoverable Reserves........................ & $1,255,351$ & $1,279,011$ & $1,365,188$ & $1,828,072$ & $1,452,789$ & $1,739,114$ & -1.8 & -3.6 & -3.5 \\
\hline 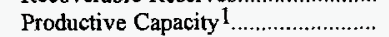 & 189,225 & 203,173 & 213,427 & 204,805 & 195,352 & 185,985 & -6.9 & -8 & .2 \\
\hline 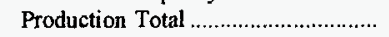 & 152,425 & 153,739 & 161,642 & 156,299 & 161,068 & 165,192 & -.8 & -1.4 & -.9 \\
\hline 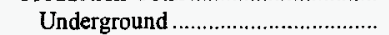 & 94,306 & 94,207 & 95,414 & 92,207 & 96,053 & 92,882 & .1 & -.4 & .2 \\
\hline Surface & 58,119 & 59,532 & 66,227 & 64,092 & 65,016 & 72,310 & -2.4 & -2.8 & -2.4 \\
\hline Capacity Utilization ${ }^{2} \ldots \ldots \ldots \ldots \ldots \ldots \ldots \ldots$ & 80.38 & 75.49 & 75.54 & 76.11 & 82.15 & 88.03 & 6.5 & -.5 & -1.0 \\
\hline \multicolumn{10}{|l|}{ Ratio of Recoverable } \\
\hline Reserves to Production ......................... & 8.2 & 8.3 & 8.4 & 11.7 & 9.0 & 10.5 & -1.0 & -2.2 & -2.7 \\
\hline Number of Miners & 18,826 & 21,125 & 23,368 & 24,063 & 24,624 & 32,590 & -10.9 & -6.5 & -5.9 \\
\hline Productivity Total 2 & 3.80 & 3.57 & 3.25 & 3.25 & 3.20 & 2.69 & 6.6 & 4.4 & 3.9 \\
\hline Underground & 3.53 & 3.25 & 2.89 & 2.93 & 2.91 & 2.40 & 8.6 & 5.0 & 4.4 \\
\hline 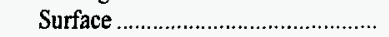 & 4.35 & 4.23 & 3.96 & 3.84 & 3.75 & 3.18 & 2.9 & 3.8 & 3.5 \\
\hline Ptoducer/Distributor Stocks .............. & 4,460 & 4,777 & 5,025 & 3,216 & 3,796 & - & -6.6 & 4.1 & - \\
\hline \multicolumn{10}{|l|}{ Distribution (thousand short tons) } \\
\hline Distribution Total ................................ & 152,891 & 151,466 & 159,130 & 160,395 & 161,860 & NA & .9 & -1.4 & NA \\
\hline 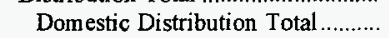 & 143,748 & 141,771 & 151,963 & 150,874 & 147,825 & NA & 1.4 & -.7 & $\mathrm{NA}$ \\
\hline 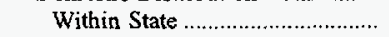 & 25,700 & 27,140 & 26,719 & 28,136 & 27,941 & NA & -5.3 & -2.1 & $\mathrm{NA}$ \\
\hline 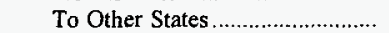 & 118,047 & 114,631 & 125,244 & 122,738 & 119,883 & NA & 3.0 & -.4 & NA \\
\hline Foreign Distribution Total.............. & 9,143 & 9,695 & 7,167 & 9,521 & 14,036 & NA & -5.7 & -10.2 & NA \\
\hline 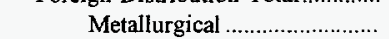 & 5,303 & R 3,640 & R 3,120 & $R_{4,415}$ & R 5,916 & $\mathrm{NA}$ & 45.7 & -2.7 & NA \\
\hline Steam & 3,841 & R 6,055 & $R_{4,047}$ & $\mathrm{R}_{5,106}$ & $R_{8,120}$ & NA & -36.6 & -17.1 & NA \\
\hline Canada Total & 1,178 & 777 & 1,099 & 1,416 & 2,078 & NA & 51.5 & -13.2 & NA \\
\hline 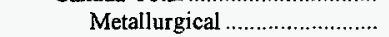 & 1,178 & 777 & 1,073 & 1,356 & 1,373 & $\mathrm{NA}$ & 51.5 & -3.8 & $\mathrm{NA}$ \\
\hline Steam & - & - & 26 & 60 & 705 & $\mathrm{NA}$ & - & - & $\mathrm{NA}$ \\
\hline Overseas Total ${ }^{3}$ & 7,966 & 8,918 & 6,067 & 8,106 & 11,958 & NA & -10.7 & -9.7 & NA \\
\hline 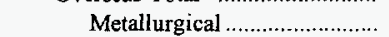 & 4,125 & R 2,863 & R 2,047 & $R_{3,059}$ & $\mathbf{R}_{4,544}$ & NA & 44.1 & -2.4 & $\mathrm{NA}$ \\
\hline Steam & 3,841 & R 6,055 & $R_{4,021}$ & R 5,047 & R 7,414 & $\mathrm{NA}$ & -36.6 & -15.2 & $\mathrm{NA}$ \\
\hline \multicolumn{10}{|l|}{ Demand (thousand short tons) } \\
\hline 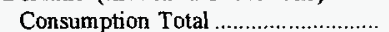 & 40,863 & 39,516 & 38,090 & 39,095 & 34,704 & 32,023 & 3.4 & 4.2 & 2.7 \\
\hline Electric Utility & 37,072 & 35,707 & 34,564 & 35,264 & 31,715 & 28,569 & 3.8 & 4.0 & 2.9 \\
\hline 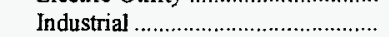 & 2,322 & 2,250 & 1,994 & 2,392 & 1,648 & 1,869 & 3.2 & 8.9 & 2.4 \\
\hline 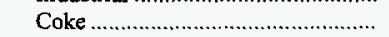 & $w$ & $w$ & $w$ & W & $w$ & $w$ & $w$ & $w$ & $w$ \\
\hline Residential/Commercial .................... & w & w & $w$ & $\mathbf{w}$ & w & w & w & $w$ & $w$ \\
\hline Consumer Stocks Total ........................ & $w$ & $w$ & $w$ & $w$ & w & w & $w$ & w & w \\
\hline 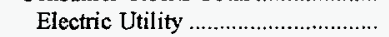 & 4,121 & 4,472 & 4,466 & 3,990 & 5,415 & 7,679 & -7.8 & -6.6 & -6.7 \\
\hline 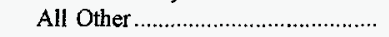 & w & w & $w$ & $w$ & w & w & $\mathrm{w}$ & w & w \\
\hline \multicolumn{10}{|c|}{ Coal Prices (nominal dollars per short ton) } \\
\hline Mine Total & $\$ 23.91$ & $\$ 24.79$ & $\$ 24.88$ & $\$ 24.77$ & $\$ 24.50$ & $\$ 26.15$ & -3.6 & -.6 & -1.0 \\
\hline 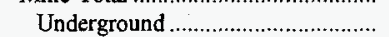 & 24.66 & 25.18 & 26.14 & 25.07 & 25.18 & 26.80 & -2.0 & -.5 & -9 \\
\hline 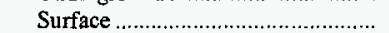 & 22.68 & 24.19 & 23.07 & 24.35 & 23.50 & 25.32 & -6.2 & -.9 & -1.2 \\
\hline \multicolumn{10}{|l|}{ Consumer } \\
\hline 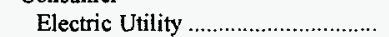 & 24.43 & 25.71 & 27.16 & 27.29 & 27.01 & 28.94 & -5.0 & -2.5 & -1.9 \\
\hline 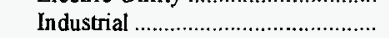 & 44.02 & 44.09 & 43.22 & 42.30 & 43.78 & 43.12 & -.2 & .1 & .2 \\
\hline Coke & w & w & w & $w$ & w & w & w & $w$ & $\mathbf{w}$ \\
\hline
\end{tabular}

1 For 1987, the Form ElA-7A solicited data on "Daily Productive Capacity." To obtain annual productive capacity for a mine in 1987, each mine's daily productive capacity was multiplied by the number of days worked during the year.

2 Capacity utilization (percent) is the ratio of total production to annual productive capacity as reported by mining companies on Form EIA-7A. Productivity (short tons per miner per hour) is calculated by dividing total coal production by the total direct labor hours worked by all employees engaged in production, preparation, processing, development, maintenance, repair, and shop or yard work at mining operations.

3 Includes Mexico.

Witheld to avoid disclosure of individual company data.

NA Not available.

R Revised Data.

Notes: Excludes silt, culm, refuse bank, slurry dam, and dredge operations except for Pennsylvania anthracite. Consumption Total does not include coal consumed by independent power producers. Totals may not equal sum of components due to independent rounding.

Sources: Energy Information Administration, Form EIA.-3, "Quarterly Coal Consumption Report - Manufacturing Plants"; Form EIA-5, "Coke Plant Report - Quarterly"; Form ELA-6, "Coal Distribution Report"; Form EIA-7A, "Coal Production Report"; Form EIA-759, "Monthly Power Plant Report"; and U.S. Department of Commerce, Bureau of the Census, "Monthly Report EM 545" and "Monthly Report IM 145." 
Table A7. Montana Coal Statistics, 1987, 1992-1996

\begin{tabular}{|c|c|c|c|c|c|c|c|c|c|}
\hline \multirow{2}{*}{ Category } & \multirow{2}{*}{1996} & \multirow{2}{*}{1995} & \multirow{2}{*}{1994} & \multirow{2}{*}{1993} & \multirow{2}{*}{1992} & \multirow{2}{*}{1987} & \multirow{2}{*}{$\begin{array}{c}\text { Percent } \\
\text { Change } \\
1995-1996\end{array}$} & \multicolumn{2}{|c|}{$\begin{array}{l}\text { Average Annual } \\
\text { Percent Change }\end{array}$} \\
\hline & & & & & & & & $1992-1996$ & $1987-1996$ \\
\hline \multicolumn{10}{|l|}{ Supply (thousand short tons) } \\
\hline 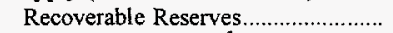 & $1,308,793$ & $1,250,866$ & $1,282,525$ & $1,284,806$ & $1,351,609$ & $1,610,532$ & 4.6 & -0.8 & -2.3 \\
\hline Productive Capacity 1 ............................ & 56,175 & 51,597 & 51,104 & 50,849 & 48,582 & 42,848 & 8.9 & 3.7 & 3.0 \\
\hline 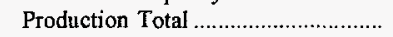 & 37,891 & 39,451 & 41,640 & 35,917 & 38,889 & 34,399 & -3.9 & -.6 & 1.1 \\
\hline 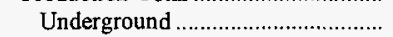 & 147 & 10 & 3 & 10 & 10 & - & NM & 97.7 & - \\
\hline Surface & 37,744 & $39,44 !$ & 41,636 & 35,907 & 38,879 & 34,399 & -4.3 & -.7 & 1.0 \\
\hline Capacity Utilization 2 ........................ & 67.45 & 76.44 & 81.47 & 70.64 & 80.03 & 80.28 & -11.8 & -4.2 & -1.9 \\
\hline \multicolumn{10}{|l|}{ Ratio of Recoverable } \\
\hline Reserves to Production .......................... & 34.5 & 31.7 & 30.8 & 35.8 & 34.8 & 46.8 & 8.9 & -.1 & -3.3 \\
\hline 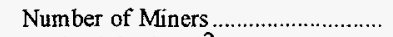 & 705 & 722 & 705 & 660 & 715 & 847 & -2.3 & -.3 & -2.0 \\
\hline 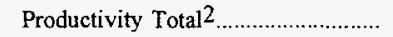 & 21.88 & 21.06 & 21.92 & 19.49 & 20.16 & 18.70 & 3.9 & 2.1 & 1.8 \\
\hline 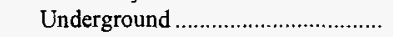 & 3.50 & - & - & 1.06 & - & - & - & - & - \\
\hline 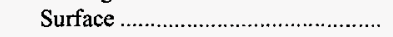 & 22.34 & 21.06 & 21.92 & 19.59 & 20.16 & 18.70 & 6.1 & 2.6 & 2.0 \\
\hline Producer/Distributor Stocks .............. & 580 & 718 & 635 & 876 & 694 & - & -19.3 & -4.4 & - \\
\hline \multicolumn{10}{|l|}{ Distribution (thousand short tons) } \\
\hline Distribution Total & 38,288 & 39,620 & 41,916 & 35,916 & 38,866 & NA & -3.4 & -.4 & NA \\
\hline Domestic Distribution Total........... & 37,770 & 39,362 & 41,672 & 35,795 & 38,804 & NA & -4.0 & -.7 & $\mathrm{NA}$ \\
\hline 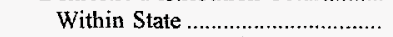 & 7,844 & 9,477 & 10,581 & 9,115 & 11,159 & NA & -17.2 & -8.4 & NA \\
\hline 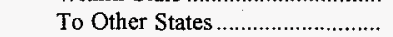 & 29,926 & 29,885 & 31,092 & 26,680 & 27,645 & NA & .1 & 2.0 & NA \\
\hline Foreign Distribution Total ............. & 518 & 259 & 243 & 121 & 62 & NA & 100.2 & 70.2 & NA \\
\hline Steam & 518 & 259 & 243 & 121 & 62 & NA & 100.2 & 70.2 & NA \\
\hline 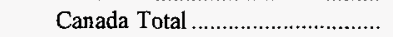 & 316 & 259 & 90 & 54 & - & NA & 22.1 & - & $\mathrm{NA}$ \\
\hline Steam & 316 & 259 & 90 & 54 & - & NA & 22.1 & - & NA \\
\hline 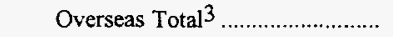 & 202 & - & 153 & 67 & 62 & NA & - & 34.6 & $\mathrm{NA}$ \\
\hline Steam & 202 & - & 153 & 67 & 62 & NA & - & 34.6 & NA \\
\hline \multicolumn{10}{|l|}{ Demand (thousand short tons) } \\
\hline 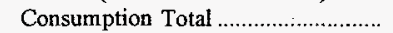 & 8,032 & 10,005 & 11,089 & 9,247 & 11,040 & 7,730 & -19.7 & -7.6 & .4 \\
\hline 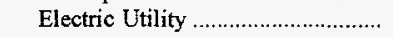 & 7,897 & 9,373 & 10,513 & 8,869 & 10,768 & 7,530 & -15.8 & -7.4 & .5 \\
\hline 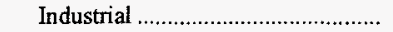 & w & $\mathbf{w}$ & $w$ & w & w & $\mathbf{w}$ & w & w & w \\
\hline Residential/Commercial ................... & w & w & w & $w$ & w & w & w & w & $\mathbf{w}$ \\
\hline Consumer Stocks Total ......................... & w & w & w & w & $w$ & w & w & w & w \\
\hline 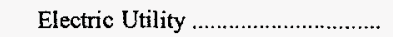 & 509 & 511 & 517 & 721 & 735 & 851 & -.5 & -8.8 & -5.6 \\
\hline 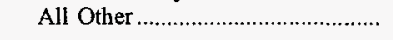 & w & w & w & w & w & w & $\mathbf{w}$ & w & w \\
\hline \multicolumn{10}{|c|}{ Coal Prices (nominal dollars per short ton) } \\
\hline 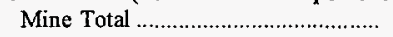 & $\$ 9.96$ & $\$ 9.62$ & $\$ 10.39$ & $\$ 11.05$ & $\$ 10.20$ & $\$ 12.43$ & 3.5 & -.6 & -2.4 \\
\hline 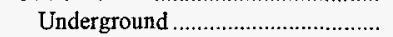 & w & $\mathbf{w}$ & - & w & - & - & - & - & - \\
\hline 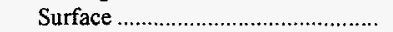 & w & $w$ & 10.39 & w & 10.20 & 12.43 & $\mathbf{w}$ & $w$ & $w$ \\
\hline \multicolumn{10}{|l|}{ Consumer } \\
\hline 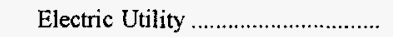 & $\$ 11.90$ & $\$ 11.47$ & 11.79 & $\$ 11.78$ & 12.14 & 11.12 & 3.8 & -.5 & .7 \\
\hline 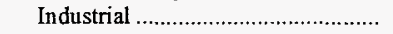 & w & $w$ & w & w & $\mathbf{w}$ & w & $\mathbf{w}$ & w & w \\
\hline
\end{tabular}

1 For 1987, the Form EIA-7A solicited data on "Daily Productive Capacity." To obtain annual productive capacity for a mine in 1987, each mine's daily productive capacity was multiplied by the number of days worked during the year.

2 Capacity utilization (percent) is the ratio of total production to annual productive capacity as reported by mining companies on Form EIA-7A. Productivity (short tons per miner per hour) is calculated by dividing total coal production by the total direct labor hours worked by all employees engaged in production, preparation, processing, development, maintenance, repair, and shop or yard work at mining operations.

3 Includes Mexico.

w Witheld to avoid disclosure of individual company data.

NM Not meaningful as value is greater than 500 percent.

NA Not available.

Notes: Excludes silt, culm, refuse bank, slurry dam, and dredge operations except for Pennsylvania anthracite. Consumption Total does not include coal consumed by independent power producers. Totals may not equal sum of components due to independent rounding.

Sources: Energy Information Administration, Form EIA-3, "Quarterly Coal Consumption Report - Manufacturing Plants"; Form EIA-5, "Coke Plant Report : Quarterly"; Form EIA-6, "Coal Distribution Report"; Form EIA-7A, "Coal Production Report"; Form EIA-759, "Monthly Power Plant Report"; and U.S. Department of Commerce, Bureau of the Census, "Monthly Report EM 545" and "Monthly Report IM 145." 
Table A8. New Mexico Coal Statistics, 1987, 1992-1996

\begin{tabular}{|c|c|c|c|c|c|c|c|c|c|}
\hline \multirow{2}{*}{ Category } & \multirow{2}{*}{1996} & \multirow{2}{*}{1995} & \multirow{2}{*}{1994} & \multirow{2}{*}{1993} & \multirow{2}{*}{1992} & \multirow{2}{*}{1987} & \multirow{2}{*}{$\begin{array}{c}\text { Percent } \\
\text { Change } \\
1995-1996\end{array}$} & \multicolumn{2}{|c|}{$\begin{array}{l}\text { Average Annual } \\
\text { Percent Change }\end{array}$} \\
\hline & & & & & & & & 1992-1996 & $1987-1996$ \\
\hline \multicolumn{10}{|l|}{ Supply (thousand short tons) } \\
\hline Recoverable Reserves............................. & $1,436,359$ & $1,479,956$ & $1,457,523$ & $1,472,927$ & $1,494,541$ & $1,557,487$ & -2.9 & -1.0 & -0.9 \\
\hline Productive Capacity 1 ........................... & 32,695 & 32,760 & 32,807 & 33,360 & 29,512 & 33,295 & -.2 & 2.6 & -.2 \\
\hline 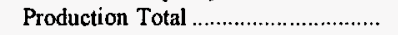 & 24,067 & 26,813 & 28,041 & 28,268 & 24,549 & 19,131 & -10.2 & -.5 & 2.6 \\
\hline 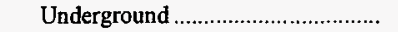 & - & 640 & 950 & 719 & 93 & 620 & -100.0 & - & - \\
\hline 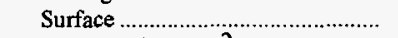 & 24,067 & 26,173 & 27,091 & 27,549 & 24,456 & 18,512 & -8.0 & -.4 & 2.9 \\
\hline Capacity Utilization ${ }^{2} \ldots \ldots \ldots \ldots \ldots \ldots \ldots$ & 73.61 & 81.85 & 85.47 & 84.74 & 83.18 & 57.46 & -10.1 & -3.0 & 2.8 \\
\hline Ratio of Recoverable & & & & & & & & & \\
\hline Reserves to Production ..................... & 59.7 & 55.2 & 52.0 & 52.1 & 60.9 & 81.4 & 8.1 & -.5 & -3.4 \\
\hline 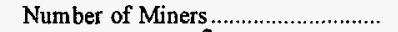 & 1,347 & 1,747 & 1,786 & 1,762 & 1,683 & 1,658 & -22.9 & -5.4 & -2.3 \\
\hline 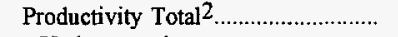 & 8.45 & 6.92 & 6.77 & 6.68 & 6.68 & 5.74 & 22.1 & 6.0 & 4.4 \\
\hline 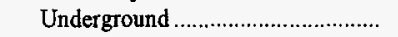 & - & 2.68 & 2.57 & 1.63 & .40 & 1.74 & -100.0 & - & - \\
\hline Surface & 8.45 & 7.19 & 7.18 & 7.26 & 7.11 & 6.23 & 17.4 & 4.4 & 3.4 \\
\hline Producer/Distributor Stocks .............. & 1,890 & 2,015 & 1,467 & 2,343 & 1,648 & - & -6.2 & 3.5 & - \\
\hline \multicolumn{10}{|l|}{ Distribution (thousand short tons) } \\
\hline 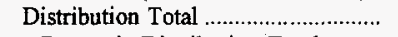 & 25,043 & 26,154 & 28,570 & 27,942 & 24,827 & NA & -4.3 & .2 & NA \\
\hline Domestic Distribution Total ........... & 25,035 & 25,640 & 28,540 & 27,942 & 24,823 & NA & -2.4 & .2 & NA \\
\hline 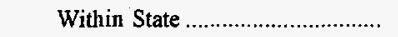 & 15,009 & 14,630 & 15,464 & 14,938 & 14,829 & $\mathrm{NA}$ & 2.6 & 3 & NA \\
\hline To Other States .................................. & 10,026 & 11,010 & 13,076 & 13,004 & 9,993 & NA & -8.9 & .1 & NA \\
\hline Foreign Distribution Total............. & 9 & 514 & 30 & - & 5 & NA & -98.3 & 16.5 & NA \\
\hline 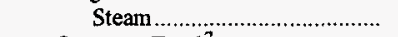 & 9 & 514 & 30 & - & 5 & NA & -98.3 & 16.5 & NA \\
\hline Overseas Total ${ }^{3}$ & 9 & 514 & 30 & - & 5 & NA & -98.3 & 16.5 & NA \\
\hline Steam & 9 & 514 & 30 & - & 5 & NA & -98.3 & 16.5 & NA \\
\hline \multicolumn{10}{|l|}{ Demand (thousand short tons) } \\
\hline Consumption Total & 15,297 & 15,221 & 15,374 & 15,012 & 14,832 & 14,395 & .5 & .8 & .7 \\
\hline 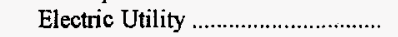 & 15,215 & 15,137 & 15,297 & 14,942 & 14,775 & 14,340 & .5 & .7 & .6 \\
\hline 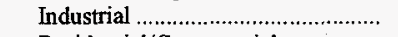 & $w$ & w & $w$ & $w$ & $w$ & w & $\mathbf{w}$ & $w$ & w \\
\hline Residential/Commercial................... & $w$ & $\mathbf{w}$ & $\mathbf{w}$ & $w$ & $w$ & $w$ & $w$ & $w$ & $w$ \\
\hline Consumer Stocks Total ...................... & $\mathbf{w}$ & $\mathbf{w}$ & $\mathbf{w}$ & w & w & $\mathbf{w}$ & w & w & $\mathbf{w}$ \\
\hline 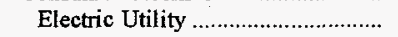 & 814 & 967 & 1,462 & 1,506 & 1,570 & 1,246 & -15.8 & -15.1 & -4.6 \\
\hline 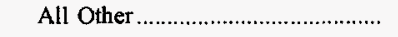 & w & w & w & w & w & w & w & w & w \\
\hline \multicolumn{10}{|c|}{ Coal Prices (nominal dollars per short ton) } \\
\hline Mine Total & $\$ 24.66$ & $\$ 23.80$ & $\$ 23.29$ & $\$ 22.96$ & $\$ 23.14$ & $\$ 21.78$ & 3.6 & 1.6 & 1.4 \\
\hline 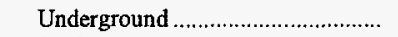 & - & $w$ & w & w & w & $w$ & $w$ & $w$ & $w$ \\
\hline 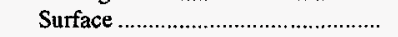 & 24.66 & $\mathbf{w}$ & $\mathbf{w}$ & w & $w$ & w & w & w & w \\
\hline \multicolumn{10}{|l|}{ Consumer } \\
\hline 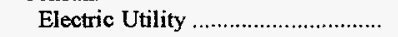 & 26.04 & $\$ 25.59$ & $\$ 25.48$ & $\$ 24.61$ & $\$ 23.83$ & $\$ 21.87$ & 1.8 & 2.2 & 2.0 \\
\hline 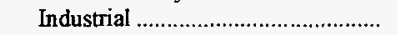 & $\mathbf{w}$ & w & w & $\mathbf{w}$ & w & w & $\mathbf{w}$ & $\mathbf{w}$ & $w$ \\
\hline
\end{tabular}

1 For 1987, the Form EIA-7A solicited data on "Daily Productive Capacity." To obtain annual productive capacity for a mine in 1987, each mine's daily productive capacity was multiplied by the number of days worked during the year.

2 Capacity utilization (percent) is the ratio of total production to annual productive capacity as reported by mining companies on Form EIA-7A. Productivity (short tons per miner per hour) is calculated by dividing total coal production by the total direct labor hours worked by all employees engaged in production, preparation, processing, development, maintenance, repair, and shop or yard work at mining operations.

3 Includes Mexico.

W Withheld to avoid disclosure of individual company data.

NA Not available.

Notes: Excludes silt, culm, refuse bank, slurry dam, and dredge operations except for Pennsylvania anthracite. Consumption Total does not include coal consumed by independent power producers. Totals may not equal sum of components due to independent rounding.

Sources: Energy Information Administration, Form ElA-3, "Quarterly Coal Consumption Report - Manufacturing Plants"; Form ElA-5, "Coke Plant Report - Quarterly"; Form EIA-6, "Coal Distribution Report"; Form EIA-7A, "Coal Production Report"; Form EIA-759, "Monthly Power Plant Report"; and U.S. Department of Commerce, Bureau of the Census, "Monthly Report EM 545" and "Monthly Report IM 145." 
Table A9. North Dakota Coal Statistics, 1987, 1992-1996

\begin{tabular}{|c|c|c|c|c|c|c|c|c|c|}
\hline \multirow{2}{*}{ Category } & \multirow{2}{*}{1996} & \multirow{2}{*}{1995} & \multirow{2}{*}{1994} & \multirow{2}{*}{1993} & \multirow{2}{*}{1992} & \multirow{2}{*}{1987} & \multirow{2}{*}{$\begin{array}{c}\text { Percent } \\
\text { Change } \\
\text { 1995-1996 }\end{array}$} & \multicolumn{2}{|c|}{$\begin{array}{l}\text { Average Annual } \\
\text { Percent Change }\end{array}$} \\
\hline & & & & & & & & $1992-1996$ & $1987-1996$ \\
\hline \multicolumn{10}{|l|}{ Supply (thousand short tons) } \\
\hline Recoverable Reserves.............. & $1,301,400$ & $1,667,596$ & $1,694,548$ & $1,411,026$ & $1,335,164$ & $1,384,343$ & -21.9 & -0.6 & -0.7 \\
\hline 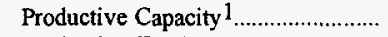 & 32,184 & 34,464 & 35,920 & 36,371 & 36,986 & 39,166 & -6.6 & -3.4 & -2.1 \\
\hline 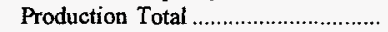 & 29,861 & 30,112 & 32,286 & 31,973 & 31,744 & 25,142 & -.8 & -1.5 & 1.9 \\
\hline Surface & 29,861 & 30,112 & 32,286 & 31,973 & 31,744 & 25,142 & -.8 & -1.5 & 1.9 \\
\hline Capacity Utilization ${ }^{2} \ldots \ldots \ldots \ldots \ldots \ldots \ldots \ldots$ & 92.78 & 87.37 & 89.88 & 87.89 & 85.83 & 64.19 & 6.2 & 2.0 & 4.2 \\
\hline Ratio of Recoverable & & & & & & & & & \\
\hline Reserves to Production ...................... & 43.6 & 55.4 & 52.5 & 44.1 & 42.1 & 55.1 & -21.3 & .9 & -2.6 \\
\hline 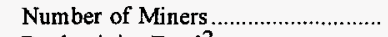 & 640 & 716 & 645 & 782 & 744 & 961 & -10.6 & -3.7 & -4.4 \\
\hline 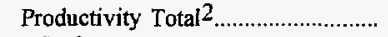 & 17.20 & 16.80 & 18.84 & 17.66 & 18.12 & 13.46 & 2.4 & -1.3 & 2.8 \\
\hline 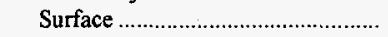 & 17.20 & 16.80 & 18.84 & 17.66 & 18.12 & 13.46 & 2.4 & -1.3 & 2.8 \\
\hline Producer/Distributor Stocks .............. & 1,574 & 1,797 & 1,812 & 1,607 & 1,614 & - & -12.4 & -.6 & - \\
\hline \multicolumn{10}{|l|}{ Distribution (thousand short tons) } \\
\hline 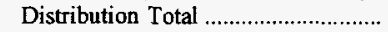 & 30,025 & 30,118 & 32,056 & 32,372 & 31,702 & NA & -.3 & -1.3 & NA \\
\hline Domestic Distribution Total........... & 30,025 & 30,118 & 32,056 & 32,372 & 31,702 & NA & -.3 & -1.3 & NA \\
\hline Within State .................................. & 30,025 & 28,838 & 29,731 & 30,215 & 29,573 & NA & 4.1 & .4 & NA \\
\hline 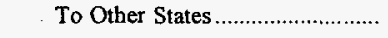 & - & 1,281 & 2,325 & 2,157 & 2,129 & NA & -100.0 & - & NA \\
\hline \multicolumn{10}{|l|}{ Demand (thousand short tons) } \\
\hline 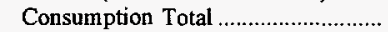 & 30,511 & 30,237 & 30,363 & 30,302 & 30,301 & 24,101 & .9 & .2 & 2.6 \\
\hline 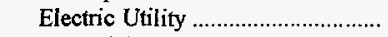 & 23,640 & 22,680 & 23,248 & 23,290 & 23,192 & 17,434 & 4.2 & .5 & 3.4 \\
\hline 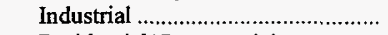 & w & w & w & w & w & $w$ & w & w & w \\
\hline Residential/Commercial .................... & $w$ & w & $\mathbf{w}$ & $w$ & w & w & w & $w$ & $\mathbf{w}$ \\
\hline Consumer Stocks Total ....................... & w & w & $\mathbf{w}$ & w & w & w & $\mathbf{w}$ & w & $\mathbf{w}$ \\
\hline 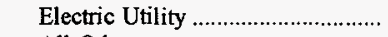 & 1,642 & 1,858 & 2,406 & 2,417 & 2,194 & 3,586 & -11.6 & -7.0 & -8.3 \\
\hline All Other & w & w & w & w & $\mathbf{w}$ & $\mathbf{w}$ & $\mathbf{w}$ & $\mathbf{w}$ & w \\
\hline \multicolumn{10}{|c|}{ Coal Prices (nominal dollars per short ton) } \\
\hline Mine Total & $\$ 8.01$ & $\$ 7.99$ & $\$ 7.62$ & $\$ 7.63$ & $\$ 7.48$ & $\$ 7.91$ & .2 & 1.7 & .1 \\
\hline Surface & 8.01 & 7.99 & 7.62 & 7.63 & 7.48 & 7.91 & .2 & 1.7 & .1 \\
\hline \multicolumn{10}{|l|}{ Consumer } \\
\hline 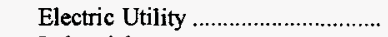 & 9.72 & 9.65 & 9.28 & 9.38 & 9.45 & 10.46 & .7 & .7 & -.8 \\
\hline 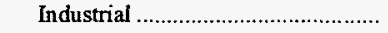 & w & w & w & $\mathbf{w}$ & w & w & $w$ & w & $w$ \\
\hline
\end{tabular}

1 For 1987, the Form EIA-7A solicited data on "Daily Productive Capacity." To obtain annual productive capacity for a mine in 1987, each mine's daily productive capacity was multiplied by the number of days worked during the year.

2 Capacity utilization (percent) is the ratio of total production to annual productive capacity as reported by mining companies on Form EIA-7A. Productivity (short tons per miner per hour) is calculated by dividing total coal production by the total direct labor hours worked by all employees engaged in production, preparation, processing, development, maintenance, repair, and shop or yard work at mining operations.

witheld to avoid disclosure of individual company data.

NA Not available.

Notes: Excludes silt, culm, refuse bank, slurry dam, and dredge operations except for Pennsylvania anthracite. Consumption Total does not include coal consumed by independent power producers. Totals may not equal sum of components due to independent rounding.

Sources: Energy Information Administration, Form EIA-3, "Quarterly Coal Consumption Report - Manufacturing Plants"; Form EIA-5, "Coke Plant Report - Quarterly"; Form EIA-6, "Coal Distribution Report"; Form EIA-7A, "Coal Production Report"; Form EIA-759, "Monthly Power Plant Report"; and U.S. Department of Commerce, Bureau of the Census, "Monthly Report EM 545" and "Monthly Report IM 145." 
Table A10. Ohio Coal Statistics, 1987, 1992-1996

\begin{tabular}{|c|c|c|c|c|c|c|c|c|c|}
\hline \multirow{2}{*}{ Category } & \multirow{2}{*}{1996} & \multirow{2}{*}{1995} & \multirow{2}{*}{1994} & \multirow{2}{*}{1993} & \multirow{2}{*}{1992} & \multirow{2}{*}{1987} & \multirow{2}{*}{$\begin{array}{c}\text { Percent } \\
\text { Change } \\
1995-1996\end{array}$} & \multicolumn{2}{|c|}{$\begin{array}{l}\text { Average Annual } \\
\text { Percent Change }\end{array}$} \\
\hline & & & & & & & & 1992-1996 & $1987-1996$ \\
\hline \multicolumn{10}{|l|}{ Supply (thousand short tons) } \\
\hline 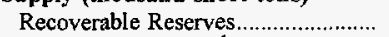 & 414,759 & 467,984 & 479,235 & 519,768 & 575,973 & 754,690 & -11.4 & -7.9 & -6.4 \\
\hline 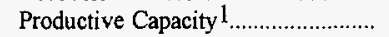 & 37,584 & 34,011 & 43,925 & 42,236 & 41,329 & 46,337 & 10.5 & -2.3 & -2.3 \\
\hline 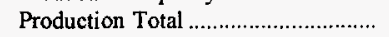 & 28,572 & 26,118 & 29,897 & 28,816 & 30,403 & 35,788 & 9.4 & -1.5 & -2.5 \\
\hline 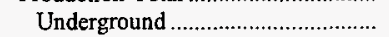 & 15,912 & 13,077 & 13,607 & 10,437 & 12,031 & 12,617 & 21.7 & 7.2 & 2.6 \\
\hline Surface & 12,660 & 13,041 & 16,290 & 18,379 & 18,371 & 23,171 & -2.9 & -8.9 & -6.5 \\
\hline Capacity Utilization ${ }^{2} \ldots \ldots \ldots \ldots \ldots \ldots \ldots \ldots$ & 75.88 & 76.55 & 67.87 & 67.94 & 73.19 & 76.72 & -.9 & .9 & -.1 \\
\hline \multicolumn{10}{|l|}{ Ratio of Recoverable } \\
\hline Reserves to Production........................ & 14.5 & 17.9 & 16.0 & 18.0 & 18.9 & 21.1 & -19.0 & -6.4 & -4.1 \\
\hline 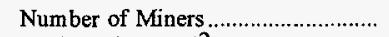 & 3,232 & 3,386 & 3,983 & 3,866 & 4,515 & 7,827 & -4.5 & -8.0 & -9.4 \\
\hline Productivity Total 2 & 3.95 & 3.62 & 3.42 & 3.46 & 3.04 & 2.28 & 9.1 & 6.8 & 6.3 \\
\hline 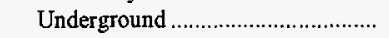 & 4.19 & 3.81 & 3.51 & 3.27 & 3.01 & 1.80 & 9.9 & 8.6 & 9.8 \\
\hline Surface & 3.69 & 3.46 & 3.34 & 3.58 & 3.06 & 2.67 & 6.9 & 4.8 & 3.7 \\
\hline Producer/Distributor Stocks ............... & 532 & 1,374 & 833 & 550 & 1,087 & - & -61.3 & -16.4 & - \\
\hline Imports ${ }^{3}$ & 1 & 1 & 2 & 2 & 13 & - & 3.1 & -42.3 & - \\
\hline \multicolumn{10}{|l|}{ Distribution (thousand short tons) } \\
\hline 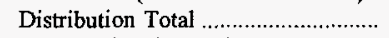 & 28,881 & 24,345 & 28,749 & 28,315 & 29,550 & NA & 18.6 & -.6 & NA \\
\hline Domestic Distribution Total ........... & 28,609 & 24,318 & 28,688 & 28,315 & 29,549 & NA & 17.6 & -.8 & $\mathrm{NA}$ \\
\hline 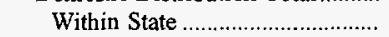 & 24,478 & 20,228 & 23,907 & 24,370 & 26,941 & $\mathrm{NA}$ & 21.0 & -2.4 & $\mathrm{NA}$ \\
\hline 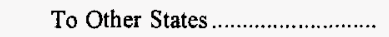 & 4,131 & 4,090 & 4,782 & 3,944 & 2,608 & NA & 1.0 & 12.2 & NA \\
\hline Foreign Distribution Total .............. & 271 & $\therefore \quad 28$ & 61 & - & 2 & $\mathrm{NA}$ & $\mathrm{NM}$ & 257.2 & NA \\
\hline Steam & 271 & 28 & 61 & - & 2 & NA & NM & 257.2 & NA \\
\hline 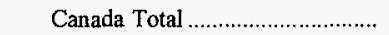 & 3 & 13 & - & - & * & $\mathrm{NA}$ & -78.7 & 60.2 & $\mathrm{NA}$ \\
\hline Steam & 3 & 13 & - & - & $*$ & NA & -78.7 & 60.2 & $\mathrm{NA}$ \\
\hline Overseas Total 4 & 269 & 15 & 61 & - & 1 & NA & $\mathrm{NM}$ & 282.7 & NA \\
\hline Steam & 269 & 15 & 61 & - & 1 & $\mathrm{NA}$ & $\mathrm{NM}$ & 282.7 & NA \\
\hline \multicolumn{10}{|l|}{ Demand (thousand short tons) } \\
\hline 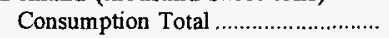 & 59,835 & 515,580 & 56,711 & 59,031 & 58,671 & 59,350 & 5.8 & .5 & .1 \\
\hline 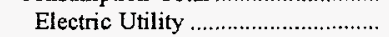 & 53,543 & 49,785 & 49,326 & 51,456 & 50,358 & 47,520 & 7.5 & 1.5 & 1.3 \\
\hline 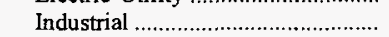 & 3,794 & 3,609 & 3,794 & 4,100 & 3,970 & 5,657 & 5.1 & -1.1 & -4.3 \\
\hline 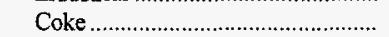 & 1,842 & 2,777 & 3,092 & 2,892 & 3,755 & 5,361 & -33.7 & -16.3 & -11.2 \\
\hline Residential/Commercial ................... & 656 & 409 & 498 & 584 & 588 & 812 & 60.4 & 2.8 & -2.3 \\
\hline 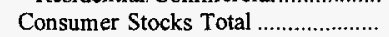 & 5,431 & 5,936 & 7,815 & 7,630 & 10,804 & 11,164 & -8.5 & -15.8 & -7.7 \\
\hline Electric Utility & 5,232 & 5,661 & 7,499 & 7,249 & 10,395 & 9,959 & -7.6 & -15.8 & -6.9 \\
\hline 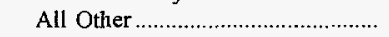 & 199 & 275 & 316 & 381 & 408 & 1,205 & -27.6 & -16.4 & -18.1 \\
\hline \multicolumn{10}{|c|}{ Coal Prices (nominal dollars per short ton) } \\
\hline Mine Total & $\$ 24.85$ & $\$ 25.97$ & $\$ 29.13$ & $\$ 28.04$ & $\$ 26.93$ & $\$ 30.80$ & -4.3 & -2.0 & -2.3 \\
\hline 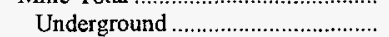 & 25.98 & 28.98 & 31.61 & 30.73 & 30.11 & 37.20 & -10.4 & -3.6 & -3.9 \\
\hline Surface & 23.43 & 22.92 & 27.04 & 26.51 & 24.84 & 27.28 & 2.2 & -1.4 & -1.7 \\
\hline \multicolumn{10}{|l|}{ Consumer } \\
\hline 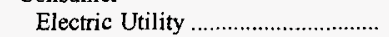 & 32.31 & 34.44 & 34.70 & 34.05 & 34.40 & 37.15 & -6.2 & -1.5 & -1.5 \\
\hline Industrial & 35.28 & 35.18 & 35.75 & 34.82 & 35.24 & 33.98 & .3 & $*$ & .4 \\
\hline Coke & 44.98 & 42.18 & 42.02 & 45.07 & 46.68 & 46.04 & 6.6 & -.9 & -.3 \\
\hline
\end{tabular}

1 For 1987, the Form EIA-7A solicited data on "Daily Productive Capacity." To obtain annual productive capacity for a mine in 1987, each mine's daily productive capacity was multiplied by the number of days worked during the year.

2 Capacity utilization (percent) is the ratio of total production to annual productive capacity as reported by mining companies on Form EIA-7A. Productiv ity (short tons per miner per hour) is calculated by dividing total coal production by the total direct labor hours worked by all employees engaged in production, preparation, processing, development, maintenance, repair, and shop or yard work at mining operations.

3 Imports for 1992 through 1996 include imports to electric utilities, manufacturing plants and coke plants. Imports for 1987 include only imports to electric utilities.

4 Includes Mexico.

* Data round to zero.

NM Not meaningful as value is greater than 500 percent.

NA Not available.

Notes: Excludes silt, culm, refuse bank, slurry dam, and dredge operations except for Pennsylvania anthracite. Totals may not equal sum of components due to independent rounding.

Sources: Energy Information Administration, Form ElA-3, "Quarterly Coal Consumption Report - Manufacturing Plants"; Form ElA-5, "Coke Plant Report - Quarterly"; Form EIA-6, "Coal Distribution Report"; Form EIA-7A, "Coal Production Report"; Form EIA-759, "Monthly Power Plant Report"; and U.S. Department of Commerce, Bureau of the Census, "Monthly Report EM 545" and "Monthly Report IM 145." 
Table A11. Pennsylvania Coal Statistics, 1987, 1992-1996

\begin{tabular}{|c|c|c|c|c|c|c|c|c|c|}
\hline \multirow{2}{*}{ Category } & \multirow{2}{*}{1996} & \multirow{2}{*}{1995} & \multirow{2}{*}{1994} & \multirow{2}{*}{1993} & \multirow{2}{*}{1992} & \multirow{2}{*}{1987} & \multirow{2}{*}{$\begin{array}{c}\text { Percent } \\
\text { Change } \\
1995-1996\end{array}$} & \multicolumn{2}{|c|}{$\begin{array}{l}\text { Average Annual } \\
\text { Percent Change }\end{array}$} \\
\hline & & & & & & & & 1992-1996 & $1987-1996$ \\
\hline \multicolumn{10}{|l|}{ Supply (thousand short tons) } \\
\hline Recoverable Reserves.......................... & 796,035 & 736,601 & 912,870 & 939,706 & 937,427 & $1,368,540$ & 8.1 & -4.0 & -5.8 \\
\hline Productive Capacity 1 ............................ & 81,684 & 77,187 & 80,975 & 82,148 & 82,968 & 86,765 & 5.8 & -4 & -.7 \\
\hline 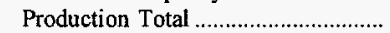 & 67,942 & 61,576 & 62,237 & 59,700 & 68,981 & 70,423 & 10.3 & -.4 & -.4 \\
\hline 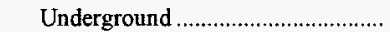 & 47,247 & 41,409 & 39,974 & 36,934 & 45,112 & 38,450 & 14.1 & 1.2 & 2.3 \\
\hline Surface & 20,694 & 20,167 & 22,263 & 22,766 & 23,868 & 31,974 & 2.6 & -3.5 & -4.7 \\
\hline $\begin{array}{l}\text { Capacity Utilization } 2 \\
\text { Ratio of Recoverable }\end{array}$ & 82.53 & 78.81 & 75.89 & 71.79 & 82.20 & 80.04 & 4.7 & .1 & .3 \\
\hline Reserves to Production....................... & 11.7 & 12.0 & 14.7 & 15.7 & 13.6 & 19.4 & -2.0 & -3.6 & -5.5 \\
\hline 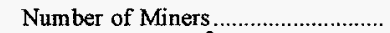 & 9,021 & 8,968 & 9,975 & 10,940 & 12,659 & 17,287 & .6 & -8.1 & -7.0 \\
\hline Productivity Total ${ }^{2} \ldots \ldots \ldots \ldots \ldots \ldots \ldots \ldots \ldots$ & 3.36 & 3.23 & 2.98 & 2.80 & 2.67 & 1.97 & 4.1 & 5.9 & 6.1 \\
\hline 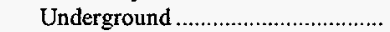 & 3.74 & 3.49 & 3.18 & 2.91 & 2.81 & 1.81 & 7.1 & 7.4 & 8.4 \\
\hline Surface & 2.72 & 2.79 & 2.67 & 2.63 & 2.45 & 2.23 & -2.4 & 2.6 & 2.3 \\
\hline Producer/Distributor Stocks ............... & 3,113 & 2,487 & 2,787 & 1,826 & 2,903 & - & 25.2 & 1.8 & - \\
\hline Imports $^{3} \ldots \ldots \ldots$ & 80 & 87 & - & - & - & - & -7.7 & - & - \\
\hline \multicolumn{10}{|l|}{ Distribution (thousand short tons) } \\
\hline 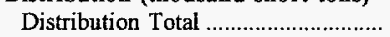 & 69,128 & 62,240 & 61,508 & 58,990 & 67,649 & $\mathrm{NA}$ & 11.1 & .5 & NA \\
\hline Domestic Distribution Total ........... & 59,882 & 53,961 & 55,207 & 53,482 & 61,208 & NA & 11.0 & -.5 & NA \\
\hline 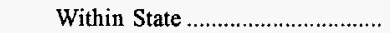 & 39,222 & 36,147 & 35,189 & 33,456 & 37,696 & $\mathrm{NA}$ & 8.5 & 1.0 & $\mathrm{NA}$ \\
\hline 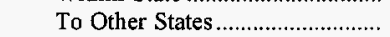 & 20,660 & 17,814 & 20,018 & 20,026 & 23,512 & NA & 16.0 & -3.2 & $\mathrm{NA}$ \\
\hline 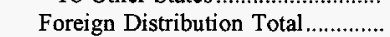 & 9,246 & 8,279 & 6,301 & 5,508 & 6,440 & NA & 11.7 & 9.5 & NA \\
\hline 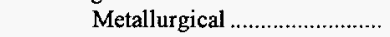 & 1,642 & 1,467 & 1,624 & 1,919 & 1,301 & NA & 11.9 & 6.0 & NA \\
\hline Steam & 7,604 & 6,812 & 4,677 & 3,589 & 5,139 & NA & 11.6 & 10.3 & $\mathrm{NA}$ \\
\hline Canada Total & 1,050 & 713 & 844 & 597 & 1,599 & NA & 47.4 & -10.0 & $\mathrm{NA}$ \\
\hline Metallurgical ............................ & - & 4 & - & 10 & - & NA & -100.0 & - & NA \\
\hline Steam & 1,050 & 708 & 844 & 587 & 1,599 & NA & 48.2 & -10.0 & NA \\
\hline Overseas Total 4 & 8,196 & 7,566 & 5,457 & 4,911 & 4,841 & $\mathrm{NA}$ & 8.3 & 14.1 & $\mathrm{NA}$ \\
\hline 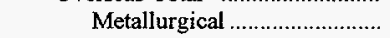 & 1,642 & 1,463 & 1,624 & 1,909 & 1,301 & NA & 12.2 & 6.0 & NA \\
\hline 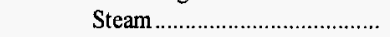 & 6,554 & 6,103 & 3,833 & 3,002 & 3,540 & NA & 7.4 & 16.6 & $\mathrm{NA}$ \\
\hline \multicolumn{10}{|l|}{ Demand (thousand short tons) } \\
\hline Consumption Total & 57,226 & 55,326 & 54,094 & 56,158 & 56,074 & 55,305 & 3.4 & .5 & .4 \\
\hline 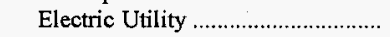 & 41,076 & 39,252 & 38,044 & 40,257 & 40,407 & 41,263 & 4.6 & .4 & * \\
\hline 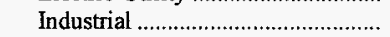 & 4,466 & 4,027 & 4,044 & 4,311 & 4,173 & 4,112 & 10.9 & 1.7 & .9 \\
\hline Coke & 10,689 & 10,858 & 10,849 & 10,333 & 9,868 & 8,395 & -1.5 & 2.0 & 2.7 \\
\hline Residential/Commercial.................. & 995 & 1,188 & 1,156 & 1,257 & 1,626 & 1,535 & -16.3 & -11.6 & -4.7 \\
\hline Consumer Stocks Total ...................... & 8,860 & 10,303 & 12,060 & 12,265 & 15,976 & 16,121 & -14.0 & -13.7 & -6.4 \\
\hline Electric Utility & 7,880 & 9,244 & 11,000 & 11,110 & 14,866 & 15,034 & -14.8 & -14.7 & -6.9 \\
\hline 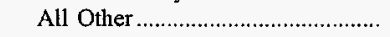 & 980 & 1,059 & 1,060 & 1,155 & 1,110 & 1,087 & -7.5 & -3.1 & -1.1 \\
\hline \multicolumn{10}{|c|}{ Coal Prices (nominal dollars per short ton) } \\
\hline 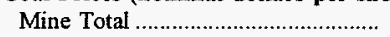 & $\$ 25.78$ & $\$ 26.78$ & $\$ 26.18$ & $\$ 26.50$ & $\$ 28.61$ & $\$ 29.81$ & -3.7 & -2.6 & -1.6 \\
\hline 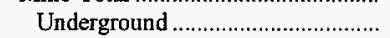 & 25.79 & 27.09 & 26.59 & 27.35 & 30.23 & 32.70 & -4.8 & -3.9 & -2.6 \\
\hline Surface & 25.76 & 26.14 & 25.43 & 25.09 & 25.48 & 26.27 & -1.4 & 3 & -.2 \\
\hline \multicolumn{10}{|l|}{ Consumer } \\
\hline 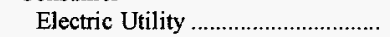 & 34.06 & 33.48 & 35.39 & 35.73 & 36.81 & 35.43 & 1.7 & -1.9 & -.4 \\
\hline 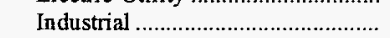 & 33.84 & 34.07 & 33.66 & 34.04 & 35.70 & 36.62 & -.7 & -1.3 & -.9 \\
\hline Coke & 45.16 & 46.11 & 46.25 & 46.41 & 46.49 & 42.54 & -2.0 & -.7 & .7 \\
\hline
\end{tabular}

1 For 1987, the Form EIA-7A solicited data on "Daily Productive Capacity." To obtain annual productive capacity for a mine in 1987, each mine's daily productive capacity was multiplied by the number of days worked during the year.

2 Capacity utilization (percent) is the ratio of total production to annual productive capacity as reported by mining companies on Form EIA-7A. Productivity (short tons per miner per hour) is calculated by dividing total coal production by the total direct labor hours worked by all employees engaged in production, preparation, processing, development, maintenance, repair, and shop or yard work at mining operations.

3 Imports for 1992 through 1996 include imports to electric utilities, manufacturing plants and coke plants. Imports for 1987 include only imports to electric utilities.

4 Includes Mexico

* Data round to zero.

NA Not available.

Notes: Excludes silt, culm, refuse bank, slurry dam, and dredge operations except for Pennsylvania anthracite. Totals may not equal sum of components due to independent rounding.

Sources: Energy Information Administration, Form EIA-3, "Quarterly Coal Consumption Report - Manufacturing Plants"; Form EIA-5, "Coke Plant Report - Quarterly"; Form EIA-6, "Coal Distribution Report"; Form EIA-7A, "Coal Production Report"; Form EIA-759, "Monthly Power Plant Report"; and U.S. Department of Commerce, Bureau of the Census, "Monthly Report EM 545" and "Monthly Report IM 145." 
Table A12. Texas Coal Statistics, 1987, 1992-1996

\begin{tabular}{|c|c|c|c|c|c|c|c|c|c|}
\hline \multirow{2}{*}{ Category } & \multirow{2}{*}{1996} & \multirow{2}{*}{1995} & \multirow{2}{*}{1994} & \multirow{2}{*}{1993} & \multirow{2}{*}{1992} & \multirow{2}{*}{1987} & \multirow{2}{*}{$\begin{array}{c}\text { Percent } \\
\text { Change } \\
\text { 1995-1996 }\end{array}$} & \multicolumn{2}{|c|}{$\begin{array}{l}\text { Average Annual } \\
\text { Percent Change }\end{array}$} \\
\hline & & & & & & & & 1992-1996 & $1987-1996$ \\
\hline \multicolumn{10}{|l|}{ Supply (thousand short tons) } \\
\hline 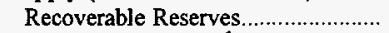 & 878,486 & 939,901 & $1,026,309$ & $1,104,864$ & $1,188,240$ & $1,214,513$ & -6.5 & -7.3 & -3.5 \\
\hline 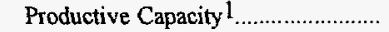 & 59,604 & 54,758 & 55,856 & 57,115 & 58,541 & 51,636 & 8.8 & .4 & 1.6 \\
\hline 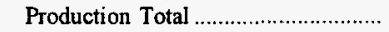 & 55,164 & 52,684 & 52,346 & 54,567 & 55,071 & 50,529 & 4.7 & $*$ & 1.0 \\
\hline Surface & 55,164 & 52,684 & 52,346 & 54,567 & 55,071 & 50,529 & 4.7 & $*$ & 1.0 \\
\hline Capacity Utilization ${ }^{2} \ldots \ldots \ldots \ldots \ldots \ldots \ldots$ & 92.55 & 96.21 & 93.72 & 95.54 & 94.07 & 97.86 & -3.8 & -.4 & -.6 \\
\hline Ratio of Recoverable & & & & & & & & & \\
\hline Reserves to Production ........................ & 15.9 & 17.8 & 19.6 & 20.3 & 21.6 & 24.0 & -10.7 & -7.3 & -4.5 \\
\hline Number of Miners & 1,550 & 1,590 & 1,733 & 1,841 & 2,001 & 3,319 & -2.5 & -6.2 & -8.1 \\
\hline 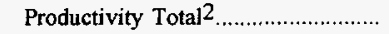 & 10.13 & 9.10 & 8.82 & 8.42 & 7.34 & 6.45 & 11.3 & 8.4 & 5.1 \\
\hline 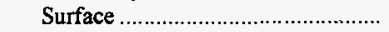 & 10.13 & 9.10 & 8.82 & 8.42 & 7.34 & 6.45 & 11.3 & 8.4 & 5.1 \\
\hline Producer/Distributor Stocks .............. & 1,254 & 864 & 1,430 & 1,237 & 543 & - & 45.1 & 23.3 & - \\
\hline Imports $^{3}$ & 16 & - & 153 & 156 & 80 & 94 & - & -33.5 & -18.1 \\
\hline \multicolumn{10}{|l|}{ Distribution (thousand short tons) } \\
\hline 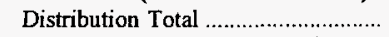 & 49,655 & 52,832 & 52,256 & 54,224 & 54,447 & NA & -6.0 & -2.3 & NA \\
\hline Domestic Distribution Total........... & 49,538 & 52,812 & 52,256 & 54,224 & 54,447 & $\mathrm{NA}$ & -6.2 & -2.3 & NA \\
\hline Within State & 49,538 & 52,812 & 52,256 & 54,224 & 54,447 & NA & -6.2 & -2.3 & NA \\
\hline Foreign Distribution Total.............. & 117 & 20 & - & - & - & NA & 494.0 & - & NA \\
\hline 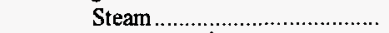 & 117 & 20 & - & - & - & NA & 494.0 & - & NA \\
\hline 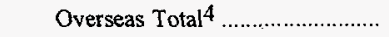 & 117 & 20 & - & - & - & NA & 494.0 & - & NA \\
\hline Stearn & 117 & 20 & - & - & - & NA & 494.0 & - & NA \\
\hline \multicolumn{10}{|l|}{ Demand (thousand short tons) } \\
\hline 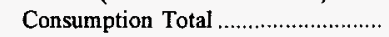 & 98,997 & 92,612 & 93,829 & 96,809 & 91,568 & 82,915 & 6.9 & 2.0 & 2.0 \\
\hline 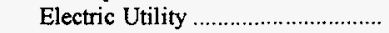 & 94,189 & 88,358 & 88,479 & 92,135 & 87,333 & 78,802 & 6.6 & 1.9 & 2.0 \\
\hline 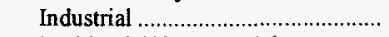 & 4,808 & 4,255 & 5,350 & 4,667 & 4,225 & 4,083 & 13.0 & 3.3 & 1.8 \\
\hline Residential/Commercial................... & - & - & * & 6 & $w$ & $w$ & - & $w$ & $w$ \\
\hline Consumer Stocks Total ........................ & 10,481 & 10,829 & 9,793 & 9,172 & w & $\mathbf{w}$ & -3.2 & $\mathbf{w}$ & $\mathbf{w}$ \\
\hline 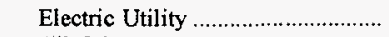 & 10,290 & 10,628 & 9,578 & 8,125 & 10,143 & 8,992 & -3.2 & .4 & 1.5 \\
\hline 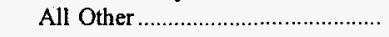 & 190 & 201 & 215 & 1,047 & $w$ & w & -5.6 & $w$ & $\mathbf{w}$ \\
\hline \multicolumn{10}{|c|}{ Coal Prices (nominal dollars per short ton) } \\
\hline 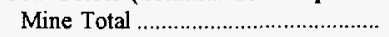 & $\$ 12.17$ & $\$ 12.16$ & $\$ 12.38$ & $\$ 12.87$ & $\$ 12.42$ & $\$ 12.10$ & * & -.5 & .1 \\
\hline 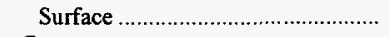 & 12.17 & 12.16 & 12.38 & 12.87 & 12.42 & 12.10 & $*$ & -.5 & .1 \\
\hline \multicolumn{10}{|l|}{ Consumer } \\
\hline Electric Utility ................................... & 19.26 & 19.65 & 19.84 & 20.91 & 21.58 & 22.48 & -1.9 & -2.8 & -1.7 \\
\hline 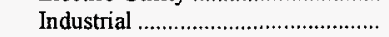 & 18.99 & 18.76 & 19.54 & 17.58 & 18.14 & 19.19 & 1.2 & 1.1 & -.1 \\
\hline
\end{tabular}

1 For 1987, the Form EIA-7A solicited data on "Daily Productive Capacity." To obtain annual productive capacity for a mine in 1987, each mine's daily productive capacity was multiplied by the number of days worked during the year.

2 Capacity utilization (percent) is the ratio of total production to annual productive capacity as reported by mining companies on Form EIA-7A. Productivity (short tons per miner per hour) is calculated by dividing total coal production by the total direct labor hours worked by all employees engaged in production, preparation, processing, development, maintenance, repair, and shop or yard work at mining operations.

3 Imports for 1992 through 1996 include imports to electric utilities, manufacturing plants and coke plants. Imports for 1987 include only imports to electric utilities.

4 Includes Mexico.

- Data round to zero.

Witheld to avoid disclosure of individual company data.

NA Not available.

Notes: Excludes silt, culm, refuse bank, slurry dam, and dredge operations except for Pennsylvania anthracite. Totals may not equal sum of components due to independent rounding.

Sources: Energy Information Administration, Form EIA-3, "Quarterly Coal Consumption Report - Manufacturing Plants"; Form EIA-5, "Coke Plant Report - Quarterly"; Form EIA-6, "Coal Distribution Report"; Form EIA-7A, "Coal Production Report"; Form EIA-759, "Monthly Power Plant Report"; and U.S. Department of Commerce, Bureau of the Census, "Monthly Report EM S45" and "Monthly Report IM 145." 


\begin{tabular}{|c|c|c|c|c|c|c|c|c|c|}
\hline \multirow{2}{*}{ Category } & \multirow{2}{*}{1996} & \multirow{2}{*}{1995} & \multirow{2}{*}{1994} & \multirow{2}{*}{1993} & \multirow{2}{*}{1992} & \multirow{2}{*}{1987} & \multirow{2}{*}{$\begin{array}{c}\text { Percent } \\
\text { Change } \\
1995-1996\end{array}$} & \multicolumn{2}{|c|}{$\begin{array}{l}\text { Average Annual } \\
\text { Percent Change }\end{array}$} \\
\hline & & & & & & & & 1992-1996 & $1987-1996$ \\
\hline \multicolumn{10}{|l|}{ Supply (thousand short tons) } \\
\hline Recoverable Reserves................ & 284,433 & 374,750 & 423,349 & 446,729 & 488,109 & 489,634 & -24.1 & -12.6 & -5.8 \\
\hline Productive Capacity 1 ............................ & 30,230 & 30,888 & 27,640 & 25,933 & 25,534 & 22,976 & -2.1 & 4.3 & 3.1 \\
\hline 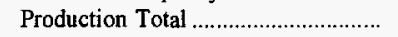 & 27,507 & 25,167 & 24,399 & 21,847 & 21,339 & 16,508 & 9.3 & 6.5 & 5.8 \\
\hline 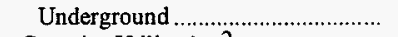 & 27,507 & 25,167 & 24,399 & 21,847 & 21,339 & 16,508 & 9.3 & 6.5 & 5.8 \\
\hline Capacity Utilization $2 \ldots \ldots \ldots \ldots \ldots \ldots \ldots$ & 90.97 & 81.48 & 88.27 & 84.22 & 83.57 & 71.85 & 11.6 & 2.1 & 2.6 \\
\hline \multicolumn{10}{|l|}{ Ratio of Recoverable } \\
\hline Reserves to Production ......................... & 10.3 & 14.9 & 17.3 & 20.4 & 22.9 & 29.7 & -30.5 & -18.0 & -11.0 \\
\hline Number of Miners & 1,804 & 1,893 & 1,675 & 1,769 & 1,997 & 2,544 & -4.7 & -2.5 & -3.7 \\
\hline Productivity Total $2 \ldots \ldots \ldots \ldots \ldots \ldots \ldots \ldots$ & 7.23 & 7.02 & 6.59 & 5.96 & 5.46 & 3.86 & 3.0 & 7.2 & 7.2 \\
\hline 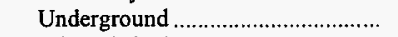 & 7.24 & 7.02 & 6.59 & 5.96 & 5.46 & 3.86 & 3.1 & 7.3 & 7.2 \\
\hline Producer/Distributor Stocks .............. & 1,337 & 1,946 & 1,301 & 1,203 & 1,827 & - & -31.3 & -7.5 & - \\
\hline \multicolumn{10}{|l|}{ Distribution (thousand short tons) } \\
\hline 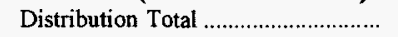 & 23,868 & 25,521 & 23,225 & 22,243 & 21,052 & NA & -6.5 & 3.2 & NA \\
\hline Domestic Distribution Total .......... & 18,563 & 21,591 & 20,527 & 19,283 & 18,792 & NA & -14.0 & -.3 & NA \\
\hline 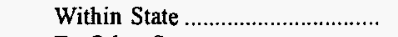 & 9,389 & 12,755 & 13,586 & 13,418 & 13,035 & NA & -26.4 & -7.9 & NA \\
\hline 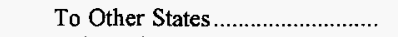 & 9,174 & 8,836 & 6,941 & 5,866 & 5,758 & $\mathrm{NA}$ & 3.8 & 12.3 & NA \\
\hline Foreign Distribution Total .............. & 5,305 & 3,930 & 2,698 & 2,959 & 2,260 & NA & 35.0 & 23.8 & NA \\
\hline Metallurgical ............................ & 187 & - & - & - & - & NA & - & - & NA \\
\hline Steam & 5,118 & 3,930 & 2,698 & 2,959 & 2,260 & NA & 30.2 & 22.7 & $\mathrm{NA}$ \\
\hline 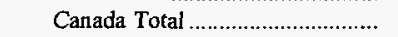 & - & - & - & 346 & - & NA & - & - & NA \\
\hline Steam & - & - & - & 346 & - & NA & - & - & NA \\
\hline Overseas Total ${ }^{3}$ & 5,305 & 3,930 & 2,698 & 2,613 & 2,260 & NA & 35.0 & 23.8 & NA \\
\hline 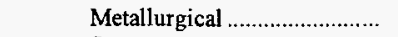 & 187 & - & - & - & - & NA & - & - & NA \\
\hline 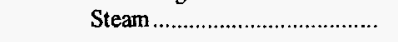 & 5,118 & 3,930 & 2,698 & 2,613 & 2,260 & $\mathrm{NA}$ & 30.2 & 22.7 & $\mathrm{NA}$ \\
\hline \multicolumn{10}{|l|}{ Demand (thousand short tons) } \\
\hline 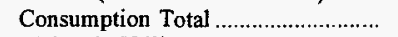 & 15,237 & 15,307 & 16,216 & 15,848 & 15,719 & 11,807 & -.4 & -.8 & 2.9 \\
\hline 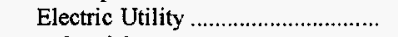 & 13,584 & 13,325 & 14,269 & 13,995 & 13,857 & 11,175 & 1.9 & -.5 & 2.2 \\
\hline 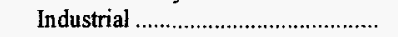 & 512 & 915 & 835 & 727 & 525 & 507 & -44.1 & -.6 & .1 \\
\hline Coke & w & w & $w$ & $w$ & w & w & w & $\mathbf{w}$ & $\mathbf{w}$ \\
\hline Residential/Commercial .................. & $\mathrm{w}$ & $\mathbf{w}$ & w & w & w & w & $\mathbf{w}$ & $\mathbf{w}$ & w \\
\hline 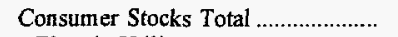 & w & w & w & w & w & w & w & w & w \\
\hline 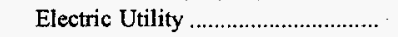 & 1,526 & 2,250 & 2,753 & 3,264 & 3,153 & 3,133 & -32.2 & -16.6 & -7.7 \\
\hline 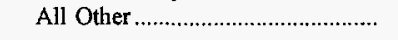 & w & $w$ & $w$ & w & $w$ & w & $\mathbf{w}$ & w & w \\
\hline \multicolumn{10}{|c|}{ Coal Prices (nominal dollars per short ton) } \\
\hline 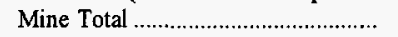 & $\$ 21.63$ & $\$ 19.10$ & $\$ 19.27$ & $\$ 20.81$ & $\$ 21.11$ & $\$ 25.70$ & 13.3 & 6 & -1.9 \\
\hline 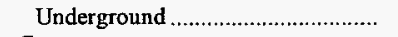 & 21.63 & 19.10 & 19.27 & 20.81 & 21.11 & 25.70 & 13.3 & 6 & -1.9 \\
\hline \multicolumn{10}{|l|}{ Consumer } \\
\hline 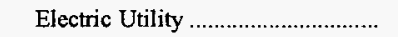 & 24.66 & 25.27 & 26.10 & 27.34 & 27.54 & 29.09 & -2.4 & -2.7 & -1.8 \\
\hline 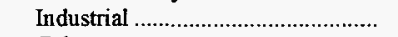 & 19.10 & 19.74 & 26.57 & 26.51 & 25.35 & 23.85 & -3.2 & -6.8 & -2.4 \\
\hline Coke & $w$ & $\mathbf{w}$ & $w$ & w & w & w & w & w & w \\
\hline
\end{tabular}

1 For 1987, the Form EIA-7A solicited data on "Daily Productive Capacity." To obtain annual productive capacity for a mine in 1987, each mine's daily productive capacity was multiplied by the number of days worked during the year.

2 Capacity utilization (percent) is the ratio of total production to annual productive capacity as reported by mining companies on Form EIA-7A. Productivity (short tons per miner per hour) is calculated by dividing total coal production by the total direct labor hours worked by all employees engaged in production, preparation, processing, development, maintenance, repair, and shop or yard work at mining operations.

3 Includes Mexico.

Witheld to avoid disclosure of individual company data.

NA Not available.

Notes: Excludes silt, culm, refuse bank, slurry dam, and dredge operations except for Pennsylyania anthracite. Totals may not equal sum of components due to independent rounding.

Sources: Energy Information Administration, Form EIA-3, "Quarterly Coal Consumption Report - Manufacturing Plants"; Form EIA-5, "Coke Plant Report - Quarterly"; Form EIA-6, "Coal Distribution Report"; Form EIA-7A, "Coal Production Report"; Form EIA-759, "Monthly Power Plant Report"; and U.S. Department of Commerce, Bureau of the Census, "Monthly Report EM 545" and "Monthly Report IM 145." 
Table A14. Virginia Coal Statistics, 1987, 1992-1996

\begin{tabular}{|c|c|c|c|c|c|c|c|c|c|}
\hline \multirow{2}{*}{ Category } & \multirow{2}{*}{1996} & \multirow{2}{*}{1995} & \multirow{2}{*}{1994} & \multirow{2}{*}{1993} & \multirow{2}{*}{1992} & \multirow{2}{*}{1987} & \multirow{2}{*}{$\begin{array}{c}\text { Percent } \\
\text { Change } \\
1995-1996\end{array}$} & \multicolumn{2}{|c|}{$\begin{array}{l}\text { Average Annual } \\
\text { Percent Change }\end{array}$} \\
\hline & & & & & & & & $1992-1996$ & 1987-1996 \\
\hline \multicolumn{10}{|l|}{ Supply (thousand short tons) } \\
\hline Recoverable Reserves......................... & 188,344 & 203,159 & 236,773 & 335,883 & 365,823 & 507,545 & -7.3 & -15.3 & -10.4 \\
\hline Productive Capacity 1 ......................... & 41,593 & 43,037 & 46,462 & 50,879 & 54,471 & 49,676 & -3.3 & -6.5 & -1.9 \\
\hline 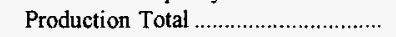 & 35,590 & 34,099 & 37,129 & 39,317 & 43,024 & 44,543 & 4.4 & -4.6 & -2.5 \\
\hline 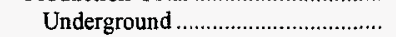 & 25,568 & 25,372 & 28,054 & 30,166 & 34,715 & 36,943 & .8 & -7.4 & -4.0 \\
\hline Surface & 10,022 & 8,727 & 9,075 & 9,151 & 8,308 & 7,599 & 14.8 & 4.8 & 3.1 \\
\hline $\begin{array}{l}\text { Capacity Utilization }{ }^{2} \\
\text { Ratio of Recoverable }\end{array}$ & 85.34 & 79.07 & 79.61 & 77.07 & 78.70 & 88.93 & 7.9 & 2.0 & -.4 \\
\hline Reserves to Production ......................... & 5.3 & 6.0 & 6.4 & 8.5 & 8.5 & 11.4 & -11.2 & -11.2 & -8.2 \\
\hline Number of Miners .................................. & 6,241 & 6,919 & 8,121 & 8,339 & 9,138 & 12,047 & -9.8 & -9.1 & -7.0 \\
\hline Productivity Total 2 ............................ & 2.72 & 2.50 & 2.51 & 2.41 & 2.37 & 2.08 & 8.4 & 3.5 & 3.0 \\
\hline 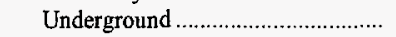 & 2.44 & 2.25 & 2.27 & 2.19 & 2.20 & 2.01 & 8.6 & 2.7 & 2.2 \\
\hline 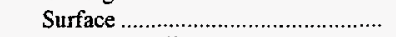 & 3.79 & 3.73 & 3.73 & 3.55 & 3.50 & 2.52 & 1.7 & 2.0 & 4.6 \\
\hline Producer/Distributor Stocks .............. & 1,644 & 1,649 & 1,180 & 1,389 & 1,714 & - & -.3 & -1.0 & - \\
\hline \multicolumn{10}{|l|}{ Distribution (thousand short tons) } \\
\hline 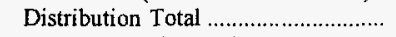 & 36,208 & 34,024 & 38,548 & 41,639 & 45,728 & NA & 6.4 & -5.7 & NA \\
\hline Domestic Distribution Total........... & 22,776 & 24,283 & 26,866 & 27,388 & 28,504 & NA & -6.2 & -5.4 & NA \\
\hline 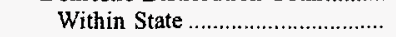 & 7,231 & 5,657 & 6,867 & 6,076 & 6,082 & $\mathrm{NA}$ & 27.8 & 4.4 & NA \\
\hline 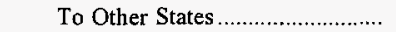 & 15,545 & 18,625 & 19,999 & 21,312 & 22,422 & NA & -16.5 & -8.8 & NA \\
\hline Foreign Distribution Total............. & 13,432 & 9,742 & 11,683 & 14,251 & 17,224 & NA & 37.9 & -6.0 & NA \\
\hline 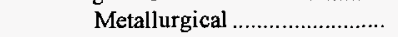 & 12,760 & 8,921 & 11,155 & 13,747 & 14,389 & $\mathrm{NA}$ & 43.0 & -3.0 & NA \\
\hline Steam & 671 & 821 & 527 & 503 & 2,835 & NA & -18.2 & -30.2 & $\mathrm{NA}$ \\
\hline 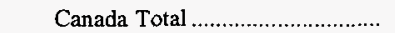 & 387 & 445 & 786 & 1,229 & 1,331 & NA & -13.0 & -26.5 & NA \\
\hline 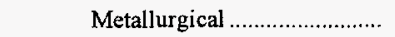 & 387 & 445 & 786 & 1,229 & - & $\mathrm{NA}$ & -13.0 & - & $\mathrm{NA}$ \\
\hline Steam & - & - & - & - & 1,331 & NA & - & - & $\mathrm{NA}$ \\
\hline Overseas Total ${ }^{3}$ & 13,044 & 9,297 & 10,897 & 13,021 & 15,893 & NA & 40.3 & -4.8 & NA \\
\hline 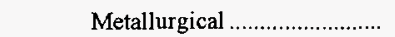 & 12,373 & R 8,475 & R 10,370 & ${ }^{R} 12,518$ & $\mathrm{R}_{14,389}$ & NA & 46.0 & -3.7 & $\mathrm{NA}$ \\
\hline Steam ........................................ & 671 & R 821 & $R_{527}$ & $\mathrm{R}_{503}$ & $R_{1,505}$ & NA & -18.2 & -18.3 & $\mathrm{NA}$ \\
\hline \multicolumn{10}{|l|}{ Demand (thousand short tons) } \\
\hline 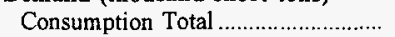 & 14,983 & 13,378 & 12,792 & 13,584 & 13,418 & 13,227 & 12.0 & 2.8 & 1.4 \\
\hline 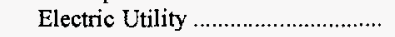 & 10,994 & 9,543 & 8,670 & 9,447 & 8,661 & 8,297 & 15.2 & 6.1 & 3.2 \\
\hline 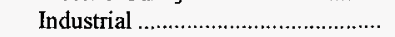 & 2,613 & 2,585 & 2,838 & 2,863 & 3,592 & 3,662 & 1.1 & -7.6 & -3.7 \\
\hline Coke & $w$ & $w$ & $w$ & $w$ & $w$ & $w$ & $w$ & w & w \\
\hline Residential/Commercial ................. & $w$ & $w$ & $w$ & w & $w$ & $\mathbf{w}$ & $\mathbf{w}$ & $\mathbf{w}$ & $w$ \\
\hline Consumer Stocks Total ..................... & w & w & $w$ & w & w & w & w & w & $\mathbf{w}$ \\
\hline 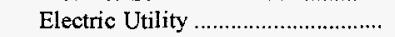 & 1,010 & 1,098 & 2,064 & 1,418 & 1,922 & 2,300 & -7.9 & -14.8 & -8.7 \\
\hline 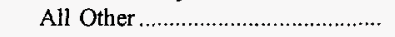 & $w$ & w & w & w & w & w & w & w & w \\
\hline \multicolumn{10}{|c|}{ Coal Prices (nominal dollars per short ton) } \\
\hline 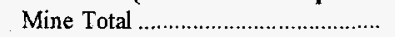 & $\$ 28.45$ & $\$ 28.47$ & $\$ 26.84$ & $\$ 26.80$ & $\$ 27.55$ & $\$ 27.42$ & -.1 & .8 & .4 \\
\hline Underground & 29.46 & 29.20 & 27.33 & 27.26 & 27.88 & 27.94 & .9 & 1.4 & 6 \\
\hline Surface & 25.88 & 26.34 & 25.33 & 25.29 & 26.17 & 24.90 & -1.8 & -.3 & .4 \\
\hline \multicolumn{10}{|l|}{ Consumer } \\
\hline 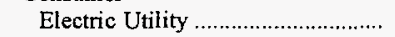 & 35.73 & 36.90 & 37.05 & 37.57 & 37.81 & 40.52 & -3.2 & -1.4 & -1.4 \\
\hline 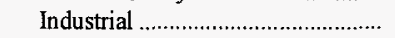 & 43.51 & 42.50 & 41.56 & 41.27 & 40.97 & 38.92 & 2.4 & 1.5 & 1.2 \\
\hline 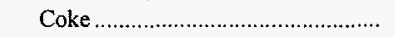 & w & $w$ & w & $w$ & w & w & $w$ & $w$ & $\mathbf{w}$ \\
\hline
\end{tabular}

1 For 1987, the Form EIA-7A solicited data on "Daily Froductive Capacity." To obtain annual productive capacity for a mine in 1987, each mine's daily productive capacity was multiplied by the number of days worked during the year.

2 Capacity utilization (percent) is the ratio of total production to annual productive capacity as reported by mining companies on Form EIA-7A. Productivity (short tons per miner per hour) is calculated by dividing total coal production by the total direct labor hours worked by all employees engaged in production, preparation, processing, development, maintenance, repair, and shop or yard work at mining operations.

3 Includes Mexico.

Withheld to avoid disclosure of individual company data

NA Not available.

R Revised Data.

Notes: Excludes silt, culm, refuse bank, slurry dam, and dredge operations except for Pennsylvania anthracite. Totals may not equal sum of components due to independent rounding.

Sources: Energy Information Administration, Form ElA-3 "Quarterly Coal Consumption Report - Manufacturing Plants"; Form EIA-5, "Coke Plant Report - Quarterly"; Form EIA-6, "Coal Distribution Report"; Form EIA-7A, "Coal Production Report"; Form EIA-759, "Monthly Power Plant Report"; and U.S. Department of Commerce, Bureau of the Census, "Monthly Report EM 545" and "Monthly Report IM 145." 
Table A15. West Virginia Coal Statistics, 1987, 1992-1996

\begin{tabular}{|c|c|c|c|c|c|c|c|c|c|}
\hline \multirow{2}{*}{ Category } & \multirow{2}{*}{1996} & \multirow{2}{*}{1995} & \multirow{2}{*}{1994} & \multirow{2}{*}{1993} & \multirow{2}{*}{1992} & \multirow{2}{*}{1987} & \multirow{2}{*}{$\begin{array}{c}\text { Percent } \\
\text { Change } \\
1995-1996\end{array}$} & \multicolumn{2}{|c|}{$\begin{array}{l}\text { Average Annual } \\
\text { Percent Change }\end{array}$} \\
\hline & & & & & & & & 1992-1996 & 1987-1996 \\
\hline \multicolumn{10}{|l|}{ Supply (thousand short tons) } \\
\hline Recoverable Reserves & $1,731,154$ & $1,731,400$ & $1,829,630$ & $1,930,903$ & $2,043,062$ & $2,547,607$ & $*$ & -4.0 & -4.2 \\
\hline Productive Capacity 1 ........................... & 217,409 & 204,837 & 201,684 & 191,706 & 198,083 & 158,356 & 6.1 & 2.3 & 3.6 \\
\hline 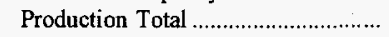 & 170,433 & 162,997 & 161,776 & 130,525 & 162,164 & 136,676 & 4.6 & 1.3 & 2.5 \\
\hline Underground & 115,585 & 110,029 & 111,679 & 87,997 & 115,212 & 107,463 & 5.0 & .1 & .8 \\
\hline Surface & 54,848 & 52,968 & 50,097 & 42,528 & 46,952 & 29,213 & 3.5 & 4.0 & 7.3 \\
\hline $\begin{array}{l}\text { Capacity Utilization } 2 \\
\text { Ratio of Recoverable }\end{array}$ & 78.32 & 79.50 & 80.07 & 67.91 & 81.70 & 85.84 & -1.5 & -1.0 & -1.0 \\
\hline Reserves to Production ....................... & 10.2 & 10.6 & 11.3 & 14.8 & 12.6 & 18.6 & -4.4 & -5.2 & -6.5 \\
\hline 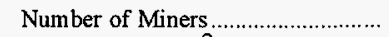 & 20,121 & 21,334 & 21,861 & 22,979 & 26,017 & 29,458 & -5.7 & -6.2 & -4.1 \\
\hline Productivity Total ${ }^{2}$ & 3.91 & 3.74 & 3.69 & 3.27 & 3.27 & 2.47 & 4.4 & 4.5 & 5.3 \\
\hline 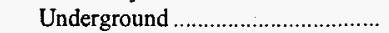 & 3.50 & 3.40 & 3.38 & 2.92 & 2.99 & 2.31 & 2.9 & 4.0 & 4.7 \\
\hline 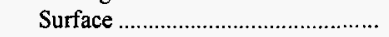 & 5.18 & 4.74 & 4.62 & 4.35 & 4.27 & 3.29 & 9.4 & 5.0 & 5.2 \\
\hline Producer/Distributor Stocks .............. & 4,947 & 6,176 & 6,692 & 4,059 & 7,405 & - & -19.9 & -9.6 & - \\
\hline \multicolumn{10}{|l|}{ Distribution (thousand short tons) } \\
\hline 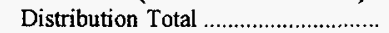 & 169,200 & 165,187 & 158,985 & 135,818 & 163,723 & NA & 2.4 & .8 & NA \\
\hline Domestic Distribution Total ........... & 127,156 & 120,866 & 122,779 & 102,659 & 112,917 & NA & 5.2 & 3.0 & NA \\
\hline Within State & 29,220 & 29,018 & 30,891 & 22,887 & 26,644 & NA & .7 & 2.3 & $\mathrm{NA}$ \\
\hline 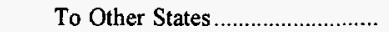 & 97,936 & 91,848 & 91,888 & 79,772 & 86,273 & NA & 6.6 & 3.2 & NA \\
\hline Foreign Distribution Total ............... & 42,044 & 44,321 & 36,205 & 33,159 & 50,806 & NA & -5.1 & -4.6 & NA \\
\hline 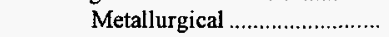 & 31,717 & 34,633 & 31,603 & 26,504 & 33,413 & $\mathrm{NA}$ & -8.4 & -1.3 & NA \\
\hline Steam & 10,327 & 9,688 & 4,602 & 6,655 & 17,393 & NA & 6.6 & -12.2 & NA \\
\hline 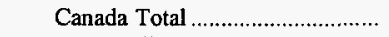 & 7,222 & 5,784 & 5,644 & 4,108 & 8,911 & NA & 24.9 & -5.1 & NA \\
\hline 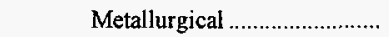 & 6,907 & 5,759 & 5,605 & 4,071 & 8,021 & $\mathrm{NA}$ & 19.9 & -3.7 & NA \\
\hline Steam & 315 & 25 & 40 & 37 & 890 & NA & $\mathrm{NM}$ & -22.8 & NA \\
\hline Overseas Total ${ }^{3} \ldots \ldots \ldots \ldots \ldots \ldots \ldots \ldots$ & 34,822 & 38,537 & 30,561 & 29,052 & 41,895 & $\mathrm{NA}$ & -9.6 & -4.5 & NA \\
\hline 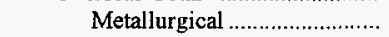 & 24,810 & 28,874 & 25,998 & 22,433 & 25,392 & NA & -14.1 & -.6 & $\mathrm{NA}$ \\
\hline Steam & 10,012 & 9,663 & 4,563 & 6,618 & 16,504 & NA & 3.6 & -11.8 & NA \\
\hline \multicolumn{10}{|l|}{ Demand (thousand short tons) } \\
\hline 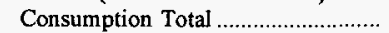 & 36,139 & 34,489 & 34,767 & 32,046 & 32,019 & 34,815 & 4.8 & 3.1 & .4 \\
\hline 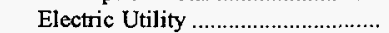 & 32,775 & 30,657 & 30,318 & 27,782 & 28,050 & 30,605 & 6.9 & 4.0 & 8 \\
\hline 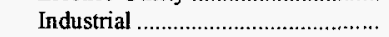 & 1,630 & 1,984 & 2,637 & 2,406 & 2,266 & 2,326 & -17.8 & -7.9 & -3.9 \\
\hline Coke & w & w & $w$ & w & $w$ & $w$ & w & $\mathbf{w}$ & w \\
\hline Residential/Commercial................. & $w$ & $\mathbf{w}$ & w & w & $\mathbf{w}$ & w & $w$ & $w$ & w \\
\hline 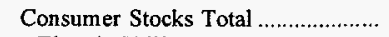 & $w$ & w & $w$ & $\mathbf{w}$ & $w$ & $w$ & $\mathbf{w}$ & $w$ & $w$ \\
\hline 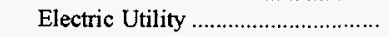 & 4,372 & 4,744 & 4,479 & 4,001 & 7,863 & 9,401 & -7.8 & -13.6 & -8.1 \\
\hline All Other & w & w & w & w & w & w & $\mathbf{w}$ & w & w \\
\hline \multicolumn{10}{|c|}{ Coal Prices (nominal dollars per short ton) } \\
\hline 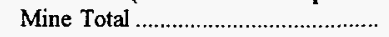 & $\$ 26.58$ & $\$ 27.18$ & $\$ 27.42$ & $\$ 27.58$ & $\$ 28.15$ & $\$ 29.15$ & -2.2 & -1.4 & -1.0 \\
\hline 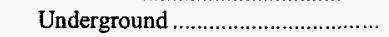 & 27.31 & 27.77 & 27.93 & 28.54 & 29.14 & 29.86 & -1.6 & -1.6 & -1.0 \\
\hline 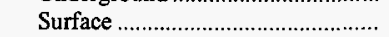 & 25.04 & 25.95 & 26.29 & 25.57 & 25.72 & 26.51 & -3.5 & -.7 & -6 \\
\hline \multicolumn{10}{|l|}{ Consumer } \\
\hline 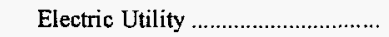 & 30.93 & 31.61 & 34.70 & 35.42 & 36.88 & 67.41 & -2.1 & -4.3 & -101.5 \\
\hline Industrial & 33.37 & 33.61 & 32.73 & 32.91 & 31.93 & 31.39 & -.7 & 1.1 & .7 \\
\hline 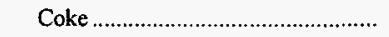 & w & w & w & w & w & w & w & w & $w$ \\
\hline
\end{tabular}

1 For 1987, the Form EIA-7A solicited data on "Daily Productive Capacity." To obtain annual productive capacity for a mine in 1987, each mine's daily productive capacity was multiplied by the number of days worked during the year.

2 Capacity utilization (percent) is the ratio of total production to annual productive capacity as reported by mining companies on Form EIA-7A. Productivity (short tons per miner per hour) is calculated by dividing total coal production by the total direct labor hours worked by all employees engaged in production, preparation, processing, development, maintenance, repair, and shop or yard work at mining operations.

3 Includes Mexico.

* Data round to zero.

Witheld to avoid disclosure of individual company data.

NM Not meaningful as value is greater than 500 percent.

NA Not available.

Notes: Excludes silt, culm, refuse bank, slurry dam, and dredge operations except for. Pennsylvania anthracite. Totals may not equal sum of compo-

nents due to independent rounding.

Sources: Energy Information Administration, Form ELA-3, "Quarterly Coal Consumption Report - Manufacturing Plants"; Form EIA-5, "Coke Plant Report - Quarterly"; Form EIA-6, "Coal Distribution Report"; Form EIA-7A, "Coal Production Report"; Form EIA-759, "Monthly Power Plant Report"; and U.S Department of Commerce, Bureau of the Census, "Monthly Report EM 545" and "Monthly Report IM 145." 
Table A16. Wyoming Coal Statistics, 1987, 1992-1996

\begin{tabular}{|c|c|c|c|c|c|c|c|c|c|}
\hline \multirow{2}{*}{ Category } & \multirow{2}{*}{1996} & \multirow{2}{*}{1995} & \multirow{2}{*}{1994} & \multirow{2}{*}{1993} & \multirow{2}{*}{1992} & \multirow{2}{*}{1987} & \multirow{2}{*}{$\begin{array}{c}\text { Percent } \\
\text { Change } \\
\text { 1995-1996 }\end{array}$} & \multicolumn{2}{|c|}{$\begin{array}{l}\text { Average Annual } \\
\text { Percent Change }\end{array}$} \\
\hline & & & & & & & & 1992-1996 & 1987-1996 \\
\hline \multicolumn{10}{|l|}{ Supply (thousand short tons) } \\
\hline 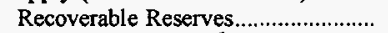 & $6,591,293$ & $6,723,963$ & $6,999,460$ & $6,830,720$ & $6,750,718$ & $6,596,139$ & -2.0 & -0.6 & $*$ \\
\hline Productive Capacity 1 ............................ & 350,908 & 337,184 & 321,046 & 277,875 & 253,312 & 247,476 & 4.1 & 8.5 & 3.9 \\
\hline Production Total & 278,440 & 263,822 & 237,092 & 210,129 & 190,172 & 146,850 & 5.5 & 10.0 & 7.4 \\
\hline 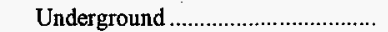 & 2,641 & 2,008 & 2,735 & - $\quad 2,136$ & 2,511 & 107 & 31.5 & 1.3 & 42.8 \\
\hline Surface & 275,799 & 261,814 & 234,357 & 207,993 & 187,661 & 146,743 & 5.3 & 10.1 & 7.3 \\
\hline 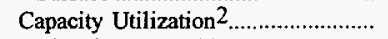 & 79.35 & 78.24 & 73.85 & 75.62 & 75.07 & 59.34 & 1.4 & 1.4 & 3.3 \\
\hline \multicolumn{10}{|l|}{ Ratio of Recoverable } \\
\hline Reserves to Production .......................... & 23.7 & 25.5 & 29.5 & 32.5 & 35.5 & 44.9 & -7.1 & -9.6 & -6.9 \\
\hline 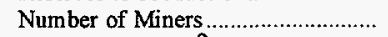 & 2,814 & 3,142 & 3,291 & 3,159 & 3,326 & 3,191 & -10.4 & -4.1 & -1.4 \\
\hline Productivity Total 2 & 32.06 & 30.06 & 26.05 & 24.46 & 21.50 & 17.91 & 6.6 & 10.5 & 6.7 \\
\hline 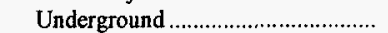 & 9.18 & 5.97 & 5.07 & 3.56 & 4.19 & 2.71 & 53.6 & 21.7 & 14.5 \\
\hline 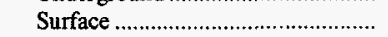 & 32.84 & 31.02 & 27.37 & 26.03 & 22.76 & 17.98 & 5.9 & 9.6 & 6.9 \\
\hline Producer/Distributor Stocks .............. & 1,504 & 1,997 & 1,592 & 998 & 2,794 & - & -24.7 & -14.3 & - \\
\hline \multicolumn{10}{|l|}{ Distribution (thousand short tons) } \\
\hline 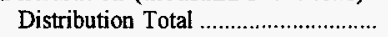 & 279,117 & 263,601 & 235,540 & 211,713 & 190,260 & NA & 5.9 & 10.0 & NA \\
\hline Domestic Distribution Total ........... & 276,723 & 261,333 & 234,016 & 210,739 & 188,983 & NA & 5.9 & 10.0 & NA \\
\hline 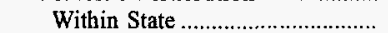 & 26,253 & 26,521 & 28,334 & 25,519 & 26,388 & NA & -1.0 & -.1 & NA \\
\hline 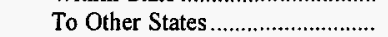 & 250,470 & 234,812 & 205,682 & 185,220 & 162,595 & $\mathrm{NA}$ & 6.7 & 11.4 & NA \\
\hline Foreign Distribution Total............. & 2,395 & 2,269 & 1,524 & 974 & 1,277 & NA & 5.5 & 17.0 & NA \\
\hline Steam & 2,395 & 2,269 & 1,524 & 974 & 1,277 & NA & 5.5 & 17.0 & NA \\
\hline Canada Total & 443 & 32 & - & - & - & $\mathrm{NA}$ & $\mathrm{NM}$ & - & NA \\
\hline Steam & 443 & 32 & - & - & - & $\mathrm{NA}$ & NM & - & $\mathrm{NA}$ \\
\hline Overseas Total 3 & 1,952 & 2,237 & 1,524 & 974 & 1,277 & NA & -12.8 & 11.2 & $\mathrm{NA}$ \\
\hline Steam & 1,952 & 2,237 & 1,524 & 974 & 1,277 & $\mathrm{NA}$ & -12.8 & 11.2 & $\mathrm{NA}$ \\
\hline \multicolumn{10}{|l|}{ Demand (thousand short tons) } \\
\hline 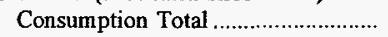 & 26,646 & 25,933 & 27,459 & 26,171 & 27,339 & 24,399 & 2.8 & -.6 & 1.0 \\
\hline 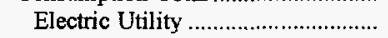 & 24,430 & 23,850 & 25,350 & 24,111 & 25,114 & 22,408 & 2.4 & -.7 & 1.0 \\
\hline Industrial & 1,835 & 1,937 & 1,867 & 1,873 & 2,126 & $w$ & -5.3 & -3.6 & w \\
\hline 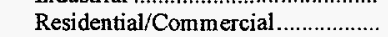 & 382 & 146 & 242 & 187 & $\mathrm{w}$ & $w$ & 162.0 & w & w \\
\hline 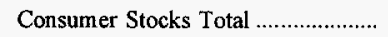 & 2,269 & 2,936 & 2,553 & 1,841 & 2,242 & w & -22.7 & .3 & $\mathrm{w}$ \\
\hline 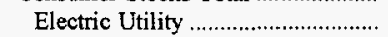 & 2,198 & 2,857 & 2,476 & 1,728 & 2,153 & 2,805 & -23.1 & .5 & -2.7 \\
\hline 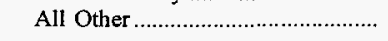 & 71 & 79 & 77 & 113 & 89 & w & -10.7 & -5.6 & $\mathbf{w}$ \\
\hline \multicolumn{10}{|c|}{ Coal Prices (nominal dollars per short ton) } \\
\hline 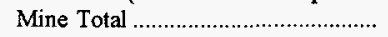 & $\$ 6.41$ & $\$ 6.58$ & $\$ 6.83$ & $\$ 7.32$ & $\$ 8.14$ & $\$ 9.80$ & -2.6 & -5.8 & -4.6 \\
\hline 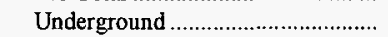 & $w$ & $w$ & $\mathbf{w}$ & w & w & w & w & w & w \\
\hline 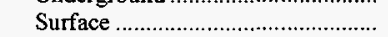 & $w$ & $\mathrm{w}$ & $w$ & $\mathbf{w}$ & $w$ & $w$ & $\mathbf{w}$ & $\mathbf{w}$ & $w$ \\
\hline \multicolumn{10}{|l|}{ Consumer } \\
\hline 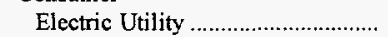 & $\$ 14.30$ & $\$ 14.29$ & $\$ 14.09$ & $\$ 14.03$ & $\$ 13.42$ & $\$ 15.27$ & .1 & 1.6 & -.7 \\
\hline 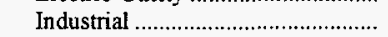 & 22.32 & 22.72 & 22.87 & 23.43 & 23.67 & w & -1.8 & -1.4 & w \\
\hline
\end{tabular}

1 For 1987, the Form EIA-7A solicited data on "Daily Productive Capacity." To obtain annual productive capacity for a mine in 1987, each mine's daily productive capacity was multiplied by the number of days worked during the year.

2 Capacity utilization (percent) is the ratio of total production to annual productive capacity as reported by mining companies on Form ElA-7A. Productivity (short tons per miner per hour) is calculated by dividing total coal production by the total direct labor hours worked by all employees engaged in production, preparation, processing, development, maintenance, repair, and shop or yard work at mining operations.

3 Includes Mexico.

* Data round to zero.

w Withheld to avoid disclosure of individual company data.

NM Not meaningful as value is greater than 500 percent

NA Not available.

Notes: Excludes silt, culm, refuse bank, slurry dam, and dredge operations except for Pennsylvania anthracite. Totals may not equal sum of components due to independent rounding.

Sources: Energy Information Administration, Form E]A-3, "Quarterly Coal Consumption Report - Manufacturing Plants"; Form EIA-5, "Coke Plant Report - Quarterly"; Form EIA-6, "Coal Distribution Report"; Form EIA-7A, "Coal Production Report"; Form EIA-759, "Monthly Power Plant Report"; and U.S. Department of Commerce, Bureau of the Census, "Monthly Report EM 545" and "Monthly Report IM 145." 
Table A17. All Other States Coal Statistics, 1987, 1992-1996

\begin{tabular}{|c|c|c|c|c|c|c|c|c|c|}
\hline \multirow{2}{*}{ Category } & \multirow{2}{*}{1996} & \multirow{2}{*}{1995} & \multirow{2}{*}{1994} & \multirow{2}{*}{1993} & \multirow{2}{*}{1992} & \multirow{2}{*}{1987} & \multirow{2}{*}{$\begin{array}{c}\text { Percent } \\
\text { Change } \\
1995-1996\end{array}$} & \multicolumn{2}{|c|}{$\begin{array}{l}\text { Average Annual } \\
\text { Percent Change }\end{array}$} \\
\hline & & & & & & & & $1992-1996$ & $1987-1996$ \\
\hline \multicolumn{10}{|l|}{ Supply (thousand short tons) } \\
\hline 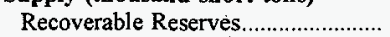 & 427,163 & 420,998 & 476,538 & 506,419 & 514,739 & 803,845 & 1.5 & -4.5 & -6.8 \\
\hline 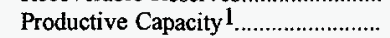 & 23,441 & 24,495 & 23,451 & 23,567 & 28,144 & 35,803 & -4.3 & -4.5 & -4.6 \\
\hline 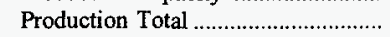 & 19,674 & 19,911 & 19,672 & 18,848 & 22,284 & 28,878 & -1.2 & -3.1 & -4.2 \\
\hline Underground & 5,227 & 4,880 & 4,880 & 4,523 & 4,367 & 7,329 & 7.1 & 4.6 & -3.7 \\
\hline Surface & 14,447 & 15,031 & 14,792 & 14,325 & 17,917 & 21,549 & -3.9 & -5.2 & -4.3 \\
\hline Capacity Utilization 2 .......................... & 83.55 & 81.02 & 83.52 & 79.48 & 78.65 & 80.17 & 3.1 & 1.5 & .4 \\
\hline \multicolumn{10}{|l|}{ Ratio of Recoverable } \\
\hline Reserves to Production........................ & 21.7 & 21.1 & 24.2 & 26.9 & 23.1 & 27.8 & 2.7 & -1.5 & -2.7 \\
\hline Number of Miners & 2,394 & 2,312 & 2,373 & 2,478 & 2,918 & 5,362 & 3.5 & -4.8 & -8.6 \\
\hline 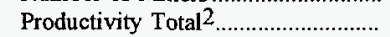 & 3.73 & 3.96 & 3.82 & 3.59 & 3.40 & 2.58 & -6.0 & 2.3 & 4.1 \\
\hline 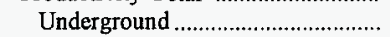 & 2.94 & 3.03 & 2.86 & 3.05 & 2.40 & 1.98 & -3.1 & 5.2 & 4.5 \\
\hline Surface & 4.13 & 4.41 & 4.30 & 3.80 & 3.78 & 2.88 & -6.2 & 2.3 & 4.1 \\
\hline Producer/Distributor Stocks ............... & 292 & 784 & 598 & 297 & 289 & - & -62.8 & .3 & - \\
\hline Imports ${ }^{3}$ & 5,267 & 5,084 & 5,327 & 4,605 & 1,713 & 671 & 3.6 & 32.4 & 25.7 \\
\hline \multicolumn{10}{|l|}{ Distribution (thousand short tons) } \\
\hline 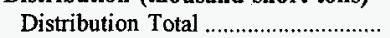 & 19,828 & 19,080 & ${ }^{R} 18,813$ & ${ }^{R} 19,056$ & R 21,904 & NA & 3.9 & -2.5 & NA \\
\hline Domestic Distribution Total ............ & 18,285 & 17,930 & 17,767 & 17,914 & 20,654 & NA & 2.0 & -3.0 & NA \\
\hline 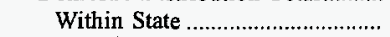 & 28 & 26 & 25 & 100 & 143 & NA & 5.2 & -33.6 & NA \\
\hline 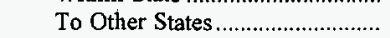 & 18,257 & 17,904 & 17,743 & 17,814 & 20,511 & $\mathrm{NA}$ & 2.0 & -2.9 & $\mathrm{NA}$ \\
\hline Foreign Distributiọn Total............... & 1,544 & 1,150 & 1,046 & 1,142 & 1,250 & NA & 34.2 & 5.4 & NA \\
\hline Metallurgical & - & 38 & 109 & 51 & - & NA & -100.0 & - & NA \\
\hline Steam & 1,544 & 1,112 & 937 & 1,092 & 1,250 & NA & 38.8 & 5.4 & $\mathrm{NA}$ \\
\hline 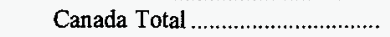 & $*$ & 1 & 3 & 1 & - & $\mathrm{NA}$ & -82.9 & - & NA \\
\hline Steam & $*$ & 1 & 3 & 1 & - & NA & -82.9 & - & NA \\
\hline Overseas Total ${ }^{4}$ & 1,544 & 1,149 & 1,042 & 1,142 & 1,250 & NA & 34.3 & 5.4 & NA \\
\hline 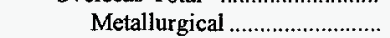 & - & 38 & 109 & 51 & - & $\mathrm{NA}$ & -100.0 & - & NA \\
\hline 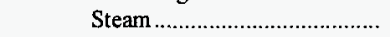 & 1,544 & 1,112 & 934 & 1,091 & 1,250 & NA & 38.9 & 5.4 & NA \\
\hline \multicolumn{10}{|l|}{ Demand (thousand short tons) } \\
\hline Consumption Total ............................... & 400,049 & 382,060 & 371,817 & 365,045 & 350,253 & 334,403 & 4.7 & 3.4 & 2.0 \\
\hline 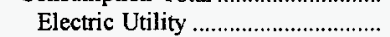 & 365,146 & 344,785 & 334,773 & 329,279 & 315,259 & 296,439 & 5.9 & 3.7 & 2.3 \\
\hline 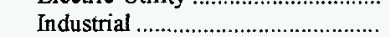 & 29,720 & 31,653 & 31,795 & 31,970 & 31,728 & 30,998 & -6.1 & -1.6 & -.5 \\
\hline 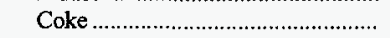 & 2,686 & 2,784 & 2,723 & 1,243 & 1,148 & 4,603 & -3.5 & 23.7 & -5.8 \\
\hline Residential/Commercial ................... & 2,497 & 2,838 & 2,526 & 2,552 & 2,119 & 2,363 & -12.0 & 4.2 & 6 \\
\hline 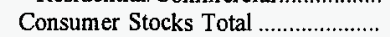 & 60,142 & 62,368 & 57,506 & 48,850 & 68,257 & 78,115 & -3.6 & -3.1 & -2.9 \\
\hline 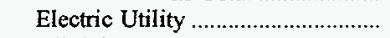 & 55,838 & 58,348 & 53,253 & 45,411 & 64,006 & 71,867 & -4.3 & -3.3 & -2.8 \\
\hline 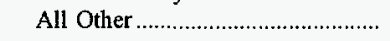 & 4,304 & 4,021 & 4,252 & 3,439 & 4,251 & 6,247 & 7.0 & .3 & -4.0 \\
\hline \multicolumn{10}{|c|}{ Coal Prices (nominal dollars per short ton) } \\
\hline Mine Total & $\$ 24.06$ & $\$ 22.80$ & $\$ 23.19$ & $\$ 23.14$ & $\$ 24.39$ & $\$ 25.88$ & 5.5 & -.3 & -.8 \\
\hline 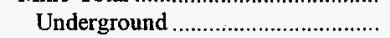 & 25.78 & 26.25 & 26.97 & 26.72 & 27.26 & 27.79 & -1.8 & -1.4 & -.8 \\
\hline 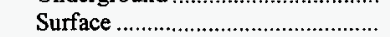 & 23.44 & 21.67 & 21.94 & 22.02 & 23.69 & 25.23 & 8.1 & -.3 & -.8 \\
\hline \multicolumn{10}{|l|}{ Consumer } \\
\hline 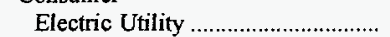 & 27.46 & 28.22 & 29.70 & 30.68 & 31.79 & 34.55 & -2.7 & -3.6 & -2.5 \\
\hline 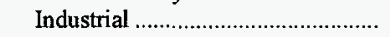 & 38.22 & 38.51 & 38.72 & 38.95 & 39.35 & 40.68 & -.8 & -.7 & -.7 \\
\hline 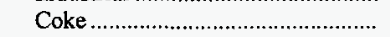 & 51.21 & 51.67 & 50.89 & 58.19 & 53.10 & 51.49 & -.9 & -.9 & $*$ \\
\hline
\end{tabular}

1 For 1987, the Form EIA-7A solicited data on "Daily Productive Capacity." To obtain annual productive capacity for a mine in 1987, each mine's daily productive capacity was multiplied by the number of days worked during the year.

2 Capacity utilization (percent) is the ratio of total production to annual productive capacity as reported by mining companies on Form EIA-7A. Productivity (short tons per miner per hour) is calculated by dividing total coal production by the total direct labor hours worked by all employees engaged in production, preparation, processing, development, maintenance, repair, and shop or yard work at mining operations.

3 Imports for 1992 through 1996 include imports to electric utilities, manufacturing plants and coke plants. Imports for 1987 include only imports to electric utilities.

4 Includes Mexico.

* Data round to zero.

w Withheld to avoid disclosure of individual company data.

NA Not available.

R Revised Data.

Notes: Other States include Alaska, Arkansas, California, Iowa, Kansas, Louisiana, Maryland, Missouri, Oklahoma, Tennessee, and Washington, Excludes silt, culm, refuse bank, slurry dam, and dredge operations except for Pennsylvania anthracite. Totals may not equal sum of components due to independent rounding.

Sources: Energy Information Administration, Form EIA-3, "Quarterly Coal Consumption Report - Manufacturing Plants"; Form EIA-5, "Coke Plant Report - Quarterly"; Form EIA-6, "Coal Distribution Report"; Form EIA-7A, "Coal Production Report"; Form EIA-759, "Monthly Power Plant Report"; and U.S. Department of Commerce, Bureau of the Census, "Monthly Report EM 545" and "Monthly Report IM 145." 
Table A18. Total U.S. Coal Statistics, 1987, 1992-1996

\begin{tabular}{|c|c|c|c|c|c|c|c|c|c|}
\hline \multirow{2}{*}{ Category } & \multirow{2}{*}{1996} & \multirow{2}{*}{1995} & \multirow{2}{*}{1994} & \multirow{2}{*}{1993} & \multirow{2}{*}{1992} & \multirow{2}{*}{1987} & \multirow{2}{*}{$\begin{array}{c}\text { Percent } \\
\text { Change } \\
\text { 1995-1996 }\end{array}$} & \multicolumn{2}{|c|}{$\begin{array}{l}\text { Average Annual } \\
\text { Percent Change }\end{array}$} \\
\hline & & & & & & & & 1992-1996 & $1987-1996$ \\
\hline \multicolumn{10}{|l|}{ Supply (thousand short tons) } \\
\hline 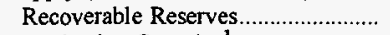 & $19,427,980$ & $20,105,197$ & $21,016,526$ & $21,535,283$ & $21,626,971$ & $24,241,218$ & -3.4 & -2.6 & -2.4 \\
\hline Productive Capacity 1 ........................... & $1,324,712$ & $1,299,054$ & $1,320,656$ & $1,261,873$ & $1,241,054$ & $1,183,404$ & 2.0 & 1.6 & 1.3 \\
\hline Production Total .................................. & $1,063,856$ & $1,032,974$ & $1,033,504$ & 945,424 & 997,545 & 918,762 & 3.0 & 1.6 & 1.6 \\
\hline 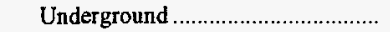 & 409,849 & 396,249 & 399,103 & 351,053 & 407,239 & 372,874 & 3.4 & .1 & 1.0 \\
\hline 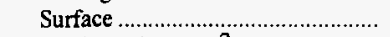 & 654,007 & 636,725 & 634,401 & 594,371 & 590,306 & 545,888 & 2.7 & 2.6 & 2.0 \\
\hline Capacity Utilization ${ }^{2}$ & 80.21 & 79.40 & 78.11 & 74.77 & 80.20 & 77.29 & 1.0 & $*$ & .4 \\
\hline Ratio of Recoverable & & & & & & & & & \\
\hline Reserves to Production & 18.3 & 19.5 & 20.3 & 22.8 & 21.7 & 26.4 & -6.2 & -4.2 & -4.0 \\
\hline 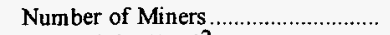 & 83,462 & 90,252 & 97,500 & 101,322 & 110,196 & 142,667 & -7.5 & -6.7 & -5.8 \\
\hline Productivity Total ${ }^{2} \ldots \ldots \ldots \ldots \ldots \ldots \ldots \ldots$ & 5.69 & 5.38 & 4.98 & 4.70 & 4.36 & 3.30 & 5.8 & 6.9 & 6.3 \\
\hline Underground ....................................... & 3.57 & 3.39 & 3.19 & 2.95 & 2.93 & 2.20 & 5.6 & 5.1 & 5.5 \\
\hline Surface & 9.05 & 8.48 & 7.67 & 7.23 & 6.59 & 4.98 & 6.7 & 8.3 & 6.9 \\
\hline Producer/Distributor Stocks .............. & 28,648 & 34,444 & 33,219 & 25,284 & 33,993 & 34,090 & -16.8 & -4.2 & -1.9 \\
\hline Imports $^{3}$ & 6,476 & 6,317 & 6,599 & 5,496 & 1,806 & 765 & 2.5 & 37.6 & 26.8 \\
\hline \multicolumn{10}{|l|}{ Distribution (thousand short tons) } \\
\hline 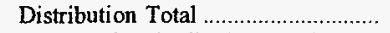 & $1,059,892$ & $1,(1) 30,330$ & $1,022,523$ & 959,445 & 998,647 & NA & 2.9 & 1.5 & NA \\
\hline Domestic Distribution Total ........... & 967,693 & 940,423 & 949,843 & 883,934 & 897,267 & NA & 2.9 & 1.9 & NA \\
\hline 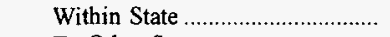 & 340,005 & 36,821 & 353,765 & 339,034 & 355,232 & NA & .9 & -1.1 & NA \\
\hline 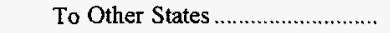 & 627,688 & $6,03,602$ & 596,078 & 544,900 & 542,035 & NA & 4.0 & 3.7 & NA \\
\hline Foreign Distribution Total............. & 92,199 & 89,907 & 72,680 & 75,510 & 101,380 & NA & 2.5 & -2.3 & NA \\
\hline Metallurgical .......................... & 56,162 & 54,077 & 52,206 & 52,591 & 61,432 & NA & 3.9 & -2.2 & NA \\
\hline Steam & 36,037 & 35,830 & 20,474 & 22,919 & 39,948 & NA & .6 & -2.5 & NA \\
\hline 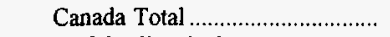 & 10,599 & 8,023 & 8,467 & 7,751 & 13,919 & NA & 32.1 & -6.6 & NA \\
\hline 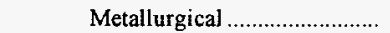 & 8,472 & 6,986 & 7,464 & 6,666 & 9,394 & NA & 21.3 & -2.5 & NA \\
\hline Steam & 2,127 & 1,037 & 1,003 & 1,085 & 4,525 & NA & 105.0 & -17.2 & NA \\
\hline Overseas Total ${ }^{4} \ldots$ & 81,600 & 81,884 & 64,214 & 67,759 & 87,461 & $\mathrm{NA}$ & -.3 & -1.7 & NA \\
\hline 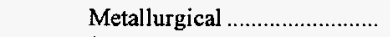 & 47,690 & 47,091 & 44,743 & 45,925 & 52,038 & NA & 1.3 & -2.2 & NA \\
\hline Steam & 33,910 & 34,793 & 19,471 & 21,834 & 35,423 & NA & -2.5 & -1.1 & NA \\
\hline \multicolumn{10}{|l|}{ Demand (thousand short tons) } \\
\hline 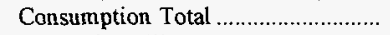 & 983,334 & 940,880 & 930,201 & 925,944 & 892,421 & 836,860 & 4.5 & 2.4 & 1.8 \\
\hline 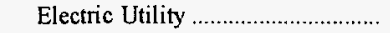 & 874,681 & 829,007 & 817,270 & 813,508 & 779,860 & 717,894 & 5.5 & 2.9 & 2.2 \\
\hline 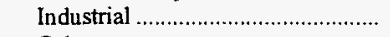 & 70,941 & 73,055 & 75,179 & 74,892 & 74,042 & 75,175 & -2.9 & -1.1 & -.6 \\
\hline 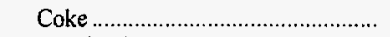 & 31,706 & 33,011 & 31,740 & 31,323 & 32,366 & 36,877 & -3.9 & -.5 & -1.7 \\
\hline Residential/Commercial .................... & 6,006 & 5,807 & 6,013 & 6,221 & 6,153 & 6,914 & 3.4 & -.6 & -1.5 \\
\hline Consumer Stocks Total ......................... & 123,024 & 134,639 & 136,139 & 120,458 & 163,692 & 185,459 & -8.6 & -6.9 & -4.4 \\
\hline 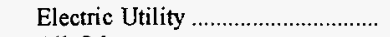 & 114,669 & 126,304 & 126,897 & 111,341 & 154,130 & 170,797 & -9.2 & -7.1 & -4.3 \\
\hline 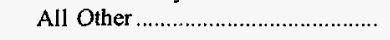 & 8,355 & 8,334 & 9,243 & 9,117 & 9,562 & 14,662 & .3 & -3.3 & -6.0 \\
\hline \multicolumn{10}{|c|}{ Coal Prices (nominal dollars per short ton) } \\
\hline 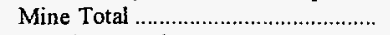 & $\$ 18.50$ & $\$ 18.83$ & $\$ 19.41$ & $\$ 19.85$ & $\$ 21.03$ & $\$ 23.07$ & -1.8 & -3.1 & -2.4 \\
\hline 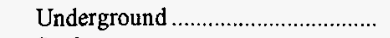 & 25.96 & 26.18 & 26,39 & 26.92 & 27.83 & 29.63 & -.9 & -1.7 & -1.5 \\
\hline 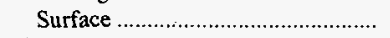 & 13.82 & 14.25 & 15.02 & 15.67 & 16.34 & 18.58 & -3.0 & -4.1 & -3.2 \\
\hline \multicolumn{10}{|l|}{ Consumer } \\
\hline 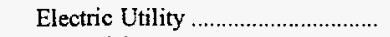 & 26.45 & 27.01 & 28.03 & 28.58 & 29.36 & 31.83 & -2.1 & -2.6 & -2.0 \\
\hline 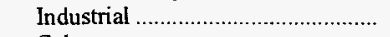 & 32.32 & 32.42 & 32.55 & 32.23 & 32.78 & 33.71 & -.3 & -.3 & -.5 \\
\hline 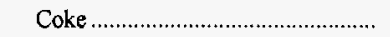 & 47.33 & 47.34 & 46.56 & 47.44 & 47.92 & 46.55 & $*$ & -.3 & .2 \\
\hline
\end{tabular}

1 For 1987, the Form EIA-7A solicited data on "Daily Froductive Capacity." To obtain annual productive capacity for a mine in 1987, each mine's daily productive capacity was multiplied by the number of days worked during the year.

2 Capacity utilization (percent) is the ratio of total production to annual productive capacity as reported by mining companies on Form EIA-7A. Productivity (short tons per miner per hour) is calculated by dividing total coal production by the total direct labor hours worked by all employees engaged in production, preparation, processing, development, maintenance, repzir, and shop or yard work at mining operations.

3 Imports for 1992 through 1996 include imports to electric utilities, manufacturing plants and coke plants. Imports for 1987 include only imports to electric utilities.

4 Includes Mexico.

* Data round to zero.

NA Not available.

Notes: Excludes silt, culm, refuse bank, slurry dam, and dredge operations except for Pennsylvania anthracite. Consumption Total does not include coal consumed by independent power producers. Totals may not equal sum of components due to independent rounding.

Sources: Energy Information Administration, Form ElA-3, "Quarterly Coal Consumption Report - Manufacturing Plants"; Form EIA-5, "Coke Plant Report - Quarterly"; Form EIA-6, "Coal Distribution Report"; Form EIA-7A, "Coal Production Report"; Form EIA-759, "Monthly Power Plant Report"; and U.S. Department of Commerce, Bureau of the Census, "Monthly Report EM 545" and "Monthly Report IM 145." 


\section{Appendix B}

\section{Metric Tables}

In response to requests from international users of U.S. coal statistics, certain summary data have been converted from the customary short tons to metric. This enables U.S. statistics to be compared with data published by countries using the metric system. The conversion to metric tons is made by multiplying short tons by 0.907185 .

The data converted to metric tons are from Tables ES3, 1, 16, 25, 35, 48, 67, 68, 76, 80, 92, 94, 96, 98, and 99.

Table B1. Trends in U.S. Coal Production, Imports, Consumption, Exports, and Stocks, 1987, 1992-1996

(Million Metric Tons)

\begin{tabular}{|c|c|c|c|c|c|c|c|c|c|}
\hline \multirow{2}{*}{ Activity } & \multirow{2}{*}{1996} & \multirow{2}{*}{1995} & \multirow{2}{*}{1994} & \multirow{2}{*}{1993} & \multirow{2}{*}{1992} & \multirow{2}{*}{1987} & \multirow{2}{*}{$\begin{array}{c}\text { Percent } \\
\text { Change } \\
\text { 1995-1996 }\end{array}$} & \multicolumn{2}{|c|}{ Average Annual Percent Change } \\
\hline & & & & & & & & 1992-1996 & $1987-1996$ \\
\hline Production & 965 & 937 & 938 & 858 & 905 & 833 & 3.0 & 1.6 & 1.6 \\
\hline Imports & 6 & 7 & 7 & 7 & 3 & 2 & -1.0 & 17.0 & 16.9 \\
\hline Producer and Distributor Stocks 1 & 26 & 31 & 30 & 23 & 31 & 31 & -16.8 & -4.2 & -1.9 \\
\hline Consumption & 892 & 854 & 844 & 840 & 810 & 759 & 4.5 & 2.4 & 1.8 \\
\hline Exports & 82 & 80 & 65 & 68 & 93 & 72 & 2.2 & -3.1 & 1.4 \\
\hline Consumer Stocks' & 112 & 122 & 124 & 109 & 148 & 168 & -8.6 & -6.9 & -4.4 \\
\hline
\end{tabular}

1 Reported as of the last day of the quarter.

Note: Consumption does not include coal consumed by independent power producers.

Sources: - Production: Energy Information Administration (ELA), Form EIA-7A, “Coal Production Report"; U.S. Department of Labor, Mine Safety and Health Administration, Form 7000-2, "Quarterly Mine Employment and Coal Production Report"; and State Mining Agency Coal Production Reports. - Inports: U.S. Department of Commerce, Bureau of the Census, "Monthly Report IM 145." - Producer and Distributor Stocks: EIA, Form EIA-6, "Coal Distribution Report." - Exports: U.S. Department of Commerce, Bureau of the Census, "Monthly Report EM 545."

- Consumption and Consumer Stocks: EIA, Form EIA-759, "Monthly Power Plant Report"; Form EIA-3, Quarterly Coal Consumption Report - Manufacturing Plants"; Form EIA-5, "Coke Plant Report - Quarterly"; and Form EIA-6, "Coal Distribution Report." 
Table B2. Coal Production by State, 1987, 1992-1996

(Thousand Metric Tons)

\begin{tabular}{|c|c|c|c|c|c|c|c|c|c|}
\hline \multirow{2}{*}{$\begin{array}{l}\text { Coal-Producing } \\
\text { State and Region }\end{array}$} & \multirow{2}{*}{1996} & \multirow{2}{*}{1995} & \multirow{2}{*}{1994} & \multirow{2}{*}{1993} & \multirow{2}{*}{1992} & \multirow{2}{*}{1987} & \multirow{2}{*}{$\begin{array}{c}\text { Percent } \\
\text { Change } \\
\text { 1995-1996 }\end{array}$} & \multicolumn{2}{|c|}{ Average Annual Percent Change } \\
\hline & & & & & & & & $1992-1996$ & $1987-1996$ \\
\hline Alabama & 22,351 & 22,353 & 21,106 & 22,469 & 23,401 & 23,169 & * & -1.1 & -0.4 \\
\hline 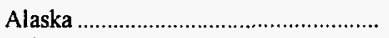 & 1,343 & 1,540 & 1,422 & 1,452 & 1,392 & 1,354 & -12.8 & -.9 & -.1 \\
\hline Arizona & 9,473 & 10,838 & 11,844 & 11,043 & 11,351 & 10,323 & -12.6 & -4.4 & -9 \\
\hline 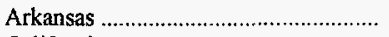 & 19 & 26 & 46 & 40 & 53 & 76 & -28.0 & -22.6 & -14.3 \\
\hline California & - & - & - & - & 94 & 42 & - & - & - \\
\hline Colorado & 22,576 & 23,324 & 22,955 & 19,854 & 17,442 & 13,082 & -3.2 & 6.7 & 6.3 \\
\hline 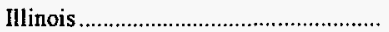 & 42,325 & 43,708 & 47,896 & 37,283 & 54,301 & 53,665 & -3.2 & -6.0 & -2.6 \\
\hline Indiana & 26,916 & 23,593 & 28,057 & 26,576 & 27,639 & 31,033 & 14.1 & -.6 & -1.6 \\
\hline Iowa & - & - & 42 & 159 & 262 & 425 & - & - & - \\
\hline 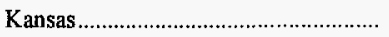 & 211 & 258 & 258 & 310 & 329 & 1,834 & -18.4 & -10.5 & -21.4 \\
\hline 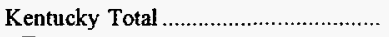 & 138,278 & 139,470 & 146,639 & 141,792 & 146,119 & 149,859 & -.8 & -1.4 & -.9 \\
\hline 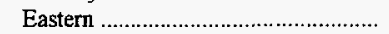 & 106,096 & 107,539 & 112,897 & 109,036 & 108,302 & 108,777 & -1.3 & -.5 & -.3 \\
\hline Western & 32,182 & 31,931 & 33,742 & 32,756 & 37,817 & 41,082 & .8 & -3.9 & -2.7 \\
\hline Louisiana & 2,922 & 3,374 & 3,141 & 2,843 & 2,939 & 2,495 & -13.4 & -.1 & 1.8 \\
\hline 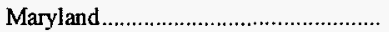 & 3,713 & 3,327 & 3,295 & 3,044 & 3,031 & 3,595 & 11.6 & 5.2 & .3 \\
\hline Missouri & 644 & 497 & 761 & 592 & 2,618 & 3,894 & 29.6 & -29.6 & -18.1 \\
\hline 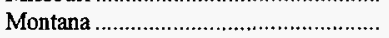 & 34,374 & 35,789 & 37,775 & 32,583 & 35,280 & 31,207 & -3.9 & -.6 & 1.1 \\
\hline New Mexico & 21,834 & 24,324 & 25,438 & 25,644 & 22,270 & 17,356 & -10.2 & -.5 & 2.6 \\
\hline 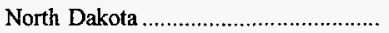 & 27,089 & 27,317 & 29,289 & 29,005 & 28,797 & 22,809 & -.8 & -1.5 & 1.9 \\
\hline Ohio & 25,920 & 23,694 & 27,122 & 26,141 & 27,581 & 32,466 & 9.4 & -1.5 & -2.5 \\
\hline 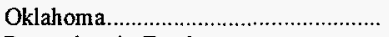 & 1,543 & 1,702 & 1,733 & 1,595 & 1,580 & 2,603 & -9.3 & -.6 & -5.6 \\
\hline 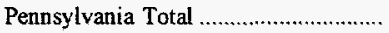 & 61,636 & 55,860 & 56,460 & 54,159 & 62,578 & 63,887 & 10.3 & -.4 & -.4 \\
\hline Anthracite & 4,310 & 4,248 & 4,192 & 3,906 & 3,160 & 3,230 & 1.5 & 8.1 & 3.3 \\
\hline 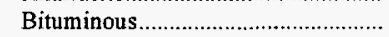 & 57,325 & 51,613 & 52,268 & 50,253 & 59,419 & 60,657 & 11.1 & -.9 & -.6 \\
\hline 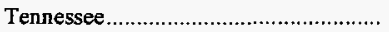 & 3,312 & $2 ; 922$ & 2,709 & 2,764 & 3,154 & 5,844 & 13.3 & 1.2 & -6.1 \\
\hline 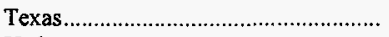 & 50,044 & 47,794 & 47,488 & 49,502 & 49,960 & 45,839 & 4.7 & * & 1.0 \\
\hline 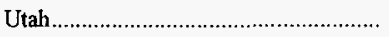 & 24,954 & 22,831 & 22,135 & 19,819 & 19,359 & 14,976 & 9.3 & 6.5 & 5.8 \\
\hline Virginia & 32,286 & 30,934 & 33,683 & 35,668 & 39,030 & 40,409 & 4.4 & -4.6 & -2.5 \\
\hline Washington & 4,142 & 4,416 & 4,439 & 4,300 & 4,764 & 4,036 & -6.2 & -3.4 & .3 \\
\hline 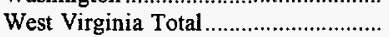 & 154,614 & 147,869 & 146,760 & 118,411 & 147,112 & 123,991 & 4.6 & $\begin{array}{r}-5.4 \\
1.3\end{array}$ & 2.5 \\
\hline 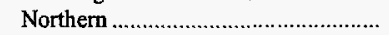 & 41,649 & 41,834 & 44,739 & 30,665 & 45,379 & 47,176 & -.4 & -2.1 & -1.4 \\
\hline Southem & 112,965 & 106,034 & 102,022 & 87,746 & 101,734 & 76,814 & 6.5 & 2.6 & 4.4 \\
\hline 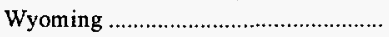 & 252,597 & 239,336 & 215,086 & 190,626 & 172,522 & 133,220 & 5.5 & 10.0 & 7.4 \\
\hline Appalachian Total ${ }^{1} \ldots \ldots \ldots \ldots \ldots \ldots \ldots \ldots$ & 409,928 & 394,499 & 404,033 & 371,690 & 414,189 & 402,138 & 3.9 & -.3 & .2 \\
\hline Interior Total ${ }^{1}$ & 156,805 & $152, ; 384$ & 163,164 & 151,658 & 177,499 & 182,946 & 2.6 & $-\mathbf{3 . 0}$ & -1.7 \\
\hline Western Total ${ }^{1}$ & 398,381 & 389,715 & 370,382 & 334,327 & 313,270 & 248,404 & 2.2 & 6.2 & 5.4 \\
\hline East of Miss. River & 511,351 & 493,732 & 513,729 & 468,306 & 533,946 & 527,917 & 3.6 & -1.1 & -.3 \\
\hline West of Miss. River ............................... & 453,763 & 443,367 & 423,851 & 389,369 & 371,012 & $\mathbf{3 0 5 , 5 7 0}$ & 2.3 & 5.2 & 4.5 \\
\hline U.S. Total.. & 965,114 & 937,098 & 937,580 & 857,675 & 904,958 & 833,487 & 3.0 & 1.6 & 1.6 \\
\hline
\end{tabular}

1 For a definition of coal-producing regions, see Appendix C.

* Data round to zero.

Notes: Coal production excludes silt, culm, refuse bank, slurry dam, and dredge operations except for Pennsylvania anthracite. Totals may not equal sum of components due to independent rounding.

Sources: Energy Information Administration, Form EIA-7A, "Coal Production Report"; State Mining Agency Coal Production Reports; and/or U.S. Department of Labor, Mine Safety and Health Administration, Form 7000-2, "Quarterly Mine Employment and Coal Production Report." 
Table B3. Productive Capacity of Coal Mines by State, 1987, 1992-1996 (Thousand Metric Tons)

\begin{tabular}{|c|c|c|c|c|c|c|c|c|c|}
\hline \multirow{2}{*}{$\begin{array}{l}\text { Coal-Producing } \\
\text { State and Region }\end{array}$} & \multirow{2}{*}{1996} & \multirow{2}{*}{1995} & \multirow{2}{*}{1994} & \multirow{2}{*}{1993} & \multirow{2}{*}{1992} & \multirow{2}{*}{19871} & \multirow{2}{*}{$\begin{array}{c}\text { Percent } \\
\text { Change } \\
\text { 1995-1996 }\end{array}$} & \multicolumn{2}{|c|}{ Average Annual Percent Change } \\
\hline & & & & & & & & 1992-1996 & $1987-1996$ \\
\hline Alabama & 29,174 & 29,526 & 29,982 & 25,325 & 27,048 & 26,480 & -1.2 & 1.9 & 1.1 \\
\hline 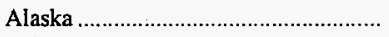 & $\mathrm{w}$ & w & $w$ & $w$ & w & $w$ & $w$ & $w$ & w \\
\hline Arizona & w & w & $w$ & $\mathrm{w}$ & $w$ & $w$ & $w$ & w & $w$ \\
\hline 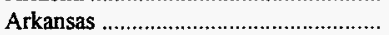 & - & w & w & $\mathbf{w}$ & $w$ & $w$ & $w$ & $\mathbf{w}$ & $\mathbf{w}$ \\
\hline 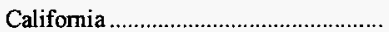 & - & - & - & - & $w$ & w & w & w & $\mathbf{w}$ \\
\hline 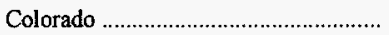 & 26,608 & 29,425 & 28,191 & 27,252 & 23,449 & 19,342 & -9.6 & 3.2 & 3.6 \\
\hline 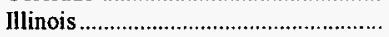 & 55,998 & 51,371 & 62,971 & 62,886 & 68,753 & 68,812 & 9.0 & -5.0 & -2.3 \\
\hline Indiana & 32,263 & 31,984 & 35,318 & 39,875 & 39,000 & 41,135 & 9 & -4.6 & -2.7 \\
\hline Iowa & - & - & w & w & w & w & $\mathrm{w}$ & $w$ & w \\
\hline 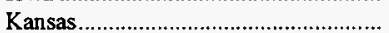 & $w$ & w & $\mathbf{w}$ & $\mathbf{w}$ & $\mathbf{w}$ & $w$ & $w$ & $w$ & w \\
\hline Kentucky Total & 171,662 & 184,316 & 193,618 & 185,796 & 177,220 & 168,722 & -6.9 & -.8 & .2 \\
\hline Eastern & 132,169 & 137,993 & 146,720 & 142,717 & 135,212 & 116,913 & -4.2 & -.6 & 1.4 \\
\hline Western & 39,493 & 46,323 & 46,897 & 43,079 & 42,008 & 51,810 & -14.7 & -1.5 & -3.0 \\
\hline 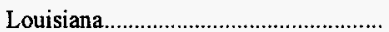 & $w$ & $w$ & $w$ & w & w & $w$ & w & w & $w$ \\
\hline Maryland & 4,477 & 3,999 & 3,930 & 3,563 & 3,539 & 4,216 & 11.9 & 6.0 & .7 \\
\hline 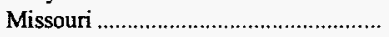 & 949 & 980 & 1,097 & w & w & w & -3.2 & w & -16.6 \\
\hline Montana & 50,961 & 46,808 & 46,361 & 46,129 & 44,073 & 38,871 & 8.9 & 3.7 & 3.0 \\
\hline 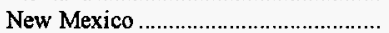 & 29,660 & 29,719 & 29,762 & 30,264 & 26,773 & 30,205 & -.2 & 26 & -2 \\
\hline North Dakota & 29,197 & 31,265 & 32,586 & 32,995 & 33,553 & 35,531 & -6.6 & -3.4 & -2.1 \\
\hline Ohio & 34,096 & 30,854 & 39,848 & 38,315 & 37,493 & 42,036 & 10.5 & -2.3 & -2.3 \\
\hline 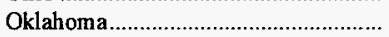 & 1,797 & 2,320 & 2,042 & 2,197 & 2,256 & 3,464 & -22.5 & -5.5 & -7.0 \\
\hline Pennsylvania Total & 74,102 & 70,023 & 73,460 & 74,524 & 75,267 & 78,712 & 5.8 & -.4 & -.7 \\
\hline Anthracite & 4,993 & 5,939 & 5,240 & 5,267 & 3,758 & 3,512 & -15.9 & 7.4 & 4.0 \\
\hline 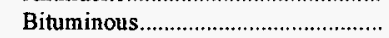 & 69,109 & 64,083 & 68,220 & 69,256 & 71,509 & 75,201 & 7.8 & -.8 & -.9 \\
\hline 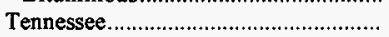 & 3,637 & 3,402 & 3,092 & 3,414 & 3,567 & 6,627 & 6.9 & .5 & -6.4 \\
\hline Texas & 54,072 & 49,676 & 50,672 & 51,814 & 53,108 & 46,843 & 8.8 & .4 & 1.6 \\
\hline Utah & 27,424 & 28,021 & 25,075 & 23,526 & 23,164 & 20,843 & -2.1 & 4.3 & 3.1 \\
\hline Virginia & 37,733 & 39,042 & 42,150 & 46,156 & 49,415 & 45,065 & -3.3 & -6.5 & -1.9 \\
\hline Washington & $\mathbf{w}$ & w & $w$ & $w$ & w & w & w & w & $w$ \\
\hline West Virginia Total & 197,230 & 185,825 & 182,964 & 173,913 & 179,697 & 143,658 & 6.1 & 2.3 & $\cdot 3.6$ \\
\hline Northern & 49,534 & 51,124 & 53,792 & 54,445 & 56,981 & 54,095 & -3.1 & -3.4 & -1.0 \\
\hline Southern & 147,696 & 134,701 & 129,172 & 119,468 & 122,716 & 89,563 & 9.6 & 4.7 & 5.7 \\
\hline Wyoming & 318,338 & 305,888 & 291,248 & 252,084 & 229,801 & 224,506 & 4.1 & 8.5 & 3.9 \\
\hline Appalachian Total 2 .. & 512,618 & 500,664 & 522,147 & 507,927 & 511,239 & 463,708 & $\mathbf{2 . 4}$ & .1 & 1.1 \\
\hline Interior Total 2 & 188,385 & 186,329 & 203,116 & 204,968 & 213,225 & 222,826 & 1.1 & -3.0 & -1.8 \\
\hline Western Total 2 & $\mathbf{5 0 0 , 7 5 7}$ & 491,489 & 472,817 & 431,858 & 401,401 & 387,033 & 1.9 & 5.7 & 2.9 \\
\hline East of Miss. River ................................ & 640,372 & 630,341 & 667,333 & 653,767 & 661,000 & 625,465 & 1.6 & -.8 & .3 \\
\hline West of Miss. River................................ & 561,387 & 548,141 & 530,746 & 490,986 & 464,865 & 448,102 & 2.4 & 4.8 & 2.5 \\
\hline 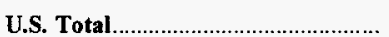 & $1,201,759$ & $1,178,482$ & $1,198,079$ & $1,144,753$ & $1,125,866$ & $1,073,567$ & 2.0 & 1.6 & 1.3 \\
\hline
\end{tabular}

1 For 1987, Form EIA-7A solicited data on "Daily Productive Capacity." To obtain annual productive capacity for a mine in 1987, each mine's daily productive capacity was multiplied by the number of days worked during the year.

2 For a definition of coal-producing regions, see Appendix C.

Notes: Productive capacity is the maximum amount of coal that can be produced as reported by mining companies on Form EIA-7A. Excludes silt culm, refuse bank, slurry dam, and dredge operations except for Pennsylvania anthracite. Excludes mines producing less than 10,000 short tons, which are not required to provide these data.

Source: Energy Information Administration, Form EIA-7A, "Coal Production Report." 
Table B4. Recoverable Coal Reserves at Producing Mines by State, 1987, 1992-1996 (Million Metric Tons)

\begin{tabular}{|c|c|c|c|c|c|c|c|c|c|}
\hline \multirow{2}{*}{$\begin{array}{l}\text { Coal-Producing } \\
\text { State and Region }\end{array}$} & \multirow{2}{*}{1996} & \multirow{2}{*}{1995} & \multirow{2}{*}{1994} & \multirow{2}{*}{1993} & \multirow{2}{*}{1992} & \multirow{2}{*}{1987} & \multirow{2}{*}{$\begin{array}{c}\text { Percent } \\
\text { Change } \\
1995-1996\end{array}$} & \multicolumn{2}{|c|}{ Average Annual Percent Change } \\
\hline & & & & & & & & $1992-1996$ & $1987-1996$ \\
\hline Alabama & 410 & 463 & 415 & 387 & 424 & 488 & -11.5 & -0.9 & -1.9 \\
\hline Alaska & $\mathbf{w}$ & w & w & $\mathbf{w}$ & w & w & w & w & $\mathbf{w}$ \\
\hline 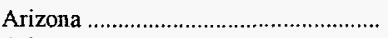 & $w$ & w & w & $\mathbf{w}$ & $\mathbf{w}$ & w & $w$ & $w$ & $w$ \\
\hline 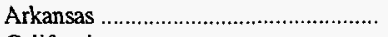 & - & $w$ & $w$ & $\mathbf{w}$ & $w$ & w & w & $\mathbf{w}$ & $\mathbf{w}$ \\
\hline 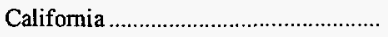 & - & - & - & - & w & w & w & w & w \\
\hline 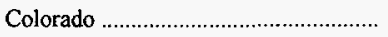 & 582 & 528 & 614 & 552 & 552 & 606 & -7.3 & 1.3 & -.4 \\
\hline 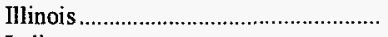 & 808 & 800 & 874 & 965 & 1,088 & 1,298 & 1.0 & -7.1 & -5.1 \\
\hline Indiana & 350 & 294 & 276 & 344 & 367 & 467 & 19.3 & -1.2 & -3.1 \\
\hline Iowa & - & - & $w$ & w & w & 33 & w & w & w \\
\hline 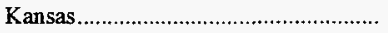 & w & w & $\mathbf{w}$ & w & w & w & w & w & w \\
\hline 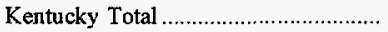 & 1,139 & 1,160 & 1,238 & 1,658 & 1,318 & 1,578 & -1.8 & -3.6 & -3.5 \\
\hline Eastern & 742 & 592 & 734 & 1,222 & 866 & 1,011 & 7.3 & -3.8 & -3.4 \\
\hline 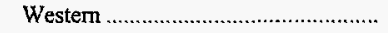 & 396 & 468 & 505 & 436 & 452 & 566 & -15.3 & -3.2 & -3.9 \\
\hline 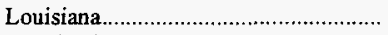 & w & w & $\mathbf{w}$ & w & w & $w$ & w & w & $w$ \\
\hline Maryland & 64 & 52 & 80 & 60 & 53 & 68 & 23.1 & 4.7 & -.7 \\
\hline Missouri & 2 & 2 & 11 & w & w & 140 & 13.1 & w & -36.4 \\
\hline Montana & 1,187 & 1,135 & 1,163 & 1,166 & 1,226 & 1,461 & 4.6 & -.8 & -2.3 \\
\hline New Mexico & 1,303 & 1,343 & 1,322 & 1,336 & 1,356 & 1,413 & -2.9 & -1.0 & -.9 \\
\hline 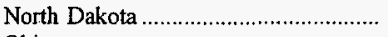 & 1,181 & 1,513 & 1,537 & 1,280 & 1,211 & 1,256 & -21.9 & -.6 & -.7 \\
\hline Ohio & 376 & 425 & 435 & 472 & 523 & 685 & -11.4 & -7.9 & -6.4 \\
\hline Oklahoma & 17 & 17 & 39 & 42 & 44 & 45 & -1.3 & -21.1 & -10.2 \\
\hline Pennsylvania Total ................................ & 722 & 668 & 828 & 852 & 850 & 1,242 & $8 . \mathrm{L}$ & -4.0 & -5.8 \\
\hline Anthracite & 81 & 45 & 34 & 59 & 64 & 55 & 81.4 & 6.3 & 4.5 \\
\hline 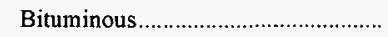 & 641 & 623 & 794 & 793 & 787 & 1,187 & 2.8 & -5.0 & -6.6 \\
\hline 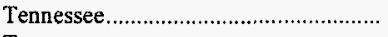 & 54 & 62 & 38 & 27 & 39 & 93 & -12.9 & 8.3 & -6.0 \\
\hline Texas & 797 & 853 & 931 & 1,002 & 1,078 & 1,102 & -6.5 & -7.3 & -3.5 \\
\hline 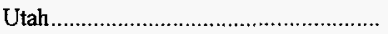 & 258 & 340 & 384 & 405 & 443 & 444 & -24.1 & -12.6 & -5.8 \\
\hline 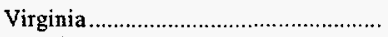 & 171 & 184 & 215 & 305 & 332 & 460 & -7.3 & -15.3 & -10.4 \\
\hline Washington & w & w & $\mathbf{w}$ & w & w & $\mathbf{w}$ & $\mathbf{w}$ & $w$ & $w$ \\
\hline West Virginia Total ................................ & 1,570 & 1,571 & 1,660 & 1,752 & 1,853 & 2,311 & $*$ & -4.0 & -4.2 \\
\hline 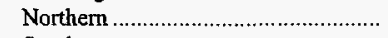 & 673 & 709 & 781 & 747 & 871 & 1,153 & -5.2 & -6.3 & -5.8 \\
\hline 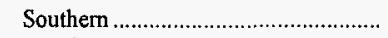 & 898 & 361 & 879 & 1,005 & 982 & 1,158 & 4.3 & -2.2 & -2.8 \\
\hline 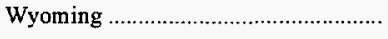 & 5,980 & 6,100 & 6,350 & 6,197 & 6,124 & 5,984 & -2.0 & -.6 & * \\
\hline Appalachian Total 1 & 4,110 & 4,117 & 4,404 & 5,076 & 4,941 & 6,358 & -.2 & -4.5 & -4.7 \\
\hline 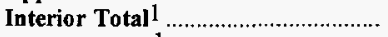 & 2,501 & 2,572 & 2,784 & 2,994 & 3,228 & 3,815 & -2.8 & -6.2 & -4.6 \\
\hline Western Total ${ }^{1}$ & 11,014 & 11,550 & 11,878 & 11,466 & 11,450 & 11,818 & -4.6 & -1.0 & -.8 \\
\hline 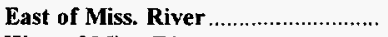 & 5,665 & 5,679 & 6,059 & 6,822 & 6,848 & 8,690 & -.2 & -4.6 & -4.6 \\
\hline West of Miss. River ............................. & 11,960 & 12,560 & 13,007 & 12,715 & 12,772 & 13,302 & -4.8 & -1.6 & -1.2 \\
\hline U.S. Total & 17,625 & 18,239 & 19,066 & 19,536 & 19,620 & 21,991 & -3.4 & -2.6 & -2.4 \\
\hline
\end{tabular}

1 For a definition of coal-producing regions, see Appenclix C.

* Data round to zero.

Notes: Recoverable reserves represent the quantity of coal that can be recovered (i.e., mined) from existing coal reserves at reporting mines. Excludes silt, culm, refuse bank, slurry dam, and dredge operations except for Pennsylvania anthracite. Excludes mines producing less than 10,000 short tons, which are not required to provide these data. Totals may not equal sum of components due to independent rounding.

Sources: Energy Information Administration, Form EIA-7A, "Coal Production Report"; State Mining Agency Coal Production Reports; and/or U.S. Department of Labor, Mine Safety and Health Administration, Form 7000-2, "Quarterly Mine Employment and Coal Production Report." 
Table B5. U.S. Coal Imports by Continent and Country of Origin, 1987, 1992-1996 (Metric Tons)

\begin{tabular}{|c|c|c|c|c|c|c|c|c|c|}
\hline \multirow{2}{*}{$\begin{array}{c}\text { Continent and Country } \\
\text { of Origin }\end{array}$} & \multirow{2}{*}{1996} & \multirow{2}{*}{1995} & \multirow{2}{*}{1994} & \multirow{2}{*}{1993} & \multirow{2}{*}{1992} & \multirow{2}{*}{1987} & \multirow{2}{*}{$\begin{array}{c}\text { Percent } \\
\text { Change } \\
1995-1996\end{array}$} & \multicolumn{2}{|c|}{ Average Annual Percent Change } \\
\hline & & & & & & & & 1992-1996 & $1987-1996$ \\
\hline North America Total............................. & $1,300,730$ & $1,219,813$ & $1,137,082$ & 955,786 & 926,102 & 498,312 & 6.6 & 8.9 & 11.2 \\
\hline 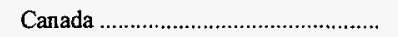 & $1,294,100$ & $1,197,249$ & $1,136,881$ & 953,696 & 926,102 & 459,727 & 8.1 & 8.7 & 12,2 \\
\hline 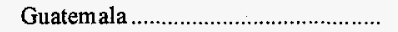 & - & - & - & 4 & - & - & - & - & - \\
\hline Mexico & 6,630 & 196 & 201 & 2,086 & - & 38,585 & NM & - & -17.8 \\
\hline Netherlands Antilles ............................... & - & 22,368 & - & - & - & - & -100.0 & - & - \\
\hline South America Total ............................ & $3,619,341$ & $4,157,892$ & $4,463,532$ & $4,912,696$ & $2,088,277$ & 895,361 & -12.9 & 14.7 & 16.8 \\
\hline Colombia & $2,292,279$ & $2,482,908$ & $3,075,043$ & $3,734,915$ & $1,599,502$ & 868,084 & -7.7 & 9.4 & 11.4 \\
\hline 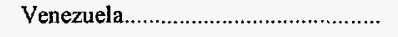 & $1,327,062$ & $1,674,984$ & $1,388,489$ & $1,177,781$ & 488,775 & 27,277 & -20.8 & 28.4 & 54.0 \\
\hline Europe Total & 2,369 & 474 & 36 & 56 & 81 & 218 & 399.8 & 132.5 & 30.3 \\
\hline Belgium \& Luxembourg ...................... & 2,243 & - & - & - & - & - & - & - & - \\
\hline 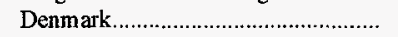 & - & 214 & - & 54 & - & - & -100.0 & - & - \\
\hline Poland & - & - & 36 & 2 & - & - & - & - & - \\
\hline Spain & 90 & - & - & - & - & 40 & - & - & 9.4 \\
\hline 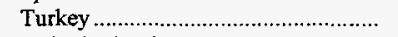 & 36 & - & - & - & - & - & - & - & - \\
\hline United Kingdom & - & 260 & - & - & 81 & 178 & -100.0 & -100.0 & -100.0 \\
\hline Asia Total & $1,392,520$ & 923,980 & $1,046,493$ & 642,359 & 338,513 & - & 50.7 & 42.4 & - \\
\hline 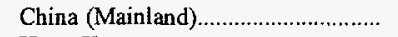 & - & 48 & 101 & - & 258 & - & -100.0 & -100.0 & - \\
\hline 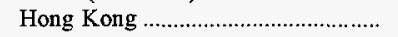 & 1 & - & - & - & 1 & - & - & - & - \\
\hline 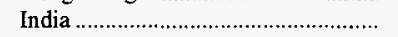 & - & - & - & - & 60,014 & - & - & -100.0 & - \\
\hline 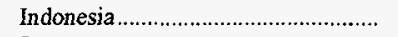 & $1,392,517$ & 923,908 & $1,025,543$ & 642,359 & 229,779 & - & 50.7 & 56.9 & - \\
\hline 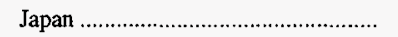 & 2 & 24 & 1 & - & - & - & -91.7 & - & - \\
\hline 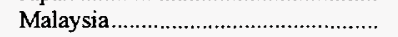 & - & - & - & - & 48,461 & - & - & -100.0 & - \\
\hline Vietnam & - & - & 20,848 & - & - & - & - & - & - \\
\hline Oceania \& Australia Total................. & 149,498 & 230,554 & 91,002 & 95,664 & 91,613 & 191,088 & -35.1 & 13.0 & -2.7 \\
\hline 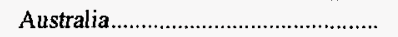 & 149,498 & 192,054 & 83,646 & 90,787 & 91,613 & 191,088 & -22.1 & 13.0 & -2.7 \\
\hline 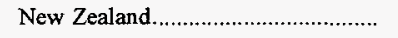 & - & 38,500 & 7,356 & 4,877 & - & - & -100.0 & - & - \\
\hline 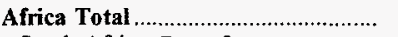 & - & - & 141,931 & 23,967 & 5,227 & - & - & -100.0 & - \\
\hline 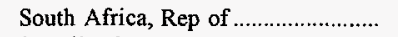 & - & - & 135,849 & 18,433 & 5,227 & - & - & -100.0 & - \\
\hline Swaziland & - & - & 6,082 & 5,534 & - & - & - & - & - \\
\hline Total & $6,464,458$ & $6,532,713$ & $6,880,076$ & $6,630,528$ & $3,449,813$ & $1,584,979$ & -1.0 & 17.0 & 16.9 \\
\hline
\end{tabular}

NM Not meaningful as value is greater than 500 percent.

Note: Coal imports include coal to Puerto Rico and the Virgin Islands.

Source: U.S. Department of Commerce, Bureau of the Census, "Monthly Report IM 145." 
Table B6. Coal Mining Productivity by State, 1987, 1992-1996

(Metric Tons of Coal Produced per Miner per Hour)

\begin{tabular}{|c|c|c|c|c|c|c|c|c|c|}
\hline \multirow{2}{*}{$\begin{array}{l}\text { Coal-Producing } \\
\text { State and Region }\end{array}$} & \multirow{2}{*}{1996} & \multirow{2}{*}{1995} & \multirow{2}{*}{1994} & \multirow{2}{*}{1993} & \multirow{2}{*}{1992} & \multirow{2}{*}{1987} & \multirow{2}{*}{$\begin{array}{c}\text { Percent } \\
\text { Change } \\
\text { 1995-1996 }\end{array}$} & \multicolumn{2}{|c|}{ Average Annual Percent Change } \\
\hline & & & & & & & & 1992-1996 & $1987-1996$ \\
\hline Alabama & 2.00 & 2.03 & 2.04 & 2.14 & 2.26 & 1.79 & -1.6 & -3.0 & 1.3 \\
\hline Alaska & 6.18 & 6.77 & 6.30 & 6.71 & 6.29 & 6.21 & -8.8 & -.4 & $*$ \\
\hline Arizona & 5.72 & 5.75 & 6.08 & 5.63 & 5.71 & 6.13 & -.6 & $*$ & -.8 \\
\hline 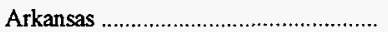 & - & 1.33 & 1.38 & 1.26 & 1.52 & 1.64 & -100.0 & - & - \\
\hline California & - & - & - & - & 10.09 & 4.71 & - & - & - \\
\hline 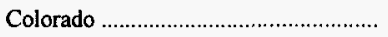 & 6.64 & 5.57 & 5.62 & 5.30 & 4.78 & 3.83 & 19.2 & 8.5 & 6.3 \\
\hline 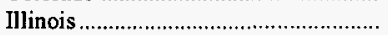 & 3.79 & 3.51 & 3.26 & 2.93 & 3.10 & 2.29 & 8.2 & 5.2 & 5.8 \\
\hline Indiana & 4.52 & 4.24 & 3.88 & 4.04 & 3.71 & 3.24 & 6.4 & 5.0 & 3.8 \\
\hline lowa & - & - & 1.38 & .65 & 1.03 & 1.27 & - & - & - \\
\hline 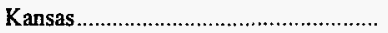 & 1.97 & 2.01 & 1.75 & 2.09 & 2.00 & 3.40 & -2.4 & -.5 & -5.9 \\
\hline 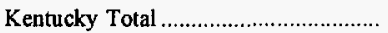 & 3.45 & 324 & 2.95 & 2.94 & 2.90 & 2.44 & 6.6 & 4.4 & 3.9 \\
\hline Eastern & 3.34 & 3.14 & 2.94 & 2.88 & 2.82 & 2.35 & 6.1 & 4.3 & 4.0 \\
\hline 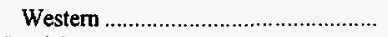 & 3.89 & 3.60 & 2.97 & 3.16 & 3.17 & 2.70 & 7.9 & 5.3 & 4.1 \\
\hline 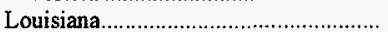 & 9.86 & 121.02 & 11.79 & 11.02 & 11.27 & 13.84 & -18.0 & -3.3 & -3.7 \\
\hline 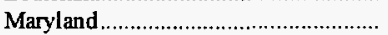 & 3.74 & 3.46 & 3.34 & 3.10 & 2.64 & 3.07 & 8.2 & 9.1 & 2.2 \\
\hline Missouri & 3.16 & 2.31 & 3.26 & 1.67 & 2.81 & 2.07 & 36.9 & 3.0 & 4.8 \\
\hline 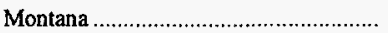 & 19.85 & 19.10 & 19.89 & 17.68 & 18.29 & 16.96 & 3.9 & 2.1 & 1.8 \\
\hline 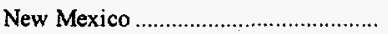 & 7.66 & 6.27 & 6.14 & 6.06 & 6.06 & 5.21 & 22.1 & 6.0 & 4.4 \\
\hline 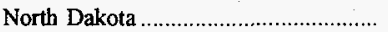 & 15.61 & 15.24 & 17.09 & 16.02 & 16.43 & 12.21 & 2.4 & -1.3 & 2.8 \\
\hline Ohio & 3.59 & 3.29 & 3.10 & 3.14 & 2.76 & 2.07 & 9.1 & 6.8 & 6.3 \\
\hline Oklahoma & 2.37 & 2.69 & 2.43 & 2.54 & 1.97 & 1.72 & -12.1 & 4.7 & 3.6 \\
\hline Pennsylvaria Total & 3.05 & 2.93 & 2.71 & 2.54 & 2.43 & 1.79 & 4.1 & 5.9 & 6.1 \\
\hline Anthracite & 1.74 & 1.89 & 1.75 & 1.68 & 1.21 & 1.03 & -8.0 & 9.5 & 6.0 \\
\hline Bituminous & 3.23 & 3.06 & 2.82 & 2.64 & 2.55 & 1.85 & 5.4 & 6.0 & 6.3 \\
\hline 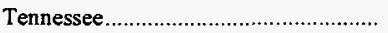 & 1.99 & 2.14 & 2.03 & 2.24 & 1.98 & 1.52 & -6.8 & .1 & 3.1 \\
\hline Texas & 9.19 & 8.25 & 8.00 & 7.63 & 6.66 & 5.85 & 11.3 & 8.4 & 5.1 \\
\hline 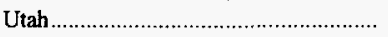 & 6.56 & 6.37 & 5.98 & 5.41 & 4.96 & 3.50 & 3.0 & 7.2 & 7.2 \\
\hline Virginia & 2.46 & 2.27 & 2.28 & 2.18 & 2.15 & 1.89 & 8.4 & 3.5 & 3.0 \\
\hline Washington & 3.60 & 3.67 & 3.73 & 3.62 & 4.09 & 2.78 & -1.8 & -3.1 & 2.9 \\
\hline 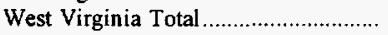 & 3.55 & 3.40 & 3.35 & 2.97 & 2.97 & 2.24 & 4.4 & 4.5 & 5.3 \\
\hline 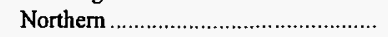 & 3.68 & 3.38 & 3.29 & 2.70 & 2.88 & 2.37 & 8.9 & 6.3 & 5.0 \\
\hline Southem & 3.50 & 3.40 & 3.37 & 3.07 & 3.01 & 2.16 & 2.8 & 3.8 & 5.5 \\
\hline Wyoming & 29.08 & 27.27 & 23.63 & 22.19 & 19.50 & 16.25 & 6.6 & 10.5 & 6.7 \\
\hline Appalachian Total 1 ............................ & 3.16 & 3.01 & 2.90 & 2.73 & 2.67 & 2.09 & 4.8 & 4.3 & 4.7 \\
\hline Interior Total 1 & 4.89 & 4.51 & 4.02 & 4.01 & 3.79 & 3.02 & 8.4 & 6.6 & 5.5 \\
\hline Western Totall & 15.79 & 14.22 & 13.23 & 12.27 & 11.55 & 9.45 & 11.0 & 8.1 & 5.9 \\
\hline 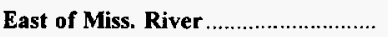 & 3.30 & 3.13 & 2.98 & 2.82 & 2.78 & 2.19 & 5.3 & 4.3 & 4.6 \\
\hline West of Miss. River............................... & 14.21 & 12.87 & 11.99 & 11.01 & 10.01 & 7.92 & 10.4 & 9.2 & 6.7 \\
\hline 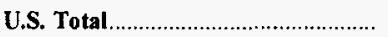 & 5.16 & 4.88 & 4.51 & 4.26 & 3.96 & 2.99 & 5.8 & 6.9 & 6.3 \\
\hline
\end{tabular}

1 For a definition of coal-producing regions, see Appendix C.

* Data round to zero.

Notes: Productivity is calculated by dividing total coal production by the total direct labor hours worked by all mine employees. Excludes silt, culm, refuse bank, slurry dam, and dredge operations except for Pennsylvania anthracite. Excludes mines producing less than 10,000 short tons, which are not required to provide these data.

Sources: Energy Information Administration, Form ElA-7A, "Coal Production Report"; State Mining Agency Coal Production Reports; and/or U.S. Department of Labor, Mine Safety and Health Administration, Form 7000-2, "Quarterly Mine Employment and Coal Production Report." 
Table B7. Coal Consumption by Census Division and State, 1987, 1992-1996 (Thousand Metric Tons)

\begin{tabular}{|c|c|c|c|c|c|c|c|c|c|}
\hline \multirow{2}{*}{ Census Division and State } & \multirow{2}{*}{1996} & \multirow{2}{*}{1995} & \multirow{2}{*}{1994} & \multirow{2}{*}{1993} & \multirow{2}{*}{1992} & \multirow{2}{*}{1987} & \multirow{2}{*}{$\begin{array}{c}\text { Percent } \\
\text { Change } \\
1995-1996\end{array}$} & \multicolumn{2}{|c|}{ Average Annual Percent Change } \\
\hline & & & & & & & & $1992-1996$ & $1987-1996$ \\
\hline New England Total & 6,372 & 6,043 & 5,945 & 5,883 & 6,620 & 6,139 & 5.4 & -0.9 & 0.4 \\
\hline Connecticut & 845 & 822 & 782 & 715 & 770 & 739 & 2.7 & 2.3 & 1.5 \\
\hline 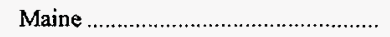 & 212 & 256 & 421 & 407 & 777 & 247 & -17.0 & -27.7 & -1.7 \\
\hline 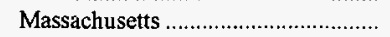 & 4,062 & 3,732 & 3,567 & 3,457 & 3,862 & 4,071 & 8.8 & 1.3 & * \\
\hline 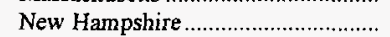 & 1,249 & 1,229 & 1,167 & 1,295 & 1,189 & 1,067 & 1.6 & 1.2 & 1.8 \\
\hline 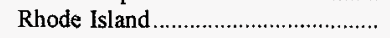 & 3 & 2 & 3 & 3 & 4 & 4 & 21.1 & -9.6 & -3.9 \\
\hline 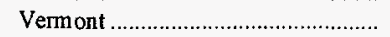 & 2 & 3 & 4 & 5 & 18 & 11 & -38.2 & -45.8 & -19.8 \\
\hline Middle Atlantic Total.......................... & 64,379 & 62,108 & 61,268 & 63,856 & 64,790 & 63,695 & 3.6 & -.1 & .1 \\
\hline 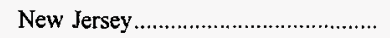 & 2,179 & 1,881 & 1,786 & 2,135 & 2,130 & 3,116 & 15.8 & .6 & -3.9 \\
\hline 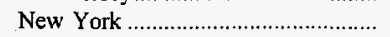 & 10,284 & 10,036 & 10,409 & 10,776 & 11,790 & 10,407 & 2.5 & -3.3 & -.1 \\
\hline 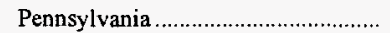 & 51,915 & 50,191 & 49,073 & 50,945 & 50,870 & 50,172 & 3.4 & .5 & .4 \\
\hline East North Central Total .................. & 207,746 & 197,496 & 193,401 & 191,082 & 182,035 & 183,100 & 5.2 & 3.3 & 1.4 \\
\hline 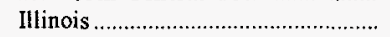 & 40,307 & 35,946 & 35,450 & 34,595 & 28,667 & 32,279 & 12.1 & 8.9 & 2.5 \\
\hline Indiana & 58,079 & 56,818 & 54,427 & 54,751 & 53,310 & 46,616 & 2.2 & 2.2 & 2.5 \\
\hline 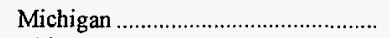 & 33,288 & 32,479 & 32,363 & 29,226 & 28,626 & 32,536 & 2.5 & 3.8 & 3 \\
\hline Ohio & 54,282 & 51,328 & 51,447 & 53,552 & 53,225 & 53,841 & 5.8 & .5 & .1 \\
\hline 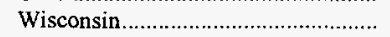 & 21,790 & 20,925 & 19,714 & 18,957 & 18,208 & 17,828 & 4.1 & 4.6 & 2.3 \\
\hline 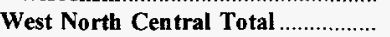 & 123,961 & 118,867 & 113,935 & 109,715 & 104,785 & 92,169 & 4.3 & 4.3 & 3.3 \\
\hline Iowa & 19,206 & 18,721 & 17,546 & 17,407 & 16,322 & 13,781 & 2.6 & 4.1 & 3.8 \\
\hline 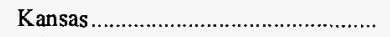 & 17,313 & 14,987 & 15,566 & 15,772 & 12,906 & 13,784 & 15.5 & 7.6 & 2.6 \\
\hline 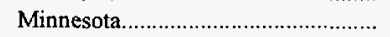 & 17,476 & 17,189 & 16,991 & 16,620 & 15,354 & 13,158 & 1.7 & 3.3 & 3.2 \\
\hline Missouri & 31,191 & 28,806 & 25,096 & 21,211 & 22,843 & 22,465 & 8.3 & 8.1 & 3.7 \\
\hline 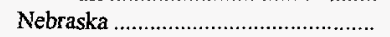 & 9,415 & 9,431 & 8,437 & 8,769 & 7,450 & 6,118 & -.2 & 6.0 & 4.9 \\
\hline 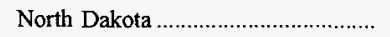 & 27,679 & 27,431 & 27,545 & 27,490 & 27,488 & 21,864 & .9 & .2 & 2.6 \\
\hline 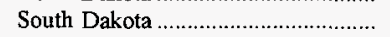 & 1,680 & 2,302 & 2,754 & 2,445 & 2,422 & 998 & -27.0 & -8.7 & 5.9 \\
\hline South Atlantic Total. & 150,180 & 140,849 & 137,833 & 136,604 & 130,796 & 132,965 & 6.6 & 3.5 & 1.4 \\
\hline 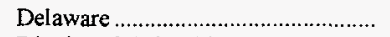 & 1,775 & 1,825 & 2,020 & 2,219 & 1,605 & 2,458 & -2.7 & 2.5 & -3.5 \\
\hline 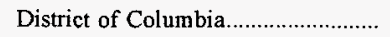 & 21 & 5 & 43 & 46 & 46 & 63 & 309.0 & -17.6 & -11.5 \\
\hline Florida & 25,803 & 24,064 & 23,661 & 23,977 & 23,921 & 21,450 & 7.2 & 1.9 & 2.1 \\
\hline 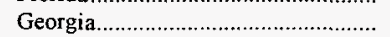 & 28,266 & 28,384 & 26,539 & 24,567 & 23,116 & 26,423 & -.4 & 5.1 & .8 \\
\hline 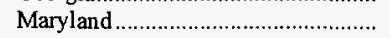 & 10,311 & 10,159 & 9,518 & 9,315 & 8,811 & 10,261 & 1.5 & 4.0 & $*$ \\
\hline 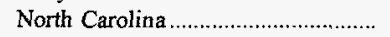 & 25,060 & 21,849 & 21,121 & 23,369 & 21,840 & 18,112 & 14.7 & 3.5 & 3.7 \\
\hline 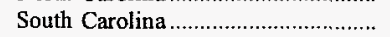 & 12,566 & 11,139 & 11,787 & 11,715 & 10,238 & 10,615 & 12.8 & 5.3 & 1.9 \\
\hline 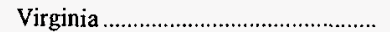 & 13,592 & 12,136 & 11,605 & 12,323 & 12,173 & 11,999 & 12.0 & 2.8 & 1.4 \\
\hline 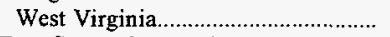 & 32,785 & 31,288 & 31,540 & 29,072 & 29,047 & 31,584 & 4.8 & 3.1 & .4 \\
\hline East South Central Total.................... & 100,199 & 96,008 & 90,074 & 94,371 & 85,097 & 80,016 & 4.4 & 4.2 & 2.5 \\
\hline Alabama & 33,613 & 31,124 & 28,552 & 29,980 & 28,585 & 24,160 & 8.0 & 4.1 & 3.7 \\
\hline Kentucky & 37,070 & 35,849 & 34,555 & 35,467 & 31,483 & 29,050 & 3.4 & 4.2 & 2.7 \\
\hline 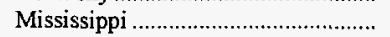 & 5,254 & 4,178 & 3,887 & 3,656 & 3,161 & 4,396 & 25.7 & 13.5 & 2.0 \\
\hline 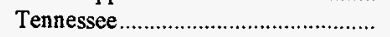 & 24,262 & 24,856 & 23,079 & 25,268 & 21,868 & 22,409 & -2.4 & 2.6 & 9 \\
\hline 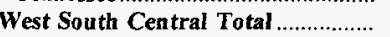 & 132,877 & 126,195 & 125,419 & 127,729 & 122,660 & 107,816 & 5.3 & 2.0 & 2.3 \\
\hline 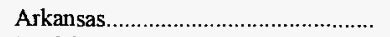 & 13,441 & 12,284 & 11,427 & 10,384 & 11,374 & 10,946 & 9.4 & 4.3 & 2.3 \\
\hline 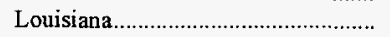 & 11,371 & 12,118 & 12,791 & 12,407 & 12,405 & 9,426 & -6.2 & -2.1 & 2.1 \\
\hline Oklahoma & 18,257 & 17,777 & 16,080 & 17,115 & 15,812 & 12,225 & 2.7 & 3.7 & 4.5 \\
\hline 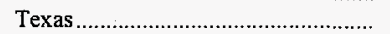 & 89,809 & 84,017 & 85,121 & 87,823 & 83,069 & 75,219 & 6.9 & 2.0 & 2.0 \\
\hline Mountain Total....................................... & 97,274 & 97,906 & 104,957 & 100,401 & 101,752 & 85,390 & -.6 & -1.1 & 1.4 \\
\hline Arizona & 15,234 & 15,134 & 17,763 & 17,228 & 16,252 & 12,134 & 6 & -1.6 & 2.6 \\
\hline Colorado & 15,623 & 15,395 & 15,853 & 15,485 & 15,146 & 13,614 & 1.5 & .8 & 1.5 \\
\hline Idaho & 360 & 421 & 484 & 1479 & 485 & 448 & -14.5 & -7.2 & -2.4 \\
\hline 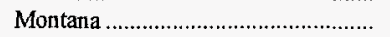 & 7,286 & 9,076 & 10,060 & 8,389 & 10,015 & 7,012 & -19.7 & -7.6 & 4 \\
\hline Nevada & 6,898 & 6,659 & 7,229 & 7,081 & 7,338 & 6,278 & 3.6 & -1.5 & 1.0 \\
\hline New Mexico & 13,877 & 13,809 & 13,947 & 13,619 & 13,455 & 13,059 & .5 & .8 & .7 \\
\hline 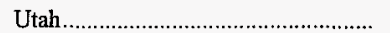 & 13,823 & 13,886 & 14,711 & 14,377 & 14,260 & 10,711 & -.4 & -.8 & 2.9 \\
\hline Wyoming & 24,173 & 23,526 & 24,910 & 23,742 & 24,801 & 22,134 & 2.8 & -.6 & 1.0 \\
\hline 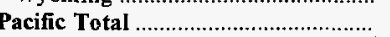 & 9,079 & 8,081 & 11,034 & 10,362 & 11,055 & 7,524 & 12.3 & -4.8 & 2.1 \\
\hline 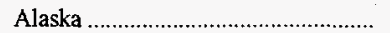 & 640 & 740 & 722 & 783 & 718 & 249 & -13.4 & -2.8 & 11.1 \\
\hline 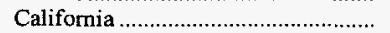 & 2,102 & 2,375 & 2,266 & 2,226 & 2,559 & 1,754 & -11.5 & -4.8 & 2.0 \\
\hline 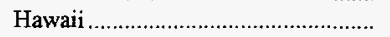 & 154 & 174 & 78 & 66 & 42 & 57 & -11.6 & 37.9 & 11.6 \\
\hline Oregon & 1,029 & 1,020 & 2,249 & 1,904 & 1,927 & 186 & .8 & -14.5 & 20.9 \\
\hline 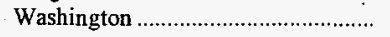 & 5,155 & 3,772 & 5,718 & 5,383 & 5,808 & 5,279 & 36.6 & -2.9 & -.3 \\
\hline U.S. Total & 892,066 & $\mathbf{8 5 3 , 5 5 2}$ & 843,865 & 840,003 & 809,591 & 759,187 & 4.5 & 2.4 & 1.8 \\
\hline
\end{tabular}

* Data tound to zero.

Note: U.S. Total does not include coal consumed by independent power producers. Totals may not equal sum of components due to independent rounding.

Sources: Energy Information Administration, Form EIA-759, "Monthly Power Plant Report"; Form EIA-3, "Quarterly Coal Consumption Report - Manufacturing Plants"; Form EIA-5, "Coke Plant Report - Quarterly"; and Form EIA-6, "Coal Distribution Report." 
Table B8. Year-End Consumer Coal Stocks by Census Division and State, 1987, 1992-1996 (Thousand Metric Tons)

\begin{tabular}{|c|c|c|c|c|c|c|c|c|c|}
\hline \multirow{2}{*}{ Census Division and State } & \multirow{2}{*}{1996} & \multirow{2}{*}{1995} & \multirow{2}{*}{1994} & \multirow{2}{*}{1993} & \multirow{2}{*}{1992} & \multirow{2}{*}{1987} & \multirow{2}{*}{$\begin{array}{c}\text { Percent } \\
\text { Change } \\
\text { 1995-1996 }\end{array}$} & \multicolumn{2}{|c|}{ Average Annual Percent Change } \\
\hline & & & & & & & & $1992-1996$ & $1987-1996$ \\
\hline New England Total............................... & 1,177 & 879 & 1,013 & 897 & 1,137 & 958 & 33.9 & 0.9 & 2.3 \\
\hline 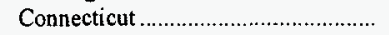 & $w$ & w & $w$ & w & w & w & $\mathbf{w}$ & $w$ & w \\
\hline Maine & $\mathbf{w}$ & w & $\mathrm{w}$ & w & w & $\mathbf{w}$ & w & $\mathbf{w}$ & $\mathbf{w}$ \\
\hline 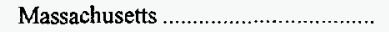 & $w$ & $w$ & w & $w$ & w & $\mathbf{w}$ & w & w & w \\
\hline 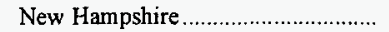 & $w$ & $w$ & $w$ & $w$ & $w$ & $\mathrm{w}$ & w & $\mathbf{w}$ & w \\
\hline 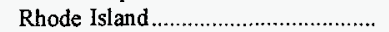 & - & - & - & - & - & w & - & - & - \\
\hline 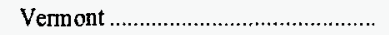 & - & - & - & - & - & w & - & - & - \\
\hline Middle Atlantic Total........................... & 9,887 & 11,253 & 12,762 & 12,755 & 17,678 & 17,779 & -12.1 & -13.5 & -6.3 \\
\hline 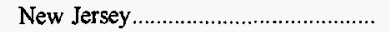 & $w$ & $w$ & w & w & w & w & w & w & w \\
\hline New York & w & w & w & w & w & w & w & w & w \\
\hline 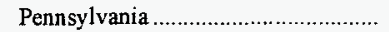 & 8,037 & 9,346 & 10,940 & 11,127 & 14,493 & 14,624 & -14.0 & -13.7 & -6.4 \\
\hline East North Central Total ................... & 27,962 & 30,679 & 32,507 & 27,363 & 39,609 & 47,024 & -8.8 & -8.3 & -5.6 \\
\hline 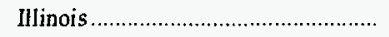 & $w$ & w & $w$ & $w$ & w & $\mathrm{w}$ & w & w & w \\
\hline 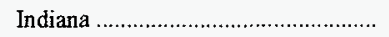 & 7,219 & 8,435 & 10,620 & 7,074 & 11,347 & 13,606 & -14.4 & -10.7 & -6.8 \\
\hline 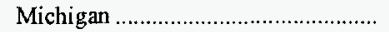 & w & $\mathbf{w}$ & w & w & w & w & w & $\mathbf{w}$ & w \\
\hline Ohio & 4,927 & 5,385 & 7,090 & 6,922 & 9,801 & 10,128 & -8.5 & -15.8 & -7.7 \\
\hline 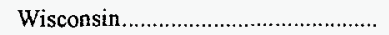 & 4,044 & 3,317 & $w$ & $w$ & $w$ & $\mathrm{w}$ & 21.9 & w & w \\
\hline West North Central Total ................... & 16,634 & 16,976 & $\mathbf{w}$ & $\mathbf{w}$ & $\mathbf{w}$ & $\mathbf{w}$ & -2.0 & $\mathbf{w}$ & $\mathbf{w}$ \\
\hline Iowa & 4,186 & 4,1934 & 3,790 & 3,465 & 4,406 & 4,621 & 3.8 & -1.3 & -1.1 \\
\hline 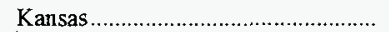 & 2,709 & 3,501 & 2,380 & 1,836 & 2,503 & 3,018 & -22.6 & 2.0 & -1.2 \\
\hline 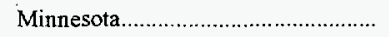 & 1,577 & 1,800 & 2,026 & 1,134 & 2,043 & 3,109 & -12.4 & -6.3 & -7.3 \\
\hline 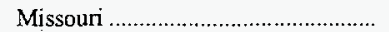 & 4,826 & 4,335 & w & $w$ & $w$ & $\mathbf{w}$ & 11.3 & w & w \\
\hline 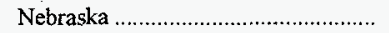 & $w$ & w & w & w & $w$ & w & w & $\mathbf{w}$ & w \\
\hline 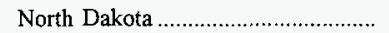 & $\mathbf{w}$ & w & $\mathrm{w}$ & $\mathbf{w}$ & $w$ & w & w & w & $w$ \\
\hline 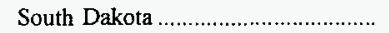 & $w$ & $w$ & $\mathrm{w}$ & w & w & w & w & $\mathbf{w}$ & $\mathrm{w}$ \\
\hline South Atlantic Total............................. & 17,766 & 17,378 & 22,160 & 17,320 & 26,565 & 29,066 & -.6 & -9.6 & -5.3 \\
\hline Delaware & $w$ & w & $w$ & $w$ & $w$ & $w$ & $w$ & $w$ & $w$ \\
\hline Florida & 3,120 & 2,965 & 3,550 & 3,212 & 3,739 & 2,992 & 5.3 & -4.4 & .5 \\
\hline Georgia & 3,491 & 3,435 & 4,394 & 2,655 & 4,387 & 6,089 & 1.6 & -5.5 & -6.0 \\
\hline 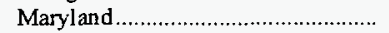 & $w$ & $\mathbf{w}$ & w & $w$ & $w$ & $\mathbf{w}$ & w & $\mathbf{w}$ & w \\
\hline North Carolina ........................................ & 2,424 & 2,590 & 3,917 & 2,776 & 4,277 & 4,349 & -6.4 & -13.2 & -6.3 \\
\hline 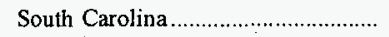 & 1,976 & 1,990 & 2,298 & 1,717 & 2,223 & 2,156 & -.7 & -2.9 & -1.0 \\
\hline 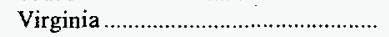 & w & w & $w$ & $w$ & w & w & w & w & w \\
\hline 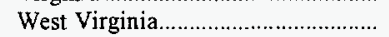 & $w$ & w & w & w & w & w & w & w & w \\
\hline East South Central Total.................... & 8,447 & 9,925 & 10,221 & 8,416 & 13,063 & 17,757 & -14.9 & -10.3 & -7.9 \\
\hline 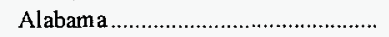 & 2,594 & 3,310 & 3,748 & 2,538 & 4,109 & 4,547 & -21.6 & -10.8 & -6.0 \\
\hline 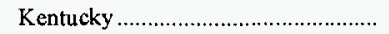 & w & w & $w$ & $w$ & w & w & w & w & $\mathbf{w}$ \\
\hline 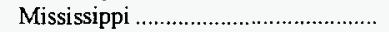 & w & w & w & w & $w$ & $w$ & w & w & w \\
\hline Tennessee & 1,362 & 1,709 & w & w & $w$ & w & -20.3 & w & w \\
\hline West South Central Total .................. & 18,048 & 18,656 & 14,478 & 13,703 & $\mathbf{w}$ & $\mathbf{w}$ & $-\mathbf{3 . 3}$ & $\mathbf{w}$ & $\mathbf{w}$ \\
\hline 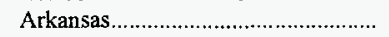 & 2,468 & 2,558 & 1,612 & 1,706 & 1,444 & 2,140 & -3.5 & 14,3 & 1.6 \\
\hline 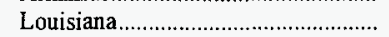 & 2,251 & 2,422 & 1,744 & 1,814 & 1,592 & 2,559 & -7.0 & 9.0 & -1.4 \\
\hline Oklahoma & 3,821 & 3,352 & 2,238 & 1,862 & 2,867 & 3,436 & -.8 & 7.4 & 1.2 \\
\hline 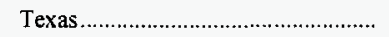 & 9,508 & 9,824 & 8,884 & 8,320 & w & w & -3.2 & $w$ & $\mathrm{w}$ \\
\hline 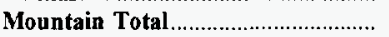 & $\mathbf{w}$ & w & $w$ & $\mathbf{w}$ & $\mathbf{w}$ & $\mathbf{w}$ & $\mathbf{w}$ & $\mathbf{w}$ & $\mathbf{w}$ \\
\hline 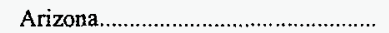 & 1,837 & 2,751 & 2,941 & 3,372 & 3,263 & 3,483 & -33.2 & -13.4 & -6.9 \\
\hline 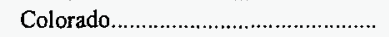 & 2,773 & 3,340 & 2,853 & 3,133 & 3,120 & $w$ & -17.0 & -2.9 & w \\
\hline Idaho & 70 & 07 & 71 & 78 & 92 & 105 & -34.4 & -6.5 & -4.4 \\
\hline 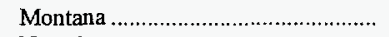 & $\mathbf{w}$ & $\mathbf{w}$ & w & w & w & w & w & $\mathbf{w}$ & w \\
\hline 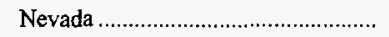 & $w$ & $w$ & $\mathbf{w}$ & w & $w$ & w & w & w & w \\
\hline 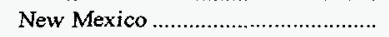 & $\mathbf{w}$ & $\mathbf{w}$ & $w$ & $w$ & $w$ & $w$ & w & $\mathbf{w}$ & w \\
\hline 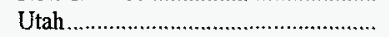 & w & w & w & w & w & w & w & $w$ & w \\
\hline 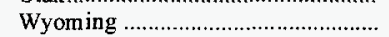 & 2,058 & 2,664 & 2,316 & 1,670 & 2,034 & 2,601 & -22.7 & 3 & -2.6 \\
\hline 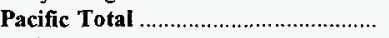 & 1,156 & 2,3146 & 796 & 833 & 1,387 & 1,334 & -50.7 & -4.4 & -1.6 \\
\hline 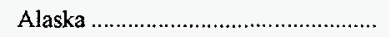 & 1 & 1 & 2 & 4 & w & $w$ & - & $w$ & $w$ \\
\hline 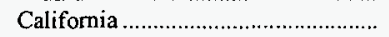 & 136 & 21 & 114 & 84 & 99 & 157 & 12.4 & 8.3 & -1.6 \\
\hline 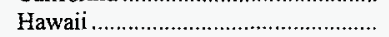 & w & $w$ & w & $w$ & $w$ & w & w & w & w \\
\hline Oregon & w & $w$ & $w$ & $w$ & $w$ & w & $w$ & $w$ & $\mathbf{w}$ \\
\hline 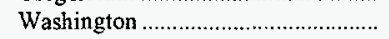 & 778 & 1,786 & 516 & 416 & 667 & 618 & -56.4 & 3.9 & 2.6 \\
\hline 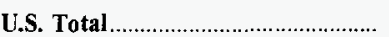 & 111,606 & $122,1.42$ & 123,504 & 109,278 & 148,499 & 168,246 & -8.6 & -6.9 & -4.4 \\
\hline
\end{tabular}

w Withheld to avoid disclosure of individual company data.

Notes: Totals may not equal sum of components due to independent rounding. Stocks for Residential and Commercial Sector are not inclúded. Sources: Energy Information Administration, Form EIA-759, "Monthly Power Plant Report"; Form EIA-5, "Coke Plant Report - Quarterly"; and Form EIA-3, "Quarterly Coal Consumption Report - Manufacturing Plants." 
Table B9. U.S. Coal Exports by Destination, 1987, 1992-1996 (Thousand Metric Tons)

\begin{tabular}{|c|c|c|c|c|c|c|c|c|c|}
\hline \multirow{2}{*}{$\begin{array}{l}\text { Continent and Country } \\
\text { of Destination }\end{array}$} & \multirow{2}{*}{1996} & \multirow{2}{*}{1995} & \multirow{2}{*}{1994} & \multirow{2}{*}{1993} & \multirow{2}{*}{1992} & \multirow{2}{*}{1987} & \multirow{2}{*}{$\begin{array}{c}\text { Percent } \\
\text { Change } \\
\text { 1995-1996 }\end{array}$} & \multicolumn{2}{|c|}{ Average Annual Percent Change } \\
\hline & & & & & & & & 1992-1996 & $1987-1996$ \\
\hline North America Total........................... & 12,346 & 9,444 & 8,623 & 8,363 & 13,908 & 14,936 & 30.7 & -2.9 & -2.1 \\
\hline Canada 1 & 10,912 & 8,552 & 8,340 & 8,064 & 13,734 & 14,721 & 27.6 & -5.6 & -3.3 \\
\hline 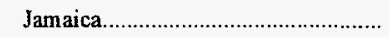 & 22 & 75 & 23 & 26 & 24 & - & -71.1 & -2.9 & - \\
\hline Mexico & 1,369 & 790 & 218 & 226 & 70 & 96 & 73.3 & 110.6 & 34.3 \\
\hline Other $^{2}$ & 44 & 27 & 41 & 47 & 80 & 120 & 62.5 & -13.8 & -10.5 \\
\hline South America Total............................ & 6,808 & 6,321 & 5,394 & 5,216 & 6,141 & 6,096 & 7.7 & 2.6 & 1.2 \\
\hline 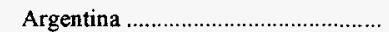 & 276 & 310 & 411 & 476 & 304 & 679 & -11.1 & -2.4 & -9.5 \\
\hline 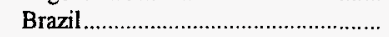 & 5,933 & 5,761 & 4,973 & 4,715 & 5,778 & 5,289 & 3.0 & .7 & 1.3 \\
\hline Chile & 521 & 206 & * & $*$ & 25 & 85 & 152.9 & 114.5 & 22.4 \\
\hline Other ${ }^{2}$ & 79 & 44 & 11 & 25 & 34 & 44 & 78.2 & 23.7 & 6.8 \\
\hline Europe Total & 42,813 & 44,107 & 32,500 & 34,088 & 51,941 & 30,989 & -2.9 & -4.7 & 3.6 \\
\hline 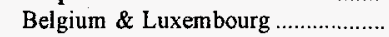 & 4,145 & 4,084 & 4,455 & 4,744 & 6,528 & 4,153 & 1.5 & -10.7 & * \\
\hline Bulgaria & 1,258 & 1,214 & 1,123 & 822 & 546 & - & 3.6 & 23.2 & - \\
\hline Denmark & 1,194 & 1,905 & 432 & 305 & 3,477 & 847 & -37.3 & -23.4 & 3.9 \\
\hline Finland & 638 & 1,187 & 342 & 229 & 186 & 156 & -46.2 & 36.1 & 17.0 \\
\hline France & 3,495 & 3,319 & 2,608 & 3,603 & 7,312 & 2,618 & 5.3 & -16.8 & 3.3 \\
\hline Germany, FR & 957 & 1,772 & 293 & 461 & 910 & 433 & -46.0 & 1.3 & 9.2 \\
\hline Ireland & 694 & 829 & 883 & 894 & 1,314 & 1,279 & -16.3 & -14.8 & -6.6 \\
\hline Italy & 8,350 & 8,222 & 6,843 & 6,276 & 8,476 & 8,652 & 1.6 & -.4 & -.4 \\
\hline 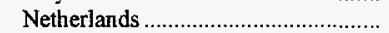 & 6,403 & 6,624 & 4,421 & 5,046 & 8,299 & 3,713 & -3.3 & -6.3 & 6.2 \\
\hline Norway & 77 & 109 & 79 & 92 & 107 & 157 & -29.2 & -7.9 & -7.6 \\
\hline 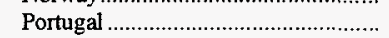 & 1,635 & 1,590 & 958 & 1,353 & 1,342 & 1,167 & 2.8 & 5.1 & 3.8 \\
\hline 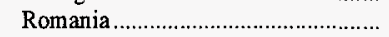 & 1,372 & 1,800 & 1,409 & 653 & 683 & 996 & -23.8 & 19.0 & 3.6 \\
\hline Spain & 3,713 & 4,221 & 3,748 & 3,687 & 4,114 & 2,227 & -12.0 & -2.5 & 5.8 \\
\hline 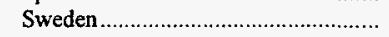 & 970 & 1,014 & 636 & 668 & 1,057 & 606 & -4.3 & -2.1 & 5.4 \\
\hline 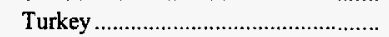 & 1,966 & 1,825 & 1,211 & 1,456 & 1,805 & 700 & 7.7 & 2.1 & 12.1 \\
\hline United Kingdom & 5,621 & 4,288 & 3,051 & 3,730 & 5,076 & 2,348 & 31.1 & 2.6 & 10.2 \\
\hline Yugoslavia, FR & - & 59 & - & 71 & 659 & 807 & -100.0 & -100.0 & -100.0 \\
\hline Other ${ }^{2}$ & 324 & 47 & 7 & $*$ & 49 & 129 & NM & 60.1 & 10.8 \\
\hline 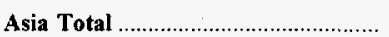 & 16,311 & 17,323 & 16,290 & 17,690 & 18,633 & 18,330 & -5.8 & -3.3 & -1.3 \\
\hline China (Taiwan) & 2,215 & 2,298 & 3,061 & 3,117 & 3,230 & 4,326 & -3.6 & -9.0 & -7.2 \\
\hline 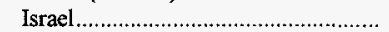 & 1,090 & 690 & 784 & 770 & 748 & 220 & 58.0 & 9.9 & 19.4 \\
\hline 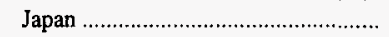 & 9,551 & 10,693 & 9,215 & 10,776 & 11,162 & 10,054 & -10.7 & -3.8 & -.6 \\
\hline 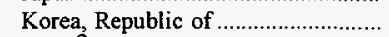 & 3,423 & 3,640 & 3,228 & 3,008 & 3,041 & 3,651 & -6.0 & 3.0 & -.7 \\
\hline Other ${ }^{2}$ & 32 & 2 & 3 & 20 & 454 & 79 & $\mathrm{NM}$ & -48.3 & -9.3 \\
\hline Oceania \& Australia Total................ & 1 & * & * & 1 & * & 1 & 322.4 & 31.6 & -6.0 \\
\hline Other ${ }^{2}$ & 1 & * & * & 1 & $*$ & 1 & 322.4 & 31.6 & -6.0 \\
\hline Africa Total & 3,796 & 3,133 & 1,927 & 2,245 & 2,378 & 1,865 & 21.2 & 12.4 & 8.2 \\
\hline Algeria & 160 & 200 & 322 & 371 & 555 & 826 & -19.8 & -26.7 & -16.6 \\
\hline 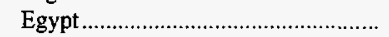 & 941 & 1,120 & 951 & 788 & 770 & 424 & -16.0 & 5.2 & 9.3 \\
\hline Morocco & 1,497 & 1,099 & 76 & 533 & 668 & 457 & 36.2 & 22.3 & 14.1 \\
\hline South Africa, Rep of & 1,197 & 713 & 578 & 515 & 385 & 56 & 67.9 & 32.8 & 40.5 \\
\hline Other $^{2}$ & - & - & - & 38 & - & 103 & - & - & -100.0 \\
\hline Total & 82,075 & 80,329 & 64,735 & 67,603 & 93,001 & 72,219 & 2.2 & -3.1 & 1.4 \\
\hline
\end{tabular}

1 Based on the U.S. - Canada Free Trade Agreement, as of January 1990, the U.S. Department of Commerce began reporting statistics on U.S. exports to Canada based on information on imports provided monthly by the Canadian government.

2 Includes countries with exports less than or equal to 50,000 short tons (45,359 metric tons) in 1995 .

* Data round to zero.

Not meaningful as value is greater than 500 percent.

Note: Total may not equal sum of components due to independent rounding.

Source: U.S. Department of Commerce, Bureau of the Census, "Monthly Report EM 545." 
Table B10. Average Mine Price of Coal by State, 1987, 1992-1996 (Nominal Dollars per Metric Ton)

\begin{tabular}{|c|c|c|c|c|c|c|c|c|c|}
\hline \multirow{2}{*}{$\begin{array}{l}\text { Coal-Producing } \\
\text { State and Region }\end{array}$} & \multirow{2}{*}{1996} & \multirow{2}{*}{1995} & \multirow{2}{*}{1994} & \multirow{2}{*}{1993} & \multirow{2}{*}{1992} & \multirow{2}{*}{1987} & \multirow{2}{*}{$\begin{array}{c}\text { Percent } \\
\text { Change } \\
1995-1996\end{array}$} & \multicolumn{2}{|c|}{ Average Annual Percent Change } \\
\hline & & & & & & & & $1992-1996$ & $1987-1996$ \\
\hline Alabama & $\$ 43.52$ & $\$ 42.38$ & $\$ 44.22$ & $\$ 46.67$ & $\$ 45.00$ & $\$ 45.66$ & 2.7 & -0.8 & -0.5 \\
\hline 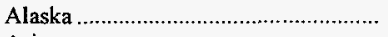 & $\mathbf{w}$ & $w$ & w & $\mathbf{w}$ & $w$ & w & $\mathbf{w}$ & $\mathbf{w}$ & w \\
\hline Arizona & $w$ & w & w & $\mathbf{w}$ & w & w & $\mathbf{w}$ & $\mathbf{w}$ & w \\
\hline 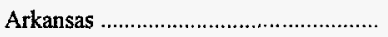 & - & $w$ & $w$ & w & $\mathbf{w}$ & w & $w$ & w & $w$ \\
\hline 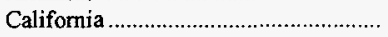 & - & - & - & - & w & w & w & w & w \\
\hline Colorado & $\$ 19.77$ & $\$ 21.23$ & $\$ 21.78$ & $\$ 22.44$ & $\$ 23.51$ & $\$ 25.99$ & -6.9 & -4.2 & -3.0 \\
\hline Illinois & 25.07 & 25.41 & 25.50 & 27.86 & 30.49 & 32.59 & -1.3 & -4.8 & -2.9 \\
\hline Indiana & 22.31 & 23.93 & 24.56 & 25.23 & 25.81 & 27.09 & -6.8 & -3.6 & -2.1 \\
\hline Iowa & - & - & $\mathbf{w}$ & $w$ & $w$ & 28.54 & w & w & $\mathbf{w}$ \\
\hline 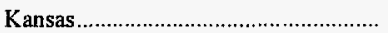 & w & w & w & w & w & 27.05 & w & $\mathbf{w}$ & w \\
\hline Kentucky Total & $\$ 26.36$ & $\$ 27.33$ & $\$ 27.42$ & $\$ 27.31$ & $\$ 27.01$ & 28.83 & -3.6 & -.6 & -1.0 \\
\hline Eastern & 27.54 & 28.66 & 27.83 & 28.11 & 27.55 & 29.45 & -3.9 & * & -.7 \\
\hline Western & 22.47 & 22.88 & 26.05 & 24.64 & 25.46 & 27.20 & -1.8 & -3.1 & -2.1 \\
\hline 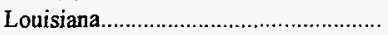 & w & w & w & w & w & w & w & w & w \\
\hline 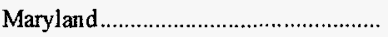 & $\$ 26.90$ & $\$ 27.22$ & $\$ 29.04$ & $\$ 27.79$ & $\$ 27.99$ & $\$ 26.79$ & -1.2 & -1.0 & * \\
\hline 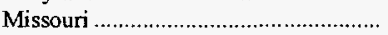 & 25.70 & 20.84 & 24.01 & $w$ & w & 31.87 & 23.3 & $w$ & -2.4 \\
\hline 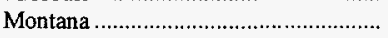 & 10.98 & 10.61 & 11.46 & $\$ 12.18$ & $\$ 11.24$ & 13.70 & 3.5 & -.6 & -2.4 \\
\hline New Mexico & 27.18 & 26.24 & 25.68 & 25.31 & 25.51 & 24,01 & 3.6 & 1.6 & 1.4 \\
\hline 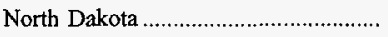 & 8.83 & 8.81 & 8.40 & 8.41 & 8.25 & 8.72 & .2 & 1.7 & .1 \\
\hline Ohio & 27.39 & 28.62 & 32.11 & 30.91 & 29.69 & 33.95 & -4.3 & -2.0 & -2.3 \\
\hline 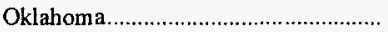 & 29.25 & 26.60 & 28.18 & 27.46 & 28.40 & 35.08 & 9.9 & .7 & -2.0 \\
\hline 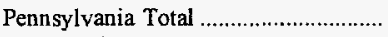 & 28.42 & 29.52 & 28.86 & 29.21 & 31.53 & 32.85 & -3.7 & -2.6 & -1.6 \\
\hline 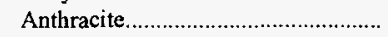 & 40.54 & 43.85 & 39.76 & 36.31 & 37.74 & 48.11 & -7.5 & 1.8 & -1.9 \\
\hline 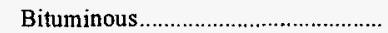 & 27.54 & 28.41 & 28.05 & 28.70 & 31.24 & 32.14 & -3.0 & -3.1 & -1.7 \\
\hline 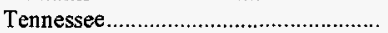 & 30.63 & 29.70 & 29.95 & 30.01 & 29.88 & 30.48 & 3.1 & .6 & * \\
\hline 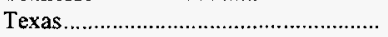 & 13.41 & 13.41 & 13.65 & 14.19 & 13.69 & 13.33 & $*$ & -.5 & .1 \\
\hline 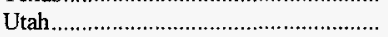 & 23.85 & 21.06 & 21.24 & 22.94 & 23.27 & 28.33 & 13.3 & .6 & -1.9 \\
\hline 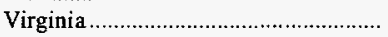 & 31.36 & 31.38 & 29.59 & 29.55 & 30.37 & 30.23 & -.1 & .8 & .4 \\
\hline 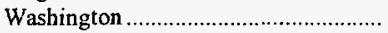 & w & w & w & w & w & $w$ & w & w & w \\
\hline 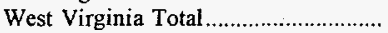 & $\$ 2.9 .30$ & $\$ 29.96$ & $\$ 30.23$ & $\$ 30.40$ & $\$ 31.03$ & $\$ 32.13$ & -2.2 & -1.4 & -1.0 \\
\hline 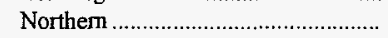 & 27.41 & 27.46 & 29.51 & 30.96 & 32.00 & 31.60 & -.2 & -3.8 & -1.6 \\
\hline 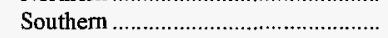 & 30.00 & 3094 & 30.54 & 30.20 & 30.60 & 32.45 & -3.1 & -.5 & -.9 \\
\hline Wyoming & 7.06 & 7.25 & 7.53 & 8.06 & 8.97 & 10.80 & -2.6 & -5.8 & -4.6 \\
\hline Appalachian Total ${ }^{1}$ & 29.52 & 30.25 & 30.16 & 30.47 & 30.81 & 32.19 & -2.4 & -1.1 & -.9 \\
\hline 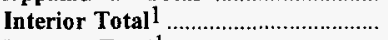 & 20.29 & 20.73 & 21.90 & 22.08 & 23.82 & 25.38 & -2.1 & -3.9 & -2.4 \\
\hline Western Total ${ }^{2}$ & 11.06 & 11.19 & 11.65 & 12.28 & 12.78 & 14.60 & -1.1 & -3.5 & -3.0 \\
\hline East of Miss. River & 28.33 & 29.04 & 29.15 & 29.56 & 30.13 & 31.54 & $-2,5$ & -1.5 & -1.2 \\
\hline West of Miss. River ........................... & 11.46 & 11.56 & 12.03 & 12.68 & 13.21 & 14.93 & -.8 & -3.5 & -2.9 \\
\hline U.S. Total & 20.39 & 20.76 & 21.40 & 21.88 & 23.18 & 25.43 & -1.8 & -3.1 & -2.4 \\
\hline
\end{tabular}

1 For a definition of coal-producing regions, see Appendix C

* Data round to zero.

Notes: Average mine price is calculated by dividing the total free on board (f.o.b.) mine value of the coal produced by the total production. A measure of dispersion of these average prices at the State level (interquartile range) is given in Appendix D, Table D2. Excludes silt, culm, refuse bank, slurry dam, and dredge operations except for Pennsylvania anthracite. Excludes mines producing less than 10,000 short tons, which are not required to provide these data.

Sources: Energy Information Administration, Form ElA-7A, "Coal Production Report”; State Mining Agency Coal Production Reports; and/or U.S. Department of Labor, Mine Safety and Health Administration, Form 7000-2, "Quarterly Mine Employment and Coal Production Report." 
Table B11. Average Price of Coal Delivered to Electric Utilities by Census Division and State, 1987, 1992-1996

(Nominal Dollars per Metric Ton)

\begin{tabular}{|c|c|c|c|c|c|c|c|c|c|}
\hline \multirow{2}{*}{ Census Division and State } & \multirow{2}{*}{1996} & \multirow{2}{*}{1995} & \multirow{2}{*}{1994} & \multirow{2}{*}{1993} & \multirow{2}{*}{1992} & \multirow{2}{*}{1987} & \multirow{2}{*}{$\begin{array}{c}\text { Percent } \\
\text { Change } \\
\text { 1995-1996 }\end{array}$} & \multicolumn{2}{|c|}{ Average Annual Percent Change } \\
\hline & & & & & & & & 1992-1996 & 1987-1996 \\
\hline New England Total............................. & $\$ 48.01$ & $\$ 47.78$ & $\$ 47.19$ & $\$ 47.78$ & $\$ 49.76$ & $\$ 51.76$ & 0.5 & -0.9 & -0.8 \\
\hline 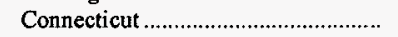 & 55.17 & 54.37 & 51.20 & 49.38 & 56.55 & 70.06 & 1.5 & -.6 & -2.6 \\
\hline 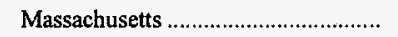 & 47.00 & 47.00 & 47.40 & 47.83 & 48.62 & 46.71 & $*$ & -.8 & .1 \\
\hline New Hampshire .................................. & 46.55 & 45.93 & 43.72 & 46.73 & 49.26 & 56.73 & 1.3 & -1.4 & -2.2 \\
\hline 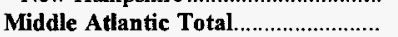 & 38.67 & 38.17 & 40.04 & 40.41 & 41.41 & 40.34 & 1.3 & -1.7 & -.5 \\
\hline 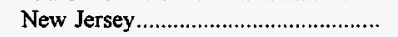 & 50.18 & 52.00 & 53.45 & 52.36 & 51.39 & 51.59 & -3.5 & -.6 & -.3 \\
\hline 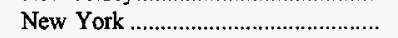 & 40.95 & 40.63 & 41.49 & 42.58 & 42.57 & 43.49 & .8 & -1.0 & -.7 \\
\hline 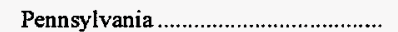 & 37.55 & 36.91 & 39.01 & 39.39 & 40.58 & 39.05 & 1.7 & -1.9 & -.4 \\
\hline East North Central Total ..................... & 31.18 & 32.70 & 33.69 & 34.15 & 35.33 & 40.37 & -4.6 & -3.1 & -2.8 \\
\hline 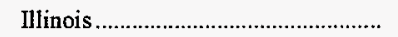 & 35.43 & 35.91 & 36.04 & 38.92 & 40.85 & 47.18 & -1.3 & -3.5 & -3.1 \\
\hline Indiana & 27.20 & 28.59 & 29.54 & 29.47 & 30.75 & 35.19 & -4.9 & -3.0 & -2.8 \\
\hline Michigan & 32.34 & 34.12 & 36.26 & 36.57 & 37.73 & 44.70 & -5.2 & -3.8 & -3.5 \\
\hline Ohio & 35.61 & 37.96 & 38.25 & 37.54 & 37.92 & 40.95 & -6.2 & -1.5 & -1.5 \\
\hline 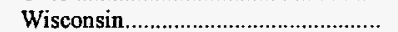 & 21.55 & 23.40 & 25.50 & 25.31 & 28.57 & 31.25 & -7.9 & -6.8 & -4.0 \\
\hline West North Central Total ................... & 17.12 & 17.75 & 18.47 & 18.60 & 20.86 & 23.40 & -3.6 & -4.8 & $-3,4$ \\
\hline lowa & 17.96 & 18.88 & 19.17 & 19.32 & 21.58 & 25.15 & -4.8 & -4.5 & -3.7 \\
\hline 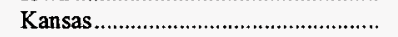 & 19.30 & 19.66 & 19.68 & 19.49 & 23.14 & 24.49 & -1.8 & -4.4 & -2.6 \\
\hline 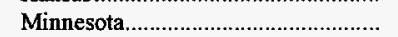 & 20.94 & 22.18 & 22.14 & 22.12 & 23.10 & 23.24 & -5.6 & -2.4 & -1.1 \\
\hline 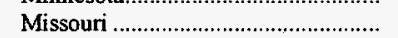 & 19.08 & 20.00 & 23.58 & 26.90 & 30.39 & 33.02 & -4.6 & -11.0 & -5.9 \\
\hline 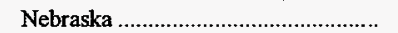 & 13.64 & 14.18 & 14.45 & 14.24 & 14.07 & 17.92 & -3.8 & -.8 & -3.0 \\
\hline 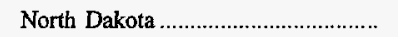 & 10.71 & 10.64 & 10.23 & 10.34 & 10.42 & 11.53 & .7 & .7 & -.8 \\
\hline 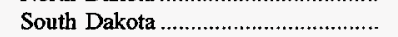 & 18.67 & 15.82 & 14.44 & 14.66 & 15.07 & 16.48 & 18.0 & 5.5 & 1.4 \\
\hline South Atlantic Total............................... & 40.43 & 42.17 & $\mathbf{4 3 . 5 7}$ & 44.98 & 45.50 & 45.75 & -4.1 & -2.9 & -1.4 \\
\hline Delaware & 45.76 & 46.60 & 46.28 & 48.53 & 49.95 & 52.18 & -1.8 & -2.2 & -1.4 \\
\hline 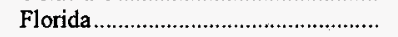 & 46.74 & 48.42 & 48.18 & 48.04 & 49.63 & 49.58 & -3.5 & -1.5 & -.6 \\
\hline 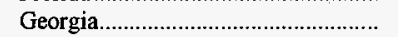 & 40.28 & 42.57 & 43.89 & 47.72 & 47.80 & 48.02 & -5.4 & -4.2 & -1.9 \\
\hline 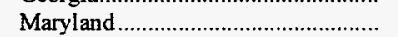 & 42.43 & 42.99 & 43.92 & 44.95 & 44.84 & 44.06 & -1.3 & -1.4 & -.4 \\
\hline 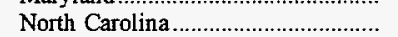 & 40.65 & 44.72 & 46.04 & 46.69 & 47.40 & 49.51 & -9.1 & -3.8 & -2.2 \\
\hline South Carolina & 41.38 & 42.83 & 43.92 & 44.28 & 43.14 & 48.51 & -3.4 & -1.0 & -1.8 \\
\hline 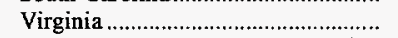 & 39.38 & 40.68 & 40.84 & 41.41 & 41.67 & 44.66 & -3.2 & -1.4 & -1.4 \\
\hline 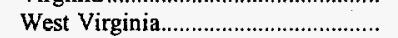 & 34.09 & 34.84 & 38.25 & 39.05 & 40.65 & 74.31 & -2.1 & -4.3 & -101.5 \\
\hline East South Central Total. & 32.35 & 33.16 & 35.75 & 36.71 & 36.43 & 39.62 & -2.4 & -2.9 & -2.2 \\
\hline 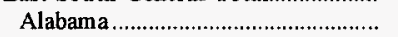 & 40.11 & 40.78 & 44.55 & 46.91 & 45.93 & 51.86 & -1.6 & -3.3 & -2.8 \\
\hline Kentucky & 26.93 & 28.34 & 29.94 & 30.08 & 29.77 & 31.90 & -5.0 & -2.5 & -1.9 \\
\hline 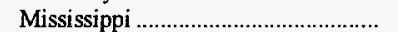 & 36.71 & 37.92 & 39.18 & 44.66 & 44.02 & 54.63 & -3.2 & -4.4 & -4.3 \\
\hline 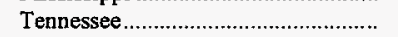 & 30.46 & 30.80 & 33.75 & 34.11 & 34.18 & 36.09 & -1.1 & -2.8 & -1.9 \\
\hline West South Central Total .................. & 22.19 & 22.78 & 22.91 & 24.40 & 24.86 & 26.37 & -2.6 & -2.8 & -1.9 \\
\hline 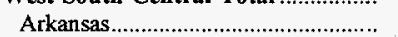 & 28.83 & 30.85 & 30.76 & 32.52 & 31.79 & 29.70 & -6.5 & -2.4 & -.3 \\
\hline 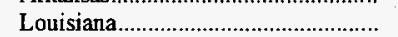 & 27.27 & 27.70 & 27.61 & 28.27 & 27.48 & 29.58 & -1.5 & -.2 & -.9 \\
\hline Oklahoma & 18.51 & 18.74 & 19.29 & 23.50 & 23.66 & 30.38 & -1.2 & -5.9 & -5.3 \\
\hline 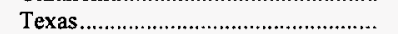 & 21.24 & 21.66 & 21.86 & 23.05 & 23.79 & 24.78 & -1.9 & -2.8 & -1.7 \\
\hline 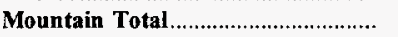 & 24.05 & 23.71 & 24.07 & 24.37 & 23.85 & 24.26 & 1.5 & .2 & -.1 \\
\hline 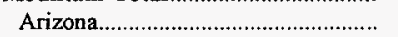 & 32.57 & 31.59 & 31.15 & 30.63 & 31.20 & 30.38 & 3.1 & 1.1 & .8 \\
\hline 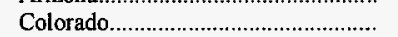 & 22.31 & 22.85 & 23.16 & 23.80 & 23.88 & 24.26 & -2.4 & -1.7 & -.9 \\
\hline Montana & 13.12 & 12.64 & 12.99 & 12.98 & 13.38 & 12.26 & 3.8 & -.5 & .7 \\
\hline Nevada & 33.55 & 31.99 & 35.68 & 35.64 & 35.63 & 34.48 & 4.9 & -1.5 & -.3 \\
\hline New Mexico & 28.71 & 28.21 & 28.08 & 27.12 & 26.27 & 24.11 & 1.8 & 2.2 & 2.0 \\
\hline 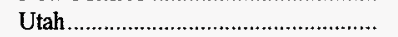 & 27.18 & 27.85 & 28.77 & 30.13 & 30.35 & 32.07 & -2.4 & -2.7 & -1.8 \\
\hline 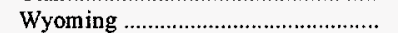 & 15.76 & 15.75 & 15.53 & 15.47 & 14.79 & 16.83 & .1 & 1.6 & -.7 \\
\hline Pacific Total & 26.42 & 25.16 & 24.18 & 23.75 & 24.44 & 29.56 & 5.0 & 2.0 & -1.2 \\
\hline Oregon & 20.73 & 20.72 & 21.14 & 21.77 & 23.40 & 26.22 & .1 & -3.0 & -2.6 \\
\hline 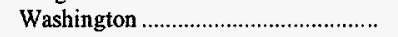 & 27.46 & 26.17 & 25.27 & 24.35 & 24.78 & 29.85 & 4.9 & 2.6 & -.9 \\
\hline U.S. Total & 29.16 & 29.77 & 30.89 & 31.51 & 32.36 & 35.09 & -2.1 & -2.6 & -2.0 \\
\hline
\end{tabular}

* Data round to zero.

Note: Average prices are based on the cost including insurance and freight.

Source: Federal Energy Regulatory Commission, FERC Form 423, "Monthly Report of Cost and Quality of Fuels for Electric Plants." 
Table B12. Average Price of Coal Delivered to Other Industrial Plants by Census Division and State, 1987, 1992-1996

(Nominal Dollars per Metric Ton)

\begin{tabular}{|c|c|c|c|c|c|c|c|c|c|}
\hline \multirow{2}{*}{ Census Division and State } & \multirow{2}{*}{1996} & \multirow{2}{*}{1995} & \multirow{2}{*}{1994} & \multirow{2}{*}{1993} & \multirow{2}{*}{1992} & \multirow{2}{*}{1987} & \multirow{2}{*}{$\begin{array}{c}\text { Percent } \\
\text { Change } \\
1995-1996\end{array}$} & \multicolumn{2}{|c|}{ Average Annual Percent Change } \\
\hline & & & & & & & & 1992-1996 & $1987-1996$ \\
\hline New England Total & $\$ 63.22$ & $\$ 6: .72$ & $\$ 61.44$ & $\$ 63.75$ & $\$ 72.43$ & $\$ 65.29$ & 0.8 & -3.3 & -0.3 \\
\hline 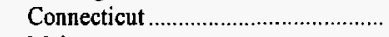 & - & - & - & - & - & w & $w$ & w & w \\
\hline 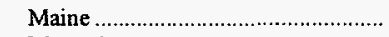 & w & $w$ & $w$ & $w$ & $w$ & $\mathbf{w}$ & $\mathbf{w}$ & w & $w$ \\
\hline 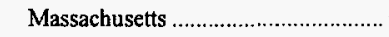 & w & $\mathbf{w}$ & w & $w$ & $w$ & $w$ & w & $w$ & $\mathbf{w}$ \\
\hline New Hampshire ................................... & - & - & - & - & - & $w$ & $w$ & $w$ & w \\
\hline Vermont & - & - & - & - & - & $w$ & w & w & $w$ \\
\hline Middle Atlantic Total.............................. & $\mathbf{w}$ & $\mathbf{w}$ & $\mathbf{w}$ & $\mathbf{w}$ & $w$ & $\mathbf{w}$ & $\mathbf{w}$ & $\mathbf{w}$ & $\mathbf{w}$ \\
\hline 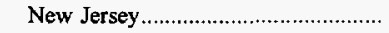 & w & w & $\mathbf{w}$ & w & $\mathbf{w}$ & w & w & w & $w$ \\
\hline 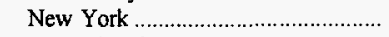 & 44.21 & 46.19 & 46.52 & 46.47 & 48.44 & 48.66 & -4.3 & -2.3 & -1.0 \\
\hline 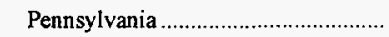 & 37.30 & 37.56 & 37.10 & 37.53 & 39.35 & 40.36 & -.7 & -1.3 & -.9 \\
\hline East North Central Total .................... & 37.96 & 38.45 & 38.27 & 38.07 & 38.64 & 39.38 & -1.3 & -.4 &.- .4 \\
\hline Mllinois & 32.72 & 32.00 & 32.11 & 32.43 & 32.23 & 36.54 & 2.3 & .4 & -1.2 \\
\hline Indiana & 35.01 & 36.53 & 34.56 & 34.07 & 34.81 & 34.11 & -4.2 & .1 & .3 \\
\hline 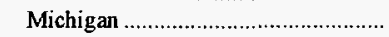 & 45.50 & 45.39 & 45.42 & 45.70 & 46.49 & 47.49 & .2 & -.5 & -.5 \\
\hline Ohio & 38.89 & 38.78 & 39.40 & 38.38 & 38.84 & 37.46 & .3 & * & .4 \\
\hline 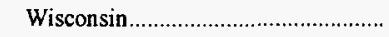 & 44.12 & 44.32 & 45.45 & 45.02 & 46.60 & 48.49 & -.4 & -3 & -1.0 \\
\hline West North Central Total ................. & 21.00 & 20.86 & 20.52 & 19.84 & 19.57 & 19.42 & .6 & 1.8 & .9 \\
\hline lowa & 32.32 & $32 ! .23$ & 31.44 & 30.87 & 29.94 & 38.44 & .3 & 1.9 & -1.9 \\
\hline 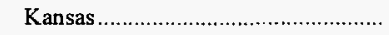 & 35.78 & 34.74 & 35.55 & 36.44 & 35.23 & 38.01 & .1 & .4 & -.7 \\
\hline 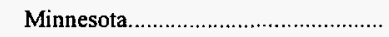 & 31.80 & 37.92 & 39.31 & 39.47 & 39.27 & 41.23 & -16.1 & -5.1 & -2.8 \\
\hline 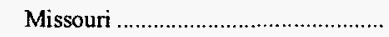 & 34.58 & 36.17 & 36.24 & 35.40 & 34.70 & 32.32 & -4.4 & -.1 & .8 \\
\hline 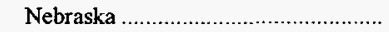 & w & $w$ & $w$ & w & w & w & w & w & $w$ \\
\hline 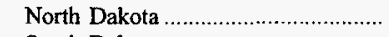 & $w$ & $w$ & $\mathbf{w}$ & $w$ & $w$ & w & $w$ & $w$ & w \\
\hline South Dakota & $w$ & $w$ & $\mathbf{w}$ & $w$ & $\mathbf{w}$ & w & $\mathbf{w}$ & $w$ & $w$ \\
\hline South Atlantic Total. & $\mathbf{w}$ & $\mathbf{w}$ & $\mathbf{w}$ & $\mathbf{w}$ & $\mathbf{w}$ & $\mathbf{w}$ & $\mathbf{w}$ & $\mathbf{w}$ & $\mathbf{w}$ \\
\hline 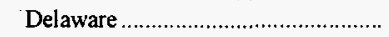 & w & w & w & w & w & w & w & w & w \\
\hline Florida & 50.36 & 51.41 & 51.37 & 53.22 & 53.20 & 51.47 & -2.0 & -1.4 & -.2 \\
\hline 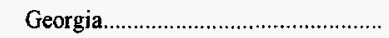 & 48.73 & $4 ! .21$ & 50.39 & 49.82 & 49.80 & 48.14 & -1.0 & -.5 & .1 \\
\hline 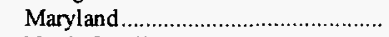 & 35.85 & 34.90 & 35.47 & 35.47 & 36.18 & 35.68 & 2.7 & -.2 & $*$ \\
\hline North Carolina ...................................... & 47.80 & $4: .72$ & 48.08 & 47.89 & 47.91 & 48.26 & .2 & * & -.1 \\
\hline South Carolina & 48.59 & 47.58 & 48.32 & 47.78 & 47.74 & 46.99 & 2.1 & .4 & .4 \\
\hline 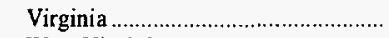 & 47.96 & 46.85 & 45.82 & 45.49 & 45.16 & 42.90 & 2.4 & 1.5 & 1.2 \\
\hline 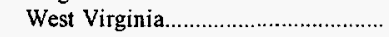 & 36.78 & $3 \% .05$ & 36.07 & 36.28 & 35.20 & 34.60 & -.7 & 1.1 & .7 \\
\hline East South Central Total. .................... & $w$ & $\mathbf{w}$ & $\mathbf{w}$ & $\mathbf{w}$ & w & $\mathbf{w}$ & $\mathbf{w}$ & $\mathbf{w}$ & $\mathbf{w}$ \\
\hline Alabama & 44.26 & 43.58 & 42.70 & 43.00 & 43.83 & 44.51 & 1.5 & .2 & -.1 \\
\hline 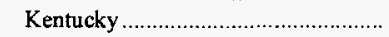 & 48.52 & 48.60 & 47.64 & 46.63 & 48.26 & 47.53 & -.2 & .1 & .2 \\
\hline 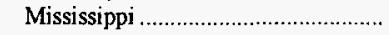 & $w$ & w & w & w & $w$ & w & w & $w$ & $w$ \\
\hline 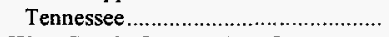 & 38.82 & 39.33 & 38.96 & 39.04 & 39.16 & 39.11 & -1.3 & -.2 & -.1 \\
\hline West South Central Total .................. & 24.02 & 24.29 & 25.29 & 23.57 & 25.13 & $\mathbf{w}$ & -1.1 & -1.1 & $\mathbf{w}$ \\
\hline 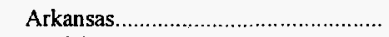 & 47.67 & 47.97 & 48.81 & 48.57 & 49.18 & 49.81 & -.6 & -.8 & -.5 \\
\hline 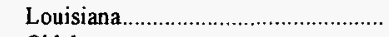 & w & w & w & w & w & w & w & w & w \\
\hline 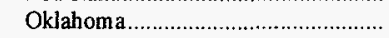 & $w$ & $\mathrm{w}$ & w & w & $w$ & 45.54 & $w$ & w & $\mathbf{w}$ \\
\hline 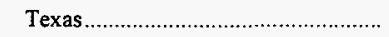 & 20.93 & 20.68 & 21.54 & 19.38 & 19.99 & 21.16 & 1.2 & 1.1 & -.1 \\
\hline Mountain Total & 29.44 & 29.83 & 31.73 & 31.43 & 31.59 & 31.67 & -1.3 & -1.7 & -.8 \\
\hline 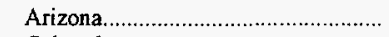 & 43.29 & 44.60 & 45.58 & 44.65 & 45.14 & 42.62 & -2.9 & -1.0 & .2 \\
\hline 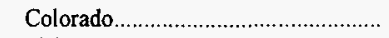 & 25.54 & 28.78 & 31.92 & 31.56 & 33.44 & 31.73 & -11.2 & -6.5 & -2.4 \\
\hline 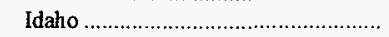 & 40.11 & 37.60 & 36.76 & 36.13 & 36.70 & 38.62 & 6.7 & 2.2 & .4 \\
\hline 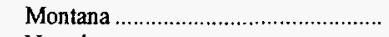 & w & w & $\mathbf{w}$ & w & w & $w$ & w & w & w \\
\hline 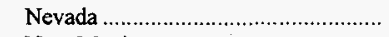 & $\mathbf{w}$ & w & w & $\mathbf{w}$ & $\mathbf{w}$ & $\mathrm{w}$ & $\mathrm{w}$ & w & $w$ \\
\hline 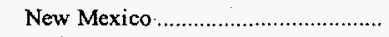 & $w$ & w & $w$ & w & w & w & w & w & $w$ \\
\hline 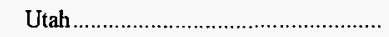 & 21.06 & 21.76 & 29.29 & 29.23 & 27.94 & 26.29 & -3.2 & -6.8 & -2.4 \\
\hline 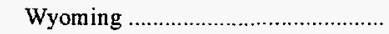 & 24.61 & 25.05 & 25.21 & 25.83 & 26.09 & 26.02 & -1.8 & -1.4 & -.6 \\
\hline 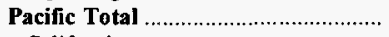 & 46.79 & 48.15 & 49.51 & 48.31 & 47.80 & 52.61 & -2.8 & -.5 & -1.3 \\
\hline California & 43.58 & 45.32 & 47.83 & 47.25 & 46.35 & 53.61 & -3.8 & -1.5 & -2.3 \\
\hline 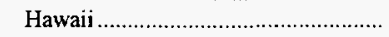 & $w$ & w & w & w & w & w & w & w & w \\
\hline Oregon & $w$ & w & $\mathbf{w}$ & w & $\mathbf{w}$ & w & w & w & w \\
\hline Washington & 64.83 & 65.20 & 64.89 & 58.54 & 62.00 & 51.18 & -.6 & 1.1 & 2.7 \\
\hline U.S. Total & 35.63 & 35.74 & 35.88 & $\mathbf{3 5 . 5 3}$ & 36.14 & 37.15 & -.3 & -.3 & -.5 \\
\hline
\end{tabular}

- Data round to zero.

w Withheld to avoid disclosure of individual company data.

Notes: Price data are for manufacturing plants only. Average prices are based on the cost including insurance, freight, and taxes. Source: Energy Information Administration, Form EIA-3, "Quarterly Coal Consumption - Manufacturing Plants." 
Table B13. Average Price of Coal Delivered to Coke Plants by Census Division and State, 1987, 1992-1996

(Nominal Dollars per Metric Ton)

\begin{tabular}{|c|c|c|c|c|c|c|c|c|c|}
\hline \multirow{2}{*}{ Census Division and State } & \multirow{2}{*}{1996} & \multirow{2}{*}{1995} & \multirow{2}{*}{1994} & \multirow{2}{*}{1993} & \multirow{2}{*}{1992} & \multirow{2}{*}{1987} & \multirow{2}{*}{$\begin{array}{c}\text { Percent } \\
\text { Change } \\
\text { 1995-1996 }\end{array}$} & \multicolumn{2}{|c|}{ Average Annual Percent Change } \\
\hline & & & & & & & & 1992-1996 & 1987-1996 \\
\hline Middle Atlantic Total......................... & $\mathbf{w}$ & $\mathbf{w}$ & $\mathbf{w}$ & $\mathbf{w}$ & $\mathbf{w}$ & $\mathbf{w}$ & $\mathbf{w}$ & $\mathbf{w}$ & $\mathbf{w}$ \\
\hline 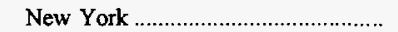 & w & w & w & $w$ & $w$ & w & w & w & $\mathbf{w}$ \\
\hline 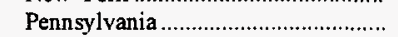 & $\$ 49.79$ & $\$ 50.83$ & $\$ 50.98$ & $\$ 51.16$ & $\$ 51.25$ & $\$ 46.89$ & -2.0 & -0.7 & 0.7 \\
\hline East North Central Total ................ & 54.61 & 54.12 & 52.06 & 54.58 & $\mathbf{5 5 . 5 3}$ & 54.01 & .9 & -.4 & .1 \\
\hline 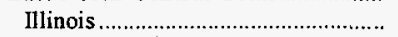 & w & w & w & $w$ & w & w & w & $\mathbf{w}$ & w \\
\hline Indiana & 57.25 & 58.13 & 56.11 & 57.64 & 59.21 & 56.70 & -1.5 & -.8 & .1 \\
\hline 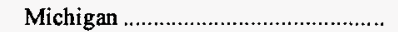 & $\mathbf{w}$ & $\mathbf{w}$ & $\mathbf{w}$ & - & - & $\mathbf{w}$ & w & w & $\mathbf{w}$ \\
\hline Ohio & 49.58 & 46.50 & 46.32 & 49.68 & 51.45 & 50.75 & 6.6 & -9 & -.3 \\
\hline West North Central Total .................. & - & - & - & - & - & $\mathbf{w}$ & - & $\mathbf{w}$ & $\mathbf{w}$ \\
\hline 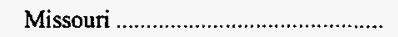 & - & - & - & - & - & w & - & $\mathbf{w}$ & $w$ \\
\hline 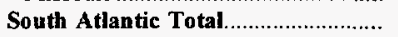 & $\mathbf{w}$ & $\mathbf{w}$ & $\mathbf{w}$ & $\mathbf{w}$ & $\mathbf{w}$ & $\mathbf{w}$ & $\mathbf{w}$ & $\mathbf{w}$ & $\mathbf{w}$ \\
\hline 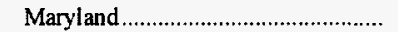 & - & - & - & - & - & $w$ & $w$ & $\mathbf{w}$ & w \\
\hline 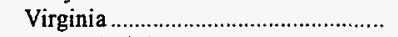 & $w$ & w & w & $\mathbf{w}$ & $w$ & w & $w$ & w & w \\
\hline 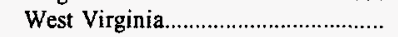 & $\mathbf{w}$ & $w$ & w & $\mathbf{w}$ & w & $w$ & $w$ & $\mathbf{w}$ & $\mathbf{w}$ \\
\hline East South Central Total................... & $w$ & $\mathbf{w}$ & $\mathbf{w}$ & $\mathbf{w}$ & $\boldsymbol{w}$ & $\mathbf{w}$ & $\mathbf{w}$ & $w$ & $\mathbf{w}$ \\
\hline Alabama & 54.42 & 53.38 & 52.31 & 52.36 & 52.70 & 50.59 & 1.9 & .8 & .8 \\
\hline 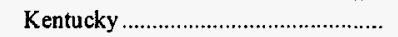 & $w$ & w & w & $w$ & w & w & $w$ & w & $\mathbf{w}$ \\
\hline 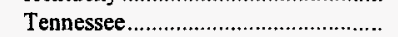 & - & - & - & - & - & $w$ & - & $w$ & $\mathbf{w}$ \\
\hline 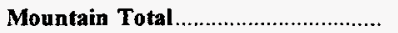 & $\mathbf{w}$ & $\mathbf{w}$ & $\mathbf{w}$ & $\mathbf{w}$ & $\mathbf{w}$ & - & $\mathbf{w}$ & $\mathbf{w}$ & $\mathbf{w}$ \\
\hline 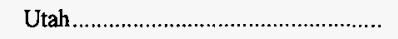 & $\mathbf{w}$ & w & $w$ & $w$ & $\mathbf{w}$ & - & $w$ & w & $\mathbf{w}$ \\
\hline U.S. Total & 52.17 & 52.18 & 51.32 & $\mathbf{5 2 . 3 0}$ & 52.82 & 51.31 & * & -.3 & .2 \\
\hline
\end{tabular}

* Data round to zero.

w Withheld to avoid disclosure of individual company data.

Note: Average prices are based on the cost including insurance, freight, and taxes.

Source: Energy Information Administration, Form EIA-5, "Coke Plant Report - Quarterly." 
Table B14. Average Price of U.S. Coal Imports by Continent and Country of Origin, 1987, 1992-1996

(Nominal Dollars per Metric Ton)

\begin{tabular}{|c|c|c|c|c|c|c|c|c|c|}
\hline \multirow{2}{*}{$\begin{array}{c}\text { Continent and Country } \\
\text { of Origin }\end{array}$} & \multirow{2}{*}{1996} & \multirow{2}{*}{1995} & \multirow{2}{*}{1994} & \multirow{2}{*}{1993} & \multirow{2}{*}{1992} & \multirow{2}{*}{1987} & \multirow{2}{*}{$\begin{array}{c}\text { Percent } \\
\text { Change } \\
\text { 1995-1996 }\end{array}$} & \multicolumn{2}{|c|}{ Average Annual Percent Chang } \\
\hline & & & & & & & & 1992-1996 & $1987-1996$ \\
\hline North America Total........................... & $\$ 38.46$ & $\$: 18.72$ & $\$ 33.96$ & $\$ 32.63$ & $\$ 32.58$ & $\$ 35.86$ & -0.7 & 4.2 & 0.8 \\
\hline 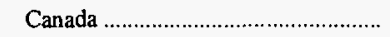 & 38.47 & 38.72 & 33.96 & 32.65 & 32.58 & 36.59 & -.6 & 4.2 & .5 \\
\hline 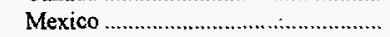 & 36.85 & - & - & 23.35 & - & 28.18 & - & - & 3.0 \\
\hline South America Total............................ & 34.44 & 36.11 & 31.97 & 30.46 & 36.42 & 36.33 & -4.6 & -1.4 & -.6 \\
\hline Colombia & 34.61 & 34.33 & 30.27 & 30.04 & 35.55 & 36.25 & .8 & -.7 & -.5 \\
\hline 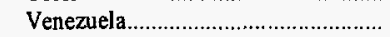 & 34.14 & 38.74 & 35.73 & 31.82 & 39.25 & 38.82 & -11.9 & -3.4 & -1.4 \\
\hline Europe Total & - & 18.27 & - & 35.65 & 37.49 & 31.57 & -100.0 & -100.0 & -100.0 \\
\hline 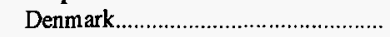 & - & - & - & 35.65 & - & - & - & - & - \\
\hline 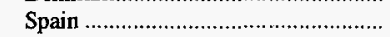 & - & - & - & - & - & 31.57 & - & - & -100.0 \\
\hline United Kingdom ............................. & - & 2.8 .27 & - & - & 37.49 & - & -100.0 & -100.0 & - \\
\hline Asia Total & 35.77 & 38.73 & $\mathbf{3 7 . 5 8}$ & 47.07 & 42.71 & - & -7.6 & -4.3 & - \\
\hline India & - & - & - & - & 26.07 & - & - & -100.0 & - \\
\hline Indonesia & 35.77 & 38.73 & 37.26 & 47.07 & 45.13 & - & -7.6 & -5.6 & - \\
\hline Malaysia & - & - & - & - & 51.87 & - & - & -100.0 & - \\
\hline Vietnam & - & - & 53.00 & - & - & - & - & - & - \\
\hline Oceania \& Australia Total ................ & 36.83 & 37.00 & 34.35 & 34.79 & 39.76 & 29.94 & -.5 & -1.9 & 2.3 \\
\hline Australia & 36.83 & 34.16 & 33.09 & 34.79 & 39.76 & 29.94 & 7.8 & -1.9 & 2.3 \\
\hline 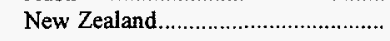 & - & 51.17 & 48.67 & - & - & - & -100.0 & - & - \\
\hline 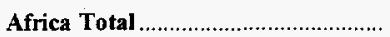 & $\dot{-}$ & - & 27.92 & 30.66 & 54.33 & - & - & -100.0 & - \\
\hline South Africa, Rep of & - & - & 27.92 & - & 54.33 & - & - & -100.0 & - \\
\hline 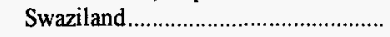 & - & - & - & 30.66 & - & - & - & - & - \\
\hline Total $^{1}$ & 35.46 & $3: 6.97$ & 33.08 & 32.46 & 36.19 & 35.37 & -4.1 & -.5 & * \\
\hline 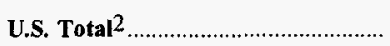 & 36.87 & 3.7 .62 & 33.30 & 32.94 & 36.88 & 35.32 & -2.0 & • & .5 \\
\hline
\end{tabular}

1 The average prices presented in this table, with the exception of U.S. Total, are considered to be representative prices for coal imports and fall within the range of $\$ 20$ to $\$ 55$ per short ton ( $\$ 18.14$ to $\$ 49.90$ per metric tor), inclusively.

2 U.S. Total is the average price of all coal imports.

* Data round to zero.

Notes: Average price is based on the customs import value. Coal imports include coal to Puerto Rico and the Virgin Islands.

Source: U.S. Department of Commerce, Bureau of the Census, "Monthly Report IM 145." 
Table B15. Average Price of U.S. Coal Exports by Destination, 1987, 1992-1996 (Nominal Dollars per Metric Ton)

\begin{tabular}{|c|c|c|c|c|c|c|c|c|c|}
\hline \multirow{2}{*}{$\begin{array}{c}\text { Continent and Country } \\
\text { of Destination }\end{array}$} & \multirow{2}{*}{1996} & \multirow{2}{*}{1995} & \multirow{2}{*}{1994} & \multirow{2}{*}{1993} & \multirow{2}{*}{1992} & \multirow{2}{*}{1987} & \multirow{2}{*}{$\begin{array}{c}\text { Percent } \\
\text { Change } \\
\text { 1995-1996 }\end{array}$} & \multicolumn{2}{|c|}{ Average Annual Percent Change } \\
\hline & & & & & & & & $1992-1996$ & $1987-1996$ \\
\hline North America Total............................. & $\$ 36,48$ & $\$ 37.53$ & $\$ 35.73$ & $\$ 37.53$ & $\$ 36.43$ & $\$ 45.37$ & -2.8 & * & -2.4 \\
\hline Canada ${ }^{1}$ & 35.53 & 36.92 & 35.36 & 37.22 & 36.36 & 45.40 & -3.8 & -0.6 & -2.7 \\
\hline 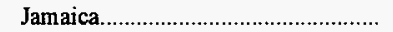 & 38.34 & 36.01 & 36.62 & 31.35 & 29.56 & - & 6.5 & 6.7 & - \\
\hline 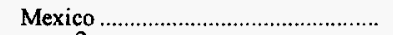 & 43.77 & 44.05 & 48.81 & 48.55 & 49.26 & 43.21 & -.6 & -2.9 & .1 \\
\hline Other $^{2}$ & 43.71 & 43.03 & 40.77 & 40.57 & 40.27 & 42.74 & 1.6 & 2.1 & .2 \\
\hline South America Total............................. & 48.29 & 47.90 & 46.61 & 48.25 & 50.15 & 48.53 & .8 & -.9 & * \\
\hline Argentina & 51.11 & 47.84 & 46.83 & 47.63 & 50.06 & 47.70 & 6.8 & .5 & .8 \\
\hline 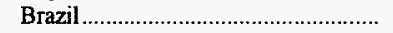 & 49.25 & 48.37 & 46.59 & 48.32 & 50.23 & 48.57 & 1.8 & -.5 & .1 \\
\hline Chile & 35.69 & 34.94 & 38.12 & 51.59 & 52.23 & 50.67 & 2.1 & -9.1 & -3.8 \\
\hline Other ${ }^{2}$ & 43.19 & 47.87 & 45.04 & 43.50 & 34.38 & 56.09 & -9.8 & 5.9 & -2.9 \\
\hline Europe Total & 46.41 & 45.10 & 46.14 & 47.45 & 46.74 & 46.70 & 2.9 & -.2 & -.1 \\
\hline Belgium \& Luxembourg ..................... & 50.41 & 47.92 & 46.55 & 47.65 & 48.51 & 45.87 & 5.2 & $1: 0$ & 1.0 \\
\hline 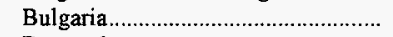 & 48.79 & 48.54 & 46.40 & 46.25 & 45.97 & - & .5 & 1.5 & - \\
\hline Denmark & 32.29 & 32.37 & 32.22 & 38.53 & 36.03 & 36.39 & -.2 & -2.7 & -1.3 \\
\hline Finland & 46.42 & 43.51 & 45.34 & 43.66 & 44.99 & 45.32 & 6.7 & .8 & .3 \\
\hline 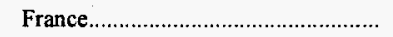 & 49.53 & 48.19 & 48.76 & 46.56 & 43.46 & 47.53 & 2.8 & 3.3 & .4 \\
\hline Germany, FR & 45.28 & 38.57 & 49.97 & 42.97 & 42.19 & 46.03 & 17.4 & 1.8 & -.2 \\
\hline 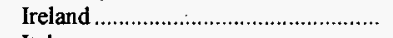 & 41.17 & 39.76 & 37.28 & 39.51 & 40.57 & 48.04 & 3.6 & 4 & -1.7 \\
\hline Italy & 49.66 & 48.66 & 47.40 & 48.86 & 50.08 & 45.84 & 2.0 & -.2 & .9 \\
\hline 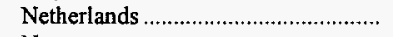 & 45.59 & 46.26 & 46.22 & 48.75 & 47.78 & 47.74 & -1.4 & -1.2 & -.5 \\
\hline 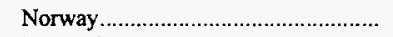 & 62.89 & 62.19 & 60.16 & 58.95 & 55.25 & 52.10 & 1.1 & 3.3 & 2.1 \\
\hline 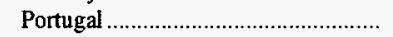 & 40.26 & 40.18 & 39.96 & 41.56 & 44.91 & 47.30 & .2 & -2.7 & -1.8 \\
\hline 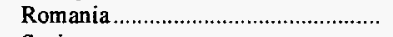 & 51.76 & 46.77 & 42.00 & 42.48 & 47.02 & 47.31 & 10.6 & 2.4 & 1.0 \\
\hline Spain & 41.41 & 38.30 & 44.23 & 47.02 & 46.24 & 48.27 & 8.1 & -2.7 & -1.7 \\
\hline Sweden & 52.36 & 53.14 & 50.22 & 50.66 & 51.44 & 47.77 & -1.5 & .4 & 1.0 \\
\hline Turkey & 48.86 & 46.97 & 45.51 & 46.94 & 50.20 & 48.67 & 4.0 & -.7 & * \\
\hline 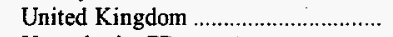 & 42.88 & 45.11 & 49.67 & 50.64 & 50.26 & 48.78 & -4.9 & -3.9 & -1.4 \\
\hline Yugoslavia, FR & - & 41.95 & - & 43.65 & 47.21 & 47.21 & -100.0 & -100.0 & -100.0 \\
\hline Other ${ }^{2} \ldots \ldots \ldots \ldots \ldots \ldots$ & 41.92 & 61.67 & 42.04 & 44.91 & 46.17 & 47.28 & -32.0 & -2.4 & -1.3 \\
\hline Asia Total & 43.62 & 43.10 & 42.58 & 44.63 & 47.02 & 47.81 & 1.2 & -1.8 & -1.0 \\
\hline 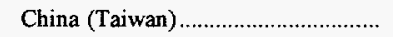 & 40.63 & 40.73 & 42.60 & 43.53 & 45.45 & 44.03 & -.2 & -2.8 & -.9 \\
\hline 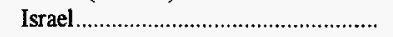 & 40.12 & 39.45 & 36.63 & 38.35 & 43.11 & 40.30 & 1.7 & -1.8 & $*$ \\
\hline Japan & 43.44 & 43.14 & 42.47 & 44.88 & 47.30 & 50.28 & .7 & -2.1 & -1.6 \\
\hline 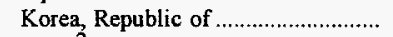 & 47.10 & 45.17 & 44.35 & 46.43 & 49.53 & 45.78 & 4.3 & -1.3 & .3 \\
\hline Other $^{2}$ & 53.89 & 33.52 & 41.05 & 48.75 & 40.85 & 59.84 & 60.8 & 7.2 & -1.1 \\
\hline Oceania \& Australia Total ................. & 44.89 & 43.82 & 44.04 & 37.99 & 38.08 & 42.00 & 2.4 & 4.2 & .7 \\
\hline Other ${ }^{2}$ & 44.89 & 43.82 & 44.04 & 37.99 & 38.08 & 42.00 & 2.4 & 4.2 & .7 \\
\hline 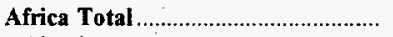 & 48.90 & 47.47 & 48.05 & 46.90 & 47.37 & 45.55 & 3.0 & .8 & .8 \\
\hline Algeria & 55.37 & 52.69 & 47.66 & 48.85 & 51.10 & 48.20 & 5.1 & 2.0 & 1.5 \\
\hline 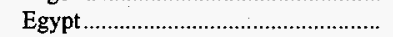 & 58.83 & 54.42 & 47.55 & 49.45 & 50.80 & 46.39 & 8.1 & 3.7 & 2.7 \\
\hline Morocco & 37.40 & 36.37 & 38.62 & 37.32 & 37.26 & 39.19 & 2.8 & .1 & -.5 \\
\hline 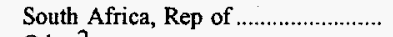 & 54.61 & 52.23 & 50.34 & 51.67 & 52.68 & 53.18 & 4.6 & .9 & .3 \\
\hline Other $^{2}$ & - & - & - & 44.68 & - & 45.00 & - & - & -100.0 \\
\hline Total $^{3}$ & 44.67 & 44.13 & 43.98 & 45.55 & 45.51 & 46.84 & 1.2 & -.5 & -.5 \\
\hline U.S. Total ${ }^{4} .$. & 44.93 & 44.39 & 44.02 & 45.65 & 45.57 & 47.15 & 1.2 & -.3 & -.5 \\
\hline
\end{tabular}

1 Based on the U.S. - Canada Free Trade Agreement, as of January 1990, the U.S. Department of Commerce began reporting statistics on U.S. exports to Canada based on information on imports provided monthly by the Canadian government.

2 Includes countries with exports less than or equal to 50,000 short tons in 1995 .

3 The average prices presented in this table, with the exception of U.S. Total, are considered to be representative prices for coal exports and fall within the range of $\$ 20$ to $\$ 60$ per short ton ( $\$ 18.14$ to $\$ 54.43$ per metric ton), inclusively.

4 U.S. Total is the average price of all coal exports.

* Data round to zero.

Note: Average price is based on the free alongside ship (f.a.s.) value.

Source: U.S. Department of Commerce, Bureau of the Census, "Monthly Report EM 545." 



\section{References}

Figure C1. Coal-Bearing Areas of the United States

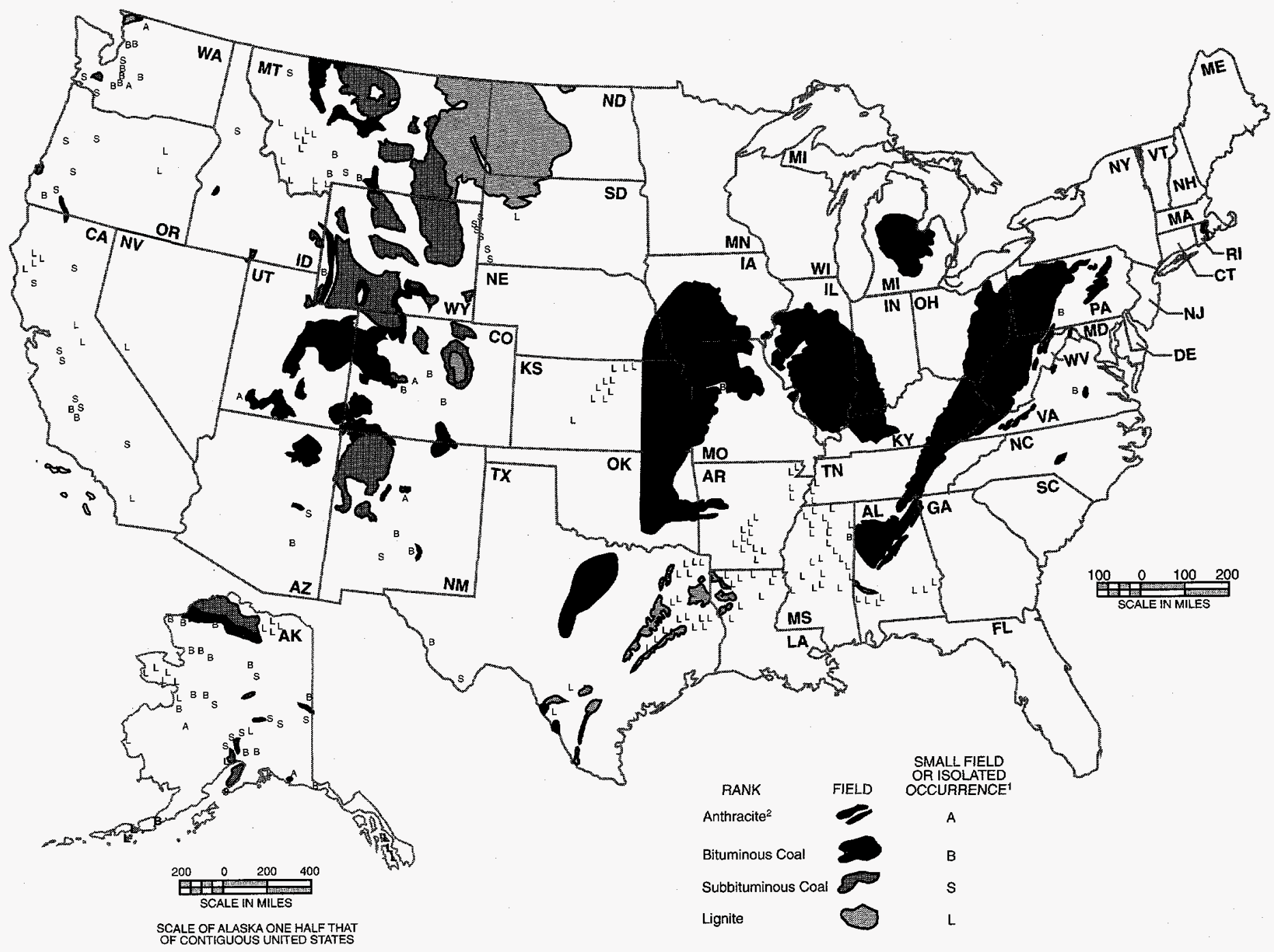




\section{Coal-Producing Regions}

\section{Appalachian}

Alabama, Georgia, Eastern Kentucky, Maryland, Ohio, Pennsylvania, Tennessee, Virginia, West Virginia Interior

Arkansas, Illinois, Indiana, lowa, Kansas, Western Kentucky, Louisiana, Missouri, Oklahoma, Texas

\section{Western}

Alaska, Arizona, California, Colorado, Montana, New Mexico, North Dakota, Utah, Washington, Wyoming

Iable C1. Classification of Coals by Bank

\section{Principles}

The lower rank of coals can be classified based on heat content. The heat content of the higher rank coals is generally above 14,000 Btu per pound for each coal rank group (except for meta-anthracite, which trends slightly lower), and heat content ranges vary within a relatively narrow range. Since heat content is not a dependable criterion for these higher rank coals, their rank categories are instead described by degree of metamorphism, or "coalification," a property that is measured by fixed carbon content. Finally, the agglomerating character of bituminous coals is a critical attribute for certain coal consumers, and thus agglomerating character has come to define the distinctions between certain adjacent coal groups. Some high-volatile $C$ bituminous and subbituminous $A$ coals can be distinguished only on the basis of agglomerating character.

\begin{tabular}{|c|c|c|c|c|}
\hline Coal Rank & Coal Group & \multicolumn{3}{|c|}{ Basis of Classification } \\
\hline \multirow{2}{*}{\multicolumn{2}{|c|}{ Coal Classified by Fixed Carkion }} & \multicolumn{2}{|c|}{ Fixed Carbon Percentage ${ }^{1}$} & \multirow[b]{2}{*}{$\begin{array}{c}\text { Agglomerating } \\
\text { Character }\end{array}$} \\
\hline & & $\begin{array}{l}\text { Equal to or } \\
\text { Greater than }\end{array}$ & Less than & \\
\hline I. Anthracite & 1. Meta-anthracite & 98 & -- & Non-agglomerating \\
\hline \multirow{5}{*}{ II. Bituminous } & 2. Anthracite & 92 & 98 & Non-agglomerating \\
\hline & 3. Semianthracite ${ }^{2}$ & 86 & 92 & Non-agglomerating \\
\hline & 1. Low-volatile bituminous & 78 & 86 & Commonly agglomerating ${ }^{3}$ \\
\hline & 2. Medium-volatile bituminous & $69^{4}$ & 78 & Commonly agglomerating ${ }^{3}$ \\
\hline & 3. High-volatile A bituminous & -- & $69^{4}$ & Commonly agglomerating ${ }^{3}$ \\
\hline \multirow{2}{*}{\multicolumn{2}{|c|}{ Coals Classified by Heat Content }} & \multicolumn{2}{|c|}{ Heat Content in Btu per Pound ${ }^{5}$} & \multirow[b]{2}{*}{$\begin{array}{l}\text { Agglomerating } \\
\text { Character }\end{array}$} \\
\hline & & $\begin{array}{l}\text { Equal to or } \\
\text { Greater than }\end{array}$ & Less than & \\
\hline \multirow[t]{3}{*}{ II. Bituminous } & 4. High-volatile $B$ bituminous & 13,000 & 14,000 & Commonly agglomerating ${ }^{3}$ \\
\hline & 5. High-volatile $\mathrm{C}$ bituminous & 11,500 & 13,000 & Commonly agglomerating ${ }^{3}$ \\
\hline & 6. High-volatile $\mathrm{C}$ bituminous & 10,500 & 11,500 & Agglomerating \\
\hline \multirow[t]{3}{*}{ III. Subbituminous } & 1. Subbituminous $A$ & 10,500 & 11,500 & Non-agglomerating \\
\hline & 2. Subbituminous B & 9,500 & 10,500 & Non-agglomerating \\
\hline & 3. Subbituminous $\mathrm{C}$ & 8,300 & 9,500 & Non-agglomerating \\
\hline \multirow[t]{2}{*}{ IV. Lignitic } & 1. Lignite A & 6,300 & 8,300 & Non-agglomerating \\
\hline & 2. Lignite $B$ & -- & 6,300 & Non-agglomerating \\
\hline
\end{tabular}

${ }^{1}$ Percentages are based on dry mineral-matter-free coal. Volatile matter (not shown) is the complement of fixed carbon; that is, the percentages of fixed carbon and volatile matter sum to too percent. As fixed carbon percentage decreases, therefore, volatile matter percentage increases by the same amount.

${ }^{2}$ If agglomerating, classify in low-volatile group of the bituminous class.

${ }^{3}$ There may be nonagglomerating varieties in the bituminous class, most notably in the high-volatile $\mathrm{C}$ bituminous group.

${ }^{4}$ Coals having 69 percent or more fixed carbon are classified according to fixed carbon, regardless of Btu value. Coals with less than 69 percent fixed carbon, but with 14,000 or more Btu per pound, are classified as high-volatile A bituminous.

${ }^{5}$ Calorific values in Btu per pound, on a moist mineral-matter-free basis.

Note: Terms in this table are defined in the Gloissary.

Source: Adapted from American Society for Tesiting and Materiais 1988, Standard Classification of Coal by Rank, ASTM Designation D 388-91a. 


\section{Coal Reports and Feature Articles}

\section{Coal Reports}

- Weekly Coal Production, DOE/EIA-0218 (97-35).

- Coal Data: A Reference, DOE/EIA-0064 (93), February 1995.

- State Coal Profiles, DOE/EIA-0576, January 1994.

- Quarterly Coal Report, DOE/EIA-0121(97/1Q).

- The Changing Structure of the U.S. Coal Industry: An Update, DOE/EIA-0513(93), July 1993.

- U.S. Coal Reserves: A Review and Update DOE/EIA-0529(95), August 1996.

- The U.S. Coal Industry, 1970-1990: Two Decades of Change, DOA/EIA-0559, November 1992.

- Trends in Contract Coal Transportation, 1979-1987, DOE/EIA-0549, September 1991.

- Electric Power Monthly, DOE/EIA-0226(97/09), September 1997.

- Electric Power Annual, DOE/EIA-0348(96), Vol. 1, September 1997.

- Longwall Mining, DOE/EIA-TR-0588 March 1995.

- Monthly Energy Review, DOE/EIA-0035(97/07) July 1997

- Electric Utility Phase I Acid Rain Compliance Strategies for the Clean Air Act Amendments of 1990, DOE/EIA-0582, March 1994.

- Cost and Quality of Fuels for Electric Utility Plants 1994, DOE/EIA-0191(94), July 1995.

\section{Feature Articles}

- "U.S. Coal Supply and Demand: 1996 Review," Mining Engineering, May 1997, Vol.49,No.5, May 1997, pp.43-50.

- "Carbon Dioxide Emission Factors for Coal," Quarterly Coal Report, DOE/EIA-0121 (94/1Q), August 1994.

- "Federal and Indian Coal Lands: A Growing Source of Energy and Revenue," Coal Production 1992, DOE/EIA-0118(92), October 1993.

- "Coal Supply and Demand in 1993: A Review, 1993," Mining Engineering, May 1994, pp.433-436.

- "Wyoming Coal: An Overview," Coal Production 1991, DOE/EIA-0118(91), October 1992.

- "Profile of New Coal Mines in the 1980's," Coal Production 1990, DOE/EIA-0118), September 1991.

- "The Comparability of Resource and Reserve Data for Crude Oil, Natural Gas, Coal, and Uranium," Quarterly Coal Report OctoberDecember 1994, DOE/EIA-0121 (94/4Q) May 1995.

- "Annual Review 1995: Coal Overview," Mining Engineering, Vol. 48, No. 5, May 1996, pp. 41-46.

- "Coal Geology, Reserves and Production in Northern and Central Appalachia," Mining Engineering, Special Edition, December 1995. 
Table C2. Approximate Heat Content of Coal

(Million Btu per Short Ton)

\begin{tabular}{|c|c|c|c|c|c|c|c|}
\hline $\begin{array}{l}\text { Coal Rank } \\
\text { Sector }\end{array}$ & 1983 & 1984 & 1985 & 1986 & 1987 & 1988 & 1989 \\
\hline \multicolumn{8}{|l|}{ Anthracite } \\
\hline Production & 22.734 & 23.107 & 22.428 & 23.084 & 23.108 & 23.266 & 23.385 \\
\hline 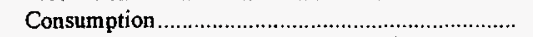 & 21.583 & 22.322 & 20.817 & 21.512 & 22.435 & 22.423 & 22.623 \\
\hline 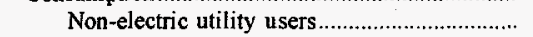 & 24.536 & 25.128 & 23.031 & 24.399 & 26.293 & 26.021 & 27.196 \\
\hline 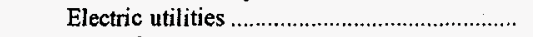 & 16.516 & 17.018 & 16.784 & 15.578 & 15.962 & 17.312 & 16.310 \\
\hline 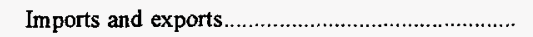 & 25.400 & 25.400 & 25.400 & 25.400 & 25.400 & 25.400 & 25.400 \\
\hline
\end{tabular}

Bituminous Coal and Lignite

\begin{tabular}{|c|c|c|c|c|c|c|c|}
\hline 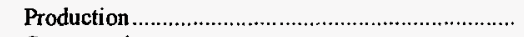 & 22.048 & 22.005 & 21.867 & 21.908 & 21.918 & 21.817 & 21.759 \\
\hline 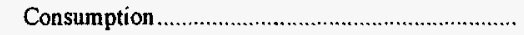 & 21.576 & 21.570 & 21.368 & 21.462 & 21.514 & 21.324 & 21.268 \\
\hline 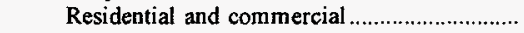 & 22.438 & 22.406 & 22.568 & 22.669 & 22.800 & 23.135 & 22.917 \\
\hline Coke plants & 26.800 & 26.800 & 26.800 & 26.800 & 26.800 & 26.800 & 26.800 \\
\hline \multicolumn{8}{|l|}{ Other industrial and } \\
\hline 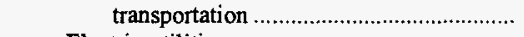 & 22.680 & 22.525 & 22.013 & 22.185 & 22.360 & 22.341 & 22.324 \\
\hline 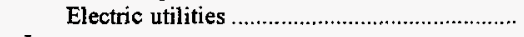 & 21.141 & 21.108 & 20.965 & 21.091 & 21.143 & 20.905 & 20.854 \\
\hline Imports & 25.000 & 25.000 & 25.000 & 25.000 & 25.000 & 25.000 & 25.000 \\
\hline 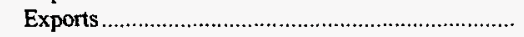 & 26.300 & 26.410 & 26.320 & 26.308 & 26.304 & 26.308 & 26.166 \\
\hline \multirow[t]{2}{*}{ 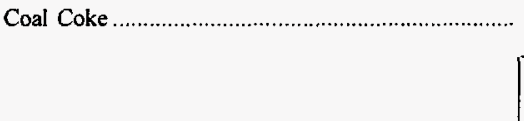 } & 24.800 & 24.800 & 24.800 & 24.800 & 24.800 & 24.800 & 24.800 \\
\hline & 1990 & 1991 & 1992 & 1993 & 1994 & 1995 & 1996 \\
\hline
\end{tabular}

Anthracite

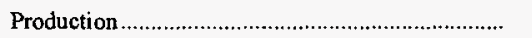

21.668

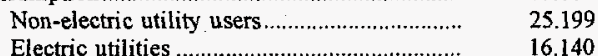

Electric utilities.

16.140
25.400

22.573
21.410
25.268
15.858
25.400

22.572

21.423

24.617

16.944

25.400

22.573
21.262
24.096
16.534
25.400

22.572

20.828

22.572

20.808

22.573

15.858
25.400

25.037

14.680

24.696

14.572

20.860

24.872

14.568

Imports and exports......e.c...

Bituminous Coal and Lignite

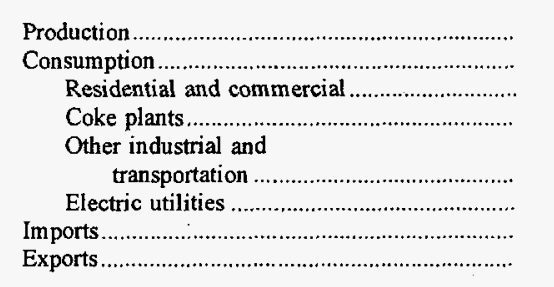

$\begin{array}{lllllll}21.819 & 21.678 & 21.643 & 21.383 & 21.347 & 21.271 & 21.272 \\ 21.330 & 21.146 & 21.142 & 20.983 & 21.011 & 20.845 & 20.852 \\ 22.578 & 22.635 & 22.768 & 22.749 & 22.683 & 22.767 & 22.785 \\ 26.800 & 26.800 & 26.800 & 26.800 & 26.800 & 26.800 & 26.800 \\ & & & & & & \\ 22.444 & 22.448 & 22.242 & 22.111 & 22.046 & 21.931 & 21.887 \\ 20.935 & 20.761 & 20.792 & 20.644 & 20.681 & 20.502 & 20.509 \\ 25.000 & 25.000 & 25.000 & 25.000 & 25.000 & 25.000 & 25.000 \\ 26.207 & 26.192 & 26.165 & 26.341 & 26.335 & 26.187 & 26.212\end{array}$

Coal Coke

24.800

24.800

24.800

24.800

24.800

24.800

24.800

Note: Values for 1996 are preliminary.

Source: Calculated by Energy Information Administration. SeeMonthly Energy Review DOE/EIA-0035 Appendix A for detailed description. 


\section{Explanatory Notes}

\section{Data Sources}

All data in this report were collected by the Energy Information Administration (EIA), U.S. Department of Energy (DOE), except: import and export data, which were collected by the Bureau of the Census (Census Bureau), U.S. Department of Commerce; supplemental export data which were collected by King's Publishing Corporation, Knoxville, Tennessee; Federal and Indian land leasing data which were collected by the U.S. Department of the Interior (Bureau of Land Management and Minerals Management Service); and miner injury and fatality data which were collected by the U.S. Department of Labor (Mine Safety and Health Administration).

\section{Coal Surveys}

As early as the $1880^{\prime}$ s, the U.S. Geological Survey began collecting coal data under a voluntary reporting system. The responsibility for gathering this information was transferred to the Bureau of Mines in the 1920 's, initially under the U.S. Department of Commerce and later under the U.S. Department of the Interior, which published the data in its Minerals Yearbook. Except for a brief period from 1937 to 1943, when bituminous coal data were collected under the mandatory authority of the Bituminous Coal Act, the Bureau of Mines continued to conduct voluntary coal surveys until the Department of Energy was created.

\section{Coal Production Report (Form EIA-7A)}

The Energy Information Administration (EIA) began collecting annual coal production data on October 1 , 1977. The 1995 coal production and identification data in this report were collected on Form EIA-7A, "Coal Production Report," from companies that produced, processed, or prepared coal in 1995. All other data collected on Form EIA-7A are reported for only those companies that owned a mining operation that produced, processed, or prepared 10,000 short tons or more of coal in 1995 and preparation plants with 5,000 or more employee hours.

So that the EIA may fulfill its data collection functions as specified in the Federal Energy Administration Act of 1974 (Public Law 93-275), response to this survey is mandatory. EIA compares respondents to this survey with lists of mining operations maintained by various State coal mining/licensing agencies and the Mine Safety and Health Administration (MSHA), U.S. Department of Labor, to identify new respondents. No sampling procedures are used. In 1995 , there were 2,278 mining operations that produced, processed, or prepared 10,000 or more short tons of coal. Of these mining operations, 77.6 percent $(1,767)$ responded to the EIA-7A survey. In 1995, there were 1,716 mines that produced 10,000 or more short tons of coal. Of these mines, 70.2 percent $(1,205)$ responded to the EIA-7A survey; they accounted for 987 million short tons, or 95.6 percent of the 1995 production total. All of the data were collected by mail and were edited to ensure that they were complete and accurate.

As in all surveys, data from Form EIA-7A, "Coal Production Report," are subject to various sources of error: (1) coverage (the list of respondents may not be complete or, on the other hand, there may be double counting), (2) nonresponse (all units that are surveyed may not respond or may not provide all the information requested), (3) respondents (respondents may commit errors in reporting the data), (4) processing (the data collection agency may lose or incorrectly transcribe the submissions), (5) concept (the data collection elements may not measure the items they were intended to measure), and (6) adjustment (errors may be made in estimating values for missing data).

Because the annual coal production survey (Form EIA-7A) is not a sample survey, the estimates shown 
Table D1. Sources of Data for Total U.S. Coal Production and Number of Mining Operations (Thousand Short Tons)

\begin{tabular}{|c|c|c|c|c|c|c|}
\hline \multirow{3}{*}{$\begin{array}{l}\text { Coal-Producing } \\
\text { State }\end{array}$} & \multicolumn{2}{|c|}{ Received } & \multicolumn{2}{|c|}{ Generated Data Sources } & \multicolumn{2}{|c|}{ Total } \\
\hline & \multicolumn{2}{|c|}{ Form EIA-7A } & \multicolumn{2}{|c|}{$\begin{array}{c}\text { Derived From } \\
\text { Mine Safety and } \\
\text { Health Administration Data }\end{array}$} & \multirow{2}{*}{$\begin{array}{l}\text { Number of } \\
\text { Operations }\end{array}$} & \multirow{2}{*}{ Production } \\
\hline & $\begin{array}{l}\text { Number of } \\
\text { Operations }\end{array}$ & Production & $\begin{array}{l}\text { Number of } \\
\text { Operations }\end{array}$ & Production & & \\
\hline Alabama & 46 & 23,677 & 16 & 960 & 62 & 24,637 \\
\hline 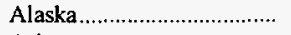 & 1 & $1,4: 31$ & - & - & 1 & 1,481 \\
\hline Arizona & 3 & 10,442 & - & - & 3 & 10,442 \\
\hline 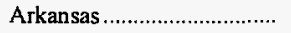 & - & - & 5 & 21 & 5 & 21 \\
\hline 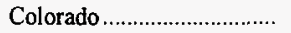 & 17 & 24,757 & 1 & 129 & 18 & 24,886 \\
\hline 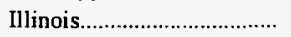 & 32 & 46,419 & 5 & 237 & 37 & 46,656 \\
\hline Indiana & 40 & 29,657 & 1 & 3 & 41 & 29,670 \\
\hline 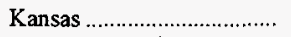 & 1 & 2.32 & - & - & 1 & 232 \\
\hline Kentucky Total......................... & 484 & $139,0.45$ & 223 & 13,379 & 707 & 152,425 \\
\hline 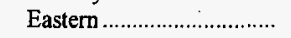 & 429 & 105,211 & 201 & 11,740 & 630 & 116,951 \\
\hline Western & 55 & 33,835 & 22 & 1,639 & 77 & 35,474 \\
\hline 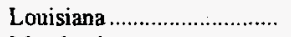 & 2 & $3,2: 21$ & - & - & 2 & 3,221 \\
\hline 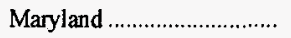 & 14 & 4,058 & 5 & 35 & 19 & 4,093 \\
\hline 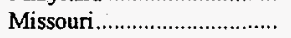 & 3 & 395 & 2 & 315 & 5 & 710 \\
\hline Montana & 8 & 37,891 & - & - & 8 & 37,891 \\
\hline New Mexico ........................... & 6 & 24,067 & - & - & 6 & 24,067 \\
\hline North Dakota......................... & 6 & 29,861 & - & - & 6 & 29,861 \\
\hline Ohio & 97 & 28,248 & 22 & 324 & 119 & 28,572 \\
\hline 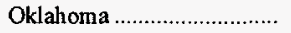 & 10 & 1,687 & 2 & 14 & 12 & 1,701 \\
\hline Pennsylvania Total ............... & 304 & 64,094 & 205 & 3,847 & 509 & 67,942 \\
\hline 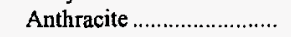 & 78 & 3,342 & 91 & 1,409 & 169 & 4,751 \\
\hline 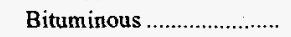 & 226 & 60,752 & 114 & 2,438 & 340 & 63,190 \\
\hline 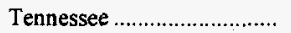 & 31 & 3,581 & 7 & 69 & 38 & 3,651 \\
\hline 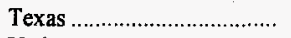 & 13 & 55,164 & - & - & 13 & 55,164 \\
\hline Utah & 12 & 26,926 & 4 & 581 & 16 & 27,507 \\
\hline 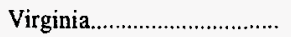 & 159 & 29,862 & 91 & 5,727 & 250 & 35,590 \\
\hline Washington ............................ & 3 & 4,565 & - & - & 3 & 4,565 \\
\hline West Virginia Total ........... & 361 & 158,132 & 162 & 12,251 & 523 & 170,433 \\
\hline 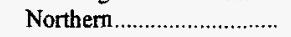 & 91 & 45,136 & 36 & 774 & 127 & 45,910 \\
\hline 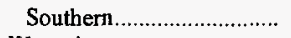 & 270 & 113,046 & 126 & 11,476 & 396 & 124,523 \\
\hline Wyoming $\ldots \ldots \ldots \ldots$ & 27 & 278,440 & - & - & 27 & 278,440 \\
\hline 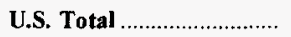 & 1,680 & $1,025,9644$ & 751 & 37,892 & 2,431 & $1,063,856$ \\
\hline
\end{tabular}

Notes: Coal production excludes silt, culm, refuse bank, slurry dam, and dredge production except for Pennsylvania anthracite. Number of mining operations includes preparation plants. All available State mining agency production data were reviewed, but none were included in this report because production data reported on Form EIA-7A to the Energy Information Administration and on Form 7000-1 to the Mine Safety and Health Administration were found to be complete. Totals may not equal sum of components because of independent rounding.

Sources: Energy Information Administration, Form ElA-7A, "Coal Production Report"; U.S. Department of Labor, Mine Safety and Health Administration, Form 7000-2, "Quarterly Mine Employment and Coal Production Report."

in this report are not subject to sampling error. ${ }^{1}$ It is not possible to present estimates of nonsampling error, but precautionary steps were taken at each stage of the survey design to minimize the possible occurrence of these errors. These steps are described below.

The forms are logged within 24 hours of receipt and assigned to a team of data editors consisting of Coal Data Systems Branch personnel. The editors screen the forms for legibility, completeness, and consistency. Names and address changes are updated in the files. The reported data are compared with data from previous years and with secondary sources such as Mine Safety and Health Administration and State mining agency reports. Inconsistencies are identified and the respondents contacted for clarification. Computer edits are generated to identify keypunch errors, errors made by coders, out-of-range codes, and unlikely data combinations. Errors are corrected to conform to the data on the submissions or revised after telephone conversations with company representatives. All changes to reported data are documented. EIA maintains data from the Form EIA-7A on an automated database at its computing facility in Washington, D.C. The survey forms are filed by EIA identification number organized by State and county.

The survey forms were sent via regular mail in January with a due date of March 1, 1996. Nonresponse letters were mailed March 8 to those mining operations that had not submitted their forms. Subsequent to the letter, attempts were made to contact, by telephone, those nonrespondents whose reported 1994 production was 100,000 short tons or more. 
Some forms could not be delivered. Where possible, address corrections were obtained. Some mining operations that had reported in earlier surveys or operated only in 1995 were no longer in business. Unobtainable data were derived from a secondary source: the Form 7000-2, "Quarterly Mine Employment and Coal Production Report," collected by the Mine Safety and Health Administration. Missing production quantity data were derived from the secondary source for 585 mines, which produced 45 million short tons, or 4.4 percent of total production. The received and generated data sources for total U.S. coal production are shown in Table D1. Of the mines whose production exceeded 10,000 or more short tons, missing production quantity data were derived from the secondary source for 511 mines, representing 4.3 percent (44.4 million short tons).

Since 1992, California has not reported coal production as the courts determined that the product mined in that State did not meet the standard classification for coal.

Missing coalbed classification, coalbed thickness, and coal rank/group data were estimated using State mining agency production reports, geological data, and previous years' reports for these mines.

When a mine had a missing production value, its production was multiplied by the county-level average mine price to estimate the value. County-level average mine prices were calculated by dividing the total value for the appropriate disposition (open/captive) and type of mining (underground or surface) by the corresponding total production. All missing production was classified as open market unless information was available to classify it as captive. Of those mines whose production was 10,000 or more short tons, value data were estimated for 562 mines, representing 5.2 percent ( 53.8 million short tons) of the production total.

When an underground mine had unreported mining method, it was assumed that the mining method was conventional.

Employment data include direct labor hours, number of production days worked, average length of a production shift, average number of miners per shift, and average number of shifts per day. Of those mines whose production exceeded 10,000 or more short tons, one or more of these data elements were derived from secondary sources or estimated for 532 mines representing 4.4 percent ( 45.2 million short tons) of their production total. Of the mining operations that produced, processed, or prepared 10,000 or more short tons, one or more of these data elements were derived from secondary sources or estimated for 868 mining operations, or 38.1 percent of the total operations in this category.

Missing direct labor hours were estimated using county-level or State-level productivity-per-hour averages. The averages were calculated by dividing the total production at the county or State level by total direct labor hours at the same level for the appropriate type of mining (underground or surface). The quantity of production for the mine with missing data was divided by this productivity average to derive direct labor hours.

Missing average number of production days worked and length of a production shift were derived by using the corresponding county-level or State-level average for the appropriate type of mining (underground or surface).

Missing average number of miners per shift was estimated as the average number of miners working daily. The average number of miners working daily was calculated by multiplying the average length of a production shift by the number of production days worked, and then dividing this number into the total direct labor hours.

Missing average number of shifts per day was estimated as one shift per day.

Missing recoverable coal reserves data were estimated by using the mine's 1994 recoverable reserves minus the mine's 1995 production. If this calculation could not be made, the mine's projected production for 1996 was used. If recoverable coal reserves for 1994 and 1995 and projected production for 1996 were all missing, no estimate was made. In 1995, recoverable reserves were reported by or estimated for 1,186 mines, representing 976.9 million short tons, or 69.1 percent of the mines whose production exceeded 10,000 or more short tons.

Missing recovery percentage data were estimated by using 1995 recovery percentage averages at the State level for the appropriate type of mining (underground or surface).

Missing productive capacity data were estimated by assuming productive capacity was equal to 1995 production. If productive capacity was reported as less than annual production, productive capacity was equated to 1995 production. There were 627 in-scope mines with production of 177.8 million short tons for which 1995 production was used as a proxy for productive capacity,-resulting in 100 percent capacity utilization.

These mines included the MSHA generated mines, mines with productive capacity less than 1995 production, and mines that did not report productive capacity and could not be contacted. If these mines were excluded from the calculation of percent utilization, the U.S. total becomes 76.13 versus 79.40 , when those mines are included.

In 1995, there were 26 mines that produced 1.2 million short tons of refuse bituminous and subbituminous coal. Those operations are not included in this report. In 1995, there were 2.3 million short tons of anthracite refuse produced and included in this report. An additional 4 million short tons of anthracite refuse was recovered and used by nonutility power producers in Pennsylvania is not included in this report. 
In order to protect the confidentiality of individual respondent's data, a policy was implemented to ensure that the reporting of survey data on mine prices and recoverable reserves in this publication would not associate those data with a particular company. The final phase in the data quality assurance and control procedures is determining which data must be suppressed (withheld) during publication to provide the necessary confidentiality for mines or companies that represent a significant portion of a reported data cell. All withholding analysis is done based upon production volumes. These procedures are performed as follows:

1. Primary Withholding Based on the Number of Respondents in a Cell -- All cells with three or fewer active coal mining operations are suppressed.

2. Residual Withholding Dominance Rule Phase 1 All cells containing between 4 and 10 active coal mining operations are examined. A cell is suppressed if any single respondent accounts for 75 percent or more of the volume for all respondents included in that cell.

3. Residual Withholding Dominance Rule Phase 2 All cells in which two active coal mining operations represent 90 percent or more of the volume for all respondents included in that cell are suppressed.

4. Complementary Suppression -- All tables are reviewed to identify cells which should have data withheld to prevent disclosure of already suppressed cells. An example of cells to be withheld during complementary suppression is underground price, if the surface price is withheld during primary or residual suppression. Because the total price is published, if the underground price is not withheld, the surface price could be calculated using the total price, the underground price, and the underground, surface, and total volumes.

5. Most complementary suppression involves type of mining considerations. Other complementary suppression is based on regional level data. A State or region must be withheld during complementary suppression because an already withheld State could be calculated using other States and the regional total. Cells are also selected for complementary suppression that represent the smallest volumes or that were withheld in prior years.

6. Inter-table effects are also examined regarding complementary suppression. For example, States that are withheld in one State table can influence the complementary suppression of an associated State table. This analysis is very similar to that done at the regional level, except that two tables are involved rather than one. Finally, similar tables are reviewed to ensure that all like suppressed cells are consistently withheld (suppressed) in all tables in which they appear.

The withholding/suppression of data is performed as an adjunct to the quality assurance (QA) procedures. The work is performed by survey editors, and the QA staff and is reviewed by the survey manager before being submitted to division-level QA review.
All sensitive cells identified in withholding analysis are denoted with the symbol/letter "w." The use of the symbol/letter applies to primary, complementary and inter-table suppressions as well as all withheld data. The symbol/letter " $w$ " is footnoted as follows: "w Withheld to avoid disclosure of individual company data."

The interquartile range is a measure of dispersion of State-level average mine prices. Two States may have the same average mine price, but the spread about this price may be totally different. For a fixed average mine price, a larger interquartile range suggests a broader distribution of coal prices than a smaller interquartile range. The summary statistics (Table D2) given in this publication are weighted by production. The interquartile range (weighted by production) is computed in the following manner:

- Each cell is sorted according to average mine price, from the lowest to the highest.

- For each cell, the corresponding mine's production according to increasing average mine price is divided by that particular cell's total production and multiplied by 100 . These percentages are then added as a cumulative percentage of production.

- The first quartile (Q1) is the associated mine price for which the cumulative percentage of production first passes 25 percent. Thus, at least 25 percent of that cell's total production is identified with prices at or lower than Q1.

- The third quartile (Q3), is the associated mine price for which the cumulative percentage of production first passes 75 percent. Thus, at least 75 percent of that cell's total production is identified with prices at or lower than Q3.

The interquartile range is $\mathrm{Q} 3-\mathrm{Q} 1$.

\section{Quarterly Coal Consumption Report - Manufacturing Plants (Form EIA-3)}

Form EIA-3 is used to survey U.S. manufacturers that consume 1,000 tons or more of coal per year for all uses other than coke production. These data were collected on a monthly basis until 1980, when the reporting cycle was revised to a quarterly schedule. Data on manufacturers' coal stocks, receipts, prices, and consumption are reported.

Through the end of 1988 , all manufacturers that consumed coal were required to file Form EIA-3. Beginning with the first quarter of 1989 , only those manufacturers that consumed 1,000 or more tons in the past year were required to report. In 1995, 730 manufacturers responded to the EIA-3 survey. The response rate for the current year was 100 percent. In order to identify undercoverage problems, the data from this survey are compared with shipments to manufacturers reported on EIA's "Coal Distribution Report," Form EIA-6. At present, the coal receipts reported by manufacturers on Form EIA-3 cover approximately 97 percent of the coal shipments to 
manufacturers on Form EIA-6. Consequently, the coal consumption data gathered on the Form EIA-3 do not represent the total consumption at manufac-

Table D2. Interquartile Range and Average Mine Price by State and Mine Type, 1996 (Dollars per Short Ton)

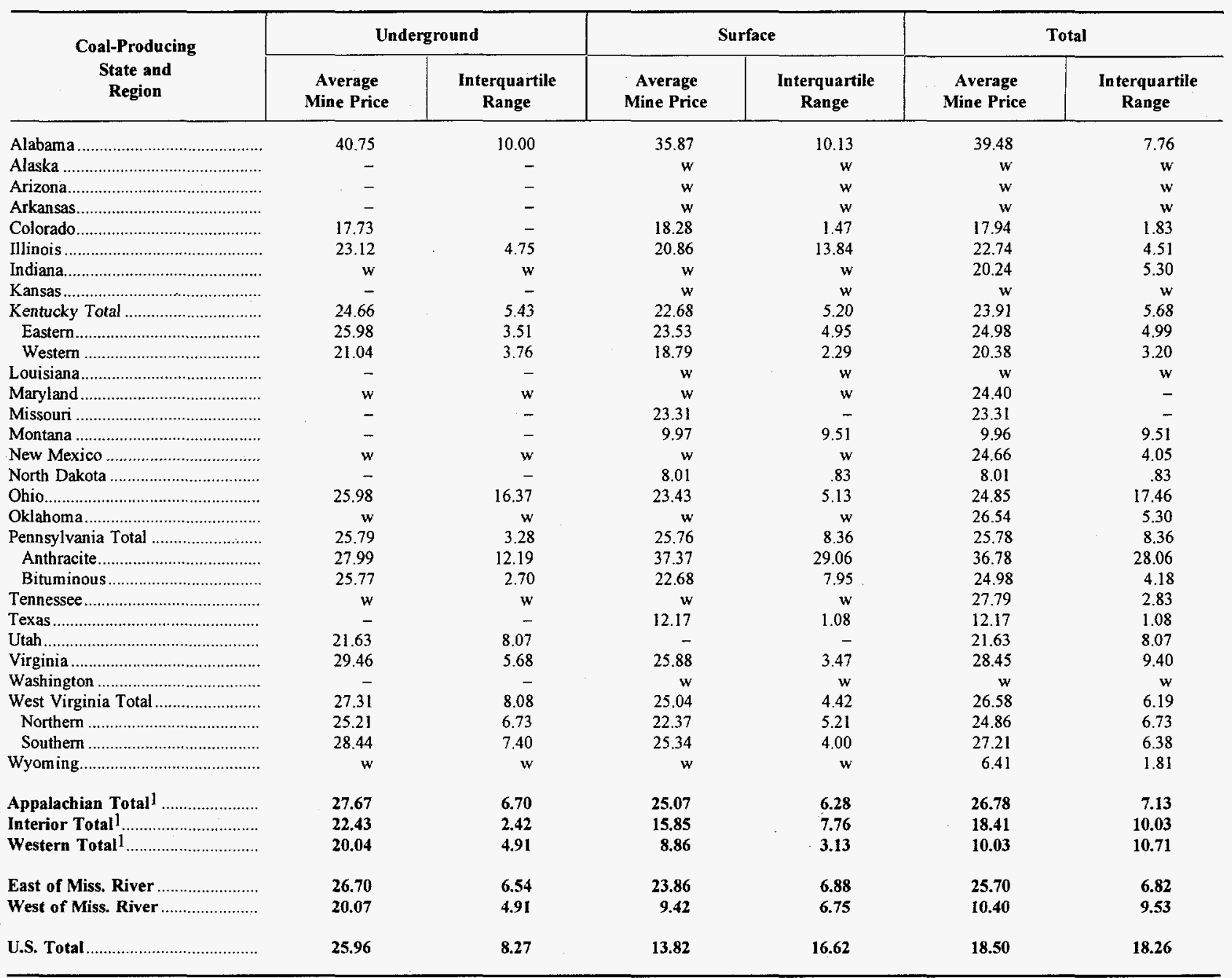

1 For a definition of coal-producing regions, see Appendix C.

w Withheld to avoid disclosure of individual company data.

Note: Excludes silt, culm, refuse bank, slurry dam, and dredge operations except for Pennsylvania anthracite. Excludes mines producing less than 10,000 short tons of coal during the year. Average Mine Price is calculated by dividing the total free on board (f.o.b) mine value of the coal produced by the total production.

Sources: Energy Information Administration, Form EIA-7A, "Coal Production Report"; State Mining Agency Coal Production Reports; and/or U.S. Department of Labor, Mine Safety and Health Administration, Form 7000-2, "Quarterly Mine Employment and Coal Production Report."

turing plants. See Technical Note 5 for data adjustment procedures for coal consumption for the other industrial sector.

Current year data from this survey are preliminary and unrevised in the January - March, April - June, and July - September issues of the Quarterly Coal Report (DOE/EIA-0121). In the October - December issue, any revisions necessary for the entire year are applied and the data are considered final.

The respondent list of manufacturers for Form EIA-3 is compared with lists of coal-consuming manufacturing plants from State Air Quality and Energy
Offices. When new respondents are found, they are added to the survey mailing list.

\section{Annual Coal Quality Report - Manufacturing Plants (Form EIA-3A)}

Form EIA-3A contains questions on the origin of coal (State or Country), the quantity of coal receipts, the Btu, sulfur and ash content of the coal receipts, and the basis used to determine the coal quality data. The threshold for the annual collection will be the same as for the EIA-3: manufacturing plants that consume in excess of 1,000 short tons of coal per per year. In 
1995,730 manufacturers responded to the EIA-3A survey. The response rate for the current year was 100 percent.

\section{Coke Plant Report (Form EIA-5)}

Form EIA-5, a quarterly report of coal receipts, carbonization, and stocks, and of coke and breeze production, distribution, and stocks, is used to survey all U.S. coke plants.

Coke plants were surveyed monthly and a supplemental survey was taken annually until 1981, when the reporting cycle was revised to a quarterly schedule with an annual supplemental survey. In 1985, collection of the annual supplement was ended.

In 1995 , there were 28 respondents to the EIA-5 survey, and the response rate was 100 percent. The respondent list for this survey is updated by continuous monitoring of the industry literature.

Current year data from this survey are preliminary and unrevised in the January - March, April - June, and July - September issues of the Quarterly Coal Report (DOE/EIA-0121). In the October - December issue, any revisions necessary for the entire year are applied and the data are considered final.

\section{Annual Coal Quality Report - Coke Plants (Form EIA-5A)}

This form contains questions on the origin of coal (State or country), the quantity of coal receipts, the volatile matter, sulfur and ash content, and the basis used to determine the coal quality data. There is no threshold for this form. In 1995, there were 28 respondents to the EIA-5A survey, and the response rate was 100 percent.

\section{Coal Distribution Report (Form EIA-6)}

Form EIA-6 is used to survey all U.S. companies (producers and/or distributors) that own or purchase and distribute more than 50,000 short tons annually. ${ }^{2}$ Data on coal production and purchases, distribution by consumer category, and method of transportation are reported.

In 1995 , there were about 1,100 respondents to the EIA-6 survey. Until the end of 1988 , coal distribution companies were required to report production on a Bureau of Mines district basis. For the year 1989 , respondents were required to report on a BOM district/State basis. Beginning with the first quarter of 1990 , respondents were required to report on a State basis. The response rate for the current quarter was 100 percent. The annual production total reported on Form EIA-6 exceeds 99 percent of total production as reported by all mines on Form EIA-7A, "Coal Production Report," due to the difference in reporting thresholds. The data gathered on the Form EIA- 6 only represent the domestic coal distributed during the quarter. Therefore, imported coal distributed during the quarter is not included.

Current year data from this survey are preliminary and unrevised in the January - March, April - June, and July - September issued of the Quarterly Coal Report (DOE/EIA-0121). In the October - December issue, any revisions necessary for the entire year are applied and the data are considered final.

The respondent list for this survey is updated by comparing it with lists of coal producers from the Mine Safety and Health Administration (MSHA), U.S. Department of Labor, and from similar lists maintained by various State agencies. Also, new respondents are frequently identified on Form EIA-6 itself when other companies are named as sources of coal purchases.

\section{Electric Utility Surveys}

Coal data appear in this report from three surveys of electric utilities --from all generating electric utilities and from fossil-fueled plants.

The Census Bureau collected and published the results of a census taken every 5 years from 1902 to 1937 on the electric light and power industries and some data on industrial production of electric energy. The U.S. Geological Survey collected data on capacity and generation of electric utilities from 1920 to 1936, when this activity was turned over to the Federal Power Commission (FPC).

All data are presented as reported on the surveys. No estimates or other adjustments are made for missing data. The data are maintained in a computer system and are edited to ensure that they are reasonable, consistent, and complete. For additional information from these surveys and for other electric utility data, see the EIA publication, Electric Power Monthly (DOE/EIA-0226).

\section{Monthly Power Plant Report (Form EIA-759)}

Form EIA-759 (which, until 1982, was called FPC Form 4) is used to survey all generating electric utilities. The Federal Power Act and FPC Order Number 141 define the legislative authority to collect power production data. Consumption and stocks of coal and other fuels at each plant are reported. The respondents to Form EIA-759, approximately 3,000 plants, account for 100 percent of total electric utility generation.

2 For the States of Arkansas, Maryland, Oklahoma, and the anthracite portion of Pennsylvania, the threshold is 10,000 tons. 
Current year data from this survey are considered final.

\section{Monthly Report of Cost and Quality of Fuels for Electric Plants (FERC Form 423)}

Federal Energy Regulatory Commission (FERC) Form 423 is used to survey all fossil-fueled plants with a total steam-generating capacity of 50 megawatts or more. It is submitted by approximately 230 electric utilities. (Before 1983, this form was called FPC Form 423 , and all fossil-fueled plants with a total generating capacity of 25 megawatts or more were surveyed.) In 1972, the FPC issued Order Number 453, which included the legislative authority to create FERC Form 423. Cost, quality, and source of fuels (by State or country of origin), including coal, are reported. Current year data from this survey are considered final.

\section{Steam-Electric Plant Operation and Design Report (Form EIA-767)}

The Form EIA-767 is a mandatory restricted-universe census of all electric power plants with a total existing or planned organic- or nuclear-fueled steamelectric generator nameplate rating of 10 or more megawatts. The entire form is filed by approximately 700 power plants with a nameplate capacity of 100 or more megawatts. An additional 200 power plants with a nameplate capacity between 10 and 100 megawatts submit information only on fuel consumption/quality, boiler/generator configuration, and flue-gas desulfurization equipment, if applicable. The Form EIA-767 is used to collect data annually on plant operations and equipment design (including boiler, generator, cooling system, flue gas desulfurization, flue gas particulate collectors, and stack data). Data from Form EIA-767 are used for economic, regulatory, and environmental analyses conducted by the DOE, the FERC, the Environmental Protection Agency, and the Department of Commerce.

Form EIA-767 data for 1995 are preliminary. Data for prior years are final.

\section{Annual Nonutility Power Producer Report (Form EIA-867)}

The Form EIA-867 is a restricted-universe census used to collect annual data from all existing and planned nonutility power producers in the United States. In 1992, the reporting threshold of the Form EIA-867 was lowered to include all facilities with a combined nameplate capacity of 1 or more megawatts. Previously data were collected every 3 years from facilities with a nameplate capacity between 1 and 5 megawatts. For the purpose of this data collection, a nonutility power producer is an enterprise that has electric generating capacity and is not an electric utility. They include qualifying cogenerators, qualifying small power producers, and other nonutility generators (including independent power producers) without a designated franchised service area. The form is used to collect data on the installed capacity, energy consumption, generation, and electric energy sales to electric utilities from approximately 2,000 facilities.

Form EIA-867, coal consumption data for 1989 through 1995 are: $876,1600,6000,10000,12344$, 15140 and 20800 thousand short tons.

\section{Export and Import Data}

Export and import data (except imports to electric utilities, manufacturing plants and coke plants, which are reported on the FERC Form 423, EIA-3A, and EIA-5A, respectively.) are obtained from the Census Bureau--export data from the monthly EM 545 (formerly EM 522) report, import data from the monthly IM 145 report. The Census Bureau compiles these data monthly from documents filed with the U.S. Customs Service as required by law. They include shippers' export declaration forms, import entry forms, and warehouse withdrawal forms. No sampling procedures are used. The Census Bureau publication Guide to Foreign Trade Statistics describes the foreign trade statistics program, including the EM 545 and IM 145 monthly reports. Data from these surveys are considered final at the time of publication.

Foreign distribution of U.S. coal, major exporting State, and destination, along with foreign distribution of metallurgical and steam coal (Tables 62, 63, and 64, respectively), was determined using EIA-6 distribution data by origin State, and coal export data from King's COALBASE (King Publishing Corporation, Knoxville, Tennessee) which gives the metallurgical and steam breakdown as well as the country destination data. The percentage of metallurgical and steam coal for each country of destination are applied to the EIA-6 export figures for each State of origin to derive coal distribution data that link State of origin to countries of destination by type of coal. The King's destination country data are considered to be more accurate than the Census country-of-destination data because it account for transhipments through intermediate countries to final destination countries, whereas the Census data would designate the destination as the intermediate country.

Copies of the survey forms and instructions used to collect data appearing in this publication can be obtained by calling EIA's National Energy Information Center at (202) 586-8800. 


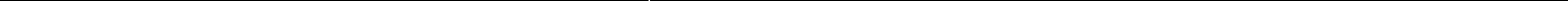




\section{Technical Notes}

\section{Differences in Related Coal Data}

Coal Production versus Coal Distribution. Coal production represents newly mined coal. Coal distribution represents shipments of newly mined coal and coal from producer/distributor stockpiles (previously mined coal).

Coal Distribution versus Coal Receipts. Differences in coal distribution data and coal receipts data are due to the time lag between distribution and receipt of coal shipments, and due to the survey threshold differences. In addition, coal distributed includes only domestic coal, whereas receipts include imported coal.

Foreign Distribution of U.S. Coal versus U.S. Coal Exports. Foreign distribution of U.S. coal does not equal U.S. coal exports because there are differences in reporting time and survey thresholds.

Receipts of Imported Coal versus U.S. Coal Imports. Receipts of imported coal at electric utilities and manufacturing and coke plants does not equal U.S. coal imports due to reporting time differences. In addition, it does not include receipts at independent power producers.

\section{Other Industrial Plants and Manufacturing}

The other industrial plants end-use sector includes the manufacturing, agriculture, forestry and fishing, mining, and construction industries. Manufacturing accounts for approximately 97 percent of the coal receipts and consumption and 100 percent of the coal stocks in the other industrial plants sector as reported herein. Data sources for the other industrial plants sector and the manufacturing sector are Forms EIA-6 and EIA-3, respectively. The source statement in each table identifies the survey used to collect coal data for the other industrial plants sector, and the following technical notes describe the methodology used when data were derived.

\section{Residential and Commercial}

To reduce the reporting burden to coal users, the EIA does not conduct any survey of coal data from residential and commercial users of coal. Shipments of coal to this sector, reported by producers and distributors of coal on Form EIA-6, are equated to coal receipts and consumption by the residential and commercial sector, assuming no stock changes.

\section{Consumer Prices}

Prices are derived for each end-use sector as follows:

Electric Utilities. Prices are reported for each plant in cents-per-million Btu on FERC Form 423. The price per ton of coal is calculated at each plant using centsper-million Btu and the average Btu content per pound of coal for the appropriate rank of coal. The average prices appearing in the tables (e.g., across all States) are calculated by summing the dollar value at each plant (short tons of coal multiplied by price per short ton) and dividing by the corresponding total tons. For more information about prices of coal at electric utilities, see the EIA publication, Electric Power Monthly (DOE/EIA-0226).

Coke Plants. Respondents are asked to report the number of tons of coal received (or coke distributed) on Form EIA-5 and the total value of that coal (or coke) in dollars. Average prices are calculated by summing the reported values (e.g., across all States) and dividing by the corresponding total tons.

Other Industrial Plants. Respondents (manufacturing plants only) are asked to report the number of tons of coal received on Form EIA-3 and the total value of that coal in dollars. Average prices are calculated by summing the reported values across all States and dividing by the corresponding total tons.

Residential and Commercial. Data are not collected. See Technical Note 3. 


\section{Consumption}

\section{Annual Data}

Annual coal consumption data are sums of quarterly or monthly data described below except for nonutility power producers whose coal consumption is not included in this report. These data are however, reported on Form EIA-867 and published in the Electric Power Annual (DOE/EIA-0348).

Electric Utilities. Consumption is reported on Form EIA-759.

Nonutility Electric Generating Facilities. Consumption is reported on Form EIA-867.

Coke Plants. Consumption is reported on Form EIA-5.

Other Industrial Plants. In deriving a quarterly estimate for coal consumption for the other industrial plants sector, the first step is to equate consumption to beginning stocks plus receipts minus ending stocks. In terms of an equation, consumption can be expressed as $\mathrm{C}=\mathrm{Sb}+\mathrm{R}-\mathrm{Se}$, where $\mathrm{Sb}=$ beginning stocks, $\mathrm{R}=$ receipts, and $\mathrm{Se}=$ ending stocks.

Therefore, consumption is $\mathrm{C}=(\mathrm{Sb}-\mathrm{Se}$ (change in stocks)) $+\mathrm{R}$. Next, stock change at the State level is equated to the stock change for that State as reported on Form EIA-3, receipts at the State level are derived as described in Section 3 , and a computed consumption is derived using the same equation for each State. Finally, the quarterly consumption $(C)$ at the State level is equated to the maximum of the computed consumption at the State level, as previously described, and the quarterly consumption for that State as reported on Form EIA-3. This process ensures that State-level consumption for the other industrial plants sector is always greater than or equal to the manufacturing sector consumption for that State. Total quarterly consumption for the other industrial plants sector is computed by summing the quarterly State-level consumption figures.

Residential and Commercial. Shipments to the residential and commercial sector as reported on Form EIA-6 are defined as consumption as well as receipts for this end-use sector.

EIA publishes monthly estimates of coal consumption in the Monthly Energy Review (DOE/EIA-(1035).

Monthly coal consumption at electric utility plants is derived directly from Form EIA-759. Prior to 1980 , monthly coal consumption at coke plants was derived directly from Form EIA-5. For 1981 through 1987, it was derived from the quarterly coal consumption reported on Form EIA-5, using the ratios of monthly to quarterly consumption in 1979 , the last year that coke plant data were collected monthly on Form EIA-5. These ratios by month (January - December) are $0.3377,0.3200,0.3423 ; 0.3529,0.3462,0.3009$; $0.3364,0.3347,0.3289$; and $0.3273,0.3301,0.3426$.
Starting with 1988 , monthly coal consumption at coke plants is derived from quarterly coal consumption reported on Form EIA-5, using ratios derived from monthly data on raw steel production published by the American Iron and Steel Institute (AISI) on Form AIS7. The ratio is the proportion of monthly raw steel production from open hearth and basic oxygen process furnaces to the quarterly raw steel production from those furnace types.

Prior to 1978 , coal consumption for the other industrial plants sector (i.e., industrial users minus coke plants) was derived by using monthly data reported on Form EIA-3 to modify baseline coal consumption figures from the most recent Census of Manufactures or Annual Survey of Manufactures, Bureau of the Census, U.S. Department of Commerce. For 1978 through 1987, data from Forms EIA-3 and EIA-6 are used to compute monthly coal consumption for the other industrial plants sector.

Given the quarterly consumption for the other industrial plants sector (C), the monthly consumption for the sector $(\mathrm{Cm})$ is estimated for each month in the quarter as $\mathrm{Cm}=(\mathrm{Cm} 3 / \mathrm{C} 3) \times \mathrm{C}$ where $\mathrm{Cm} 3 / \mathrm{C} 3$ is the ratio of monthly to quarterly coal consumption as reported on Form EIA-3. For the 1978 coal consumption figures, the ratios used are based on 1978 EIA-3 data. For 1979 through 1987, the ratios used are based on the 1979 EIA-3 data. These 1979 ratios by month (January - December) are 0.3593, 0.3264, 0.3143; $0.3485,0.3332,0.3183 ; 0.3317,0.3407,0.3276$; and $0.3045,0.3253,0.3702$.

Starting with 1988, monthly coal consumption for the other industrial plants sector is derived from quarterly coal consumption reported on Form EIA-3 using monthly ratios derived from the industrial production indices published by the Board of Governors of the Federal Reserve System. Six major industry groups' indices are used as the basis for calculating the monthly ratios. These groups are foods (Standard Industrial Classification (SIC) 20), paper and products (SIC 26), chemicals and products (SIC 28), petroleum products (SIC 29), clay, glass, stone products (SIC 32 ), and primary metals (SIC 33).

The monthly ratios are computed as the monthly sum of weighted indices as a proportion of the quarterly sum of weighted indices, using the 1985 proportion as the weight.

Prior to 1980 , monthly coal consumption for the residential and commercial sector was derived by using monthly data reported on Form EIA-2, "Monthly Coal Report -- Retail Dealers and Upper Lake Docks," to modify baseline coal consumption figures developed by the Bureau of Mines, U.S. Department of the Interior.

For 1980 , the quarterly coal consumption figures in the residential and commercial sector are converted to monthly coal consumption figures using the ratios of monthly to quarterly coal deliveries to this sector in 1979 as reported on Form EIA-2. These 1979 ratios by month (January-December) are $0.4002,0.3502$, 
$0.2496 ; \quad 0.4805,0.2901,0.2294 ; 0.3126,0.2952$, 0.3922 ; and $0.2931,0.3101,0.3968$. The 1981 and 1982 monthly coal consumption figures were derived using the 1979 ratios but were also modified according to heating/cooling degree-days. For 1983 through 1987, coal consumption figures are converted to monthly coal consumption figures using only the ratios of monthly to quarterly coal deliveries to this sector in 1979.

Starting with 1988 , monthly coal consumption figures are derived using the monthly national average population weighted heating/cooling degree-days obtained from the National Oceanic and Atmospheric Administration. The ratio is the proportion of the monthly national sum of heating and cooling degree-days to the quarterly sum.

\section{Stocks}

Annual stocks are calculated at the end of the year or the end of the fourth quarter. Coal stocks are derived for each end-use sector as follows:

Electric Utilities. Stocks are reported on Form EIA-759.

Coke Plants. Stocks are reported on Form EIA-5.

Other Industrial Plants. Stocks are reported on Form EIA-3, i.e., stocks at manufacturing plants only. Technical Note 2 discusses the difference between other industrial plants and manufacturing plants.

Residential and Commercial. Data are not available. See Technical Note 3.

Producer and Distributor. Stocks are reported on Form EIA-6.

\section{Methods of Transportation}

Rail: Shipments of coal moved to consumers by rail, either private or public/commercial. Included is coal hauled to or away from a railroad siding by truck.

Water Transportation: Shipments of coal moved by one of the three methods--river, Great Lakes, or tidewater piers and coastal ports. Included in these shipments is coal hauled to or from water loading facilities by other means of transportation.

River: Shipments of coal moved to consumers via river by barge, except shipments to Great Lakes coal loading docks or tidewater piers or coastal ports.

Great Lakes: Shipments of coal moved to consumers via the Great Lakes. These shipments are moved via the Great Lakes coal loading docks, which are iden- tified by name and locations as follows: Superior Midwest Energy Terminal, Superior, Wisconsin; Bessemer \& Lake Erie Coal Storage \& Transfer Facility, Conneaut, Ohio; B\&O Railroad Coal Loading Dock, Lorain, Ohio; C\&O Railroad Presque Isle Docks, Toledo, Ohio; Lakefront Dock \& Railroad Terminal Company Coal Loading Dock, Toledo, Ohio; N\&W Sandusky Coal Pier No. 3, Sandusky, Ohio; ConRail Coal Transfer Facilities, Ashtabula, Ohio; Rail to Water Transfer Corporation Dock, Chicago, Illinois.

Tidewater Piers and Coastal Ports: Shipments of coal moved to tidewater piers and coastal ports for further shipments to consumers via coastal water or ocean. The tidewater piers are identified by name and location as follows: B\&O Curtis Bay Coal Piers, Baltimore, Maryland; C\&O Coal Piers Nos. $14 \& 15$, Newport News, Virginia; N\&W Lamberts Point Coal Piers Nos. 5 \& 6, Norfolk, Virginia; Alabama State Docks Bulk Handling Plant, Mobile, Alabama; Alabama State Docks/McDuffie Terminals, Mobile, Alabama; Canton Coal Piers, Baltimore Harbor on the Chesapeake Bay; Greenwich Coal Pier, Greenwich Point, Philadelphia, Pennsylvania, on Delaware River; Port Richmond Pier, Pier 18 Port Richmond, Philadelphia, Pennsylvania, on the Delaware River; Galveston Regional Coal Distribution Center, Pelican Island, Galveston, Texas; International Marine Terminals/Plaquemines Parish Terminal, Mile 57 AHP-Mississippi River, approximately 30 miles south of New Orleans; Energy Terminals of Houston, Inc., a Subsidiary of Soros Associates, Houston, Texas. Coastal Ports are those located at Charleston, South Carolina; New York, New York; San Diego, California; Los Angeles, California; and Seattle, Washington.

Truck: Shipments of coal moved to consumers by truck.

Tramway, Conveyor, or Slurry Pipeline: Shipments of coal moved to consumers by tramway, conveyor, or slurry pipeline.

\section{Census Export and Import Data}

Export and import data are obtained from the Bureau of the Census, U.S. Department of Commerce, where they are compiled monthly from documents filed with the U.S. Customs Service, as required by law.

Each coal shipment is reported in short tons with corresponding total dollar values. EIA converts all value data obtained from the Census Bureau to average price data by dividing the dollar value by the quantity.

Based on an analysis and sample validation of the Census Bureau import and export data conducted by the EIA, it was determined that some of the coal and coke data collected from the Census Bureau may be misleading or incorrect (particularly those data associ- 
ated with very small quantities or very high prices). Because of this, a methodology was developed to edit the Census Bureau price data.

Prior to 1989 , certain data cells had been suppressed for publication purposes only: (1) average import coal prices of $\$ 50.00$ or more per short ton; (2) average export coal prices of $\$ 60.00$ or more per short ton; (3) average coke prices of $\$ 200.00$ or more per short ton; (4) all percent changes of 500 percent or more.

Beginning with 1989, coal export data were categorized as metallurgical coal and steam coal, rather than as bituminous steam coal, lignite, anthracite, and bituminous metallurgical coal.

In addition, coal export tables were revised to present those countries to which the United States exported more than 50,000 short tons in the prior calendar year. The remaining countries in each continent were aggregated in an "other" category. This reduces the number of empty cells and highlights the major importers of U.S. coal. All coke export and import, and coal import countries and quantities are displayed.

The following methodology was used to derive the typical average prices as presented in the price tables. For all coal, a price distribution was derived from the prior calendar year export price data. Since extreme price variations in the Census Bureau data are the exception rather than the rule, the price distribution was used to identify a typical price range. The price distribution, from low to high, along with the frequency of each price (quantity) was analyzed to determine the representative prices. The extreme prices at both ends of the distribution were eliminated to arrive at a price range that covered at least 90 percent of the exports. This price range was considered to include typical or representative prices. Considering the records that fell within the typical price range, the weighted average price was calculated by country of destination and type of coal.

The same procedure was used to determine the typical average prices of coal imports. In addition to the average prices based on the above methodology, a U.S. total row is presented in the price tables, which represents the average price using all the Census Bureau data.

For reporting purposes, the month of exportation reflects the month in which the shipment leaves the United States. The month of importation generally is based on the month in which the U.S. Customs Service releases the merchandise to the importer. For both sets of data, however, there can exist a small carry-over from the actual month of exportation or importation to a subsequent month, usually the succeeding month. A number of factors in processing account for this, e.g., late receipt of a document for an end-of-month shipment, or rejection of a shipment by the computer due to failure to meet established edit criteria. These limitations should be considered when making comparisons.

Based on the U.S. - Canada Free Trade Agreement, as of January 1990, the U.S. Department of Commerce began reporting statistics on U.S. exports to Canada based on information on imports provided monthly by the Canadian government.

Comparing Census reported imported coal figures in Table 34 with EIA reported imported coal receipts at electric utilities, manufacturers, and coke plants for 1994 shows a difference of about 1.8 million short tons. The main reason for this is that the EIA receipts data do not cover imported coal received by nonutility power producers who are not in the manufacturing sector.

\section{Revisions}

All data published in this report are considered final. The Office of Coal, Nuclear, Electric and Alternate Fuels has adopted the following policy with respect to the revision and correction of recurrent data in energy publications:

1. Annual survey data collected by this office are published either as preliminary or final when first appearing in a data report. Data initially released as preliminary will be so noted in the report. These data will be revised, if necessary, and declared final in the next publication of the data.

2. All monthly and quarterly survey data collected by this office are published as preliminary. These data are revised only after the completion of the 12-month cycle of the data. No revisions are made to the published data before this.

3. After data are published as final, corrections will be made only in the event of a greater than one percent difference at the national level. Corrections for differences that are less than the 1-percent threshold are left to the discretion of the Office Director.

\section{Price Data and Taxes}

F.O.B. mine coal prices and prices of coal delivered to or received by end-use consumers (electric utility plants, manufacturing plants, and coke plants) as reported in this publication include relevant local, State and Federal excise and sales taxes. 
Table D3. Implicit Price Deflator,

1987-1996

\begin{tabular}{c|c}
\hline Year & $\begin{array}{c}\text { Implicit Price Deflator } \\
(\mathbf{1 9 9 2}=\mathbf{1 0 0 )}\end{array}$ \\
\hline 1987 & 83.1 \\
1988 & 86.1 \\
1989 & 89.7 \\
1990 & 93.6 \\
1991 & 97.3 \\
1992 & 100.0 \\
1993 & 102.6 \\
$1994^{\mathrm{R}}$ & 104.9 \\
$1995^{\mathrm{R}}$ & 107.6 \\
1996 & 109.7 \\
\hline
\end{tabular}

\footnotetext{
${ }^{\mathbf{R}}$ Revised data.

Source: Bureau of Economic Analysis, U.S. Department of Commerce, Survey of Current Business.
} 


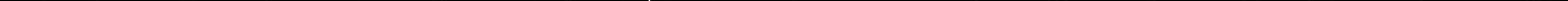




\section{Glossary}

Agglomerating Character: Agglomeration describes the caking properties of coal. Agglomerating character is determined by examination and testing of the residue when a small powdered sample is heated to 950 degrees centigrade under specified conditions. If the sample is "agglomerating," the residue will be coherent, show swelling or cell structure, and be capable of supporting a 500-gram weight without pulverizing.

Anthracite: A hard, black lustrous coal, often referred to as hard coal, containing a high percentage of fixed carbon and a low percentage of volatile matter. Comprises three groups classified according to the following ASTM Specification D388-91a, on a dry mineral-matter-free ( $\mathrm{mmf}$ ) basis:

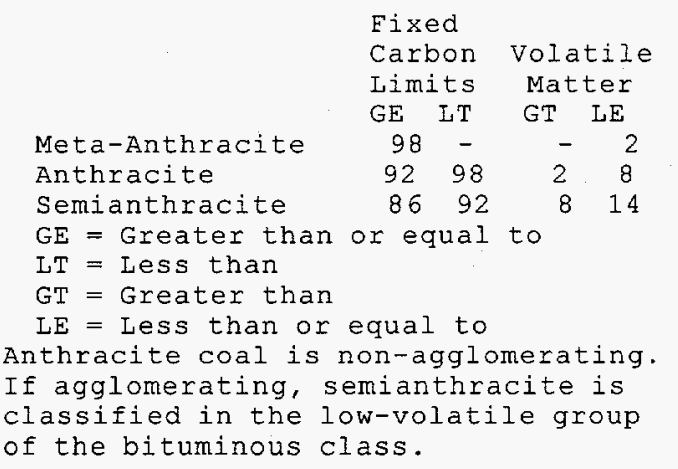

Ash: Impurities consisting of silica, iron, alumina, and other incombustible matter that are contained in coal. Ash increases the weight of coal, adds to the cost of handling, and can affect the burning characteristics. Ash content is measured as a percent by weight of coal on an "as received" or a "dry" (moisture-free, usually part of a laboratory analysis) basis.

Auger Mine: A surface mine where coal is recovered through the use of a large-diameter drill driven into a coalbed in a hillside. It usually follows contour surface mining, particularly when the overburden is too costly to excavate.

\section{Average Annual Percent Change:}

$$
\sqrt[n]{\frac{V_{n}}{V_{0}}}-1
$$

Where: $V_{0}=$ the value for the base period.

$V_{n}=$ the value for the $n^{\text {th }}$ period.

$\mathrm{n}=$ the number of periods.
Average Daily Production: The ratio of the total production at a mining operation to the total number of production days worked at the operation.

Average Length of a Shift: The arithmetic mean number of hours worked during a production shift. Overtime is included if usually worked during the year.

Average Mine Price: The ratio of the total value of the coal produced at the mine to the total production tonnage. (See F.O.B. mine price.)

Average Number of Employees per Shift: The arithmetic mean number of employees working during a production shift. Includes all employees except office workers. (See direct labor hours.)

Average Number of Miners Working Daily: The arithmetic mean number of miners working each day at a mining operation. Includes maintenance as well as production work performed.

Average Number of Shifts per Day: The arithmetic mean number of shifts each day at a mining operation. Includes maintenance as well as production shifts.

Average Production per Miner per Day: The product of the average production per miner per hour at a mining operation and the average length of a production shift at the operation.

Average Production per Miner per Hour: The ratio of the total production at a mining operation to the total direct labor hours worked at the operation.

Average Production per Miner per Shift: Calculated by multiplying average production per miner per hour by the average length of a miner shift.

Average Quality of Coal: Refers to individual measurements such as heat value, fixed cabon, moisture, ash, sulfur, phosphorus, major, minor, and trace elements, coking properties, petrologic properties, and particular organic constituents. The individual quality elements may be aggregated in various ways to classify coal for such special purposes as metallurgical, gas, petrochemical, and blending usages.

Average Recovery Percentage: Average recovery percentage represents the percentage of coal that can be recovered from coal reserves at reporting mines, averaged for all mines in the reported geographic area.

Bituminous Coal: The most common coal. It is dense and black (often with well-defined bands of 
bright and dull material). Its moisture content is usually less than 20 percent. It is used for generating electricity, making coke, and for space heating. Comprises five groups classified according to ASTM Specification D-388-91a, on a dry mineral-matter-free $\mathrm{mmf}$ basis for fixed-carbon and volatile matter and a moist $\mathrm{mmf}$ basis for calorific value. Coals having 69 percent or more fixed carbon on the dry, mineralmatter-free basis shall be classified according to fixed carbon, regardless of calorific (heating) value. Highvolatile $\mathrm{C}$ bituminous coal is agglomerating, but other bituminous coals are commonly agglomerating. However, it is recognized that there may be nonagglomerating varieties in these groups of the bituminous class, and there are notable exceptions in the high-volatile $\mathrm{C}$ bituminous group. Coals with less than 69 percent fixed carbon, but with 14,000 or more Btu per pound, are classified as high-volatile A bituminous.

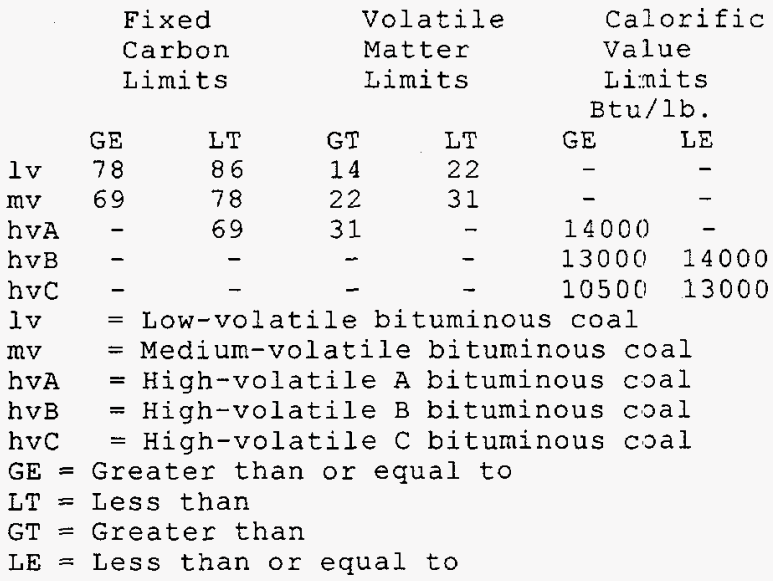

Btu (British thermal unit): The amount of heat needed to raise the temperature of 1 pound of water by 1 degree fahrenheit. The Btu is a convenient measure by which to compare the energy content of various fuels:

Cannel Coal: A variety of bituminous coal that is noncaking, contains a high percentage of volatile matter, ignites easily, and burns with a luminous smokey flame.

Capacity Utilization: Capacity utilization is computed by dividing production by productive capacity and multiplying by 100 .

Captive Coal: Coal produced and consumed by the mine operator, a subsidiary, or parent company (for example, steel companies and electric utilities).

Carbon Dioxide: $\mathrm{CO}_{2}$ A colorless, odorless, incombustible gas formed during combustion in fossil-fuel electric generation plants.

Census Divisions: The nine geographic divisions of the United States established by the Bureau of the Census, U.S. Department of Commerce for statistical analysis. The boundaries of Census divisions coincide with State boundaries. In some cases, the Pacific
Division is subdivided into the Pacific Contiguous and Pacific Noncontiguous areas.

CIF: See Cost, Insurance, Freight.

Coal Carbonized: The amount of coal decomposed into solid coke and gaseous products by heating in a coke oven in a limited air supply or in the absence of air.

\section{Coal (Coke): See Coke (coal).}

Coal Mining Productivity: Coal mining productivity is calculated by dividing total coal production by the total direct labor hours worked by all mine employees.

Coal Preparation: The process of sizing and cleaning coal to meet market specifications by removing impurities such as rock, sulfur, etc. May include crushing, screening, or mechanical cleaning.

Coal-Producing Regions: A geographic classification of coal-producing States. The States in the Appalachian Region are Alabama, Georgia, eastern Kentucky, Maryland, Ohio, Pennsylvania, Tennessee, Virginia, and West Virginia. The States in the Interior Region are Arkansas, Illinois, Indiana, Iowa, Kansas, western Kentucky, Louisiana, Missouri, Oklahoma, and Texas. The States in the Western Region are Alaska, Arizona, California, Colorado, Montana, New Mexico, North Dakota, Utah, Washington, and Wyoming.

Coal-Producing States: The States where mined and/or purchased coal originates are defined as follows: Alabama, Alaska, Arizona, Arkansas, California, Colorado, Illinois, Indiana, Iowa, Kansas, Kentucky Eastern, Kentucky Western, Louisiana, Maryland, Missouri, Montana, New Mexico, North Dakota, Ohio, Oklahoma, Pennsylvania anthracite, Pennsylvania bituminous, Tennessee, Texas, Utah, Virginia, Washington, West Virginia Northern, West Virginia Southern, and Wyoming. The following Coal-Producing States are split in origin of coal, as defined below:

- Kentucky, Eastern All mines located in counties other than the Western Kentucky counties.

- Kentucky, Western All mines in the following counties in Western Kentucky: Butler, Caldwell, Christian, Crittenden, Daviess, Edmonson, Grayson, Hancock, Henderson, Hopkins, Logan, McLean, Muhlenberg, Ohio, Simpson, Todd, Union, Warren, and Webster.

- Pennsylvania Anthracite All mines in the following counties: Carbon, Columbia, Dauphin, Lackawanna, Lebanon, Luzerne, Northumberland, Schuylkill, Sullivan, and Susquehanna. All anthracite mines in Bradford County.

- Pennslyvania Bituminous All mines located in counties other than the Pennsylvania anthracite counties and all bituminous mines in Bradford County.

- West Virginia, Northern All mines in the following counties (formerly defined as Coal- 
Producing Districts 1, 3, \& 6): Barbour, Brooke, Braxton, Calhoun, Doddridge, Gilmer, Grant, Hancock, Harrison, Jackson, Lewis, Marion, Marshall, Mineral, Monongalia, Ohio, Pleasants, Preston, Randolph, Ritchie, Roane, Taylor, Tucker, Upshur, Webster, Wetzel, Wirt, and Wood.

- West Virginia, Southern All mines in the following counties (formerly defined as CoalProducing Districts 7 \& 8): Boone, Cabell, Clay, Fayette, Greenbrier, Kanawha, Lincoln, Logan, Mason, McDowell, Mercer, Mingo, Monroe, Nicholas, Pocahontas, Putnam, Raleigh, Summers, Wayne, and Wyoming.

Coal Rank/Group: A classification of coal based on fixed carbon, volatile matter, calorific (heating) value, and agglomerating character. Coal is ranked progressively from lignite (least carbonaceous) to anthracite (most carbonaceous). The rank of coal can also determined by measuring the reflectance of vitrinite, one of several organic components of coal. The lower rank coal can be classified based on heat content. The heat content of the higher rank coals is generally above 14 thousand Btu per pound for each coal rank group (except for meta-anthracite, which trends slightly lower), and heat content ranges vary within a relatively narrow range. Since heat content is not a dependable criterion for these higher rank coals, their rank categories are instead described by degree of metamorphism, or "coalification," a property that is measured by fixed carbon content. Finally, the agglomerating character of bituminous coals is a critical attribute for certain coal consumers, and thus agglomerating character has come to define the distinctions between certain adjacent coal groups. Some high-volatile $C$ bituminous and subbituminous $A$ coals can be distinguished only on the basis of agglomerating character. Percentages are based on dry mineral-matter-free coal. Volatile matter (not shown) is the complement of fixed carbon; that is, the percentages of fixed carbon and volatile matter sum to 100 percent. As fixed carbon percentage decreases, therefore, volatile matter percentage increases by the same amount.

Coal Stocks: The supply of coal at a mine, plant, or utility at the end of the reporting period.

Coalbed: A bed or stratum of coal. Also called a coal seam.

Cogenerator: A generating facility that produces electricity and another form of useful thermal energy (such as heat or steam) used for industrial, commercial, heating, and cooling purposes. To receive status as a qualifying facility (QF) under the Public Utility Regulatory Policies Act (PURPA), the facility must produce electric energy and "another form of useful thermal energy through the sequencial use of energy," and meet certain ownership, operating, and efficiency criteria established by the Federal Energy Regulatory Commission (FERC). (See the Code of Federal Regulation, Title 18, Part 292.)

Coke (coal): In general, coke is made from bituminous coal (or blends of bituminous coal) from which the volatile constituents are driven off by baking in an oven at temperatures as high as 2,000 degrees Fahrenheit, so that the fixed carbon and ash are fused together. Coke is hard and porous, has a gray, submetallic luster, and is strong enough to support a load of iron ore in a blast furnace. It is used both as a fuel and a reducing agent in smelting iron ore in a blast furnace. Coke has a heating value of 24.8 million Btu per short ton.

Coke Plants: Plants where coal is carbonized in slot or beehive ovens for the manufacture of coke.

Continuous Mining: A form of room-and-pillar mining in which a continuous mining machine extracts and removes coal from the working face in one operation; no blasting is required.

Conventional Mining: The oldest form of room-andpillar mining which consists of a series of operations that involve cutting the coalbed so it breaks easily when blasted with explosives or high-pressure air, and then loading the broken coal.

Cost, Insurance, Freight (CIF): A type of sale in which the buyer of the product agrees to pay a unit price that includes the F.O.B. value of the product at the point of origin plus all costs of insurance and transportation. This type of transaction differs from a "delivered" purchase in that the buyer accepts the quantity as determined at the loading port (as certified by the Bill of Loading and Quality Report) rather than pay on the basis of the quantity and quality ascertained at the unloading port. It is similar to the terms of an F.O.B. sale, except that the seller, as a service for which he is compensated, arranges for transportation and insurance.

Crude Oil: A mixture of hydrocarbons that exists in liquid phase in underground reservoirs and remains liquid at amospheric pressure after passing through surface-separating facilities. Included are lease condensate and liquid hydrocarbons produced from tar sands, gilsonite and oil shale. Drip gases are also included, but topped crude (residual oil) and other unfinished oils are excluded. Liquids produced at natural gas processing plants and mixed with crude oil are likewise excluded where identifiable. Crude oil is considered as either domestic or foreign, according to the following: or from its "outer continental shelf" as defined in 43 U.S.C. 1331. States. Imported Athabasca hydrocarbons are included.

Culm: Waste from Pennsylvania anthracite preparation plants, consisting of coarse rock fragments containing as much as 30 percent small-sized coal; sometimes defined as including very fine coal particles called silt. Its heat value ranges from 8 to 17 million Btu per short ton.

Customs District: Customs districts, as defined by the Bureau of the Census, U.S. Department of Commerce, "Monthly Report EM 545," are as follows

- Eastern: Bridgeport, CT, Washington, DC, Boston, MA, Baltimore, MD, Portland, ME, Buffalo, NY, New York City, NY, Ogdensburg, 
NY, Philadelphia, PA, Providence, RI, Norfolk, VA, St. Albans, VT.

- Southern: Mobile, AL, Savannah, GA, Miami, FL, Tampa, FL, New Orleans, LA, Wilmington, NC, San Juan, PR, Charleston, SC: Dallas-Fort Worth, TX, El Paso, TX, Houston-Gallveston, TX, Laredo, TX, Virgin Islands.

- Western: Anchorage, AK, Nogales, AZ, Los Angeles, CA, San Diego, CA, San Fracisco, CA, Honolulu, HI, Great Falls, MT, Portland, OR, Seattle, WA.

- Northern: Chicago, IL, Detroit, MI, Duluth, MN, Minneapolis, MN, St. Louis, MO, Pembina, ND, Cleveland, $\mathrm{OH}$, Milwaukee, WI.

Demonstrated Reserve Base: A collective term for the sum of coal in both measured and indicated resource categories of reliability which represents 100 percent of the coal in these categories in place as of a certain date. Includes beds of bituminous coal and anthracite 28 inches or more thick and beds of subbituminous coal 60 inches or more thick that occur at depths to 1 thousand feet. Includes beds of lignite 60 inches or more thick that can be surface mined. Includes also thinner and/or deeper beds that presently are being mined or for which there is evidence that they could be mined commercially at this time. Represents that portion of the identified coal resource from which reserves are calculated.

Depletion: The subtraction of both the tonnage produced and the tonnage lost to mining from identified resources to determine the remaining tonnage as of a certain time.

Depletion Factor: The multiplier applied to the tonnage produced to compute depletion. This multiplier takes into account both the tonnage recovered and the tonnage lost due to mining. The depletion factor is the reciprocal of the recovery factor in relation to a given quantity of production.

Direct Labor Hours: Direct labor houris worked by all mining employees at a mining operation during the year. Includes hours worked by those employees engaged in production, preparation, development, maintenance, repair, shop or yard work, management, and technical or engineering work. Excludes office workers. Excludes vacation and leave hours.

Distillate Fuel Oil: A general classification for one of the petroleum fractions produced in conventional distillation operations. Included are products known as No.1, No.2, and No.4 fuel oils and No.1, No.2, and No.4 diesel fuels. It is used primarily for space heating, on-and-off-highway diesel engine fuel (including railroad engine fuel and fuel for agricultural machinery), and electric power generation.

Dredge Mining: A method of recovering coal from rivers or streams.

Drift Mine: An underground mine that has a horizontal entry dug to a coalbed in a hillside.
Dry (Coal) Basis: Coal quality data calculated to a theoretical basis in which no moisture is associated with the sample. This basis is determined by measuring the weight loss of a sample when its inherent moisture is driven off under controlled conditions of low temperature air-drying followed by heating to just above the boiling point of water (104 to 110 degrees centigrade).

Electricity: A form of energy generated by friction, induction, or chemical change that is caused by the presence and motion of elementary charged particles of which matter consists.

Electricity Generation: The process of producing electric energy or transforming other forms of energy into electric energy. Also the amount of electric energy produced or expressed in watthours (Wh).

Electricity Generation, Gross: The total amount of electric energy produced by the generating station or stations, measured at the generator terminals.

Electricity Generation, Net: Gross generation less electricity consumed at the generating plant for station use. Electricity required for pumping at pumped-storage plants is regarded as plant use and is deducted from gross generation.

Electric Power Plant: A station containing prime movers, electric generators, and auxiliary equipment for converting mechanical, chemical, and/or fission energy into electric energy.

Electric Utility: A corporation, person, agency, authority, or other legal entity or instrumentality that owns and/or operates facilities within the United States, its territories, or Puerto Rico for the generation, transmission, distribution, or sale of electric energy primarily for use by the public and files forms listed in the Code of Federal Regulations, Title 18, Part 141. Facilities that qualify as cogenerators or small power producers under the Public Utility Regulatory Policies Act (PURPA) and exempt wholesale generators under Energy Policy Act of 1992 are not considered electric utilities. See definition of nonutility power producer.

Electric Utility Sector: The electric utility sector consists of privately and publicly owned establishments that generate, transmit, distribute, or sell electricity primarily for use by the public and that meet the definition of an electric utility. Nonutility power producers are not included in the electric utility sector.

Emissions: The pollutants dischaged into the atmosphere in exhaust gases. For coal-burning plants, these emissions are primarily Carbon Dioxide $\left(\mathrm{CO}_{2}\right)$, Nitrogen Oxide $\left(\mathrm{NO}_{x}\right)$, and Sulfur Dioxide $\left(\mathrm{SO}_{2}\right)$.

Energy: The capacity for doing work as measured by the capability of doing work (potential energy) or the conversion of this capability to motion (kinetic energy). Energy has several forms, some of which are easily convertible and can be changed to another form useful for work. Most of the world's convertible 
energy comes from fossil fuels that are burned to produce heat that is then used as a transfer medium to mechanical or other means in order to accomplish tasks. Electrical energy is usually measured in kilowatthours, while heat energy is usually measured in British thermal units.

Energy Consumption: The use of energy as a source of heat or power or as an input in the manufacturing process.

Exports: Shipments of goods from the 50 States and the District of Columbia to foreign countries, Puerto Rico, the Virgin Islands, and other U.S. possessions and territories.

Fahrenheit: A temperature scale on which the boiling point of water is at 212 degrees above zero on the scale and the freezing point is at 32 degrees above zero at standard atmospheric pressure.

F.A.S. Value: Free alongside ship value. The value of a commodity at the port of exportation, generally including the purchase price plus all charges incurred in placing the commodity alongside the carrier at the port of exportation in the country of exportation.

Federal Energy Regulatory Commission (FERC): A quasi-independent regulatory agency within the Department of Energy having jurisdiction over interstate electricity sales, wholesale electric rates, hydroelectric licensing, natural gas pricing, oil pipeline rates, and gas pipeline certification.

Federal Coal Lease: A lease granted to a mining company to produce coal from land owned and administered by the Federal Government in exchange for royalties and other revenues.

Federal Power Act: Enacted in 1920, and amended in 1935, the Act consists of three parts. The first part incorporated the Federal Water Power Act administered by the former Federal Power Commission, whose activities were confined almost entirely to licensing non-Federal hydroelectric projects. Parts II and III were added with the passage of the Public Utility Act. These parts extended the Act's jurisdiction to include regulating the interstate transmission of electrical energy and rates for its sale as wholesale in interstate commerce. The Federal Energy Regulatory Commission is now charged with the administration of this law.

Federal Power Commission: The predecessor agency of the Federal Energy Regulatory Commission. The Federal Power Commission (FPC) was created by an Act of Congress under the Federal Water Power Act on June 10, 1920. It was charged originally with regulating the electric power and natural gas industries. The FPC was abolished on September 20,1977, when the Department of Energy was created. The functions of the FPC were divided between the Department of Energy and the Federal Energy Regulatory Commission.

FERC: The Federal Energy Regulatory Commission.
Fixed Carbon: The nonvolatile matter in coal minus the ash. Fixed carbon is the solid residue other than ash obtained by prescribed methods of destructive distillation of a coal. Fixed carbon is the part of the total carbon that remains when coal is heated in a closed vessel until all volatile matter is driven off.

Flue Gas Desulfurization Unit (Scrubber): Equipment used to remove sulfur oxides from the combustion gases of a boiler plant before discharge to the atmosphere. Chemicals, such as lime, are used as the scrubbing media.

Flue Gas Particulate Collectors: Equipment used to remove fly ash from the combustion gases of a boiler plant before discharge to the atmosphere. Particulate collectors include electrostatic precipitators, mechanical collectors (cyclones), fabric filters (baghouses), and wet scrubbers.

F.O.B. Mine Price: The free on board mine price. This is the price paid for coal at the mining operation site. It excludes freight or shipping and insurance costs.

Foreign-Controlled Firms: Foreign-controlled firms are U.S. coal producers with more than 50 percent of their stock or assets owned by a foreign firm.

Fossil-Fuel Electric Generation: Electric generation in which the prime mover is a turbine rotated by highpressure steam produced in a boiler by heat from burning fossil fuels.

Geothermal Energy: Energy from the internal heat of the earth, which may be residual heat, friction heat, or a result of radioactive decay. The heat is found in rocks and fluids at various depths and can be extracted by drilling and/or pumping.

Greenhouse Effect: The increasing mean global surface temperature of the earth caused by gases in the atmosphere (including carbon dioxide, methane, nitrous oxide, ozone, and chlorofluorocarbon). The greenhouse effect allows solar radiation to penetrate but absorbs the infrared radiation returning to space.

Gross Domestic Product (GDP): The total value of goods and services produced by labor and property in the United States. As long as the labor and property are located in the United States, the supplier (that is, the workers and, for property, the owners) may be either U.S. residents or residents of foreign countries.

Hand Loading: An underground loading method by which coal is removed from the working face by manual labor through the use of a shovel for conveyance to the surface. Though rapidly disappearing, it is still used in very small-tonnage mines.

Highwall: the unexcavated face of exposed overburden and coal in a surface mine.

High-Volatile A Bituminous Coal: See Bituminous coal. 
High-Volatile B Bituminous Coal: See Bituminous coal.

High-Volatile C Bituminous Coal: See Bituminous coal.

High-Volatile (specific sub-group unknown): See Bituminous coal.

Hydroelectric Power: The harnessing of flowing water to produce mechanical or electrical energy.

Implicit Price Deflator: The implicit price deflator, published by the U.S. Department of Commerce, Bureau of Economic Analysis, is used to convert nominal figures to real figures.

Imports: Receipts of goods into the 50 States and the District of Columbia from foreign countries and from Puerto Rico, the Virgin Islands, and other U.S. possessions and territories.

Indian Coal Lease: A lease granted to a mining company to produce coal from Indian lands in exchange for royalties and other reventues; obtained by direct negotiatian with the Indians, but subject to approval and administration by the U.S. Department of the Interior.

Industrial Sector: The industrial sector comprises manufacturing industries which make up the largest part of the sector, along with mining, construction, agriculture, fisheries, and forestry. Establishments in the sector range from steel mills, to small farms, to companies assembling electronic components. The SIC codes used to classify establishments as industrial are 1 through 39.

Interquartile Range: The interquartile range is the range within which the middle 50 percent of observations are concentrated. See Appendix D, Section "Interquartile Range."

Jet Fuel: The term includes kerosene-type jet fuel and naphtha-type jet fuel. Kerosene-type jet fuel is a kerosene-quality product used primarily for commercial turbojet and turboprop aircraft engines. Naphthatype jet fuel is a fuel in the heavy naphthas range used primarily for military turbojet and turboprop aircraft engines.

Lease Condensate: A natural gas liquid recovered from gas well gas (associated and non-associated) in lease separators or natural gas field facilities. Lease condensate consists primarily of pentanes and heavier hydrocarbons.

Lignite: A brownish-black coal of low rank with high inherent moisture and volatile matter (used almost exclusively for electric power generation). Similar coal in Europe and Australia are also referred to as brown coal. Lignite comprises two groups classified according to the following ASTM Specifiaction D-388-91a for calorific values on a moist mineralmatter-free basis:

$\begin{array}{lcc} & \text { Limits } & \text { Gtu/lb. } \\ \text { Lignite A } & 6300 & \text { LT } \\ \text { Iignite B } & - & 6300 \\ \text { GE = Greater than or equal to } & 6300 \\ \text { LT = Less than } & & \\ \text { Lignite is non-agglomerating. }\end{array}$

Lignite A: See Lignite.

Lignite B: See Lignite.

Longwall Mining: A form of underground coal mining which is gaining in importance in the United States and can be used at greater depths than roomand-pillar mining. In longwall mining, a cutting machine is pulled back and forth across a panel of coal 300 to 600 feet wide and as much as a mile long, with the broken coal moved by conveyor. Longwall mining is done under movable roof supports that are advanced as the bed is cut. The roof in the mined-out area is allowed to fall as the mining advances.

Low-Volatile Bituminous Coal: See Bituminous Coal.

Major Coal-Producing States: Any State that produces more than 12 million short tons of coal during the year.

Manufacturing (except coke plants): Those industrial users/plants, not including coke plants, that are engaged in the mechanical or chemical transformation of materials or substances into new (i.e., finished or semifinished) products. Includes coal used for gasification/liquifaction.

Medium-Volatile Bituminous Coal: See Bituminous Coal.

Merchant Coke Plant: A coke plant where coke is produced primarily for sale on the commercial (open) market.

Meta-Anthracite: See Anthracite.

Metallurgical Coal: Coal that meets the requirements for making coke. It must be low in ash and sulfur and form a coke that is capable of supporting the charge of iron ore and limestone in a blast furnace. A blend of two or more bituminous coals is usually required to make coke.

Metric Ton: A unit of weight equal to 2,204.6 pounds.

Mine Type: See Surface Mine and Underground Mine.

Mineral-Matter-Free Basis: Mineral matter in coal is the parent material in coal from which ash is derived, and which comes from minerals present in the original plant materials that formed the coal, or from extraneous sources such as sediments and precipitates from mineralized water is called the mineral matter. Mineral matter in coal cannot be analytically determined and is commonly calculated using data on 
ash and ash-forming constituents. Coal analyses are calculated to the mineral-matter-free basis by adjusting formulas used in calculations in order to deduct the weight of mineral matter from the total coal.

Moist (Coal) Basis: "Moist" coal contains its natural inherent or bed moisture, but does not include water adhering to the surface. Coal analyses expressed on a moist basis are performed or adjusted so as to describe the data when the coal contains only that moisture which exists in the bed in its natural state of deposition, and when the coal has not lost any moisture due to drying.

Naphtha: A genetic term applied to a petroleum fraction with an approximate boiling range between 122 and 400 degrees fahrenheit.

Natural Gas: A mixture of hydrocarbons and small quantities of various nonhydrocarbons existing in the gaseous phase or in solution with crude oil in underground reservoirs.

Natural Gas (Dry): The marketable portion of natural gas production, which is obtained by subtracting extraction losses, including natural gas liquids removed at natural gas processing plants, from total production.

Natural Gas Plant Liquids (NGPL): Natural gas liquids recovered from natural gas in processing plants and, in some situations, from natural gas field facilities, as well as those extracted by fractionators. Natural gas plant liquids are defined according to the published specifications of the Gas Processors Association and the American Society for Testing and Materials as follows: ethane, propane, normal butane, isobutane, pentanes plus, and other products from natural gas processing plants (i.e., products meeting the standards for finished petroleum products produced at natural gas processing plants, such as finished motor gasoline, finished avaiation gasoline, special naphthas, kerosene, distillate fuel oil, and miscellaneous products).

Nitrogen Oxide: $N O_{x}$. A gas formed in hightemperature environments when nitrogen and oxygen are present together. This typically occurs in a combustion chamber such as those in fossil-fuel burning electric utilities. Nitrogen oxide emissions are a contributor to acid rain.

Nominal Price: The price paid for a product or service at the time of the transaction. The nominal price, which is expressed in current dollars, is not adjusted to remove the effect of changes in the purchasing power of the dollar.

Nonutility Power Producers: A corporation, person, agency, authority, or other legal entity or instrumentality that owns electric generating capacity and is not an electric utility. Nonutility power producers include qualifying cogenerators, qualifying small- power producers, and other nonutility generators (including independent power producers) without a designated franchised service area and which do not file forms listed in the Code of Federal Regulations, Title 18, Part 141. (See Electric Utility.)

Nuclear Electric Power: Electricity generated by an electric power plant whose turbines are driven by steam generated in a reactor by heat from the fissioning of nuclear fuel.

Number of Mines: The number of mines, or mines collocated with preparation plants or tipples, located in a particular geographic area (State or region). If a mine is mining coal across two counties within a State, or across two States, then it is counted as two operations. This is done so that EIA can separate production by State and county.

Number of Mining Operations: The number of mining operations includes preparation plants with greater than 5,000 total direct labor hours. Mining operations that consist of a mine and preparation plant or a preparation plant only will be counted as two operations, if the preparation plant processes both underground and surface coal. Exluded are silt, culm, refuse bank, slurry dam, and dredge operations except for Pennsylvania anthracite. Excludes mines producing less than 10,000 short tons of coal during the year, and preparation plants with less than 5,000 employee hours.

Open Market Coal: Coal sold in the open market, i.e., coal sold to companies other than the reporting company's parent company or an operating subsidiary of the parent company.

Operating Subsidiary: A company which is controlled through the ownership of voting stock, or a corporate joint venture in which a corporation is owned by a small group of businesses as a separate and specific business or project for the mutual benefit of the members of the group.

Other Industrial Plant: Industrial users, not including coke plants, engaged in the mechanical or chemical transformation of materials or substances into new products (manufacturing); and companies engaged in the agriculture, mining, or construction industries.

\section{Other Unions: See Union Type.}

Overburden: Any material, consolidated or unconsolidated, that overlies a coal deposit.

Parent Company: A company which solely or jointly owns the reporting company and which is not itself a subsidiary of, or owned by, another company.

Percent Utilization: The ratio of total production to productive capacity, times 100 .

Petroleum: Petroleum includes residential and distillate fuel oils, crude oil, and all other petroleum fuels, excluding petroleum coke.

Petroleum Coke: A residue that is the final product of the condensation process in cracking. The product 
is either marketable petroleum coke or catalyst petroleum coke.

Petroleum Products: Products obtained from the processing of crude oil (including lease condensate), natural gas, and other hydrocarbon compounds. Petroleum products include unfinished oils, liquefied petroleum gases, pentanes plus, aviation gasoline, motor gasoline, naphtha-type jet fuel, kerosene-type jet fuel, kerosene, distillate fuel oil, residual fuel oil, petrochemical feedstocks, special naphthas, lubricants, waxes, petroleum coke, asphalt, road oil, still gas, and miscellaneous products.

Photovoltiac and Solar Thermal Energy (as used at electric utilities): Energy radiated by the sun as electromagnetic waves (electromagnetic radiation) that is converted at electric utilities into electricity by means of solar (photovoltiac) cells or concentrating (focusing) collectors.

Preparation Plant: A mining facility at which coal is crushed, screened, and mechanically cleaned.

Producer and Distributor Coal Stocks: Producer and distributor coal stocks consist of coal held in stock by producers/distributors at the end of a reporting period.

Productive Capacity: The maximum anount of coal that a mining operation can produce or process during a period with the existing mining equipment and/or preparation plant in place, assuming that the labor and materials sufficient to utilize the plant and equipment are available, and that the market exists for the maximum production.

Quadrillion Btu: $10^{15}$ Btu.

Real Price: A price that has been adjusted to remove the effect of changes in the purchasing power of the dollar. Real prices, which are expressed in constant dollars, usually reflect buying power relative to a base year.

Recoverable Coal Reserves at Mines: The quantity of coal that can be recovered (i.e., mined) from existing coal reserves, as reported on Form EIA-7A.

Recoverable Reserves of Coal: An estimate of the amount of coal that can be recovered (mined) from the accessible reserves of the demonstrated reserve base.

Recovery Percentage: The percentage of coal that can be recovered from the coal deposits at existing mines.

Refuse Bank: A repository for waste material generated by the coal cleaning process.

Refuse Mine: A surface mine where coal is recovered from previously mined coal. It may also be known as a silt bank, culm bank, refuse bank, slurry dam, or dredge operation.

Report Year: The calendar year beginning at 12:00 a.m. January 1 and ending at 11:59 p.m. December 31 .
Residential and Commercial Sector: Housing units; wholesale and retail businesses (except coal wholesale dealers); health institutions (hospitals); social and educational institutions (schools and universities); and Federal, State, and local governments (military installations, prisons, office buildings).

Residual Fuel Oil: The heavier oils that remain after the distillate fuel oils and and lighter hydrocarbons are distilled away in refinery operations and that conform to ASTM Specifications D396 and 975. Included are No. 5, a residual fuel oil of medium viscosity; Navy Special, for use in steam-powered vessels in government service and in shore power plants; and No. 6, which includes Bunker C fuel oil and is used for commercial and industrial heating, electricity generation, and to power ships. Imports of residual fuel oil include imported crude oil burned as fuel.

Room-and-Pillar Mining: The most common method of underground mining in which the mine roof is supported mainly by coal pillars left at regular intervals. Rooms are places where the coal is mined; pillars are areas of coal left between the rooms. Room-and-pillar mining is done either by conventional or continuous mining.

Royalties: Payments, in money or kind, of a stated share of production from mineral deposits, by the lessee to the lessor. Royalties may be an established minimum, a sliding-scale, or a step-scale. A step-scale royalty rate increases by steps as the average production on the lease increases. A sliding-scale royalty rate is based on average production and applies to all production from the lease.

Sales Volume: The reported output from Federal and/or Indian lands, the basis of royalties. It is approximately equivalent to production, which includes coal sold, and coal added to stockpiles.

Scoop Loading: An underground loading method by which coal is removed from the working face by a tractor unit equipped with a hydraulically operated bucket attached to the front; also called a front-end loader.

Semianthracite: See Anthracite.

Shaft Mine: An underground mine that reaches the coalbed by means of a vertical shaft. In addition to the passages providing entry to the coalbed, a network of other passages are also dug, some to provide access to various parts of the mine and some for ventilation.

Short Ton: A unit of weight equal to 2,000 pounds.

Shortwall Mining: A form of underground mining that involves the use of a continuous mining machine and movable roof supports to shear coal panels 150 to 200 feet wide and more than half a mile long. Although similar to longwall mining, shortwall mining is generally more flexible because of the smaller working area. Productivity is lower than with 
longwall mining because the coal is hauled to the mine face by shuttle cars as opposed to conveyors.

SIC: See Standard Industrial Classification.

Silt: Waste from Pennsylvania anthracite preparation plants, consisting of coarse rock fragments containing as much as 30 percent small-sized coal; sometimes defined as including very fine coal particles called silt. Its heat value ranges from 8 to 17 million Btu pèr short ton. Synonymous with culm.

Silt, Culm Refuse Bank, or Slurry Dam Mining: A mining operation producing coal from these sources of coal. (See refuse mine.)

Slope Mine: An underground mine in which the entry is driven at an angle to reach the coal deposit.

Slurry Dam: A repository for the silt or culm from a preparation plant.

Solar Energy: The radiant energy of the sun, which can be converted into other forms of energy, such as heat or electricity.

Solar Thermal Collector: A devise designed to receive solar radiation and convert it into thermal energy. Normally, a solar thermal collector includes a frame, glazing, and an absorber, together with appropriate insulation. The heat collected by the solar thermal collector may be used immediately or stored for later use.

Standard Industrial Classification (SIC): A set of codes developed by the Office of Management and Budget which categorizes industries to groups with similar economic activities.

Steam Coal: All noncoking coal.

Stocks: The supply of coal or coke at a mine, plant, or utility at the end of the reporting period.

Strategic Petroleum Reserve (SPR): Petroleum stocks maintained by the Federal Government for use during periods of major supply interruption.

Strip (Surface) Mining: A method used on flat terrain to recover coal by mining long strips successively. The material excavated from the strip being mined is deposited in the strip previously mined.

Subbituminous Coal: A dull black coal of rank intermediate between lignite and bituminous, consisting of subbituminous A coal, subbituminous B coal, and subbituminous $C$ coal, classified according to the following ASTM Specification D-388-91a on a moist mineral-matter-free basis:

\begin{tabular}{lcc} 
& \multicolumn{2}{c}{ Calorific } \\
& Value \\
& Limits \\
& Btu/lb. \\
& GE & LT \\
& 10500 & 11500 \\
Subbituminous A Coal & 9500 & 10500 \\
Subbituminous B Coal & 8300 & 9500 \\
Subbituminous C Coal & GE = Greater than or equal to & \\
LT = Less than & & \\
Subbituminous coal is non-agglomerating.
\end{tabular}

Subbituminous A Coal: See Subbituminous Coal.

Subbituminous B Coal: See Subbituminous Coal.

Subbituminous C Coal: See Subbituminous Coal.

Sulfur: One of the elements present in varying quantities in coal that contributes to environmental degradation when coal is burned. In terms of sulfur content by weight, coal is generally classified as low (less than or equal to 1 percent), medium (greater than 1 percent and less than or equal to 3 percent), and high (greater than 3 percent). Sulfur content is measured as a percent by weight of coal on an "as received" or a "dry" (moisture-free, usually part of a laboratory analysis) basis.

Sulfur Dioxide: $\mathrm{SO}_{2}$. A caustic, corrosive gas that is a by-product of combustion and emissions from fossil-fuel burning electric utility plants. The primary agent in the production of acid rain.

Supplemental Gaseous Fuels: Any gaseous substance that, introduced into or commingled with natural gas, increases the volume available for disposition. Such substances include, but are not limited to, propane-air, refinery gas, coke oven gas, still gas, manufactured gas, biomass gas, or air or inert gases added for Btu stabilization.

Surface Mine: A coal-producing mine that is usually within a few hundred feet of the surface. Earth and rock above or around the coal (overburden) is removed to expose the coalbed, which is then mined with surface excavation equipment such as draglines, power shovels, bulldozers, loaders, and augers. It may also be known as an area, contour, open-pit, strip, or auger mine.

Tipple: A central facility used in loading coal for transportation by rail or truck.

Transportation Sector: The transportation sector consists of private and public vehicles that move people and commodities. Included are automobiles, trucks. buses, motorcycles, railroads and railways (including streetcars), aircraft, ships, barges, and natural gas pipelines.

Underground Mine: A mine where coal is produced by tunneling into the earth to the coalbed, which is then mined with underground mining equipment such as cutting machines and continuous, longwall, and shortwall mining machines. Underground mines are classified according to the type of opening used to 
reach the coal, i.e., drift (level tunnel), slope (inclined tunnel), or shaft (vertical tunnel).

Unfinished Oils: All oils requiring further refinery processing, except those requiring only mechanical blending. Includes naphthas and lighter oils, kerosene and light gas oils, heavy gas oils, and residuum.

Union Type: Union type consists of United Mine Workers of America (UMWA), and the following "Other Union" types: Southern Labor Union (SLU), Appalachian Miners of America (AMA), Scotia Employees Association (SEA), International Union of Operation Engineers (IUOE), Utility Workers of America (UWA), Progressive Mine Workers Association (PMWA), International Brotherhood of Electrical Workers (IBEW), International Chemical Workers Union (ICWU), Redstone Workers Association (RWA), Chariton Valley Independent Union (CVIU), American Federation of Labor - Congress of Industrial Organization (AFL-CIO), Labors International (LABO), Crow Hollow Miners (CROW), Coal Strippers (COAL), United Steel Workers (USW), Independent Miners Association (IMA), Independent Union (INUN), Independence Miners, Brokers, and Truckers Association (IMBT), Council of Southern Mountains (CSM), International Brotherhood of Teamsters, Chauffeurs, Warehousemen and Helpers Union (TEAM), Thompson Creek Workers Association (TCWA), United Brotherhood of Clay Workers (UBCW), Wilmot Employees Independent Union (WEIU), Independent Strip Miners Union (ISMU), Independent Miners (IM), Independent Workers (IW), Coal Strippers Union (CSU), Independent Miners Union (IMU), Independent Coal Workers (ICW), Independent Strip Mining Workers (ISMW), Independent Strip Union (ISU), Association of Bituminous Contractors (ABC), Arch Minerals Employees Associ- ation (AMEA), United Paperworkers International Union (UPIU), Welch Miners Union (WMU), Falcon Coal Employees Association (FCEA), Justus Employees Association (JEA), International Construction Union (ICU), Brotherhood of Miners (BOM), Western Energy Workers (WEW), Carlin Independent Union (CIU), International Association of United Workers Union (IAWU), and Stove, Furnace and Allied Appliance Workers International Union of N. A. (SFAW).

U.S. Coal Exports: Amount of U.S. coal shipped to foreign destinations, as reported in the U.S. Department of Commerce, Bureau of Census, "Monthly Report EM 545."

U.S. Coal Imports: Amount of foreign coal shipped to the United States, as reported in the U.S. Department of Commerce, Bureau of the Census, "Monthly Report IM 145."

Wind Energy (as used at electric utilities): The kinetic energy of wind converted at electric utilities into mechanical energy by wind turbines (i.e., blades rotating from the hub) that drive generators to produce electricity for distribution.

Wood and Waste (as used at electric utilities):

Wood energy, garbage, bagasse, sewerage gas, and other industrial, agricultural, and urban refuse used to generate electricity for distribution.

Volatile Matter: Those products, exclusive of moisture, given off by a material as gas or vapor. Volatile matter is determined by heating the coal to 950 degrees centigrade under carefully controlled conditions and measuring the weight loss, excluding weight of moisture driven off at 105 degrees centigrade. 